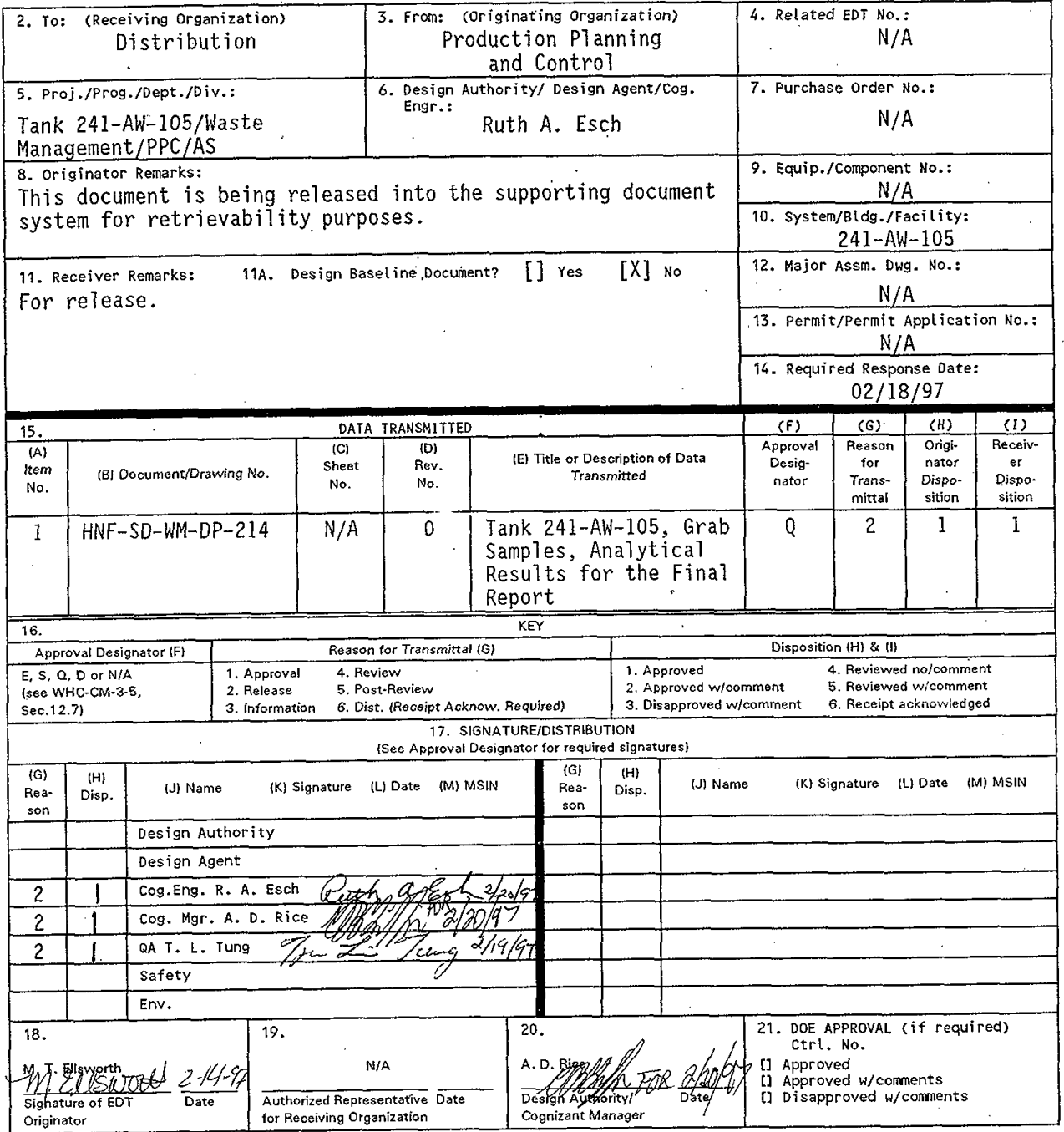




\title{
Tank 214-AW-105, Grab Samples, Analytical Results for the Final Report
}

\author{
Ruth A. Esch
}

Rust Federal Services of Hanford, Inc., Richland, WA 99352

U.S. Department of Energy Contract DE-AC06-96RL13200
EDT/ECN: EDT-620387
Org Code: $7576 \mathrm{~B}$
B\&R Code: EW3120074
UC: 2070
Charge Code: $M 0378$
Total Pages: 17461744 KB
$2 / 26 / 97$

Key Words: Tank 241-AW-105, 241-AW-105, AW-105, AW Farm, Grab Samples, Analytical Results, Final Report

Abstract: $N / A$

TRADEMARK DISCLAIMER. Reference herein to any specific comercial product, process, or service by trade name, trademark, manufacturer, or otherwise, does not necessarily constitute or imply its endorsement, recomendation, or favoring by the United States Government or any agency thereof or its contractors or subcontractors.

Printed in the United States of America. To obtain copies of this document, contact: Document Control Services, P.0. Box 950, Mailstop H6-08, Richland WA 99352, Phone (509) 372-2420; Fax (509) 376-4989.
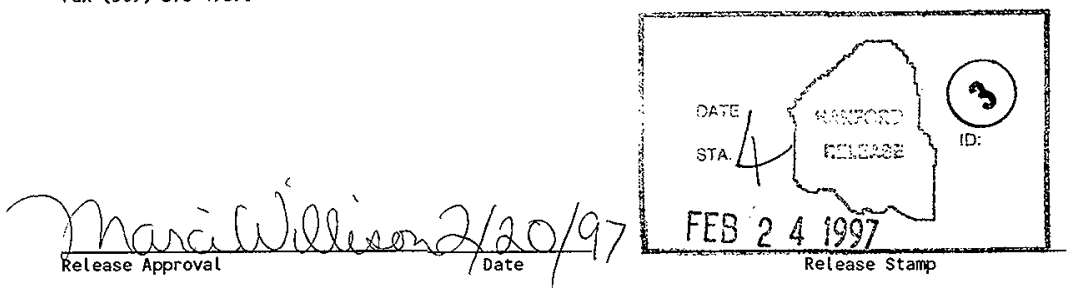

\section{Approved for Public Release}


HNF-SD-WM-DP-214, REV. 0

ANALYTICAL SERVICES

TANK 241-AW-105, GRAB SAMPLES, ANALYTICAL RESULTS FOR THE FINAL REPORT

\author{
Project Coordinator: RUTH A. ESCH \\ Prepared for the U.S. Department of Energy \\ office of Environmental Restoration \\ and Waste Management \\ - by \\ 222-S Laboratory \\ Rust Federal Services of Hanford Inc. \\ P.0. Box 700 \\ Richland, Washington
}


HNF-SD-WM-DP-214, REV. 0

\section{THIS PAGE WAS INTENTIOANLLY LEFT BLANK}


WHC-SD-WM-DP-214, REV. 0

TABLE OF CONTENTS

Narrative ...................... 1

AW-105 Sample Breakdown (Attachment 1) . . . . . . . 18

Sample Data Summary . . . . . . . . . . . . . . . 25

Chain of Custody Forms . . . . . . . . . . . . 63

Breakdown Analysis ................... 85

Sample Preparation .................. 123

Fusion Digestion Work 7 ist \# $13318(5052,5053,5054) \ldots . . . .125$

Fusion Digestion Work 7 ist \#13319 $(5084,5085,5086) \ldots \ldots . . .126$

Water Digestion Worklist \# $13320(5055,5056) \ldots . . . . . . .127$

Water Digestion Workl ist \# $13321(5057,5087) \ldots . . . . . . .128$

Water Digestion Work 1 ist \# $13322(5088,5089) \ldots \ldots . . . . .129$

Inorganic Analyses . . . . . . . . . . . . . . 130

Differential Scanning Calorimetry (DSC)

DSC Workl ist \# $13070(5004,5005) \ldots \ldots . \ldots . . . \ldots 132$

DSC Work ist \# $13072(5006) \ldots \ldots . . . . . . . . .138$

DSC Work i ist \# $13135(5010,5011) \ldots \ldots . \ldots . . . . . .143$

DSC Work list \# $13136(5012) \ldots \ldots . . . . . . . . . .149$

DSC Workl ist \# $13912(5049,5050) \ldots \ldots \ldots \ldots . . \ldots 153$

DSC Work ist \# $13913(5051,5081)$. . . . . . . . . . . 160

DSC Work 1 ist \# $13914(5082,5083) \ldots \ldots . . \ldots . . . . .167$

DSC Workl ist \# $13915(5019,5020) \ldots \ldots \ldots 173$

DSC Work ist \# $13916(5021,5075)$. . . . . . . . . . . . 180

DSC Work list \# $13917(5076,5077) \ldots \ldots$. . . . . . . . . 186

DSC Work list \# $14911(5021,5075,5076,5077) \ldots \ldots 192$

Thermogravimetric Analys is (TGA)

TGA Workl ist \# $13068(5004,5005)$. . . . . . . . . . 194

TGA Worklist \# 13069 (5006) . . . . . . . . . . . . . . 200

TGA Workl ist \# $13132(5010,5011) \ldots . . . . . . . .205$

TGA Work1 ist \# $13133(5012) \ldots . . . . . . . . . . .211$

TGA Workl ist \# $13918(5049,5050) \ldots . . . . . . . .215$

TGA Work1 ist \# $13919(5051,5081) \ldots . . . . . . . . . .222$

TGA Work1 ist \# $13920(5082,5083) \ldots . . . . . . . . . .229$

TGA Workl ist \# $13921(5019,5020) \ldots . . . \ldots 235$

TGA Workl ist \# $13922(5021,5075) \ldots . . . . . . . . . .242$

TGA Work 1 ist \# $13923(5076,5077) \ldots . . . . . . . .248$ 
HNF-SD-WM-DP-214, REV. 0

\section{TABLE OF CONTENTS (Continued)}

Specific Gravity Analysis (SpG)

SpG Worklist \# $13073(5004) \ldots \ldots . . . . . . . . .254$

SpG Workl ist \# $13137(5010,5011,5012) \ldots \ldots . . \ldots . . . . .258$

SpG Work1 ist \# $13781(5008) \ldots \ldots . . \ldots . . . . . .267$

Ph Analysis (Ph)

Ph Workl ist \#13063 $(5004,5005,5006) \ldots \ldots . . . . . . . .277$

Ph Work1 ist \# $13204(5010,5011,5012) \ldots \ldots . . . . . .278$

Ph Work1 ist \# $13844(5049,5050,5051,5081,5082,5083) \ldots 279$

Ph Work list \# $13854(5019,5020,5021,5075,5076,5077) \ldots . . .280$

Hydroxide Analys is (OH)

OH Workl ist \# $13062(5004,5005,5006) \ldots \ldots . . . . . . .281$

OH Work list \# $13203(5010,5011,5012) \ldots \ldots . . . . . . .290$

OH Workl ist \# $13842(5049,5050,5051,5081,5082,5083) \ldots . . . .302$

$\mathrm{OH}$ Work list \# 14364 (5008) . . . . . . . . 323

$\mathrm{OH}$ Work 1 ist \# $14789(5055,5056,5057,5087,5088,5089) \ldots 331$

Ion Chromatography Analysis (IC)

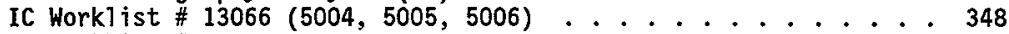

IC Work list \# 13571 (5012) . . . . . . . . . . . . . . . 359

IC Work list \#13780 (5008) . . . . . . . . . . . . . . 367

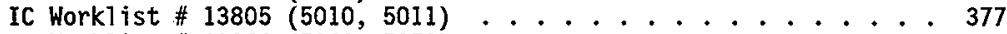

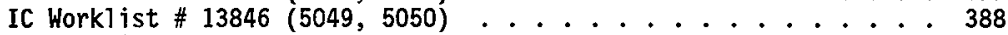

IC Workl ist \# 13847 (5081) . . . . . . . . . . . . . . . 399

IC Work list \# $13849(5082,5083) \ldots . . . . . . . . . .407$

IC Work list \# $14191(5055,5056) \ldots \ldots . \ldots . . \ldots 24$

IC Work 1 ist \# $14193(5087,5057) \ldots \ldots . . \ldots . . . . . .438$

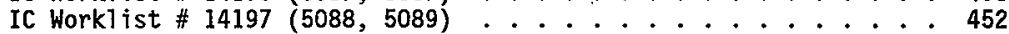

IC Work list \# $14522(5051)$. . . . . . . . . . . . . . . 463

IC Workl ist \# 15292 (5088) . . . . . . . . . . . . . 472

Inductive Coupled Plasma Analysis (ICP)

ICP Worklist \# $13282(5004,5005) \ldots \ldots 48$

ICP Workl ist \# $13283(5010,5011,5012) \ldots . . . . . . . .4488$

ICP Worklist \# $13582(5050,5051) \ldots . . . . . . . . . .495$

ICP Worklist \# $13583(5081,5082) \ldots . . . . . . . . . .503$

ICP Workl ist \# $14128(5052,5053,5054) \ldots . . . . . . . . . .510$

ICP Workl ist \# $14129(5084,5085,5086) \ldots \ldots . . . . . . . .533$

ICP Workl ist \# $14790(5049) \ldots \ldots . . . . . . . .5540$

Total Inoganic Carbon/Total Organic Carbon Analyses (TICTOC)

TICTOC Work list \# $13856(5019,5020,5021) \ldots \ldots 546$

TICTOC Workl ist \# $13858(5075,5076,5077) \ldots . . . . . . .581$

Total Organic Carbon (TOC)

TOC Workl ist \# $13065(5004,5006) \ldots \ldots \ldots 618$

TOC Workl ist \# $13779(5008) \ldots \ldots . . . . . . . . .6635$

TOC Workl ist \# $13867(5081,5050,5051) \ldots . . . . . . .649$ 
HNF-SD-WM-DP-214, REV. 0

TABLE OF CONTENTS (Continued)

TOC Work1ist \# $13869(5082,5083) \ldots . . . . . . . .670$

TOC Worklist \# $14541(5010,5011,5012) \ldots . . . . . . . .687$

TOC Workl ist \# 14796 (5049) ............... 708

TOC WorkT ist \# $15377(5008,5049) \ldots . . . . . . . .725$

Total Inorganic Carbon (TIC)

TIC Work1 ist \# $13064(5004,5006,5008) \ldots . . . . . . . . .742$

TIC Work 1 ist \# $13128(5010,5011,5012) \ldots . . . . . . . .7766$

TIC Work1 ist \# $13865(50815050,5051) \ldots . . . . . . .788$

TIC Workl ist \# $13866(5082,5083) \ldots . . . . . . . . .811$

TIC Worklist \# 14795 (5049) . . . . . . . . . . . . 829

Radiochemical Analyses . . . . . . . . . . . . . 843

Total Alpha Analyses (AT)

AT Work1 ist \# $13054(5007,5008,5009) \ldots . . . . . . . .845$

AT Work1 ist \# $13160(5013,5014,5015) \ldots \ldots . \ldots . . . . . .856$

AT Work1 jst \# $13829(5081,5082,5083) \ldots . . . . . . . .887$

AT Work1 jst \# $14181(5053,5054) \ldots . . . . . . . . .878$

AT Work1 ist \# $14182(5085,5086)$. . . . . . . . . . . . 884

AT Workl ist \# $14493(5050,5051) \ldots . . . . . . . .892$

AT Work7 ist \# 14398 (5084) . . . . . . . . . . . . . . . . . 901

AT Worklist \# $14399(5052,5054) \ldots \ldots 908$

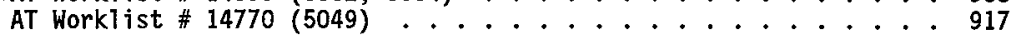

Gamma Energy Analysis (GEA)

GEA Work 1 ist \# $13074(5007,5008,5009) \ldots \ldots . \ldots . \ldots . . . . .922$

GEA Work l ist \# $13163(5013,5014,5015) \ldots . . \ldots 958$

GEA Work 1 ist \# $13852(5050,5051,5081,5082,5083) \ldots 993$

GEA Work 1 ist \# $14187(5052,5053,5054) \ldots . . . . . . . . .1044$

GEA Work l ist \# $14188(5084,5085,5086) \ldots . . \ldots 1075$

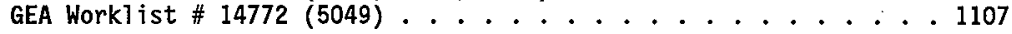

Strontium Analysis (Sr90)

Sr90 Workl ist \# $13076(5007,5008,5009) \ldots \ldots . . . . . . .1125$

Sr90 Workl ist \# $13161(5013,5014) \ldots \ldots . . . . . . . . .1135$

Sr90 Workl ist \# $14183(5052,5053,5054) \ldots . . . . . . . .1143$

Sr90 Workl ist \# $14184(5084,5085,5086) \ldots . . . . . . . . .1153$

Sr90 Worklist \# 14768 (5015) . . . . . . . . . . . . 1163

Sr90 Worklist \# $15113(5049) \ldots \ldots$. . . . . . . . . . . . . 1169

Sr90 Workl ist \# $15177(5051,5082) \ldots \ldots . . . . . . . .1175$

Sr90 Worklist \# $15379(5050,5081,5083) \ldots \ldots . . . . .1185$

Americium 241 (AM-241)

AM241 Workl ist \# $13127(5007,5008,5009) \ldots \ldots . . . . . .1195$

AM241 Workl ist \# $13162(5013,5014,5015) \ldots \ldots . . . . . . .1230$

AM241 Worklist \# $13833(5050) \ldots \ldots . . . . . . . . .1265$

AM241 Worklist \# $13834(5083) \ldots . . . . . . . . . . . .1286$

AM241 WorkTist \# $14185(5053,5054) \ldots \ldots . . . . . . . .1305$ 
HNF-SD-WM-DP-214, REV. 0

TABLE OF CONTENTS (Continued)

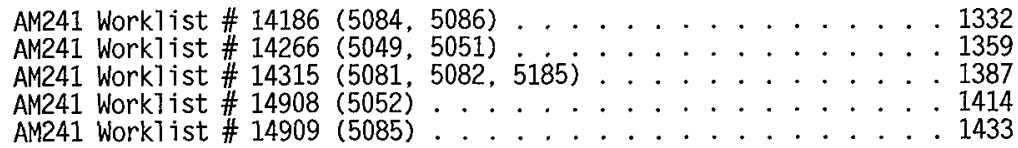

Plutonium Analysis (Pu239)

Pu239 Worklist \# $13075(5007,5008,5009) \ldots \ldots . \ldots . . . . .1452$

Pu239 Worklist \# $13164(5013,5014,5015)$. . . . . . . . . . . . . 1487

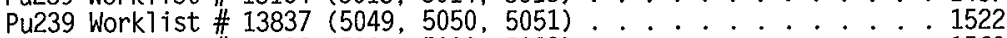

Pu239 Worklist \# $13839(5081,5082,5083)$. . . . . . . . . . . . . 1560

Pu239 Work list \# $14190(5052,5053,5054) \ldots . . . . . . . . . .1596$

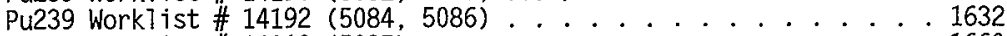

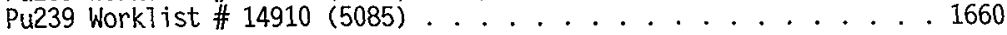

Organic Analysis . . . . . . . . . . . . . . . 1679

This Document consists of pages 1 through 1738 and pages $i 1,2,19,26,64$, $86,124,131$, and 844 were intentionaliy left blank.

\section{TRADEMARKS:}

Perkin-Elmer is a Registered Trademark of Research and Manufacturing Company, Inc. Mettler is a Registered Trademark of Mettler Electronics 
HNF-SD-WM-DP-214, REV. 0

\section{THIS PAGE WAS INTENTIOANLLY LEFT BLANK}




\section{HNF-SD-WM-DP-214, REV. 0}

\section{2-S ANALYTICAL SERVICES}

\section{TANK 241-AW-105, GRAB SAMPLES, 5AW-96-1 THROUGH 5AW-96-20 ANALYTICAL RESULTS FOR THE FINAL REPORT}

This document is the final report for tank $241-\mathrm{AW}-105$ grab samples. Twenty grabs samples were collected from risers $10 \mathrm{~A}$ and $15 \mathrm{~A}$ on August 20 and 21, 1996, of which eight were designated for the $\mathrm{K}$ Basin sludge compatibility and mixing studies, as identified in Table 1 . This document presents the analytical results for the remaining twelve samples. Analyses were performed in accordance with the Compatibility Grab Sampling and Analysis Plan (TSAP) (Sasaki, 1996) and the Data Quality Objectives for Tank Farms Waste Compatibility Program (DQO) (Fowler, 1995).

The results for the previous sampling of this tank were reported in WHC-SD-WM-DP-149, Rev. 0, 60-Day Waste Compatibility Safety Issue and Final Results for Tank 241-AW-105, Grab Samples 5AW-95-1, 5AW-95-2 and 5AW-95-3.

Three supernate samples exceeded the TOC notification limit (30,000 $\mu \mathrm{g} \mathrm{C} / \mathrm{g}$ dry weight). Appropriate notifications were made. No immediate notifications were required for any other analyte.

The TSAP requested analyses for polychlorinated biphenyls (PCB) for all liquids and centrifuged solid subsamples. The PCB analysis of the liquid samples has been delayed and will be presented in a revision to this document.

\section{Appearance and Sample Handling}

Attachment 1 is provided as a cross-reference for relating the tank farm customer identification numbers with the 222-S Laboratory sample numbers and the portion of sample analyzed. Due to a field error in the requested sampling depth for sample 5AW-96-13, the tank coordinator directed the laboratory to analyze sample 5AW-96-14.

Table 1 provides the available appearance information for all twenty samples. 
HNF-SD-WM-DP-214, REV. 0

Table 1: Appearance Information for Tank 241-AW-105 Grab Samples

\begin{tabular}{|c|c|c|c|c|c|}
\hline 8) & 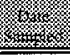 & 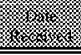 & 被 & & 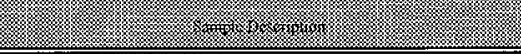 \\
\hline SAW-96-1 & $8 / 20 / 96$ & $8 / 26 / 96$ & 511 & Trace & Clear yellow liquid; no organic layer; trace amount of solids \\
\hline $5 A W-96-2$ & $8 / 20 / 96$ & $8 / 27 / 96$ & 529 & Trace & Clear yellow liquid; no organic layer; trace amount of solids \\
\hline SAW-96-3 & $8 / 20 / 96$ & $8 / 21 / 96$ & 529 & $\mathrm{n} / \mathrm{a}$ & Sample designated for K Basin sludge mixing studies \\
\hline SAW-96-4 & $8 / 20 / 96$ & $8 / 27 / 96$ & 548 & Trace & Clear yellow liquid; no organic layer; trace amount of solids \\
\hline $5 A W-96-5$ & $8 / 20 / 96$ & $8 / 27 / 96$ & 571 & $\sim 22 \%$ & $\begin{array}{l}\text { Clear yellow liquid with approximately } 22 \% \text { settled solids. Solids were a } \\
\text { yellow soft sludge material with some small black particulates. }\end{array}$ \\
\hline $5 A W-96-6$ & $8 / 20 / 96$ & $8 / 26 / 96$ & 588 & $n / a$ & Sample designated for K Basin sludge mixing studies \\
\hline SAW-96-7 & $8 / 20 / 96$ & $8 / 26 / 96$ & 588 & $\sim 90 \%$ & $\begin{array}{l}\text { Liquid color was indistinguishable. Solids were a yellow/gray soft sludge } \\
\text { material with some small black particulates. }\end{array}$ \\
\hline 5AW-96-8 & $8 / 20 / 96$ & $8 / 21 / 96$ & 588 & $\mathrm{n} / \mathrm{a}$ & Sample designated for $\mathrm{K}$ Basin sludge mixing studies \\
\hline $5 A W-96-9$ & $8 / 20 / 96$ & $8 / 27 / 96$ & 589 & $\sim 90 \%$ & $\begin{array}{l}\text { Liquid color was indistinguishable. Solids were a nonhomogeneous } \\
\text { mixture of light yellow and gray soft sludge material with some small black } \\
\text { particulates. }\end{array}$ \\
\hline $5 A W-96-10$ & $8 / 21 / 96$ & $8 / 22 / 96$ & 511 & Trace & Clear yellow liquid; no organic layer, trace amount of solids \\
\hline $5 A W-96-11$ & $8 / 21 / 96$ & $8 / 22 / 96$ & 529 & Trace & Clear yellow liquid; no organic layer, trace amount of solids \\
\hline $5 A W-96-12$ & $8 / 21 / 96$ & $8 / 23 / 96$ & 529 & n/a & Sample designated for $\mathrm{K}$ Basin sludge mixing studies \\
\hline $5 A W-96-13$ & $8 / 21 / 96$ & $8 / 23 / 96$ & 529 & $\mathbf{n} / \mathbf{a}$ & Sample designated for K Basin sludge mixing studies \\
\hline SAW-96-14 & $8 / 21 / 96$ & $8 / 23 / 96$ & 548 & Trace & Clear yellow tiquid; no organic layer; trace amount of solids \\
\hline $5 A W-96-15$ & $8 / 21 / 96$ & $8 / 23 / 96$ & 571 & $-90 \%$ & $\begin{array}{l}\text { Liquid color was indistinguishable. Solids were a nonhomogeneous } \\
\text { mixture of dark yellow and gray soft sludge material with some small black } \\
\text { particulates. }\end{array}$ \\
\hline $5 A W-96-16$ & $8 / 21 / 96$ & $8 / 23 / 96$ & 593 & $\mathrm{n} / \mathrm{a}$ & Sample designated for $\mathrm{K}$ Basin sludge mixing studies \\
\hline SAW-96-17 & $8 / 21 / 96$ & $8 / 23 / 96$ & 593 & $-90 \%$ & $\begin{array}{l}\text { Liquid color was indistinguishable. Solid's were a nonhomogeneous } \\
\text { mixture composed mostly of dark gray/black soft sludge material with some } \\
\text { yellow sludge swirled throughout. }\end{array}$ \\
\hline $5 A W-96-18$ & $8 / 21 / 96$ & $8 / 23 / 96$ & 593 & $\mathbf{n} / \mathbf{a}$ & Sample designated for $\mathrm{K}$ Basin sludge mixing studies \\
\hline $5 A W-96-19$ & $8 / 21 / 96$ & $8 / 23 / 96$ & 593 & $\mathrm{n} / \mathrm{a}$ & Sample designated for $\mathrm{K}$ Basin siudge mixing studies \\
\hline $5 A W-96-20$ & $8 / 21 / 96$ & $8 / 26 / 96$ & 600 & $-93 \%$ & $\begin{array}{l}\text { Liquid color was indistinguishable. Solids were a nonhomogeneous } \\
\text { mixture composed mostly of a somewhat even mixture of yellow and gray } \\
\text { soft sludge material swirled together. There were some larger "chunks" of } \\
\text { material scattered throughout - some black and some nearly white. }\end{array}$ \\
\hline
\end{tabular}

* Sample depth is measured from the top of the riser to the mouth of the sample bottle

$\mathrm{n} / \mathrm{a} \quad$ This information was not available 
HNF-SD-WM-DP-214, REV. 0

The samples were all subsampled for analysis. For the supernates, 20-mL aliquots were created for direct analysis. After the supernate was removed, the settled sludges were centrifuged to separate the solid and liquid portions for separate analysis. The solids were designated as centrifuged solids and the liquid as interstitial liquid. Bulk density and volume percent solids data were reported for the wet sludge and were designated as sludge for the sample portion for the report.

The weights and volumes of the three separated portions of the sludge samples are presented in Table 2.

Table 2: Sludge Separation Information for Tank 241-AW-105 Grab Samples

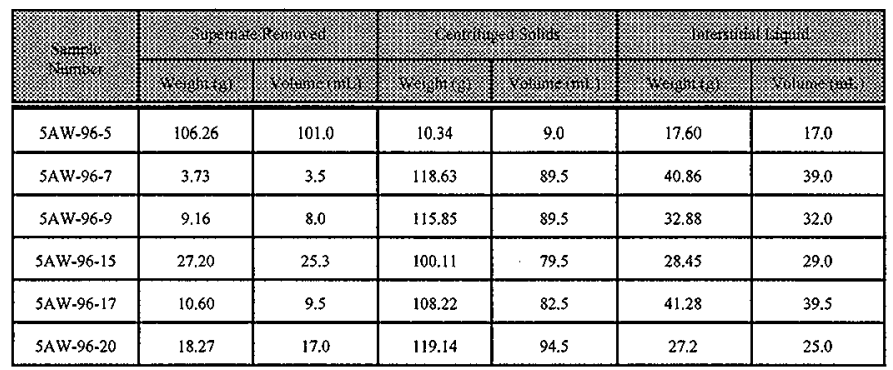

\section{Analytical Results Summary}

The data summary table (Table 3 ) included in this report compiles the analytical results that comply with the applicable DQO.

\section{Compatibility Program Concerns}

\section{DSC - Differential Scanning Calorimetry - Energetics Decision Rule}

The DSC analyses were performed in duplicate on direct subsamples. The standard recoveries for this analysis were within the required limits.

There was no exothermic energy observed in any of the supernate or interstitial liquid samples, thus the dry weight calculations were not applicable. A very small exotherm was observed for 4 of the 6 centrifuged solid samples. Exothermic energy based on the dry weight was calculated for each. The average of the thermogravimetric analysis (TGA) results for each subsample was used in the dry weight correction for that subsample. No results exceeded the notification limit. 


\section{HNF-SD-WM-DP-214, REV. 0}

Two centrifuged solid sample portions had high $(>20 \%)$ relative percent differences (RPD) between sample and duplicate results. These high results were attributed to low exothermic energy and the heterogeneous nature of the sludges. No reruns were requested.

For waste compatibility energetics decision concerns, since there was no exothermic energy observed for the liquid sample portions, the exotherm/endotherm ratio calculation was not applicable. For safe waste transfers this ratio must be less than 1. For the centrifuged solid portions, since the dry weight exothermic energy was well below the notification limit and the average percent water was approximately $70 \%$, it follows that the exotherm/endotherm ratio will be less than 1 , therefore calculations were not performed.

\section{Nitrate $\left(\mathrm{NO}_{3}{ }^{-}\right)$, Hydroxide $\left(\mathrm{OH}^{-}\right)$and Nitrite $\left(\mathrm{NO}_{2}^{-}\right)$- Corrosion Decision Rule}

The results with respect to the waste compatibility corrosion rules are presented in Table 4 (pp. 57-62). These rules are only applicable to liquid sample portions. The analyses were performed on direct liquid aliquots. The table presents the $\mathrm{NO}_{3}+\mathrm{OH}^{-}$, and $\mathrm{NO}_{2}{ }^{-}$results in $\mu \mathrm{g} / \mathrm{mL}$ and molarity (M) units. The spreadsheet compares the results to the concentration ranges specified in the waste compatibility DQO. A "YES" will appear in the appropriate space for the condition that is met. Only one of three sets of conditions must be met for $\mathrm{OH}^{-}$and $\mathrm{NO}_{2}^{-}$based on the range that the $\mathrm{NO}_{3}{ }^{-}$concentration falls in. If "NO" appears in the space under a condition, that condition is not met and a notification is required. No notifications were required.

The standard recoveries, appropriate spike recoveries and RPDs for these analyses were all within the required limits with the exception of a high spike recovery (128.3\%) for $\mathrm{NO}_{3}^{-}$for sample 5AW-96-2 (S96T005008). This high recovery was attributed to the high sample concentration with respect to the amount of spike added. Although a small amount of $\mathrm{NO}_{3}{ }^{-}$was found in the method blank for this analysis, it was insignificant with respect to the sample results. Since the results for the sample were consistent with those obtained for the other supernate samples no rerun was requested.

\section{Sp.G. - Specific Gravity - Flammable Gas Accumulation Decision Rule}

The Sp.G. of the liquid portion of the waste is used for determination of the potential to cause an accumulation of flammable gases. The flammable gas decision rule requires that the weighted mean Sp.G. not exceed 1.41. The results ranged from 1.01 to 1.04 for the supernates, giving a weighted mean of 1.022 . The bulk density was used as a close approximation of the Sp.G. for the interstitial liquids. These results ranged from 0.980 to 1.09 , giving a weighted mean of 1.04 . These results do not exceed the 1.41 limit to allow waste transfer. The standard recovery and RPDs for the Sp.G. analysis were within the required limits. 


\section{HNF-SD-WM-DP-214, REV. 0}

${ }^{239 / 240} \mathrm{Pu}$ - Plutonium-239/240 - Criticality Decision Rule

${ }^{239 / 240} \mathrm{Pu}$ concentration is used to evaluate criticality safety for waste transfers. The analysis was performed on direct aliquots for the supernate and interstitial liquid samples. The centrifuged solids were prepared for analysis by a fusion digestion, indicated by an " $F$ " in the aliquot class (A\#) column in Table 3.

The results were all below the safety concern immediate notification limits of $61.5 \mu \mathrm{Ci} / \mathrm{mL}$ for the supernate and interstitial liquid subsamples and $41 \mu \mathrm{Ci} / \mathrm{g}$ for the centrifuged solid subsamples.

The results for the liquid portions were well below the criticality prevention limit of 0.80 $\mu \mathrm{Ci} / \mathrm{mL}$ stated in the compatibility DQO. This limit was calculated to be $0.61 \mu \mathrm{Ci} / \mathrm{g}$ based on the highest bulk density determined for the centrifuged solids. All of the centrifuged solid results exceeded this limit, with the exception of sample 5AW-96-5 (S96T005052; average result = $0.453 \mu \mathrm{Ci} / \mathrm{g})$. There was no immediate notification requirement for this limit violation.

The standard recoveries for this analysis were within the required limits. The RPD for all subsamples met the $\pm 20 \%$ criterion, with the exception of sample 5AW-96-17 centrifuged solid portion (S96T005085) which had a RPD of $21.5 \%$ after one rerun. Because of the observed nonhomogeneous nature of this sample, no further reruns were requested.

\section{${ }^{241}$ Am - Americium-241 - Criticality Decision Rule}

${ }^{241} \mathrm{Am}$ analysis is also used to evaluate criticality safety for waste transfers. The analysis was performed on direct liquid portions for the supernate and interstitial liquid samples. The centrifuged solids were prepared for analysis by a fusion digestion, indicated by an " $\mathrm{F}$ " in the A\# column in Table 3.

The results ranged from $<5.27 \mathrm{e}-6$ to $<7.94 \mathrm{e}-6 \mu \mathrm{Ci} / \mathrm{mL}$ for the supernates; from $<3.33 \mathrm{e}-5$ to $6.09 \mathrm{e}-4 \mu \mathrm{Ci} / \mathrm{mL}$ for the interstitial liquids and from $2.87 \mathrm{e}-1$ to $1.07 \mu \mathrm{Ci} / \mathrm{g}$ for the centrifuged solids. The standard recoveries for this analysis were within the required limits. The RPDs for all samples met the required criterion with the exception of sample 5AW-96-17 centrifuged solid portion (S96T005085), with a RPD of $45.7 \%$ after one rerun. Because of the observed heterogeneity, the very high result of $1.07 \mu \mathrm{Ci} / \mathrm{g}$ for this sample may be considered a statistical outlier. No further reruns were requested.

\section{Inorganic Analyses}

\section{TGA - Thermogravimetric Analysis}

TGA analyses were performed on direct aliquots. Standard recoveries and RPDs were within the required limits. Typically, the percent moisture of the sample is reported as the total weight loss 


\section{HNF-SD-WM-DP-214, REV. 0}

that occurred below $200^{\circ} \mathrm{C}$. In some cases, this weight loss occured in more than one weight loss step. More information may be obtained by reviewing the raw data.

\section{pH}

The $\mathrm{pH}$ analysis was performed on direct aliquots. The results ranged from 11.66 to 12.49 for the centrifuged solids; from 13.24 to 13.55 for the interstitial liquids and from 13.38 to 13.64 for the supernates. Results for $\mathrm{pH}$ greater than 12.5 should be considered estimates because the highest calibration buffer available is 12.5 and $\mathrm{pH}$ electrode performance degrades at high $\mathrm{pH}$. Standard recovery and relative percent difference (RPD) criteria were met for all samples.

\section{$\mathrm{OH}^{-}$- Hydroxide}

In addition to the $\mathrm{OH}^{-}$analysis on the liquid aliquots (discussed previously with respect to corrosion concerns), this analysis was also performed on water digested aliquots of the centrifuged solids. This preparation is indicated by a "W" in the A\# column in Table 3 . There was no free hydroxide found in these water preps. The standard recoveries met the requested criterion. RPDs are not applicable for results that are all less than the detection limit.

\section{IC - Ion Chromatography}

The ion chromatography (IC) analysis was performed on direct aliquots for the supernates and interstitial liquids. The results for the $\mathrm{NO}_{2}{ }^{-}$and $\mathrm{NO}_{3}{ }^{-}$were discussed previously with respect to the corrosion rules, the results for the remaining analytes will be discussed here. The centrifuged solids were prepared for analysis by a water digestion, indicated by an "W" in the A\# column in Table 3. Bromide and oxalate results were included for infomational purposes only as part of the method of analysis. Since these analytes were not requested in the TSAP, the QC results were not reviewed.

The standard recovery, spike recovery and RPDs for this analysis were all within the required limits with the exception of the RPD for fluoride ( $\mathrm{F}^{-}$) for sample 5AW-96-1 (22.4\%) and chloride $\left(\mathrm{Cl}^{-}\right)$for sample $5 \mathrm{AW}-96-20$ centrifuged solid (29.2\%). Since the reported results were consistent with those of the other samples and the RPD was not significantly higher than the requested $20 \%$, no rerun was requested.

The phosphate results for the centrifuged solids appeared to show considerable variations with two samples giving positive results ranging from 378 to $486 \mu \mathrm{g} / \mathrm{g}$, and the remaining samples reported with large "less than" values in the range of $<472$ to $<1,117 \mu \mathrm{g} / \mathrm{g}$. This variability may be due to the heterogeneous nature of the sample. However, since the reported results were near the detection limit (DL) (approximately $2 \mathrm{X} \mathrm{DL}$ ) and the samples required a large dilution due to high $\mathrm{NO}_{3}^{-}$, this variability may be attributed to decreased precision near the DL.

Low concentrations of $\mathrm{Cl}^{-}$and $\mathrm{NO}_{3}^{-}$were found in the preparation blank for the centrifuged solid 


\section{HNF-SD-WM-DP-214, REV. 0}

analysis. Since the levels were insignificant with respect to the sample results, no reprep was requested.

\section{ICP - Inductively Coupled Plasma Spectrophotometry}

The ICP analysis was performed on acid diluted aliquots for the supernates and the interstitial liquids. This dilution is indicated by an " $\mathrm{D}$ " in the A\# column in Table 3. The centrifuged solids were prepared for analysis by fusion digest, indicated by an " $F$ " in the A\# column in Table 3 .

Aluminum ( $\mathrm{Al}$ ), iron ( $\mathrm{Fe}$ ) and sodium (Na) were the only analytes requested in the TSAP. The other analyte results were provided as additional information as part of the method of analysis. Since these analytes were not requested in the TSAP, the $\mathrm{QC}$ results were not reviewed. The standard recoveries and the spike recoveries for the centrifuged solid analyses were all within the required limits.

The RPDs were all less than the required $20 \%$ with the exception of $\mathrm{Fe}$ in the centrifuged solid samples; 5AW-96-7 RPD $=87.9 \%, 5 \mathrm{AW}-96-15 \mathrm{RPD}=46.0 \%$ and $5 \mathrm{AW}-96-20 \mathrm{RPD}=45.2 \%$. These high RPDs were attributed to the large dilution required because of the high concentration of potassium in the fusion digested samples. The tank coordinator was contacted, and no reprep or reruns were requested. The RPD for aluminum (Al) for sample 5AW-96-9 centrifuged solids was high (29.8\%), but no rerun was requested because the sample results were very near the DL.

For the supernates and interstitial liquids, the sodium (Na) results were more than four times the concentration of the spike added. In some cases this caused a poor spike recovery. With analyte concentrations higher than $1000 \mu \mathrm{g} / \mathrm{mL}$ it is difficult to add sufficient spike to perform a meaningful analysis. Therefore, the assessment of the accuracy of the measurement for these analytes was made by comparison of the sample results to those of a serial dilution of the sample. The serial dilution was performed by preparing and analyzing an additional 5-fold dilution of the sample. The result obtained from this analysis should be within $\pm 10 \%$ of the undiluted sample result. The results of this comparison, as presented in Table 5, indicate the accuracy of these analyses was acceptable.

Sodium was detected in the preparation blank for the centrifuged solid analyses. Since the concentration was below the method DL, no repreps or reruns were requested.

\section{TOC - Total Organic Carbon}

TOC analysis was performed, on direct aliquots, by the furnace oxidation method for the supernates and interstitial liquids and by the persulfate oxidation method for the centrifuged solids. The results based on the dry weight of the sample are presented in Table 6 . Three of the supernate sample results exceeded the notification limit of $30,000 \mu \mathrm{gC} / \mathrm{g}$ (dry weight basis); 5AW-96-1, 5AW-96-4 and 5AW-96-10 (duplicate result only). Appropriate notifications were made. 


\section{Table 5}

\section{ICP Serial Dilution Results for Tank 241-AW-105 Risers 10A and 15A}

\begin{tabular}{|c|c|c|c|c|}
\hline Sample ID & Analyte & $\begin{array}{c}\text { Sample Result }(\mu \mathrm{g} / \mathrm{mL}) \\
\text { Undiluted }\end{array}$ & $\begin{array}{l}\text { Serial Dilution Result } \\
(\mu \mathrm{g} / \mathrm{mL})\end{array}$ & $\begin{array}{c}\text { Percent } \\
\text { Difference }\end{array}$ \\
\hline \multicolumn{5}{|l|}{ S96T005004 } \\
\hline $5 \mathrm{AW}-96-1$ & $\mathrm{Na}$ & $1.26 \mathrm{E}+04$ & $1.27 \mathrm{E}+04$ & 1.0 \\
\hline \multicolumn{5}{|l|}{ Supernate } \\
\hline \multicolumn{5}{|l|}{ S96T005005 } \\
\hline $5 \mathrm{AW}-96-2$ & $\mathrm{Na}$ & $1.27 \mathrm{E}+04$ & $1.28 \mathrm{E}+04$ & 0.5 \\
\hline \multicolumn{5}{|l|}{ Supérnate } \\
\hline \multicolumn{5}{|l|}{ S96T005006 } \\
\hline $5 \mathrm{AW}-96-4$ & $\mathrm{Na}$ & $1.30 \mathrm{E}+04$ & $1.32 \mathrm{E}+04$ & 1.5 \\
\hline \multicolumn{5}{|l|}{ Supernate } \\
\hline \multicolumn{5}{|l|}{ S96T005010 } \\
\hline $5 \mathrm{AW}-96-10$ & $\mathrm{Na}$ & $1.27 E+04$ & 1.30E+04 & 2.4 \\
\hline \multicolumn{5}{|l|}{ Supernate } \\
\hline \multicolumn{5}{|l|}{ S96T005011 } \\
\hline $5 \mathrm{AW}-96-11$ & $\mathrm{Na}$ & $1.29 \mathrm{E}+04$ & $1.30 \mathrm{E}+04$ & 0.8 \\
\hline \multicolumn{5}{|l|}{ Supernate } \\
\hline \multicolumn{5}{|l|}{ S96T005012 } \\
\hline $5 \mathrm{AW}-96-14$ & $\mathrm{Na}$ & $1.87 \mathrm{E}+04$ & $1.90 \mathrm{E}+04$ & 1.8 \\
\hline \multicolumn{5}{|l|}{ Supernate } \\
\hline \multicolumn{5}{|l|}{ S96T005049 } \\
\hline $5 \mathrm{AW}-96-5$ & $\mathrm{Na}$ & $2.13 E+04$ & $2.14 \mathrm{E}+04$ & 0.5 \\
\hline \multicolumn{5}{|l|}{ Interstitial Liq. } \\
\hline \multicolumn{5}{|l|}{ S96T005081 } \\
\hline 5AW-96-15 & $\mathrm{Na}$ & $2.77 \mathrm{E}+04$ & $2.79 \mathrm{E}+04$ & 0.6 \\
\hline Interstitial Liq. & & & & \\
\hline
\end{tabular}

Percent Difference $=[($ ABS $($ Sample - Serial Dilution $)) /$ Sample $] \times 100$ 
HNF-SD-WM-DP-214, REV. 0

Table 6

Total Organic Carbon Results (dry weight)

Tank 241-AW-105

\begin{tabular}{|c|c|c|c|c|c|c|}
\hline Cuistomer W & $\begin{array}{l}\text { Segment } \\
\text { Portion }\end{array}$ & $\begin{array}{l}\text { LabCore } \\
\mathbf{I D}\end{array}$ & $\begin{array}{l}\text { Sample } \\
\text { Resuilt }\end{array}$ & $\begin{array}{l}\text { Duplicate } \\
\text { Result }\end{array}$ & $\begin{array}{l}\text { Triplicate } \\
\text { Result }\end{array}$ & Mean \\
\hline & a & क्सेमn & ing C/g & igC/g & $\mu \mathrm{gC} / \mathrm{g}$ & $\mathrm{HgClg}$ \\
\hline 5 AW-96-1 & Supernate & S96T005004 & $9.65 \mathrm{E}+04$ & $9.99 \mathrm{E}+04$ & & $9.82 \mathrm{E}+04$ \\
\hline 5 AW-96-2 & Supernate & S96T005008 & $2.21 \mathrm{E}+03$ & $2.30 \mathrm{E}+03$ & & $2.25 \mathrm{E}+03$ \\
\hline $5 A W-96-2 \quad 1$ & Supernate & S96T005008 1 & $2.44 \mathrm{E}+03$ & $2.25 E+03$ & & $2.34 \mathrm{E}+03$ \\
\hline 5 AW-96-4 & Supernate & S96T005006 & $8.36 \mathrm{E}+04$ & $9.25 E+04$ & & $8.81 \mathrm{E}+04$ \\
\hline 5 AW-96-10 & Supernate & S96T005010 & $2.98 \mathrm{E}+04$ & $3.02 E+04$ & & $3.00 \mathrm{E}+04$ \\
\hline $5 A W-96-11$ & Supernate & S96T005011 & $2.74 \mathrm{E}+04$ & $2.70 E+04$ & & $2.72 E+04$ \\
\hline $5 A W-96-14$ & Supernate & S96T005012 & $2.62 E+04$ & $2.68 \mathrm{E}+04$ & & $2.65 \mathrm{E} \div 04$ \\
\hline \multicolumn{3}{|c|}{ W"Bस } & $\mu \mathrm{gCg}$ & $\mu \mathrm{gG} / \mathrm{g}$ & $\mu \mathrm{gC/g}$ & $\mu \mathrm{gC} / \mathrm{gh}$ \\
\hline $5 \mathrm{AW}-96-5$ & Interstitia! Liq. & S96T005049 & $1.25 \mathrm{E}+04$ & $7.73 \mathrm{E}+03$ & $7.07 \mathrm{E}+03$ & $9.09 \mathrm{E} \div 03$ \\
\hline $5 A W-96-51$ & Interstitial Liq. & S96T005049 1 & $9.23 E+03$ & $9.23 E+03$ & & $9.23 E \div 03$ \\
\hline $5 A W-96-7$ & Interstitial Liq. & S96T005050 & $2.14 \mathrm{E}+04$ & $2.09 \mathrm{E}+04$ & & $2.11 E+04$ \\
\hline 5 AW-96-9 & Interstitial Liq. & S96T005051 & $1.95 E+04$ & $1.90 \mathrm{E}+04$ & & $1.92 E+04$ \\
\hline $5 A W-96-15$ & Interstitial Liq. & S96T005081 & $1.93 E+04$ & $1.72 E+04$ & & $1.82 E \div 04$ \\
\hline 5 AW-96-17 & Interstitial Liq. & S96T005082 & $1.25 E+04$ & $1.23 E+04$ & & $1.24 \mathrm{E}+04$ \\
\hline 5 AW-96-20 & Interstitial Liq. & S96T005083 & $1.71 \mathrm{E}+04$ & $1.62 \mathrm{E}+04$ & & $1.66 \mathrm{E} \div 04$ \\
\hline \multicolumn{3}{|c|}{ 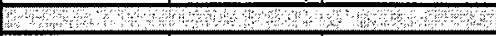 } & $\mu \mathrm{gC} / \mathrm{g}$ & $1 \mathrm{gC} / \mathrm{g}$ & 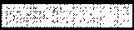 & $\mathrm{\mu gC/g}$ \\
\hline $5 A W-96-5$ & Cent. Sol. & S96T005019 & $9.10 \mathrm{E}+03$ & $8.70 \mathrm{E}+03$ & & $8.90 \mathrm{E}+03$ \\
\hline 5AW-96-7 & Cent. Sol. & S96T005020 & $5.13 E+03$ & $4.98 \mathrm{E}+03$ & & $5.05 E+03$ \\
\hline 5 AW-96-9 & Cent. Sol. & S96T005021 & $4.90 \mathrm{E}+03$ & $5.08 \mathrm{E}+03$ & & $4.99 \mathrm{E}+03$ \\
\hline $5 A W-96-15$ & Cent. Sol. & S96T005075 & $5.69 \mathrm{E}+03$ & $5.25 E+03$ & & $5.47 \mathrm{E}+03$ \\
\hline 5 AW- $96-17$ & Cent. Sol. & S96T005076 & $6.11 \mathrm{E}+03$ & $6.84 E+03$ & & $6.48 \mathrm{E}+03$ \\
\hline 5 AW-96-20 & Cent. Sol. & S96T005077 & $5.65 \mathrm{E}+03$ & $4.57 \bar{E}+03$ & & $5.11 \mathrm{E}+03$ \\
\hline
\end{tabular}




\section{HNF-SD-WM-DP-214, REV. 0}

The standard recoveries and spike recoveries were within the required limits. Two samples had high RPDs; sample 5AW-96-20 centrifuged solid (21.2\%), and 5AW-96-5 interstitial liquid $(47.0 \%)$. Since the results for sample 5AW-96-20 centrifuged solid were consistent with the results obtained from the same portion of the other sludge samples and this sample was reported to be heterogeneous at the time of sample breakdown, no rerun was requested.

As a result of the high RPD, a triplicate aliquot was analyzed for sample 5AW-96-5. A replicate analysis was also performed because the results from the original analysis for this sample were much lower than those obtained for the other interstitial liquids from the same riser (10A). The results from both analyses are presented in Table 7. A comparison of the mean values from the two analyses indicates that the original results obtained were valid numbers, but the high RPD may have been due to suspended solids in the sample. The results for the replicate analysis are also presented in Table 3, denoted with a "1" following the sample number.

\section{Table 7: TOC Results for Sample 5AW-96-5 Interstitial Liquid}

\begin{tabular}{|c|c|c|c|c|c|}
\hline 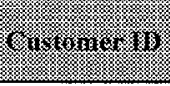 & ing & 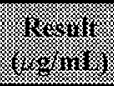 & 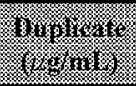 & 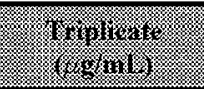 & 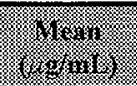 \\
\hline $5 \mathrm{AW}-96-5$ & S96T005049 & 951 & 589 & 539 & 693 \\
\hline $5 A W-96-5$ & S96T005049 1 & 704 & 704 & & 704 \\
\hline $5 A W-96-5$ & S96T005049 & & & Overall Mean $\rightarrow$ & 697.4 \\
\hline
\end{tabular}

The results for sample 5AW-96-2 supernate (S96T005008) were much lower (nearly fifty times lower) than the other two supernate samples from riser 10A. An additional sample and duplicate analysis was requested to verify the results. These results, denoted in Table 3 with a " 1 " following the sample number, indicated that the first set of results were correct.

\section{TIC - Total Inorganic Carbon}

TIC analysis was performed on direct aliquots. The standard recoveries, spike recoveries and RPDs were within the required limits.

\section{Radionuclide Analyses}

\section{${ }^{90} \mathrm{Sr}$ - Strontium-90}

${ }^{90} \mathrm{Sr}$ analysis was performed on direct aliquots for the supernates and interstitial liquids. The centrifuged solids were prepared for analysis by a fusion digestion, indicated by an "F" in the A\# column in Table 3 . The standard recoveries were all within the required limits. 


\section{HNF-SD-WM-DP-214, REV . 0}

${ }^{90} \mathrm{Sr}$ was detected in most of the method and preparation blank analyses. However, in all cases the results were insignificant with respect to the sample concentration and no reruns or repreparation s were requested.

The RPDs for the centrifuged solid samples and five of the six supernates were within the required limit (20\%). However, due to suspended solids observed in sample 5AW-96-14 supernate and all six of the interstitial liquids, it was very difficult to obtain reproducible results for these samples. Historical data indicate that ${ }^{90} \mathrm{Sr}$ forms fine solids upon cooling of the waste after sampling. Some of the observed suspended solids may be of this form thus making it difficult to obtain reproducible sample and duplicate aliquots. More than one rerun was requested for each sample. Only two of the six interstitial liquids had acceptable RPDs for the final reported results. No further reruns were requested.

\section{GEA - Gamma Energy Analysis}

GEA analysis was performed for ${ }^{137} \mathrm{Cs}$ on direct aliquots for the supernates and interstitial liquids. The centrifuged solids were prepared for analysis by a fusion digestion, indicated by an "F" in the A\# column in Table 3. The results for ${ }^{60} \mathrm{Co}$ are included for information purposes as part of the method. However, since this analyte was not requested in the TSAP, the QC results were not reviewed. The standard recoveries and RPDs for ${ }^{137} \mathrm{Cs}$ were within the required limits.

${ }^{137} \mathrm{Cs}$ was reported as detected in the method blank for the analysis of sample $5 \mathrm{AW}-96-5$ interstitial liquid. However, since the result was insignificant with respect to the sample result, no rerun was requested.

\section{AT - Total Alpha Activity}

AT analysis was performed on direct aliquots for the supernates and interstitial liquids. The centrifuged solids were prepared for analysis by a fusion digestion, indicated by an " $\mathrm{F}$ " in the A\# column in Tabie 3 . The standard recoveries were within the required limits.

The spike recoveries were within the required limits, with the exception of the following low spike recoveries: sample 5AW-96-5 centrifuged solids $(66.3 \%)$; $5 \mathrm{AW}-96-7$ interstitial liquid (70.75\%); 5AW-96-15 interstitial liquid (73.82\%); and 5AW-96-1 supernate (74.65\%). The poor spike recoveries were possibly due to matrix interference from solids in the samples. The spike recoveries above $70 \%$ were within the method control limit for the standard and, therefore, were accepted with no additional reruns. Sample 5AW-96-5 was already rerun once and additional reruns would not improve the results because of the solids content of the sample. 


\section{HNF-SD-WM-DP-214, REV. 0}

The RPDs were within the required limits, with the exception of three of the interstitial liquid samples: 5AW-96-9 (46.7\%); 5AW-96-15 (44.2\%); and 5AW-96-17 (58.0\%). The high RPDs are possibly due to matrix interference from solids in the samples and low sample activity. One of the samples (5AW-96-9) was analyzed more than once, with poor results each time. It was decided that additional reruns on the remaining samples would not improve the reproducibility.

Alpha activity was detected in the preparation blank for sample 5AW-96-7 centrifuged solids, but was insignificant with respect to the sample results. Alpha activity was detected in the method blank for three of the six interstitial liquids at a concentration near that of the samples. However, the sample data indicated that there was no contamination and no rerun was requested.

\section{Organic Analyses}

\section{PCB - Polychlorinated Biphenyls Analysis}

PCB analyses were performed on direct aliquots for the centrifuged solid subsamples. The results are presented in Table 8. The samples were prepared by addition of anhydrous sodium sulfate for drying, followed by two extractions with hexane. The combined extracts from each sample were condensed to $2 \mathrm{~mL}$ each under a nitrogen blanket.

A decachlorobiphenyl surrogate standard was added to the method blank, samples and spikes. All recoveries were within the acceptable limits, ranging from 79\%-112\%. Arochlor 1260 was added to the subsample from 5AW-96-9 (S96T005048) for the matrix spike and matrix spike duplicate analyses. The recoveries were acceptable at $95.4 \%$ and $95.2 \%$, respectively. The duplicate analysis was performed on the subsample from 5AW-96-15 (S96T005078). Since there were no PCB results reported, the RPD calculation is not applicable (n/a). The Arochlor 1260 standard was also used for the final calibration verification analysis and met the method criteria.

No PCB patterns were observed on any of the samples. The results are reported as undetected at the method detection limit. This detection limit was calculated based on the estimated quantitation limit and the sample size used. A more detailed discussion of results may be found in the narrative included with the raw data.

\section{Procedures}

Table 9 lists the analytical procedures used for performing the safety screening analyses. Abbreviations for analyses are defined in the table notes. 
HNF-SD-WM-DP-214, REV. 0

Table 8

Tank 241-AW-105

Polychlorinated Biphenyls Analysis Results

Risers 10A and 15A

Sample Portion: Centrifuged Solids

\begin{tabular}{|c|c|c|c|c|c|c|c|c|}
\hline Gus toulerin & Shamp $1 \%$ & Gonits & Sitandard $\%$ & W & W Result & Wuplicate & Find & Detelm \\
\hline $5 \mathrm{AW}-96-5$ & S96T005046 & $\mu \mathrm{g} / \mathrm{g}$ & 101.7 & $\mathrm{U} 0.993$ & $\mathrm{U} 0.993$ & $\mathrm{n} / \mathrm{a}$ & $\mathrm{n} / \mathrm{a}$ & 0.993 \\
\hline $5 A W-96-7$ & S96T005047 & $\mu \mathrm{g} / \mathrm{g}$ & 101.7 & U 0.993 & U 0.888 & $\mathrm{n} / \mathrm{a}$ & $\mathrm{n} / \mathrm{a}$ & 0.888 \\
\hline $5 A W-96-9$ & S96T005048 & $\mu \mathrm{g} / \mathrm{g}$ & 101.7 & U 0.993 & $\mathrm{U} 0.955$ & $\mathrm{n} / \mathrm{a}$ & $\mathrm{n} / \mathrm{a}$ & 0.955 \\
\hline $5 \mathrm{AW}-96-15$ & S96T005078 & $\mu \mathrm{g} / \mathrm{g}$ & 101.7 & U 0.993 & U 0.903 & U 0.903 & $\mathrm{n} / \mathrm{a}$ & 0.903 \\
\hline $5 \mathrm{AW}-96-17$ & S96T005079 & $\mu \mathrm{g} / \mathrm{g}$ & 101.7 & U 0.993 & U 0.897 & $\mathrm{n} / \mathrm{a}$ & $\mathrm{n} / \mathrm{a}$ & 0.897 \\
\hline $5 A W-96-20$ & S96T005080 & $\mu \mathrm{g} / \mathrm{g}$ & 101.7 & U 0.993 & $\mathrm{U} 0.955$ & $\overline{\mathrm{n} / \mathrm{a}}$ & $\mathrm{n} / \mathrm{a}$ & 0.955 \\
\hline
\end{tabular}

$\mathrm{U}=$ Analyte was undetected at the method detection limit based on a $500 \mu \mathrm{g} / \mathrm{L}$ standard.

\begin{tabular}{|c|c|c|c|c|c|c|}
\hline Goustome iD & 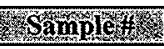 & Unis & 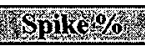 & Spile Dap & Ho & Shmogated \\
\hline $5 \mathrm{AW}-96-5$ & S96T005046 & $\mu \mathrm{g} / \mathrm{g}$ & $\mathrm{n} / \mathrm{a}$ & $\mathrm{n} / \mathrm{a}$ & $\mathrm{n} / \mathrm{a}$ & 103.0 \\
\hline $5 \mathrm{AW}-96-7$ & S96T005047 & $\mu \mathrm{g} / \mathrm{g}$ & $\mathrm{n} / \mathrm{a}$ & $\mathrm{n} / \mathrm{a}$ & $\mathrm{n} / \mathrm{a}$ & 119.6 \\
\hline $5 A W-96-9$ & S96T005048 & $\mu \mathrm{g} / \mathrm{g}$ & 95.4 & 95.2 & 0.18 & 109.6 \\
\hline $5 \mathrm{AW}-96-15$ & S96T005078 & $\mu \mathrm{g} / \mathrm{g}$ & $\mathrm{n} / \mathrm{a}$ & $\mathrm{n} / \mathrm{a}$ & $n / a$ & 85.9 \\
\hline $5 \mathrm{AW}-96-17$ & S96T005079 & $\mu \mathrm{g} / \mathrm{g}$ & $\mathrm{n} / \mathrm{a}$ & $\mathrm{n} / \mathrm{a}$ & $\mathrm{n} / \mathrm{a}$ & 100.3 \\
\hline $5 \mathrm{AW}-96-20$ & S96T005080 & $\mu \mathrm{g} / \mathrm{g}$ & $\mathrm{n} / \mathrm{a}$ & $\mathrm{n} / \mathrm{a}$ & $\mathrm{n} / \mathrm{a}$ & 103.9 \\
\hline
\end{tabular}




\section{HNF-SD-WM-DP-214, REV. 0}

\section{Table 9: Analytical Procedures}

\begin{tabular}{|c|c|c|}
\hline DSC & Direct Analysis & $\begin{array}{l}\text { LA-514-113 Rev. C-1 } \\
\text { LA-514-114 Rev. D-0 }\end{array}$ \\
\hline $\mathrm{TGA}$ & Direct Analysis & $\begin{array}{l}\text { LA-514-114 Rev. D-0 } \\
\text { LA-560-112 Rev. C-0 }\end{array}$ \\
\hline pH & Direct Analysis & $\begin{array}{l}\text { Liquid - LA-212-106 Rev. B-0 } \\
\text { Solid - LA-212-105 Rev. B-0 }\end{array}$ \\
\hline Sp.G. & Direct Analyses & LA-510-112 Rev. C-3 \\
\hline $\mathrm{OH}^{-}$ & Direct Analysis & LA-211-102 Rev. C-0 \\
\hline IC & $\begin{array}{l}\text { Liquid - Direct Analysis } \\
\text { Solid - LA-504-101 Rev. E-0 + }\end{array}$ & LA-533-105 Rev. D-1 \\
\hline ICP & $\begin{array}{l}\text { Liquid - Acid Dilution } \\
\text { Solid - LA-549-141 Rev. F-0 ++ }\end{array}$ & LA-505-161 Rev. B-1 \\
\hline TOC & Direct Analysis & $\begin{array}{l}\text { Liquid - LA-344-105 Rev. D-1 } \\
\text { Solid - LA-342-100 Rev. E-0 }\end{array}$ \\
\hline TIC & Direct Arnalysis & L.A.342-100 Rev. E-0 * \\
\hline Bulk Density & Direct Analysis & LO-160-103 Rev, B-0 \\
\hline Vol \% Solids & Direct Analysis & $\begin{array}{l}\text { Centrif. - LA-519-132 Rev. D-0 } \\
\text { Visual - LA-519-151 Rev. F-1 }\end{array}$ \\
\hline \multicolumn{3}{|c|}{ Radionulelide analyses } \\
\hline Total Alpha & $\begin{array}{c}\text { Liquid - Direct Analysis } \\
\text { Solid - LA-549-141 Rev. F-0 ++ }\end{array}$ & LA-508-101 Rev. E-0 \\
\hline GEA & $\begin{array}{c}\text { Liquid - Direct Analysis } \\
\text { Solid - LA-549-141 Rev, F=0 ++ }\end{array}$ & LA-548-121 Rev. E-0 \\
\hline${ }^{\infty} \mathrm{Sr}$ & $\begin{array}{c}\text { Liquid - Direct Analysis } \\
\text { Solid - LA-S49-141 Rev. F-0++ }\end{array}$ & LA-220-101 Rev. D-1 \\
\hline${ }^{241} \mathrm{Am}$ & $\begin{array}{c}\text { Liquid - Direct Analysis } \\
\text { Solid - LA-549-141 Rev. F-0 ++ }\end{array}$ & LA-953-103 Rev. B-0 \\
\hline${ }^{239240} \mathrm{Pu}$ & $\begin{array}{l}\text { Liquid - Direct Analysis } \\
\text { Solid-LA-549-141 Rev. F-0 ++ }\end{array}$ & LA-943-128 Rev. B-0 \\
\hline \multicolumn{3}{|c|}{ Oganúc Analyses } \\
\hline $\mathrm{PCB}$ & Direct Analysis & LA-523-434 Rev. A-0 \\
\hline
\end{tabular}




\section{HNF-SD-WM-DP-214, REV. 0}

Notes: * - The TIC analysis was performed by LA-342-100 rather than LA-622-102, as requested in the TSAP, because the instrumentation was unavailable to perform the requested procedure.

$+\quad$ Water digestion procedure

+. Fusion digestion procedure

Abbreviations:

$\mathrm{DSC}=$ differential scanning calorimetry

TOC = total organic carbon

TGA = thermogravimetric analysis

TIC = total inorganic carbon

Sp.G. = specific gravity

$\mathrm{OH}=$ hydroxide

IC = ion chromatography

ICP = inductively coupled plasma

PCB = polychlorinated biphenyls

GEA = gamma energy analysis

${ }^{90} \mathrm{Sr} \quad=$ strontium 90

${ }^{241} \mathrm{Am}=$ americium 241

${ }^{239 / 240} \mathrm{Pu}=$ plutonium $239 / 240$

\section{$\underline{\text { References }}$}

Fowler, K.D., 1995, Data Quality Objectives for Tank Farms Waste Compatibility Program, WHC-SD-WM-DQO-001, Rev. 1, Westinghouse Hanford Company, Richland, WA 99352.

Sasaki, L. M., 1996, Compatibility Grab Sampling and Analysis Plan, WHC-SD-TSAP-037, Rev. 2D, Westinghouse Hanford Company, Richland, WA 99352 
HNF-SD-WM-DP-214, REV. 0

ATTACHMENT I

AW-105 SAMPLE BREAKDOWN

$18 \div$ 
HNF-SD-WM-DP-214, REV. 0

THIS PAGE WAS INTENTIOANLLY LEFT BLANK 


\section{Attachment 1 (1 of 5) AW-105 GRAB SAMPLE BREAKDOWN}

Riser $10 \mathrm{~A}$
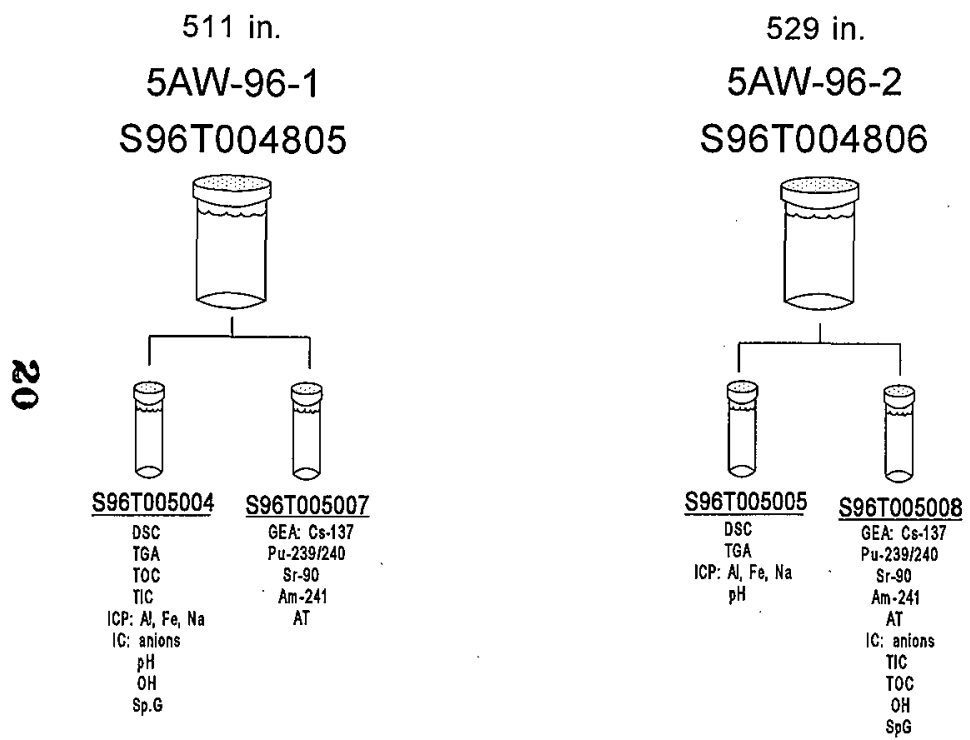

548 in

5AW-96-4

S96T004808

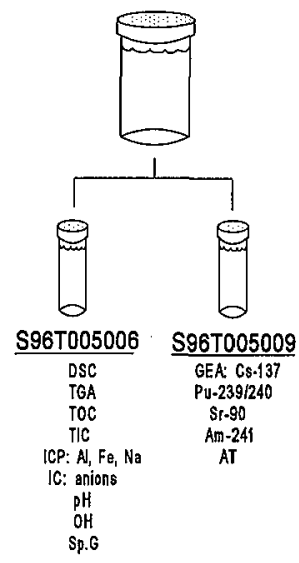




\section{Attachment 1 ( 2 of 5 ) AW-105 GRAB SAMPLE BREAKDOWN}

\section{Riser $15 \mathrm{~A}$}

511 in.

5AW-96-10

S96T004814

3
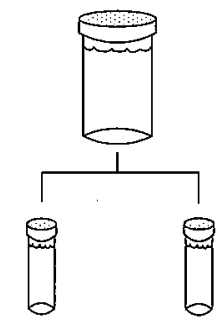

S96T005010

DSC

TGA
TOC
TIC

$I C P: \mathrm{Al}_{1} \mathrm{Fa}_{1} \mathrm{Na}$

1C: anions

PH

Sp.6
529 in.

5AW-96-11

S96T004815

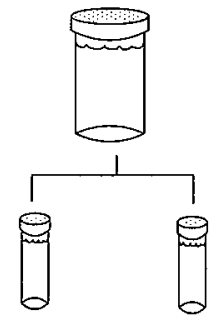

S96T005011

S96T005013

GEA: : : -137

U.2391240

Sr-90

AT

Pu-239/240

$5 \mathrm{~s}-90$

Am-241

AT

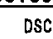

DSC

TOA

TIC

ICP: $A_{1} \mathrm{FE}_{1} \mathrm{Na}$

IC: anions

$\mathrm{PH}$

Sp.G
548 in.

5AW-95-14 S96T004818

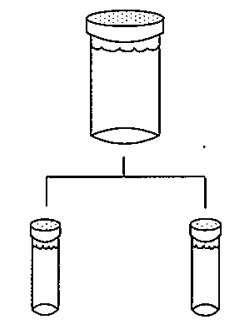

S96T005012

GEA Cs.137

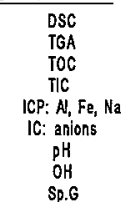

$\$ 96 T 005015$

GER: Gs-137

Pu-239/240

Si -90

AT 


\section{Attachment 1 (3 of 5) AW-105 GRAB SAMPLE BREAKDOWN \\ Riser $10 \mathrm{~A}$}

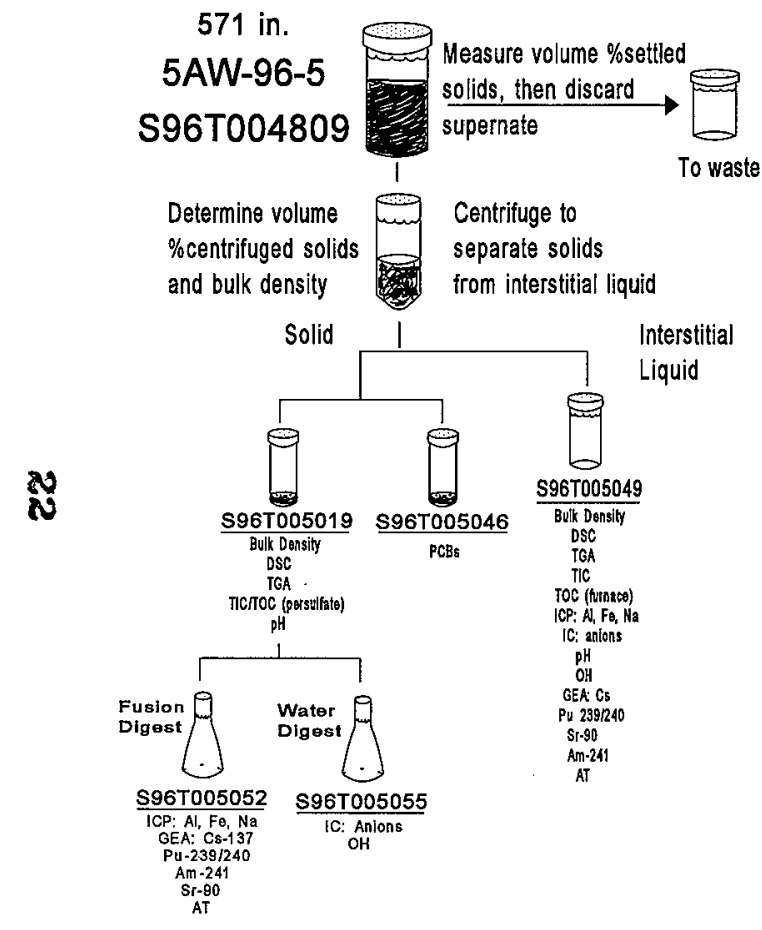

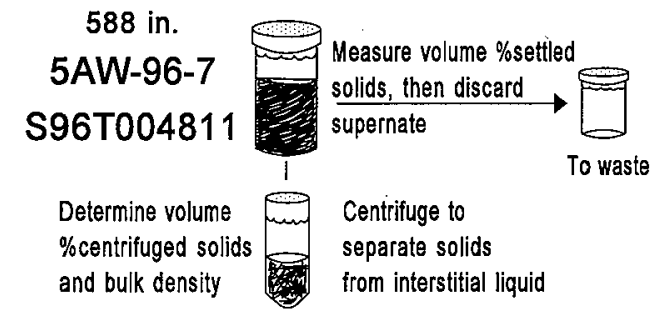

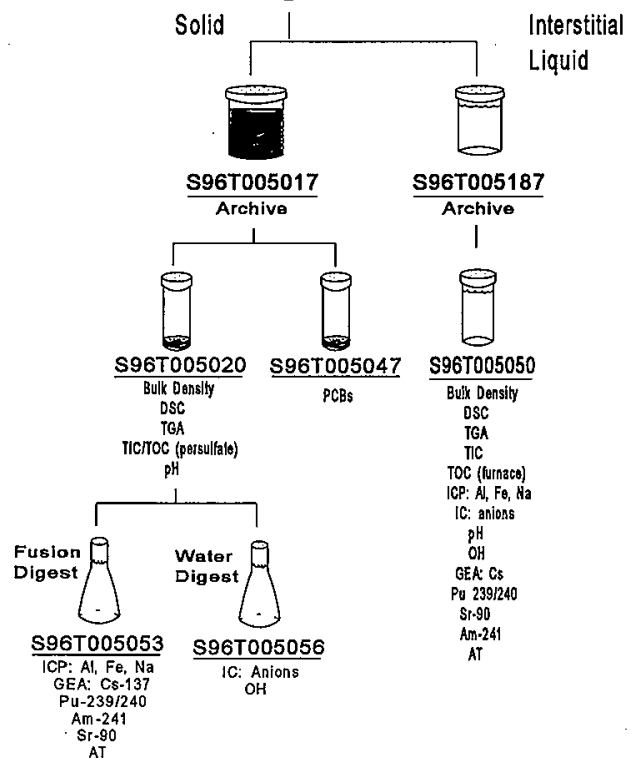




\section{Attachment 1 (4 of 5) AW-105 GRAB SAMPLE BREAKDOWN}

\section{Riser 10A}

589 in.

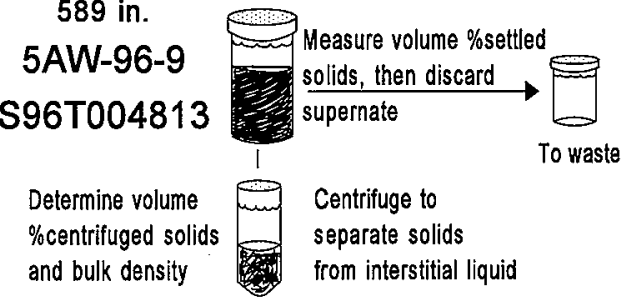

a

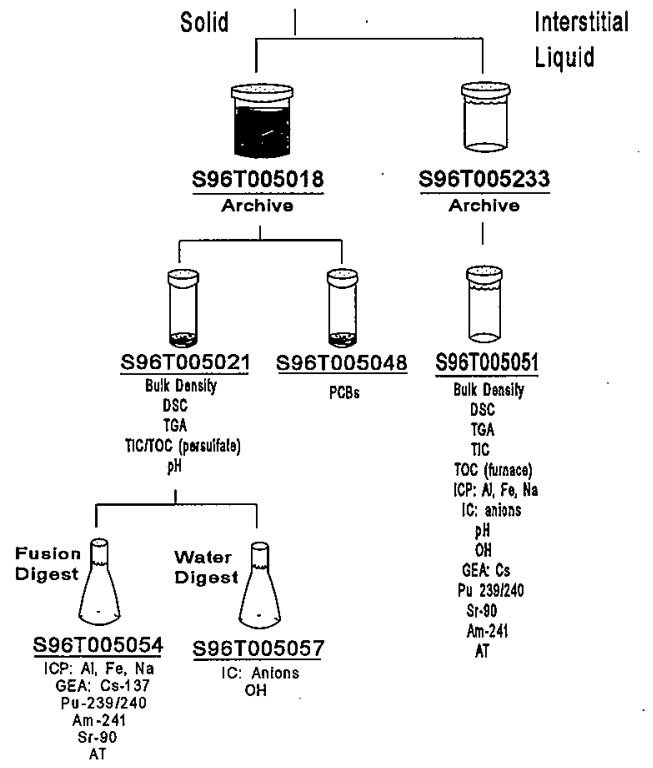

Riser 15A
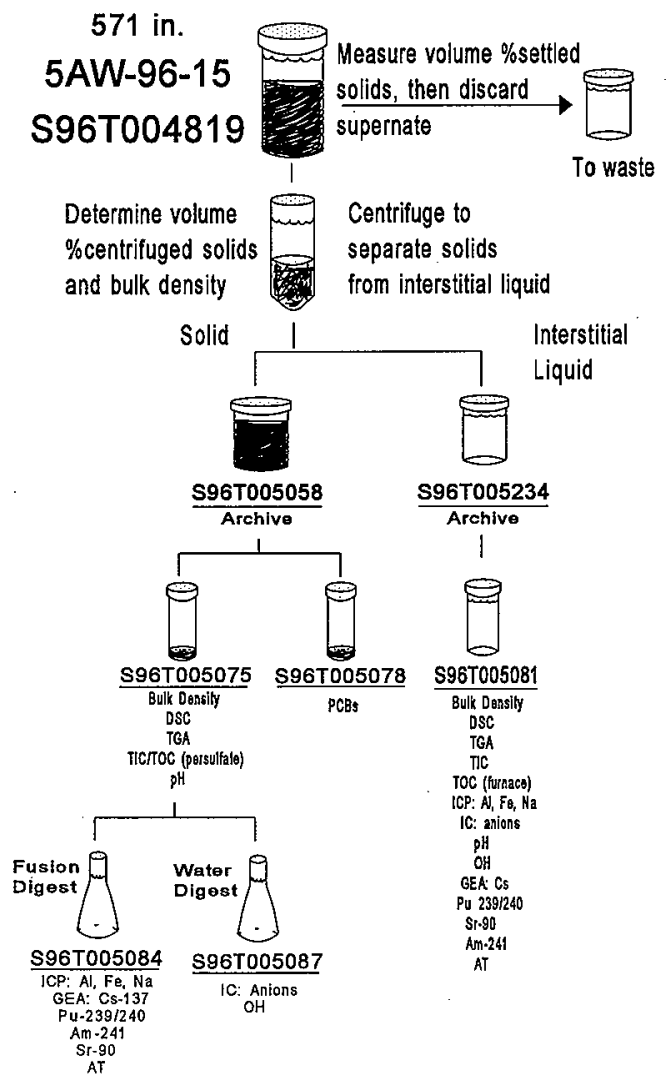


\section{Attachment 1 (5 of 5) AW-105 GRAB SAMPLE BREAKDOWN \\ Riser $15 \mathrm{~A}$}
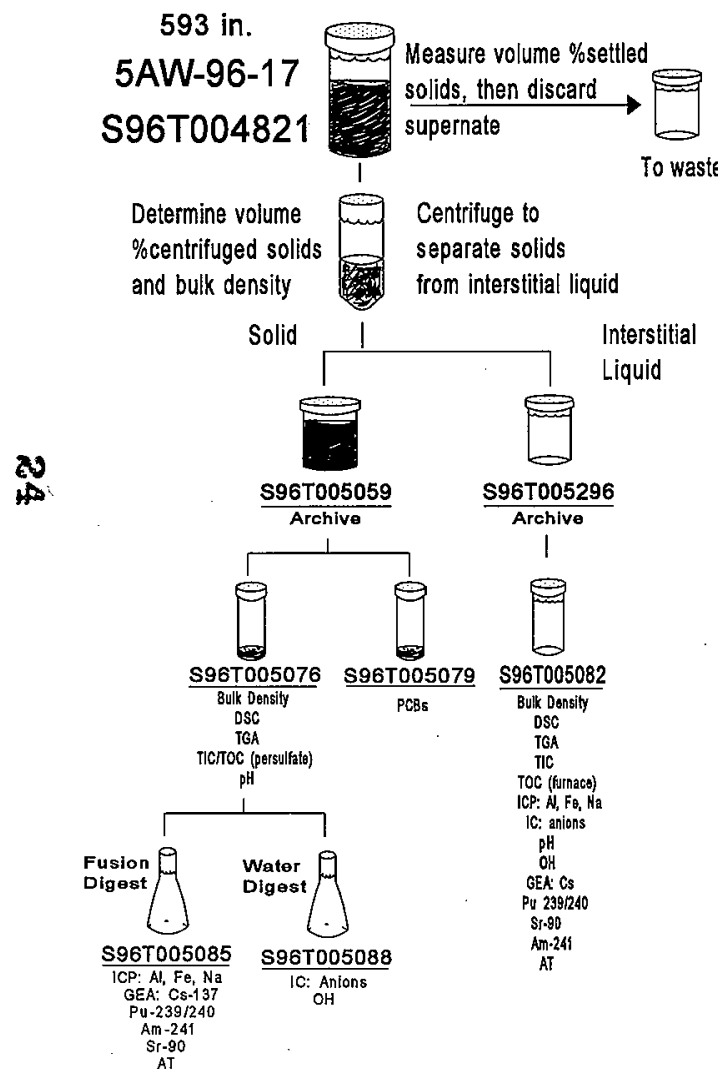
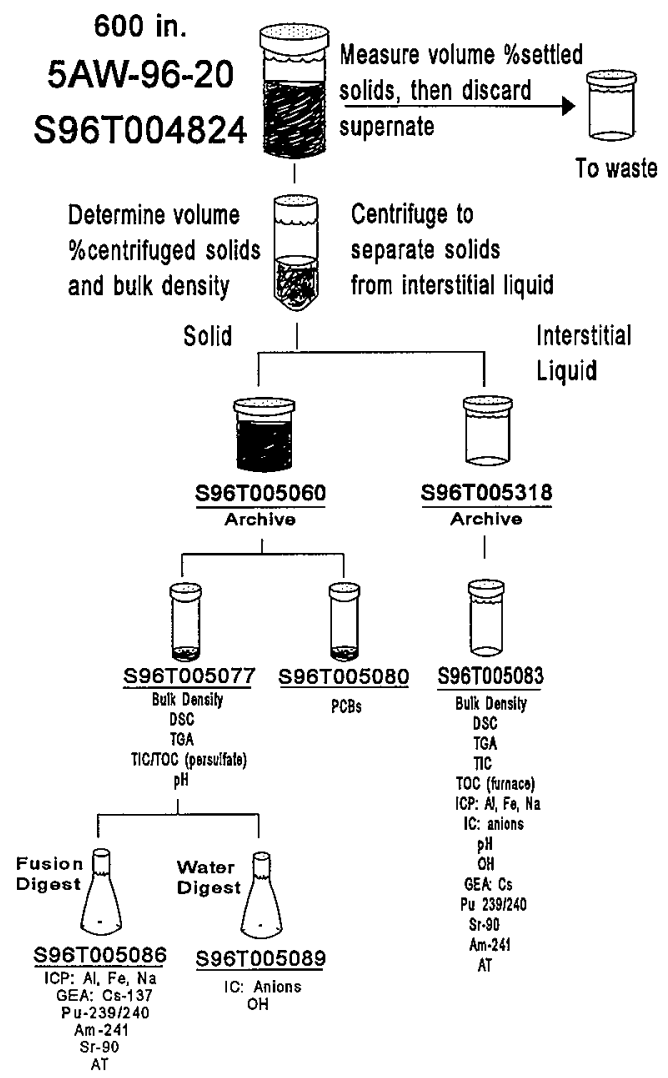
HNF-SD-WM-DP-214, REV. 0

Sample Data Summary 
HNF-SD-HM-DP-214, REV. 0

THIS PAGE HAS INTENTIOANLLY LEFT BLANK

26 
RISER: 10A

SEGMENT \#: 5AW-96-1

SEGMENT PORTION: Supernate

\begin{tabular}{|c|c|c|c|c|c|c|c|c|c|c|c|c|}
\hline Sample\# & R A\# & Analyte & Unit & Standard \% & Blank & Result & Dupl icate & Average & RPD \% & Spk Rec \% & Det Limit & Count Err\% \\
\hline S96T005004 & & DSC Exotherm using Mettler & Joules/g & 100.2 & $\mathrm{n} / \mathrm{a}$ & $0.00 \mathrm{e}+00$ & $0.00 \mathrm{e}+00$ & $0.00 \mathrm{e}+00$ & 0.00 & $\mathrm{n} / \mathrm{a}$ & $n / a$ & $n / a$ \\
\hline $596 \mathrm{~T} 005004$ & & OH- by Pot. Titration & $\mathrm{ug} / \mathrm{mL}$ & 98.56 & $<42.00$ & $3.27 \mathrm{e}+03$ & $3.37 \mathrm{e}+03$ & $3.32 \mathrm{e}+03$ & 3.01 & $\mathrm{n} / \mathrm{a}$ & 625.0 & $n / a$ \\
\hline$\$ 967005004$ & & pH Direct & $\mathrm{pH}$ & 99.86 & $\mathrm{n} / \mathrm{a}$ & 13.38 & 13.39 & 13.39 & 0.07 & $\mathrm{n} / \mathrm{a}$ & $1.00 e-02$ & $n / a$ \\
\hline S96T005004 & & Specific Gravity & Sp.G. & 100.4 & $\mathrm{n} / \mathrm{a}$ & 1.021 & 1,015 & 1.018 & 0.52 & $\mathrm{n} / \mathrm{a}$ & $1.00 e^{-03}$ & $n / a$ \\
\hline 5961005004 & & \% water by TGA using Mettler & $\%$ & 98.95 & $n / a$ & 95.04 & 95.72 & 95.38 & 0.71 & $\mathrm{n} / \mathrm{a}$ & $n / a$ & $n / a$ \\
\hline $596 \mathrm{~T} 005004$ & & TIC by Acid/Coul ometry & $\mathrm{ug} / \mathrm{mL}$ & 97.00 & 5.400 & $1.70 \mathrm{e}+02$ & 204.0 & 187.0 & 18.2 & 101.1 & 5.000 & $n / a$ \\
\hline S96T005004 & & Tot. Organic Carbon by coul. & $\mathrm{ug} / \mathrm{mL}$ & 98.00 & 5.000 & $4.54 \mathrm{e}+03$ & $4.70 \mathrm{e}+03$ & $4.62 \mathrm{e}+03$ & 3.46 & 115.4 & 55.00 & $n / a$ \\
\hline S96T005004 & D & Silver-ICP-Acid Dil. & $\mu \mathrm{g} / \mathrm{mL}$ & 99.20 & $<1.00 \mathrm{e}-02$ & $9.94 e^{-01}$ & 1.020 & 1.007 & 2.58 & 83.90 & $4.10 e-01$ & $n / a$ \\
\hline S96T005004 & D & Aluminium-ICP-Acid Dil. & $\mathrm{ug} / \mathrm{mL}$ & 98.00 & $<5.00 \mathrm{e}-02$ & 15.60 & 16.10 & 15.85 & 3.15 & 98.40 & 2.050 & $\mathrm{n} / \mathrm{a}$ \\
\hline S96T005004 & D & Arsenic-ICP-Acid Dil. & $\mathrm{gg} / \mathrm{mL}$ & 102.4 & $<1.00 \mathrm{e}-01$ & 4.100 & $<4.10 \mathrm{e} 0$ & n/a & $\mathrm{n} / \mathrm{a}$ & 105.0 & 4.100 & $n / a$ \\
\hline S96T005004 & D & Boron-ICP-Acid Dil. & $\mathrm{ug} / \mathrm{mL}$ & 99.20 & $<5,00 \mathrm{e}-02$ & 3.180 & 3.240 & 3.210 & 1.87 & 97.00 & 2.050 & $\mathrm{n} / \mathrm{a}$ \\
\hline $596 \mathrm{T005004}$ & $\mathrm{D}$ & Barium-ICP-Acid Dil. & ug/mL & 99.00 & $<5.00 \mathrm{e}-02$ & $<\quad 2.050$ & $<2.05 \mathrm{e} 0$ & $\mathrm{n} / \mathrm{a}$ & $\mathrm{n} / \mathrm{a}$ & 96.80 & 2.050 & $n / a$ \\
\hline$\$ 96 T 005004$ & $\mathrm{D}$ & Beryllium-ICP-Acid Dil. & $\mathrm{ug} / \mathrm{mL}$ & 101.6 & $<5.00 \mathrm{e}-03$ & $<2.05 \mathrm{e}-01$ & $<2.05 e-1$ & $n / a$ & $n / a$ & 100.0 & $2.05 e-01$ & $n / a$ \\
\hline$\$ 96 \mathrm{~T} 005004$ & D & Bismuth-ICP-Acid Dil. & $\mathrm{ug} / \mathrm{mL}$ & 101.8 & $<1.00 \mathrm{e}-01$ & $\leq \quad 4.100$ & $<4.10 \mathrm{e} 0$ & $\mathrm{n} / \mathrm{a}$ & $n / a$ & 100.0 & $\quad 4.100$ & $\mathrm{n} / \mathrm{a}$ \\
\hline$\$ 967005004$ & D & Calcium-ICP-Acid Díl. & $\mu \mathrm{g} / \mathrm{mL}$ & 100.0 & $<1.00 \mathrm{e}-01$ & $<\quad 4.100$ & $<4.10 \mathrm{e} 0$ & $n / a$ & $\mathrm{n} / \mathrm{a}$ & 108.0 & 4.100 & $\mathrm{n} / \mathrm{a}$ \\
\hline S96T005004 & D & Cadmium-ICP-Acid Dil. & $\mathrm{ug} / \mathrm{mL}$ & 99.60 & $<5.00 \mathrm{e}-03$ & $<2.05 \mathrm{e}-01$ & $<2.05 e^{-1}$ & $n / a$ & $n / a$ & 98.80 & $2.05 e^{-01}$ & $n / a$ \\
\hline $596 \mathrm{~T} 005004$ & D & Cerium-ICP-Acid Dil. & $\underline{\mathrm{ng}} / \mathrm{mL}$ & 100.0 & $<1.00 e-01$ & $<\quad 4.100$ & $<4.10 \mathrm{e} 0$ & $\mathrm{n} / \mathrm{a}$ & $\mathrm{n} / \mathrm{a}$ & 99.30 & 4.100 & $n / a$ \\
\hline S96T005004 & D & Cobalt-1CP-Acid Dil. & ug $/ \mathrm{mL}$ & 99.80 & $<2.00 \mathrm{e}-02$ & $<8.20 \mathrm{e}-01$ & $<8.20 \mathrm{e}^{-1}$ & $\mathrm{n} / \mathrm{a}$ & $\mathrm{n} / \mathrm{a}$ & 98.70 & $8.20 \mathrm{e}-01$ & $\mathrm{n} / \mathrm{a}$ \\
\hline 5961005004 & D & Chromium-ICP-Acid Dil. & $\mathrm{ug} / \mathrm{mL}$ & 100.2 & $\leq 1.00 \mathrm{e}-02$ & $6.40 \mathrm{e}-01$ & $5.73 e-01$ & $6.07 \mathrm{e}-01$ & 11.0 & 99.10 & $4.10 \mathrm{e}-01$ & $n / a$ \\
\hline $596 \mathrm{~T} 005004$ & D & Copper-ICP-Acid Dil. & $\mathrm{ug} / \mathrm{mL}$ & 103.2 & $<1.00 \mathrm{e}-02$ & $<4.10 \mathrm{e}-01$ & $<4.10 \mathrm{e}-1$ & $n / a$ & $\mathrm{n} / \mathrm{a}$ & 103.0 & $4.10 \mathrm{e}-01$ & $\mathrm{n} / \mathrm{a}$ \\
\hline$\$ 967005004$ & D & Iron-ICP-ACid Dil. & $\mathrm{ug} / \mathrm{mL}$ & 100.0 & $<5.00 e-02$ & $\leq \quad 2.050$ & $<2.05 \mathrm{e} 0$ & $\mathrm{n} / \mathrm{a}$ & $n / a$ & 98.80 & 2.050 & $\mathrm{n} / \mathrm{a}$ \\
\hline$\$ 96 \mathrm{~T} 005004$ & D & Potassium-ICP-Acid Dil. & $\mathrm{ug} / \mathrm{mL}$ & 96.00 & $<5.00 e^{-01}$ & $1.23 \mathrm{e}+03$ & $1.26 \mathrm{e}+03$ & $1.24 \mathrm{e}+03$ & 2.41 & 140.0 & 20.50 & $n / a$ \\
\hline$\$ 96 T 005004$ & $\bar{D}$ & Lanthanum-ICP-Acid Dil. & $\mathrm{ug} / \mathrm{mL}$ & 101.6 & $<5.00 e-02$ & $<\quad 2.050$ & $<2.05 \mathrm{e} 0$ & $n / a$ & $\mathrm{n} / \mathrm{a}$ & 101.0 & 2.050 & $\mathrm{n} / \mathrm{a}$ \\
\hline 5967005004 & D & Lithium-ICP-Acid Dil. & $\mathrm{ug} / \mathrm{mL}$ & 101.8 & $<1,00 \mathrm{e}-02$ & $<4.10 \mathrm{e}-01$ & $<4.10 e^{-1}$ & $n / a$ & $\mathrm{n} / \mathrm{a}$ & 103.0 & $4.10 \mathrm{e}-01$ & $\mathrm{n} / \mathrm{a}$ \\
\hline S96T005004 & D & Magnes i um-ICP-Acid DiL. & $\mathrm{ug} / \mathrm{mL}$ & 93.40 & $<1.00 \mathrm{e}-01$ & $<4.100$ & $<4.10 \mathrm{e} 0$ & $n / a$ & $\mathrm{n} / \mathrm{a}$ & 93.40 & 4.100 & $n / a$ \\
\hline$\$ 96 T 005004$ & D & Manganese-1CP-Acid Dil. & $\mathrm{ug} / \mathrm{mL}$ & 97.20 & $\leq 1.00 \mathrm{e}-02$ & $\angle 4.10 \mathrm{e}-01$ & $<4.10 \mathrm{e}^{-1}$ & $\mathrm{n} / \mathrm{a}$ & $\mathrm{n} / \mathrm{a}$ & 94.40 & $4.10 \mathrm{e}-01$ & $\mathrm{n} / \mathrm{a}$ \\
\hline$\$ 967005004$ & D & Molybdenum-ICP-Acid Dil. & ugg/mL & 100.2 & $<5.00 \mathrm{e}-02$ & $\leq \quad 2.050$ & $<2.05 \mathrm{e} 0$ & $n / a$ & $\mathrm{n} / \mathrm{a}$ & 101.0 & 2.050 & $n / a$ \\
\hline$\$ 967005004$ & $\mathrm{D}$ & Sodium-ICP-Acid Dil. & $\mathrm{ug} / \mathrm{mL}$ & 98.20 & $<1.00 \mathrm{e}-01$ & $1.26 \mathrm{e}+04$ & $1.27 \mathrm{e}+04$ & $1.26 \mathrm{e}+04$ & 0.79 & 543.0 & 4.100 & $n / a$ \\
\hline$\$ 967005004$ & $D$ & Neodymium-ICP-Acid Dil. & $\mathrm{ug} / \mathrm{mL}$ & 102.0 & $<1.00 \mathrm{e}-01$ & $\leq \quad 4.100$ & $\angle 4.10 \mathrm{e} 0$ & $\mathrm{n} / \mathrm{a}$ & $\mathrm{n} / \mathrm{a}$ & 101.0 & 4.100 & $\mathrm{n} / \mathrm{a}$ \\
\hline 5967005004 & D & Nickel-ICP-Acid Díl. & $\mathrm{ug} / \mathrm{mL}$ & 98.60 & $<2.00 \mathrm{e}-02$ & $<8.20 \mathrm{e}-01$ & $<8.20 \mathrm{e}^{-1}$ & $n / a$ & $n / a$ & 98.20 & $8.20 e^{-01}$ & $n / a$ \\
\hline 5967005004 & D & Phosphorus-ICP-Acid Dil. & $4 \mathrm{~g} / \mathrm{mL}$ & 102.2 & $<2.00 e^{-01}$ & 14.40 & 13.80 & 14.10 & 4.26 & 105.0 & 8.200 & $\mathrm{n} / \mathrm{a}$ \\
\hline 5967005004 & D & Lead-1CP-Acid Dil. & $\mathrm{ug} / \mathrm{mL}$ & 99.80 & $<1.00 \mathrm{e}-01$ & 4.100 & $<4.10 \mathrm{e} 0$ & $\mathrm{n} / \mathrm{a}$ & $n / a$ & 97.60 & 4.100 & $\mathrm{n} / \mathrm{a}$ \\
\hline 5967005004 & D & Sulfur-ICP-Acid Dil. & $\mathrm{ug} / \mathrm{mL}$ & 98.60 & $<1.00 \mathrm{e}-01$ & 46.00 & 46.50 & 46.25 & 1.08 & 99.60 & 4.100 & $n / a$ \\
\hline$\$ 96 T 005004$ & D & Ant imony-ICP-Acid Dil. & $\mathrm{ug} / \mathrm{mL}$ & 95.00 & $<6.00 \mathrm{e}-02$ & 2.460 & $<2.4600$ & $\mathrm{n} / \mathrm{a}$ & $\mathrm{n} / \mathrm{a}$ & 95.80 & 2.460 & $n / a$ \\
\hline S96T005004 & D & Selenium-ICP-ACid Dil. & $\mathrm{ug} / \mathrm{mL}$ & 95.60 & $<1.00 e-01$ & 4.100 & $<4.10 \mathrm{e} 0$ & $n / a$ & $\mathrm{n} / \mathrm{a}$ & 100.0 & 4.100 & $n / a$ \\
\hline$\$ 96 \mathrm{T005004}$ & $\bar{D}$ & Silicon-ICP-Acid Dil. & $\mathrm{ug} / \mathrm{mL}$ & 100.2 & $<5.00 \mathrm{e}-02$ & 45.60 & 46.30 & 45.95 & 1.52 & 104.0 & 2.050 & $\mathrm{n} / \mathrm{a}$ \\
\hline$\$ 96 T 005004$ & D & Samarium-ICP-Acid Dil. & $\mathrm{ug} / \mathrm{mL}$ & 98.20 & $<1.00 \mathrm{e}-01$ & $<\quad 4.100$ & $<4.10 \mathrm{e} 0$ & $n / a$ & $n / a$ & 95.50 & 4.100 & $\mathrm{n} / \mathrm{a}$ \\
\hline 5967005004 & $\bar{D}$ & stront i um-ICP-Acid Dil. & $\mathrm{ug} / \mathrm{mL}$ & 98.80 & $<1.00 \mathrm{e}-02$ & $<4.10 \mathrm{e}-01$ & $<4.10 \mathrm{e}-1$ & $\mathrm{n} / \mathrm{a}$ & $\mathrm{n} / \mathrm{a}$ & 97.50 & $4.10 \mathrm{e}-01$ & $\mathrm{n} / \mathrm{a}$ \\
\hline $596 T 005004$ & D & Titanium-ICP-Acid Dil. & $\mathrm{ug} / \mathrm{mL}$ & 98.20 & $<1.00 \mathrm{e}-02$ & $<4.10 \mathrm{e}-01$ & $<4.10 e-1$ & $\mathrm{n} / \mathrm{a}$ & $\mathrm{n} / \mathrm{a}$ & 97.70 & $4.10 \mathrm{e}-01$ & $\mathrm{n} / \mathrm{a}$ \\
\hline 5967005004 & D & Thal lium-ICP-Acid Dil. & $\mathrm{ug} / \mathrm{mL}$ & 96.20 & $<2.00 \mathrm{e}-01$ & $<\quad 8.200$ & $<8.20 \mathrm{e} 0$ & $n / a$ & $\mathrm{n} / \mathrm{a}$ & 94.10 & 8.200 & $n / a$ \\
\hline $596 T 005004$ & D & Uranium-ICP-Acid Dil. & $\mathrm{ug} / \mathrm{mL}$ & 96.20 & $<5.00 \mathrm{e}-01$ & 20.50 & $<2.05 \mathrm{e} 1$ & $n / a$ & $n / a$ & 113.5 & 20.50 & $n / a$ \\
\hline 5961005004 & D & Vanadium-ICP-Acid DilL. & $\mathrm{ug} / \mathrm{mL}$ & 99.60 & $<5.00 e-02$ & 2.050 & $<2.05 \mathrm{e}^{0}$ & $n / a$ & $\mathrm{n} / \mathrm{a}$ & 98.60 & 2.050 & $\mathrm{n} / \mathrm{a}$ \\
\hline$\$ 96 \mathrm{~T} 005004$ & D & Zinc-1CP-Acid Dil. & $\mathrm{ug} / \mathrm{mL}$ & 100.6 & $<1.00 \mathrm{e}-02$ & 1.290 & 1.280 & 1.285 & 0.78 & 100.0 & $4.10 \mathrm{e}-01$ & $n / a$ \\
\hline$\$ 967005004$ & ID & Zirconium-ICP-Acid Dil. & $\mathrm{ug} / \mathrm{mL}$ & 98.80 & $<1.00 \mathrm{e}-02$ & $<4.10 \mathrm{e}-01$ & $<4.10 e^{-1}$ & $\mathrm{n} / \mathrm{a}$ & $\mathrm{n} / \mathrm{a}$ & 97.50 & $4.10 \mathrm{e}-01$ & $n / a$ \\
\hline
\end{tabular}




\begin{tabular}{|c|c|c|c|c|c|c|c|c|c|c|c|c|}
\hline Sample\# & R|A\# & Analyte & Unit & Standard \% & Blank & Result & Duplicate & Average & RPD \% & Spk Rec \% & Det Limit & Count Err\% \\
\hline 5967005004 & & Fluoride-IC-Dionex $4000 / 4500$ & $\mathrm{ug} / \mathrm{mL}$ & 103.9 & $<1.30 \mathrm{e}-02$ & $1.59 \mathrm{e}+02$ & 127.0 & 142.9 & 22.4 & 83.39 & 7.878 & $\mathrm{n} / \mathrm{a}$ \\
\hline 5967005004 & & Chloride-IC-Dionex $4000 / 4500$ & $\mathrm{ug} / \mathrm{mL}$ & 103.8 & $<1.70 \mathrm{e}-02$ & $2.22 \mathrm{e}+02$ & 230.0 & 225.8 & 3.54 & 102.2 & 10.30 & $n / a$ \\
\hline S96T005004 & & Nitrite-IC - Dionex $4000 / 4500$ & $\mathrm{ug} / \mathrm{mL}$ & 100.4 & $<1.07 \mathrm{e}-01$ & $1.09 \mathrm{e}+03$ & $1.12 \mathrm{e}+03$ & $1.10 \mathrm{e}+03$ & 2.71 & 101.5 & 64.84 & $n / a$ \\
\hline 5967005004 & & Bromide by Ion Chromatograph & Ug/mL & 104.8 & $<1.26 e^{-01}$ & $<76.36$ & $<7.64 \mathrm{e} 1$ & $n / a$ & $\mathrm{n} / \mathrm{a}$ & 104.6 & 76.36 & $\mathrm{n} / \mathrm{a}$ \\
\hline S96TO & & Nitrate by IC-Dionex $4000 / 4500$ & $\mathrm{ug} / \mathrm{mL}$ & 104.2 & $<1.40 \mathrm{e}-01$ & $2.39 \mathrm{e}+04$ & $2.41 \mathrm{e}+04$ & $2.40 e+04$ & 0.83 & 102.4 & 84.84 & $\mathrm{n} / \mathrm{a}$ \\
\hline S96TOd & & Phosphate-IC-Dionex $4000 / 4500$ & $\mathrm{ug} / \mathrm{mL}$ & 100.5 & $<1.19 \mathrm{e}-01$ & $<72.05$ & $<7.21 \mathrm{e} 1$ & $n / a$ & $n / a$ & 100.6 & 72.05 & $\mathrm{n} / \mathrm{a}$ \\
\hline$\$ 967005004$ & & Sulfate by IC-Dionex $4000 / 4500$ & ug/mL & 103.0 & $<1.36 e^{-01}$ & $1.67 e+02$ & 166.0 & 166.3 & 0.60 & 102.2 & 82.36 & $\mathrm{n} / \mathrm{a}$ \\
\hline 596T0 & & Oxalate by IC-D jonex $4000 / 4500$ & $\mathrm{ug} / \mathrm{mL}$ & 101.9 & $<1.05 \mathrm{e}-01$ & $<63.63$ & $<6.36 \mathrm{e} 1$ & $n / a$ & n/a & 104.2 & 63.63 & $\mathrm{n} / \mathrm{a}$ \\
\hline S961 & & Strontium-89/90 High Level & $\mathrm{uCi} / \mathrm{mL}$ & 110.0 & $2.51 e^{-05}$ & $2.11 \mathrm{e}-02$ & $2.01 \mathrm{e}-02$ & $2.06 \mathrm{e}-02$ & 4.85 & $n / a$ & $8.37 e-05$ & $1.95 \mathrm{E}+00$ \\
\hline $596 \mathrm{~T}$ & & PU-239/240 by TRU-SPEC Resin & $\mathrm{uCi} / \mathrm{mL}$ & 101.3 & $<3.72 \mathrm{e}-06$ & $<3.73 e-06$ & $<3.75 \bar{E}-6$ & $\mathrm{n} / \mathrm{a}$ & $n / a$ & $\mathrm{n} / \mathrm{a}$ & $3.73 \mathrm{e}-06$ & $1.00 \mathrm{E}+02$ \\
\hline S96T005007 & & Cobalt -60 by GEA & $\mathrm{UCi} / \mathrm{mL}$ & 99.32 & $<2.39 \mathrm{e}-05$ & $1.08 \mathrm{e}-04$ & $1.07 e^{-04}$ & $1.07 \mathrm{e}-04$ & 0.93 & $\mathrm{n} / \mathrm{a}$ & $\mathrm{n} / \mathrm{a}$ & 37.6 \\
\hline S96T005007 & & Cesium- 137 by GEA & $\mathrm{UCi} / \mathrm{mL}$ & 102.3 & $<2.89 \mathrm{e}-05$ & $7.39 \mathrm{e}-01$ & $7.36 \mathrm{e}-01$ & $7.38 \mathrm{e}-01$ & 0.41 & $\mathrm{n} / \mathrm{a}$ & $n / a$ & 0.250 \\
\hline S96T & & Am-241 by Extraction & $\mathrm{uCi} / \mathrm{mL}$ & 84.87 & $<5.57 \mathrm{e}-06$ & $<5.27 e-06$ & $<6.08 \mathrm{E}-6$ & $n / a$ & $n / a$ & $n / a$ & $5.27 e-06$ & $1.00 \mathrm{E}+02$ \\
\hline 5961005007 & & Alpha in Liquid Samples & $\mathrm{uCi} / \mathrm{mL}$ & 88.75 & $<5.40 \mathrm{e}-05$ & $<6.59 \mathrm{e}-05$ & $<4.09 E-5$ & $\mathrm{n} / \mathrm{a}$ & $\mathrm{n} / \mathrm{a}$ & 74.65 & $9.99 e^{-05}$ & $5.00 \mathrm{E}+02$ \\
\hline
\end{tabular}


RISER: 1OA

SEGMENT \#: 5AW-96-2

SEGMENT PORTION: Supernate

\begin{tabular}{|c|c|c|c|c|c|c|c|c|c|c|c|c|}
\hline Sample\# & $\mathrm{R}$ A\# & Analyte & Unit & Standard \% & Blank & Result & Duplicate & Average & RPD \% & Spk Rec \% & Det Limit & Count Err\% \\
\hline$\$ 96 T 005005$ & & DSC Exotherm using Mettler & Joules/g & 100.2 & $\mathrm{n} / \mathrm{a}$ & $0.00 \mathrm{e}+00$ & $0.00 \mathrm{e}+00$ & $0.00 \mathrm{e}+00$ & 0.00 & $n / a$ & $\mathrm{n} / \mathrm{a}$ & $n / a$ \\
\hline S96T005005 & & pH Direct & $\mathrm{pH}$ & 99.86 & $\mathrm{n} / \mathrm{a}$ & 13.43 & 13.44 & 13.43 & 0.07 & $n / a$ & $1.00 \mathrm{e}-02$ & $\mathrm{n} / \mathrm{a}$ \\
\hline$\$ 967005005$ & & $\%$ Water by TGA using Mettler & $\%$ & 98.95 & $n / a$ & 95.67 & 95.63 & 95.65 & 0.04 & $n / a$ & $\mathrm{n} / \mathrm{a}$ & $n / a$ \\
\hline S96T005005 & $\mathrm{D}$ & Silver-ICP-Acid Dil. & $\mathrm{ug} / \mathrm{mL}$ & 99.20 & $<1.00 \mathrm{e}-02$ & 1.070 & 1.120 & 1.095 & 4.57 & 83.80 & $4.10 e^{-01}$ & $n / a$ \\
\hline S96T005005 & D & Aluminium-1CP-Acid Dil. & $\mathrm{ug} / \mathrm{mL}$ & 98.00 & $<5.00 \mathrm{e}-02$ & 16.50 & 16.90 & 16.70 & 2.40 & 97.90 & 2.050 & $n / a$ \\
\hline S96T005005 & D & Arsenic-ICP-Acid Dil. & $\mathrm{ug} / \mathrm{mL}$ & 102.4 & $<1.00 \mathrm{e}-01$ & 4.100 & $<4.10 \mathrm{e} 0$ & $n / a$ & $\dot{n} / \mathrm{a}$ & 105.0 & 4.100 & $n / a$ \\
\hline \$96T005005 & $\bar{D}$ & Boron-ICP-Acid Dil. & $\mathrm{ug} / \mathrm{mL}$ & 99.20 & $<5.00 \mathrm{e}-02$ & 5.440 & 5.460 & 5.450 & 0.37 & 96.80 & 2.050 & $\mathrm{n} / \mathrm{a}$ \\
\hline S96T005005 & D & Barium-ICP-Acid Dil. & $\mathrm{ug} / \mathrm{mL}$ & 99.00 & $<5.00 \mathrm{e}-02$ & 2.050 & $<2.05 \mathrm{e} 0$ & $n / a$ & $n / a$ & 98.10 & 2.050 & $\mathrm{n} / \mathrm{a}$ \\
\hline S96T005005 & $D$ & Beryllium-ICP-Acid Dil. & $\mathrm{ug} / \mathrm{mL}$ & 101.6 & $<5.00 e-03$ & $<2.05 \mathrm{e}-01$ & $<2.05 \mathrm{e}-1$ & $\mathrm{n} / \mathrm{a}$ & $n / a$ & 100.0 & $2.05 e-01$ & $n / a$ \\
\hline 5967005005 & D & Bismuth-ICP-Acid Dil. & $\mathrm{ug} / \mathrm{mL}$ & 101.8 & $<1.00 \mathrm{e}-01$ & $<\quad 4.100$ & $<4.10 \mathrm{e} 0$ & $n / a$ & $\mathrm{n} / \mathrm{a}$ & 101.0 & 4.100 & $\mathrm{n} / \mathrm{a}$ \\
\hline$\$ 967005005$ & D & Calcium-ICP-Acid Dil. & $\mathrm{ug} / \mathrm{mL}$ & 100.0 & $<1.00 \mathrm{e}-01$ & 6.180 & 6.230 & 6.205 & 0.81 & 101.0 & 4.100 & $n / a$ \\
\hline 5967005005 & D & Cadmium-ICP-ACid Dil. & $\underline{u g} / \mathrm{mL}$ & 99.60 & $<5.00 e-03$ & $<2.05 e^{-01}$ & $<2.05 \mathrm{e}-1$ & $n / a$ & $\mathrm{n} / \mathrm{a}$ & 100.0 & $2.05 e-01$ & $n / a$ \\
\hline S96T005005 & D & Cerium-ICP-Acid Dil, & $\mathrm{ug} / \mathrm{mL}$ & 100.0 & $<1.00 \mathrm{e}-01$ & $<\quad 4.100$ & $<4.10 \mathrm{e} 0$ & $n / a$ & $n / a$ & 100.0 & 4.100 & $\mathrm{n} / \mathrm{a}$ \\
\hline S96T005005 & D & Cobalt-ICP-Acid Dil. & $\mathrm{ug} / \mathrm{mL}$ & 99.80 & $<2.00 \mathrm{e}-02$ & $<8.20 e^{-01}$ & $<8.20 \mathrm{e}-1$ & $n / a$ & $n / a$ & 99.80 & $8.20 \mathrm{e}-01$ & $n / a$ \\
\hline S96T005005 & D & Chromjum-ICP-Acid Dil. & $\mathrm{ug} / \mathrm{mL}$ & 100.2 & $<1.00 \mathrm{e}-02$ & $6.15 e-01$ & $6.31 \mathrm{e}-01$ & $6.23 \mathrm{e}^{-01}$ & 2.57 & 100.0 & $4.10 e-01$ & $n / a$ \\
\hline S96T005005 & $\bar{D}$ & Copper-ICP-Acid Dil. & $\mathrm{ug} / \mathrm{mL}$ & 103.2 & $<1.00 \mathrm{e}-02$ & $<4.10 \mathrm{e}-01$ & $<4.10 \mathrm{e}-1$ & $\mathrm{n} / \mathrm{a}$ & $n / a$ & 102.0 & $4.10 \mathrm{e}-01$ & $\mathrm{n} / \mathrm{a}$ \\
\hline S96T005005 & D & Iron-ICP-Acid Dil, & ug/mL & 100.0 & $<5.00 \mathrm{e}-02$ & $<\quad 2.050$ & $<2.05 \mathrm{e} 0$ & $\mathrm{n} / \mathrm{a}$ & $\mathrm{n} / \mathrm{a}$ & 99.60 & 2.050 & $n / a$ \\
\hline S96T005005 & D & Potassium-ICP-Acid Dil. & $\mathrm{ug} / \mathrm{mL}$ & 96.00 & $<5.00 \mathrm{e}-01$ & $1.27 \mathrm{e}+03$ & $1.29 \mathrm{e}+03$ & $1.28 \mathrm{e}+03$ & 1.56 & 155.0 & 20.50 & $n / a$ \\
\hline 5967005005 & D & Lanthanum-ICP-Acid Dil. & $\mathrm{ug} / \mathrm{mL}$ & 101.6 & $<5.00 \mathrm{e}-02$ & $<2.050$ & $<2.05 \mathrm{e} 0$ & $n / a$ & $\mathrm{n} / \mathrm{a}$ & 101.0 & 2.050 & $n / a$ \\
\hline S96T005005 & D & Lithium-ICP-Acid Dil. & $\underline{\mathrm{ug}} / \mathrm{mL}$ & 101.8 & $<1.00 \mathrm{e}-02$ & $<4.10 \mathrm{e}-01$ & $<4.10 \mathrm{e}-1$ & $n / a$ & $\mathrm{n} / \mathrm{a}$ & 102.0 & $4.10 e^{-01}$ & $n / a$ \\
\hline S96T005005 & D & Magnes ium-ICP-Acid Dil. & $\mathrm{ug} / \mathrm{mL}$ & 93.40 & $<1.00 \mathrm{e}-01$ & $<\quad 4.100$ & $<4.10 \mathrm{e} 0$ & $\mathrm{n} / \mathrm{a}$ & $n / a$ & 93.70 & 4.100 & $\mathrm{n} / \mathrm{a}$ \\
\hline$\$ 96 T 005005$ & D & Manganese-ICP-Acid Dil. & $\mathrm{ug} / \mathrm{mL}$ & 97.20 & $<1.00 \mathrm{e}^{-02}$ & $<4.10 e^{-01}$ & $<4.10 e-1$ & $n / a$ & $\mathrm{n} / \mathrm{a}$ & 95.50 & $4.10 \mathrm{e}-01$ & $\mathrm{n} / \mathrm{a}$ \\
\hline S96T005005 & $\bar{D}$ & Molybdenum-ICP-Acid Dil. & $\mathrm{ug} / \mathrm{mL}$ & 100.2 & $<5.00 e-02$ & $<2.050$ & $<2.05 \mathrm{e} 0$ & $n / a$ & $\mathrm{n} / \mathrm{a}$ & 102.0 & 2.050 & $n / a$ \\
\hline S96T005005 & $\bar{D}$ & Sodi um-ICP-Acid Dil. & $\mathrm{ug} / \mathrm{mL}$ & 98.20 & $<1.00 \mathrm{e}-01$ & $1.27 e+04$ & $1.29 \mathrm{e}+04$ & $1.28 \mathrm{e}+04$ & 1.56 & 310.0 & 4.100 & $n / a$ \\
\hline S96T005005 & D & Neodymium-ICP-Acid Dil. & $\mathrm{ug} / \mathrm{mL}$ & 102.0 & $<1.00 \mathrm{e}-01$ & $<4.100$ & $<4.10 \mathrm{e} 0$ & $\mathrm{n} / \mathrm{a}$ & $\mathrm{n} / \mathrm{a}$ & 101.0 & 4.100 & $n / a$ \\
\hline$\$ 967005005$ & $\bar{D}$ & Nickel-1CP-Acid Dil. & $\mathrm{ug} / \mathrm{mL}$ & 98.60 & $<2.00 \mathrm{e}-02$ & $<8.20 \mathrm{e}-01$ & $<8.20 e^{-1}$ & $n / a$ & $\mathrm{n} / \mathrm{a}$ & 98.40 & $8.20 e-01$ & $\mathrm{n} / \mathrm{a}$ \\
\hline S96T005005 & D & Phosphorus-ICP-Acid Dil. & $\mathrm{ug} / \mathrm{mL}$ & 102.2 & $<2.00 \mathrm{e}-01$ & 13.40 & 13.40 & 13.40 & 0.00 & 105.0 & 8.200 & $\mathrm{n} / \mathrm{a}$ \\
\hline S96T005005 & D & Lead-ICP-Acid Dil. & $\mathrm{ug} / \mathrm{mL}$ & 99.80 & $<1.00 e-01$ & 4.100 & $<4.10 \mathrm{e} 0$ & $n / a$ & $\mathrm{n} / \mathrm{a}$ & 101.0 & 4.100 & $\mathrm{n} / \mathrm{a}$ \\
\hline S96T005005 & D & Sulfur-ICP-Acid Dil. & $\mathrm{ug} / \mathrm{mL}$ & 98.60 & $<1.00 \mathrm{e}-01$ & 45.90 & 46.00 & 45.95 & 0.22 & 103.0 & 4.100 & $n / a$ \\
\hline S96T005005 & D & Antimony-ICP-Acid Dil. & $\mathrm{ug} / \mathrm{mL}$ & 95.00 & $<6.00 e-02$ & 2.460 & $<2.46 \mathrm{e} 0$ & $n / a$ & $n / a$ & 95.80 & 2.460 & $n / a$ \\
\hline S96T005005 & D & Seleni um-ICP-Acid Dil. & $\mathrm{ug} / \mathrm{mL}$ & 95.60 & $<1.00 \mathrm{e}-01$ & 4.100 & $<4.10 \mathrm{e} 0$ & $n / a$ & $\mathrm{n} / \mathrm{a}$ & 98.60 & 4.100 & $\mathrm{n} / \mathrm{a}$ \\
\hline S96T005005 & D & Silicon-ICP-Acid Dil. & $\mathrm{ug} / \mathrm{mL}$ & 100.2 & $<5.00 \mathrm{e}-02$ & 51.30 & 51.50 & 51.40 & 0.39 & 104.0 & 2.050 & $\mathrm{n} / \mathrm{a}$ \\
\hline S961005005 & D & Samarium-ICP-Acid Dil. & $\mathrm{ug} / \mathrm{mL}$ & 98.20 & $<1.00 \mathrm{e}-01$ & $<4.100$ & $<4.10 \mathrm{e} 0$ & $n / a$ & $\mathrm{n} / \mathrm{a}$ & 97.00 & 4.100 & $\mathrm{n} / \mathrm{a}$ \\
\hline S96T005005 & D & Strontium-ICP-Acid Dil. & $\mathrm{ug} / \mathrm{mL}$ & 98.80 & $<1.00 \mathrm{e}-02$ & $<4.10 \mathrm{e}-01$ & $<4.10 \mathrm{e}-1$ & $n / a$ & $\mathrm{n} / \mathrm{a}$ & 98.20 & $4.10 \mathrm{e}-01$ & $n / a$ \\
\hline 5961005005 & D & Titanium-ICP-Acid Dil. & $\mathrm{ug} / \mathrm{mL}$ & 98.20 & $<1.00 \mathrm{e}-02$ & $<4.10 e^{-01}$ & $<4.10 e^{-1}$ & $\mathrm{n} / \mathrm{a}$ & $n / a$ & 98.60 & $4.10 e-01$ & $\mathrm{n} / \mathrm{a}$ \\
\hline S96T005005 & $\overline{\mathbf{D}}$ & Thall ium-ICP-Acid Dil. & $\mathrm{ug} / \mathrm{mL}$ & 96.20 & $<2.00 e-01$ & $<8.200$ & $<8.20 \mathrm{e} 0$ & $\mathrm{n} / \mathrm{a}$ & $\mathrm{n} / \mathrm{a}$ & 94.10 & 8.200 & $\mathrm{n} / \mathrm{a}$ \\
\hline$\$ 9670$ & D & Uranium-ICP-Acid Dil. & ug/mL & 96.20 & $<5.00 e-01$ & 20.50 & $<2.05 \mathrm{e} 1$ & $n / a$ & $\mathrm{n} / \mathrm{a}$ & 116.0 & 20.50 & $\mathrm{n} / \mathrm{a}$ \\
\hline S96TO & D & Vanadium-ICP-Acid Dil. & $\mathrm{ug} / \mathrm{mL}$ & 99.60 & $<5.00 e^{-02}$ & 2.050 & $<2.05 \mathrm{e} 0$ & $n / a$ & $\mathrm{n} / \mathrm{a}$ & 99.60 & 2.050 & $\mathrm{n} / \mathrm{a}$ \\
\hline S96T005005 & D & Zinc-ICP-Acid Dil. & ug/mL & 100.6 & $<1.00 \mathrm{e}-02$ & 1.570 & 1.580 & 1.575 & 0.63 & 101.0 & $4.10 \mathrm{e}-01$ & $n / a$ \\
\hline S96T005005 & D & Zirconfum-ICP-Acid Dil. & $\mathrm{ug} / \mathrm{mL}$ & 98.80 & $<1.00 \mathrm{e}-02$ & $<4.10 \mathrm{e}-01$ & $<4.10 \mathrm{e}^{-1}$ & $\mathrm{n} / \mathrm{a}$ & $\mathrm{n} / \mathrm{a}$ & 98.50 & $4.10 \mathrm{e}-01$ & $n / a$ \\
\hline$\$ 967005008$ & & OH- by Pot. Titration & $\mathrm{ug} / \mathrm{mL}$ & 95.55 & $<43.00$ & $3.68 \mathrm{e}+03$ & $3.89 e+03$ & $3.78 \mathrm{e}+03$ & 5.55 & $n / a$ & 125.0 & $\mathrm{n} / \mathrm{a}$ \\
\hline S96T005008 & & Specific Gravity & Sp:G. & 101.4 & $n / a$ & 1.019 & 1.020 & 1.019 & 0.10 & $n / a$ & $1.00 e-03$ & $\mathrm{n} / \mathrm{a}$ \\
\hline S96T005008 & & TIC by Acid/Coulometry & $\mathrm{ug} / \mathrm{mL}$ & 97.00 & 5.400 & $1.87 \mathrm{e}+02$ & 190.0 & 188.5 & 1.59 & $\mathrm{n} / \mathrm{a}$ & 5.000 & $n / a$ \\
\hline 5967005008 & & Tot. Organic Carbon by coul. & $\mathrm{ug} / \mathrm{mL}$ & 90.33 & $4.00 \mathrm{e}-01$ & 97.90 & 102.0 & 99.95 & 4.10 & 97.70 & 5.500 & $\mathrm{n} / \mathrm{a}$ \\
\hline
\end{tabular}




\begin{tabular}{|c|c|c|c|c|c|c|c|c|c|c|c|c|}
\hline Samole\# & A\# & Analyte & Unit & Standard \% & Blank & Result & Duplicate & Average & RPD \% & Spk Rec \% & Det Limit & Count Err\% \\
\hline$\$ 9610050081$ & & Tot. Organic Carbon by coul. & $\mathrm{ug} / \mathrm{mL}$ & 98.67 & $6.00 e-01$ & $1.08 \mathrm{e}+02$ & 99.60 & 103.8 & 8.09 & 85.50 & 5.500 & $n / a$ \\
\hline $596 \mathrm{~T} 005008$ & & Strontium- $89 / 90$ High Level & $\mathrm{UCi} / \mathrm{mL}$ & 110.0 & $2.51 \mathrm{e}-05$ & $3.02 \mathrm{e}-02$ & $3.12 e-02$ & $3.07 \mathrm{e}-02$ & 3.26 & $n / a$ & $5.81 e-05$ & $1.35 E+00$ \\
\hline 5967005008 & & PU-239/240 by TRU-SPEC Resin & $\mathrm{uCi} / \mathrm{mL}$ & 101.3 & $<3.72 \mathrm{e}-06$ & $\angle 4.00 e^{-06}$ & $<3.60 \mathrm{E}-6$ & $n / a$ & $n / a$ & $n / a$ & $4.00 e-06$ & $1.00 \mathrm{E}+02$ \\
\hline 5961005008 & & Fluoride-1C-Dionex $4000 / 4500$ & ug $/ \mathrm{mL}$ & 101.7 & $<1.20 \mathrm{e}-02$ & $1.55 \mathrm{e}+02$ & 154.0 & 154.3 & 0.65 & 96.95 & 13.33 & $n / a$ \\
\hline 5967005008 & & Chloride-IC-Dionex $4000 / 4500$ & ug $/ \mathrm{mL}$ & 96.20 & $<1.70 \mathrm{e}-02$ & $2.62 \mathrm{e}+02$ & 231.0 & 246.6 & 12.6 & 91.65 & 18.89 & $\mathrm{n} / \mathrm{a}$ \\
\hline$\$ 96 \mathrm{~T} 005008$ & & Nitrite-IC - Dionex $4000 / 4500$ & $\mathrm{ug} / \mathrm{mL}$ & 98.70 & $<1.08 \mathrm{e}-01$ & $1.20 \mathrm{e}+03$ & $1.13 \mathrm{e}+03$ & $1.17 \mathrm{e}+03$ & 6.01 & 97.41 & 120.0 & $n / a$ \\
\hline 5967005008 & & Bromide by Ion Chromatograph & $\mathrm{ug} / \mathrm{mL}$ & 101.7 & $<1.25 \mathrm{e}-01$ & $<1.39 e+02$ & $<1.39 \mathrm{e} 2$ & $n / a$ & $n / a$ & 106.1 & 138.9 & $n / a$ \\
\hline S96T005008 & & Nitrate by IC-Dionex $4000 / 4500$ & $\mathrm{ug} / \mathrm{mL}$ & 103.9 & $2.73 \mathrm{e}-01$ & $2.43 e+04$ & $2.46 \mathrm{e}+04$ & $2.45 \mathrm{e}+04$ & 1.23 & 128.3 & 154.4 & $\mathrm{n} / \mathrm{a}$ \\
\hline S96T005008 & & Phosphate-IC-Dionex $4000 / 4500$ & $\mathrm{ug} / \mathrm{mL}$ & 101.3 & $<1.20 \mathrm{e}-01$ & $<1.33 \mathrm{e}+02$ & $<1.33 \mathrm{e} 2$ & $n / a$ & $\mathrm{n} / \mathrm{a}$ & 103.1 & 133.3 & $\mathrm{n} / \mathrm{a}$ \\
\hline S96T005008 & & Sulfate by IC-Dionex $4000 / 4500$ & $u g / m L$ & 101.4 & $<1.38 \mathrm{e}-01$ & $2.30 \mathrm{e}+02$ & 225.0 & 227.5 & 2.20 & 103.0 & 153.31 & $n / a$ \\
\hline S96T005008 & & Oxalate by IC-Dionex $4000 / 4500$ & ug $/ \mathrm{mL}$ & 103.6 & $<1.05 e-01$ & $<1.17 \mathrm{e}+02$ & $<1.17 \mathrm{e} 2$ & $n / a$ & $\mathrm{n} / \mathrm{a}$ & 107.2 & 116.7 & $\mathrm{n} / \mathrm{a}$ \\
\hline \$96T005008 & & Cobal $t-60$ by GEA & $\mathrm{uCi} / \mathrm{mL}$ & 99.32 & $<2.39 e-05$ & $1.06 e^{-04}$ & $9.63 e-05$ & $1.01 \mathrm{e}-04$ & 9.59 & $\mathrm{n} / \mathrm{a}$ & $\mathrm{n} / \mathrm{a}$ & 28.9 \\
\hline S96T005008 & & Cesiunn- 137 by GEA & UCi/mL & 102.3 & $<2.89 e-05$ & $7.55 e-01$ & $7.44 \mathrm{e}-01$ & $7.50 \mathrm{e}-01$ & 1.47 & $\mathrm{n} / \mathrm{a}$ & $\mathrm{n} / \mathrm{a}$ & 0.250 \\
\hline $596 \mathrm{~T} 005008$ & & Am-241 by Extraction & $\mathrm{uCi} / \mathrm{mL}$ & 84.87 & $<5.57 e-06$ & $<7.94 \mathrm{e}-06$ & $<6.75 \mathrm{E}-6$ & $n / a$ & $\mathrm{n} / \mathrm{a}$ & $\mathrm{n} / \mathrm{a}$ & $7.94 \mathrm{e}^{-06}$ & $1.00 \mathrm{E}+02$ \\
\hline S961005008 & & Alpha in Liquid samples & $\mathrm{UCi} / \mathrm{mL}$ & 88.75 & $<5.40 \mathrm{e}-05$ & $<8.18 e-05$ & $\angle 4.09 E-5$ & $\mathrm{n} / \mathrm{a}$ & $\mathrm{n} / \mathrm{a}$ & $\mathrm{n} / \mathrm{a}$ & $9.99 e^{-05}$ & $5.00 E+02$ \\
\hline
\end{tabular}


RISER: $10 \mathrm{~A}$

SEGNENT \#: 5AW-96-4

SEGMENT PORTION: Supernate

\begin{tabular}{|c|c|c|c|c|c|c|c|c|c|c|c|c|}
\hline Sample\# & R A\# & \# Analyte & Unit & Standard \% & Blank & Result & Duplicate & Average & RPD \% & Spk Rec \% & Det Limit & Count Err\% \\
\hline $596 T 005006$ & & DSC Exotherm on Perkin Elmer & Joules/g & 95.89 & $\mathrm{n} / \mathrm{a}$ & $0.00 \mathrm{e}+00$ & $0.00 \mathrm{e}+00$ & $0.00 \mathrm{e}+00$ & 0.00 & $n / a$ & $\mathrm{n} / \mathrm{a}$ & $n / a$ \\
\hline $596 \mathrm{T005006}$ & & OH- by Pot. Titration & $\mathrm{ug} / \mathrm{mL}$ & 98.56 & $<42.00$ & $3.27 \mathrm{e}+03$ & $3.19 \mathrm{e}+03$ & $3.23 e+03$ & 2.48 & $\mathrm{n} / \mathrm{a}$ & 625.0 & $n / a$ \\
\hline$\$ 967005006$ & & ph Direct & $\mathrm{pH}$ & 99.86 & $n / a$ & 13.47 & 13.48 & 13.48 & 0.07 & $\mathrm{n} / \mathrm{a}$ & $1.00 \mathrm{e}-02$ & $n / a$ \\
\hline $596 \mathrm{~T} 005006$ & & Specific Gravity & Sp.6. & 99.17 & $\mathrm{n} / \mathrm{a}$ & 1.012 & 1.010 & 1.011 & 0.20 & $n / a$ & $1.00 \mathrm{e}-03$ & $\mathrm{n} / \mathrm{a}$ \\
\hline $596 \mathrm{~T} 005006$ & & \% Water by TGA on Perkin Elmer & $\%$ & 99.11 & $\mathrm{n} / \mathrm{a}$ & 94.61 & 94.53 & 94.57 & 0.08 & $n / a$ & $n / a$ & $\mathrm{n} / \mathrm{a}$ \\
\hline$\$ 961005006$ & & TIC by Acid/Coul ometry & $\mathrm{ug} / \mathrm{mL}$ & 97.00 & 5.400 & $1.78 \mathrm{e}+02$ & .193 .0 & 185.5 & 8.09 & $\mathrm{n} / \mathrm{a}$ & 5.000 & $n / a$ \\
\hline $596 \mathrm{~T} 005006$ & & Tot. Organic Carbon by coul. & $\mathrm{ug} / \mathrm{mL}$ & 98.00 & 5.000 & $4.59 \mathrm{e}+03$ & $5.08 \mathrm{e}+03$ & $4.84 \mathrm{e}+03$ & $10 . \overline{1}$ & $\mathrm{n} / \mathrm{a}$ & 55.00 & $n / a$ \\
\hline S96T005006 & $\mathrm{D}$ & Silver-ICP-Acid Dil. & $\mathrm{ug} / \mathrm{mL}$ & 99.20 & $<1.00 \mathrm{e}-02$ & 1.140 & 1.060 & 1.100 & 7.27 & 83.20 & $4.10 \mathrm{e}-01$ & $\mathrm{n} / \mathrm{a}$ \\
\hline$\$ 961005006$ & $D$ & Aluminium-ICP-Acid Dil. & $\mathrm{ug} / \mathrm{mL}$ & 98.00 & $<5.00 \mathrm{e}-02$ & 17.40 & 17.00 & 17.20 & 2.33 & 97.10 & 2.050 & $\mathrm{n} / \mathrm{a}$ \\
\hline $596 \mathrm{~T} 005006$ & D & Arsenic-ICP-Acid Dil. & $\mathrm{ug} / \mathrm{mL}$ & 102.4 & $<1.00 \mathrm{e}-01$ & $\leq 4.100$ & $<4.10 \mathrm{e} 0$ & $n / a$ & $\mathrm{n} / \mathrm{a}$ & 104.0 & 4.100 & $\mathrm{n} / \mathrm{a}$ \\
\hline 5967005006 & D & Boron-ICP-Acid Dil. & $\mathrm{ug} / \mathrm{mL}$ & 99.20 & $<5.00 \mathrm{e}-02$ & 2.980 & 2.860 & 2.920 & 4.11 & 96.80 & 2.050 & $\mathrm{n} / \mathrm{a}$ \\
\hline $596 T 005006$ & D & Barium-ICP-Acid Dil. & $\mathrm{ug} / \mathrm{mL}$ & 99.00 & $<5.00 e-02$ & $\leq 2.050$ & $<2.05 \mathrm{e} 0$ & $n / a$ & $n / a$ & 97.50 & 2.050 & $\mathrm{n} / \mathrm{a}$ \\
\hline$\$ 967005006$ & D & Beryllium-ICP-Acid Dil. & $\underline{\mathrm{ug}} / \mathrm{mL}$ & 101.6 & $<5.00 \mathrm{e}-03$ & $<2.05 e^{-01}$ & $<2.05 e-1$ & $\mathrm{n} / \mathrm{a}$ & $n / a$ & 101.0 & $2.05 e-01$ & $\mathrm{n} / \mathrm{a}$ \\
\hline 5961005006 & D & B ismuth-icP-Acid Dil. & $\mathrm{ug} / \mathrm{mL}$ & 101.8 & $<1.00 \mathrm{e}-01$ & $<\quad 4.100$ & $<4.10 \mathrm{e} 0$ & $\mathrm{n} / \mathrm{a}$ & $\mathrm{n} / \mathrm{a}$ & 100.0 & 4.100 & $n / a$ \\
\hline 5961005006 & D & Calcium-ICP-Acid Dil. & $\mathrm{ug} / \mathrm{mL}$ & 100.0 & $<1.00 \mathrm{e}-01$ & $\leq \quad 4.100$ & $<4.10 \mathrm{e} 0$ & $n / a$ & $n / a$ & 109.0 & 4.100 & $\mathrm{n} / \mathrm{a}$ \\
\hline 5967005006 & D & Cadmium-ICP-Acid Dil. & $\mathrm{ug} / \mathrm{mL}$ & 99.60 & $<5.00 e-03$ & $<2.05 e-01$ & $<2.05 e^{-1}$ & $n / a$ & $\mathrm{n} / \mathrm{a}$ & 100.0 & $2.05 e-01$ & n/a \\
\hline S96T005006 & ID & Cerium-ICP-Acid Dil. & $\mathrm{ug} / \mathrm{mL}$ & 100.0 & $<1.00 \mathrm{e}-01$ & $\leq \quad 4.100$ & $<4.10 \mathrm{e} 0$ & $\mathrm{n} / \mathrm{a}$ & $n / a$ & 99.40 & 4.100 & $\mathrm{n} / \mathrm{a}$ \\
\hline $596 T 005006$ & $D$ & Cobalt-ICP-Acid Dil. & ug $/ \mathrm{mL}$ & 99.80 & $\leq 2.00 \mathrm{e}-02$ & $<8.20 e^{-01}$ & $<8.20 \mathrm{e}-1$ & $n / a$ & $\mathrm{n} / \mathrm{a}$ & 99.20 & $8.20 e-01$ & $\mathrm{n} / \mathrm{a}$ \\
\hline S96T005006 & D & Chromium-ICP-Acid Dil. & $\mathrm{ug} / \mathrm{mL}$ & 100.2 & $<1.00 e+02$ & $6.51 \mathrm{e}-01$ & $6.52 \mathrm{e}-01$ & $6.51 e-01$ & 0.15 & 100.0 & $4.10 \mathrm{e}-01$ & $n / a$ \\
\hline S96T005006 & D & Copper-ICP-Acid Dil. & $\mathrm{ug} / \mathrm{mL}$ & 103.2 & $<1.00 e-02$ & $<4.10 \mathrm{e}-01$ & $<4.10 \mathrm{e}-1$ & $\mathrm{n} / \mathrm{a}$ & $n / a$ & 103.0 & $4.10 \mathrm{e}-01$ & $\mathrm{n} / \mathrm{a}$ \\
\hline 5961005006 & D & Iron-ICP-Acid Dil. & $\mathrm{ug} / \mathrm{mL}$ & 100.0 & $<5.00 \mathrm{e}-02$ & $<\quad 2.050$ & $<2.05 \mathrm{e} 0$ & $\mathrm{n} / \mathrm{a}$ & $\mathrm{n} / \mathrm{a}$ & 99.20 & 2.050 & $n / a$ \\
\hline S96T005006 & $\overline{\mathbf{D}}$ & Potass ium-ICP-Acid Dil. & $\mathrm{ug} / \mathrm{mL}$ & 96.00 & $<5.00 e-01$ & $1.32 \mathrm{e}+03$ & $1.32 e+03$ & $1.32 \mathrm{e}+03$ & 0.00 & 92.10 & 20.50 & $n / a$ \\
\hline 5967005006 & D & Lanthanum-ICP-Acid Dil. & $\mathrm{ug} / \mathrm{mL}$ & 101.6 & $<5.00 \mathrm{e}-02$ & $<\quad 2.050$ & $<2.05 \mathrm{e} 0$ & $\mathrm{n} / \mathrm{a}$ & $n / a$ & 101.0 & 2.050 & $\mathrm{n} / \mathrm{a}$ \\
\hline$\$ 967005006$ & D & Lithium-ICP-Acid Dil. & $\mathrm{ug} / \mathrm{mL}$ & 101.8 & $<1.00 e-02$ & $<4.10 \mathrm{e}-01$ & $<4.10 \mathrm{e}-1$ & $n / a$ & $\mathrm{n} / \mathrm{a}$ & 102.0 & $4.10 \mathrm{e}-01$ & $\mathrm{n} / \mathrm{a}$ \\
\hline \$9670005006 & D & Magnesium-ICP-Acid Dil. & $\mathrm{ug} / \mathrm{mL}$ & 93.40 & $<1.00 e-01$ & $\leq 4.100$ & $<4.10 \mathrm{e} 0$ & $n / a$ & $n / a$ & 92.50 & 4.100 & $n / a$ \\
\hline S96T005006 & D & Manganese-ICP-Acid Dil. & $\mathrm{ug} / \mathrm{mL}$ & 97.20 & $<1.00 \mathrm{e}-02$ & $<4.10 e-01$ & $<4.10 e^{-1}$ & $n / a$ & $n / a$ & 95.20 & $4.10 \mathrm{e}^{-01}$ & $n / a$ \\
\hline $596 \mathrm{~T} 005006$ & $\bar{D}$ & Molybdenum-ICP-Acid Dil. & $\mathrm{ug} / \mathrm{mL}$ & 100.2 & $\angle 5.00 \mathrm{e}-02$ & $<\quad 2.050$ & $<2.05 e 0$ & $\mathrm{n} / \mathrm{a}$ & $\mathrm{n} / \mathrm{a}$ & 102.0 & 2.050 & $n / a$ \\
\hline S96T005006 & D & Sodium-ICP-Acid Dil. & $\mathrm{ug} / \mathrm{mL}$ & 98.20 & $\leq 1.00 \mathrm{e}-01$ & $1.30 \mathrm{e}+04$ & $1.30 \mathrm{e}+04$ & $1.30 \mathrm{e}+04$ & 0.00 & $-1.720 \mathrm{e} 2$ & 4.100 & $\mathrm{n} / \mathrm{a}$ \\
\hline 5961005006 & $\bar{D}$ & Neodymium-ICP-Acid DiL. & $\mathrm{ug} / \mathrm{mL}$ & 102.0 & $<1.00 \mathrm{e}-01$ & $\leq 4.100$ & $<4.10 \mathrm{e} 0$ & $n / a$ & $\mathrm{n} / \mathrm{a}$ & 101.0 & 4.100 & $\mathrm{n} / \mathrm{a}$ \\
\hline S96T005006 & D & Nickel-ICP-Acid Dil. & $\mathrm{ug} / \mathrm{mL}$ & 98.60 & $<2.00 e-02$ & $<8.20 \mathrm{e}-01$ & $<8.20 \mathrm{e}-1$ & $\mathrm{n} / \mathrm{a}$ & $\mathrm{n} / \mathrm{a}$ & 98.80 & $8.20 e^{-01}$ & $\mathrm{n} / \mathrm{a}$ \\
\hline 596T005006 & D & Phosphorus-ICP-Acid Dil. & $\mathrm{ug} / \mathrm{mL}$ & 102.2 & $<2.00 e-01$ & 12.90 & 13.70 & 13.30 & 6.02 & 109.0 & 8.200 & $n / a$ \\
\hline S96T005006 & D & Lead-ICP-Acid Dil, & ug/mL & 99.80 & $<1.00 e-01$ & 4.100 & $<4.10 \mathrm{e} 0$ & $n / a$ & $\mathrm{n} / \mathrm{a}$ & 101.0 & 4.100 & $\mathrm{n} / \mathrm{a}$ \\
\hline $596 T 005006$ & $\bar{D}$ & Sulfur-ICP-Acid Dil. & $\mathrm{ug} / \mathrm{mL}$ & 98.60 & $<1.00 e+01$ & 46.70 & 46.50 & 46.60 & 0.43 & 99.10 & 4.100 & $n / a$ \\
\hline S96T005006 & $D$ & Antimony-ICP-Acid Dil. & ug/mL & 95.00 & $\angle 6.00 \mathrm{e}-02$ & 2.460 & $<2.46 \mathrm{e} 0$ & $n / a$ & $\mathrm{n} / \mathrm{a}$ & 97.40 & 2.460 & $n / a$ \\
\hline$\$ 961005006$ & $\bar{D}$ & Selenium-ICP-Acid Dil. & $\mathrm{ug} / \mathrm{mL}$ & 95.60 & $<1.00 e-01$ & 4.100 & $<4.10 \mathrm{e} 0$ & $n / a$ & $\mathrm{n} / \mathrm{a}$ & 97.90 & 4.100 & $\mathrm{n} / \mathrm{a}$ \\
\hline S96T005006 & $D$ & Silicon-ICP-Acid Dil. & $\mathrm{ug} / \mathrm{mL}$ & 100.2 & $\angle 5.00 e-02$ & 47.70 & 47.40 & 47.55 & 0.63 & 104.0 & 2.050 & $\mathrm{n} / \mathrm{a}$ \\
\hline S961005006 & D & Samarium-ICP-Acid Dil. & $\mathrm{ug} / \mathrm{mL}$ & 98.20 & $<1.00 \mathrm{e}-01$ & $<\quad 4.100$ & $<4.10 \mathrm{e} 0$ & $n / a$ & $\mathrm{n} / \mathrm{a}$ & 96.80 & 4.100 & $\mathrm{n} / \mathrm{a}$ \\
\hline 5967005006 & $D$ & Strontium-ICP-Acid Dil. & $\mathrm{ug} / \mathrm{mL}$ & 98.80 & $<1.00 \mathrm{e}-02$ & $<4.10 \mathrm{e}-01$ & $<4.10 \mathrm{e}-1$ & $\mathrm{n} / \mathrm{a}$ & $n / a$ & 97.80 & $4.10 \mathrm{e}-01$ & $n / a$ \\
\hline $596 \mathrm{~T} 005006$ & $D$ & Titanium-ICP-Acid Dil. & $\mathrm{ug} / \mathrm{mL}$ & 98.20 & $\leq 1.00 \mathrm{e}-02$ & $<4.10 \mathrm{e}-01$ & $<4.10 e^{-1}$ & $\mathrm{n} / \mathrm{a}$ & $n / a$ & 98.30 & $4.10 e-01$ & $n / a$ \\
\hline 5961005006 & $D$ & Thallium-ICP-Acid Dil. & $\mathrm{ug} / \mathrm{mL}$ & 96.20 & $<2.00 \mathrm{e}-01$ & $<8.200$ & $<8.20 \mathrm{e}$ & $\mathrm{n} / \mathrm{a}$ & $n / a$ & 95.60 & 8.200 & $\mathrm{n} / \mathrm{a}$ \\
\hline S96T005006 & $\mathrm{D}$ & Uranium-ICP-Acid Dil. & $\mathrm{ug} / \mathrm{mL}$ & 96.20 & $<5.00 e-01$ & 20.50 & $<2.05 \mathrm{e} 1$ & $\mathrm{n} / \mathrm{a}$ & $\mathrm{n} / \mathrm{a}$ & 115.5 & 20.50 & $\mathrm{n} / \mathrm{a}$ \\
\hline S96T005006 & D & Vanadi um-ICP-Acid Dil. & $\mathrm{ug} / \mathrm{mL}$ & 99.60 & $<5.00 e-02$ & 2.050 & $<2.05 \mathrm{e} 0$ & $\mathrm{n} / \mathrm{a}$ & $\mathrm{n} / \mathrm{a}$ & 99.40 & 2.050 & $\mathrm{n} / \mathrm{a}$ \\
\hline$\$ 96 T 005006$ & D & Zinc-ICP-Acid Dil. & $\mathrm{ug} / \mathrm{mL}$ & 100.6 & $<1.00 \mathrm{e}-02$ & 1.340 & 1.320 & 1.330 & 1.50 & 101.0 & $4.10 \mathrm{e}-01$ & $n / a$ \\
\hline \$96T005006 & D & Zirconium-ICP-Acid Dil. & ug/mL & 98.80 & $<1.00 e-02$ & $<4.10 e-01$ & $<4.10 \mathrm{e}-1$ & $\mathrm{n} / \mathrm{a}$ & $\mathrm{n} / \mathrm{a}$ & 98.20 & $4.10 \mathrm{e}-01$ & $\mathrm{n} / \mathrm{a}$ \\
\hline
\end{tabular}




\begin{tabular}{|c|c|c|c|c|c|c|c|c|c|c|c|c|}
\hline Sample\# & R $|A \#|$ & Analyte & Unit & Standard \% & Blank & Result & Dupl icate & Average & RPD \% & Spk Rec \% & Det Limit & Count Err\% \\
\hline \$96T005006 & & Fluoride-IC-Dionex $4000 / 4500$ & ug $/ \mathrm{mL}$ & 103.9 & $<1.30 \mathrm{e}-02$ & $1.74 \mathrm{e}+02$ & 166.0 & 170.2 & 4.71 & $n / a$ & 7.878 & $\mathrm{n} / \mathrm{a}$ \\
\hline S96T005006 & & Chloride-IC-Dionex $4000 / 4500$ & $\mathrm{ug} / \mathrm{mL}$ & 103.8 & $<7.70 \mathrm{e}-02$ & $2.21 \mathrm{e}+02$ & 216.0 & 218.3 & 2.29 & $\mathrm{n} / \mathrm{a}$ & 10.30 & $n / a$ \\
\hline \$96T005006 & & Nitrite-IC - Dionex $4000 / 4500$ & $\mathrm{ug} / \mathrm{mL}$ & 100.4 & $<1.07 \mathrm{e}-01$ & $1.12 \mathrm{e}+03$ & $1.10 \mathrm{e}+03$ & $1.11 \mathrm{e}+03$ & 1.80 & $n / a$ & 64.84 & $n / a$ \\
\hline$\$ 967005006$ & & Bromide by Ion Chromatograph & $\mathrm{ug} / \mathrm{mL}$ & 104.8 & $<1.26 \mathrm{e}-01$ & $<76.36$ & $<7.64 \mathrm{e} 1$ & $\mathrm{n} / \mathrm{a}$ & $\mathrm{n} / \mathrm{a}$ & $\mathrm{n} / \mathrm{a}$ & 76.36 & $\mathrm{n} / \mathrm{a}$ \\
\hline S96T005006 & & Nitrate by IC-Dionex $4000 / 4500$ & $\mathrm{ug} / \mathrm{mL}$ & 104.2 & $<1.40 \mathrm{e}-01$ & $2.41 \mathrm{e}+04$ & $2.41 \mathrm{e}+04$ & $2.41 \mathrm{e}+04$ & 0.00 & $\mathrm{n} / \mathrm{a}$ & 84.84 & $\mathrm{n} / \mathrm{a}$ \\
\hline S96T005006 & & Phosphate-IC-Dionex $4000 / 4500$ & $\mathrm{ug} / \mathrm{mL}$ & 100.5 & $<1.19 e^{-01}$ & $\leq 72.05$ & $<7.21 \mathrm{e} 1$ & $n / a$ & $n / a$ & $\mathrm{n} / \mathrm{a}$ & 72.05 & $n / a$ \\
\hline \$96T005006 & & Sul fate by IC-Dionex $4000 / 4500$ & $\mathrm{ug} / \mathrm{mL}$ & 103.0 & $<1.36 \mathrm{e}^{-01}$ & $1.75 e+02$ & 189.0 & 182.1 & 7.69 & $\mathrm{n} / \mathrm{a}$ & 82.36 & $n / a$ \\
\hline S96T005006 & & oxalate by IC-Dionex $4000 / 4500$ & ug/mL & 101.9 & $<1.05 \mathrm{e}-01$ & $<\quad 63.63$ & $<6.36 \mathrm{e} 1$ & $\mathrm{n} / \mathrm{a}$ & $\mathrm{n} / \mathrm{a}$ & $\mathrm{n} / \mathrm{a}$ & 63.63 & $\mathrm{n} / \mathrm{a}$ \\
\hline S96T005009 & & Stront ium- $89 / 90 \mathrm{High}$ Level & $\mathrm{uCi} / \mathrm{mL}$ & 110.0 & $2.51 e^{-05}$ & $1.61 \mathrm{e}-02$ & $1.59 \mathrm{e}-02$ & $1.60 \mathrm{e}-02$ & 1.25 & $n / a$ & $4.99 \mathrm{e}-05$ & $1.72 \mathrm{E}+00$ \\
\hline S96T005009 & & Pu-239/240 by TRU-SPEC Resin & $\mathrm{uCi} / \mathrm{mL}$ & 101.3 & $<3.72 e^{-06}$ & $<4.08 \mathrm{e}-06$ & $<3.74 \mathrm{E}-6$ & $\mathrm{n} / \mathrm{a}$ & $\mathrm{n} / \mathrm{a}$ & $n / a$ & $4.08 \mathrm{e}-06$ & $1.00 E+02$ \\
\hline 5967005009 & & Cobalt -60 by GEA & $\mathrm{uCi} / \mathrm{mL}$ & 99.32 & $<2.39 \mathrm{e}-05$ & $<6.31 e^{-05}$ & $1.16 \mathrm{e}-04$ & $n / a$ & $\mathrm{n} / \mathrm{a}$ & $\mathrm{n} / \mathrm{a}$ & $6.31 \mathrm{e}-05$ & $n / a$ \\
\hline S96T0 & & Cesi um- 137 by GEA & $\mathrm{uCi} / \mathrm{mL}$ & 102.3 & $<2.89 e^{-05}$ & $7.88 \mathrm{e}-01$ & $8.00 \mathrm{e}-01$ & $7.94 e^{-01}$ & 1.51 & $\mathrm{n} / \mathrm{a}$ & $n / a$ & 0.250 \\
\hline$\$ 96 \mathrm{~T} 005009$ & & Am-241 by Extraction & $\mathrm{uCi} / \mathrm{mL}$ & 84.87 & $<5.57 e-06$ & $\angle 6.00 \mathrm{e}-06$ & $<6.02 \mathrm{E}-6$ & $\mathrm{n} / \mathrm{a}$ & $\mathrm{n} / \mathrm{a}$ & $\mathrm{n} / \mathrm{a}$ & $6.00 \mathrm{e}-06$ & $1.00 \mathrm{E}+02$ \\
\hline \$96T005009 & & Alpha in Liquid Samples & UCi/mL & 88.75 & $<5.40 \mathrm{e}-05$ & $<5.79 \mathrm{e}-05$ & $<6.59 \mathrm{E}-5$ & $\mathrm{n} / \mathrm{a}$ & $\mathrm{n} / \mathrm{a}$ & $n / a$ & $9.99 \mathrm{e}-05$ & $5.00 \mathrm{E}+02$ \\
\hline
\end{tabular}


RISER: $10 \mathrm{~A}$

SEGMENT \#: 5AW-96-5

SEGMENT PORTION: Centrifuged SOlids (Grab Sample)

\begin{tabular}{|c|c|c|c|c|c|c|c|c|c|c|c|c|}
\hline Sample\# & A\# & Analyte & Unit & Standard \% & Blank & Result & Duplicate & Average & RPD \% & Spk Rec \% & Det Limit & Count Err\% \\
\hline S96T005019 & & Bulk Density of Sample & $\mathrm{g} / \mathrm{mL}$ & $\mathrm{n} / \mathrm{a}$ & $n / a$ & 1.150 & $n / a$ & $n / a$ & $\mathrm{n} / \mathrm{a}$ & $\mathrm{n} / \mathrm{a}$ & $5.00 e-01$ & $\mathrm{n} / \mathrm{a}$ \\
\hline S96T005019 & & DSC Exotherm on Perkin Elmer & Joules/g & 97.12 & $n / a$ & $0.00 \mathrm{e}+00$ & $0.00 \mathrm{e}+00$ & $0.00 \mathrm{e}+00$ & 0.00 & $n / a$ & $n / a$ & $n / a$ \\
\hline 5967005019 & & pH on SSI Samples & $\mathrm{pH}$ & 99.62 & $\mathrm{n} / \mathrm{a}$ & 11.66 & 11.70 & 11.68 & 0.34 & $\mathrm{n} / \mathrm{a}$ & $1.00 e-02$ & $n / a$ \\
\hline \$96T005019 & & $\%$ water by TGA on Perkin Elmer & $\%$ & 100.7 & $\mathrm{n} / \mathrm{a}$ & 80.79 & 79.42 & 80.11 & 1.71 & $n / a$ & $n / a$ & $\mathrm{n} / \mathrm{a}$ \\
\hline 5967005019 & & TIC by Acid/Coulometry & ug/g & 100.8 & $7.00 \mathrm{e}-01$ & $1.12 \mathrm{e}+03$ & $1.09 \mathrm{e}+03$ & $1.10 \mathrm{e}+03$ & 2.71 & 102.0 & 5.000 & $\mathrm{n} / \mathrm{a}$ \\
\hline 5967005019 & & Toc by Persulfate/coulometry & $\mathrm{ug} / \mathrm{g}$ & 89.67 & 13.50 & $1.81 \mathrm{e}+03$ & $1.73 e+03$ & $1.77 \mathrm{e}+03$ & 4.52 & 89.90 & 40.00 & $\mathrm{n} / \mathrm{a}$ \\
\hline \$96T005052 & $F$ & Stront ium-89/90 High Level & $u C i / g$ & 104.3 & $1.70 \mathrm{e}-02$ & 19.60 & 19.30 & 19.45 & 1.54 & $n / a$ & $4.00 \mathrm{e}-03$ & $6.15 \mathrm{E}-01$ \\
\hline S96T005052 & $F$ & PU-239/240 by TRU-SPEC Resin & uCi/g & 97.31 & $<3.09 \mathrm{e}-03$ & $4.58 \mathrm{e}-01$ & $4.47 \mathrm{e}-01$ & $4.53 e-01$ & 2.43 & $n / a$ & $2.20 \mathrm{e}-02$ & $1.56 \mathrm{E}+00$ \\
\hline \$96T005052 & $F$ & Silver-ICP-Fusion & $\mathrm{ug} / \mathrm{g}$ & 97.80 & $<1.00 \mathrm{e}-02$ & $<3.55 \mathrm{e}+02$ & $<3.63 \mathrm{e} 2$ & $\mathrm{n} / \mathrm{a}$ & $\mathrm{n} / \mathrm{a}$ & 81.50 & 355.0 & $\mathrm{n} / \mathrm{a}$ \\
\hline \$96T005052 & $F$ & Aluminium -ICP-Fusion & $u g / g$ & $97.00^{\circ}$ & $<5.00 e-02$ & $<1.78 \mathrm{e}+03$ & $<1.81 \mathrm{e}^{3}$ & $n / a$ & $\mathrm{n} / \mathrm{a}$ & 97.70 & $1.78 \mathrm{e}+03$ & $n / a$ \\
\hline \$96T005052 & $F$ & Arsenic-ICP-Fusion & $\mathrm{ug} / \mathrm{g}$ & 100.8 & $<1.00 \mathrm{e}-01$ & $<3.55 \mathrm{e}+03$ & $<3.63 \mathrm{e} 3$ & $\mathrm{n} / \mathrm{a}$ & $\mathrm{n} / \mathrm{a}$ & 97.00 & $3.55 \mathrm{e}+03$ & $\mathrm{n} / \mathrm{a}$ \\
\hline 5967005052 & $F$ & Boron-ICP-Fusion & ug/g $/ g$ & 99.20 & $<5.00 e^{-02}$ & $<1.78 \mathrm{e}+03$ & $<1.81 \mathrm{e}^{3}$ & $\mathrm{n} / \mathrm{a}$ & $\mathrm{n} / \mathrm{a}$ & 95.80 & $1.78 \mathrm{e}+03$ & $\mathrm{n} / \mathrm{a}$ \\
\hline 5967005052 & $F$ & Barium -ICP-Fusion & ug/g & 99.20 & $<5.00 e-02$ & $<1.78 \mathrm{e}+03$ & $<1.81 \mathrm{e}^{3}$ & $\mathrm{n} / \mathrm{a}$ & $\mathrm{n} / \mathrm{a}$ & 96.70 & $1.78 \mathrm{e}+03$ & $\mathrm{n} / \mathrm{a}$ \\
\hline 5967005052 & $F$ & Beryllífum-ICP-fusion & ug/g & 101.0 & $<5.00 e-03$ & $51.78 \mathrm{e}+02$ & $\leq 1.81 \mathrm{e} 2$ & $\mathrm{n} / \mathrm{a}$ & $n / a$ & 97.80 & 178.0 & $n / a$ \\
\hline 5967005052 & $\mathrm{~F}$ & Bismuth -ICP-Fusion & $49 / 9$ & 100.6 & $<1.00 \mathrm{e}-01$ & $<3.55 e+03$ & $<3.63 \mathrm{e} 3$ & $\mathrm{n} / \mathrm{a}$ & $\mathrm{n} / \mathrm{a}$ & 96.70 & $3.55 e+03$ & $n / a$ \\
\hline$\$ 967005052$ & $F$ & Calcium-ICP-Fusion & $49 / 9$ & 95.40 & $<1.00 \mathrm{e}-01$ & $9.41 \mathrm{e}+03$ & $9.18 \mathrm{e}+03$ & $9.30 \mathrm{e}+03$ & 2.47 & 90.50 & $3.55 \mathrm{e}+03$ & $n / a$ \\
\hline S96T005052 & $F$ & Cadmium-ICP-Fusion & $\mathrm{ug} / \mathrm{g}$ & 98.60 & $<5.00 \mathrm{e}-03$ & $<1.78 \mathrm{e}+02$ & $<1.81 \mathrm{e} 2$ & $n / a$ & $n / a$ & 96.90 & 178.0 & $n / a$ \\
\hline S96T0005052 & $F$ & Cerium-ICP-Fusion & ug/g & 97.60 & $<1.00 \mathrm{e}-01$ & $<3.55 e+03$ & $<3.63 \mathrm{e} 3$ & $\mathrm{n} / \mathrm{a}$ & $\mathrm{n} / \mathrm{a}$ & 94.80 & $3.55 \mathrm{e}+03$ & $n / a$ \\
\hline S96T005052 & $F$ & Cobalt -ICP-Fusion & $\mathrm{ug} / \mathrm{g}$ & 98.60 & $<2.00 e^{-02}$ & $<7.11 e+02$ & $<7.26 \mathrm{e} 2$ & $\mathrm{n} / \mathrm{a}$ & $n / a$ & 96.50 & 709.0 & $\mathrm{n} / \mathrm{a}$ \\
\hline S96T005052 & $F$ & Chromium -ICP-Fusion & $\mathrm{ug} / \mathrm{g}$ & 99.00 & $<1.00 \mathrm{e}-02$ & $<3.55 \mathrm{e}+02$ & $<3.63 \mathrm{e} 2$ & $\mathrm{n} / \mathrm{a}$ & $n / a$ & 97.50 & 355.0 & $n / a$ \\
\hline 5967005052 & $F$ & \begin{tabular}{|c|} 
Copper -ICP-Fusion \\
\end{tabular} & ug/g & 99.40 & $<1.00 \mathrm{e}-02$ & $<3.55 \mathrm{e}+02$ & $<3.63 \mathrm{e} 2$ & $n / a$ & $n / a$ & 97.80 & 355.0 & $\mathrm{n} / \mathrm{a}$ \\
\hline S961005052 & $F$ & Iron-ICP-Fusion & $\mathrm{ug} / \mathrm{g}$ & 99.40 & $<5.00 \mathrm{e}-02$ & $3.71 \mathrm{e}+03$ & $3.77 \mathrm{e}+03$ & $3.74 \mathrm{e}+03$ & 1.60 & 99.70 & $1.78 \mathrm{e}+03$ & $n / a$ \\
\hline $596 \mathrm{~T} 005052$ & $F$ & Lanthanum -ICP-Fusion & $\mathrm{ug} / \mathrm{g}$ & 99.40 & $<5.00 \mathrm{e}-02$ & $<1.78 \mathrm{e}+03$ & $<1.81 \mathrm{e} 3$ & $\mathrm{n} / \mathrm{a}$ & $n / a$ & 98.40 & $1.78 \mathrm{e}+03$ & $n / a$ \\
\hline S96T005052 & $\mathrm{F}$ & Lithium-ICP-Fusion & ug/g & 97.80 & $<1.00 \mathrm{e}-02$ & $<3.55 e+02$ & $<3.63 \mathrm{e} 2$ & $n / a$ & $\mathrm{n} / \mathrm{a}$ & 90.70 & 355.0 & $\mathrm{n} / \mathrm{a}$ \\
\hline S96T005052 & $F$ & Magnesium-ICP-Fusion & $\mathrm{ug} / \mathrm{g}$ & 95.20 & $<1.00 e-01$ & $<3.55 \mathrm{e}+03$ & $<3.63 \mathrm{e} 3$ & $n / a$ & $n / a$ & 92.30 & $3.55 \mathrm{e}+03$ & $\mathrm{n} / \mathrm{a}$ \\
\hline 5967005052 & $F$ & Manganese -ICP-Fusion & $\mathrm{ug} / \mathrm{g}$ & 98.20 & $<1.00 \mathrm{e}-02$ & $<3.55 \mathrm{e}+02$ & $<3.63 \mathrm{e} 2$ & $\mathrm{n} / \mathrm{a}$ & n/a & 97.60 & 355.0 & $n / a$ \\
\hline \$96T005052 & $\mathrm{F}$ & Molybdenum - ICP-Fusion & $\mathrm{ug} / \mathrm{g}$ & 99.00 & $<5.00 \mathrm{e}-02$ & $<1.78 \mathrm{e}+03$ & $<1.81 \mathrm{e} 3$ & $n / a$ & $n / a$ & 96.40 & $1.78 \mathrm{e}+03$ & $\mathrm{n} / \mathrm{a}$ \\
\hline S967005052 & $\mathrm{F}$ & Sodium-ICP-Fusion & $\mathrm{ug} / \mathrm{g}$ & 95.80 & 2.250 & $7.80 e+04$ & $7.88 \mathrm{e}+04$ & $7.84 \mathrm{e}+04$ & 9.02 & 83.70 & $3.55 \mathrm{e}+03$ & $n / a$ \\
\hline S96T005052 & $F$ & Neodymium -ICP-Fusion & ugg/g & 101.2 & $<1.00 e^{-01}$ & $<3.55 \mathrm{e}+03$ & $<3.63 \mathrm{e} 3$ & $\mathrm{n} / \mathrm{a}$ & $n / a$ & 100.0 & $3.55 \mathrm{e}+03$ & $\mathrm{n} / \mathrm{a}$ \\
\hline S96T0 & $F$ & Phosphorus -ICP-Fusion & $\mathrm{ug} / \mathrm{g}$ & 99.20 & $<2.00 \mathrm{e}-01$ & $<7.11 \mathrm{e}+03$ & $<7.26 \mathrm{e}^{3}$ & $n / a$ & $n / a$ & 107.0 & $7.09 \mathrm{e}+03$ & $n / a$ \\
\hline S96T005052 & $F$ & Lead -ICP-Fusion & ug/g & 98.60 & $<1.00 e-01$ & $<3.55 e+03$ & $<3.63 \mathrm{e} 3$ & $\mathrm{n} / \mathrm{a}$ & $\mathrm{n} / \mathrm{a}$ & 98.50 & $3.55 e+03$ & $\mathrm{n} / \mathrm{a}$ \\
\hline S96T005052 & $\mathrm{F}$ & Sulfur - ICP-Fusion & $u g / g$ & 98.60 & $<1.00 e-01$ & $<3.55 \mathrm{e}+03$ & $<3.63 \mathrm{e} 3$ & $n / a$ & $\mathrm{n} / \mathrm{a}$ & 94.50 & $3.55 \mathrm{e}+03$ & $n / a$ \\
\hline$\$ 967005052$ & $F$ & Ant imony -ICP-Fusion & $u g / g$ & 90.20 & $<6.00 e-02$ & $<2.13 e+03$ & $2.66 \mathrm{e}+03$ & $n / a$ & $n / a$ & 89.50 & $2.13 e+03$ & $\mathrm{n} / \mathrm{a}$ \\
\hline S96T005052 & $F$ & Selenium-ICP-Fusion & $\mathrm{ug} / \mathrm{g}$ & 105.0 & $<1.00 e-01$ & $<3.55 \mathrm{e}+03$ & $<3.63 \mathrm{e} 3$ & $n / a$ & $n / a$ & 101.0 & $3.55 \mathrm{e}+03$ & $n / a$ \\
\hline 5967005052 & $F$ & Silicon-ICP-Fusion & $4 \mathrm{~g} / \mathrm{g}$ & 98.40 & $<5.00 e-02$ & $1.83 \mathrm{e}+03$ & $<1.81 \mathrm{e} 3$ & $n / a$ & $n / a$ & 97.90 & $1.78 \mathrm{e}+03$ & $\mathrm{n} / \mathrm{a}$ \\
\hline S96T005052 & $F$ & Samarium -ICP-Fusion & $\mathrm{ug} / \mathrm{g}$ & 101.0 & $<1.00 \mathrm{e}-01$ & $<3.55 \mathrm{e}+03$ & $<3.63 \mathrm{e} 3$ & $n / a$ & n/a & 97.40 & $3.55 \mathrm{e}+03$ & $\mathrm{n} / \mathrm{a}$ \\
\hline S96T005052 & $F$ & Strontium-ICP-Fusion & ug/g & 98.40 & $<1.00 \mathrm{e}-02$ & $<3.55 e+02$ & $<3.63 \mathrm{e} 2$ & $n / a$ & $n / a$ & 96.60 & 355.0 & $\mathrm{n} / \mathrm{a}$ \\
\hline S96T005052 & $F$ & Titanium-ICP-Fusion & $u g / 9$ & 96.00 & $\leq 1.00 e^{-02}$ & $<3.55 e+02$ & $<3.63 \mathrm{e} 2$ & $\mathrm{n} / \mathrm{a}$ & $n / a$ & 93.80 & 355.0 & $n / a$ \\
\hline 5961005052 & F & Thallium -ICP-Fusion & ug/g & 97.20 & $<2.00 e-01$ & $<7.11 e+03$ & $<7.26 \mathrm{e} 3$ & $n / a$ & $\mathrm{n} / \mathrm{a}$ & 96.60 & $7.09 e+03$ & $\mathrm{n} / \mathrm{a}$ \\
\hline S96T005052 & $\mathbf{F}$ & Uranium-ICP-Fusion & $u g / g$ & 97.10 & $<5.00 \mathrm{e}-01$ & $6.17 e+04$ & $5.63 \mathrm{e}+04$ & $5.90 \mathrm{e}+04$ & 9.15 & 96.00 & $1.78 \mathrm{e}+04$ & $n / a$ \\
\hline $596 T 005052$ & $\mathbf{F}$ & Vanadium-ICP-Fusion & $u g / g$ & 99.80 & $\angle 5.00 e-02$ & $<1.78 \mathrm{e}+03$ & $<1.81 \mathrm{e} 3$ & $n / a$ & $\mathrm{n} / \mathrm{a}$ & 98.50 & $1.78 \mathrm{e}+03$ & $\mathrm{n} / \mathrm{a}$ \\
\hline 5967005052 & $\mathrm{~F}$ & Zine-ICP-Fusion & ug $/ g$ & 99.40 & $1.60 \mathrm{e}-02$ & $<3.55 \mathrm{e}+02$ & 913.0 & $n / a$ & $\mathrm{n} / \mathrm{a}$ & 99.60 & 355.0 & $n / a$ \\
\hline 5961005052 & & Zirconium-ICP-Fusion & ug/g & 98.00 & $\leq 1.00 \mathrm{e}-02$ & $5.83 e+02$ & 558.0 & 570.5 & 4.38 & 97.20 & 355.0 & $n / a$ \\
\hline S96T005052 & $F$ & Cobalt -60 by GEA & uCi $/ 9$ & 97.35 & $<2.77 \mathrm{e}-02$ & $<3.55 e^{-02}$ & $<3.81 \mathrm{e}-2$ & $n / a$ & $n / a$ & $n / a$ & $3.50 \mathrm{e}-02$ & $n / a$ \\
\hline
\end{tabular}




\begin{tabular}{|c|c|c|c|c|c|c|c|c|c|c|c|c|c|}
\hline Sample\# & $\mathrm{R} \mid \mathrm{A}$ & A\# & Analyte & Unit & Standard \% & $B \operatorname{Lank}$ & Result & Duplicate & Average & RPD \% & Spk Rec \% & Det Limit & Count Err\% \\
\hline \$96T005052 & & $\mathrm{F}$ & Ces ium- 137 by GEA & UCi/g & 95.75 & $<6.17 e-02$ & 6.328 & 6.580 & 6.454 & 3.87 & $n / a$ & $n / a$ & 2.89 \\
\hline S96T005052 & & $\mathrm{F}$ & Am-241 by Extraction & uCi $/ \mathrm{g}$ & 115.1 & $<8.33 e-03$ & $3.24 \mathrm{e}-01$ & $2.87 \mathrm{e}-01$ & $3.05 e-01$ & 12.1 & $n / a$ & $2.90 \mathrm{e}-02$ & $1.77 \mathrm{E}+00$ \\
\hline 5967005052 & & $F$ & Alpha of Digested Solid & $4 C_{i} / g$ & 81.39 & $<4.70 \mathrm{e}-03$ & $6.20 e-01$ & $5.68 e-01$ & $5.94 e^{-01}$ & 8.75 & 66.30 & $9.00 e^{-03}$ & $8.69 \mathrm{E}+00$ \\
\hline S96T005055 & & $\mathrm{W}$ & OH-by Pot. Titration & $\mathrm{ug} / \mathrm{g}$ & 97.36 & $<42.00$ & $<42.00$ & $<42$ & $\mathrm{n} / \mathrm{a}$ & $\mathrm{n} / \mathrm{a}$ & n/a & 41.70 & $n / a$ \\
\hline S96r005055 & & $\mathbf{W}$ & Fluoride-IC-Dionex $4000 / 4500$ & $u g / g$ & 90.51 & $<1.20 \mathrm{e}-02$ & $1.41 \mathrm{e}+03$ & $1.38 \mathrm{e}+03$ & $1.40 \mathrm{e}+03$ & 2.15 & 106.1 & 22.11 & $n / a$ \\
\hline S96T005055 & & W & Chloride-IC-Dionex $4000 / 4500$ & $40 / 9$ & 91.52 & $2.00 \mathrm{e}-02$ & $2.08 \mathrm{e}+02$ & 203.0 & 205.6 & 2.43 & 93.92 & 31.32 & $n / a$ \\
\hline \$96T005055 & & $\mathbf{W}$ & Nitrite-IC - Dionex $4000 / 4500$ & $u g / g$ & 91.67 & $<1.08 \mathrm{e}-01$ & $1.31 \mathrm{e}+03$ & $1.26 \mathrm{e}+03$ & $1.28 \mathrm{e}+03$ & 3.89 & 93.52 & 198.9 & $\mathrm{n} / \mathrm{a}$ \\
\hline S96T005055 & $\mathbf{W}$ & W & Bromide by Ion Chromatograph & $\mathrm{ug} / \mathrm{g}$ & 94.57 & $<1.25 e-01$ & $<2.30 \mathrm{e}+02$ & $<2.28 \mathrm{e} 2$ & $n / a$ & $n / a$ & 98.13 & 230.3 & $\mathrm{n} / \mathrm{a}$ \\
\hline $596 \mathrm{TO0} 05055$ & & $\overline{\mathrm{W}}$ & Nitrate by IC-Dionex $4000 / 4500$ & ug/g & 100.0 & $1.79 \mathrm{e}-01$ & $2.62 \mathrm{e}+04$ & $2.64 e+04$ & $2.63 \mathrm{e}+04$ & 0.76 & 117.2 & 256.1 & $n / a$ \\
\hline 5967005055 & & $\overline{\mathrm{W}}$ & Phosphate-IC-D ionex $4000 / 4500$ & $u g / g$ & 95.78 & $<1.20 e-01$ & $4.45 e+02$ & 486.0 & 465.6 & 8.81 & 98.53 & 221.1 & $\mathrm{n} / \mathrm{a}$ \\
\hline $596 \mathrm{~T} 005055$ & W & $\vec{W}$ & Sulfate by IC-Dionex $4000 / 4500$ & $\mathrm{ug} / \mathrm{g}$ & 101.6 & $<1.38 \mathrm{e}-01$ & $4.34 \mathrm{e}+02$ & 415.0 & 424.5 & 4.48 & 104.4 & 254.2 & $n / a$ \\
\hline 5967005055 & & $\mathrm{~W}$ & oxalate by IC-Dionex $4000 / 4500$ & $\mathrm{ug} / \mathrm{g}$ & 99.24 & $<1.05 e-01$ & $<1.93 \mathrm{e}+02$ & $<1.92 \mathrm{e} 2$ & $n / a$ & $\mathrm{n} / \mathrm{a}$ & 102.7 & 193.4 & $n / a$ \\
\hline
\end{tabular}

\begin{tabular}{|c|c|c|c|c|c|c|c|c|c|c|c|c|}
\hline Sample\# & A\# & Analyte & Unit & Standard \% & 8 lank & Result & Duplicate & Average & RPD \% & Spk Rec \% & Det Limit & Count Err\% \\
\hline S96T005049 & & Bulk Density of Sample & $\mathrm{g} / \mathrm{mL}$ & $\mathrm{n} / \mathrm{a}$ & $n / a$ & 1.040 & $\mathrm{n} / \mathrm{a}$ & n/a & $n / a$ & $n / a$ & $5.00 \mathrm{e}-01$ & $n / a$ \\
\hline S96r005049 & & DSC Exotherm on Perkin Elmer & Joules/g & 92.30 & n/a & $0.00 \mathrm{e}+00$ & $0.00 \mathrm{e}+00$ & $0.00 \mathrm{e}+00$ & 0.00 & $\mathrm{n} / \mathrm{a}$ & $n / a$ & $\mathrm{n} / \mathrm{a}$ \\
\hline S96T005049 & & $\mathrm{OH}-$ by Pot. Titration & $\mathrm{ug} / \mathrm{mL}$ & 106.4 & $\angle 42.00$ & $5.41 \mathrm{e}+03$ & $5.48 \mathrm{e}+03$ & $5.44 \mathrm{e}+03$ & 1.29 & $\mathrm{n} / \mathrm{a}$ & 500.0 & $\mathrm{n} / \mathrm{a}$ \\
\hline$\$ 96 \mathrm{~T} 005049$ & & pH Direct & $\mathrm{pH}$ & 100.0 & $\mathrm{n} / \mathrm{a}$ & 13.24 & 13.26 & 13.25 & 0.15 & $n / a$ & $1.00 e-02$ & $n / a$ \\
\hline$\$ 967005049$ & & $\%$ Water by TGA on Perkin Elmer & $\%$ & 99.27 & $\mathrm{n} / \mathrm{a}$ & 92.61 & 92.74 & 92.67 & 0.14 & $n / a$ & $n / a$ & $n / a$ \\
\hline $596 \mathrm{~T} 005049$ & & TIC by Acid/Coulometry & $\mathrm{ug} / \mathrm{mL}$ & 99.50 & 2.800 & $3.92 \mathrm{e}+02$ & 459.0 & 425.5 & 15.7 & 110.8 & 5.000 & $\mathrm{n} / \mathrm{a}$ \\
\hline S96T005049 & & Tot. Organic Carbon by Coul. & ug/mL & 103.0 & 1.200 & $9.51 \mathrm{e}+02$ & 589.0 & 770.0 & 47.0 & 93.00 & 55.00 & $\mathrm{n} / \mathrm{a}$ \\
\hline$\$ 9610050491$ & & Tot. Organic Carbon by coul. & $\mathrm{ug} / \mathrm{mL}$ & 98.67 & $6.00 \mathrm{e}-01$ & $7.04 e+02$ & 704.0 & 704.0 & 0.00 & $n / a$ & 55.00 & n/a \\
\hline S96T005049 & & Strontium-89/90 High Level & $\mathrm{u} \mathrm{i} \mathrm{i} / \mathrm{mL}$ & 118.6 & $6.49 e-05$ & $2.94 \mathrm{e}-01$ & $9.60 \mathrm{e}-02$ & $1.95 \mathrm{e}-01$ & 102 & $\mathrm{n} / \mathrm{a}$ & $2.86 \mathrm{e}^{-05}$ & $3.92 E-01$ \\
\hline S96T005049 & & PU-239/240 by TRU-SPEC Resin & uCi $/ \mathrm{mL}$ & 90.60 & $<7.39 e-06$ & $1.18 \mathrm{e}-03$ & $1.00 \mathrm{e}-03$ & $1.09 \mathrm{e}-03$ & 16.5 & $\mathrm{n} / \mathrm{a}$ & $5.75 e-05$ & $1.32 E+00$ \\
\hline S96T005049 & D & Silver-ICP-Acid Dil. & $\mathrm{ug} / \mathrm{mL}$ & 99.80 & $<1.00 \mathrm{e}-02$ & 1.260 & 1.620 & 1.440 & 25.0 & 97.90 & 1.010 & $\mathrm{n} / \mathrm{a}$ \\
\hline$\$ 967005049$ & İ & Aluminium-ICP-Acid Dil. & $\mathrm{ug} / \mathrm{mL}$ & 99.00 & $<5.00 e-02$ & $1.29 \mathrm{e}+02$ & 135.0 & 132.0 & 4.55 & 97.30 & 5.050 & $n / a$ \\
\hline S96T005049 & D & Arsenic-ICP-Acid Dil. & $\mathrm{ug} / \mathrm{mL}$ & 104.6 & $<1.00 \mathrm{e}-01$ & $<\quad 10.10$ & $<1.01 \mathrm{e} 1$ & $n / a$ & n/a & 108.0 & 10.10 & $n / a$ \\
\hline 5967005049 & D & Boron-ICP-Acid Dil. & ug/mL & 100.4 & $<5.00 e-02$ & 9.100 & 9.190 & 9.145 & 0.98 & 99.30 & 5.050 & $n / a$ \\
\hline S967005049 & D & Barium-ICP-Acid Dil. & $\underline{u g} / \mathrm{mL}$ & 100.0 & $<5.00 e-02$ & $<5.050$ & $<5.05 \mathrm{e} 0$ & $n / a$ & $\mathrm{n} / \mathrm{a}$ & 99.20 & 5.050 & $n / a$ \\
\hline 5967005049 & D & Beryllium-ICP-Acid Dil. & $\mathrm{ug} / \mathrm{mL}$ & 104.8 & $<5.00 \mathrm{e}-03$ & $<5.05 e-01$ & $<5.05 e^{-1}$ & $n / a$ & $\mathrm{n} / \mathrm{a}$ & 103.0 & $5.05 \mathrm{e}-01$ & $\mathrm{n} / \mathrm{a}$ \\
\hline $596 \mathrm{~T} 005049$ & D & Bismuth-ICP-ACid Dil. & $\mathrm{ug} / \mathrm{mL}$ & 100.6 & $<1.00 \mathrm{e}-01$ & $\leq \quad 10.10$ & $<1.01 \mathrm{e} 1$ & $n / a$ & $\mathrm{n} / \mathrm{a}$ & 101.0 & 10.10 & $\mathrm{n} / \mathrm{a}$ \\
\hline S96T005049 & D & Calcfim-ICP-Acid Dil. & $\mathrm{ug} / \mathrm{mL}$ & 99.00 & $<1.00 \mathrm{e}-01$ & 16.60 & 96.90 & 56.75 & 141 & 97.40 & 10.10 & $n / a$ \\
\hline 5967005049 & ID & Cadmi Um-ICP-Acid Dil. & $\underline{u g} / \mathrm{mL}$ & 101.0 & $<5.00 \mathrm{e}-03$ & $<5.05 e-01$ & $7.17 \mathrm{e}-01$ & $n / a$ & $\mathrm{n} / \mathrm{a}$ & 102.0 & $5.05 e^{-01}$ & $n / a$ \\
\hline S96T005049 & $\mathrm{D}$ & Cerium-ICP-Acid Dil. & $\mathrm{ug} / \mathrm{mL}$ & 101.2 & $<1.00 e-01$ & $\leq \quad 10.10$ & $<1.01 \mathrm{e} 1$ & $\mathrm{n} / \mathrm{a}$ & $\mathrm{n} / \mathrm{a}$ & 101.0 & 10.10 & $\mathrm{n} / \mathrm{a}$ \\
\hline 5967005049 & D & Cobalt-ICP-Acid Dil. & $\mu \mathrm{g} / \mathrm{mL}$ & 100.6 & $<2.00 \mathrm{e}-02$ & 2.020 & $<2.02 \mathrm{e} 0$ & $\mathrm{n} / \mathrm{a}$ & $\mathrm{n} / \mathrm{a}$ & 101.0 & 2.020 & $n / a$ \\
\hline 5967005049 & D & Chromium-ICP-Acid Dil. & $\mathrm{ug} / \mathrm{mL}$ & 101.8 & $<1.00 e-02$ & 6.720 & 8.540 & 7.630 & 23.9 & 102.0 & 1.010 & $\mathrm{n} / \mathrm{a}$ \\
\hline 5967005049 & D & Copper-ICP-ACid Dil. & $\mathrm{ug} / \mathrm{mL}$ & 105.2 & $<1.00 \mathrm{e}-02$ & 1.010 & $<1.01 \mathrm{e} 0$ & $n / a$ & $\mathrm{n} / \mathrm{a}$ & 105.0 & 1.010 & $n / a$ \\
\hline S96T005049 & DD & Iron-ICP-Acid Dil. & $\mathrm{ug} / \mathrm{mL}$ & 101.8 & $<5.00 \mathrm{e}-02$ & $<\quad 5.050$ & 34.90 & $n / a$ & $\mathrm{n} / \mathrm{a}$ & 105.0 & 5.050 & $\mathrm{n} / \mathrm{a}$ \\
\hline 5967005049 & D & Potassium-icP-Acid Dil. & $\mathrm{ug} / \mathrm{mL}$ & 100.0 & $<5.00 e-01$ & $4.15 e+03$ & $4.15 e+03$ & $4.15 \mathrm{e}+03$ & 0.00 & 86.90 & 50.50 & $n / a$ \\
\hline S967005049 & $\mathrm{D}$ & Lanthanum-ICP-Acid Dil. & $\mathrm{ug} / \mathrm{mL}$ & 103.4 & $<5.00 \mathrm{e}-02$ & $<\quad 5.050$ & $<5.05 \mathrm{e} 0$ & $n / a$ & $\mathrm{n} / \mathrm{a}$ & 104.0 & 5.050 & $n / a$ \\
\hline$\$ 967005049$ & D & Lithium-ICP-Acid Dil. & $\underline{u g} / \mathrm{mL}$ & 103.6 & $<1.00 \mathrm{e}-02$ & 1.010 & $<1.01 \mathrm{e} 0$ & $n / a$ & $n / a$ & 103.0 & 1.010 & $n / a$ \\
\hline 5967005049 & D & Magnesium-ICP-Acid Dil. & $\mathrm{ug} / \mathrm{mL}$ & 97.60 & $<1.00 \mathrm{e}-01$ & 10.10 & $<1.01 \mathrm{e} 1$ & $n / a$ & $n / a$ & 96.00 & 10.10 & $n / a$ \\
\hline 5967005049 & D & Manganese-ICP-Acid Dil. & $\mathrm{ug} / \mathrm{mL}$ & 98.80 & $<1.00 \mathrm{e}-02$ & 1.010 & 1.510 & $\mathrm{n} / \mathrm{a}$ & $n / a$ & 97.50 & 1.010 & $\mathrm{n} / \mathrm{a}$ \\
\hline 5967005049 & D & Molybdenum-ICP-Acid Dil. & ug/mL & 101.2 & $<5.00 \mathrm{e}-02$ & 5.050 & $<5.05 \mathrm{e} 0$ & $n / a$ & $n / a$ & 103.0 & 5.050 & $\mathrm{n} / \mathrm{a}$ \\
\hline S961005049 & $D$ & Sodium-ICP-Acid Di!... & $\mathrm{ug} / \mathrm{mL}$ & 99.80 & $<1.00 \mathrm{e}-01$ & $2.13 e+04$ & $2.11 \mathrm{e}+04$ & $2.12 \mathrm{e}+04$ & 0.94 & $-1.890 \mathrm{e} 1$ & 10.10 & $n / a$ \\
\hline S96T005049 & $\bar{D}$ & Neodymium-ICP-Acid Dil. & $\mathrm{ug} / \mathrm{mL}$ & 103.8 & $<1.00 \mathrm{e}-01$ & $<\quad 10.10$ & $\leq 1.01 \mathrm{e} 1$ & n/a & $n / a$ & 104.0 & 10.10 & n/a \\
\hline 5967005049 & D & Nickel-ICP-Acid Dil. & $\mu \mathrm{g} / \mathrm{mL}$ & 100.2 & $<2.00 \mathrm{e}-02$ & 2.020 & $\leq 2.02 \mathrm{e} 0$ & $n / a$ & $n / a$ & 101.0 & 2.020 & $\mathrm{n} / \mathrm{a}$ \\
\hline $5967005049^{\circ}$ & ID & Phosphorus-ICP-Acid Dil, & $\mathrm{ug} / \mathrm{mL}$ & 105.0 & $<2.00 e-01$ & 89.30 & 121.0 & 105.2 & 30.1 & 101.0 & 20.20 & $\mathrm{n} / \mathrm{a}$ \\
\hline 5967005049 & $D$ & Lead-ICP-Acid Dil. & $\mathrm{ug} / \mathrm{mL}$ & 100.0 & $<1.00 \mathrm{e}-01$ & 10.10 & $<1.01 \mathrm{e} 1$ & $n / a$ & $\mathrm{n} / \mathrm{a}$ & 101.0 & 10.10 & $\mathrm{n} / \mathrm{a}$ \\
\hline
\end{tabular}




\begin{tabular}{|c|c|c|c|c|c|c|c|c|c|c|c|c|}
\hline Sample\# & A\# & Analyte & Unit & Standard \% & Blank & Result & Dupl icate & Average & RPD $\%$ & Spk Rèc \% & Det Limit & Count Err\% \\
\hline S967005049 & $D$ & Sulfur-ICP-Acid Dil. & $\mathrm{ug} / \mathrm{mL}$ & 99.20 & $<1.00 \mathrm{e}-01$ & $1.47 e+02$ & 153.0 & 150.0 & 4.00 & 111.0 & 10.10 & $n / a$ \\
\hline$\$ 967005049$ & D & Ant imony-ICP-Acid Dil. & $\mathrm{ug} / \mathrm{mL}$ & 95.20 & $<6.00 \mathrm{e}-02$ & $<6.060$ & $<6.06 \mathrm{e} 0$ & $n / a$ & $n / a$ & 95.80 & 6.060 & $n / a$ \\
\hline$\$ 967005049$ & D & Selenium-ICP-ACid Dil. & $\mathrm{ug} / \mathrm{mL}$ & 97.60 & $<1.00 \mathrm{e}-01$ & 10.10 & $<1.01 \mathrm{e} 1$ & $\mathrm{n} / \mathrm{a}$ & $n / a$ & 101.0 & 10.10 & $n / a$ \\
\hline 5967005049 & D & Silicon-ICP-Acid Dil. & $\mathrm{ug} / \mathrm{mL}$ & 98.40 & $<5.00 \mathrm{e}-02$ & 94.90 & 113.0 & 104.0 & 17.4 & 102.0 & 5.050 & $n / a$ \\
\hline$\$ 967005049$ & $\frac{D}{D}$ & Samarím-ICP-Acid Dil. & $\mathrm{ug} / \mathrm{mL}$ & 100.0 & $<1.00 \mathrm{e}-01$ & 10.10 & $<1.01 \mathrm{e} 1$ & $\mathrm{n} / \mathrm{a}$ & $\mathrm{n} / \mathrm{a}$ & 99.20 & 10.10 & $\mathrm{n} / \mathrm{a}$ \\
\hline$\$ 967005049$ & D & Stront ium-ICP-Acid Dil. & $\mathrm{ug} / \mathrm{mL}$ & 101.2 & $<1.00 \mathrm{e}-02$ & 1.010 & $<1.01 \mathrm{e} 0$ & $\mathrm{n} / \mathrm{a}$ & $n / a$ & 101.0 & 1.010 & $n / a$ \\
\hline$\$ 967005049$ & D & Titanium-ICP-ACid Dill. & $\mathrm{ug} / \mathrm{mL}$ & 98.80 & $<1.00 \mathrm{e}-02$ & 1.010 & $<1.01 \mathrm{e} 0$ & $\mathrm{n} / \mathrm{a}$ & $n / a$ & 99.10 & 1.010 & $n / a$ \\
\hline 5967005049 & $D$ & Thallium-ICP-Acid Dil. & Ug/mL & 96.00 & $<2.00 \mathrm{e}-01$ & 20.20 & $<2.02 \mathrm{e} 1$ & $n / a$ & $n / a$ & 96.50 & 20.20 & $n / a$ \\
\hline S967005049 & D & Uranium-ICP-Acid DiL. & $\mathrm{ug} / \mathrm{mL}$ & 97.50 & $<5.00 \mathrm{e}-01$ & 76.70 & 464.0 & 270.4 & 143 & 91.50 & 50.50 & $\mathrm{n} / \mathrm{a}$ \\
\hline 5967005049 & D & Vanadium-ICP-ACid DiL. & $\mathrm{ug} / \mathrm{mL}$ & 101.4 & $<5.00 \mathrm{e}-02$ & 5.050 & $<5.05 \mathrm{e} 0$ & $\mathrm{n} / \mathrm{a}$ & n/a & 102.0 & 5.050 & $\mathrm{n} / \mathrm{a}$ \\
\hline S967005049 & D & Zinc-ICP-Acid Dil. & $\mathrm{ug} / \mathrm{mL}$ & 101.2 & $<1.00 e-02$ & 5.880 & 7.050 & 6.465 & 18.1 & 102.0 & 1.010 & $\mathrm{n} / \mathrm{a}$ \\
\hline S96T005049 & $\frac{P}{D}$ & Zirconjum-ICP-Acid Dil. & $\mathrm{ug} / \mathrm{mL}$ & 99.60 & $<1.00 \mathrm{e}-02$ & 1.590 & 12.50 & 7.045 & 155 & 99.50 & 1.010 & $\mathrm{n} / \mathrm{a}$ \\
\hline S967005049 & & Fluoride-IC-Dionex $4000 / 4500$ & $\mathrm{ug} / \mathrm{mL}$ & 89.15 & $<1.20 \mathrm{e}-02$ & $1.51 \mathrm{e}+03$ & $1.49 \mathrm{e}+03$ & $1.50 e+03$ & 1.33 & 93.73 & 13.33 & $n / a$ \\
\hline$\$ 967005049$ & & Chloride-IC-Dionex $4000 / 4500$ & $\mathrm{ug} / \mathrm{mL}$ & 92.91 & $<1.70 \mathrm{e}-02$ & $2.48 \mathrm{e}+02$ & 257.0 & 252.3 & 3.56 & 92.03 & 18.89 & $n / a$ \\
\hline S967005049 & & Nitrite-1C - Dionex $4000 / 4500$ & $\mathrm{ug} / \mathrm{mL}$ & 91.67 & $<1.08 \mathrm{e}-01$ & $1.52 \mathrm{e}+03$ & $1.53 e+03$ & $1.52 e+03$ & 0.66 & 93.15 & 120.0 & $\mathrm{n} / \mathrm{a}$ \\
\hline S96T005049 & & Bromide by Ion chromatograph & ug/mL & 94.40 & $<1.25 \mathrm{e}-01$ & $<1.39 \mathrm{e}+02$ & $<1.39 \mathrm{e} 2$ & $n / a$ & $\mathrm{n} / \mathrm{a}$ & 91.00 & 138.9 & $\mathrm{n} / \mathrm{a}$ \\
\hline S96T005049 & & Nitrate by IC-Dionex $4000 / 4500$ & $\mathrm{ug} / \mathrm{mL}$ & 100.3 & $2.13 e-01$ & $3.39 \mathrm{e}+04$ & $3.35 \mathrm{e}+04$ & $3.37 \mathrm{e}+04$ & 1.19 & 96.63 & 154.4 & $n / a$ \\
\hline S96T005049 & & Phosphate-1C-Dionex $4000 / 4500$ & $\mathrm{ug} / \mathrm{mL}$ & 95.96 & $<1.20 \mathrm{e}-01$ & $<1.33 \mathrm{e}+02$ & $<1.33 \mathrm{e} 2$ & $\mathrm{n} / \mathrm{a}$ & $\mathrm{n} / \mathrm{a}$ & 98.72 & 133.3 & $n / a$ \\
\hline S96T005049 & & Sulfate by IC-Dionex $4000 / 4500$ & $\mathrm{ug} / \mathrm{mL}$ & 101.9 & $<1.38 \mathrm{e}-01$ & $4.12 \mathrm{e}+02$ & 399.0 & 405.2 & 3.21 & 101.4 & 153.3 & $\mathrm{n} / \mathrm{a}$ \\
\hline S96T005049 & & Oxalate by IC-Dionex $4000 / 4500$ & $\mathrm{ug} / \mathrm{mL}$ & 100.0 & $<1.05 \mathrm{e}-01$ & $<1.17 e+02$ & $<1.17 \mathrm{e} 2$ & $\mathrm{n} / \mathrm{a}$ & $\mathrm{n} / \mathrm{a}$ & 103.8 & 116.7 & $n / a$ \\
\hline S96T005049 & & Cobal $t-60$ by GEA & $\mathrm{uCi} / \mathrm{mL}$ & 101.4 & $<2.15 e-05$ & $2.07 \mathrm{e}-03$ & $2.11 \mathrm{e}-03$ & $2.09 e-03$ & 1.91 & $n / a$ & $n / a$ & 12.6 \\
\hline \$96T005049 & & Cesium- 137 by GEA & $u \mathrm{Ci} / \mathrm{mL}$ & 102.3 & $3.69 e-05$ & 6.460 & 6.660 & 6.560 & 3.05 & $\mathrm{n} / \mathrm{a}$ & $\mathrm{n} / \mathrm{a}$ & 0.170 \\
\hline S96T005049 & & Am-241 by Extraction & $\mathrm{uCi} / \mathrm{mL}$ & 90.76 & $<6.400-05$ & $<6.44 \mathrm{e}-05$ & $7.75 e-05$ & n/a & n/a & $n / a$ & $6.44 e-05$ & $5.67 E+00$ \\
\hline S96T005049 & & Alpha in Liquid Samples & $u C \mathrm{i} / \mathrm{mL}$ & 93.51 & $<4.00 \mathrm{e}-04$ & $3.97 e-03$ & $3.90 \mathrm{e}-03$ & $3.93 \mathrm{e}-03$ & 1.78 & $\mathrm{n} / \mathrm{a}$ & $1.00 \mathrm{e}-03$ & $2.76 E+01$ \\
\hline
\end{tabular}

Sludge (from Liquid Grab Sample): Sludge (from Liquid Grab Sample)

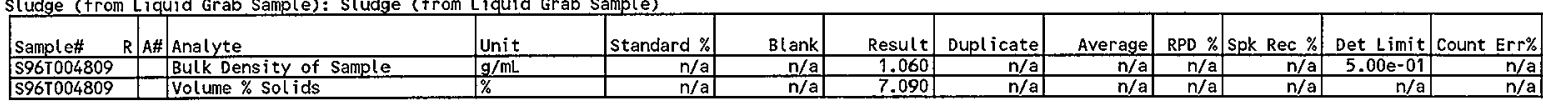

n/a


RISER: $10 \mathrm{~A}$

SEGMENT \#: 5 AW-96-7

SEGMENT PORTION: Centrifuged Solids (Grab Sample)

\begin{tabular}{|c|c|c|c|c|c|c|c|c|c|c|c|c|}
\hline Samole\# & A\# & Analyte & Unit & Standard \% & B lank & Result & Duplicate & Average & RPD \% & Spk Rec \% & Det Limit & Count Err\% \\
\hline S96T005020 & & Bulk Density of Sample & $\mathrm{g} / \mathrm{mL}$ & $n / a$ & $\mathrm{n} / \mathrm{a}$ & 1.320 & $\mathrm{n} / \mathrm{a}$ & $n / a$ & $n / a$ & $n / a$ & $5.00 \mathrm{e}-01$ & $n / a$ \\
\hline 5967005020 & & DSC Exotherm on Perkin Elmer & Joules $/ \mathrm{g}$ & 97.12 & n/a & $0.00 \mathrm{e}+00$ & $0.00 \mathrm{e}+00$ & $0.00 \mathrm{e}+00$ & 0.00 & $\mathrm{n} / \mathrm{a}$ & $n / a$ & $n / a$ \\
\hline 5967005020 & & pH on SST Samples & $\mathrm{pH}$ & 99.62 & $\mathrm{n} / \mathrm{a}$ & 12.26 & 12.25 & 12.25 & 0,08 & $\mathrm{n} / \mathrm{a}$ & $1.00 \mathrm{e}-02$ & $\mathrm{n} / \mathrm{a}$ \\
\hline 5967005020 & & $\%$ Water by TGA on Perkin Elmer & $\%$ & 100.7 & $\mathrm{n} / \mathrm{a}$ & 64.68 & 66.26 & 65.47 & 2.41 & $\mathrm{n} / \mathrm{a}$ & $\mathrm{n} / \mathrm{a}$ & $n / a$ \\
\hline S967005020 & & TIC by Acid/Coulometry & $u g / g$ & 100.8 & $7.00 \mathrm{e}-01$ & $9.69 \mathrm{e}+02$ & 870.0 & 919.5 & 10.8 & $\mathrm{n} / \mathrm{a}$ & 5.000 & $n / a$ \\
\hline S96T005020 & & TOC by Persulfate/Coulometry & $\mathrm{ug} / \mathrm{g}$ & 89.67 & 13.50 & $1.77 \mathrm{e}+03$ & $1.72 \mathrm{e}+03$ & $1.74 \mathrm{e}+03$ & 2.87 & $n / a$ & 40.00 & $\mathrm{n} / \mathrm{a}$ \\
\hline 5967005053 & F & Strontium- $89 / 90$ High Level & $\mathrm{uCi} / \mathrm{g}$ & 104.3 & $1.70 e^{-02}$ & 65.20 & 65.00 & 65.10 & 0.31 & $\mathrm{n} / \mathrm{a}$ & $3.00 \mathrm{e}-03$ & $2.54 E-01$ \\
\hline S96T005053 & $F$ & PU-239/240 by TRU-SPEC Resin & $\mathrm{uCj} / \mathrm{g}$ & 97.31 & $<3.09 e-03$ & 1.640 & 1.670 & 1.655 & 1.81 & $\mathrm{n} / \mathbf{a}$ & $7.10 \mathrm{e}-02$ & $9.36 \mathrm{E}+00$ \\
\hline $596 \mathrm{T005053}$ & $F$ & Silver-ICP-Fusion & $\mathrm{ug} / \mathrm{g}$ & 97.80 & $<1.00 \mathrm{e}-02$ & $4.24 \mathrm{e}+02$ & 366.0 & 395.0 & 14.7 & $n / a$ & 194.0 & $n / a$ \\
\hline S96T005053 & $F$ & Aluminium-ICP-Fusion & ug/g & 97.00 & $<5.00 e-02$ & $1.22 \mathrm{e}+03$ & 999.0 & $1.11 \mathrm{e}+03$ & 19.9 & $n / a$ & 968.0 & $\mathrm{n} / \mathrm{a}$ \\
\hline S96T005053 & $F$ & Arsenic-ICP-Fusion & $\mathrm{ug} / \mathrm{g}$ & 100.8 & $<1.00 e-01$ & $<1.94 \mathrm{e}+03$ & $<1.94 \mathrm{e} 3$ & $\mathrm{n} / \mathbf{a}$ & $\mathrm{n} / \mathrm{a}$ & $\mathrm{n} / \mathrm{a}$ & $1.94 \mathrm{e}+03$ & $\mathrm{n} / \mathrm{a}$ \\
\hline S96r005053 & $F$ & Boron-ICP-Fusion & ug/g & 99.20 & $<5.00 e-02$ & $<9.69 \mathrm{e}+02$ & $<9.70 \mathrm{e} 2$ & $\mathrm{n} / \mathrm{a}$ & $\mathrm{n} / \mathrm{a}$ & $n / a$ & 968.0 & $\mathrm{n} / \mathrm{a}$ \\
\hline S96T005053 & $\mathrm{F}$ & Barium-ICP-Fusion & ug/g & 99.20 & $<5.00 \mathrm{e}-02$ & $49.69 e+02$ & $<9.70 \mathrm{e} 2$ & $n / a$ & $n / a$ & $n / a$ & 968.0 & $n / a$ \\
\hline $596 \mathrm{~T} 005053$ & $F$ & Beryllium -ICP-Fusion & $\mathrm{ug} / \mathrm{g}$ & 101.0 & $45.00 e^{-03}$ & $<\quad 96.90$ & $<9.70 \mathrm{e} 1$ & $n / a$ & $\mathrm{n} / \mathrm{a}$ & $n / a$ & 96.80 & $n / a$ \\
\hline \$96 T005053 & $F$ & Bismuth -ICP-Fusion & ug/g & 100.6 & $<1.00 \mathrm{e}-01$ & $<1.94 e+03$ & $<1.94 \mathrm{e} 3$ & $n / a$ & $\mathrm{n} / \mathrm{a}$ & $n / a$ & $1.94 \mathrm{e}+03$ & $n / a$ \\
\hline $596 \mathrm{~T} 005053$ & $F$ & Calcium-ICP-Fusion & $\mathrm{ug} / \mathrm{g}$ & 95.40 & $<1.00 \mathrm{e}-01$ & $<1.94 \mathrm{e}+03$ & $\leq 1.94 \mathrm{e} 3$ & $\mathrm{n} / \mathrm{a}$ & $\mathrm{n} / \mathrm{a}$ & $\mathrm{n} / \mathrm{a}$ & $1.94 \mathrm{e}+03$ & $n / a$ \\
\hline S96T005053 & $F$ & Cadmium-ICP-Fusion & $4 \mathrm{~g} / \mathrm{g}$ & 98.60 & $<5.00 e-03$ & $1.10 e+02$ & $<9.70 \mathrm{e} 1$ & $n / a$ & $n / a$ & $n / a$ & 96.80 & $\mathrm{n} / \mathrm{a}$ \\
\hline S96T005053 & $F$ & Cerium-ICP-Fusion & $\mathrm{ug} / \mathrm{g}$ & 97.60 & $\angle 1.00 \mathrm{e}-01$ & $<1.94 \mathrm{e}+03$ & $<1.94 \mathrm{e}^{3}$ & $\mathrm{n} / \mathrm{a}$ & $\mathrm{n} / \mathrm{a}$ & $\mathrm{n} / \mathrm{a}$ & $1.94 \mathrm{e}+03$ & $n / a$ \\
\hline s96r005053 & $F$ & Cobalt -ICP-Fusion & $\mathrm{ug} / \mathrm{g}$ & 98.60 & $<2.00 \mathrm{e}-02$ & $<3.88 \mathrm{e}+02$ & $\leq 3.88 \mathrm{e} 2$ & $n / a$ & $n / a$ & $\mathrm{n} / \mathrm{a}$ & 388.0 & $n / a$ \\
\hline 5967005053 & $F$ & Chromium-ICP-Fusion & ug/g & 99.00 & $<1.00 \mathrm{e}-02$ & $2.31 e+02$ & $<1.94 \mathrm{e} 2$ & $\mathrm{n} / \mathrm{a}$ & $n / a$ & $n / a$ & 194.0 & $n / a$ \\
\hline S96T005053 & $F$ & Copper -ICP-Fusion & $\mathrm{ug} / \mathrm{g}$ & 99.40 & $<1.00 \mathrm{e}-02$ & $<1.94 \mathrm{e}+02$ & $<1.94 \mathrm{e} 2$ & $n / a$ & $\mathrm{n} / \mathrm{a}$ & $n / a$ & 194.0 & $\mathrm{n} / \mathrm{a}$ \\
\hline \$96T005053 & $F$ & Iron-ICP-Fusion & $\mathrm{ug} / \mathrm{g}$ & 99.40 & $<5.00 e-02$ & $1.27 e+03$ & $3.26 \mathrm{e}+03$ & $2.26 \mathrm{e}+03$ & 87.9 & $\mathrm{n} / \mathrm{a}$ & 968.0 & $\mathrm{n} / \mathrm{a}$ \\
\hline 5967005053 & $F$ & Lanthanum -ICP-Fusion & $\mathrm{ug} / \mathrm{g}$ & 99.40 & $<5.00 \mathrm{e}-02$ & $1.56 \mathrm{e}+03$ & $1.58 \mathrm{e}+03$ & $1.57 \mathrm{e}+03$ & 1.27 & $\mathrm{n} / \mathrm{a}$ & 968.0 & $n / a$ \\
\hline $596 T 005053$ & $\bar{F}$ & Lithium -ICP-Fusion & $\mathrm{ug} / \mathrm{g}$ & 97.80 & $<1.00 e-02$ & $<1.94 \mathrm{e}+02$ & $<1.94 \mathrm{e} 2$ & $\mathrm{n} / \mathrm{a}$ & $\mathrm{n} / \mathrm{a}$ & $n / a$ & 194.0 & $n / a$ \\
\hline 5967005053 & $F$ & Magnesium -ICP-Fusion & ug $/ \mathrm{g}$ & 95.20 & $<7.00 \mathrm{e}-01$ & $<1.94 \mathrm{e}+03$ & $<1.94 \mathrm{e} 3$ & $\mathrm{n} / \mathrm{a}$ & $\mathrm{n} / \mathrm{a}$ & $\mathrm{n} / \mathrm{a}$ & $1.94 e+03$ & $n / a$ \\
\hline S96T005053 & $F$ & Manganese - ICP-Fusion & $\mathrm{ug} / \mathrm{g}$ & 98.20 & $<1.00 e-02$ & $9.88 \mathrm{e}+02$ & 967.0 & 977.5 & 2.15 & $\mathrm{n} / \mathrm{a}$ & 194.0 & $n / a$ \\
\hline $596 T 005053$ & $F$ & Molybdenum - ICP-Fusion & ug/g & 99.00 & $<5.00 \mathrm{e}-02$ & $<9.69 \mathrm{e}+02$ & $<9.70 \mathrm{e} 2$ & $n / a$ & $n / a$ & $n / a$ & 968.0 & $\mathrm{n} / \mathrm{a}$ \\
\hline$\$ 967005053$ & $\mathrm{~F}$ & Sodium -ICP-Fusion & $\mathrm{ug} / \mathrm{g}$ & 95.80 & 2.250 & $9.87 e+04$ & $9.14 \mathrm{e}+04$ & $9.50 \mathrm{e}+04$ & 7.68 & $\mathrm{n} / \mathrm{a}$ & $1.94 \mathrm{e}+03$ & $n / a$ \\
\hline S96T005053 & $F$ & Neodymium-ICP-Fusion & $\mathrm{ug} / \mathrm{g}$ & 101.2 & $<1.00 \mathrm{e}-01$ & $<1.94 \mathrm{e}+03$ & $<1.94 \mathrm{e} 3$ & $n / a$ & $\mathrm{n} / \mathrm{a}$ & $n / a$ & $1.94 \mathrm{e}+03$ & $\mathrm{n} / \mathrm{a}$ \\
\hline S96T005053 & $F$ & Phosphorus -ICP-Fusion & ug/g & 99.20 & $\angle 2.00 \mathrm{e}-01$ & $<3.88 \mathrm{e}+03$ & $<3.88 \mathrm{e} 3$ & $\mathrm{n} / \mathrm{a}$ & $\mathrm{n} / \mathrm{a}$ & $n / a$ & $3.88 \mathrm{e}+03$ & $n / a$ \\
\hline$\$ 96 T 005053$ & $\bar{F}$ & Lead -ICP-Fusion & $\mathrm{ug} / \mathrm{g}$ & 98.60 & $<1.00 e-01$ & $<1.94 \mathrm{e}+03$ & $<1.94 \mathrm{e} 3$ & $n / a$ & $n / a$ & $\mathrm{n} / \mathrm{a}$ & $1.94 \mathrm{e}+03$ & $n / a$ \\
\hline $596 T 005053$ & $F$ & Sulfur -ICP-Fusion & $\mathrm{ug} / \mathrm{g}$ & 98.60 & $\leq 1.00 \mathrm{e}-01$ & $<1.94 \mathrm{e}+03$ & $<1.94 \mathrm{e} 3$ & $\mathrm{n} / \mathrm{a}$ & $n / a$ & $\mathrm{n} / \mathrm{a}$ & $1.94 \mathrm{e}+03$ & $n / a$ \\
\hline $596 T 005053$ & $F$ & Antimony -ICP-Fusion & $\mathrm{ug} / \mathrm{g}$ & 90.20 & $<6.00 e-02$ & $<1.16 \mathrm{e}+03$ & $1.30 \mathrm{e}+03$ & $\mathrm{n} / \mathrm{a}$ & $n / a$ & $\mathrm{n} / \mathrm{a}$ & $1.16 \mathrm{e}+03$ & $\mathrm{n} / \mathrm{a}$ \\
\hline S96T005053 & $F$ & Selenium-ICP-Fusion & $u g / g$ & 105.0 & $<1.00 \mathrm{e}-01$ & $<1.94 \mathrm{e}+03$ & $<1.94 \mathrm{e} 3$ & $\mathrm{n} / \mathrm{a}$ & $\mathrm{n} / \mathrm{a}$ & $n / a$ & $1.94 \mathrm{e}+03$ & $n / a$ \\
\hline S967005053 & $F$ & Silicon-ICP-Fusion & $\mathrm{ug} / 9$ & 98.40 & $55.00 \mathrm{e}-02$ & $1.03 e+03$ & $1.18 \mathrm{e}+03$ & $1.10 \mathrm{e}+03$ & 13.6 & $n / a$ & 968.0 & $n / a$ \\
\hline 5961005053 & $F$ & Samarium-ICP-Fusion & $u g / g$ & 101.0 & $<1.00 \mathrm{e}-01$ & $<1.94 \mathrm{e}+03$ & $<1.94 \mathrm{e} 3$ & $n / a$ & $\mathrm{n} / \mathrm{a}$ & $\mathrm{n} / \mathrm{a}$ & $1.94 \mathrm{e}+03$ & $\mathrm{n} / \mathrm{a}$ \\
\hline S96T005053 & $F$ & Strontium -ICP-Fusion & ug $/ g$ & 98.40 & $<1.00 \mathrm{e}-02$ & $<1.94 \mathrm{e}+02$ & $<1.94 \mathrm{e} 2$ & $n / a$ & $n / a$ & $n / a$ & 194.0 & $n / a$ \\
\hline $596 \mathrm{~T} 005053$ & $F$ & Titanium+ICP-Fusion & $u g / g$ & 96.00 & $<1.00 \mathrm{e}-02$ & $<1.94 \mathrm{e}+02$ & $<1.94 \mathrm{e} 2$ & $n / a$ & $\mathrm{n} / \mathrm{a}$ & $\mathrm{n} / \mathrm{a}$ & 194.0 & $\mathrm{n} / \mathrm{a}$ \\
\hline 596T005053 & $F$ & Thall lium - ICP-Fusion & $\mathrm{ug} / \mathrm{g}$ & 97.20 & $<2,00 \mathrm{e}-01$ & $<3.88 \mathrm{e}+03$ & $<3.88 \mathrm{e} 3$ & $\mathrm{n} / \mathrm{a}$ & $\mathrm{n} / \mathrm{a}$ & $\mathrm{n} / \mathrm{a}$ & $3.88 e+03$ & $n / a$ \\
\hline $596 \mathrm{Y} 005053$ & $F$ & Uranium -ICP-Fusion & ug/g & 97.10 & $<5.00 \mathrm{e}-01$ & $4.29 \mathrm{e}+04$ & $4.47 e+04$ & $4.38 \mathrm{e}+04$ & 4.11 & $n / a$ & $9.68 \mathrm{e}+03$ & $\mathrm{n} / \mathrm{a}$ \\
\hline S96r005053 & $F$ & Vanadium-ICP-Fusion & $\mathrm{ug} / \mathrm{g}$ & 99.80 & $<5.00 \mathrm{e}-02$ & $<9.69 \mathrm{e}+02$ & $<9.70 \mathrm{e} 2$ & $n / a$ & $n / a$ & $\mathrm{n} / \mathrm{a}$ & 968.0 & $n / a$ \\
\hline $596 \mathrm{~T} 005053$ & $F$ & Zinc -ICP-Fusion & $\mathrm{ug} / \mathrm{g}$ & 99.40 & $1.60 \mathrm{e}-02$ & $2.58 \mathrm{e}+02$ & 403.0 & 330.5 & 43.9 & $\mathrm{n} / \mathrm{a}$ & 194.0 & $\mathrm{n} / \mathrm{a}$ \\
\hline$\$ 961005053$ & $F$ & Zirconium -ICP-Fusion & ug/g & 98.00 & $<1.00 \mathrm{e}-02$ & $8.44 \mathrm{e}+104$ & $8.40 \mathrm{e}+04$ & $8.42 \mathrm{e}+04$ & 0.48 & $\mathrm{n} / \mathrm{a}$ & 194.0 & $\mathrm{n} / \mathrm{a}$ \\
\hline $596 \mathrm{~T} 005053$ & $\mathrm{~F}$ & Cobal $t-60$ by GEA & $\mathrm{uCi} / \mathrm{g}$ & 97.35 & $<2.77 e-02$ & $9.60 e^{-02}$ & $7.43 e^{-02}$ & $8.51 \mathrm{e}^{-02}$ & 25.5 & $n / a$ & $\mathrm{n} / \mathrm{a}$ & 28.5 \\
\hline
\end{tabular}




\begin{tabular}{|c|c|c|c|c|c|c|c|c|c|c|c|c|}
\hline Sample\# & $\mathbf{R}$ A\# & Analyte & Unit & Standard \% & Blank & Result & Dupl icate & Average & RPD \% & Spk Rec \% & Det Limit & Count Err\% \\
\hline $596 \mathrm{~T} 005053$ & $\mathrm{~F}$ & Cesium- 137 by GEA & $u C i / g$ & 95.75 & $<6.17 e-02$ & 20.66 & 20.60 & 20.63 & 0.48 & $n / a$ & $n / a$ & 1.65 \\
\hline 5967005053 & $\mathrm{~F}$ & Am-241 by Extraction & $\mathrm{LCi} / \mathrm{g}$ & 89.92 & $<5.46 \mathrm{e}-03$ & $3.87 e-01$ & $3.81 \mathrm{e}-01$ & $3.84 e^{-01}$ & 1.56 & $\mathrm{n} / \mathrm{a}$ & $5.10 e^{-02}$ & $1.72 E+00$ \\
\hline S96T005053 & $F$ & Alpha of Digested solid & uCi $/ \mathrm{g}$ & 88.75 & $3.00 \mathrm{e}-03$ & 1.740 & 1.830 & 1.785 & 5.04 & $\mathrm{n} / \mathrm{a}$ & $2.10 \mathrm{e}-02$ & $5.55 \mathrm{E}+00$ \\
\hline $596 \mathrm{~T} 005056$ & W & OH- by Pot. Titration & $u g / g$ & 97.36 & $<42.00$ & 42.00 & $<42$ & $\mathrm{n} / \mathrm{a}$ & $\mathrm{n} / \mathrm{a}$ & $\mathrm{n} / \mathrm{a}$ & 41.70 & $n / a$ \\
\hline S96T0 & $\mathbf{W}$ & Fluoride-IC-Dionex $4000 / 4500$ & $u g / g$ & 90.51 & $<1.20 \mathrm{e}-02$ & $3.84 \mathrm{e}+04$ & $3.48 \mathrm{e}+04$ & $3.66 \mathrm{e}+04$ & 9.84 & $\mathrm{n} / \mathrm{a}$ & 116.3 & $n / a$ \\
\hline S96T005056 & W & Chloride-IC-Dionex $4000 / 4500$ & $u g / g$ & 91.52 & $2.00 \mathrm{e}-02$ & 1.75 et 02 & $<1.66 \mathrm{e} 2$ & $n / a$ & $n / a$ & $\mathrm{n} / \mathrm{a}$ & 164.8 & $n / a$ \\
\hline $596 \mathrm{~T} 005056$ & $\mathbf{W}$ & Nitrite-IC - Dionex $4000 / 4500$ & $\mathrm{ug} / \mathrm{g}$ & 91.67 & $<1.08 \mathrm{e}-01$ & $3.68 \mathrm{e}+03$ & $3.52 \mathrm{e}+03$ & $3.60 \mathrm{e}+03$ & 4.44 & $\mathrm{n} / \mathrm{a}$ & $1.05 \mathrm{e}+03$ & $n / a$ \\
\hline 5967005056 & W & Bromide by Ion chromatograph & ug/g & 94.57 & $<1.25 \mathrm{e}^{-01}$ & $<1.21 e+03$ & $<1.22 \mathrm{e}^{3}$ & $\mathrm{n} / \mathrm{a}$ & $\mathrm{n} / \mathrm{a}$ & $\mathrm{n} / \mathrm{a}$ & $1.21 \mathrm{e}+03$ & $\mathrm{n} / \mathrm{a}$ \\
\hline$\$ 96 T 005056$ & W & Nitrate by IC-Dionex $4000 / 4500$ & $49 / 9$ & 100.0 & $1.79 \mathrm{e}-01$ & $1.86 e+04$ & $1.69 \mathrm{e}+04$ & $1.78 \mathrm{e}+04$ & 9.58 & $\mathrm{n} / \mathrm{a}$ & $1.35 \mathrm{e}+03$ & $n / a$ \\
\hline 5967005056 & $\overline{\mathrm{w}}$ & Phosphate-1C-Dionex $4000 / 4500$ & $u g / g$ & 95.78 & $<1.20 \mathrm{e}-01$ & $<1.16 e+03$ & $<1.17 \mathrm{e} 3$ & $\mathrm{n} / \mathrm{a}$ & $\mathrm{n} / \mathrm{a}$ & $\mathrm{n} / \mathrm{a}$ & $1.16 \mathrm{e}+03$ & $\mathrm{n} / \mathrm{a}$ \\
\hline 5967005056 & W & Sulfate by IC-Dionex $4000 / 4500$ & ug /g & 101.6 & $<1.38 \mathrm{e}-01$ & $1.54 \mathrm{e}+03$ & $1.73 e+03$ & $1.63 \mathrm{e}+03$ & 11.6 & $n / a$ & $1.34 \mathrm{e}+03$ & $n / a$ \\
\hline$\$ 967005056$ & W & Oxalate by IC-Dionex $4000 / 4500$ & $\mathrm{ug} / \mathrm{g}$ & 99.24 & $<1.05 e-01$ & $<1.02 e+03$ & $<1.03 \mathrm{e} 3$ & $n / a$ & $\mathrm{n} / \mathrm{a}$ & $n / a$ & $1.02 \mathrm{e}+03$ & \\
\hline
\end{tabular}

\section{Interstitial Liquid: Interstitial Liquid}

\begin{tabular}{|c|c|c|c|}
\hline Sample\# & $\mathbf{R}$ & A\# & Analyte \\
\hline $596 \mathrm{~T} 005050$ & & & Bulk Density of Sample \\
\hline$\$ 96 \mathrm{~T} 005050$ & & & DSC Exotherm on Perkin Elmer \\
\hline$\$ 96 \mathrm{~T} 005050$ & & & OH- by Pot. Titration \\
\hline $596 T 005050$ & & & pH Direct \\
\hline $596 \mathrm{~T} 005050$ & & & $\%$ Water by TGA on Perkin Elmer \\
\hline S96T005050 & & & TIC by Acid/Coulometry \\
\hline$\$ 961005050$ & & & Tot. Organic Carbon by coul. \\
\hline 5961005050 & & & Strontium-89/90 Hīgh Level \\
\hline$\$ 967005050$ & & & PU-239/240 by TRU-SPEC Res in \\
\hline$\$ 967005050$ & & & Silver-ICP-Acid Dil. \\
\hline 5967005050 & & & Aluminium-ICP-Acid Dil. \\
\hline$\$ 967005050$ & & & Arsenic-ICP-Acid Dil. \\
\hline 5967005050 & & & Boron-ICP-Acid Dil. \\
\hline$\$ 967005050$ & & & Barium-ICP-Acid Dil. \\
\hline$\$ 967005050$ & & & Beryllium-ICP-Acid Dil. \\
\hline $596 \mathrm{~T} 005050$ & & & Bismuth-ICP-Acid Dil. \\
\hline 5967005050 & & & Calcium-ICP-Acid Dil. \\
\hline 5967005050 & & & Cadmium-ICP-Acid Dil. \\
\hline 5967005050 & & & Cerium-ICP-Acid Dil. \\
\hline $596 \mathrm{~T} 005050$ & & & Cobalt-ICP-Acid Dil. \\
\hline 5967005050 & & & Chromium-ICP-Acid Dil. \\
\hline 5961005050 & & & Copper-ICP-ACid Dil. \\
\hline 5967005050 & & & Iron-ICP-Acid Dil. \\
\hline $596 \mathrm{~T} 005050$ & & & Potassium-ICP-Acid Dil. \\
\hline $596 \mathrm{~T} 005050$ & & & Lanthanum- ICP-Acid Dil. \\
\hline $596 \mathrm{~T} 005050$ & & & Lithium-ICP-Acid Dil. \\
\hline $596 r 005050$ & & & Magnesium-ICP-Acid DiE. \\
\hline $596 \mathrm{~T} 005050$ & & & Manganese-ICP-Acid DiE. \\
\hline $596 \mathrm{~T} 005050$ & & & Molybdenum-ICP-Acid Dil \\
\hline $596 \mathrm{~T} 005050$ & & & Sodium-ICP-Acid Dil. \\
\hline $596 \mathrm{~T} 005050$ & & & Neodymiun-ICP-Acid Dil. \\
\hline S96T005050 & $\bar{D}$ & & Mickel-ICP-Ácid Dil. \\
\hline $596 \mathrm{~T} 005050$ & & & Phosphorus-ICP-Acid Dil. \\
\hline$\$ 96 \mathrm{~T} 005050$ & & D & Lead-ICP-Acid Dil. \\
\hline
\end{tabular}

\begin{tabular}{l|l|l}
5961005050 & D & Lead-ICP-Acid Dil. \\
\hline 5961005050 & D & Sul fur-ICP-Acid Dil
\end{tabular}

\begin{tabular}{|c|c|c|c|c|c|c|c|c|c|}
\hline Unit & Standard \% & Blank & Resul t & Dupl icate & Average & RPD \% & Spk Rec \% & Det Limit & Count Err\% \\
\hline $\mathrm{g} / \mathrm{mL}$ & $n / a$ & $n / a$ & 1.050 & $n / a$ & $n / a$ & $n / a$ & $\mathrm{n} / \mathrm{a}$ & $5.00 \mathrm{e}-01$ & $n / a$ \\
\hline Joules $/ g$ & 92.30 & $\mathrm{n} / \mathrm{a}$ & $0.00 \mathrm{e}+00$ & $0.00 e+00$ & $0.00 \mathrm{e}+00$ & 0.00 & $n / a$ & $n / a$ & $\mathrm{n} / \mathrm{a}$ \\
\hline $\mathrm{ug} / \mathrm{mL}$ & 106.4 & $<42.00$ & $8.64 e+03$ & $8.53 \mathrm{e}+03$ & $8.58 \mathrm{e}+03$ & 1.28 & $\mathrm{n} / \mathrm{a}$ & 500.0 & $n / a$ \\
\hline $\mathrm{pH}$ & 100.0 & $\mathrm{n} / \mathrm{a}$ & 13.45 & 13.49 & 13.47 & 0.30 & $n / a$ & $1.00 \mathrm{e}-02$ & n/a \\
\hline$\%$ & 99.27 & $n / a$ & 88.66 & 88.43 & 88.55 & 0.26 & $n / a$ & $n / a$ & $n / a$ \\
\hline $\mathrm{ug} / \mathrm{mL}$ & 100.2 & $6.00 \mathrm{e}-01$ & $8.50 \mathrm{e}+02$ & 841.0 & 845.5 & 1.06 & $n / a$ & 5.000 & $n / a$ \\
\hline $\mathrm{ug} / \mathrm{mL}$ & 106.3 & 1.200 & $2.57 \mathrm{e}+03$ & $2.51 \mathrm{e}+03$ & $2.54 \mathrm{e}+03$ & 2.36 & $\mathrm{n} / \mathrm{a}$ & 55.00 & $n / a$ \\
\hline $\mathrm{UC \textrm {i }} / \mathrm{mL}$ & 97.14 & $5.89 e^{-05}$ & $1.72 \mathrm{e}-03$ & $2.32 \mathrm{e}-03$ & $2.02 e-03$ & 29.7 & $n / a$ & $1.18 \mathrm{e}-05$ & $3.26 \mathrm{E}+00$ \\
\hline $\mathrm{UCi} / \mathrm{mL}$ & 90.60 & $<7.39 \mathrm{e}-06$ & $1.82 \mathrm{e}-03$ & $1.91 \mathrm{e}-03$ & $1.86 \mathrm{e}-03$ & 4.83 & $n / a$ & $1.82 \mathrm{e}-04$ & $1.77 \mathrm{E}+00$ \\
\hline $\mathrm{ug} / \mathrm{mL}$ & 99.20 & $<1.00 \mathrm{e}-02$ & 2.730 & 2.820 & 2.775 & 3.24 & 95.60 & 1.010 & $\mathrm{n} / \mathrm{a}$ \\
\hline $\mathrm{Ug} / \mathrm{mL}$ & 99.80 & $<5.00 e^{-02}$ & $6.96 \mathrm{e}+02$ & 713.0 & 704.5 & 2.41 & 109.0 & 5.050 & $n / a$ \\
\hline $\mathrm{ug} / \mathrm{mL}$ & 102.4 & $<1.00 \mathrm{e}-01$ & $<\quad 10.10$ & $<1.01 \mathrm{e} 1$ & $\mathrm{n} / \mathrm{a}$ & $\mathrm{n} / \mathrm{a}$ & 114.0 & 10.10 & $\mathrm{n} / \mathrm{a}$ \\
\hline $\mathrm{Ug} / \mathrm{mL}$ & 99.80 & $<5.00 \mathrm{e}-02$ & 5.930 & 6.180 & 6.055 & 4.13 & 98.30 & 5.050 & $\mathrm{n} / \mathrm{a}$ \\
\hline $\mathrm{ug} / \mathrm{mL}$ & 100.6 & $<5.00 \mathrm{e}-02$ & 5.050 & $<5.0500$ & $n / a$ & $\mathrm{n} / \mathrm{a}$ & 101.0 & 5.050 & $n / a$ \\
\hline $\mathrm{ug} / \mathrm{mL}$ & 102.4 & $<5.00 \mathrm{e}-03$ & 1.360 & 1.380 & 1.370 & 1.46 & 102.0 & $5.05 e-01$ & $\mathrm{n} / \mathrm{a}$ \\
\hline $\mathrm{ug} / \mathrm{mL}$ & 100.4 & $<1.00 \mathrm{e}-01$ & 10.10 & $<1.01 \mathrm{e} 1$ & $n / a$ & $\mathrm{n} / \mathrm{a}$ & 100.0 & 10.10 & $n / a$ \\
\hline $\mathrm{ug} / \mathrm{mL}$ & 100.0 & $<1.00 \mathrm{e}-01$ & $<\quad 10.10$ & $<1.01 \mathrm{e}$ & $n / a$ & $\mathrm{n} / \mathrm{a}$ & 103.0 & 10.10 & $n / a$ \\
\hline $\mathrm{ug} / \mathrm{mL}$ & 100.6 & $<5.00 \mathrm{e}-03$ & $<5.05 \mathrm{e}-01$ & $<5.05 \mathrm{e}-1$ & $n / a$ & n/a & 101.0 & $5.05 e^{-01}$ & $n / a$ \\
\hline $\mathrm{ug} / \mathrm{mL}$ & 99.00 & $<1.00 \mathrm{e}-01$ & $<\quad 10.10$ & $<1.01 \mathrm{e} 1$ & n/a & $\mathrm{n} / \mathrm{a}$ & 100.0 & 10.10 & $n / a$ \\
\hline $\mathrm{ug} / \mathrm{mL}$ & 101.2 & $<2.00 e^{-02}$ & 2.020 & $<2.02 \mathrm{e} 0$ & $\mathrm{n} / \mathrm{a}$ & $\mathrm{n} / \mathrm{a}$ & 102.0 & 2.020 & $n / a$ \\
\hline $\mathrm{ug} / \mathrm{mL}$ & 101.2 & $<1.00 \mathrm{e}-02$ & 28.10 & 28.60 & 28,35 & 1.76 & 103.0 & 1.010 & $\mathrm{n} / \mathrm{a}$ \\
\hline $\mathrm{ug} / \mathrm{mL}$ & 104.0 & $<1.00 \mathrm{e}-02$ & 1.010 & $<1.01 \mathrm{e} 0$ & $\mathrm{n} / \mathrm{a}$ & $\mathrm{n} / \mathrm{a}$ & 105.0 & 1.030 & $\mathrm{n} / \mathrm{a}$ \\
\hline $\mathrm{ug} / \mathrm{mL}$ & 100.6 & $<5.00 \mathrm{e}-02$ & 5.050 & $<5.05 \mathrm{e} 0$ & $n / a$ & $\mathrm{n} / \mathrm{a}$ & 102.0 & 5.050 & $\mathrm{n} / \mathrm{a}$ \\
\hline $\mathrm{ug} / \mathrm{mL}$ & 94.40 & $<5.00 e^{-01}$ & $1.08 \mathrm{e}+04$ & $1.11 \mathrm{e}+04$ & $1.10 \mathrm{e}+04$ & 2.74 & 333.0 & 50.50 & $\mathrm{n} / \mathrm{a}$ \\
\hline $\mathrm{ug} / \mathrm{mL}$ & 100.8 & $<5.00 \mathrm{e}-02$ & $<\quad 5.050$ & 5.950 & $\mathrm{n} / \mathrm{a}$ & $\mathrm{n} / \mathrm{a}$ & 108.0 & 5.050 & n/a \\
\hline $\mathrm{Lg} / \mathrm{mL}$ & 101.2 & $<1.00 \mathrm{e}-02$ & 1.010 & $<1.01 \mathrm{e} 0$ & $n / a$ & $n / a$ & 104.0 & 1.010 & $\mathrm{n} / \mathrm{a}$ \\
\hline $\mathrm{ug} / \mathrm{mL}$ & 98.40 & $<1.00 \mathrm{e}-01$ & 10.10 & $<1.01 \mathrm{e} 1$ & $\mathrm{n} / \mathrm{a}$ & $\mathrm{n} / \mathrm{a}$ & 95.20 & 10.10 & $\mathrm{n} / \mathrm{a}$ \\
\hline $\mathrm{Ug} / \mathrm{mL}$ & 98.00 & $<1.00 \mathrm{e}-02$ & 1.010 & $<1.0100$ & n/a & $n / a$ & 97.20 & 1.010 & $\mathrm{n} / \mathrm{a}$ \\
\hline $\mathrm{ug} / \mathrm{mL}$ & 101.0 & $<5.00 \mathrm{e}-02$ & $<5.050$ & $<5.05 \mathrm{e} 0$ & $\mathrm{n} / \mathrm{a}$ & $\mathrm{n} / \mathrm{a}$ & 105.0 & 5.050 & $n / a$ \\
\hline $\mathrm{ug} / \mathrm{mL}$ & 98.20 & $<1.00 \mathrm{e}-01$ & $3.66 \mathrm{e}+04$ & $3.76 \mathrm{e}+04$ & $3.71 \mathrm{e}+04$ & 2.70 & 686.0 & 10.10 & $\mathrm{n} / \mathrm{a}$ \\
\hline $\mathrm{ug} / \mathrm{mL}$ & 103.8 & $<1.00 \mathrm{e}-01$ & $<\quad 10.10$ & $<1.01 \mathrm{e} 1$ & $n / a$ & $\mathrm{n} / \mathrm{a}$ & 108.0 & 10.10 & $n / a$ \\
\hline $\mathrm{ug} / \mathrm{mL}$ & 100.2 & $<2.00 \mathrm{e}-02$ & 2.610 & 2.540 & 2.575 & 2.72 & 99.90 & 2.020 & $n / a$ \\
\hline $\mathrm{ug} / \mathrm{mL}$ & 98,60 & $<2.00 \mathrm{e}-01$ & $4.43 e+02$ & 448.0 & 445.5 & 1.12 & 102.0 & 20.20 & $\mathrm{n} / \mathrm{a}$ \\
\hline $\mathrm{ug} / \mathrm{mL}$ & 99.40 & $<1.00 \mathrm{e}-01$ & $<\quad 10.10$ & $<1.01 \mathrm{e} 1$ & $n / a$ & $\mathrm{n} / \mathrm{a}$ & 100.0 & 10.10 & $n / a$ \\
\hline $\mathrm{ug} / \mathrm{mL}$ & 99.20 & $<1.00 \mathrm{e}-01$ & $5.86 e+02$ & 591.0 & 588.5 & 0.85 & 106.0 & 10.10 & $\mathrm{n} / \mathrm{a}$ \\
\hline
\end{tabular}




\begin{tabular}{|c|c|c|c|c|c|c|c|c|c|c|c|c|}
\hline Sample\# & RlA\# & Analyte & Unit & Standard \% & $\mathrm{B}$ lank & Result & Dupl icate & Average & RPD \% & Spk Rec \% & Det Limit & Count Err\% \\
\hline $596 T 005050$ & $D$ & Antimony-ICP-Acid Dil. & $\mathrm{ug} / \mathrm{mL}$ & 94.80 & $<6.00 \mathrm{e}-02$ & 6.060 & $<6,06 \mathrm{e} 0$ & $\mathrm{n} / \mathrm{a}$ & $n / a$ & 98.70 & 6.060 & $n / a$ \\
\hline S96T005050 & $D$ & Selenium-ICP-Acid Dil. & $\mathrm{ug} / \mathrm{g} / \mathrm{mL}$ & 98.80 & $<1,00 \mathrm{e}-01$ & 10.10 & $<1.01 \mathrm{e} 1$ & $n / a$ & $\mathrm{n} / \mathrm{a}$ & 105.0 & 10.10 & $\mathrm{n} / \mathrm{a}$ \\
\hline S96T005050 & D & Silicon-ICP-Acid Dil. & $\mathrm{ug} / \mathrm{mL}$ & 98.80 & $<5.00 \mathrm{e}-02$ & 56.90 & 58.60 & 57.75 & 2.94 & 104.0 & 5.050 & $n / a$ \\
\hline S96T005050 & D & Samar ium-ICP-Acid Dil. & ug/mL & 100.6 & $<1.00 \mathrm{e}-01$ & 10.10 & $<1.01 \mathrm{e} 1$ & $n / a$ & $\mathrm{n} / \mathrm{a}$ & 101.0 & 10.10 & $n / a$ \\
\hline S96T005050 & D & Strontium-ICP-Acid Dil. & $\mathrm{ug} / \mathrm{mL}$ & 99.60 & $<1.00 \mathrm{e}-02$ & 1.010 & $<1.01 \mathrm{e} 0$ & $\mathrm{n} / \mathrm{a}$ & $\mathrm{n} / \mathrm{a}$ & 101.0 & 1.010 & n/a \\
\hline $596 \mathrm{TO005050}$ & D & Titanium-ICP-Acid Dil. & $\mathrm{ug} / \mathrm{mL}$ & 97.80 & $<1.00 \mathrm{e}-02$ & 1.010 & $<1.01 \mathrm{e} 0$ & $n / a$ & $n / a$ & 99.20 & 1.010 & $\mathrm{n} / \mathrm{a}$ \\
\hline S96T005050 & D & Thallium-ICP-Acid Dil. & $\mathrm{ug} / \mathrm{mL}$ & 97.20 & $<2.00 \mathrm{e}-01$ & $\leq \quad 20.20$ & $<2.02 \mathrm{e} 1$ & $\mathrm{n} / \mathrm{a}$ & $\mathrm{n} / \mathrm{a}$ & 97.20 & 20.20 & $\mathrm{n} / \mathrm{a}$ \\
\hline S96T005050 & $D$ & Uranium-ICP-Acid Dil. & $\mathrm{ug} / \mathrm{mL}$ & 101.0 & $<5.00 \mathrm{e}-01$ & $1.23 \mathrm{e}+02$ & 151.0 & 137.0 & 20.4 & 103.5 & 50.50 & $n / a$ \\
\hline S96T005050 & D & Vanadium-ICP-Acid Dil. & $\mathrm{ug} / \mathrm{mL}$ & 101.4 & $<5.00 \mathrm{e}-02$ & $<5.050$ & $<5.05 \mathrm{e} 0$ & $\mathrm{n} / \mathrm{a}$ & $\mathrm{n} / \mathrm{a}$ & 102.0 & 5.050 & $n / a$ \\
\hline$\$ 961005050$ & D & Zinc-ICP-Acid Dil. & $\mathrm{ug} / \mathrm{mL}$ & 101.6 & $<1.00 \mathrm{e}-02$ & 6.970 & 7.180 & 7.075 & 2.97 & 103.0 & 1.010 & $n / a$ \\
\hline S96T005050 & D & Zirconium-ICP-Acid Dil. & $\mathrm{ug} / \mathrm{mL}$ & 99.40 & $<1.00 \mathrm{e}-02$ & 49.50 & 62.50 & 56.00 & 23.2 & 103.0 & 1.010 & $n / a$ \\
\hline S96T005050 & & Fluoride-IC-Dionex $4000 / 4500$ & $\mathrm{ug} / \mathrm{mL}$ & 89.15 & $<1.20 \mathrm{e}-02$ & $9.61 \mathrm{e}+03$ & $9.61 \mathrm{e}+03$ & $9.61 \mathrm{e}+03$ & 0.00 & $n / a$ & 13.33 & $\mathrm{n} / \mathrm{a}$ \\
\hline 5967005050 & & Chloride-IC-Dionex $4000 / 4500$ & $\mathrm{ug} / \mathrm{mL}$ & 92.91 & $<7.70 \mathrm{e}-02$ & $3.15 \mathrm{e}+02$ & 312.0 & 313.4 & 0.96 & $\mathrm{n} / \mathrm{a}$ & 18.89 & $\mathrm{n} / \mathrm{a}$ \\
\hline 5967005050 & & Nitrite-IC - Dionex $4000 / 4500$ & $\mathrm{ug} / \mathrm{mL}$ & 91.67 & $<1.08 \mathrm{e}-01$ & $4.64 \mathrm{e}+03$ & $4.64 \mathrm{e}+03$ & $4.64 \mathrm{e}+03$ & 0.00 & $\mathrm{n} / \mathrm{a}$ & 120.0 & $n / a$ \\
\hline S96T005050 & & Bromide by Ion Chromatograph & ug/mL & 94.40 & $<1.25 e^{-01}$ & $<1.39 \mathrm{e}+02$ & $<1.39 \mathrm{e}^{2}$ & $\mathrm{n} / \mathrm{a}$ & $\mathrm{n} / \mathrm{a}$ & $n / a$ & 138.9 & $\mathrm{n} / \mathbf{a}$ \\
\hline S96T005050 & & Nitrate by IC-Dionex $4000 / 4500$ & $\mathrm{ug} / \mathrm{mL}$ & 100.3 & $2.13 e-01$ & $2.68 \mathrm{e}+04$ & $2.69 \mathrm{e}+04$ & $2.69 \mathrm{e}+04$ & 0.37 & $n / a$ & 154.4 & $\mathrm{n} / \mathrm{a}$ \\
\hline S96T005050 & & Phosphate-IC-Dionex $4000 / 4500$ & ug $/ \mathrm{mL}$ & 95.96 & $<1.20 \mathrm{e}-01$ & $2.89 e+02$ & .324 .0 & 306.6 & 11.4 & n/a & 133.3 & $n / a$ \\
\hline S96T005050 & & Sulfate by IC-Dionex $4000 / 4500$ & $\mathrm{ug} / \mathrm{mL}$ & 101.9 & $<1.38 \mathrm{e}-01$ & $1.55 \mathrm{e}+03$ & $1.58 \mathrm{e}+03$ & $1.56 e+03$ & 1.92 & $n / a$ & 153.3 & n/a \\
\hline S96TO005050 & & Oxalate by IC-Dionex $4000 / 4500$ & $\mathrm{ug} / \mathrm{mL}$ & 100.0 & $<7.05 e-01$ & $3.20 \mathrm{e}+02$ & 311.0 & 315.6 & 2.85 & $n / a$ & 116.7 & $\mathrm{n} / \mathrm{a}$ \\
\hline \$96T005050 & & Cobalt -60 by GEA & $\mathrm{UCi} / \mathrm{mL}$ & 99.36 & $<2.93 \mathrm{e}-04$ & $2.57 e-02$ & $2.59 e^{-02}$ & $2.58 \mathrm{e}-02$ & 0.78 & $n / a$ & $n / a$ & 5.85 \\
\hline S96T005050 & & Cesium- 137 by GEA & $\mathrm{uCi} / \mathrm{mL}$ & 100.2 & $<7.59 \mathrm{e}-04$ & 26.70 & 26.20 & 26.45 & 1.89 & $\mathrm{n} / \mathrm{a}$ & $\mathrm{n} / \mathrm{a}$ & 0.150 \\
\hline S96T005050 & & Am-241 by Extraction & $\mathrm{uCi} / \mathrm{mL}$ & 87.39 & $<1.54 e^{-05}$ & $2.63 \mathrm{e}-04$ & $2.89 \mathrm{e}-04$ & $2.76 \mathrm{e}-04$ & 9.42 & $n / a$ & $3.25 e^{-05}$ & $1.58 \mathrm{E}+00$ \\
\hline$\$ 96 T 005050$ & & Alpha in Liquid Samples & $\mathrm{uCi} / \mathrm{mL}$ & 100.0 & $<5.10 \mathrm{e}-04$ & $3.03 e-03$ & $2.55 e^{-03}$ & $2.79 e^{-03}$ & 17.2 & 70.75 & $1.00 \mathrm{e}-03$ & $3.24 E+01$ \\
\hline
\end{tabular}

Sludge (from Liquid Grab Sample): sludge (from Liquid Grab Sample)

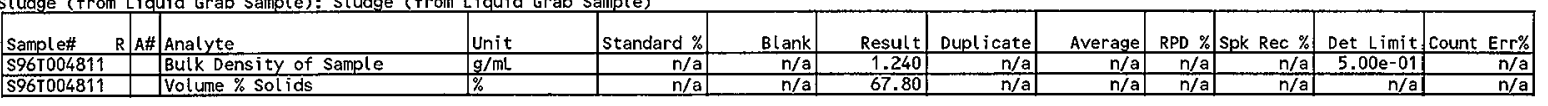


RISER: $10 \mathrm{~A}$

SEGMENT \#: 5AW-96-9

SEGMENT PORTION: Centrifuged Solids (Grab Sample)

\begin{tabular}{|c|c|c|c|c|c|c|c|c|c|c|c|c|}
\hline Sample\# & $\mathbf{R}$ A\# & Analyte & Unit & Standard \% & Blank & Result & Duplicate & Average & RPD \% & Spk Rec \% & Det Limit & Count Err\% \\
\hline S961005021 & & Bulk Density of Sample & $\mathrm{g} / \mathrm{mL}$ & $n / a$ & $n / a$ & 1.290 & $\mathrm{n} / \mathrm{a}$ & $\mathrm{n} / \mathrm{a}$ & $n / a$ & $n / a$ & $5.00 \mathrm{e}-01$ & $n / a$ \\
\hline$\$ 961005021$ & & DSC Exotherm using Mettler & Joules/g & 96.66 & $n / a$ & 14.10 & $0.00 \mathrm{e}+00$ & 7.050 & 200 & $\mathrm{n} / \mathrm{a}$ & $\mathrm{n} / \mathrm{a}$ & $n / a$ \\
\hline 5967005021 & & DSC Exotherm Dry Calculated & Joules/g Dry & $n / a$ & $n / a$ & 42.10 & $0.00 \mathrm{e}+00$ & 21.05 & 200 & $\mathrm{n} / \mathrm{a}$ & $n / a$ & $\mathrm{n} / \mathrm{a}$ \\
\hline 5967005021 & & pH on SST Samples & $\mathrm{pH}$ & 99.62 & $\mathrm{n} / \mathrm{a}$ & 12.44 & 12,47 & 12.46 & 0.24 & $\mathrm{n} / \mathrm{a}$ & $1.00 e-02$ & $\mathrm{n} / \mathrm{a}$ \\
\hline 5967005021 & & $\%$ Water by TGA using Mettler & $\frac{10}{\%}$ & 99.12 & $n / a$ & 66.46 & 66.61 & 66.53 & 0.23 & $\mathrm{n} / \mathrm{a}$ & $\mathrm{n} / \mathrm{a}$ & $n / a$ \\
\hline 5967005021 & & TIC by Acid/Coulometry & ug/g & 100.8 & $7.00 \mathrm{e}-01$ & $9.21 \mathrm{e}+02$ & 827.0 & 874.0 & 10.8 & $n / a$ & 5.000 & $n / a$ \\
\hline \$96T005021 & & TOC by Persulfate/coul onetry & ug/g & 89.67 & 13.50 & $1.64 \mathrm{e}+03$ & $1.70 e+03$ & $1.67 \mathrm{e}+03$ & 3.59 & $n / a$ & 40.00 & $n / a$ \\
\hline $596 T 005054$ & $F$ & Strontium-89/90 High Level & $\mathrm{uCi} / \mathrm{g}$ & 104.3 & $1.70 \mathrm{e}-02$ & 50.70 & 50.00 & 50.35 & 1.39 & $\mathrm{n} / \mathrm{a}$ & $3.00 \mathrm{e}-03$ & $3.01 \mathrm{E}-01$ \\
\hline$\$ 967005054$ & $F$ & Pu-239/240 by TRU-SPEC Resin & $\mathrm{uCi} / \mathrm{g}$ & 97.31 & $<3.09 e-03$ & 1.770 & 1.730 & 1.750 & 2.29 & $\mathrm{n} / \mathrm{a}$ & $7.40 \mathrm{e}-02$ & $1.63 E+00$ \\
\hline 5967005054 & $\mathrm{~F}$ & Silver-ICP-Fusion & $\mathrm{ug} / \mathrm{g}$ & 97.80 & $<1.00 \mathrm{e}-02$ & $3.42 \mathrm{e}+02$ & 421.0 & 381.5 & 20.7 & $\mathrm{n} / \mathrm{a}$ & 201.0 & $\mathrm{n} / \mathrm{a}$ \\
\hline$\$ 967005054$ & $\mathrm{~F}$ & Aluminium-ICP-Fusion & ug/g & 97.00 & $<5.00 e-02$ & $1.03 e+03$ & $1.39 \mathrm{e}+03$ & $1.21 \mathrm{e}+03$ & 29.8 & $n / a$ & $1.01 \mathrm{e}+03$ & $\mathrm{n} / \mathrm{a}$ \\
\hline $596 \mathrm{~T} 005054$ & $\mathrm{~F}$ & Arsenic-ICP-Fusion & $\mathrm{ug} / \mathrm{g}$ & 100.8 & $<1.00 \mathrm{e}-01$ & $<2.01 \mathrm{e}+03$ & $<2.05 \mathrm{e} 3$ & $n / a$ & $n / a$ & $\mathrm{n} / \mathrm{a}$ & $2.01 \mathrm{e}+03$ & $\mathrm{n} / \mathrm{a}$ \\
\hline S96T005054 & $F$ & Boron-ICP-Fusion & $\mathrm{ug} / \mathrm{g}$ & 99.20 & $<5.00 e-02$ & $<1.01 \mathrm{e}+03$ & $<1.02 \mathrm{e} 3$ & $\mathrm{n} / \mathrm{a}$ & $n / a$ & n/a & $1.01 \mathrm{e}+03$ & $n / a$ \\
\hline 5967005054 & $F$ & Barium -ICP-Fusion & $\mathrm{gg} / \mathrm{g}$ & 99.20 & $<5.00 e-02$ & $<1.01 \mathrm{e}+03$ & $<1.02 \mathrm{e} 3$ & $\mathrm{n} / \mathrm{a}$ & $\mathrm{n} / \mathrm{a}$ & $\mathrm{n} / \mathrm{a}$ & $1.01 \mathrm{e}+03$ & $\mathrm{n} / \mathrm{a}$ \\
\hline S961005054 & $\mathrm{F}$ & Beryllium -ICP-Fusion & ug/g & 101.0 & $<5.00 \mathrm{e}-03$ & $<1.01 e+02$ & $<1.02 \mathrm{e} 2$ & $\mathrm{n} / \mathrm{a}$ & $\mathrm{n} / \mathrm{a}$ & $\mathrm{n} / \mathrm{a}$ & 101.0 & $\mathrm{n} / \mathrm{a}$ \\
\hline S96T005054 & $F$ & Bismuth-ICP-Fusion & ug/g & 100.6 & $<7.00 \mathrm{e}-01$ & $<2.01 e+03$ & $<2.05 \mathrm{e} 3$ & $\mathrm{n} / \mathrm{a}$ & $n / a$ & $\mathrm{n} / \mathrm{a}$ & $2.01 \mathrm{e}+03$ & $\mathrm{n} / \mathrm{a}$ \\
\hline S96T005054 & $F$ & Calcium-ICP-Fusion & ug/g & 95.40 & $<1.00 \mathrm{e}-01$ & $<2.01 e+03$ & $<2.05 \mathrm{e} 3$ & $n / a$ & $\mathrm{n} / \mathrm{a}$ & $\mathrm{n} / \mathrm{a}$ & $2.01 \mathrm{e}+03$ & $n / a$ \\
\hline 5967005054 & $F$ & Cadmium-ICP-Fusion & ug/g & 98.60 & $<5.00 e-03$ & $<1.01 \mathrm{e}+02$ & 126.0 & $n / a$ & $n / a$ & $\mathrm{n} / \mathrm{a}$ & 101.0 & $\mathrm{n} / \mathrm{a}$ \\
\hline$\$ 967005054$ & $\mathrm{~F}$ & Cerium-ICP-Fusion & ug/g & 97.60 & $<1.00 e-01$ & $<2.01 e+03$ & $<2.05 \mathrm{e} 3$ & $\mathrm{n} / \mathrm{a}$ & $n / a$ & $n / a$ & $2.01 \mathrm{e}+03$ & $\mathrm{n} / \mathrm{a}$ \\
\hline S967005054 & $F$ & Cobalt -ICP-Fusion & ug/g & 98.60 & $<2.00 e-02$ & $<4.03 e+02$ & $<4.10 \mathrm{e} 2$ & $\mathrm{n} / \mathrm{a}$ & $\mathrm{n} / \mathrm{a}$ & $\mathrm{n} / \mathrm{a}$ & 403.0 & $\mathrm{n} / \mathrm{a}$ \\
\hline 5967005054 & $F$ & Chromium -ICP-Fusion & ug/g & 99.00 & $<1.00 e^{-02}$ & $<2.01 e+02$ & 267.0 & $\mathrm{n} / \mathrm{a}$ & $\mathrm{n} / \mathrm{a}$ & $n / a$ & 201.0 & $n / a$ \\
\hline$\$ 967005054$ & $F$ & Copper -ICP-Fusion & $\mathrm{ug} / \mathrm{g}$ & 99.40 & $<1.00 e-02$ & $<2.01 e+02$ & $<2.05 \mathrm{e} 2$ & $n / a$ & $n / a$ & $\mathrm{n} / \mathrm{a}$ & 201.0 & $n / a$ \\
\hline$\$ 967005054$ & $F$ & Iron-ICP-Fusion & $\mathrm{ug} / \mathrm{g}$ & 99.40 & $<5.00 \mathrm{e}-02$ & $1.34 \mathrm{e}+03$ & $1.35 \mathrm{e}+03$ & $1.34 \mathrm{e}+03$ & 0.74 & $\mathrm{n} / \mathrm{a}$ & $1.01 \mathrm{e}+03$ & $\mathrm{n} / \mathrm{a}$ \\
\hline S9670005054 & $F$ & Lanthanum -ICP-Fusion & ug/g & 99.40 & $<5.00 \mathrm{e}-02$ & $1.47 e+03$ & $1.46 \mathrm{e}+03$ & $1.46 \mathrm{e}+03$ & 0.68 & $\mathrm{n} / \mathrm{a}$ & $1.01 \mathrm{e}+03$ & $\mathrm{n} / \mathrm{a}$ \\
\hline$\$ 96 T 005054$ & F & Lithium -ICP-Fusion & $u g / 9$ & 97.80 & $<1.00 \mathrm{e}-02$ & $<2.01 e+02$ & $<2.05 \mathrm{e} 2$ & $n / a$ & $n / a$ & $\mathrm{n} / \mathrm{a}$ & 201.0 & $n / a$ \\
\hline \$96T005054 & $\mathbf{F}$ & Magnesium -ICP-Fusion & $u g / g$ & 95.20 & $<1.00 \mathrm{e}-01$ & $<2.01 e+03$ & $<2.05 \mathrm{e}$ & $n / a$ & $n / a$ & $n / a$ & $2.01 \mathrm{e}+03$ & $\mathrm{n} / \mathrm{a}$ \\
\hline 5967005054 & $F$ & Manganese -iCP-Fusion & ug/g & 98.20 & $<1.00 \mathrm{e}-02$ & $8.29 e+02$ & 856.0 & 842.5 & 3.20 & $n / a$ & 201.0 & $n / a$ \\
\hline S96T005054 & $\bar{F}$ & Molybdenum-ICP-Fusion & $u g / g$ & 99.00 & $<5.00 e-02$ & $<1.01 \mathrm{e}+03$ & $<1.02 \mathrm{e} 3$ & $n / a$ & $\mathrm{n} / \mathrm{a}$ & $n / a$ & $1.01 \mathrm{e}+03$ & $n / a$ \\
\hline 5967005054 & $F$ & Sodium-ICP-Fusion & ug/g & 95.80 & 2.250 & $9.14 e+04$ & $9.08 \mathrm{e}+04$ & $9.11 \mathrm{e}+04$ & 0.66 & $\mathrm{n} / \mathrm{a}$ & $2.01 \mathrm{e}+03$ & $\mathrm{n} / \mathrm{a}$ \\
\hline \$96T005054 & $F$ & Neodymium -ICP-Fusion & $\mathrm{ug} / \mathrm{g}$ & 101.2 & $<1.00 \mathrm{e}-01$ & $<2.01 e+03$ & $<2.05 \mathrm{e} 3$ & $n / a$ & $\mathrm{n} / \mathrm{a}$ & $\mathrm{n} / \mathrm{a}$ & $2.01 \mathrm{e}+03$ & $\mathrm{n} / \mathrm{a}$ \\
\hline S96T005054 & $F$ & Phosphorus - ICP-Fusion & $u g / 9$ & 99.20 & $<2.00 \mathrm{e}-01$ & $<4.03 e+03$ & $<4.10 e^{3}$ & $\mathrm{n} / \mathrm{a}$ & $\mathrm{n} / \mathrm{a}$ & $\mathrm{n} / \mathrm{a}$ & $4.03 \mathrm{e}+03$ & $\mathrm{n} / \mathrm{a}$ \\
\hline $596 T 005054$ & $\bar{F}$ & Lead - ICP-Fusion & ug/g & 98.60 & $<1.00 \mathrm{e}-01$ & $<2.01 e+03$ & $<2.05 \mathrm{e} 3$ & $\mathrm{n} / \mathrm{a}$ & $\mathrm{n} / \mathrm{a}$ & $\mathrm{n} / \mathrm{a}$ & $2.01 \mathrm{e}+03$ & $n / a$ \\
\hline$\$ 967005054$ & $F$ & Sulfur -ICP-Fusion & $\mathrm{ug} / \mathrm{g}$ & 98.60 & $<1.00 \mathrm{e}-01$ & $<2.01 \mathrm{e}+03$ & $<2.05 \mathrm{e} 3$ & $\mathrm{n} / \mathrm{a}$ & $\mathrm{n} / \mathrm{a}$ & $\mathrm{n} / \mathrm{a}$ & $2.01 \mathrm{e}+03$ & $\mathrm{n} / \mathrm{a}$ \\
\hline S96T005054 & $\bar{F}$ & Antimony-ICP-Fusion & $\mathrm{ug} / \mathrm{g}$ & 90.20 & $<6.00 \mathrm{e}-02$ & $<1.21 \mathrm{e}+03$ & $1.47 \mathrm{e}+03$ & $\mathrm{n} / \mathrm{a}$ & $\mathrm{n} / \mathrm{a}$ & $\mathrm{n} / \mathrm{a}$ & $1.21 \mathrm{e}+03$ & $\mathrm{n} / \mathrm{a}$ \\
\hline S967005054 & $F$ & Selenium -ICP-Fusion & $u g / g$ & 105.0 & $<1.00 \mathrm{e}-01$ & $<2.01$ e+03 & $<2.05 \mathrm{e} 3$ & $\mathrm{n} / \mathrm{a}$ & $\mathrm{n} / \mathrm{a}$ & $\mathrm{n} / \mathrm{a}$ & $2.01 e+03$ & $\mathrm{n} / \mathrm{a}$ \\
\hline S96T005054 & $F$ & Silicon-ICP-Fusion & $\mathrm{ug} / \mathrm{g}$ & 98.40 & $<5.00 \mathrm{e}-02$ & $1.01 e+03$ & $<1.02 \mathrm{e} 3$ & $n / a$ & $\mathrm{n} / \mathrm{a}$ & $n / a$ & $1.01 \mathrm{e}+03$ & $\mathrm{n} / \mathrm{a}$ \\
\hline S96r005054 & $F$ & Samarium -ICP-Fusion & $\mathrm{ug} / \mathrm{g}$ & 101.0 & $<1.00 \mathrm{e}-01$ & $<2.01 e+03$ & $<2.05 \mathrm{e} 3$ & $\mathrm{n} / \mathrm{a}$ & $\mathrm{n} / \mathrm{a}$ & $n / a$ & $2.01 \mathrm{e}+03$ & $n / a$ \\
\hline \$96T005054 & $F$ & Strontium - ICP-Fusion & ug $/ \mathrm{g}$ & 98.40 & $<1.00 \mathrm{e}-02$ & $<2.01 \mathrm{e}+02$ & $<2.05 \mathrm{e} 2$ & $\mathrm{n} / \mathrm{a}$ & $n / a$ & $n / a$ & 201.0 & $n / a$ \\
\hline \$967005054 & $F$ & Titanium-ICP-Fusion & ug/g & 96.00 & $<1.00 \mathrm{e}-02$ & $<2.01 e+02$ & $<2.05 e 2$ & $\mathrm{n} / \mathrm{a}$ & $\mathrm{n} / \mathrm{a}$ & $\mathrm{n} / \mathrm{a}$ & 201.0 & $n / a$ \\
\hline 5967005054 & $F$ & Thallium-ICP-Fusion & $\mathrm{ug} / \mathrm{g}$ & 97.20 & $<2.00 \mathrm{e}-01$ & $<4.03 e+03$ & $<4.10 \mathrm{e} 3$ & $n / a$ & $\mathrm{n} / \mathrm{a}$ & $\mathrm{n} / \mathrm{a}$ & $4.03 e+03$ & $\mathrm{n} / \mathrm{a}$ \\
\hline 5967005054 & $F$ & Uranium-ICP-Fusion & ug/g & 97.10 & $<5.00 \mathrm{e}-01$ & $5.60 \mathrm{e}+04$ & $5.30 \mathrm{e}+04$ & $5.45 e+04$ & 5.50 & $\mathrm{n} / \mathrm{a}$ & $1.01 \mathrm{e}+04$ & $n / a$ \\
\hline \$96T005054 & $F$ & Vanadium -ICP-Fusion & $u g / g$ & 99.80 & $<5.00 \mathrm{e}-02$ & $<1.01 \mathrm{e}+03$ & $<1.02 \mathrm{e} 3$ & $n / a$ & $\mathrm{n} / \mathrm{a}$ & $\mathrm{n} / \mathrm{a}$ & $1.01 \mathrm{e}+03$ & $n / a$ \\
\hline$\$ 967005054$ & $F$ & Zinc-ICP-Fusion & ug/g & 99.40 & $1.60 \mathrm{e}-02$ & $5.61 \mathrm{e}+02$ & 258.0 & 409.5 & 74.0 & $n / a$ & 201.0 & $n / a$ \\
\hline S967005054 & $\mathbf{F}$ & Zirconium -ICP-Fusion & ug/g & 98.00 & $<1.00 \mathrm{e}=02$ & $6.93 \mathrm{e}+04$ & $6.98 \mathrm{e}+04$ & $6.96 \mathrm{e}+04$ & 0.72 & $\mathrm{n} / \mathrm{a}$ & 201.0 & $\mathrm{n} / \mathrm{a}$ \\
\hline
\end{tabular}




\begin{tabular}{|c|c|c|c|c|c|c|c|c|c|c|c|c|}
\hline Sample\# & $\mathbf{R} \mid \mathrm{A \#}$ & Analyte & Unit & Standard \% & Blank & Result $t$ & Duplicate & Average & RPD \% & Spk Rec \% & Det Limit & Count Err\% \\
\hline $596 T 005054$ & $\mathrm{~F}$ & Cobal $t-60$ by GEA & $u C i / g$ & 97.35 & $<2.77 \mathrm{e}-02$ & $7.46 \mathrm{e}-02$ & $5.55 \mathrm{e}^{-02}$ & $6.51 \mathrm{e}-02$ & 29.4 & $\mathrm{n} / \mathrm{a}$ & $\mathrm{n} / \mathrm{a}$ & 36.2 \\
\hline S961005054 & $F$ & Ces $i$ um- 137 by GEA & $\mathrm{uCi} / \mathrm{g}$ & 95.75 & $<6.17 \mathrm{e}-02$ & 18.86 & 19.20 & 19.03 & 1.57 & $\mathrm{n} / \mathrm{a}$ & $\mathrm{n} / \mathrm{a}$ & 1.76 \\
\hline 5967005054 & $\mathrm{~F}$ & Am-241 by Extraction & $u C i / g$ & 89.92 & $<5.46 e-03$ & $3.71 \mathrm{e}-01$ & $3.55 \mathrm{e}-01$ & $3.63 \mathrm{e}-01$ & 4.41 & $n / a$ & $5.30 \mathrm{e}-02$ & $1.84 E+00$ \\
\hline$\$ 961005054$ & $F$ & Alpha of Digested solid & uCi/g & 81.39 & $<4.70 \mathrm{e}-03$ & 2.390 & 2.360 & 2.375 & 1.26 & $\mathrm{n} / \mathrm{a}$ & $5.00 e-03$ & $3.34 \mathrm{E}+00$ \\
\hline $596 \mathrm{~T} 005057$ & $W$ & OH- by Pot. Titration & $\mathrm{ug} / \mathrm{g}$ & 97.36 & $<42.00$ & $<42.00$ & $<42$ & $n / a$ & $\mathrm{n} / \mathrm{a}$ & $\mathrm{n} / \mathrm{a}$ & 41.70 & $n / a$ \\
\hline S96T005057 & W & Fluoride-IC-Dionex $4000 / 4500$ & $\mathrm{ug} / \mathrm{g}$ & 90.68 & $<1.20 \mathrm{e}-02$ & $2.82 \mathrm{e}+04$ & $2.86 \mathrm{e}+04$ & $2.84 \mathrm{e}+04$ & 1.41 & $n / a$ & 47.21 & $n / a$ \\
\hline S96T005057 & W & Chloride-IC-Dionex $4000 / 4500$ & ug $/ 9$ & 92.66 & $2.00 e-02$ & $1.86 \mathrm{e}+02$ & 208.0 & 196.8 & 11.2 & $\mathrm{n} / \mathrm{a}$ & 66.89 & $n / a$ \\
\hline$\$ 967005057$ & $W$ & Nitrite-IC - Dionex $4000 / 4500$ & ug $/ g$ & $92 . \overline{96}$ & $<1.08 \mathrm{e}-01$ & $2.99 \mathrm{e}+03$ & $2.99 e+03$ & $2.99 \mathrm{e}+03$ & 0.00 & $\mathrm{n} / \mathrm{a}$ & 425.0 & $\mathrm{n} / \mathrm{a}$ \\
\hline $596 \mathrm{TO}$ & W & Bromide by Ion Chromatograph & $\mathrm{ug} / \mathrm{g}$ & 94.57 & $<1.25 \mathrm{e}-01$ & $<4.92 \mathrm{e}+02$ & $<4.94 \mathrm{e} 2$ & $n / a$ & $\mathrm{n} / \mathrm{a}$ & $n / a$ & 491.8 & $n / a$ \\
\hline $596 T 005057$ & W & Nitrate by IC-0 ionex $4000 / 4500$ & ug $/ g$ & 100.0 & $1.70 \mathrm{e}-01$ & $1.83 \mathrm{e}+04$ & $1.83 e+04$ & $1.83 \mathrm{e}+04$ & 0.00 & $\mathrm{n} / \mathrm{a}$ & 546.8 & $n / a$ \\
\hline $596 \mathrm{~T} 005057$ & W & Phosphate-IC-D ionex $4000 / 4500$ & $u g / g$ & 95.23 & $<1.20 \mathrm{e}-01$ & $<4.72 \mathrm{e}+02$ & $<4.74 \mathrm{e} 2$ & $n / a$ & $\mathrm{n} / \mathrm{a}$ & $\mathrm{n} / \mathrm{a}$ & 472.1 & $n / a$ \\
\hline S96T005057 & $\ddot{W}$ & Sulfate by IC-Dionex $4000 / 4500$ & $\mathrm{ug} / \mathrm{g}$ & 101.3 & $<1.38 \mathrm{e}-01$ & $1.19 \mathrm{e}+03$ & $1.26 \mathrm{e}+03$ & $1.22 \mathrm{e}+03$ & 5.71 & $n / a$ & 543.1 & $n / a$ \\
\hline$\$ 96 T 005057$ & W & Oxalate by IC-Dionex $4000 / 4500$ & ug/g & 99.81 & $<1.05 \mathrm{e}-01$ & $4.46 \mathrm{e}+02$ & $<4.15 \mathrm{e} 2$ & $n / a$ & $\mathrm{n} / \mathrm{a}$ & $\mathrm{n} / \mathrm{a}$ & 413.1 & \\
\hline
\end{tabular}

S96T005057 W Dxalate by IC-Dionex $4000 / 4500 \mathrm{ug} / \mathrm{g}$

\begin{tabular}{|c|c|c|c|}
\hline Sample\# & R A\# & Analyte & Unjt \\
\hline S96T005051 & & Bulk Density of Sample & $\mathrm{g} / \mathrm{mL}$ \\
\hline S96T005051 & & DSC Exotherm on Perkin Elmer & Joules $/ g$ \\
\hline 9961005051 & & OH- by Pot. Titration & $\mathrm{ug} / \mathrm{mL}$ \\
\hline S96T005051 & & pH Direct & $\mathrm{pH}$ \\
\hline 5967005051 & & $\%$ Water by TGA on Perkin Elmer & $\%$ \\
\hline 5967005051 & & TIC by Acid/Coulometry & $\mathrm{ug} / \mathrm{mL}$ \\
\hline S967005051 & & Tot. Organic Carbon by coul. & $\mathrm{ug} / \mathrm{mL}$ \\
\hline S96T005051 & & Strontium-89/90 High Level & $\mathrm{uCi} / \mathrm{mL}$ \\
\hline S96T005051 & & PU-239/240 by TRU-SPEC Resin & $\mathrm{uCi} / \mathrm{mL}$ \\
\hline S96T005051 & $\mathrm{D}$ & Silver-ICP-Acid Dil. & $\mathrm{ug} / \mathrm{mL}$ \\
\hline S96T005051 & D & Aluminium-ICP-Acid Dil. & $\mathrm{ug} / \mathrm{mL}$ \\
\hline S96T005051 & $D$ & Arsenic-ICP-Acid Dil. & $\mathrm{ug} / \mathrm{mL}$ \\
\hline S96T005051 & $\bar{D}$ & Boron-ICP-Acid Dil. & ug $/ \mathrm{mL}$ \\
\hline 596T005051 & $\bar{D}$ & Barium-iCP-Acid Dil. & $\mathrm{ug} / \mathrm{mL}$ \\
\hline S967005051 & D & Beryllium-ICP-Acid Dil. & $\mathrm{ug} / \mathrm{mL}$ \\
\hline S96T005051 & D & Bismuth-ICP-Acid Dil. & $\mathrm{ug} / \mathrm{mL}$ \\
\hline S96T005051 & D & Calcium-ICP-Acid Dil. & $\mathrm{ug} / \mathrm{mL}$ \\
\hline S96r005051 & D & Cadmium-ICP-Acid Dil. & $\mathrm{ug} / \mathrm{mL}$ \\
\hline S96T005051 & ID & Cerium-ICP-Acid Dil. & $\mathrm{ug} / \mathrm{mL}$ \\
\hline S96T005051 & D & Cobalt-ICP-Acid Dil. & $\mathrm{ug} / \mathrm{mL}$ \\
\hline 5967005051 & D & Chromium-ICP-Acid Dil. & $\mathrm{ug} / \mathrm{mL}$ \\
\hline S967005051 & D & Copper-ICP-Acid Dil. & $\mathrm{ug} / \mathrm{mL}$ \\
\hline S96T005051 & D & Iron-ICP-Acid Dil. & $\mathrm{ug} / \mathrm{mL}$ \\
\hline S $96 T 005051$ & D & Potassium-ICP-Acid Dil. & $\mathrm{ug} / \mathrm{mL}$ \\
\hline 5967005051 & D & Lanthanum-ICP-Acid Dil. & $\mathrm{ug} / \mathrm{mL}$ \\
\hline S96T005051 & $\overline{\mathrm{D}}$ & Lithium-ICP-Acid Dil. & ug/mL \\
\hline S96T005051 & D & Magnesium-ICP-Acid Dil. & $\mathrm{ug} / \mathrm{mL}$ \\
\hline \$967005051 & D & Manganese-ICP-ACid Dil. & $\mathrm{ug} / \mathrm{mL}$ \\
\hline S 967005051 & TD & Molybdenum-ICP-Acid Dil. & $\mathrm{ug} / \mathrm{mL}$ \\
\hline S96T005051 & D & Sodjum-ICP-Acid Dil. & ug/mL \\
\hline 5967005051 & D & Neodymium-ICP-Acid DiL. & $\mathrm{ug} / \mathrm{mL}$ \\
\hline \$96T005051 & $\bar{D}$ & Nickel-ICP-Acid Dil. & $\mathrm{ug} / \mathrm{mL}$ \\
\hline 5967005051 & D & Phosphorus-ICP-Acid Dil. & $\mathrm{ug} / \mathrm{mL}$ \\
\hline 5967005051 & & Iead-ICP-ACId Dil & \\
\hline
\end{tabular}

\begin{tabular}{|c|c|c|c|c|c|c|c|c|}
\hline Standard \% & B Lank & Result & Dupl icate & Average & RPD \% & Spk Rec \% & Det Limit & Count Err\% \\
\hline $\mathrm{n} / \mathrm{a}$ & $n / a$ & 1.030 & $\mathrm{n} / \mathrm{a}$ & $n / a$ & $n / a$ & $n / a$ & $5.00 \mathrm{e}-01$ & $\mathrm{n} / \mathrm{a}$ \\
\hline 93.95 & $n / a$ & $0.00 \mathrm{e}+00$ & $0.00 \mathrm{e}+00$ & $0.00 \mathrm{e}+00$ & 0.00 & $n / a$ & $\mathrm{n} / \mathrm{a}$ & $n / a$ \\
\hline 106.4 & $<42.00$ & $9.41 \mathrm{e}+03$ & $9.50 \mathrm{e}+03$ & $9.46 \mathrm{e}+03$ & 0.95 & $n / a$ & 500.0 & $n / a$ \\
\hline 100.0 & $n / a$ & 13.48 & 13.55 & 13.52 & 0.52 & $n / a$ & $1.00 \mathrm{e}-02$ & $\mathrm{n} / \mathrm{a}$ \\
\hline 99.27 & $\mathrm{n} / \mathrm{a}$ & 88.68 & 89.02 & 88.85 & 0.38 & $n / a$ & $n / a$ & $\mathrm{n} / \mathrm{a}$ \\
\hline 100.2 & $6.00 e-01$ & $7.45 \mathrm{e}+02$ & 741.0 & 743.0 & 0.54 & $\mathrm{n} / \mathrm{a}$ & 5.000 & n/a \\
\hline 106.3 & 1.200 & $2.24 \mathrm{e}+03$ & $2.18 e+03$ & $2.21 \mathrm{e}+03$ & 2.71 & $n / a$ & 55.00 & $n / a$ \\
\hline 108.6 & $<1.23 \mathrm{e}-05$ & $6.84 \mathrm{e}-01$ & $6.23 e-01$ & $6.53 \mathrm{e}-01$ & 9.33 & $\mathrm{n} / \mathrm{a}$ & $2.34 e^{-05}$ & $2.16 \mathrm{E}-01$ \\
\hline 90.60 & $<7.39 e-06$ & $5.85 e-03$ & $5.30 \mathrm{e}-03$ & $5.58 \mathrm{e}-03$ & 9.87 & $n / a$ & $1.00 \mathrm{e}-03$ & $1.80 E+00$ \\
\hline 99.20 & $<1.00 \mathrm{e}-02$ & 2.610 & 2.620 & 2.615 & 0.38 & $\mathrm{n} / \mathrm{a}$ & 1.010 & $\mathrm{n} / \mathrm{a}$ \\
\hline 99.80 & $<5.00 e-02$ & $6.60 \mathrm{e}+02$ & 667.0 & 663.5 & 1.06 & $n / a$ & 5.050 & $n / a$ \\
\hline 102.4 & $<1.00 \mathrm{e}-01$ & $\leq \quad 10.10$ & $<1.01 \in 1$ & $\mathrm{n} / \mathrm{a}$ & $\mathrm{n} / \mathrm{a}$ & $\mathrm{n} / \mathrm{a}$ & 10.10 & $\mathrm{n} / \mathrm{a}$ \\
\hline 99.80 & $<5.00 \mathrm{e}-02$ & $\leq \quad 5.050$ & $<5.05 \mathrm{e} 0$ & $n / a$ & $\mathrm{n} / \mathrm{a}$ & $\mathrm{n} / \mathrm{a}$ & 5.050 & $\mathrm{n} / \mathrm{a}$ \\
\hline 100.6 & $<5.00 e-02$ & 5.050 & $<5.05 \mathrm{e} 0$ & $\mathrm{n} / \mathrm{a}$ & $n / a$ & $n / a$ & 5.050 & $n / a$ \\
\hline 102.4 & $\angle 5.00 \mathrm{e}-03$ & 1.200 & 1.210 & 1.205 & 0.83 & $\mathrm{n} / \mathrm{a}$ & $5.05 e-01$ & $n / a$ \\
\hline 100.4 & $\langle 1.00 \mathrm{e}-01$ & 10.10 & $<1.01 \mathrm{e} 1$ & $n / a$ & $\mathrm{n} / \mathrm{a}$ & $\mathrm{n} / \mathrm{a}$ & 10.10 & $\mathrm{n} / \mathrm{t}$ \\
\hline 100.0 & $<1.00 e^{-01}$ & $\leq \quad 10.10$ & $<1.01 \mathrm{e} 1$ & $\mathrm{n} / \mathrm{a}$ & $\mathrm{n} / \mathrm{a}$ & $\mathrm{n} / \mathrm{a}$ & 10.10 & $n / a$ \\
\hline 100.6 & $<5.00 \mathrm{e}-03$ & $<5.05 e-01$ & $<5.05 e-1$ & $n / a$ & $n / a$ & $n / a$ & $5.05 \mathrm{e}-01$ & $n / a$ \\
\hline 99.00 & $<1.00 \mathrm{e}-01$ & $<\quad 10.10$ & $<1.01 \mathrm{e} 1$ & $n / a$ & $\mathrm{n} / \mathrm{a}$ & $\mathrm{n} / \mathrm{a}$ & 10.10 & $n / t$ \\
\hline 101.2 & $<2.00 \mathrm{e}-02$ & $<\quad 2.020$ & $<2.02 e 0$ & $\mathrm{n} / \mathrm{a}$ & $n / a$ & $n / a$ & 2.020 & $n / a$ \\
\hline 101.2 & $<1.00 \mathrm{e}-02$ & 30.40 & 30.50 & 30.45 & 0.33 & $n / a$ & 1.010 & $\mathrm{n} / \mathrm{a}$ \\
\hline 104.0 & $<1.00 \mathrm{e}-02$ & 1.010 & $<1.01 \mathrm{e}$ & $\mathrm{n} / \mathrm{a}$ & $n / a$ & $n / a$ & 1.010 & $\mathrm{n} / \mathrm{a}$ \\
\hline 100.6 & $<5.00 \mathrm{e}-02$ & 5.050 & $<5.05 \mathrm{e} 0$ & $\mathrm{n} / \mathrm{a}$ & $n / a$ & $n / a$ & 5.050 & $n / a$ \\
\hline 94.40 & $\angle 5.00 \mathrm{e}-01$ & $1.02 e+04$ & $1.04 \mathrm{e}+04$ & $1.03 \mathrm{e}+04$ & 1.94 & $\mathrm{n} / \mathrm{a}$ & 50.50 & $n / a$ \\
\hline 100.8 & $<5.00 e-02$ & $<\quad 5.050$ & $<5.05 \mathrm{e} 0$ & $\mathrm{n} / \mathrm{a}$ & $\mathrm{n} / \mathrm{a}$ & $n / a$ & 5.050 & $\mathrm{n} / \mathrm{a}$ \\
\hline 101.2 & $<1.00 \mathrm{e}-02$ & 1.010 & $<1.01 \mathrm{e} 0$ & $\mathrm{n} / \mathrm{a}$ & $n / a$ & $n / a$ & 1.010 & $n /$ \\
\hline 98.40 & $<1.00 \mathrm{e}-01$ & 10.10 & $<1.01 \mathrm{e}$ & $n / a$ & $\mathrm{n} / \mathrm{a}$ & $n / a$ & 10.10 & $n / a$ \\
\hline 98.00 & $<1.00 \mathrm{e}-02$ & 1.010 & $\leq 1.01=0$ & $\mathrm{n} / \mathrm{a}$ & $\mathrm{n} / \mathrm{a}$ & $\mathrm{n} / \mathrm{a}$ & 1.010 & $n / a$ \\
\hline 101.0 & $<5.00 \mathrm{e}-02$ & $<5.050$ & $<5.05 e 0$ & $n / a$ & $\mathrm{n} / \mathrm{a}$ & $\mathrm{n} / \mathrm{a}$ & 5.050 & $n / a$ \\
\hline 98.20 & $<1.00 \mathrm{e}-01$ & $3.71 \mathrm{e}+04$ & $3.74 e+04$ & $3.72 \mathrm{e}+04$ & 0.81 & $n / a$ & 10.10 & $n / 2$ \\
\hline 103.8 & $<1.00 \mathrm{e}-01$ & $<\quad 10.10$ & $<1.01 \mathrm{e} 1$ & $\mathrm{n} / \mathrm{a}$ & $\mathrm{n} / \mathrm{a}$ & $\mathrm{n} / \mathrm{a}$ & 10.10 & $\mathrm{n} / \mathrm{a}$ \\
\hline 100.2 & $<2.00 \mathrm{e}-02$ & 2.400 & $<2.0200$ & $\mathrm{n} / \mathrm{a}$ & $n / a$ & $\mathrm{n} / \mathrm{a}$ & 2.020 & $n / a$ \\
\hline 98.60 & $<2.00 \mathrm{e}-01$ & $4.17 e+02$ & 416.0 & 416.5 & 0.24 & $\mathrm{n} / \mathrm{a}$ & 20.20 & $\mathrm{n} / \mathrm{a}$ \\
\hline 99.40 & $<1.00 \mathrm{e}-01$ & $\leq \quad 10.10$ & $<1.01 \mathrm{e} 1$ & $\mathrm{n} / \mathrm{a}$ & $n / a$ & $\mathrm{n} / \mathrm{a}$ & 10.10 & $n / a$ \\
\hline
\end{tabular}




\begin{tabular}{|c|c|c|c|c|c|c|c|c|c|c|c|c|}
\hline Sample\# $R$ & A\# & Analyte & Unit & Standard \% & B Lank & Result & Dupl icate & Average & RPD \% & Spk Rec \% & Det Limit & Count Err\% \\
\hline $596 \mathrm{~T} 005051$ & D & Sulfur-ICP-Acid Dil. & $\mathrm{ug} / \mathrm{mL}$ & 99.20 & $<1.00 \mathrm{e}-01$ & $5.40 \mathrm{e}+02$ & 543.0 & 541.5 & 0.55 & $n / a$ & 10.10 & $n / a$ \\
\hline S961005051 & D & Antimony-ICP-Acid Dil. & $\mathrm{ug} / \mathrm{mL}$ & 94.80 & $<6.00 \mathrm{e}-02$ & $<6.060$ & $<6.06 \mathrm{e} 0$ & $\mathrm{n} / \mathbf{a}$ & $n / a$ & $n / a$ & 6.060 & $\mathrm{n} / \mathrm{a}$ \\
\hline 5967005051 & D & Selenium-ICP-Acid Dil. & $\mathrm{ug} / \mathrm{mL}$ & 98.80 & $<1.00 \mathrm{e}-01$ & 10.10 & $<1.01 \mathrm{e} 1$ & $\mathrm{n} / \mathrm{a}$ & $n / a$ & $\mathrm{n} / \mathrm{a}$ & 10.10 & $n / a$ \\
\hline 5967005051 & D & Silicon-ICP-Acid Dil. & $\mathrm{ug} / \mathrm{mL}$ & 98.80 & $<5.00 \mathrm{e}-02$ & 52.40 & 55.20 & 53.80 & 5.20 & $\mathrm{n} / \mathrm{a}$ & 5.050 & $n / a$ \\
\hline S96T005051 & D & Samarium-ICP-Acid Dil. & $\mathrm{ug} / \mathrm{mL}$ & 100.6 & $<1.00 \mathrm{e}-01$ & 10.10 & $<1.01 \mathrm{e} 1$ & $n / a$ & $\mathrm{n} / \mathrm{a}$ & $\mathrm{n} / \mathrm{a}$ & 10.10 & $n / a$ \\
\hline S96T005051 & D & Stront ium-ICP-ACid Dill. & $\mathrm{ug} / \mathrm{mL}$ & 99.60 & $<1.00 e^{-02}$ & 1.010 & $<1.01 \mathrm{e0}$ & $\mathrm{n} / \mathrm{a}$ & $\mathrm{n} / \mathrm{a}$ & $\mathrm{n} / \mathrm{a}$ & 1.010 & $\mathrm{n} / \mathrm{a}$ \\
\hline$\$ 96 T 005051$ & D & Titanium-ICP-Acid Dil. & $\mathrm{ug} / \mathrm{mL}$ & 97.80 & $<1.00 \mathrm{e}-02$ & 1.010 & $<1.01 \mathrm{e} 0$ & $\mathrm{n} / \mathrm{a}$ & $\mathrm{n} / \mathrm{a}$ & $n / a$ & 1.010 & $n / a$ \\
\hline 5967005051 & D & Thal I i um-ICP-Acid Dil. & $\mathrm{gg} / \mathrm{mL}$ & 97.20 & $<2.00 \mathrm{e}-01$ & 20.20 & $<2.02 \mathrm{e} 1$ & $\mathrm{n} / \mathrm{a}$ & $\mathrm{n} / \mathrm{a}$ & $\mathrm{n} / \mathrm{a}$ & 20.20 & $n / a$ \\
\hline S96T005051 & D & Uranium-ICP-Acid Dil. & $\mathrm{ug} / \mathrm{mL}$ & 101.0 & $<5.00 \mathrm{e}-01$ & 50.50 & $<5.05 \mathrm{e} 1$ & $\mathrm{n} / \mathrm{a}$ & $\mathrm{n} / \mathrm{a}$ & $\mathrm{n} / \mathrm{a}$ & 50.50 & $n / a$ \\
\hline $596 r 005051$ & D & Vanadium-ICP-Acid Dil. & $\mathrm{ug} / \mathrm{mL}$ & 101.4 & $<5.00 e^{-02}$ & 5.050 & $<5.05 \mathrm{e} 0$ & $\mathrm{n} / \mathrm{a}$ & $\mathrm{n} / \mathrm{a}$ & $\mathrm{n} / \mathrm{a}$ & 5.050 & $\mathrm{n} / \mathrm{a}$ \\
\hline S96T005051 & D & Zinc-ICP-Acid Dil. & $\mathrm{ug} / \mathrm{mL}$ & 101.6 & $<1.00 \mathrm{e}-02$ & 10.30 & 10.30 & 10.30 & 0.00 & $n / a$ & 1.010 & $\mathrm{n} / \mathrm{a}$ \\
\hline S96T005051 & D & Zirconium-ICP-Acid Dil. & $\mathrm{ug} / \mathrm{mL}$ & 99.40 & $<1.00 e^{-02}$ & 13.70 & 6.720 & 10.21 & 68.4 & $n / a$ & 1.010 & $\mathrm{n} / \mathrm{a}$ \\
\hline \$967005051 & & Fluoride-IC-Dionex $4000 / 4500$ & $\mathrm{Lg} / \mathrm{mL}$ & 98.47 & $<1.20 \mathrm{e}^{-02}$ & $1.25 e+04$ & $1.26 \mathrm{e}+04$ & $1.26 \mathrm{e}+04$ & 0.80 & 92.03 & 25.45 & $\mathrm{n} / \mathrm{a}$ \\
\hline S96T005051 & & Chloride-IC-Dionex $4000 / 4500$ & $\mathrm{ug} / \mathrm{mL}$ & 97.72 & $<1.70 \mathrm{e}-02$ & $3.99 \mathrm{e}+02$ & 420.0 & 409.4 & 5.13 & 95.57 & 36.06 & $\mathrm{n} / \mathrm{a}$ \\
\hline S96T005051 & & Nitrite-IC - Dionex $4000 / 4500$ & $\mathrm{ug} / \mathrm{mL}$ & 97.96 & $<1.08 \mathrm{e}-01$ & $4.70 \mathrm{e}+03$ & $4.68 \mathrm{e}+03$ & $4.69 \mathrm{e}+03$ & 0.43 & 100.7 & 229.1 & $\mathrm{n} / \mathrm{a}$ \\
\hline S96T005051 & & Bromide by Ion chromatograph & $\mathrm{ug} / \mathrm{mL}^{2}$ & 104.1 & $<1.25 \mathrm{e}-01$ & $<2.65 \mathrm{e}+02$ & $<2.65 \mathrm{e} 2$ & $\mathrm{n} / \mathrm{a}$ & $\mathrm{n} / \mathrm{a}$ & 103.4 & 265.1 & $\mathrm{n} / \mathrm{a}$ \\
\hline \$96T005051 & & Nitrate by IC-0ionex $4000 / 4500$ & $\mathrm{ug} / \mathrm{mL}$ & 103.9 & $2.03 e-01$ & $2.77 e+04$ & $2.81 e+04$ & $2 . \overline{79}++04$ & 1.43 & 110.9 & 294.8 & $\mathrm{n} / \mathrm{a}$ \\
\hline S96T005051 & & Phosphate-IC-D ionex $4000 / 4500$ & $\mathrm{ug} / \mathrm{mL}$ & 103.3 & $<1.20 \mathrm{e}-01$ & $5.90 \mathrm{e}+02$ & 599.0 & 594.2 & 1.51 & 102.2 & 254.5 & $n / a$ \\
\hline $596700505 ?$ & & Sulfate by IC-Dionex $4000 / 4500$ & $\mathrm{ug} / \mathrm{mL}$ & 102.5 & $<1.38 \mathrm{e}-01$ & $1.61 \mathrm{e}+03$ & $1.65 e+03$ & $1.63 \mathrm{e}+03$ & 2.45 & 102.9 & 292.7 & $n / a$ \\
\hline$\$ 967005051$ & & Oxalate by IC-Djonex $4000 / 4500$ & $\mathrm{ug} / \mathrm{mL}$ & 105.3 & $<1.05 \mathrm{e}-01$ & $3.27 \mathrm{e}+02$ & $<2.23 \mathrm{e} 2$ & $\mathrm{n} / \mathrm{a}$ & $n / a$ & 106.5 & 222.7 & $\mathrm{n} / \mathrm{a}$ \\
\hline $596 \mathrm{~T} 005051$ & & Cobal $t-60$ by GEA & $\mathrm{uCi} / \mathrm{mL}$ & 99.36 & $<2.93 e-04$ & $2.45 \mathrm{e}-02$ & $2.32 \mathrm{e}-02$ & $2.38 \mathrm{e}-02$ & 5.45 & $n / a$ & $n / a$ & 5.94 \\
\hline $596 \mathrm{r} 005051$ & & Cesium- 137 by GEA & $\mathrm{UCi} / \mathrm{mL}$ & 100.2 & $<7.59 \mathrm{e}-04$ & 25.00 & 25.00 & 25.00 & 0.00 & $\mathrm{n} / \mathrm{a}$ & $n / a$ & 0.160 \\
\hline 5961005051 & & Am-241 by Extraction & $\mathrm{uCi} / \mathrm{mL}$ & 90.76 & $<6.40 e-05$ & $<6.13 \mathrm{e}-05$ & $<5.88 \mathrm{E}-5$ & $n / a$ & $n / a$ & $\mathrm{n} / \mathrm{a}$ & $6.13 \mathrm{e}-05$ & $1.00 \mathrm{E}+02$ \\
\hline S96T005051 & & Alpha in Liquid Samples & $\mathrm{uCi} / \mathrm{mL}$ & 100.0 & $<5.10 \mathrm{e}-04$ & $1.05 e^{-03}$ & $1.69 \mathrm{e}-03$ & $1 . \overline{37 \mathrm{e}-03}$ & 46.7 & $\mathrm{n} / \mathrm{a}$ & $1.00 \mathrm{e}-03$ & $7.14 \mathrm{E}+01$ \\
\hline
\end{tabular}

Sludge (from Liquid Grab Sample): Sludge (from Liquid Grab Sample)

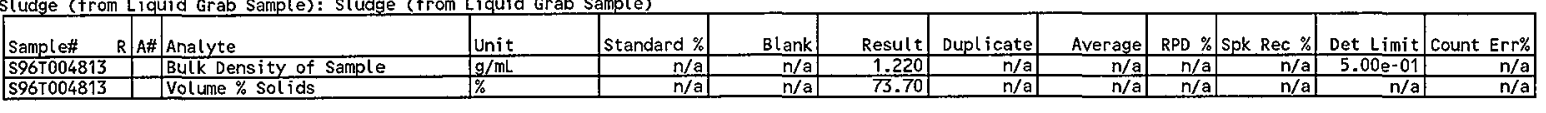


RISER: $15 \mathrm{~A}$

SEGMENT \#: 5AW-96-10

SEGMENT PORTION: Supernate

\begin{tabular}{|c|c|c|c|c|c|c|c|c|c|c|c|c|}
\hline Sample\# & $\mathrm{R} \mid \dot{A} \#$ & \# Analiyte & Unit & Standard \% & Blank & Result & Duplicate & Average & RPD \% & Spk Rec $\%$ & Det Limit & Count Err\% \\
\hline 5967005010 & & DSC Exotherm using Mettler & Joules/g & 92.44 & $n / a$ & $0.00 \mathrm{e}+00$ & $0.00 \mathrm{e}+00$ & $0.00 \mathrm{e}+00$ & 0.00 & $n / a$ & $n / a$ & $n / a$ \\
\hline S96T005010 & & oh- by Pot. Titration & $\mathrm{ug} / \mathrm{mL}$ & 99.76 & $<42.00$ & $2.90 \mathrm{e}+03$ & $2.85 \mathrm{e}+03$ & $2.88 \mathrm{e}+03$ & 1.74 & $\mathrm{n} / \mathrm{a}$ & 625.0 & $\mathrm{n} / \mathrm{a}$ \\
\hline S96r005010 & & pH Direct & $\mathrm{pH}$ & 99.86 & $n / a$ & 13.41 & 13.42 & 13.41 & 0.07 & $n / a$ & $1.00 \mathrm{e}-02$ & $\mathrm{n} / \mathrm{a}$ \\
\hline S96T005010 & & Specific Gravity & Sp.G. & 99.60 & $n / a$ & 1.016 & 1.027 & 1.022 & 1.08 & $\mathrm{n} / \mathrm{a}$ & $1.00 \mathrm{e}-02$ & $\mathrm{n} / \mathrm{a}$ \\
\hline $596 \mathrm{~T} 005010$ & & $\%$ Water by TGA using Mettler & $\%$ & 99.64 & $n / a$ & 95.10 & 94.98 & 95.04 & 0.13 & $\mathrm{n} / \mathrm{a}$ & $n / a$ & $n / a$ \\
\hline S967005010 & & TIC by Acid/Coulometry & $\mathrm{ug} / \mathrm{mL}$ & 106.1 & 2.500 & $4.32 \mathrm{e}+02$ & 437.0 & 434.5 & 1.15 & 88.10 & 5.000 & $\mathrm{n} / \mathrm{a}$ \\
\hline $596 \mathrm{~T} 005010$ & & Tot. Organic Carbon by Coul. & $\mathrm{ug} / \mathrm{mL}$ & 103.7 & 1.200 & $1.51 \mathrm{e}+03$ & $1.53 e+03$ & $1.52 \mathrm{e}+03$ & 1.32 & 103.0 & 55.00 & $n / a$ \\
\hline 5967005010 & D & Silver-ICP-Acid Dil. & $\mathrm{ug} / \mathrm{mL}$ & 99.20 & $<1.00 \mathrm{e}-02$ & 1.040 & 1.070 & 1.055 & 2.84 & 80.50 & $4.10 \mathrm{e}-01$ & $n / a$ \\
\hline 5961005010 & D & Aluminium-ICP-Acid Dil. & $\mathrm{ug} / \mathrm{mL}$ & 98.00 & $<5.00 \mathrm{e}-02$ & 16.00 & 16.20 & 16.10 & 1.24 & 98.50 & 2.050 & $n / a$ \\
\hline$\$ 967005010$ & D & Arsenic-ICP-Acid Dil. & $\mathrm{ug} / \mathrm{mL}$ & 103.2 & $<1.00 e-01$ & 4.100 & $<4.10 \mathrm{e} 0$ & $n / a$ & $n / a$ & 108.0 & 4.100 & $\mathrm{n} / \mathrm{a}$ \\
\hline S96T005010 & D & Boron-ICP-Acid Dil. & $\mathrm{ug} / \mathrm{mL}$ & 101.4 & $<5.00 e-02$ & 2.850 & 2.870 & 2.860 & 0.70 & 900.0 & 2.050 & $\mathrm{n} / \mathrm{a}$ \\
\hline$\$ 967005010$ & D & Bari IUm-ICP-Acid Dil. & $\mathrm{ug} / \mathrm{mL}$ & 100.6 & $<5.00 \mathrm{e}-02$ & 2.050 & $<2.05 \mathrm{ee}$ & $n / a$ & $n / a$ & 99.80 & 2.050 & $n / a$ \\
\hline S96T005010 & D & Beryllíum-ICP-Acid Dil. & $\mathrm{ug} / \mathrm{mL}$ & 102.2 & $<5.00 \mathrm{e}-03$ & $<2.05 e-01$ & $<2.05 e^{-1}$ & $\mathrm{n} / \mathrm{a}$ & $n / a$ & 102.0 & $2.05 e^{-01}$ & $\mathrm{n} / \mathrm{a}$ \\
\hline $596 T 005010$ & D & Bismuth-ICP-Acid Dil, & $\mathrm{ug} / \mathrm{mL}$ & 101.8 & $<1.00 \mathrm{e}-01$ & $<4.100$ & $<4.1000$ & $\mathrm{n} / \mathrm{a}$ & $\mathrm{n} / \mathrm{a}$ & 107.0 & 4.100 & $n / a$ \\
\hline S96T005010 & D & Calcium-ICP-Acid Dil. & $\underline{\mathrm{ug} / \mathrm{mL}}$ & 100.2 & $<1.00 \mathrm{e}-01$ & $\leq 4.100$ & $<4.10 \mathrm{e} 0$ & $\mathrm{n} / \mathrm{a}$ & $\mathrm{n} / \mathrm{a}$ & 112.0 & 4.100 & $n / a$ \\
\hline S96T005010 & D & Cadmi Um-ICP-Acid DiL. & $\mathrm{ug} / \mathrm{mL}$ & 100.2 & $<5.00 \mathrm{e}-03$ & $<2.05 e-01$ & $<2.05 e-1$ & $\mathrm{n} / \mathrm{a}$ & n/a & 102.0 & $2.05 e-01$ & $n / a$ \\
\hline$\$ 965005010$ & D & Cerium-ICP-Acid Díl. & $\mathrm{ug} / \mathrm{mL}$ & 100.0 & $<1.00 \mathrm{e}-01$ & $<4.100$ & $<4.10 \mathrm{e} 0$ & $\mathrm{n} / \mathrm{a}$ & $n / a$ & 101.0 & 4.100 & $\mathrm{n} / \mathrm{a}$ \\
\hline S96T005010 & D & Cobalt-ICP-Acid Dil. & $\mathrm{ug} / \mathrm{mL}$ & 102.0 & $<2.00 e-02$ & $<8.20 \mathrm{e}-01$ & $<8.20 e^{-1}$ & $n / a$ & $\mathrm{n} / \mathrm{a}$ & 103.0 & $8.20 \mathrm{e}-01$ & $\mathrm{n} / \mathrm{a}$ \\
\hline \$96T005010 & D & Chromium-ICP-Acid Dil. & $\mathrm{ug} / \mathrm{mL}$ & 101.0 & $<1.00 \mathrm{e}-02$ & $6.10 \mathrm{e}-01$ & $6.48 \mathrm{e}-01$ & $6.29 \mathrm{e}-01$ & 6.04 & 103.0 & $4.10 \mathrm{e}-01$ & $n / a$ \\
\hline S96T005010 & $\bar{D}$ & Copper-ICP-Acid Dil. & $\mathrm{ug} / \mathrm{mL}$ & 105.0 & $<1.00 e^{-02}$ & $<4.10 e^{-01}$ & $<4.10 \mathrm{e}-1$ & $\mathrm{n} / \mathrm{a}$ & $\mathrm{n} / \mathrm{a}$ & 105.0 & $4.10 \mathrm{e}-01$ & $\mathrm{n} / \mathrm{a}$ \\
\hline 5961005010 & D & Iron-ICP-Acid Dil. & $\mathrm{ug} / \mathrm{mL}$ & 100.6 & $<5.00 \mathrm{e}-02$ & $<2.050$ & $<2.05 \mathrm{e} 0$ & $n / a$ & $\mathrm{n} / \mathrm{a}$ & 101.0 & 2.050 & $n / a$ \\
\hline S96T005010 & D & Potassium-ICP-Acid Dil. & $\underline{u g} / \mathrm{mL}$ & 97.40 & $<5.00 \mathrm{e}-01$ & $1.27 \mathrm{e}+03$ & $1.27 \mathrm{e}+03$ & $1.27 \mathrm{e}+03$ & 0.00 & 98.80 & 20.50 & $n / a$ \\
\hline S96T005010 & $\frac{D}{D}$ & Lanthanum-ICP-Acid Dil. & $\mathrm{ug} / \mathrm{mL}$ & 101.8 & $<5.00 e-02$ & $<2.050$ & $<2.05 \mathrm{e} 0$ & $n / a$ & $\mathrm{n} / \mathrm{a}$ & 102.0 & 2.050 & $\mathrm{n} / \mathrm{a}$ \\
\hline S96T005010 & D & Lithium-ICP-ACid Dil. & $\mathrm{ug} / \mathrm{mL}$ & 101.0 & $<1.00 \mathrm{e}-02$ & $<4.10 \mathrm{e}-01$ & $<4.10 \mathrm{e}-1$ & $\mathrm{n} / \mathrm{a}$ & $\mathrm{n} / \mathrm{a}$ & 102.0 & $4.10 \mathrm{e}-01$ & $n / a$ \\
\hline $596 r 005010$ & D & Magnesium-ICP-Acid Dil. & $\underline{u g} / \mathrm{mL}$ & 96.20 & $<1.00 e-01$ & $\leq 4.100$ & $<4.10 \mathrm{e} 0$ & $\mathrm{n} / \mathrm{a}$ & $\mathrm{n} / \mathrm{a}$ & 96.80 & 4.100 & $n / a$ \\
\hline \$96T005010 & D & Manganese-ICP-Acid Dil. & $\mathrm{ug} / \mathrm{mL}$ & 97.80 & $<1.00 \mathrm{e}-02$ & $<4.10 \mathrm{e}-01$ & $<4.10 e^{-1}$ & $n / a$ & $n / a$ & 96.90 & $4.10 e-01$ & $n / a$ \\
\hline$\$ 961005010$ & $D$ & Molybdenum-ICP-Acid Dil. & $\mathrm{ug} / \mathrm{mL}$ & 102.2 & $<5.00 e^{-02}$ & $\leq \quad 2.050$ & $<2.05 \mathrm{e} 0$ & $\mathrm{n} / \mathrm{a}$ & $\mathrm{n} / \mathrm{a}$ & 105.0 & 2.050 & $n / a$ \\
\hline $596 T 005010$ & ID & Sodium-ICP-Acid Dil. & $\mathrm{ug} / \mathrm{mL}$ & 97.80 & $<1.00 e-01$ & $1.27 e+04$ & $1.28 \mathrm{e}+04$ & $1.28 \mathrm{e}+04$ & 0.78 & 88.00 & 4.100 & $\mathrm{n} / \mathrm{a}$ \\
\hline $596 \mathrm{~T} 005010$ & D & Neodymium-ICP-Acid Dil. & $\mathrm{ug} / \mathrm{mL}$ & 102.2 & $<1.00 e-01$ & $<\quad 4.100$ & $<4.10 \mathrm{e} 0$ & $n / a$ & $\mathrm{n} / \mathrm{a}$ & 102.0 & 4.100 & $n / a$ \\
\hline S967005010 & D & Nickel-ICP-Acid Dil. & $\mathrm{ug} / \mathrm{mL}$ & 100.0 & $<2.00 e-02$ & $<8.20 e-01$ & $<8.20 e^{-1}$ & $\mathrm{n} / \mathrm{a}$ & $\mathrm{n} / \mathrm{a}$ & 101.0 & $8.20 \mathrm{e}-01$ & $n / a$ \\
\hline 5967005010 & D & Phosphorus-ICP-Acid Dil. & $\mathrm{ug} / \mathrm{mL}$ & 99.00 & $<2.00 e-01$ & 13.30 & 13.00 & 13.15 & 2.28 & 102.0 & 8.200 & $\mathrm{n} / \mathrm{a}$ \\
\hline 5967005010 & $\frac{1}{D}$ & Lead-ICP-Acid Dil. & $\mathrm{ug} / \mathrm{mL}$ & 100.0 & $<1.00 e-01$ & 4.100 & $<4.10 \mathrm{e} 0$ & $n / a$ & $\mathrm{n} / \mathrm{a}$ & 102.0 & 4.100 & $n / a$ \\
\hline$\$ 967005010$ & D & Sulfur-ICP-ACid Dil. & $\mathrm{ug} / \mathrm{mL}$ & 98.20 & $<1.00 \mathrm{e}-01$ & 46.50 & 45.90 & 46.20 & 1.30 & 101.0 & 4.100 & $\mathrm{n} / \mathrm{a}$ \\
\hline 5967005010 & D & Ántimony-ICP-Acid Dil. & $\mathrm{ug} / \mathrm{mL}$ & 96.80 & $<6.00 \mathrm{e}-02$ & 2.460 & $<2.46 \mathrm{e} 0$ & $n / a$ & $n / a$ & 98.50 & 2.460 & $n / a$ \\
\hline 5967005010 & D & Selenium-ICP-Acid Dil. & $\mathrm{ug} / \mathrm{mL}$ & 96.00 & $<1.00 e-01$ & 4.100 & $<4.10 \mathrm{e} 0$ & $\mathrm{n} / \mathrm{a}$ & $n / a$ & 103.0 & 4.100 & $n / a$ \\
\hline S96T005010 & $\mathrm{ID}$ & Silicon-ICP-Acid Dil. & $\mathrm{ug} / \mathrm{mL}$ & 99.60 & $<5.00 e-02$ & 39.60 & 40.10 & 39.85 & 1.25 & 105.0 & 2.050 & $n / a$ \\
\hline S96T005010 & T & Samar ium-ICP-Acid Dil. & $\mathrm{ug} / \mathrm{mL}$ & 98.40 & $<1.00 e-01$ & 4.100 & $<4.10 \mathrm{e} 0$ & $\mathrm{n} / \mathrm{a}$ & $\mathrm{n} / \mathrm{a}$ & 97.50 & 4.100 & $\mathrm{n} / \mathrm{a}$ \\
\hline$\$ 96 \mathrm{~T} 005010$ & D & Strontium-ICP-Acid Dil. & $\mathrm{ug} / \mathrm{mL}$ & 99.20 & $<1.00 \mathrm{e}-02$ & $<4.10 \mathrm{e}-01$ & $<4.10 e-1$ & $\mathrm{n} / \mathrm{a}$ & $\mathrm{n} / \mathrm{a}$ & 99.60 & $4.10 e-01$ & $\mathrm{n} / \mathrm{a}$ \\
\hline S96T005010 & D & Títaníum-ICP-Acid Dil. & $\mathrm{ug} / \mathrm{mL}$ & 99.40 & $<1.00 \mathrm{e}-02$ & $<4.10 \mathrm{e}-01$ & $<4.10 \mathrm{e}-1$ & $n / a$ & $\mathrm{n} / \mathrm{a}$ & 100.0 & $4.10 e-01$ & $\mathrm{n} / \mathrm{a}$ \\
\hline 5967005010 & D & Thallium-ICP-Acid Dil. & $\mathrm{ug} / \mathrm{mL}$ & 96.80 & $<2.00 e^{-01}$ & $<8.200$ & $<8.20 \mathrm{e} 0$ & $n / a$ & $n / a$ & 96.70 & 8.200 & $n / a$ \\
\hline 5967005010 & $\mathrm{D}$ & Uranium-ICP-Acid Dil. & $\mathrm{ug} / \mathrm{mL}$ & 97.30 & $<5.00 e-01$ & 20.50 & $<2.05 \mathrm{e} 1$ & $n / a$ & $n / a$ & 116.5 & 20.50 & $n / a$ \\
\hline 5967005010 & $D$ & Vanadi um-ICP-Acid Dil. & $\mathrm{ug} / \mathrm{mL}$ & 101.2 & $<5.00 e-02$ & 2.050 & $<2.05 \mathrm{e} 0$ & $\mathrm{n} / \mathrm{a}$ & n/a & 102.0 & 2.050 & $\mathrm{n} / \mathrm{a}$ \\
\hline $596 r 005010$ & $\bar{D}$ & Zinc-ICP-Acid Dil. & $\mathrm{ug} / \mathrm{mL}$ & 101.8 & $<1.00 \mathrm{e}-02$ & 2.610 & 2.670 & 2.640 & 2.27 & 104.0 & $4.10 e-01$ & $\mathrm{n} / \mathrm{a}$ \\
\hline 5961005010 & D & Zirconílum-ICP-Acid Dil. & $\mathrm{ug} / \mathrm{mL}$ & 100.4 & $<1.00 \mathrm{e}-02$ & $<4.10 \mathrm{e}-01$ & $<4.10 \mathrm{e}-1$ & $\mathrm{n} / \mathrm{a}$ & $\mathrm{n} / \mathrm{a}$ & 101.0 & $4.10 \mathrm{e}-01$ & $\mathrm{n} / \mathrm{a}$ \\
\hline
\end{tabular}




\begin{tabular}{|c|c|c|c|c|c|c|c|c|c|c|c|c|}
\hline Sample\# & R A\# & Analyte & Unit & Standard \% & Blank & Result & Dupl icate & Average & RPD \% & Spk Rec \% & Det Limit & Count Err\% \\
\hline$\$ 967005010$ & & Fluoride-IC-Dionex $4000 / 4500$ & $\mathrm{ug} / \mathrm{mL}$ & 90.68 & $<1.20 \mathrm{e}^{-02}$ & $1.31 \mathrm{e}+02$ & 129.0 & 129.9 & 1.54 & 86.10 & 13.33 & $n / a$ \\
\hline S967005010 & & Chloride-IC-Dionex $4000 / 4500$ & $\mathrm{ug} / \mathrm{mL}$ & 97.34 & $<1.70 \mathrm{e}-02$ & $2.14 \mathrm{e}+02$ & 210.0 & 211.8 & 1.89 & 96.33 & 18.89 & $n / a$ \\
\hline$\$ 967005010$ & & Nitrite-IC - Dionex $4000 / 4500$ & $\mathrm{ug} / \mathrm{mL}$ & 91.85 & $<7.08 \mathrm{e}-01$ & $1.09 \mathrm{e}+03$ & $1.07 \mathrm{e}+03$ & $1.08 \mathrm{e}+03$ & 1.85 & 86.67 & 120.0 & $n / a$ \\
\hline S96T005010 & & Bromide by Ion chromatograph & $\mathrm{ug} / \mathrm{mL}$ & 98.98 & $<1.25 e^{-01}$ & $<1.39 \mathrm{e}+02$ & $<1.39 \mathrm{e} 2$ & n/a & $\mathrm{n} / \mathrm{a}$ & 87.27 & 138.9 & $n / a$ \\
\hline S96TC & & Nitrate by IC-Dionex $4000 / 4500$ & $\mathrm{ug} / \mathrm{mL}$ & 101.3 & $<1.39 \mathrm{e}-01$ & $2.46 \mathrm{e}+04$ & $2.48 \mathrm{e}+04$ & $2.47 \mathrm{e}+04$ & 0.81 & 110.6 & 154.4 & $\mathrm{n} / \mathrm{a}$ \\
\hline S96T005010 & & Phosphate-[C-Dionex $4000 / 4500$ & $\mathrm{ug} / \mathrm{mL}$ & 97.43 & $<1.20 \mathrm{e}-01$ & $<1.33 e+02$ & $<1.33 \mathrm{e} 2$ & $n / a$ & $\mathrm{n} / \mathrm{a}$ & 90.46 & 133.3 & $\mathrm{n} / \mathrm{a}$ \\
\hline$\$ 967005010$ & & sulfate by IC-Dionex $4000 / 4500$ & $\mathrm{ug} / \mathrm{mL}$ & 102.4 & $<1.38 \mathrm{e}-01$ & $2.21 \mathrm{e}+02$ & 187.0 & 204.1 & 16.7 & 94.29 & 153.3 & $n / a$ \\
\hline S96T005010 & & Oxalate by IC-Dionex $4000 / 4500$ & $\mathrm{ug} / \mathrm{mL}$ & 101.1 & $<1.05 e-01$ & $<1.17 \mathrm{e}+02$ & $<1.17 \mathrm{e} 2$ & $n / a$ & $\mathrm{n} / \mathrm{a}$ & 93.84 & 116.7 & $n / a$ \\
\hline \$967 & & Strontium-89/90 High Level & uci/mL & 106.4 & $1.16 \mathrm{e}-04$ & $2.11 e^{-02}$ & $1.93 \mathrm{e}-02$ & $2.02 \mathrm{e}-02$ & 8.91 & $n / a$ & $2.80 e^{-05}$ & $1.49 E+00$ \\
\hline S96T0 & & Pu-239/240 by TRU-SPEC Resin & $\mathrm{UCi} / \mathrm{mL}$ & 100.7 & $<4.45 e^{-06}$ & $<4.58 \mathrm{e}-06$ & $<4.15 \mathrm{E}-6$ & $n / a$ & $\mathrm{n} / \mathrm{a}$ & $n / a$ & $4.58 \mathrm{e}-06$ & $7.12 \mathrm{E}+00$ \\
\hline S96T005013 & & Cobal $t-60$ by GEA & $\mathrm{uCi} / \mathrm{mL}$ & 98.07 & $<3.85 e^{-05}$ & $1.30 \mathrm{e}-04$ & $1.04 e-04$ & $1.17 \mathrm{e}-04$ & 22.2 & $\mathrm{n} / \mathrm{a}$ & $n / a$ & 21.6 \\
\hline S96TO & & Cesium- 137 by GEA & $\mathrm{uCi} / \mathrm{mL}$ & 106.6 & $<7.27 \mathrm{e}-05$ & $7.19 e-01$ & $7.14 \mathrm{e}-01$ & $7.16 \mathrm{e}-01$ & 0.70 & $n / a$ & $\mathrm{n} / \mathrm{a}$ & 0.290 \\
\hline \$96T & & Am-241 by Extraction & $\mathrm{uCi} / \mathrm{mL}$ & 100.8 & $<6.64 e^{-06}$ & $<6.57 \overline{e-06}$ & $<5.58 \mathrm{E}-6$ & $n / a$ & $\mathrm{n} / \mathrm{a}$ & $n / a$ & $6.57 \mathrm{e}-06$ & $1.00 E+02$ \\
\hline \$96T005013 & & Alpha in Liquid samples & $\mathrm{uCi} / \mathrm{mL}$ & 105.2 & $<7.07 \mathrm{e}-05$ & $<7.87 e-05$ & $<7.07 E-5$ & $\mathrm{n} / \mathrm{a}$ & n/a & 87.74 & $9.44 e^{-05}$ & $1.62 E+02$ \\
\hline
\end{tabular}


Table 3: Data Sumary Report AW-105 GRAB1

RISER: $15 \mathrm{~A}$

SEGMENT \#: 5AW-96-11

SEGMENT PORTION: Supernate

\begin{tabular}{|c|c|c|c|c|c|c|c|c|c|c|c|c|}
\hline Sample\# & $R \mid A$ & A\# Analyte & Unit & Standard \% & $B$ Lank & Result & Duplicate & Average & RPD \% & Spk Rec \% & Det Limit & Count Err\% \\
\hline \$96T005011 & & DSC Exotherm using Mettler & Joules $/ \mathrm{g}$ & 92.44 & $\mathrm{n} / \mathrm{a}$ & $0.00 \mathrm{e}+00$ & $0.00 \mathrm{e}+00$ & $0.00 \mathrm{e}+00$ & 0.00 & $\mathrm{n} / \mathrm{a}$ & $\mathrm{n} / \mathrm{a}$ & $n / a$ \\
\hline S96T005011 & & OH- by Pot. Titration & $\mathrm{ug} / \mathrm{mL}$ & 99.76 & $<42.00$ & $3.15 \mathrm{e}+03$ & $3.37 e+03$ & $3.26 e+03$ & 6.75 & $\mathrm{n} / \mathrm{a}$ & 625.0 & $n / a$ \\
\hline S967005011 & & pH Direct & $\mathrm{pH}$ & 99.86 & $n / a$ & 13.46 & 13.46 & 13.46 & 0.00 & $\mathrm{n} / \mathrm{a}$ & $1.00 \mathrm{e}-02$ & $n / a$ \\
\hline$\$ 96 T 005011$ & & Specific Gravity & Sp.G. & 99.60 & $\mathrm{n} / \mathrm{a}$ & 1.021 & 1.025 & 1.023 & 0.39 & $n / a$ & $1.00 \mathrm{e}-02$ & $\mathrm{n} / \mathrm{a}$ \\
\hline S96T005011 & & $\%$ Water by TGA using Mettler & $\%$ & 99.64 & $\mathrm{n} / \mathrm{a}$ & 94.79 & 94.92 & 94.86 & 0.14 & $n / a$ & $\mathrm{n} / \mathrm{a}$ & n/a \\
\hline \$96T005011 & & TIC by Acid/coulometry & $\mathrm{ug} / \mathrm{mL}$ & 106.1 & 2.500 & $3.67 \mathrm{e}+02$ & 398.0 & 382.5 & 8.10 & $n / a$ & 5.000 & $\mathrm{n} / \mathrm{a}$ \\
\hline \$96T005011 & & Tot. Organic Carbon by Coul. & $\mathrm{ug} / \mathrm{mL}$ & 103.7 & 1.200 & $1.44 \mathrm{e}+03$ & $1.42 \mathrm{e}+03$ & $1.43 \mathrm{e}+03$ & 1.40 & n/a & 55.00 & n/a \\
\hline$\$ 961005011$ & D & Silver-ICP-Acid Dil. & $\mathrm{ug} / \mathrm{mL}$ & 99.20 & $<1.00 \mathrm{e}-02$ & 1.160 & 1.150 & 1.155 & 0.87 & 81.10 & $4.10 \mathrm{e}-01$ & $n / a$ \\
\hline 5967005011 & D & Aluminium-ICP-Acid Dil. & ug $/ \mathrm{mL}$ & 98.00 & $<5.00 \mathrm{e}^{-02}$ & 16.90 & 16.90 & 16.90 & 0.00 & 98.30 & 2.050 & $n / a$ \\
\hline$\$ 967005011$ & D & Arsenic-ICP-Acid Dil, & ug/mL & 103.2 & $<1.00 \mathrm{e}-01$ & $<\quad 4.100$ & $<4.10 \mathrm{e} 0$ & $n / a$ & $\mathrm{n} / \mathrm{a}$ & 107.0 & 4.100 & $\mathrm{n} / \mathrm{a}$ \\
\hline$\$ 967005011$ & $\bar{D}$ & Boron-ICP-Acid Dil. & ugg/mL & 101.4 & $<5.00 \mathrm{e}-02$ & 2.680 & 2.660 & 2.670 & 0.75 & 98.70 & 2.050 & $\mathrm{n} / \mathrm{a}$ \\
\hline \$96T005011 & D & Barium-ICP-Acid Dil. & $\mathrm{ug} / \mathrm{mL}$ & 100.6 & $<5.00 \mathrm{e}-02$ & $<2.050$ & $<2.05 \mathrm{e} 0$ & $n / a$ & $n / a$ & 99.10 & 2.050 & $\mathrm{n} / \mathrm{a}$ \\
\hline \$96T005011 & D & Beryllium-ICP-Acid Dil. & $\mathrm{ug} / \mathrm{mL}$ & 102.2 & $<5.00 \mathrm{e}-03$ & $<2.05 e-01$ & $<2.05 e^{-1}$ & $n / a$ & $\mathrm{n} / \mathrm{a}$ & 101.0 & $2.05 e-01$ & $n / a$ \\
\hline 5967005011 & $\bar{D}$ & Bismuth-ICP-Acid Dil. & ug/mL & 101.8 & $<1.00 \mathrm{e}-01$ & $<4.100$ & $<4.10 \mathrm{e} 0$ & $n / a$ & $\mathrm{n} / \mathrm{a}$ & 101.0 & 4.100 & $n / a$ \\
\hline$\$ 967005011$ & D & Calcium-ICP-Acid Dil. & $\mathrm{ug} / \mathrm{mL}$ & 100.2 & $<1.00 \mathrm{e}-01$ & 4.170 & 4.190 & 4.180 & 0.48 & 103.0 & 4.100 & $n / a$ \\
\hline$\$ 967005011$ & D & Cadmi un-ICP-Acid Dil. & ug/mL & 100.2 & $<5.00 \mathrm{e}-03$ & $<2.05 e^{-01}$ & $<2.05 \mathrm{e}-1$ & $\mathrm{n} / \mathrm{a}$ & $n / a$ & 102.0 & $2.05 e-01$ & $n / a$ \\
\hline S96T005011 & $\bar{D}$ & Cerium-ICP-Acid Dil. & $\mathrm{ug} / \mathrm{mL}$ & 100.0 & $<1.00 \mathrm{e}-01$ & $<4.100$ & $<4.10 \mathrm{e} 0$ & $\mathrm{n} / \mathrm{a}$ & $\mathrm{n} / \mathrm{a}$ & 99.50 & 4.100 & $\mathrm{n} / \mathrm{a}$ \\
\hline S96T005011 & D & Cobalt-ICP-Acid Dil. & $\mathrm{ug} / \mathrm{mL}$ & 102.0 & $<2.00 \mathrm{e}-02$ & $<8.20 \mathrm{e}-01$ & $<8.20 e^{-1}$ & $n / a$ & $n / a$ & 103.0 & $8.20 e-01$ & $n / a$ \\
\hline S96T005011 & D & Chromium-ICP-Acid Dil. & $\mathrm{ug} / \mathrm{mL}$ & 101.0 & $<1.00 \mathrm{e}-02$ & $6.53 e-01$ & $6.43 e-01$ & $6.48 \mathrm{e}-01$ & 1.54 & 102.0 & $4.10 \mathrm{e}-01$ & $n / a$ \\
\hline S96r005011 & D & Copper-ICP-Acid Dil. & $\mathrm{ug} / \mathrm{mL}$ & 105.0 & $<1.00 \mathrm{e}-02$ & $<4.10 \mathrm{e}-01$ & $<4.10 \mathrm{e}-1$ & $n / a$ & $n / a$ & 105.0 & $4.10 \mathrm{e}-01$ & n/a \\
\hline 5967005011 & D & Iron-ICP-Acid Dil. & $\mathrm{ug} / \mathrm{mL}$ & 100.6 & $<5.00 e-02$ & $<2.050$ & $<2.05 \mathrm{e} 0$ & $n / a$ & $\mathrm{n} / \mathrm{a}$ & 101.0 & 2.050 & $\mathrm{n} / \mathrm{a}$ \\
\hline$\$ 967005011$ & D & Potassium-icp-Acid Dil. & $\mathrm{ug} / \mathrm{mL}$ & 97.40 & $<5.00 e-01$ & $1.3 \% \mathrm{e}+03$ & $1.31 \mathrm{e}+03$ & $1.31 \mathrm{e}+03$ & 0.00 & 86.40 & 20.50 & $n / a$ \\
\hline \$96T005011 & D & Lanthanum-ICP-Acid Dil, & ug $/ \mathrm{mL}$ & 101.8 & $<5.00 \mathrm{e}^{-02}$ & $<2.050$ & $<2.05 \mathrm{e} 0$ & $n / a$ & $n / a$ & 102.0 & 2.050 & $n / a$ \\
\hline 5967005011 & D & Li thium-ICP-Acid Dil. & $\mathrm{ug} / \mathrm{mL}$ & 101.0 & $<1.00 \mathrm{e}-02$ & $<4.10 \mathrm{e}-01$ & $<4.10 \mathrm{e}-1$ & $\mathrm{n} / \mathrm{a}$ & $\mathrm{n} / \mathrm{a}$ & 102.0 & $4.10 \mathrm{e}-01$ & $n / a$ \\
\hline $596 T 005011$ & $\bar{D}$ & Magnes ium-ICP-Acid Dil. & $\mathrm{ug} / \mathrm{mL}$ & 96.20 & $<1.00 \mathrm{e}-01$ & $<4.100$ & $<4.10 \mathrm{e} 0$ & $\mathrm{n} / \mathrm{a}$ & $\mathrm{n} / \mathrm{a}$ & 95.60 & 4.100 & $n / a$ \\
\hline S967005011 & D & Manganese-ICP-Acid DiL. & $\mathrm{ug} / \mathrm{mL}$ & 97.80 & $<1.00 e-02$ & $<4.10 \mathrm{e}-01$ & $<4.10 \mathrm{e}-1$ & $n / a$ & $n / a$ & 96.10 & $4.10 e^{-01}$ & $\mathrm{n} / \mathrm{a}$ \\
\hline S96T005011 & D & Molybdenum-ICP-Acid Dil. & $\mathrm{ug} / \mathrm{mL}$ & 102.2 & $<5.00 \mathrm{e}-02$ & $<\quad 2.050$ & $<2.05 \mathrm{e} 0$ & $\mathrm{n} / \mathrm{a}$ & $\mathrm{n} / \mathrm{a}$ & 105.0 & 2.050 & $\mathrm{n} / \mathrm{a}$ \\
\hline S96T005011 & D & Sodium-ICP-Acid Dil. & $\mathrm{ug} / \mathrm{mL}$ & 97.80 & $<1.00 \mathrm{e}-01$ & $1.29 e+04$ & $1.29 \mathrm{e}+04$ & $1.29 \mathrm{e}+04$ & 0.00 & 17.10 & 4.100 & $\mathrm{n} / \mathrm{a}$ \\
\hline \$96T005011 & D & Neodymium-ICP-Acid Dil. & ug $/ \mathrm{mL}$ & 102.2 & $<1.00 \mathrm{e}-01$ & 4.100 & $<4.10 \mathrm{e} 0$ & $\mathrm{n} / \mathrm{a}$ & $\mathrm{n} / \mathrm{a}$ & 102.0 & 4.100 & $\mathrm{n} / \mathrm{a}$ \\
\hline \$96T005011 & D & Nickel-ICP-Acid Dil. & $\mathrm{ug} / \mathrm{mL}$ & 100.0 & $<2,00 \mathrm{e}-02$ & $<8.20 \mathrm{e}-01$ & $<8.20 e^{-1}$ & $n / a$ & $\mathrm{n} / \mathrm{a}$ & 101.0 & $8.20 \mathrm{e}-01$ & n/a \\
\hline 5967005011 & D & Phosphorus-ICP-Acid Dil. & $\mathrm{ug} / \mathrm{mL}$ & 99.00 & $<2.00 \mathrm{e}-01$ & 11.60 & 12.30 & 11.95 & 5.86 & 103.0 & 8.200 & $\mathrm{n} / \mathrm{a}$ \\
\hline S96T005011 & $\bar{D}$ & Lead-ICP-Acid Dil. & $\mathrm{ug} / \mathrm{mL}$ & 100.0 & $<1.00 \mathrm{e}-01$ & 4.100 & $<4.1000$ & $n / a$ & $n / a$ & 103.0 & 4.100 & $n / a$ \\
\hline S96T005011 & $\bar{D}$ & Sul fur-ICP-Acid Dil. & $\underline{u g} / \mathrm{mL}$ & 98.20 & $<1.00 \mathrm{e}-01$ & 47.20 & 47.40 & 47.30 & 0.42 & 98.40 & 4.100 & $\mathrm{n} / \mathrm{a}$ \\
\hline S96T005011 & $\mathrm{D}$ & Ant imony-ICP-Acid Dil. & $\mathrm{ug} / \mathrm{mL}$ & 96.80 & $<6.00 e^{-02}$ & 2.460 & $<2.46 \mathrm{e} 0$ & $n / a$ & $\mathrm{n} / \mathrm{a}$ & 99.60 & 2.460 & $\mathrm{n} / \mathrm{a}$ \\
\hline \$96T005011 & D & Selenjum-ICP-Acid Dil. & $\mathrm{ug} / \mathrm{mL}$ & 96.00 & $<1.00 \mathrm{e}-01$ & 4.100 & $<4.10 \mathrm{e} 0$ & $n / a$ & $\mathrm{n} / \mathrm{a}$ & 103.0 & 4.100 & $\mathrm{n} / \mathrm{a}$ \\
\hline S96T005011 & D & Silicon-ICP-Acid Dil. & $\mathrm{ug} / \mathrm{mL}$ & 99.60 & $<5.00 e-02$ & 39.10 & 39.00 & 39.05 & 0.26 & 103.0 & 2.050 & $n / a$ \\
\hline S96T005011 & D & Samarium-ICP-Acid Dil. & $\mathrm{ug} / \mathrm{mL}$ & 98.40 & $<1.00 \mathrm{e}-01$ & $<4.100$ & $<4.10 \mathrm{e} 0$ & $\mathrm{n} / \mathrm{a}$ & $\mathrm{n} / \mathrm{a}$ & 96.50 & 4.100 & $\mathrm{n} / \mathrm{a}$ \\
\hline 5967005011 & $D$ & Strontium-ICP-Acid Dil. & ug/mL & 99.20 & $<1,00 \mathrm{e}-02$ & $<4.10 \mathrm{e}-01$ & $<4.10 e^{-1}$ & $\mathrm{n} / \mathrm{a}$ & $\mathrm{n} / \mathrm{a}$ & 98.80 & $4.10 \mathrm{e}-01$ & $n / a$ \\
\hline S96T005011 & D & Titanium-1CP-Acid Dil. & $\mathrm{ug} / \mathrm{mL}$ & 99.40 & $<1.00 \mathrm{e}-02$ & $<4.10 e-01$ & $<4.10 \mathrm{e}-1$ & $\mathrm{n} / \mathrm{a}$ & $\mathrm{n} / \mathrm{a}$ & 99.70 & $4.10 \mathrm{e}-01$ & $\mathrm{n} / \mathrm{a}$ \\
\hline \$96T005011 & $D$ & Thall ium-ICP-ACid Dil. & $\mathrm{ug} / \mathrm{mL}$ & 96.80 & $<2.00 \mathrm{e}-01$ & $\leq 8.200$ & $<8.20 \mathrm{e} 0$ & $\mathrm{n} / \mathrm{a}$ & n/a & 95.50 & 8.200 & $\mathrm{n} / \mathrm{a}$ \\
\hline \$96T005011 & D & Uranium-ICP-Acid Dil. & $u g / m L$ & 97.30 & $<5.00 \mathrm{e}-01$ & 20.50 & $<2.05 \mathrm{e} 1$ & n/a & n/a & 115.5 & 20.50 & $n / a$ \\
\hline$\$ 96 \mathrm{~T} 005011$ & D & Vanadium-ICP-Acid Dil. & $\mathrm{ug} / \mathrm{mL}$ & 101.2 & $<5.00 \mathrm{e}-02$ & 2.050 & $<2.05 \mathrm{e} 0$ & $\mathrm{n} / \mathrm{a}$ & $n / a$ & 102.0 & 2.050 & $\mathrm{n} / \mathrm{a}$ \\
\hline S96T005011 & D & Zinc-ICP-Acid Dil. & $\mathrm{ug} / \mathrm{mL}$ & 101.8 & $<1.00 \mathrm{e}-02$ & 2.820 & 2.800 & 2.810 & 0.71 & 103.0 & $4.10 \mathrm{e}-01$ & $\mathrm{n} / \mathrm{a}$ \\
\hline $596 \mathrm{~T} 005011$ & D & Zirconium-ICP-Acid Dil. & $\mathrm{ug} / \mathrm{mL}$ & 100.4 & $<1.00 e-02$ & $<4.10 \mathrm{e}-01$ & $<4.10 \mathrm{e}-1$ & $\mathrm{n} / \mathrm{a}$ & $\mathrm{n} / \mathrm{a}$ & 99.90 & $4.10 \mathrm{e}-01$ & $n / a$ \\
\hline
\end{tabular}




\begin{tabular}{|c|c|c|c|c|c|c|c|c|c|c|c|c|}
\hline Sample\# & $R$ A\# & Analyte & Unit & Standard \% & Blank & Result & Dupl icate & Average & RPD $\%$ & Spk Rec \% & Det Limit & Count Err\% \\
\hline S96T005011 & & Fluoride-IC-Dionex $4000 / 4500$ & $\mathrm{ug} / \mathrm{mL}$ & 90.68 & $<1.20 \mathrm{e}-02$ & $1.59 \mathrm{e}+02$ & 153.0 & 156.2 & 3.85 & $n / a$ & 13.33 & $n / a$ \\
\hline$\$ 96 T 005011$ & & Chloride-IC-Dionex $4000 / 4500$ & $\mathrm{ug} / \mathrm{mL}$ & 97.34 & $<1.70 \mathrm{e}-02$ & $2.52 \mathrm{e}+02$ & 248.0 & 249.8 & 1.60 & $\mathrm{n} / \mathrm{a}$ & 18.89 & $\mathrm{n} / \mathrm{a}$ \\
\hline S96T005011 & & Nitrite-1C - Dionex $4000 / 4500$ & $\mathrm{ug} / \mathrm{mL}$ & 91.85 & $<1.08 e^{-01}$ & $1.28 \mathrm{e}+03$ & $1.25 \mathrm{e}+03$ & $1.27 \mathrm{e}+03$ & 2.37 & $\mathrm{n} / \mathrm{a}$ & 120.0 & $n / a$ \\
\hline S96T005011 & & Bromide by Ion Chromatograph & $\mathrm{ug} / \mathrm{mL}$ & 98.98 & $<1.25 e^{-01}$ & $<1.39 \mathrm{e}+02$ & $\leq 1.39 \mathrm{e} 2$ & $n / a$ & $n / a$ & $\mathrm{n} / \mathrm{a}$ & 138.9 & $n / a$ \\
\hline S96T005011 & & witrate by IC-Dionex $4000 / 4500$ & ug/mL & 101.3 & $<1.39 \mathrm{e}-01$ & $2.92 \mathrm{e}+04$ & $2.85 e+04$ & $2.88 \mathrm{e}+04$ & 2.43 & $n / a$ & 154.4 & $\mathrm{n} / \mathrm{a}$ \\
\hline \$96T005011 & & Phosphate-IC-Dionex $4000 / 4500$ & $\mathrm{ug} / \mathrm{mL}$ & 97.43 & $<1.20 \mathrm{e}-01$ & $<1.33 \mathrm{e}+02$ & $<1.33 \mathrm{e} 2$ & $n / a$ & $n / a$ & $\mathrm{n} / \mathrm{a}$ & 133.3 & $\mathrm{n} / \mathrm{a}$ \\
\hline S96T005011 & & Sulfate by IC-Dionex $4000 / 4500$ & $\mathrm{ug} / \mathrm{mL}$ & 102.4 & $<1.38 \mathrm{e}-01$ & $2.14 e+02$ & 214.0 & 213.8 & 0.00 & $n / a$ & 153.3 & $\mathrm{n} / \mathrm{a}$ \\
\hline S96T005011 & & Oxalate by IC-Dionex $4000 / 4500$ & $\mathrm{ug} / \mathrm{mL}$ & 101.1 & $<1.05 \mathrm{e}-01$ & $<1.17 \mathrm{e}+02$ & $<1.17 \mathrm{e} 2$ & $\mathrm{n} / \mathrm{a}$ & $\mathrm{n} / \mathrm{a}$ & $\mathrm{n} / \mathrm{a}$ & 116.7 & $n / a$ \\
\hline \$96T005014 & & Strontium-89/90 High Level & $\mathrm{UCi} / \mathrm{mL}$ & 106.4 & $1.16 \mathrm{e}-04$ & $2.41 \mathrm{e}-02$ & $2.62 \mathrm{e}-02$ & $2.51 \mathrm{e}-02$ & 8.35 & $\mathrm{n} / \mathrm{a}$ & $2.91 \mathrm{e}^{-05}$ & $1.42 E+00$ \\
\hline S96T005014 & & PU-239/240 by KRU-SPEC Resin & $\mathrm{uCi} / \mathrm{mL}$ & 100.7 & $<4.45 \mathrm{e}-06$ & $<4.26 e-06$ & $<4.44 \mathrm{E}-6$ & $n / a$ & $\mathrm{n} / \mathrm{a}$ & $n / a$ & $4.26 \mathrm{e}-06$ & $1.00 E+02$ \\
\hline S96T00 & & Cobal $t-60$ by GEA & $\mathrm{uCi} / \mathrm{mL}$ & 98.07 & $<3.85 e^{-05}$ & $7.85 \mathrm{e}-05$ & $1.14 \mathrm{e}-04$ & $9.63 e-05$ & 36.9 & $n / a$ & $\mathrm{n} / \mathrm{a}$ & 37.1 \\
\hline \$96T00 & & Cesium-137 by GEA & $\mathrm{UCi} / \mathrm{mL}$ & 106.6 & $<7.27 \mathrm{e}-05$ & $7.45 \bar{e}-01$ & $7.55 e^{-01}$ & $7.50 \mathrm{e}-01$ & 1.33 & $\mathrm{n} / \mathrm{a}$ & $\mathrm{n} / \mathrm{a}$ & 0.280 \\
\hline 5967005014 & & Am-241 by Extraction & $\mathrm{uCi} / \mathrm{mL}$ & 100.8 & $<6.64 \mathrm{e}-06$ & $<6.08 \mathrm{e}-06$ & $<6.14 \mathrm{E}-6$ & $\mathrm{n} / \mathrm{a}$ & $\mathrm{n} / \mathrm{a}$ & $\mathrm{n} / \mathrm{a}$ & $6.08 \mathrm{e}-06$ & $1.00 E+02$ \\
\hline 5967005014 & & Alpha in Liquid Samples & $\mathrm{UCi} / \mathrm{mL}$ & 105.2 & $<7.07 \mathrm{e}-05$ & $<5.34 \mathrm{e}-05$ & $<6.00 E-5$ & $n / a$ & $n / a$ & $\mathrm{n} / \mathrm{a}$ & $9.44 \mathrm{e}-05$ & $5.00 E+02$ \\
\hline
\end{tabular}


Table 3: Data Summary Report AW-105 GRAB1

RISER: 15A

SEGMENT \#: 5AW-96-14

SEGMENT PORTION: Supernate

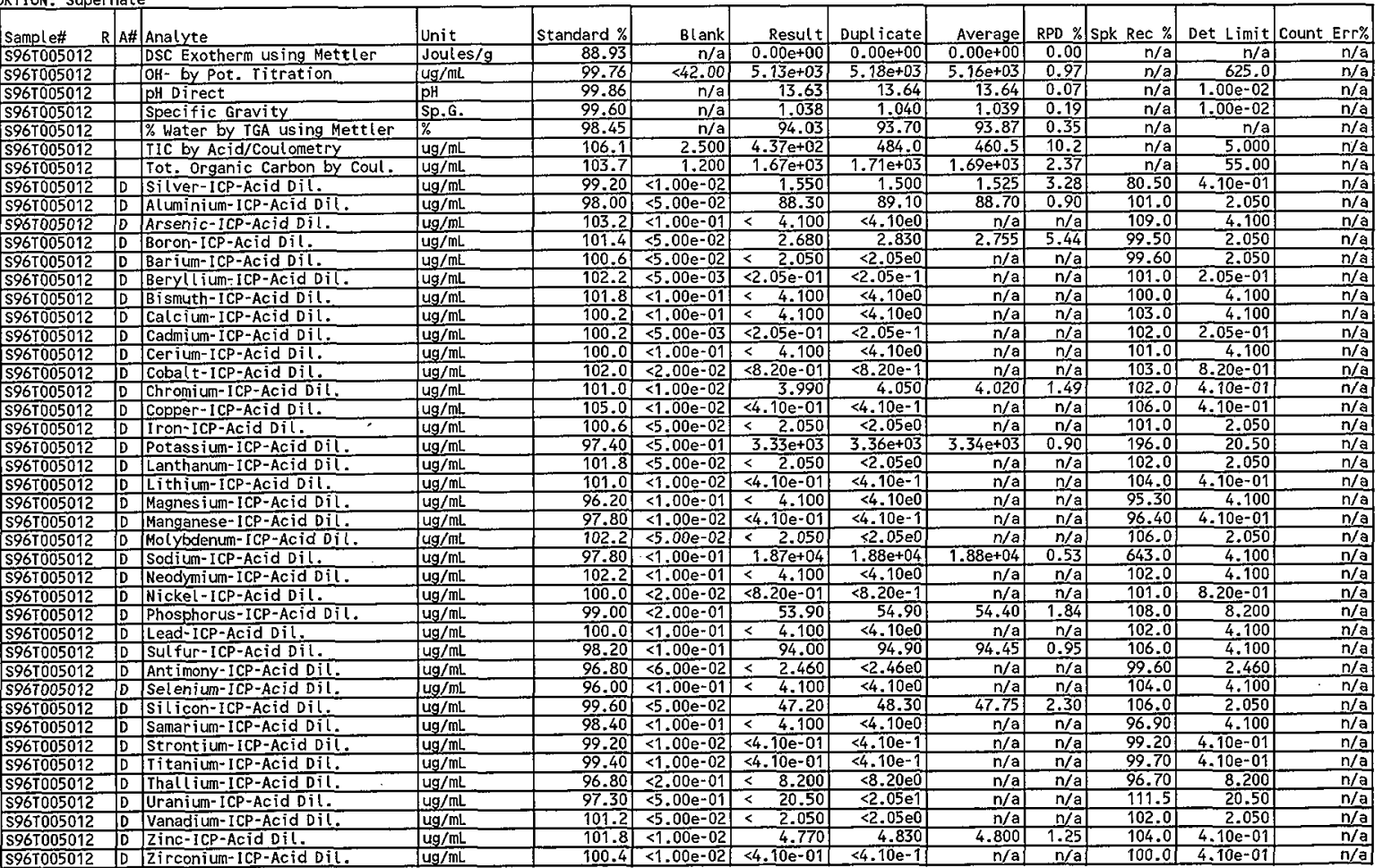




\begin{tabular}{|c|c|c|c|c|c|c|c|c|c|c|c|c|}
\hline Sample\# & A\# & Analyte & Unit & Standard \% & Blank & Result $t$ & Duplicate & Average & RPD \% & Spk Rec \% & Det Limit & Count Err\% \\
\hline$\$ 967005012$ & & Fluoride-IC-Dionex $4000 / 4500$ & $\mathrm{ug} / \mathrm{mL}$ & 97.97 & $<1.20 \mathrm{e}-02$ & $1.06 e+03$ & $1.16 \mathrm{e}+03$ & $1.11 \mathrm{e}+03$ & 9.01 & $\mathrm{n} / \mathrm{a}$ & 61.81 & $\mathrm{n} / \mathrm{a}$ \\
\hline 5967005012 & & Chloride-IC-Dionex $4000 / 4500$ & $\mathrm{ug} / \mathrm{mL}$ & 100.4 & $<1.70 \mathrm{e}-02$ & $3.08 \mathrm{e}+02$ & 307.0 & 307.4 & 0.33 & $\mathrm{n} / \mathrm{a}$ & 87.57 & $\mathrm{n} / \mathrm{a}$ \\
\hline S967005012 & & Nitrite-IL - Dionex $4000 / 4500$ & ug/mL & 97.97 & $<1.08 \mathrm{e}-01$ & $1.66 \mathrm{e}+03$ & $1.76 \mathrm{e}+03$ & $1.71 \mathrm{e}+03$ & 5.85 & $\mathrm{n} / \mathrm{a}$ & 556.3 & $n / a$ \\
\hline S96T005012 & & Bromide by Ion Chromatograph & $\mathrm{ug} / \mathrm{mL}$ & 97.11 & $<1.25 \mathrm{e}-01$ & $<6.44 \mathrm{e}+02$ & $<6.44 \mathrm{e} 2$ & $n / a$ & $\mathrm{n} / \mathrm{a}$ & $n / a$ & 643.9 & $\mathrm{n} / \mathrm{a}$ \\
\hline$\$ 967005012$ & & Nitrate by IC-Dionex $4000 / 4500$ & $\mathrm{ug} / \mathrm{mL}$ & 98.82 & $1.40 \mathrm{e}-01$ & $2.80 \mathrm{e}+04$ & $3.02 \mathrm{e}+04$ & $2.91 \mathrm{e}+04$ & 7.56 & $\mathrm{n} / \mathrm{a}$ & 716.0 & $\mathrm{n} / \mathrm{a}$ \\
\hline \$96T005012 & & Phosphate-IC-Dionex $4000 / 4500$ & ug/mL & 91.74 & $<1.20 e^{-01}$ & $<6.18 \mathrm{e}+02$ & $<6.18 \mathrm{e} 2$ & $n / a$ & $n / a$ & $\mathrm{n} / \mathrm{a}$ & 618.1 & $\mathrm{n} / \mathrm{a}$ \\
\hline 5967005012 & & Sulfate by IC-D ionex $4000 / 4500$ & $\mathrm{ug} / \mathrm{mL}$ & 96.99 & $\angle 1.38 \mathrm{e}=01$ & $<7.11 \mathrm{e}+02$ & $<7.11 \mathrm{e} 2$ & $\mathrm{n} / \mathrm{a}$ & $\mathrm{n} / \mathrm{a}$ & $n / a$ & 710.8 & $\mathrm{n} / \mathrm{a}$ \\
\hline S96T005012 & & Oxalate by IC-Dionex $4000 / 4500$ & $\mathrm{ug} / \mathrm{mL}$ & 100.0 & $<1.05 \mathrm{e}=01$ & $<5.41 \mathrm{e}+02$ & $<5.41 \mathrm{e} 2$ & $\mathrm{n} / \mathrm{a}$ & $\mathrm{n} / \mathrm{a}$ & $n / a$ & 540.9 & $n / a$ \\
\hline$\$ 96 \mathrm{~T} 005015$ & & Strontium-89/90 High Level & $\mathrm{uCi} / \mathrm{mL}$ & 105.0 & $1.60 \mathrm{e}=06$ & $7.33 e-04$ & $4.38 \mathrm{e}-04$ & $5.86 e-04$ & 50.4 & $\mathrm{n} / \mathrm{a}$ & $2.85 e^{-06}$ & $2.46 \mathrm{E}+00$ \\
\hline S967005015 & & PU-239/240 by TRU-SPEC Resin & $\mathrm{uCi} / \mathrm{mL}$ & 100.7 & $\angle 4.45 e=06$ & $<4.80 \mathrm{e}-06$ & $<4.28 \mathrm{E}-6$ & $n / a$ & $\mathrm{n} / \mathrm{a}$ & $\mathrm{n} / \mathrm{a}$ & $4.80 \mathrm{e}-06$ & $1.00 E+01$ \\
\hline 5967005015 & & Cobal $t-60$ by GEA & $\mathrm{uCi} / \mathrm{mL}$ & 98.07 & $<3.85 e^{-05}$ & $9.66 e-04$ & $1.16 e-03$ & $1.06 e^{-03}$ & 18.3 & $n / a$ & $n / a$ & 17.2 \\
\hline 5967005015 & & Cesium- 137 by GEA & $\mathrm{uCi} / \mathrm{mL}$ & 106.6 & $<7.27 \mathrm{e}-05$ & 4.710 & 4.810 & 4.760 & 2.10 & n/a & $n / a$ & 0.160 \\
\hline S96T005015 & & Am-241 by Extraction & uCi $/ \mathrm{mL}$ & 100.8 & $<6.64 \mathrm{e} \times 06$ & $<6.59 e-06$ & $<6.68 \mathrm{E}-6$ & $\mathrm{n} / \mathrm{a}$ & $\mathrm{n} / \mathrm{a}$ & $\mathrm{n} / \mathrm{a}$ & $6.59 \mathrm{e}-06$ & $1.00 \mathrm{OOE}+02$ \\
\hline S96T005015 & & Alpha in Liquid Samples & uCi $/ \mathrm{mL}$ & 105.2 & $<7.07 \mathrm{e} .05$ & $\leq 1.85 e-04$ & $<3.15 E-4$ & $\mathrm{n} / \mathrm{a}$ & $\mathrm{n} / \mathrm{a}$ & $\mathrm{n} / \mathrm{a}$ & $3.78 e-04$ & $5.00 \mathrm{E}+02$ \\
\hline
\end{tabular}


RISER: $15 \mathrm{~A}$

SEGMENT \#: 5AW-96-15

SEGMENT PORTION: Centrifuged Solids (Grab Sample)

\begin{tabular}{|c|c|c|c|}
\hline Sample\# & $\mathrm{R} \mid \mathrm{A \#}$ & Analyte & Unit \\
\hline$\$ 967005075$ & & Bulk Density of Sample & $\mathrm{g} / \mathrm{mL}$ \\
\hline 5967005075 & & DSC Exotherm using Mettler & Joules $/ g$ \\
\hline S96T005075 & & DSC Exotherm Dry Calculated & Joules/g Dry \\
\hline$\$ 967005075$ & & pH on SST Samples. & $\mathrm{pH}$ \\
\hline 5967005075 & & $\%$ Water by TGA using Mettler & $\%$ \\
\hline$\$ 967005075$ & & IIC by Acid/Coul ometry & ug/g \\
\hline 5967005075 & & TOC by Persulfate/Coul ometry & $\mathrm{ug} / \mathrm{g}$ \\
\hline \$967005084 & $\mathbf{F}$ & Stront ium- $89 / 90 \mathrm{High}$ Level & $\mathrm{uci} / \mathrm{g}$ \\
\hline 5967005084 & $\bar{F}$ & Pu-239/240 by TRU-SPEC Resin & $u C i / g$ \\
\hline 5967005084 & $\bar{F}$ & Silver -ICP-Fusion & $4 \mathrm{~g} / \mathrm{g}$ \\
\hline S96T005084 & $\mathbf{F}$ & Aluminium-ICP-Fusion & $\mathrm{ug} / \mathrm{g}$ \\
\hline S96T005084 & $\mathbf{F}$ & Arsenic-ICP-fusion & $\underline{4} g / g$ \\
\hline 5967005084 & $\mathbf{F}$ & Boron-ICP-Fusion & ug/g \\
\hline 5967005084 & $\mathbf{F}$ & Barium -ICP-Fusion & $\mathrm{ug} / \mathrm{g}$ \\
\hline$\$ 967005084$ & $\bar{F}$ & Beryllium-ICP-Fusion & ug $/ g$ \\
\hline S96T005084 & $F$ & Bismuth -ICP-Fusion & $u g / g$ \\
\hline S967005084 & $\bar{F}$ & Calcium-ICP-Fusion & $\mathrm{ug} / \mathrm{g}$ \\
\hline 5967005084 & $F$ & Cadmium-ICP-Fusion & $\mathrm{ug} / \mathrm{g}$ \\
\hline \$96T005084 & $\mathrm{F}$ & Cerium -ICP-Fusion & $4 \mathrm{~g} / \mathrm{g}$ \\
\hline S96T005084 & $\mathbf{F}$ & Cobalt -ICP-Fusion & ㄴg/g \\
\hline S96T005084 & $F$ & Chromium - ICP-Fusion & $u g / g$ \\
\hline$\$ 967005084$ & $\bar{F}$ & Copper -ICP-Fusion & $u g / g$ \\
\hline 5967005084 & $F$ & Jron-ICP-Fusion & $\mathrm{ug} / \mathrm{g}$ \\
\hline \$96T005084 & $\mathbf{F}$ & Lanthanum -ICP-Fusion & $\mathrm{ug} / \mathrm{g}$ \\
\hline S96T005084 & $\mathbf{F}$ & Lithium -ICP-Fusion & $4 \mathrm{~g} / \mathrm{g}$ \\
\hline 5967005084 & $\mathbf{F}$ & Magnesium-ICP-Fusion & $4 \mathrm{~g} / \mathrm{g}$ \\
\hline S96T005084 & $\mathbf{F}$ & Manganese - IcP-Fusion & $4 \mathrm{~g} / \mathrm{g}$ \\
\hline$\$ 967005084$ & $\bar{F}$ & Molybdenum -ICP-Fusion & $4 \mathrm{~g} / \mathrm{g}$ \\
\hline S967005084 & $\bar{F}$ & Sodium-ICP-Fusion & $4 \mathrm{~g} / \mathrm{g}$ \\
\hline$\$ 967005084$ & $\mathrm{~F}$ & Neodymiun -ICP-Fusion & $\mathrm{ug} / \mathrm{g}$ \\
\hline $596 \mathrm{~T} 005084$ & $F$ & Phosphorus -ICP-Fusion & $4 g / g$ \\
\hline S967005084 & $F$ & Lead-ICP-Fusion & $49 / 9$ \\
\hline S96T005084 & $\mathbf{F}$ & Sulfur - ICP-Fusion & ug/g \\
\hline 5967005084 & $\mathrm{~F}$ & Ant imony - ICP-Fusion & $4 \mathrm{~g} / \mathrm{g}$ \\
\hline$\$ 967005084$ & $\mathbf{F}$ & Selenium-ICP-Fusion & $u g / g$ \\
\hline$\$ 967005084$ & $F$ & Silicon-ICP-Fusion & $4 \mathrm{~g} / \mathrm{g}$ \\
\hline 5967005084 & $\bar{F}$ & Samarium-ICP-Fusion & $\mathrm{ug} / \mathrm{g}$ \\
\hline$\$ 96 \mathrm{~T} 005084$ & $\bar{F}$ & Strontium -ICP-Fusion & ug $/ \mathrm{g}$ \\
\hline$\$ 967005084$ & $F$ & Titanium-ICP-Fusion & $\mathrm{ug} / \mathrm{g}$ \\
\hline 5967005084 & $F$ & Thal lium - ICP-Fusion & $\mathrm{ug} / \mathrm{g}$ \\
\hline S96T005084 & $\bar{F}$ & Uranium - ICP-Fus ion & $4 \mathrm{~g} / \mathrm{g}$ \\
\hline 5967005084 & $F$ & Vanadium -JCP-Fus ion & ug/g \\
\hline 5967005084 & $\mathbf{F}$ & Zinc-ICP-Fusion & $4 \mathrm{~g} / \mathrm{g}$ \\
\hline
\end{tabular}

standard \% Blank $\quad$ Result Dupl icate $1.260 \quad \mathrm{n} / \mathrm{a}$ \begin{tabular}{ll|l}
$\mathrm{n} / \mathrm{a}$ & $\mathrm{n} / \mathrm{a}$ & 66.70
\end{tabular}

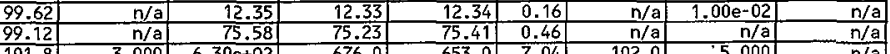

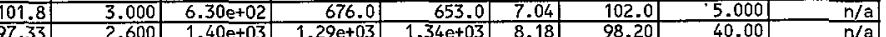
\begin{tabular}{|r|r|r|r|r|r|r|r|r|}
97.35 & 2.600 & $1.40 \mathrm{e}+03$ & $1.29 \mathrm{e}+03$ & $1.34 \mathrm{e}+03$ & 8.18 & 98.20 & 40.00 & $\mathrm{n} / \mathrm{a}$ \\
\hline
\end{tabular} \begin{tabular}{|r|r|r|r|r|r|r|r|r|}
\hline 80.00 & $1.23 \mathrm{e}-01$ & 30.50 & 32.20 & 31.35 & 5.42 & $n / a$ & $1.40 \mathrm{e}-02$ & $8.14 \mathrm{E}-01$ \\
\hline 112.1 & $<1.61 \mathrm{e}-02$ & 3.470 & 3.770 & 3.620 & 8.29 & $n / a$ & $1.48 \mathrm{e}-01$ & $1.37 \mathrm{E}+00$ \\
\hline
\end{tabular}

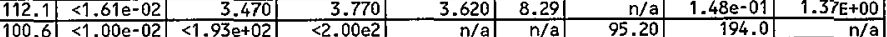

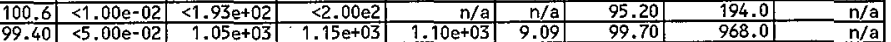

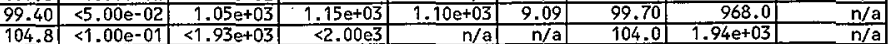

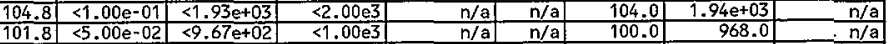
\begin{tabular}{rrrrr|r|r|r|r|}
102.0 & $<5.00 \mathrm{e}-02$ & $<9.67 \mathrm{e}+02$ & $<1.00 \mathrm{e}$ & $n / a$ & $n / a$ & 101.0 & 968.0 & $\mathrm{n} / \mathrm{a}$ \\
\hline 104 & $<5.00 \mathrm{e}-03$ & $<96.70$ & $<1.00 \mathrm{e}$ & $\mathrm{n} / \mathrm{a}$ & $\mathrm{n} / \mathrm{a}$ & 103.0 & 96.80 & $\mathrm{n} / \mathrm{a}$ \\
\hline
\end{tabular}

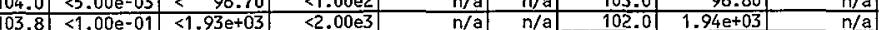

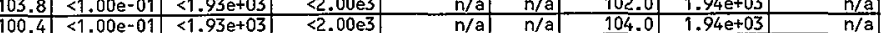
\begin{tabular}{ll|l|l|}
\hline 00.4 & $<1.00 \mathrm{e}-01$ & $<1.93 \mathrm{e}+03$ & $<2.00 \mathrm{e}$ \\
\hline
\end{tabular}

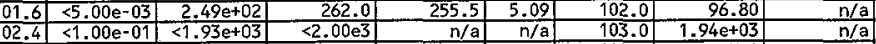

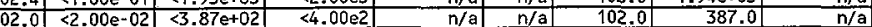

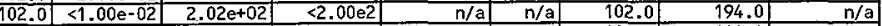

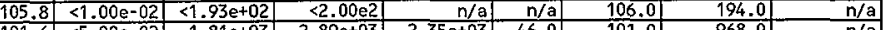
\begin{tabular}{|l|l|r|r|r|r|r|r|r|}
101.6 & $<5.00 \mathrm{e}-02$ & $1.81 \mathrm{e}+03$ & $2.89 \mathrm{e}+03$ & $2.35 \mathrm{e}+03$ & 46.0 & 101.0 & 968.0 & $\mathrm{n} / \mathrm{a}$ \\
\hline
\end{tabular} \begin{tabular}{|l|l|l|l|l|l|r|r|r|}
\hline 103.4 & $<5.00 \mathrm{e}-02$ & $1.05 \mathrm{e}+03$ & $1.15 \mathrm{e}+03$ & $1.10 \mathrm{e}+03$ & 9.09 & 104.0 & 968.0 & $\mathrm{n} / \mathrm{a}$ \\
\hline
\end{tabular}

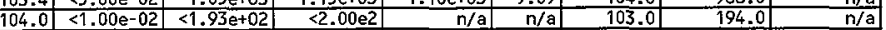
\begin{tabular}{llll}
104.0 & $<1.00 \mathrm{e}-02$ & $<1.93 \mathrm{e}+02$ & $<2.00 \mathrm{e}$ \\
\hline
\end{tabular} \begin{tabular}{r|r|r|r|}
98.00 & $<1.00 \mathrm{e}-01$ & $<1.93 \mathrm{e}+03$ & $<2.00 \mathrm{e}$ \\
\hline 99.60 & $<1.00 \mathrm{e}-02$ & $7.52 \mathrm{e}+02$ & 789.0
\end{tabular} \begin{tabular}{r|r|r|r|}
99.60 & $<1.00 \mathrm{e}-02$ & $7.52 \mathrm{e}+02$ & 789.0 \\
\hline 101.8 & $<5.00 \mathrm{e}-02$ & $<9.67 \mathrm{e}+02$ & $<1.00 \mathrm{e}$
\end{tabular} \begin{tabular}{|r|r|r|r|r|r|r|r|r|}
\hline & $\mathrm{n} / \mathrm{a}$ & $\mathrm{n} / \mathrm{a}$ & 102.0 & 968.0 & $\mathrm{n} / \mathrm{a}$ \\
\hline
\end{tabular}

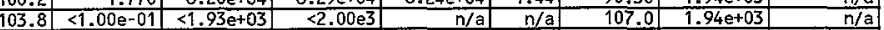
\begin{tabular}{|l|l|r|r|r|r|r|r|r|}
\hline 102.0 & $<2.00 \mathrm{e}-01$ & $<3.87 \mathrm{e}+03$ & $<4.00 \mathrm{e} 3$ & $\mathrm{n} / \mathrm{a}$ & $\mathrm{n} / \mathrm{a}$ & 107.0 & $3.87 \mathrm{e}+03$ & $\mathrm{n} / \mathrm{a}$ \\
\hline
\end{tabular}

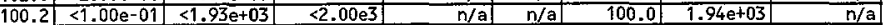

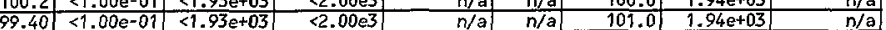

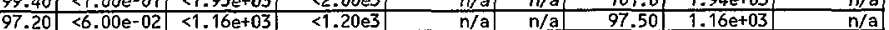
\begin{tabular}{r|r|r|r|r|r|r|r|r|}
\hline 9.20 & $<6.00 \mathrm{e}^{-02}$ & $<1.16 \mathrm{e}+03$ & $<1.20 \mathrm{e}$ & $\mathrm{n} / \mathrm{a}$ & $\mathrm{n} / \mathrm{a}$ & 97.50 & $1.16 \mathrm{e}+03$ & $\mathrm{n} / \mathrm{a}$ \\
\hline 98.40 & $<1.00 \mathrm{e}^{-01}$ & $<1.93 \mathrm{e}^{+03}$ & $<2.00 \mathrm{e}$ & $\mathrm{n} / \mathrm{a}$ & $\mathrm{n} / \mathrm{a}$ & 102.0 & $1.94 \mathrm{e}+03$ & $\mathrm{n} / \mathrm{a}$ \\
\hline
\end{tabular}

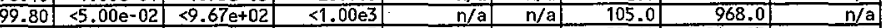
$\begin{array}{lllll}00.4 & <1.00 \mathrm{e}-01 & <1.93 \mathrm{e}+03 & <2.00 \mathrm{e}\end{array}$ \begin{tabular}{ll|l|l|l|}
100.6 & $<1.00 \mathrm{e}-02$ & $<1.93 \mathrm{e}+02$ & $<2.00 \mathrm{e}$
\end{tabular} \begin{tabular}{ll|l|l|}
99.40 & $<1.00 \mathrm{e}-02$ & $<1.93 \mathrm{e}+02$ & $<2.00 \mathrm{e} 2$
\end{tabular} \begin{tabular}{ll|l|l|}
98.20 & $<2.00 \mathrm{e}-01<3.87 \mathrm{e}+03$ & $<4.00 \mathrm{e} 3$
\end{tabular} \begin{tabular}{|c|c|c|c|}
98.20 & $<5.00 \mathrm{e}-01$ & $9.27 \mathrm{e}+04$ & $9.75 \mathrm{e}+04$ \\
\hline
\end{tabular} \begin{tabular}{l|l|l|l|}
101.8 & $<5.00 \mathrm{e}-02$ & $<9.67 \mathrm{e}+02$ & $<1.00 \mathrm{e} 3$ \\
101.4 & $<1.00 \mathrm{e}-02$ & $<1.93 \mathrm{e}+02$ & $<2.00 \mathrm{e}$
\end{tabular}

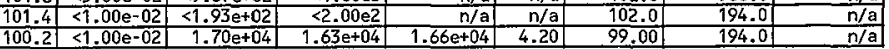




\begin{tabular}{|c|c|c|c|c|c|c|c|c|c|c|c|c|}
\hline Sample\# & $\mathbf{R} \mid \mathbf{A}$ & Analyte & Unjt & Standard \% & Blank & Result & Dupl icate & Average & RPD \% & Spk Rec \% & Det Limit & Count Er \\
\hline S96T005084 & $F$ & Cobal $t-60$ by GEA & uci/g & $9 \overline{3.56}$ & $<7.37 \mathrm{e}-03$ & $3.63 e-02$ & $3.54 e^{-02}$ & $3.58 \mathrm{e}-02$ & 2.51 & $\mathrm{n} / \mathrm{a}$ & $n / a$ & \\
\hline$\$ 967005084$ & $F$ & Cesium-137 by GEA & uci/g & 98.85 & $<1.29 \mathrm{e}-02$ & 13.58 & 13.80 & 13.69 & 1.46 & $n / a$ & $n / a$ & 0.67 \\
\hline 5084 & $F$ & Am-241 by Extraction & $\mathrm{uCi} / \mathrm{g}$ & 95.80 & $<2.82 e-02$ & $4.54 \mathrm{e}-01$ & $4.89 \mathrm{e}-01$ & $4.72 e^{-01}$ & 7.42 & $n / a$ & $5.60 e-02$ & $1.78 \mathrm{E}+00$ \\
\hline S961 & $F$ & Alpha of Digested solid & $\mathrm{uCi} / \mathrm{g}$ & 104.8 & $<5.33 \mathrm{e}-03$ & 4.080 & 3.640 & 3.860 & 11.4 & 86.91 & $1.30 e-02$ & $2.59 E+00$ \\
\hline S96T & W & OH- by Pot. Titration & $\mathrm{ug} / \mathrm{g}$ & 97.36 & $<42.00$ & $<\quad 42.00$ & $<42$ & $n / a$ & $n / a$ & $n / a$ & 41.70 & $\mathrm{n} /$ \\
\hline S96T & $\mathrm{W}$ & Fluoride-1C-D ionex $4000 / 4500$ & $\mathrm{ug} / \mathrm{g}$ & 90.68 & $<1.20 \mathrm{e}-02$ & $4.03 e+03$ & $4.11 \mathrm{e}+03$ & $4.07 e+03$ & 1.97 & 107.5 & 26.33 & $\mathrm{n}$ \\
\hline S96 & $W$ & Chlor ide-IC-Dionex $4000 / 4500$ & ug/g & 92.66 & $2.00 e-02$ & $2.21 \mathrm{e}+02$ & 228.0 & 224.4 & 3.12 & 89.87 & 37.31 & $n$ \\
\hline S961 & W & Nitrite-IC - Dionex $4000 / 4500$ & $\mathrm{ug} / \mathrm{g}$ & 92.96 & $<1.08 e-01$ & $2.22 \mathrm{e}+03$ & $2.28 \mathrm{e}+03$ & $2.25 e+03$ & 2.67 & 91.85 & 237.1 & \\
\hline $596 \mathrm{TO}$ & W & Bromide by Ion Chromatograph & $\mathrm{ug} / \mathrm{g}$ & 94.57 & $<1.25 e-01$ & $<2.74 \mathrm{e}+02$ & $<2.78 \mathrm{e} 2$ & $n / a$ & $n / a$ & 95.42 & 274.3 & \\
\hline \$96T & $W$ & Nitrate by IC-Dionex $4000 / 4500$ & ug/g & 100.0 & $1.70 \mathrm{e}-01$ & $2.30 \mathrm{e}+04$ & $2.31 \mathrm{e}+04$ & $2.30 \mathrm{e}+04$ & 0.43 & 103.2 & 305.0 & \\
\hline S961 & $\mathrm{W}$ & Phosphate-IC-Dionex $4000 / 4500$ & ug $/ \mathrm{g}$ & 95.23 & $<1.20 \mathrm{e}-01$ & $4.11 \mathrm{e}+02$ & 378.0 & 394.7 & 8.37 & 93.58 & 263.3 & $\mathrm{n}$ \\
\hline $596 T 005087$ & $\mathrm{~W}$ & Sul fate by IC-Dionex $4000 / 4500$ & ug/g & 101.3 & $<1.38 \mathrm{e}-01$ & $8.80 \mathrm{e}+02$ & 851.0 & 865.6 & 3.35 & 100.2 & 302.8 & \\
\hline$\$ 964005087$ & W & Oxalate by IC-Dionex $4000 / 4500$ & ug $/ \mathrm{g}$ & 99.81 & $<1.05 \mathrm{e}-01$ & $2.36 \mathrm{e}+02$ & $<2.33 \mathrm{e} 2$ & $n / a$ & $n / a$ & 97.15 & 230.4 & \\
\hline
\end{tabular}

Interstitial Liquid: Interstitial Liquid

\begin{tabular}{|c|c|c|c|c|c|c|c|c|c|c|c|c|}
\hline Sample\# & A\# & Analyte & Unit & Standard \% & Blank & Result & Duplicate & Average & RPD \% & Spk Rec \% & Det Limit & Count Err\% \\
\hline \$96T005081 & & Bulk Density of Sample & $\mathrm{g} / \mathrm{mL}$ & $\mathrm{n} / \mathrm{a}$ & $\mathrm{n} / \mathrm{a}$ & $9.80 \mathrm{e}-01$ & $\mathrm{n} / \mathrm{a}$ & $\mathrm{n} / \mathrm{a}$ & $n / a$ & $n / a$ & $5.00 \mathrm{e}-01$ & n/a \\
\hline $596 \mathrm{~T} 005081$ & & DSC Exotherm on Perkin Elmer & Joules $/ \mathrm{g}$ & 93.95 & $\mathrm{n} / \mathrm{a}$ & $0.00 \mathrm{e}+00$ & $0.00 \mathrm{e}+00$ & $0.00 e+00$ & 0.00 & $n / a$ & $n / a$ & $n / a$ \\
\hline $596 \mathrm{~T} 005081$ & & OH- by Pot. Titration & $\mathrm{ug} / \mathrm{mL}$ & 106.4 & $\leq 42.00$ & $7.62 \mathrm{e}+03$ & $7.44 \mathrm{e}+03$ & $7.53 \mathrm{e}+03$ & 2.39 & $n / a$ & 500.0 & $\mathrm{n} / \mathrm{a}$ \\
\hline \$967005081 & & pH Direct & pH & 100.0 & $n /$ a & 13.46 & 13.48 & 13.47 & 0.15 & $\mathrm{n} / \mathrm{a}$ & $1.00 \mathrm{e}-02$ & $n / a$ \\
\hline $596 \mathrm{~T} 005081$ & & $\%$ Water by TGA on Perk in Elmer & $\%$ & 99.27 & $\mathrm{n} / \mathrm{a}$ & 90.54 & 90.78 & 90.66 & 0.26 & $n / a$ & $\mathrm{n} / \mathrm{a}$ & $n / a$ \\
\hline 5967005081 & & TIC by Acid/Coulometry & $\mathrm{ug} / \mathrm{mL}$ & 100.2 & $6.00 \mathrm{e}-01$ & $5.76 \mathrm{e}+02$ & 586.0 & 581.0 & 1.72 & 103.1 & 5.000 & $n / a$ \\
\hline S96T005081 & & Tot. Organic Carbon by Coul. & $\mathrm{ug} / \mathrm{mL}$ & 106.3 & 1.200 & $1.77 \mathrm{e}+03$ & $1.57 \mathrm{e}+03$ & $1.67 \mathrm{e}+03$ & 12.0 & 82.50 & 55.00 & $n / a$ \\
\hline S96T005081 & & Stront ium- $89 / 90 \mathrm{High}$ Level & $\mathrm{uCi} / \mathrm{mL}$ & 97.14 & $5.89 \mathrm{e}-05$ & $6.91 e-04$ & $5.30 \mathrm{e}-04$ & $6.10 e-04$ & 26.4 & $\mathrm{n} / \mathrm{a}$ & $1.18 \mathrm{e}-05$ & $5.23 E+00$ \\
\hline $596 \mathrm{~T} 005081$ & & Pu-239/240 by TRU-SPEC Res in & $\mathrm{uCj} / \mathrm{mL}$ & 89.93 & $<6.50 \mathrm{e}-06$ & $4.77 \mathrm{e}-03$ & $4.36 \mathrm{e}-03$ & $4.56 \mathrm{e}-03$ & 8.98 & $n / a$ & $1.80 \mathrm{e}-04$ & $1.17 E+00$ \\
\hline 5961005081 & D & Silver-ICP-Acid Dil. & $\mathrm{ug} / \mathrm{mL}$ & 100.8 & $<1.00 e-02$ & 2.080 & 1.900 & 1.990 & 9.05 & 91.00 & 1.010 & $\mathrm{n} / \mathrm{a}$ \\
\hline$\$ 967005081$ & D & Aluminium-ICP-Acid Dil. & ug/mL & 98.20 & $<5.00 \mathrm{e}-02$ & $4.64 e+02$ & 461.0 & 462.5 & 0.65 & 90.30 & 5.050 & $n / a$ \\
\hline 5967005081 & D & Arsenic-ICP-Acíd Dil. & $\mathrm{ug} / \mathrm{mL}$ & 102.8 & $\leq 1.00 \mathrm{e}-01$ & $<\quad 10.10$ & $<1.01 \mathrm{e} 1$ & $n / a$ & $n / a$ & 110.0 & 10.10 & $n / a$ \\
\hline 596T005081 & D & Boron-ICP-ACid Dil. & $\mathrm{ug} / \mathrm{mL}$ & 100.2 & $\angle 5.00 \mathrm{e}-02$ & $\leq \quad 5.050$ & $<5.05 e 0$ & $\mathrm{n} / \mathrm{a}$ & $\mathrm{n} / \mathrm{a}$ & 101.0 & 5.050 & $n / a$ \\
\hline S96T005081 & D胥 & Barium-ICP-Acid Dil. & $\mathrm{ug} / \mathrm{mL}$ & 100.0 & $<5.00 \mathrm{e}-02$ & $<5.050$ & $<5.0500$ & $\mathrm{n} / \mathrm{a}$ & $n / a$ & 99.00 & 5.050 & $\mathrm{n} / \mathrm{a}$ \\
\hline S96T005081 & D & Beryllium-ICP-Acid Dil. & $\mathrm{ug} / \mathrm{mL}$ & 102.0 & $<5.00 \mathrm{e}-03$ & $<5.05 e-01$ & $<5.05 e^{-1}$ & $\mathrm{n} / \mathrm{a}$ & $n / a$ & 102.0 & $5.05 e-01$ & $n / a$ \\
\hline S96T005081 & D & Bismuth-ICP-Acid Dil. & $\mathrm{ug} / \mathrm{mL}$ & 103.2 & $<1.00 \mathrm{e}-01$ & $<\quad 10.10$ & $<1.01 \mathrm{e} 1$ & $\mathrm{n} / \mathrm{a}$ & $\mathrm{n} / \mathrm{a}$ & 104.0 & 10.10 & $\mathrm{n} / \mathrm{a}$ \\
\hline S96r005081 & D & Calcium-ICP-Acid Dil. & $\mathrm{ug} / \mathrm{mL}$ & 101.6 & $<1.00 \mathrm{e}-01$ & $<\quad 10.10$ & $<1.01 \mathrm{e} 1$ & $\mathrm{n} / \mathrm{a}$ & n/a & 103.0 & 10.10 & $\mathrm{n} / \mathrm{a}$ \\
\hline \$96T005081 & D & Cadmi um-ICP-Acid Dil. & $\mathrm{ug} / \mathrm{mL}$ & 101.4 & $<5.00 \mathrm{e}-03$ & $<5.05 e-01$ & $<5.05 e-1$ & $\mathrm{n} / \mathrm{a}$ & $\mathrm{n} / \mathrm{a}$ & 101.0 & $5.05 \mathrm{e}-01$ & $n / a$ \\
\hline S96T005081 & D & Cerium-ICP-Acid Dil. & $\mathrm{ug} / \mathrm{mL}$ & 100.2 & $<1.00 \mathrm{e}-01$ & $<10.10$ & $<1.01 \mathrm{e} 1$ & $\mathrm{n} / \mathrm{a}$ & $\mathrm{n} / \mathrm{a}$ & 102.0 & 10.10 & $\mathrm{n} / \mathrm{a}$ \\
\hline \$96T005081 & $\mathrm{D}$ & Cobalt-ICP-Acid Dil. & ug $/ \mathrm{mL}$ & 102.0 & $<2.00 \mathrm{e}-02$ & 2.020 & $<2.02 \mathrm{e} 0$ & $\mathrm{n} / \mathrm{a}$ & $\mathrm{n} / \mathrm{a}$ & 101.0 & 2.020 & $n / a$ \\
\hline 5967005081 & $\bar{D}$ & Chromium-ICP-Acid Dil. & $\mathrm{ug} / \mathrm{mL}$ & 102.2 & $<1.00 \mathrm{e}-02$ & 15.20 & 15.10 & 15.15 & 0.66 & 101.0 & 1.010 & $n / a$ \\
\hline S96T005081 & D & Copper-ICP-Acid Dil. & $\mathrm{ug} / \mathrm{mL}$ & 103.4 & $<1.00 \mathrm{e}-02$ & 1.010 & $<1.01 \mathrm{e} 0$ & $\mathrm{n} / \mathrm{a}$ & $\mathrm{n} / \mathrm{a}$ & 104.0 & 1.010 & $\mathrm{n} / \mathrm{a}$ \\
\hline S96T005081 & D & Iron-ICP-Acid Dil. & $\mathrm{ug} / \mathrm{mL}$ & 701.2 & $<5.00 \mathrm{e}-02$ & $<5.050$ & $<5.05 \mathrm{e} 0$ & $\mathrm{n} / \mathrm{a}$ & $\mathrm{n} / \mathrm{a}$ & 101.0 & 5.050 & $n / a$ \\
\hline S96T005081 & D & Potassium-ICP-Acid Dib. & $\mathrm{ug} / \mathrm{mL}$ & 99.00 & $<5.00 \mathrm{e}-01$ & $7.36 \mathrm{e}+03$ & $7.36 \mathrm{e}+03$ & $7.36 \mathrm{e}+03$ & 0.00 & $-2.880 \mathrm{e} 0$ & 50.50 & $n / a$ \\
\hline S96T005081 & $\bar{D}$ & Lanthanum-ICP-Acid Dil. & $\mathrm{ug} / \mathrm{mL}$ & 101.4 & $<5.00 \mathrm{e}-02$ & $\leq 5.050$ & $<5.05 e 0$ & $n / a$ & $n / a$ & 103.0 & 5.050 & n/a \\
\hline$\$ 967005081$ & D & Lithium-ICP-ACid Dil. & $\mathrm{ug} / \mathrm{mL}$ & 100.6 & $<1.00 e^{-02}$ & 1.010 & $<1.01 \mathrm{e} 0$ & $\mathrm{n} / \mathrm{a}$ & $\mathrm{n} / \mathrm{a}$ & 101.0 & 1.010 & $\mathrm{n} / \mathrm{a}$ \\
\hline 596T005081 & $D$ & Magnes jum-ICP-Acid Dil. & $\mathrm{ug} / \mathrm{mL}$ & 97.00 & $<1.00 \mathrm{e}-01$ & 10.70 & $<1.01 \mathrm{e}$ & $\mathrm{n} / \mathrm{a}$ & $\mathrm{n} / \mathrm{a}$ & 95.90 & 10.10 & $n / a$ \\
\hline S96T005081 & D & Manganese-ICP-Acid Dil. & $\mathrm{ug} / \mathrm{mL}$ & 99.20 & $<1.00 \mathrm{e}-02$ & 1.010 & $<1.01 \mathrm{e} 0$ & $\mathrm{n} / \mathrm{a}$ & $\mathrm{n} / \mathrm{a}$ & 96.50 & 1.010 & $n / a$ \\
\hline S96T005081 & D & Molybdenum-ICP-Acid Dil. & $\mathrm{ug} / \mathrm{mL}$ & 101.6 & $<5.00 \mathrm{e}-02$ & $<5.050$ & $<5.05 \mathrm{e} 0$ & $\mathrm{n} / \mathrm{a}$ & $\mathrm{n} / \mathrm{a}$ & 103.0 & 5.050 & $n / a$ \\
\hline S96T005081 & $\mathrm{D}$ & Sodium-ICP-Acid DiL. & $\mathrm{ug} / \mathrm{mL}$ & 98.20 & $<1.00 \mathrm{e}-01$ & $2.77 e+04$ & $2.77 e+04$ & $2.77 \mathrm{e}+04$ & 0.00 & $-2.620 \mathrm{e} 2$ & 10.10 & $\mathrm{n} / \mathrm{a}$ \\
\hline S967005081 & 0 & Neodymi um-ICP-Acid Dil. & $\mathrm{ug} / \mathrm{mL}$ & 101.8 & $<1.00 \mathrm{e}-01$ & $<\quad 10.10$ & $<1.01 \mathrm{e} 1$ & $n / a$ & $n / a$ & 102.0 & 10.10 & $n / a$ \\
\hline S967005081 & D & Nickel-ICP-Acid Dil. & $\mathrm{ug} / \mathrm{mL}$ & 101.2 & $<2.00 e^{-02}$ & $<2.020$ & $<2.02 \mathrm{e} 0$ & $\mathrm{n} / \mathrm{a}$ & $\mathrm{n} / \mathrm{a}$ & 101.0 & 2.020 & $\mathrm{n} / \mathrm{a}$ \\
\hline S96T005081 & D & Phosphorus-ICP-Acid Dil. & $\mathrm{ug} / \mathrm{mL}$ & 103.6 & $<2.00 \mathrm{e}-01$ & $2.50 \mathrm{e}+02$ & 247.0 & 248.5 & 1.21 & 101.0 & 20.20 & $n / a$ \\
\hline & & lead-ICP-Acid Dil & $\mathrm{ug} / \mathrm{mL}$ & 101.4 & $<1.00 \mathrm{e}-01$ & $<\quad 10.10$ & $<1.01 \mathrm{e} 1$ & $n / a$ & $\mathrm{n} / \mathrm{a}$ & 100,0 & 10.10 & $\mathrm{n} / \mathrm{a}$ \\
\hline
\end{tabular}




\begin{tabular}{|c|c|c|c|c|c|c|c|c|c|c|c|c|}
\hline Sample\# & R $\mathbf{A} \#$ & Analyte & Unit & Standard \% & BLank & Result & Duplicate & Average & RPD \% & Spk Rec \% & Det Limit & Count Err\% \\
\hline $596 T 005081$ & D & Sulfur-ICP-Acid Dil. & $\mathrm{ug} / \mathrm{mL}$ & 100.6 & $<1.00 \mathrm{e}-01$ & $3.51 \mathrm{e}+02$ & 348.0 & 349.5 & 0.86 & 103.0 & 10.10 & $n / a$ \\
\hline S96T005081 & D & Ant imony-ICP-Acid Dil. & ug/mL & 96.60 & $<6.00 \mathrm{e}-02$ & $<\quad 6.060$ & $<6.06 \mathrm{e} 0$ & $n / a$ & $\mathrm{n} / \mathrm{a}$ & 97.00 & 6.060 & $n / a$ \\
\hline S961005081 & D & Selenium-ICP-Acid Dil. & $\mathrm{ug} / \mathrm{mL}$ & 96.00 & $<1.00 \mathrm{e}-01$ & 10.10 & $<1.01 \mathrm{e} 1$ & $n / a$ & $n / a$ & 100.0 & 10.10 & $\mathrm{n} / \mathrm{a}$ \\
\hline s96r005081 & D & Silicon-ICP-Acid Dil. & $\mathrm{ug} / \mathrm{mL}$ & 99.60 & $<5.00 \mathrm{e}-02$ & 31.00 & 35.80 & 33.40 & 14.4 & 112.0 & 5.050 & $\mathrm{n} / \mathrm{a}$ \\
\hline$\$ 967005081$ & $\bar{D}$ & Samarium-ICP-Acid Dil. & $\mathrm{ug} / \mathrm{mL}$ & 98.00 & $<1.00 e-01$ & 10.10 & $\leq 1.01 \mathrm{e} 1$ & $n / a$ & $\mathrm{n} / \mathrm{a}$ & 98.40 & 10.10 & $n / a$ \\
\hline$\$ 96 T 005081$ & $D$ & Strontium-ICP-Acid Dil. & $\mathrm{ug} / \mathrm{mL}$ & 98.80 & $\leq 1.00 \mathrm{e}-02$ & 1.010 & $<1.01 \mathrm{e} 0$ & $\mathrm{n} / \mathrm{a}$ & $n / a$ & 99.00 & 1.010 & $\mathrm{n} / \mathrm{a}$ \\
\hline S96T005081 & $D$ & Titanium-ICP-ACid Dil. & $\mathrm{ug} / \mathrm{mL}$ & 97.80 & $<1.00 \mathrm{e}-02$ & 1.010 & $<1.01 \mathrm{e} 0$ & $\mathrm{n} / \mathrm{a}$ & $\mathrm{n} / \mathrm{a}$ & 97.80 & 1.010 & $\mathrm{n} / \mathrm{a}$ \\
\hline S96T005081 & D & Thallium-ICP-Acid Dil. & $\mathrm{ug} / \mathrm{mL}$ & 98.20 & $<2.00 \mathrm{e}-01$ & 20.20 & $<2.02 \mathrm{e} 1$ & $\mathrm{n} / \mathrm{a}$ & $n / a$ & 98.00 & 20.20 & $\mathrm{n} / \mathrm{a}$ \\
\hline \$96T005081 & D & Uranium-ICP-Acid Dil. & $\mathrm{ug} / \mathrm{ml}$ & 96.10 & $<5.00 e-01$ & 58.40 & $<5.05 \mathrm{e} 1$ & $\mathrm{n} / \mathrm{a}$ & $\mathrm{n} / \mathrm{a}$ & 78.50 & 50.50 & $n / a$ \\
\hline 5967005081 & D & Vanadium-ICP-Acid Dil. & $\mathrm{ug} / \mathrm{mL}$ & 101.2 & $<5.00 \mathrm{e}^{-02}$ & 5.050 & $<5.0500$ & $n / a$ & $n / a$ & 101.0 & 5.050 & $\mathrm{n} / \mathrm{a}$ \\
\hline \$96T005081 & D & Zinc-ICP-Acid Dil. & $\mathrm{ug} / \mathrm{mL}$ & 102.4 & $<1.00 e^{-02}$ & 3.750 & 3.700 & 3.725 & 1.34 & 102.0 & 1.010 & $n / a$ \\
\hline S96T005081 & D & Zirconium-ICP-Acid Dil. & $\mathrm{ug} / \mathrm{mL}$ & 99.00 & $<1.00 \mathrm{e}-02$ & 4.210 & 2.460 & 3.335 & 52.5 & 96.60 & 1.010 & $\mathrm{n} / \mathrm{a}$ \\
\hline S96T005081 & & Fluoride-IC-Dionex $4000 / 4500$ & $\mathrm{ug} / \mathrm{mL}$ & 90.51 & $<1.20 e^{-02}$ & $4.23 e+03$ & $4.23 e+03$ & $4.23 e+03$ & 0.00 & $n / a$ & 5.412 & $n / a$ \\
\hline $596 T 005081$ & & Chloride-[C-Dionex $4000 / 4500$ & $\mathrm{ug} / \mathrm{mL}$ & 90.89 & $<1.70 \mathrm{e}^{-02}$ & $3.98 \mathrm{e}+02$ & 393.0 & 395.6 & 1.26 & $n / a$ & 7.667 & $n / a$ \\
\hline$\$ 967005081$ & & Nitrite-IC - Dionex $4000 / 4500$ & $\mathrm{ug} / \mathrm{mL}$ & 90.37 & $<1.08 \mathrm{e}-01$ & $3.01 e+03$ & $2.93 e+03$ & $2.97 \mathrm{e}+03$ & 2.69 & $\mathrm{n} / \mathrm{a}$ & 48.71 & $\mathrm{n} / \mathrm{a}$ \\
\hline$\$ 967005081$ & & Bromide by Ion Chromatograph & $\mathrm{ug} / \mathrm{mL}$ & 97.11 & $<1.25 \mathrm{e}-01$ & $<\quad 56.38$ & $<5.64 \mathrm{e} 1$ & $\mathrm{n} / \mathrm{a}$ & $n / a$ & $n / a$ & 56.38 & $n / a$ \\
\hline S96T005081 & & Nitrate by IC-Dionex $4000 / 4500$ & $\mathrm{ug} / \mathrm{mL}$ & 100.7 & $<1.39 \mathrm{e}-01$ & $2.93 e+04$ & $2.91 e+04$ & $2.92 e+04$ & 0.68 & $n / a$ & 62.69 & $n / a$ \\
\hline S96T005081 & & Phosphate-IC-Dionex $4000 / 4500$ & $\mathrm{ug} / \mathrm{mL}$ & 97.61 & $<1.20 \mathrm{e}-01$ & $2.28 e+02$ & 223.0 & 225.4 & 2.22 & $n / a$ & 54.12 & $\mathrm{n} / \mathrm{a}$ \\
\hline S96T005081 & & Sulfate by IC-Dionex $4000 / 4500$ & $\mathrm{ug} / \mathrm{mL}$ & 101.1 & $<1.38 \mathrm{e}-01$ & $9.51 \mathrm{e}+02$ & 949.0 & 949.9 & 0.21 & $\mathrm{n} / \mathrm{a}$ & 62.24 & $n / a$ \\
\hline S96T005081 & & Oxalate by IC-Dionex $4000 / 4500$ & ugg/mL & 100.4 & $<1.05 \mathrm{e}-01$ & $1.96 \mathrm{e}+02$ & 232.0 & 213.9 & 16.8 & $n / a$ & 47.35 & $\mathrm{n} / \mathrm{a}$ \\
\hline S96T005081 & & Cobalt -60 by GEA & $\mathrm{uCi} / \mathrm{mL}$ & 99.36 & $<2.93 e-04$ & $1.21 e-02$ & $1.12 \mathrm{e}-02$ & $1.17 e-02$ & 7.73 & $n / a$ & $n / a$ & 7.80 \\
\hline$\$ 967005081$ & & Cesium- 137 by GEA & $\mathrm{uCi} / \mathrm{mL}$ & 100.2 & $<7.59 \mathrm{e}^{-04}$ & 16.40 & 15.40 & 15.90 & 6.29 & $\mathrm{n} / \mathrm{a}$ & $\mathrm{n} / \mathrm{a}$ & 0.190 \\
\hline 5967005081 & & Am-241 by Extraction & $\mathrm{uCi} / \mathrm{mL}$ & 91.60 & $<4.02 \mathrm{e}-05$ & $<3.33 e-05$ & $\leq 3.66 \mathrm{E}-5$ & $n / a$ & $n / a$ & $\mathrm{n} / \mathrm{a}$ & $3.33 \mathrm{e}-05$ & $5.76 E+00$ \\
\hline S967005081 & & Alpha in Liquid Samples & $\mathrm{UCi} / \mathrm{mL}$ & 85.71 & $1.00 \mathrm{e}-03$ & $8.80 \mathrm{e}-04$ & $1.38 \mathrm{e}-03$ & $1.13 \mathrm{e}-03$ & 44.2 & 73.82 & $3.52 e-04$ & $5.88 \mathrm{E}+01$ \\
\hline
\end{tabular}

sludge (from Liquid Grab Sample): Sludge (from Liquid Grab Sample)

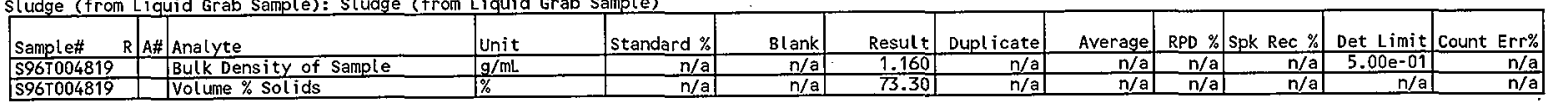


Table 3: Data Sumary Report AW- 105 GRAB 1

RISER: $15 \mathrm{~A}$

SEGMENT \#: 5AW-96-17

SEGMENT PORTION: Centrifuged Sol ids (Grab Sample)

\begin{tabular}{|c|c|c|c|c|c|c|c|c|c|c|c|c|}
\hline Sample\# & R A\# & Analyte & Unit & Standard \% & Blank & Result $t$ & Duplicate & Average & RPD \% & Spk Rec \% & Det Limit & Count Err\% \\
\hline \$96T005076 & & Bulk Density of Sample & $\mathrm{g} / \mathrm{mL}$ & $n / a$ & $n / a$ & 1.310 & $n / a$ & $n / a$ & $\mathrm{n} / \mathrm{a}$ & $n / a$ & $5.00 \mathrm{e}-01$ & n/a \\
\hline S96T005076 & & DSC Exotherm using Mettler & Joules $/ \mathrm{g}$ & 101.9 & $n / a$ & 31.60 & 49.50 & 40.55 & 44.1 & $\mathrm{n} / \mathrm{a}$ & $n / a$ & $n / a$ \\
\hline $596 r 005076$ & & DSC Exotherm Dry Calculated & Joules/g Dry & $\mathrm{n} / \mathrm{a}$ & $\mathrm{n} / \mathrm{a}$ & 92.80 & 145.0 & 118.9 & 43.9 & $\mathrm{n} / \mathrm{a}$ & $\mathrm{n} / \mathrm{a}$ & $n / a$ \\
\hline 5967005076 & & pH on SST Samples & $\mathrm{pH}$ & 99.62 & $\mathrm{n} / \mathrm{a}$ & 12.35 & 12.27 & 12.31 & 0.65 & $n / a$ & $1.00 e-02$ & $\mathrm{n} / \mathrm{a}$ \\
\hline S96T005076 & & \% Water by TGA using Mettler & $\%$ & 99.54 & $n / a$ & 65.12 & 66.79 & 65.96 & 2.53 & $n / a$ & $\mathrm{n} / \mathrm{a}$ & $n / a$ \\
\hline$\$ 967005076$ & & TIC by Acid/Coul ometry & ug $/ g$ & 101.8 & 3.000 & $8.08 \mathrm{e}+02$ & 812.0 & 810.0 & 0.49 & $n / a$ & 5.000 & $\mathrm{n} / \mathrm{a}$ \\
\hline 5967005076 & & TOC by Persulfate/Coulometry & $\mathrm{ug} / \mathrm{g}$ & 97.33 & 2.600 & $2.08 \mathrm{e}+03$ & $2.33 \mathrm{e}+03$ & $2.20 \mathrm{e}+03$ & 11.3 & $n / a$ & 40.00 & $\mathrm{n} / \mathrm{a}$ \\
\hline S96T005085 & $\mathbf{F}$ & stront jum-89/90 High Level & $\mathrm{uCi} / \mathrm{g}$ & 80.00 & $1.23 \mathrm{e}-01$ & 16.10 & 18.60 & 17.35 & 14.4 & $n / a$ & $1.50 \mathrm{e}-02$ & $1.18 \mathrm{E}+00$ \\
\hline $596 T 005085$ & $\mathbf{F}$ & Pu-239/240 by TRU-SPEC Resin & $\mathrm{uCi} / \mathrm{g}$ & 99.33 & $<8,48 \mathrm{e}-02$ & 3.760 & 3.030 & 3.395 & 21.5 & $n / a$ & $2.29 \mathrm{e}-01$ & $1.51 \mathrm{E}+00$ \\
\hline$\$ 961005085$ & $F$ & Silver-ICP-Fusion & $u g / g$ & 100.6 & $<1.00 e-02$ & $3.20 \mathrm{e}+02$ & 296.0 & 308.0 & 7.79 & $\mathrm{n} / \mathrm{a}$ & 198.0 & $n / a$ \\
\hline S96r005085 & $\mathrm{F}$ & Aluminium-ICP-Fusion & ug $/ 9$ & 99.40 & $55.00 \mathrm{e}-02$ & $1.69 \mathrm{e}+03$ & $1.67 \mathrm{e}+03$ & $1.68 \mathrm{e}+03$ & 1.19 & $n / a$ & 988.0 & $n / a$ \\
\hline $596 \mathrm{~T} 005085$ & $F$ & Arseníc-ICP-Fusion & $\mathrm{ug} / \mathrm{g}$ & 104.8 & $<1.00 \mathrm{e}-01$ & $<1.98 \mathrm{e}+03$ & $<2.02 e^{3}$ & $n / a$ & $n / a$ & $n / a$ & $1.98 \mathrm{e}+03$ & $n / a$ \\
\hline \$96T005085 & $F$ & Boron-ICP-Fusion & ug/g & 101.8 & $<5.00 \mathrm{e}-02$ & $49.88 \mathrm{e}+02$ & $<1.01 \mathrm{e} 3$ & $\mathrm{n} / \mathrm{a}$ & $n / a$ & $n / a$ & 988.0 & $n / a$ \\
\hline 5967005085 & $\mathbf{F}$ & Barium-ICP-Fusion & $\mathrm{ug} / \mathrm{g}$ & 102.0 & $<5.00 \mathrm{e}-02$ & $<9.88 \mathrm{e}+02$ & $<1.01 \mathrm{e3}$ & $\mathrm{n} / \mathrm{a}$ & $\mathrm{n} / \mathrm{a}$ & $n / a$ & 988.0 & $\mathrm{n} / \mathrm{a}$ \\
\hline 5967005085 & $F$ & Beryllium-ICP-Fusion & $\mathrm{ug} / \mathrm{g}$ & 104.0 & $<5.00 e^{-03}$ & $<\quad 98.80$ & $\leq 1.01 \mathrm{e} 2$ & $\mathrm{n} / \mathrm{a}$ & $n / a$ & $\mathrm{n} / \mathrm{a}$ & 98.80 & $n / a$ \\
\hline $596 r 005085$ & $F$ & Bismuth -ICP-Fusion & $\mathrm{ug} / \mathrm{g}$ & 103.8 & $<1.00 \mathrm{e}-01$ & $<1.98 \mathrm{e}+03$ & $<2.02 \mathrm{e} 3$ & $n / a$ & $\mathrm{n} / \mathrm{a}$ & $\mathrm{n} / \mathrm{a}$ & $1.98 \mathrm{e}+03$ & $n / a$ \\
\hline \$96T005085 & $F$ & Calcium-ICP-Fusion & $\mathrm{ug} / \mathrm{g}$ & 100.4 & $<1.00 \mathrm{e}-01$ & $<1,98 \mathrm{e}+03$ & $<2.02 \mathrm{e} 3$ & $\mathrm{n} / \mathrm{a}$ & $\mathrm{n} / \mathrm{a}$ & $\mathrm{n} / \mathrm{a}$ & $1.98 \mathrm{e}+03$ & $\mathrm{n} / \mathrm{a}$ \\
\hline S96T005085 & $F$ & Cadmium-ICP-Fusion & ug/g & 101.6 & $<5.00 e^{-03}$ & $1.04 \mathrm{e}+02$ & 107.0 & 105.5 & 2.84 & $n / a$ & 98.80 & $\mathrm{n} / \mathrm{a}$ \\
\hline S96T005085 & $F$ & Cerium-ICP-Fusion & $u g / 9$ & 102.4 & $<1.00 \mathrm{e}-01$ & $<1.98 \mathrm{e}+03$ & $<2.02 \mathrm{e}^{3}$ & $n / a$ & $\mathrm{n} / \mathrm{a}$ & $\mathrm{n} / \mathrm{a}$ & $1.98 \mathrm{e}+03$ & $n / a$ \\
\hline 5967005085 & $F$ & Cobalt -ICP-Fusion & ug/g & 102.0 & $<2.00 e^{-02}$ & $<3.95 \mathrm{e}+02$ & $<4.04 \mathrm{e} 2$ & $\mathrm{n} / \mathrm{a}$ & $n / a$ & $n / a$ & 395.0 & $\mathrm{n} / \mathrm{a}$ \\
\hline$\$ 961005085$ & $F$ & Chromium - ICP-Fusion & ug/g & 102.0 & $<1.00 \mathrm{e}-02$ & $7.52 \mathrm{e}+02$ & $7 \overline{12.0}$ & 732.0 & 5.46 & $\mathrm{n} / \mathrm{a}$ & 198.0 & $\mathrm{n} / \mathrm{a}$ \\
\hline $596 \mathrm{~T} 005085$ & $F$ & Copper -ICP-Fusion & ug/g & 105.8 & $<1.00 \mathrm{e}-02$ & $<1.98 \mathrm{e}+02$ & $<2.02 \mathrm{e} 2$ & $\mathrm{n} / \mathrm{a}$ & $n / a$ & $\mathrm{n} / \mathrm{a}$ & 198.0 & $n / a$ \\
\hline$\$ 967005085$ & $F$ & Iron-ICP-Fusion & $\mathrm{ug} / \mathrm{g}$ & 101.6 & $<5.00 \mathrm{e}-02$ & $4.85 \mathrm{e}+03$ & $4.30 \mathrm{e}+03$ & $4.58 \mathrm{e}+03$ & 12.0 & $n / a$ & $988 . \overline{0}$ & $n / a$ \\
\hline 5967005085 & $\frac{F}{F}$ & Lanthanum -ICP-Fusion & ug/g & 103.4 & $<5.00 \mathrm{e}-02$ & $2.37 \mathrm{e}+03$ & $2.20 \mathrm{e}+03$ & $2.28 \mathrm{e}+03$ & 7.44 & $n / a$ & 988.0 & $n / a$ \\
\hline 5961005085 & $F$ & Lithium-ICP-Fusion & $\mathrm{ug} / \mathrm{g}$ & 104.0 & $<1.00 \mathrm{e}-02$ & $<1.98 \mathrm{e}+02$ & $<2 . \overline{02 \mathrm{e} 2}$ & $\mathrm{n} / \mathrm{a}$ & $\mathrm{n} / \mathrm{a}$ & $\mathrm{n} / \mathrm{a}$ & 198.0 & $\mathrm{n} / \mathrm{a}$ \\
\hline $596 \mathrm{~T} 005085$ & $\mathrm{~F}$ & Magnesium -ICP-Fusion & ug $/ \mathrm{g}$ & 98.00 & $<1.00 \mathrm{e}-01$ & $<1, \overline{98} \mathrm{e}+03$ & $<2.02 \mathrm{e} 3$ & $n$ n/a & $n / a$ & $\mathrm{n} / \mathrm{a}$ & $1.98 e^{+03}$ & $\mathrm{n} / \mathrm{a}$ \\
\hline 5961005085 & $F$ & Manganese -ICP-Fusion & ug/g & 99.60 & $<1.00 e^{-02}$ & $4.28 \mathrm{e}+03$ & $4.00 \mathrm{e}+03$ & $4.14 \mathrm{e}+03$ & 6.76 & $\mathrm{n} / \mathrm{a}$ & 198.0 & $n / a$ \\
\hline 5961005085 & $\mathrm{~F}$ & Molybdenum -ICP-Fusion & ugg/g & 101.8 & $<5.00 \mathrm{e}-02$ & $<9.88 \mathrm{e}+02$ & $<1.01 \mathrm{e} 3$ & $\mathrm{n} / \mathrm{a}$ & $n / a$ & $n / a$ & 988.0 & $\mathrm{n} / \mathrm{a}$ \\
\hline $596 \mathrm{~T} 005085$ & $F$ & Sodium-ICP-Fusion & ug/g & 100.2 & 1.770 & $8.04 \mathrm{e}+04$ & $7.84 \mathrm{e}+04$ & $7.94 \mathrm{e}+04$ & 2.52 & $n / a$ & $1.98 \mathrm{e}+03$ & $n / a$ \\
\hline 5961005085 & $F$ & Neodymium -ICP-Fusion & ug/g & 103.8 & $<1.00 e^{-01}$ & $<1.98 \mathrm{e}+03$ & $<2.02 \mathrm{e} 3$ & $\mathrm{n} / \mathrm{a}$ & $\mathrm{n} / \mathrm{a}$ & $n / a$ & $1.98 \mathrm{e}+03$ & $n / a$ \\
\hline S96T005085 & $F$ & Phosphorus-ICP-Fusion & ug/g & 102.0 & $<2.00 \mathrm{e}-01$ & $<3.95 \mathrm{e}+03$ & $<4.04 \mathrm{e}^{3}$ & $n / a$ & $\mathrm{n} / \mathrm{a}$ & $n / a$ & $3.95 e+03$ & $n / a$ \\
\hline 5961005085 & $F$ & Lead -ICP-Fusion & ug/g & 100.2 & $<7.00 \mathrm{e}-01$ & $<1.98 e+03$ & $<2.02 \mathrm{e} 3$ & $n / a$ & $n / a$ & $n / a$ & $1.98 \mathrm{e}+03$ & $n / a$ \\
\hline 5961005085 & $F$ & Sulfur -ICP-Fusion & $\mathrm{ug} / \mathrm{g}$ & 99.40 & $<1.00 \mathrm{e}-01$ & $<1.98 \mathrm{e}+03$ & $<2.02 \mathrm{e} 3$ & $\mathrm{n} / \mathrm{a}$ & $\mathrm{n} / \mathrm{a}$ & $\mathrm{n} / \mathrm{a}$ & $1.98 \mathrm{e}+03$ & $n / a$ \\
\hline 5961005085 & $\mathrm{~F}$ & Ant imony - ICP-Fusion & ug/g $/ 9$ & 97.20 & $<6.00 \mathrm{e}-02$ & $<1.19 \mathrm{e}+03$ & $<1.21 \mathrm{e} 3$ & $n / a$ & $\mathrm{n} / \mathrm{a}$ & $\mathrm{n} / \mathrm{a}$ & $1.18 \mathrm{e}+0 \overline{3}$ & $n / a$ \\
\hline $596 T 005085$ & $F$ & Selenium -ICP-Fusion & ug/9 & 98.40 & $<1.00 e^{-01}$ & $<1.98 \mathrm{e}+03$ & $<2.02 \mathrm{e} 3$ & $n / a$ & $\mathrm{n} / \mathrm{a}$ & $n / a$ & $1.98 \mathrm{e}+0 \overline{3}$ & $n / a$ \\
\hline 5961005085 & $F$ & Silicon -ICP-Fusion & ug/g & 99.80 & $\leq 5.00 e-02$ & $1.23 \mathrm{e}+03$ & $1.28 e+03$ & $1.26 \mathrm{e}+03$ & 3.98 & $\mathrm{n} / \mathrm{a}$ & 988.0 & $\mathrm{n} / \mathrm{a}$ \\
\hline S96T005085 & $F$ & Samarium -ICP-Fusion & $\mathrm{ug} / \mathrm{g}$ & 100.4 & $<1.00 \mathrm{e}-01$ & $<1.98 \mathrm{e}+03$ & $<2.02 \mathrm{e} 3$ & $n / a$ & $n / a$ & $n / a$ & $1.98 \mathrm{e}+03$ & $n / a$ \\
\hline 5965005085 & $F$ & Strontium -ICP-Fusion & ug/g & 100.6 & $<1.00 \mathrm{e}-02$ & $<1.98 \mathrm{e}+02$ & $<2.02 \mathrm{e} 2$ & $\mathrm{n} / \mathrm{a}$ & $n / a$ & $n / a$ & 198.0 & $\mathrm{n} / \mathrm{a}$ \\
\hline S96T005085 & $F$ & Titanium-ICP-Fusion & $u g / g$ & 99.40 & $<1.00 \mathrm{e}-02$ & $<1.98 \mathrm{e}+02$ & $<2,02 \mathrm{e} 2$ & $n / a$ & $n / a$ & $n / a$ & 198.0 & $n / a$ \\
\hline 5961005085 & $F$ & Thallium-ICP-Fusion & $\mathrm{ug} / \mathrm{g}$ & 98.20 & $<2.00 \mathrm{e}-01$ & $<3.95 e^{+03}$ & $<4.04 \mathrm{e} 3$ & $n / a$ & $n / a$ & $\mathrm{n} / \mathrm{a}$ & $3.95 \mathrm{e}+03$ & $\mathrm{n} / \mathrm{a}$ \\
\hline S96T005085 & $F$ & Uranium -ICP-Fusion & ug/g & 98.20 & $<5.00 \mathrm{e}-01$ & $4.39 \mathrm{e}+04$ & $4.18 \mathrm{e}+04$ & $4.28 \mathrm{e}+04$ & 4.90 & $\mathrm{n} / \mathrm{a}$ & $9.88 \mathrm{e}+03$ & $\mathrm{n} / \mathrm{a}$ \\
\hline S967005085 & $F$ & Vanadium -ICP-Fusion & ug/g & 101.8 & $<5.00 e-02$ & $<9.88 \mathrm{e}+02$ & $<1.01 \mathrm{e} 3$ & $\mathrm{n} / \mathrm{a}$ & $n / a$ & $\mathrm{n} / \mathrm{a}$ & 988.0 & $\mathrm{n} / \mathrm{a}$ \\
\hline S96T005085 & $F$ & Zinc-ICP-Fusion & $\mathrm{ug} / \mathrm{g}$ & 101.4 & $<1.00 e^{-02}$ & $<1.98 \mathrm{e}+02$ & $<2,02 \mathrm{e} 2$ & $n / a$ & $n / a$ & $n / a$ & 198.0 & $\mathrm{n} / \mathrm{a}$ \\
\hline S967005085 & & 2irconium -ICP-Fusion & $\mathrm{gg} / \mathrm{g}$ & 100.2 & $<1.00 \mathrm{e}-02$ & $7.89 e+04$ & $7.67 \mathrm{e}+04$ & $7.78 \mathrm{e}+04$ & 2.83 & $n / a$ & 198.0 & $n / a$ \\
\hline
\end{tabular}




\begin{tabular}{|c|c|c|c|c|c|c|c|c|c|c|c|c|}
\hline Sample\# & R|A\# & Analyte & Unit & Standard \% & Blank & Result & Duplicate & Average & RPD \% & Spk Rec \% & Det Limit & Count Err\% \\
\hline $596 T 005085$ & $\begin{array}{ll}F \\
\end{array}$ & Cobalt -60 by GEA & uci $/ \mathrm{g}$ & 93.56 & $<7.37 e^{-03}$ & $<1.06 \mathrm{e}^{-01}$ & $<1.03 \mathrm{e}^{-1}$ & $\mathrm{n} / \mathrm{a}$ & $n / a$ & $n / a$ & $1.06 \mathrm{e}^{-01}$ & $n / a$ \\
\hline \$96r005085 & $f F$ & Cesium -137 by GEA & $\mathrm{UCi} / \mathrm{g}$ & 98.85 & $<1.29 e^{-02}$ & 20.39 & 20.20 & 20.30 & 0.99 & $\mathrm{n} / \mathrm{a}$ & $\mathrm{n} / \mathrm{a}$ & 1.80 \\
\hline 5967005085 & $F$ & Am-241 by Extraction & $\mathrm{uCi} / \mathrm{s}$ & 121.8 & $<1.31 e^{-01}$ & 1.070 & $6.72 e-01$ & $8.71 \mathrm{e}-01$ & 45.7 & $n / a$ & $2.03 e-01$ & $2.36 \mathrm{E}+00$ \\
\hline S96T005085 & $F$ & Alpha of Digested Solid & $\mathrm{uCi} / \mathrm{g}$ & 91.78 & $<6.94 \mathrm{e}-03$ & 1.720 & 1.680 & 1.700 & 2.35 & $\mathrm{n} / \mathrm{a}$ & $9.00 e-03$ & $3.88 \mathrm{E}+00$ \\
\hline S96T005088 & $\mathrm{W}$ & OH- by Pot. Titration & $\mathrm{ug} / \mathrm{g}$ & 97.36 & $<42.00$ & $<\quad 42.00$ & $<42$ & $\mathrm{n} / \mathrm{a}$ & $n / a$ & $n / a$ & 41.70 & $n / a$ \\
\hline S96T0 & $\mathrm{W}$ & Fluoride-IC-Dionex $4000 / 4500$ & ug/g & 99.83 & $<1.20 \mathrm{e}-02$ & $2.02 \mathrm{e}+04$ & $1.99 \mathrm{e}+04$ & $2.00 \mathrm{e}+04$ & 1.50 & 99.15 & 58.50 & $\mathrm{n} / \mathrm{a}$ \\
\hline S96T005088 & $\mathrm{W}$ & Chloride-IC-Dionex $4000 / 4500$ & ug/g & 103.2 & $<1.70 e^{-02}$ & $1.13 e+02$ & 120.0 & 116.5 & 6.01 & 111.1 & 66.95 & $\mathrm{n} / \mathrm{a}$ \\
\hline S96T005088 & W & Nitrite-IC - Dionex $4000 / 4500$ & $4 \mathrm{~g} / \mathrm{g}$ & 95.00 & $<1.08 \mathrm{e}^{-01}$ & $3.49 \mathrm{e}+03$ & $3.39 \mathrm{e}+03$ & $3.44 \mathrm{e}+03$ & 2.91 & 96.67 & 526.5 & $\mathrm{n} / \mathrm{a}$ \\
\hline S96TC & W & Bromide by Ion Chromatograph & ug/g & 103.1 & $<1.25 \mathrm{e}-01$ & $<6.09 \mathrm{e}+02$ & $<6.08 \mathrm{e} 2$ & $n / a$ & $\mathrm{n} / \mathrm{a}$ & 102.9 & 609.4 & $\mathrm{n} / \mathrm{a}$ \\
\hline 5967005088 & W & Nitrate by IC-Dionex $4000 / 4500$ & ug/g & 102.7 & $2.13 e^{-01}$ & $1.88 \mathrm{e}+04$ & $1.85 e+04$ & $1.87 \mathrm{e}+04$ & 1.61 & 105.4 & 677.6 & $n / a$ \\
\hline S96T005088 & $W$ & Phosphate-IC-Dionex $4000 / 4500$ & $\mathrm{ug} / \mathrm{g}$ & 102.2 & $<1.20 \mathrm{e}=01$ & $<5.85 \mathrm{e}+02$ & $<5.83 \mathrm{e} 2$ & $n / a$ & $\mathrm{n} / \mathrm{a}$ & 103.7 & 585.0 & $\mathrm{n} / \mathrm{a}$ \\
\hline S96T005088 & $\mathrm{W}$ & Sulfate by IC-Dionex $4000 / 4500$ & $\mathrm{ug} / \mathrm{g}$ & 101.3 & $<1.38 \mathrm{e}-01$ & $1.68 \mathrm{e}+03$ & $1.69 \mathrm{e}+03$ & $1.68 \mathrm{e}+03$ & 0.59 & 100.5 & 672.9 & $n / a$ \\
\hline$\$ 967005088$ & w & Oxalate by IC-Dionex $4000 / 4500$ & $u g / g$ & 100.2 & $<1.05 \mathrm{e}^{-01}$ & $<5.12 \mathrm{e}+02$ & $<5.10 \mathrm{e} 2$ & $n / a$ & $n / a$ & 105.9 & 511.9 & $n / a$ \\
\hline
\end{tabular}

\begin{tabular}{|c|c|c|c|c|c|c|c|c|c|c|c|c|}
\hline Sample\# & R A\# & Analyte & Unit & Standard \% & Blank & Result & Duplicate & Average & RPD \% & Spk Rec \% & Det Limit & Count Err\% \\
\hline S96T005082 & & Bulk Density of Sample & $\mathrm{g} / \mathrm{mL}$ & $\mathrm{n} / \mathrm{a}$ & $n / a$ & 1.040 & $n / a$ & $\mathrm{n} / \mathrm{a}$ & $n / a$ & $n / a$ & $5.00 \mathrm{e}-01$ & $\mathrm{n} / \mathrm{a}$ \\
\hline $596 T 005082$ & & DSC Exotherm using Mettler & Joules/g & 97.01 & $n / a$ & $0.00 \mathrm{e}+00$ & $0.00 \mathrm{e}+00$ & $0.00 \mathrm{e}+00$ & 0.00 & $\mathrm{n} / \mathrm{a}$ & $\mathrm{n} / \mathrm{a}$ & $n / a$ \\
\hline$\$ 967005082$ & & $\mathrm{OH}^{-}$by Pot. Titration & $\mathrm{ug} / \mathrm{mL}$ & 106.4 & $<42.00$ & $9.04 \mathrm{e}+03$ & $9.39 \mathrm{e}+03$ & $9.22 \mathrm{e}+03$ & 3.80 & $\mathrm{n} / \mathrm{a}$ & 500.0 & $\mathrm{n} / \mathrm{a}$ \\
\hline S96T005082 & & pü Direct & $\mathrm{pH}$ & 100.0 & $n / a$ & 13.42 & 13.43 & 13.43 & 0.07 & $\mathrm{n} / \mathrm{a}$ & $1.00 e-02$ & $n / a$ \\
\hline 5967005082 & & $\%$ Water by TGA using Mettler & $\%$ & 99.38 & $n / a$ & 90.16 & 74.58 & 82.37 & 18.9 & $n / a$ & $n / a$ & $n / a$ \\
\hline S96T005082 & & TIC by Acid/coulometry & $\mathrm{ug} / \mathrm{mL}$ & 102.0 & $3.00 \mathrm{e}-01$ & $7.75 \mathrm{e}+02$ & 792.0 & 783.5 & 2.17 & 103.0 & 5.000 & $\mathrm{n} / \mathrm{a}$ \\
\hline 5967005082 & & Tot. Organic Carbon by coul. & ug/mL & 106.0 & 1.200 & $2.29 \mathrm{e}+03$ & $2.25 \mathrm{e}+03$ & $2.27 \mathrm{e}+03$ & 1.76 & 84.40 & 55.00 & $n / a$ \\
\hline S96T005082 & & Stront ium-89/90 High Level & uCi $/ \mathrm{mL}$ & 108.6 & $<1.23 \mathrm{e}-05$ & $1.47 \mathrm{e}-01$ & $1.24 \mathrm{e}-01$ & $1.36 \mathrm{e}-01$ & 17.0 & $\mathrm{n} / \mathrm{a}$ & $2.36 e-05$ & $4.69 E-01$ \\
\hline S96T005082 & & Pu-239/240 by TRU-SPEC Res in & $\mathrm{uCj} / \mathrm{mL}$ & 89.93 & $<6.50 \mathrm{e}-06$ & $2.86 \mathrm{e}-03$ & $2.59 e^{-03}$ & $2.72 \mathrm{e}-03$ & 9.91 & $n / a$ & $1.22 \mathrm{e}-04$ & $1.19 \mathrm{E}+00$ \\
\hline S96T005082 & D & Silver-ICP-Acid Dil. & $\mathrm{ug} / \mathrm{mL}$ & 100.8 & $<1.00 e^{-02}$ & 2.650 & 2.680 & 2.665 & 1.13 & $\mathrm{n} / \mathrm{a}$ & 1.010 & $n / a$ \\
\hline$\$ 96 T 005082$ & $D$ & Aluminium-ICP-Acid Dil. & $\mathrm{ug} / \mathrm{mL}$ & 98.20 & $<5.00 e-02$ & $7.02 \mathrm{e}+02$ & 707.0 & 704.5 & 0.71 & $n / a$ & 5.050 & $n / a$ \\
\hline $596 T 005082$ & D & Arsenic-ICP-Acid Dil. & $\mathrm{ug} / \mathrm{mL}$ & 102.8 & $<1.00 e^{-01}$ & $<\quad 10.10$ & $<1.01 \mathrm{e} 1$ & $n / a$ & $\mathrm{n} / \mathrm{a}$ & $\mathrm{n} / \mathrm{a}$ & 10.10 & $n / a$ \\
\hline $596 \mathrm{~T} 005082$ & $\frac{D}{D}$ & Boron-ICP-ACid Dil. & ugg/mL & 100.2 & $<5.00 e-02$ & 5.050 & $<5.05 \mathrm{e} 0$ & $n / a$ & $n / a$ & $\mathrm{n} / \mathrm{a}$ & 5.050 & $n / a$ \\
\hline S96T005082 & $D$ & Barium-ICP-Acid Dil. & $\mathrm{ug} / \mathrm{mL}$ & 100.0 & $<5.00 \mathrm{e}-02$ & 5.050 & $<5.05 \mathrm{e} 0$ & $n / a$ & $n / a$ & $n / a$ & 5.050 & $n / a$ \\
\hline S967005082 & D & Beryllium-ICP-Acid Dil. & $\mathrm{ug} / \mathrm{mL}$ & 102.0 & $<5.00 \mathrm{e}-03$ & 1.030 & 1.040 & 1.035 & 0.97 & $n / a$ & $5.05 e-01$ & $n / a$ \\
\hline \$96T005082 & D & Bismuth-ICP-Acid Dil. & $\mathrm{ug} / \mathrm{mL}$ & 103.2 & $<1.00 \mathrm{e}-01$ & 10.10 & $<1.01 \mathrm{e} 1$ & $\mathrm{n} / \mathrm{a}$ & $\mathrm{n} / \mathrm{a}$ & $\mathrm{n} / \mathrm{a}$ & 10.10 & $n / a$ \\
\hline $596 T 005082$ & D & Calcium-ICP-Acid Dil. & $\mathrm{ug} / \mathrm{mL}$ & 101.6 & $<1.00 e-01$ & $<\quad 10.10$ & $<1.01 \mathrm{e} 1$ & $\mathrm{n} / \mathrm{a}$ & $\mathrm{n} / \mathrm{a}$ & $\mathrm{n} / \mathrm{a}$ & 10.10 & $n / a$ \\
\hline 5967005082 & D & Cadmium-ICP-Acid Dil. & $\mathrm{ug} / \mathrm{mL}$ & 101.4 & $<5.00 \mathrm{e}-03$ & $<5.05 e-01$ & $<5.05 e-1$ & $\mathrm{n} / \mathrm{a}$ & $n / a$ & $\mathrm{n} / \mathrm{a}$ & $5.05 e-01$ & $n / a$ \\
\hline S96T005082 & $D$ & Cerium-ICP-Acid Dil. & $\mathrm{ug} / \mathrm{mL}$ & 100.2 & $<1.00 \mathrm{e}-01$ & $<\quad 10.10$ & $<1.01 \mathrm{e} 1$ & $\mathrm{n} / \mathrm{a}$ & $n / a$ & $\mathrm{n} / \mathrm{a}$ & 10.10 & $\mathrm{n} / \mathrm{a}$ \\
\hline 596T005082 & $\bar{D}$ & Cobalt-ICP-Acid Dil. & $\mathrm{ug} / \mathrm{mL}$ & 102.0 & $<2.00 e^{-02}$ & 2.020 & $<2.02 \mathrm{e} 0$ & $n / a$ & $n / a$ & $\mathrm{n} / \mathrm{a}$ & 2.020 & $\mathrm{n} / \mathrm{a}$ \\
\hline S96r005082 & D & Chromium-ICP-Acid Díl. & $\mathrm{ug} / \mathrm{mL}$ & 102.2 & $<1.00 \mathrm{e}-02$ & 32.60 & 33.30 & 32.95 & 2.12 & $n / a$ & 1.010 & $n / a$ \\
\hline$\$ 961005082$ & D & Copper-ICP-Acid Dil. & $\mathrm{ug} / \mathrm{mL}$ & 103.4 & $<1.00 \mathrm{e}-02$ & 1.030 & $<1.01 \mathrm{e} 0$ & $\mathrm{n} / \mathrm{a}$ & $\mathrm{n} / \mathrm{a}$ & $\mathrm{n} / \mathrm{a}$ & 1.010 & $\mathrm{n} / \mathrm{a}$ \\
\hline \$967005082 & D & Iron-ICP-Acid Dil. & $\mathrm{ug} / \mathrm{mL}$ & 101.2 & $<5.00 e^{-02}$ & $<\quad 5.050$ & $<5.05 \mathrm{e} 0$ & $n / a$ & $\mathrm{n} / \mathrm{a}$ & $\mathrm{n} / \mathrm{a}$ & 5.050 & $\mathrm{n} / \mathrm{a}$ \\
\hline $596 \mathrm{~T} 005082$ & $\bar{D}$ & Potassium-ICP-Acid Dil. & $\mathrm{ug} / \mathrm{mL}$ & 99.00 & $<5.00 e^{-01}$ & $1.04 \mathrm{e}+04$ & $1.05 e+04$ & $1.04 \mathrm{e}+04$ & 0.96 & $\mathrm{n} / \mathrm{a}$ & 50.50 & $n / a$ \\
\hline S96T005082 & D & Lanthanum-ICP-ACid Dil. & $\mathrm{ug} / \mathrm{mL}$ & 101.4 & $<5.00 \mathrm{e}^{-02}$ & $<\quad 5.050$ & $<5.05 \mathrm{e} 0$ & $n / a$ & $\mathrm{n} / \mathrm{a}$ & $\mathrm{n} / \mathrm{a}$ & 5.050 & $n / a$ \\
\hline S96T005082 & $\bar{D}$ & Lithium-ICP-Acid Dil. & $\mathrm{ug} / \mathrm{mL}$ & 100.6 & $51.00 \mathrm{e}-02$ & 1.010 & $<1.01 \mathrm{e} 0$ & $\mathrm{n} / \mathrm{a}$ & $\mathrm{n} / \mathrm{a}$ & $\mathrm{n} / \mathrm{a}$ & 1.010 & $n / a$ \\
\hline \$96T005082 & $\bar{D}$ & Magnes ium-ICP-Acid Dil. & $\mathrm{ug} / \mathrm{mL}$ & 97.00 & $<1.00 \mathrm{e}-01$ & 10.10 & $<1.01 \mathrm{e} 1$ & $\mathrm{n} / \mathrm{a}$ & $\mathrm{n} / \mathrm{a}$ & $n / a$ & 10.10 & $n / a$ \\
\hline S96T005082 & D & Manganese-ICP-Acid Dil. & $\mathrm{ug} / \mathrm{mL}$ & 99.20 & $<1.00 \mathrm{e}-02$ & 1.090 & $\leq 1.0100$ & $\mathrm{n} / \mathrm{a}$ & $\mathrm{n} / \mathrm{a}$ & $n / a$ & 1.010 & $\mathrm{n} / \mathrm{a}$ \\
\hline S96T005082 & D & Molybdenum-ICP-Acid Dil. & $\mathrm{ug} / \mathrm{mL}$ & 101.6 & $<5.00 \mathrm{e}-02$ & $<\quad 5.050$ & $<5.05 \mathrm{e}$ & $\mathrm{n} / \mathrm{a}$ & $\mathrm{n} / \mathrm{a}$ & $\mathrm{n} / \mathrm{a}$ & 5.050 & $\mathrm{n} / \mathrm{a}$ \\
\hline \$96T005082 & D & Sodium-ICP-ACid Dil. & $\mathrm{ug} / \mathrm{mL}$ & 98.20 & $<1.00 e^{-01}$ & $3.69 \mathrm{e}+04$ & $3.71 \mathrm{e}+04$ & $3.70 \mathrm{e}+04$ & 0.54 & $\mathrm{n} / \mathrm{a}$ & 10.10 & $n / a$ \\
\hline S96T005082 & D & Neodymium-ICP-Acid Dil. & $\mathrm{ug} / \mathrm{mL}$ & 101.8 & $<1.00 e^{-01}$ & $<10.10$ & $<1.01 \mathrm{e} 1$ & $n / a$ & $n / a$ & $n / a$ & 10.10 & $\mathrm{n} / \mathrm{a}$ \\
\hline S96T005082 & D & Nickel-ICP+Acid Dil. & $\mathrm{ug} / \mathrm{mL}$ & 101.2 & $<2.00 \mathrm{e}-02$ & 3.130 & 3.250 & 3.190 & 3.76 & $n / a$ & 2.020 & $\mathrm{n} / \mathrm{a}$ \\
\hline \$96T005082 & D & Phosphorus-ICP-Acid Dil. & $\mathrm{Ug} / \mathrm{mL}$ & 103.6 & $<2.00 e^{-01}$ & $3.92 \mathrm{e}+02$ & 392.0 & 392.0 & 0.00 & $\mathrm{n} / \mathrm{a}$ & 20.20 & $n / a$ \\
\hline S96T005082 & D & Lead-ICP-Acid Dil. & $\mathrm{ug} / \mathrm{mL}$ & 101.4 & $<1.00 \mathrm{e}-01$ & $<\quad 10.10$ & $<1.01 \mathrm{e} 1$ & $\mathrm{n} / \mathrm{a}$ & $\mathrm{n} / \mathrm{a}$ & $n / a$ & 10.10 & $n / a$ \\
\hline
\end{tabular}




\begin{tabular}{|c|c|c|c|c|c|c|c|c|c|c|c|c|}
\hline Sample\# & $\mathbf{R}$ A\# & Analyte & Unit & Standard $\%$ & Blank) & Result & Duplicatel & Average & $\mathrm{RPD} \%$ & Spk Rec \% & Det Limit & Count Err\% \\
\hline $596 \mathrm{r} 005082$ & $\frac{D}{D}$ & Sulfur-ICP-Acid Dil. & $\mathrm{ug} / \mathrm{mL}$ & 100.6 & $<1.00 \mathrm{e}-01$ & $5.31 \mathrm{e}+02$ & 531.0 & 531.0 & 0.00 & $\mathrm{n} / \mathrm{a}$ & 10.10 & $\mathrm{n} / \mathrm{a}$ \\
\hline$\$ 96 \mathrm{~T} 005082$ & D & Antimony-ICP-Acid Dil. & ug/mL & 96.60 & $<6.00 e-02$ & $<6.060$ & $<6.06 \mathrm{e} 0$ & $\mathrm{n} / \mathrm{a}$ & $\mathrm{n} / \mathrm{a}$ & $\mathrm{n} / \mathrm{a}$ & 6.060 & $\mathrm{n} / \mathrm{a}$ \\
\hline 5961005082 & D & Selenium-ICP-Acid Dil. & $\mathrm{ug} / \mathrm{mL}$ & 96.00 & $<1.00 \mathrm{e}-01$ & 10.10 & $<1.01 \mathrm{e} 1$ & $\mathrm{n} / \mathrm{a}$ & $\mathrm{n} / \mathrm{a}$ & $\mathrm{n} / \mathrm{a}$ & 10.10 & $n / a$ \\
\hline $596 T 005082$ & D & Silicon-ICP-Acid Dil. & $\mathrm{ug} / \mathrm{mL}$ & 99.60 & $<5.00 e-02$ & 49.20 & 53.00 & 51.10 & 7.44 & $\mathrm{n} / \mathrm{a}$ & 5.050 & $n / a$ \\
\hline 5967005082 & D & Samarium-ICP-Acid Dil. & $\underline{\mathrm{ug}} / \mathrm{mL}$ & 98.00 & $<1.00 e^{-01}$ & 10.10 & $<1.01 \mathrm{e} 1$ & $n / a$ & $\mathrm{n} / \mathrm{a}$ & $\mathrm{n} / \mathrm{a}$ & 10.10 & $\mathrm{n} / \mathrm{a}$ \\
\hline $596 \mathrm{~T} 005082$ & D & Stront i um-ICP-Acid Dil. & $\mathrm{ug} / \mathrm{mL}$ & 98.80 & $<1.00 \mathrm{e}-02$ & 1.010 & $<1.0100$ & $\mathrm{n} / \mathrm{a}$ & $\mathrm{n} / \mathrm{a}$ & $\mathrm{n} / \mathrm{a}$ & 1.010 & $\mathrm{n} / \mathrm{a}$ \\
\hline S96T005082 & $D$ & Titanium-ICP-Acid Dil. & $\mathrm{ug} / \mathrm{mL}$ & 97.80 & $<1.00 \mathrm{e}-02$ & 1.010 & $<1.01 \mathrm{e} 0$ & $\mathrm{n} / \mathrm{a}$ & $n / a$ & $n / a$ & 1.010 & $n / a$ \\
\hline 5967005082 & D & Thall ium-ICP-Acid Dil. & $\mathrm{ug} / \mathrm{mL}$ & 98.20 & $<2.00 \mathrm{e}^{-01}$ & 20.20 & $<2.02 \mathrm{e} 1$ & $n / a$ & $\mathrm{n} / \mathrm{a}$ & $n / a$ & 20.20 & $n / a$ \\
\hline$\$ 967005082$ & D & Uranium-ICP-Acid Dil. & $\mathrm{ug} / \mathrm{ml}$ & 96.10 & $<5.00 \mathrm{e}-01$ & 78.90 & 55.80 & 67.35 & 34.3 & $\mathrm{n} / \mathrm{a}$ & 50.50 & $\mathrm{n} / \mathrm{a}$ \\
\hline S96T005082 & D & Vanadium-ICP-Acid Dil. & $\mathrm{ug} / \mathrm{mL}$ & 101.2 & $<5.00 e-02$ & 5.050 & $<5.05 \mathrm{e} 0$ & $n / a$ & $\mathrm{n} / \mathrm{a}$ & $\mathrm{n} / \mathrm{a}$ & 5.050 & $\mathrm{n} / \mathrm{a}$ \\
\hline $596 \mathrm{~T} 005082$ & D & Zinc-ICP-Acid Dil. & $\mathrm{ug} / \mathrm{mL}$ & 102.4 & $<1.00 e^{-02}$ & 3.820 & 3.960 & 3.890 & 3.60 & $n / a$ & 1.010 & $\mathrm{n} / \mathrm{a}$ \\
\hline S967005082 & $\frac{1}{0}$ & Zirconium-ICP-Acid DiL. & $\mathrm{ug} / \mathrm{mL}$ & 99.00 & $<1.00 \mathrm{e}-02$ & 18.90 & 13.30 & 16.10 & 34.8 & $n / a$ & 1.010 & $\mathrm{n} / \mathrm{a}$ \\
\hline$\$ 967005082$ & & Fluoride-I'C-Dionex $4000 / 4500$ & $\mathrm{ug} / \mathrm{mL}$ & 93.39 & $<1.20 \mathrm{e}-02$ & $1.04 \mathrm{e}+04$ & $1.05 \mathrm{e}+04$ & $1.05 \mathrm{e}+04$ & 0.96 & 94.24 & 25.45 & $n / a$ \\
\hline $596 \mathrm{~T} 005082$ & & Chloride-IC-Dionex $4000 / 4500$ & $\mathrm{ug} / \mathrm{mL}$ & 90.25 & $<1.70 \mathrm{e}-02$ & $3.24 \mathrm{e}+02$ & 323.0 & 323.6 & 0.31 & 89.37 & 18.89 & $\mathrm{n} / \mathrm{a}$ \\
\hline$\$ 967005082$ & & Nitrite-IC - Dionex $4000 / 4500$ & $\mathrm{ug} / \mathrm{mL}$ & 90.74 & $<1.08 \mathrm{e}^{-01}$ & $4.71 \mathrm{e}+03$ & $4.73 \mathrm{e}+03$ & $4.72 e+03$ & 0.42 & 95.56 & 120.0 & $\mathrm{n} / \mathrm{a}$ \\
\hline \$96T005082 & & Bromide by Ion chromatograph & ug/mL & 97.45 & $<1.25 \mathrm{e}-01$ & $<1.39 \mathrm{e}+02$ & $<1.39 \mathrm{e} 2$ & $\mathrm{n} / \mathrm{a}$ & $\mathrm{n} / \mathrm{a}$ & 92.36 & 138.9 & n/a \\
\hline 5967005082 & & Nitrate by IC-Dionex $4000 / 4500$ & $\mathrm{ug} / \mathrm{mL}$ & 99.16 & $1.71 \mathrm{e}-01$ & $2.70 \mathrm{e}+04$ & $2.69 e+04$ & $2.70 e+04$ & 0.37 & 106.6 & 294.8 & n/a \\
\hline 59670 & & Phosphate-IC-Dionex $4000 / 4500$ & $\mathrm{ug} / \mathrm{mL}$ & 97.80 & $<1.20 \mathrm{e}-01$ & $3.69 \mathrm{e}+02$ & 414.0 & 391.6 & 11.5 & 97.06 & 133.3 & $\mathrm{n} / \mathrm{a}$ \\
\hline S96T005082 & & Sulfate by IC-Dionex $4000 / 4500$ & ug $/ \mathrm{mL}$ & 100.5 & $<1.38 \mathrm{e}-01$ & $1.54 \mathrm{e}+03$ & $1.49 \mathrm{e}+03$ & $1.51 \mathrm{e}+03$ & 3.30 & 101.3 & 153.3 & $\mathrm{n} / \mathrm{a}$ \\
\hline S967005082 & & Oxalate by IC-Dionex $4000 / 4500$ & $\mathrm{ug} / \mathrm{mL}$ & 100.6 & $<1.05 \mathrm{e}-01$ & $3.21 \mathrm{e}+02$ & 388.0 & 354.4 & 18.9 & 101.7 & 116.7 & $\mathrm{n} / \mathrm{a}$ \\
\hline S96T005082 & & Cobal $t-60$ by GEA & $\mathrm{uCi} / \mathrm{mL}$ & 99.36 & $<2.93 e-04$ & $2.13 \mathrm{e}-02$ & $2.17 \mathrm{e}-02$ & $2.15 e-02$ & 1.86 & $\mathrm{n} / \mathrm{a}$ & $n / a$ & 6.28 \\
\hline$\$ 967005082$ & & Cesium- 137 by GEA & $\mathrm{uCi} / \mathrm{mL}$ & 100.2 & $<7.59 \mathrm{e}-04$ & 24.80 & 25.40 & 25.10 & 2.39 & $\mathrm{n} / \mathrm{a}$ & $\mathrm{n} / \mathrm{a}$ & 0.160 \\
\hline S96T005082 & & Am-241 by Extraction & $\mathrm{uCi} / \mathrm{mL}$ & 91.60 & $<4.02 e-05$ & $<4.13 e-05$ & $\angle 4.49 \mathrm{E}-5$ & $\mathrm{n} / \mathrm{a}$ & $\mathrm{n} / \mathrm{a}$ & $\mathrm{n} / \mathrm{a}$ & $4.13 e-05$ & $7.24 E+00$ \\
\hline \$96T005082 & & Alpha in Liquid Samples & $u C \mathbf{i} / \mathrm{mL}$ & 85.71 & $1.00 \mathrm{e}-03$ & $1.78 \mathrm{e}-03$ & $9.80 e-04$ & $1.38 \mathrm{e}-03$ & 58.0 & n/a & $3.52 e-04$ & $4.08 \mathrm{E}+01$ \\
\hline
\end{tabular}
Sludge (from Liquid Grab Sample): sludge (from Liquid Grab Sample)

en

ample\# R A\# Analyte \begin{tabular}{|l|l|l|}
\hline S967004821 & Bulk Density of Sample & \% \\
\hline S9604821 & Volume \% Solids & $\%$ \\
\hline
\end{tabular}

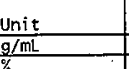

Standard
Biank n/a

Result ouplicate 1.220
67.60

Duplicate n/a

\begin{tabular}{l|l|l|l|l|} 
Average & RPD $\%$ & Spk Rec $\%$ & Det Limit & Count Err\% \\
\hline
\end{tabular} $\mathrm{n} / \mathrm{a}$ $\mathrm{n} / \mathrm{a}$ $\mathrm{n}$ \begin{tabular}{r|r} 
Rec \% & Det Limit \\
\hline n/a & $5.00 e^{-01}$ \\
\hline n/a & $n / a$
\end{tabular} 
Table 3: Data Summary Report AW-105 GRAB1

RISER: 15A

SEGMENT \#: 5 AW-96-20

SEGMENT PORTION: Centrifuged Solids (Grab Sample)

\begin{tabular}{|c|c|c|c|c|c|c|c|c|c|c|c|c|}
\hline Sample\# & $\mathrm{R}$ A\# & Analyte & Unit & Standard \% & Blank & Result & Duplicate & Average & RPD \% & Spk Rec \% & Det Limit & Count Err\% \\
\hline \$96T005077 & & Bulk Density of Sample & $g / m L$ & $n / a$ & $n / a$ & 1.260 & $n / a$ & $n / a$ & $n / a$ & $n / a$ & $5.00 \mathrm{e}-01$ & $\mathbf{n} / \mathbf{a}$ \\
\hline S96T005077 & & DSC Exotherm using Mettler & Joules/g & 101.9 & $\mathrm{n} / \mathrm{a}$ & 22.20 & 21.50 & 21.85 & 3.20 & $n / a$ & $n / a$ & $n / a$ \\
\hline \$96T005077 & & DSC Exotherm Dry Calculated & Joules/g Dry & $\mathrm{n} / \mathrm{a}$ & $\mathrm{n} / \mathrm{a}$ & 70.90 & 68.70 & 69.80 & 3.15 & $\mathrm{n} / \mathrm{a}$ & $\mathrm{n} / \mathrm{a}$ & $\mathrm{n} / \mathrm{a}$ \\
\hline 5967005077 & & pH on SST Samples & $\mathrm{pH}$ & 99.62 & $n / a$ & 12.49 & 12.48 & 12.48 & 0.08 & $\mathrm{n} / \mathrm{a}$ & $1.00 \mathrm{e}-02$ & $\mathrm{n} / \mathrm{a}$ \\
\hline S96T005077 & & $\%$ Water by TGA using Mettler & $\%$ & 99.54 & $n / a$ & 66.19 & 71.20 & 68.69 & 7.29 & $\mathrm{n} / \mathrm{a}$ & $n / a$ & $\mathrm{n} / \mathrm{a}$ \\
\hline$\$ 967005077$ & & TIC by Acid/coulometry & ugg/g & 101.8 & 3.000 & $6.35 \mathrm{e}+02$ & 766.0 & 700.5 & 18.7 & $\mathrm{n} / \mathrm{a}$ & 5.000 & $n / a$ \\
\hline S96T005077 & & Toc by Persulfate/Coulometry & $\mathrm{ug} / \mathrm{g}$ & 97.33 & 2.600 & $1.77 e+03$ & $1.43 e+03$ & $1.60 e+03$ & 21.2 & $\mathrm{n} / \mathrm{a}$ & 40.00 & $\mathrm{n} / \mathrm{a}$ \\
\hline$\$ 96 T 005086$ & $\mathbf{F}$ & Strontium- $89 / 90 \mathrm{High}$ Level & $u c i / g$ & 80.00 & $1.23 \mathrm{e}-01$ & 31.40 & 29.50 & 30.45 & 6.24 & $\mathrm{n} / \mathrm{a}$ & $1.50 \mathrm{e}-02$ & $8.39 \mathrm{E}-01$ \\
\hline \$967005086 & $\mathbf{F}$ & PU-239/240 by IRU-SPEC Resin & $\mathrm{uCi} / \mathrm{g}$ & 112.1 & $<1.61 \mathrm{e}-02$ & 3.280 & 3.240 & 3.260 & $1 . \overline{23}$ & $\mathrm{n} / \mathrm{a}$ & $1.42 e-01$ & $1.36 \mathrm{E}+00$ \\
\hline 5967005086 & $\mathbf{F}$ & Silver-ICP-Fusion & $\mathrm{ug} / \mathrm{g}$ & 100.6 & $<1.00 \mathrm{e}-02$ & $<2.03 e+02$ & $<1.99 \mathrm{e} 2$ & $\mathrm{n} / \mathrm{a}$ & $\mathrm{n} / \mathrm{a}$ & $\mathrm{n} / \mathrm{a}$ & 203.0 & $n / a$ \\
\hline S96T005086 & $F$ & Aluminium-ICP-Fusion & $\mathrm{ug} / \mathrm{g}$ & 99.40 & $<5.00 \mathrm{e}-02$ & $1.13 \mathrm{e}+03$ & $1.17 e+03$ & $1.15 e+03$ & 3.48 & $\mathrm{n} / \mathrm{a}$ & $1.02 \mathrm{e}+03$ & $\mathrm{n} / \mathrm{a}$ \\
\hline S96T005086 & $F$ & Arsenic -ICP-Fusion & ug/g & 104.8 & $<1.00 \mathrm{e}-01$ & $<2.03 e+03$ & $<1.99 \mathrm{a} 3$ & $n / a$ & $n / a$ & $n / a$ & $2.03 \mathrm{e}+03$ & $n / a$ \\
\hline S96T005086 & $F$ & Boron -ICP-Fusion & $\mathrm{ug} / \mathrm{g}$ & 101.8 & $<5.00 \mathrm{e}-02$ & $<1.02 \mathrm{e}+03$ & $<9.97 \mathrm{e} 2$ & $n / a$ & $n / a$ & $\mathrm{n} / \mathrm{a}$ & $1.02 \mathrm{e}+03$ & $\mathrm{n} / \mathrm{a}$ \\
\hline 5967005086 & $F$ & Barium-iCP-Fusion & ug $/ \mathrm{g}$ & 102.0 & $<5.00 \mathrm{e}-02$ & $<1.02 e+03$ & $<9.97 \mathrm{e} 2$ & $n / a$ & $n / a$ & $\mathrm{n} / \mathrm{a}$ & $1.02 \mathrm{e}+03$ & $\mathrm{n} / \mathrm{a}$ \\
\hline \$96T005086 & $\mathbf{F}$ & Beryllium -ICP-Fusion & $\mathrm{ug} / \mathrm{g}$ & 104.0 & $<5.00 \mathrm{e}-03$ & $<1.02 e+02$ & $<9.97 \mathrm{e} 1$ & $n / a$ & $\mathrm{n} / \mathrm{a}$ & $\mathrm{n} / \mathrm{a}$ & 102.0 & $\mathrm{n} / \mathrm{a}$ \\
\hline $596 T 005086$ & $F$ & Bismuth -ICP-Fusion & $\mathrm{ug} / \mathrm{g}$ & 103.8 & $<1.00 \mathrm{e}-01$ & $<2.03 \mathrm{e}+03$ & $<1.99 \mathrm{e} 3$ & $n / a$ & $\mathrm{n} / \mathrm{a}$ & $n / a$ & $2.03 e+03$ & $\mathrm{n} / \mathrm{a}$ \\
\hline$\$ 967005086$ & $\mathbf{F}$ & Calcium-ICP-Fusion & $\mathrm{ug} / \mathrm{g}$ & 100.4 & $<1.00 \mathrm{e}-01$ & $<2.03 \mathrm{e}+03$ & $<1.99 \mathrm{e} 3$ & $n / a$ & $\mathrm{n} / \mathrm{a}$ & $n / a$ & $2.03 e+03$ & $n / a$ \\
\hline 5967005086 & $F$ & Cadmiun -ICP-Fusion & $\mathrm{ug} / \mathrm{g}$ & 101.6 & $<5.00 \mathrm{e}-03$ & $2.38 \mathrm{e}+02$ & 246.0 & 242.0 & 3.31 & $\mathrm{n} / \mathrm{a}$ & 102.0 & $n / a$ \\
\hline S96T005086 & $F$ & Cerium-ICP-Fusion & $\mathrm{ug} / \mathrm{g}$ & 102.4 & $<1.00 \mathrm{e}-01$ & $<2.03 \mathrm{e}+03$ & $<1.99 \mathrm{e} 3$ & $n / a$ & $\mathrm{n} / \mathrm{a}$ & $\mathrm{n} / \mathrm{a}$ & $2.03 \mathrm{e}+03$ & $\mathrm{n} / \mathrm{a}$ \\
\hline S96T005086 & $\bar{F}$ & Cobalt -ICP-Fusion & $\mathrm{ug} / \mathrm{g}$ & 102.0 & $<2.00 \mathrm{e}-02$ & $<4.07 \mathrm{e}+02$ & $<3.99 \mathrm{e} 2$ & $n / a$ & $\mathrm{n} / \mathrm{a}$ & $\mathrm{n} / \mathrm{a}$ & 407.0 & $\mathrm{n} / \mathrm{a}$ \\
\hline S96T005086 & $F$ & Chromium -ICP-Fusion & $\mathrm{ug} / \mathrm{g}$ & 102.0 & $<1.00 \mathrm{e}-02$ & $2.14 \mathrm{e}+02$ & 203.0 & 208.5 & 5.28 & $\mathrm{n} / \mathrm{a}$ & 203.0 & $\mathrm{n} / \mathrm{a}$ \\
\hline S96T005086 & $F$ & Copper-ICP-Fusion & $\mathrm{ug} / \mathrm{g}$ & 105.8 & $<1,00 e-02$ & $<2.03 e+02$ & $<1.99 \mathrm{e} 2$ & $\mathrm{n} / \mathrm{a}$ & $\mathrm{n} / \mathrm{a}$ & $\mathrm{n} / \mathrm{a}$ & 203.0 & $\mathrm{n} / \mathrm{a}$ \\
\hline S96T005086 & $F$ & Iron-ICP-Fusion & $\mathrm{ug} / \mathrm{g}$ & 101.6 & $<5.00 e-02$ & $2.06 \mathrm{e}+03$ & $1.30 \mathrm{e}+03$ & $1.68 \mathrm{e}+03$ & 45.2 & $\mathrm{n} / \mathrm{a}$ & $1.02 e+03$ & $n / a$ \\
\hline S96T005086 & $F$ & Lanthanum -ICP-Fusion & $\underline{\underline{g} / \mathrm{g}}$ & 103.4 & $<5.00 \mathrm{e}-02$ & $1.07 e+03$ & $1.01 \mathrm{e}+03$ & $1.04 \mathrm{e}+03$ & 5.77 & $n / a$ & $1.02 \mathrm{e}+03$ & $n / a$ \\
\hline$\$ 967005086$ & $F$ & Lithium-ICP-Fusion & $\log / 9$ & 104.0 & $<1.00 \mathrm{e}-02$ & $<2.03 e+02$ & $<1.99 \mathrm{e} 2$ & $n / a$ & $n / a$ & $n / a$ & 203.0 & $n / a$ \\
\hline \$967005086 & $F$ & Magnesium-ICP-Fusion & $\mathrm{ug} / \mathrm{g}$ & 98.00 & $<1.00 \mathrm{e}-01$ & $<2.03 \mathrm{e}+03$ & $<1.99 \mathrm{e} 3$ & $n / a$ & $n / a$ & $\mathrm{n} / \mathrm{a}$ & $2.03 \mathrm{e}+03$ & $n / a$ \\
\hline 5967005086 & $\mathbf{F}$ & Manganese -ICP-Fusion & $\mathrm{ug} / \mathrm{g}$ & 99.60 & $<1.00 \mathrm{e}-02$ & $7.40 \mathrm{e}+02$ & 745.0 & 742.5 & 0.67 & $n / a$ & 203.0 & $\mathrm{n} / \mathrm{a}$ \\
\hline S96T005086 & $F$ & Molybdenum -ICP-Fusion & $\mathrm{ug} / \mathrm{g}$ & 101.8 & $<5.00 \mathrm{e}-02$ & $<1.02 \mathrm{e}+03$ & $<9.97 \mathrm{e} 2$ & $\mathrm{n} / \mathrm{a}$ & $\mathrm{n} / \mathrm{a}$ & $\mathrm{n} / \mathrm{a}$ & $1,02 \mathrm{e}+03$ & $n / a$ \\
\hline S96T005086 & $F$ & Sodium-ICP-Fusion & $\mathrm{ug} / \mathrm{g}$ & 100.2 & 1.770 & $6.75 e+04$ & $7.16 \mathrm{e}+04$ & $6.96 \mathrm{e}+04$ & 5.90 & $n / a$ & $2.03 \mathrm{e}+03$ & $\mathrm{n} / \mathrm{a}$ \\
\hline S96T005086 & $F$ & Neodymium -ICP-Fusion & $\mathrm{ug} / \mathrm{g}$ & 103.8 & $<1.00 \mathrm{e}-01$ & $<2.03 e+03$ & $<1.99 \mathrm{e} 3$ & $\mathrm{n} / \mathrm{a}$ & $\mathrm{n} / \mathrm{a}$ & $n / a$ & $2.03 e+03$ & $\mathrm{n} / \mathrm{a}$ \\
\hline S96T005086 & $\mathrm{F}$ & Phosphorus -ICP-Fusion & $\mathrm{ng} / \mathrm{g}$ & 102.0 & $<2.00 \mathrm{e}-01$ & $<4.07 \mathrm{e}+03$ & $<3.99 \mathrm{e} 3$ & $n / a$ & $\mathrm{n} / \mathrm{a}$ & $n / a$ & $4.07 \mathrm{e}+03$ & $\mathrm{n} / \mathrm{a}$ \\
\hline S967005086 & $F$ & Lead -ICP-Fusion & $\mathrm{ug} / \mathrm{g}$ & 100.2 & $<1.00 \mathrm{e}-01$ & $<2.03 e+03$ & $<1.99 \mathrm{e} 3$ & $n / a$ & $\mathrm{n} / \mathrm{a}$ & $\mathrm{n} / \mathrm{a}$ & $2.03 e+03$ & $n / a$ \\
\hline \$96T005086 & $F$ & Sulfur-ICP-Fusion & ug/g & 99.40 & $<1.00 \mathrm{e}-01$ & $<2.03 e+03$ & $<1.99 \mathrm{e}^{3}$ & $n / a$ & $\mathrm{n} / \mathrm{a}$ & $\mathrm{n} / \mathrm{a}$ & $2.03 e+03$ & $n / a$ \\
\hline S967005086 & $F$ & Antimony -ICP-Fusion & $\mathrm{ug} / \mathrm{g}$ & 97.20 & $<6.00 \mathrm{e}-02$ & $<1.22 \mathrm{e}+03$ & $<1.20 \mathrm{e} 3$ & $n / a$ & $\mathrm{n} / \mathrm{a}$ & $\mathrm{n} / \mathrm{a}$ & $1.22 \mathrm{e}+03$ & $\mathrm{n} / \mathrm{a}$ \\
\hline 5967005086 & $\mathbf{F}$ & Selenium-1CP-Fusion & $\mathrm{ug} / \mathrm{g}$ & 98.40 & $<1.00 \mathrm{e}-01$ & $<2.03 e+03$ & $<1.99 \mathrm{e} 3$ & $n / a$ & $\mathrm{n} / \mathrm{a}$ & n/a & $2.03 \mathrm{e}+03$ & $\mathrm{n} / \mathrm{a}$ \\
\hline$\$ 967005086$ & $\mathbf{F}$ & siticon -ICP-Fusion & $\mathrm{ug} / \mathrm{g}$ & 99.80 & $<5.00 e-02$ & $<1.02 \mathrm{e}+03$ & $<9.97 \mathrm{e} 2$ & $n / a$ & $n / a$ & $n / a$ & $1.02 \mathrm{e}+03$ & $n / a$ \\
\hline S96T005086 & $F$ & Samarium -ICP-Fusion & $\mathrm{ug} / \mathrm{g}$ & 100.4 & $<1.00 \mathrm{e}-01$ & $<2.03 e+03$ & $<1.99 \mathrm{e} 3$ & $n / a$ & $n / a$ & $\mathrm{n} / \mathrm{a}$ & $2.03 e+03$ & $\mathrm{n} / \mathrm{a}$ \\
\hline $596 T 005086$ & $F$ & Strontium -ICP-Fusion & ug/g & 100.6 & $\leq 1.00 \mathrm{e}-02$ & $<2.03 \mathrm{e}+02$ & $<1.99 \mathrm{e} 2$ & $\mathrm{n} / \mathrm{a}$ & $n / a$ & $\mathrm{n} / \mathrm{a}$ & 203.0 & $n / a$ \\
\hline S96T005086 & $F$ & Titanium-ICP-Fusion & $\mathrm{ug} / \mathrm{g}$ & 99.40 & $<1.00 \mathrm{e}-02$ & $<2.03 \mathrm{e}+02$ & $<1.99 \mathrm{e} 2$ & $n / a$ & $n / a$ & $\mathrm{n} / \mathrm{a}$ & 203.0 & $\mathrm{n} / \mathrm{a}$ \\
\hline 5967005086 & $F$ & Thallium-ICP-Fusion & $\mathrm{ug} / \mathrm{g}$ & 98.20 & $<2.00 \mathrm{e}-01$ & $<4.07 \mathrm{e}+03$ & $<3.99 \mathrm{e} 3$ & $\mathrm{n} / \mathrm{a}$ & $\mathrm{n} / \mathrm{a}$ & $\mathrm{n} / \mathrm{a}$ & $4.07 \mathrm{e}+03$ & $n / a$ \\
\hline 5967005086 & $F$ & Uranium -ICP-Fusion & $4 \mathrm{~g} / \mathrm{g}$ & 98.20 & $<5.00 \mathrm{e}-01$ & $9.43 e+04$ & $9.46 \mathrm{e}+04$ & $9 . \overline{44 e+04}$ & 0.32 & n/a & $1.02 e+04$ & $n / a$ \\
\hline S96T005086 & $\mathrm{F}$ & Vanadium -ICP-Fusion & $4 \mathrm{~g} / \mathrm{g}$ & 101.8 & $<5.00 \mathrm{e}-02$ & $<1.02 \mathrm{e}+03$ & $<9.97 \mathrm{e} 2$ & $n / a$ & $\mathrm{n} / \mathrm{a}$ & $\mathrm{n} / \mathrm{a}$ & $1.02 \mathrm{e}+03$ & $\mathrm{n} / \mathrm{a}$ \\
\hline 5967005086 & $\vec{F}$ & Zinc-ICP-Fusion & $\mathrm{ug} / \mathrm{g}$ & 101.4 & $<1.00 \mathrm{e}-02$ & $<2.03 e+02$ & $<1.99 \mathrm{e} 2$ & $n / a$ & $\mathrm{n} / \mathrm{a}$ & $\mathrm{n} / \mathrm{a}$ & 203.0 & $\mathrm{n} / \mathrm{a}$ \\
\hline 5967005086 & $\bar{F}$ & Zirconium-ICP-Fusion & ug/g & 100.2 & $<1.00 \mathrm{e}-02$ & $1.60 \mathrm{e}+04$ & $1.83 \mathrm{e}+04$ & $1 . \overline{72 e+04}$ & 13.4 & $\mathrm{n} / \mathrm{a}$ & $20 \overline{3.0}$ & n/a \\
\hline
\end{tabular}




\begin{tabular}{|c|c|c|c|c|c|c|c|c|c|c|c|c|}
\hline mple\# & $\mathbf{R}$ A\# & Analyte & Unit & Standard \% & Blank & Result & Dupl icate & Average & RPD $\%$ & Spk Rec \% & Det Limjt & Count Err\% \\
\hline 5967005086 & $F$ & Cobalt -60 by GEA & $u C i / g$ & 93.56 & $<7.37 \mathrm{e}-03$ & $3.15 \mathrm{e}-02$ & $3.06 e^{-02}$ & $3.11 \mathrm{e}-02$ & 2.90 & $n / a$ & $n / a$ & 22.9 \\
\hline $596 T 005086$ & $\mathbf{F}$ & Cesium- 137 by GEA & $\mathrm{uCi} / \mathrm{g}$ & 98.85 & $<1.29 \mathrm{e}-02$ & 13.94 & 14.20 & 14.07 & 2.14 & $\mathrm{n} / \mathrm{a}$ & $n / a$ & 0.680 \\
\hline$\$ 96 \mathrm{~T} 005086$ & $\mathbf{F}$ & Am-241 by Extraction & $\mathrm{uCi} / \mathrm{g}$ & 95.80 & $<2.82 e^{-02}$ & $4.18 \mathrm{e}-01$ & $4.32 \mathrm{e}-01$ & $4.25 \mathrm{e}-01$ & 3.29 & n/a & $5.20 \mathrm{e}-02$ & $1.78 \mathrm{E}+00$ \\
\hline$\$ 96 \mathrm{~T} 005$ & $F$ & Alpha of Digested Solid & $\mathrm{uCi} / \mathrm{g}$ & 91.78 & $<6.94 \mathrm{e}^{-03}$ & 3.690 & 3.480 & 3.585 & 5.86 & $\mathrm{n} / \mathrm{a}$ & $1.00 \mathrm{e}-02$ & $2.73 \mathrm{E}+00$ \\
\hline 5089 & $\mathbf{W}$ & OH- by Pot. Titration & $\mathrm{ug} / \mathrm{g}$ & 97.36 & $<42.00$ & $<42.00$ & $<42$ & $\mathrm{n} / \mathrm{a}$ & $\mathrm{n} / \mathrm{a}$ & $n / a$ & 41.70 & \\
\hline 5967005089 & $\mathbf{W}$ & Fluoride-IC-Dionex $4000 / 4500$ & $\mathrm{ug} / \mathrm{g}$ & 99.83 & $<1.20 \mathrm{e}-02$ & $4.15 \mathrm{e}+03$ & $4.23 \mathrm{e}+03$ & $4.19 \mathrm{e}+03$ & 1.91 & $n / a$ & 59.12 & \\
\hline$\$ 96 \mathrm{TO}$ & W & Chloride-IC-Dionex $4000 / 4500$ & $u g / g$ & 94.81 & $<1.70 e-02$ & $2.72 \mathrm{e}+02$ & 365.0 & 318.6 & 29.2 & $n / a$ & 83.77 & $n / a$ \\
\hline$\$ 96 \mathrm{TO}$ & w & Nitrite-1C - Dionex $4000 / 4500$ & $\mathrm{ug} / \mathrm{g}$ & 95.00 & $<1.08 \mathrm{e}-01$ & $2.52 \mathrm{e}+03$ & $2.60 \mathrm{e}+03$ & $2.56 \mathrm{e}+03$ & 3.12 & $\mathrm{n} / \mathrm{a}$ & 532.2 & $\mathrm{n} /$ \\
\hline$\$ 96 \mathrm{T005089}$ & W & Bromide by Ion chromatograph & $\operatorname{ugg} / 9$ & 103.1 & $\leq 1.25 e-01$ & $<6.16 \mathrm{e}+02$ & $<6.33 \mathrm{e} 2$ & $n / a$ & $\mathrm{n} / \mathrm{a}$ & $\mathrm{n} / \mathrm{a}$ & 615.9 & \\
\hline 05089 & W & Nitrate by IC-Dionex $4000 / 4500$ & ug/9 & 102.7 & $2.13 e-01$ & $2.24 \mathrm{e}+04$ & $2.31 \mathrm{e}+04$ & $2.27 \mathrm{e}+04$ & 3.08 & $\mathrm{n} / \mathrm{a}$ & 685.1 & $\mathrm{n}$ \\
\hline S96T & w & Phosphate-IC-D ionex $4000 / 4500$ & $\overline{\mathrm{ug} / \mathrm{g}}$ & 102.2 & $<1.20 \mathrm{e}-01$ & $<5.91 \mathrm{e}+02$ & $<6.07 \mathrm{e} 2$ & $\mathrm{n} / \mathrm{a}$ & $n / a$ & $\mathrm{n} / \mathrm{a}$ & 591.2 & $n /$ \\
\hline 5967005089 & W & Sul fate by IC-D ionex $4000 / 4500$ & $u g / g$ & 101.3 & $<1.38 \mathrm{e}-01$ & $1.16 \mathrm{e}+03$ & $1.31 \mathrm{e}+03$ & $1.24 e+03$ & 12.1 & $\mathrm{n} / \mathrm{a}$ & 680.2 & \\
\hline 36005089 & w & Oxalate by IC-Dionex $4000 / 4500$ & $\mathrm{ug} / \mathrm{g}$ & 100.2 & $<1.05 e-01$ & $<5.17 \mathrm{e}+02$ & $<5.31 \mathrm{e} 2$ & $\mathrm{n} / \mathrm{a}$ & $\mathrm{n} / \mathrm{a}$ & $\mathrm{n} / \mathrm{a}$ & 517.4 & \\
\hline
\end{tabular}

\begin{tabular}{|c|c|c|c|}
\hline Sampl e\# & A A & Analyte & Unit \\
\hline S96T005083 & & Bulk Density of Sample & $\mathrm{g} / \mathrm{mL}$ \\
\hline$\$ 961005083$ & & DSC Exotherm using Mettler & Joules/g \\
\hline S96T005083 & & oH- by pot. Titration & ug/mL \\
\hline S96T005083 & & pH Direct & $\mathrm{pH}$ \\
\hline S967005083 & & $\%$ Water by TGA using Mettler & $\%$ \\
\hline S96T005083 & & TIC by Acid/Coulometry & $\mathrm{ug} / \mathrm{mL}$ \\
\hline S96T005083 & & Tot. Organic Carbon by coul. & $\mathrm{ug} / \mathrm{mL}$ \\
\hline S96T005083 & & Stront ium-89/90 High Level & $\mathrm{uCj} / \mathrm{mL}$ \\
\hline S96T005083 & & Pu-239/240 by IRU-SPEC Resin & $\mathrm{uCi} / \mathrm{mL}$ \\
\hline \$96T005083 & D & Silver-ICP-Acid Dil. & $\mathrm{ug} / \mathrm{mL}$ \\
\hline \$96T005083 & D & Aluminium-ICP-Acid Dil. & $\mathrm{ng} / \mathrm{mL}$ \\
\hline S96T005083 & D & Arsenic-ICP-Acid Dil. & $\mathrm{ug} / \mathrm{mL}$ \\
\hline 5967005083 & $\frac{D}{D}$ & Boron-ICP-ACid DiL. & ug/mL \\
\hline S96T005083 & $D$ & Barium-ICP-Acid Dil. & $\mathrm{ug} / \mathrm{mL}$ \\
\hline S96T005083 & D & Beryllium-ICP-Acid Dil. & $\mathrm{ug} / \mathrm{mL}$ \\
\hline$\$ 96 T 005083$ & D & Bismuth-ICP-Acid Dil. & $\mathrm{ug} / \mathrm{mL}$ \\
\hline S96T005083 & D & Calcium-ICP-Acid Dil. & $\mathrm{ug} / \mathrm{mL}$ \\
\hline S96T005083 & D & Cadmi um-ICP-Acid Dil. & $\mathrm{ug} / \mathrm{mL}$ \\
\hline \$96T005083 & $D$ & Cerium-ICP-Acid Dil. & $\mathrm{ug} / \mathrm{mL}$ \\
\hline S967005083 & tD & Cobalt-ICP-Acid Dill. & $\mathrm{ug} / \mathrm{mL}$ \\
\hline S96T005083 & $\frac{D}{D}$ & Chromium-ICP-Acid Díl. & $\mathrm{ug} / \mathrm{mL}$ \\
\hline S96T005083 & D & Copper-ICP-Acid Dil. & $\mathrm{ug} / \mathrm{mL}$ \\
\hline \$96T005083 & D & Iron-ICP-Acid Dil. & $\mathrm{ug} / \mathrm{mL}$ \\
\hline \$96T005083 & $\bar{D}$ & Potassium-1CP-Acid Dil. & $\mathrm{ug} / \mathrm{mL}$ \\
\hline S96T005083 & D & Lanthanum-ICP-Acid Dil. & $\mathrm{ug} / \mathrm{mL}$ \\
\hline S96T005083 & $D$ & Lithium-ICP-Acid Dil. & $\mathrm{ug} / \mathrm{mL}$ \\
\hline$\$ 96 \mathrm{~T} 005083$ & D & Magnesium-ICP-Acid Dil. & $\mathrm{ug} / \mathrm{mL}$ \\
\hline S96T005083 & D & Manganese-ICP-Acid DiL. & $\mathrm{ug} / \mathrm{mL}$ \\
\hline \$967005083 & D & Molybdenum-ICP-Acid Dil. & $\mathrm{ug} / \mathrm{mL}$ \\
\hline $596 \mathrm{~T} 005083$ & D & Sodium-ICP-Acid Dil. & $\mathrm{ug} / \mathrm{mL}$ \\
\hline S967005083 & 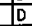 & Neodymi uin-ICP-Acid Dil. & $\mathrm{ug} / \mathrm{mL}$ \\
\hline S96T005083 & $D$ & Nickel-ICP-Acid Dil. & $\mathrm{ug} / \mathrm{mL}$ \\
\hline S96T005083 & $D$ & Phosphorus-ICP-Acid Dil. & $\mathrm{ug} / \mathrm{mL}$ \\
\hline $596 \mathrm{~T} 005083$ & & Lead-ICP-Acid Dil. & \\
\hline
\end{tabular}

standard

\begin{tabular}{|c|c|c|c|c|c|c|c|c|}
\hline & Blank & Result & Duplicate & Average & RPD \% & Spk Rec \% & Det Limit & Count Err\% \\
\hline $\mathrm{n} / \mathrm{s}$ & $n / a$ & 1.090 & $\mathrm{n} / \mathrm{a}$ & $n / a$ & $n / a$ & $n / a$ & $5.00 \mathrm{e}-01$ & $n / a$ \\
\hline 97.01 & $n / a$ & $0.00 \mathrm{e}+00$ & $0.00 e+00$ & $0.00 \mathrm{e}+00$ & 0.00 & $n / a$ & $\mathrm{n} / \mathrm{a}$ & $\mathrm{n} / \mathrm{a}$ \\
\hline 6.4 & $<42.00$ & $8.66 \mathrm{e}+03$ & $9.07 e+03$ & $8.86 e+05$ & 4.62 & $n / a$ & 500.0 & $n / a$ \\
\hline & $n / a$ & 13.34 & 13.28 & 13.31 & 0.45 & $\mathrm{n} / \mathrm{a}$ & $1.00 \mathrm{e}-02$ & $\mathrm{n} / \mathrm{a}$ \\
\hline 99.38 & $\mathrm{n} / \mathrm{a}$ & 89.29 & 90.05 & 89.67 & 0.85 & $n / a$ & $\mathrm{n} / \mathrm{a}$ & $\mathrm{n} / \mathrm{a}$ \\
\hline 102.0 & $3.00 \mathrm{e}-01$ & $6.76 \mathrm{e}+02$ & 686.0 & 681.0 & 1.47 & $n / a$ & 5.000 & $\mathrm{n} / \mathrm{a}$ \\
\hline 106.0 & 1.200 & $1.92 \mathrm{e}+03$ & $1.82 \mathrm{e}+03$ & $1.87 \mathrm{e}+03$ & 5.35 & $\mathrm{n} / \mathrm{a}$ & 55.00 & $\mathrm{n} / \mathrm{a}$ \\
\hline 97.14 & $5.89 \mathrm{e}-05$ & $8.28 \mathrm{e}-04$ & $1.77 \mathrm{e}-03$ & $1.30 \mathrm{e}-03$ & 72.5 & $n / a$ & $1.19 \mathrm{e}-05$ & $4.78 \mathrm{E}+00$ \\
\hline 39.93 & $<6.50 \mathrm{e}-06$ & $1.78 \mathrm{e}-03$ & $2.13 \mathrm{e}-03$ & $1.96 \mathrm{e}-03$ & 17.9 & $\mathrm{n} / \mathrm{a}$ & $8.55 e-05$ & $1.26 \mathrm{E}+00$ \\
\hline 100.8 & $<1.00 e-02$ & 2.670 & 2.630 & 2.650 & 1.51 & $n / a$ & 1.010 & $n / a$ \\
\hline 98.20 & $<5.00 \mathrm{e}-02$ & $5.88 \mathrm{e}+02$ & 591.0 & 589.5 & 0.51 & $\mathrm{n} / \mathrm{a}$ & 5.050 & $\mathrm{n} / \mathrm{a}$ \\
\hline 102.8 & $<1.00 e-01$ & $<\quad 10.10$ & $<1.01 \mathrm{e} 1$ & $\mathrm{n} / \mathrm{a}$ & $\mathrm{n} / \mathrm{a}$ & $\mathrm{n} / \mathrm{a}$ & 10.10 & $n / a$ \\
\hline & $<5.00 \mathrm{e}-02$ & 5.050 & $<5.05 \mathrm{e} 0$ & $\mathrm{n} / \mathrm{a}$ & $n / a$ & $\mathrm{n} / \mathrm{a}$ & 5.050 & $n / a$ \\
\hline .0 & $<5.00 \mathrm{e}-02$ & 5.050 & $<5.05 \mathrm{e} 0$ & $n / a$ & $n / a$ & $n / a$ & 5.050 & $n / a$ \\
\hline & $<5.00 \mathrm{e}-03$ & $9.95 \mathrm{e}-01$ & 1.010 & 1.002 & 1.50 & $\mathrm{n} / \mathrm{a}$ & $5.05 \mathrm{e}-01$ & $n / a$ \\
\hline 3.2 & $<1.00 \mathrm{e}-01$ & $<\quad 10.10$ & $<1.0\} \mathrm{e} 1$ & $n / a$ & $\mathrm{n} / \mathrm{a}$ & $n / a$ & 10.10 & $n / a$ \\
\hline 1.6 & $<1.00 e^{-01}$ & 10.10 & $<1.01 \mathrm{e} 1$ & $\mathrm{n} / \mathrm{a}$ & $n / a$ & $\mathrm{n} / \mathrm{a}$ & 10.10 & $\mathrm{n} / \mathrm{a}$ \\
\hline 1.4 & $<5.00 e-03$ & $<5.05 \mathrm{e}-01$ & $\leq 5.05 \mathrm{e}-1$ & $n / a$ & $n / a$ & $\mathrm{n} / \mathrm{a}$ & $5,05 e-01$ & $\mathrm{n} / \mathrm{a}$ \\
\hline & $<1.00 e-01$ & $<10.10$ & $<1.01 \mathrm{e} 1$ & $\mathrm{n} / \mathrm{a}$ & $\mathrm{n} / \mathrm{a}$ & $\mathrm{n} / \mathrm{a}$ & 10.10 & $n / a$ \\
\hline & $<2.00 \mathrm{e}-02$ & 2.020 & $<2.02 \mathrm{e} 0$ & $n / a$ & $\mathrm{n} / \mathrm{a}$ & $n / a$ & 2.020 & $n / a$ \\
\hline 2.2 & $<1.00 \mathrm{e}-02$ & 35.30 & 35.70 & 35.50 & 1.13 & $n / a$ & 1.010 & $n / a$ \\
\hline 103.4 & $<1.00 \mathrm{e}-02$ & 1.010 & $<1.01 \mathrm{e} 0$ & $n / a$ & $\mathrm{n} / \mathrm{a}$ & $n / a$ & 010 & fa \\
\hline & $<5.00 \mathrm{e}-02$ & 5.050 & $<5.05 \mathrm{e} 0$ & $n / a$ & $\mathrm{n} / \mathrm{a}$ & $\mathrm{n} / \mathrm{a}$ & 5.050 & $n / a$ \\
\hline 00 & $<5.00 \mathrm{e}-01$ & $9.29 \mathrm{e}+03$ & $9.37 e+03$ & $9.33 e+03$ & 0.86 & $\mathrm{n} / \mathrm{a}$ & 50.50 & $\mathrm{n} / \mathrm{a}$ \\
\hline 1.4 & $<5.00 e^{-02}$ & $<\quad 5.050$ & $<5.05 \mathrm{e} 0$ & $\mathrm{n} / \mathrm{a}$ & $\mathrm{n} / \mathrm{a}$ & $n / a$ & 5.050 & $n / a$ \\
\hline & $<1.00 \mathrm{e}-02$ & 1.010 & $<1.01 \mathrm{e} 0$ & $\overline{n / a}$ & $n / a$ & $n / a$ & 010 & $n / a$ \\
\hline & $<1.00 \mathrm{e}-01$ & 10.10 & $<1.01 \mathrm{e} 1$ & $\mathrm{n} / \mathrm{a}$ & $n / a$ & $\mathrm{n} / \mathrm{a}$ & 10.10 & $n / a$ \\
\hline 20 & $<1.00 e^{-02}$ & 1.010 & $<9.01 \mathrm{e} 0$ & $\mathrm{n} / \mathrm{a}$ & $n / a$ & $n / a$ & 1.010 & $n / a$ \\
\hline 1.6 & $<5.00 \mathrm{e}-02$ & 5.050 & $<5.05 \mathrm{e} 0$ & $\mathrm{n} / \mathrm{a}$ & $n / a$ & $\mathrm{n} / \mathrm{a}$ & 5.050 & $n / a$ \\
\hline & $<1.00 \mathrm{e}-01$ & $3.67 e+04$ & $3.68 \mathrm{e}+04$ & $3.68 \mathrm{e}+04$ & 0.27 & $\mathrm{n} / \mathrm{a}$ & 10.10 & $n /$ \\
\hline & $<1.00 e^{-01}$ & $<\quad 10.10$ & $<1.01 \mathrm{e} 1$ & $n / a$ & $n / a$ & $\mathrm{n} / \mathrm{a}$ & 10.10 & $n /$ \\
\hline & $<2.00 \mathrm{e}-02$ & $<\quad 2.020$ & 2.780 & $n / a$ & $n / a$ & $n / a$ & 2.020 & $n / a$ \\
\hline & $\angle 2.00 \mathrm{e}-01$ & $3.87 \mathrm{e}+02$ & 393.0 & 390.0 & 1.54 & $\mathrm{n} / \mathrm{a}$ & 20.20 & $n / a$ \\
\hline & $<1.00 e-01$ & $<\quad 10.10$ & $<101 \mathrm{e} 1$ & $n / a$ & $\mathrm{n} / \mathrm{a}$ & $\mathrm{n} / \mathrm{a}$ & 10.10 & $\mathrm{n} /$ \\
\hline
\end{tabular}




\begin{tabular}{|c|c|c|c|c|c|c|c|c|c|c|c|c|}
\hline Sample\# & $R \mid A \#$ & \# Analyte & Unit & Standard \% & Blank & Result & Duplicate & Average & RPD \% & Spk Rec \% & Det Limit & Count Err\% \\
\hline S96T005083 & D & Sulfur-ICP-Acid Dil. & $\mathrm{ug} / \mathrm{mL}$ & 100.6 & $<1.00 \mathrm{e}-01$ & $4.57 e+02$ & 459.0 & 458.0 & 0.44 & $\mathrm{n} / \mathrm{a}$ & 10.10 & $\mathrm{n} / \mathrm{a}$ \\
\hline 5967005083 & $D$ & Antimony-ICP-ACid Dil. & $\mathrm{ug} / \mathrm{mL}$ & 96.60 & $<6.00 \mathrm{e}-02$ & $<\quad 6.060$ & $<6.06 \mathrm{e} 0$ & $\mathrm{n} / \mathbf{a}$ & $n / a$ & n/a & 6.060 & $n / a$ \\
\hline S96T005083 & D & Selenium-ICP-Acid Dil. & ug/mL & 96.00 & $<1.00 \mathrm{e}-01$ & 10.10 & $<1.01 \mathrm{e} 1$ & $\mathrm{n} / \mathrm{a}$ & $\mathrm{n} / \mathrm{a}$ & $n / a$ & 10.10 & $n / a$ \\
\hline S96T005083 & D & Silicon-ICP-Acid Dil. & ug/mL & 99.60 & $<5.00 e-02$ & 52.50 & 56.00 & 54.25 & 6.45 & $\mathrm{n} / \mathrm{a}$ & 5.050 & $n / a$ \\
\hline S96T005083 & D & Samarium-ICP-Acid Dil. & ug $/ \mathrm{mL}$ & 98.00 & $<1.00 e-01$ & 10.10 & $<1.01 \mathrm{e} 1$ & $n / a$ & $\mathrm{n} / \mathrm{a}$ & $\mathrm{n} / \mathrm{a}$ & 10,10 & $n / a$ \\
\hline $596 \mathrm{~T} 005083$ & D & Strontium-ICP-Acid Dil. & $\mathrm{ug} / \mathrm{mL}$ & 98.80 & $<1.00 \mathrm{e}-02$ & 1.010 & $<1.01 \mathrm{e} 0$ & $n / a$ & $\mathrm{n} / \mathrm{a}$ & $\mathrm{n} / \mathrm{a}$ & 1.010 & $n / a$ \\
\hline 5961005083 & D & Titanium-ICP-Acid Dil. & $\mathrm{ug} / \mathrm{mL}$ & 97.80 & $<1.00 e=02$ & 1.010 & $<1.01 \mathrm{e} 0$ & $n / a$ & $\mathrm{n} / \mathrm{a}$ & $n / a$ & 1.010 & $\mathrm{n} / \mathrm{a}$ \\
\hline S96T005083 & D & Thallium-ICP-Acid Dil, & $\mathrm{ug} / \mathrm{mL}$ & 98.20 & $<2.00 \mathrm{e}-01$ & 20.20 & $<2.02 \mathrm{e} 1$ & $\mathrm{n} / \mathrm{a}$ & $\mathrm{n} / \mathrm{a}$ & $\mathrm{n} / \mathrm{a}$ & 20.20 & $n / a$ \\
\hline S967005083 & D & Uranium-ICP-Acid Dil. & $\mathrm{ug} / \mathrm{mL}$ & 96.10 & $<5.00 e^{-01}$ & 50.50 & $<5.05 \mathrm{e} 1$ & $\mathrm{n} / \mathrm{a}$ & $n / a$ & $\mathrm{n} / \mathrm{a}$ & 50.50 & $n / a$ \\
\hline S96T005083 & $\mathrm{D}$ & Vanadium-ICP-Acid Dil. & $\mathrm{ug} / \mathrm{mL}$ & 101.2 & $<5.00 e^{-02}$ & 5.050 & $<5.05 e 0$ & $n / a$ & $\mathrm{n} / \mathrm{a}$ & $n / a$ & 5.050 & $\mathrm{n} / \mathrm{a}$ \\
\hline S96T005083 & D & Zinc-ICP-Acid Dil. & $\mathrm{ug} / \mathrm{mL}$ & 102.4 & $<1,00 \mathrm{e}-02$ & 3.690 & 3.670 & 3.680 & 0.54 & $\mathrm{n} / \mathrm{a}$ & 1.010 & $n / a$ \\
\hline S96T005083 & D & Zirconium-ICP-Acid Dil. & $\mathrm{ug} / \mathrm{mL}$ & 99.00 & $<1.00 e^{-02}$ & 4.710 & 2.220 & 3.465 & 71.9 & $\mathrm{n} / \mathrm{a}$ & 1.010 & $n / a$ \\
\hline $596 T 005083$ & & Fluoride-IC-Dionex $4000 / 4500$ & $\mathrm{ug} / \mathrm{mL}$ & 93.39 & $<1.20 \mathrm{e}-02$ & $9.89 \mathrm{e}+03$ & $9.81 \mathrm{e}+03$ & $9.85 e+03$ & 0.81 & $\mathrm{n} / \mathrm{a}$ & 13.33 & $\mathrm{n} / \mathrm{a}$ \\
\hline S96T005083 & & Chlor ide-IC-Dionex $4000 / 4500$ & $\mathrm{ug} / \mathrm{mL}$ & 90.25 & $<1.70 \mathrm{e}-02$ & $2.96 \mathrm{e}+02$ & 298.0 & 296.8 & 0.67 & $\mathrm{n} / \mathrm{a}$ & 18.89 & $n / a$ \\
\hline 5967005083 & & Nitrite-IC - Dionex $4000 / 4500$ & $\mathrm{ug} / \mathrm{mL}$ & 90.74 & $<1.08 \mathrm{e}-01$ & $3.94 \mathrm{e}+03$ & $3.92 \mathrm{e}+03$ & $3.93 \mathrm{e}+03$ & 0.51 & $\mathrm{n} / \mathrm{a}$ & 120.0 & $\mathrm{n} / \mathrm{a}$ \\
\hline S96T005083 & & Bromide by Ion Chromatograph & $\mathrm{ug} / \mathrm{ml}$ & 97.45 & $<1.25 e^{-01}$ & $<1.39 e+02$ & $<1.39 \mathrm{e} 2$ & $n / a$ & $\mathrm{n} / \mathrm{a}$ & $\mathrm{n} / \mathrm{a}$ & 138.9 & $\mathrm{n} / \mathrm{a}$ \\
\hline \$96T005083 & & Nitrate by IC-Dionex $4000 / 4500$ & $\mathrm{ug} / \mathrm{mL}$ & 99.16 & $1.71 \mathrm{e}-01$ & $3.03 \mathrm{e}+04$ & $3.03 \mathrm{e}+04$ & $3.03 \mathrm{e}+04$ & 0.00 & $\mathrm{n} / \mathrm{a}$ & 154.4 & $\mathrm{n} / \mathrm{a}$ \\
\hline \$96T005083 & & Phosphate-IC-Dionex $4000 / 4500$ & $\mathrm{ug} / \mathrm{mL}$ & 97.80 & $<1.20 \mathrm{e}-01$ & $3.93 e+02$ & 404.0 & 398.6 & 2.76 & $n / a$ & 133.3 & $\mathrm{n} / \mathrm{a}$ \\
\hline \$96T005083 & & Sulfate by IC-Dionex $4000 / 4500$ & $\mathrm{ug} / \mathrm{mL}$ & 100.5 & $<1.38 \mathrm{e}-01$ & $1.24 e+03$ & $1.28 e+03$ & $1.26 \mathrm{e}+03$ & 3.17 & $n / a$ & 153.3 & $\mathrm{n} / \mathrm{a}$ \\
\hline S96T005083 & & Oxalate by IC-Dionex $4000 / 4500$ & ug/mL & 100.6 & $<1.05 \mathrm{e}^{-01}$ & $2.72 \mathrm{e}+02$ & 287.0 & 279.6 & 5.37 & $\mathrm{n} / \mathrm{a}$ & 116.7 & $\mathrm{n} / \mathrm{a}$ \\
\hline S96T005083 & & Cobal $t-60$ by GEA & $\mathrm{uci} / \mathrm{mL}$ & 99.36 & $<2.93 e-04$ & $2.01 e^{-02}$ & $1.94 \mathrm{e}-02$ & $1.98 e-02$ & 3.54 & $n / a$ & $n / a$ & 5.95 \\
\hline S96T005083 & & Cesium- 137 by GEA & $\mathrm{uCi} / \mathrm{mL}$ & 100.2 & $<7.59 \mathrm{e}-04$ & 22.50 & 21.90 & 22.20 & 2.70 & $\mathrm{n} / \mathrm{a}$ & $n / a$ & 0.160 \\
\hline S96T005083 & & Am- 241 by Extraction & uCi $/ \mathrm{mL}^{2}$ & 122.7 & $<1.18 e^{-05}$ & $5.44 e^{-04}$ & $6.09 e-04$ & $5.76 \mathrm{e}-04$ & 11.3 & $\mathrm{n} / \mathrm{a}$ & $6.82 e-05$ & $1.90 \mathrm{E}+00$ \\
\hline S96T005083 & & Alpha in Liquid Samples & $\mathrm{uCi} / \mathrm{mL}$ & 85.71 & $1.00 \mathrm{e}-03$ & $5.47 e-04$ & $6.14 e-04$ & $5.80 e-04$ & 11.5 & $n / a$ & $3.52 e-04$ & $7.33 \mathrm{E}+01$ \\
\hline
\end{tabular}

Sludge (from Liquid Grab Sample): Sludge (from Liquid Grab Sample)

\begin{tabular}{|c|c|c|c|c|c|c|c|c|c|c|c|c|}
\hline Sample\# & R AA\# & Analyte & Unit & Standard \% & Blank & Result & Dupl icate & Average & RPD \% & Spk Rec \% & Det Limit & Count Err\% \\
\hline S96T004824 & & Bulk Densjty of Sample & $\mathrm{g} / \mathrm{mL}$ & $\mathrm{n} / \mathrm{a}$ & $n / a$ & 1.200 & $\mathrm{n} / \mathrm{a}$ & $\mathrm{n} / \mathrm{a}$ & $n / a$ & n/a & $5.00 \mathrm{e}-01$ & n/a \\
\hline$\$ 967004824$ & & Volume \% Solids & $\%$ & $\mathrm{n} / \mathrm{a}$ & $n / a$ & 79.10 & $n / a$ & $n / a$ & $n / a$ & $\mathrm{n} / \mathrm{a}$ & $n / a$ & $\mathrm{n} / \mathrm{a}$ \\
\hline
\end{tabular}


HNF-SD-WM-DP-214, REV. 0
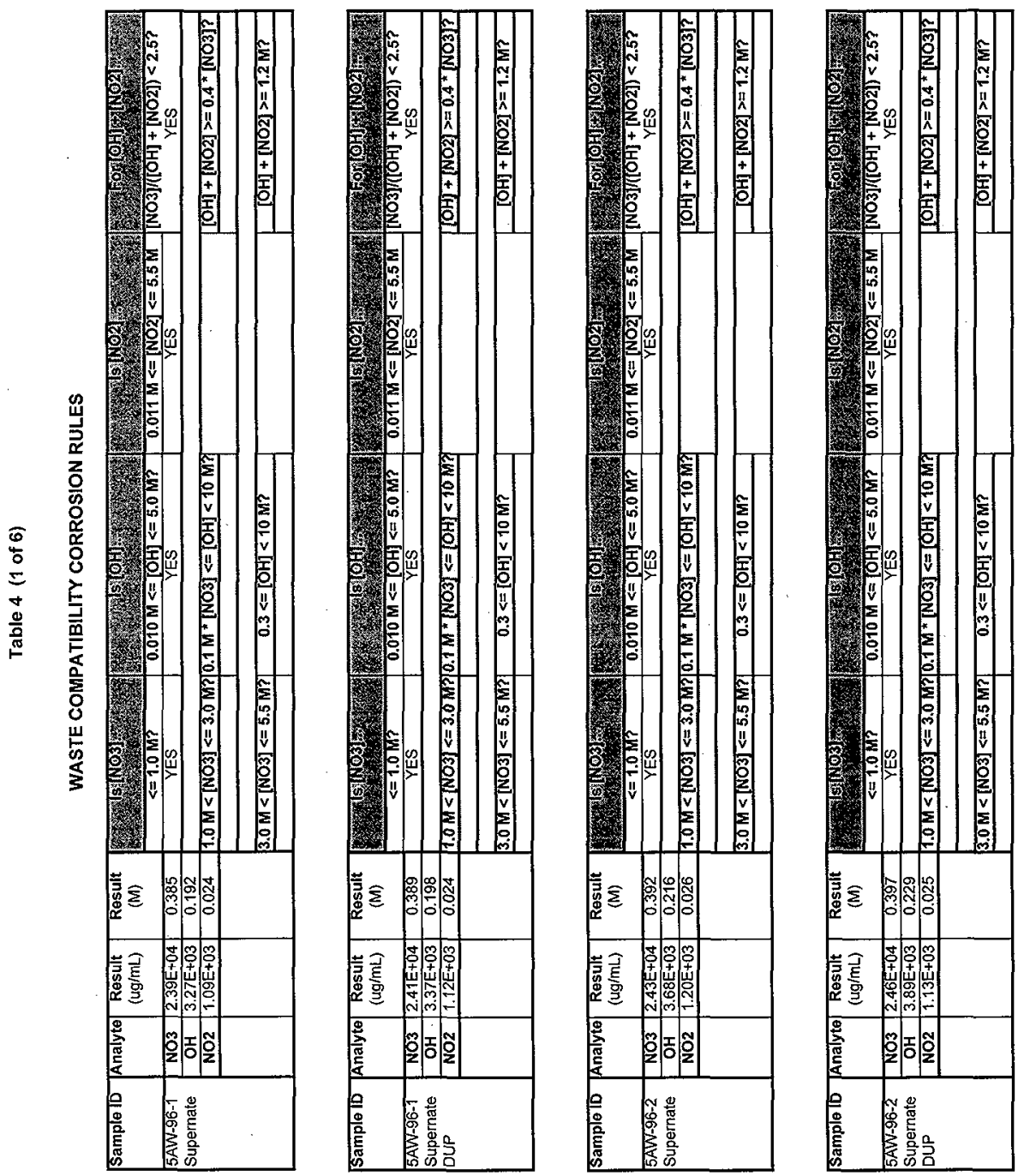
HNF-SD-WM-DP-214, REV. 0
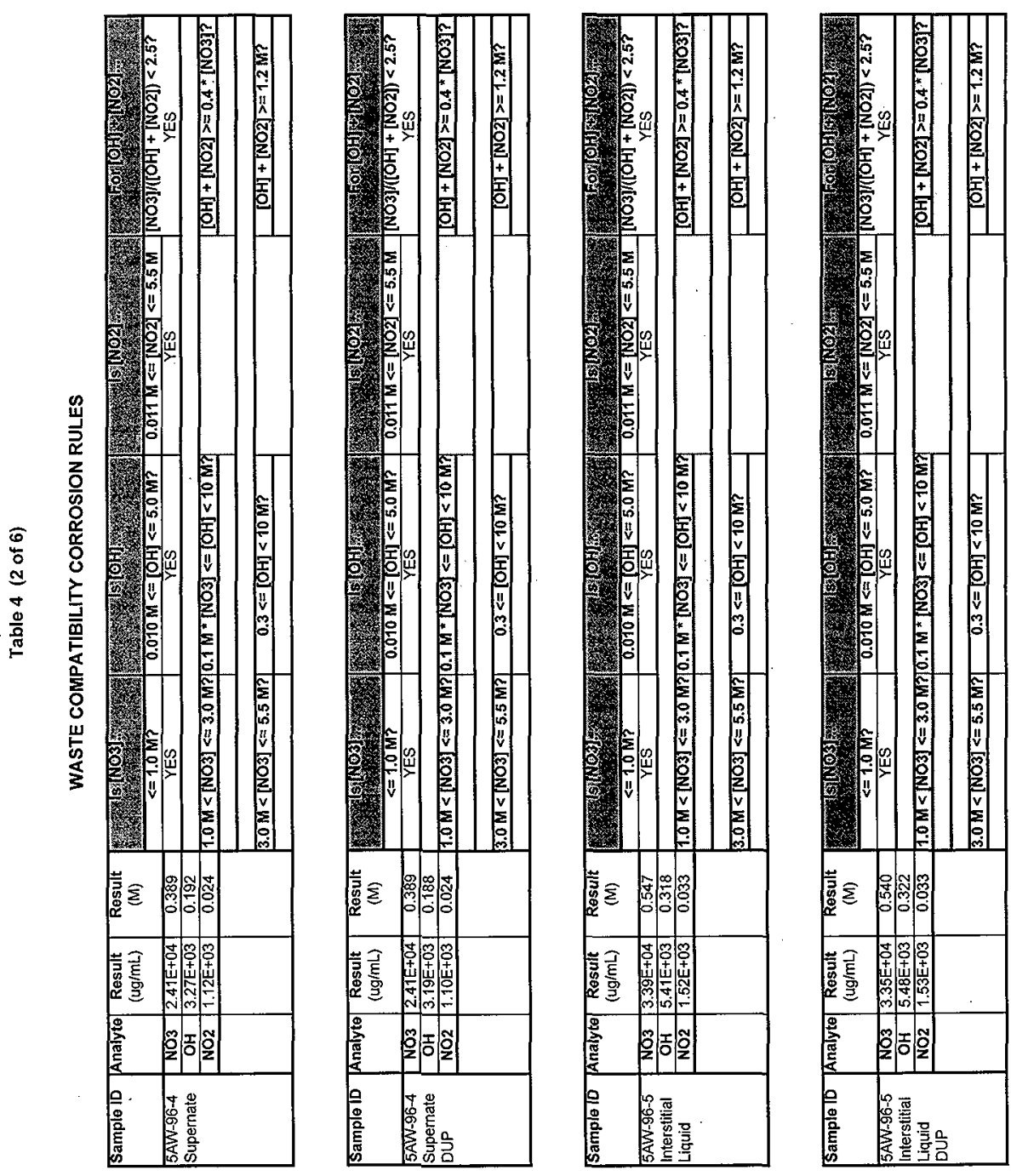
HNF-SD-WM-DP-214, REV. 0
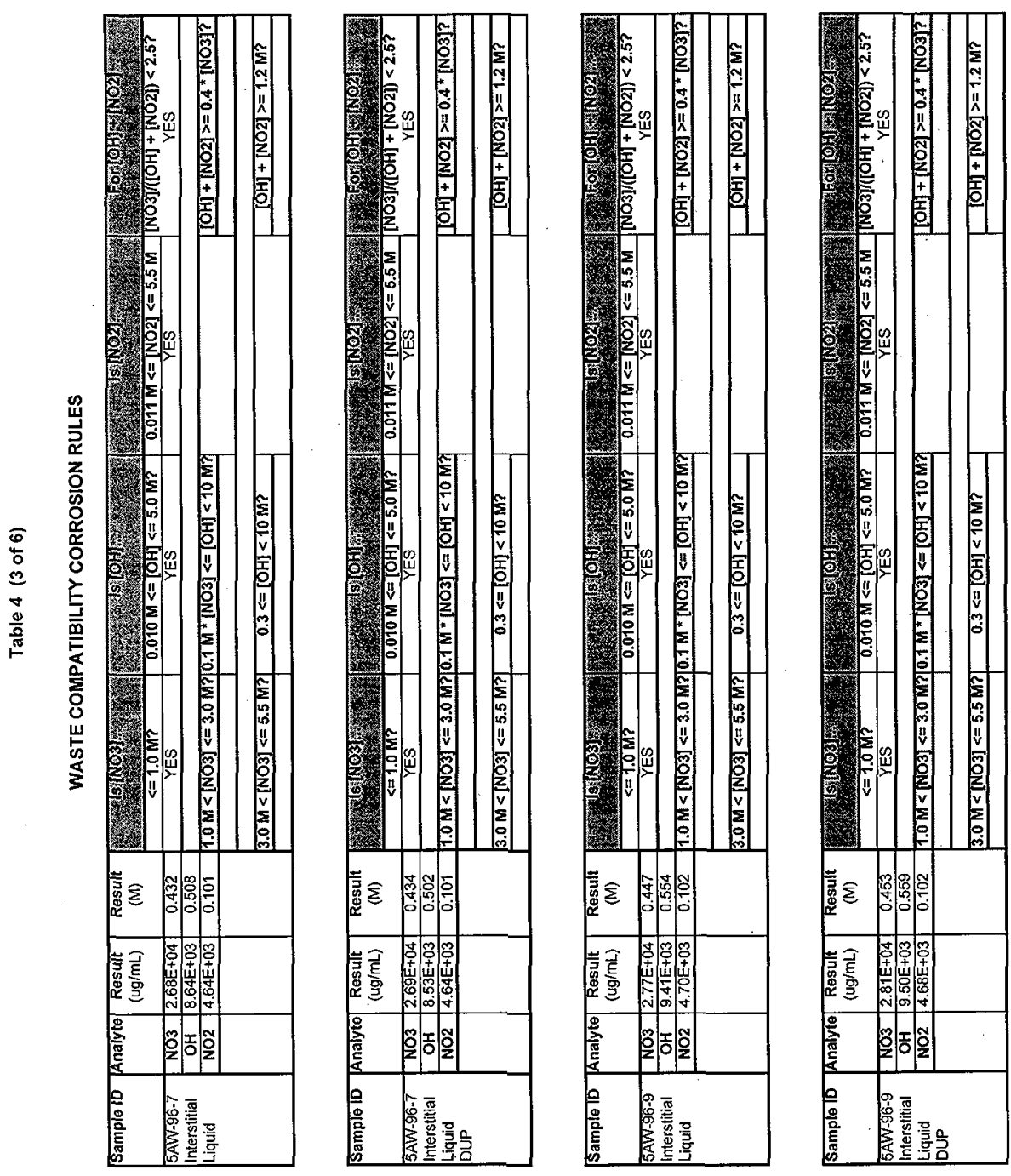
HNF-SD-WM-DP-214, REV. 0
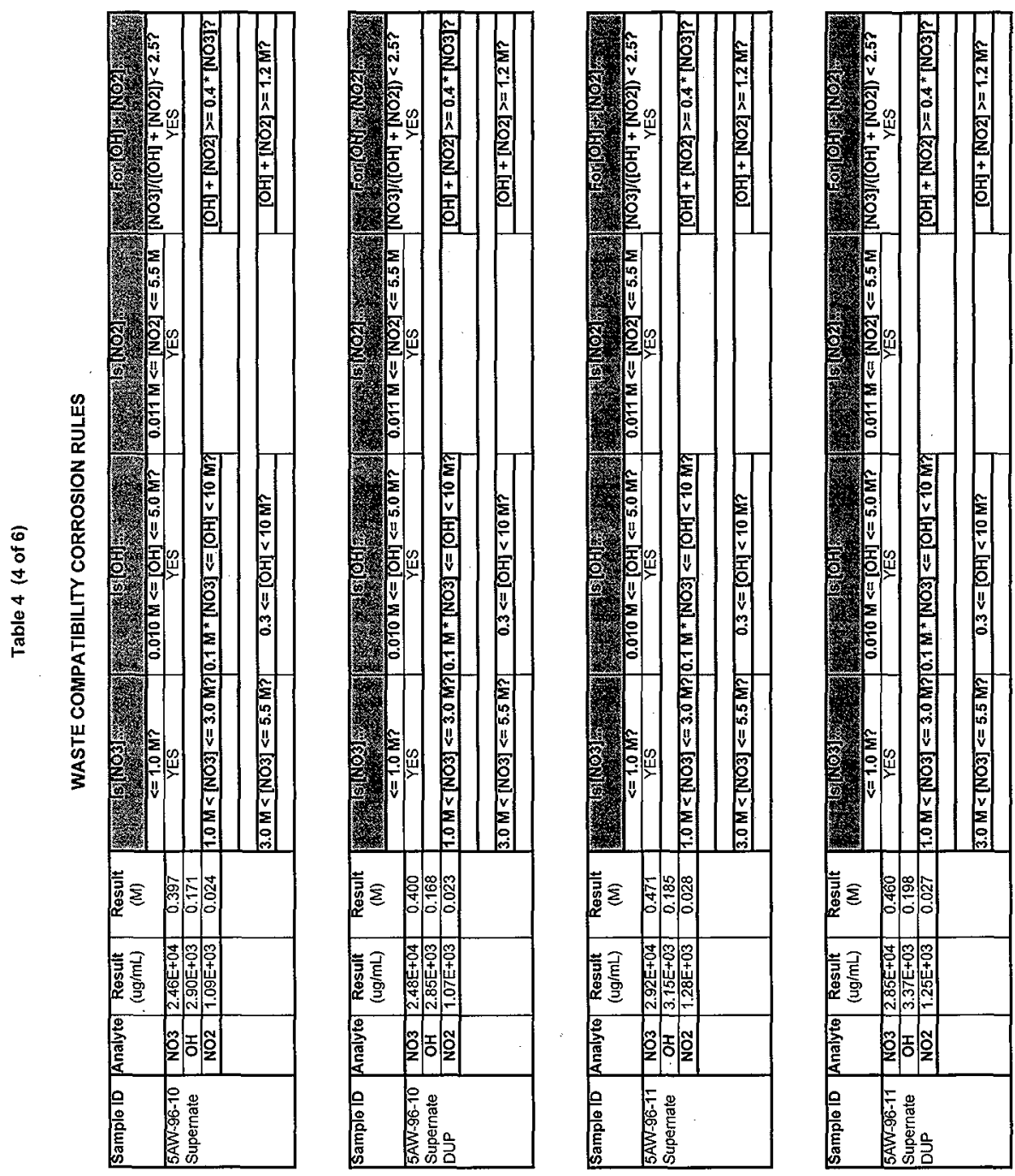
HNF-SD-WM-DP-214, REV. 0
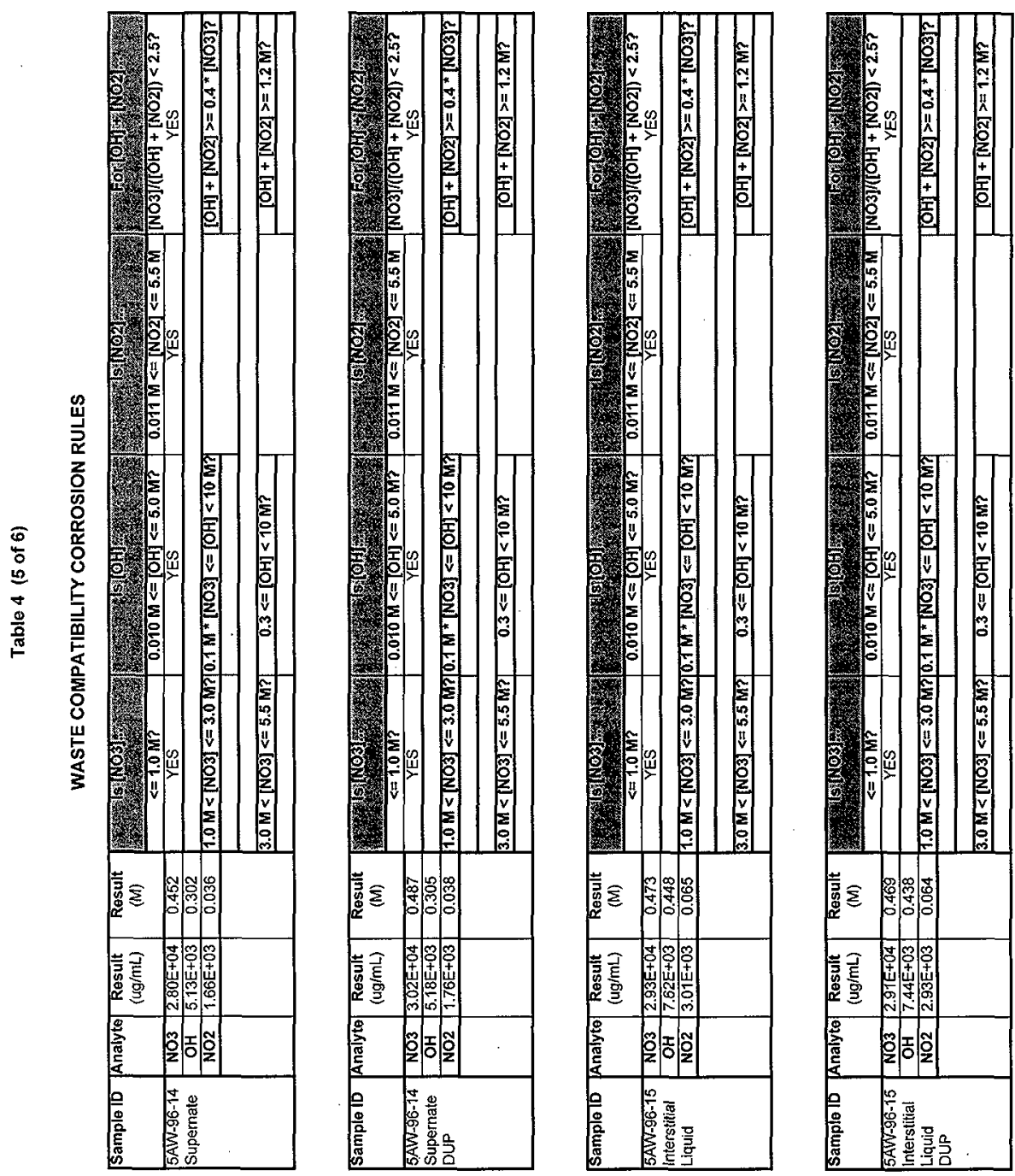
Table 4 ( 6 of 6 )

WASTE COMPATIBILITY CORROSION RULES

\begin{tabular}{|c|c|c|c|c|c|c|c|}
\hline \multirow[t]{2}{*}{ Sample IÍ } & Analyte & $\begin{array}{l}\text { Result } \\
\text { (ug/mL) }\end{array}$ & $\begin{array}{l}\text { Result } \\
\text { (M) }\end{array}$ & 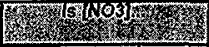 & 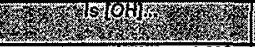 & Hox & Hor \\
\hline & & & & $<=1.0 \mathrm{M} ?$ & $0.010 \mathrm{M}<=[\mathrm{OH}]<=5.0 \mathrm{M} ?$ & $0.011 \mathrm{M} \ll[\mathrm{NO} 2] \ll 5.5 \mathrm{M}$ & {$[\mathrm{NO} 3] /([\mathrm{OH}]+[\mathrm{NO} 2])<2.5 ?$} \\
\hline \multirow{4}{*}{$\begin{array}{l}\text { 5AW-96-17 } \\
\text { Interstitial } \\
\text { Liquid }\end{array}$} & $\mathrm{NO3}$ & $2.70 E+04$ & 0.435 & YES & YES & Y'ES & YES \\
\hline & $\mathrm{OH}$ & $9.04 E+03$ & 0.532 & \multirow{2}{*}{\multicolumn{2}{|c|}{$1.0 \mathrm{M}<[\mathrm{NO}]<3.0 \mathrm{Mn}] 0.1 \mathrm{M} \mathrm{M}^{*}[\mathrm{NO} 3]<[\mathrm{OH}]<10 \mathrm{MP}$}} & & \multirow[b]{2}{*}[\mathrm{OH}]{$+[\mathrm{NO} 2]>=0.4^{*}[\mathrm{NO} 3] ?$} \\
\hline & NO2 & $4.71 \mathrm{E}+03$ & 0.102 & & & & \\
\hline & & & & $3.0 \mathrm{M}<[\mathrm{NO} 3]<=5.5 \mathrm{M} ?$ & $0.3<=[O H]<10 \mathrm{M} ?$ & & \multirow{2}{*}[\mathrm{OH}]{$+[\mathrm{NO} 2]>=1.2 \mathrm{M} ?$} \\
\hline & & & & & & & \\
\hline
\end{tabular}

\begin{tabular}{|c|c|c|c|c|c|c|c|}
\hline \multirow[t]{2}{*}{ Sample ID } & \multirow[t]{2}{*}{ |Analyto } & \multirow[t]{2}{*}{$\begin{array}{l}\text { Result } \\
\text { (ug/mL) }\end{array}$} & \multirow[t]{2}{*}{$\begin{array}{l}\text { Rosuit } \\
\text { (M) }\end{array}$} & 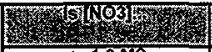 & 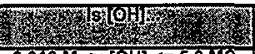 & HFy & 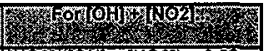 \\
\hline & & & & $<=1.0 \mathrm{M} ?$ & $0.010 \mathrm{M} \Leftrightarrow[\mathrm{OH}]<=5.0 \mathrm{~m} ?$ & $0.011 \mathrm{M}<=[\mathrm{NO} 2]<=5.5 \mathrm{M}$ & $[\mathrm{NO} 3] /(\mathrm{OH}]+[\mathrm{NO} 2])<2.57$ \\
\hline \multirow{4}{*}{$\begin{array}{l}\text { 5AW-96-17 } \\
\text { Interstitial } \\
\text { Liquid } \\
\text { DUP }\end{array}$} & $\mathrm{NO}^{\circ}$ & $2.69 E+04$ & 0.434 & YES & YES & YES & YES \\
\hline & $\frac{\mathrm{OH}}{\mathrm{NO} 2}$ & \begin{tabular}{|l}
$9.39 E+03$ \\
$4.73 E+03$ \\
\end{tabular} & 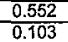 & $\mid 1.0 \mathrm{M}<[\mathrm{NO}]<=3.0 \mathrm{M} 3$ & $0.1 M^{*}\left[\mathrm{NO}^{2}\right]<[\mathrm{OH}]<10 \mathrm{M} 3$ & & {$[\mathrm{OH}]+[\mathrm{NO} 2]>=0.4 *[\mathrm{NO} 3]$ ? } \\
\hline & & & & & & & \\
\hline & & & & $3.0 \mathrm{M}<[\mathrm{NO} 3]<=5.5 \mathrm{M} ?$ & $0.3<=[\mathrm{OH}]<10 \mathrm{M} ?$ & & {$[\mathrm{OH}]+[\mathrm{NO} 2]>=1.2 \mathrm{MP}$} \\
\hline
\end{tabular}

\begin{tabular}{|c|c|c|c|c|c|c|}
\hline \multirow[t]{2}{*}{ SampleID } & \multirow[t]{2}{*}{ Analyto } & \multirow[t]{2}{*}{$\begin{array}{l}\text { Result } \\
\text { (ug/mL) }\end{array}$} & \multirow[t]{2}{*}{$\begin{array}{c}\text { Resultt } \\
\text { (M) }\end{array}$} & 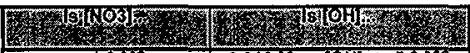 & 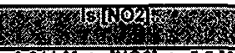 & Whorow \\
\hline & & & & 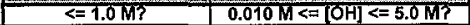 & $0.011 \mathrm{M}<=[\mathrm{NO} 2]<=5.5 \mathrm{M}$ & {$[\mathrm{NO}] /([\mathrm{OH}]+[\mathrm{NO} 2])<2.5 ?$} \\
\hline \multirow{4}{*}{$\begin{array}{l}\text { 5AW-96-20 } \\
\text { Interstitial } \\
\text { Liquid }\end{array}$} & NO3 & $3.03 E+04$ & 0.489 & YES & YES & DES \\
\hline & $\frac{\mathrm{OH}}{\mathrm{NO} 2}$ & $\frac{8.66 E+03}{3.94 E+03}$ & 0.509 & $\left.1.0 \mathrm{M}<[\mathrm{NO} 3]<=3.0 \mathrm{M}] 0.1 \mathrm{M}^{*}[\mathrm{NO} 3]<\mathrm{OH}\right]<10 \mathrm{M}$ & & \multirow{2}{*}[\mathrm{OH}]{$+[\mathrm{NO} 2]>0.4 *[\mathrm{NO} 3]\}$} \\
\hline & & & & & & \\
\hline & & & & $3.0 \mathrm{M}<[\mathrm{NO} 3]<=5.5 \mathrm{MP} \quad 0.3<=[\mathrm{OH}]<10 \mathrm{MP}$ & & {$[\mathrm{OH}]+[\mathrm{NO} 2]>=1.2 \mathrm{M} ?$} \\
\hline
\end{tabular}

\begin{tabular}{|c|c|c|c|c|c|c|c|}
\hline \multirow[t]{2}{*}{ Sample ID } & Analyto & $\begin{array}{l}\text { Result } \\
\text { (ug/mL) }\end{array}$ & $\begin{array}{l}\text { Rosult } \\
\text { (M) }\end{array}$ & 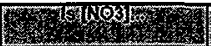 & 3. & 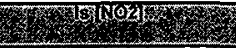 & W \\
\hline & & & & $s=1.0 \mathrm{~m} ?$ & $0.010 \mathrm{M}<=[\mathrm{OH}]<=5.0 \mathrm{M} ?$ & $0.017 \mathrm{~K} \Leftrightarrow[\mathrm{NO} 2]<=5.5 \mathrm{M}$ & \multirow{2}{*}{$\begin{array}{c}{[N O 3]([O H]+[N O 2])<2.5 ?} \\
Y E S\end{array}$} \\
\hline \multirow{4}{*}{$\begin{array}{l}5 A W-96-20 \\
\text { interstitial } \\
\text { Liquid } \\
\text { DUP }\end{array}$} & NO3 & $3.03 E+04$ & 0.489 & YES & YES & YES & \\
\hline & $\mathrm{OH}$ & $\begin{array}{l}9.07 E+03 \\
3.92 E+03\end{array}$ & $\begin{array}{l}0.534 \\
0.085\end{array}$ & \multirow{2}{*}{\multicolumn{2}{|c|}{$1.0 \mathrm{~m}<[\mathrm{NO} 3]<=3.0 \mathrm{M} 3[0.7 \mathrm{~m}:[\mathrm{NO}]<=[\mathrm{OH}]<10 \mathrm{Mr}$}} & & \multirow{2}{*}[\mathrm{OH}]{$+[\mathrm{NO} 2]>=0.4 *[\mathrm{NO} 3]_{3}^{3}$} \\
\hline & & & & & & & \\
\hline & & & & \multicolumn{2}{|c|}{$3.0 \mathrm{M}<[\mathrm{NO} 3]<=5.5 \mathrm{M} ? \quad 0.3<=[\mathrm{OH}]<10 \mathrm{M} ?$} & & {$[\mathrm{OH}]+[\mathrm{NO} 2]>=1.2 \mathrm{M} ?$} \\
\hline
\end{tabular}


HNF-SD-WM-DP-214, REV. 0

Chain of Custody Forms

63 
HNF-SD-WM-DP-214, REV. 0 
E $768-27-96 H A I N-O F-C U S T O D Y$ RECORD FOR AUGER/GRAB SAMPLING

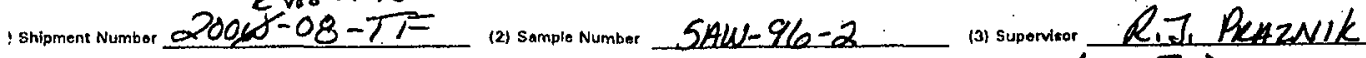
Tank AN 105

(5) Risor $\quad 10 \mathrm{~A}$

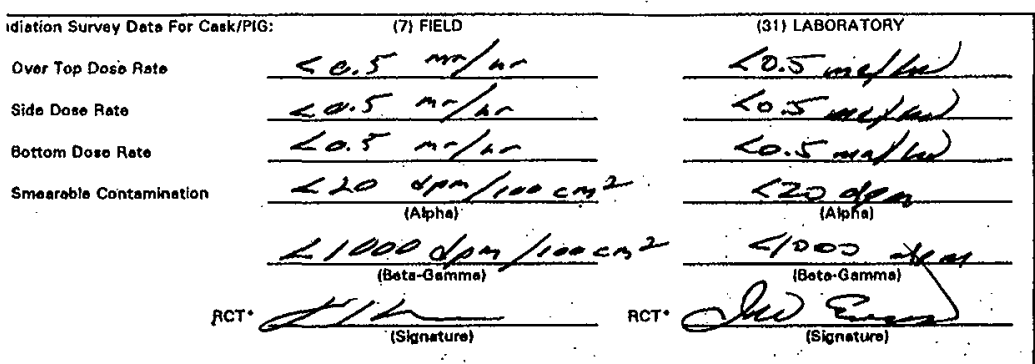

(B) Shipmont Doscriptlon

A. Work Packago Number

B. Caak/Plo Soal Number

C. Dato and Time Sample.

Pomoved from Tank

D. Expocted Liquid Contemt

E. Expooted Solld Content

f. Dose Rato Through Drill String G. Expoctod Somplo Longth (Augen)
$E S-96-00615 \%$ 10548

$\frac{\frac{8.20-96 / 1048 \mathrm{hm}}{1008}}{\frac{0.5}{125 \mathrm{ml}}}$

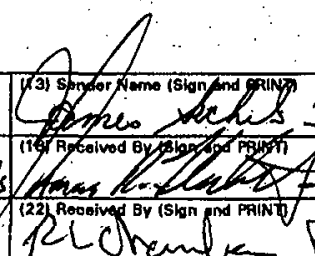

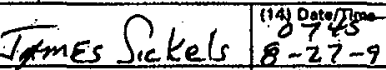
$222-5$ Sickel

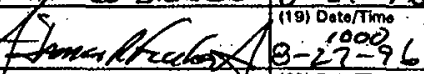
in R०ol (26) Rocoivod BY (Sion and PRINT) Pi Chatber

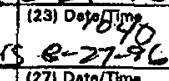

(15) Sender Commente

(20) Recaiver Commonte

(16) Sool kntact Upon Reloato

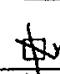

\$yor

$\square$ No

(29) Sonl hitact Upon Rocoipt?

Shipmont No.

(30) Sod Date Consiutent with this Record?

ITAIBUTION: Whito - Office of Samplo Managoment

Yollow - Reciplent of Semplo

पro:

$\square$ No

Soal No.

$\square$ No

(24) Roediver comments

(20) Racaiver Commante 
(98/10) $82 \varepsilon-1009-58$

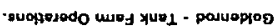

Dugfwes woo 1 ozanm - yu!d

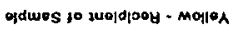

zuoutoBoudm ogdwes to os! ב

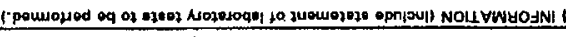

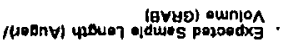

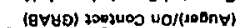

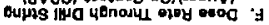
zuozuos P||OS porododx 3 HHojuos pinbly popoodxa '0 90 8001

च्य $1501 / 96 \cdot 02 \cdot 6$ bतsol

$0 / 51900-96-53$
Yuves cuon ponomisy

भdurs owll puv 0200 '0

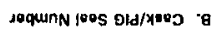

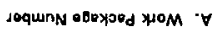

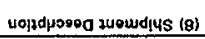

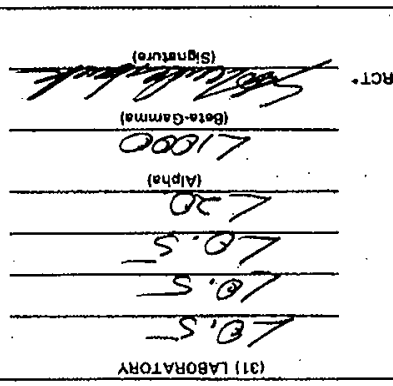

AHOLYHOQVI IIE

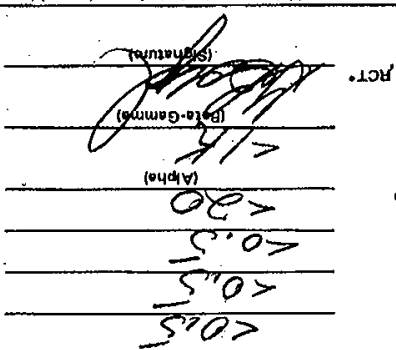

ำำ 《

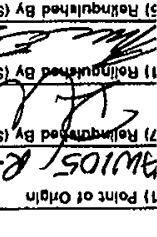

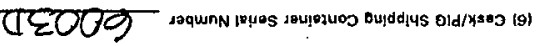

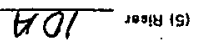

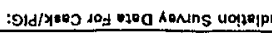
$\varepsilon-96-M H S$ soqunN श口wes (2) $=1-\$ 0-700 Z^{2}$ samnn zurudius: 


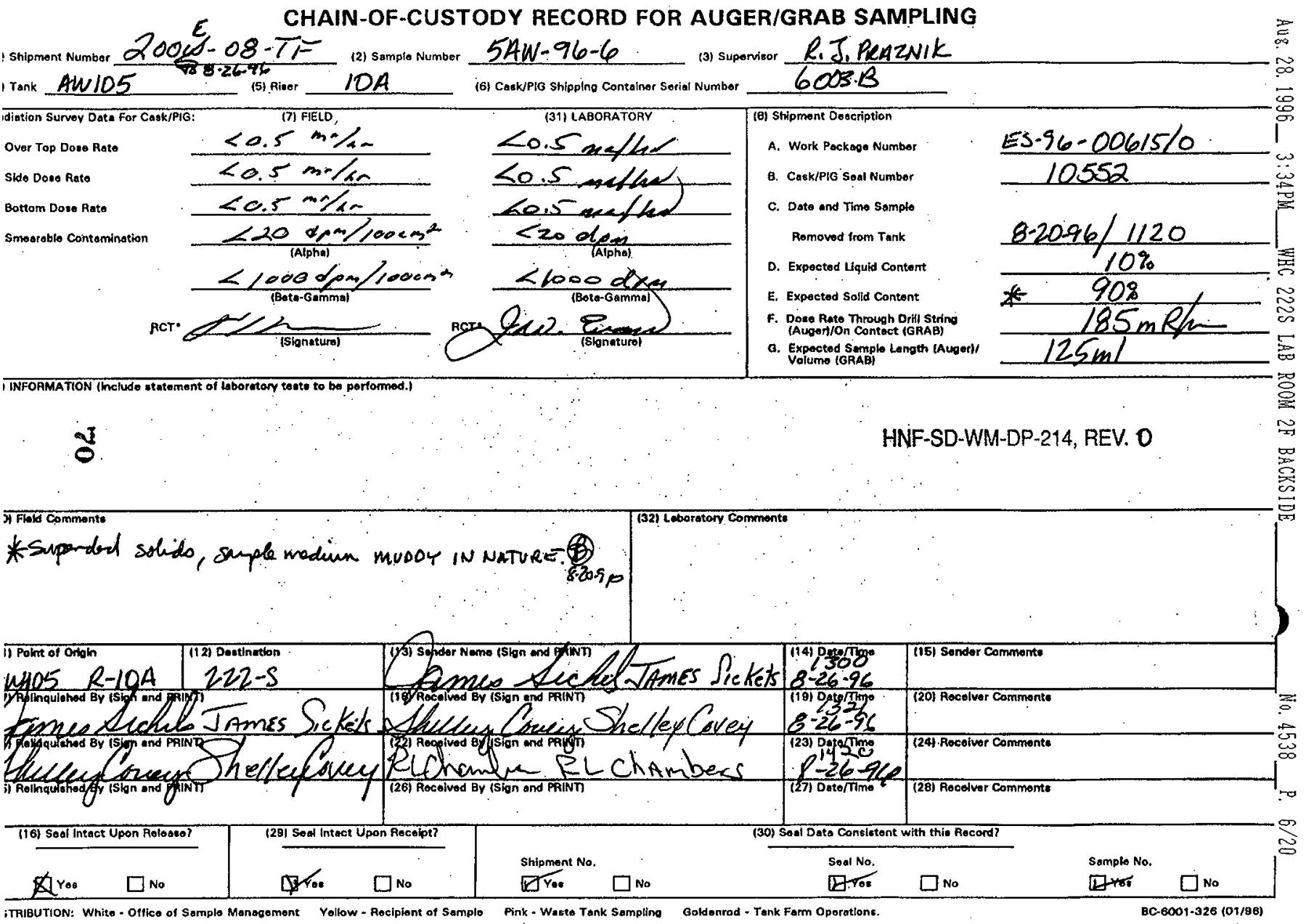


CHAIN-OF-CUSTODY RECORD FOR AUGER/GRAB SAMPLING

$200 E-08 \div T$ (2) sample Numbar $54 W-96-7$ (3) suporisor R,J, ReAzNik

I Tank $42 \mathrm{ClO}$ (5) Aiser 104 (16) Cask/PIG Shipping Container Serial Number $.6003 E$ Adiation Survey Data For Cask/PIG:
Over Top Dose Rate
Sido Doso Rote
Bottom Dose Roto
Smeorable Contaminatlon

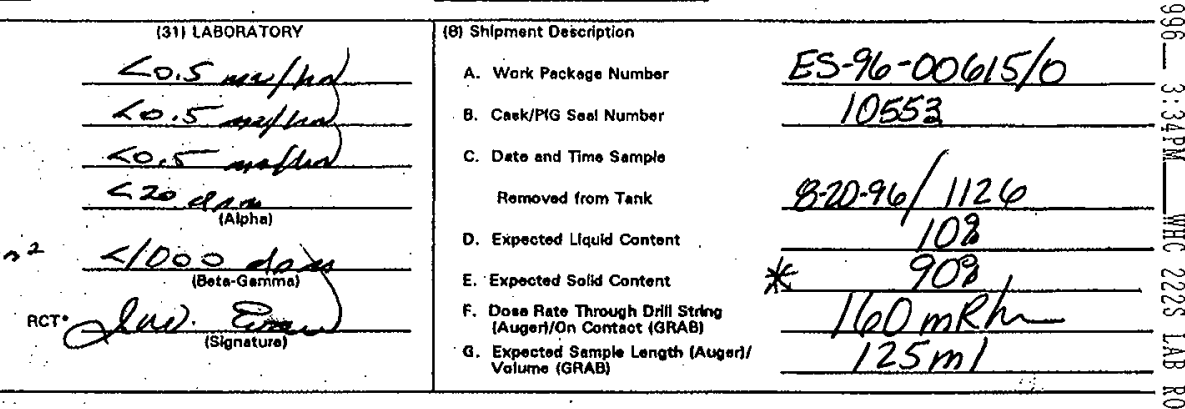

IINFORMATION (Include atatoment of leboratory teste to be performed.)

HNF-SD-WM-DP-214, REV. 0

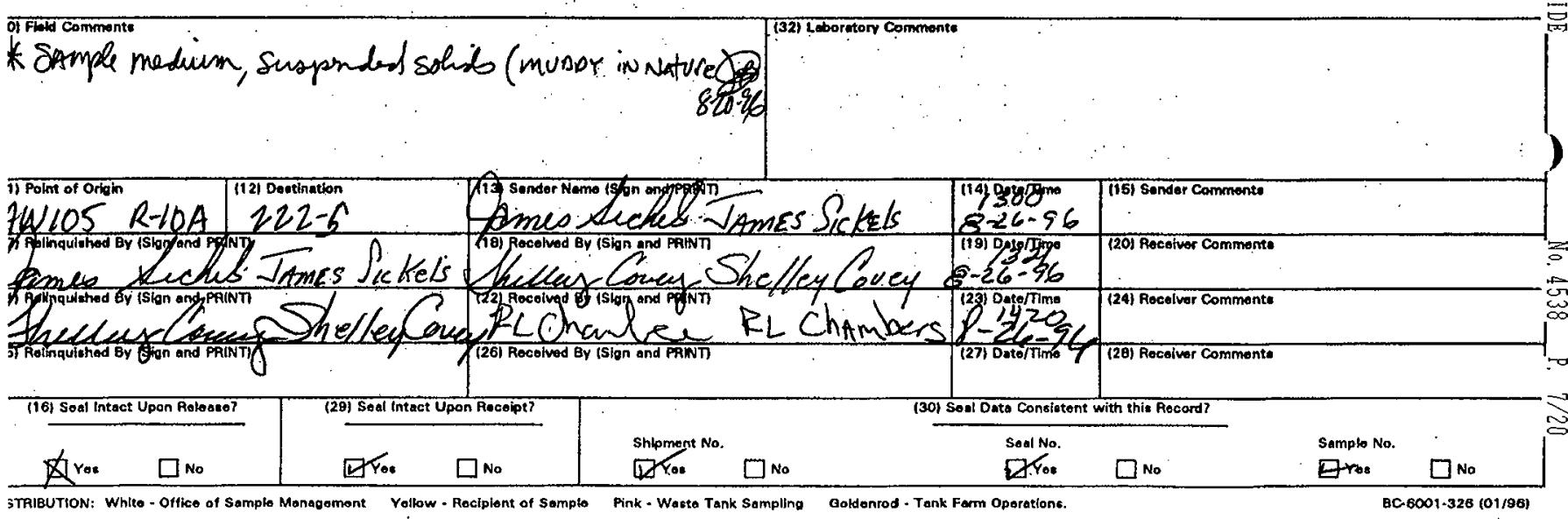


CHAIN-OF-CUSTODY RECORD FOR AUGER/GRAB SAMPLING

: Shipment Number $200 E-08=\pi$

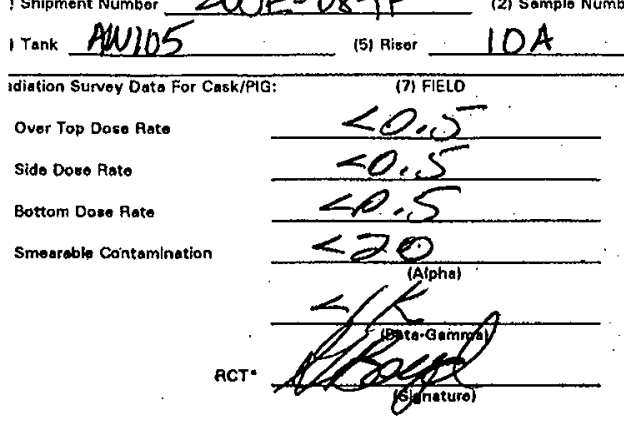

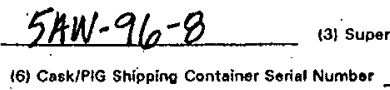

(6) Cask/PIG Shippling Container Serial Number

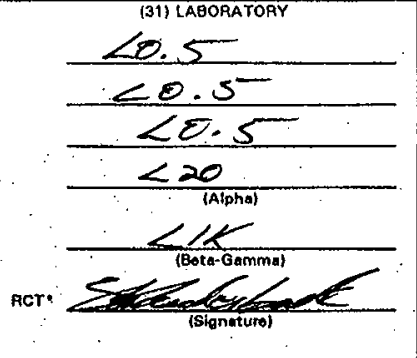

(8) Shipment

A. Work Packago Number

B. Cask/PIG Soal Number

c. Date and Timo Sample Removed Irom Tank

D. Expected Liquid Contont

E. Expected Solid Contont

F. Dowo Rato. Throuph Drill String

(Auger)/On Conteot (GRAB)

o. Expoctod Samplo Longth (Avoor)]

INFORMATION (Include etatement of laboretory toste to be porformed.)

$\sqrt{n}$

HNF-SD-WM-DP-214, REV. 0

o) Fibld Commente

KSample madinim muddy in nature. 8-20.96

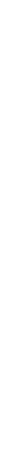

STRIBUTION: Whito - Olfice of Samplo Management Yoilow - Rociplent of Samplo Pink - Waste Tank Sampling Goldenrod - Tank Farm Operatione.

BC.6001.326 (01/96) 


\section{CHAIN-OF-CUSTODY RECORD FOR AUGER/GRAB SAMPLING}

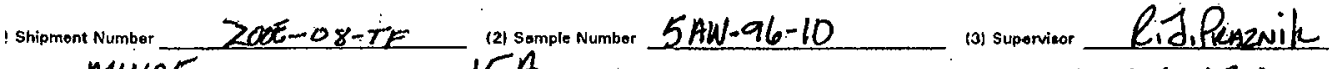

i Tonk $4110 \overline{5}$

diation Survoy Data For Cask/PIG:

Ovor Top Doso Rato

Side Dore Rete

Bottom Doso Pate

Smearsblo Contaminatior

(5) Risor $15 \mathrm{~A}$

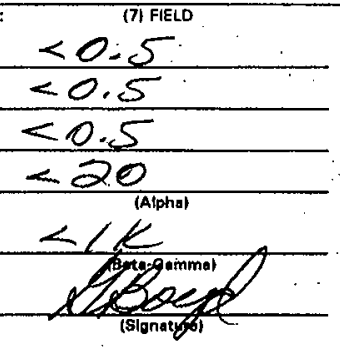

(6) CaskJPIG Shipping Conteiner Sorial Number $6003 A<B, 0$

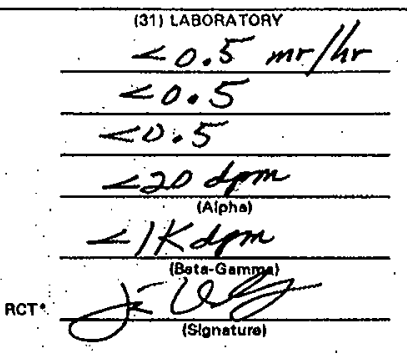

(8) Shipment Description

A. Work Packago Numbor

B. Cakk/PIO Seal Number

c. Date and Timo Sample

Removed irom rink

D. Expectod Liquid Contont

E. Expectod Solld Contont

F. Dowo Aats-Through Drill Strino

o. Expoctod Samphe Longth (Augerd! Volume (GRAB)

\section{Es-96-00615/0} 10556

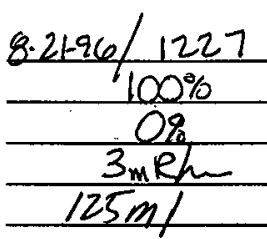

(15) Sonder Commente

(16) Soed intact Upon Reloaso?
You $\square$ No

(29) Soal intact Upon Recolpt?

Shipment No.

DPros

$\square$ No

130) Seal Date Consistont with this Rocond?

(23) Dato/Throe

$8-22-96$

ITRIBUTION: White - Otsles of Sampio Managoment Vollow - Reciplont of Samplo

Pink - Wasto Tank Sampling

DFo $\square$ No 
CHAIN-OF-CUSTODY RECORD FOR AUGER/GRAB SAMPLING

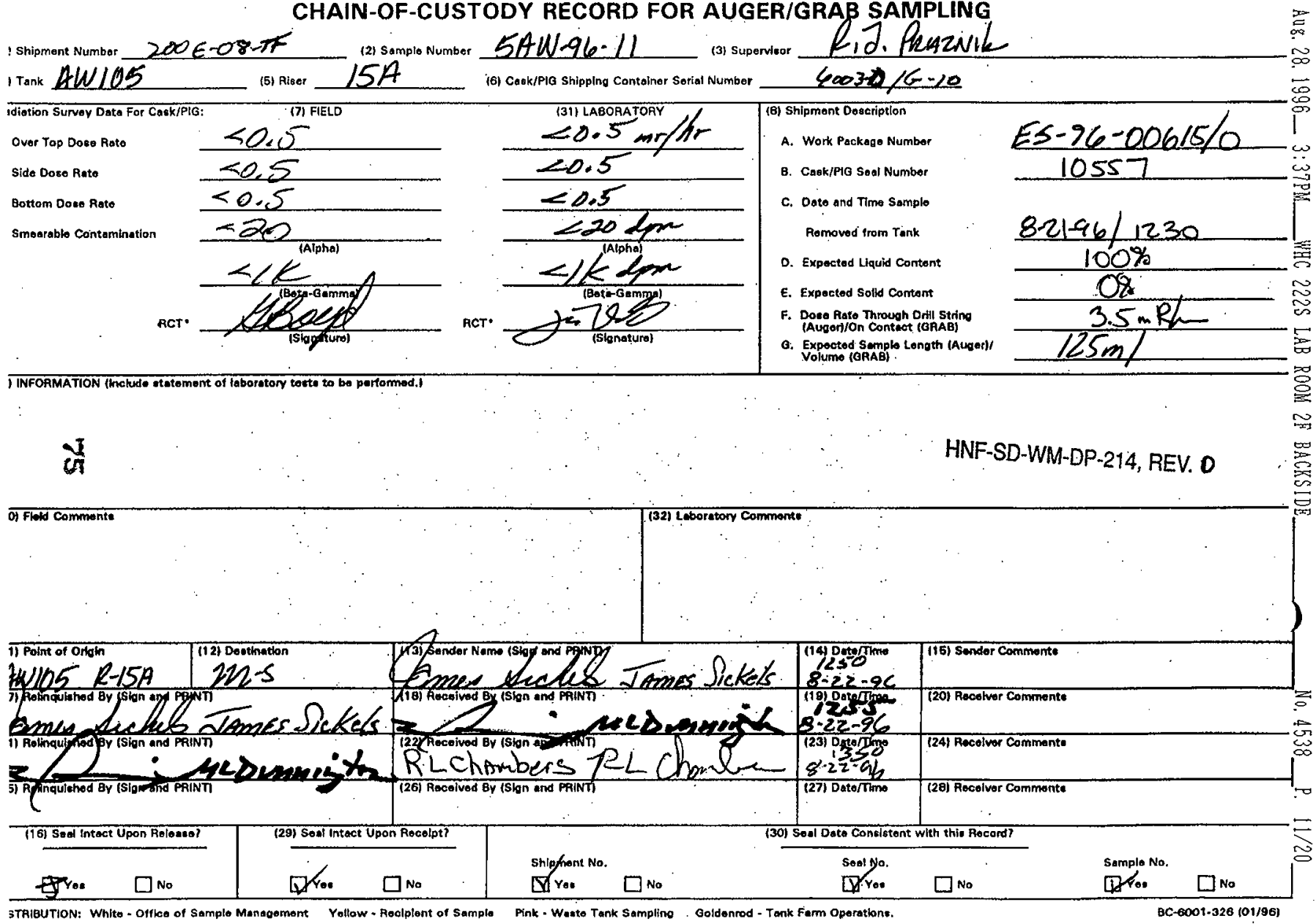


CHAIN-OF-CUSTODY RECORD FOR AUGER/GRAB SAMPLING

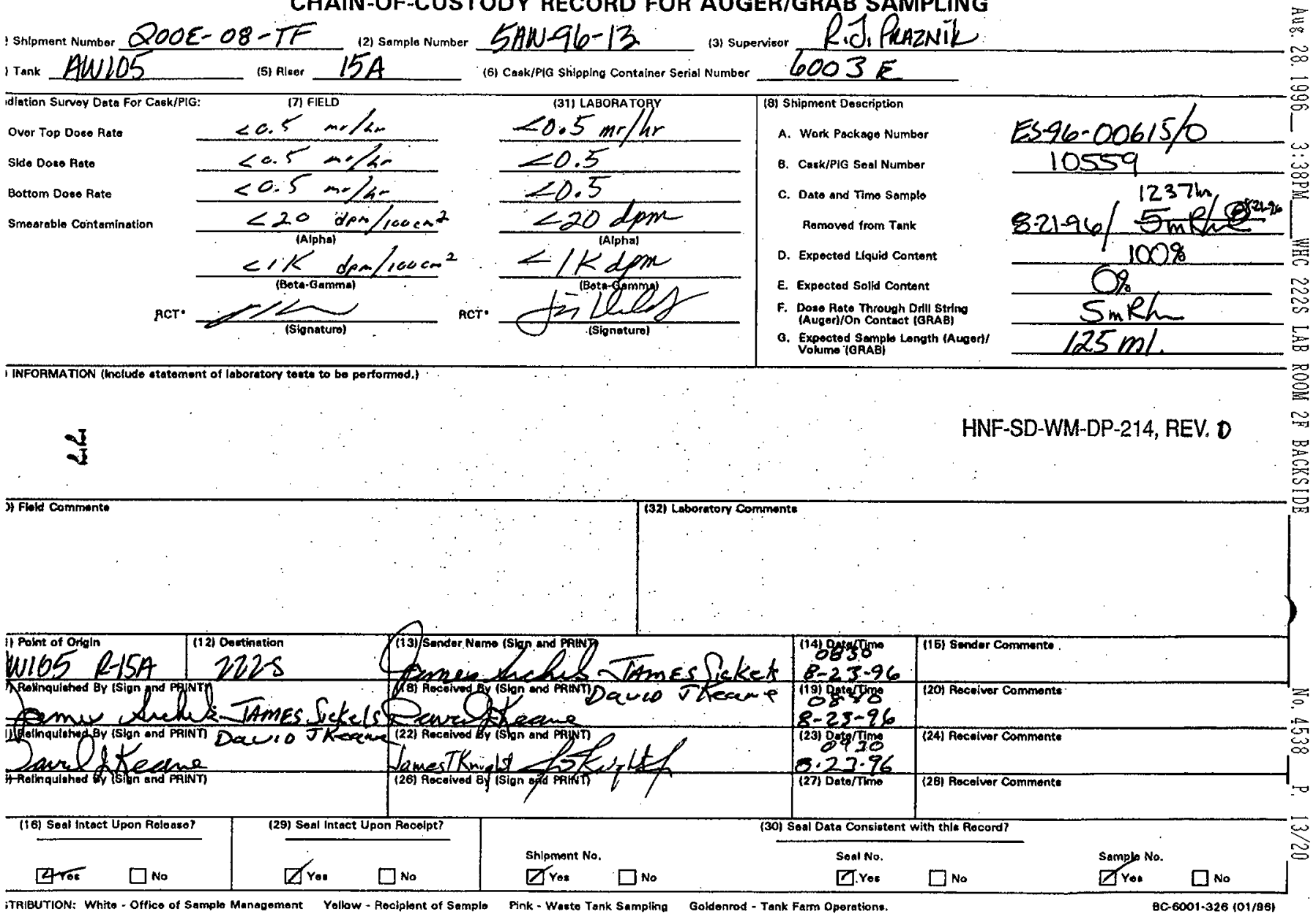


CHAIN-OF-CUSTODY RECORD FOR AUGER/GRAB SAMPLING

: Shipment Numbor $200 E-08-\pi F$ (2) sample number 5 AUW-96-15 (3) suporitor R.J. PrAZZNik 1 Tank AN105 (5) Rirer $15 A$ (6) Caak/PIG shipping container sorial Number _..

idiation Survey Dota For CakkiPIG:
Over Top Doso Rate
Sido Dovo Rato
Bottom Dose Aoto

(8) Shipment Description

A. Work Package Number

B. Cask/PIG Soal Number

c. Date and Time Samplo Romovad trom Tank

D. Expected Llauid Content

E. Expected solid Content

F. Dose Rate Throush DHt String

(Augon/On Contact (GRAB)

C. Expected Sample Length (Auger)/ volumo (BAAB)

Es-96-00615\% 10561 $8-21.96 / \frac{1246 \mathrm{~h}}{10}$ $\frac{10 \%}{90 \%}$

INFORMATION (Include itatoment of laboratory tente.to be porformed.)

HNF-SD-WM-DP-214, REV. D

ปั

0) Fiold Commonte

* Suspended Solids, mudder medimin 8.2196

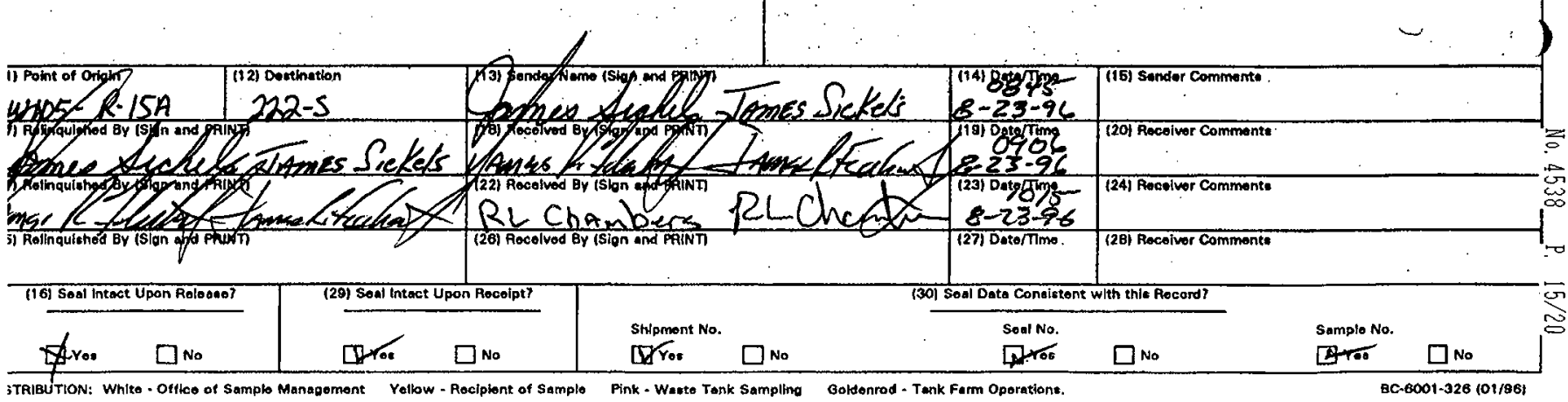


CHAIN-OF-CUSTODY RECORD FOR AUGER/GRAB SAMPLING

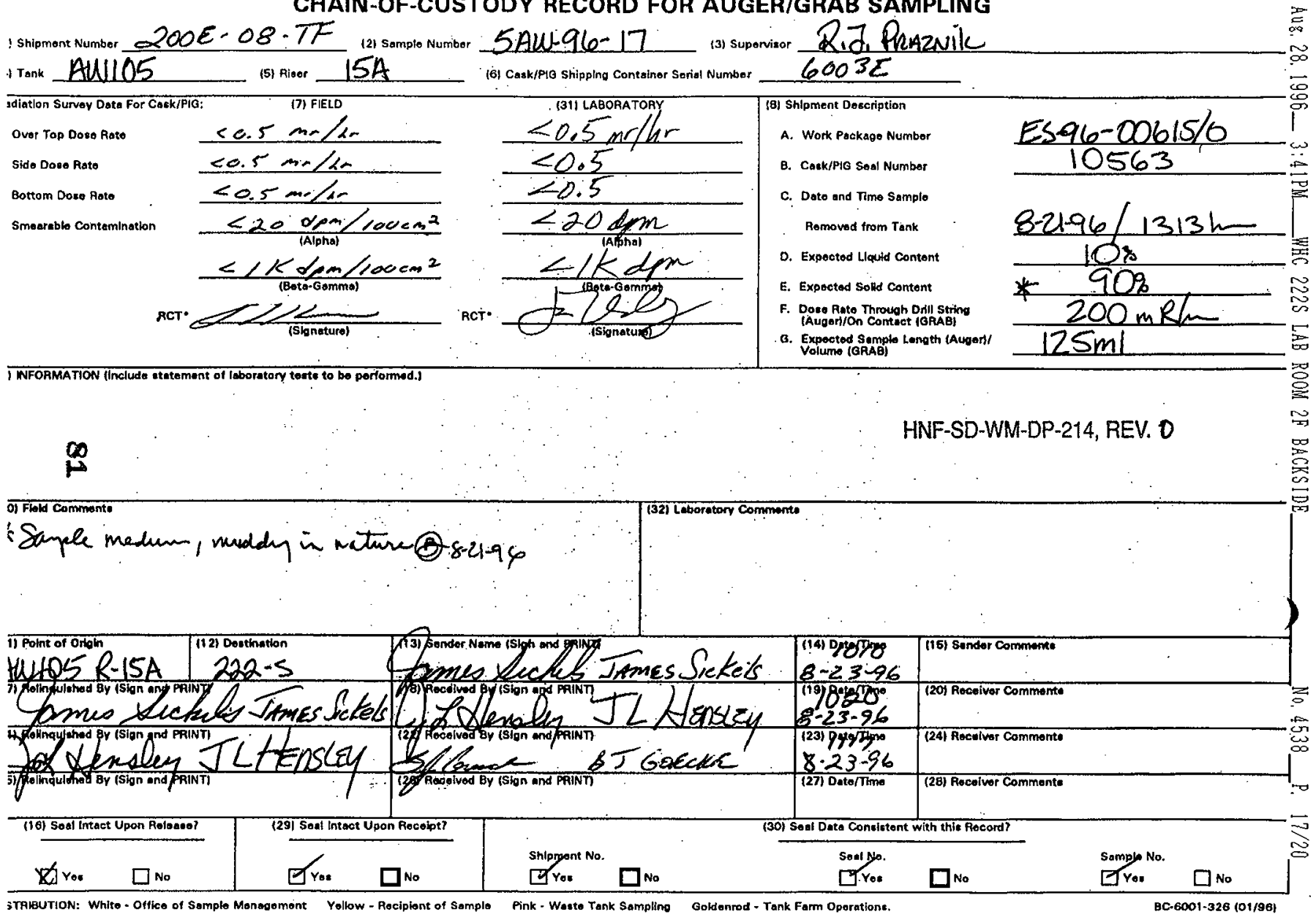


Aug. 28. 1996_ 3:429M__WHC 222S LAB ROOM 2F BACKSIDE

No. 4538 P. $19 / 20$

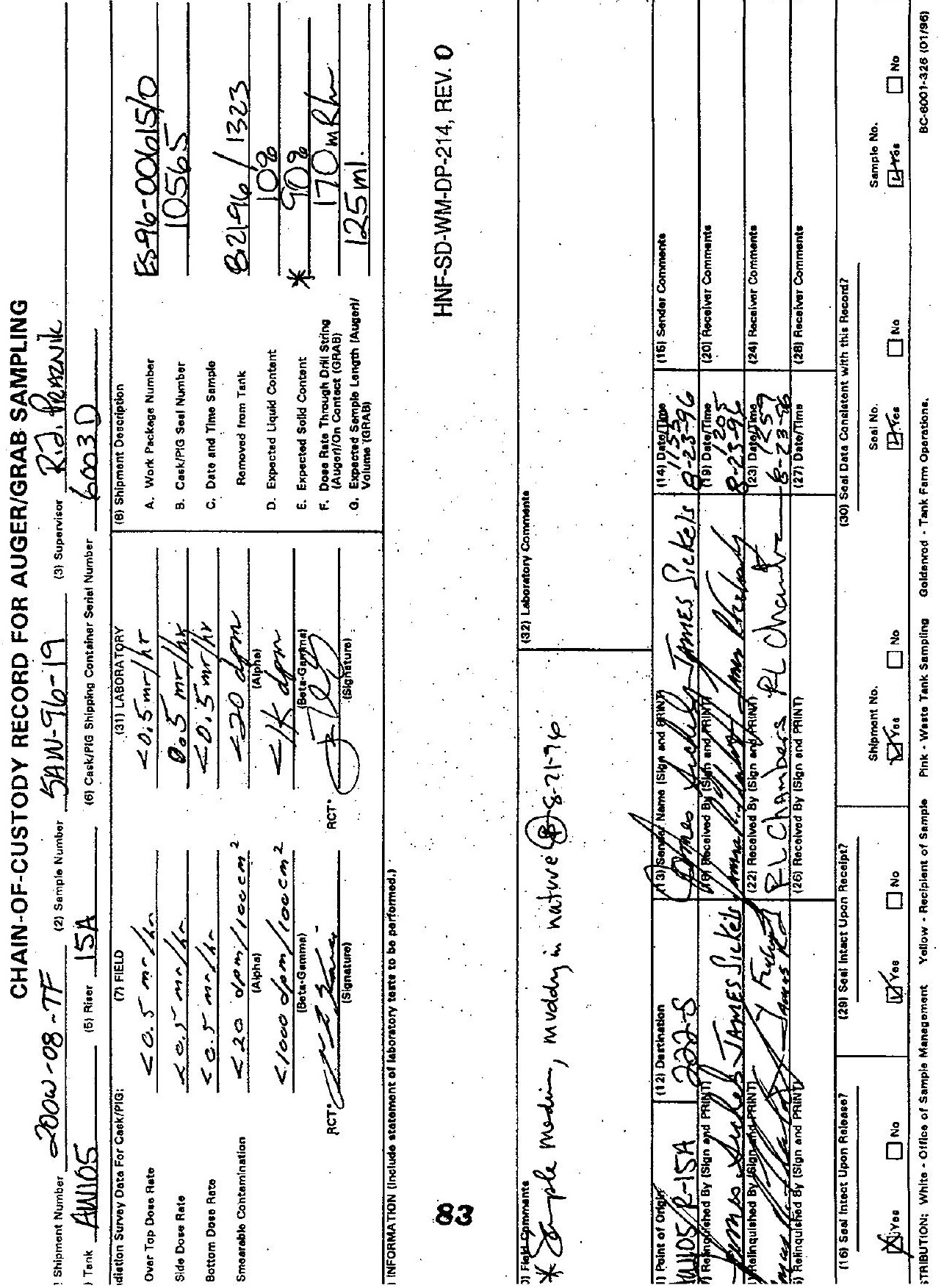




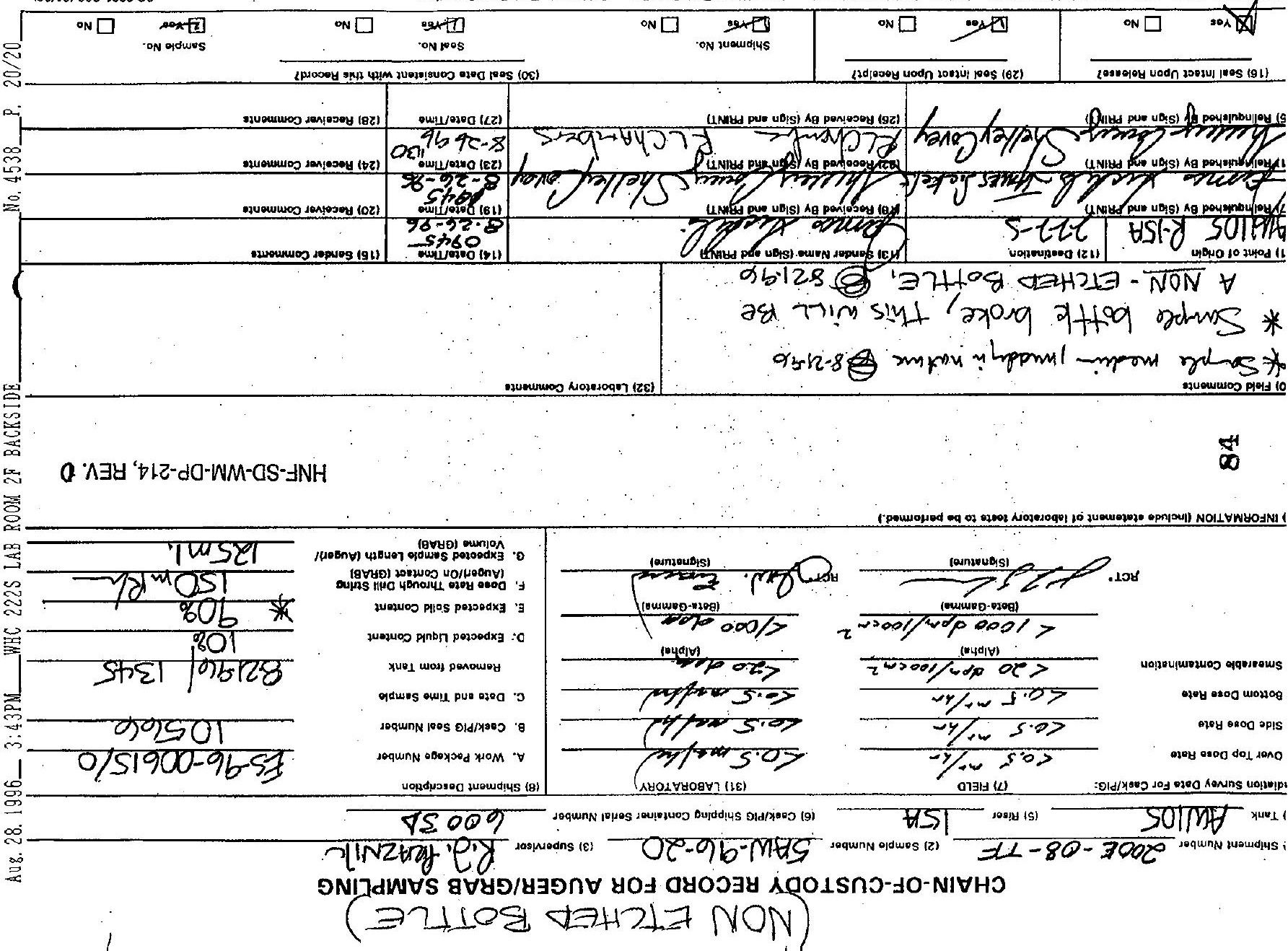


HNF-SD-WM-DP-214, REV. 0

Breakdown Analysis 
HNF-SD-WM-DP-214, REV. 0

THIS PAGE WAS INTENTIOANLLY LEFT BLANK 
Project/Tank: Aw-10S GBAB

Date:

TSAP Number:

Logbook Number:

PC Name:

$\overline{w H C-S D-W M-T S A P-037}$ Rev. $2 b$

\section{(Date of Breakdown)}

Ruth Esch

Telephone: $373-4314$ Fax: $372-1878$

\section{A. Sample Receipt and Storage}

$\mathrm{N} / \mathrm{A}$ 1. During Pig breakdown dose rate exceeded $7 \mathrm{R} / \mathrm{hr}$ or $25 \mathrm{rad} / \mathrm{hr}$. Make preparations to have the sample transferred to the $11 \mathrm{~A}$ hot cell area.

PaE 2. Place pig in short term storage. Document the location of the pig/sample.

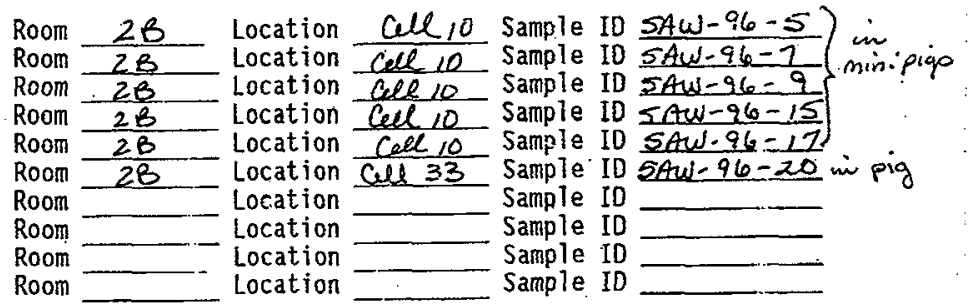

3. Make 2 copies of the chain of custody. (Hot Cell Workplan, and Logbook).

4. Attach chain of custody to the Grab Sample workp?an.

RaE 5. Prepare and attach copies of the following worksheets and associated paper work if required.
a. Grab Sample Information Worksheet
b. Breakdown - Grab Sample Worksheet
c. Subsample - Grab Sample Worksheet
d. Subsamp Je: Liquid Worksheet
e. Bulk Density: Liquid Worksheet
f. Composite Worksheet

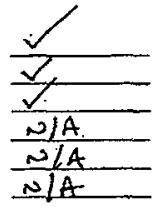

NiA

6. Transfer Grab Sample Workplan workplan to Pre-Extrusion Preparation file and contact personnel in charge of load-in and extrusions. 


\section{B. Pre-Loadin Preparation}

N/A 1. Prepare folder to consolidate papers for breakdown.

N/A

2. Update the SAMPLER/AUGER LOG-IN/LOAD-IN SHEET.

$N / A$

3. Prepare labels for pig sample if required. Labels for the pig-may be loaded in through the drop chute.

C. Pig Receipt and Preparation.

Q0)! 1. Receive pig from sample custodian and transfer to the $11 \mathrm{~A}$ hot cell area.

ilec 2. Perform the following:
a. Pig load-in (L0-161-172)
b. Sample load-out (if required)
c. Jar load-in (if requitred).

NA 3. Record date pig was loaded in on SAMPLER/AUGER LOAD-IN SHEET.

NA 4. Transfer hot cell work plan to Extrusion file.

D. Breakdown

UA. 1. Place chain of custody in the laboratory notebook.

2. Update table of contents in Taboratory notebook.

A) 1 3. Contact $P C$ and inform of sample breakdown. (Contact the $P C$ the day before breakdown)

est 4. Contact Building Shift Manager for Waste Tank Volume information (Tank 101).
a. Shift manager contacted
b. Date/Time

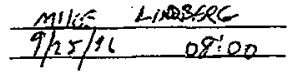

ROH 5. Record hot cell temperature and humidity a. Temperature $76.4 \%$ Humidity $20 \%$

ROL 6. Balance check with 20 and 500 gram weights:

a. $\quad 20 \mathrm{gm}=20.02 \mathrm{gms} 500 \mathrm{gm}=500.36 \mathrm{gms}$

RDV 7. Obtain the total weight of the grab sample; record on the appropriate Grab Sample Information Worksheet. 
Record appearance information on the appropriate Grab Sample
Information Worksheet.

A 9. If photography is requested by project coordinator or chemist, place the color chart next to sample.

NA 10. Take two photos of the grab samples if required by the chemist/project coordinator (Hasselblad camera):

a. Inside hot cell photography settings:
(1) F-stop is set to 4 .
(2) Aperature is set between 11 and 8 .
(3) Wind to next available film.
(4) Remove film plate.
(5) Focus camera
(6) Shoot picture

b. Record the following information:
(1) Frame \#
(2) Time taken:

WA 11. Complete Film Frame Record sheet for each picture taken.

E. Laboratory Logbook:

1. Update laboratory notebook with breakdown information.

2. Update Jar notebook for jars/vials loaded in.

\section{F. Subsampling}

- Poli 1. Assign a vial number to each subsample on the Subsampling Worksheets.

2. Breakdown and Subsample according to attached worksheets.

3. After subsampling, complete calculations on each Grab Sample Information Worksheet.

4. Record depleted jar numbers below:

SAw-96-7 SAw-96-5 SAw-96-20

SAu-96-9 S4w-96.17

QDI 5. Record jar/vials for load out on HOT CELL JAR/VIAL LOADOUT

Qj) 6. Place vials/jars for loadout next to transfer drawer in hot cell 11A-1B. 
Q 1 . clean up hot cell work area, spatulas, and sampling trays.

8. Update the Jars $/ \mathrm{Vials}$ laboratory notebook ( $\mathrm{HHC}-\mathrm{N}-754$ ) for all vials and jars created during subsampling.

BA

9. If required, update the vial number (example: $J \times X X X)$ in the labcore I.D. for each subsample.
(l) Standard Login
(2) Change Group
(3) Customer code ICP
(4) Group \# 95XXXXXX
(5) Prior samples (F6)
(5) Enter Sample number
(7) Edit the sample i.d.

RDit 10. FAX a copy of the subsampling worksheet to the PC.

ast 11. Place the copy of subsampling worksheet in PARADOX file.

12. Have chemist review and signoff.

G. Reporting/Labcore:

ONOH 1 Under PARAMETER DATA ENTRY in labcore, complete the tests
below, if generated, and sign your initials/date next to the vial on the subsampling worksheet.

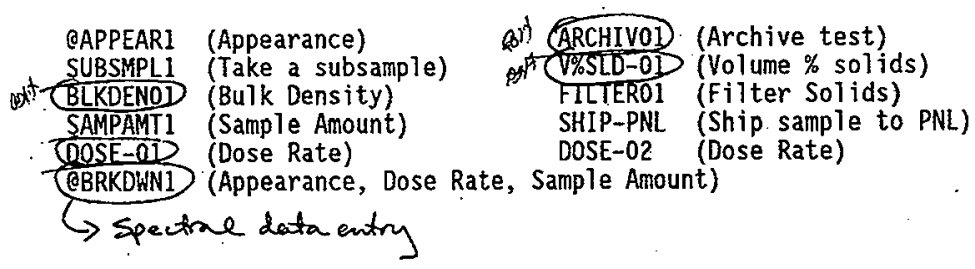


eak E. Determine bulk density of solids? Yes or No

Cac 1. Calculate bulk density of solids, if required: (BLKDENOI)
a. Sum Wgt. Solids for all cones
b. Sum vol. Solids for all cones
c. Determine bulk density of solids: $\frac{10.34}{9} \mathrm{~mL}$
Sum of Wat. Solids
Sum of Vol. Solids

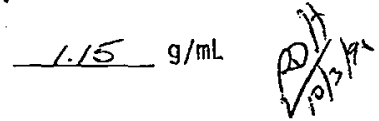
d. Other Information:
Repot BLKDNOI fo volids only as 5967005019

RE F. Determine bulk density of liquids? Yes or No

Lef 1. Calculate bulk density of liquids, if required: (BLKDEN01)
a. Wgt liquids for come $5 \mathrm{~A}$.

Wgt liquids = Sum of Net Wgt - Sum of Wgt solids
b. Vol. liquids for all cones
$17 \mathrm{~mL}$

Vol liquids = Sum of Total vol - Sum of Vol solids

c. Determine bulk density of liquids:

$$
\frac{\text { Wht. Liquids }}{\text { Vol. Liquids }} \quad 1.04 \mathrm{~g} / \mathrm{mL}
$$

d. Other Information: Repart BCKDEN 51 fon lifinds aty

edE G. Determine volume \% solids by centrifugation? Yes or No

Rese 1. Calculate volume \% solids by centrifugation, if required: (V\%SLD-01)

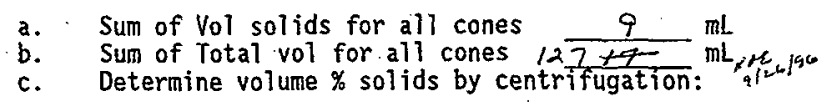

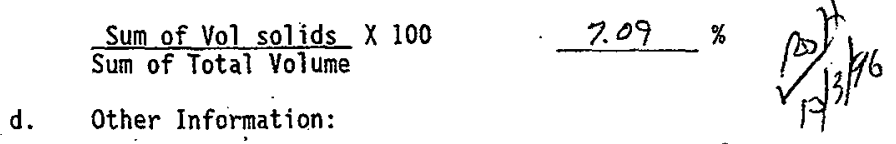

Repoot VYSCD- $\not 1$ as pample $5 \$ 67004809$

\section{2}




\section{Breakdown: Grab Sludge Worksheet}

P.C.: RA Esch

\section{Tank/}

Project: Aw-105 GRABS

Slart Time:

End Time:

Centrifugation

Time (Min.):

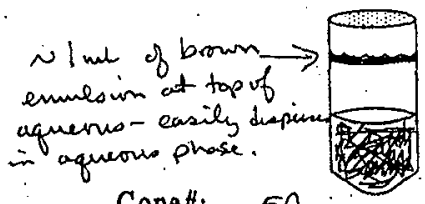

Conett: $5 A$

Wgt. Cone + Smp: $42.10 \mathrm{~g}$

Wgt. Cone + Sol: 24.20 g

Wgt. Cone: $14,16 \mathrm{~g}$

$w^{N}=1.04 \mathrm{~g}$ Net Weight: $\frac{27.94}{26 .} \mathrm{g}$

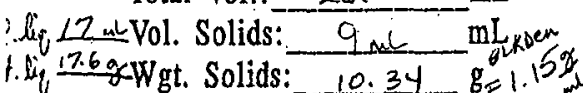

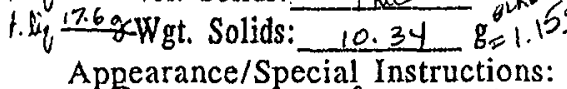

gearance/Special Instructions:
Sample ID: $5 A w-96-5$

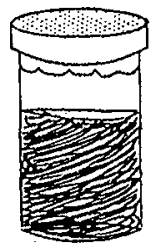

Sample ID: 596 T004809 $\mathrm{Jar} /$ Vial Size: $125 \cdot \mathrm{mL}$ Initial Weight: $26.597 \mathrm{~g}$ Final Weight: $130.04 \mathrm{~g}$ Net Weight: $135.83 \mathrm{~g}$
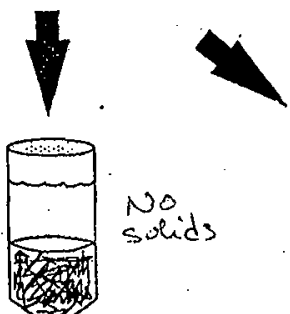

Conet: No
solids

Wgt. Cone + Smp: $66.07 \mathrm{~g}$ Wgt. Cone + Sol: $N / A g$

Wgt. Cone: $13.58 \mathrm{~g}$

Net Weight: $52,49 \mathrm{~g}$ Total Vol.: $50 \mathrm{~mL}$ Vol. Solids: $N / A \quad m L$ Wgt. Solids: $N / A$ Bulk dens. $1.05 \mathrm{~g} / \mathrm{wic}$

Date: $9 / 25 / 96$ small of soledis and biack speck. was not tiamefersed to conerer

Labcore Entry

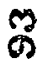
Initials:

BLKDEN01? Y or N

V\%SLD-01? $\otimes$ or $N$

small amount of suspeudnd solids werter $15 \mathrm{ml}$ conce

Cone 5

Conelf: $5 \mathrm{C}$

Wgt. Cone + Smp: $62.40 \mathrm{~g}$

Wgt. Cone + Sol: N/A g

Wgt: Cone: $\_13,21 \mathrm{~g}$

Net Weight: $48.69 \mathrm{~g}$

Total Vol.: $46.0 \mathrm{~mL}$

Vol. Solids: $\sim / A \quad m L$

Wgt. Solids:

eulk bens. $\frac{A J / A}{1.06 \mathrm{~g} / \mathrm{he} L}$ conetsane $12.76 \mathrm{~g}$

cone $7.68 \mathrm{~g}$

Net wgt $=0.08 \mathrm{gg}$

total ons. 5 unc Buth denew $=1.02$ 墕

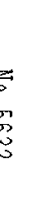

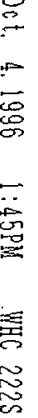

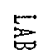

8 $\rightarrow$

, :

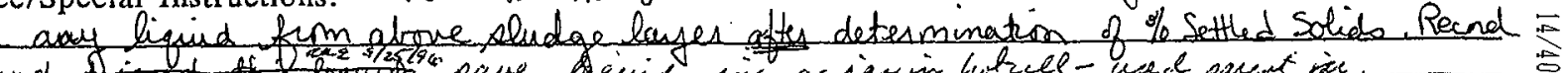

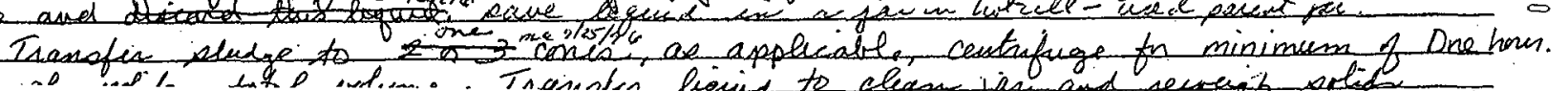




\section{Subsample: Grab Sludge Worksheet}

Date: $\frac{9 / 26 / 96}{\text { Tankl }}$
Project: Aw-105 6RAB
P.C.: RA Esch

Phonct: $373-4314$

\section{Labcore Entry} GRoup 96001213

Initials: Archive Bin \#:
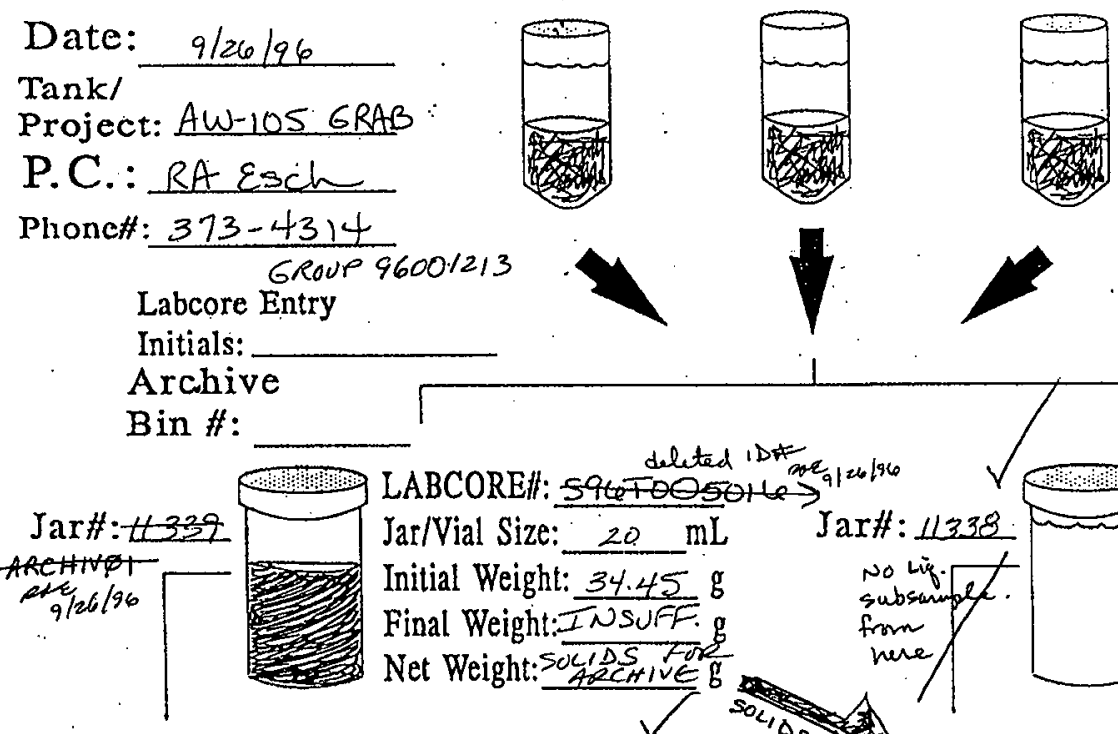

Parent

Sample ID: 5AW-96-5 $\$ 967004809$

TESTS

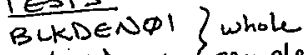
$V \%$ SLD- $\varnothing 1\}$ somple

Labcore Entry Initials: Archive Bin \#: $N / A$

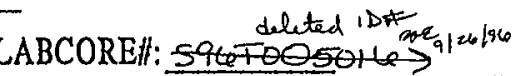
Jar/Vial Size: $20 \mathrm{~mL}$ Jar\#: 11338 . Initial Weight: $34.45 \mathrm{~g}$ Final Weight:IN SUFF. $g$ Net Weight: SuctsS tore
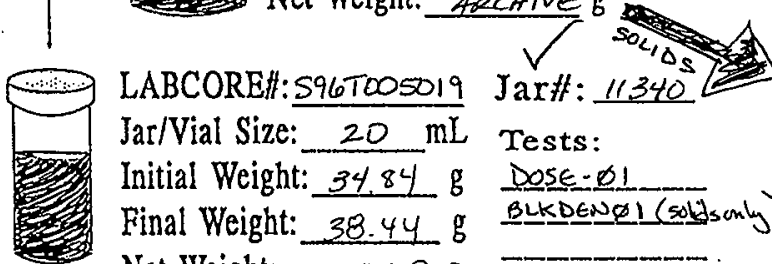
LABCOREH: $\frac{596 \text { TOO5019 }}{30 \mathrm{~mL}}$
Jar/Vial Size: $\frac{20.84}{\mathrm{~g}}$
Initial Weight: $\frac{34.84}{\mathrm{~g}}$
Final Weight: $\frac{38.44}{3.60 \mathrm{~g}}$
Net Weight: Jar\#: 11340 subsingef. from neve 1

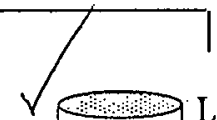
LABCORE: 596 TO0 S049:" Initial Weight: $33.52 \mathrm{~g}$ Final Weight: $50.88 \mathrm{~g}$ Net Weight: $17.36 \mathrm{~g}$ $\frac{\text { TESTS }}{\text { DOSE-DI }}$ BCKDEN $\varnothing 1$ (biquid)

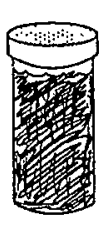

Tests:

DOSE-DI BLKDEENQDI (soldsonly) $\because$

Appearance/Special Instructions:

Subsample $8-10 \mathrm{~g}$ fin analysis! and archive remaining * 2 evid subemples fun asehive jas 
HNF-SD-WM-DP-214, REV. 0

Grab Sample Information Worksheet

Sample ID: $\frac{54 w-96-7}{\text { LABCORE \#: }}$
Tank/Project: $\frac{\text { S96T004811 }}{\text { Aw-105 GBAB }}$

Date:

$9 / 27 / 96$

(Date of Breakdown)

A. Record the sampling depth off of sample tag: 588 in.

B. Obtain the total weight of the grab sample:

Total . weight of jar + sample $=306.35$ grams

C. Description of Sample:

1. Color of liquid:

2. Is liquid clear or opaque:

3. Approximate volume (Total):

4. Are solids present: too lithe liquid to delaine color ar clarity

(Yes) or No

5. Approximate vol \% settled solids $100 \%$

6. If there is more than $2 \%$ settled solids, determine actual volume \% settled solids:

a. Height of solids:

b. Height of total. sample:

c. Volume \% settled solids:

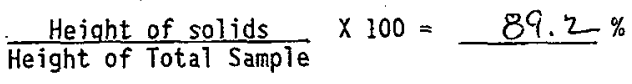

Solids au a lights. yellow/ gray creamy mixture. w/ black speck e Lover lith ar nine "core" it

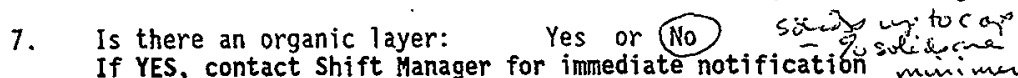
If YES, contact Shift Manager for immediate notification suliciciam to tank farms.

rave Complete

8. Other Information: Record appearance information

D. Determine bulk density of sample?

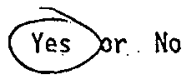

1. Calculate bulk density of sample, if required: (BLKDENO1)

a. Sum Net Weight for all cones
b. Sum Total volumes for all cones $\frac{163.22}{132} \mathrm{~g}$
$\mathrm{~mL}$

c. Determine bulk density of sample:

Sum of Net Weight

$$
1.24 \mathrm{~g} / \mathrm{mL}
$$

$(-1))^{+3}$

Sum of Total Volume

d. Other Information:

Read BCKDENDI. whole sample on $\$ 967004811$ solis

95

$07 / 91$ d 2899 ON

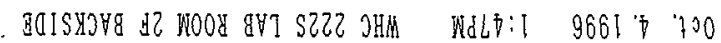


Oct. 4.1996 1:47PM WHC 222S LAB ROOM 2F BACKSIDE

No. $5632 \quad$ P. $\quad 17 / 40$

HNF-SD-WM-DP-214, REV. D

ERE. Determine buTk-density of solids?

Yes or No

1. Calculate bulk density of solids, if required: (BLKDENO1)

a. Sum Hgt. Solids for all cones $\frac{118.63}{89.5} \mathrm{~g}$
b. Sum Vol. Solids for all cones

c. Determine bulk density of solids:

$\frac{\text { Sum of Wat. Solids }}{\text { Sum of Vol. Solids }}$

$1.32-\mathrm{g} / \mathrm{mL}$

d. Other Information:

Record SCRDENQS police only $/ 396$ on $\$ 967005020$

Determine bulk density of liquids? Yes or No

edt 1. Calculate bulk density of 1 liquids, if required: (BLKDENO1)

a. Hgt liquids for ali cones $\quad 40.86 \mathrm{~g}$

Wat liquids $=$ Sum of Net Hgt $=$ Sum of Wit solids

b. Vol. liquids for all cones $\quad 39 \mathrm{~mL}$

Vol liquids = Sum of Total vol - Sum of Vol solids

c. Determine bulk density of liquids:

$\frac{\text { Wat. Liquids }}{\text { Vol. Liquids }}$

$1.05 \mathrm{~g} / \mathrm{mL}$

d. Other Information: Re and BCKJEN $\varnothing 1$ liquids only
on 596 TOOSOSO

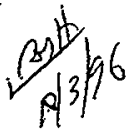

G. Determine volume \% solids by centrifugation? Yes or No

1. Calculate volume $\%$ solids by centrifugation, if required: (V\%SLD-01)

a. Sum of Vol solids for all cones $\frac{89.5}{132} \mathrm{~mL}$
b. Sum of Total vol for all cones

c. Determine volume \% solids by centrifugation:

$$
\frac{\text { Sum of Vol solids }}{\text { Sum of Total Volume }} \times 100
$$

$678 \%$

d. Other Information:

Read V\% SCD-Di on 5967004811

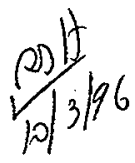

96 


\section{Subsample: Grab Sludge Worksheet}

Date: $9 / 27 / 96$

Tankl

Project: Aw-105 6RAB:

P.C. : RA غsch

Phonett: $373-4314$

Group 96001213

196 Labcore Entry
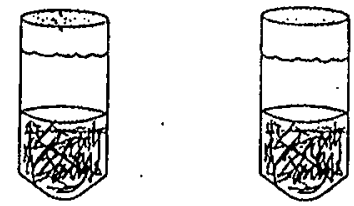

Initials:

Archive

70. $36 \mathrm{~g}=500 \mathrm{Bin} \# \mathbf{8 2}$

$20.01 \mathrm{~g}=20 \mathrm{~g} \dot{\mathrm{V}}$

Jar\#: 11385

ARCHIVøI

$\infty$

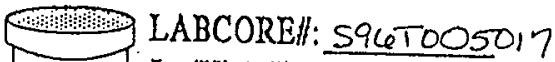
Jar/Vial Size: $60 \mathrm{~mL}$ Initial Weight: $88.12 \mathrm{~g}$ Final Weight: $163.60 \mathrm{~g}$ Net Weight:
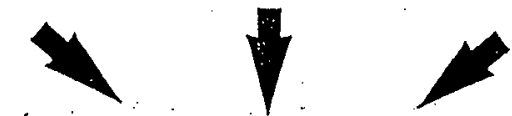

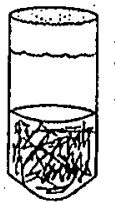

Labcore Entry

Initials:

Archive

Bin H. W $/ 2 / 21 / 56$

Bin \#: $82^{\circ}$

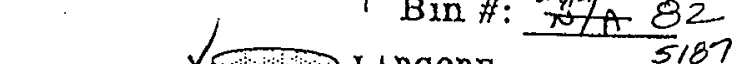

Jar\#: 1138-1

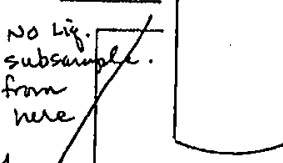
Jar/Vial Size Initial Weight: $\frac{60}{88.09} \mathrm{~g}$ Dose-\$ $\mathrm{PRE}$ Final Weight: $109.43 \mathrm{~g}$ Net Weight: $21.34 \mathrm{~g}$ (ligina) ARCHIVI LABCOREH: $\$ 967005047 \mathrm{Jar} \#: 11430^{2}$ Jar/Vial Size: $20 \quad \mathrm{~mL}$ Tests: Initial Weight: 34.11 \& Dose- $\$ 1$. Final Weight: $44,32 \mathrm{~g}$ Net Weight: $10.21 \mathrm{~g}$

Appearance/Special Instructions:

Subsample $8-10 \mathrm{~g}$ fo analysin and archive semaining

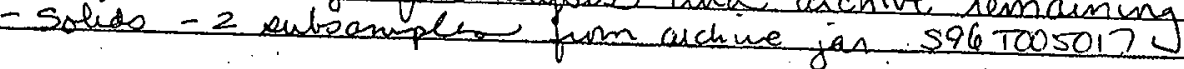


HNF-SD-WM-DP-214, REV. 0

Grab Sample Information Worksheet

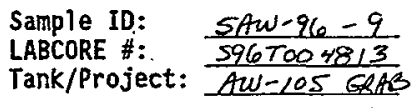

Date:

$9 / 30 / 96$

(Date of Breakdown)

A. Record the sampling depth off of sample tag: 589 in.

B. Obtain the total weight of the grab sample: a gre $9 / 27 / s 6$ Total weight of jar + sample $=\frac{301.98}{301.84}$ grams

C. Description of Sample:

1. Color of liquid:

2. Is liquid clear or opaque:

3. Approximate volume (Total):

4. Are solids present: too little liquid to determine color and clint (10) 125 or. No

5. Approximate vol \% settled solids $\sim 100 \%$

6. If there is more than $2 \%$ settled solids, determine actual volume \% settled solids:

a. Height of solids:

a. Height of solids: $\frac{75 .}{8 m}$ sids are light
b. Height of total sample: $\frac{15}{83}$ yellow mixed with
c. Volume \% settled solids: gray. $\frac{\text { Height of solids }}{\text { Height of Total Sample }} \times 100=90.4 \%$ w/blacke speckles

7. Is there an organic layer: Yes or (No) in minimum. If YES, contact Shift Manager for immediate notification to tank farms.

8. Other Information:

Rot. D. Determine bulk density of sample?

Yes or No

ear 1. Calculate bulk density of sample, if required: (BLKDENO1)

a. Sum Net Weight for all cones $157.89 \mathrm{~g}$

b. Sum Total volumes for all cones $129.5 \mathrm{~mL}$

c. Determine bulk density of sample:

Sum of Net Weight

$1.22 \mathrm{~g} / \mathrm{mL}$

Includes bligh silicic remove d from

d. Other Information: above sulky -

Record BCKDENDI fo whole sample

on 5967004813

100

$07 / 15$ d $2899^{\circ} 0 \pi$

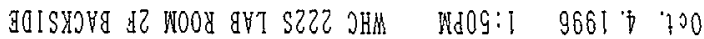


HNF-SD-WM-DP-214, REV. D

PQE E. Determine bulk density of solids?

Yes or No

Le 1. Calculate bulk density of solids, if required: (BLKDENO1)

a. Sum Witt. Solids for all cones $\quad 115.85 \mathrm{~g}$ w/exceptuon

a. Sum Wit. Solids for all cones $\frac{115.85}{8} \mathrm{~g}$ of com
b. Sum Vol. Solids for all cones
c. Determine bulk density of solids:

$\frac{\text { Sum of Wat. Solids }}{\text { Sum of Vol. Solids }}$

$1,29 \mathrm{~g} / \mathrm{mL}$

d. Other Information: Other Information:
Repeat BLEDEN 71 for solids only
on S96TODSO21

$\frac{021 t}{p 1396}$

Ra 2F. Determine bulk density of liquids? (Yes or No

Hose 1. Calculate bulk density of liquids, if required: (BLKDENO1)

a. Wt liquids for all cones $\quad 32.28 \mathrm{~g}$ w/excestion Hgt liquids = Sum of Net Wit - Sum of Wit solids of as

b. Vol. liquids for all cones, $32.0 \mathrm{~mL}$

Vol liquids = Sum of Total vol - Sum of Vol solids

c. Determine bulk density of liquids:

Wot. Liquids

$1.03 \mathrm{~g} / \mathrm{mL}$

Vol. Liquids

d. Other Information:

Recond/repent BLKDENDI for liquids only on S96T005051

ReG. Determine volume \% solids by centrifugation? Yes or No

1. Calculate volume \% solids by centrifugation, if required: (V\%SLD-01)

a. Sum of Vol solids for all cones $89.5 \mathrm{~mL}$ olepception

a. Sum of Vol solids for all cones $\frac{89.5}{\mathrm{~mL}}$ of come
b. Sum of Total vol for all cones
c. Determine volume \% solids by centrifugation:

Sum of Vol solids $\times 100$ Sum of Total Volume

d. Other Information:
$13.1 \%$

Report V\% SLD- $\$ 1$ on 5967004813

Balt 1996

101

$07 / 22$ d $2 E 9 g$ an

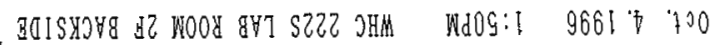




\section{Breakdown: Grab Sludge Worksheet}

P.C.: RA Esch.

Tankl

Project: Aw-105 GRAB

Start Time:

End Time:

Centrifugation

Time (Min.):

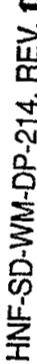

Conet: $9 A$

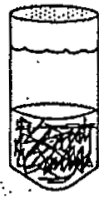

Wgt. Cone + Smp: $6 / 25 \mathrm{~g}$

Wgt. Cone + Sol: $4220 \mathrm{~g}$

Wgt. Cone:

Net Weight: $\frac{3.80}{47+5}$

Total Vol.:

47.045

39
Sample ID: $5 A \omega-96-9$

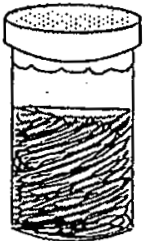

Sample ID: 596 T004813 Jar/Vial Size: $125 \mathrm{~mL}$ Initial Weight: $301.84 \mathrm{~g}$ Final Weight: $139.71 \mathrm{~g}$ Net Weight: $.162 .13 \cdot \mathrm{g}$
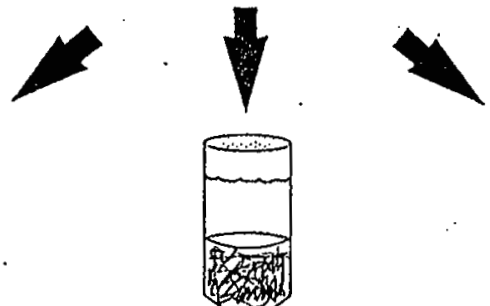

Concti:

Wgt. Cone + Smp: 63/6 g

Wgt. Conc + Sol: $53.19 \mathrm{~g}$

Wgt. Cone: g Net Weight: 18.89 g

Total Vol.: $\mathrm{mL}$ to $\mathrm{mL}$

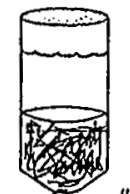

Conett:

Lif 11.5 sevol. Solids: 27.5

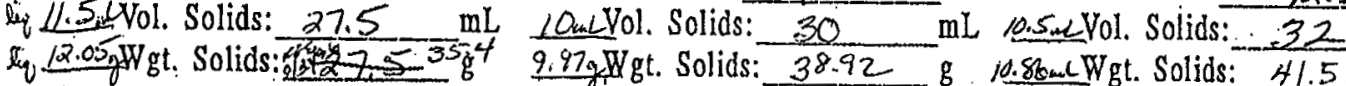

$9 \mathrm{C}$

Labcore Entry

Initials:

BLKODENO1? $\mathrm{Y}$ or $N$

V\%SLD-01? $(9$ or $\mathbf{N}$ sime areid 2 all

Ligind ferm jain the bre

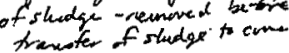

Wgt. Cone + Smp:Lee.68 g ingt Cone +Sup 16.60 Wgt. Conc + Sol: $65.82 \mathrm{~g}$,

Wgt. Cone:

Net Weight:

Total Vol::

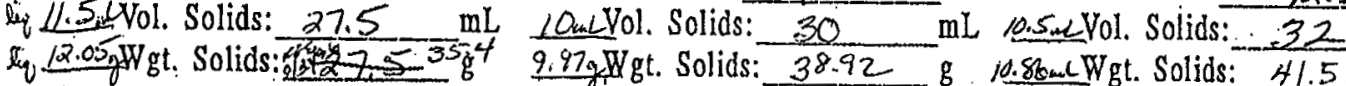
14.29 $g$ wat one 7.44 52.39 8 wet wigt. 42.5 mL To trave: 9.16 8.00 Appearance/Special Instructions:

38.92 jo. $88 \mathrm{su}$ Wgt. Solids 4153 Mil jenchder. 1,14

Beinare any ligind fim atare jlud lan

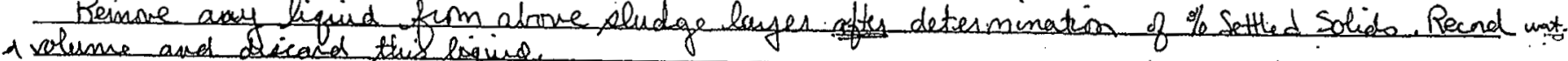

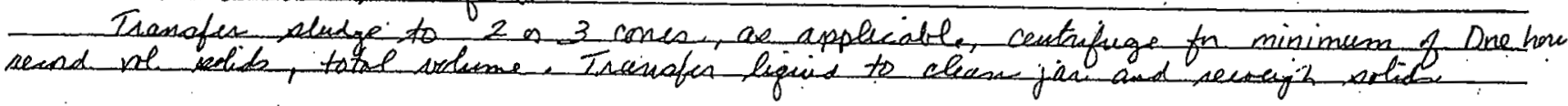




\section{Subsample: Grab Sludge Worksheet}

Date: $9 / 30 / 96$

Tank/

Project: Aw-jos GRAB

P.C.: RA Esch

Phonet: $373-4314$

GRoup $\$ 96001213$

Labcorc Entry

Initials:

Archive

JarH: 11387

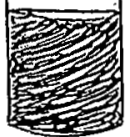

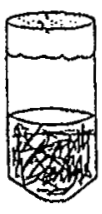
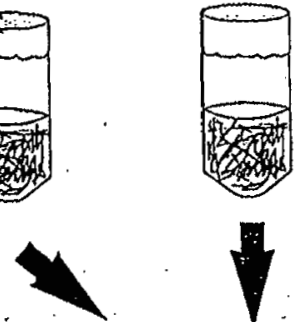
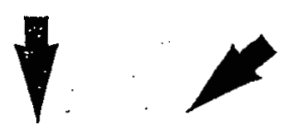

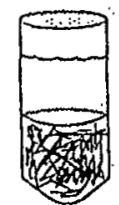

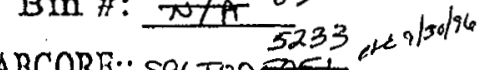

LABCOREH: 596 TOOSO18 Jar/Vial Size: $100 \mathrm{~mL}$ Initial Weight: $88.20 \mathrm{~g}$ Final Weight: $152.61 \mathrm{~g}$ Net Weight: $\frac{64.41 / 6}{8}$

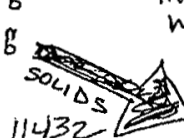

LABCORE\#: \$96T005021 Jar"1: 11432 Jar\#: 11386 Jar/Vial Sizc: $20 \mathrm{~mL}$ Tests: Initial Weight:34.44: g DosE-DI Final Weight: 44.77 g BLKDENDI (suldsonly) Net Weight: $10,33 \mathrm{~g}$
Parent.

Sample ID: $5 A W-96-9$

5967004813

TESTS BLKDENDI W whole $\checkmark \%$ sLD- $\varnothing 1\}$ somple

Labcore Entry

Initials:

Archive of 3196

Bin \#: Nta 82

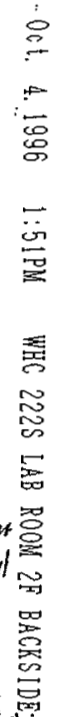
Jar\#: 11434

LABCOREH: $5967005048 \mathrm{Jar} \#$ : Initial Weight: $34.30 \mathrm{~g}$ Dose-ELFinal Weight: $44.21 \mathrm{~g}$ Net Weight: $9.91 \mathrm{~g}$

Appearance/Special Instructions:

Subsample $8-10 \mathrm{~g}$ fo analysin aud archive remaining

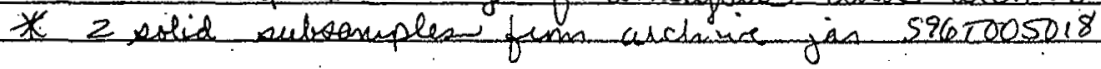


oct. 4. 1996 1:52PM WHC 2225 LAB ROOM 2 F BACKSIDE

No. $5632 \times 0$ P 25/40

量

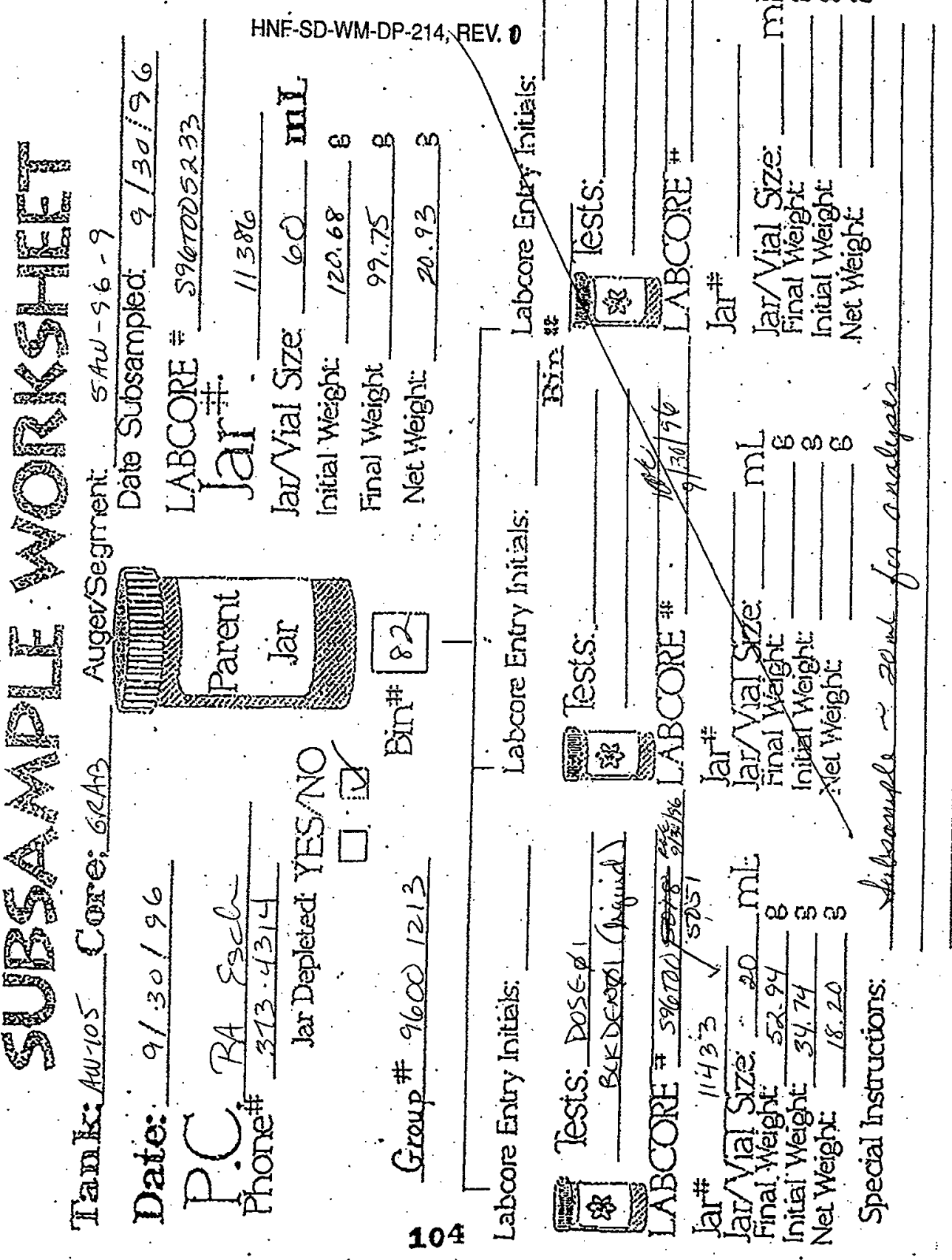


HNF-SD-WM-DP-214, REV. 0

Grab Sample Information Worksheet

Sample ID:
LABCORE \#: $\frac{5 A w-96-15}{\text { S96T004819 }}$
Tank/Project:

Date: $\quad 9 / 20$ lac $\{10 / 1 / 96$ (Date of Breakdown)

A. Record the sampling depth off of sample tag: 521 in.

B. Obtain the total weight of the grab sample:

Total weight of jar + sample $=296,00$ grams

C. Description of Sample:

1. Color of liquid:

2. Is liquid clear or opaque:

3. Approximate volume (Total):

4. Are solids present:
Yellow

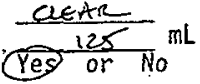

5. Approximate vol \% settled solids $\approx 90 \%$

6. If there is more than $2 \%$ settled solids, determine actual volume $\%$ settled solids:

a. Height of solids:

a. Height of solids:
b. Height of total sample: $\frac{75}{83} \mathrm{~mm}$ solids - danker
c. Volume \% settled solids: gray w/ black $\frac{\text { Height of solids }}{\text { height of Total Sample }} \times 100=-90.4 \%$ speck er

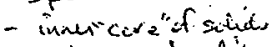
go to cop. \% solid.

7. Is there an organic layer: Yes or No is minimum If YES, contact Shift Manager for immediate notification to tank farms.

8. Other Information: Report @BRKDWNI on 5967004819

RUE D. Determine bulk density of sample? Yes or No

WL 1. Calculate bulk density of sample, if required: (BLKDENO1).

a. Sum Net Weight for all cones $155.76 \mathrm{~g}$ induing $15 \mathrm{mu}$

b. Sum Total volumes for all cones $\frac{153.8}{13.8}$. mL input le

c. Determine bulk density of sample:

Sum of Net Height.

$1.16 .9 / \mathrm{mL}$

d. Other Information:

Report BCKDENO1 whole sample

on 5967004819

105

$00 / 92$ d 2899 on

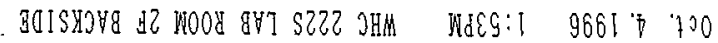


aARE. Determine bulk density of solids? Yes or No

2. Calculate bulk density of solids, if required: (BLKDENO1)
a. Sum Wgt. Solids for all cones
b. Sum Yol. Solids for all cones
c. Determine bulk density of. solids:

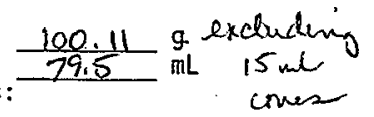
Sum of Wat. Solids
Sum of Vol. Solids
$1.26 \mathrm{~g} / \mathrm{mL}$
d. Other Information:
Bepat BCKDENQ1 solids only on. 5967005075

ear F. Determine bulk density of liquids? पPes or No

1. Calculate bulk density of liquids, if required: (BLKDENO1)
a. Wgt Tiquids for all cones $28.45 \mathrm{~g}$ excluding. Hgt liquids = Sum of Net Wgt - Sum of Hgt solids i $5 \mathrm{ml}$
b. Vol. liquids for all cones
$29: 0 \mathrm{~mL}$
cover

Vol liquids = Sum of Total vol - Sum of Vol solids

c. Determine bulk density of liquids:

$$
\frac{\text { Wat. Liquids }}{\text { Vol. Liquids }}
$$

$0.98 \mathrm{~g} / \mathrm{mL}$

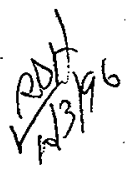

d. Other Information:

Repat BLKDENO, liquids only on 5965005081

ME. Determine volume \% solids by centrifugation?

Yes or No

ale

1. Calculate volume \%. solids by centrifugation, if required:

(V\%SLD-01)

a. Sum of Vol solids for all cones

b. Sum of Total yol for all cones

c. Determine volume $\%$ solids by centrifugation:

$\frac{\text { Sum of Vol solids }}{\text { Sum of Total Volume }} \times 100$

$73.3 \%$

d. Other Information:

Reand V\%SLD- $\$ 1$ on 5967004819

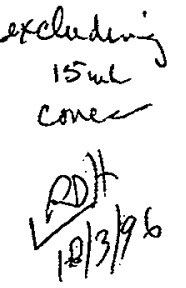

\section{6}




\section{Breakdown: Grab Sludge Worksheet}

P.C.: RA Esch

Tank I

Project: Aw-105 GBAB

Start Time:

Bnd Time:

Centrifugation

Time (Min.):
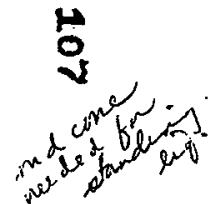

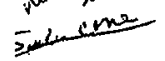

\section{SE Conett: $15 A$}

.074 g. Wgt. Cone + Smp: $34.28 \mathrm{~g}$

Wgt. Cone + Sol: $29.56 \mathrm{~g}$

$1.55 \mathrm{~g}$

$3.19 \mathrm{~g}$

$12-3 \mathrm{mic}$

$\sin 2$

Wgt. Cone: 14.19

Net Weight:

Total Vol.:

Vol. Solids:

20.09

Wgt. Solids: 15,37
Sample ID: $5 A w-96-15$

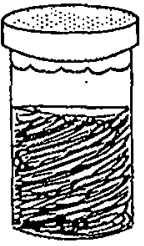

Sample ID: 596 T004819 Jar/Vial Size: $125 \mathrm{~mL}$ Initial Weight: $296,00 \mathrm{~g}$ Final Weight: $134.47 \mathrm{~g}$ Net Weight: $161.53 \mathrm{~g}$
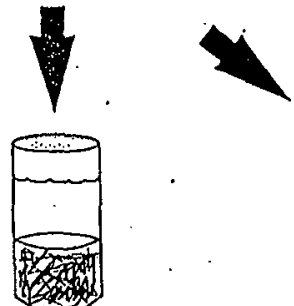

Conett: $15 B$

Wgt. Cone + Smp: $67.6 \mathrm{gg}$

W.gt. Cone + Sol: $59.06 \mathrm{~g}$. 156

Wgt. Conc:

$g$ ist. $g$ Liquid Net Weight: $\frac{13.66}{5.95} \mathrm{~g}$ uque $\mathrm{mL}$ Total Vol.: $45 \mathrm{~mL}$ $\dot{m} L S_{\mu \nu}$ L Vol. Solids: 36 $-1$

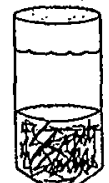

Conett: $\quad$ ISC

Labcore Entry

Initials:

BCXKDEN01? $\mathbf{Y}$ or $N$

V\%SLD-01? $Q$ or $N$ cheche wits 502g $=5700.36$. $20 \mathrm{~g}=20.01 \mathrm{~g}$ remured liq abovesld w/some sids transfer Wht Con + Smp: $6828 \mathrm{~g}$. wot ane Tsup 21449 Cone + Sol: 53,108

Wgt. Cone: 13.76 g Net Weight: $\frac{1.54 .52}{54}$ pert wight. $\frac{7.48 \mathrm{~g}}{14.01}$ Total Vol.: 46 Vol. Solids: $45.40 \mathrm{~g} 8.55 \mathrm{~g}$ Wgt. Solids: 31 $39.34 \mathrm{~g}$ inl trate ve. $\mathrm{mL}$ Bikdem. $1.08-g, d$ Appearance/Special Instructions: w voleme and dicand this liquire. Tranofer elude to 203 conea, as applecible, cesctafiege for minimsem of Dne hores. 
Date: $10 / 1 / 96$

Project: AW-105 6RAB

P.C.: RA Esch

Phonetf: $373-4314$

GRoup \#9600/215

Labcore Entry

Initials:

Archive

Bin \#:

Jar $\#: 11389$

ARCIINQ1
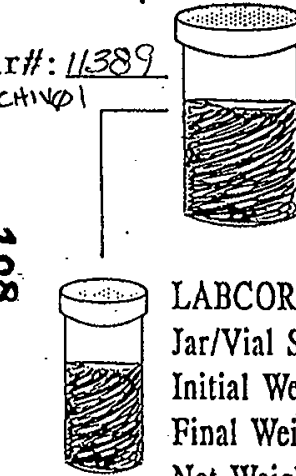

LABCOREH: 596 TOOSO58 Jar/Vial Size: $60 \mathrm{~mL}$ Initial Weight: $87.92 \mathrm{~g}$ Final Weight: $14062 \mathrm{zg}$ Net Weight:

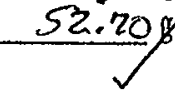

LABCORE\#:S96TOOSO75 Jar\#: $1 / 436$

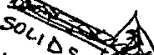

Jar/Vial Size: $20 \mathrm{~mL}$ Tests:

Initial Weight: 34,36 g Dose-01

Final Weight: 45,34

Net Weight: $10,98 \mathrm{~g}$

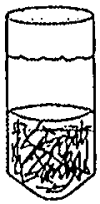

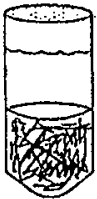

Parent

Sample ID: SAW-96-15. 5967004819

TESTS

BLKDENDI whote $V \% S(D-\varnothing)\}$ somple

Labcore Entry

Initials:

Archive

Bin \#:

N/A 82

rरe. 930 दि 5234

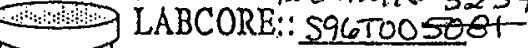

Jar\#: 11.388

BLLKDEND足 (sold'sonly)

Appearance/Special Instructions:

Subsample $8-10 \mathrm{~g}$ fn analypin and archive remaining * 2 solid subeasuples flom auchue jas 5967005058 Jar/Vial Size: $60^{\circ} \mathrm{mL}$ Initial Weight: $87.98 \mathrm{~g}$ Final Weight: $\frac{97.72}{9} \mathrm{~B}$ Net Weight: $9.74 \mathrm{~g}$

TESTS DOSE-OT subsangef. from nere D

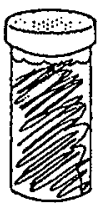

LABCORE\#: $5967005078 \mathrm{Jar} \#: 11437 \mathrm{~V}$ Jar/Vial Size: $20 \mathrm{~mL}$ Tests: Initial Weight: $34,53 \mathrm{~g}$-Dose- $\varnothing 1$ Final Weight: $45.52 \mathrm{~g}$ Net Weight: $10,99 \mathrm{~g}$ 
oct. 4. 1986 . 1:55PM.. WHC 2225 LAB ROOM 2F BACKSIDE

No. 5632 P. $30 / 40$

HNF-SD-WM-DP-214, REV. 0

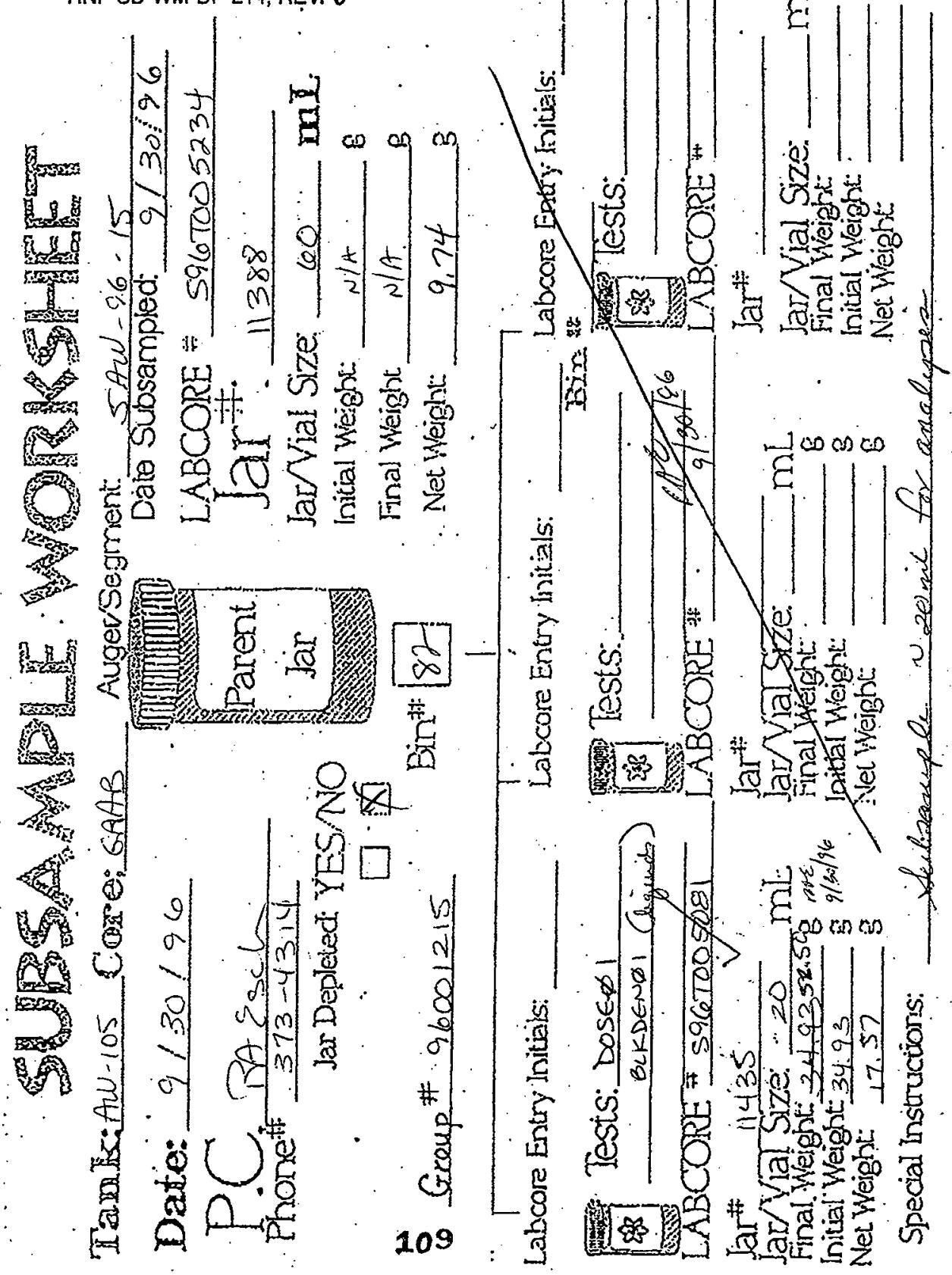


HNF-SD-WM-DP-214, REV. D

Grab Sample Information Worksheet

Sample ID: $\quad 57 w-96-17$

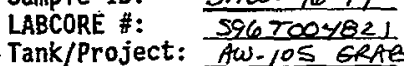

Date:

$10 / 1 / 96$ (Date of Breakdown)

A. Record the sampling depth off of sample tag: 593 in.

B. Obtain the total weight of the grab sample:

Total weight of jar + sample $=299.83$ grams

C. Description of Sample:

1. Color of liquid:

2. Is liquid clear or opaque:

3. Approximate volume (Total):

4. Are solids present:

too little liquid to

determine error and clainty

(Yes) or No

5. Approximate vol \% settled solids $\sim 100 \%$

6. If there is more than $2 \%$ settled solids, determine actual volume \% settled solids:

a. Height of solids: ${ }_{\text {b. Height of total sample: } \frac{123}{83} \mathrm{~mm}}$ solids mostly

c. Volume \% settled solids:

black w/smale

$\frac{\text { Height of solids }}{\text { Height of Total Sample }} \times 100=-88.0 \%$ amours of yellow mixed in

Vas or No goo to cape. \%o solid If YES, contact Shift Manager for immediate notification to tank farms.

8. Other Information: Report QBRKDwNi on 5967004821

Le 0 . Determine bulk density of sample? Yes or No

QR E 1. Calculate bulk density of sample, if required: (BLKDENOI)

a. Sum Net Weight for all cones $160.10 \mathrm{~g}$ includes

b. Sum Total volumes for all cones $\frac{131.5}{131} \mathrm{~mL} 15 \mathrm{mi}$ com

c. Determine bulk density of sample:

$\frac{\text { Sum of Net Weight }}{\text { Sum of Total Volume }}$

$1.22 \mathrm{~g} / \mathrm{mL}$

d. Other Information:

Report BLKDENDI whole sample

$|d| 3 \mid 9 t$

$$
\text { on } 5967004821
$$

110

$00 / 1 \varepsilon$ d $\quad 2 \varepsilon 9 g^{\circ} 0 \mathrm{~N}$

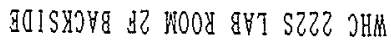

Wag: 1

$9661+700$ 
HNF-SD-WM-DP-214, REV. 0

La E.E. Determine bulk density of solids?

Yes or No

peak

1.

Calculate bulk density of solids, if required:

a.

b.

c.

Sum Wit. Solids for all cones

Sum vol. Solids for all cones

Determine bulk density of solids:

Sum of Wat. Solids

$\frac{\frac{108.22}{82.5} \mathrm{gL} \text { excluding }}{178 \mathrm{~g}_{\mathrm{mL}}}$

d. Other Information:

Report BLKDENDI solids only on 5967005026

faR F. Determine bulk density of liquids?

Yes or No

1. Calculate bulk density of liquids, if required: (BLKDENO1)

a. Hgt liquids for all cones

41.289

Wit liquids = Sum of Net Wit - Sum of Wit solids

excluderis

b. Vol. liquids for all cones $39.5 \mathrm{~mL}$

Vol liquids = Sum of Total vol - Sum of Vol solids

c. Determine bulk density. of liquids:

$\frac{\text { Wat. Liquids }}{\text { Vol. Liquids }}$

$1.04 \mathrm{~g} / \mathrm{ml}$

(7)

d. Other Information:

Repent BLKDENO1 eq quids only on 5967005082

RaE. Determine volume \% solids by centrifugation? Yes or No

120

1. Calculate volume \% solids by centrifugation, if required: (V\%SLD-01)

a. Sum of Vol solids for all cones $\frac{82.5}{122.0} \mathrm{~mL}$ exclude ing

c. Determine volume $\%$ solids by centrifugation:

Sum of Vol solids $\times 100$

Q.7.6\%

d. Other Information:

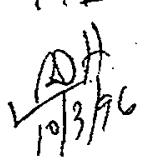

Repast V\% Sis. $\varnothing 1$ on $\$ 967004821$

111

$07 / 2 \varepsilon$ d $2 \varepsilon \rho g$ 'on 


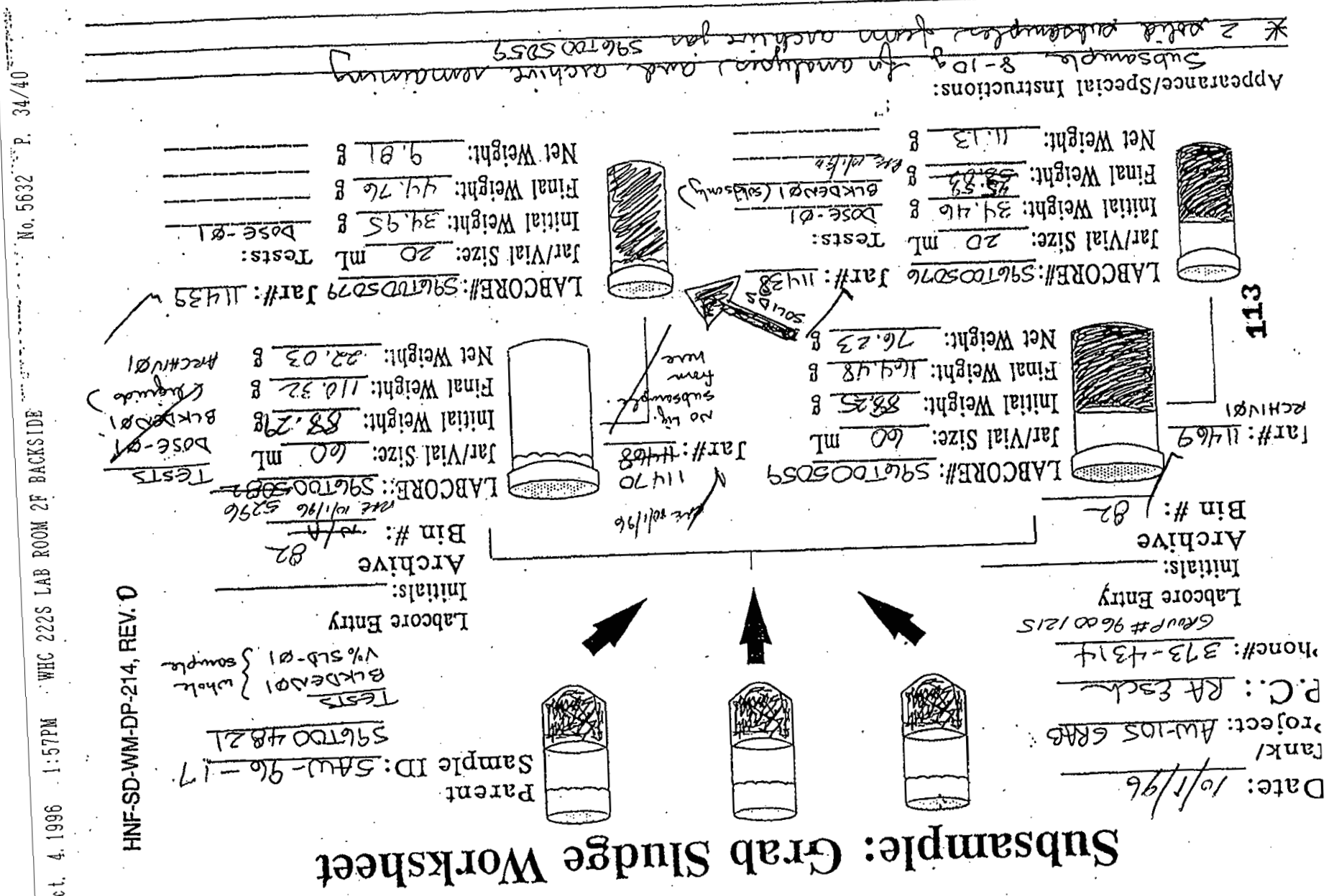


$11 / 21 / 96 \quad 09: 57$

5093731432

001

HNF-SD-WM-DP-214, REV. D

Grab Sample Information Worksheet

Sample ID:

LABCORE \#: $\frac{S W w-96-20}{\text { SqGTOOAB24 }}$
Tank/Project:

Date:

io) $2 / 26$ (Date of Breakdown)

A. Record the sampling depth off of sample tag: 1000 in.

B. Obtain the total weight of the grab sample:

Total weight of jar + sample $=302,26$ grams

C. Description of Sample:

1. Color of liquid:

2. Is liquid clear or opaque:

3. Approximate volume (Total):

4: Are solids present:

too little legend to determine Color and clarity

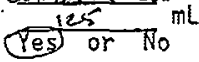

5. Approximate vol \% settled solids $100 \%$

6 . If there is more than $2 \%$ settled solids, determine actual volume \% settled solids:

a. Height of solids:

b. Height of total sample:

c. Volume $\%$ settled solids:

$\frac{75}{81}$ Solids - yellow/gray rather even colorw/ some dark lokahd and light Create $\frac{\text { Height of solids }}{\text { Height of Total Sample }} \times 100=92.6 \%$ wii S cheers.

- Solids "mound" $\frac{1}{2}$

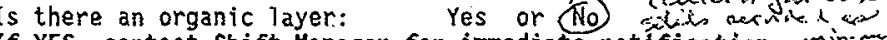
to tank farms.

8. Other Information:Repat @BRkbwist on 596 T004024

QR 2D. Determine bulk density of sample? Yes or No

Q) If 1. Calculate bulk density of sample, if required: (BLKDENO1)

a. Sum Net Weight for all cones
b. Sum Total volumes for ail cones $904.61 \mathrm{~g}$ ace

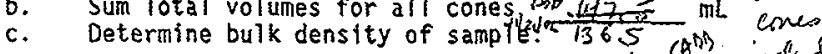
$\frac{\text { Sum of Net Weight }}{\text { Sum of Total volume }}$

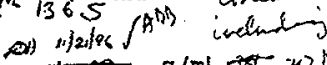

d. Other Information:

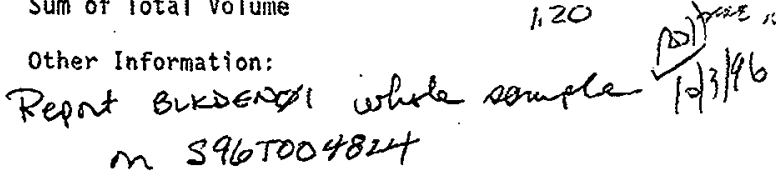

115 
HNF-SD-WM-DP-214, REV. 0

RAEE. Determine bulk density of solids? Yes or No Late 1. Calculate bulk density of solids, if required: (BLKDENOI)

a. Sum Wat. Solids for all cones $\frac{119.14}{9}$ ixcludery

b. Sum Vol. Solids for all cones 94.5 Th $\mathrm{mL}$ comes

c. Determine bulk density of solids: $10 / 2 / 96$ zeD $\{20 \mathrm{C}$

Sum of Wat. Solids

$1.26 . \mathrm{g} / \mathrm{mL}$

d. Other Information:

Repent B(kDEN \&) solids orly on 5967005017

ell F. Determine bulk density of liquids? (res or No

Pele 1. Calculate bulk density of liquids, if required: (BLKDENO1)

a. Wot liquids for all cones. $27.2 \mathrm{~g}$ Itychuliry

Wit 1 liquids = Sum of Net Wit - Sum of Wat solids coven 200 t $t$

b. Vol. liquids for all cones $25 . \mathrm{mL}$

Vol liquids $=$ Sum of Total vol - Sum of Vol solids

c. Determine bulk density of liquids:

$$
\frac{\text { Wat. Liquids }}{\text { Vol. Liquids }}
$$

$1.09-9 / \mathrm{mL}$

d. Other Information:

Repeat BCKDENDI liquids only on 5965005083

RQE2G. Determine volume \% solids by centrifugation? Yes or No

1. Calculate volume \% solids by centrifugation, if required: (V\%SLD-01)

a. Sum of Vol solids for all cones $945 \mathrm{~mL}$ extudio

c. Determine volume $\%$ solids by centrifugation: $\mathrm{mL}$. Comes

$\frac{\text { Sum of Vol solids }}{\text { Sum of Total Volume }} \times 100$

$79.1 \%$

d. Other Information:

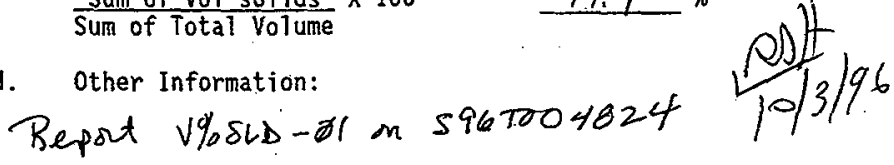

116

$07 / L \varepsilon$ d $\quad 2 \varepsilon 9 g^{\circ} \mathrm{N}$

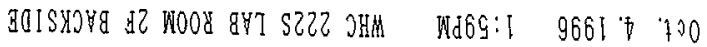


.C.: RA Esch

\section{ink $/$}

roject: Aw-105 GRABS

\section{Sianci Time:}

Ead Tine:

Centrifugation

Ti me (Min.):

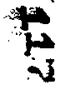

Wgt. Cone + Smp: 67.61

Wgt. Cone + Sol: SSt6se

Wgt. Cone:

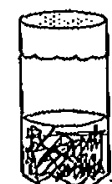

.

Net Weight: $53,29 \mathrm{~g} 200$

Total Vol. minuias
Sample ID: $5 A \omega-96-20$

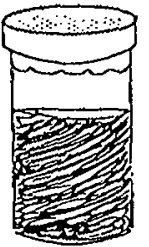

Sample ID: 5967004824 Jar/Vial Size: $125^{\prime} \mathrm{mL}$ Initial Weight: $30226 \mathrm{~g}$ Final Weight: $135,47 \mathrm{~g}$ Net Weight: $\quad 166,79 \mathrm{~g}$
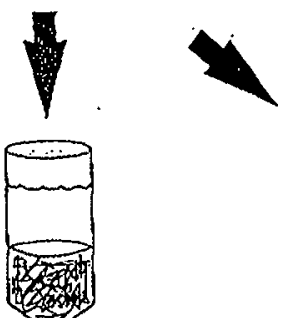

Conet: Wgt. Cone + Smp:67.39g Wgt. Cone + Sol: $59,34 \mathrm{~g}$

Wgt. Conc:

Net Weight: 53.06 Total Yol.:

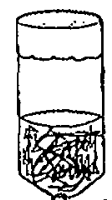

Conet:
ConeA: $20 \mathrm{~A}$

Ind Vol. Solids: 375

Vol. Solids: $36.0 \mathrm{~mL}$

Wgt. Cone + Smp:54.27g

200 Wgt. Cone + Sol:47,08 8 Lifwi. Wgt. Cone: $\frac{14.28}{39.99} \mathrm{~g}$ Total Vol.: 32.5 tesmi Vol. Solids: 260 $g$ 8.05, Wgt. Solids: $45.01 \mathrm{~g}$ 319y Wgt. Solids:

Appearance/Special Instructions:

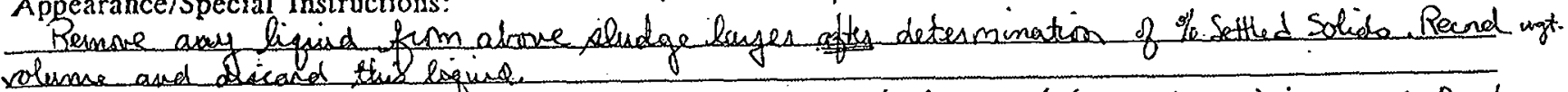

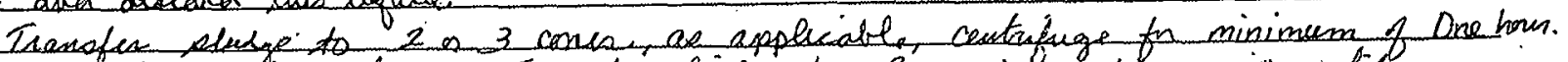

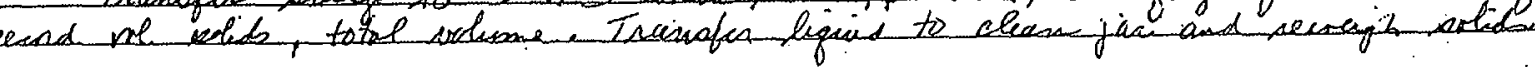

V\%SLD-01? $(9$ or $N$
Date: $/ 1 / 1 / 16$

Labcore Entry

Initials:

BLKOOEN01? Oor $\mathrm{N}^{\circ}$

hyius \& somis seis

$20 \mathrm{D} 20 \mathrm{~F}$
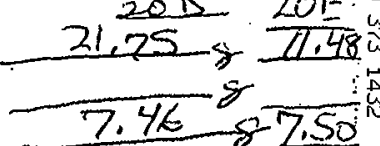

mLi

$\mathrm{mL}$

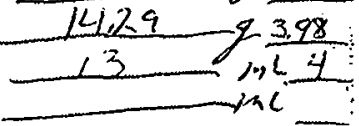




\section{Subsample: Grab Sludge Worksheet}

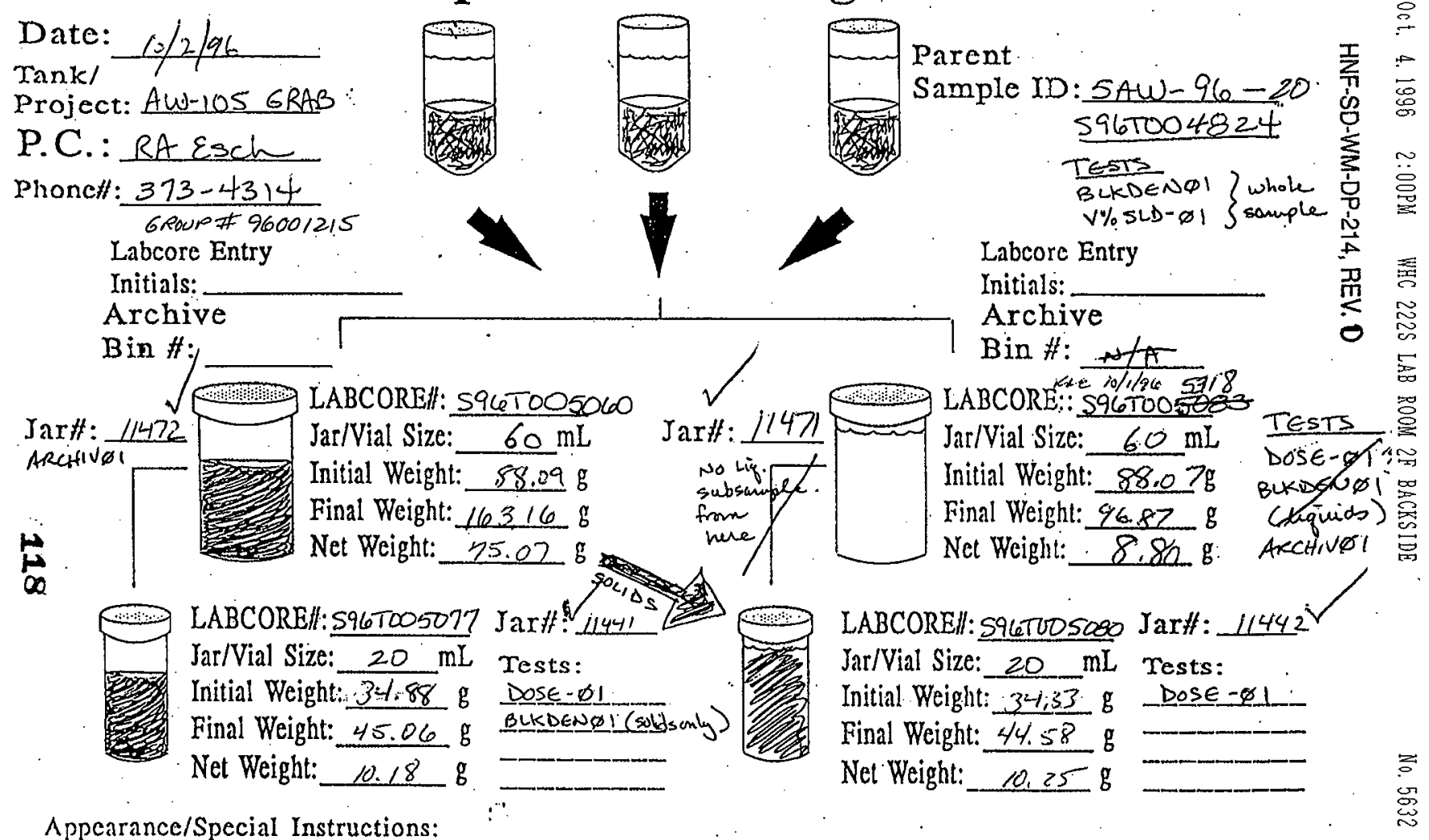

Subsample 8-10 fo analypia aud archive semaining * 2 solid subomples fum archlin jos 5967005000 . 


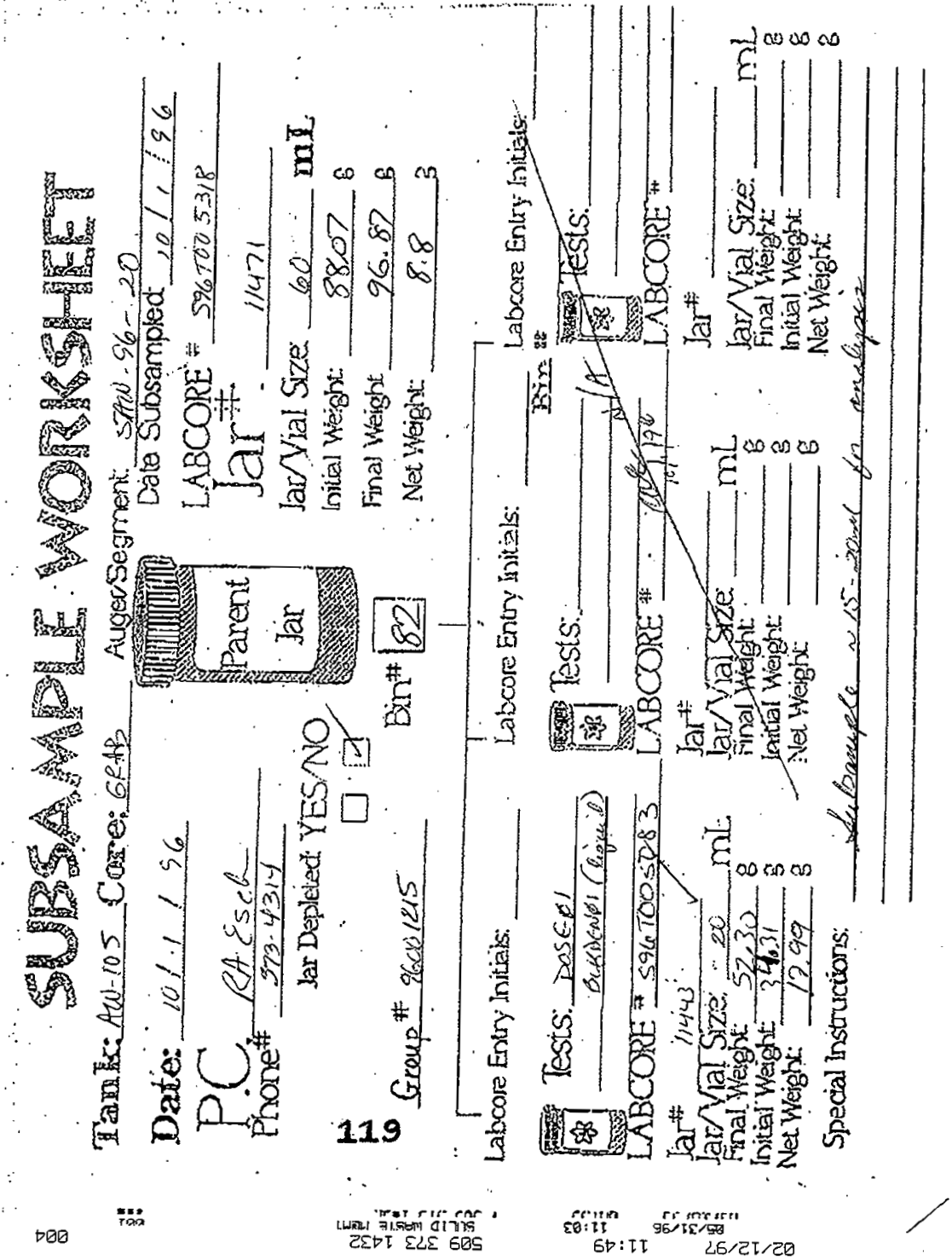




\section{Analyst: $\quad D P B \quad$ Instrument: NONE}

Book \# $N / A$

Method: LA-519-151 Rev/Mod E-2

Worklist Comment: AW105 @BRKDWN1 - rae

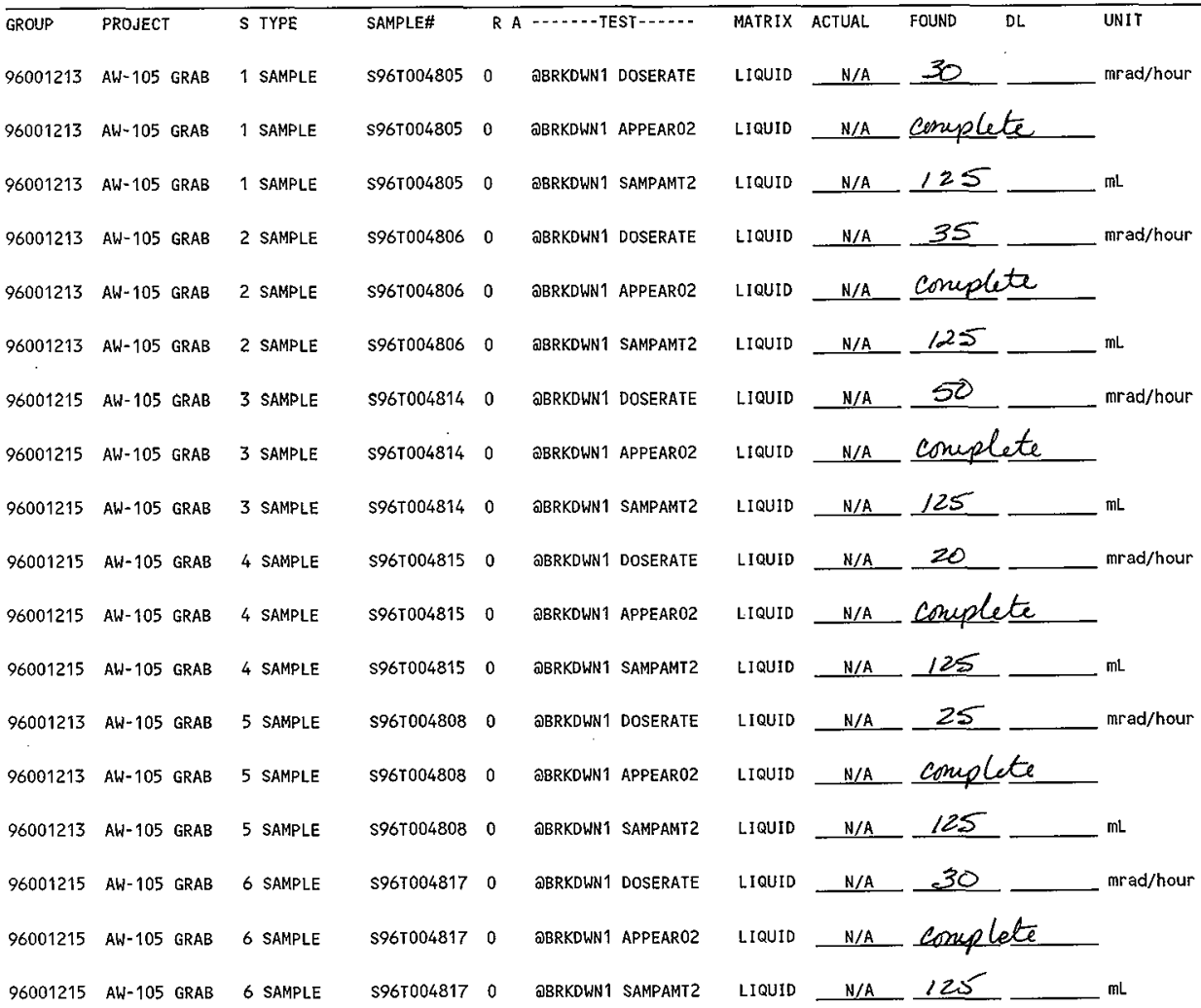

Data Entry Comments:
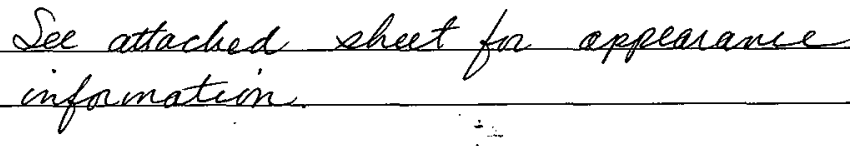

Units shown for $Q C$ (SPK \& STD) may not reflect the actual units. $D L=$ Detection Limit, $S=$ Worklist Slot Number, $R=$ Replicate Number, $A=$ Aliquot Code. 
HNF-SD-WM-DP-214, REV. D

worklistrpt Version 2.1 05/15/95

Page: 2

10/07/96 08:00

LABCORE Data Entry Template for Worklist\# 13738

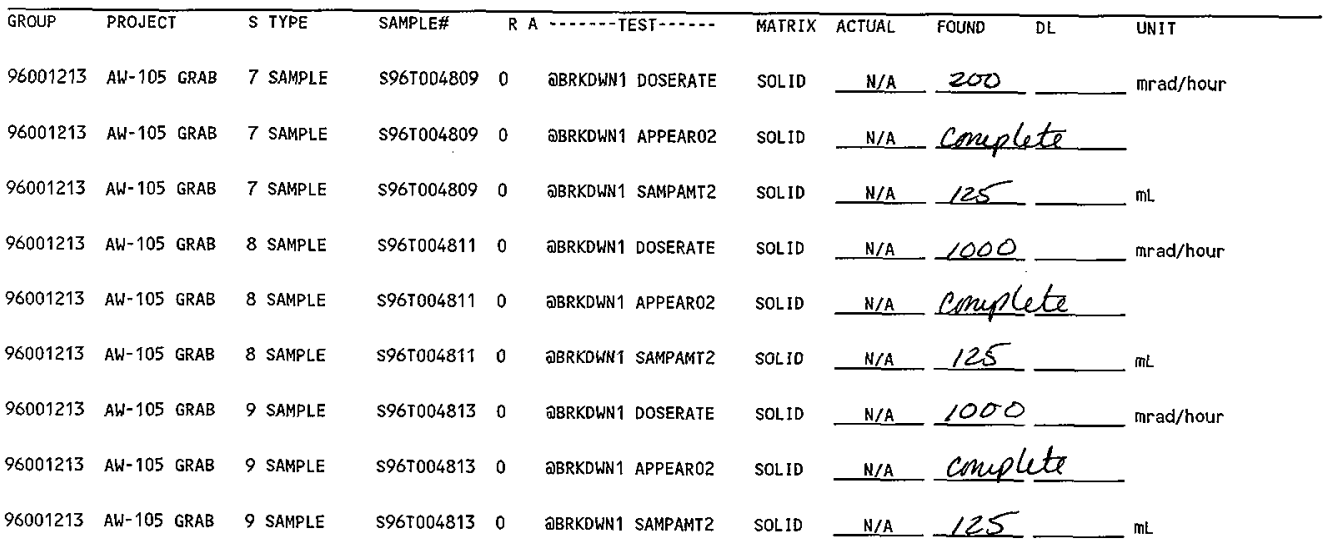

Final page for worklist \# 13738

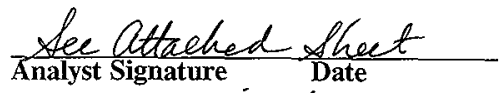

for segnatives

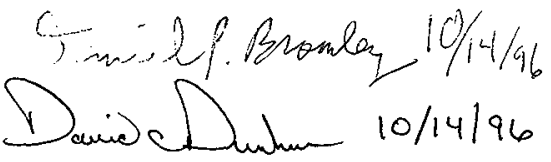

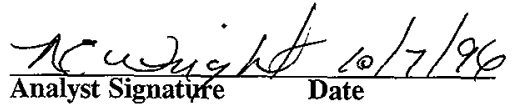

Validated

Data Entry Comments:

Units shown for $Q C$ (SPK \& STD) may not reflect the actual units. $D L=$ Detection Limit, $S=$ Worklist Slot Number, $R=$ Replicate Number, $A=$ Aliquot Code.

121 
HNF-SD-WM-DP-214, REV. D

Pig Breakdown Information $w น+13738$

\begin{tabular}{|c|c|c|c|c|c|}
\hline ABCorE Pig\# & Vol (mL) & Clarity & Color & $\%$ Solids & Dose Rate (mRad/hr) \\
\hline 61004805 5AW-96-1 & 125 & clear & yellow & $<1$ & 30 \\
\hline $4806 \quad 5 A W-96-2$ & 125 & clear & yellow & $<1$ & 35 \\
\hline 4808 5AW-96-4 & 125 & clear & light yellow & $<1$ & 25 \\
\hline $48095 \mathrm{AW}-96-5$ & 125 & clear & yellow & 20 & 200 \\
\hline $48115 \mathrm{AW}-96-7$ & 125 & opaque & brown-yellow & 95 & 1000 \\
\hline 4813 5AW-96-9 & 125 & opaque & brown-yellow & 95 & 1000 \\
\hline $48145 \mathrm{AW}-96-10$ & 125 & clear & yellow & $<1$ & 50 \\
\hline $48155 \mathrm{AW}-96-11$ & 125 & clear & light yellow & $<1$ & 20 \\
\hline 4817 5AW-96-13 & 125 & clear & light yellow & $<1$ & 30 \\
\hline
\end{tabular}

Pigs remaining to be broke down: 5AW-96-15, 5AW-96-17, 5AW-96-20

Dan P. Bromley David C. Dunham

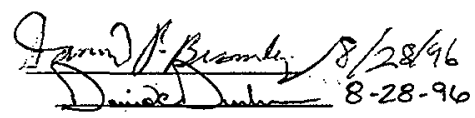


HNF-SD-WM-DP-214, REV. 0

Sample Preparations 
HNF-SD-WM-DP-214, REV. 0

THIS PAGE WAS INTENTIOANLLY LEFT BLANK 
Oct.19. 1996 5:09 AM WHC 222S LAB ROOM 2F BACKSLDE

No. 6037 P. $5 / 6$ worklistrpt Version $2.105 / 15 / 95$ HNF-SD-WM-DP-214, REV. 0

Page: I 09/25/96 09:57

LABCORE Data Entry Template for Worklist\# 13318

Analyst: Instrument: FUS01 Book \# $\mu / A$

Method: LA-549-141.Rev/Mod 7.0

Worklist Comment: TANK\#AW-105 Grab SEGMENT:5AW-96-5,5AW-96-7,5AW-96-9.FUS.new

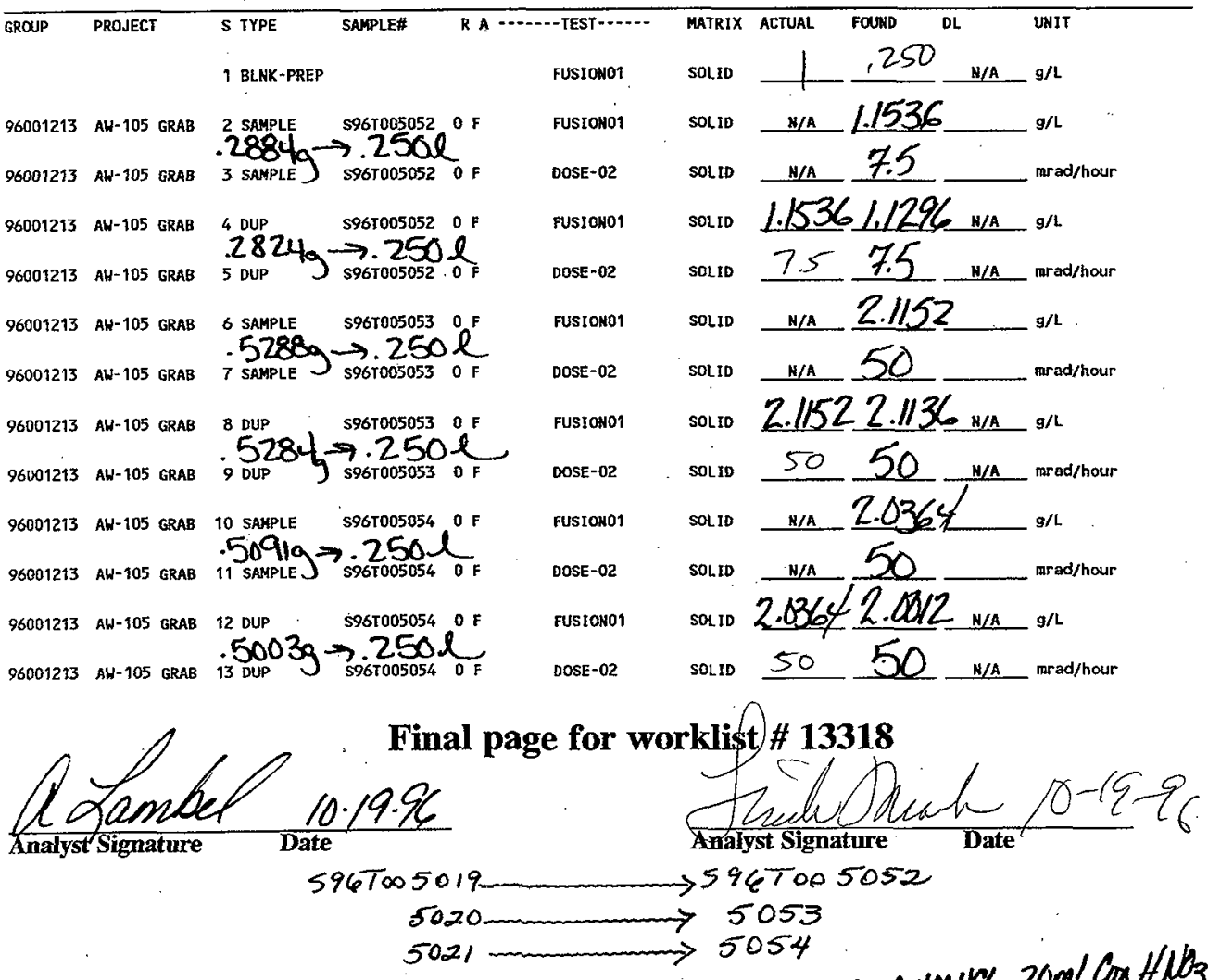

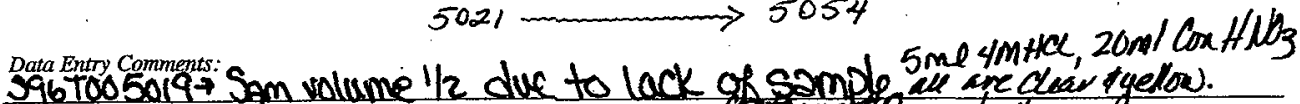
H PT

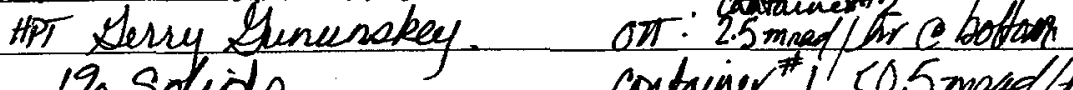
190 Solids. contoiner"I C0.5mad/hi@boton Units shown for $Q C$ (SPK \& STD) may not reflect the actual units. $D L=$ Detection Limit, $S=$ Worklist Slot Number, $R=$ Replicate Number, $A=$ Aliquot Code.

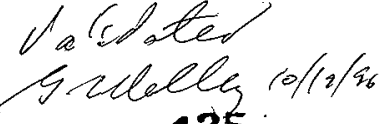
125 
Oct. 19. 1996 5:07AM THC 222S LAB ROOM 2F BACKSIDE

No. 6037 P. $1 / 6$ worklistrpt Version 2.1 05/15/95 HNF-SD-WM-DP-214, REV. 0

Page: 1 09/25/96 09:59

LABCORE Data Entry Template for Worklist\# 13319

\begin{tabular}{lll}
\hline Analyst: $\quad$ Instrument: FUS01 & Book \# $M / A$ \\
Method: LA-549-141 Rev/Mod of 0 &
\end{tabular}

Worklist Comment: TANK\#AW-105 Grab SEGMENT:5AW-96-15,17,20. FUSION. new

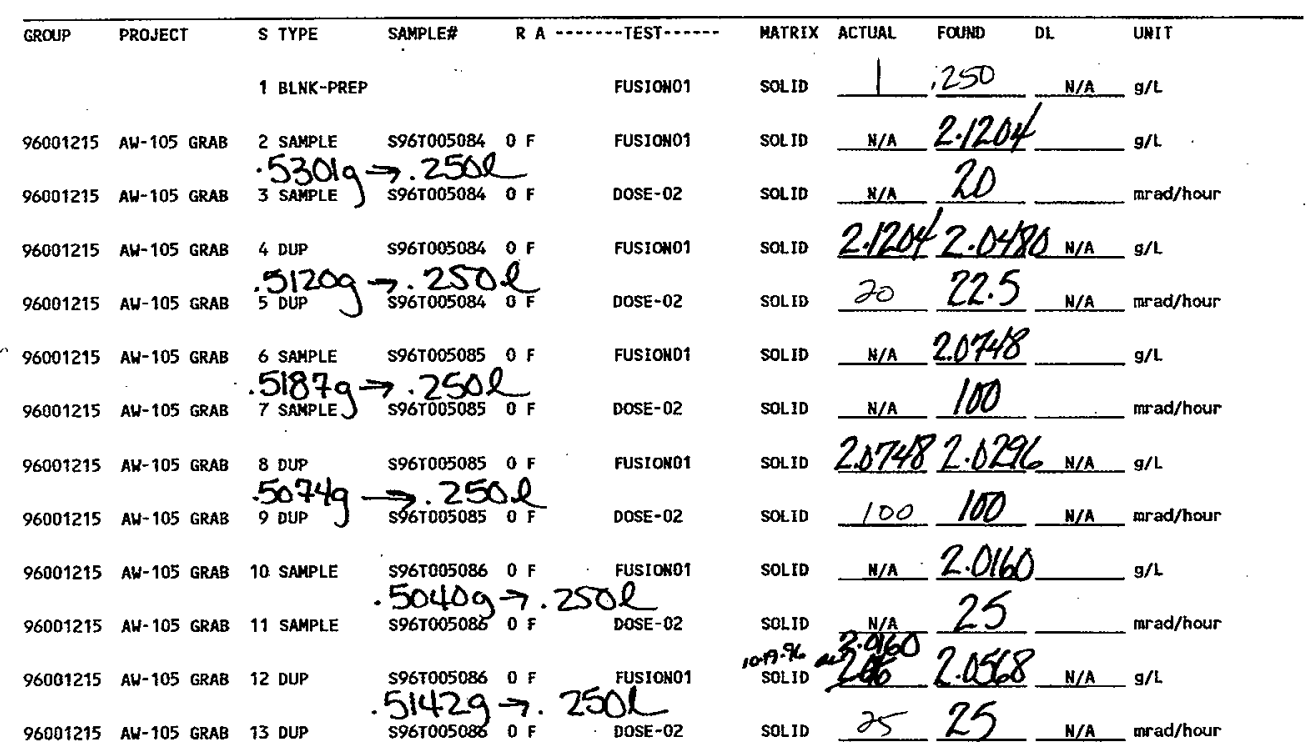

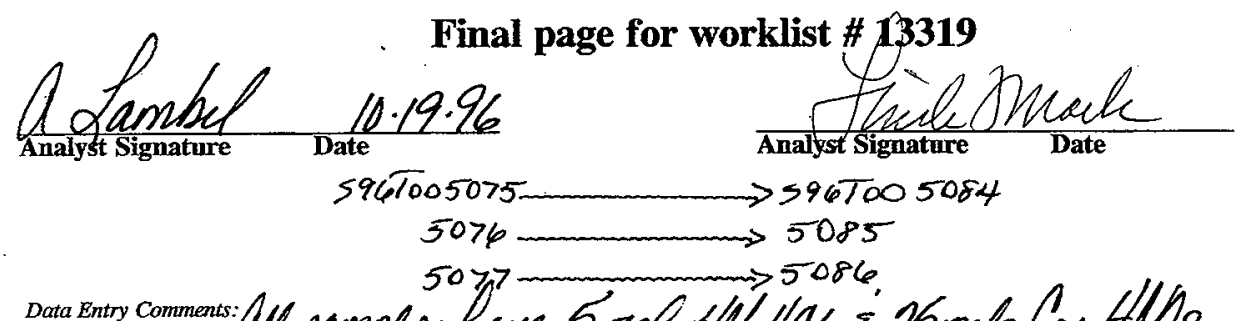

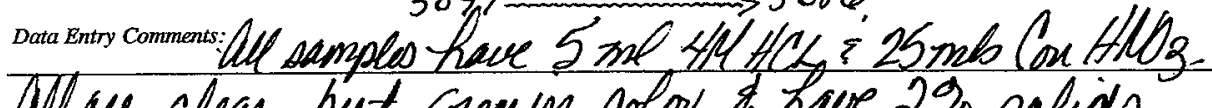
Well are clear but Grey in color \& have 29 solids

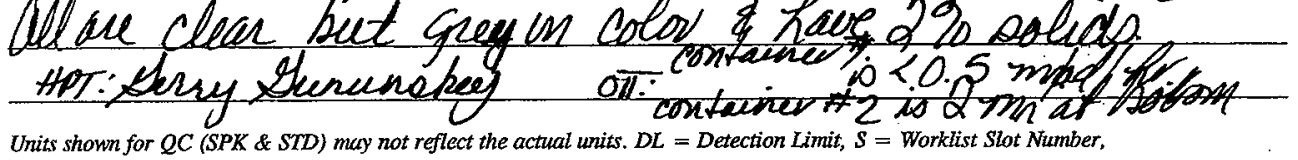
$R=$ Replicate Number, $A=$ Aliquot Code.

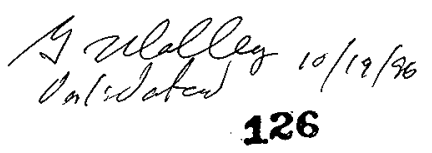


oct.19. $1996 \quad 5: 08 \mathrm{AM}$

WHO $222 S$ LAB ROOM OF BACKSIDE

No. 6037

P. $2 / 6$

workitistrpt Version 2.1 05/15/95

HNF-SD-WM-DP-214, REV. 0

Page: $\quad I$ 10/19/96 0I:05

LABCORE Data Entry Template for Worklist\# 13320

Analyst: $\quad$ \&AC Instrument: $\mathrm{H} 200 \mathrm{1}$

Book \# N $/$ A

Method: LA-504-101 Rev/Mod E.O

$$
120 \text { ar } 10 \cdot 19 \cdot 9
$$

Worklist Comment: TANK\#AW-105 GRAB SEGMENT:5AW-96-5,5AW-96-7.7USTON. new

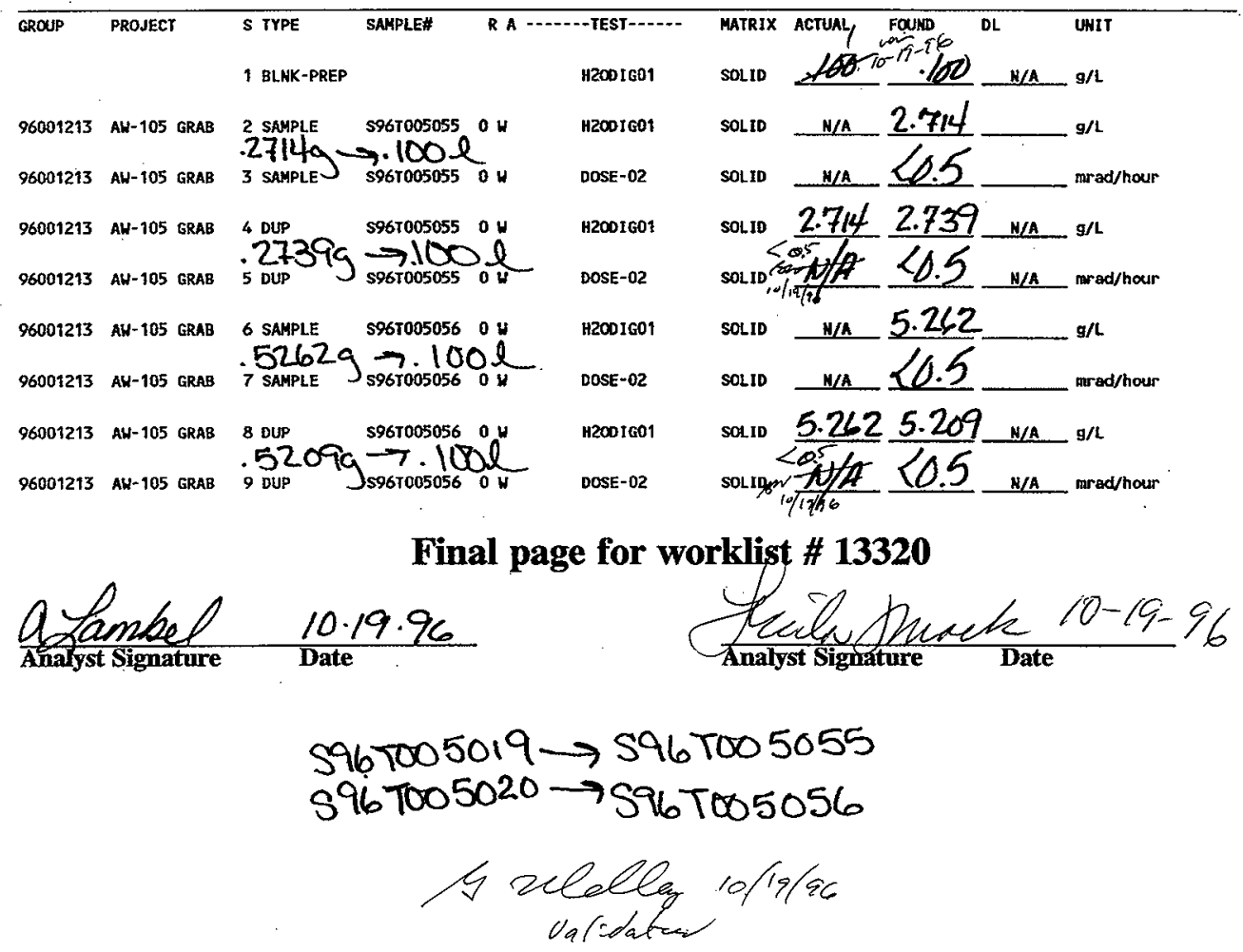

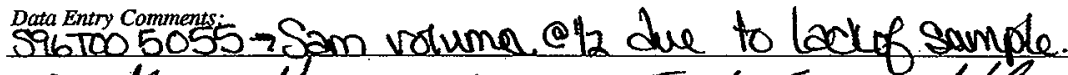
HPT: Derry funumaley oT: 20.5 moat for

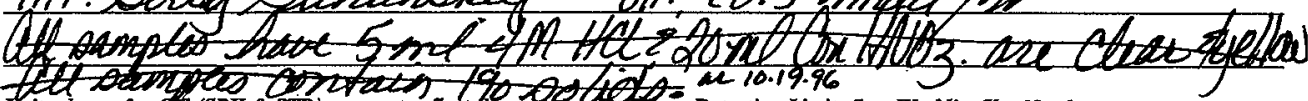
Units shown for $Q C$ (SPK \& STD) may not reflect the acturlinth. DL $=$ Detection Limit, $S=$ Worklist Slot Number,
$R=$ Replicate Number, $A=$ Aliquot Code. Kith-please correct the heeding

127 
Oct. 19.1996 5:08AM WHC 222S LAB ROOM 2F BACKSIDE

No. 6037 P. $3 / 6$

worklistrpt Version 2.1 05/15/95

HNF-SD-WM-DP-214, REV. 0

Page: 1

09/25/96 10:02

LABCORE Data Entry Template for Worklist\# 13321

Analyst: $\frac{Q A L}{\text { Instrument: } H 2001}$ Book $\frac{\mu / t}{\mathrm{H}_{2} \mathrm{O}}$

Worklist Comment: TANK\#AW-105 GRAB SEGMENT:5AW-96-9,5AW-96-15. DU ON. new

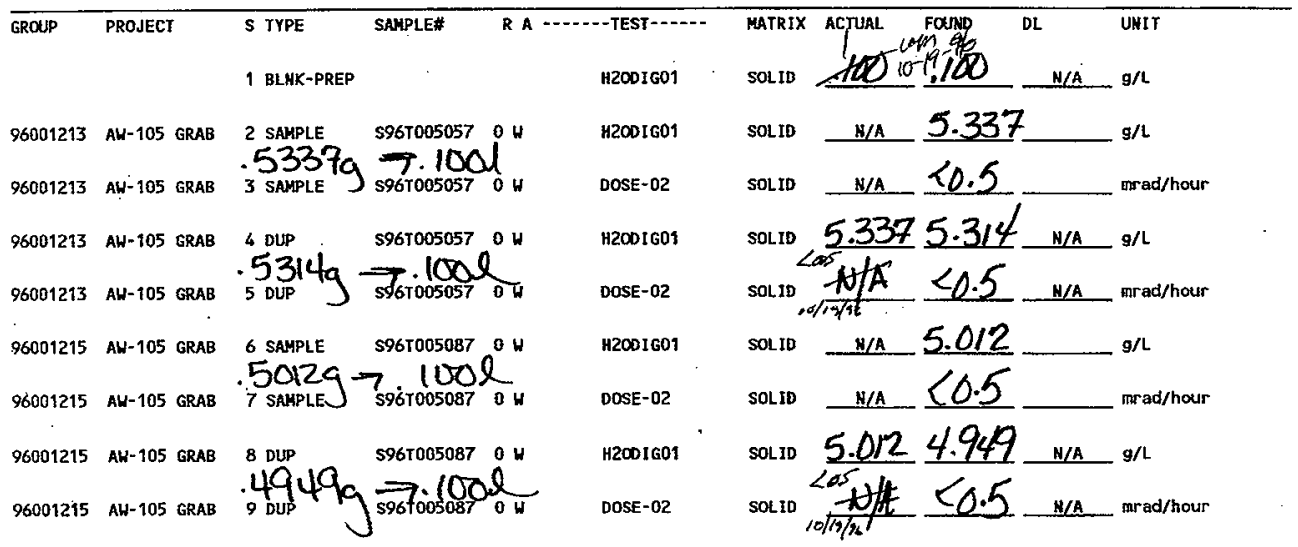

Final page for worklist/\# 13321
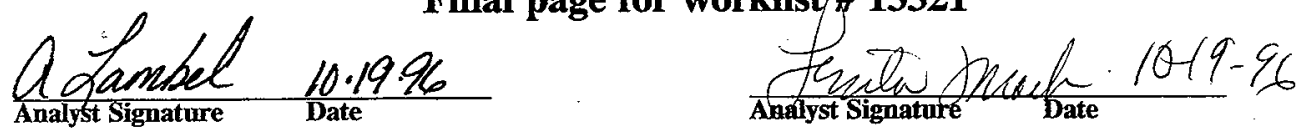

$$
5961005021
$$$$
\rightarrow \text { S96Te05057 }
$$

5075

$\rightarrow 5087$

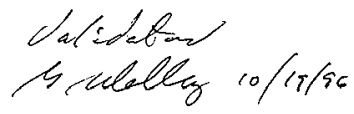

Data Entry Comments: APT: Derry Dunuskey oT: 20.5 mad far

Units shown for $Q C$ (SPK \& STD) may not reflect the actual units. $D L=$ Detection Limit, $S=$ Worklist Slot Number, $R=$ Replicate Number, $A=$ Aliquot Code.

128 
Oct .19.1996 5:09 AM

worklistrpt Version $2.105 / 15 / 95$
WHO $222 S$ LAB ROOM $2 F$ BACKSIDE

HNF-SD-WM-DP-214, REV. O

No. 6037 P. $4 / 6$ 09/25/96 10:04

LABCORE Data Entry Template for Worklist\# 13322

Analyst: $\quad$ Instrument: $\mathrm{H} 2 \mathrm{CO}$.

Book \# $N / A$

Method: LA-504-101 Rev/Mod E.U

Worklist Comment: TANK\#AW-105 GRAB SEGMENT:5AW-96-17,5AW-96-20. Hz OH. new

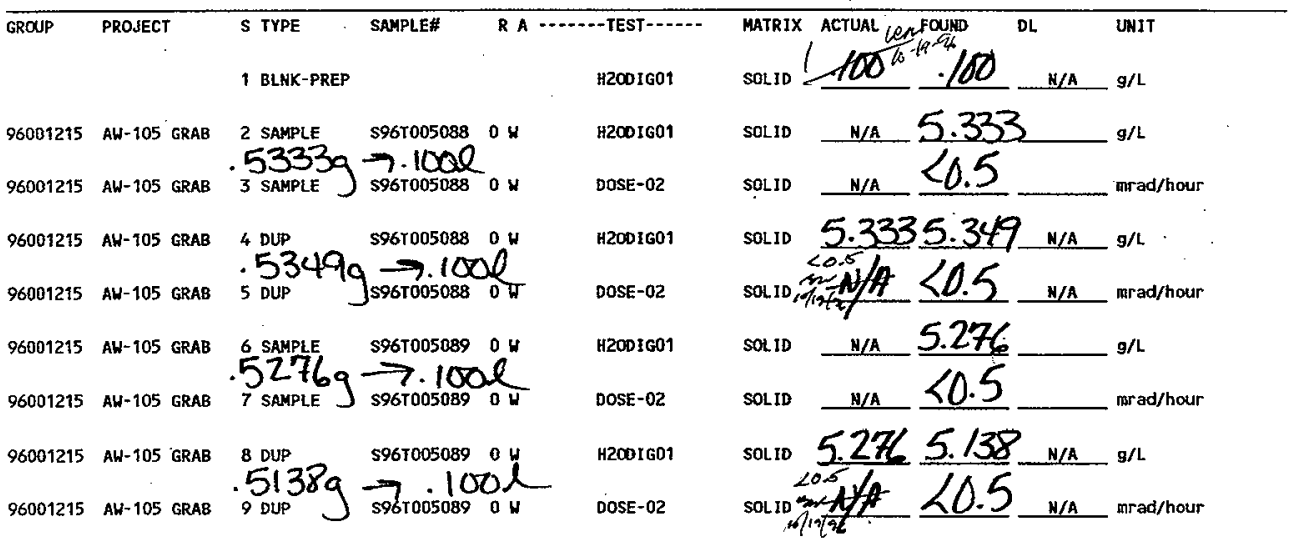

Final page for worklist $\$ \mathbf{1 3 3 2 2}$
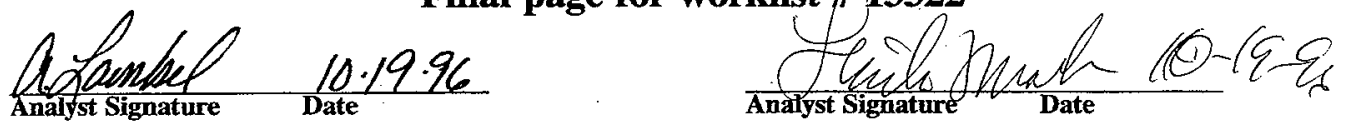

$$
\begin{aligned}
& 596 T 005076 \\
& \rightarrow 596 T 005088 \\
& 5086 \longrightarrow 5089 \\
& \text { Validation } \\
& \text { I cublleg io/ is/96 }
\end{aligned}
$$

Data Entry Comments:

H PT: Derry Zunundey

on: $<0.5$ mad the

Units shown for QC (SPK \& STD) may not reflect the actual units. $D L=$ Detection Limit, $S=$ Worklist Slot Number, $R=$ Replicate Number, $A=$ Aliquot Code.

129 
HNF-SD-WM-DP-214, REV. 0

Inorganic Analysis 
HNF-SD-HM-DP-214, REV. 0

\section{THIS PAGE WAS INTENTIOANLLY LEFT BLANK}




\section{LABCORE Data Entry Template for Worklist\# 13070}

Analyst: JLF Instrument: $\operatorname{DSCO}^{\prime}$ Book \# I2NIUB

Method: LA-514-113 Rev/Mod C - 1

Worklist Comment: AW-105 DSC, RUN UNDER N2. RCJ

\begin{tabular}{|c|c|c|c|c|c|c|c|c|c|c|}
\hline GROUP & PROJECT & $\begin{array}{l}\text { S TYPE } \\
1 \text { STD }\end{array}$ & SAMPLE\# & R A & W-TH & $\begin{array}{l}\text { MATRIX } \\
\text { LIQUID }\end{array}$ & $\begin{array}{l}\text { ACTUAL } \\
284\end{array}$ & $\begin{array}{l}\text { Found } \\
28,5\end{array}$ & $\begin{array}{l}\text { OL } \\
\quad \text { N/A }\end{array}$ & Joules/g \\
\hline 96001213 & $A H-105$ GRAB & 2 SAMPLE & s96T005004 & 0 & DSC-0t & LIQUID & N/A & $\emptyset$ & & Joules/g \\
\hline 96001213 & AL-105 GRAB & 3 DuP & S96T005004 & 0 & $D S C-01$ & LIQUID & . & $\phi$ & H/A & Joules/g \\
\hline 96001213 & AL-105 GRAB & 4 SAMPLE & \$96T005005 & 0 & DSC-01 & LIQUID & N/A & $\phi$ & & Joules/g \\
\hline 96001213 & $A H-105$ GRAB & 5 DUP & s96T005005 & 0 & DSC-01 & LIQUID & $\phi$ & $D$ & $\mathrm{~N} / \mathrm{A}$ & Joules/g \\
\hline
\end{tabular}

\section{Final page for worklist \# 13070}
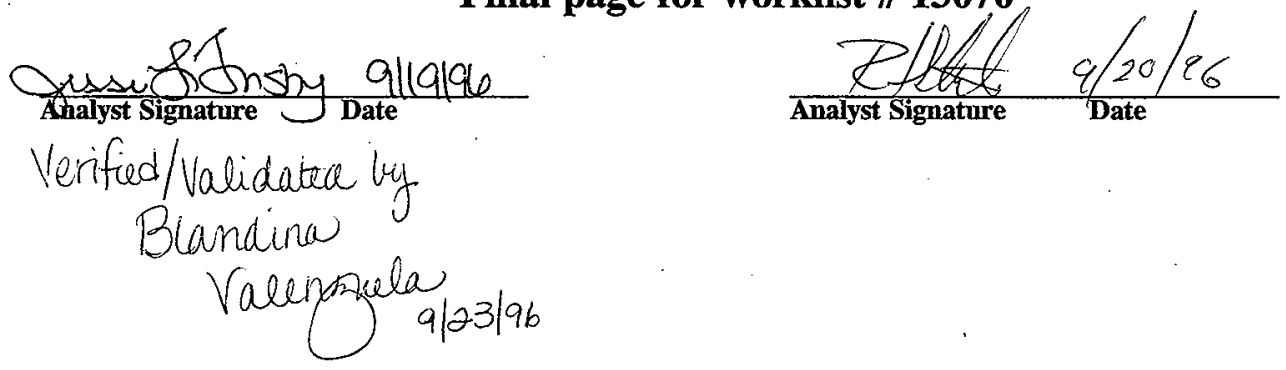

Analyst Signature $\bigcup$ Date

Analyst Signature

Date

Data Entry Comments:

Units shown for $Q C$ (SPK \& STD) may not reflect the actual units. DL = Detection Limit, $S=$ Worklist Slot Number, $R=$ Replicate Number, $A=$ Aliquot Code. 


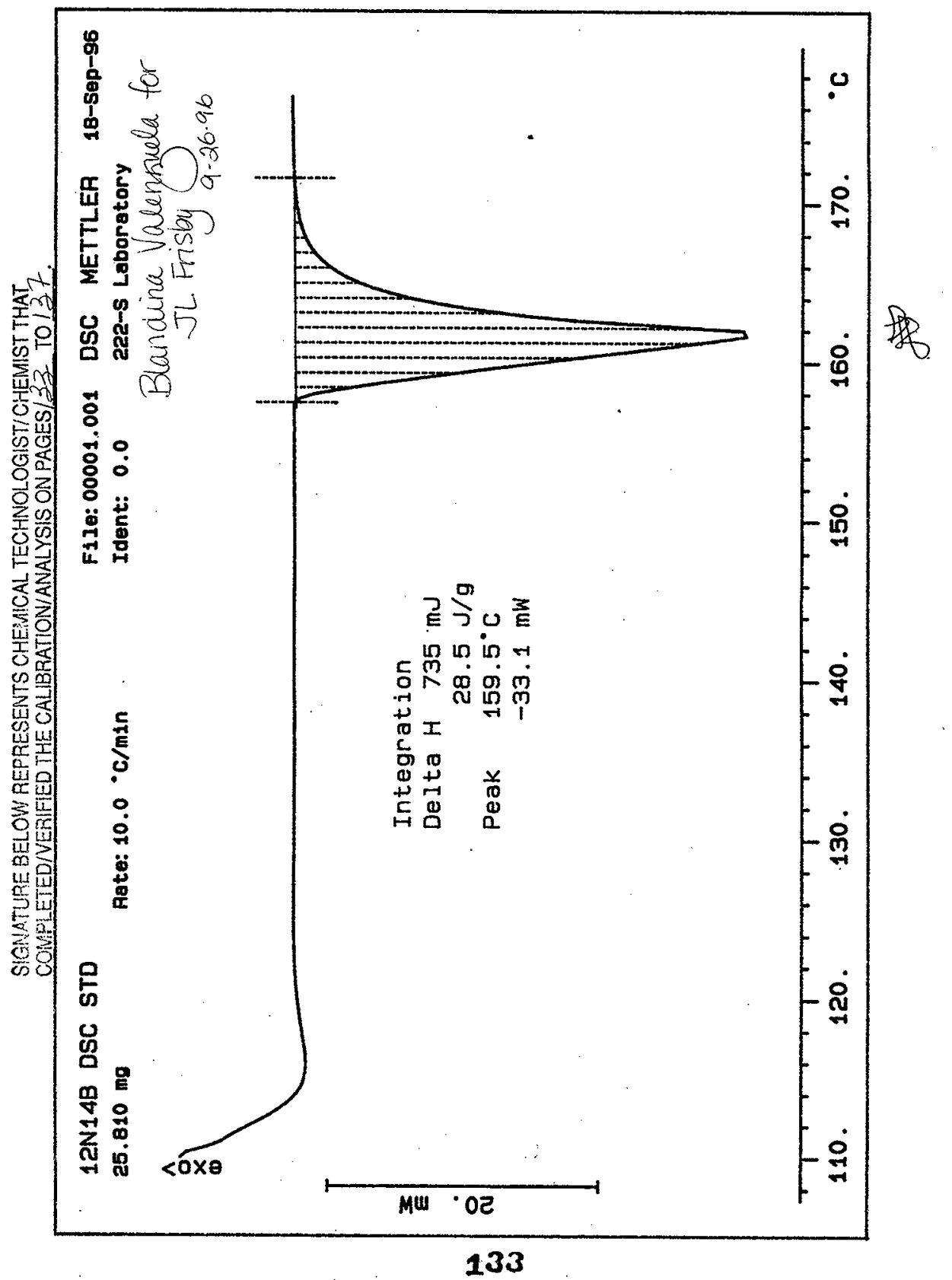




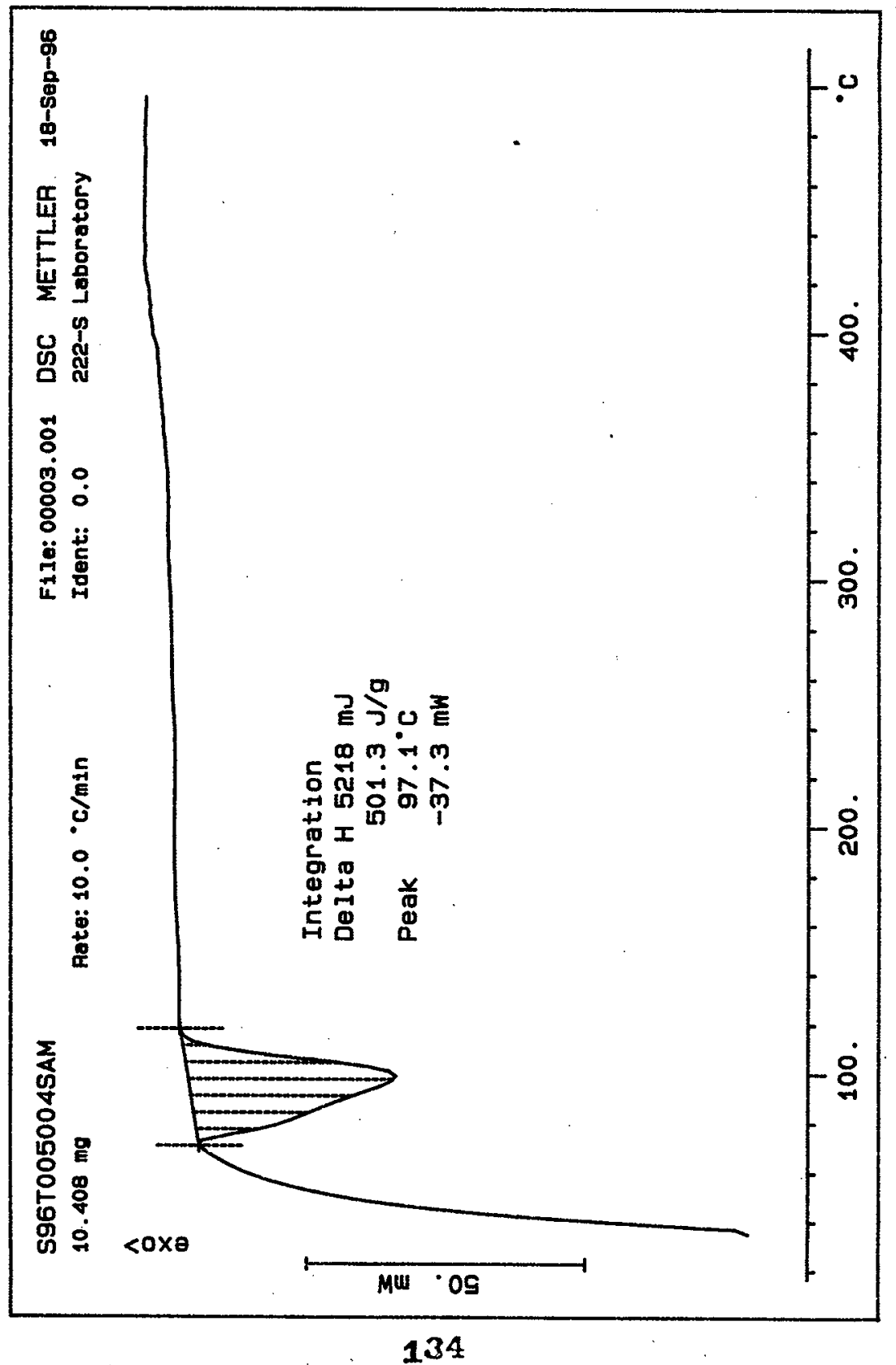


HNF-SD-WM-DP-214, REV.O

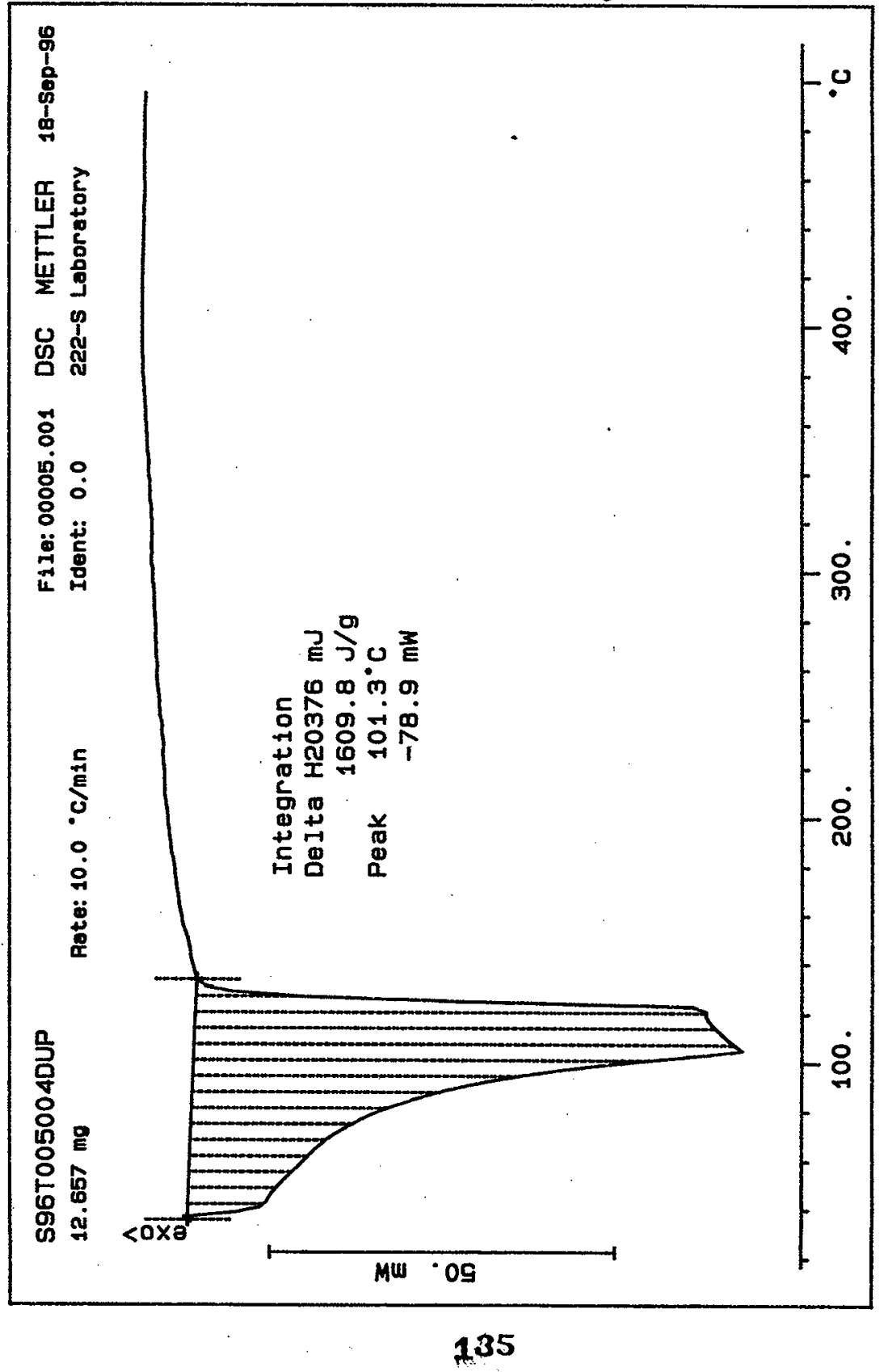




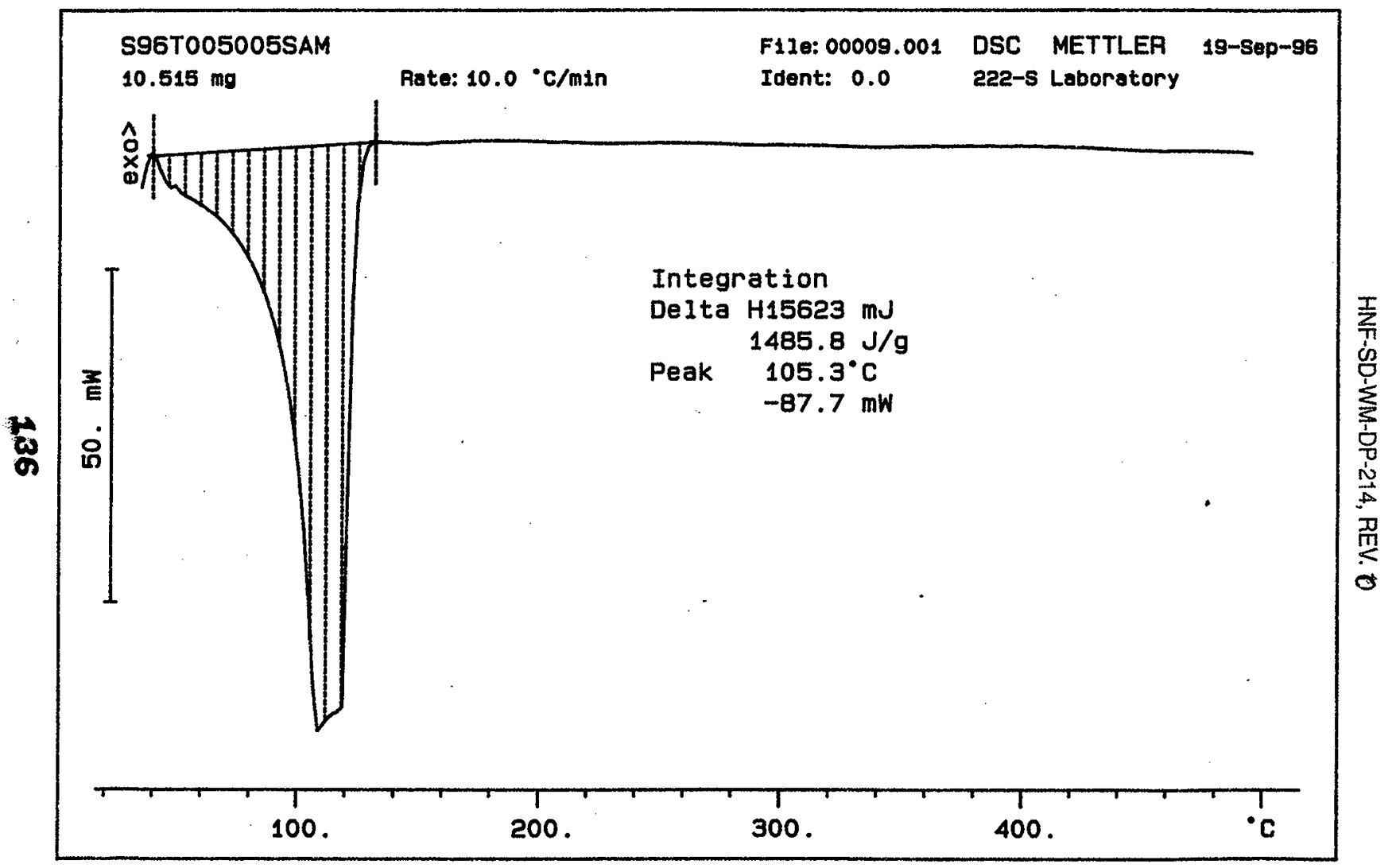




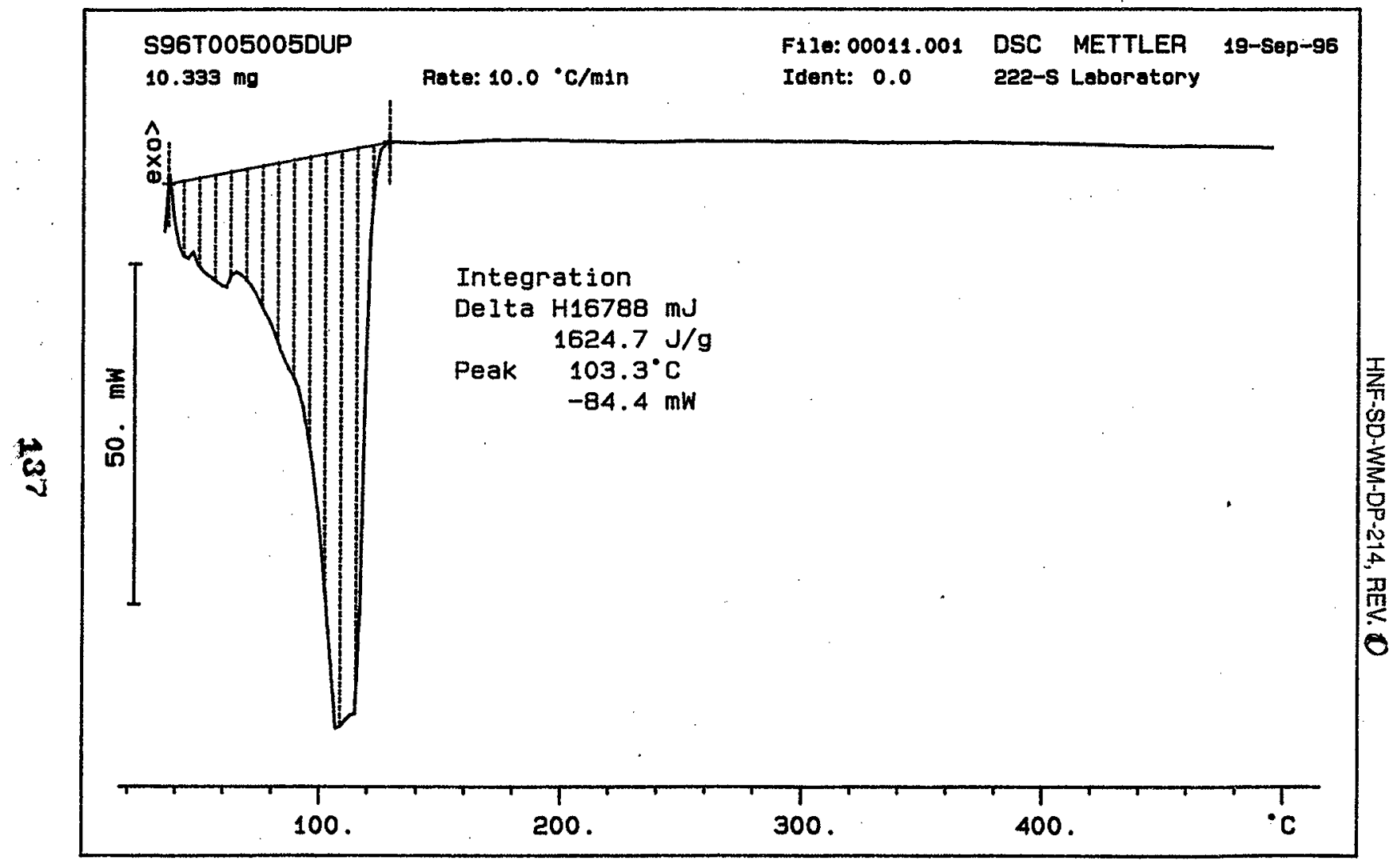




\section{LABCORE Data Entry Template for Worklist\# 13072}

Analyst: JLF $\quad$ Instrument: $\mathrm{DSCO}_{3}$ Book \#12NIAB

Method: LA-514-114 Rev/Mod C-

Mer 2/19/97

Worklist Comment: AW-105 DSC, RUN UNDER N2. RCJ

\begin{tabular}{|c|c|c|c|c|c|c|c|c|c|c|}
\hline GROUP & PROJECT & $\begin{array}{l}\text { S TYPE } \\
1 \text { STD }\end{array}$ & SAMPLE\# & R A & $\begin{array}{l}\text { DSC }-03 \\
\text { D }\end{array}$ & $\begin{array}{l}\text { MATRIX } \\
\text { LIOUID }\end{array}$ & $\begin{array}{l}\text { ACTUAL } \\
28.45 \\
\end{array}$ & $\begin{array}{l}\text { FOUND } \\
27.28 \\
\end{array}$ & N/A & Joules/g \\
\hline 96001213 & $A N-105$ GRAB & 2 SAMPLE & S96T005006 & 0 & DSC-03 & LIOUID & H/A & & & Joules/g \\
\hline 96001213 & AW- 105 GRAB & 3 DUP & S96T005006 & $\mathbf{0}$ & OSC- 03 & LICUID & & & $N / A$ & Joules/g \\
\hline
\end{tabular}

\section{Final page for worklist \# 13072}

\section{$\frac{\text { An attached }}{\text { Analyst Signature }}$}

Venfied/Validated biy

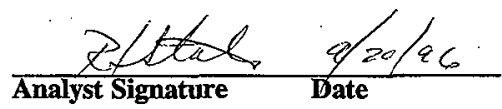

Blandina

Valengulas $9 / 23196$

Data Entry Comments:

Units shown for $Q C$ (SPK \& STD) may not reflect the actual units. $D L=$ Detection Limit, $S=$ Worklist Slot Number, $R=$ Replicate Number, $A=$ Aliquot Code. 
worklistrpt Version 2.1 05/15/95

Page: $\quad I$

09/18/96 12:22

LABCORE Data Entry Template for Worklist\# 13072

Analyst: JLF Instrument: DSC0 Book \#12N14B

Method: LA-514-113 Rev/Mod

Worklist Comment: AW-105 DSC, RUN UNDER N2. RCJ

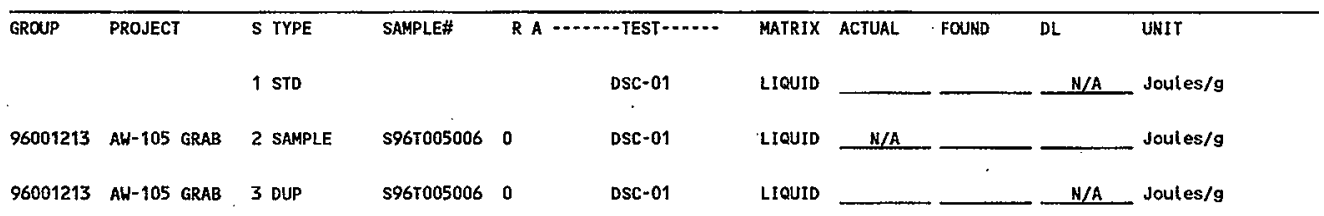

Final page for worklist \# 13072

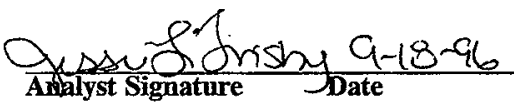

Analyst Signature Date

DSC-03 instrument

was used.

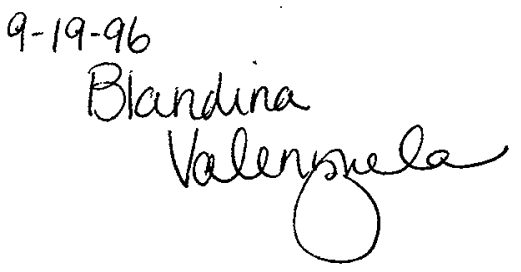

Data Entry Comments:

Units shown for $Q C$ (SPK \& STD) may not reflect the actual units. $D L=$ Detection Limit, $S=$ Worklist Slot Number, $R=$ Replicate Number, $A=$ Aliquot Code.

139 
Curve 1: DSC

F11e info:-IND091801 Wed Sep 18 17: $27: 19$ 1996

Semple Weight: $6.580 \mathrm{mg}$

12N14-B INDIUM AT 10C/MIN

CONPLETED/VERIFIED THE CALIBRATION/ANALYSIS ON PAGES 140 TO 142

- 1 12N14-B INDIUM AT 10C/MIN: IND091801

Heat flow (min)

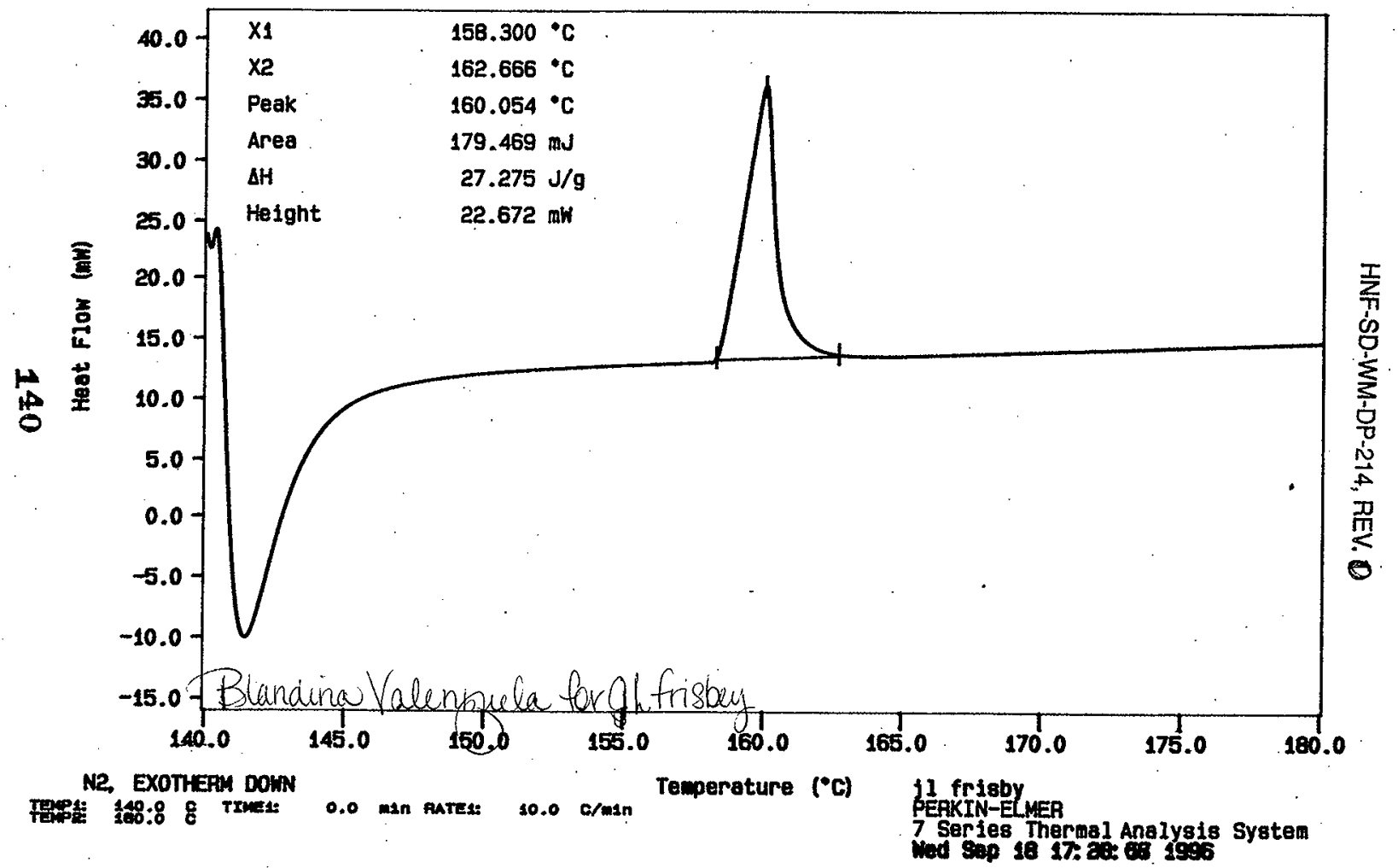


Curve 1: DSC

File info: SAMOg1803 Wed Sep 18 21: 30: 301996

Sample Weight: $12.020 \mathrm{mg}$

S96T005006SAM

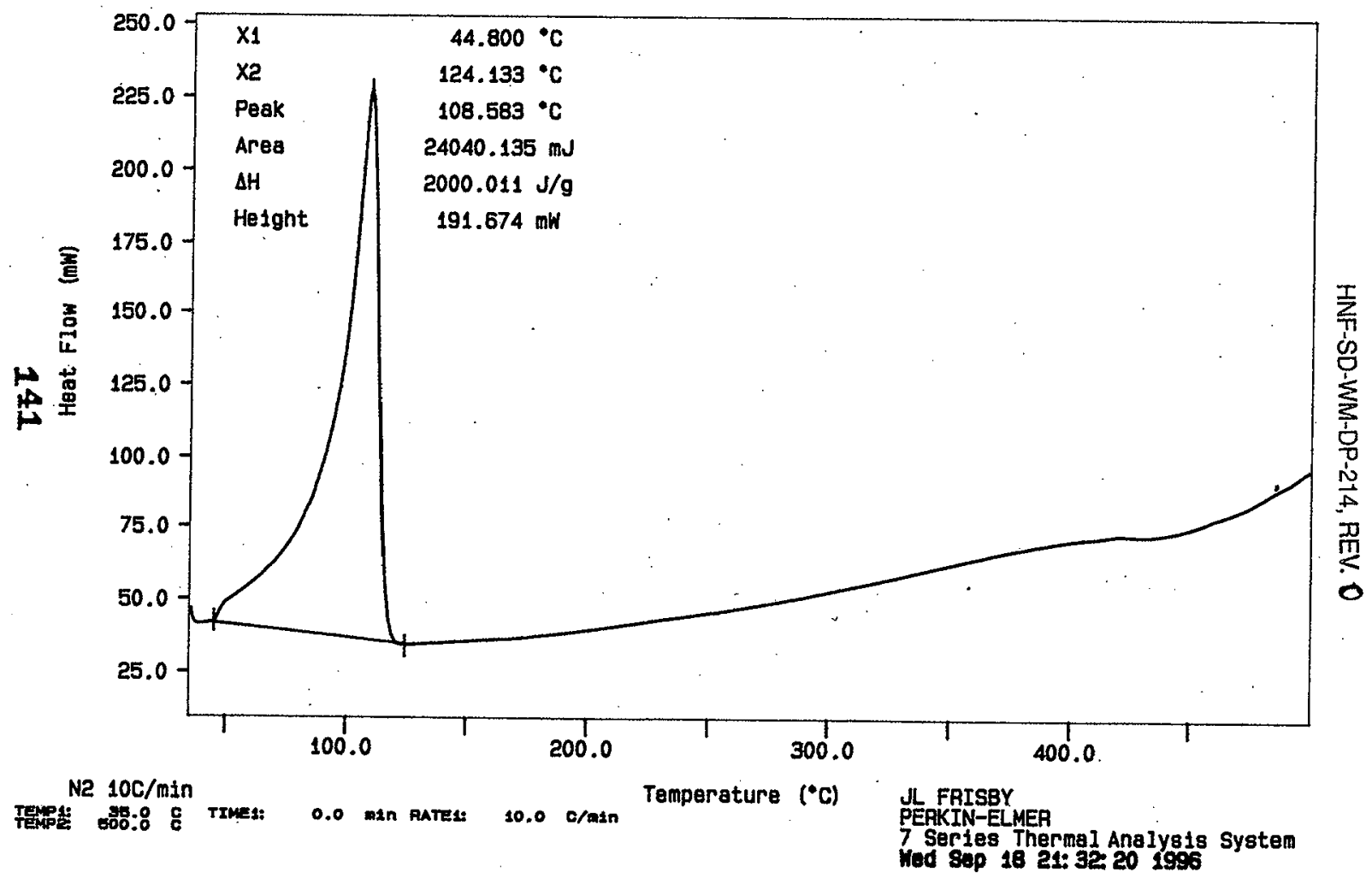


Curve 1: DSC

F11e Info: DUP091805 Hed Sep 18 22: 40: 451996

Sample Naight: 11.270 ag

sestoos006DUP

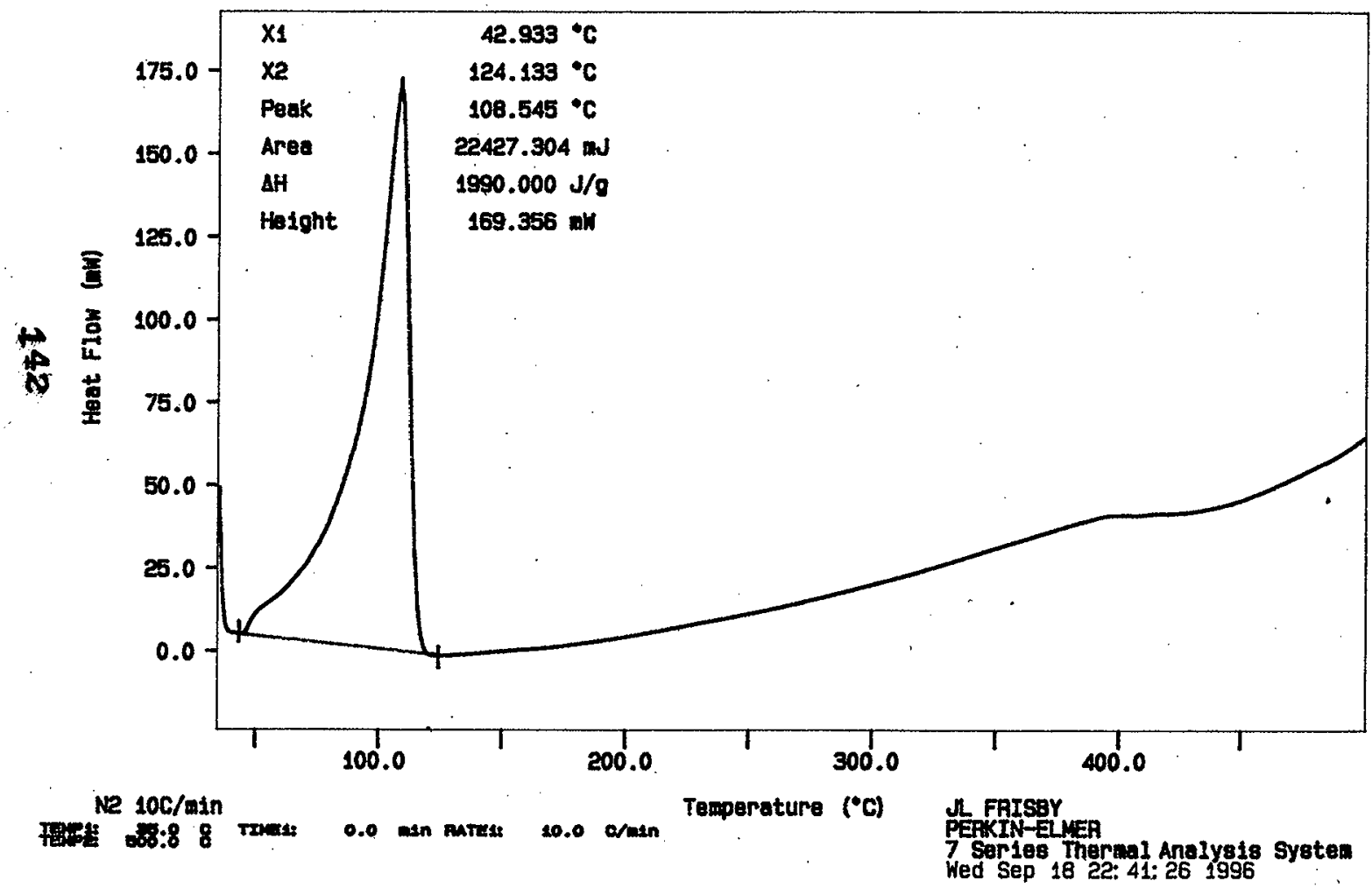




\section{LABCORE Data Entry Template for Worklist\# 13135}

\section{Analyst: $\mathrm{QAm}$ Instrument: $\mathrm{DSCO} \perp$ Book\# $12 \mathrm{~N} 14 \mathrm{~B}$}

Method: LA-514-113 Rev/Mod C-1.

Worklist Comment: AW-105 DSC,RUN UNDER N2. RCJ

\begin{tabular}{|c|c|c|c|c|c|c|c|c|c|c|c|}
\hline \multirow[t]{2}{*}{ GROUP } & \multirow{2}{*}{\multicolumn{2}{|c|}{ PROJECT }} & S TYPE & SAMPLE\# & \multicolumn{2}{|c|}{ R A $\cdots$ - . - TEST $-\cdots$} & \multirow{2}{*}{$\begin{array}{l}\text { MATRIX } \\
\text { LIQUID }\end{array}$} & \multirow{2}{*}{$\begin{array}{l}\text { ACTUAL } \\
28.45 \\
\end{array}$} & \multirow{2}{*}{$\begin{array}{l}\text { FOUND } \\
26.3 \\
\end{array}$} & \multirow{2}{*}{$\begin{array}{l}\text { DL } \\
\text { N/A }\end{array}$} & \multirow{2}{*}{$\begin{array}{l}\text { UNIT } \\
\text { Joules/g }\end{array}$} \\
\hline & & & 1 STD & & & DSC-01 & & & & & \\
\hline 96001235 & AH-105 & GRAB & 2 SAMPLE & S96T005010 & 0 & DSC-01 & LIQUID & N/A & $\varnothing$ & & Joules $/ 9$ \\
\hline 96001215 & AH -105 & GRAB & 3 DUP & $\$ 967005010$ & 0 & DSC-01 & LIQUID & $\phi$ & $\not$ & N/A & Joules/g \\
\hline 96001215 & AH- 105 & GRAB & 4 SAMPLE & S967005011 & 0 & DSC-01 & LIQUID & N/A & $\not D$ & & Joules/g \\
\hline 96001215 & AH-105 & GRAB & 5 DUP & S96T005011 & 0 & DSC-01 & LIQUID & $\phi$ & $\phi$ & $\mathrm{N} / \mathrm{A}$ & Joules $/ g$ \\
\hline
\end{tabular}

\section{Final page for worklist \# 13135}

A Aminghy $9-20-96$ Aralyst Signature Date Verified Nalidated by Blandina Valinarela

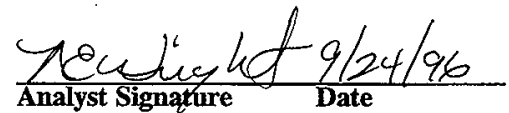

Data Entry Comments:

Units shown for QC (SPK \& STD) may not reflect the actual units. $D L=$ Detection Limit, $S=$ Worklist Slot Number, $R=$ Replicate Number, $A=$ Aliquot Code. 
SIGNATURE BELOW REPRESENTS CHE:IICAL TECHNOLOGIST/CHEMIST THHAT

COMPLETED/VERIFIED THE CALIBRATION/ANALYSIS ON PAGES 144 TO 48

Cotminpty 9-20.96

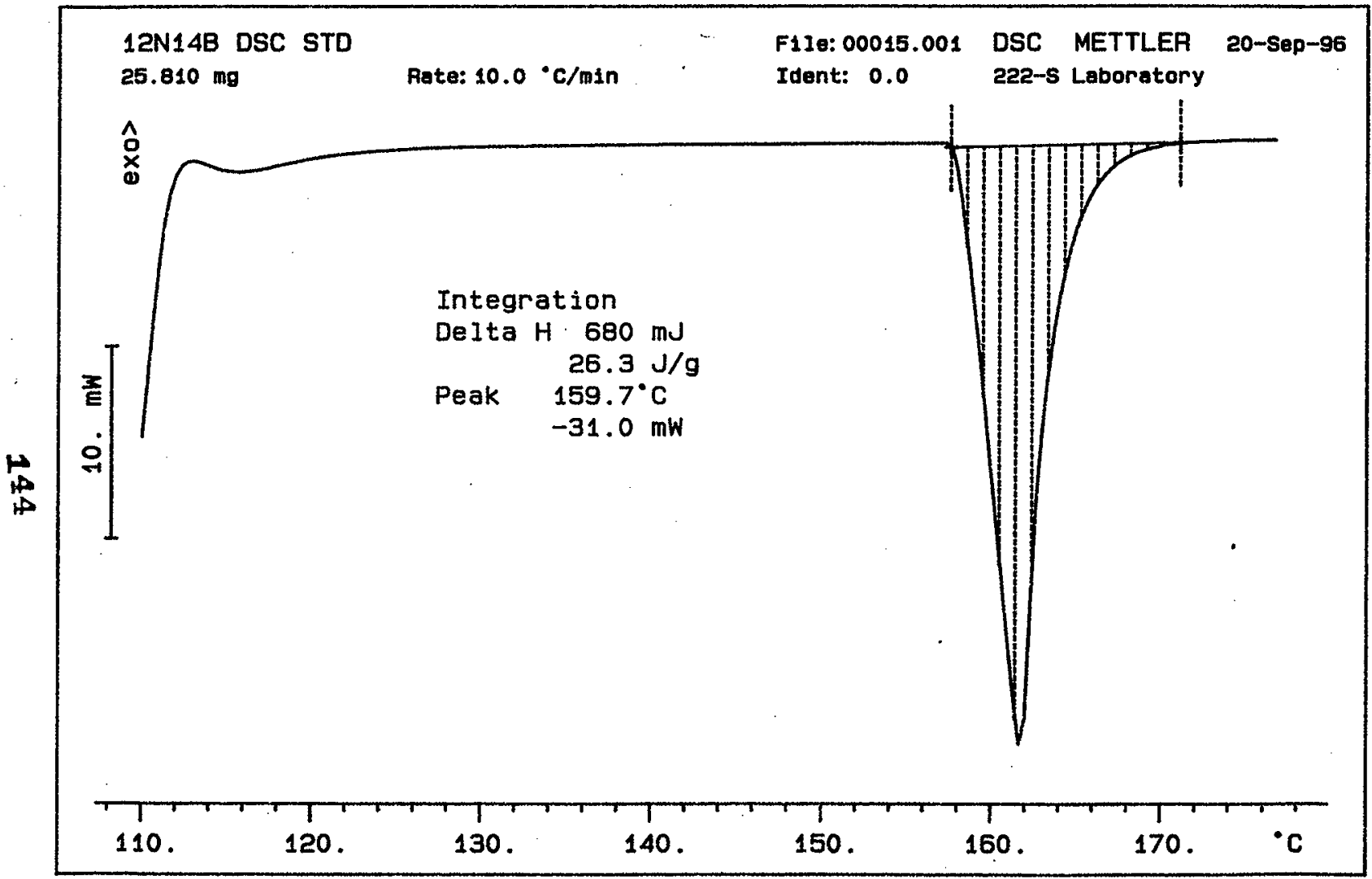




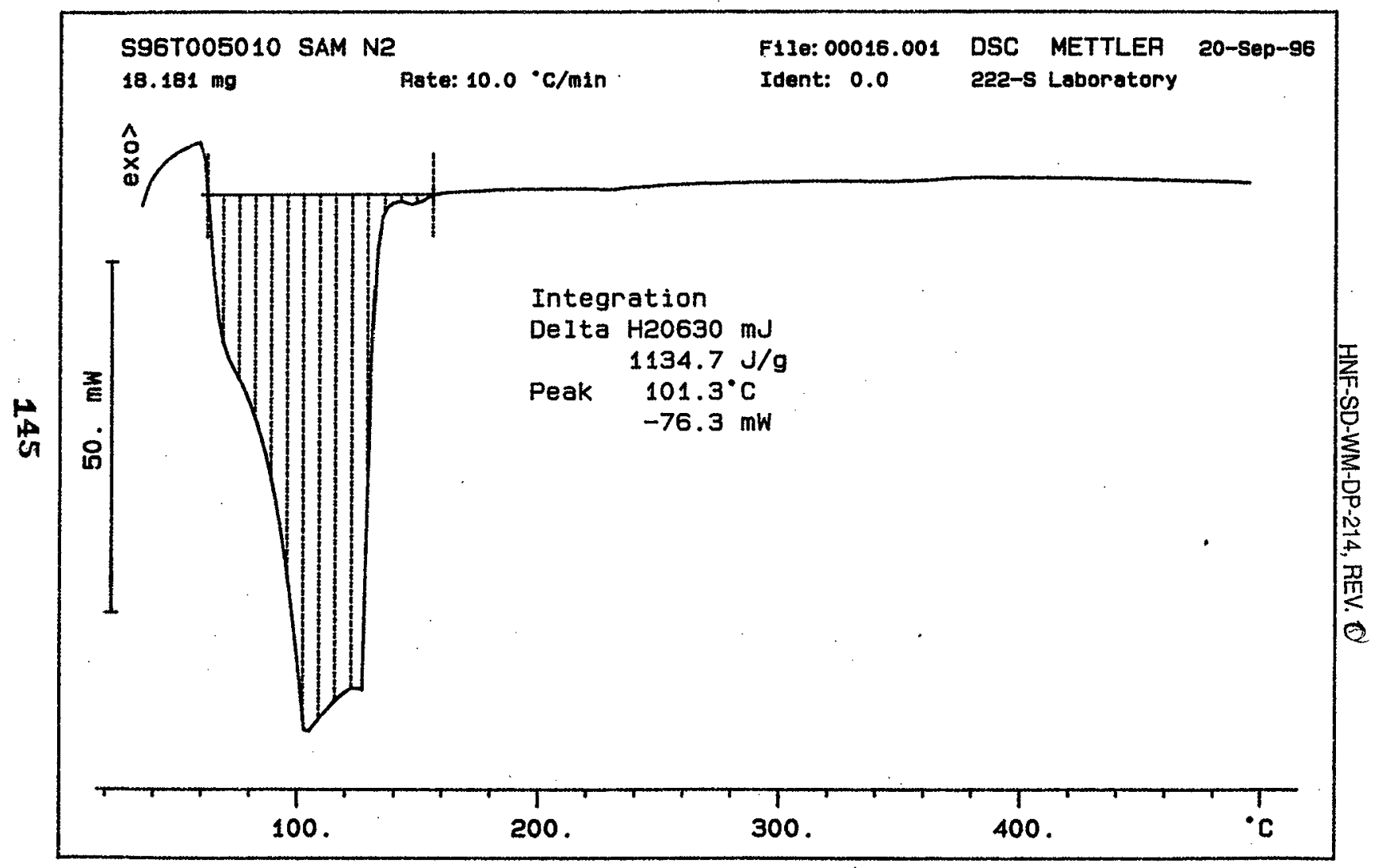




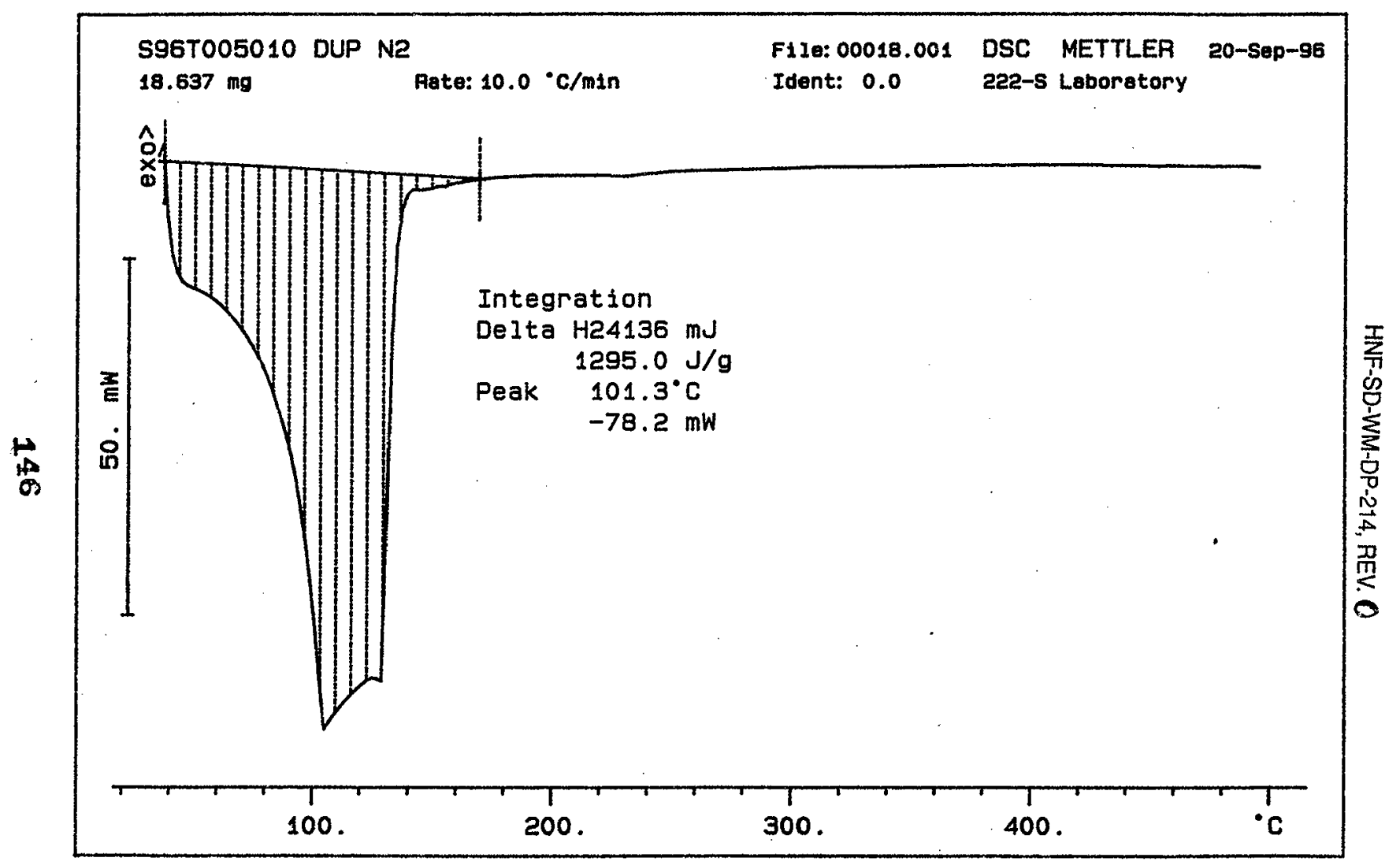




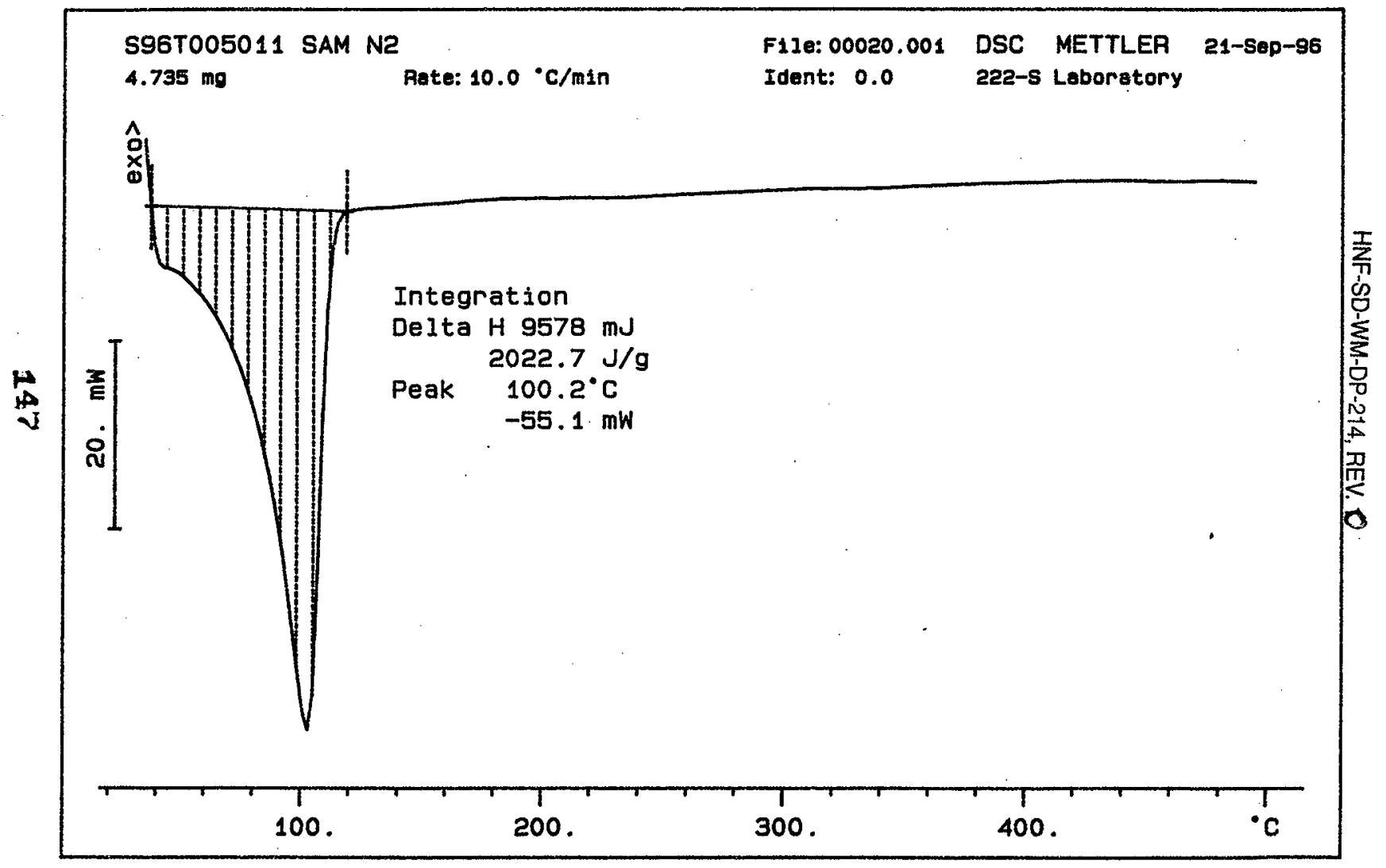




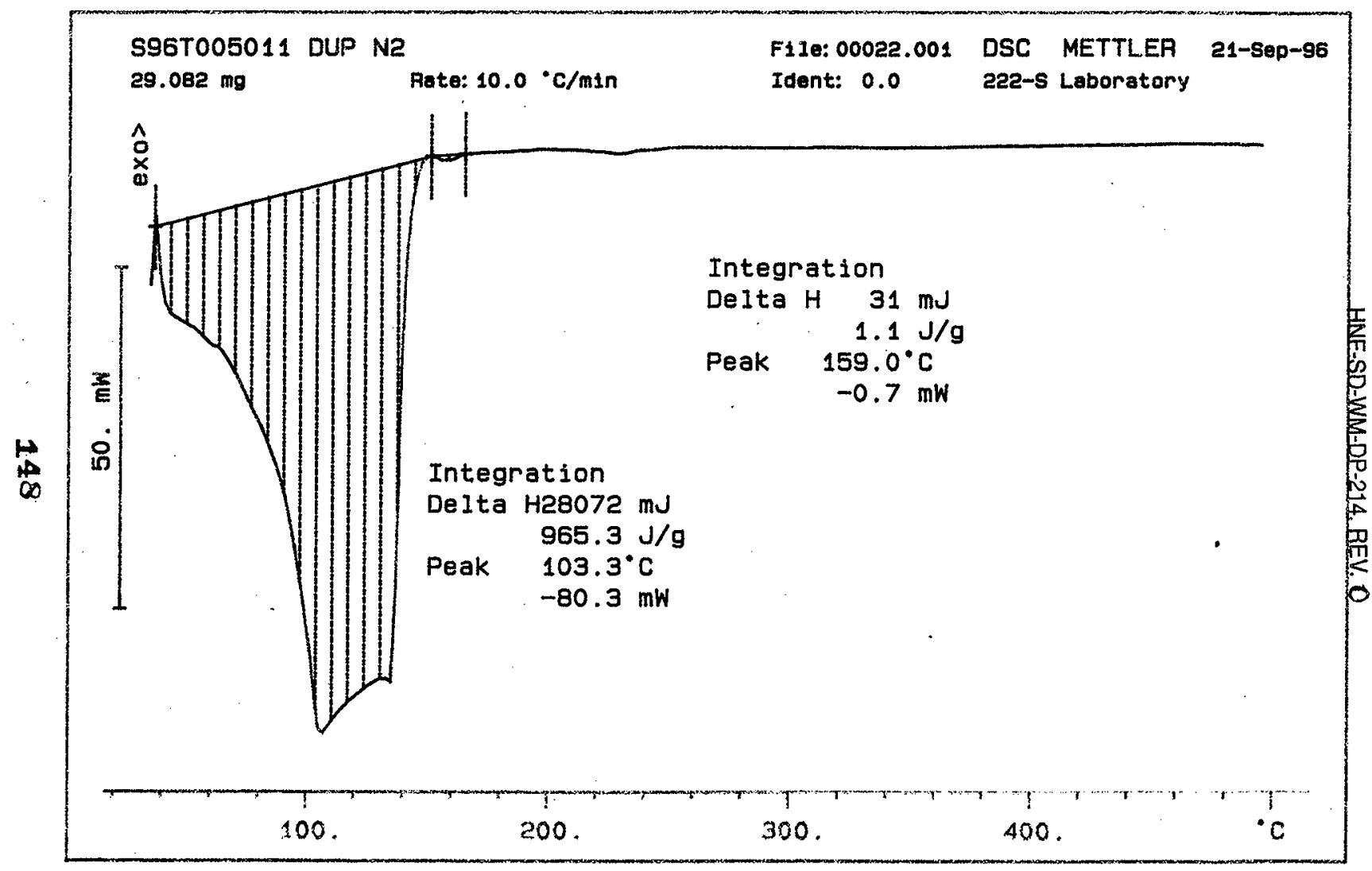


worklistrpt Version 2.1 05/15/95

HNF-SD-WM-DP-214, REV. 0

Page: $\quad I$

09/20/96 10:40

LABCORE Data Entry Template for Worklist\# 13136

Analyst: NAM Instrument: $\mathrm{DSCO} 1$ Book \#12N14B

Method: LA-514-113 Rev/Mod C-1

Worklist Comment: AW-105 DSC, RUN UNDER N2. RCJ

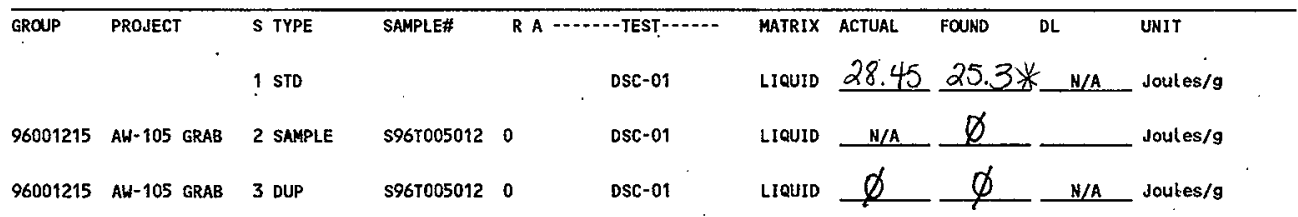

Final page for worklist \# 13136

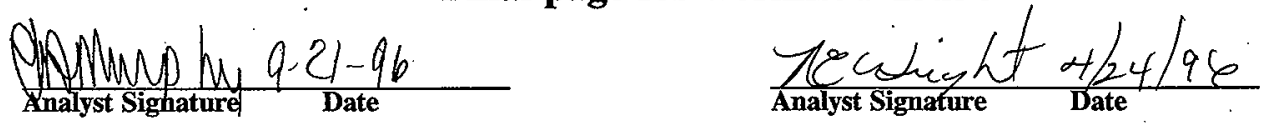

Veritid/Validutea by

Blandina

Valenzuela $9.26-96$

Data Entry Comments:

Units shown for $Q C$ (SPK \& STD) may not reflect the actual units. $D L=$ Detection Limit, $S=$ Worklist Slot Number, $R=$ Replicate Number, $A=$ Aliquot Code.

149 
SGNATURE BELOW REPRESENTS CHE:MICAL TECHNOLOGIST/CHEMIST THAT

COAPLETED/VERIFIED THE CALIBRATION/ANALYSIS ON PAGES 150 TO 152

OAtmuphy 9-21-96

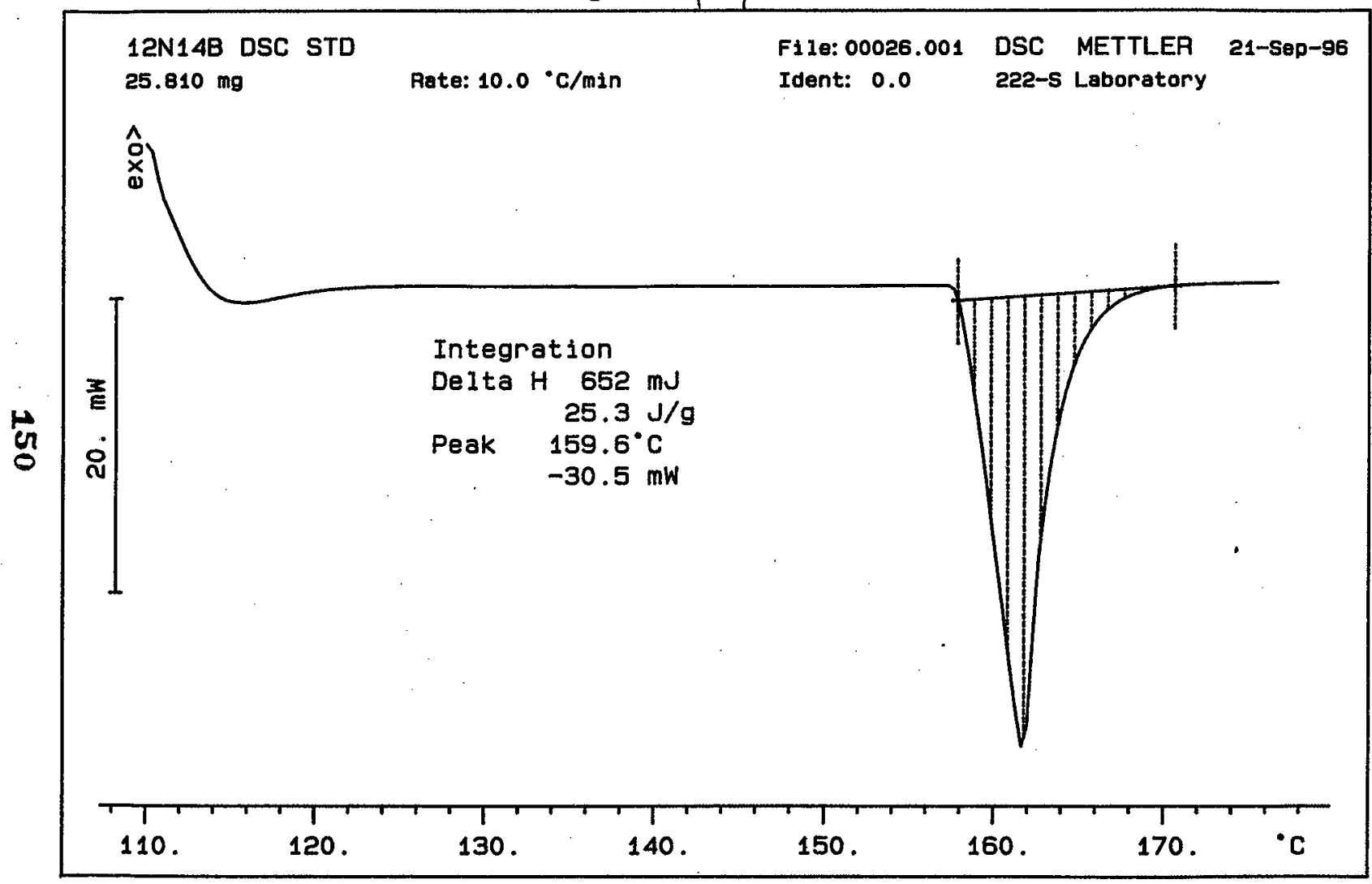




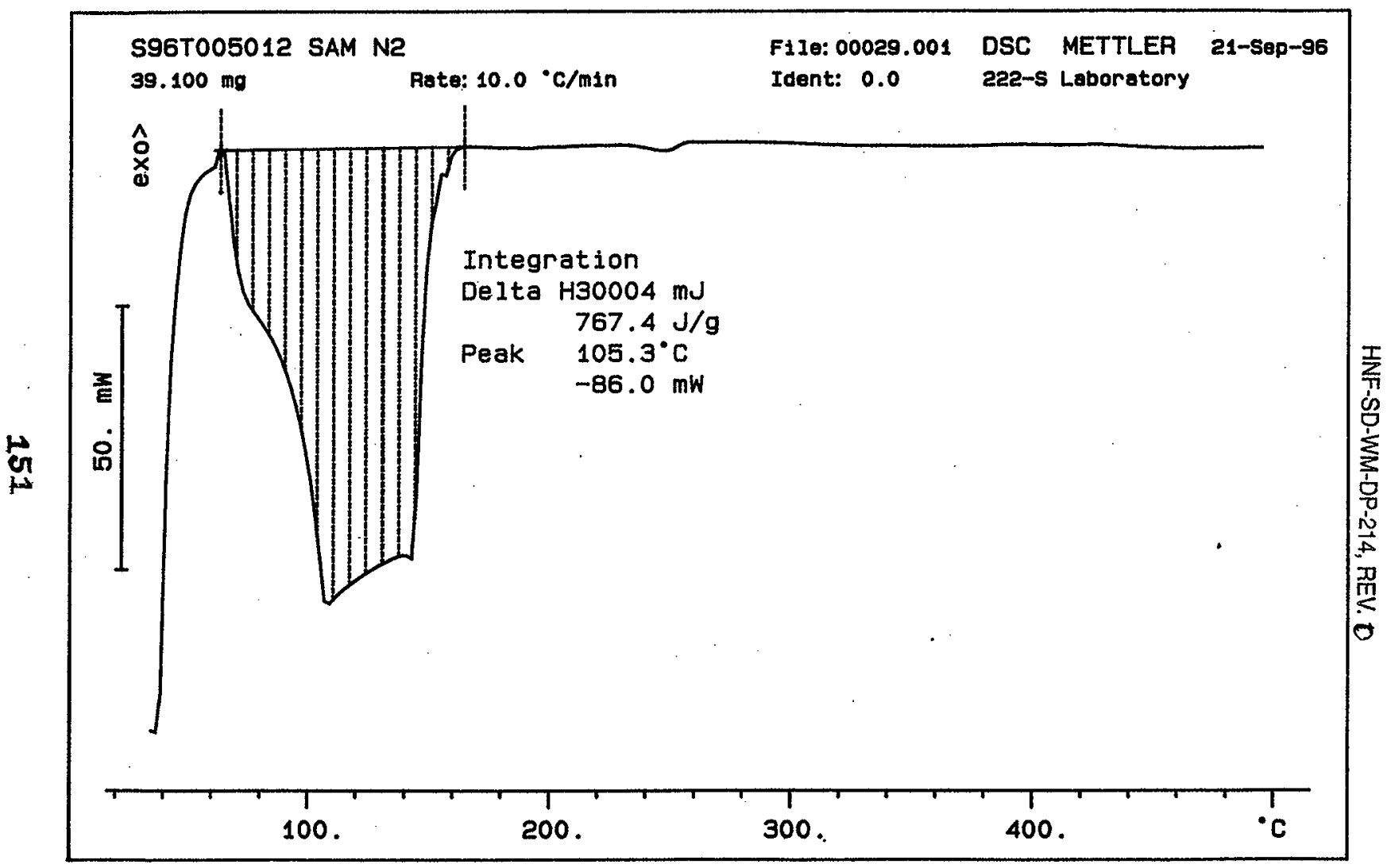




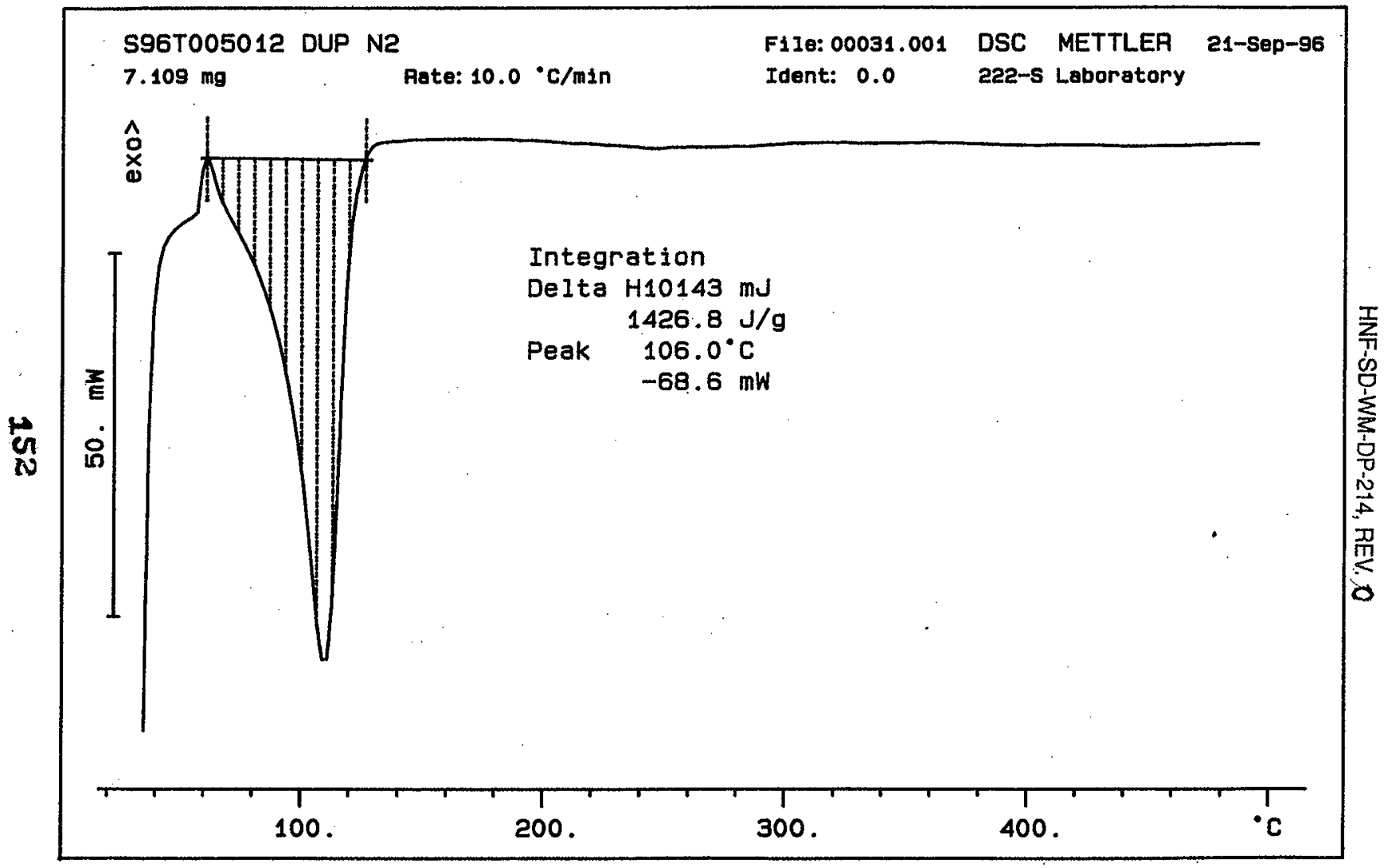




\section{LABCORE Data Entry Template for Worklist\# 13912}

Analyst: KRM Instrument: $\mathrm{DSCO}^{\circ} 3$ Book\# 12N14B.

Method: LA-514-114 Rev/Mod D-O

Worklist Comment: AW-105 Grab. Run under nitrogen.Std:INDIUM $\sim 8 \mathrm{mg}$. new

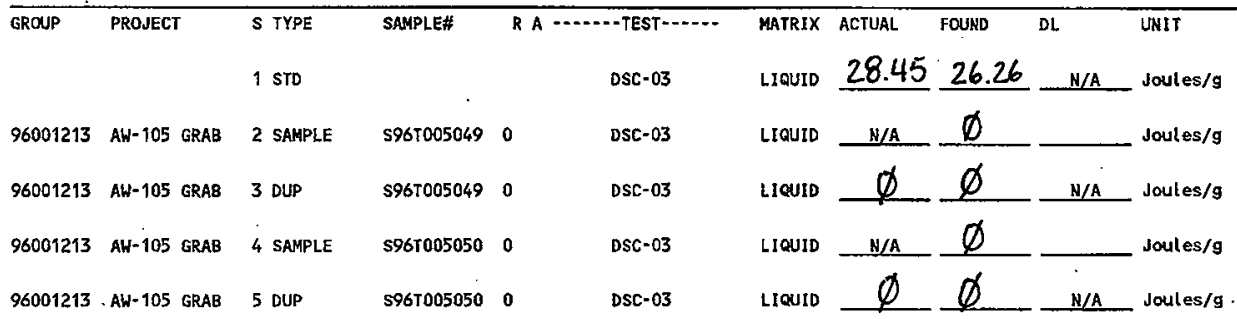

\section{Final page for worklist \# 13912}

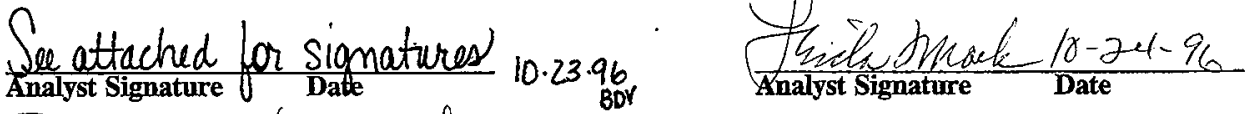

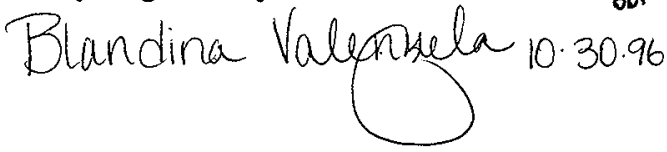

Data Entry Comments:

Units shown for $Q C$ (SPK \& STD) may not reflect the actual units. $D L=$ Detection Limit, $S=$ Worklist Slot Number, $R=$ Replicate Number, $A=$ Aliquot Code. 


\section{LABCORE Data Entry Template for Worklist\# 13912}

Analyst: $\quad K R M$ Instrument: $\mathrm{DSCO}^{\circ} \ldots$ Book \# 12 W $14 \mathrm{~B}$

Method: LA-514-113 Rev/Mod

Worklist Comment: AW-105 Grab. Run under nitrogen.Std:INDIUM $\sim 8 \mathrm{mg}$. new

\begin{tabular}{|c|c|c|c|c|c|c|c|c|c|c|c|}
\hline \multirow[t]{2}{*}{ GROUP } & \multicolumn{2}{|c|}{ PROJECT } & S TYPE & \multirow[t]{2}{*}{ SAMPLE\# } & \multicolumn{2}{|c|}{ R A $\cdots \ldots$ TEST $\cdots$} & MATRIX & \multirow[t]{2}{*}{ ACTUAL } & \multirow[t]{2}{*}{ FOUND } & \multirow[t]{2}{*}{ DL } & \multirow{2}{*}{$\begin{array}{l}\text { UNIT } \\
\text { Joules/g }\end{array}$} \\
\hline & & & 1 STD & & & DSC -01 & LIQUID & & & & \\
\hline 96001213 & $A W-105$ & GRAB & 2 SAMPLE & \$96T005049 & 0 & DSC-01 & LIQUID & $\therefore$ N/A & & & Joules/g \\
\hline 96001213 & $A W-105$ & GRAB & 3 DUP & $\$ 96 T 005049$ & 0 & DSC-01 & LIOUID & & & & Joules/g \\
\hline 96001213 & $A W=105$ & GRAB & 4 SAMPLE & S96T005050 & 0 & DSC-01 & LIOUID & $\mathrm{H} / \mathrm{A}$ & & & Joules/g \\
\hline 96001213 & AW- 105 & GRAB & 5 DUP & s96T005050 & 0 & DSC-01 & LIOUJP & & & & Joules/g \\
\hline
\end{tabular}

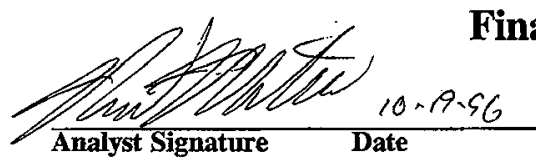

\section{Final page for worklist \# 13912}

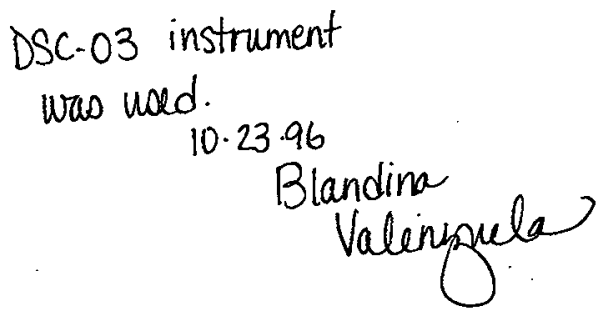

Data Entry Comments:

Units shown for QC (SPK \& STD) may not reflect the actual units. $D L=$ Detection Limit, $S=$ Worklist Slot Number, $R=$ Replicate Number, $A=$ Aliquot Code. 
Curve 1: 0SC

F1le info: IND101901 Sat Oct 19 05:03:45.1996

Sample Weight: 22.750 ag

12N14-B INDIUM AT 10C/MIN

SIGNATURE BELOW REPRESENTS CHEMICAL TECHNOLOGIST/CHEMIST THAT COMPLETED/VERIFIED THE CALIBRATION/ANALYSIS ON PAGES 155 TO $\angle 29$

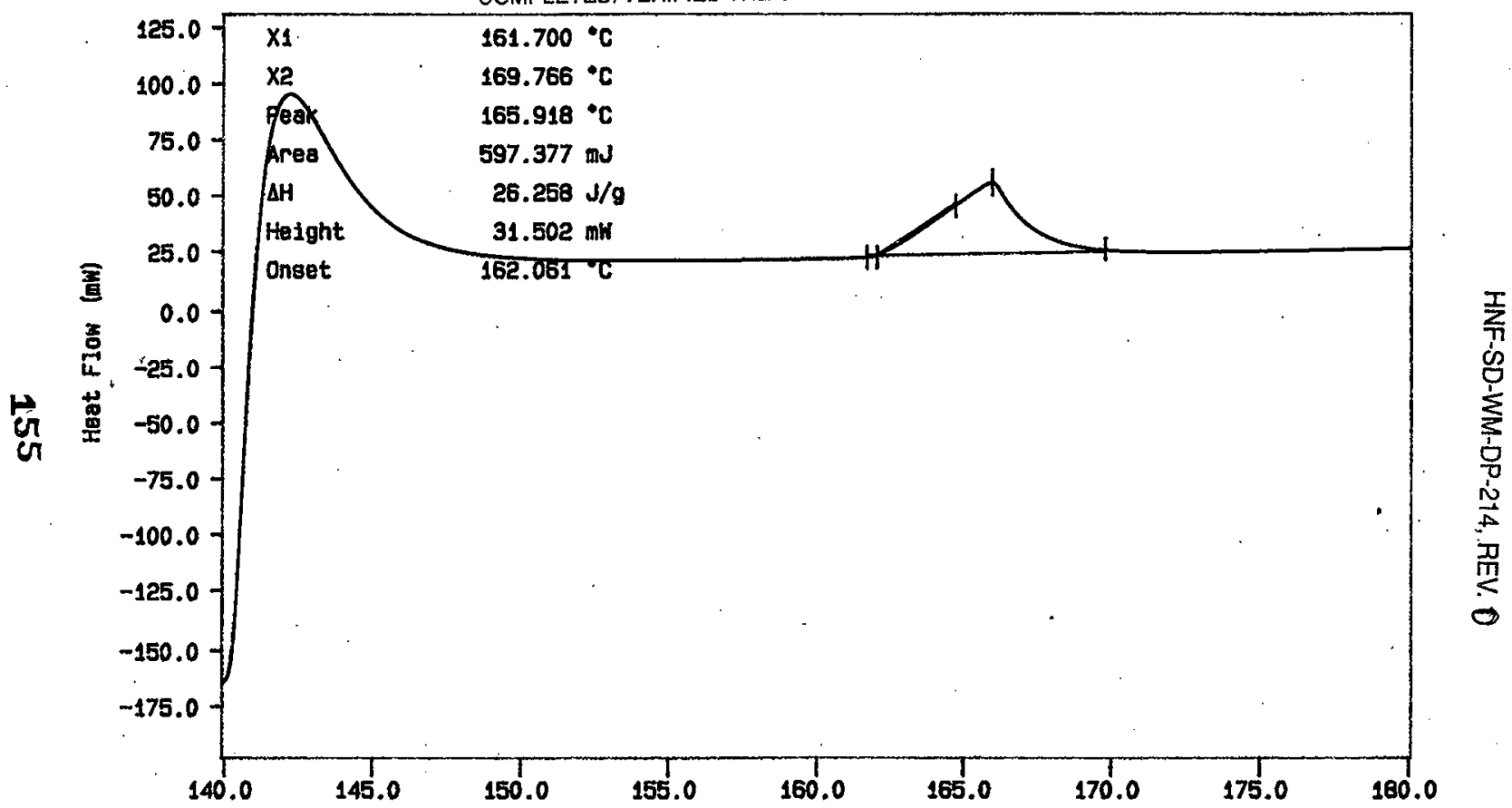

N2. EXOTHERM DOWN

Tenpe: 140:8 \& Trues:

0.0 man aates:

10.0 c/man

Temperature ('C)

KA MONTEITH

PEAKIN-ELMER

7 Series Thermal Analysis Systen
Sat Det 19 05: 06:21 1996 
Curve 1: DSC

File Info: SAM101901 Set oct 19 06: 22: 351996

Sample Weight: 10.430

S96T005049

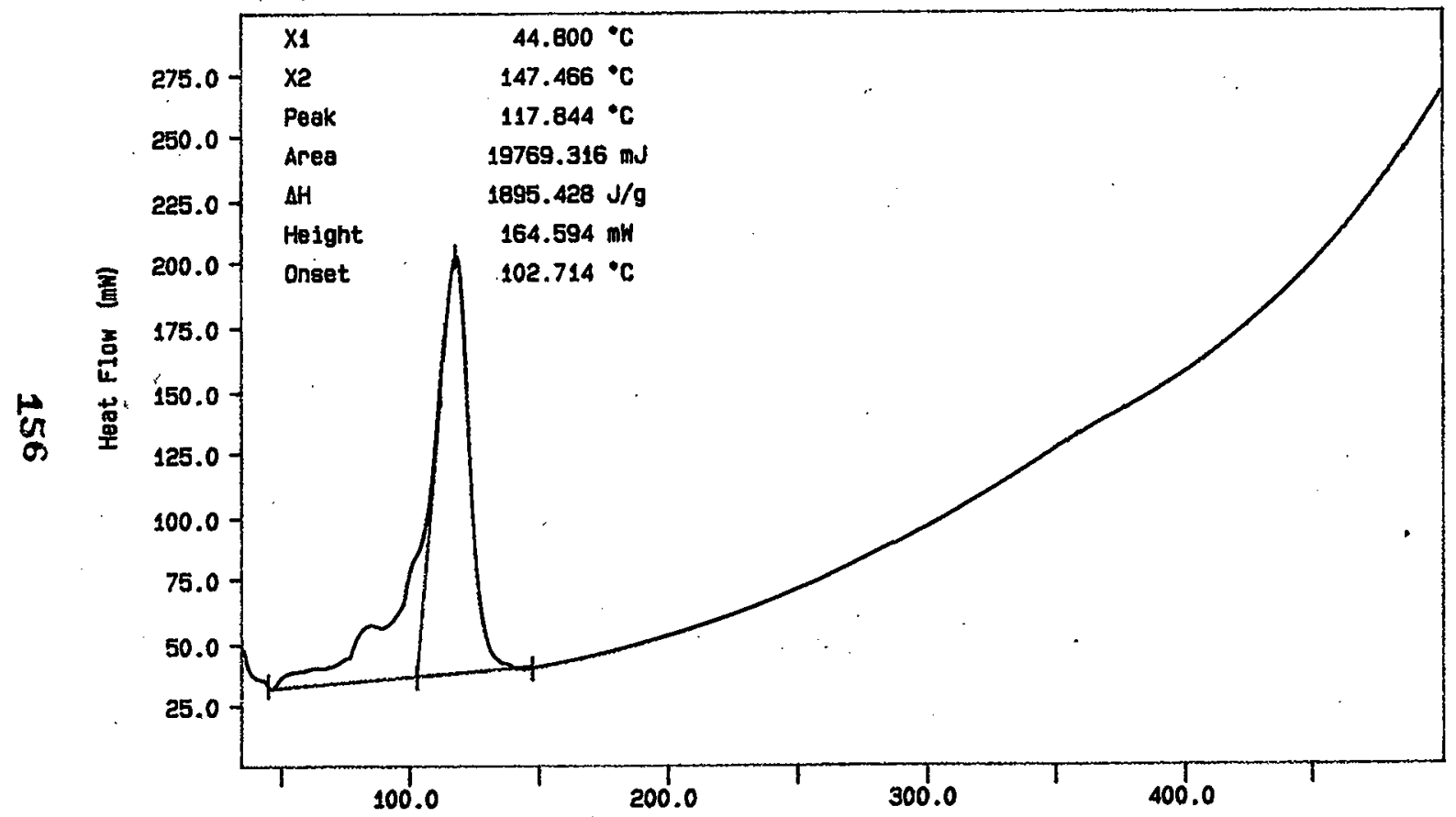

N2. $10 \mathrm{c} / \mathrm{min}$

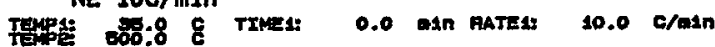
Temperature $\left({ }^{\circ} \mathrm{C}\right)$ KR MONTEITH PERKIN-ELMER 
Curve 1: 0SC

F11e Info: SAM101902 Sat Oct 19 06: 16:57 1996

Sample Weight: 10.430

mg

S96T005049 DUP

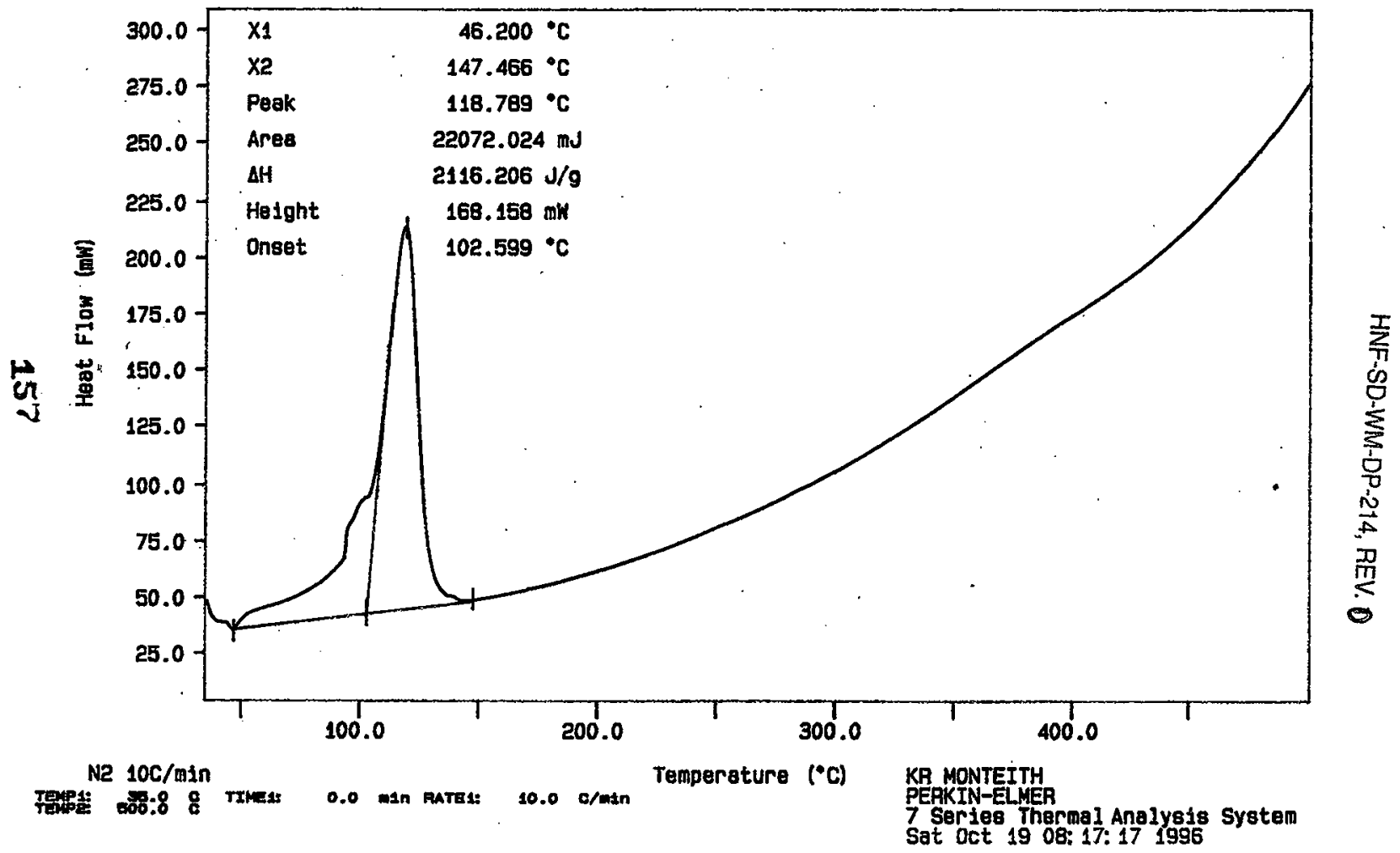


Curve 1: DSC

F1le info: SAM101903 Sat oct 19 09: $1258 \cdot 1996$

Sample Weight: $11.940 \mathrm{mg}$

s96T005050

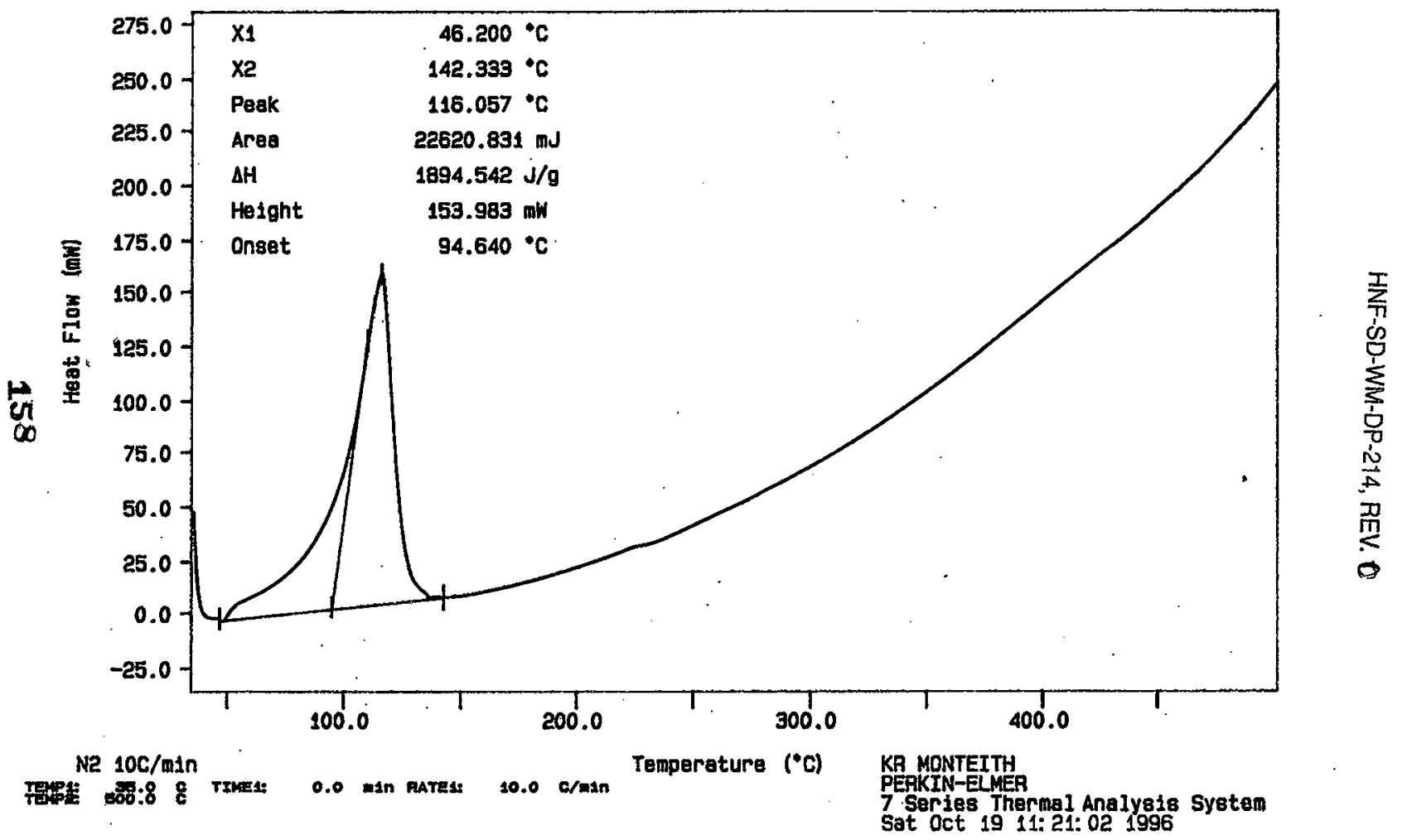


Curve 1: DSC

F士le info:. SAM101904 Sat oct 19 12: 16: 301996

Sample Neight: $11.500 \mathrm{mg}$

596T005050 DUP

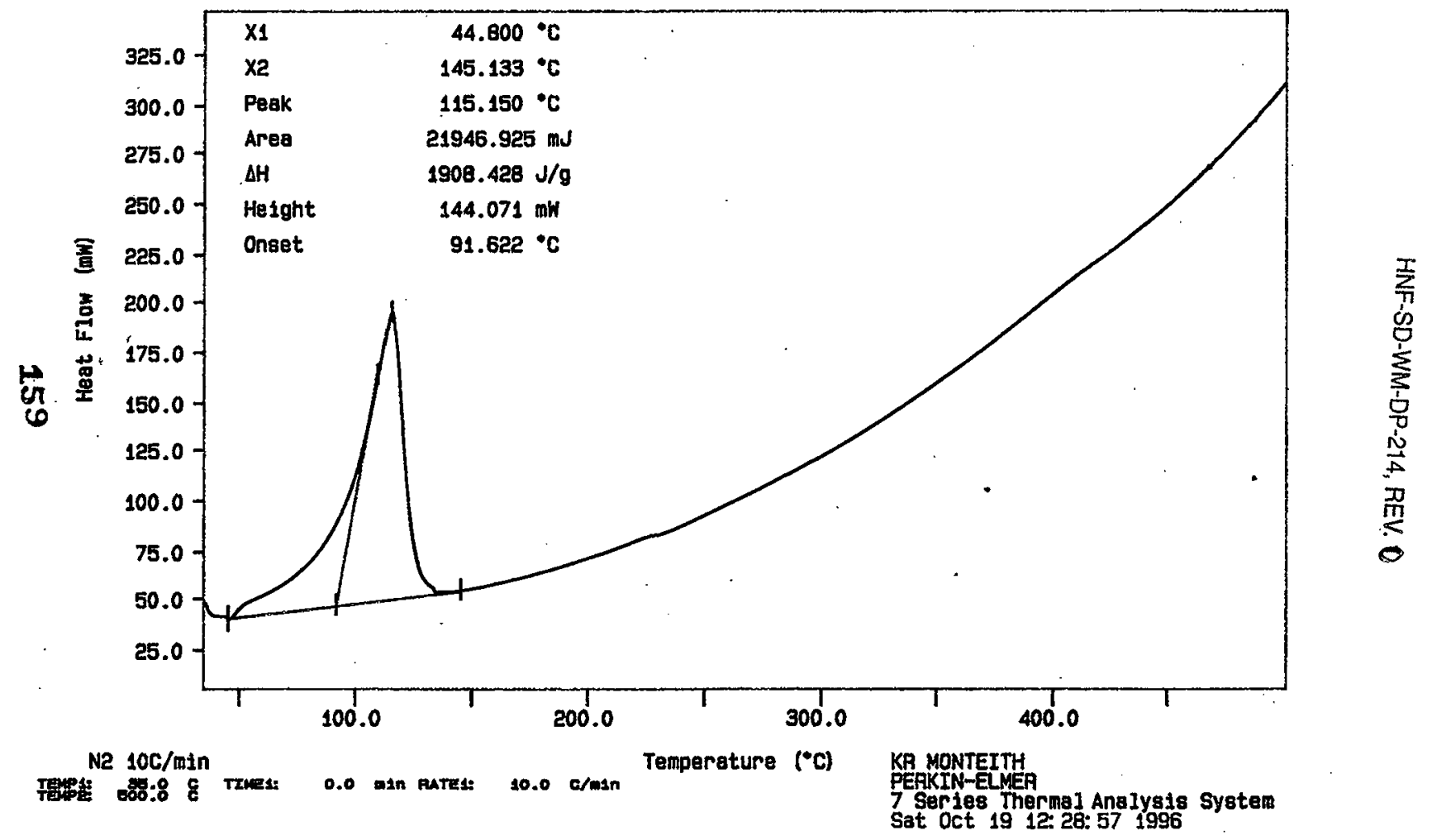


worklistrpt Version 2.1 05/15/95

HNF-SD-WM-DP-214, REV. 0

Page: 1

10/23/96 09:29

LABCORE Data Entry Template for Worklist\# 13913

Analyst: $\quad \mathrm{KRM}$ Instrument: $\mathrm{DSCO}^{\prime} 3$ Book \# $12 \mathrm{~N} 14 \mathrm{~B}$

Method: LA-514-114 Rev/Mod D-O

Worklist Comment: AW-105 Grab. Run under nitrogen.Std: INDIUM $\sim 8 \mathrm{mg}$. new .

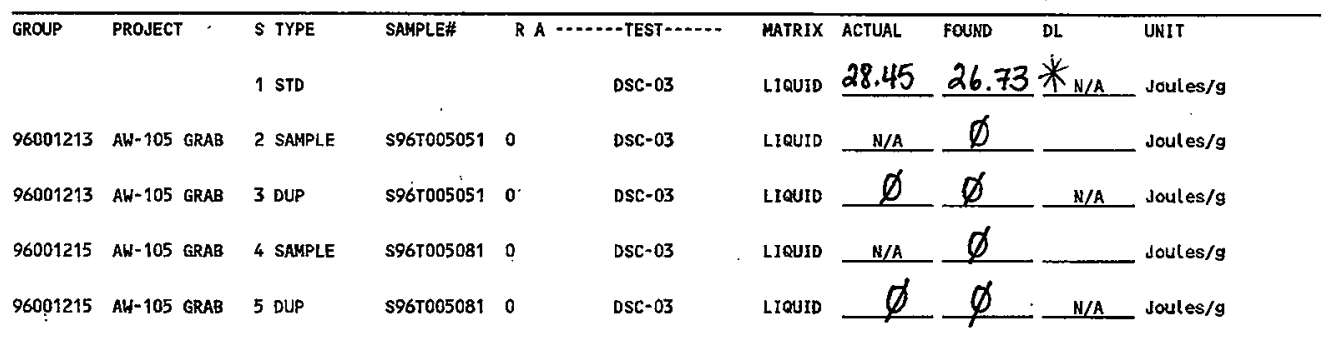

Final page for worklist \# 13913

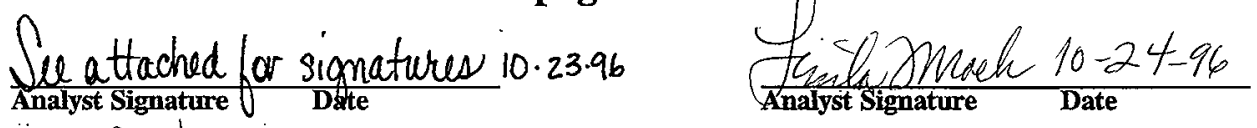

Verified Validated by

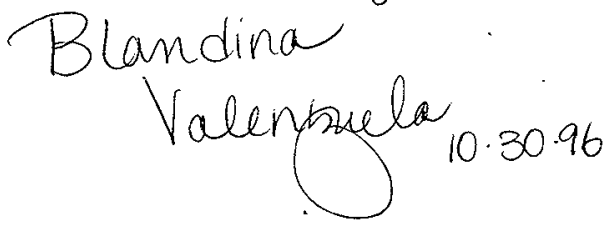

Valenosula 10.30 .96

Data Entry Comments:

Units shown for QC (SPK \& STD) may not reflect the actual units. $D L=$ Detection Limit, $S=$ Worklist Slot Number, $R=$ Replicate Number, $A=$ Aliquot Code.

160 


\section{LABCORE Data Entry Template for Worklist\# 13913}

Analyst:

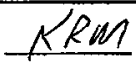

Instrument: $\mathrm{DSCO}$

Book \# $12014 B$

Method: LA-514-113 Rev/Mod

Worklist Comment: AW-105 Grab. Run under nitrogen.Std: INDIUM $\sim 8 \mathrm{mg}$. new

\begin{tabular}{|c|c|c|c|c|c|c|c|c|c|c|}
\hline \multirow[t]{2}{*}{ GROUP } & \multirow[t]{2}{*}{ PROJECT } & $S$ TYPE & \multirow[t]{2}{*}{ SAMPLE\# } & \multicolumn{2}{|c|}{ R A $\cdots$ TEST $\cdots$} & \multirow{2}{*}{$\begin{array}{l}\text { MATRIX } \\
\text { LIQUID }\end{array}$} & \multirow[t]{2}{*}{ ACTUAL } & \multirow[t]{2}{*}{ FOUND } & \multirow[t]{2}{*}{ DL } & \multirow[t]{2}{*}{ UNIT } \\
\hline & & 1 STD & & & DSC-01 & & & & & \\
\hline 96001213 & AH-105 GRAB & 2 SAMPLE & S96T005051 & 0 & DSC-01 & LIQUID & N/A & & & Joules $/ g$ \\
\hline 96001213 & AH-105 GRAB & 3 DUP & \$96r005051 & 0 & DSC-01 & LIQUID & & & & Joules/g \\
\hline 96001215 & AH-105 GRAB & 4 SAMPLE & S96r005081 & 0 & DSC-01 & LIQUID & $N / A$ & & & Joutes/g \\
\hline 96001215 & AH-105 GRAB & 5 DUP & \$96T005081 & 0 & DSC-01 & LIQUID & & & & soules/g \\
\hline
\end{tabular}

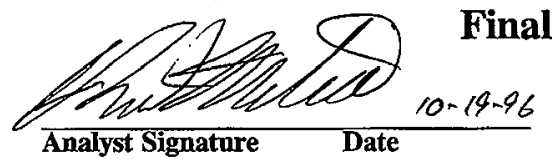

Final page for worklist \# 13913

Analyst Signature Date

\section{Analyst Signature Date}

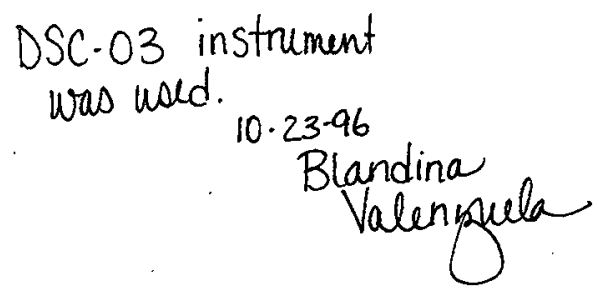

Data Entry Comments:

Units shown for QC (SPK \& STD) may not reflect the actual units. DL = Detection Limit, $S=$ Worklist Slot Number, $R=$ Replicate Number, $A=$ Aliquot Code. 
Curve 1: DSC

Fije info: IND101901 Sat oct 19 05: 03:45 1996

Sample Weight: $22.750 \mathrm{mg}$

12N14-B INDIUM AT 10C/MIN

SIGNATURE BELOW REPRESENTS CHEMICAL TECHNOLOGIST/CHEMIST THAT

COMPLETED/VERIFIED THE CALIBAATION/ANALYSIS ON PAGES $\angle 62$ TO $\angle 6 l e$.

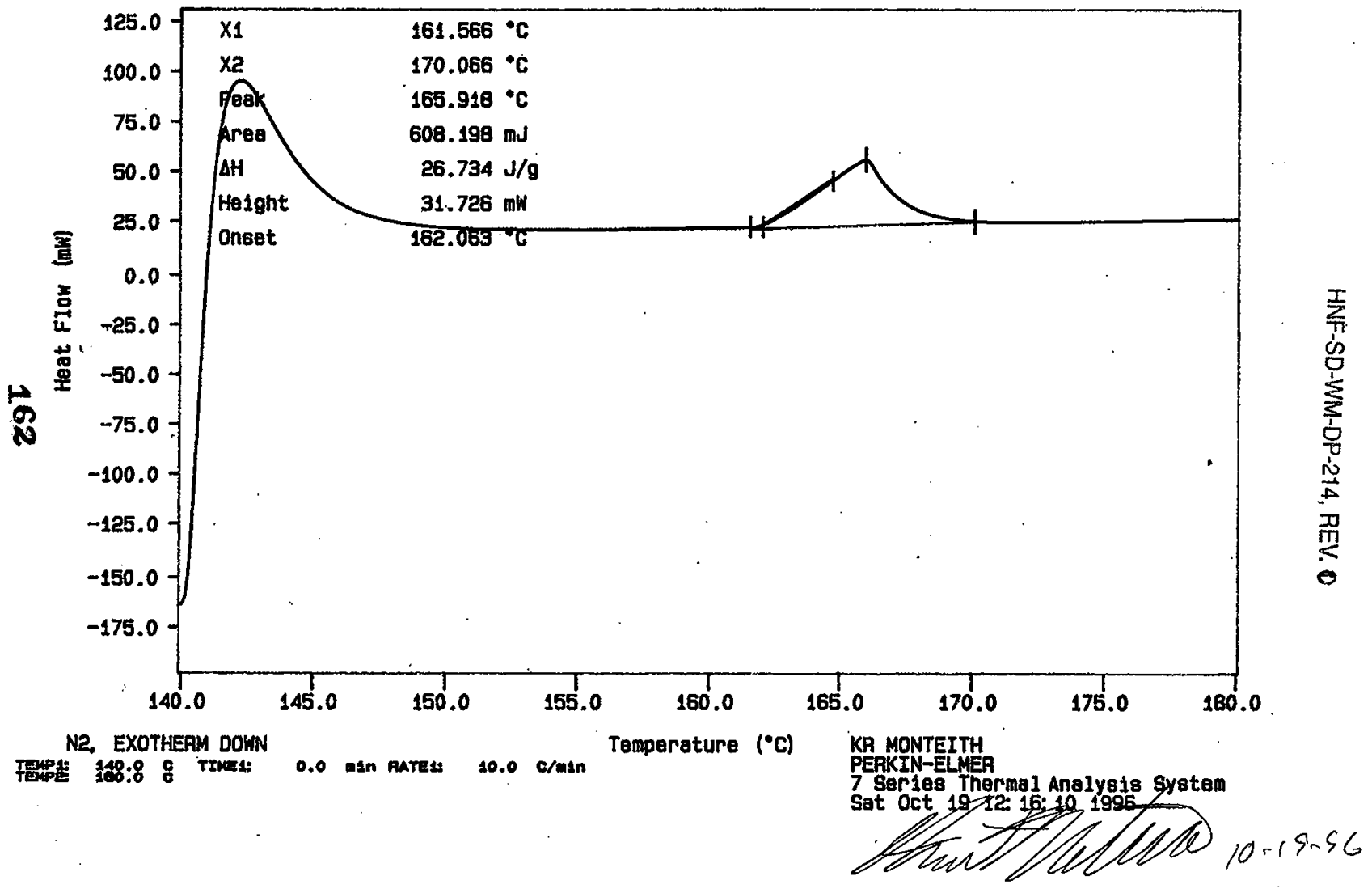


Curve 1: DSC

F 1 lo info: SAM101905 Set oct 19 13:21:20 1996

Sample Weight: 10.860

mg

S96T005051

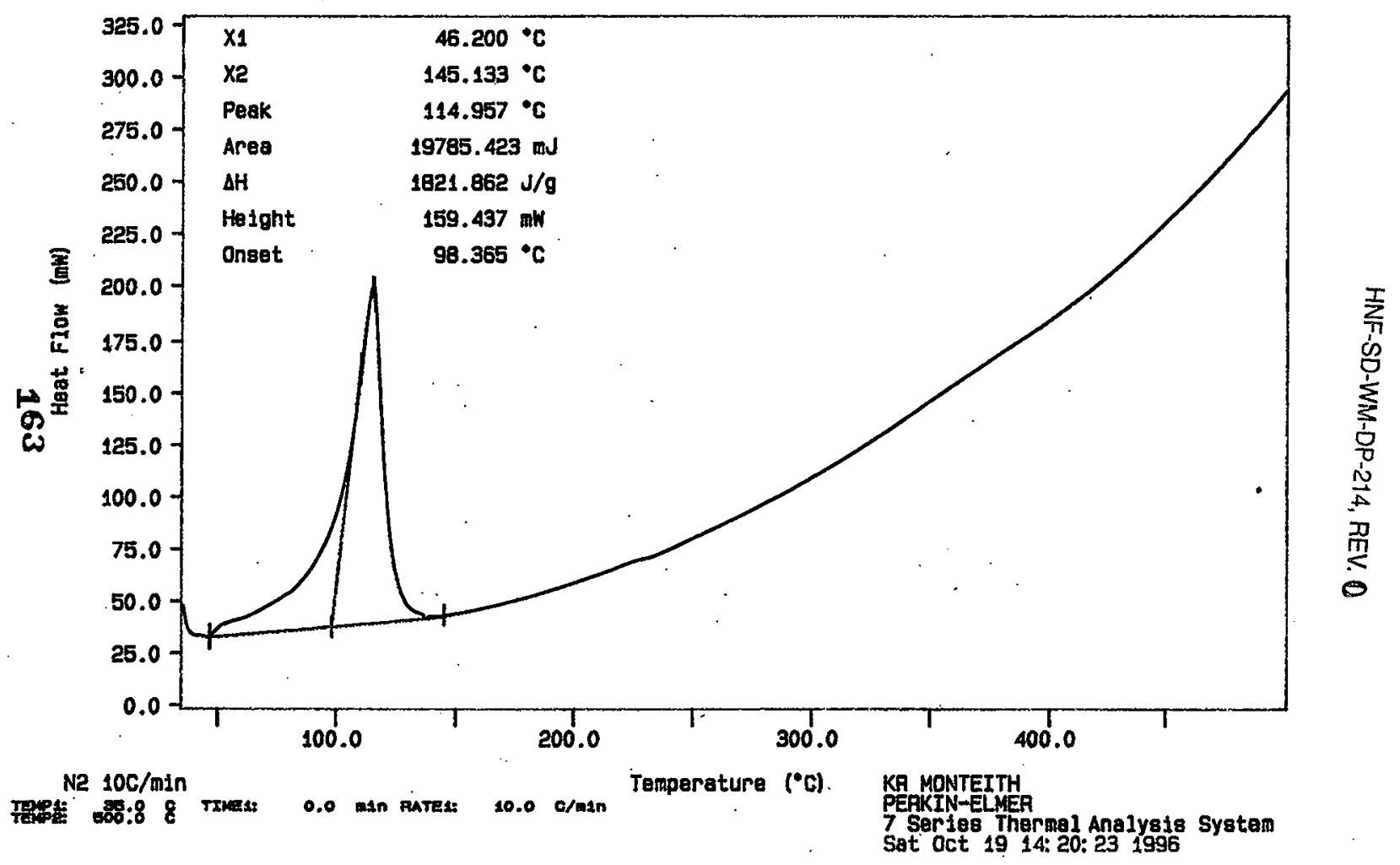


Curve 4: DSC

F1le Info: SAM101906 Sat oct 19 15: 06: 101996

Sample Weight: $10.920 \mathrm{mg}$

s96T005051 DUP

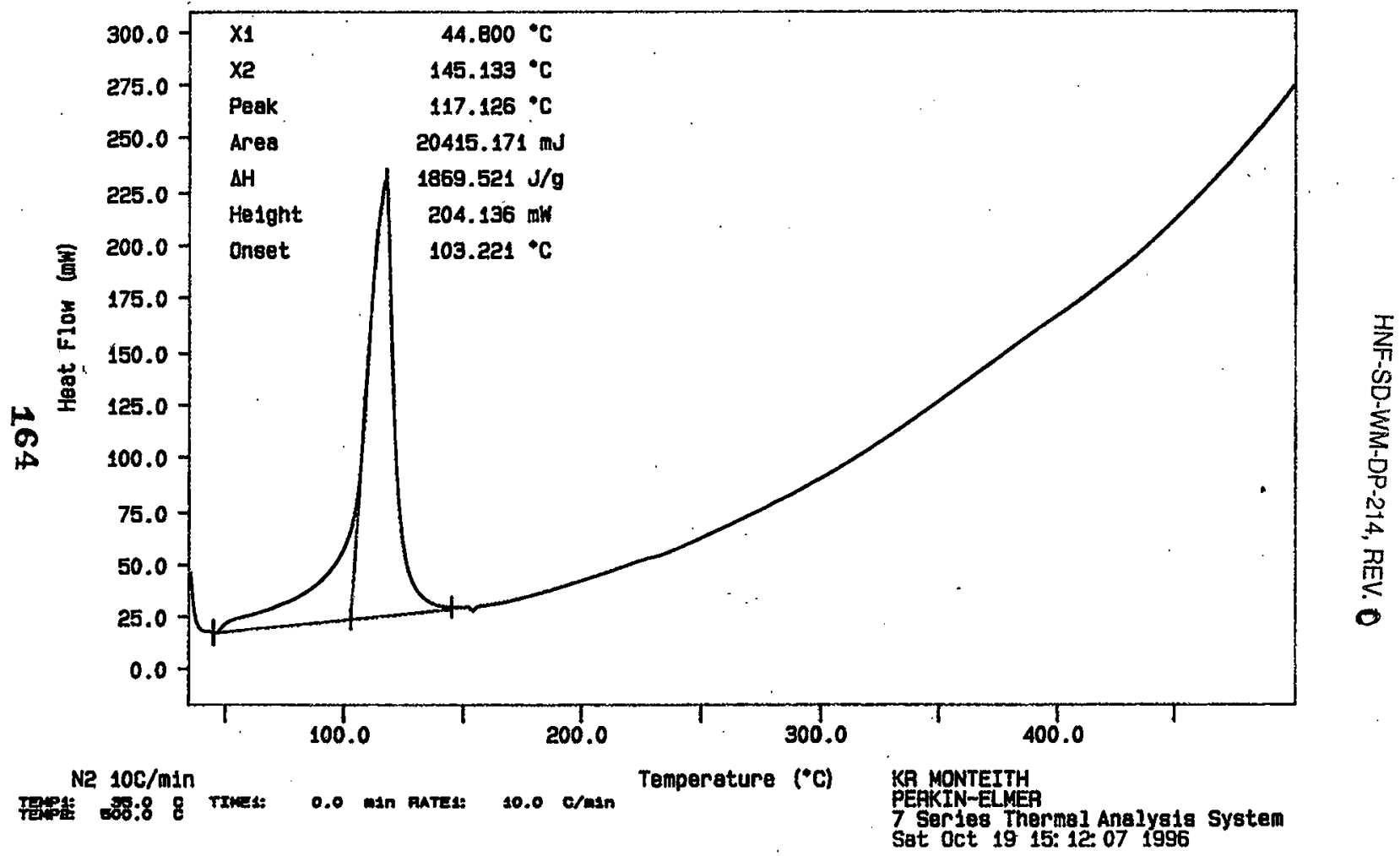


Curve 1: DSC

File info: SAM101907 Sot oct 19 15: 59: 531996

Sample Weight: $11.210 \mathrm{mg}$

S96T005081

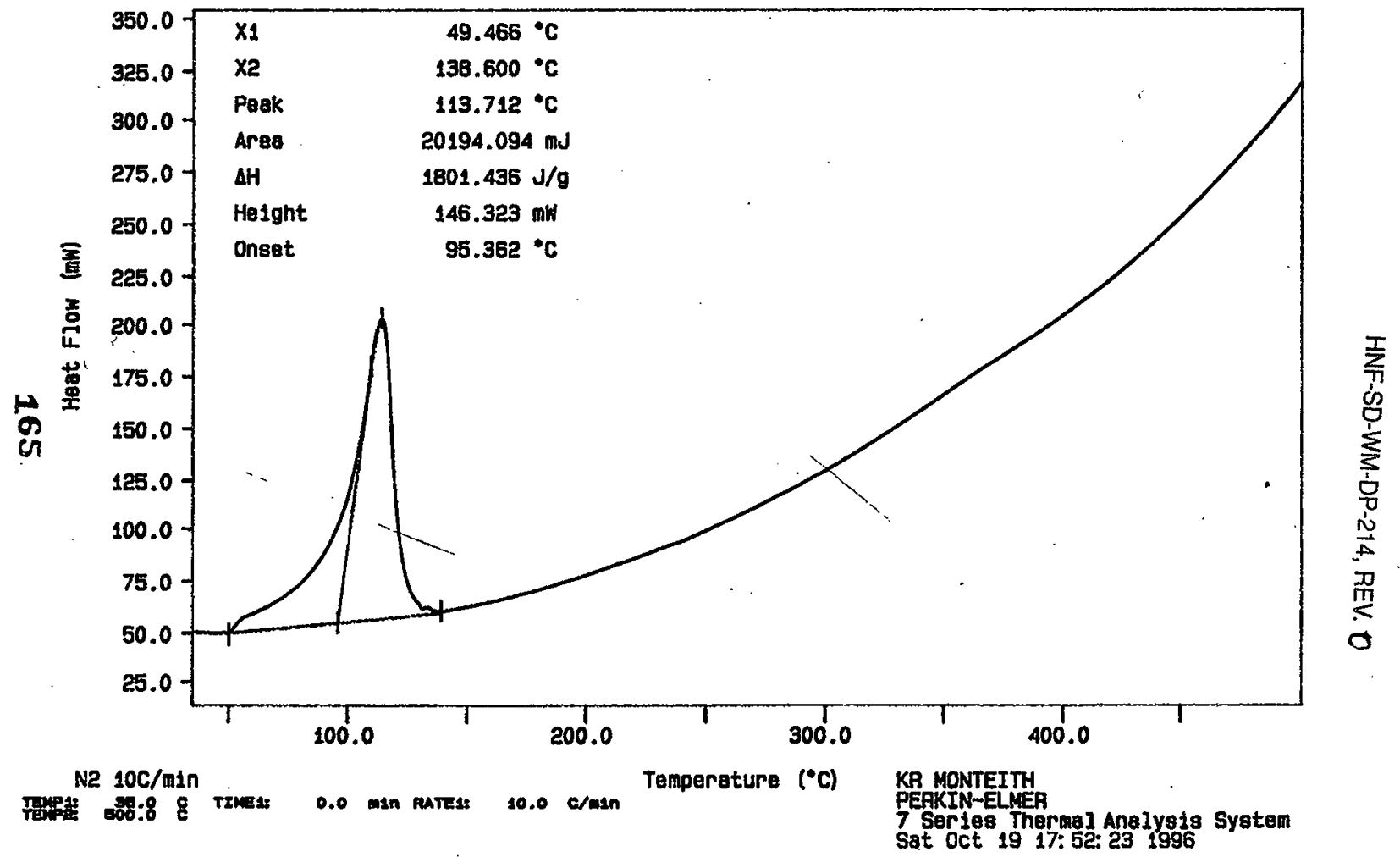


Curve 1: DSC

Flle info: SAM102001 Sun Oct 20 05: 54: 311996

Sanple Weight: 11.530 mg

S96T005061 DUP

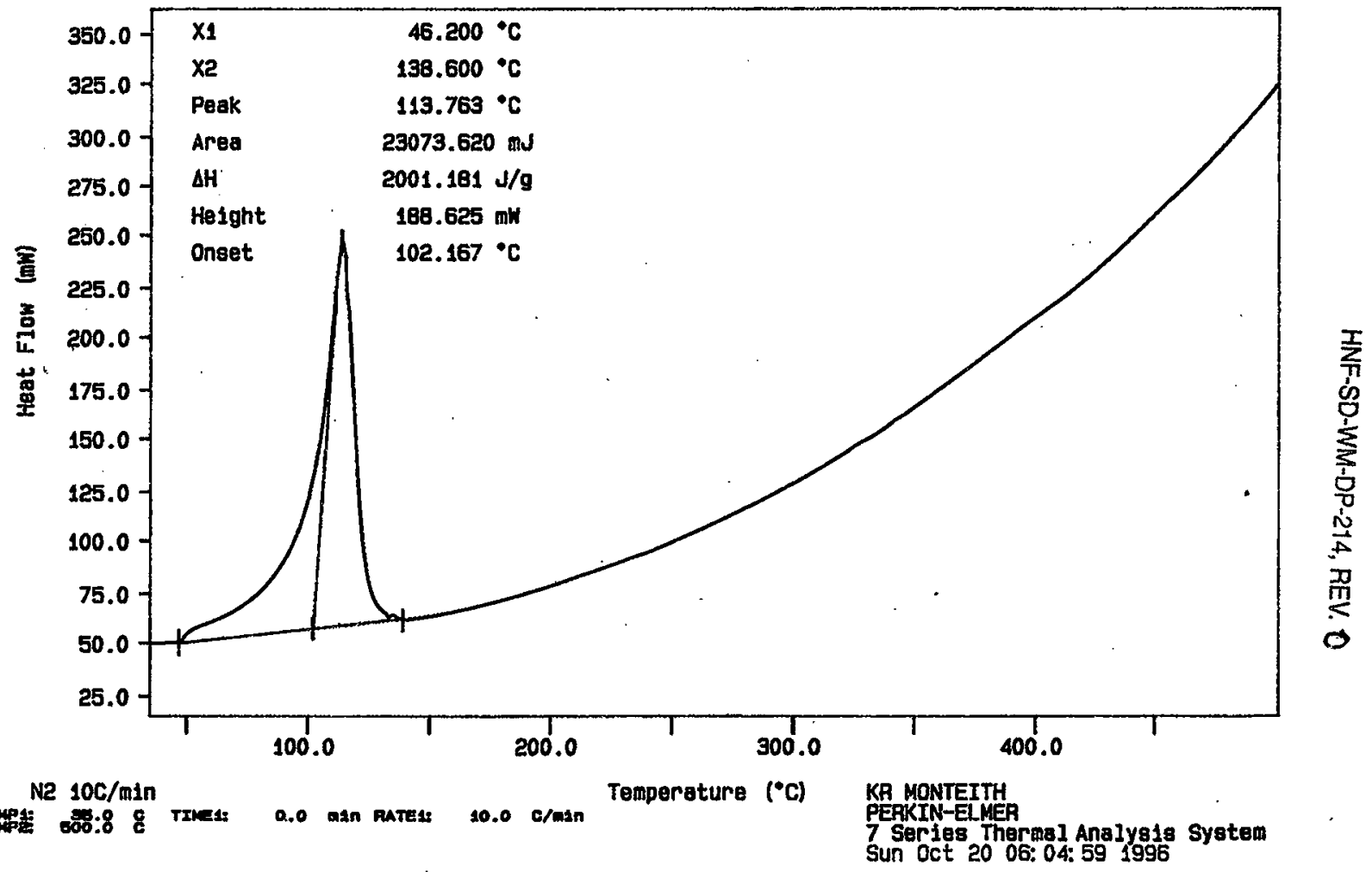




\section{LABCORE Data Entry Template for Worklist\# 13914}

Analyst: L/PM Instrument: DSCO I Book\# 12N14B

Method: LA-514-113 Rev/Mod C-1

Worklist Comment: AW-105 GRAB. Run under Nitrogen. Std: INDIUM $\sim 8 \mathrm{mg}$. new

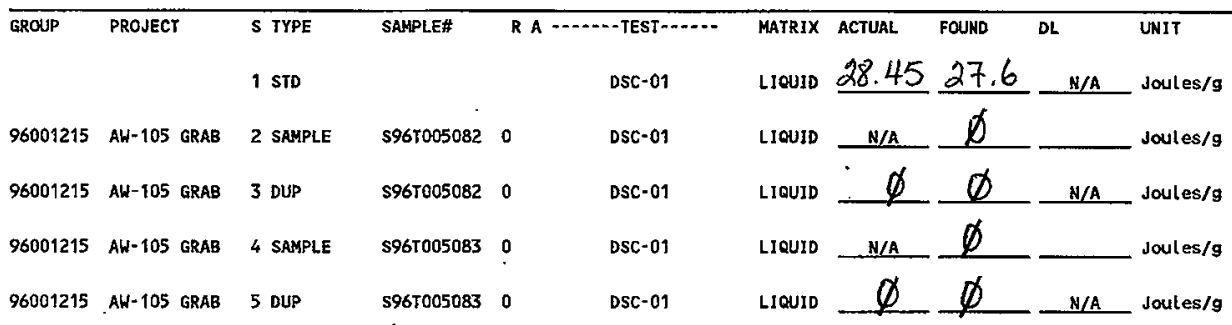

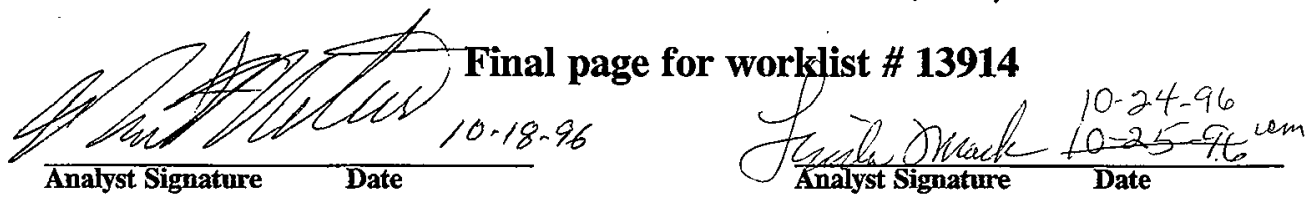

Verified/Validata by

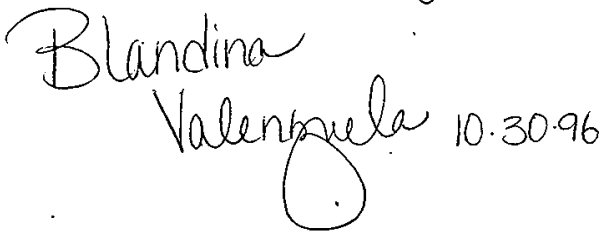

Data Entry Comments:

Units shown for $Q C$ (SPK \& STD) may not reflect the actual units. $D L=$ Detection Limit, $S=$ Worklist Slot Number, $R=$ Replicate Number, $A=$ Aliquot Code. 


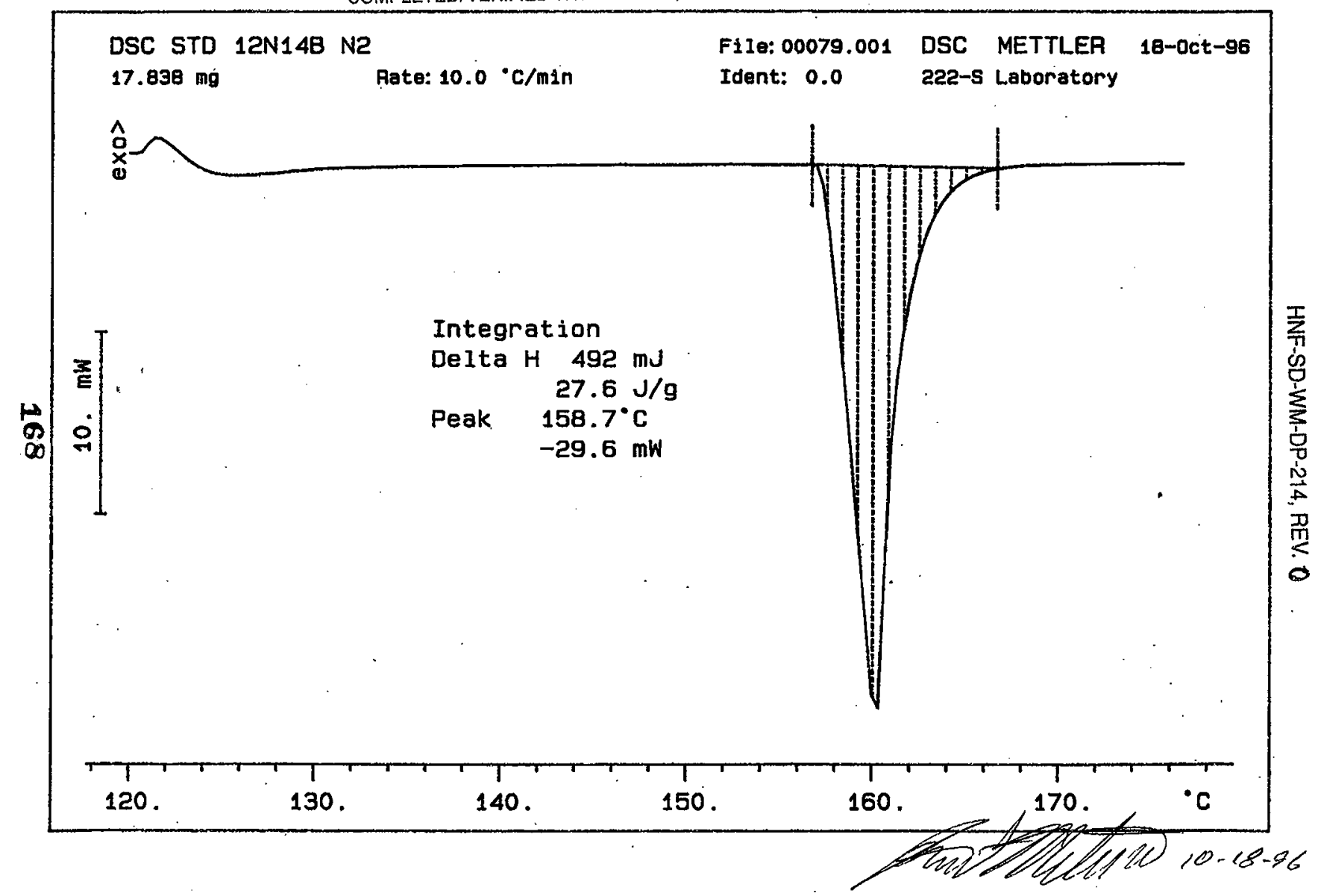




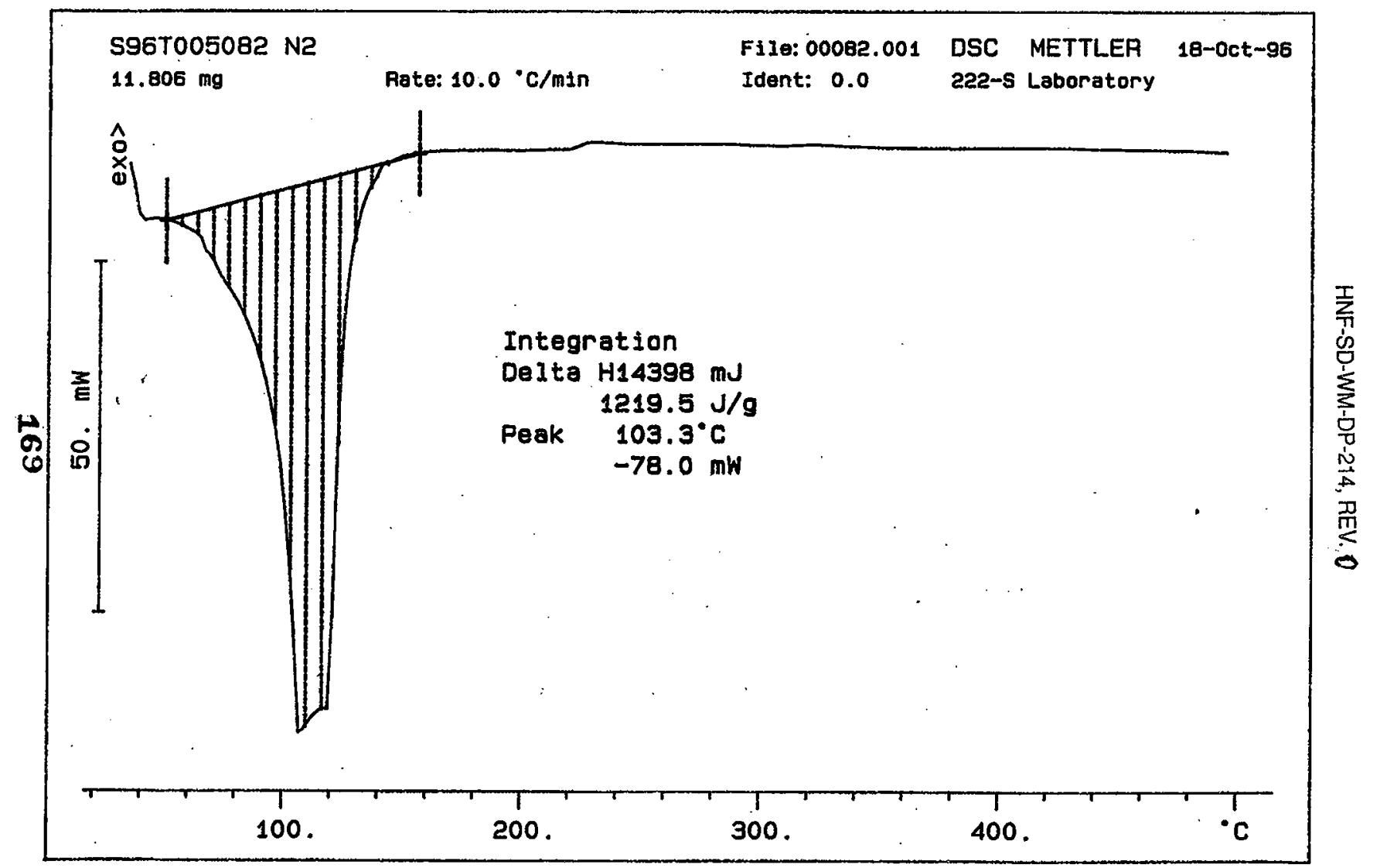




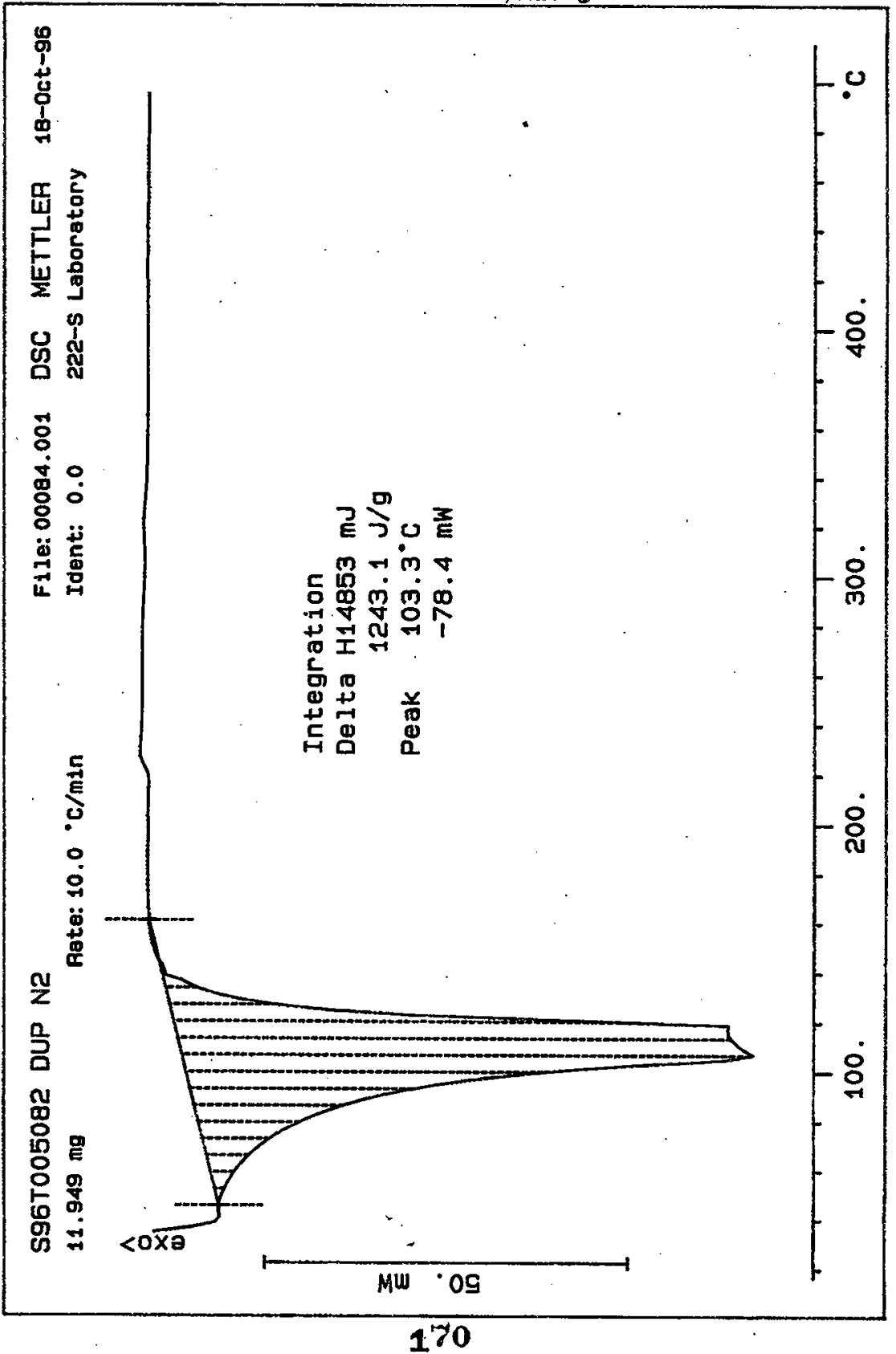


HNF-SD-WM-DP-214, REV. 0

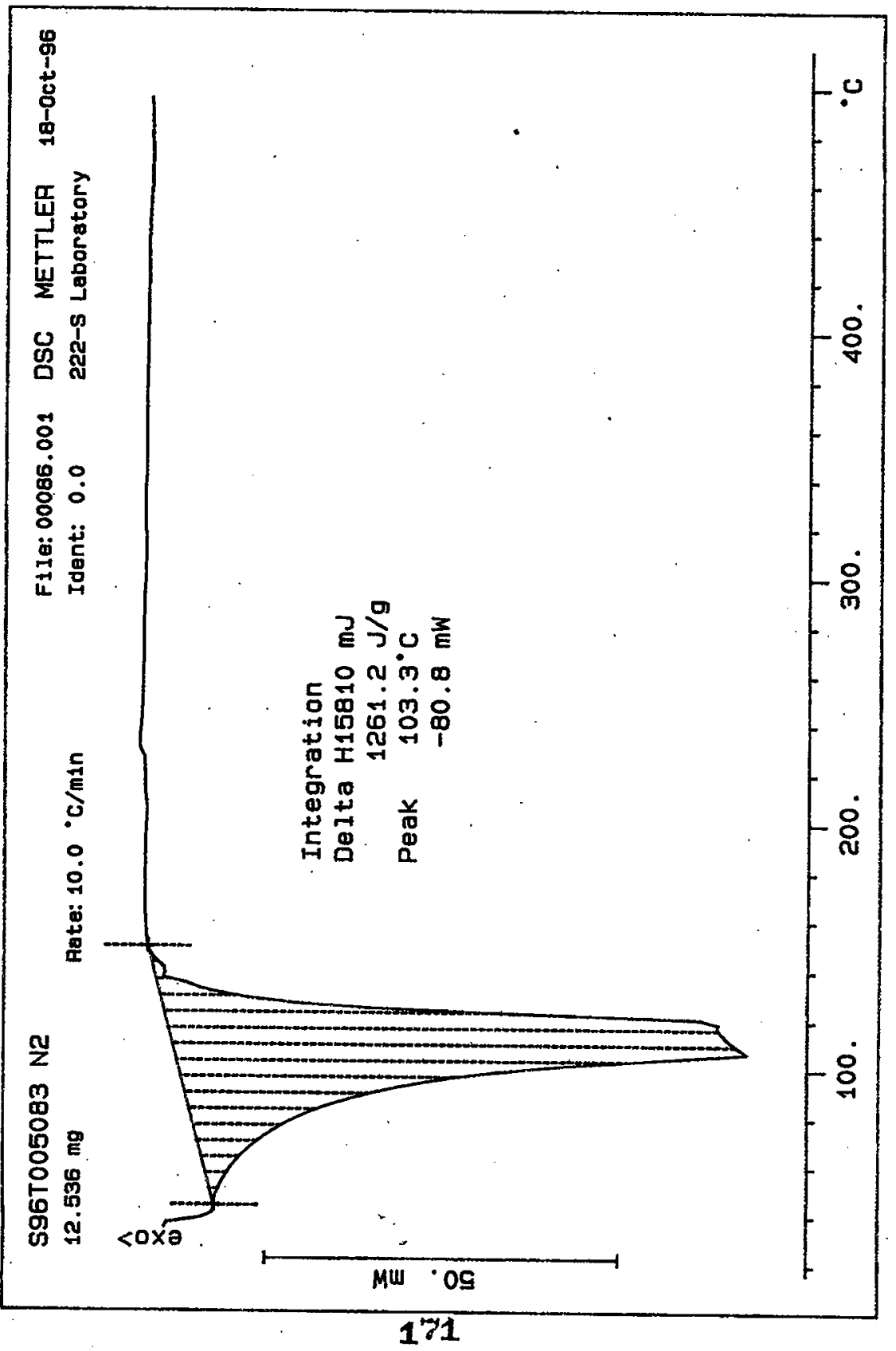


HNF-SD-WM-DP-214, REV. 0

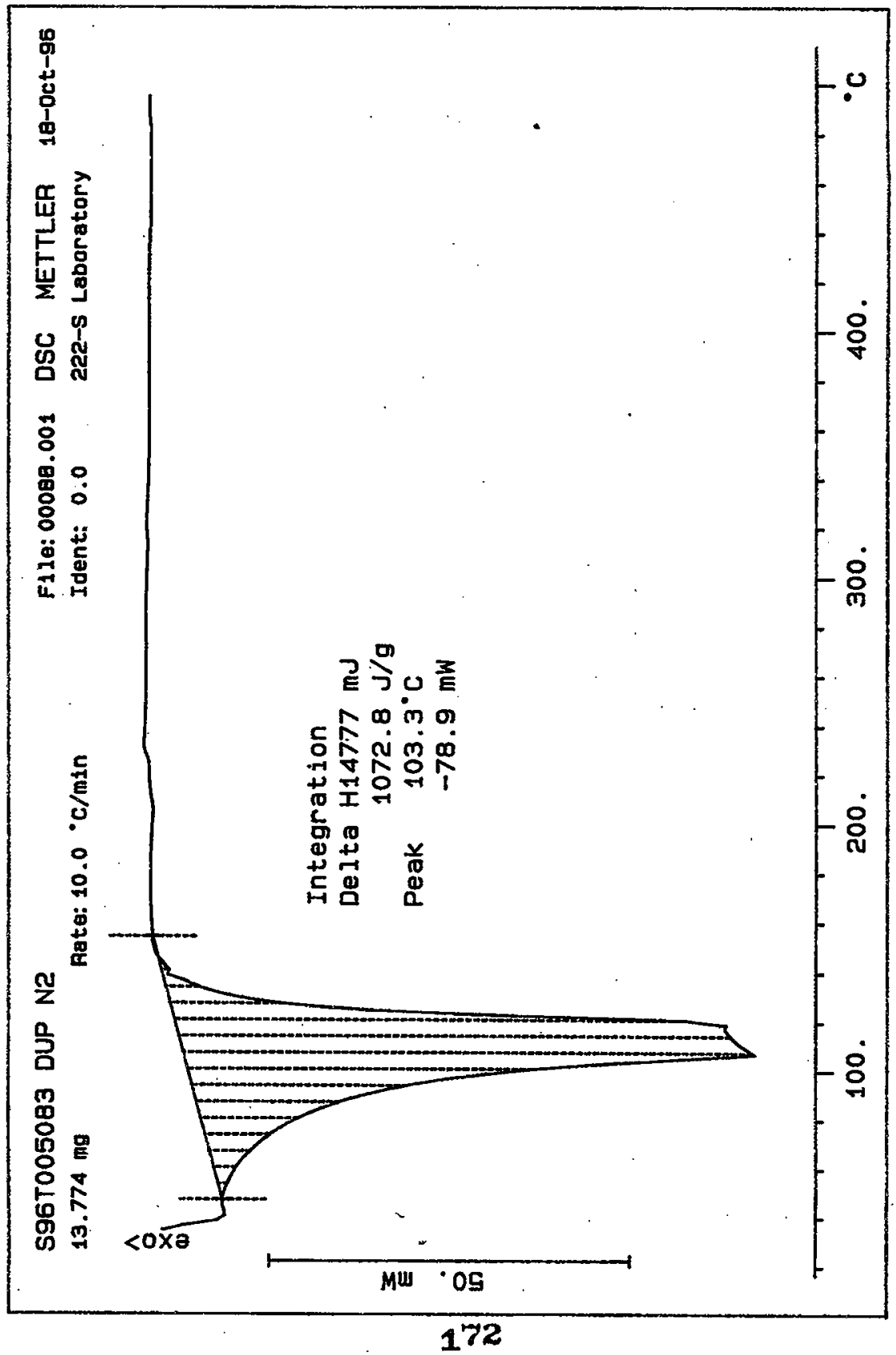




\section{LABCORE Data Entry Template for Worklist\# 13915}

Analyst: $\quad$ KRM Instrument: $D^{2} 0$ Book \# 12 N14 B

Method: LA-514-114 Rev/Mod D-O

Worklist Comment: AW-105 GRAB. Run under nitrogen. Std: INDIUM $\sim 8 \mathrm{mg}$. new

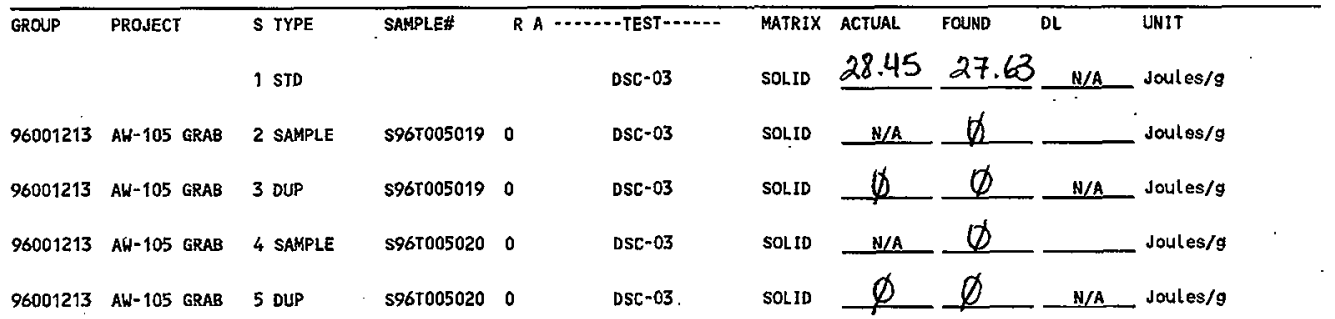

\section{Final page for worklist \# 13915}

Sele ottached for signatures $10-22-96$ Analyst Signature) Date

Verified/Nalidated by

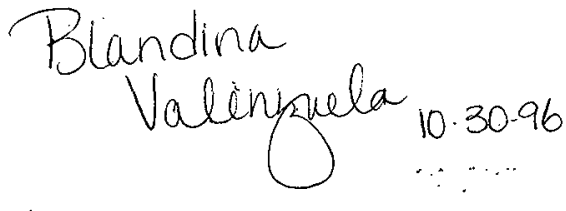

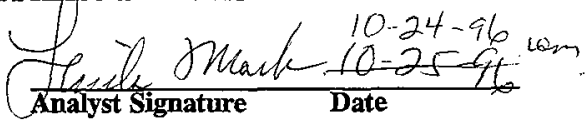

Data Entry Comments:

Units shown for $Q C$ (SPK \& STD) may not reflect the actual umits. $D L=$ Detection Limit, $S=$ Worklist Slot Number, $R=$ Replicate Number, $A=$ Aliquot Code . 


\section{LABCORE Data Entry Template for Worklist\# 13915}

Analyst: $\quad$ K RMA $\quad$ Instrument: $\mathrm{DSCO}^{\circ}$ Book \#12N14B

Method: LA-514-113 Rev/Mod

Worklist Comment: AW-105 GRAB. Run under nitrogen. Std: INDIUM. $\sim 8 \mathrm{mg}$. new

\begin{tabular}{|c|c|c|c|c|c|c|c|c|c|c|}
\hline \multirow[t]{2}{*}{ GROUP } & \multirow[t]{2}{*}{ PROJECT } & S TYPE & \multirow[t]{2}{*}{ SAMPLE\# } & \multicolumn{2}{|c|}{ R A $\cdots$ TEST $\cdots$} & \multirow{2}{*}{$\begin{array}{l}\text { MATRIX } \\
\text { SOLID }\end{array}$} & \multirow[t]{2}{*}{ ACTUAL } & \multirow[t]{2}{*}{ FOUND } & \multirow[t]{2}{*}{ DL. } & \multirow{2}{*}{$\begin{array}{l}\text { UN1T } \\
\text { Joul es/g }\end{array}$} \\
\hline & & 1 stb & & & DSC-01 & & & & & \\
\hline 96001213 & AH-105 GRAB & 2 SAMPLE & \$961005019 & 0 & DSC-01 & SOLID & $\mathrm{N} / \mathrm{A}$ & & & Joutes/g \\
\hline 96001213 & AW-105 GRAB & 3 DUP & 5961005019 & 0 & DSC=01 & SOLID & & & & Joutes/g \\
\hline 96001213 & AH-105 GRAB & 4 SAMPLE & S96T005020 & 0 & DSC-01 & SOLID & $\mathrm{N} / \mathrm{A}$ & & & Joules/g \\
\hline 96001213 & AW-105 GRAB & 5 DUP & $\$ 961005020$ & 0 & DSC-01 & SOLED & & & & Joules/g \\
\hline
\end{tabular}

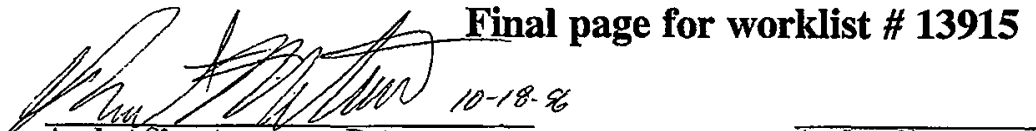

Analyst Signature Date

DSC-03 instrument

was wid.

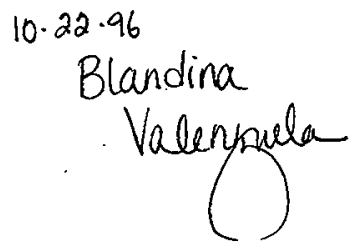

Data Entry Comments:

Units shown for $Q C$ (SPK \& STD) may not reflect the actual units. $D L=$ Detection Limit, $S=$ Worklist Slot Number, $R=$ Replicate Number, $A=$ Aliquot Code. 
Curve 1: DSC

File info: IND101801 Fri Oct 18 04: 48: 221996

Sample Weight: $22.750 \mathrm{mg}$

12N14-B INDIUM AT 10C/MIN

SIGNATURE BELOW REPRESENTS CHEMICAL TECHNOLOGIST/CHEMIST THA
COMPLETED/VERIFIED THE CALIBRATION/ANAYSS

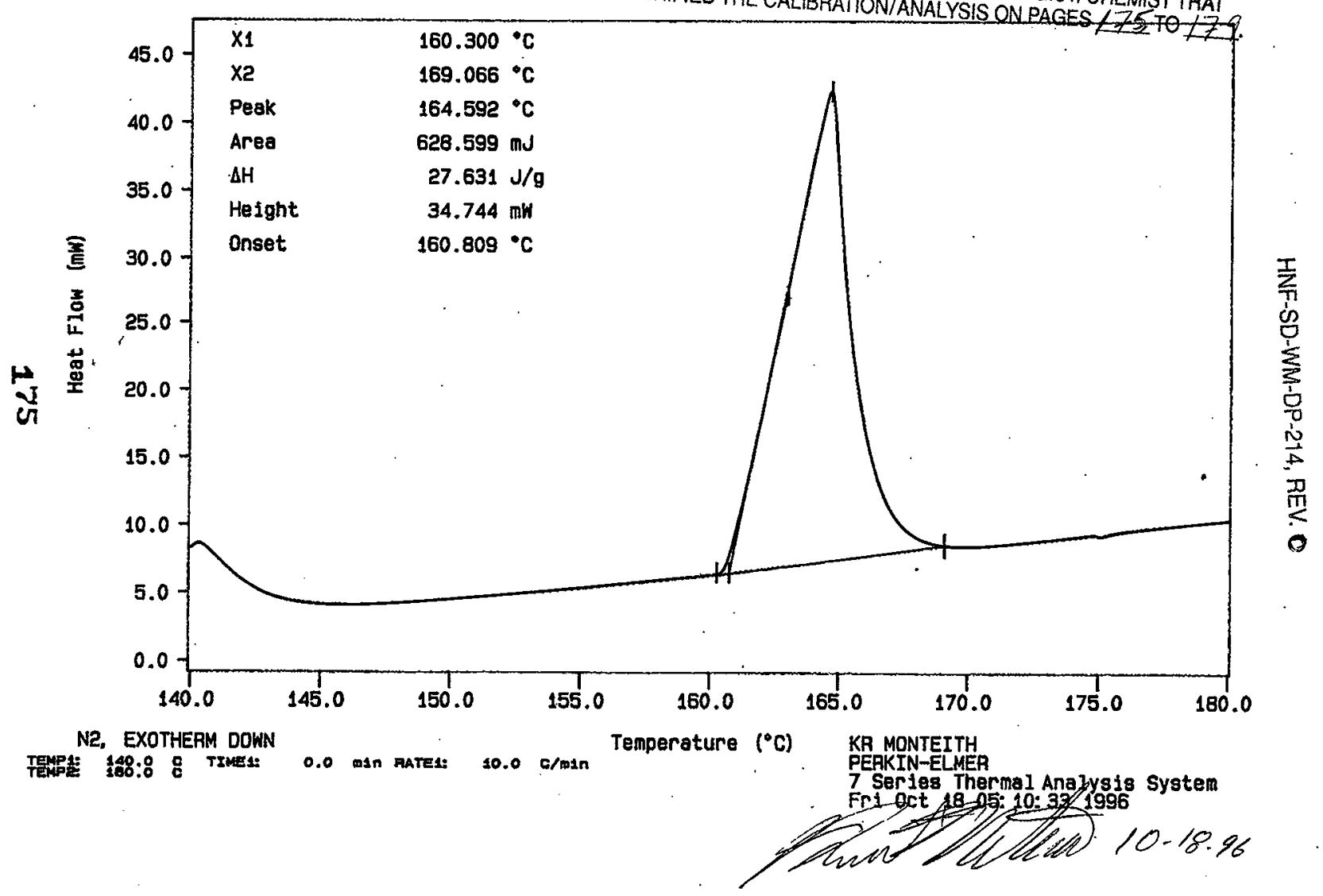


Curve 1: DSC

File info: SAM101801 Fri Oct 18 06: 34: 101996

Sample Weight: $10.780 \mathrm{mg}$

S96T005019

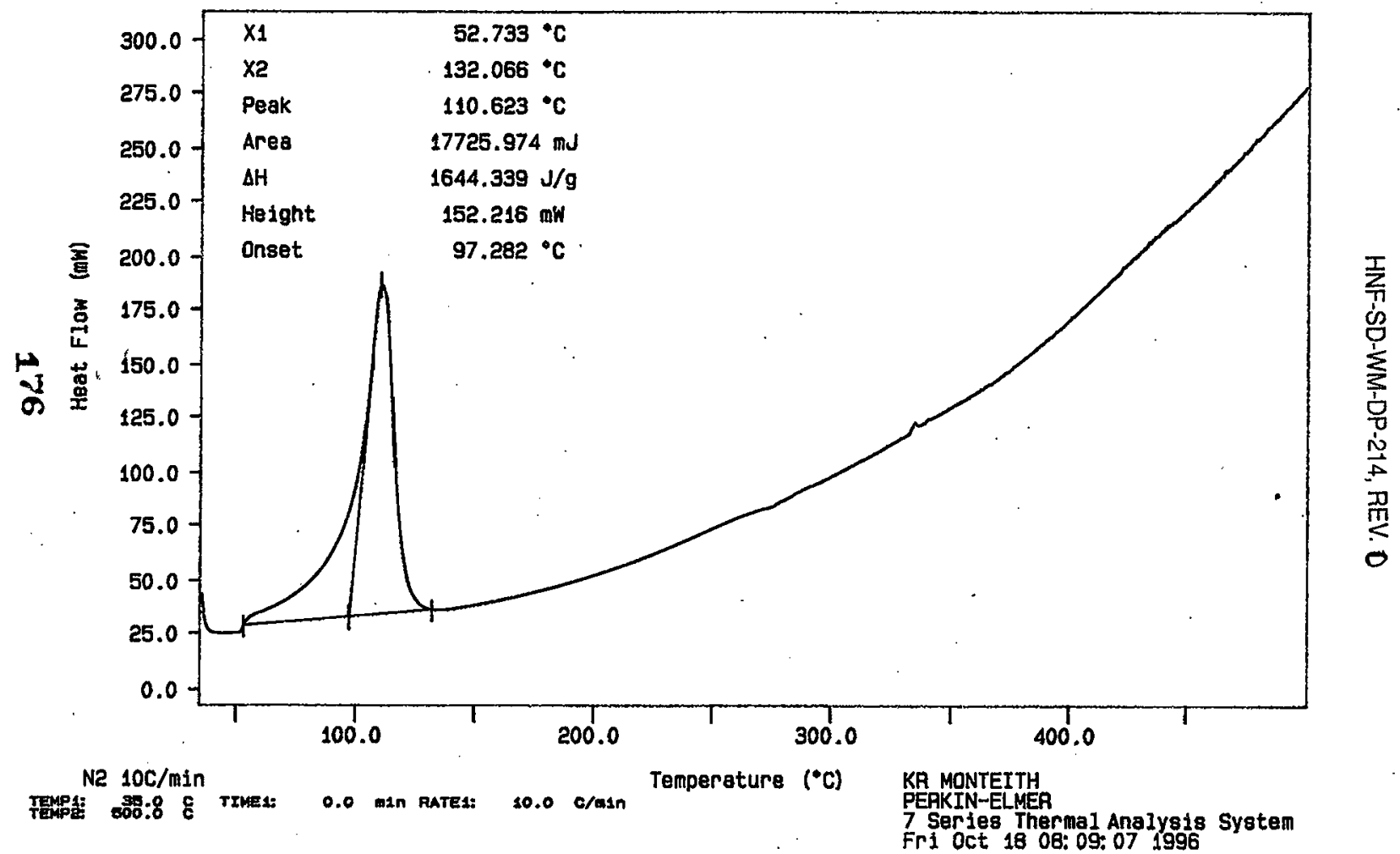


Curve 1: DSC

File info: SAM101802 Fri oct 18 09:02: 471996

Sample Weight: 23.680

$\mathrm{mg}$

S96T005019 DUP

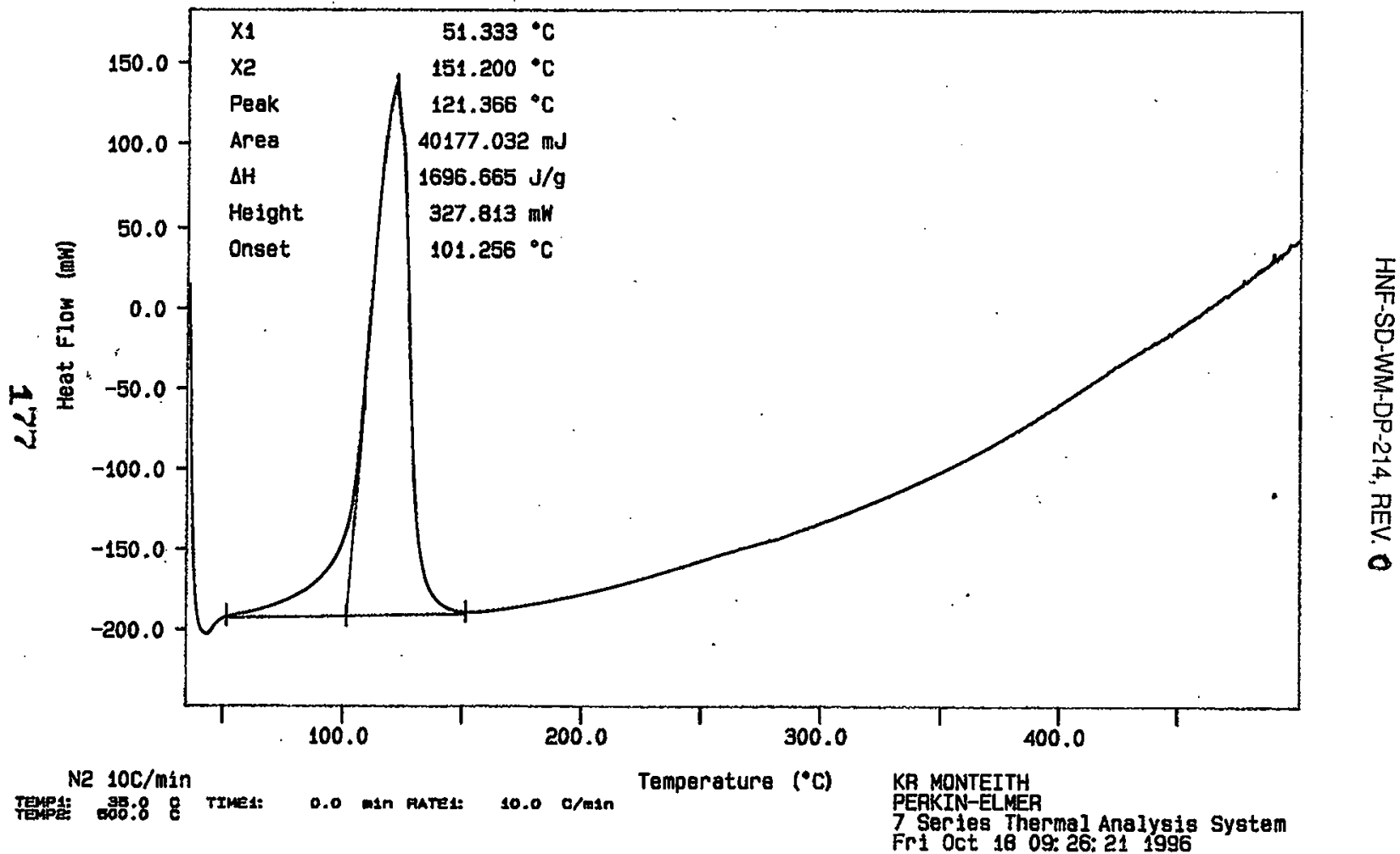


Curve 1: DSC

File Info: SAM101803 Fri Oct 18 10: 16: 351996

Sample Weight: $23.950 \mathrm{mg}$

S96T005020

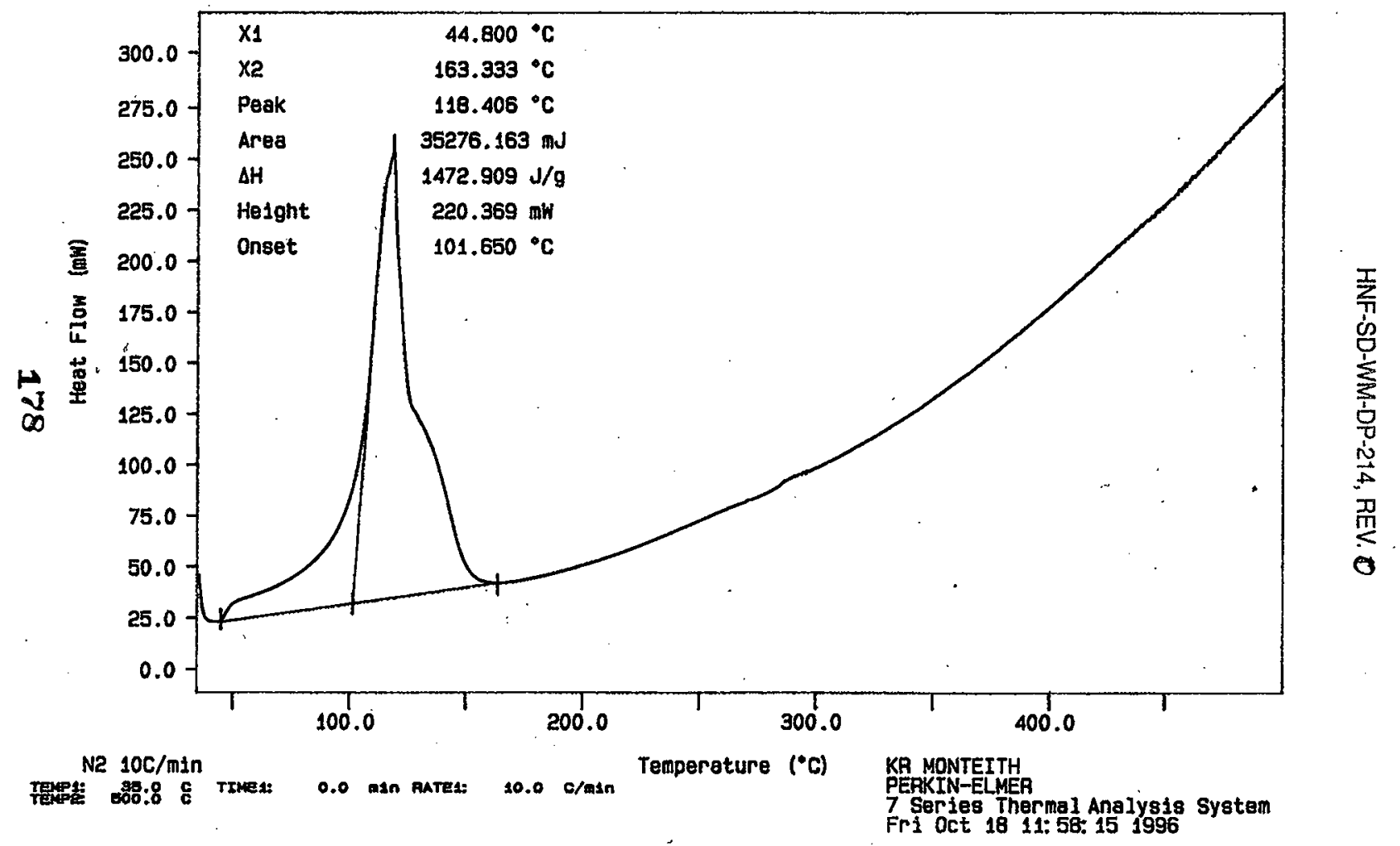


Curve 1: DSC

Fijo info: SAM101804 Fri Oct 18 12: 30: 221996

Sample Weight: $29.280 \mathrm{mg}$

S96T005020 DUP

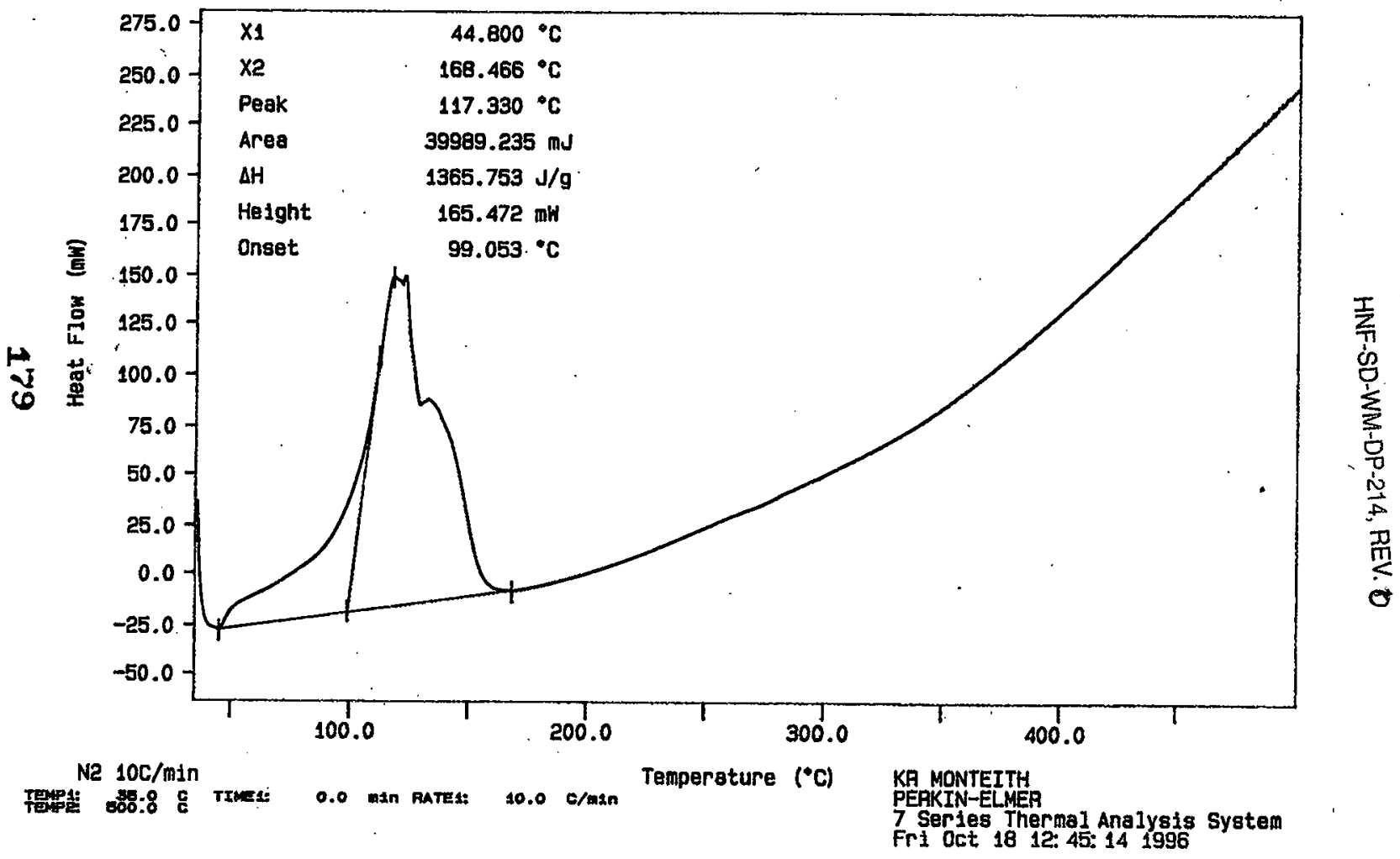




\section{LABCORE Completed Worklist Report for Worklist\# 13916}

Analyst: rga

Instrument: DSC01

Book\# 12Ni4B

Method: $L A-514-113$ Rev/Mod C -1

Worklist Comment: AW-105 GRAB. Run under nitrogen.Std: INDIUM $\sim 8 \mathrm{mg}$. new

\begin{tabular}{|c|c|c|c|c|c|}
\hline Seq Type & Sample\# $\mathbf{R} A$ & Test & Matrix & Actual & Found \\
\hline $1 \mathrm{sTD}$ & & $\mathrm{DSC}-01$ & SOLID & 28,45 & 27,54 \\
\hline 2 SAMELE & $\$ 96 T 005021$ & DSC- 01 & SOLID & $N / A$ & 14.1 \\
\hline 3 DUR & $896 \mathrm{~T} 00502 \mathrm{I}$ & $0 \mathrm{Sa-01}$ & SOLID & $14 \%$ & क \\
\hline 4 SAMPLE & $\$ 96 \mathrm{~T} 005075$ & DSC-01 & SOLID & $N / A$ & 16.4 \\
\hline SDEP & so67005075 & $\sigma_{n}$ & $\mathrm{CO}$ & & 15 \\
\hline
\end{tabular}

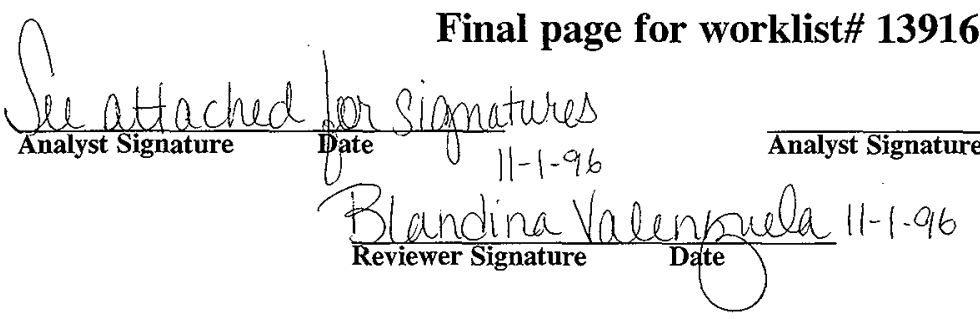




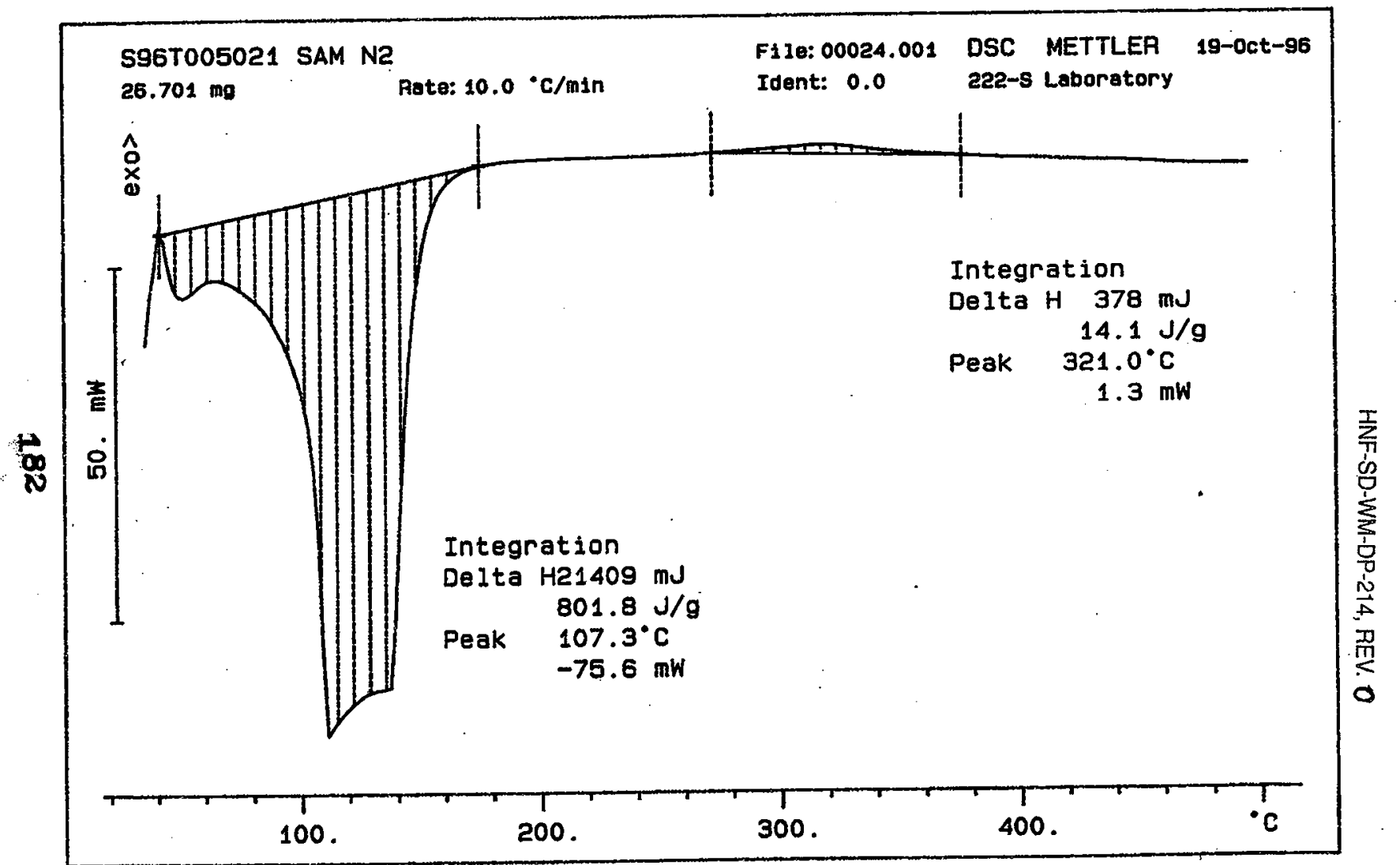


HNF-SD-WM-DP-214, REV. 0

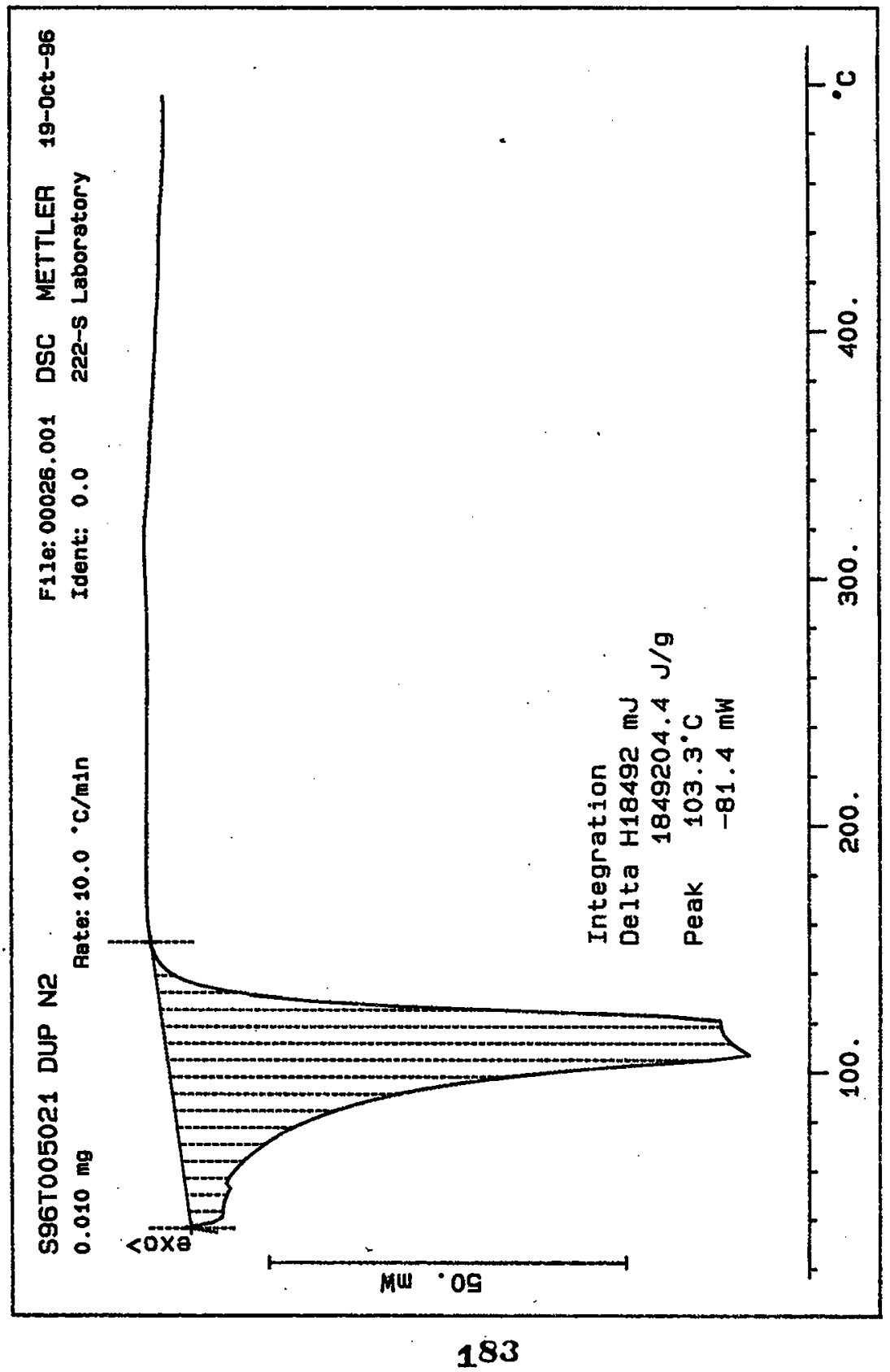




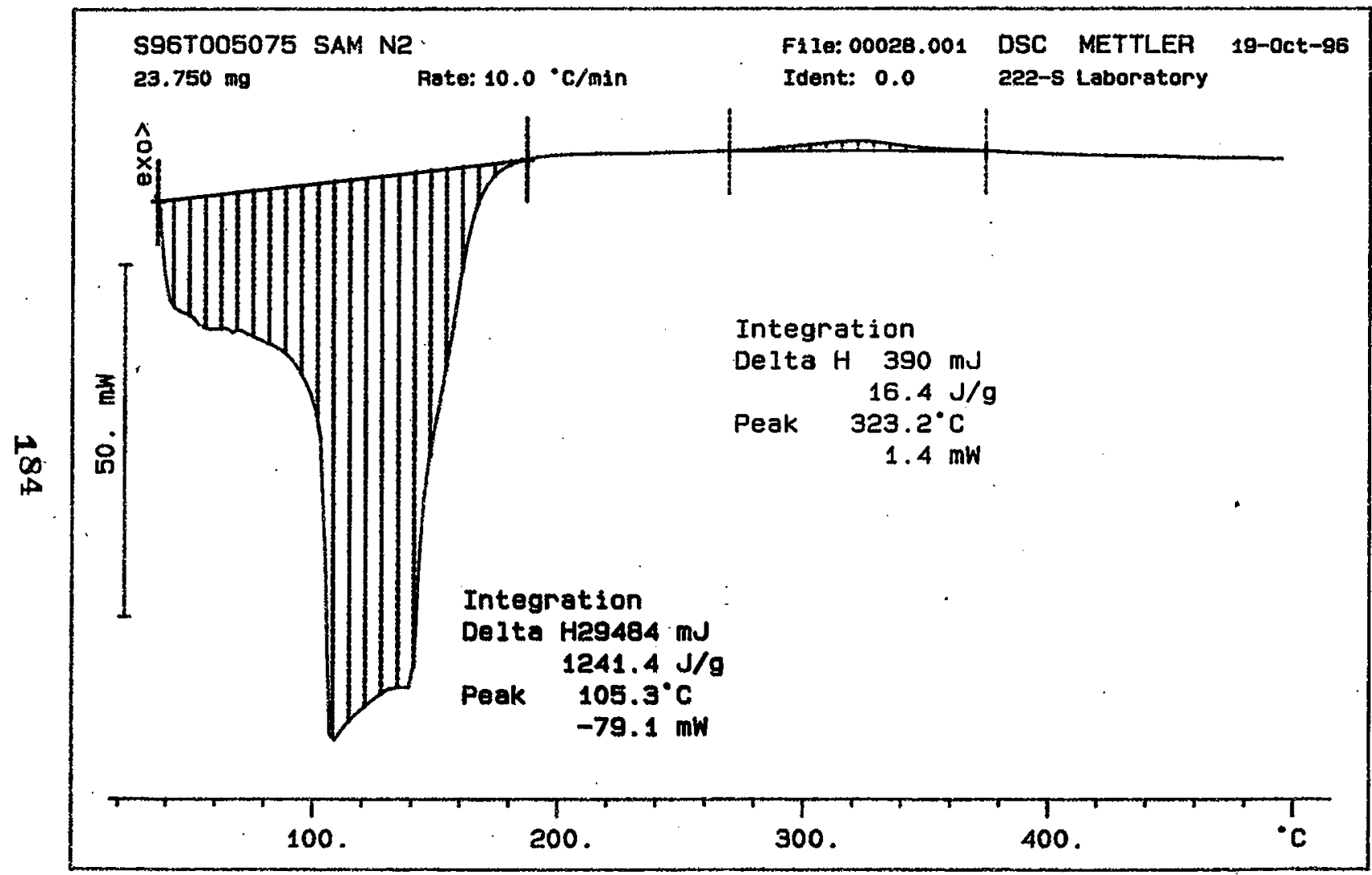


HNF-SD-WM-DP-214, REV. 0

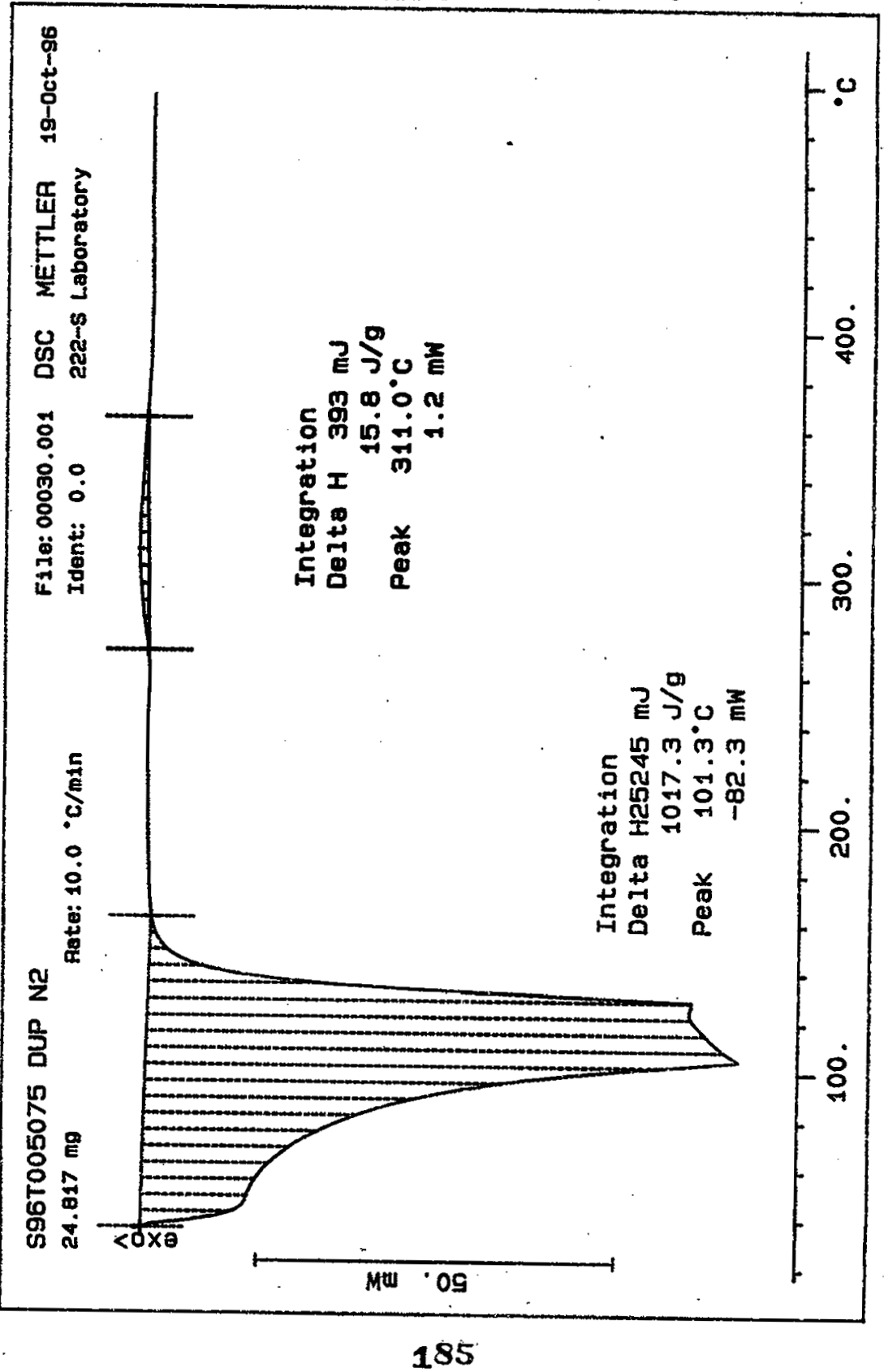




\section{LABCORE Completed Worklist Report for Worklist\# 13917}

Analyst: krm Instrument: DSC01

Book\# 12 N14B

Method: LA-514-113 Rev/Mod C-1

Worklist Comment: AW-105 Grab. Run under nitrogen. Std: INDIUM 8mg. new

\begin{tabular}{lllll}
\hline Seq Type & Sample\# RA & Test & Matrix Actual & Found DL or Yield Unit
\end{tabular}

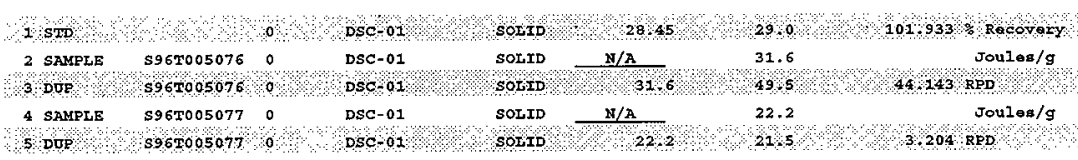

\section{Final page for worklist\# 13917}

Ale attached or siomatures 10.30 .96

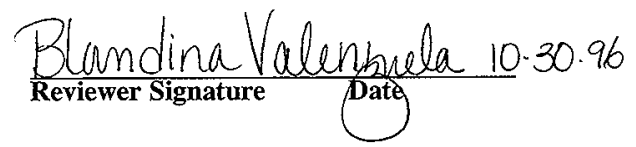


SIGNATURE BELOW REPRESENTS CHESHICAL TECHNOLOGIST/CHEMIST THAT

COMPLETED VERIFIED THE CAL IBRATIONLANALVSIS ONDAGES I IO IO

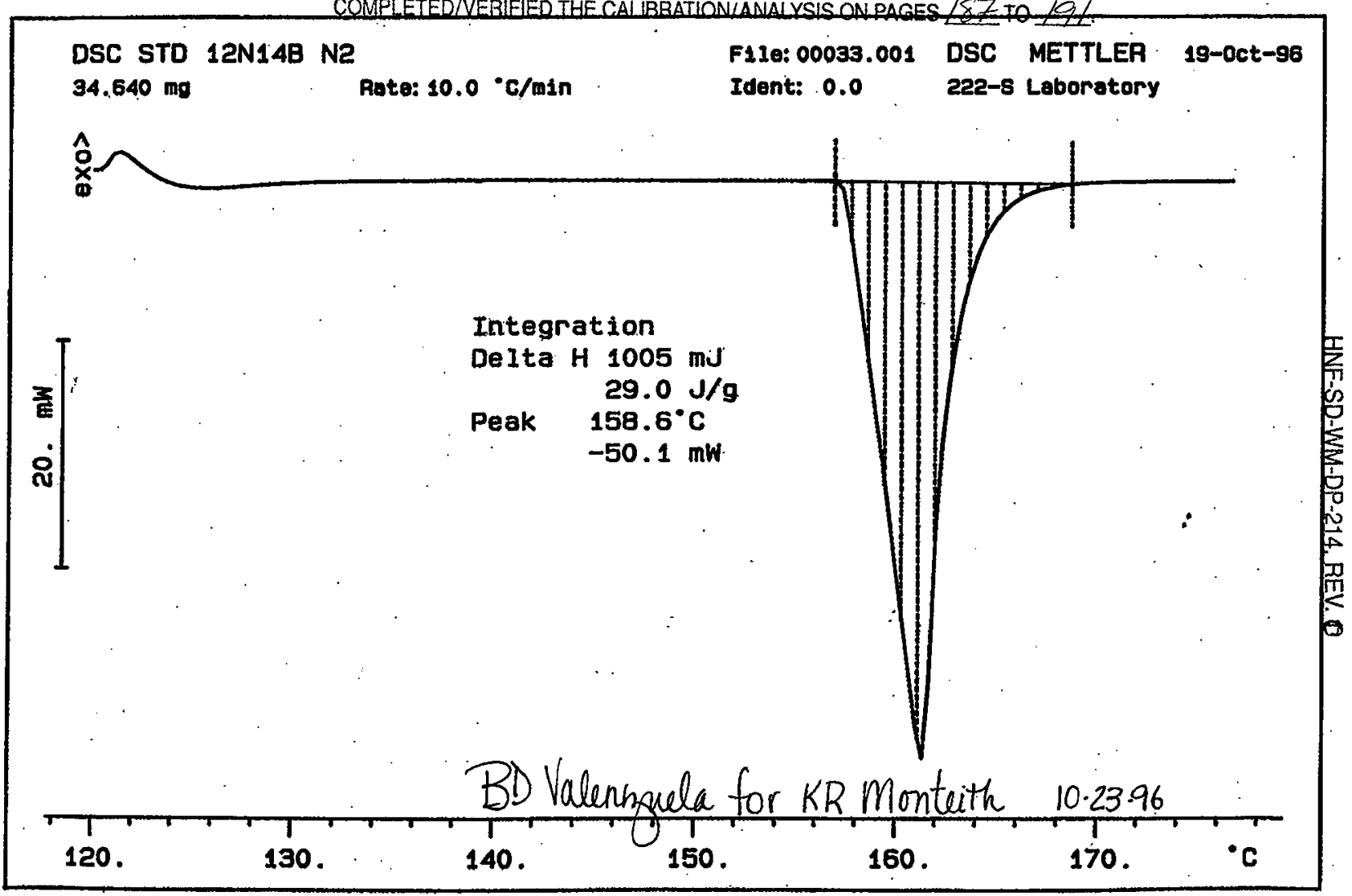




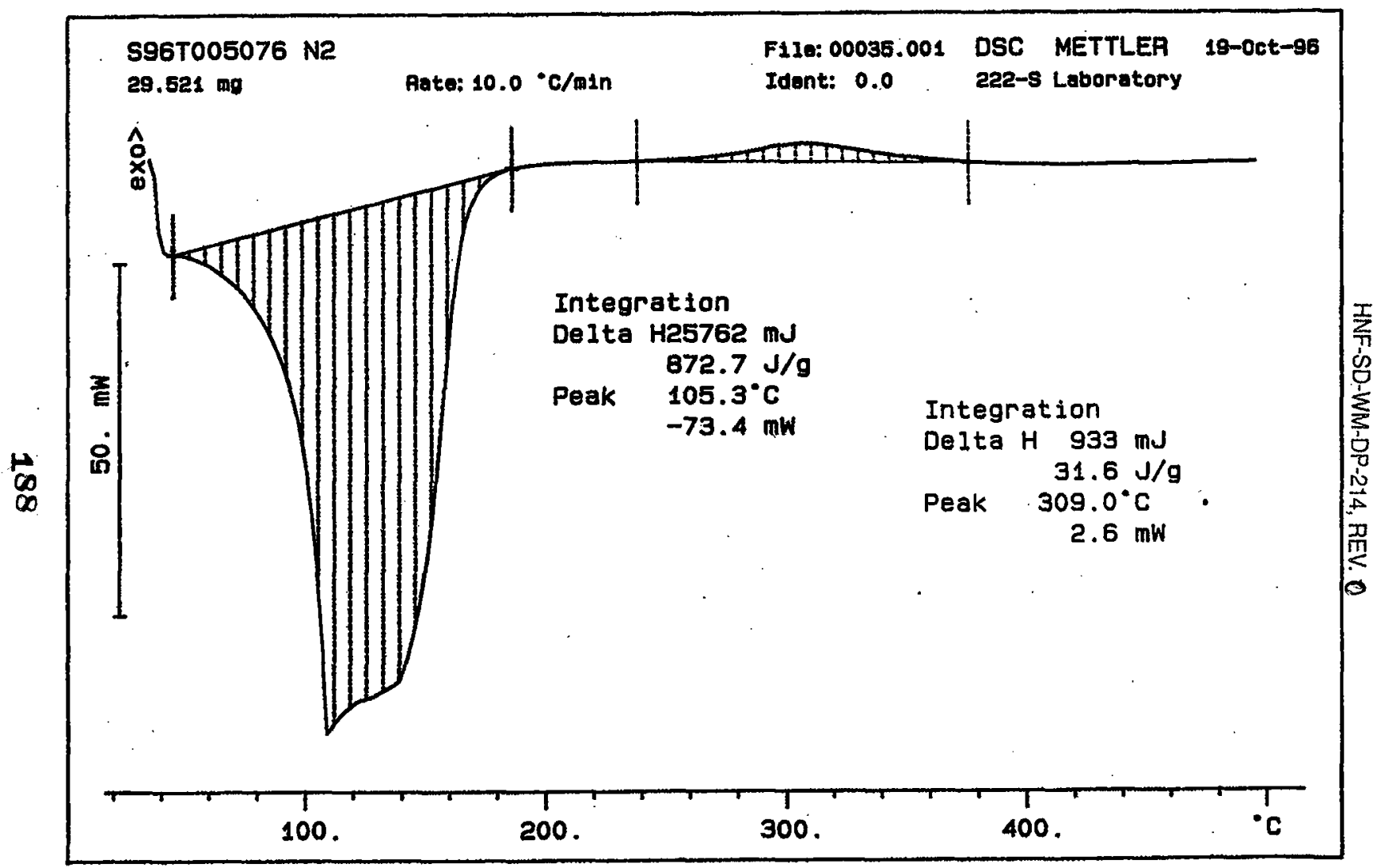




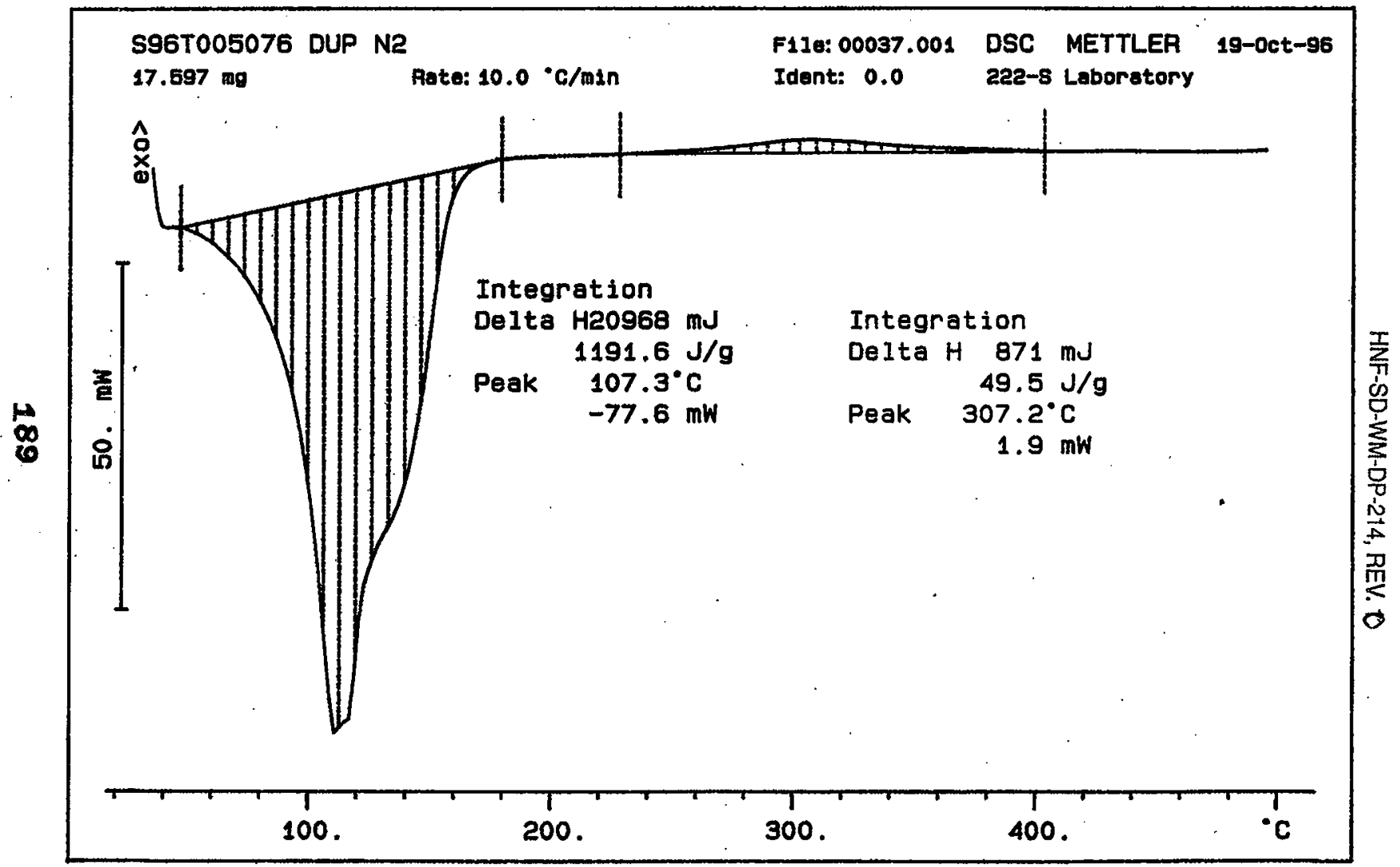




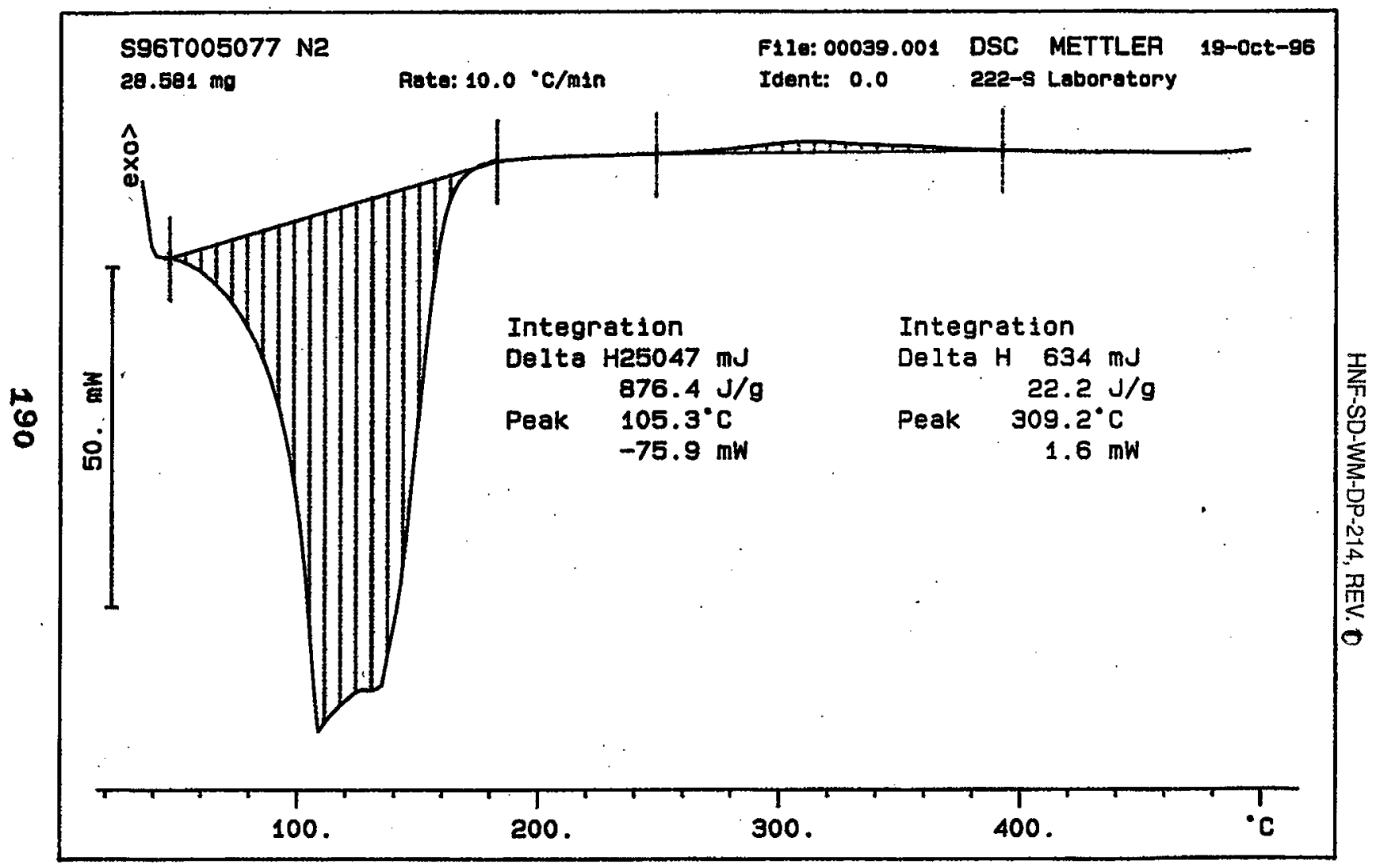


Analyst:

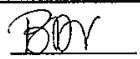

Instrument: $\mathrm{DSC} 01$

Book \#

Method: LA-514-113 Rev/Mod

Worklist Comment: Dry DSC for AW-105. bdv

\begin{tabular}{|c|c|c|c|c|c|c|c|c|c|c|}
\hline GROUP & PROJECT & S TYPE & SAMPLE\# & R A & $\cdots$ TEST $\cdots$ & MATRIX & ACTUAL & FOUND & DL & UNIT \\
\hline 96001213 & AW-105 GRAB & 1 SAMPLE & S96T005021 & 0 & DSC -02 & SOLID & N/A & 42.1 & & Joules/g Dгy \\
\hline 96001213 & AW-105 GRAB & 2 DUP & S96T005021 & 0 & DSC-02 & SOLID & 42.1 & $b$ & N/A & Joules/g Dry \\
\hline 96001215 & AW-105 GRAB & 3 SAMPLE & $596 r 005075$ & 0 & DSC-02 & SOL ID & $\mathrm{N} / \mathrm{A}$ & 66.7 & & Joules/g Dry \\
\hline 96001215 & AW-105 GRAB & 4 DUP & S96T005075 & 0 & DSC-02 & SOLID & 66.7 & 64.3 & $\mathrm{~N} / \mathrm{A}$ & Joules/g Dry \\
\hline 96001215 & $A W-105$ GRAB & 5 SAMPLE & S961005076 & 0 & DSC-02 & SOLID & N/A & 92.8 & & Joules/g Dry \\
\hline 96001215 & $A W-105$ GRAB & 6 DUP & S96T005076 & 0 & DSC-02 & SOLID & 92.8 & 145 & $\mathrm{~N} / \mathrm{A}$ & Joules/g Dry \\
\hline 96001215 & AW-105 GRAB & 7 SAMPLE & S961005077 & 0 & DSC-02 & SOLID & N/A & 70.9 & - & Joules/g Dry \\
\hline 96001215 & $A W-105$ GRAB & 8 DUP & \$96т005077 & 0 & DSC-02 & SOLID & 70.9 & 68.7 & N/A & Joules/g Dry \\
\hline
\end{tabular}

\section{Final page for worklist \# 14911}

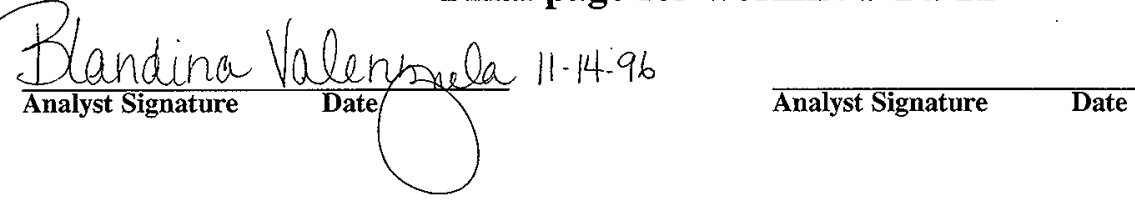

Data Entry Comments:

Units shown for $Q C$ (SPK \& STD) may not reflect the actual units. $D L=$ Detection Limit, $S=$ Worklist Slot Number, $R=$ Replicate Number, $A=$ Aliquot Code. 
HNF-SD-WM-DP-214, REV. 0

Dry DSC for Aw-105

\begin{tabular}{|c|c|c|c|}
\hline \multicolumn{4}{|c|}{ CALCULATED DRY DSC } \\
\hline SAMPLE NO. & DSC RESULT $(\mathrm{J} / \mathrm{g})$ & TGA RESULT (\% water) & DRY DSC RESULT \\
\hline 5967005021 & 14.1 & 60.54 & 42.1 \\
\hline 50210 & $\varnothing$ & 60.54 & $\varnothing$ \\
\hline 5075 & 10.4 & 75.41 & 66.7 \\
\hline 50750 & 15.8 & 75.41 & 64.3 \\
\hline 5076 & 31.6 & 65.96 & 92.8 \\
\hline 50760 & 49.5 & 65.96 & 145.4 \\
\hline 5077 & 22.2 & $68,-70$ & 70.9 \\
\hline $5077 D$ & 21.5 & 68.70 & 68,7 \\
\hline & & & \\
\hline & & & \\
\hline 5049 & 0 & 92.68 & \\
\hline 50490 & $\widehat{\mathrm{b}}$ & 92.68 & \\
\hline 5050 & 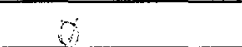 & 88.55 & \\
\hline $5050 \mathrm{~s}$ & $x$ & 89.55 & \\
\hline 5019 & $\varnothing$ & 80.11 & \\
\hline $5019 D$ & $\varnothing$ & 80.11 & \\
\hline 5020 & $\phi$ & 65.47 & \\
\hline $5020 D$ & $\emptyset$ & 65.47 & \\
\hline 5051 & $\varnothing$ & 88.85 & \\
\hline 50510 & $\varnothing$ & 88.85 & \\
\hline 5081 & $\varnothing$ & 90.66 & \\
\hline 50810 & $\varnothing$ & 90.66 & \\
\hline 5082 & $\varnothing$ & -2 & \\
\hline 50820 & $\varnothing$ & & \\
\hline 5083 & $\varnothing$ & $\longrightarrow$ & \\
\hline 50830 & $\varnothing$ & & \\
\hline
\end{tabular}




\section{LABCORE Data Entry Template for Worklist\# 13068}

Analyst: JLF

Instrument: TGAO 1 .

Book \#82084

Method: LA-560-112 Rev/Mod

B-1

Worklist Comment: AW-105 TGA, RUN UNDER N2. RCJ

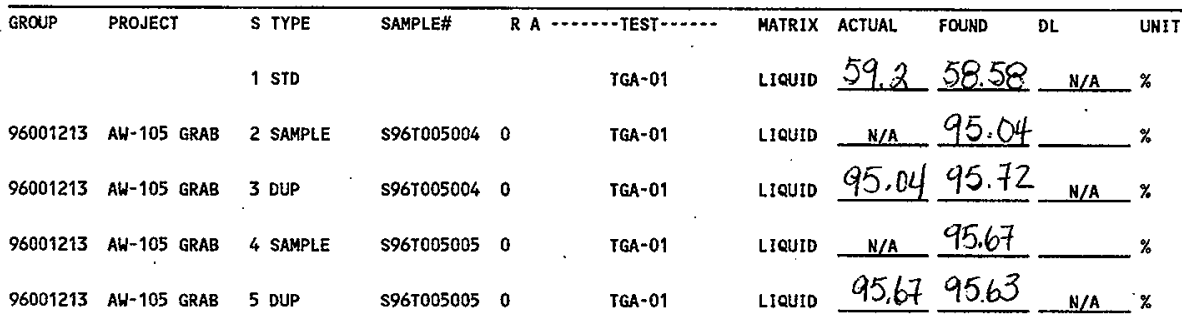

\section{Final page for worklist \# 13068}

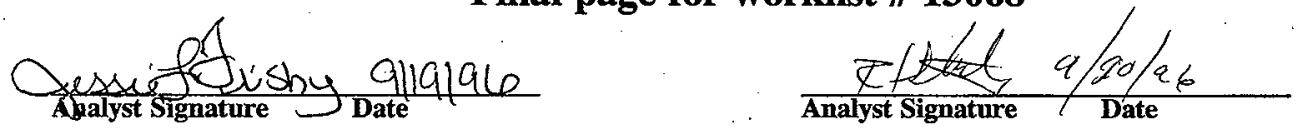

Verified/Validattic ung

Blandina

Valengmela $9123 / 96$

Data Entry Comments:

Units shown for QC (SPK \& STD) may not reflect the actual units. $D L=$ Detection Limit, $S=$ Worklist Slot Number,

$R=$ Replicate Number, $A=$ Aliquot Code. 


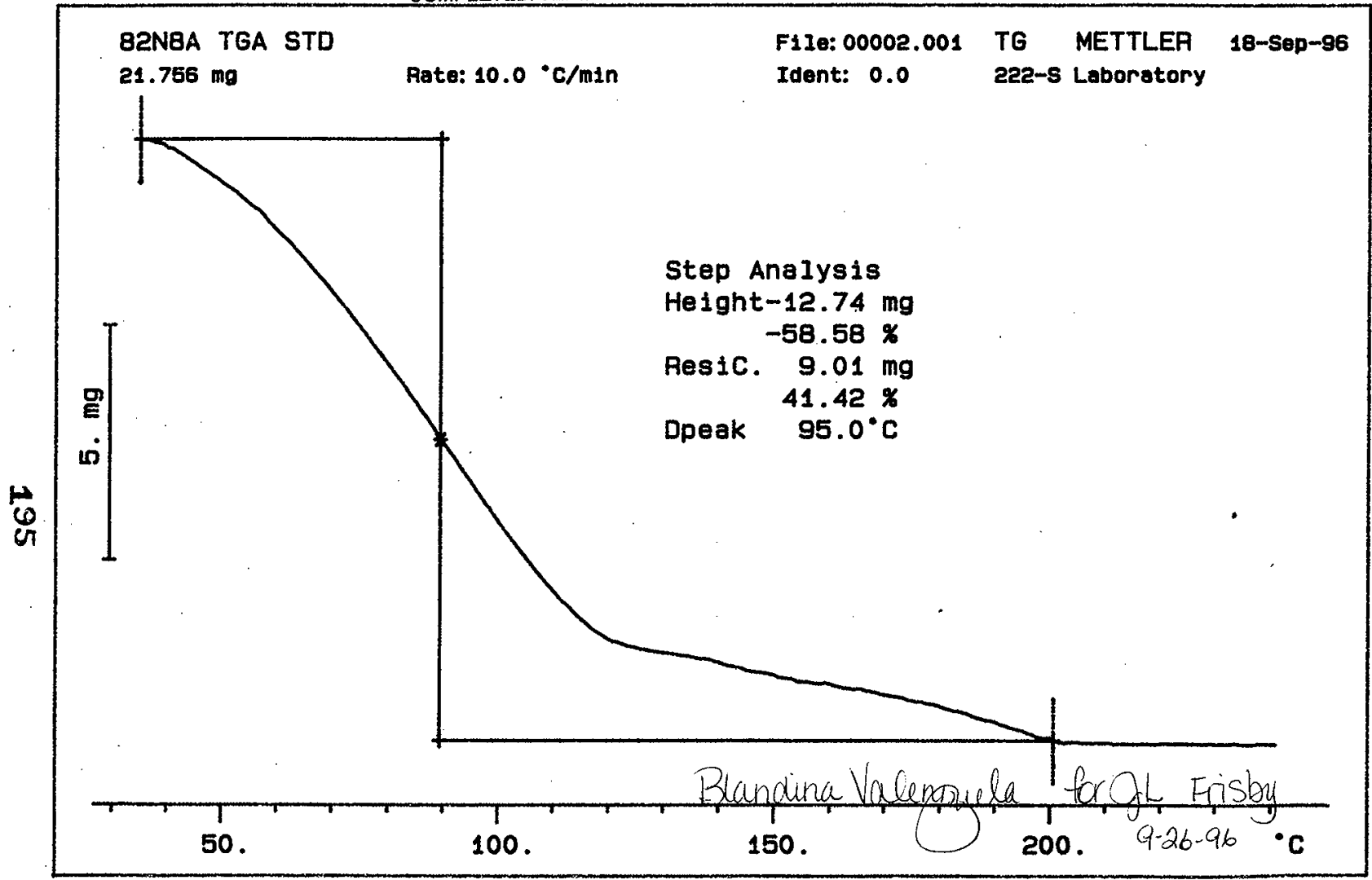




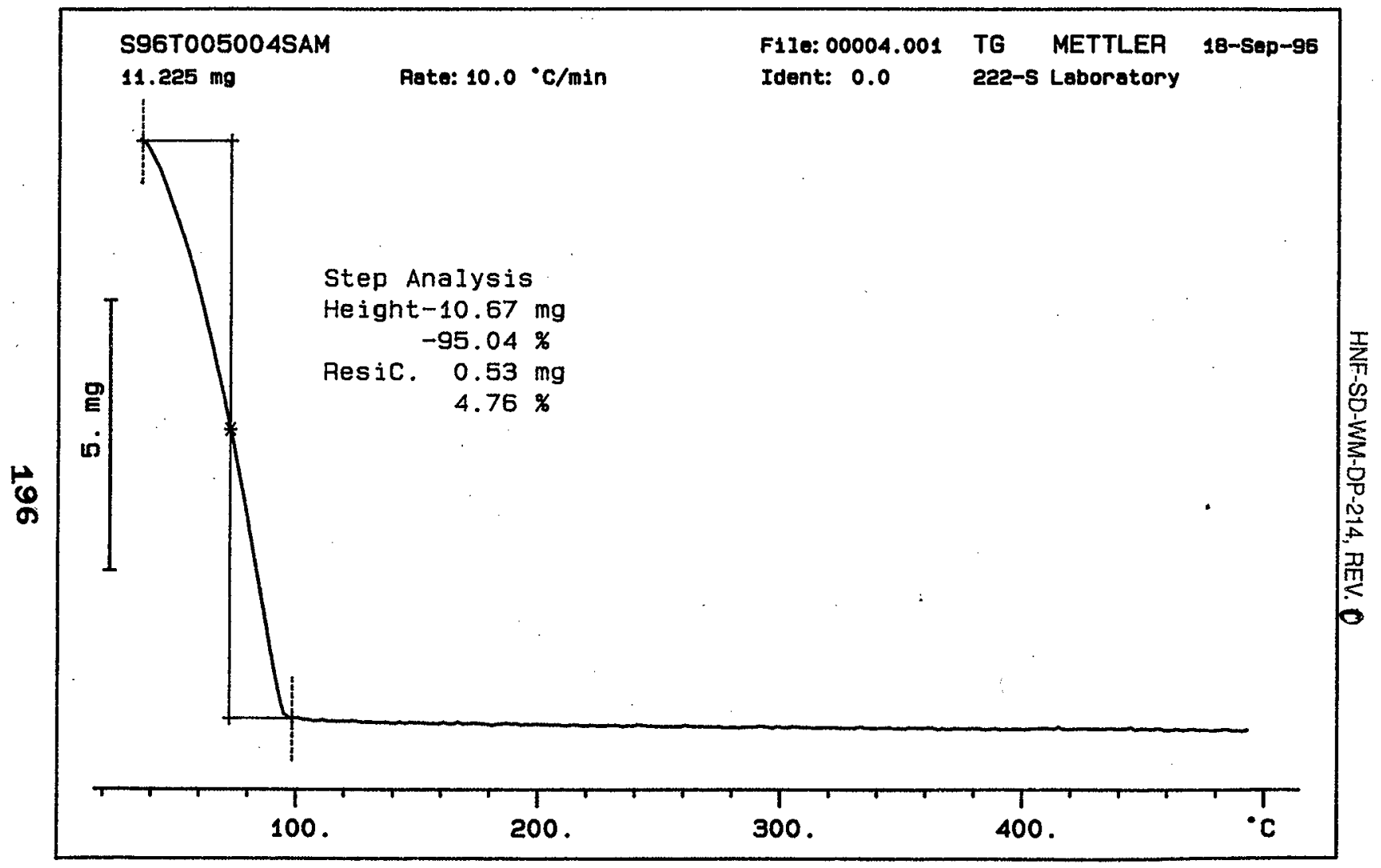




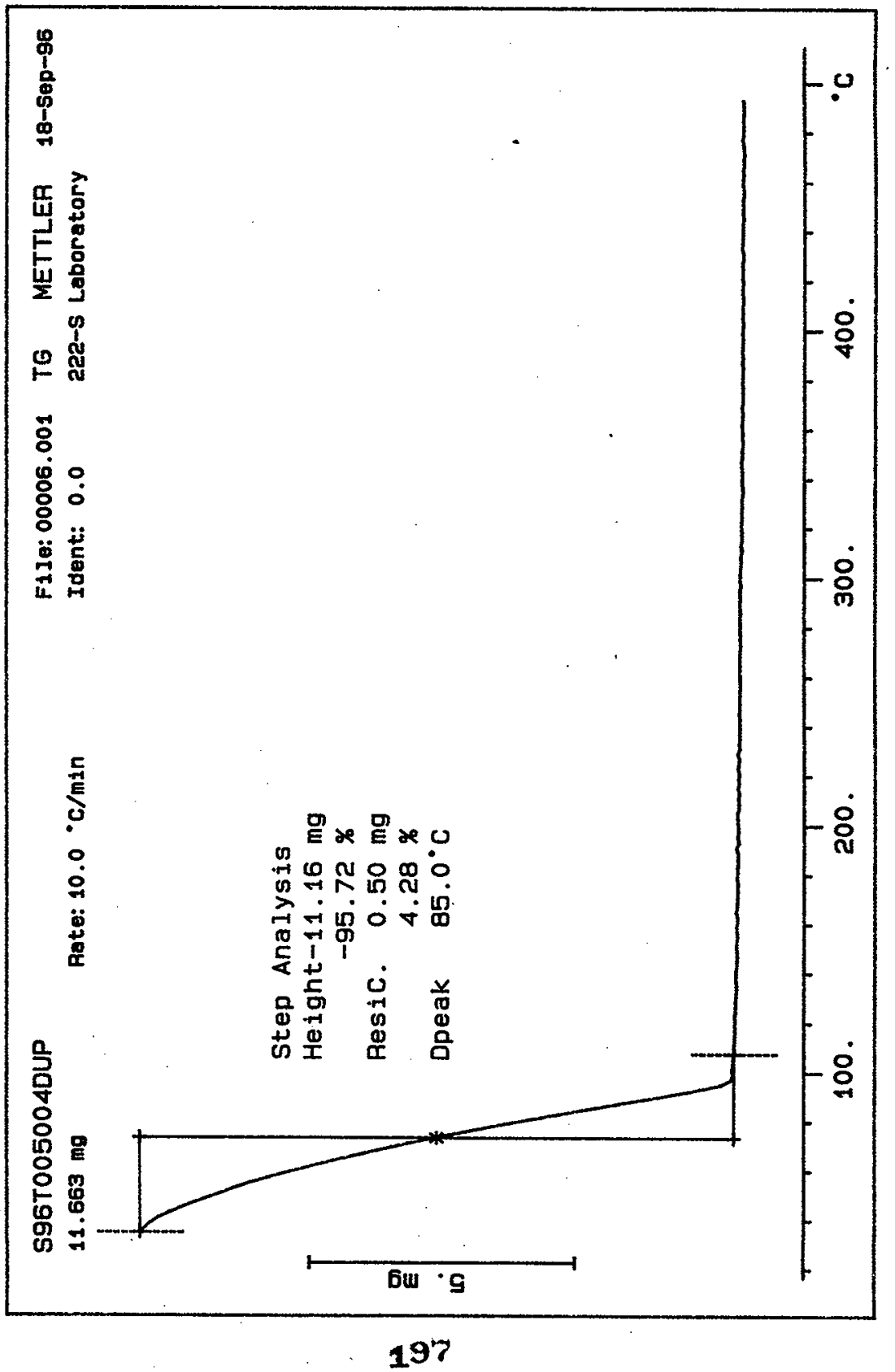




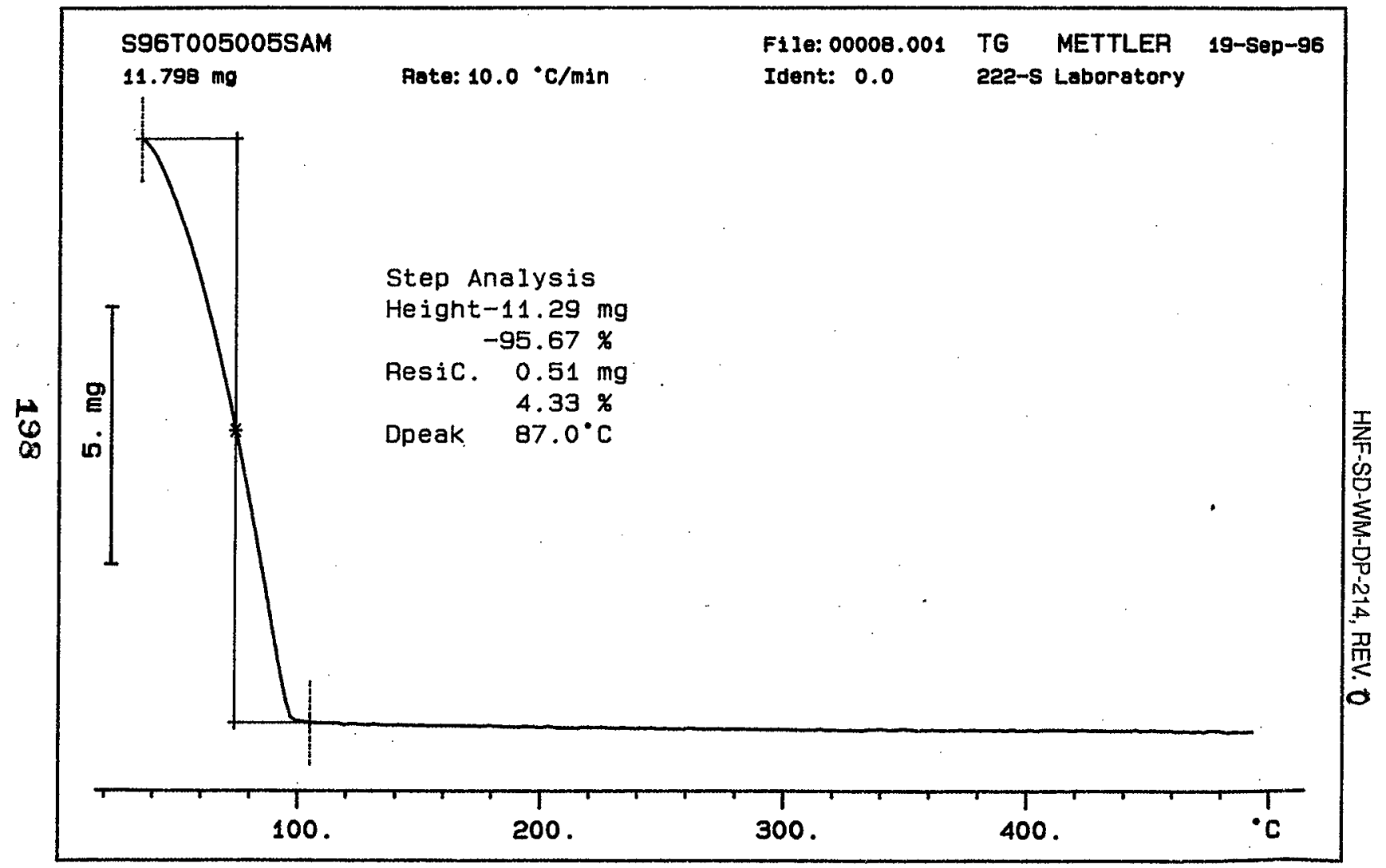




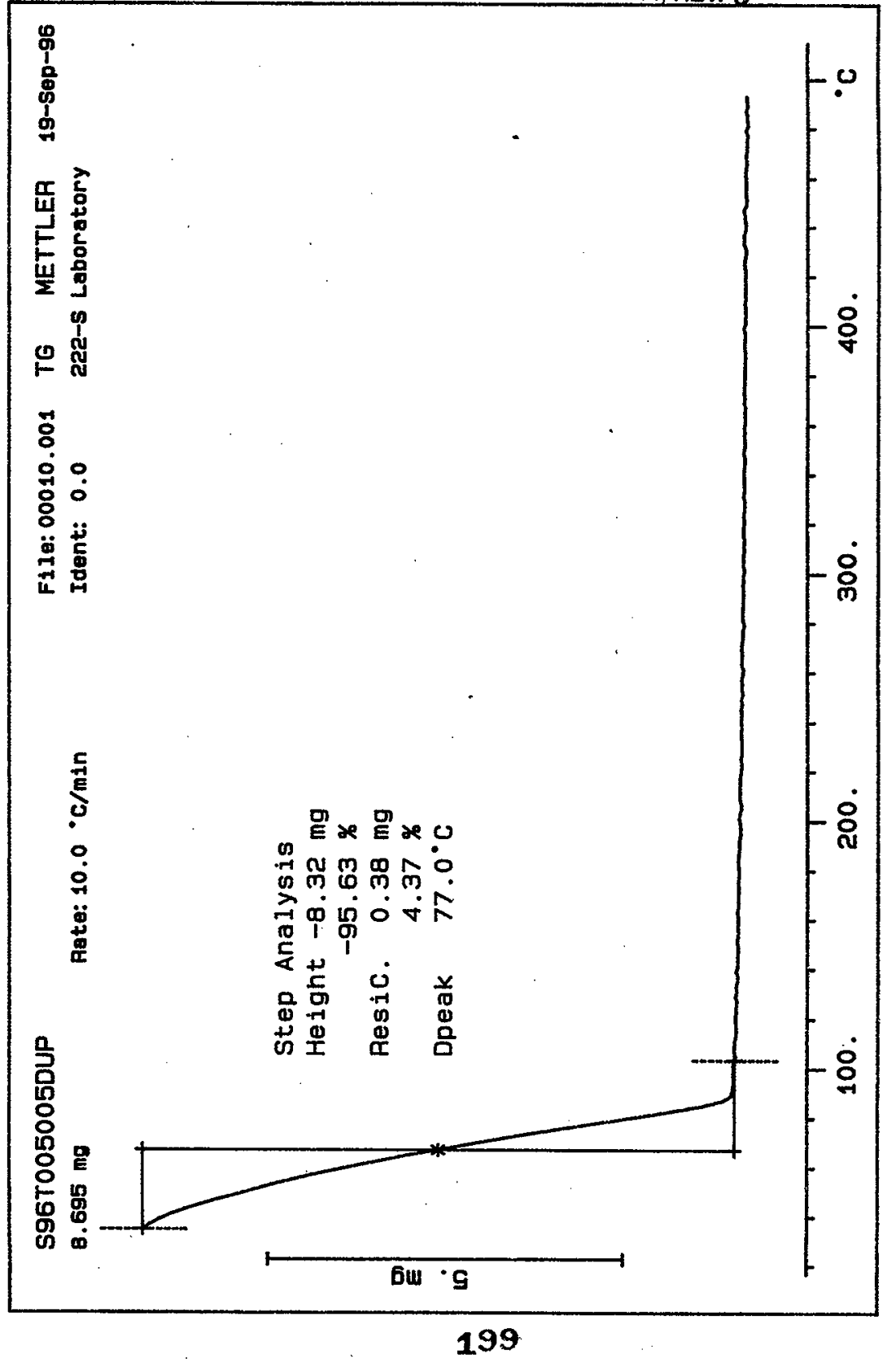


worklistrpt Version 2.1 05/15/95

HNF-SO-WM-DP-214, REV. 0

Page: 1

09/19/96 14:04

LABCORE Data Entry Template for Worklist\# 13069

Analyst: $\quad \underline{J F} \quad$ Instrument: TGA0 $\underline{3}$ Book \#82N8A

Method: LA-514-114 Rev/Mod C - 1

Worklist Comment: AW-105 TGA, RUN UNDER N2. RCJ

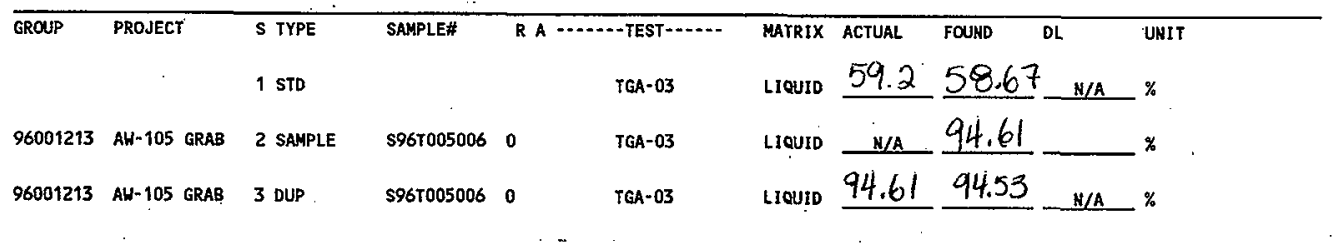

Final page for worklist \# 13069

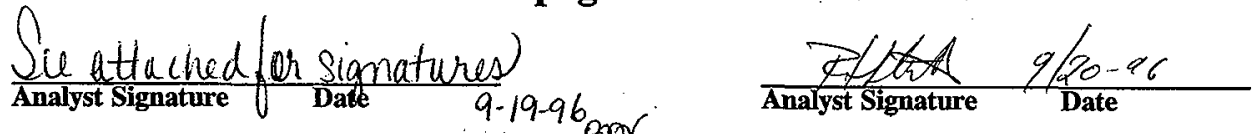

Verified/Validatea by

Blandina.

Yalengula alas /96

Data Entry Comments:

Units shown for $Q C$ (SPK \& STD) may not reflect the actual units. $D L=$ Detection Limit, $S=$ Worklist Slot Number, $R=$ Replicate Number, $A=$ Aliquot Code.

200 


\section{LABCORE Data Entry Template for Worklist\# 13069}

Analyst: $\int L F$ Instrument: TGA0 Book\# 82 N8A

Method: LA-560-112 Rev/Mod

Worklist Comment: AW-105 TGA, RUN UNDER N2. RCJ

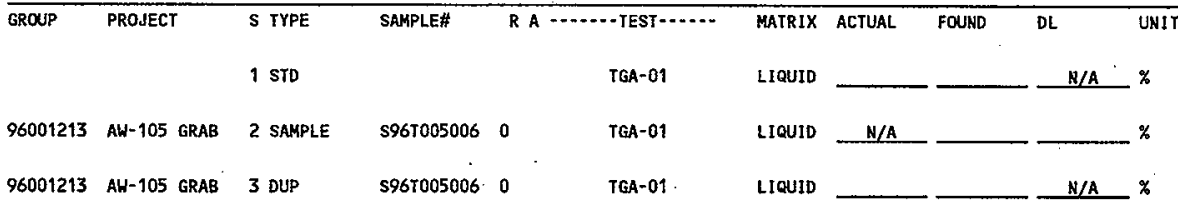

\section{Final page for worklist \# 13069}

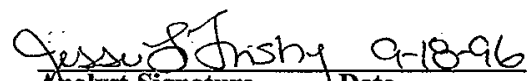

Phalyst Signature JDate

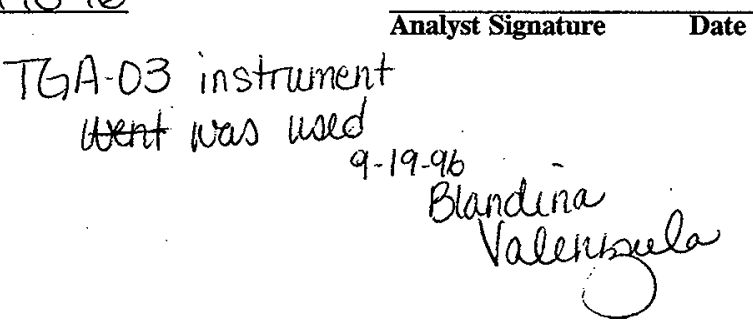

Data Entry Comments:

Units shown for $Q C$ (SPK \& STD) may not reflect the actual units. $D L=$ Detection Limit, $S=$ Worklist Slot Number, $R=$ Replicate Number, $A=$ Aliquot Code. 
Cunve 1: TGA

File info: TER091801 Wed Sep 18 18: 24:09 1996

Sample Weight: 19.153

TGA STD BENB-A

mg

SICNATUFE BELOW REPRESENTS CHEMICAL TECHNOLOGIST/CHEMIST THAT
COMPLETED/VERIFIED THE CALIBRATION/ANALYSIS ON PAGES 202 TO 204.

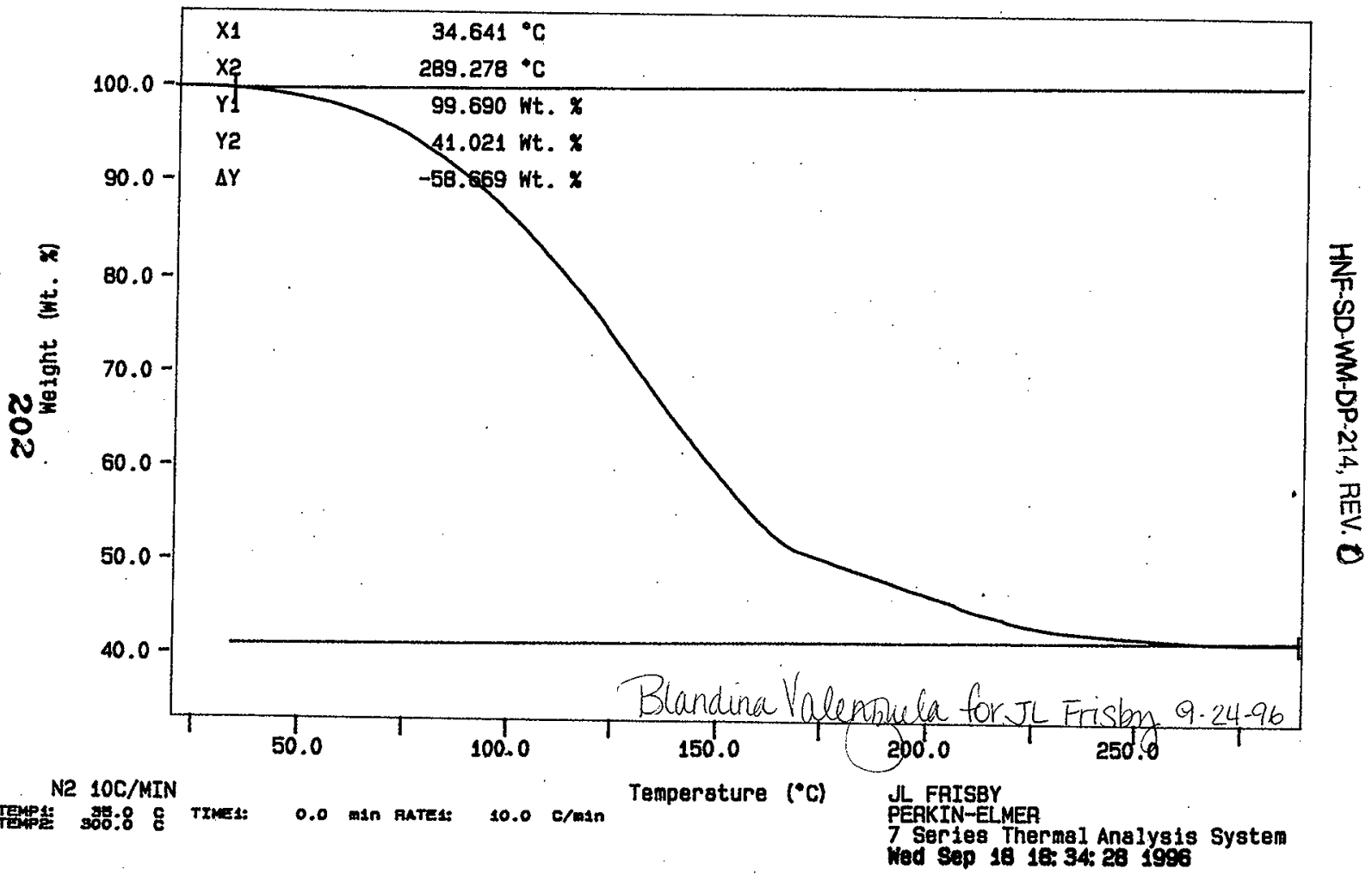


HNF-SD-WM-DP-214, REV. 0

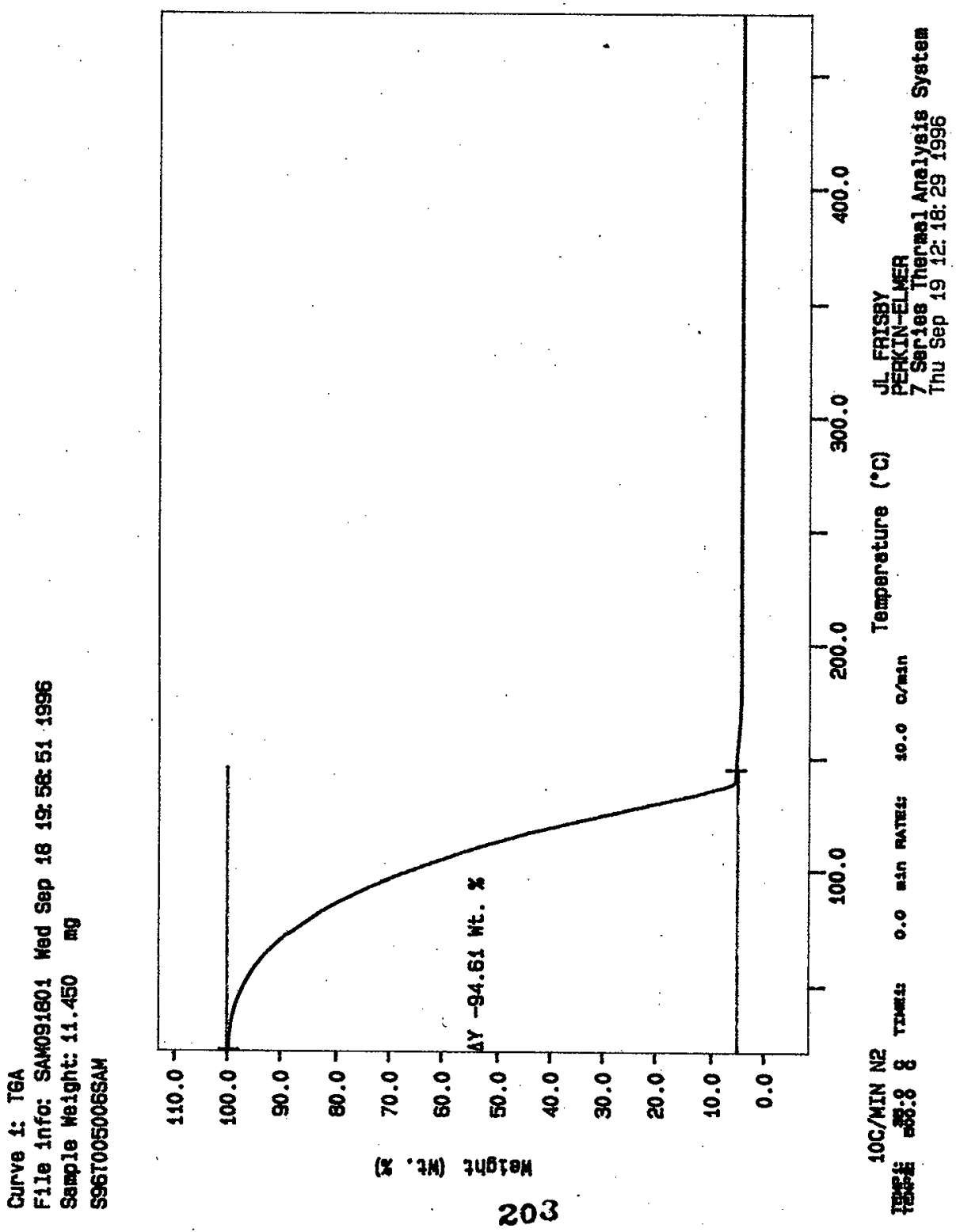


Curve 1: TGA

F1le info: DUP091804 Wed Sep 18 22: 05: 361996

Sample Weight: $9.303 \mathrm{mg}$

S96T005006DUP

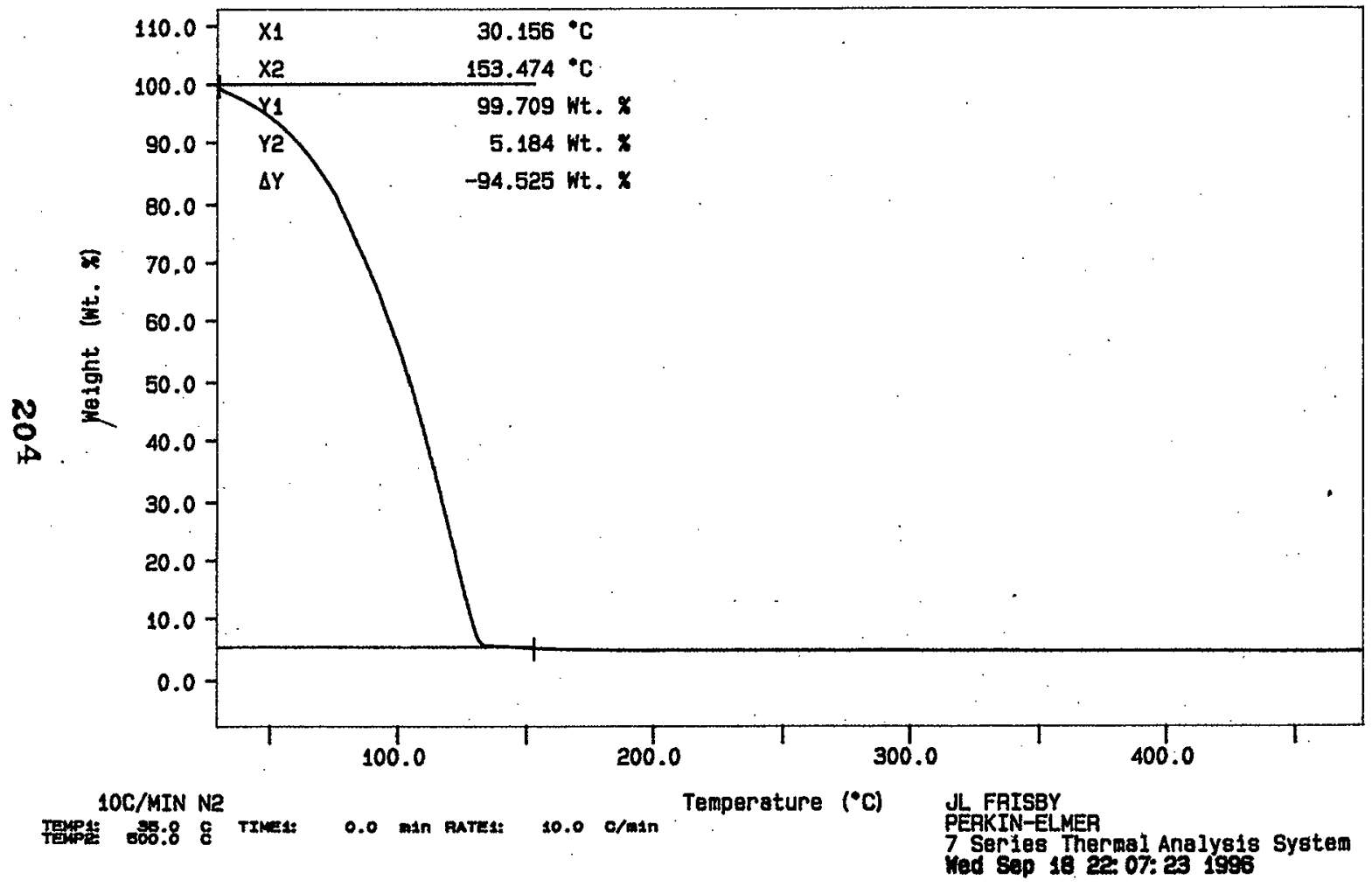


worklistrpt Version 2.1 05/15/95

HNF-SD-WM-DP-214, REV. 0

Page: $\quad 1$.

09/20/96 10:35

LABCORE Data Entry Template for Worklist\# 13132

Analyst: GAM Instrument: TGAO I Book 82 NSA

Method: LA-560-112 Rev/Mod

$B-1$

$\therefore$ Worklist Comment: AW-105 TGA, RUN UNDER N2. RCJ

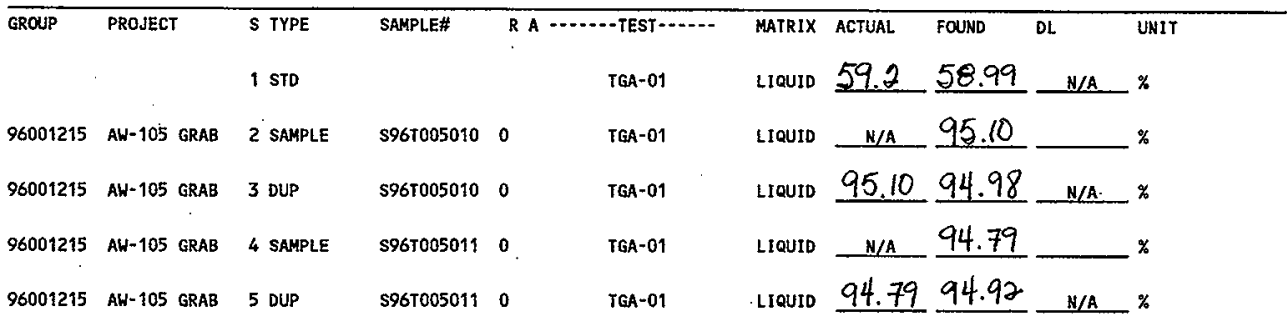

Final page for worklist \# 13132

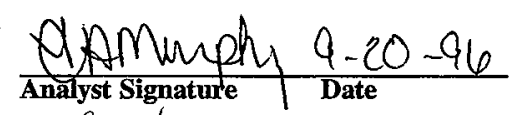

Verifid/Validated by
Bland ina

Vale sulla $9-26-96$

Data Entry Comments:

Units shown for QC (SPK \& STD) may not reflect the actual units. DL = Detection Limit, $S=$ Worklist Slot Number, $R=$ Replicate Number, $A=$ Aliquot Code.

205 


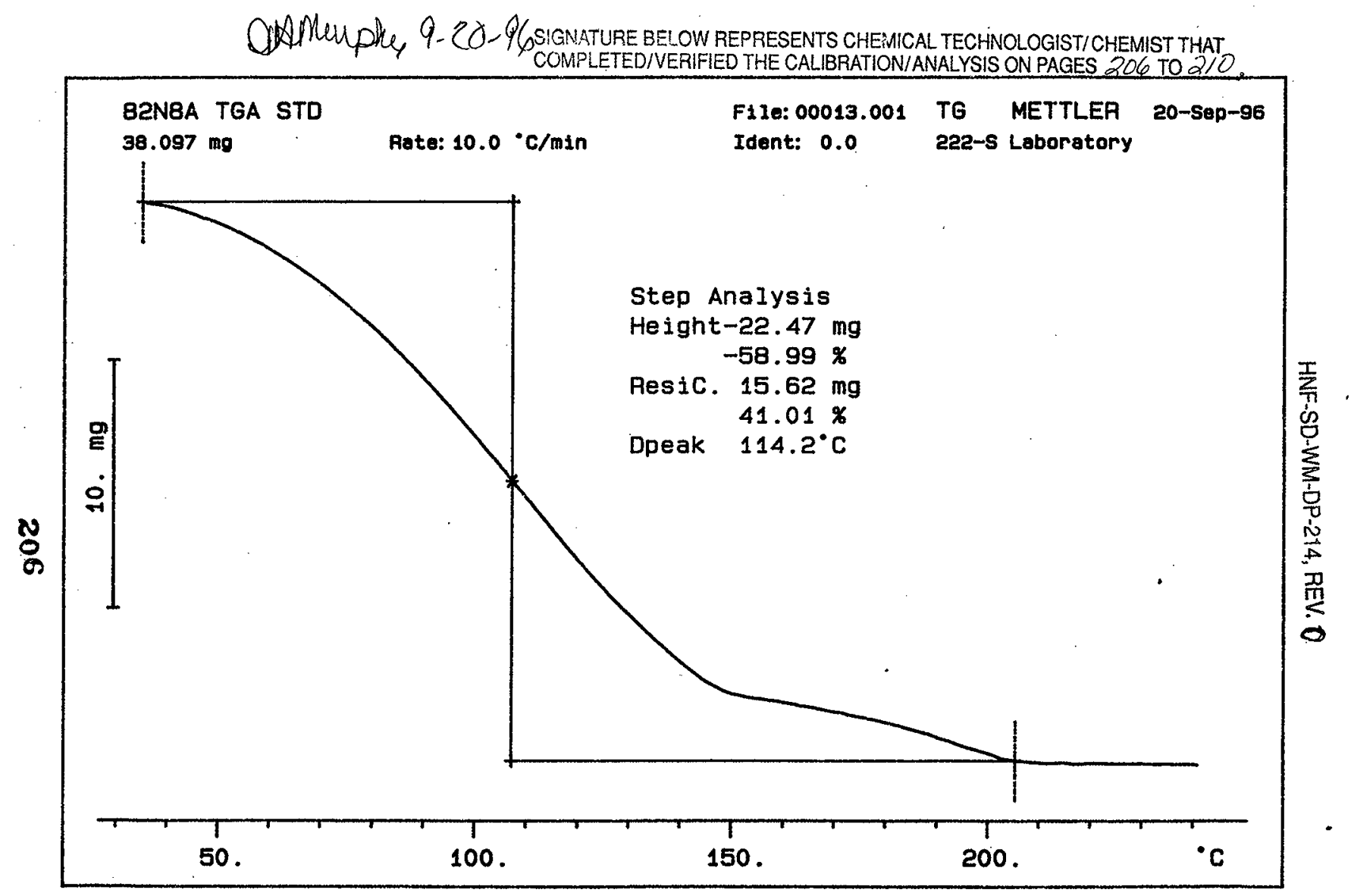




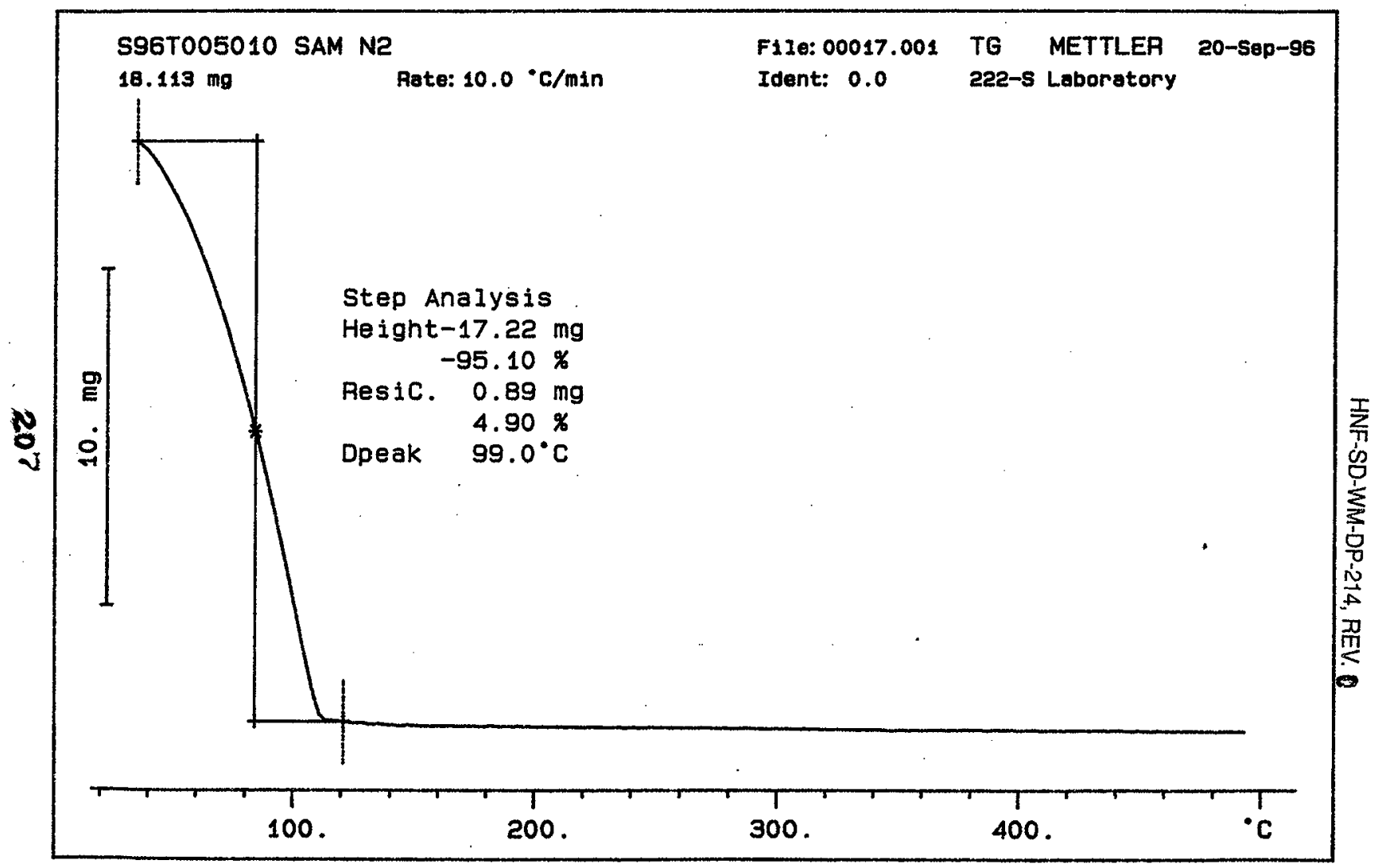




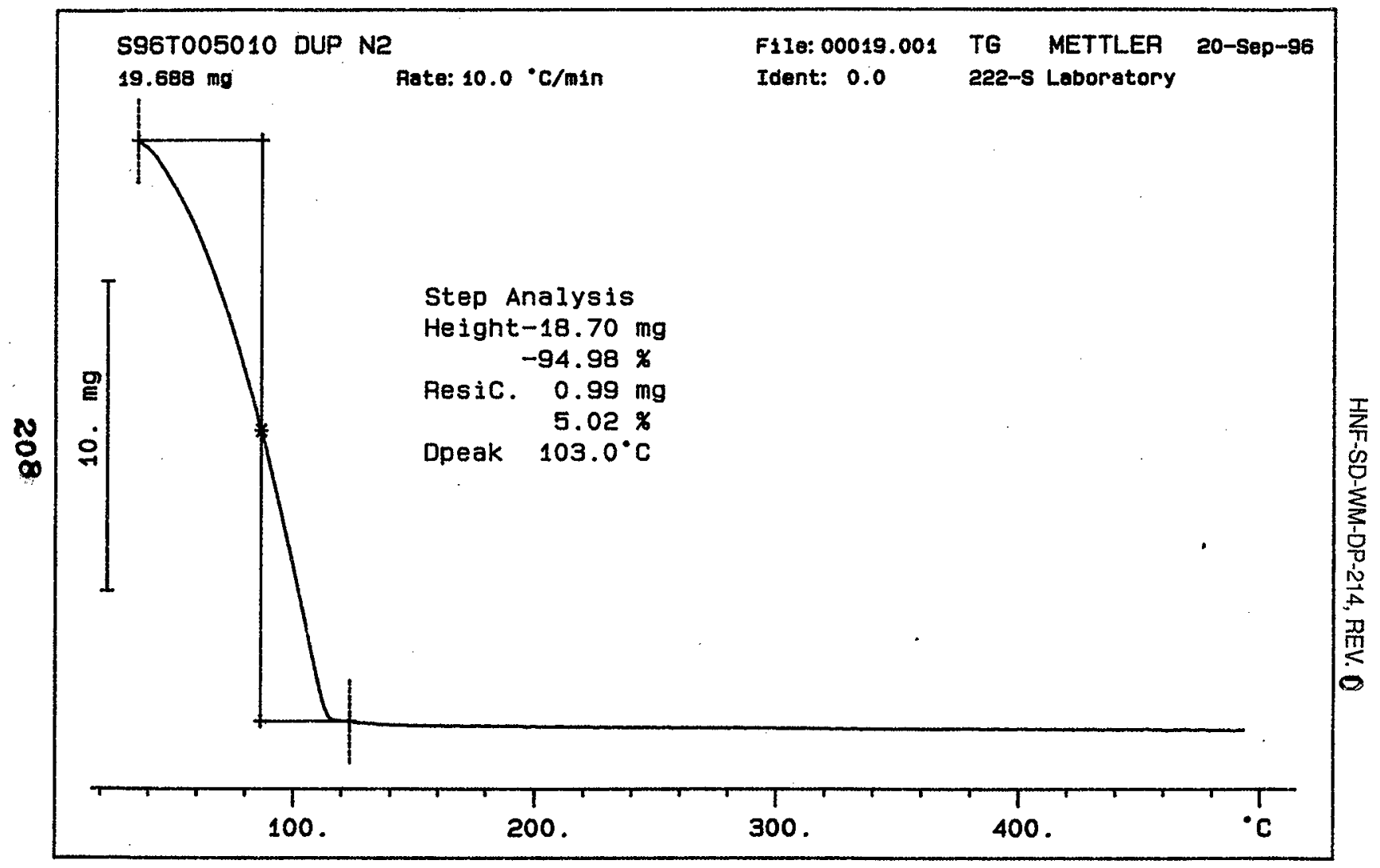




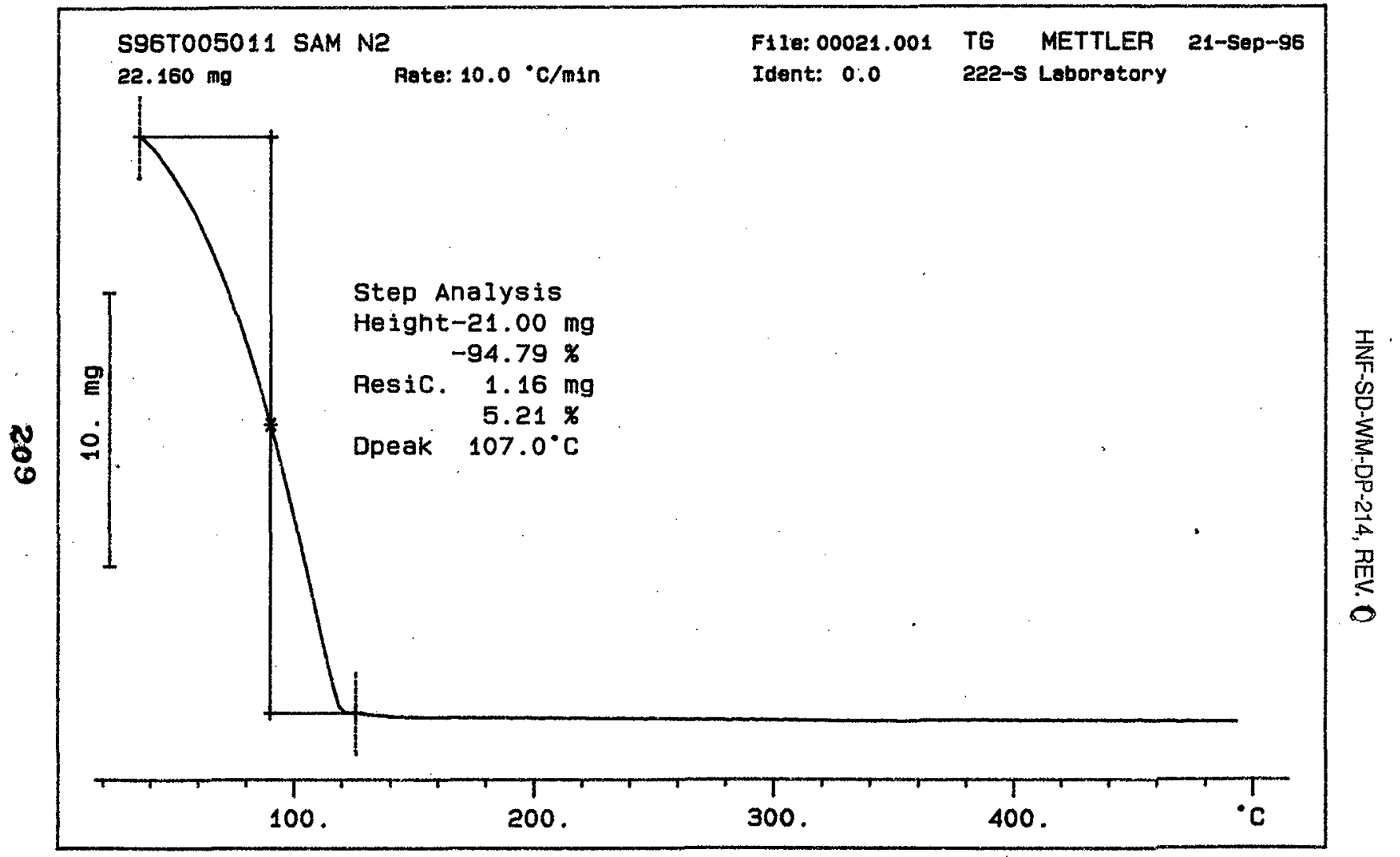




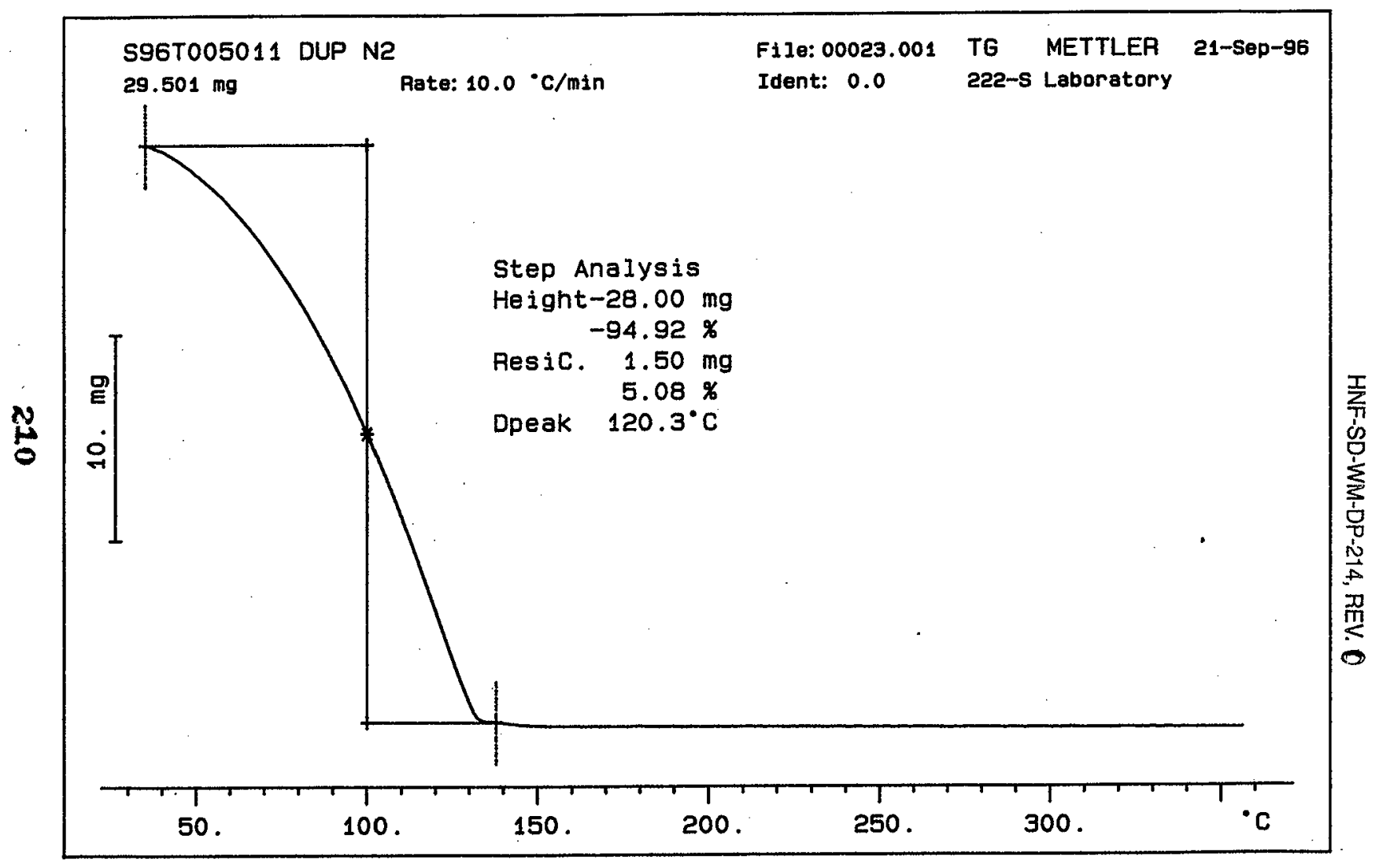


worklistrpt Version 2.1 05/15/95

HNF-SD-WM-DP-214, REV. 0

Page: 1 .

09/20/96 10:36

LABCORE Data Entry Template for Worklist\# 13133

Analyst: Instrument: TGA 1 Book \# 8208A

Method: LA-560-112 Rev/Mod $\beta-1$

Worklist Comment: AW-105 TGA, RUN UNDER N2. RCJ

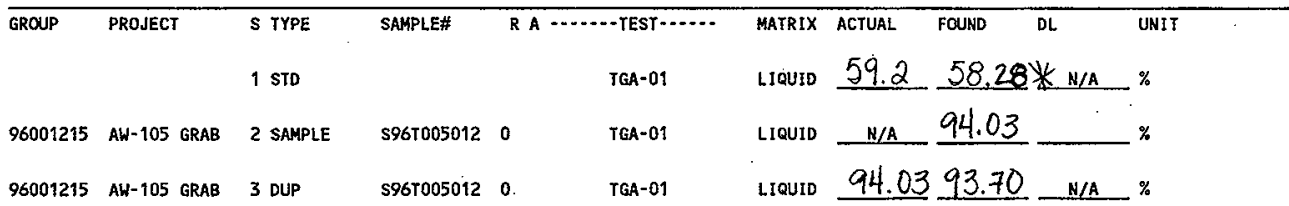

Final page for worklist \# 13133

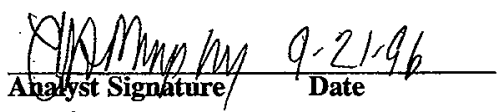

Serified/Validated by
Blandina

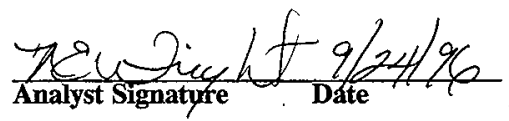

Valenzuela 9.26 .96

Data Entry Comments:

Units shown for $Q C$ (SPK \& STD) may not reflect the actual units. $D L=$ Detection Limit, $S=$ Worklist Slot Number, $R=$ Replicate Number, $A=$ Aliquot Code.

221 


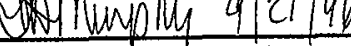

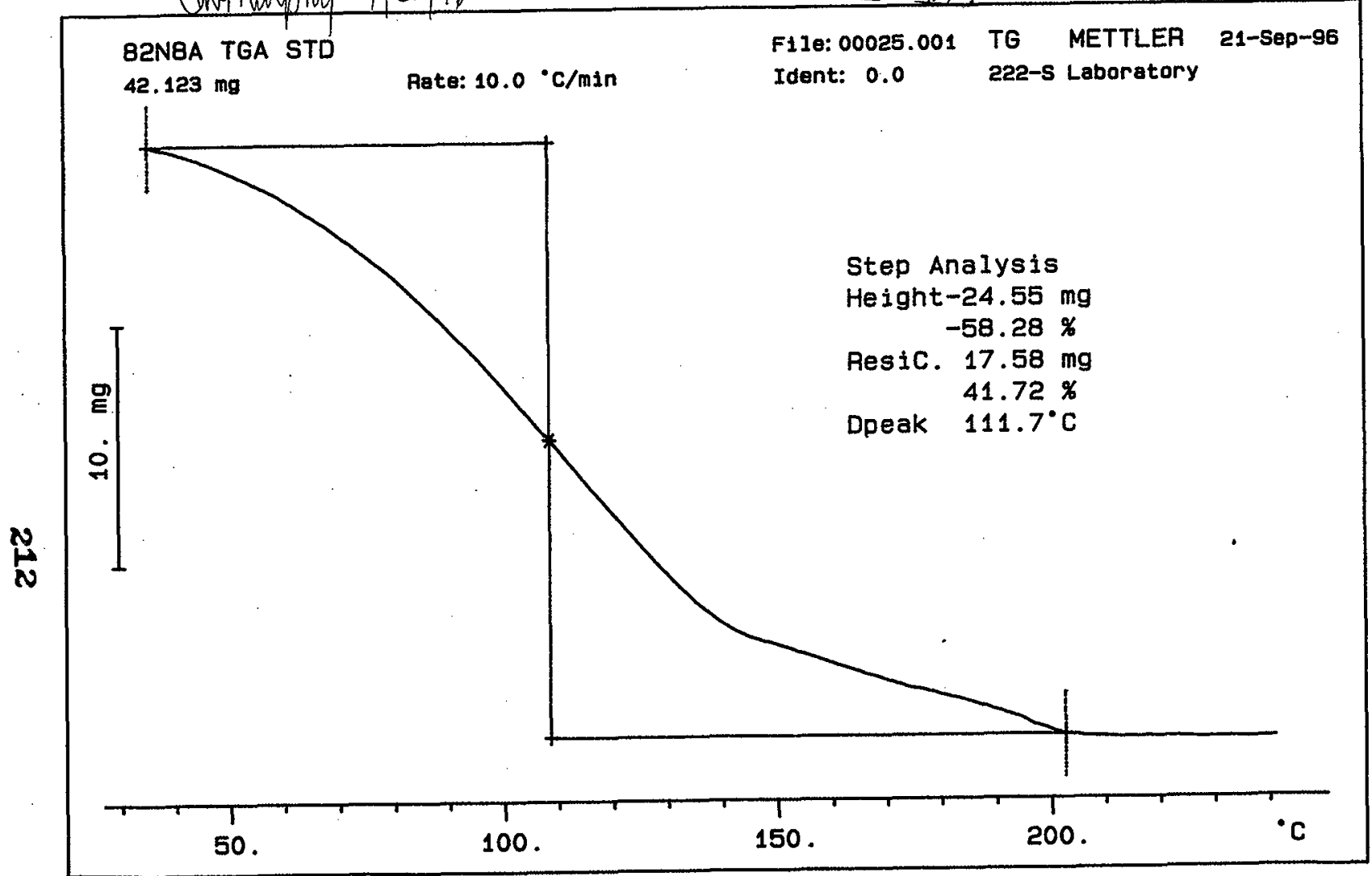




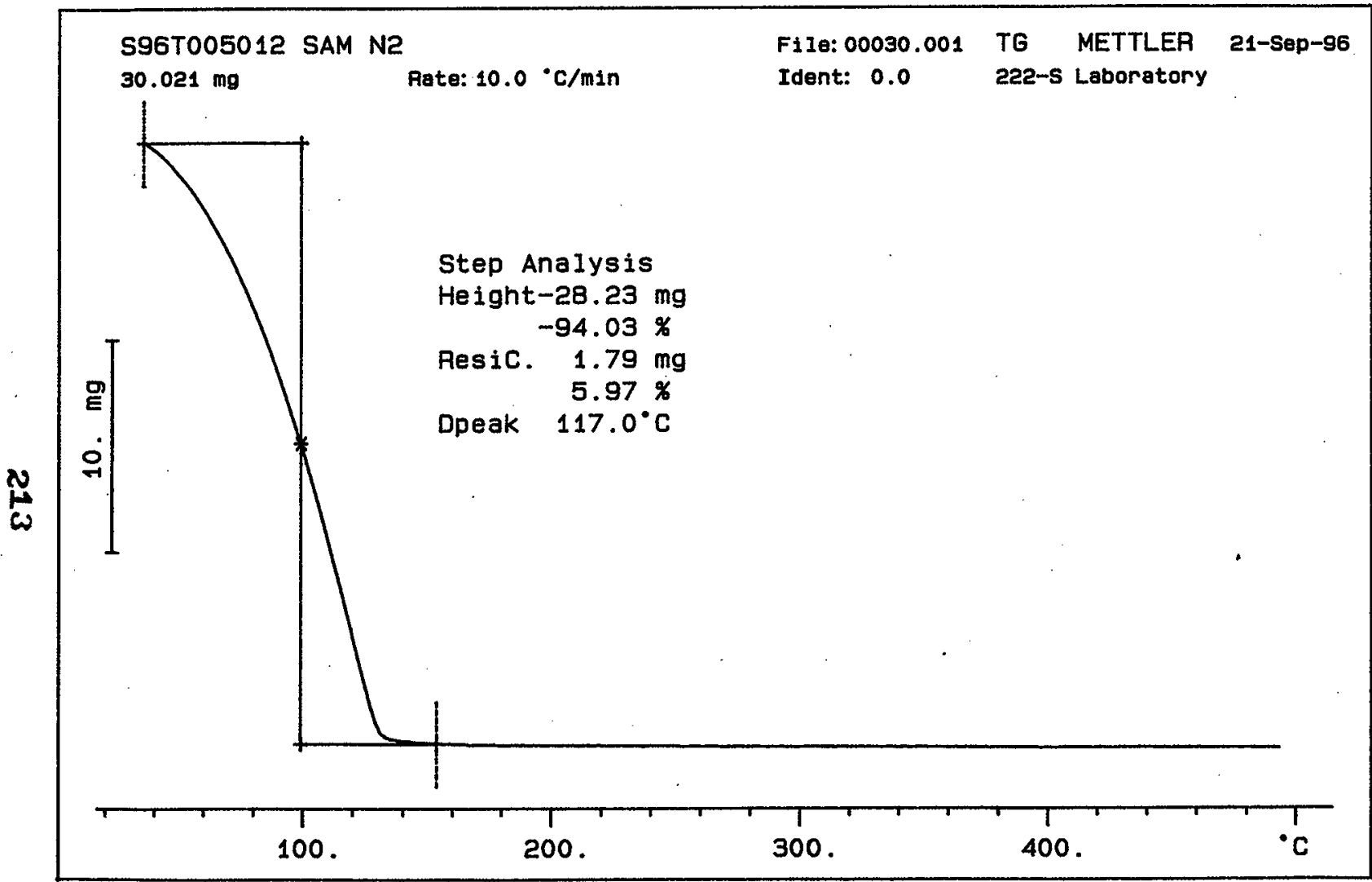




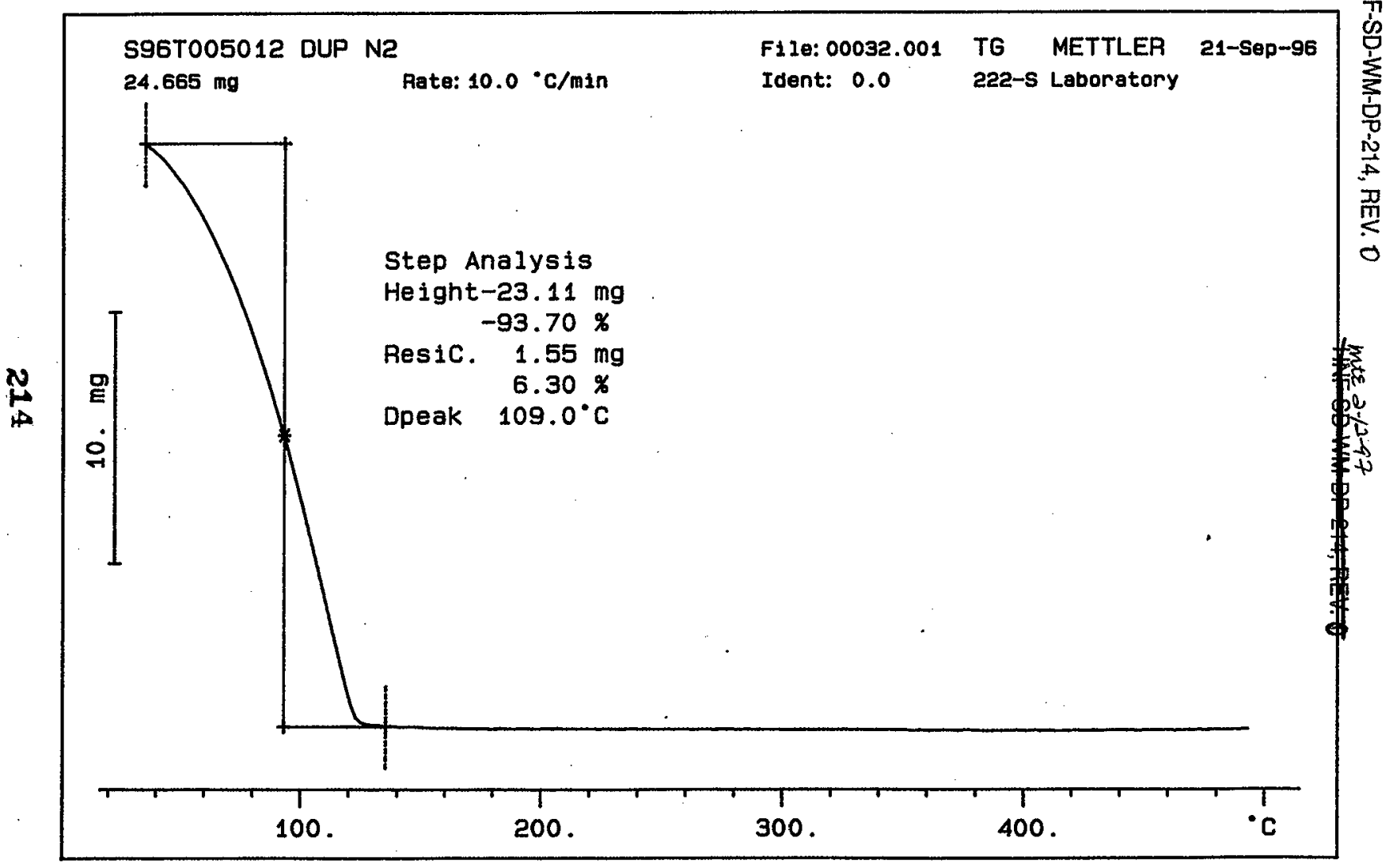




\section{LABCORE Data Entry Template for Worklist\# 13918}

Analyst: $\quad$ KRM Instrument: TGA0 3 Book \# 82N8A

Method: LA-514-114 Rev/Mod D-O

Worklist Comment: AW-105 Grab. Run under nitrogen. Std: TERLIQ .010ml. new

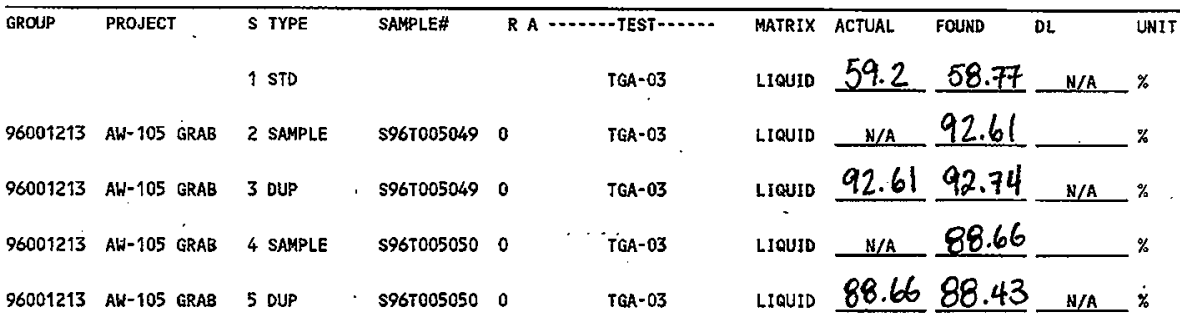

\section{Final page for worklist \# 13918}

\section{See attached for signatures 10.2396}

Verified/Validatea by

Blandina

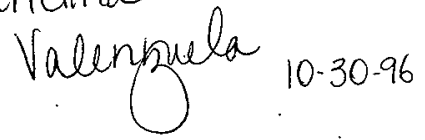

Data Entry Comments:

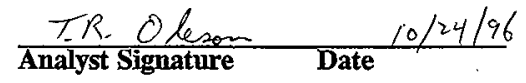




\section{LABCORE Data Entry Template for Worklist\# 13918}

Analyst: $\quad K R M$ Instrument: TGAO _ Book \# $82 N \& A$

Method: LA-560-112 Rev/Mod

Worklist Comment: AW-105 Grab. Run under nitrogen. Std: TERLIQ .010ml. new

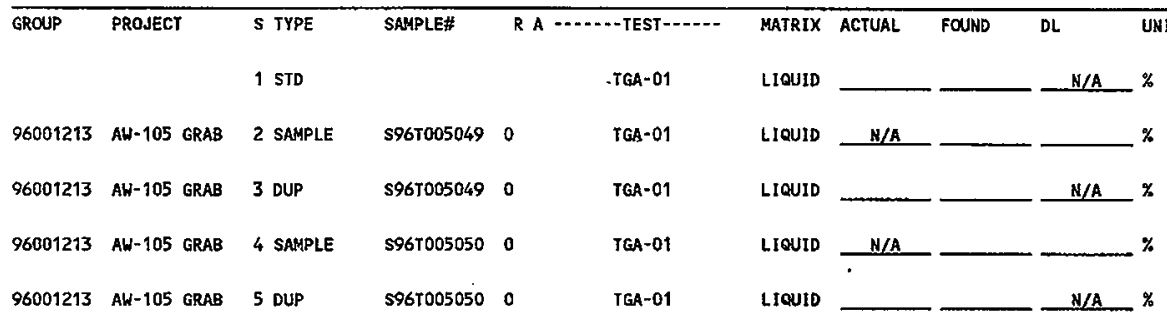

\section{Final page for worklist \# 13918}

Analyst Signature

Date

$$
10-19.96
$$

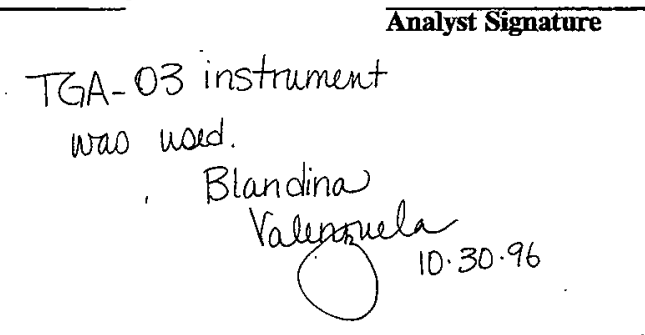

Data Entry Comments:

Units shown for QC (SPK \& STD) may not reflect the actual units. $D L=$ Detection Limit, $S=$ Worklist Slot Number, $R=$ Replicate Number, $A=$ Aliquot Code. 
Curve 1: TGA

F1le info: TER101901 Sat Oet 19 05: 28:21 1996

Sample Neight: 23.126

TGA STO 82NB-A

mg

SICNATUPE BELOW REPRESENTS CHE:ICAL TECHNOLOGIST/CHEMIST THAT
COMPLETED/VERIFIED THE CALIBRATION/ANALYSIS ON PAGES 21.7 TO 221.

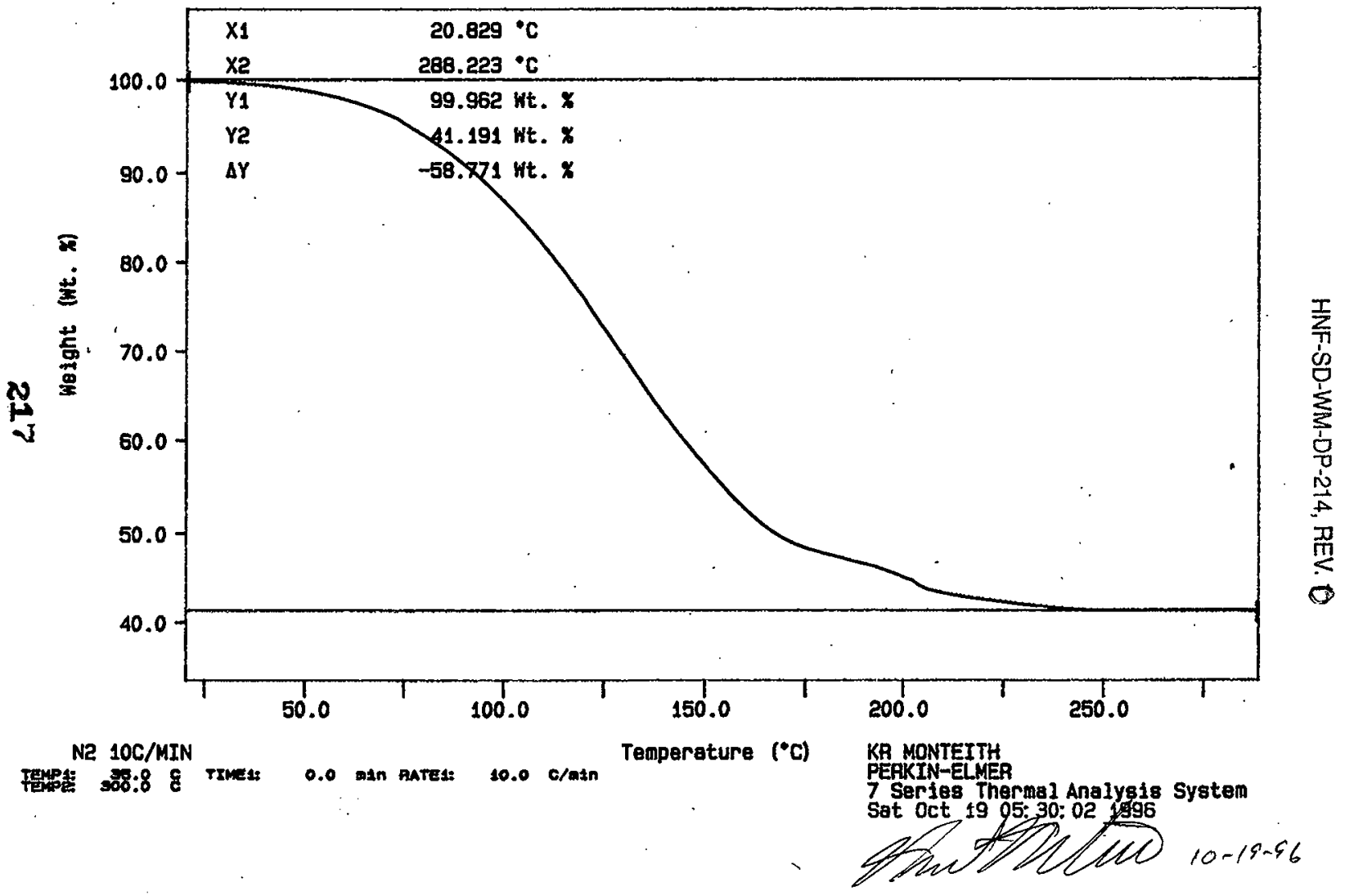


Curve 1: TGA

File info: SAM101901 Sat oct 19 06: 36: 431996

Sample Weight: 10.463 ing

S967005049

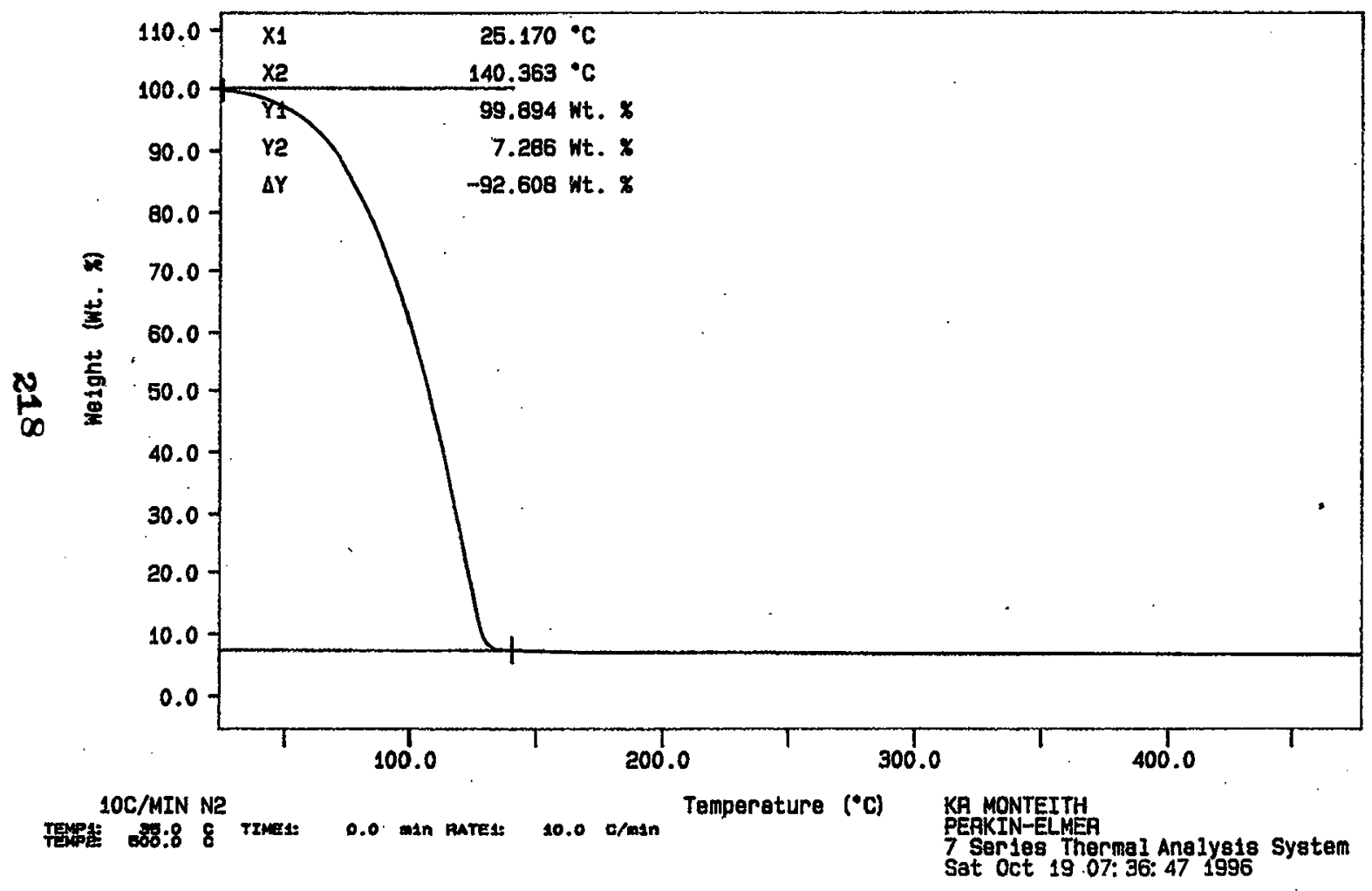


Curve 1: TGA

File Info: SAM101902 Sat Oct 19 08: 17: 151996

Sample Waight: 10.466

S96T005049 DUP

ang

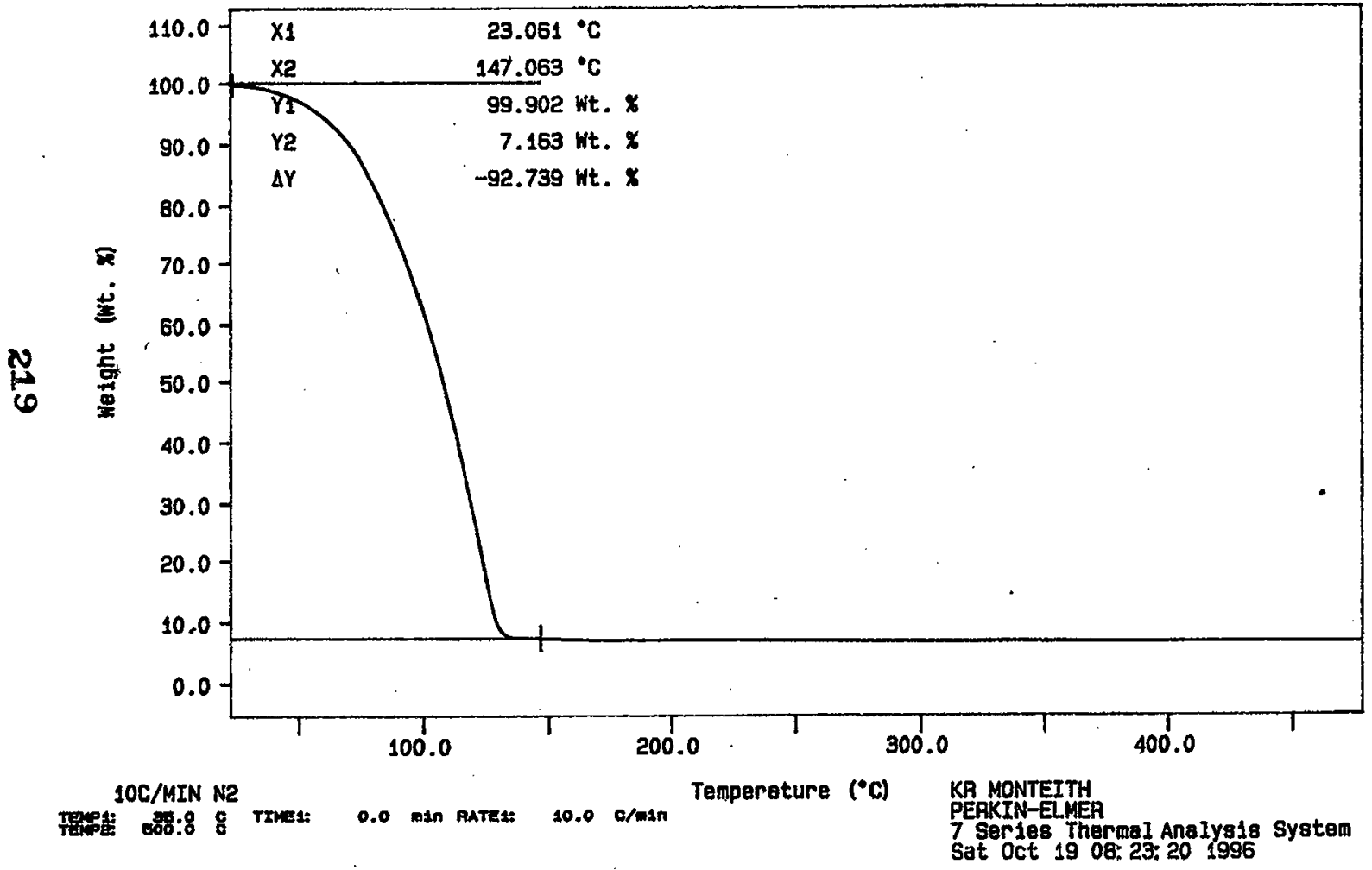


Curve 4: TGA

File Info: SAM101903 Sat Oct 19 09:23:27 1996

Sample Weight: $11.278 \mathrm{mg}$

S96T005050

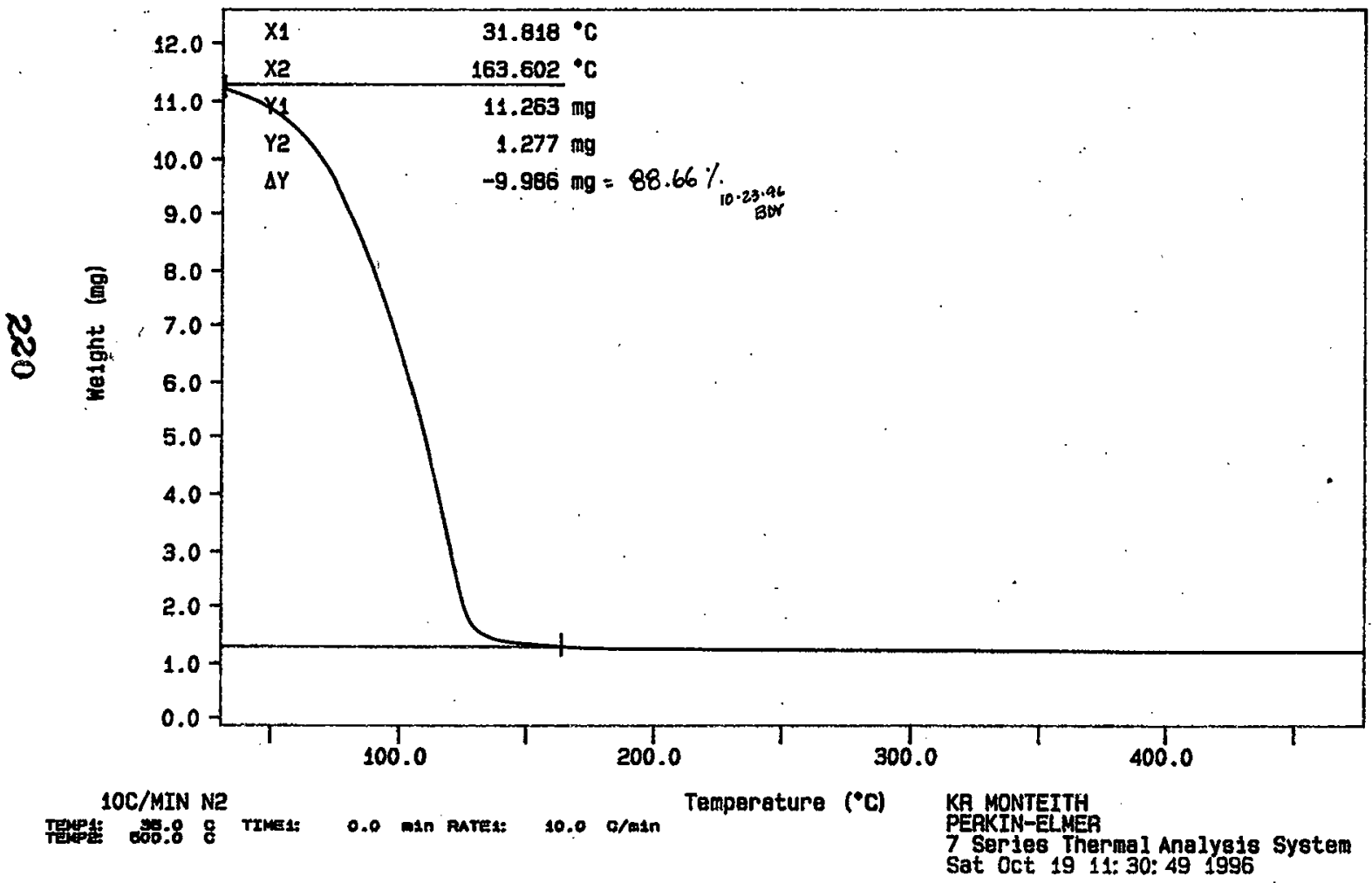


Curve 1: TGA

File info: SAM101904 Sat Oct 19 12: $16: 451996$

Sample Weight: 11.205 mg

S96T005050 DUP

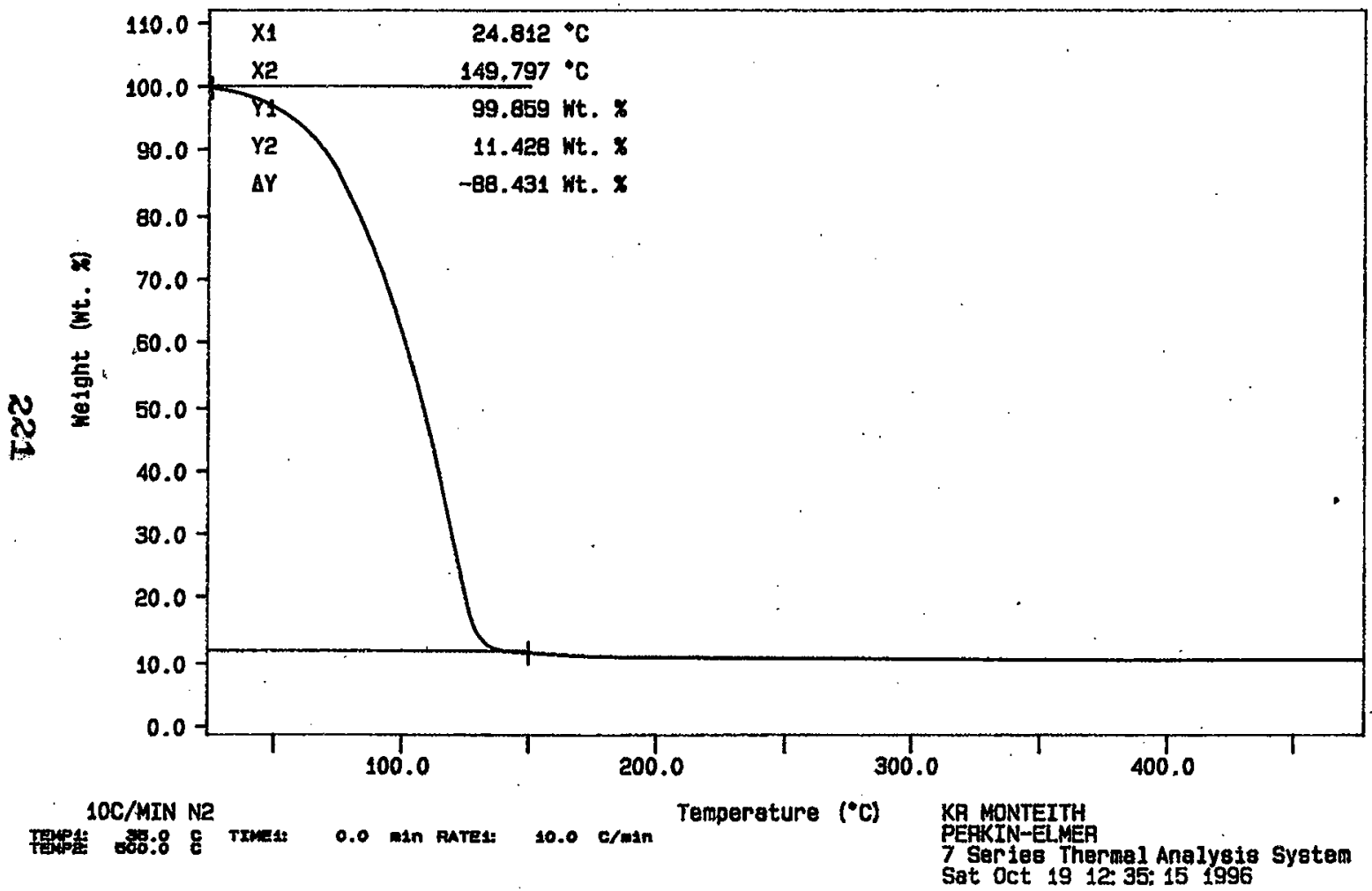




\section{LABCORE Data Entry Template for Worklist\# 13919}

Analyst: $\quad$ KRM Instrument: TGA0. 3 Book \# $82 \mathrm{~N} 8 \mathrm{~A}$

Method: LA-514-114 Rev/Mod D-O

Worklist Comment: AW-105 Grab. Run under nitrogen. Std: TERLIQ .010ml. new

\begin{tabular}{|c|c|c|c|c|c|c|c|c|c|c|c|}
\hline \multirow[t]{2}{*}{ GROUP } & \multirow[t]{2}{*}{ PROJECT } & \multirow{2}{*}{$\begin{array}{l}\text { S TYPE } \\
1 \text { STD }\end{array}$} & \multirow[t]{2}{*}{ SAMPLE\# } & \multicolumn{2}{|c|}{ R A $\cdots$ - TEST $\cdots$} & \multirow{2}{*}{$\begin{array}{l}\text { MATRIX } \\
\text { LIOUID }\end{array}$} & \multirow{2}{*}{$\begin{array}{l}\text { ACTUAL } \\
59.2 \\
\end{array}$} & \multirow{2}{*}{\multicolumn{2}{|c|}{$\begin{array}{l}\text { FOJND } \\
58.77 * \text { M/A }\end{array}$}} & \multirow{2}{*}{$\begin{array}{l}\text { UNIT } \\
\%\end{array}$} & \\
\hline & & & & & TEA-03 & & & & & & \\
\hline 96001213 & $A H-105$ GRAB & 2 SAMPLE & S96T005051 & 0 & $T G A-03$ & LIRUID & N/A & 88.68 & . & $\%$ & \\
\hline 96001213 & AW-105 GRAB & 3 DUP & S96T005051 & 0 & TGA-03 & Liquid & 88.68 & 89.02 & $\mathrm{~N} / \mathrm{A}$ & $\%$ & \\
\hline 96001215 & AW-105 GRAB & 4 SAMPLE & S96T005081 & 0 & $T \in A-03$ & Liauro & $N / A$ & 90.54 & & $\%$ & \\
\hline 96001215 & $A W-105$ GRAB & 5 DUP & S96T005081 & 0 & TGA-03 & HEUID & 90.54 & 90.78 & N/A & $\%$ & \\
\hline
\end{tabular}

\section{Final page for worklist \# 13919}

TSe attached for signatures 10.23 .96 Verifud Validated by

$\Leftrightarrow$ a

$$
\text { Valenpuela. } 10.30 .96
$$

Tink Jhack 10-24-96 Analyst Signature Date

\section{Data Entry Comments:}

Units shown for $Q C$ (SPK \& STD) may not reflect the actual units. $D L=$ Detection Limit, $S=$ Worklist Slot Number, $R=$ Replicate Number, $A=$ Aliquot Code. 


\section{LABCORE Data Entry Template for Worklist\# 13919}

Page: 1

Analyst: KRM Instrument: TGA0 _ Book \# 82N8A

Method: LA-560-112 Rev/Mod

Worklist Comment: AW-105 Grab. Run under nitrogen. Std: TERLIQ .010ml. new

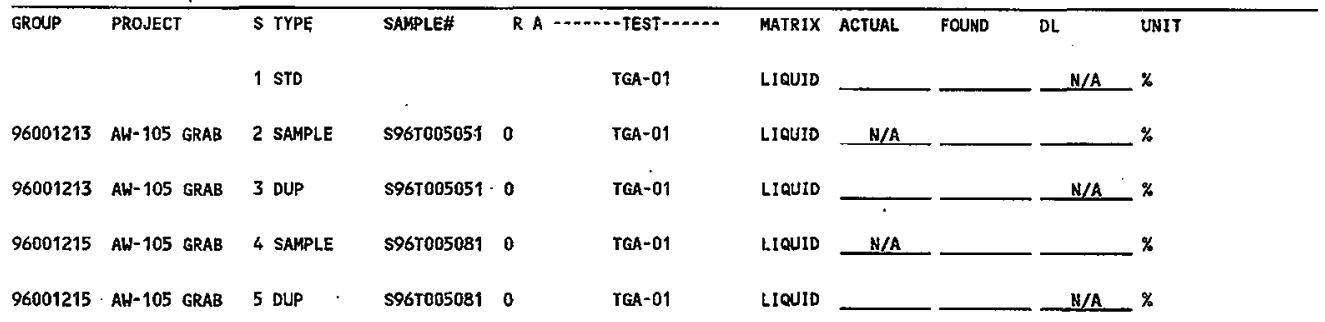

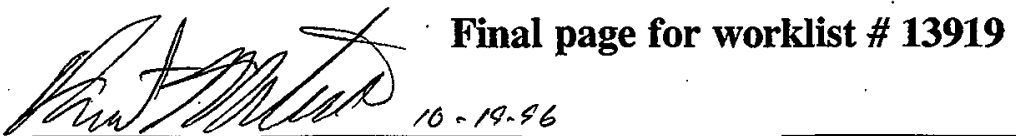

Analyst Signature Date

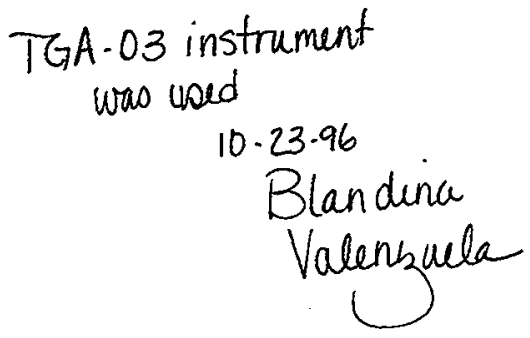

Data Entry Comments:

Units shown for $Q C$ (SPK \& STD) may not reflect the actual units. $D L=$ Detection Limit, $S=$ Worklist Slot Number, $R=$ Replicate Number, $A=$ Aliquot Code. 
Curve 1: TGA

File Info: TER101901 Sat Oct 19 05:28:21 1996

Sample Weight: $23.126 \mathrm{mg}$

TGA STD 82NB-A

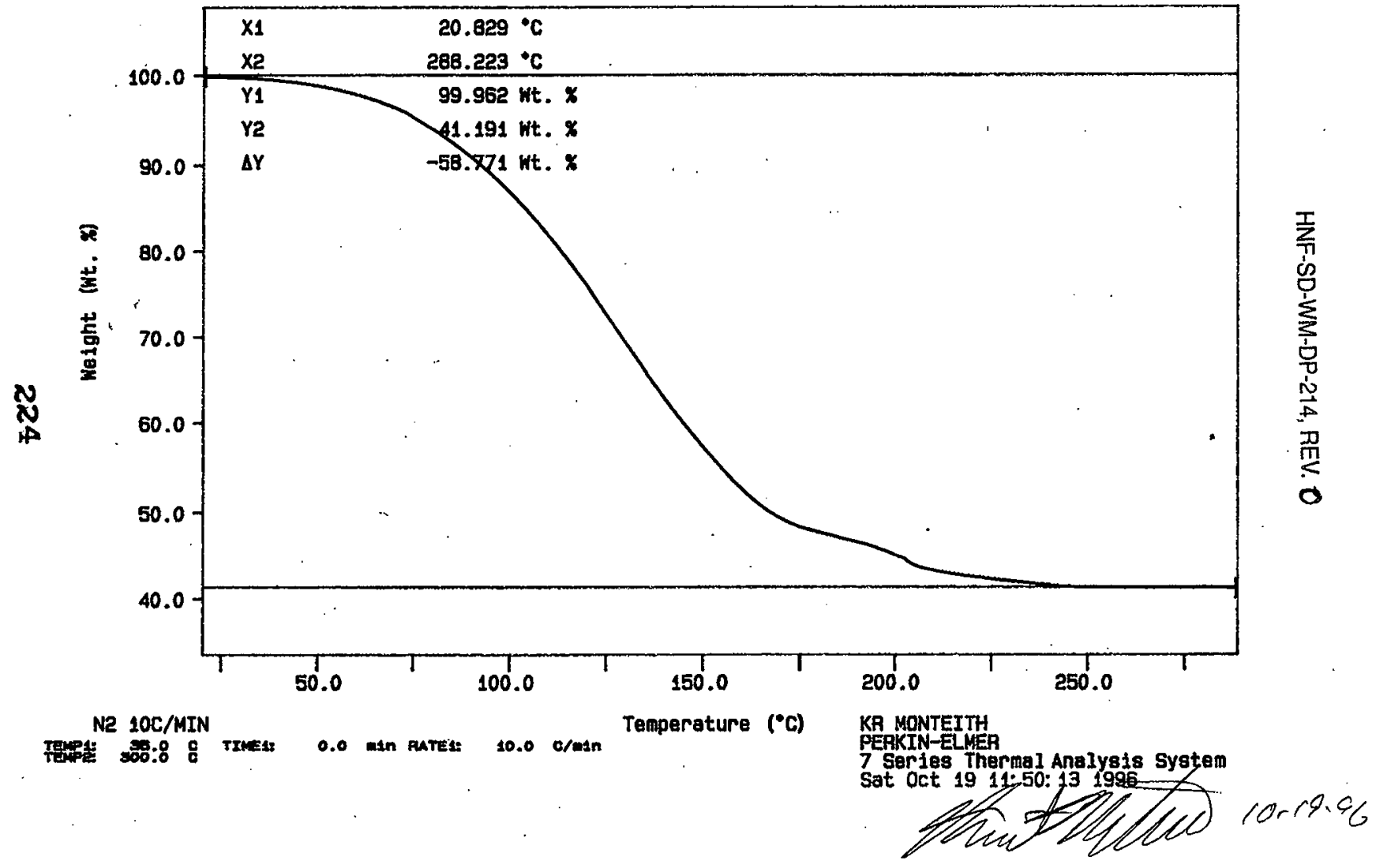


Curve 1: TEA

F11e info: SAM101905 Sat oct 19 13: 25: 311996

Semple Nelght: 11.810 mg

596r005051

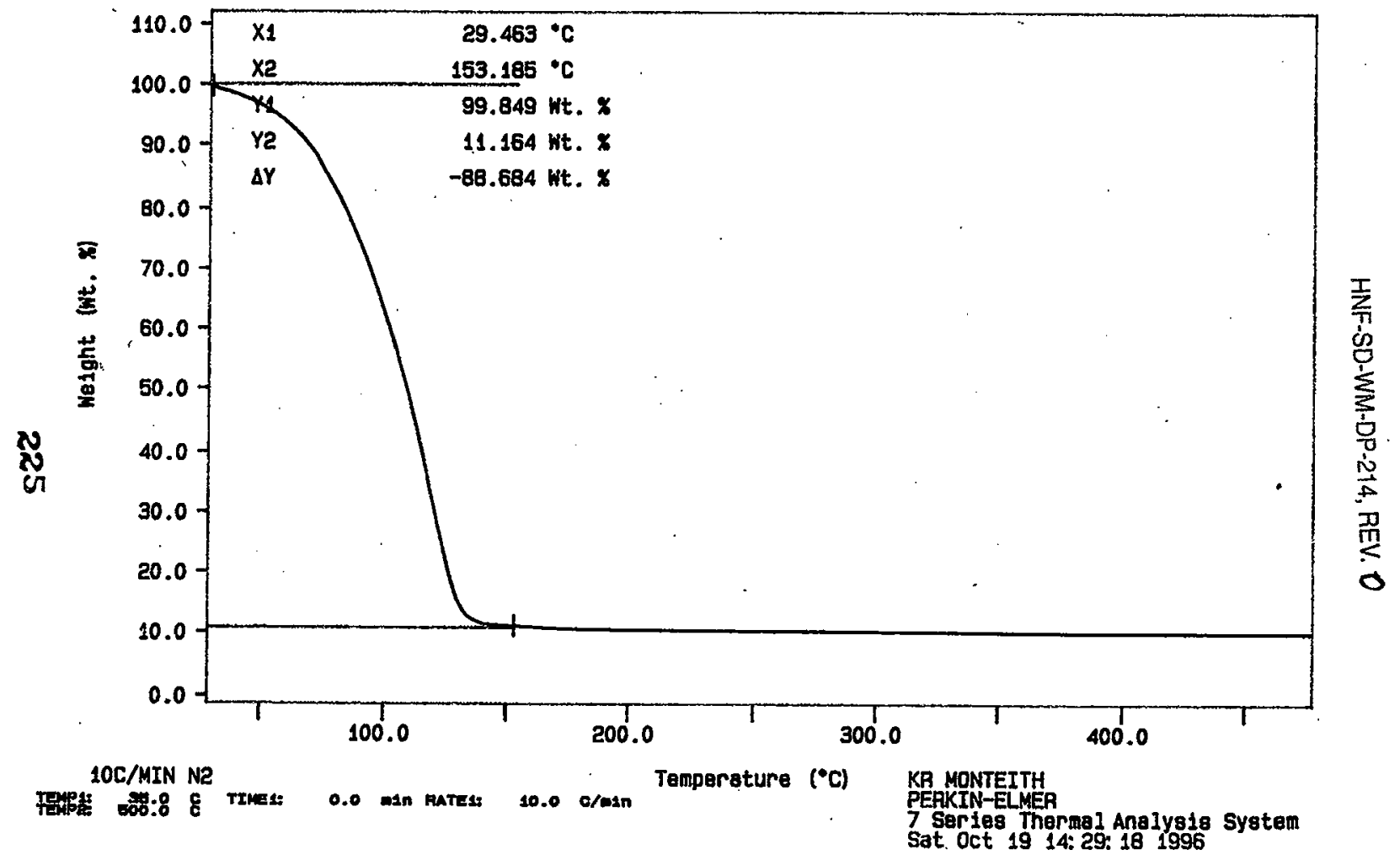


Curve 1: TEA

File info: SAN101906 Sat Oct 19 15:04:50 1996

Sample Keight: 11.577 mg

S96T005051 DUP

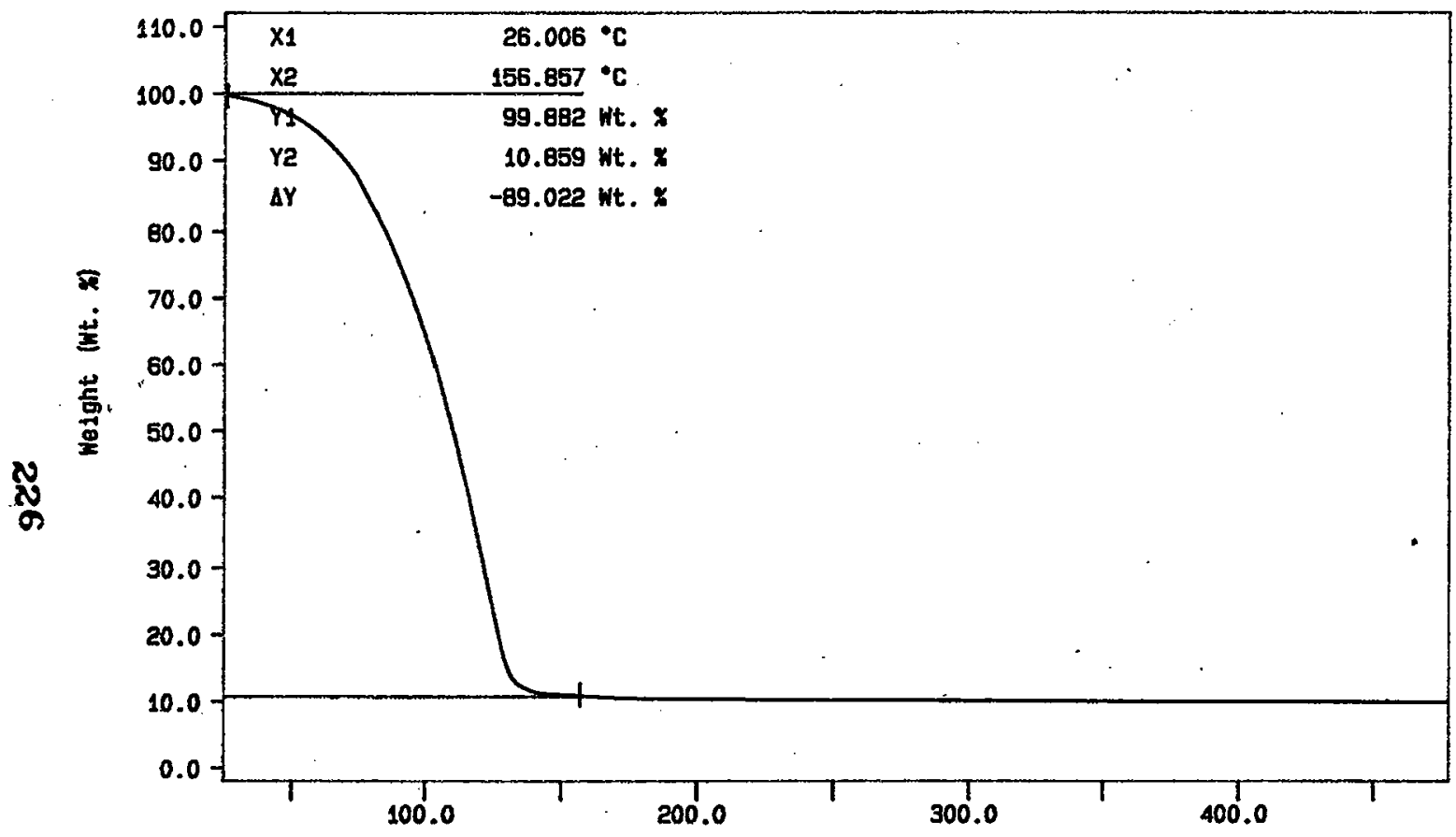

10C/MIN N2

Tepp:

\section{(n)}


Curve 1: TGA

File Info: SAM101907 Sat oct 19 16: $15: 301996$

Sample Netght: 11.150 ug

s967005081

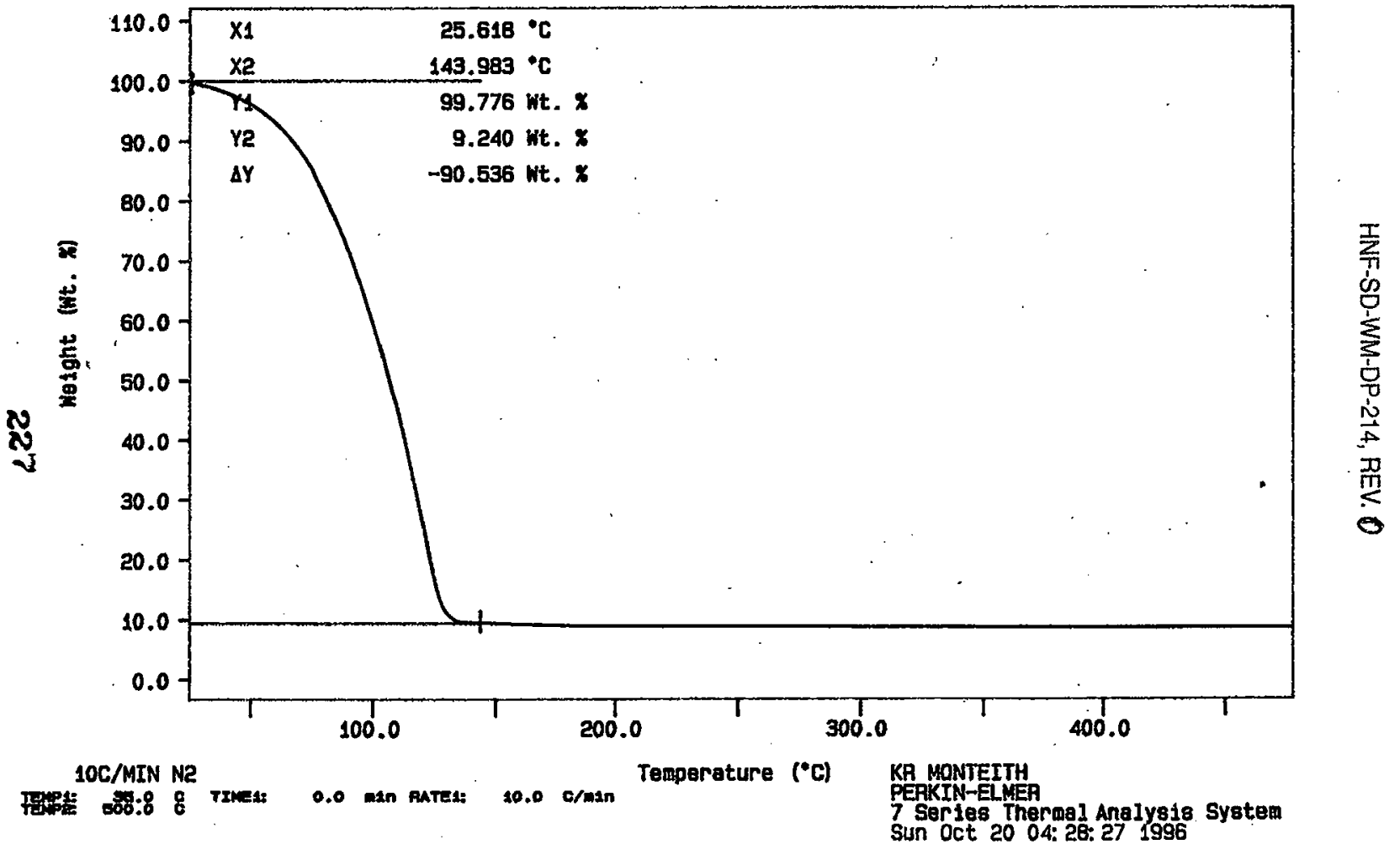


Curve 1: TGA

File Info: SAM102001 Sun Oct 20 05: 56: 221996

Sample Welght: 11.656 mg

S96T005081 DUP

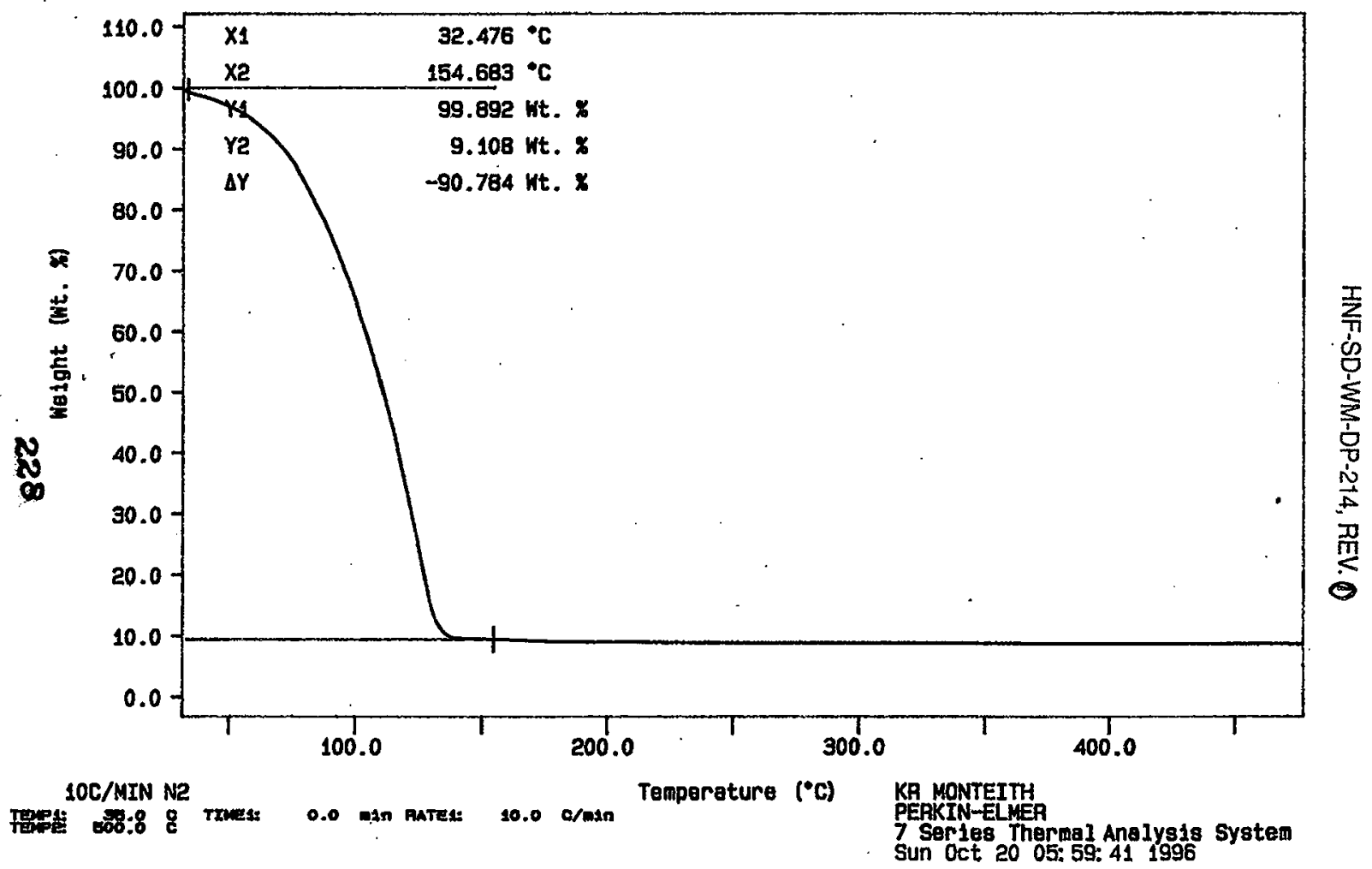




\section{LABCORE Data Entry. Template for Worklist\# 13920}

\section{Analyst: \\ Instrument: TGA0 । \\ Book \# $82 N 8$ A}

Method: LA-560-112 Rev/Mod $\mathrm{C}-\mathrm{O}$

Worklist Comment: AW-105 Grab. Run under nitrogen. Std: TERLIQ .010ml. new

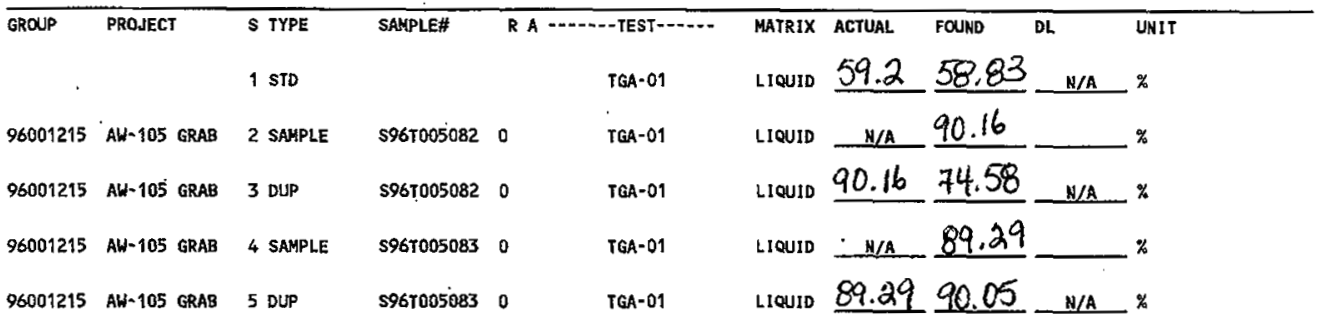

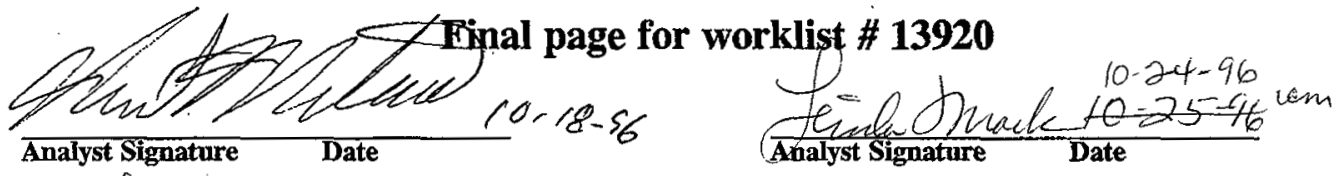

Verifid/Validated by

Blandina

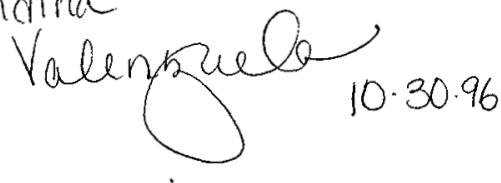

Data Entry Comments:

Units shown for $Q C$ (SPK \& STD) moy not reflect the actual units. $D L=$ Detection Limit, $S=$ Worklist slot Number,

$R=$ Replicate Number, $A=$ Aliquot Code. 
HNF-SD-WM-DP-214, REV. O

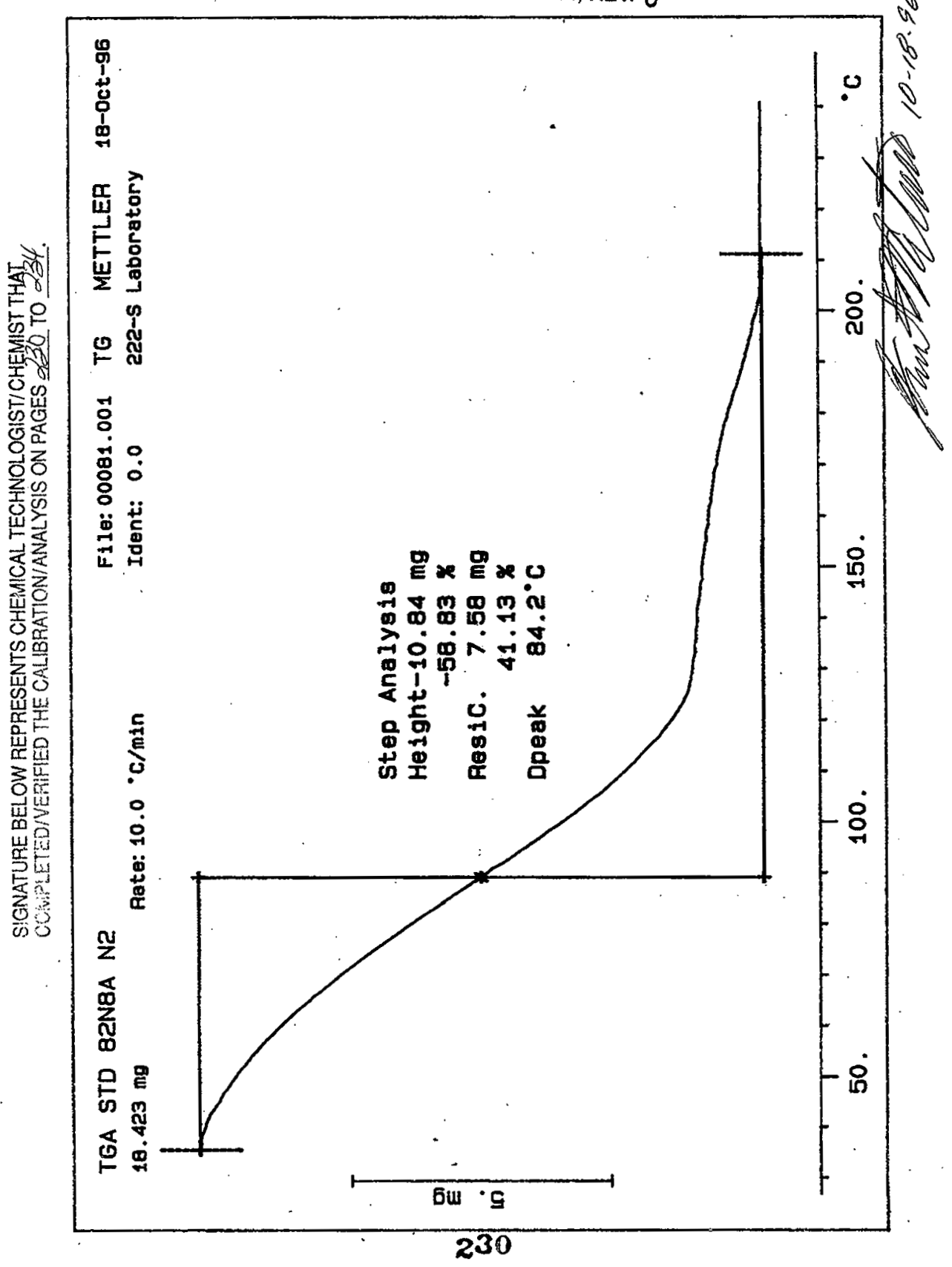


HNF-SD-WM-DP-214, REV. 0

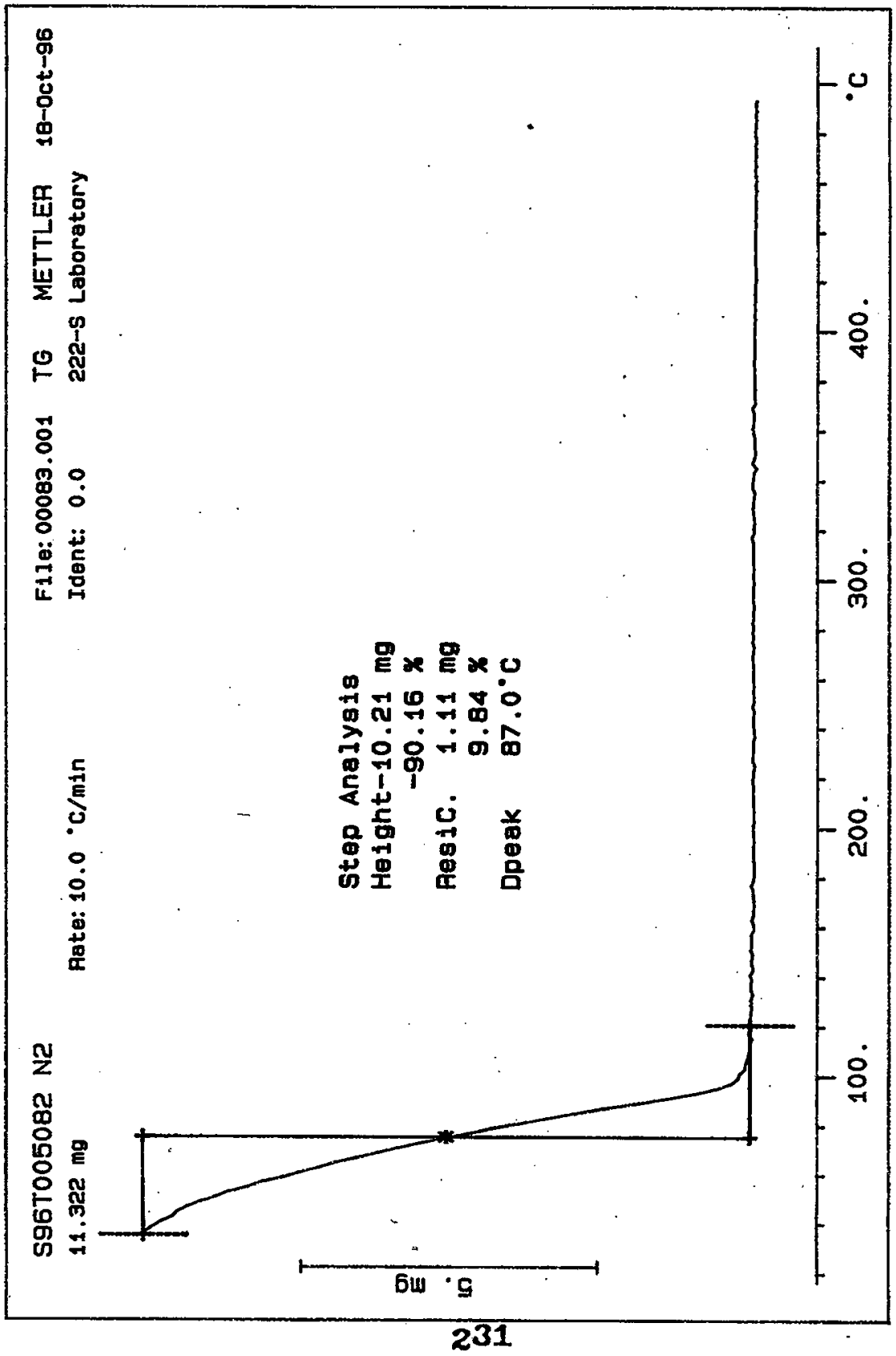




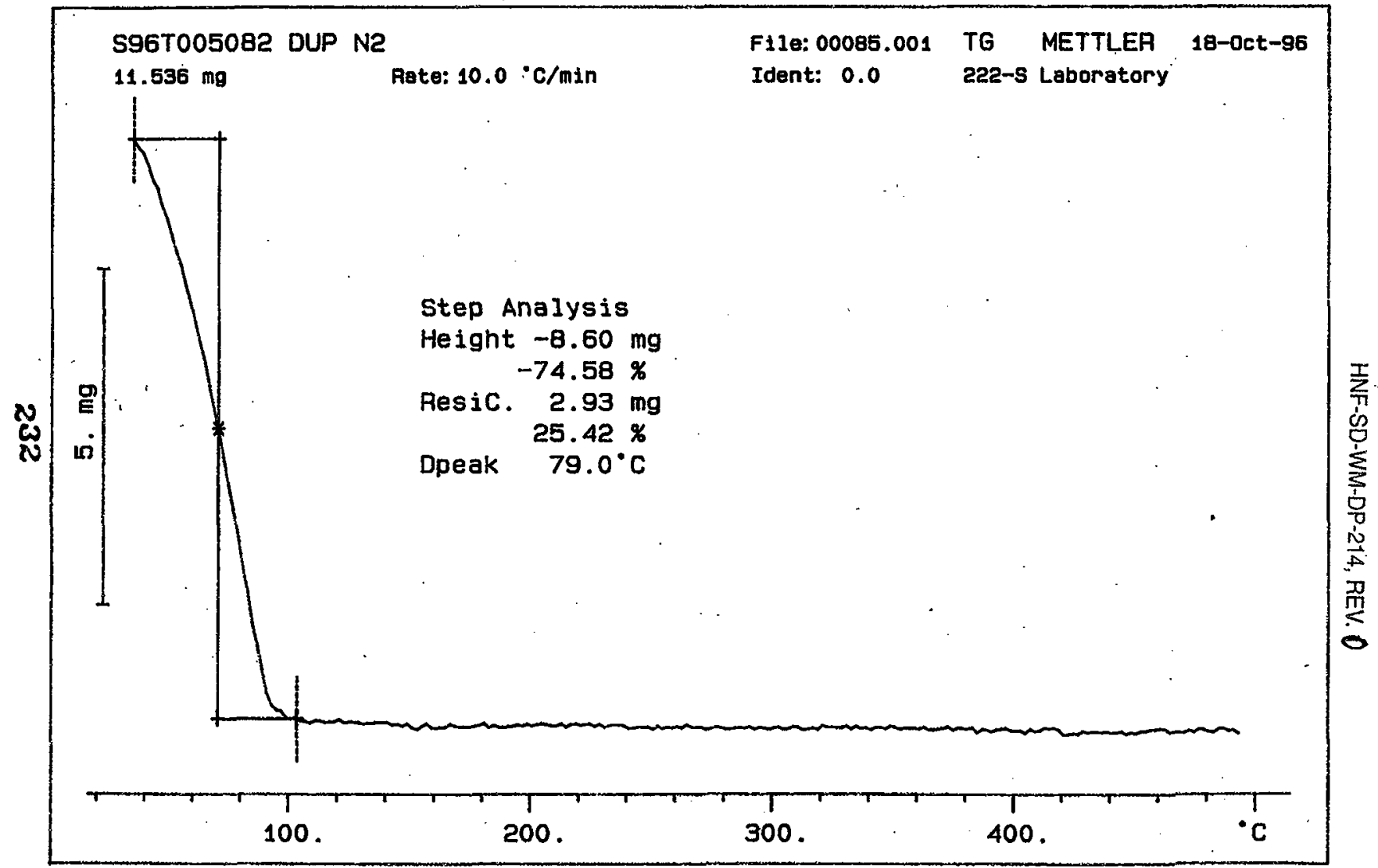




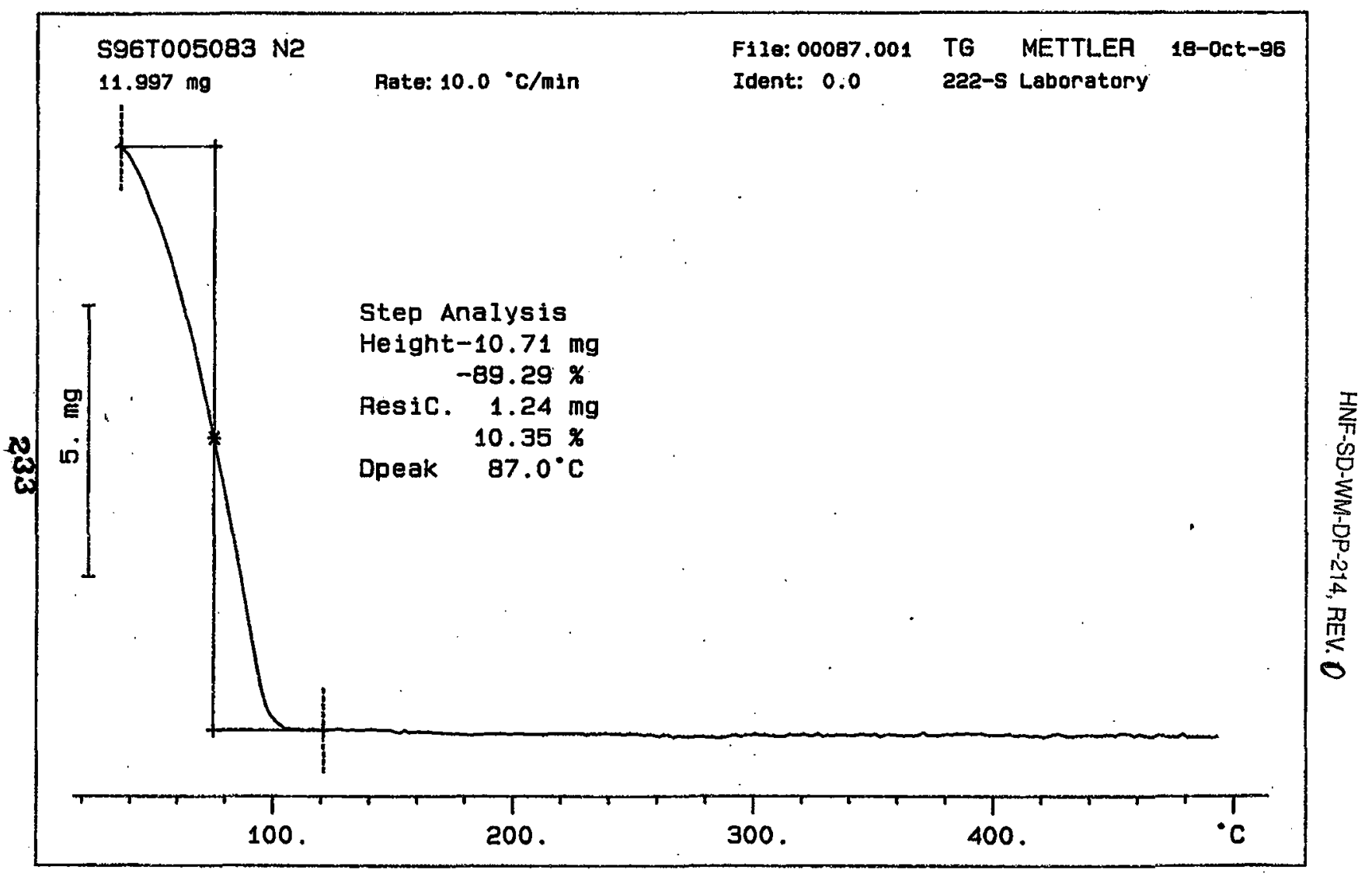




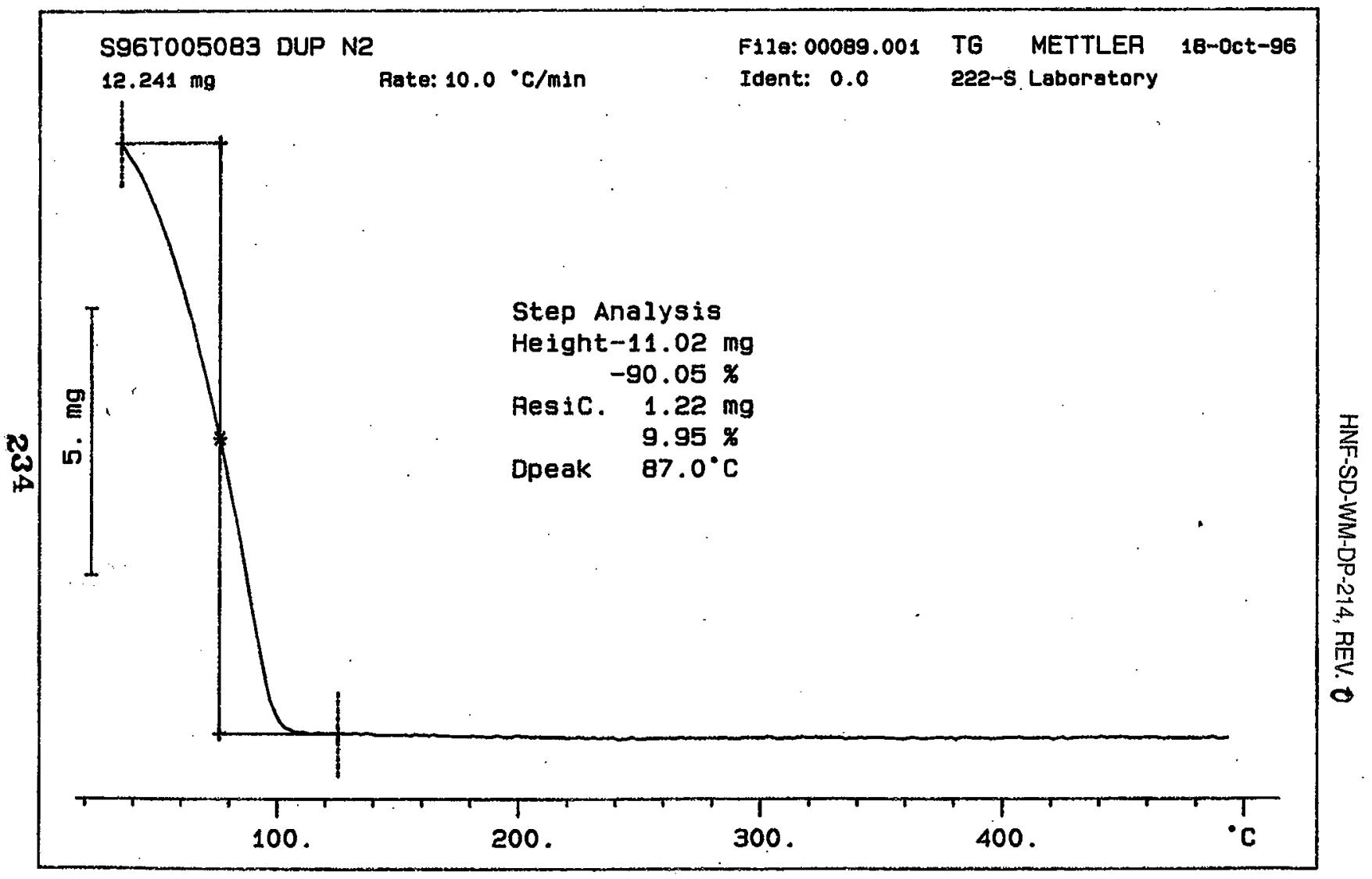


HNF-SD-WM-DP-214, REV. 0

worklistrpt Version $2.105 / 15 / 95$

Page: 1 10/23/96 11:17

LABCORE Data Entry Template for Worklist\# 13921

Analyst: $\quad \mathrm{KRm} \quad$ Instrument: $\mathrm{TGAO}^{2} 3$ Book \# $82 \mathrm{~N} 8 \mathrm{~A}$

Method: LA-514-114 Rev/Mod D. 0

Worklist Comment: AW-105 Grab. Run under nitrogen. Std: TERLIQ .01 0ml. new

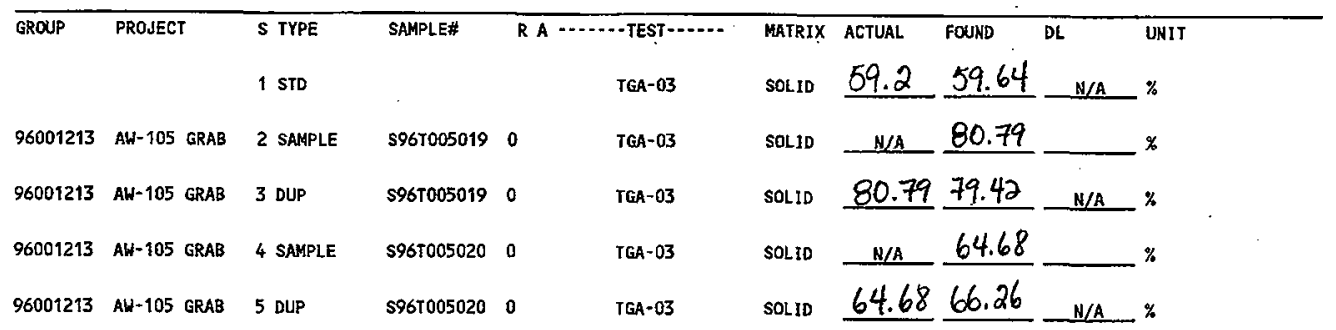

Final page for worklist \# 13921

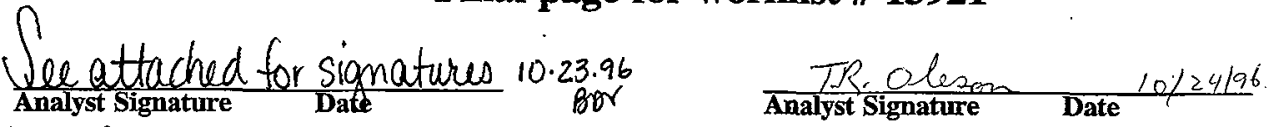

Verified/ Validated by

Blandina

Valempuela $10 \cdot 30 \cdot 96$

Data Entry Comments:

Units shown for $Q C$ (SPK \& STD) may not reflect the actual units. $D L=$ Detection Limit, $S=$ Worklist Slot Number, $R=$ Replicate Number, $A=$ Aliquot Cade.

235 


\section{LABCORE Data Entry Template for Worklist\# 13921}

\begin{tabular}{|c|c|c|}
\hline Analyst: & KAn & Instrument: TGA0 \\
\hline
\end{tabular}

Method: LA-560-112 Rev/Mod

Worklist Comment: AW-105 Grab. Run under nitrogen. Std: TERLIQ .010ml. new

\begin{tabular}{|c|c|c|c|c|c|c|c|c|c|c|}
\hline \multirow[t]{2}{*}{ GROUP } & \multirow[t]{2}{*}{ PROJECT } & S TYPE & \multirow[t]{2}{*}{ SAMPLE\# } & \multicolumn{2}{|r|}{ R A $\cdots \ldots$ TEST $\ldots \ldots$} & MATRIX & \multirow[t]{2}{*}{ ACTUAL } & \multirow[t]{2}{*}{ FOUND } & \multirow[t]{2}{*}{$D L$} & UNIT \\
\hline & & 1 STD & & & TGA-01 & SOLID & & & & $x$ \\
\hline 96001213 & $A W-105$ GRAB & 2 SAMPLE & $\$ 961005019$ & 0 & TEA-01 & SOLID & $N / A$ & & & $x$ \\
\hline 96001213 & AL-105 GRAB & 3 DUP & $\$ 967005019$ & 0 & $T C A-01$ & SOLID & & & $\mathrm{N} / \mathrm{A}$ & $\%$ \\
\hline 96001213 & AW-105 GRAB & 4 SAMPLE & $\$ 967005020$ & 0 & TGA-01 & SOLID & N/A & & & 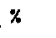 \\
\hline 96001213 & $A W-105$ GRAB & 5 DUP & \$96T005020 & 0 & TGA-01 & SOLID & & & N/A & $z$ \\
\hline
\end{tabular}

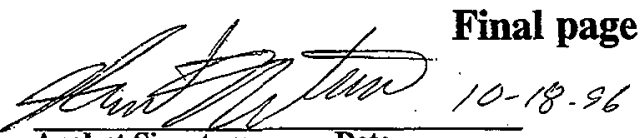

Analyst Siguature

Date

\section{Analyst Signature Date}

$$
\begin{aligned}
& \text { TGA-03 instrument } \\
& \text { was used. } \\
& 10.23 .96 \\
& \text { Blandina } \\
& \text { Valenguela }
\end{aligned}
$$

\section{Data Entry Comments:}

Units shown for QC (SPK \& STD) may not reflect the actual units. $D L=$ Detection Limit, $S=$ Worklist Slot Number, $R=$ Replicate Number, $A=$ Aliquot Code. 
Curve 1: TGA

File info: TER101801 Fri Oct 18 05: 35:08 1996

Sample Weight: $19.557 \mathrm{mg}$

TGA STD BENB-A

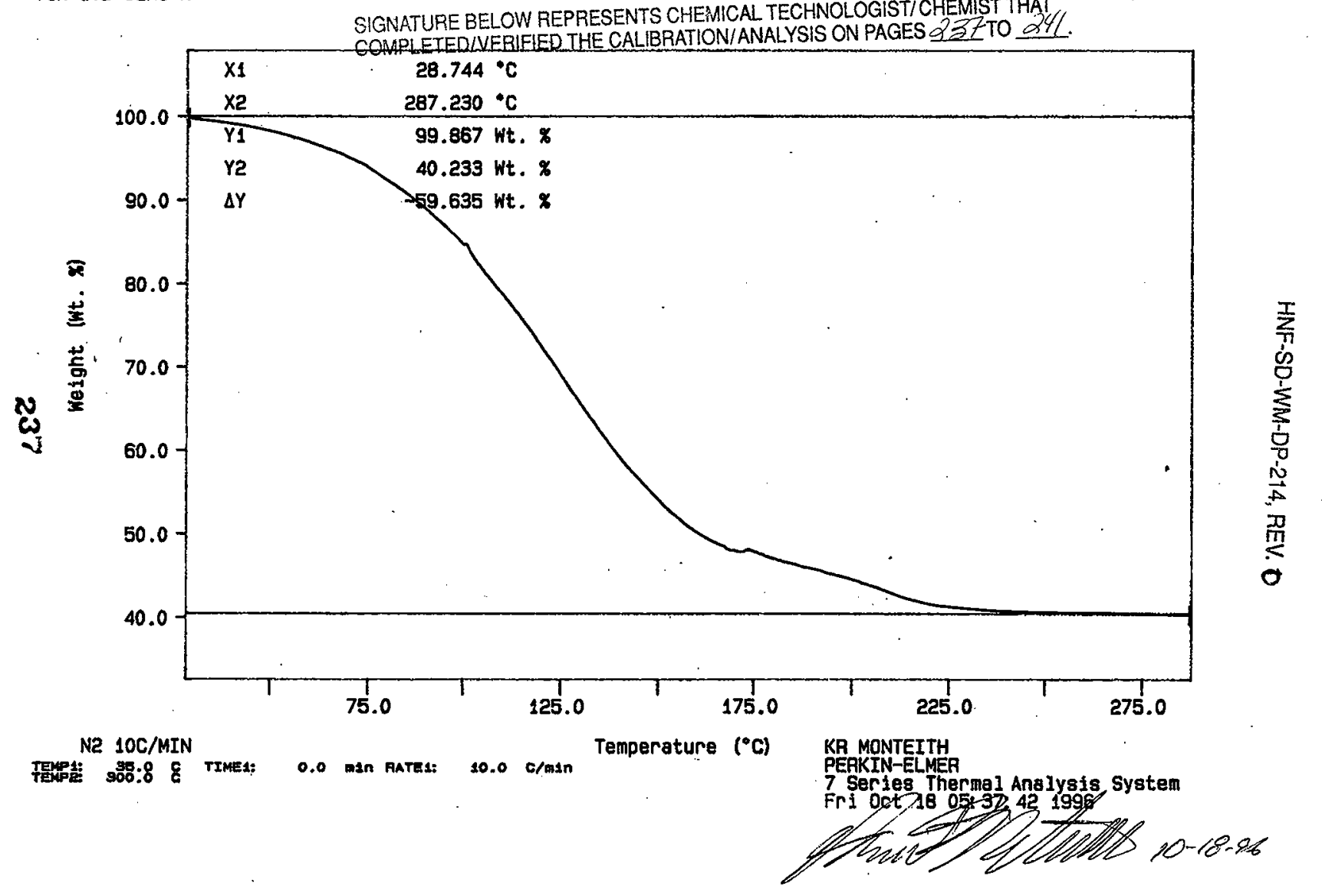


Curve 1: TGA

File info: SAM101801 Fri Oct 18 06: 41: 321996

Sample Neight: $16.463 \mathrm{mg}$

S96T005019

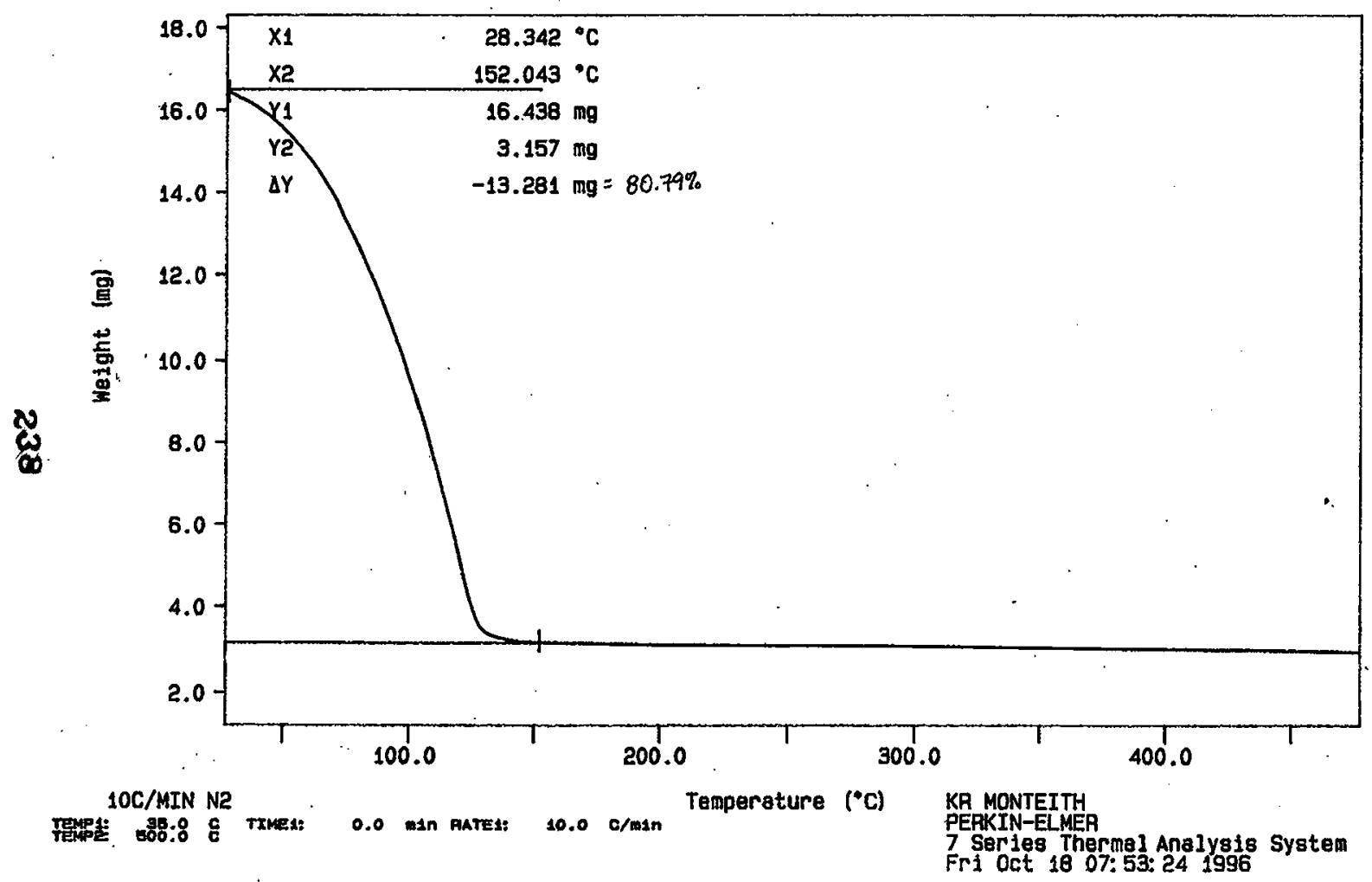


Curve 1: TGA

File info: SAM101802 Fri Oct 18 09: 25: 511996

Sample Weight: 14.567

S96T005019DUP

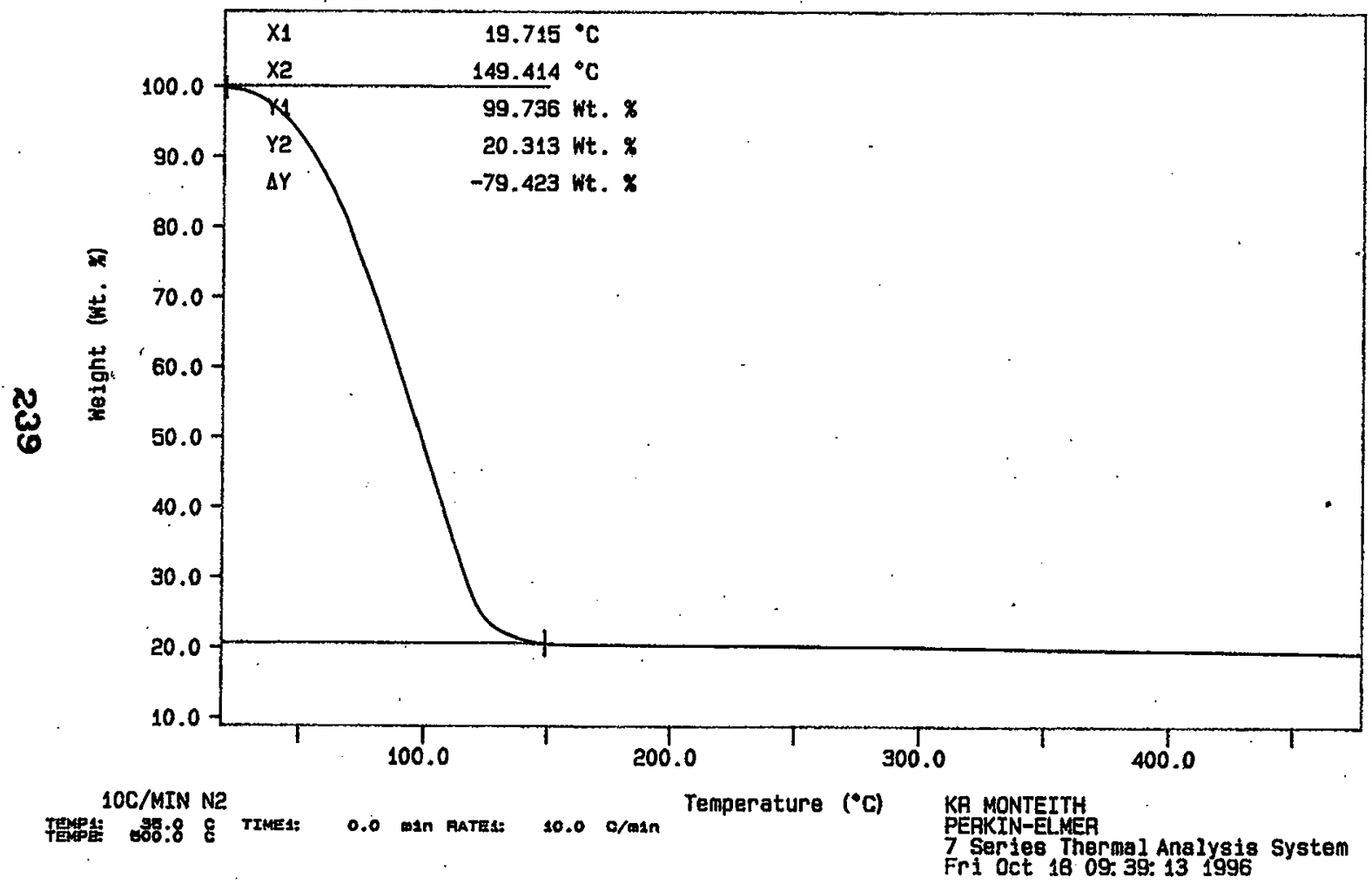


Cunve 1: TGA

File info: SAM101803 Fri oct 18 10:34: 211996

Sample Weight: $13.027 \mathrm{mg}$

\$96T005020

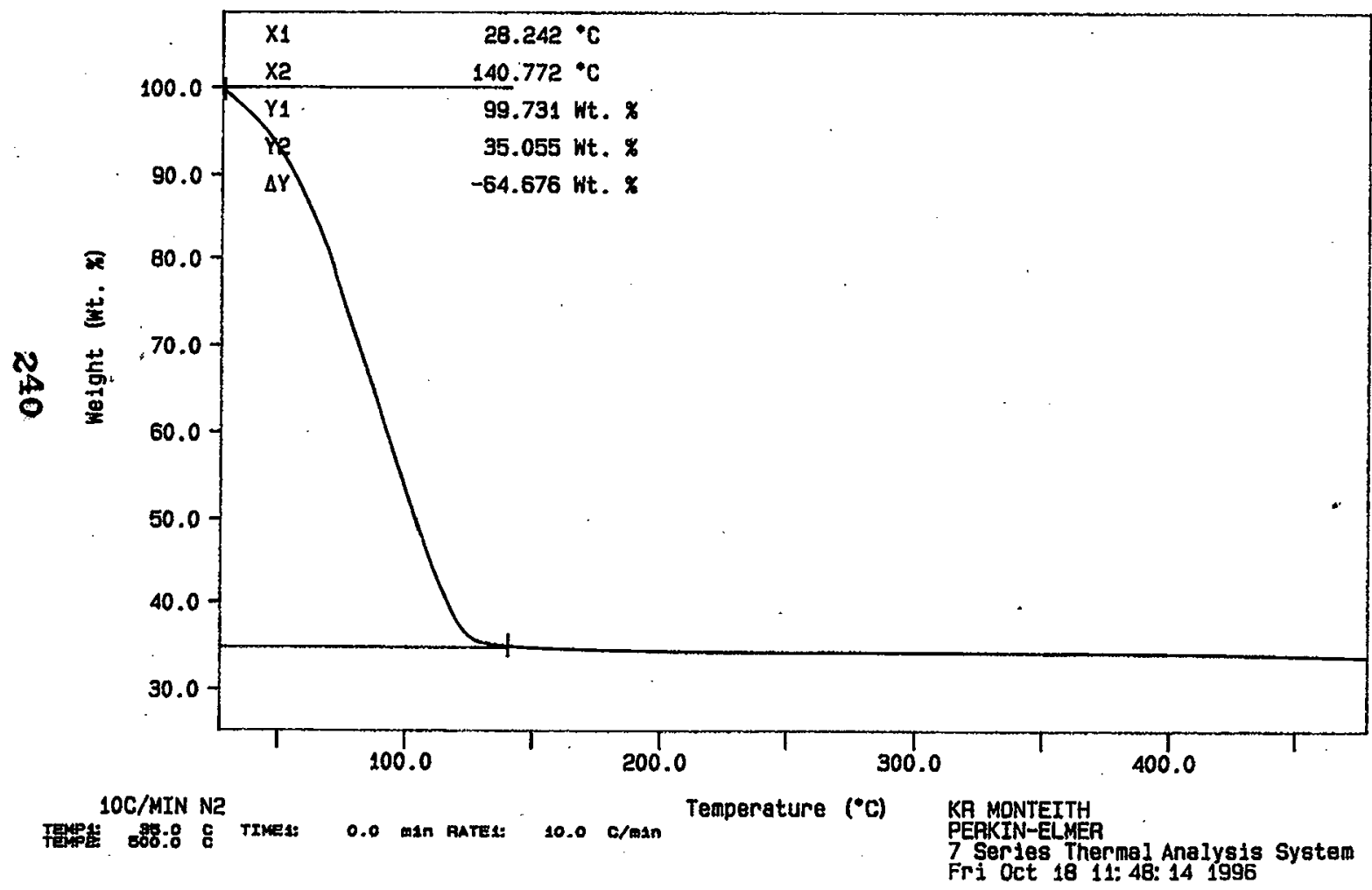


Curve 1: TGA

File info: SAM101804 Frt Oct 18 12: 33:51 1996

Sample Weight: $11.861 \mathrm{mg}$

S96T005020 DUP

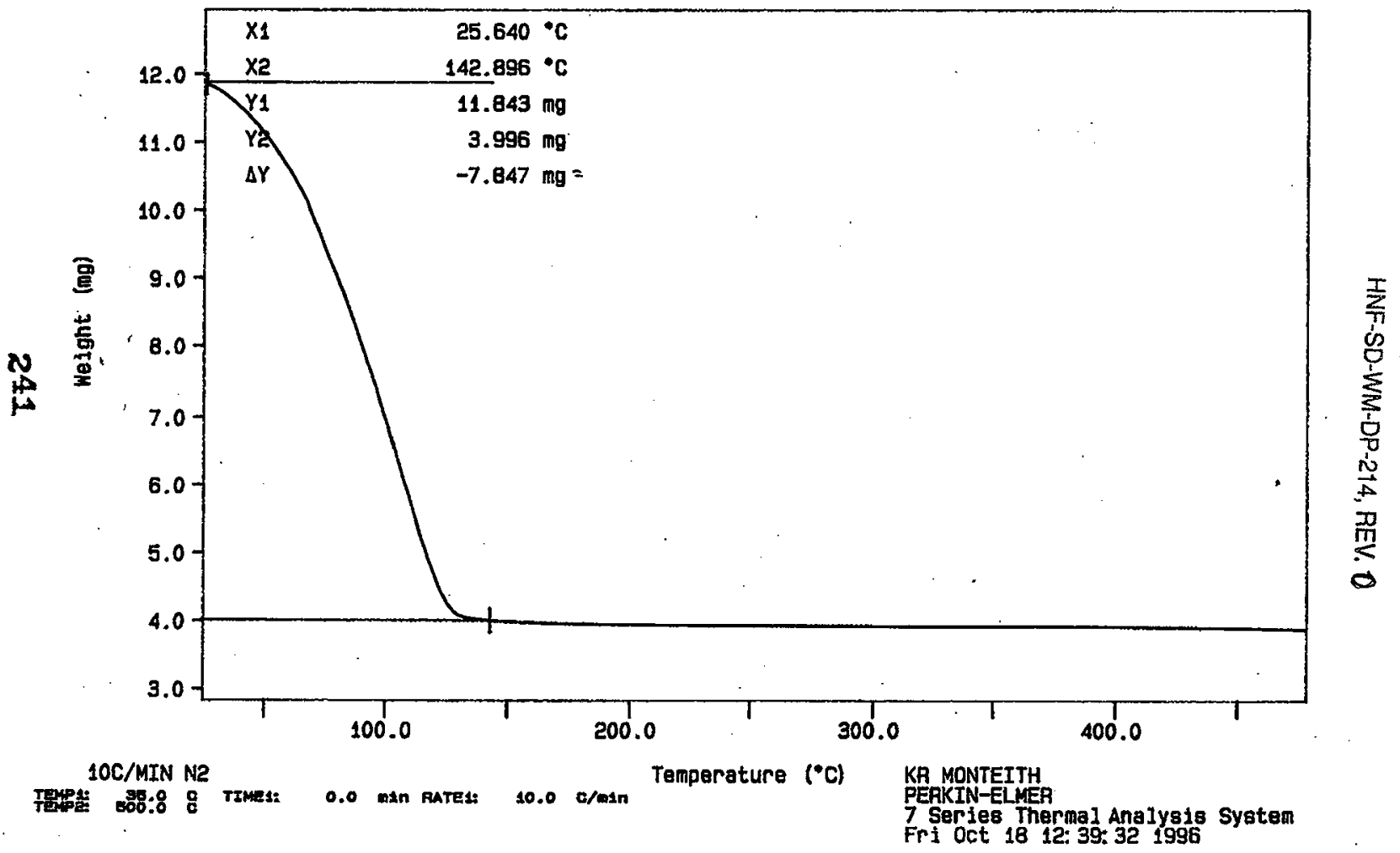




\section{LABCORE Data Entry Template for Worklist\# 13922}

Analyst: R6A Instrument: TGA 1 Book \# $2018 \mathrm{~A}$

Method: LA-560-112 Rev/Mod C-O

Worklist Comment: AW-105 Grab. Run under nitrogen. Std: TERLIQ .010ml. new

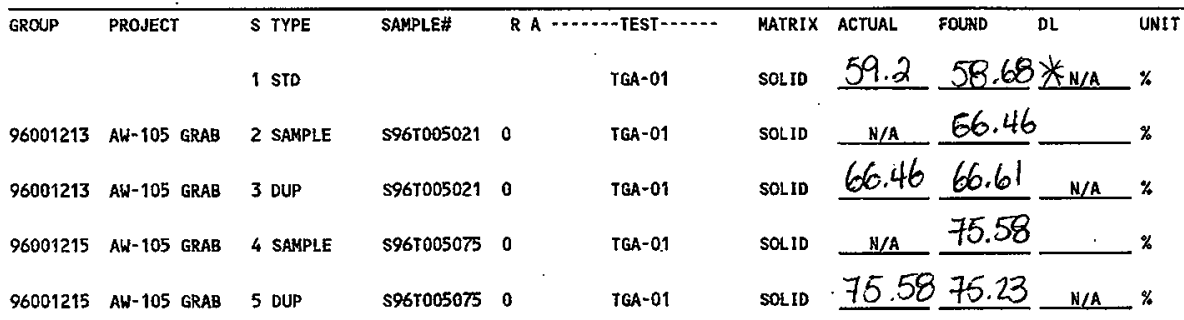

\section{Final page for worklist \# 13922}

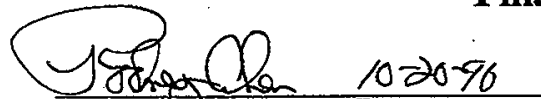

Analyst Signature Date

Verfifed/Validatea by

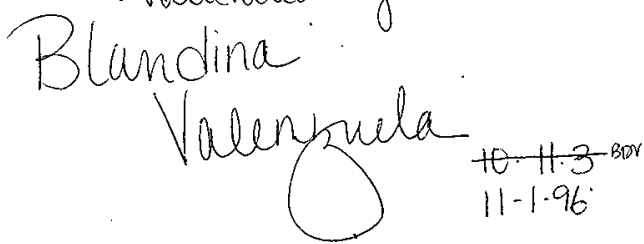

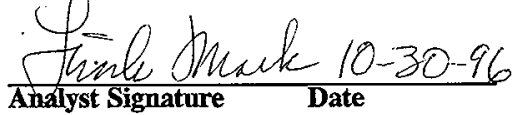

\section{Data Entry Comments:}

Units shown for $Q C$ (SPK \& STD) may not reflect the actual units. $D L=$ Detection Limit, $S=$ Worklist Slot Number, $R=$ Replicate Number, $A=$ Aliquot Code. 


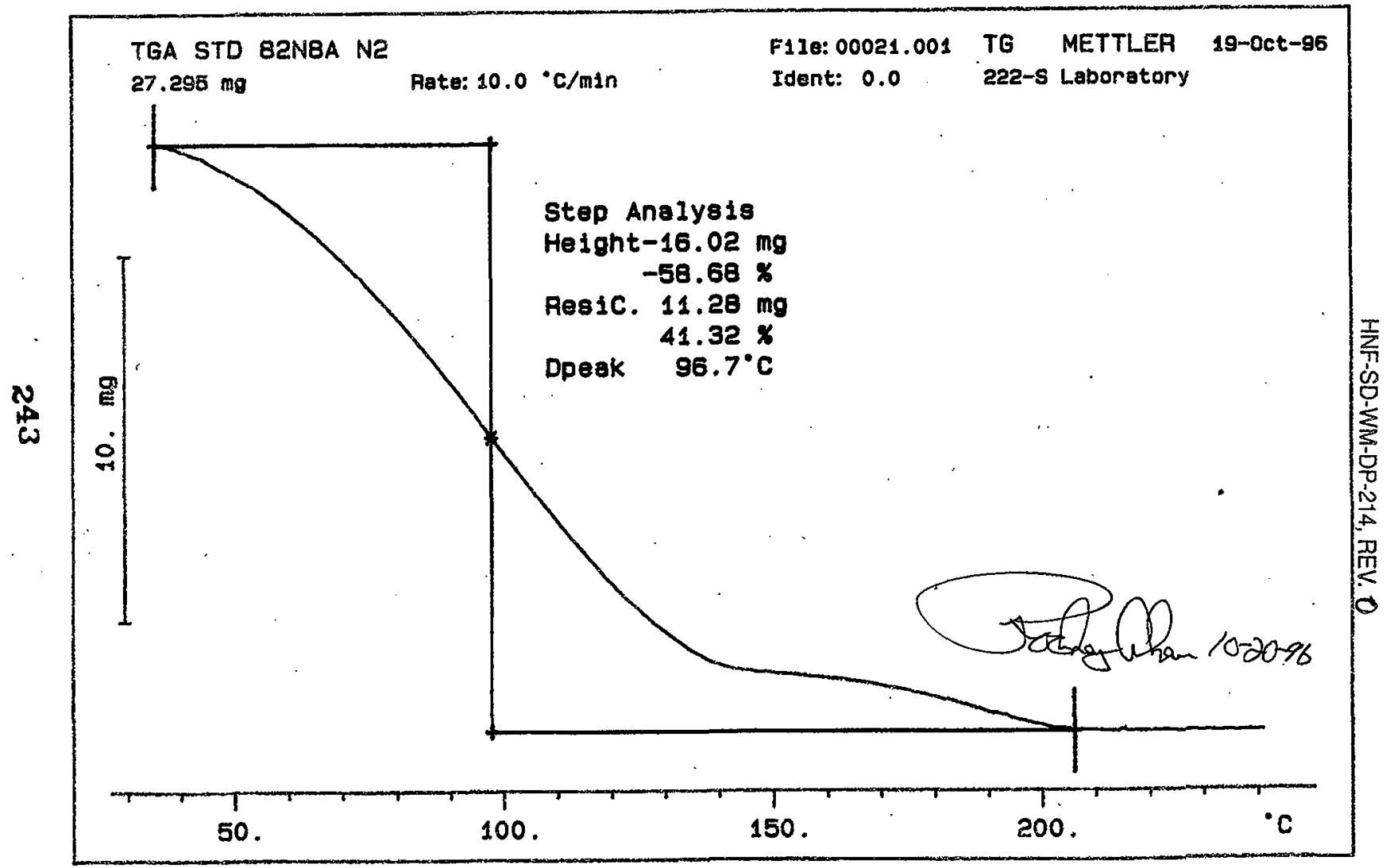


HNF-SD-WM-DP-214, REV. D

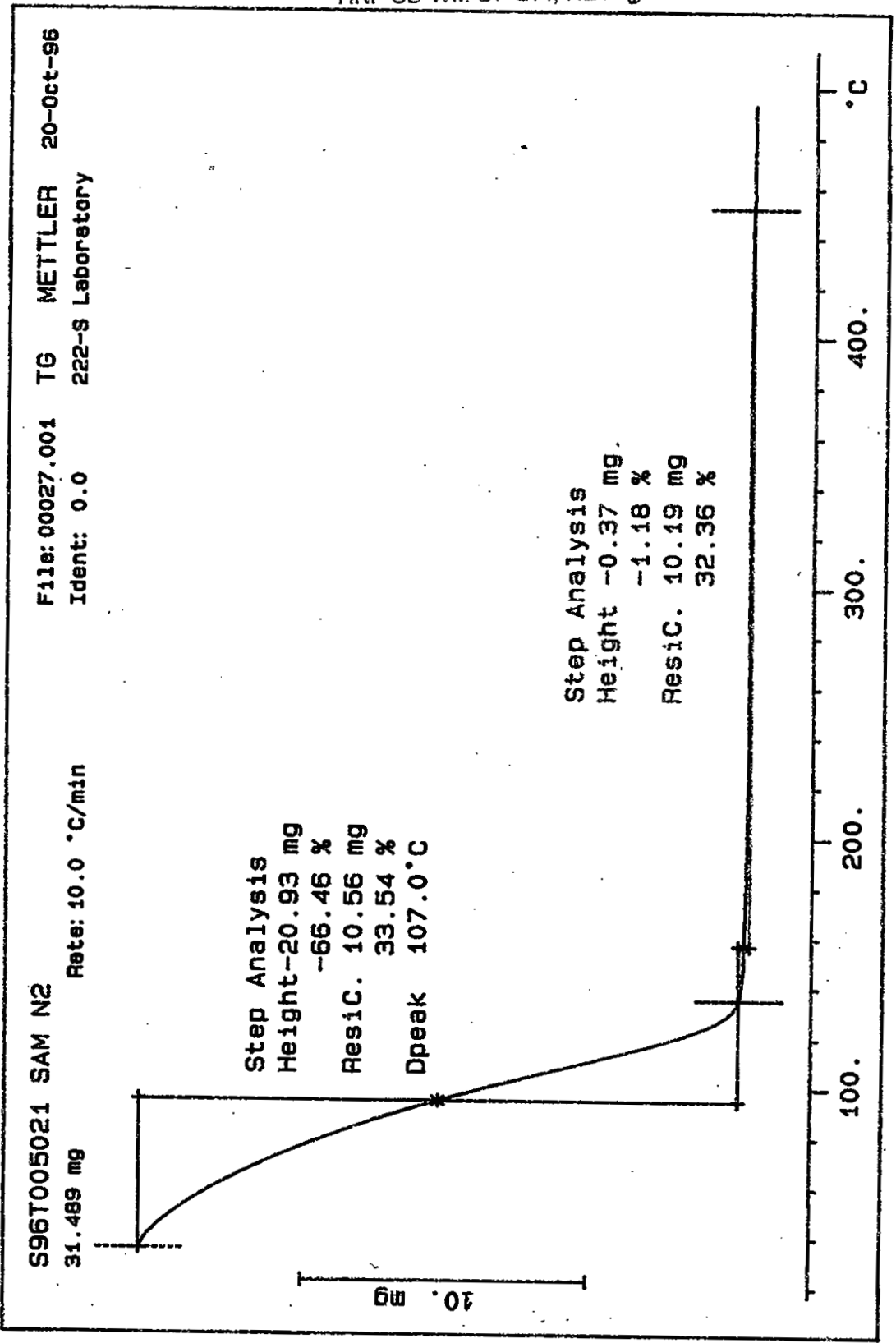

244 


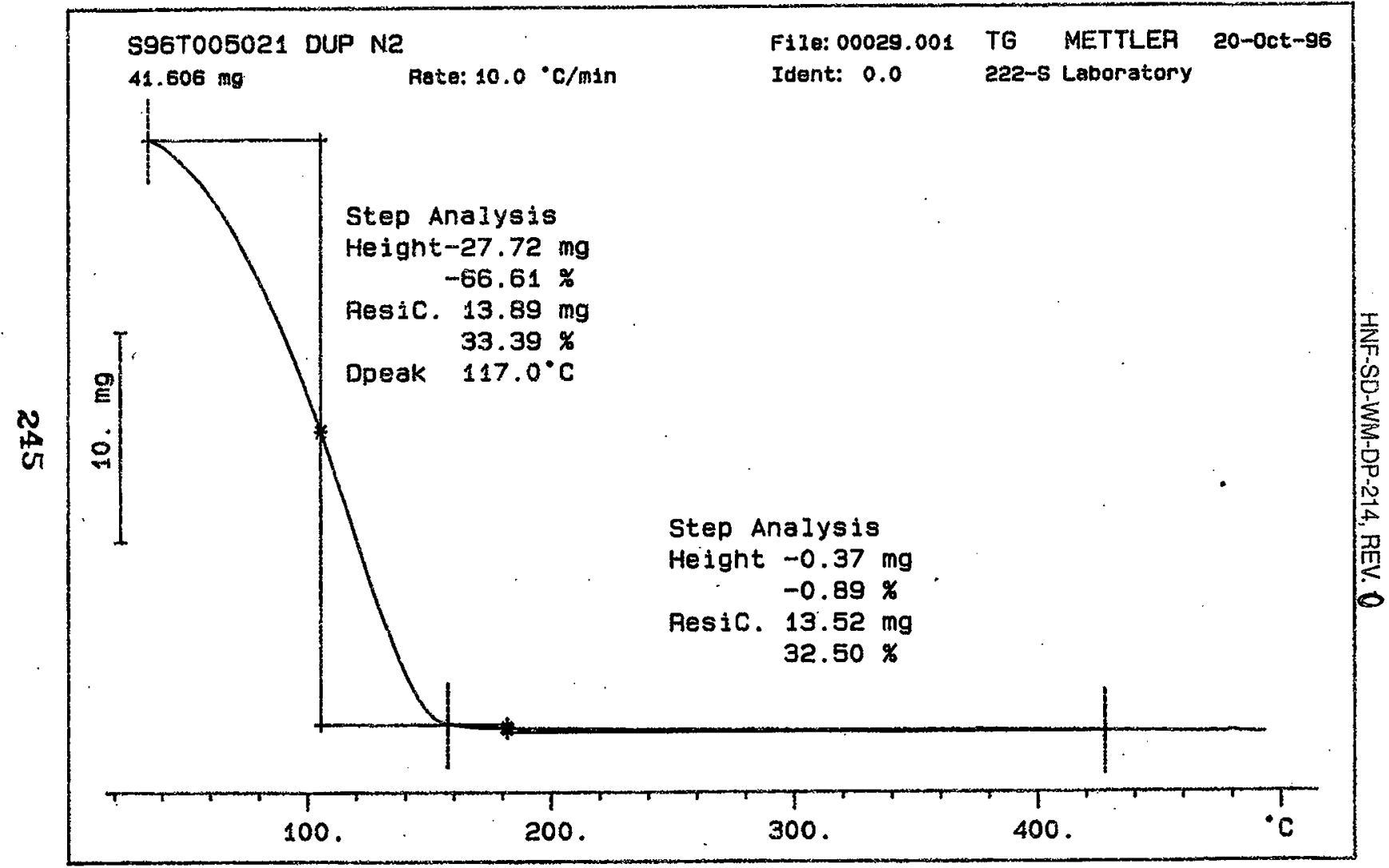


HNF-SD-WM-DP-214, REV. O

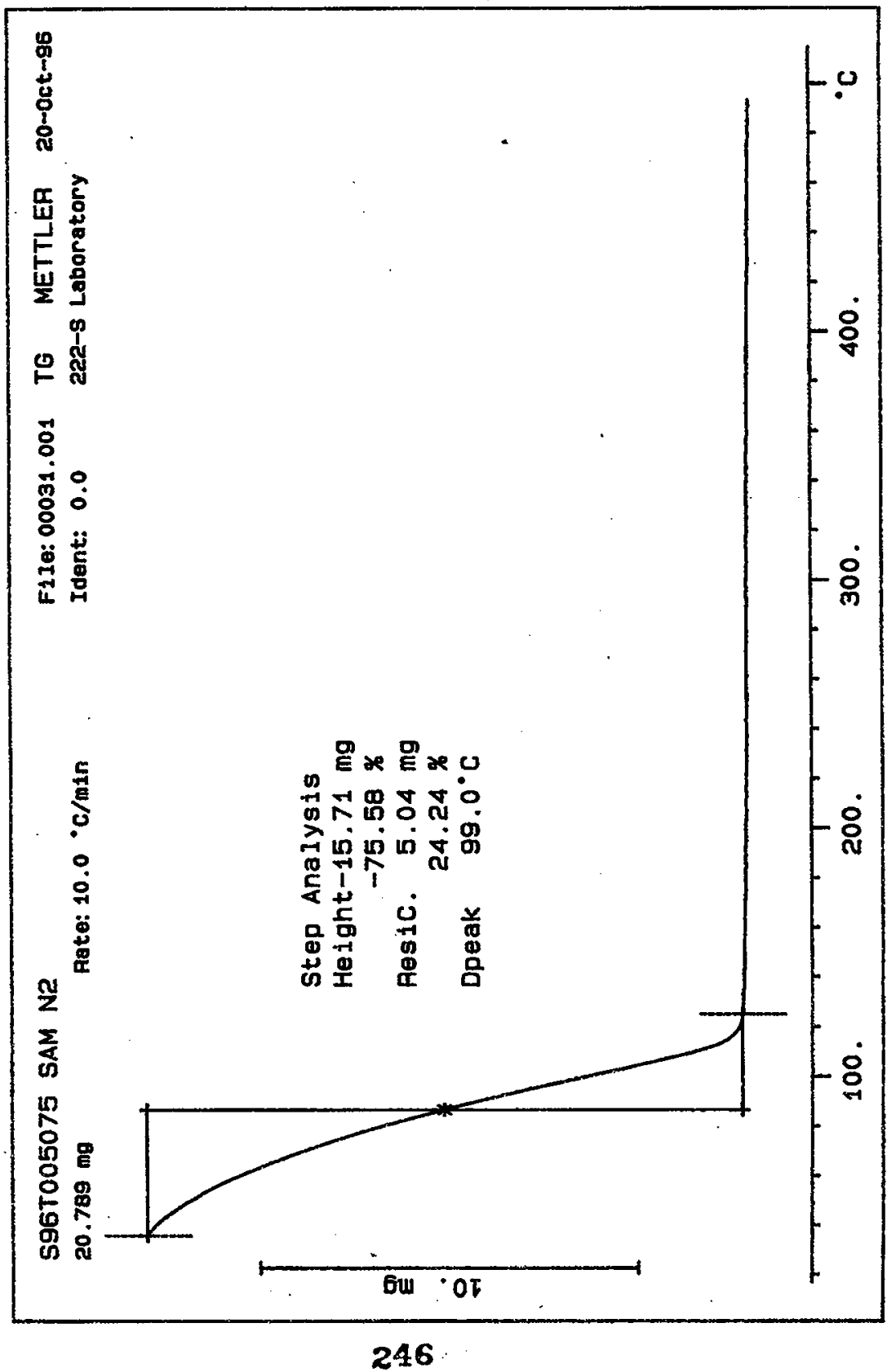


HNF-SD-WM-DP-214, REV. D

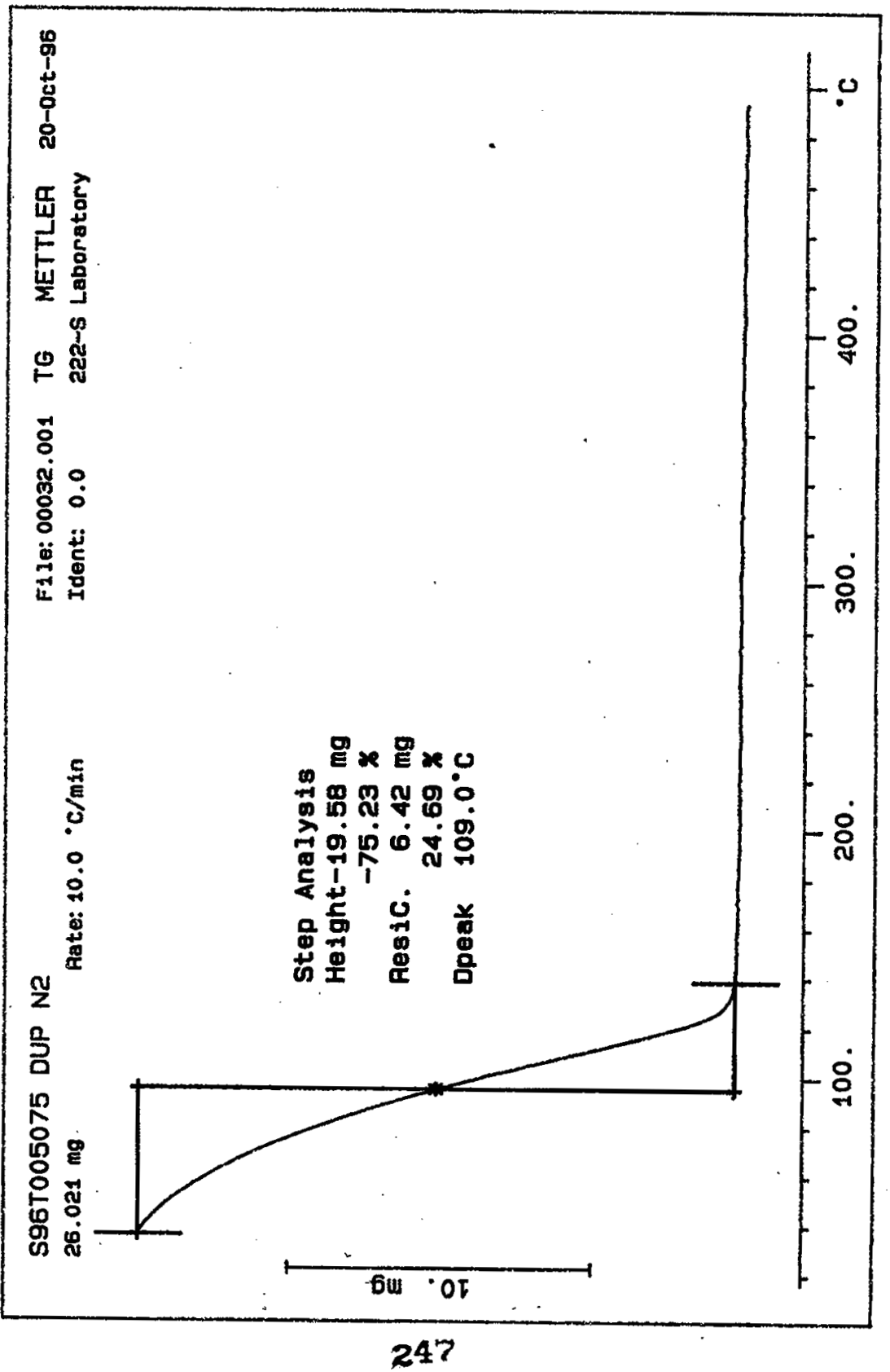




\section{LABCORE Data Entry Template for Worklist\# 13923}

Page: 1

Analyst: $\quad$ KRm Instrument: TGA0 I Book \# 820 \& A

Method: LA-560-112 Rev/Mod C-O

Worklist Comment: AW-105 Grab. Run under nitrogen. Std: TERLIQ .010ml. new

\begin{tabular}{|c|c|c|c|c|c|c|c|c|c|c|}
\hline \multirow[t]{2}{*}{ GROUP. } & \multirow[t]{2}{*}{ PROJECT } & \multirow{2}{*}{$\begin{array}{l}\text { S TYPE } \\
1 \text { STD }\end{array}$} & \multirow[t]{2}{*}{ SAMPLE\# } & \multicolumn{2}{|c|}{ R A $\cdots \cdots$ TEST - . . . } & \multirow{2}{*}{$\begin{array}{l}\text { MATRIX } \\
\text { SOLID }\end{array}$} & \multirow{2}{*}{$\begin{array}{l}\text { ACTUAL } \\
59.2 \\
\end{array}$} & \multirow{2}{*}{$\begin{array}{l}\text { FOUND } \\
58.93 \\
\end{array}$} & \multirow{2}{*}{$\begin{array}{l}\text { DL } \\
\text { M/A } \\
\end{array}$} & \multirow{2}{*}{$\begin{array}{l}\text { UNIT } \\
\%\end{array}$} \\
\hline & & & & & TGA-01 & & & & & \\
\hline 96001215 & AW-105 GRAB & 2 SAMPLE & S96T005076 & $\mathbf{0}$ & TGA-01 & SOLID & N/A & 65.12 & & $\%$ \\
\hline 96001215 & AW-105 GRAB & 3 DUP & s96T005076 & 0 & TGA -01 & SOLIO & 65.12 & 66.79 & N/A & $\%$ \\
\hline 96001215 & AW-105 GRAB & 4 SAMPLE & S96T005077 & 0 & $T G A=01$ & SOLID & $\therefore N / A$ & 66.19 & & $\%$ \\
\hline 96001215 & AL-105 GRAB & 5 DUP & s96T005077 & 0 & $T G A-01$ & SOLID & 66.19 & 71.20 & N/A & $\%$ \\
\hline
\end{tabular}
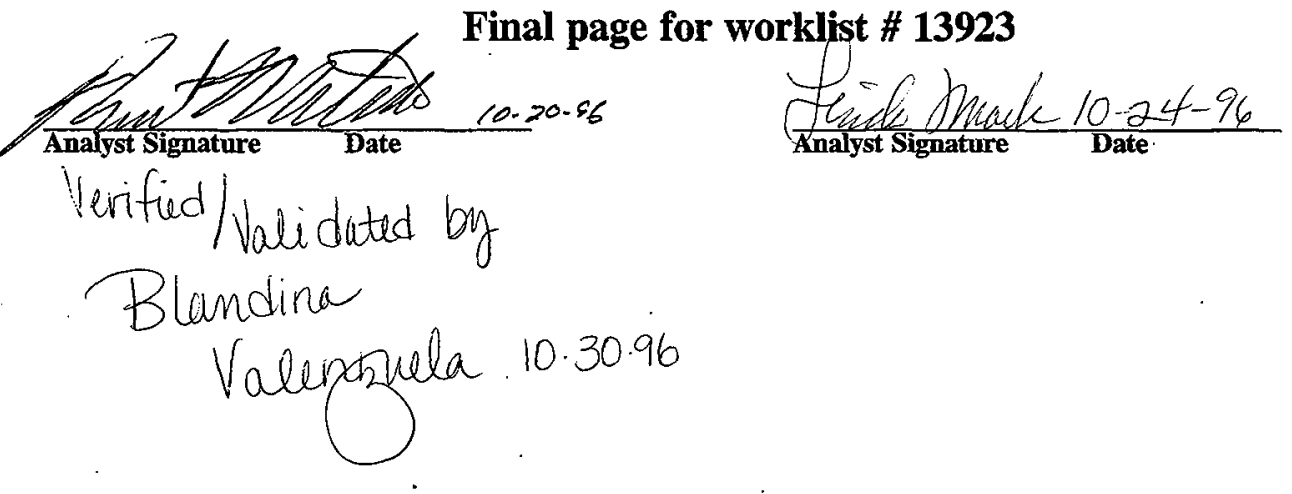

Data Entry Comments:

Units shown for $Q C$ (SPK \& STD) moy not reftect the actual units. $D L=$ Detection Limit, $S=$ Worklist Slot Number, $R=$ Replicate Number, $A=$ Aliquot Code. 


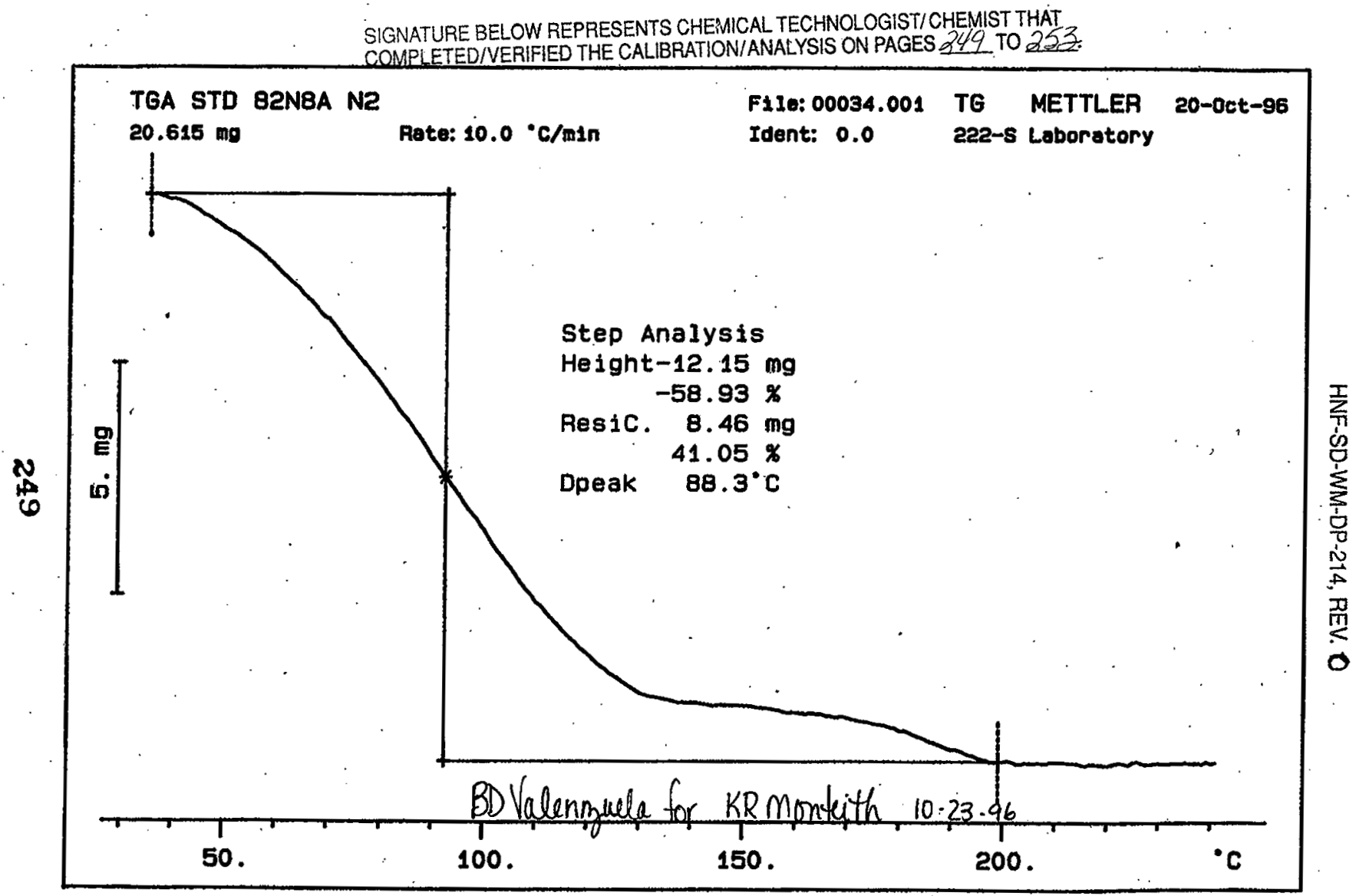


HNF-SD-WM-DP-214, REV. 0

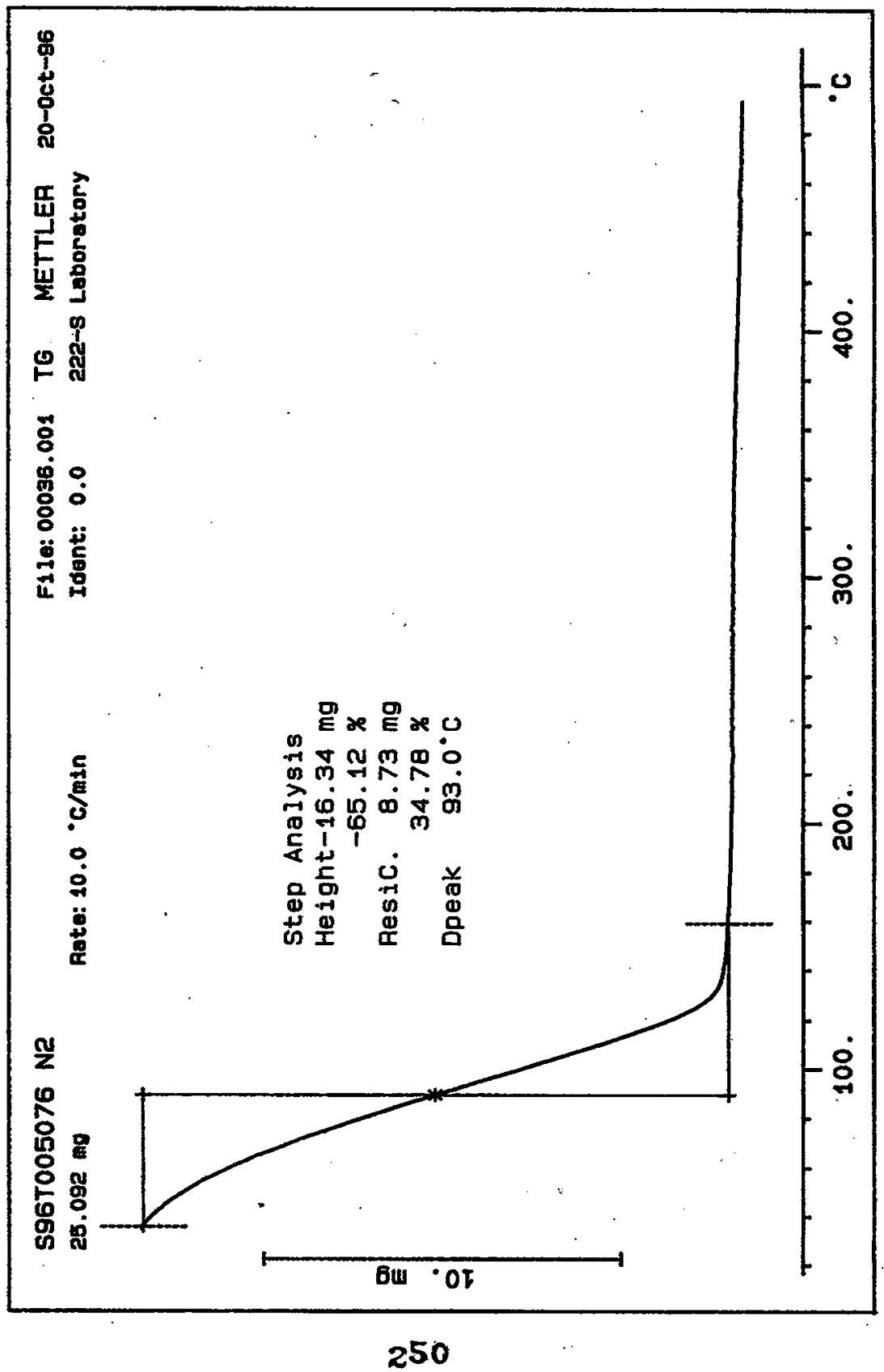




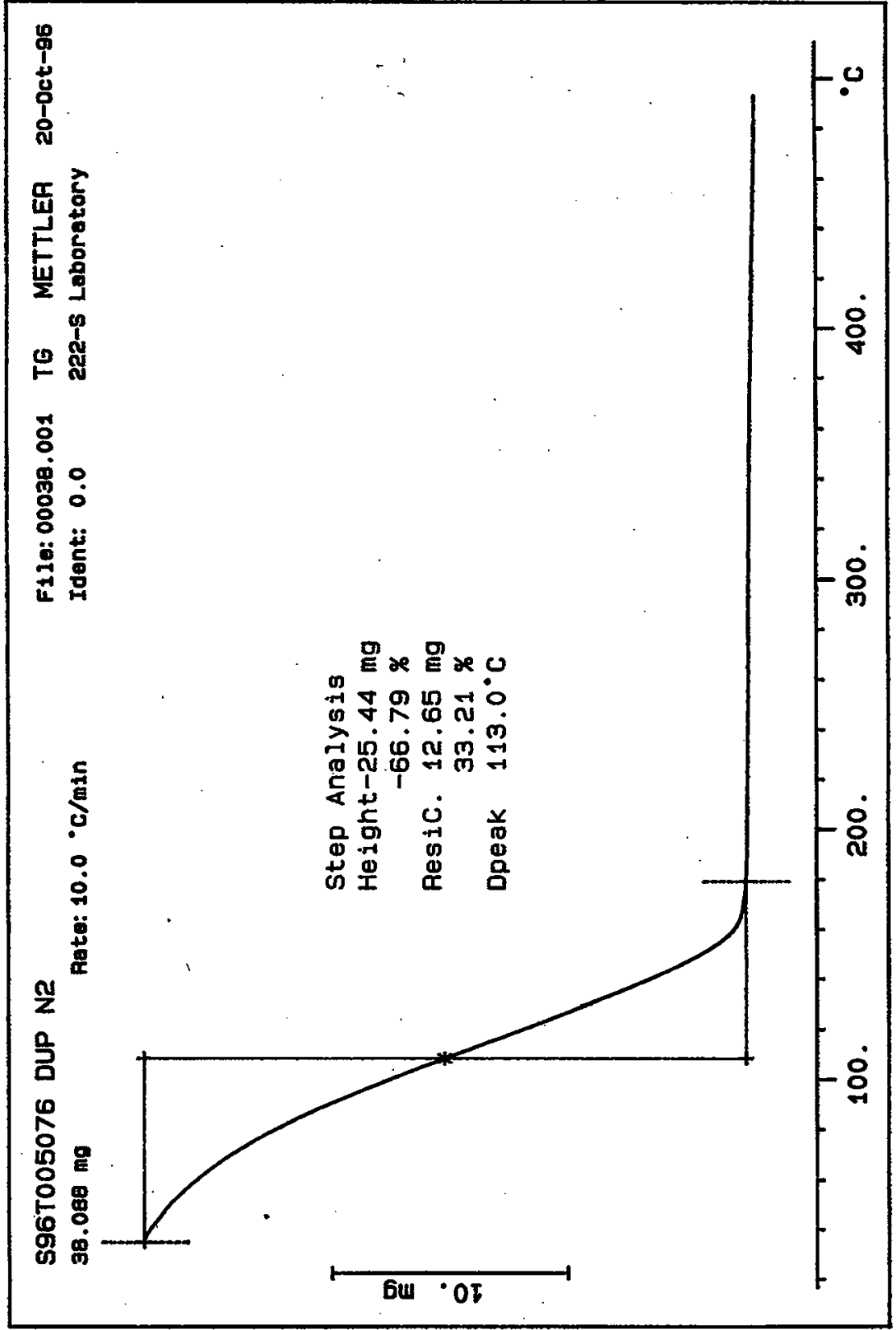

251 


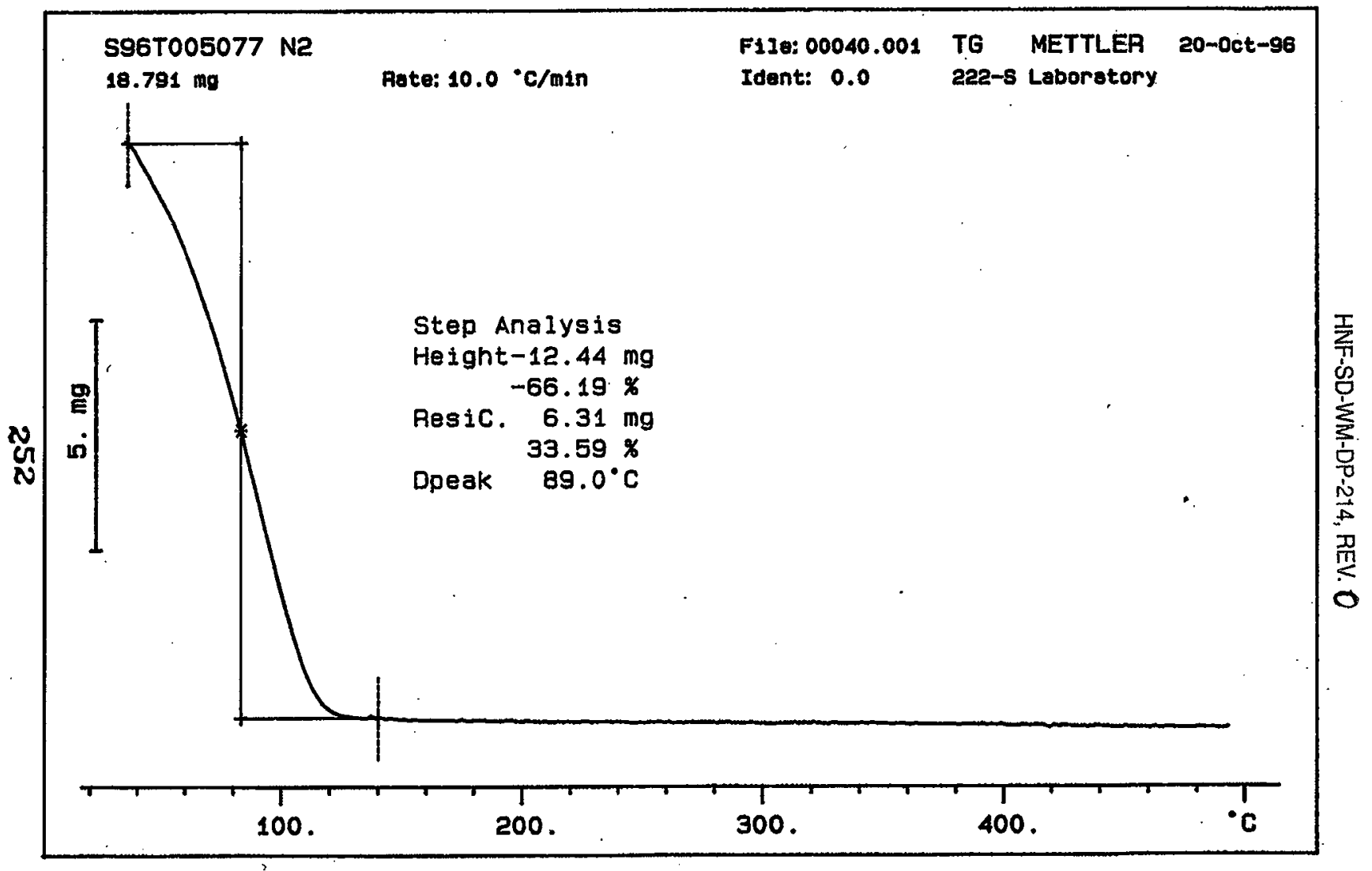




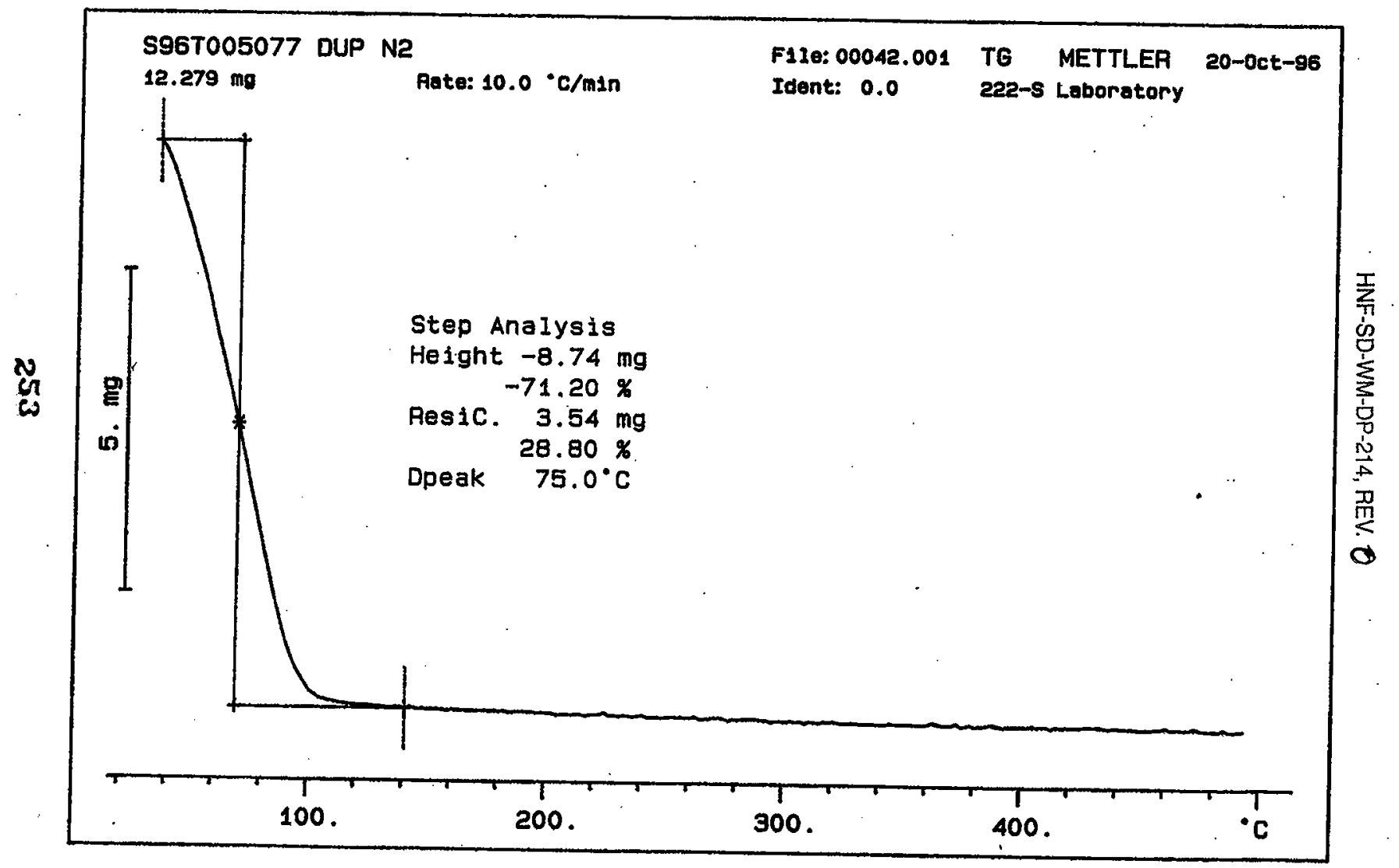




\section{LABCORE Data Entry Template for Worklist\# 13073}

Analyst: $\quad D P B \quad$ Instrument: BA001_ Book \#133N16

Method: LA-510-112 Rev/Mod $C-3$

Worklist Comment: AW-105 SPG. RCJ

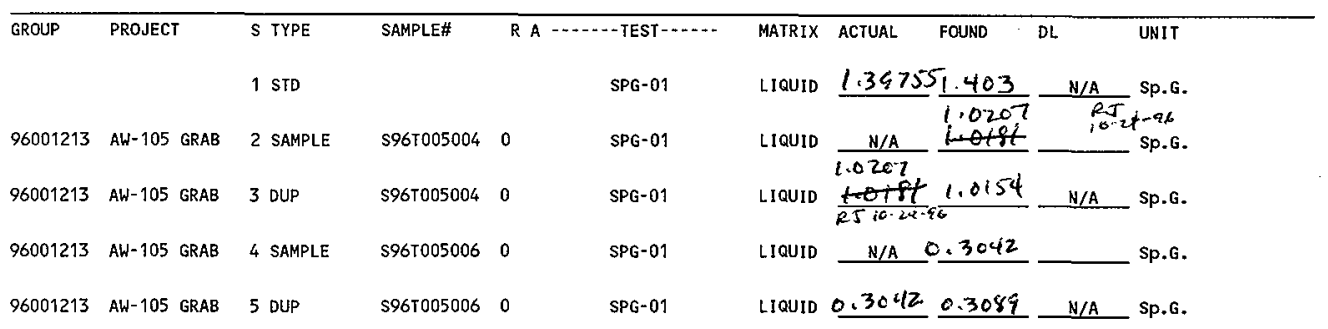

\section{Final page for worklist \# 13073}

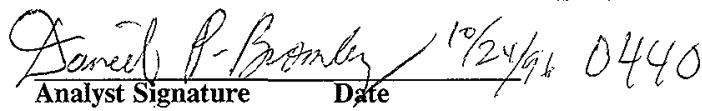
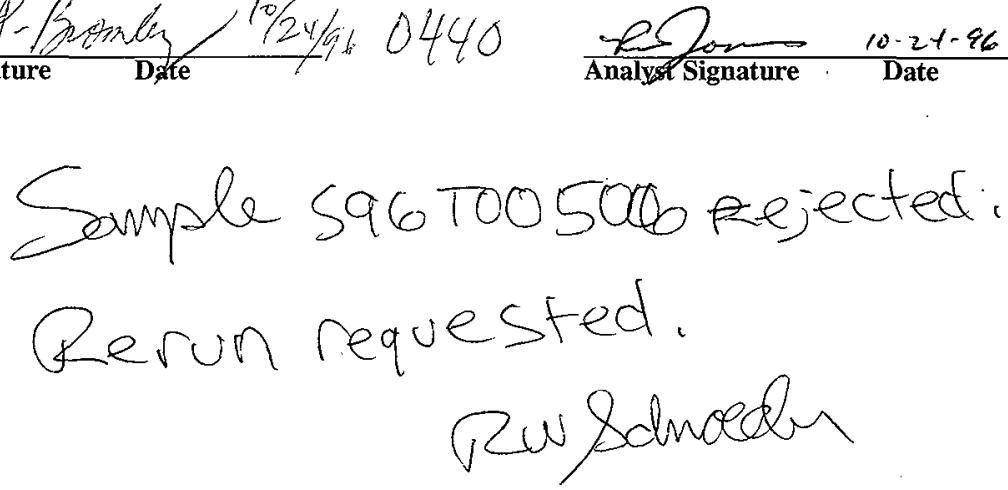

Data Entry Comments:

Units shown for $Q C$ (SPK \& STD) may not reflect the actual units. $D L=$ Detection Limit, $S=$ Worklist Slot Number, $R=$ Replicate Number, $A=$ Aliquot Code. 
SPECIFIC GRAVITY: LA-510-112 (C-3)

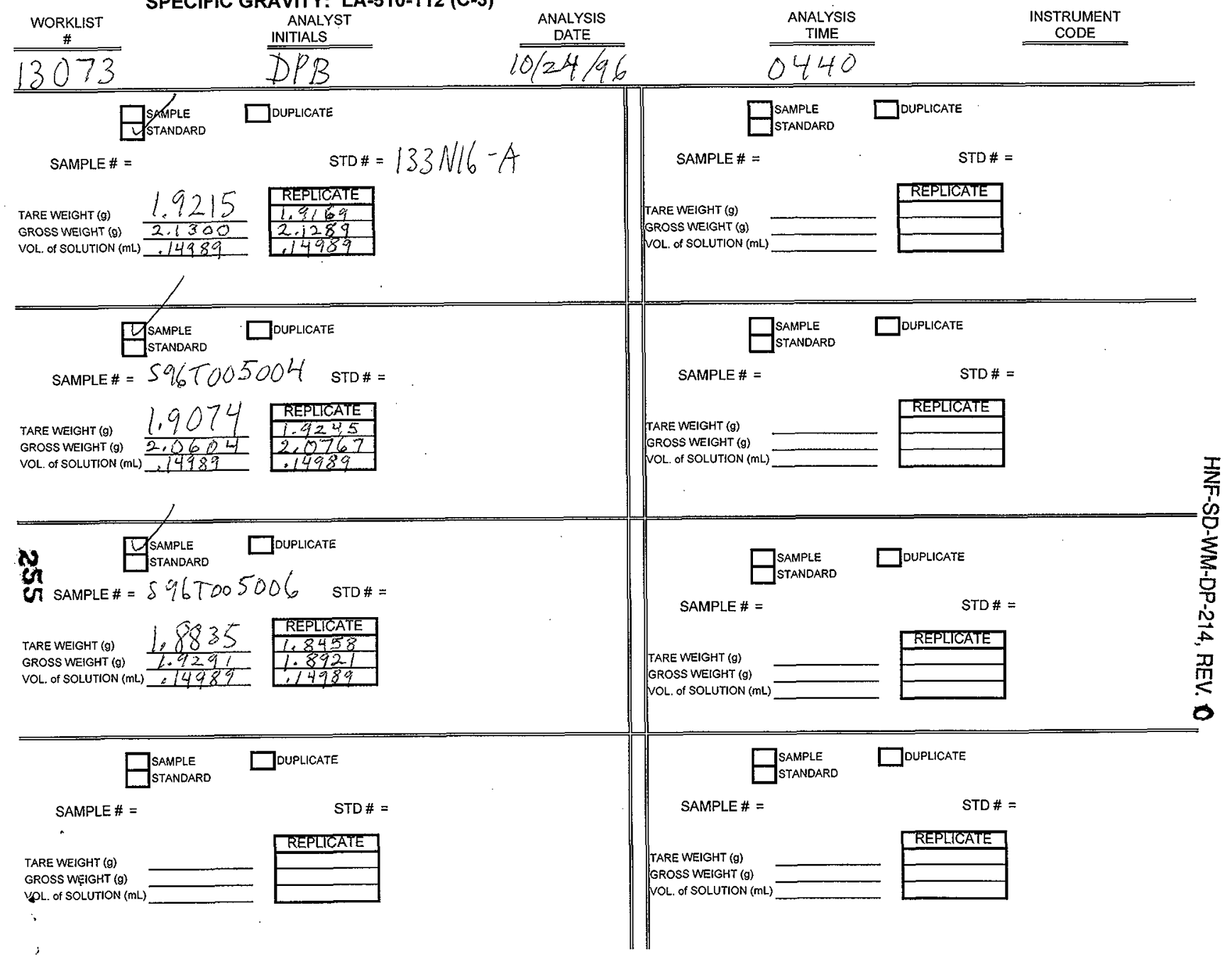




\section{SPECIFIC GRAVITY : LA-510-112 (C-3)}

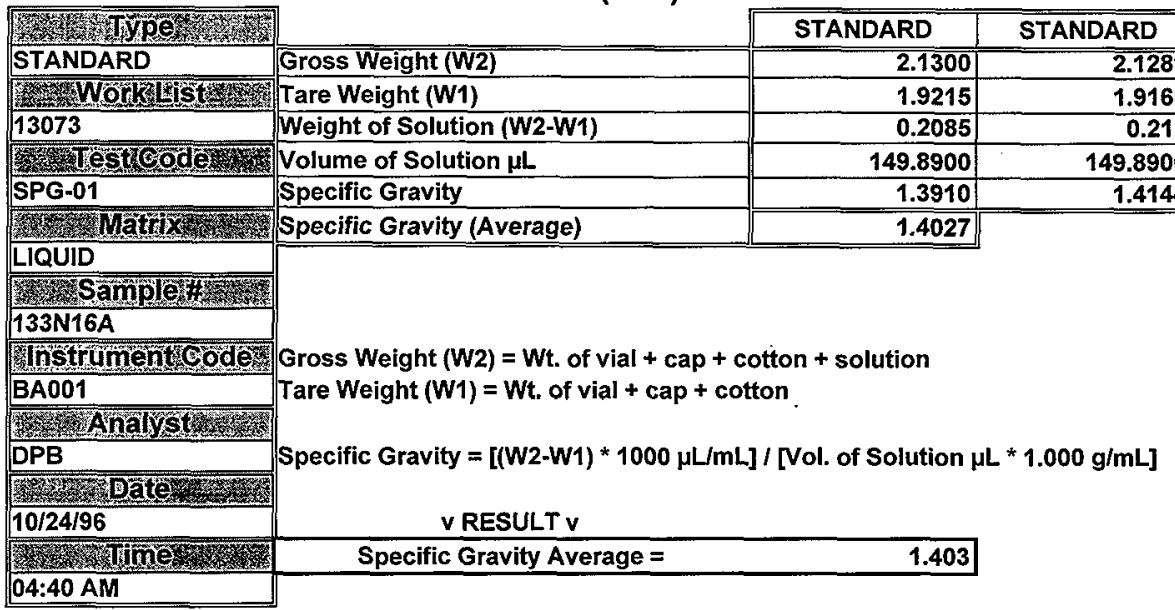

\begin{tabular}{|c|c|c|}
\hline Data Entry by: & Date: & $10 / 24 / 96$ \\
\hline Approved by: & Date: & $60 / 24 / 96$ \\
\hline Form 510112L1 Rev. 1.1 & & ge 1 of 1 \\
\hline
\end{tabular}




\section{SPECIFIC GRAVITY : LA-510-112 (C-3)}

\begin{tabular}{|c|c|c|c|}
\hline 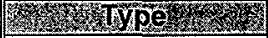 & & SAMPLE & REPLICATE \\
\hline SAMPLE & Gross Weight (W2) & 2.0604 & 2.076 \\
\hline 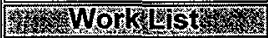 & Tare Weight (W1) & 1.9074 & 1.92 \\
\hline 13073 & Weight of Solution (W2-W1) & 0.153 & 0.15 \\
\hline Westoode & Volume of Solution $\mu \mathrm{L}$ & 149.8900 & 149.890 \\
\hline SPG-01 & Specific Gravity & 1.0207 & 1.015 \\
\hline 3 & Specific Gravity (Average) & 1.0181 & \\
\hline LIQUID & \multirow{6}{*}{\multicolumn{3}{|c|}{$\begin{array}{l}\text { Gross Weight }(W 2)=\text { Wt. of vial + cap + cotton + solution } \\
\text { Tare Weight }(W 1)=W t \text {. of vial + cap + cotton }\end{array}$}} \\
\hline 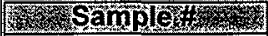 & & & \\
\hline S96T005004 & & & \\
\hline InstrumentGode & & & \\
\hline BA001 & & & \\
\hline 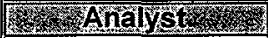 & & & \\
\hline DPB & \multirow{3}{*}{\multicolumn{3}{|c|}{ Specific Gravity $=[(\mathrm{W} 2-\mathrm{W} 1) * 1000 \mu \mathrm{L} / \mathrm{mL}] /$ [Vol. of Solution $\mu \mathrm{L} * 1.000 \mathrm{~g} / \mathrm{mL}]$}} \\
\hline WX & & & \\
\hline $10 / 24 / 96$ & & & \\
\hline Fr & Specific Gravity Average = & 1.018 & \\
\hline 04:40 AM & & & \\
\hline
\end{tabular}

\begin{tabular}{|c|c|c|c|}
\hline Data Entry by: & $32 \mathrm{CJ}$ & Date: & $10 / 24 / 96$ \\
\hline Approved by: & Ru sdinoede & Date: & $10 / 24 / 96$ \\
\hline
\end{tabular}




\section{LABCORE Data Entry Template for Worklist\# 13137}
Analyst: $\quad$ DPB
Instrument: BA001
Book $\# 133 N / 6-A$

Method: LA-510-112 Rev/Mod C-3

Worklist Comment: AW-105 SPG. RCJ

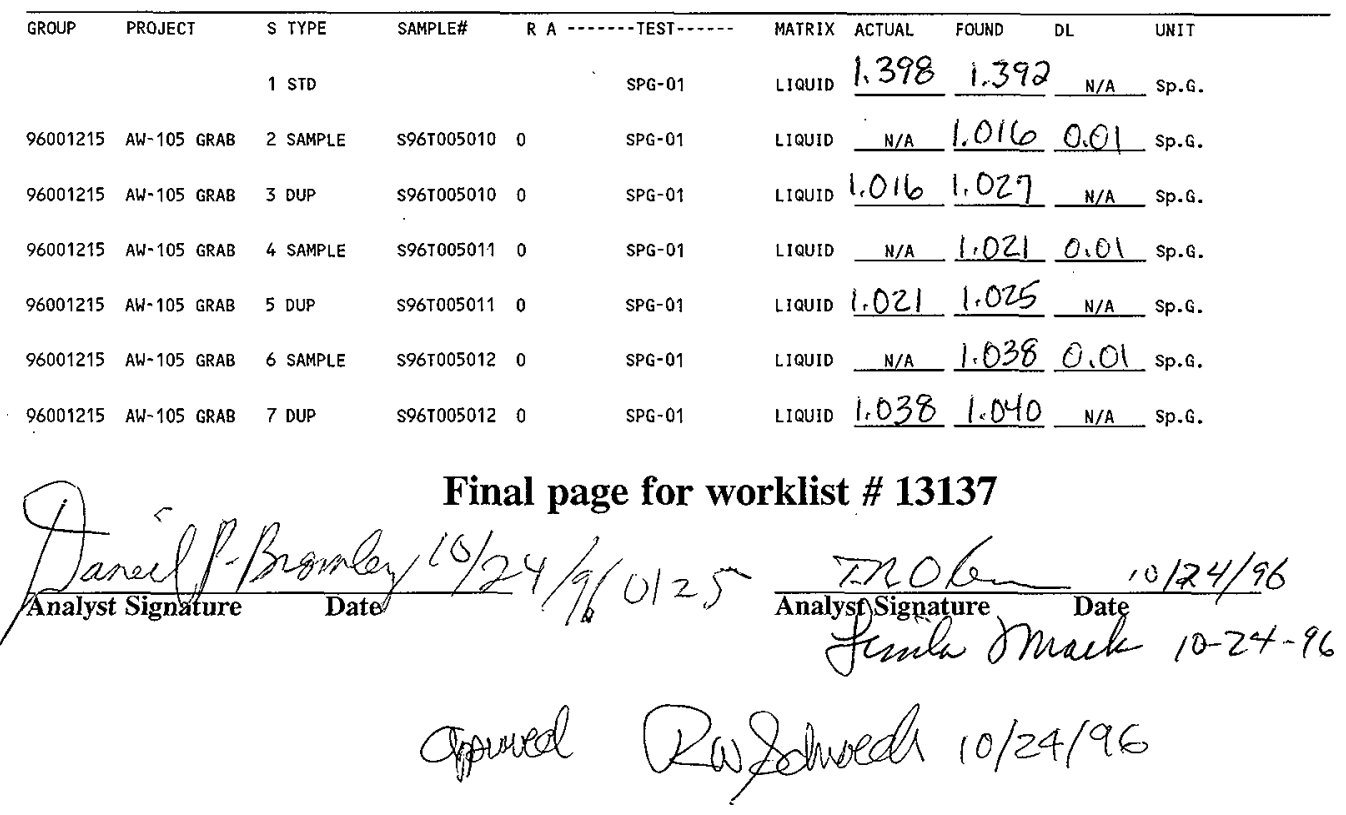

Data Entry Comments:

Units shown for $Q C$ (SPK \& STD) may not reflect the actual units. $D L=$ Detection Limit, $S=$ Worklist Slot Number, $R=$ Replicate Number, $A=$ Aliquot Code. 


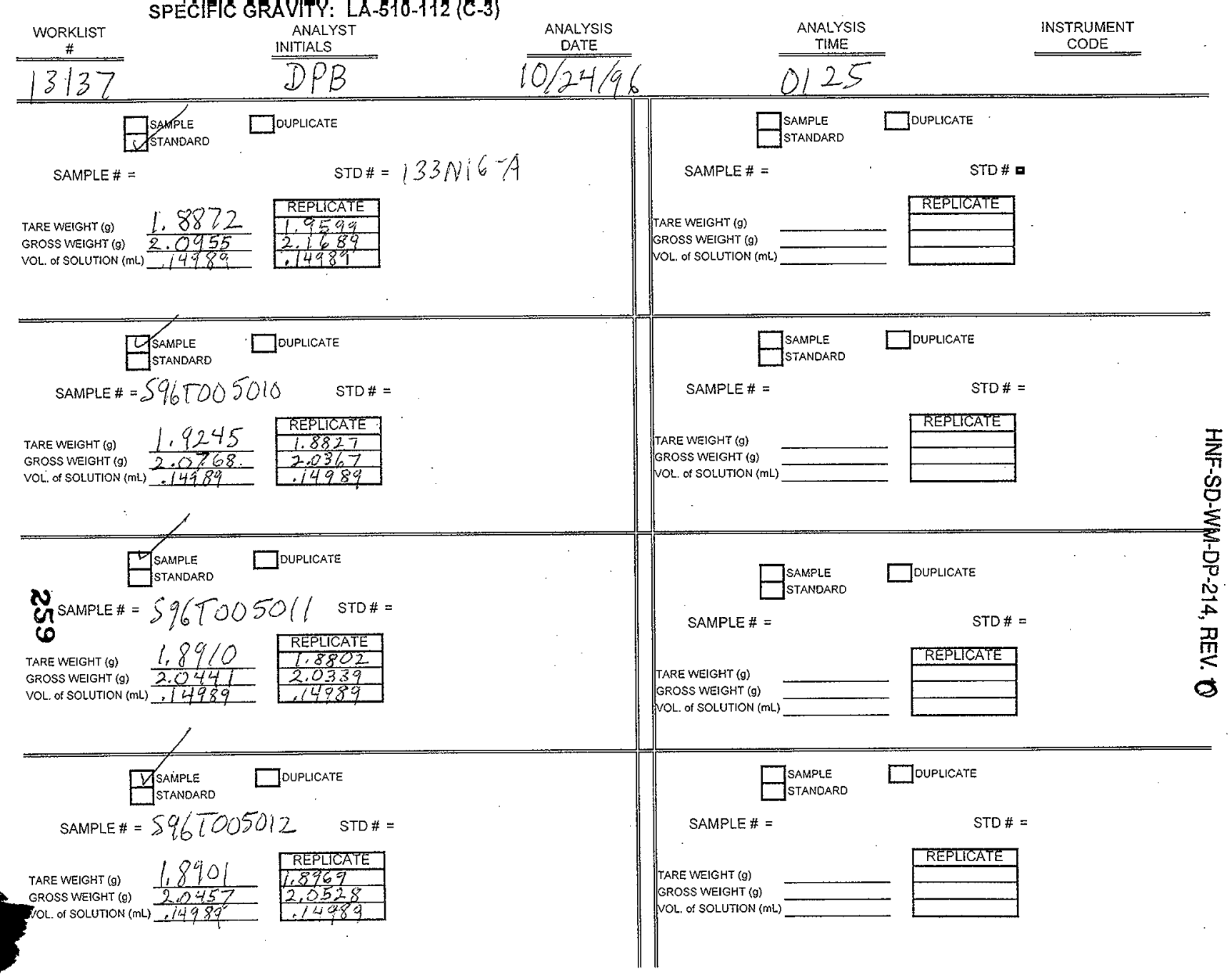


SPECIFIC GRAVITY : LA-510-112 (C-3)

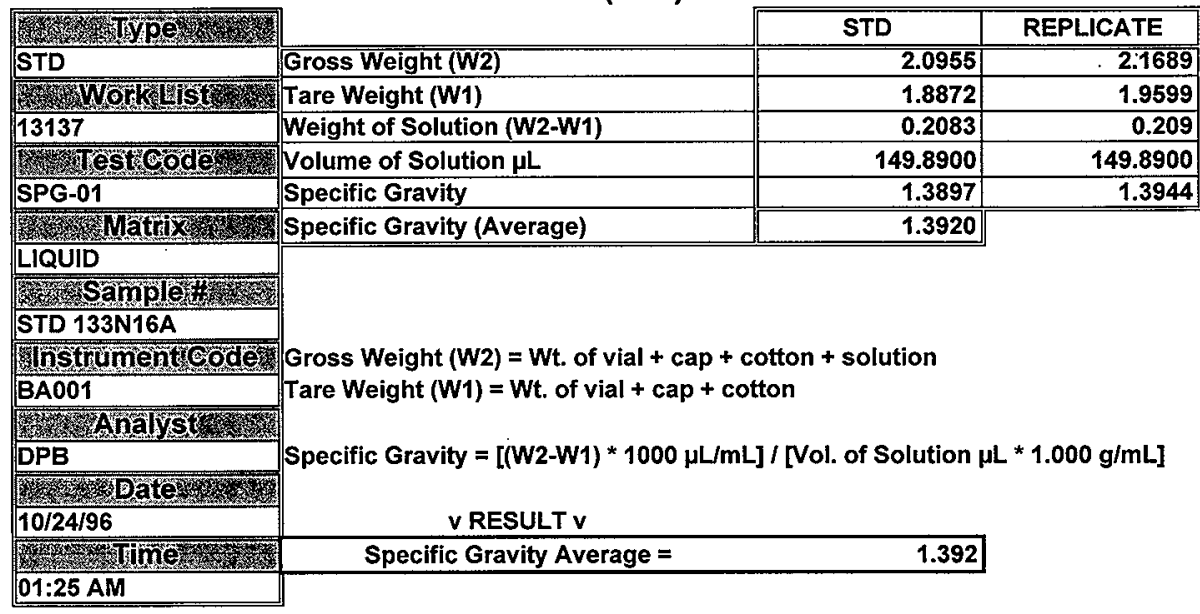

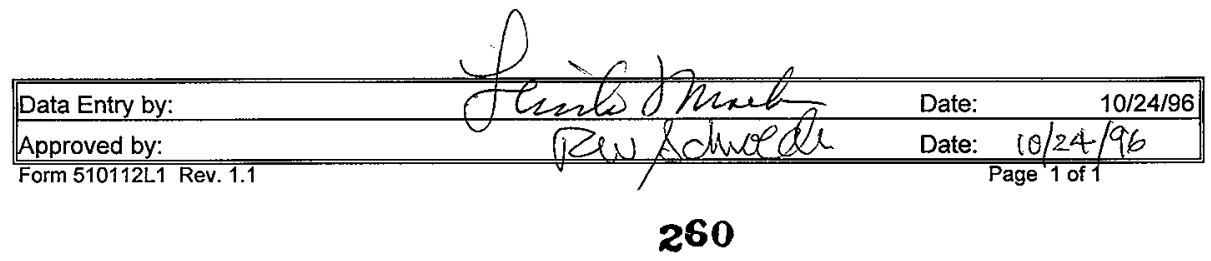




\section{SPECIFIC GRAVITY : LA-510-112 (C-3)}

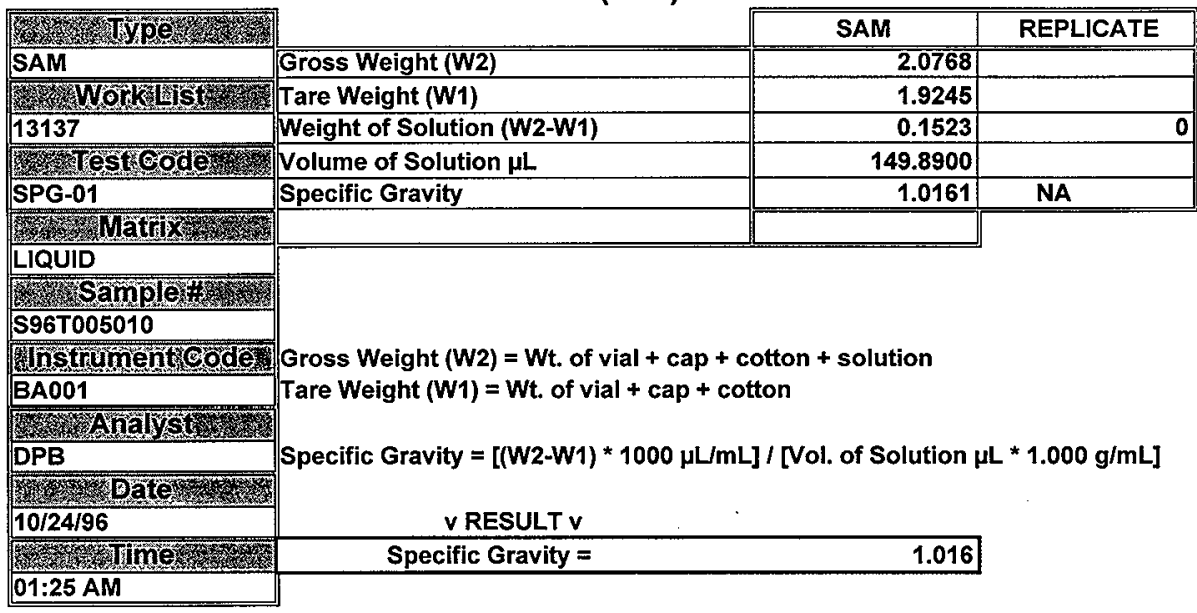

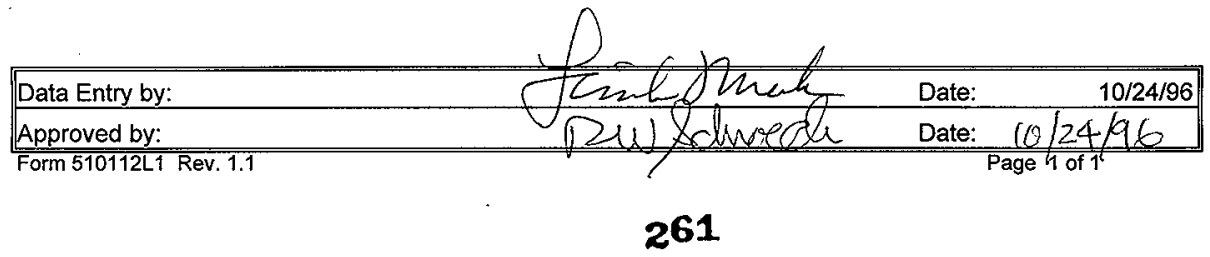




\section{SPECIFIC GRAVITY : LA-510-112 (C-3)}

\begin{tabular}{|c|c|c|c|}
\hline \multirow[b]{2}{*}{ Whypos } & \multirow{2}{*}{\multicolumn{2}{|c|}{ SAM-DUP }} & \multirow[b]{2}{*}{ REPLICATE } \\
\hline & & & \\
\hline SAM-DUP & Gross Weight (W2) & 2.0367 & \\
\hline 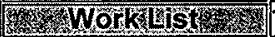 & Tare Weight (W1) & 1.8827 & \\
\hline 13137 & Weight of Solution (W2-W1) & 0.154 & 0 \\
\hline WIEstoodo & Volume of Solution $\mu \mathrm{L}$ & 149.8900 & \\
\hline SPG-01 & Specific Gravity & 1.0274 & NA \\
\hline \multicolumn{4}{|c|}{ 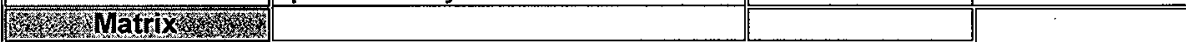 } \\
\hline \multicolumn{4}{|l|}{ LIQUID } \\
\hline \multicolumn{4}{|l|}{ Whampley } \\
\hline \multicolumn{4}{|l|}{ S96T005010 } \\
\hline 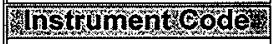 & \multirow{3}{*}{\multicolumn{3}{|c|}{$\begin{array}{l}\text { Gross Weight }(\mathrm{W} 2)=\mathrm{Wt} \text {. of vial + cap + cotton + solution } \\
\text { Tare Weight }(\mathrm{W} 1)=\mathrm{Wt} \text {. of vial + cap + cotton }\end{array}$}} \\
\hline & & & \\
\hline Alyst & & & \\
\hline \multicolumn{4}{|c|}{ DPB Specific Gravity $=[(\mathrm{W} 2-\mathrm{W} 1) * 1000 \mu \mathrm{L} / \mathrm{mL}] /[$ Vol. of Solution $\mu \mathrm{L} * 1.000 \mathrm{~g} / \mathrm{mL}]$} \\
\hline \multicolumn{4}{|c|}{ S20 } \\
\hline \multicolumn{4}{|c|}{ v RESULT $v$} \\
\hline \multirow{2}{*}{ 01:25 AM } & Specific Gravity = & 1.027 & \\
\hline & & & \\
\hline
\end{tabular}

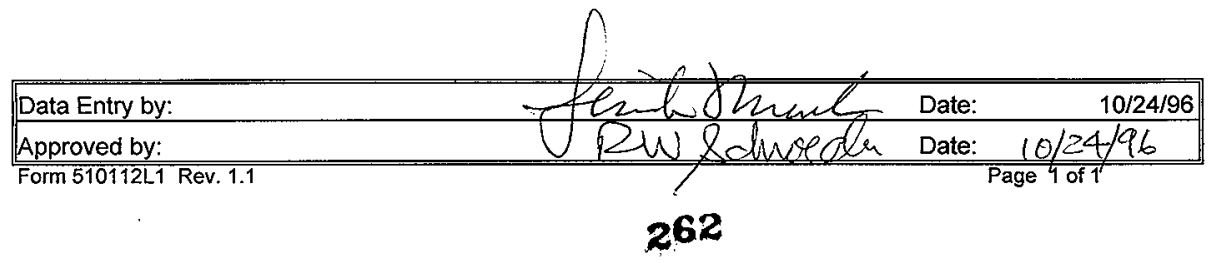




\section{SPECIFIC GRAVITY : LA-510-112 (C-3)}

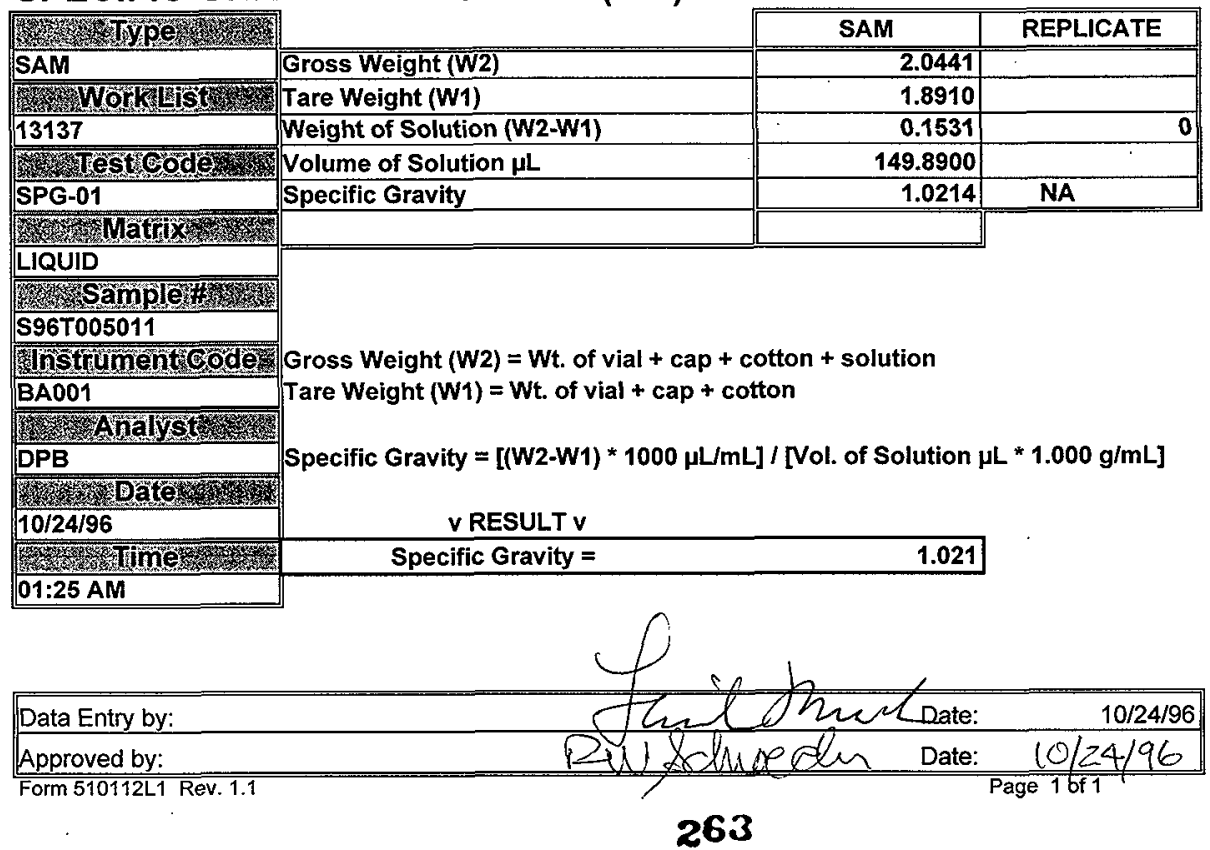




\section{SPECIFIC GRAVITY : LA-510-112 (C-3)}

\begin{tabular}{|c|c|c|c|}
\hline Hovery & & SAM-DUP & REPLICATE \\
\hline SAM-DUP & Gross Weight (W2) & 2.0339 & \\
\hline WWorJISt & Tare Weight (W1) & 1.8802 & \\
\hline 13137 & Weight of Solution (W2-W1) & 0.1537 & 0 \\
\hline Thestader & Volume of Solution $\mu L$ & 149.8900 & \\
\hline SPG-01 & Specific Gravity & 1.0254 & NA \\
\hline W & & & \\
\hline LIQUID & \multirow{6}{*}{\multicolumn{3}{|c|}{$\begin{array}{l}\text { Gross Weight }(W 2)=W t \text {. of vial + cap + cotton + solution } \\
\text { Tare Weight }(W 1)=W t \text {. of vial + cap + cotton }\end{array}$}} \\
\hline W & & & \\
\hline S96T005011 & & & \\
\hline 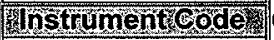 & & & \\
\hline BA001 & & & \\
\hline S- & & & \\
\hline DPB & \multirow{3}{*}{\multicolumn{3}{|c|}{ Specific Gravity $=[(\mathrm{W} 2-\mathrm{W} 1) * 1000 \mu \mathrm{L} / \mathrm{mL}] /[$ Vol. of Solution $\mu \mathrm{L} * 1.000 \mathrm{~g} / \mathrm{mL}]$}} \\
\hline (2) & & & \\
\hline $10 / 24 / 96$ & & & \\
\hline (2) & Specific Gravity = & 1.025 & \\
\hline 01:25 AM & & & \\
\hline
\end{tabular}

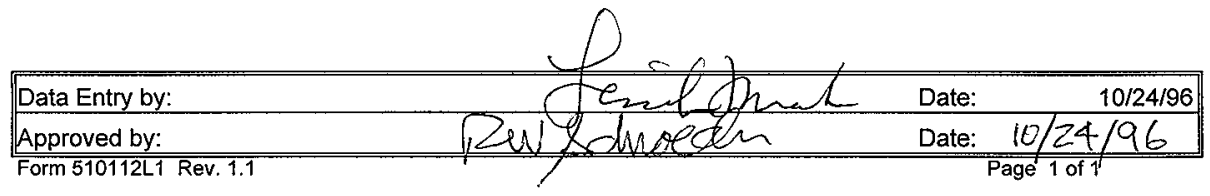


SPECIFIC GRAVITY : LA-510-112 (C-3)

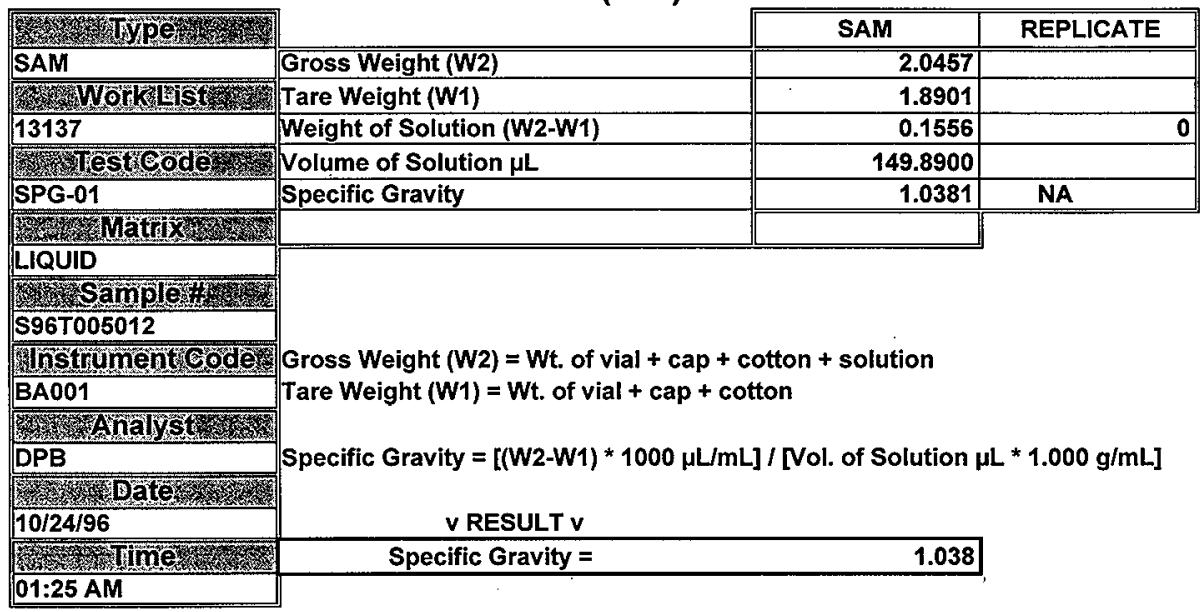

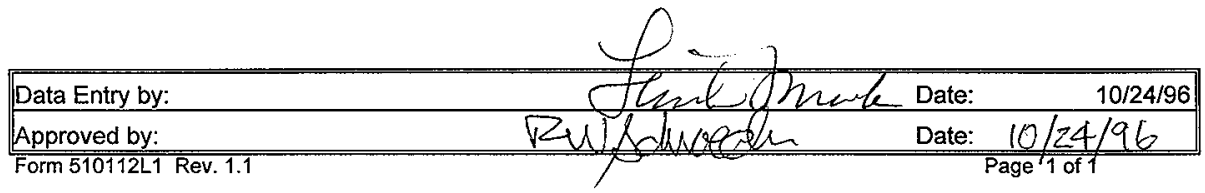


PLACE ANALYTICAL CARD IN BOX BELOW OR ATTACH TRAVELER

\section{SPECIFIC GRAVITY : LA-510-112 (C-3)}

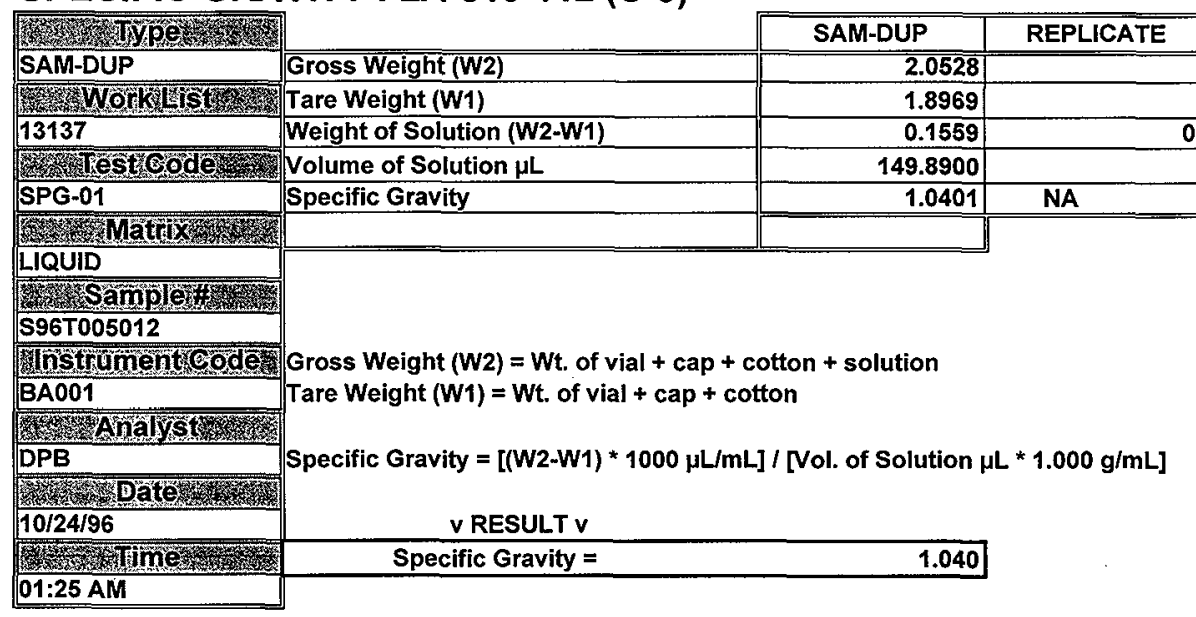

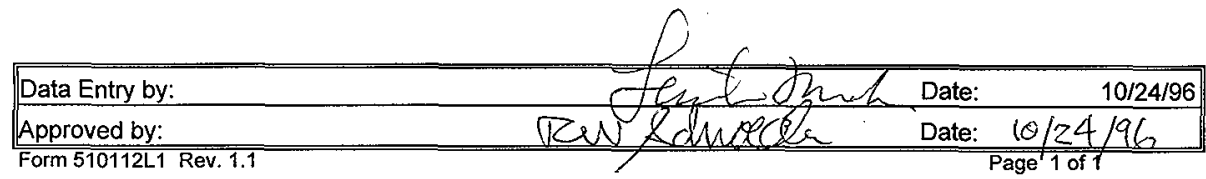




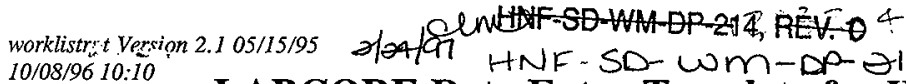
LABCORE Data Entry Template for Worklist\# 13781

Page: 1

1010819610.10

DPB Instrument: BA001

Analyst: $D P B$
Method: LA-510-112 Rev/Mod $\subset \sim 3$

Worklist Comment: AW-105 SPG. RCJ

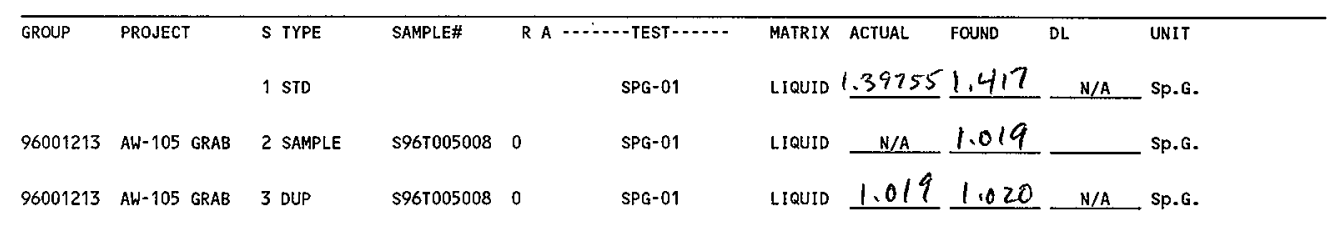

$\underbrace{}_{\text {Analyst Signature ponch }} 10 / 24 / 960515$

Final page for worklist \# 13781

Book $\# 33 \mathrm{~N} / 6-\mathrm{A}$

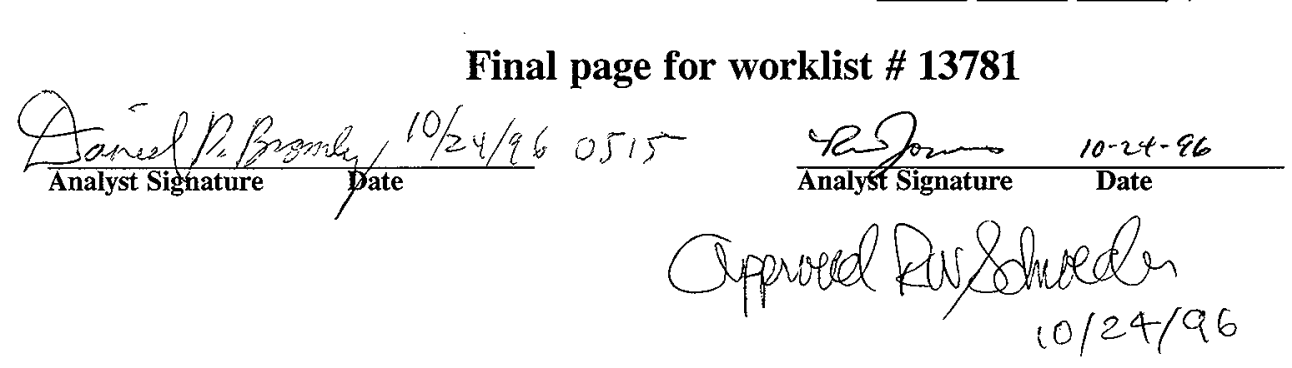

Data Entry Comments:

Units shown for QC (SPK \& STD) may not reflect the actual units. $D L=$ Detection Limit, $S=$ Worklist Slot Number, $R=$ Replicate Number, $A=$ Aliquot Code. 


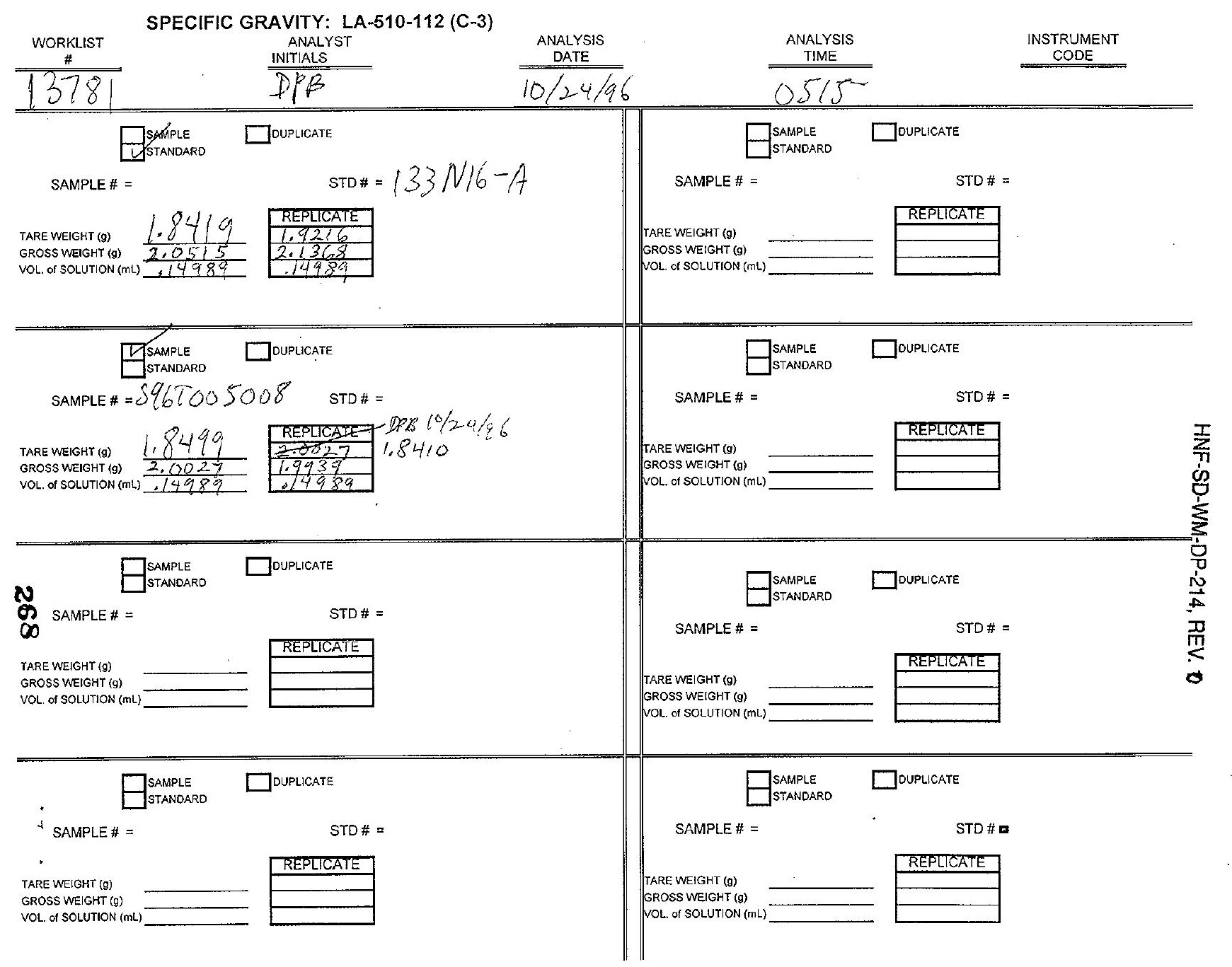




\section{SPECIFIC GRAVITY : LA-510-112 (C-3)}

\begin{tabular}{|c|c|c|c|}
\hline 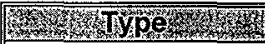 & & STANDARD & STANDARD \\
\hline STANDARD & Gross Weight (W2) & 2.0515 & 2.136 \\
\hline 3Worim & Tare Weight (W1) & 1.8419 & 1.921 \\
\hline 13781 & Weight of Solution (W2-W1) & 0.2096 & 0.215 \\
\hline Westreoders & Volume of Solution $\mu \mathrm{L}$ & 149.8900 & 149.890 \\
\hline SPG-01 & Specific Gravity & 1.3984 & 1.435 \\
\hline S- & Specific Gravity (Average) & $\mid 1.4170$ & \\
\hline LIQUID & \multirow{8}{*}{\multicolumn{3}{|c|}{$\begin{array}{l}\text { Gross Weight }(W 2)=\text { Wt. of vial }+ \text { cap }+ \text { cotton }+ \text { solution } \\
\text { Tare Weight }(W 1)=\text { Wt. of vial }+ \text { cap }+ \text { cotton } \\
\text { Specific Gravity }=[(W 2-W 1) * 1000 \mu L / m L] /[\text { Vol. of Solution } \mu \mathrm{L} * 1.000 \mathrm{~g} / \mathrm{mL}]\end{array}$}} \\
\hline Sampleztix & & & \\
\hline 133N16A & & & \\
\hline Instrumentcode & & & \\
\hline BA001 & & & \\
\hline Krivst & & & \\
\hline DPB & & & \\
\hline 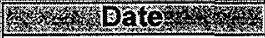 & & & \\
\hline $10 / 24 / 96$ & \multicolumn{2}{|l|}{ v RESULT v } & \\
\hline 20 & Specific Gravity Average = & 1.417 & \\
\hline 05:15 AM & & & \\
\hline
\end{tabular}

\begin{tabular}{|lll|}
\hline Data Entry by: & Date: & 10/24/96 \\
\hline Approved by: & Date: $10 / 24 / 96$ \\
\hline Form 510112L1 Rev.1.1
\end{tabular}




\section{SPECIFIC GRAVITY : LA-510-112 (C-3)}

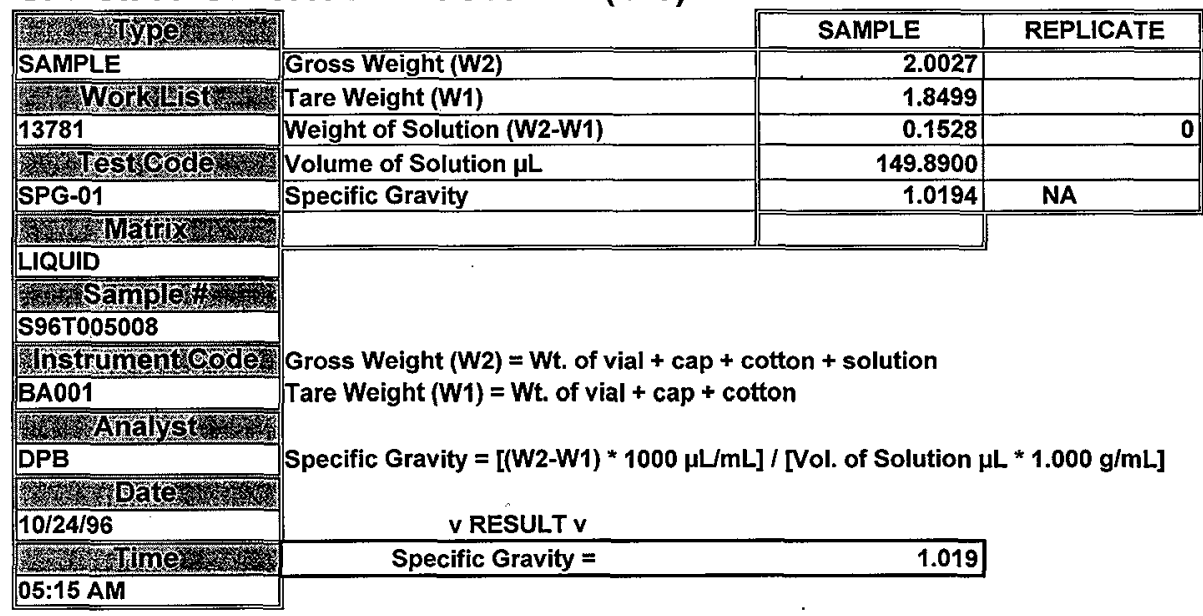

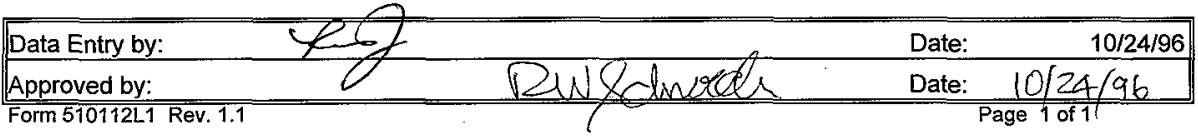




\section{SPECIFIC GRAVITY : LA-510-112 (C-3)}

\begin{tabular}{|c|c|c|c|}
\hline 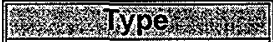 & & SAMPLE & REPLICATE \\
\hline SAMPLE & Gross Weight (W2) & 1.9939 & \\
\hline 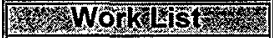 & Tare Weight (W1) & 1.8410 & \\
\hline 13781 & Weight of Solution (W2-W1) & 0.1529 & 0 \\
\hline 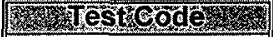 & Volume of Solution $\mu \mathrm{L}$ & 149.8900 & \\
\hline SPG-01 & Specific Gravity & 1.0201 & NA \\
\hline Wx & & & \\
\hline LIQUID & \multirow{9}{*}{\multicolumn{3}{|c|}{$\begin{array}{l}\text { Gross Weight }(W 2)=W t \text {. of vial }+ \text { cap }+ \text { cotton }+ \text { solution } \\
\text { Tare Weight }(W 1)=W t \text {. of vial }+ \text { cap }+ \text { cotton } \\
\text { Specific Gravity }=[(W 2-W 1) * 1000 \mu L / m L] /[\text { Vol. of Solution } \mu L * 1.000 \mathrm{~g} / \mathrm{mL}]\end{array}$}} \\
\hline 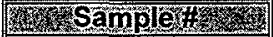 & & & \\
\hline S96T005008DUP & & & \\
\hline Instrumedeode & & & \\
\hline BA001 & & & \\
\hline 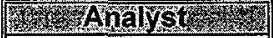 & & & \\
\hline DPB & & & \\
\hline S & & & \\
\hline $10 / 24 / 96$ & & & \\
\hline 36 & Specific Gravity = & 1.020 & \\
\hline 05:15 AM & & & \\
\hline
\end{tabular}

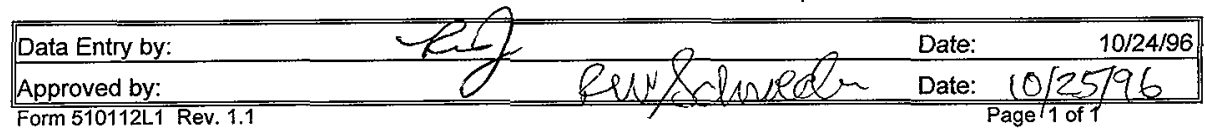




\section{LABCORE Data Entry Template for Worklist\# 14354}

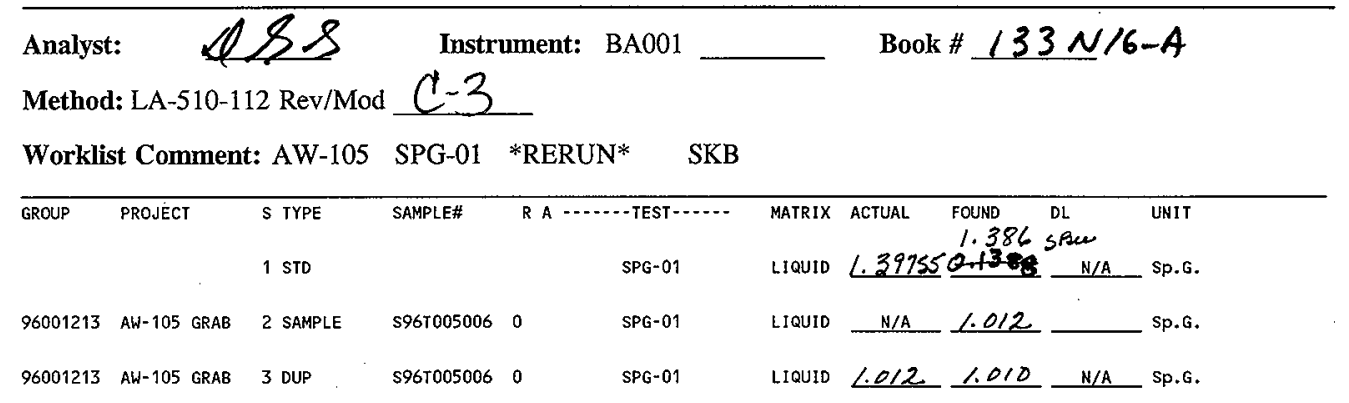

\section{Final page for worklist \# 14354}
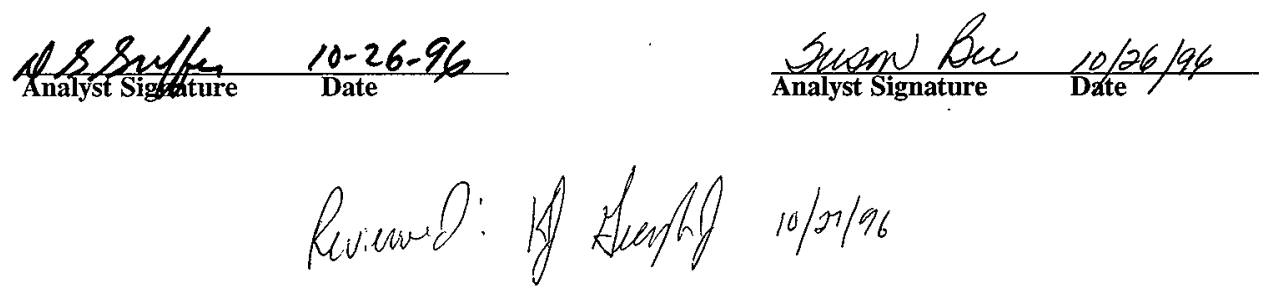

Data Entry Comments:

Units shown for $Q C$ (SPK \& STD) may not reflect the actual units. $D L=$ Detection Limit, $S=$ Worklist Slot Number,

$R=$ Replicate Number, $A=$ Aliquot Code. 
SPECIFIC GRAVITY : LA-510-112 (C-3)

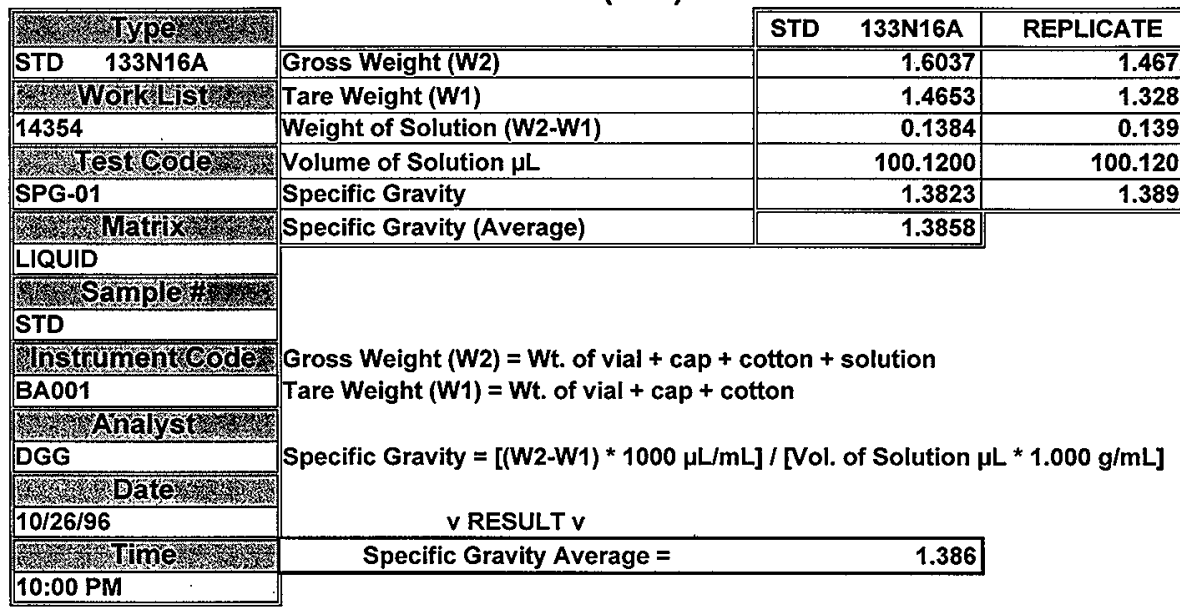

\begin{tabular}{|c|c|c|c|}
\hline Data Entry by: & & Date: & $10 / 26 / 96$ \\
\hline Approved by: & & Date: & $0 2 \longdiv { 1 2 / 9 7 }$ \\
\hline Form 510112L1 Rev. 1.1 & & & 1 of 1 \\
\hline 510112L1.WB1 & 10:04:12 PM & & 1 of 1 \\
\hline
\end{tabular}


PLACE ANALYTICAL CARD IN BOX BELOW OR ATTACH TRAVELER

\section{SPECIFIC GRAVITY : LA-510-112 (C-3)}

\begin{tabular}{|c|c|c|c|}
\hline 3y & & SAMPLE & REPLICATE \\
\hline SAMPLE & Gross Weight (W2) & 1.4504 & \\
\hline 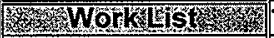 & Tare Weight (W1) & 1.3491 & \\
\hline 14354 & Weight of Solution (W2-W1) & 0.1013 & 0 \\
\hline 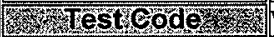 & Volume of Solution $\mu \mathrm{L}$ & 100.1200 & \\
\hline SPG-01 & Specific Gravity & 1.0118 & NA \\
\hline What & & & \\
\hline LIQUID & \multirow{6}{*}{\multicolumn{3}{|c|}{$\begin{array}{l}\text { Gross Weight }(W 2)=W t \text {. of vial + cap + cotton + solution } \\
\text { Tare Weight }(W 1)=W t \text {. of vial + cap + cotton }\end{array}$}} \\
\hline 2. 251 plo & & & \\
\hline S96T005006 & & & \\
\hline Instromougodo & & & \\
\hline BA001 & & & \\
\hline S6 & & & \\
\hline DGG & \multirow{3}{*}{\multicolumn{3}{|c|}{ Specific Gravity $=[(\mathrm{W} 2-\mathrm{W} 1) * 1000 \mu \mathrm{L} / \mathrm{mL}] /$ [Vol. of Solution $\mu \mathrm{L} . * 1.000 \mathrm{~g} / \mathrm{mL}]$}} \\
\hline 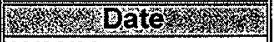 & & & \\
\hline $10 / 26 / 96$ & & & \\
\hline Syme & Specific Gravity $=$ & 1.012 & \\
\hline 10:00 PM & & & \\
\hline
\end{tabular}

Data Entry by:

Date:

$10 / 26 / 96$

Approved by:

Date: 
PLACE ANALYTICAL CARD IN BOX BELOW OR ATTACH TRAVELER

\section{SPECIFIC GRAVITY : LA-510-112 (C-3)}

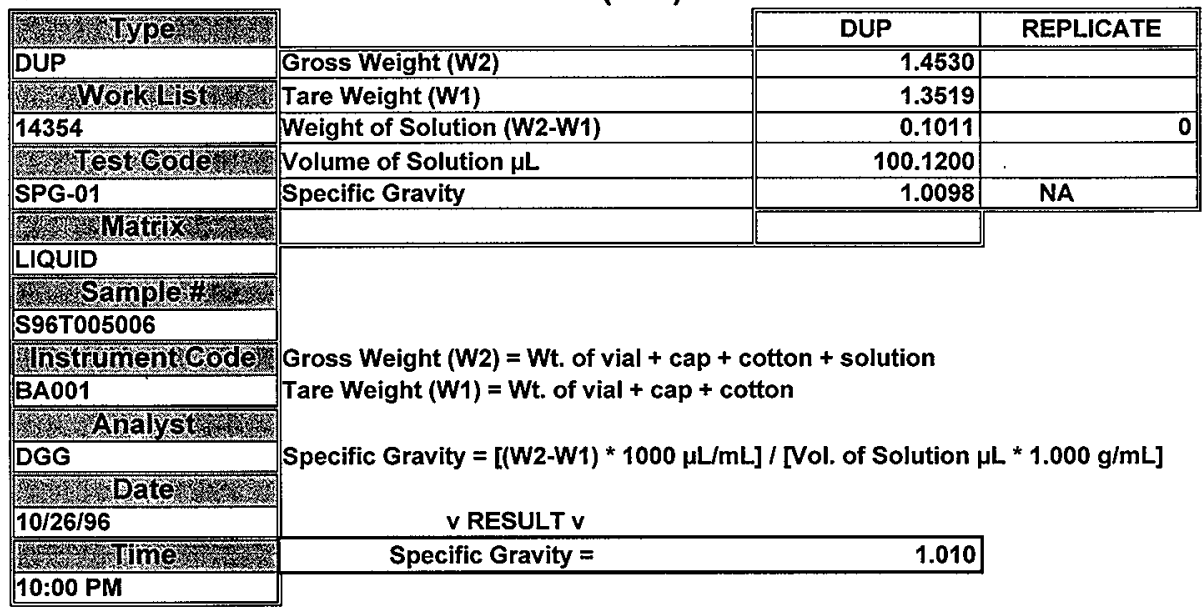

\begin{tabular}{|lll|}
\hline Data Entry by: & Date: & $10 / 26 / 96$ \\
\hline Approved by: & Date: & \\
\hline
\end{tabular}




\section{LABCORE Data Entry Template for Worklist\# 13063}

\section{Analyst: JLF Instrument: PH01_ Book \# 18 N19B}

Method: LA-212-106 Rev/Mod B-O

Worklist Comment: AW-105 PH. RCJ

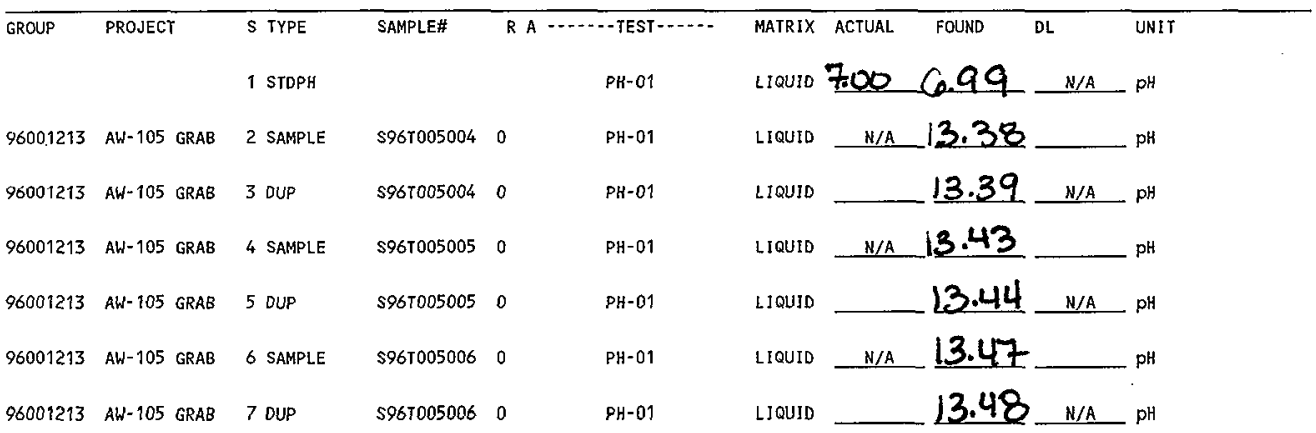

\section{Final page for worklist \# 13063}

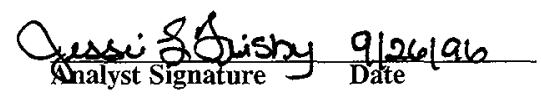

vatidated into Lalcore by
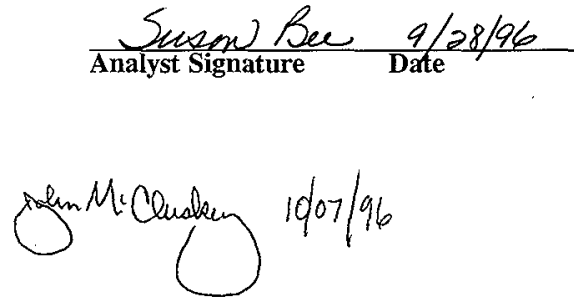

Data Entry Comments:

Units shown for $Q C$ (SPK \& STD) may not reflect the actual units. $D L=$ Detection Limit, $S=$ Worklist Slot Number, $R=$ Replicate Number, $A=$ Aliquot Code. 
Analyst: $\quad \Omega F$

Instrument: $\mathrm{PHO1}$

Book \#18N19B

Method: LA-212-106 Rev/Mod B-O

Worklist Comment: AW-105 PH. RCJ

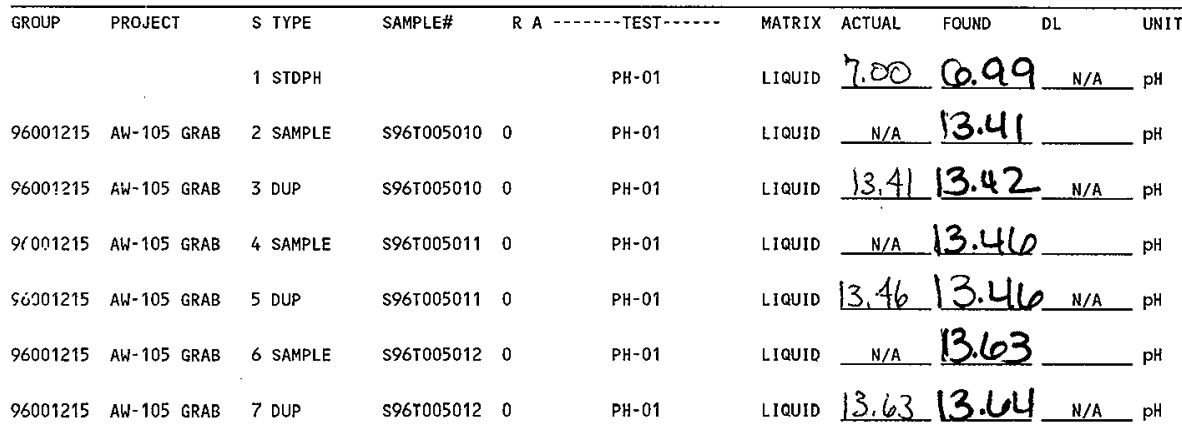

Final page for worklist \# 13204

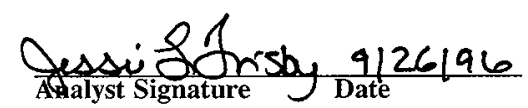

Aqalyst Signature oate

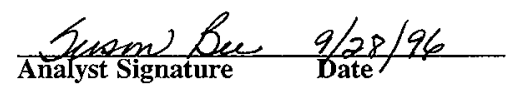

warticed into Liburese by

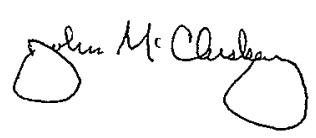

$10 / 07 / 20$

Data Entry Comments:

Units shown for QC (SPK \& STD) may not reflect the actual units. $D L=$ Detection Limit, $S=$ Worklist Slot Number,

$R=$ Replicate Number, $A=$ Aliquot Code. 
Analyst:

Aneter

Instrument: $\mathrm{PH} 01$

Book $\# 18 N 19-13$

Page: $\quad 1$

Method: LAf $12-106 \mathrm{Rev} / \mathrm{Mod}$ B-C

Worklist Comment: AW-105 PH DIRECT. RCJ

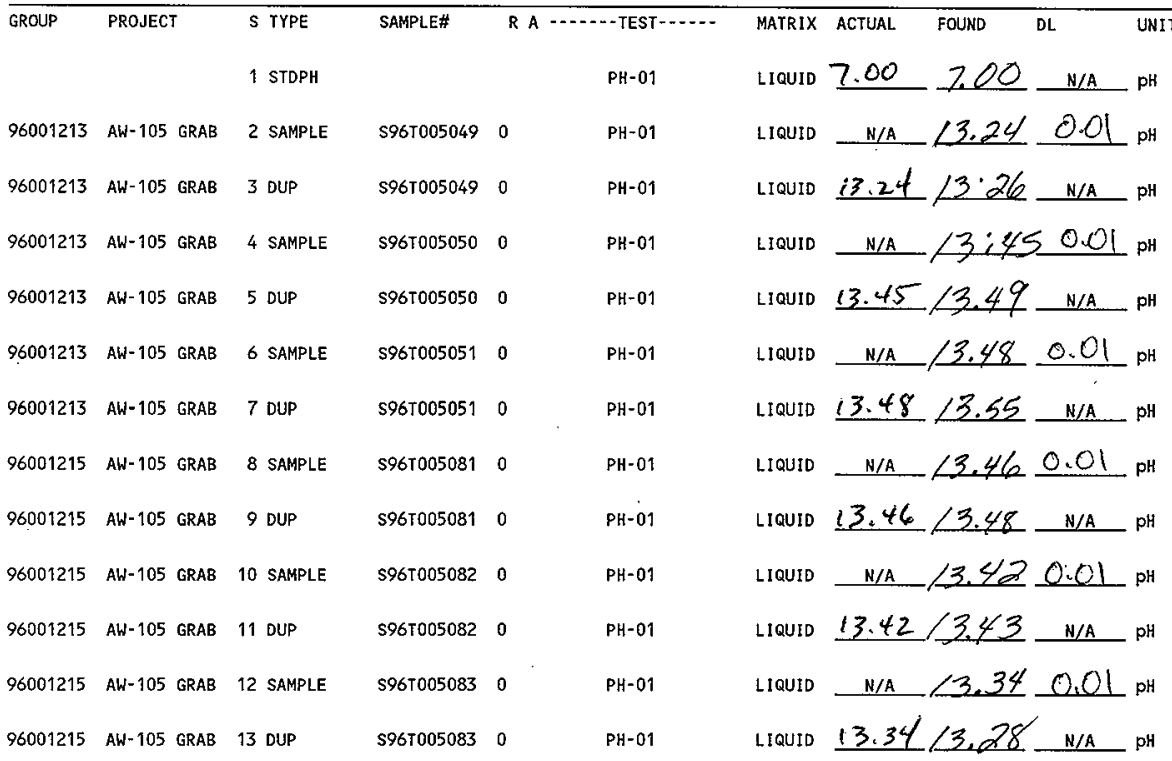

Final page for worklist \# 13844

Analyst Siguature
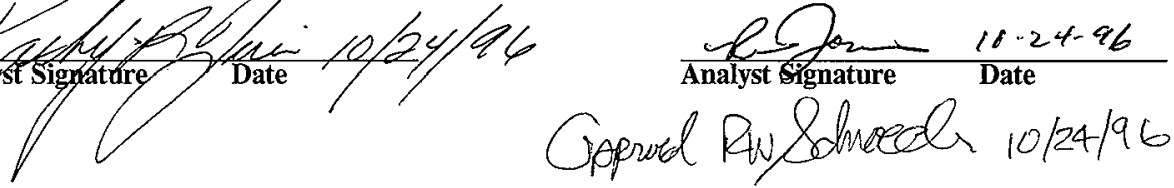

Data Entry Comments:

Units shown for $Q C$ (SPK \& STD) may not reflect the actual units. $D L=$ Detection Limit, $S=$ Worklist Slot Number,

$R=$ Replicate Number, $A=$ Aliquot Code. 


\section{LABCORE Data Entry Template for Worklist\# 13854}

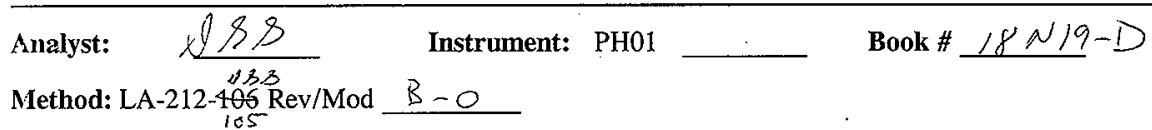

Worklist Comment: AW-105 PH-02 PER PROCEDURE. RCJ

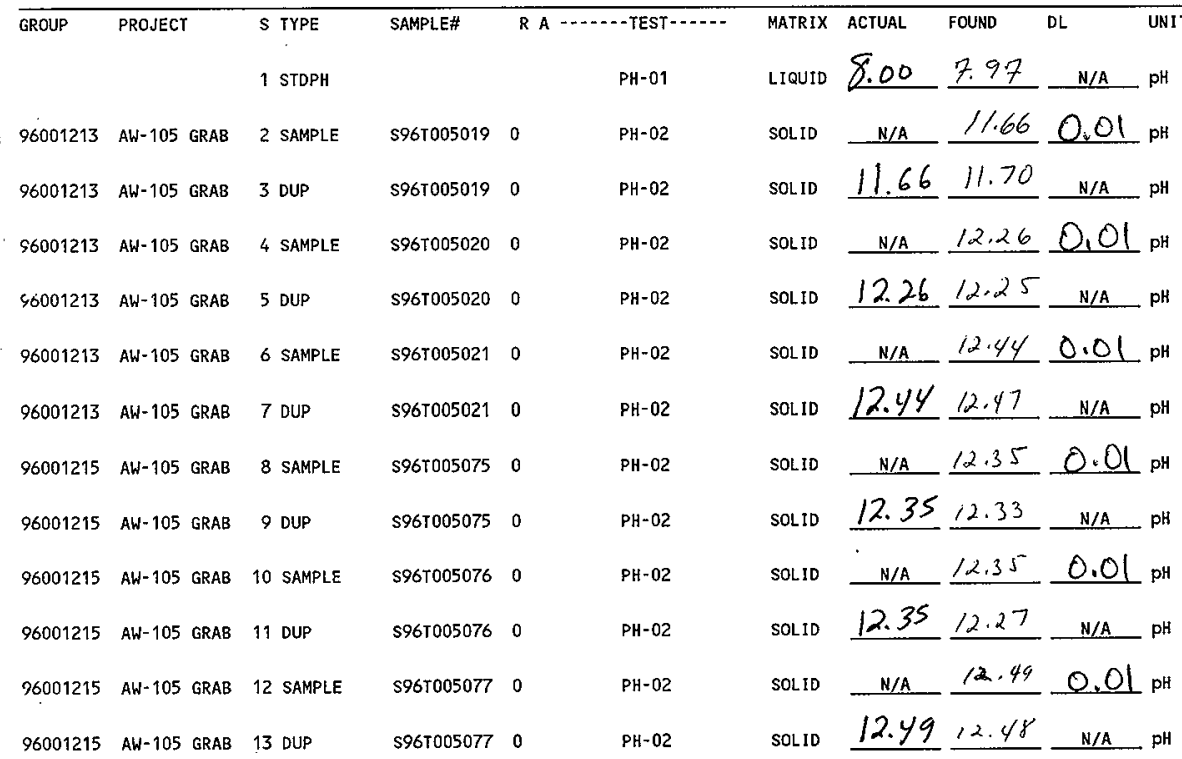

Final page for worklist \# 13854

N 8 Suthn $10-26-96$

Analyst Sighature Date

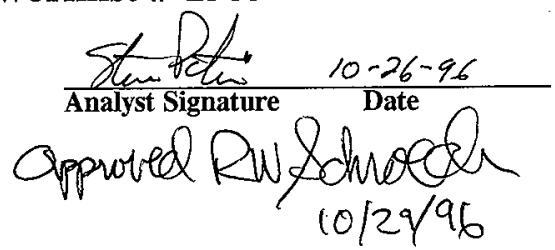

Data Entry Comments:

Units shown for $Q C$ (SPK \& STD) may not reflect the actual units. $D L=$ Detection Limit, $S=$ Worklist Slot Number, $R=$ Replicate Number, $A=$ Aliquot Code. 
worklistrpt Version $2.105 / 15 / 9.5$

HNF-SD-WM-DP-214, REV. 0

Page: $\quad I$

$09 / 18 / 9610: 50$

- LABCORE Data Entry Template for Worklist\# 13062

Analyst: RY S Instrument: PH01 Book \# 79N8

Method: LA-211-102 Rev/Mod C-O

Worklist Comment: AW-105 OH. RCJ

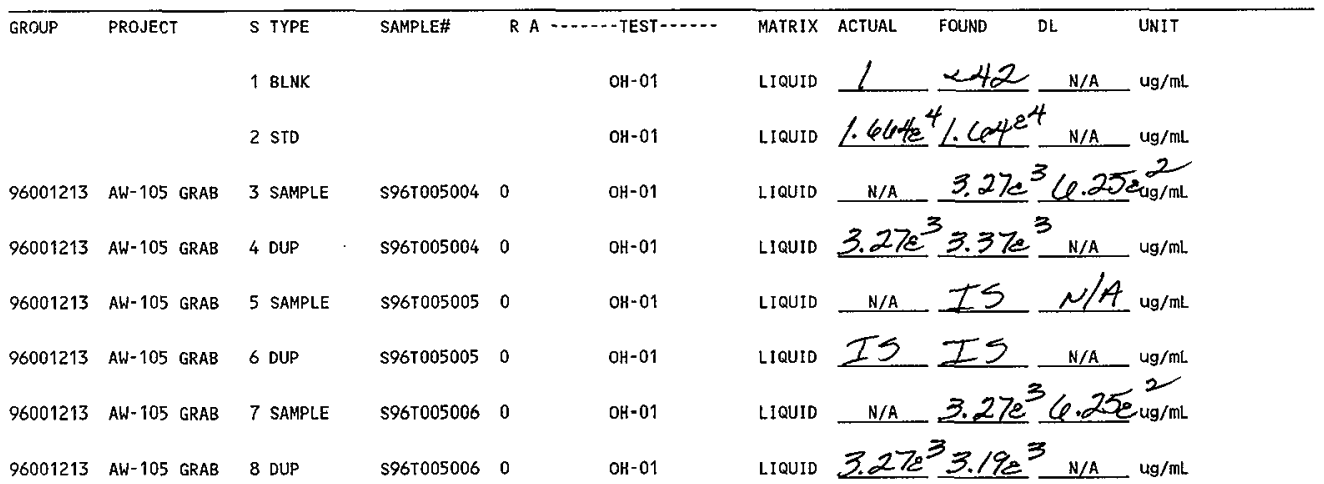

Final page for worklist \# 13062

Rae Ann Sheen 9.29.96

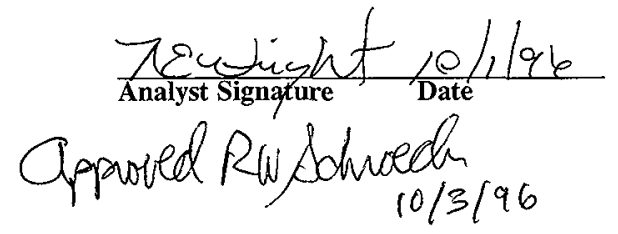

Analyst Signature

Date

Data Entry Comments: Sample $596 T 5005$ - insufficient sample contain es found empty.

Units shown for QC (SPK \& STD) may not reflect the actual units. $D L=$ Detection Limit, $S=$ Worklist Slot Number, $R=$ Replicate Number $A=$ Aliquot Code.

281 


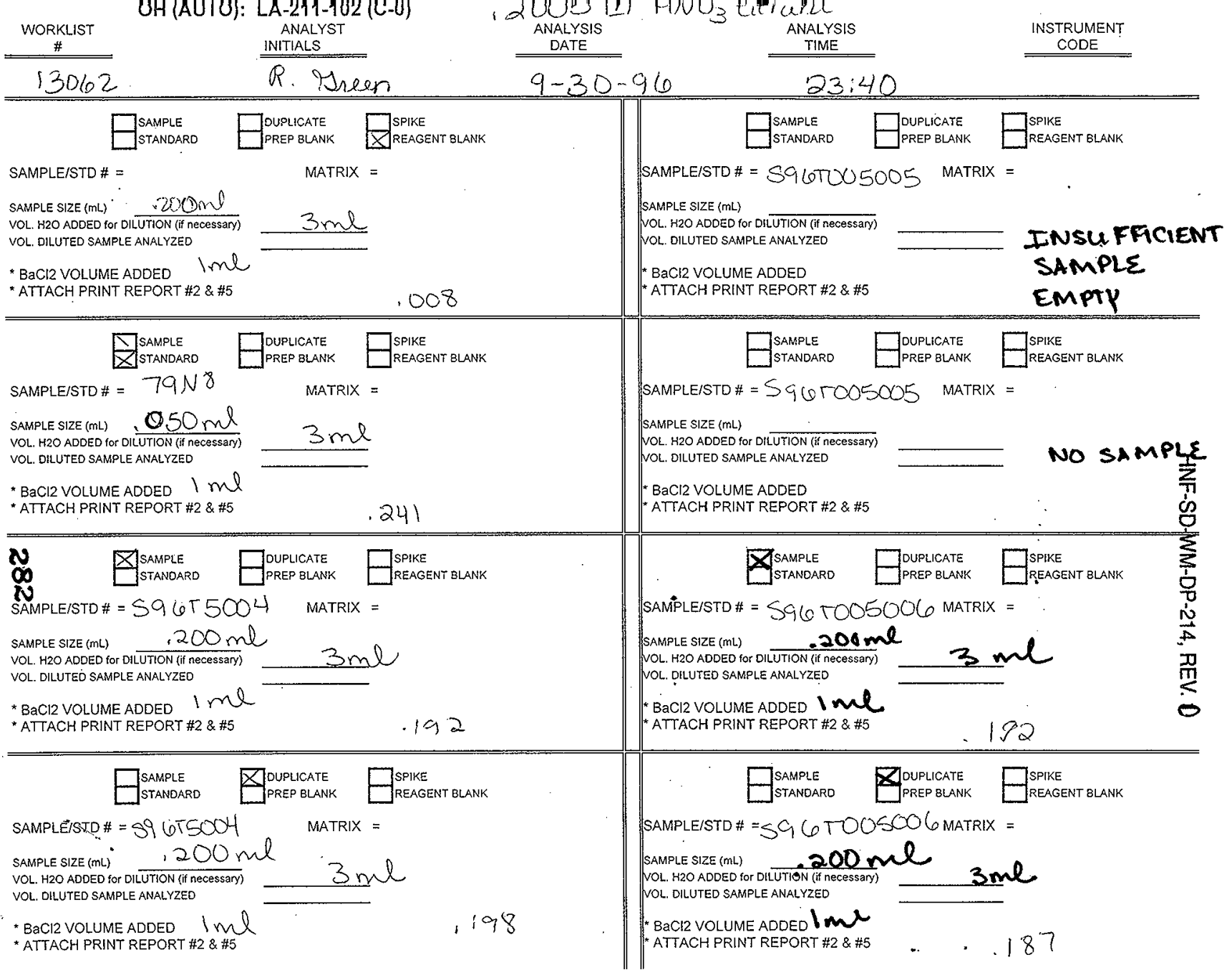




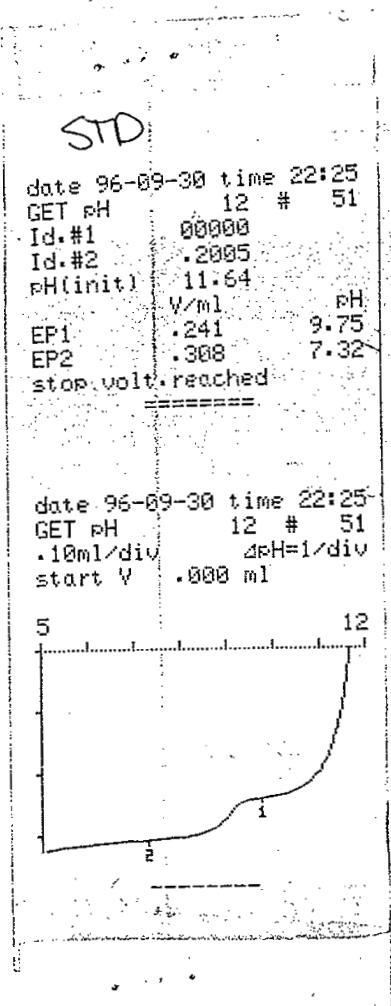

\section{Reagent Blank}

date $96-69-39$ tine 2213

GET H 12 \# 49

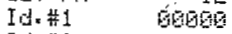

If.

phlinit) 4.24

$\begin{array}{lll} & 4 \mathrm{ml} & \mathrm{FH} \\ \text { EF1 } & .609 & 3.73\end{array}$

stop yolt. reoched

$= \pm== \pm= \pm=$

dote 95-99-30 time 22:13

DET FH I2 \# 49

-10mldiy $\triangle F=1$ diu start $y$. $600 \mathrm{in}$

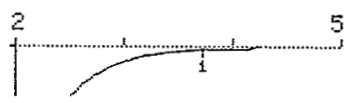

\section{OH ANALYSIS}

HNF-SD-WM-DP-214, REV, 0

5967005004

dote $95-69-39$ time $22: 35$

GET $\mathrm{BH}$ 12 \# 53

1d. \#1, $\quad 5094$

Id. $42 .+2605$

PHlint? 11.54

से 4 m

EF

$\mathrm{EF} 2,2 \mathrm{2OQ}$

stopolt reached

5.28

$====$

5967005006

date $96-69-30+1 n e 232$

GET HH $12^{\text {\# }} 58$

Id.\#1 . 5066

Id. \#2 . 2005

OHCinit . 11.51

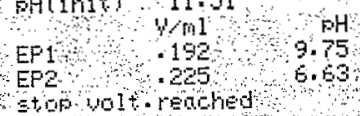

dote $96-09-30$ time 22:30

GET FH 12 \# 53

. IGmldiv $\triangle P H=1>0$

start $\quad .860 \mathrm{ml}$

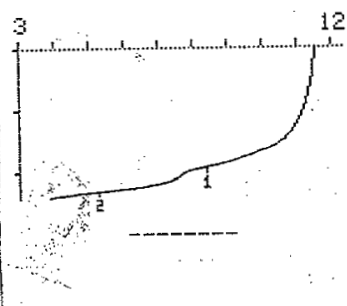

\section{S96J005004 Dup}

iote $98-89-39$ time $22: 43$

GET $\mathrm{HH} \quad \mathrm{IZ}$ \#

Id. मी $1 \quad 5004$

Id.

FH(init) $11.4 E$

$\begin{array}{lll} & Y \mathrm{~mL} & \mathrm{EH} \\ \text { EF1 } & 195 & 7.74\end{array}$

stop wol t. reoched

$== \pm== \pm=$

dote $96-69-30$ tine $22: 49$

GET FH 12 \# 54 - 1 anldir $\mathrm{ArH}=1 / \mathrm{di}$ stort $y$. 600 in

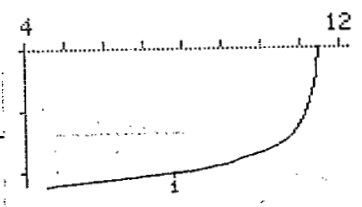

date $96-89-90$ t ime 2342

- $10 \mathrm{ml}$ div

$\Delta s H=1$ - $d i v$

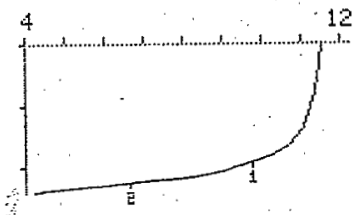

$\because$

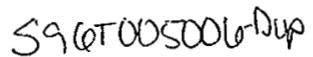

GET FH

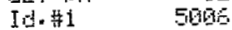

Id. +2 - 268

FH(init) 11.48

Wril

EF1 $\quad .187 \quad 10.01$

EF2 .225 6.81

stop yolt = renchoj

=ニะニ=ニニ=

dute $96-89-30$ t ime $23: 27$ GET 12 \# 59 star. $\psi^{\circ} .80 \mathrm{~m} 1$

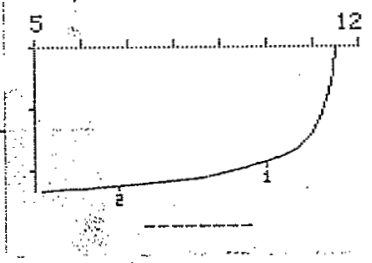

FET FH 12 स 50

stort $y$. $060 \mathrm{ml}$

date $56-59-30$ time $23: 27$ -10iriod 


\begin{tabular}{|c|c|c|}
\hline \multicolumn{2}{|c|}{ OH (AUTO) : LA-211-102 (C-0) } & BLANK \\
\hline 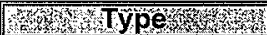 & Sample Size $(\mathrm{mL})$ SS & 3.000 \\
\hline BLANK & Concentration of $\mathrm{HNO} 3$ (Molarity) & 0.2005 \\
\hline Wonk Kist & HNO3 Titrant at $\mathrm{OH}$ end-point in $\mathrm{mL}$ & 0.008 \\
\hline 13062 & Dilution Factor DF & 1 \\
\hline 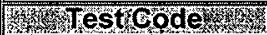 & Concentration of $\mathrm{OH}$ in Sample (Molarity) & $5.35 \mathrm{E}-04$ \\
\hline $\mathrm{OH}-01$ & $\mathrm{OH}$ in Sample in $\mu \mathrm{g} / \mathrm{mL}$ (PPM) & $9.09 \mathrm{E}+00$ \\
\hline
\end{tabular}

KX

LIQUID

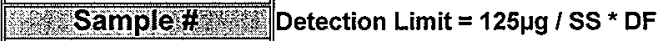

REAGENT BLANK

\begin{tabular}{|l|l|l|}
\hline Instrument Code & Detection Limit $(\mu \mathrm{g} / \mathrm{mL})$ & $4.17 \mathrm{E}+01$ \\
\hline
\end{tabular}

PH01

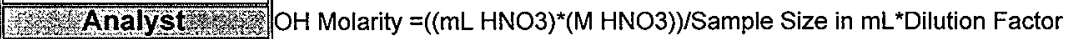

RA GREEN

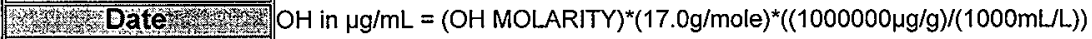

$09 / 30 / 96$

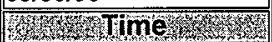

10:13 PM

\begin{tabular}{|l|r|}
\cline { 2 - 2 } \multicolumn{1}{c|}{} & \multicolumn{1}{c|}{ BLANK } \\
\hline Concentration of OH in Sample (Molarity) & $5.35 E-04$ \\
\hline OH in Sample in $\mu \mathrm{g} / \mathrm{mL}$ (PPM) & $<42$ \\
\hline
\end{tabular}

The Result is < Detection Limit

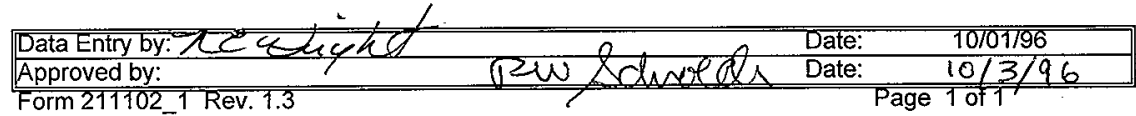

Form 211702_1 Rev. 1.3 


\begin{tabular}{|c|c|c|}
\hline \multirow{2}{*}{\multicolumn{2}{|c|}{ OH (AUTO) : LA-211-102 (C-0) }} & \multirow[b]{2}{*}{ STANDARD } \\
\hline & & \\
\hline MWY & Sample Size $(\mathrm{mL})$ SS & 0.050 \\
\hline STANDARD & Concentration of $\mathrm{HNO} 3$ (Molarity) & 0.2005 \\
\hline Work List & HNO3 Titrant at $\mathrm{OH}$ end-point in $\mathrm{mL}$ & 0.241 \\
\hline 13062 & Dilution Factor DF & 1 \\
\hline G TestCode - & Concentration of $\mathrm{OH}$ in Sample (Molarity) & $9.66 \mathrm{E}-01$ \\
\hline $\mathrm{OH}-01$ & $\mathrm{OH}$ in Sample in $\mu \mathrm{g} / \mathrm{m} \mathrm{L}$ (PPM) & $1.64 \mathrm{E}+04$ \\
\hline \multicolumn{3}{|c|}{ 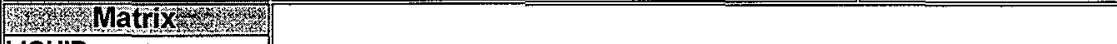 } \\
\hline \multicolumn{3}{|l|}{ LIQUID } \\
\hline \multicolumn{3}{|l|}{ Samplet } \\
\hline \multicolumn{3}{|l|}{ 79N8 } \\
\hline \multicolumn{3}{|l|}{ Instrumentcode } \\
\hline \multicolumn{3}{|l|}{$\mathrm{PHO1}$} \\
\hline Analyst this & \multirow{4}{*}{\multicolumn{2}{|c|}{$\begin{array}{l}\text { OH Molarity }=\left((\mathrm{mL} H N O 3)^{*}(\mathrm{M} \mathrm{HNO} 3)\right) / \text { Sample Size in } \mathrm{mL} \text { Dilution Factor } \\
\mathrm{OH} \text { in } \mu \mathrm{g} / \mathrm{mL}=(\mathrm{OH} \text { MOLARITY })^{*}(17.0 \mathrm{~g} / \mathrm{mole})^{*}((1000000 \mu \mathrm{g} / \mathrm{g}) /(1000 \mathrm{~mL} / \mathrm{L}))\end{array}$}} \\
\hline RA GREEN & & \\
\hline 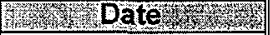 & & \\
\hline $09 / 30 / 96$ & & \\
\hline W & & STANDARD \\
\hline \multirow[t]{2}{*}{ 10:13 PM } & Concentration of $\mathrm{OH}$ in Sample (Molarity) & $9.66 \mathrm{E}-01$ \\
\hline & $\mathrm{OH}$ in Sample in $\mu \mathrm{g} / \mathrm{mL}$ (PPM) & $1.64 \mathrm{E}+04$ \\
\hline
\end{tabular}

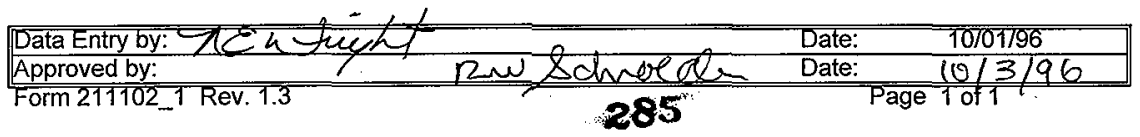




\begin{tabular}{|c|c|c|}
\hline \multicolumn{2}{|c|}{ OH (AUTO) : LA-211-102 (C-0) } & SAMPLE \\
\hline 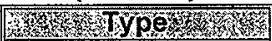 & Sample Size $(\mathrm{mL})$ SS & 0.200 \\
\hline SAMPLE & Concentration of $\mathrm{HNO} 3$ (Molarity) & 0.2005 \\
\hline Work EIst t & HNO3 Titrant at $\mathrm{OH}$ end-point in $\mathrm{mL}$ & 0.192 \\
\hline 13062 & Dilution Factor DF & 1 \\
\hline 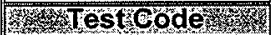 & Concentration of $\mathrm{OH}$ in Sample (Molarity) & $1.92 \mathrm{E}-01$ \\
\hline $\mathrm{OH}-01$ & OH in Sample in $\mu \mathrm{g} / \mathrm{mL}$ (PPM) & 3.27E+03 \\
\hline \multirow{2}{*}{$\begin{array}{l}\text { SIQUID } \\
\text { LITatrix }\end{array}$} & \multirow{4}{*}{ Detection Limit $=125 \mu \mathrm{g} / \mathrm{SS}$ * DF } & \\
\hline & & \\
\hline Samplet & & \\
\hline S96T005004 & & \\
\hline Instrument code & Detection Limit $(\mu \mathrm{g} / \mathrm{mL})$ & $6.25 \mathrm{E}+02$ \\
\hline $\mathrm{PHO1}$ & \multirow{3}{*}{\multicolumn{2}{|c|}{$\mathrm{OH}$ Molarity $=\left((\mathrm{mL} . \mathrm{HNO} 3)^{\star}(\mathrm{M} \mathrm{HNO})\right) /$ Sample Size in $\mathrm{mL}^{\star}$ Dilution Factor }} \\
\hline SAnalyst & & \\
\hline RA GREEN & & \\
\hline 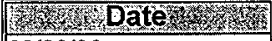 & \multirow{2}{*}{\multicolumn{2}{|c|}{ 实 $\mathrm{OH}$ in $\mu \mathrm{g} / \mathrm{mL}=(\mathrm{OH} \text { MOLARITY })^{\star}(17.0 \mathrm{~g} / \mathrm{mole})^{*}((1000000 \mathrm{~g} / \mathrm{g}) /(1000 \mathrm{~mL} / \mathrm{L}))$}} \\
\hline $09 / 30 / 96$ & & \\
\hline \multirow{2}{*}{$\begin{array}{l}\text { 10:13 PM } \\
10 \text { P }\end{array}$} & & SAMPLE \\
\hline & Concentration of $\mathrm{OH}$ in Sample (Molarity) & $1.92 \mathrm{E}-01$ \\
\hline & $\mathrm{OH}$ in Sample in $\mu \mathrm{g} / \mathrm{mL}$ (PPM) & $3.27 \mathrm{E}+03$ \\
\hline
\end{tabular}

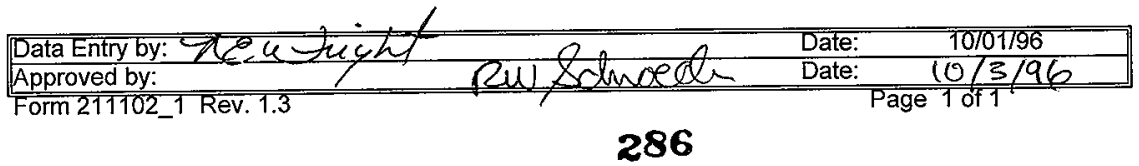




\begin{tabular}{|c|c|c|}
\hline \multicolumn{2}{|c|}{ OH (AUTO) : LA-211-102 (C-0) } & DUPLICATE \\
\hline 7rype & Sample Size $(\mathrm{mL})$ SS & 0.200 \\
\hline DUPLICATE & Concentration of HNO3 (Molarity) & 0.2005 \\
\hline Work ISts & HNO3 Titrant at $\mathrm{OH}$ end-point in $\mathrm{mL}$ & 0.198 \\
\hline 13062 & Dilution Factor DF & 1 \\
\hline TestCode & Concentration of $\mathrm{OH}$ in Sample (Molarity) & $1.98 \mathrm{E}-01$ \\
\hline $\mathrm{OH}-01$ & $\mathrm{OH}$ in Sample in $\mu \mathrm{g} / \mathrm{mL}$ (PPM) & $3.37 E+03$ \\
\hline \multirow{2}{*}{$\begin{array}{l}\text { LIQUID } \\
\text { LIQUIT) }\end{array}$} & \multirow{4}{*}{ Detection Limit $=125 \mu \mathrm{g} / \mathrm{SS}$ * DF } & \\
\hline & & \\
\hline Wample & & \\
\hline S96T005004 & & \\
\hline Instrument code & Detection Limit $(\mu \mathrm{g} / \mathrm{mL})$ & $6.25 \mathrm{E}+02$ \\
\hline PH01 & \multirow{5}{*}{\multicolumn{2}{|c|}{$\begin{array}{l}\mathrm{OH} \text { Molarity }=\left((\mathrm{mL} H N O 3)^{*}(\mathrm{M} \text { HNO3) }) / \text { Sample Size in } \mathrm{mL}^{*} \text { Dilution Factor }\right. \\
\mathrm{OH} \text { in } \mu \mathrm{g} / \mathrm{mL}=(\mathrm{OH} \text { MOLARITY })^{\star}(17.0 \mathrm{~g} / \mathrm{mole})^{*}((1000000 \mu \mathrm{g} / \mathrm{g}) /(1000 \mathrm{~mL} / \mathrm{L}))\end{array}$}} \\
\hline Analyst t & & \\
\hline RA GREEN & & \\
\hline 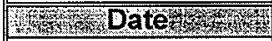 & & \\
\hline \multirow{2}{*}{0} & & \\
\hline & & DUPLICATE \\
\hline \multirow[t]{2}{*}{ 10:13 PM } & Concentration of OH in Sample (Molarity) & $1.98 \mathrm{E}-01$ \\
\hline & $\mathrm{OH}$ in Sample in $\mu \mathrm{g} / \mathrm{mL}$ (PPM) & $3.37 E+03$ \\
\hline
\end{tabular}

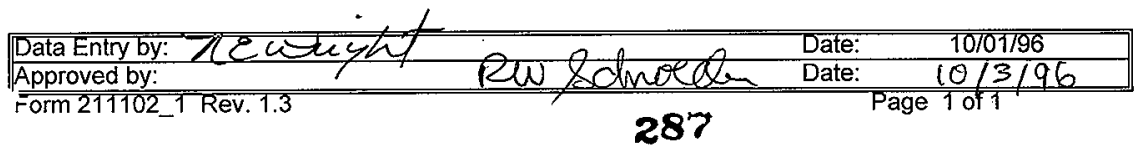


HNF-SD-WM-DP-214, REV. 0

PLACE ANALYTICAL CARD IN BOX BELOW OR ATTACH TRAVELER

\begin{tabular}{|c|c|c|}
\hline \multicolumn{2}{|c|}{ OH (AUTO) : LA-211-102 (C-0) } & SAMPLE \\
\hline 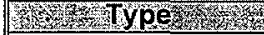 & Sample Size (mL) SS & 0.200 \\
\hline SAMPLE & Concentration of $\mathrm{HNO} 3$ (Molarity) & 0.2005 \\
\hline Work & HNO3 Titrant at $\mathrm{OH}$ end-point in $\mathrm{mL}$ & 0.192 \\
\hline 13062 & Dilution Factor DF & \\
\hline Testerde & Concentration of $\mathrm{OH}$ in Sample (Molarity) & $1.92 \mathrm{E}-01$ \\
\hline $\mathrm{OH}-01$ & $\mathrm{OH}$ in Sample in $\mu \mathrm{g} / \mathrm{mL}$ (PPM) & $3.27 \mathrm{E}+03$ \\
\hline
\end{tabular}

\%, Matrix

LIQUID

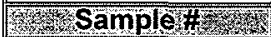

S96T005006

Instrument Code

PH01

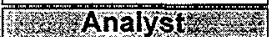

RA GREEN

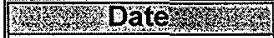

09/30/96

1.5.

10:13 PM

Detection Limit $=125 \mu \mathrm{g} / \mathrm{SS}$ * DF

Detection Limit $(\mu \mathrm{g} / \mathrm{mL})$

$6.25 E+02$

$\mathrm{OH}$ Molarity $=\left((\mathrm{mL} H N O 3)^{\star}(\mathrm{M} \mathrm{HNO})\right) /$ Sample Size in $\mathrm{mL}$ * Dilution Factor

$\mathrm{OH}$ in $\mu \mathrm{g} / \mathrm{mL}=(\mathrm{OH} \text { MOLARITY })^{*}(17.0 \mathrm{~g} / \mathrm{mole})^{*}((1000000 \mu \mathrm{g} / \mathrm{g}) /(1000 \mathrm{~mL} / \mathrm{L}))$

\begin{tabular}{|l|r|}
\cline { 2 - 2 } \multicolumn{1}{c|}{} & \multicolumn{1}{c|}{ SAMPLE } \\
\hline Concentration of OH in Sample (Molarity) & $1.92 E-01$ \\
\hline OH in Sample in $\mu \mathrm{g} / \mathrm{mL}$ (PPM) & $3.27 \mathrm{E}+03$ \\
\hline
\end{tabular}

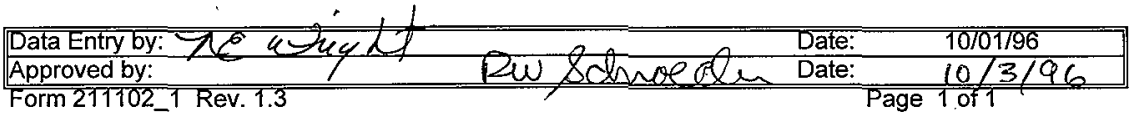




\begin{tabular}{|c|c|c|}
\hline \multirow{2}{*}{\multicolumn{2}{|c|}{ OH (AUTO) : LA-211-102 (C-0) }} & \\
\hline & & DUPLICATE \\
\hline S & Sample Size $(\mathrm{mL}) \mathrm{SS}$ & 0.200 \\
\hline DUPLICATE & Concentration of $\mathrm{HNO}_{3}$ (Molarity) & 0.2005 \\
\hline WorkList & HNO3 Titrant at $\mathrm{OH}$ end-point in $\mathrm{mL}$ & 0.187 \\
\hline 13062 & Dilution Factor DF & \\
\hline Testeode & Concentration of OH in Sample (Molarity) & 1.87E-01 \\
\hline $\mathrm{OH}-01$ & $\mathrm{OH}$ in Sample in $\mu \mathrm{g} / \mathrm{mL}$ (PPM) & $3.19 \mathrm{E}+03$ \\
\hline
\end{tabular}

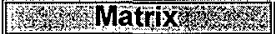

LIQUID

W Sample

S96T005006

Instrumenteode

$6.25 E+02$

PH01

7.

RA GREEN

Wh

$09 / 30 / 96$

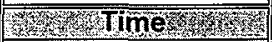

10:13 PM

$\mathrm{OH}$ Molarity $=\left((\mathrm{mL} H \mathrm{NO} 3)^{*}(\mathrm{M} H \mathrm{HO} 3)\right) /$ Sample Size in $\mathrm{mL}^{*}$ Dilution Factor

\begin{tabular}{|l|c|}
\cline { 2 - 2 } \multicolumn{1}{c|}{} & DUPLICATE \\
\hline Concentration of $\mathrm{OH}$ in Sample (Molarity) & $1.87 \mathrm{E}-01$ \\
\hline $\mathrm{OH}$ in Sample in $\mu \mathrm{g} / \mathrm{mL}$ (PPM) & $3.19 \mathrm{E}+03$ \\
\hline
\end{tabular}

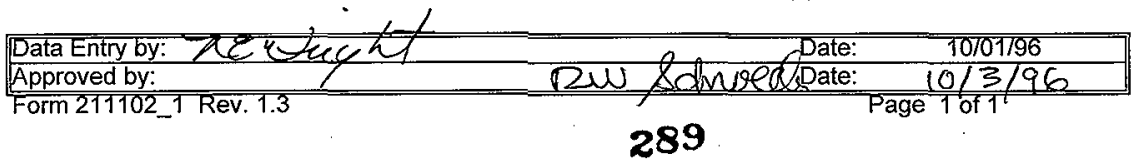




\section{LABCORE Data Entry Template for Worklist\# 13203}

Analyst: QS Instrument: PH01 Book\# 79N8

Method: LA-211-102 Rev/Mod C-O

Worklist Comment: AW-105 OH. RCJ

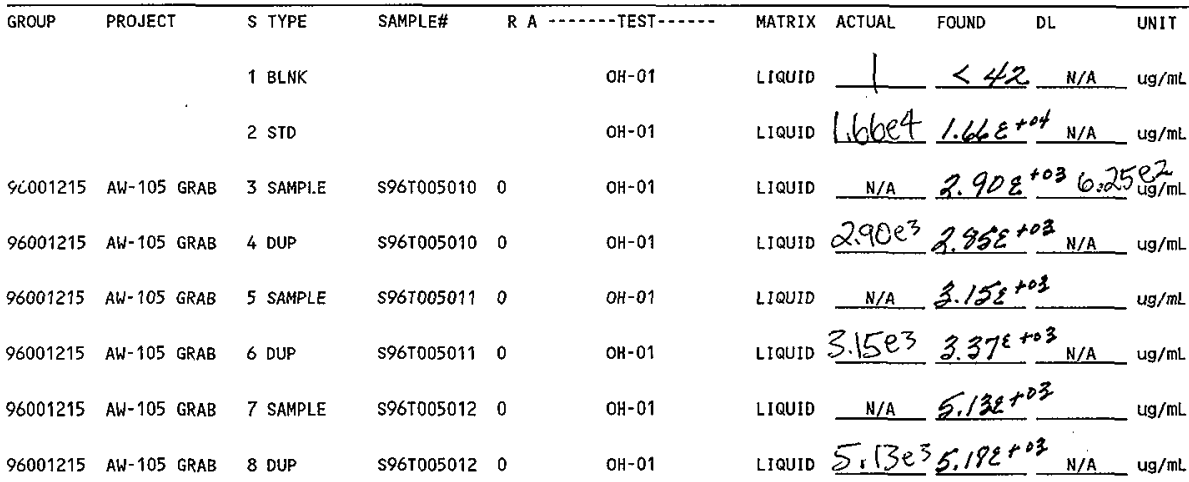

\section{Final page for worklist \# 13203}

RaeQun Jaen 10-01-96 Analyst Signature

\section{Date}

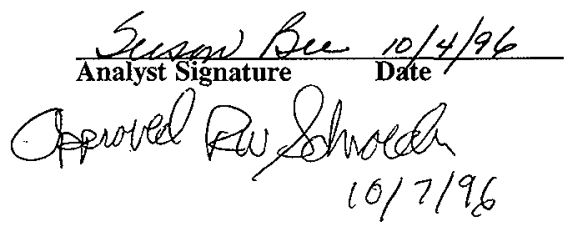

Datu Entry Comments:

Units shown for $Q C$ (SPK \& STD) may not reflect the actual units. $D L=$ Detection Limit, $S=$ Worklist Slot Number, $R=$ Replicate Number, $A=$ Aliquot Code. 


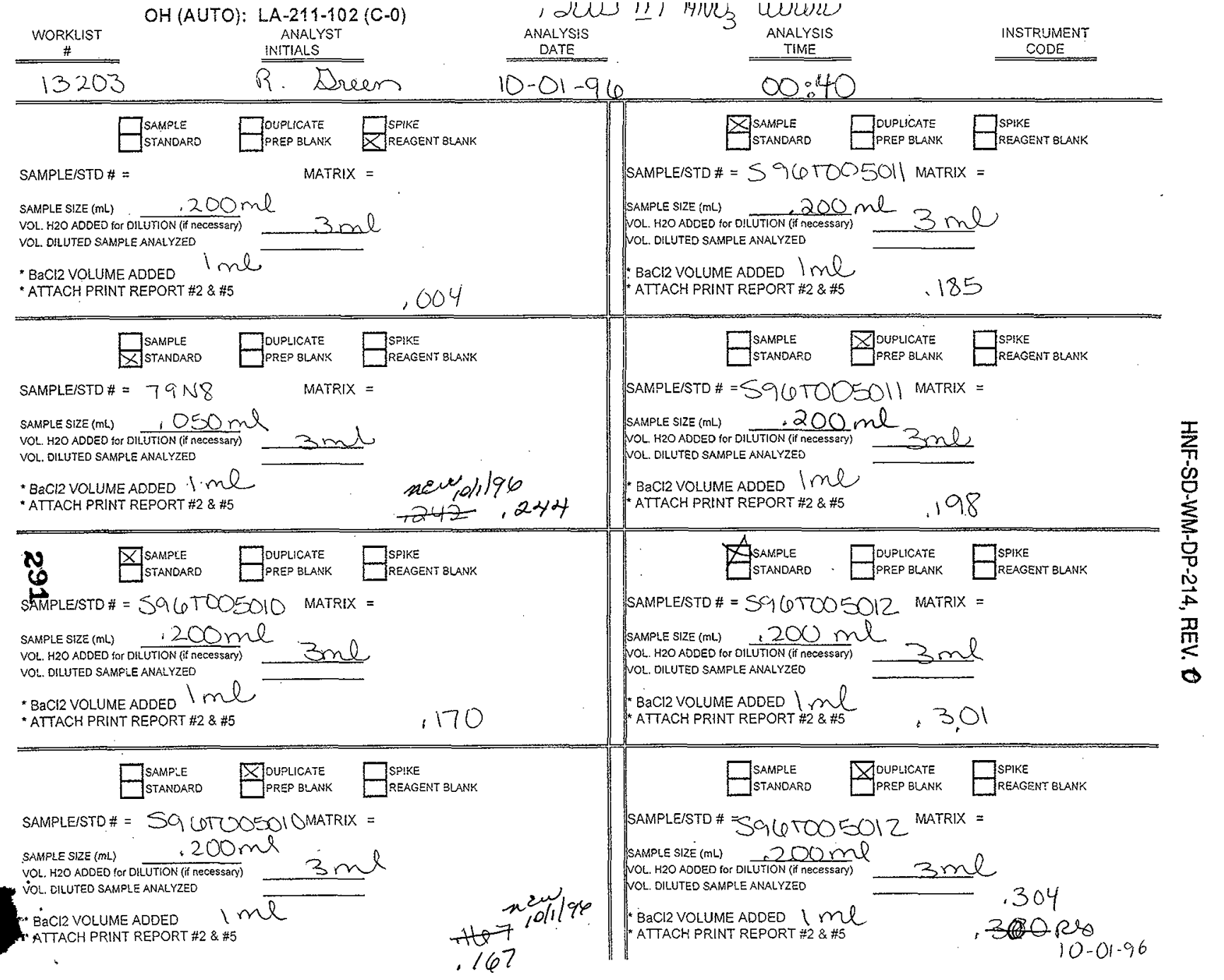




\section{HNF-SD-WM-DP-214, REV. 0 OH ANALYSIS}

\section{Peagent blante dote $96-69-30$ time $23: 30$ GET FH \& 12 : Id. 1 1 96000 Id. 2 2 209 FHint 5.94 EF1 4.92 stop volt reoglned $====$}

dote $95-99-30$ time $23: 34$ TEET FH $12 \#$ EO - IDimladiv ash=1/diu start $V, 609 \mathrm{ml}$

$\varepsilon$

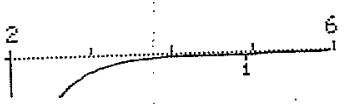

\section{Sed}

dote $96-69-30$ time $23: 39$

CIET PH 12 \# 61

Id. 1 100000

1. 42 :2005

pHinit) 11.62

$\begin{array}{lll}\text { EF1 } & .244 & 9.73 \\ \text { EF2 } & .309 & 7.60\end{array}$

stop woit. renched

$======$

date $96-09-30$ time 2399

DET $\mathrm{PH} \quad 12$ \#

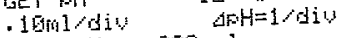
stint $y$. Bbs in

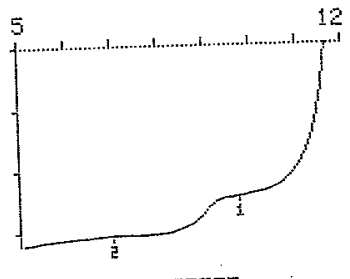
2
- 5961005010 date $96-69-30$ time 2344 EET FH 12 \# 62 Td. 11 on 5010 . Id. 2 H 2005

FHlinit $11 \times 5$

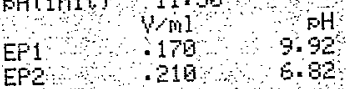
Etor wolt a reobled $== \pm==$

date 96-09-3a time $23: 44$ DET $\mathrm{FH}$ I2 - IGmlaty $\triangle \mathrm{FH}=1 / \mathrm{div}$ stat $y$. $6019 \mathrm{ml}$

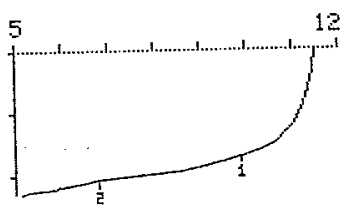

S96TOOSOIO-DUP date 96-69-39 time $23: 48$ GET FH 12 \# 63 Id. \#1 1 Id. 른 pHlinit) 11.45 $\begin{array}{llr}\text { FH(init) } & 11.451 & \\ E F 1 & .167 & 9.94 \\ E F 2 & .206 & 6.79\end{array}$ Etop yolt = retefed $=== \pm==$ = dote $56-69-30$ time $23: 48$ bET FH 12 \# 63 - 1 baldiv $A$ of start $y$. 846 il

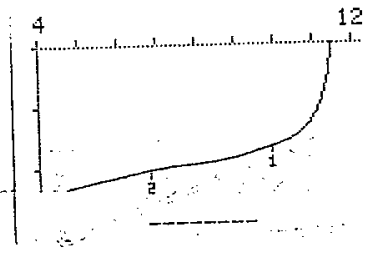

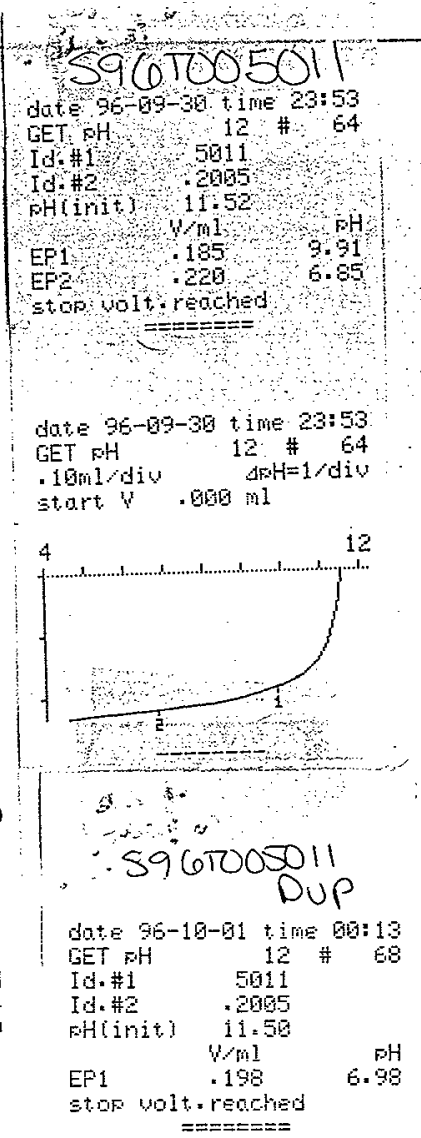

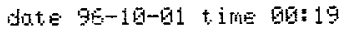
GET FH 12 \# 68

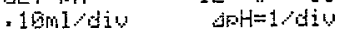
Etart $y$. 60 mi

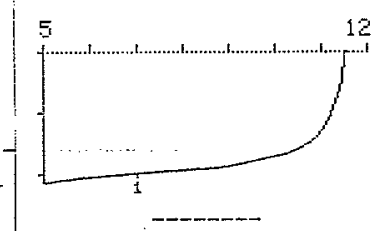




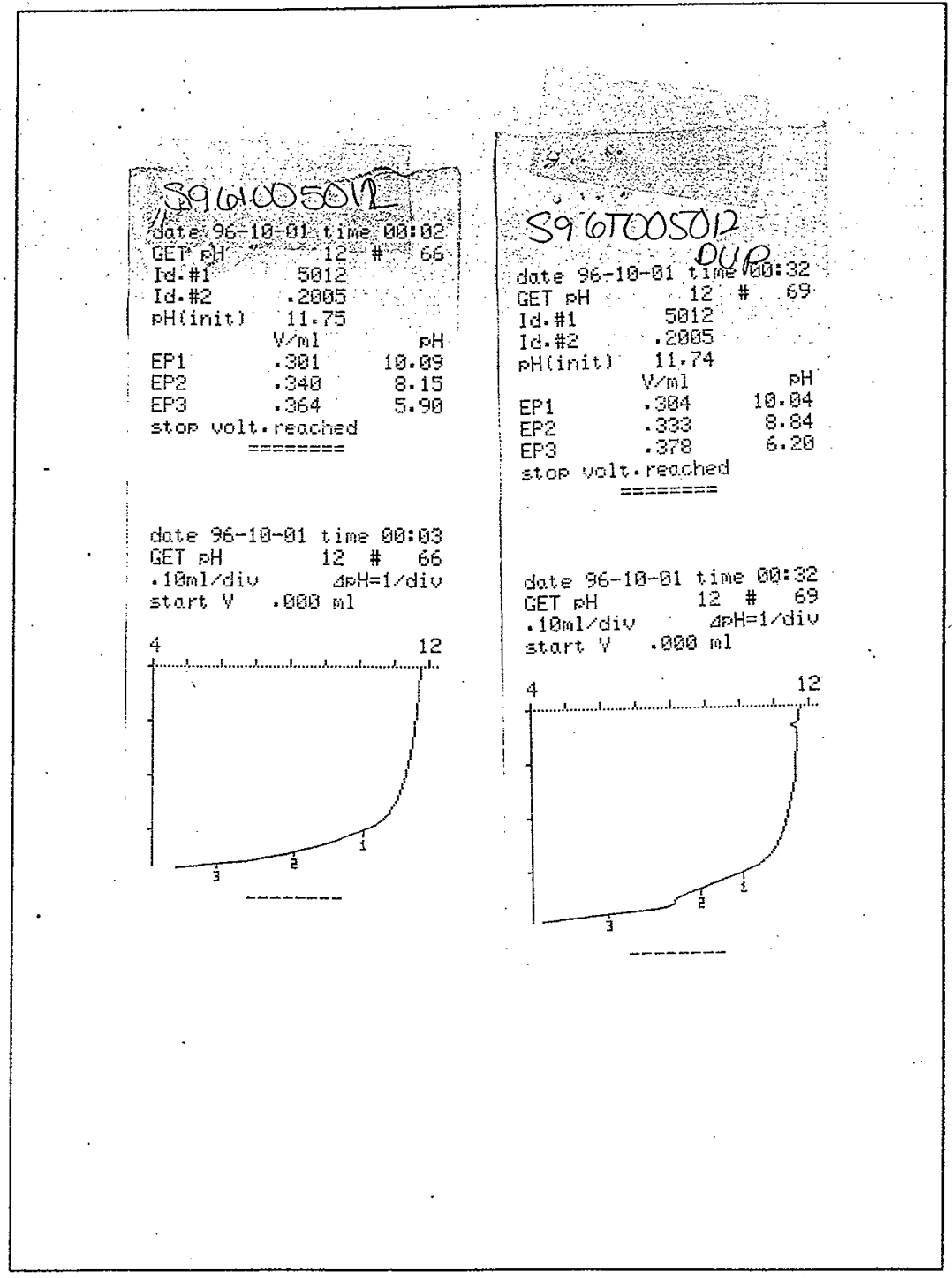




\begin{tabular}{|c|c|c|}
\hline \multicolumn{2}{|c|}{ OH (AUTO) : LA-211-102 (C-0) } & \multirow[t]{2}{*}{ BLANK } \\
\hline Wype, Thy & Sample Size $(\mathrm{mL})$ SS & \\
\hline BLANK & Concentration of HNO3 (Molarity) & 0.2005 \\
\hline Work List & HNO3 Titrant at $\mathrm{OH}$ end-point in $\mathrm{mL}$ & 0.004 \\
\hline 13203 & Dilution Factor DF & 1 \\
\hline VTesteodetrt & Concentration of $\mathrm{OH}$ in Sample (Molarity) & 2.67E-04 \\
\hline $\mathrm{OH}-01$ & $\mathrm{OH}$ in Sample in $\mu \mathrm{g} / \mathrm{mL}$ (PPM) & $4.54 \mathrm{E}+00$ \\
\hline \multirow{2}{*}{ 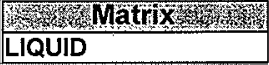 } & \multirow{4}{*}{ Detection Limit $=125 \mu \mathrm{g} / \mathrm{SS} * \mathrm{DF}$} & \\
\hline & & \\
\hline Sample\# H & & \\
\hline REAGENT BLANK & & \\
\hline Instrument Code & Detection Limit $(\mu \mathrm{g} / \mathrm{mL})$ & 4.17E+01 \\
\hline $\mathrm{PH} 01$ & \multirow{5}{*}{\multicolumn{2}{|c|}{$\begin{array}{l}\mathrm{OH} \text { Molarity }=\left((\mathrm{mL} H \mathrm{HO} 3)^{\star}(\mathrm{M} H \mathrm{NO} 3)\right) / \text { Sample Size in } \mathrm{mL} \mathrm{L}^{*} \text { Dilution Factor } \\
\mathrm{OH} \text { in } \mu \mathrm{g} / \mathrm{mL}=(\mathrm{OH} \text { MOLARITY })^{*}(17.0 \mathrm{~g} / \mathrm{mole})^{*}((1000000 \mu \mathrm{g} / \mathrm{g}) /(1000 \mathrm{~mL} / \mathrm{L}))\end{array}$}} \\
\hline Analyst & & \\
\hline RA GREEN & & \\
\hline 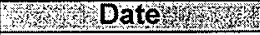 & & \\
\hline $\begin{array}{l}\text { Date } \\
10 / 01 / 96 \\
\end{array}$ & & \\
\hline \multirow{3}{*}{ 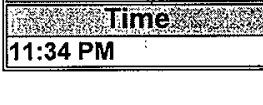 } & & BLANK \\
\hline & Concentration of $\mathrm{OH}$ in Sample (Molarity) & 2.67E-04 \\
\hline & $\mathrm{OH}$ in Sample in $\mu \mathrm{g} / \mathrm{mL}$ (PPM) & $<42$ \\
\hline
\end{tabular}

The Result is < Detection Limit

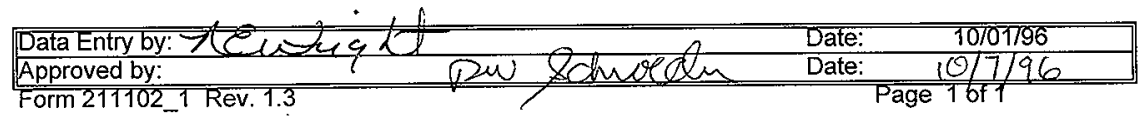




\begin{tabular}{|c|c|c|}
\hline \multicolumn{2}{|c|}{ OH (AUTO) : LA-211-102 (C-0) } & STANDARD \\
\hline WV Y Y Y & Sample Size $(\mathrm{mL}) \mathrm{SS}$ & 0.050 \\
\hline STANDARD & Concentration of $\mathrm{HNO}_{3}$ (Molarity) & 0.2005 \\
\hline Work IISt & HNO3 Titrant at $\mathrm{OH}$ end-point in $\mathrm{mL}$ & 0.244 \\
\hline 13203 & Dilution Factor DF & \\
\hline Wresteod & Concentration of $\mathrm{OH}$ in Sample (Molarity) & $9.78 \mathrm{E}-01$ \\
\hline $\mathrm{OH}-01$ & $\mathrm{OH}$ in Sample in $\mu \mathrm{g} / \mathrm{m} L$ (PPM) & $1.66 \mathrm{E}+04$ \\
\hline \multicolumn{3}{|c|}{ Matrix } \\
\hline \multicolumn{3}{|l|}{ LIQUID } \\
\hline \multirow{2}{*}{\multicolumn{3}{|c|}{ 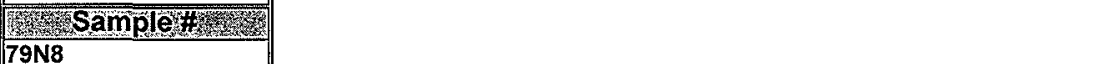 }} \\
\hline & & \\
\hline \multicolumn{3}{|l|}{ Instrument code } \\
\hline \multicolumn{3}{|l|}{ PHO1 } \\
\hline 4nalyst t & \multirow{4}{*}{\multicolumn{2}{|c|}{$\begin{array}{l}\text { OH Molarity }=\left((\mathrm{mL} \text { HNO3 })^{*}(\mathrm{M} \mathrm{HNO})\right) / \text { Sample Size in } \mathrm{mL}^{*} \text { Dilution Factor } \\
\mathrm{OH} \text { in } \mu \mathrm{g} / \mathrm{mL}=(\mathrm{OH} \text { MOLARITY })^{*}(17.0 \mathrm{~g} / \mathrm{mole})^{*}((1000000 \mu \mathrm{g} / \mathrm{g}) /(1000 \mathrm{~mL} / \mathrm{L}))\end{array}$}} \\
\hline RA GREEN & & \\
\hline Date & & \\
\hline $10 / 01 / 96$ & & \\
\hline Tx & & STANDARD \\
\hline \multirow{2}{*}{$11: 34 \mathrm{PM}$} & Concentration of $\mathrm{OH}$ in Sample (Molarity) & $9.78 \mathrm{E}-01$ \\
\hline & $\mathrm{OH}$ in Sample in $\mu \mathrm{g} / \mathrm{mL}$ (PPM) & $1.66 \mathrm{E}+04$ \\
\hline
\end{tabular}

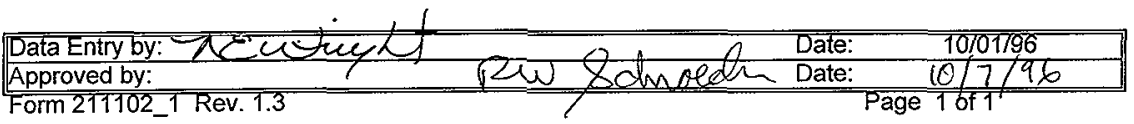




\begin{tabular}{|c|c|c|}
\hline \multicolumn{2}{|c|}{ OH (AUTO) : LA-211-102 (C-0) } & SAMPLE \\
\hline Why & Sample Size (mL) SS & 0.200 \\
\hline SAMPLE & Concentration of $\mathrm{HNO} 3$ (Molarity) & 0.2005 \\
\hline W Work I Ist & HNO3 Titrant at $\mathrm{OH}$ end-point in $\mathrm{mL}$ & 0.170 \\
\hline 13203 & Dilution Factor DF & 1 \\
\hline TSTestCode & Concentration of $\mathrm{OH}$ in Sample (Molarity) & $1.70 \mathrm{E}-01$ \\
\hline $\mathrm{OH}-01$ & OH in Sample in $\mu \mathrm{g} / \mathrm{mL}$ (PPM) & $2.90 \mathrm{E}+03$ \\
\hline \multirow{2}{*}{$\begin{array}{l}\text { WOU Matrix } \\
\text { LIQUID }\end{array}$} & \multirow[b]{3}{*}{ Detection Limit $=125 \mu \mathrm{g} / \mathrm{SS}$ * DF } & \\
\hline & & \\
\hline $\begin{array}{l}\text { Sampled } \\
\text { S96T005010 }\end{array}$ & & \\
\hline Mstrument code & Detection Limit $(\mu \mathrm{g} / \mathrm{mL})$ & $6.25 \mathrm{E}+02$ \\
\hline $\mathrm{PH} 01$ & \multirow{5}{*}{\multicolumn{2}{|c|}{$\begin{array}{l}\mathrm{OH} \text { Molarity }=\left((\mathrm{mL} \text { HNO3 })^{*}(\mathrm{M} \text { HNO3) }) / \text { Sample Size in } \mathrm{mL}{ }^{*} \text { Dilution Factor }\right. \\
\mathrm{OH} \text { in } \mu \mathrm{g} / \mathrm{mL}=(\mathrm{OH} M O L A R I T Y)^{*}(17.0 \mathrm{~g} / \mathrm{mole})^{*}((1000000 \mu \mathrm{g} / \mathrm{g}) /(1000 \mathrm{~mL} / \mathrm{L}))\end{array}$}} \\
\hline 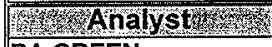 & & \\
\hline RA GREEN & & \\
\hline 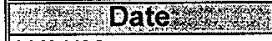 & & \\
\hline \multirow{2}{*}{ 10/01/96 } & & \\
\hline & & SAMPLE \\
\hline \multirow[t]{2}{*}{ 11:34 PM } & Concentration of $\mathrm{OH}$ in Sample (Molarity) & $1.70 \mathrm{E}-01$ \\
\hline & $\mathrm{OH}$ in Sample in $\mu \mathrm{g} / \mathrm{mL}$ (PPM) & $2.90 E+03$ \\
\hline
\end{tabular}

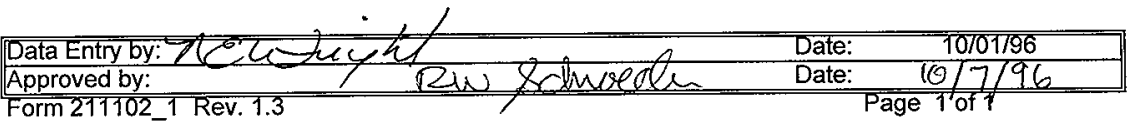


PLACE ANALYTICAL CARD IN BOX BELOW OR ATTACH TRAVELER

\begin{tabular}{|c|c|c|}
\hline \multirow{2}{*}{\multicolumn{2}{|c|}{ OH (AUTO) : LA-211-102 (C-0) }} & \multirow[b]{2}{*}{ DUPLICATE } \\
\hline & & \\
\hline 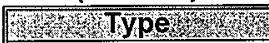 & Sample Size $(\mathrm{mL})$ SS & 0.200 \\
\hline DUPLICATE & Concentration of $\mathrm{HNO} 3$ (Molarity) & 0.2005 \\
\hline WWork & HNO3 Titrant at $\mathrm{OH}$ end-point in $\mathrm{mL}$ & 0.167 \\
\hline 13203 & Dilution Factor DF & 1 \\
\hline THESTOder & Concentration of $\mathrm{OH}$ in Sample (Molarity) & 1.67E-01 \\
\hline $\mathrm{OH}-01$ & $\mathrm{OH}$ in Sample in $\mu \mathrm{g} / \mathrm{mL}$ (PPM) & $2.85 \mathrm{E}+03$ \\
\hline \multirow{2}{*}{\multicolumn{3}{|c|}{ IOUID }} \\
\hline & & \\
\hline \multicolumn{3}{|c|}{ Detection Limit $=125 \mu \mathrm{g} / \mathrm{SS}$ * DF } \\
\hline \multicolumn{3}{|l|}{ S96T005010 } \\
\hline Instrument $C$ ode & Detection Limit $(\mu \mathrm{g} / \mathrm{mL})$ & $6.25 E+02$ \\
\hline \multicolumn{3}{|l|}{ PH01 } \\
\hline 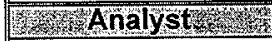 & \multirow{4}{*}{\multicolumn{2}{|c|}{$\begin{array}{l}\mathrm{OH} \text { Molarity }=\left((\mathrm{mL} \text { HNO3 })^{*}(\mathrm{M} H N O 3)\right) / \text { Sample Size in } \mathrm{mL} L^{*} \text { Dilution Factor } \\
\mathrm{OH} \text { in } \mu \mathrm{g} / \mathrm{mL}=(\mathrm{OH} \text { MOLARITY })^{*}(17.0 \mathrm{~g} / \mathrm{mole})^{\star}((1000000 \mu \mathrm{g} / \mathrm{g}) /(1000 \mathrm{~mL} / \mathrm{L}))\end{array}$}} \\
\hline \multirow{2}{*}{$\mathrm{OH}$ in $\mu \mathrm{g} / \mathrm{mL}=(\mathrm{OH} \text { MOLARITY })^{*}(17.0 \mathrm{~g} / \mathrm{mole})^{\star}((1000000 \mu \mathrm{g} / \mathrm{g}) /(1000 \mathrm{~mL} / \mathrm{L}))$} & & \\
\hline & & \\
\hline $10 / 01 / 96$ & & \\
\hline 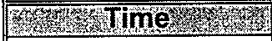 & & DUPLICATE \\
\hline \multirow[t]{2}{*}{$11: 48 \mathrm{PM}$} & Concentration of $\mathrm{OH}$ in Sample (Molarity) & $1.67 \mathrm{E}-01$ \\
\hline & $\mathrm{OH}$ in Sample in $\mu \mathrm{g} / \mathrm{mL}$ (PPM) & $2.85 \mathrm{E}+03$ \\
\hline
\end{tabular}

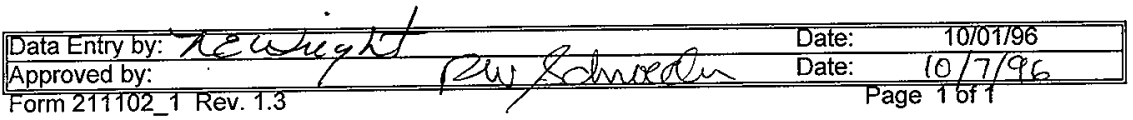




\begin{tabular}{|c|c|c|}
\hline \multirow{2}{*}{\multicolumn{2}{|c|}{ OH (AUTO) : LA-211-102 (C-0) }} & \multirow[b]{2}{*}{ SAMPLE } \\
\hline & & \\
\hline 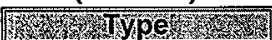 & Sample Size $(\mathrm{mL})$ sS & 0.200 \\
\hline SAMPLE & Concentration of HNO3 (Molarity) & 0.2005 \\
\hline Workist & HNO3 Titrant at $\mathrm{OH}$ end-point in $\mathrm{mL}$ & 0.185 \\
\hline 13203 & Dilution Factor DF & 1 \\
\hline KTicstoode & Concentration of $\mathrm{OH}$ in Sample (Molarity) & $1.85 \mathrm{E}-01$ \\
\hline $\mathrm{OH}-01$ & OH in Sample in $\mu \mathrm{g} / \mathrm{mL}$ (PPM) & $3.15 \mathrm{E}+03$ \\
\hline \multirow{2}{*}{$\begin{array}{l}\text { MatriX } \\
\text { LIQUID }\end{array}$} & \multirow{4}{*}{ Detection Limit $=125 \mu \mathrm{g} / \mathrm{SS}$ * DF } & \\
\hline & & \\
\hline Samplest & & \\
\hline S96T005011 & & \\
\hline Nintrumenteder & Detection Limit $(\mu \mathrm{g} / \mathrm{mL})$ & $6.25 \mathrm{E}+02$ \\
\hline \multirow{2}{*}{ 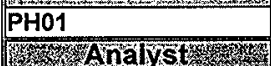 } & \multirow{5}{*}{\multicolumn{2}{|c|}{$\begin{array}{l}\mathrm{OH} \text { Molarity }=\left((\mathrm{mL} H N O 3)^{\star}(\mathrm{M} \mathrm{HNO})\right) / \text { Sample Size in } \mathrm{mL} \mathrm{L}^{\star} \text { Dilution Factor } \\
\mathrm{OH} \text { in } \mu \mathrm{g} / \mathrm{mL}=(\mathrm{OH} \text { MOLARITY })^{\star}(17.0 \mathrm{~g} / \mathrm{mole})^{\star}((1000000 \mu \mathrm{g} / \mathrm{g}) /(1000 \mathrm{~mL} / \mathrm{L}))\end{array}$}} \\
\hline & & \\
\hline EAG & & \\
\hline Dote. & & \\
\hline \multirow{4}{*}{ 10/04/96 } & & \\
\hline & & SAMPLE \\
\hline & Concentration of $\mathrm{OH}$ in Sample (Molarity) & $1.85 \mathrm{E}-01$ \\
\hline & $\mathrm{OH}$ in Sample in $\mu \mathrm{g} / \mathrm{mL}$ (PPM) & $3.15 E+03$ \\
\hline
\end{tabular}

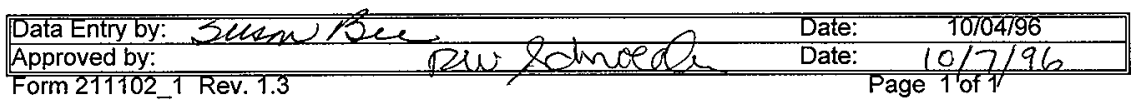




\begin{tabular}{|c|c|c|}
\hline \multicolumn{2}{|c|}{ OH (AUTO) : LA-211-102 (C-0) } & DUPLICATE \\
\hline 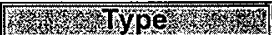 & Sample Size $(\mathrm{mL})$ SS & 0.200 \\
\hline DUPLICATE & Concentration of $\mathrm{HNO} 3$ (Molarity) & 0.2005 \\
\hline Whorkist & HNO3 Titrant at $\mathrm{OH}$ end-point in $\mathrm{mL}$ & 0.198 \\
\hline 13203 & Dilution Factor DF & 1 \\
\hline 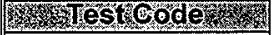 & Concentration of $\mathrm{OH}$ in Sample (Molarity) & $1.98 \mathrm{E}-01$ \\
\hline $\mathrm{OH}-01$ & OH in Sample in $\mu g / m L$ (PPM) & $3.37 E+03$ \\
\hline Natrix & \multirow[b]{3}{*}{ Detection Limit $=125 \mu \mathrm{g} / \mathrm{SS}$ * DF } & \\
\hline LIQUID & & \\
\hline $\begin{array}{l}\text { S96T005011 } \\
\text { S96t }\end{array}$ & & \\
\hline Instrumenteoder & Detection Limit $(\mu \mathrm{g} / \mathrm{mL})$ & $6.25 \mathrm{E}+02$ \\
\hline PHO1 & \multirow{4}{*}{\multicolumn{2}{|c|}{$\begin{array}{l}\mathrm{OH} \text { Molarity }=\left((\mathrm{mL} \text { HNO3 })^{*}(\mathrm{M} \text { HNO3) }) / \text { Sample Size in } \mathrm{mL}{ }^{*} \text { Dilution Factor }\right. \\
\mathrm{OH} \text { in } \mu \mathrm{g} / \mathrm{mL}=(\mathrm{OH} \text { MOLARITY })^{*}(17.0 \mathrm{~g} / \mathrm{mole})^{*}((1000000 \mu \mathrm{g} / \mathrm{g}) /(1000 \mathrm{~mL} / \mathrm{L}))\end{array}$}} \\
\hline 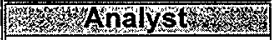 & & \\
\hline EAG & & \\
\hline $\begin{array}{l}10 \text { Dateks } \\
10 / 04 / 96\end{array}$ & & \\
\hline 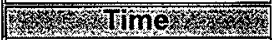 & & DUPLICATE \\
\hline & Concentration of OH in Sample (Molarity) & $\begin{array}{r}1.98 \mathrm{E}-01 \\
\end{array}$ \\
\hline & $\mathrm{OH}$ in Sample in $\mu \mathrm{g} / \mathrm{mL}$ (PPM) & $3.37 E+03$ \\
\hline
\end{tabular}

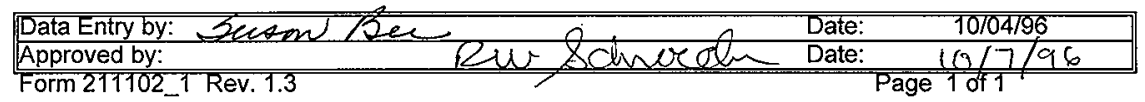




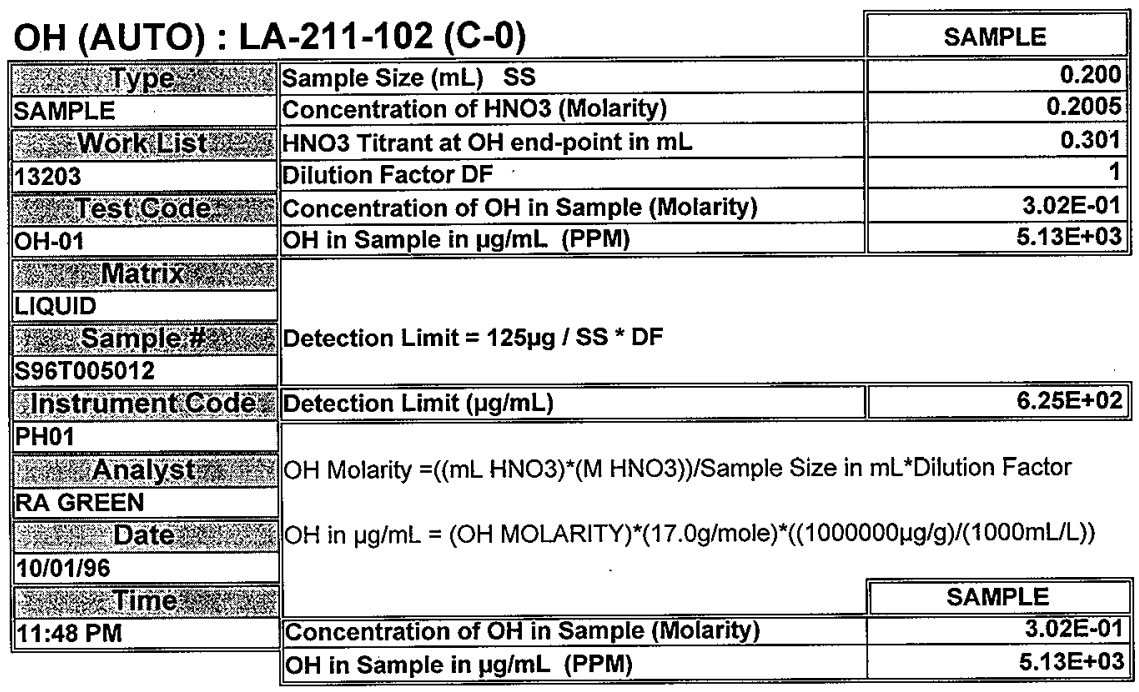

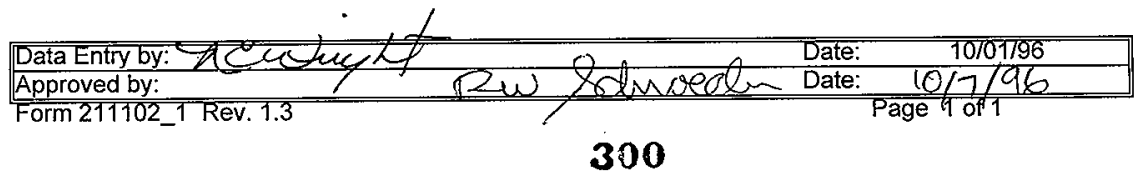




\begin{tabular}{|c|c|c|}
\hline \multicolumn{2}{|c|}{ OH (AUTO) : LA-211-102 (C-0) } & DUPLICATE \\
\hline 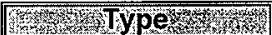 & Sample Size $(\mathrm{mL})$ SS & 0.200 \\
\hline DUPLICATE & Concentration of HNO3 (Molarity) & 0.2005 \\
\hline 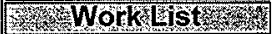 & HNO3 Titrant at $\mathrm{OH}$ end-point in $\mathrm{mL}$ & 0.304 \\
\hline 13203 & Dilution Factor DF & 1 \\
\hline Test Codery & Concentration of $\mathrm{OH}$ in Sample (Molarity) & 3.05E-01 \\
\hline $\mathrm{OH}-01$ & $\mathrm{OH}$ in Sample in $\mu \mathrm{g} / \mathrm{mL}$ (PPM) & $5.18 \mathrm{E}+03$ \\
\hline \multirow{2}{*}{$\begin{array}{l}\text { Matrix } \\
\text { LIQUID }\end{array}$} & \multirow{4}{*}{ Detection Limit $=125 \mu \mathrm{g} / \mathrm{SS}$ * DF } & \\
\hline & & \\
\hline 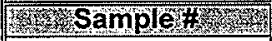 & & \\
\hline \multirow{2}{*}{\begin{tabular}{|l|} 
S96T005012 \\
Instrument $C$ od
\end{tabular}} & & \\
\hline & Detection Limit $(\mu \mathrm{g} / \mathrm{mL})$ & $6.25 \mathrm{E}+02$ \\
\hline PH01 & \multirow{5}{*}{\multicolumn{2}{|c|}{$\begin{array}{l}\mathrm{OH} \text { Molarity }=\left((\mathrm{mL} H \mathrm{NO} 3)^{*}(\mathrm{M} H \mathrm{NO})\right) / \text { Sample Size in } \mathrm{mL}^{*} \text { Dilution Factor } \\
\mathrm{OH} \text { in } \mu \mathrm{g} / \mathrm{mL}=(\mathrm{OH} \text { MOLARITY })^{*}(17.0 \mathrm{~g} / \mathrm{mole})^{*}((1000000 \mathrm{gg} / \mathrm{g}) /(1000 \mathrm{~mL} / \mathrm{L}))\end{array}$}} \\
\hline Whalyst & & \\
\hline \multirow{2}{*}{$\begin{array}{l}\text { RA GREEN } \\
\text { Siste }\end{array}$} & & \\
\hline & & \\
\hline \multirow{2}{*}{$10 / 01 / 96$} & & \\
\hline & & DUPLICATE \\
\hline \multirow[t]{2}{*}{ 11:48 PM } & Concentration of $\mathrm{OH}$ in Sample (Molarity) & $3.05 \mathrm{E}-01$ \\
\hline & $\mathrm{OH}$ in Sample in $\mu \mathrm{g} / \mathrm{mL}$ (PPM) & $5.18 \mathrm{E}+03$ \\
\hline
\end{tabular}

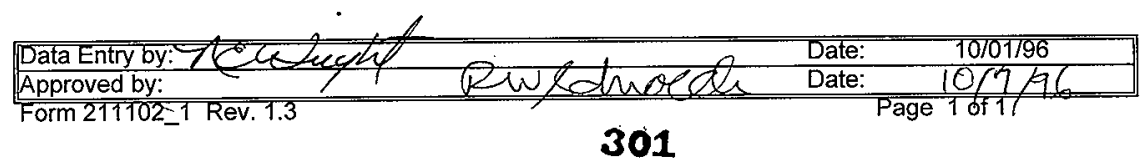




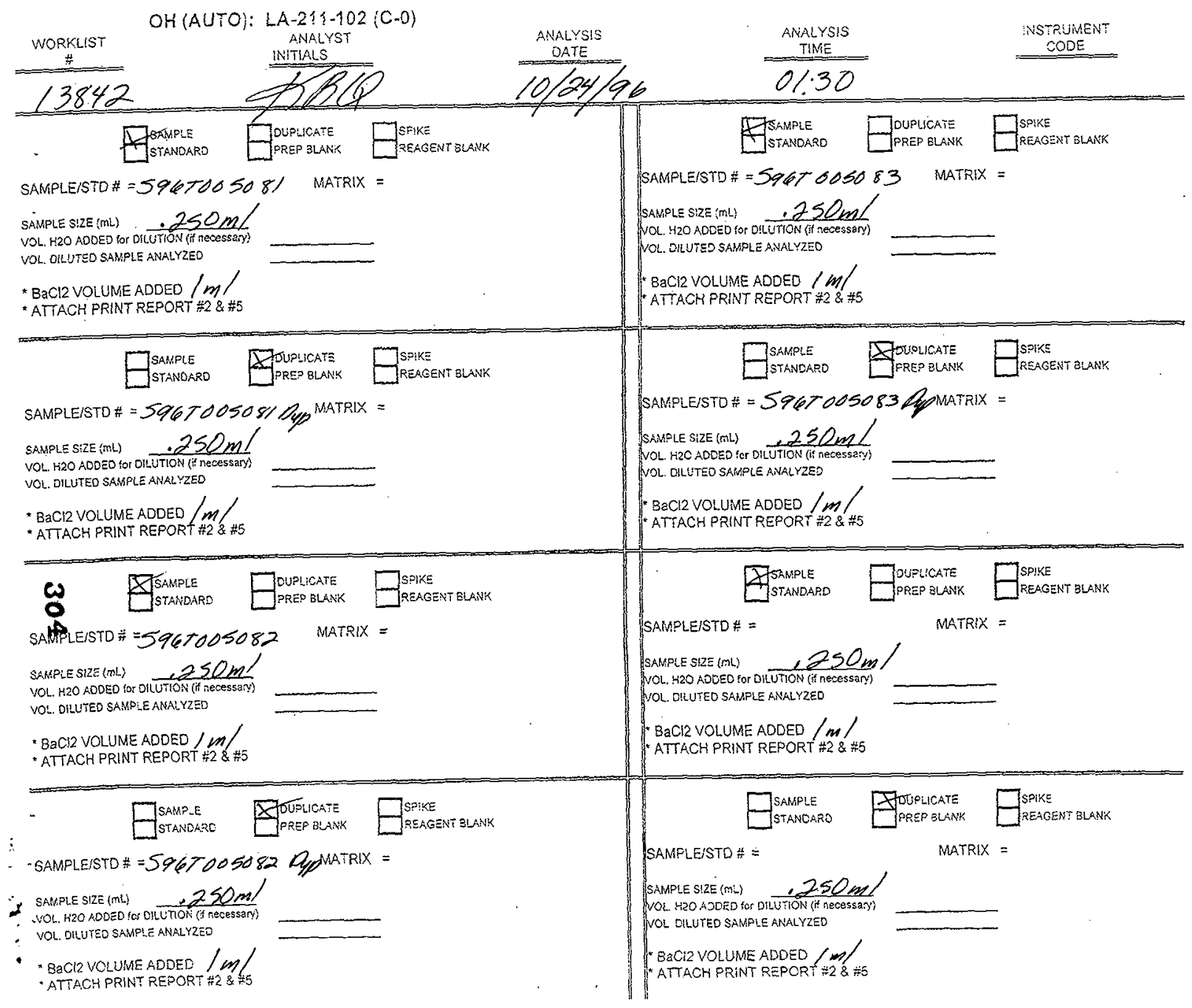






\section{HNF-SD-WM-DP-214, REV. 0}

\section{OH ANRLYSIS}

3967005081

dote 9s-19-29+ing $23: 99$

CET pH 12 \# 129

Id. 11 , 9479

Id. \#2 200

phlinit 1102

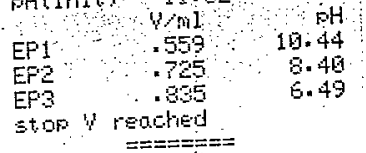

stop $======$

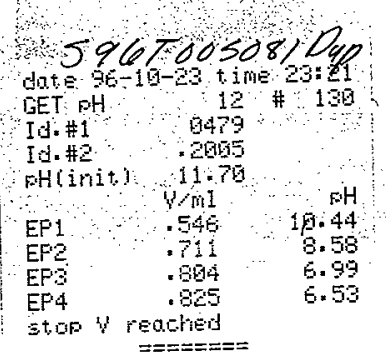

Sqmple size.050 date $96-10-23$ time 2310 CET FH - 1 Gisl ldiu start $\psi$. $600 \mathrm{ml}$
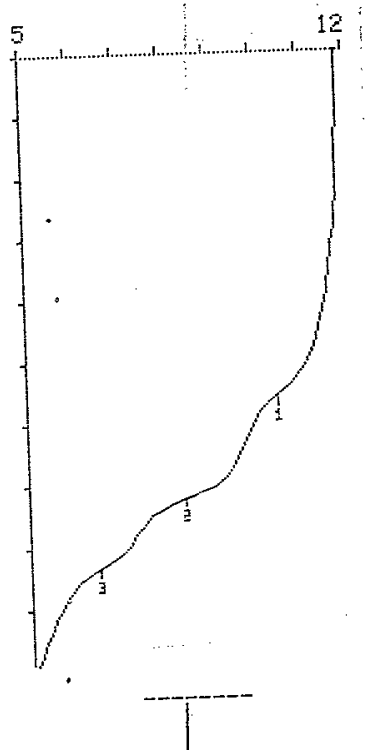

dre $96^{2}-10-23$ time 23822

GET $\mathrm{H}$. 12 \# 130

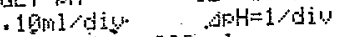
start 4001

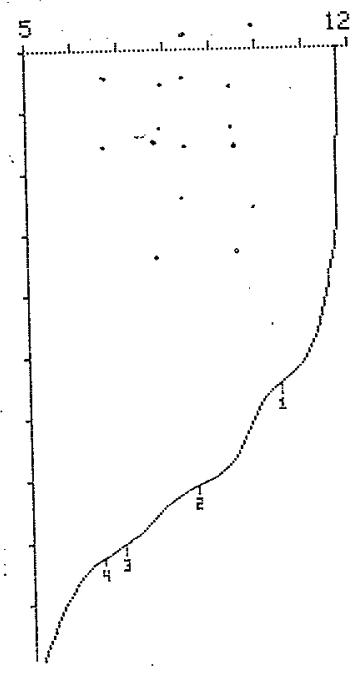

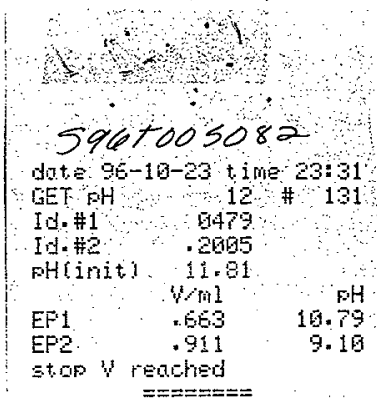

Damplesige $250 \mathrm{~m}$ inte 26-10-23 time $23: 31$ EET $\mathrm{AH} \quad 12$ \# 131 - 1 Gnifie start $Y$. 6 B日 m1

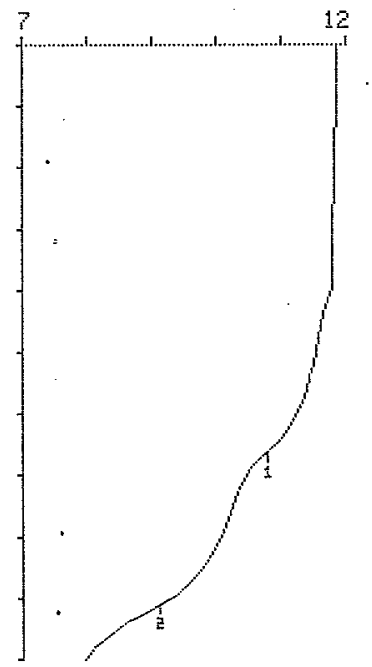




\section{OH ANALYSIS}

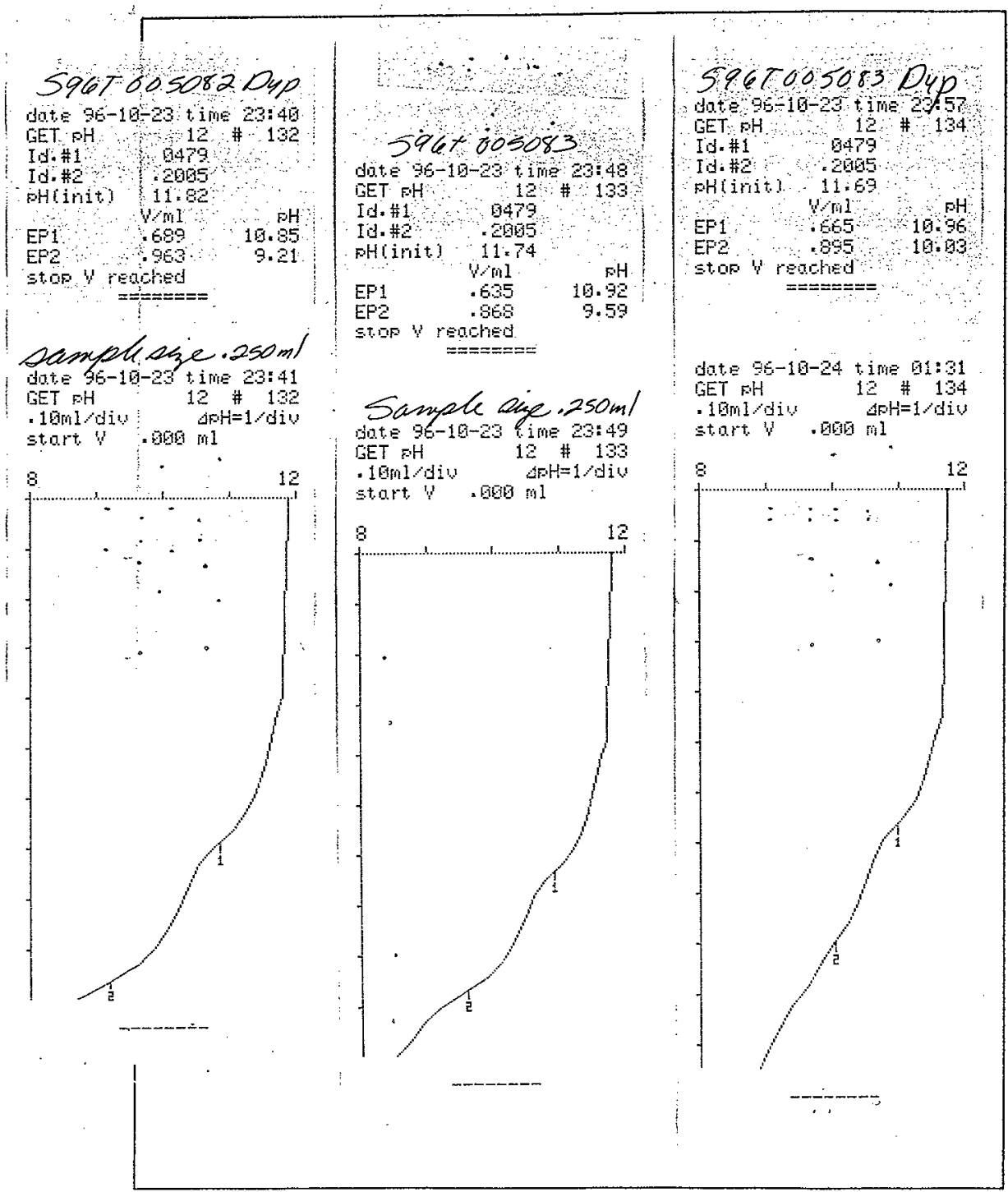




\begin{tabular}{|c|c|c|}
\hline \multirow{2}{*}{\multicolumn{2}{|c|}{ OH (AUTO) : LA-211-102 (C-0) }} & \multirow[b]{2}{*}{ STANDARD } \\
\hline & & \\
\hline 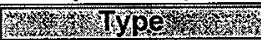 & Sample Size $(\mathrm{mL})$ SS & 0.050 \\
\hline STANDARD & Concentration of $\mathrm{HNO} 3$ (Molarity) & 0.2005 \\
\hline Whorkist & HNO3 Titrant at $\mathrm{OH}$ end-point in $\mathrm{mL}$ & 0.260 \\
\hline $13842 \quad-2$ & Dilution Factor DF & 1 \\
\hline Testeode & Concentration of $\mathrm{OH}$ in Sample (Molarity) & $1.04 E+00$ \\
\hline $\mathrm{OH}-01$ & $\mathrm{OH}$ in Sample in $\mu \mathrm{g} / \mathrm{mL}$ (PPM) & $1.77 E+04$ \\
\hline \multirow{2}{*}{\multicolumn{3}{|c|}{ Vun }} \\
\hline & & \\
\hline \multirow{2}{*}{\multicolumn{3}{|c|}{ 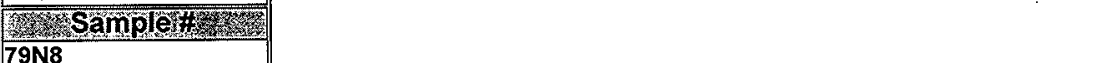 }} \\
\hline & & \\
\hline \multicolumn{3}{|l|}{ Instrument.6ode } \\
\hline \multicolumn{3}{|l|}{$\mathrm{PH} 01$} \\
\hline \multirow{2}{*}{\multicolumn{3}{|c|}{$\mathrm{OH}$ Molarity $=\left((\mathrm{mL} \text { HNO3 })^{*}(\mathrm{M} \mathrm{HNO})\right) /$ Sample Size in $\mathrm{mL}^{*}$ Dilution Factor }} \\
\hline & & \\
\hline \multicolumn{3}{|c|}{ NRL } \\
\hline \multicolumn{3}{|l|}{$10 / 24 / 96$} \\
\hline Hine & & STANDARD \\
\hline \multirow[t]{2}{*}{ 09:11 PM } & Concentration of OH in Sample (Molarity) & $1.04 \mathrm{E}+00$ \\
\hline & $\mathrm{OH}$ in Sample in $\mu \mathrm{g} / \mathrm{mL}$ (PPM) & $1.77 \mathrm{E}+04$ \\
\hline
\end{tabular}

Data Entry by: Approved by:

Form 211102_1 Rev. 1.3
$R \overline{J u}_{2}$ Date:

$10 / 24 / 96$

Date: $10 / 24 / 96$

309 


\begin{tabular}{|c|c|c|c|}
\hline OH (AUTO) : LA & A-211-102 (C-0) & BLANK & \\
\hline 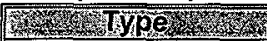 & Sample Size (mL) SS & $3.0-0.250$ & Fews $1024 / 96$ \\
\hline BLANK & Concentration of HNO3 (Molarity) & 0.2005 & \\
\hline 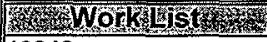 & HNO3 Titrant at $\mathrm{OH}$ end-point in $\mathrm{mL}$ & 0.008 & \\
\hline $13842 \quad \ldots \quad-\quad-2$ & Dilution Factor DF & 1 & \\
\hline Westerda & Concentration of $\mathrm{OH}$ in Sample (Molarity) & $6.42 E-03$ & Rus $0 / 24 / 0$ \\
\hline $\mathrm{OH}-01$ & OH in Sample in $\mu \mathrm{g} / \mathrm{mL}$ (PPM) & $1.09 \mathrm{E}+02$ & \\
\hline Watri X & & & \\
\hline LIQUID & & & \\
\hline RIANK & Detection Limit $=125 \mu \mathrm{g} / \mathrm{SS}$ * DF & & \\
\hline SLANK & Detection Limit $(\mu \mathrm{g} / \mathrm{mL})$ & $-5.00 E+02$ & \\
\hline PHO1 & & & $18 / 24 / 96$ \\
\hline WAnalyst & $\mathrm{OH}$ Molarity $=\left((\mathrm{mL} \text { HNO3 })^{*}(\mathrm{M}\right.$ HNO3 $\left.)\right) / \mathrm{Samp}$ & nL*Dilution Factor & \\
\hline KRQ & & & \\
\hline 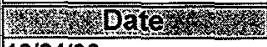 & $\mathrm{OH}$ in $\mu \mathrm{g} / \mathrm{mL}=(\mathrm{OH} \text { MOLARITY })^{*}(17.0 \mathrm{~g} / \mathrm{mole}$ & $0 \mu \mu \mathrm{g} / \mathrm{g}) /(1000 \mathrm{~mL} / \mathrm{L}))$ & \\
\hline $10 / 24 / 96$ & & & \\
\hline She & & BLANK & $\operatorname{Ros} 10 / 24 / 96$ \\
\hline 09:11 PM & $\begin{array}{l}\text { Concentration of OH in Sample (Molarity) } \\
\mathrm{OH} \text { in Sample in } \mu \mathrm{g} / \mathrm{mL} \text { (PPM) }\end{array}$ & $\begin{array}{lr} & -6.42 \mathrm{E}-03 \\
42 & -2500 \\
\end{array}$ & $\overrightarrow{R u s i d} / 24 / 96$ \\
\hline
\end{tabular}




\begin{tabular}{|c|c|c|}
\hline \multicolumn{2}{|c|}{ OH (AUTO) : LA-211-102 (C-0) } & SAMPLE \\
\hline 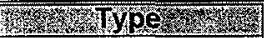 & Sample Size (mL) SS & 0.250 \\
\hline SAMPLE & Concentration of $\mathrm{HNO} 3$ (Molarity) & 0.2005 \\
\hline 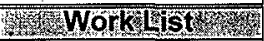 & HNO3 Titrant at $\mathrm{OH}$ end-point in $\mathrm{mL}$ & 0.397 \\
\hline 13842 & Dilution Factor DF & 1 \\
\hline Thesterde & Concentration of $\mathrm{OH}$ in Sample (Molarity) & $3.18 \mathrm{E}-01$ \\
\hline $\mathrm{OH}-01$. & $\mathrm{OH}$ in Sample in $\mu \mathrm{g} / \mathrm{mL}$ (PPM) & $5.41 \mathrm{E}+03$ \\
\hline \multirow{2}{*}{ LIQUID } & \multirow{4}{*}{ Detection Limit $=125 \mu \mathrm{g} / \mathrm{SS}$ * DF } & \\
\hline & & \\
\hline 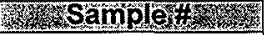 & & \\
\hline \multirow{2}{*}{$\begin{array}{l}\text { S96T0005049 } \\
\text { Instrument } 6 \text { ode }\end{array}$} & & \\
\hline & Detection Limit $(\mu \mathrm{g} / \mathrm{mL})$ & $5.00 \mathrm{E}+02$ \\
\hline PH01 & \multirow{5}{*}{\multicolumn{2}{|c|}{$\begin{array}{l}\mathrm{OH} \text { Molarity }=\left((\mathrm{mL} H \mathrm{HO} 3)^{*}(\mathrm{M} H \mathrm{HO} 3)\right) / \text { Sample Size in } \mathrm{mL}{ }^{*} \text { Dilution Factor } \\
\mathrm{OH} \text { in } \mu \mathrm{g} / \mathrm{mL}=(\mathrm{OH} \text { MOLARITY })^{*}(17.0 \mathrm{~g} / \mathrm{mole})^{*}((1000000 \mu \mathrm{g} / \mathrm{g}) /(1000 \mathrm{~mL} / \mathrm{L}))\end{array}$}} \\
\hline Syalyst & & \\
\hline \multirow{3}{*}{ 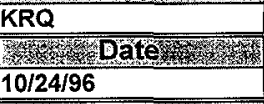 } & & \\
\hline & & \\
\hline & & \\
\hline \multirow{3}{*}{$\begin{array}{l}\text { o9:11 PM } \\
09\end{array}$} & & SAMPLE \\
\hline & Concentration of $\mathrm{OH}$ in Sample (Molarity) & $3.18 \mathrm{E}-01$ \\
\hline & $\mathrm{OH}$ in Sample in $\mu \mathrm{g} / \mathrm{mL}$ (PPM) & $5.41 E+03$ \\
\hline
\end{tabular}


PLACE ANALYTICAL CARD IN BOX BELOW OR ATTACH TRAVELER

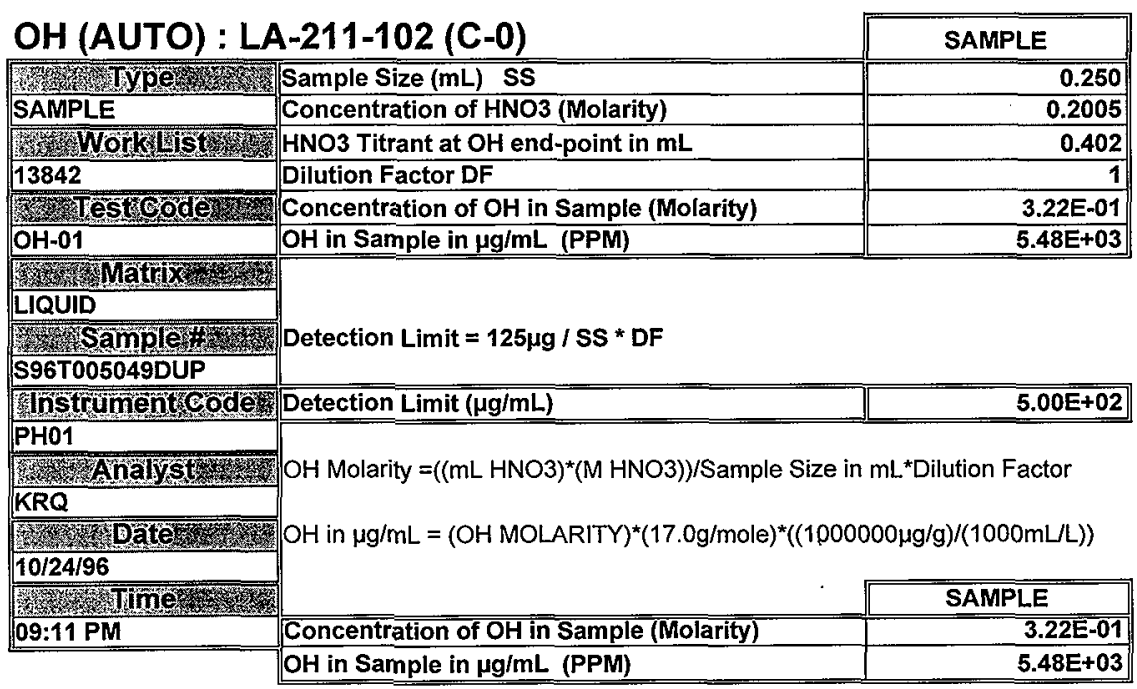

\begin{tabular}{|c|c|c|}
\hline Data Entry by: & KCJ, 0 & $10 / 24 / 96$ \\
\hline Approved by: & Ruscluneton & Date: $10 / 24 / 96$ \\
\hline
\end{tabular}


HNF-SD-WM-DP-214, REV. 0

PLACE ANALYTICAL CARD IN BOX BELOW OR ATTACH TRAVELER

\begin{tabular}{|c|c|c|}
\hline \multicolumn{2}{|c|}{ OH (AUTO) : LA-211-102 (C-0) } & SAMPLE \\
\hline 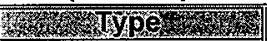 & Sample Size $(\mathrm{mL})$ SS & 0.250 \\
\hline SAMPLE & Concentration of HNO3 (Molarity) & 0.2005 \\
\hline 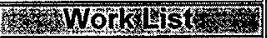 & HNO3 Titrant at $\mathrm{OH}$ end-point in $\mathrm{mL}$ & 0.634 \\
\hline 13842 & Dilution Factor DF & \\
\hline 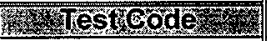 & Concentration of OH in Sample (Molarity) & 5.08E-01 \\
\hline $\mathrm{OH}-01$ & $\mathrm{OH}$ in Sample in $\mu \mathrm{g} / \mathrm{mL}$ (PPM) & $8.64 \mathrm{E}+03$ \\
\hline \multirow{2}{*}{$\begin{array}{l}\text { MatriX } \\
\text { LIQUID }\end{array}$} & \multirow{4}{*}{ Detection Limit $=125 \mu \mathrm{g} / \mathrm{SS}$ * DF } & \\
\hline & & \\
\hline \multirow{2}{*}{ 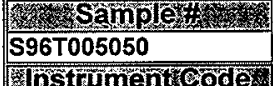 } & & \\
\hline & & \\
\hline Mistrumentcode & Detection Limit $(\mu \mathrm{g} / \mathrm{mL})$ & $5.00 \mathrm{E}+02$ \\
\hline PH01 & \multirow{5}{*}{\multicolumn{2}{|c|}{$\begin{array}{l}\mathrm{OH} \text { Molarity }=\left((\mathrm{mL} \text { HNO3 })^{*}(\mathrm{M} H \mathrm{NO} 3)\right) / \text { Sample Size in } \mathrm{mL}{ }^{*} \text { Dilution Factor } \\
\mathrm{OH} \text { in } \mu \mathrm{g} / \mathrm{mL}=(\mathrm{OH} \text { MOLARITY })^{*}(17.0 \mathrm{~g} / \mathrm{mole})^{*}((1000000 \mathrm{\mu g} / \mathrm{g}) /(1000 \mathrm{~mL} / \mathrm{L}))\end{array}$}} \\
\hline 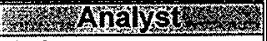 & & \\
\hline KRQ & & \\
\hline 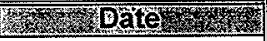 & & \\
\hline \multirow{2}{*}{$10 / 24 / 96$} & & \\
\hline & & SAMPLE \\
\hline \multirow[t]{2}{*}{ 09:11 PM } & Concentration of $\mathrm{OH}$ in Sample (Molarity) & 5.08E-01 \\
\hline & $\mathrm{OH}$ in Sample in $\mu \mathrm{g} / \mathrm{mL}$ (PPM) & $8.64 E+03$ \\
\hline
\end{tabular}

Data Entry by:

Approved by:

Form 211102_1 Rev. 1.3
RCS ing 313 


\begin{tabular}{|c|c|c|}
\hline \multicolumn{2}{|c|}{ OH (AUTO) : LA-211-102 (C-0) } & \multirow{2}{*}{$\frac{\text { SAMPLE }}{0.250}$} \\
\hline 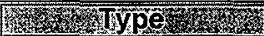 & Sample Size (mL) SS & \\
\hline SAMPLE & Concentration of HNO3 (Molarity) & 0.2005 \\
\hline 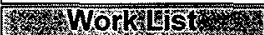 & HNO3 Titrant at $\mathrm{OH}$ end-point in $\mathrm{mL}$ & 0.626 \\
\hline 13842 & Dilution Factor DF & \\
\hline Trstede & Concentration of $\mathrm{OH}$ in Sample (Molarity) & $5.02 E-01$ \\
\hline $\mathrm{OH}-01$ & $\mathrm{OH}$ in Sample in $\mu \mathrm{g} / \mathrm{mL}$ (PPM) & $8.53 E+03$ \\
\hline \multirow{2}{*}{\multicolumn{3}{|c|}{ LIQUID }} \\
\hline & \multirow{3}{*}{\multicolumn{2}{|c|}{ Detection Limit $=125 \mu \mathrm{g} / \mathrm{SS}$ * DF }} \\
\hline Sample & & \\
\hline S96T005050DUP & & \\
\hline Lstrünetcode & Detection Limit $(\mu \mathrm{g} / \mathrm{mL})$ & $5.00 \mathrm{E}+02$ \\
\hline \multicolumn{3}{|c|}{ PH01 } \\
\hline \multirow{2}{*}{ KRQ } & \multirow{3}{*}{\multicolumn{2}{|c|}{$\begin{array}{l}\mathrm{OH} \text { Molarity }=\left((\mathrm{mL} H \mathrm{NO} 3)^{*}(\mathrm{M} \mathrm{HNO3})\right) / \text { Sample Size in } \mathrm{mL}^{*} \text { Dilution Factor } \\
\mathrm{OH} \text { in } \mu \mathrm{g} / \mathrm{mL}=(\mathrm{OH} \text { MOLARITY })^{\star}(17.0 \mathrm{~g} / \mathrm{mole})^{\star}((1000000 \mu \mathrm{g} / \mathrm{g}) /(1000 \mathrm{~mL} / \mathrm{L}))\end{array}$}} \\
\hline & & \\
\hline \multirow{2}{*}{$\begin{array}{l}10 / 24 / 96 \\
105 \text { ate }\end{array}$} & & \\
\hline & & SAMPLE \\
\hline 09:11 PM & Concentration of OH in Sample (Molarity) & $5 . \overline{5.02 E-01}$ \\
\hline & $\mathrm{OH}$ in Sample in $\mu \mathrm{g} / \mathrm{mL}$ (PPM) & $8.53 E+03$ \\
\hline
\end{tabular}

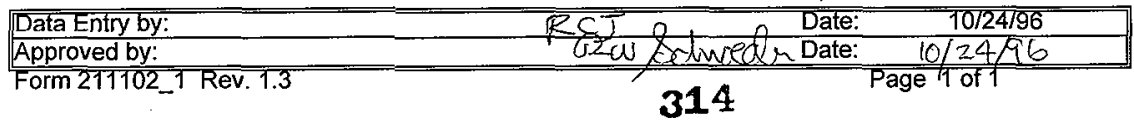




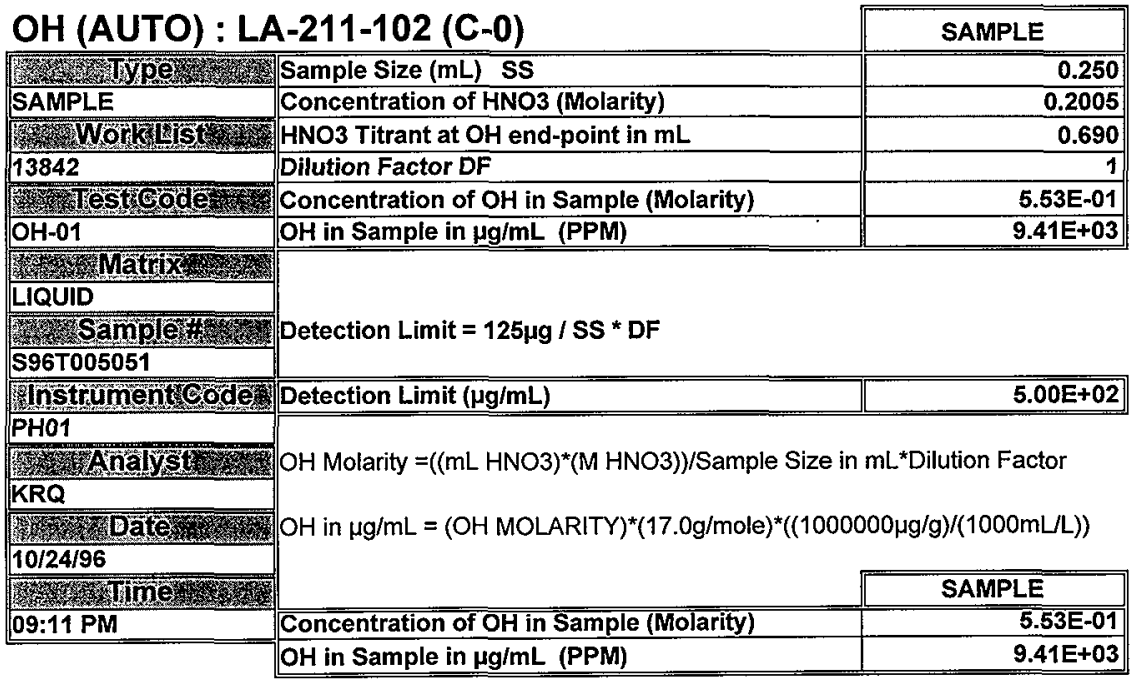




\begin{tabular}{|c|c|c|}
\hline \multirow{2}{*}{\multicolumn{2}{|c|}{ OH (AUTO) : LA-211-102 (C-0) }} & \multirow[b]{2}{*}{ SAMPLE } \\
\hline & & \\
\hline 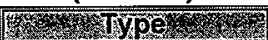 & Sample Size (mL) SS & 0.250 \\
\hline SAMPLE & Concentration of HNO3 (Molarity) & 0.2005 \\
\hline 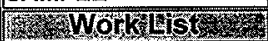 & HNO3 Titrant at $\mathrm{OH}$ end-point in $\mathrm{mL}$ & 0.697 \\
\hline 13842 & Dilution Factor DF & 1 \\
\hline Testeode & Concentration of $\mathrm{OH}$ in Sample (Molarity) & 5.59E-01 \\
\hline $\mathrm{OH}-01$ & $\mathrm{OH}$ in Sample in $\mu \mathrm{g} / \mathrm{mL}$ (PPM) & $9.50 \mathrm{E}+03$ \\
\hline \multirow{2}{*}{\multicolumn{3}{|c|}{ Matrix }} \\
\hline & \multirow{3}{*}{\multicolumn{2}{|c|}{ Detection Limit $=125 \mu \mathrm{g} / \mathrm{SS}$ * DF }} \\
\hline STrom & & \\
\hline S96T005051DUP & & \\
\hline Instrumenteode & Detection Limit $(\mu \mathrm{g} / \mathrm{mL}$ ) & $5.00 \mathrm{E}+02$ \\
\hline \multicolumn{3}{|c|}{ PH01 } \\
\hline Nolvst & \multirow{4}{*}{\multicolumn{2}{|c|}{$\begin{array}{l}\mathrm{OH} \text { Molarity }=\left((\mathrm{mL} \text { HNO3 })^{*}(\mathrm{M} \text { HNO3) }) / \text { Sample Size in mL.*Dilution Factor }\right. \\
\mathrm{OH} \text { in } \mu \mathrm{g} / \mathrm{mL}=(\mathrm{OH} \text { MOLARITY })^{*}(17.0 \mathrm{~g} / \mathrm{mole})^{*}((1000000 \mu \mathrm{g} / \mathrm{g}) /(1000 \mathrm{~mL} / \mathrm{L}))\end{array}$}} \\
\hline KRQ & & \\
\hline Th & & \\
\hline $10 / 24 / 96$ & & \\
\hline 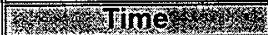 & & SAMPLE \\
\hline \multirow[t]{2}{*}{ 09:11 PM } & Concentration of $\mathrm{OH}$ in Sample (Molarity) & $\begin{array}{l}5.59 \mathrm{E}-01 \\
\end{array}$ \\
\hline & $\mathrm{OH}$ in Sample in $\mu \mathrm{g} / \mathrm{mL}$ (PPM) & $9.50 \mathrm{E}+03$ \\
\hline
\end{tabular}

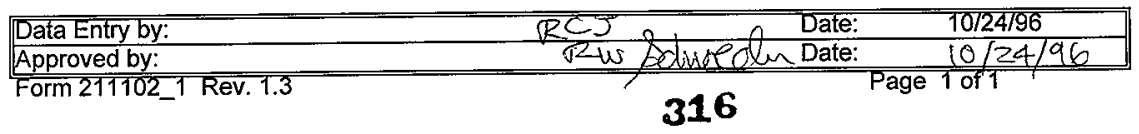


HNF-SD-WM-DP-2१4, REV. 0

PLACE ANALYTICAL CARD IN BOX BELOW OR ATTACH TRAVELER

\begin{tabular}{|c|c|c|}
\hline \multicolumn{2}{|c|}{ OH (AUTO) : LA-211-102 (C-0) } & SAMPLE \\
\hline TH & Sample Size (mL) SS & 0.250 \\
\hline SAMPLE & Concentration of $\mathrm{HNO}_{3}$ (Molarity) & 0.2005 \\
\hline 6. WorkUList & HNO3 Titrant at $\mathrm{OH}$ end-point in $\mathrm{mL}$ & 0.559 \\
\hline 13842 & Dilution Factor DF & \\
\hline Testode & Concentration of OH in Sample (Molarity) & 4.48E-01 \\
\hline $\mathrm{OH}-01$ & $\mathrm{OH}$ in Sample in $\mu \mathrm{g} / \mathrm{mL}$ (PPM) & $7.62 \mathrm{E}+03$ \\
\hline \multirow{2}{*}{$\begin{array}{l}\text { LIQUID } \\
\text { LIatix }\end{array}$} & \multirow{4}{*}{ Detection Limit $=125 \mu \mathrm{g} / \mathrm{SS} * \mathrm{DF}$} & \\
\hline & & \\
\hline 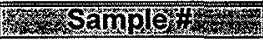 & & \\
\hline S96T005081 & & \\
\hline Unstrümenterde & Detection Limit $(\mu \mathrm{g} / \mathrm{mL})$ & $\overline{5.00 E+02}$ \\
\hline \multirow{2}{*}{ PH01 } & \multirow{5}{*}{\multicolumn{2}{|c|}{$\begin{array}{l}\mathrm{OH} \text { Molarity }=\left((\mathrm{mL} \text { HNO3 })^{*}(\mathrm{M} \text { HNO3) }) / \text { Sample Size in mL*Dilution Factor }\right. \\
\mathrm{OH} \text { in } \mu \mathrm{g} / \mathrm{mL}=(\mathrm{OH} \text { MOLARITY })^{*}(17.0 \mathrm{~g} / \mathrm{mole})^{*}((1000000 \mathrm{~g} / \mathrm{g}) /(1000 \mathrm{~mL} / \mathrm{L}))\end{array}$}} \\
\hline & & \\
\hline \multirow{3}{*}{ 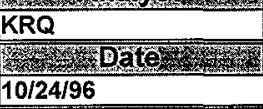 } & & \\
\hline & & \\
\hline & & SAMPLE \\
\hline \multirow{2}{*}{$\begin{array}{l}\text { Tline } \\
\text { 09:11 PM }\end{array}$} & Concentration of OH in Sample (Molarity) & $4 . \overline{48 E-01}$ \\
\hline & $\mathrm{OH}$ in Sample in $\mu \mathrm{g} / \mathrm{mL}$ (PPM) & $7.62 E+03$ \\
\hline
\end{tabular}

\begin{tabular}{|c|c|c|}
\hline Data Entry by: & $2 \mathrm{C} 3$ & $10 / 24 / 96$ \\
\hline Approved by: & दu lundeden & Date: $\quad 10 / 24 / 96$ \\
\hline
\end{tabular}




\begin{tabular}{|c|c|c|}
\hline \multicolumn{2}{|c|}{ OH (AUTO) : LA-211-102 (C-0) } & \multirow{2}{*}{ SAMPLE } \\
\hline RS & Sample Size (mL) SS & \\
\hline SAMPLE & Concentration of $\mathrm{HNO}_{3}$ (Molarity) & 0.2005 \\
\hline WorkLES & HNO3 Titrant at $\mathrm{OH}$ end-point in $\mathrm{mL}$ & 0.546 \\
\hline 13842 & Dilution Factor DF & -1 \\
\hline 2) Testeode & Concentration of $\mathrm{OH}$ in Sample (Molarity) & $4.38 \mathrm{E}-01$ \\
\hline $\mathrm{OH}-01$ & $\mathrm{OH}$ in Sample in $\mu \mathrm{g} / \mathrm{mL}$ (PPM) & $7.44 E+03$ \\
\hline \multirow{2}{*}{$\begin{array}{l}\text { MIQUID } \\
\text { LIXTIX }\end{array}$} & \multirow{4}{*}{ Detection Limit $=125 \mu \mathrm{g} / \mathrm{SS}$ * DF } & \\
\hline & & \\
\hline Y) Sampled & & \\
\hline 596T005081DUP & & \\
\hline Instrumeticode & Detection Limit $(\mu \mathrm{g} / \mathrm{mL})$ & $5.00 E+02$ \\
\hline \multirow{2}{*}{ PH01 } & \multirow{5}{*}{\multicolumn{2}{|c|}{$\begin{array}{l}\mathrm{OH} \text { Molarity }=\left((\mathrm{mL} H \mathrm{HO} 3)^{*}(\mathrm{M} H \mathrm{NO} 3)\right) / \text { Sample Size in } \mathrm{mL} \mathrm{L}^{*} \text { Dilution Factor } \\
\mathrm{OH} \text { in } \mu \mathrm{g} / \mathrm{mL}=(\mathrm{OH} \text { MOLARITY })^{*}(17.0 \mathrm{~g} / \mathrm{mole})^{*}((1000000 \mu \mathrm{g} / \mathrm{g}) /(1000 \mathrm{~mL} / \mathrm{L}))\end{array}$}} \\
\hline & & \\
\hline \multirow{2}{*}{ KRQ } & & \\
\hline & & \\
\hline $\begin{array}{l}10 / 24 / 96 \\
\end{array}$ & & \\
\hline \multirow{3}{*}{$\begin{array}{l}\text { Pa } \\
\text { 09:11 PM } \\
\end{array}$} & & SAMPLE \\
\hline & Concentration of $\mathrm{OH}$ in Sample (Molarity) & $4.38 \mathrm{E}-01$ \\
\hline & $\mathrm{OH}$ in Sample in $\mu \mathrm{g} / \mathrm{mL}$ (PPM) & $7.44 \mathrm{E}+03$ \\
\hline
\end{tabular}

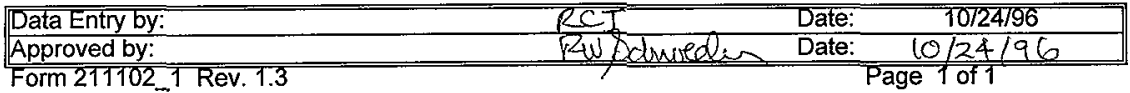




\begin{tabular}{|c|c|c|}
\hline \multicolumn{2}{|c|}{ OH (AUTO) : LA-211-102 (C-0) } & SAMPLE \\
\hline I & Sample Size (mL) SS & 0.250 \\
\hline SAMPLE & Concentration of $\mathrm{HNO} 3$ (Molarity) & 0.2005 \\
\hline 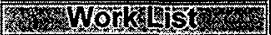 & HNO3 Titrant at OH end-point in $\mathrm{mL}$ & 0.663 \\
\hline 13842 & Dilution Factor DF & \\
\hline 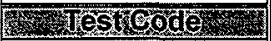 & Concentration of $\mathrm{OH}$ in Sample (Molarity) & 5.32E-01 \\
\hline $\mathrm{OH}-01$ & $\mathrm{OH}$ in Sample in $\mu \mathrm{g} / \mathrm{mL}$ (PPM) & $9.04 \mathrm{E}+03$ \\
\hline \multirow{2}{*}{ Matrox } & \multirow{4}{*}{ Detection Limit $=125 \mu \mathrm{g} / \mathrm{SS}$ * DF } & \\
\hline & & \\
\hline Sample & & \\
\hline S96T005082 & & \\
\hline \multirow{2}{*}{$\begin{array}{l}\text { Instrumenterde } \\
\text { PH01 }\end{array}$} & Detection Limit $(\mu \mathrm{g} / \mathrm{mL})$ & $5.00 \mathrm{E}+02$ \\
\hline & \multirow{5}{*}{\multicolumn{2}{|c|}{$\begin{array}{l}\mathrm{OH} \text { Molarity } \doteq\left((\mathrm{mL} \text { HNO3 })^{*}(\mathrm{M} \mathrm{HNO})\right) / \text { Sample Size in } \mathrm{mL} \mathrm{L}^{*} \text { Dilution Factor } \\
\mathrm{OH} \text { in } \mu \mathrm{g} / \mathrm{mL}=(\mathrm{OH} \text { MOLARITY })^{*}(17.0 \mathrm{~g} / \mathrm{mole})^{*}((1000000 \mu \mathrm{g} / \mathrm{g}) /(1000 \mathrm{~mL} / \mathrm{L}))\end{array}$}} \\
\hline \multirow{2}{*}{$\begin{array}{l}\text { ARalyst } \\
\text { KRQ }\end{array}$} & & \\
\hline & & \\
\hline \multirow{2}{*}{$\begin{array}{l}10 / 24 / 96 \\
1920\end{array}$} & & \\
\hline & & \\
\hline \multirow{2}{*}{ 00:11 PM } & & SAMPLE \\
\hline & Concentration of OH in Sample (Molarity) & 5.32E-01 \\
\hline $09: 11 \mathrm{PM}$ & $\mathrm{OH}$ in Sample in $\mu \mathrm{g} / \mathrm{mL}$ (PPM) & $9.04 \mathrm{E}+03$ \\
\hline
\end{tabular}

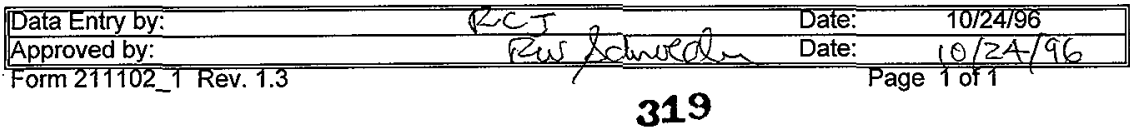




\begin{tabular}{|c|c|c|}
\hline \multicolumn{2}{|c|}{ OH (AUTO) : LA-211-102 (C-0) } & \multirow{2}{*}{ SAMPLE } \\
\hline W & Sample Size $(\mathrm{mL})$ SS & \\
\hline SAMPLE & Concentration of HNO3 (Molarity) & 0.2005 \\
\hline Whorkist & HNO3 Titrant at $\mathrm{OH}$ end-point in $\mathrm{mL}$ & 0.689 \\
\hline 13842 & Dilution Factor DF & \\
\hline 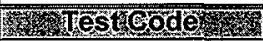 & Concentration of OH in Sample (Molarity) & 5.53E-01 \\
\hline $\mathrm{OH}-01$ & $\mathrm{OH}$ in Sample in $\mu \mathrm{g} / \mathrm{mL}$ (PPM) & $9.39 E+03$ \\
\hline \multirow{2}{*}{$\begin{array}{l}\text { MEKTIX } \\
\text { LIQUID }\end{array}$} & \multirow{4}{*}{ Detection Limit $=125 \mu \mathrm{g} / \mathrm{SS}$ * DF } & \\
\hline & & \\
\hline STampleth & & \\
\hline \multirow{2}{*}{$\begin{array}{l}\text { S96T005082DUP } \\
\text { Thstrument Crde }\end{array}$} & & \\
\hline & Detection Limit $(\mu \mathrm{g} / \mathrm{mL})$ & $5.00 \mathrm{E}+02$ \\
\hline$\frac{\mathrm{PHO1}}{\mathrm{P}}$ & \multirow{2}{*}{\multicolumn{2}{|c|}{$\mathrm{OH}$ Molarity $=\left((\mathrm{mL} H \mathrm{NO} 3)^{\star}(\mathrm{M} H \mathrm{HO})\right) /$ Sample Size in $\mathrm{mL}^{*}$ Dilution Factor }} \\
\hline Analyst & & \\
\hline \multirow{2}{*}{ KRQ } & \multirow{3}{*}{\multicolumn{2}{|c|}{$\mathrm{OH}$ in $\mu \mathrm{g} / \mathrm{mL}=(\mathrm{OH} \text { MOLARITY })^{*}(17.0 \mathrm{~g} / \mathrm{mole})^{*}((1000000 \mu \mathrm{g} / \mathrm{g}) /(1000 \mathrm{~mL} \mathrm{~L}))$}} \\
\hline & & \\
\hline \multirow{2}{*}{$10 / 24 / 96$} & & \\
\hline & & SAMPLE \\
\hline \multirow[t]{2}{*}{$09: 11 \mathrm{PM}$} & Concentration of OH in Sample (Molarity) & 5.53E-01 \\
\hline & $\mathrm{OH}$ in Sample in $\mu \mathrm{g} / \mathrm{mL}$ (PPM) & $9.39 E+03$ \\
\hline
\end{tabular}




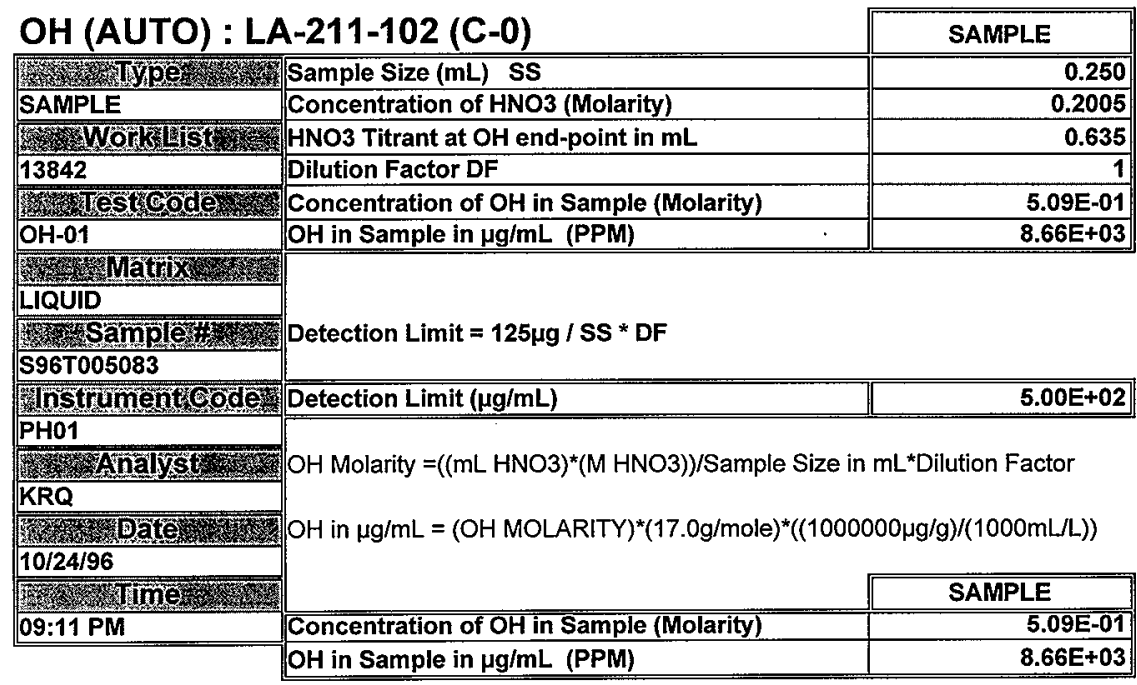




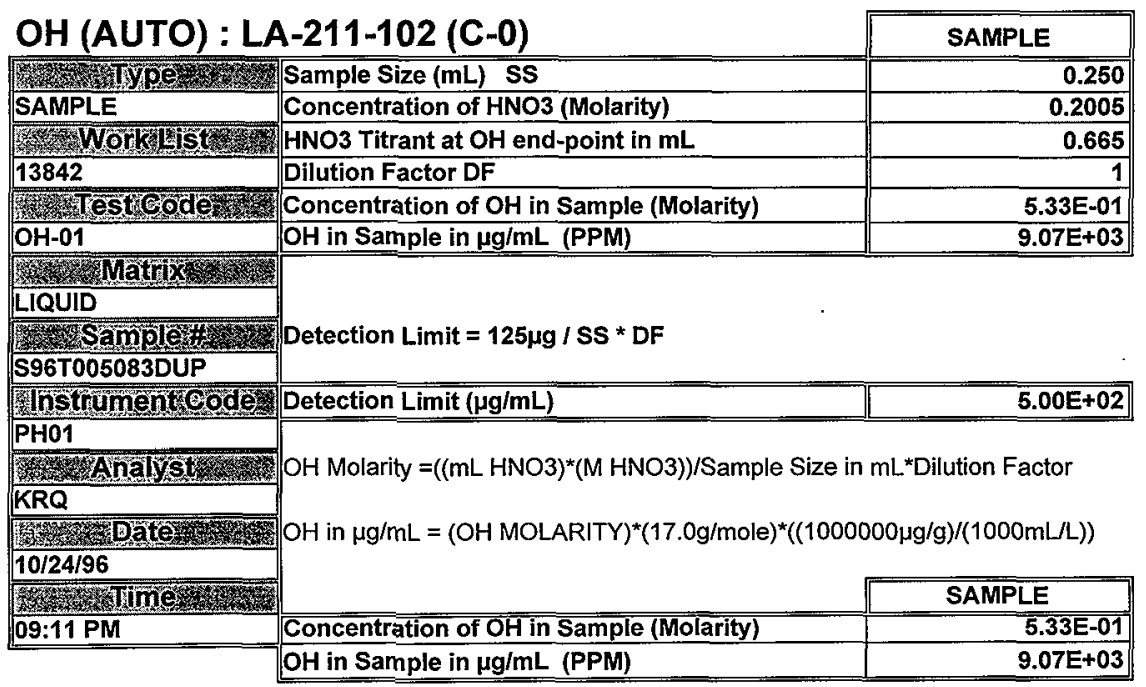




\section{LABCORE Completed Worklist Report for Worklist\# 14364}

Analyst: rdm

Instrument: PHO1

Book\# $79 \times 8$

Method: $\angle A-211-102 \quad$ Rev/Mod $\quad C-O$

Worklist Comment: OH-01 FOR AW-105 S2 Drct RAD. TEST

RTS!

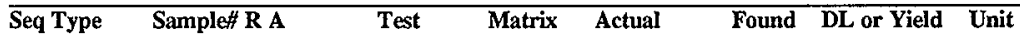

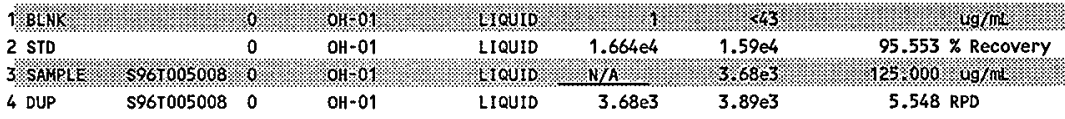

\section{Final page for worklist\# 14364}

$\frac{\text { see attached }}{\text { Analyst Signature }}$ 
$\therefore$

worklistrpt Version $2.105 / 15 / 95$

10/28/96 08:49

HNF-SD-WM-DP-214, REV. 0 .

Page: 1

\section{LABCORE Data Entry. Template for Worklist\# 14364}

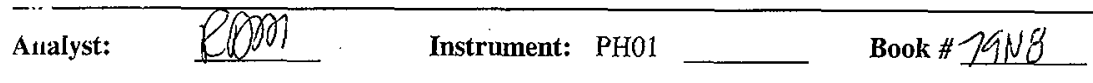

Method: LA-211-102 Rev/Mod C-O

Workilist Comment: OH-01 FOR AW-105 S2 Drct RAD. TEST

RTS!

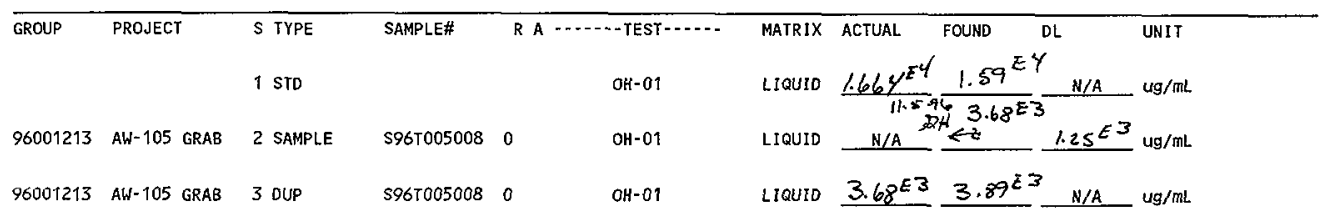

Final page for worklist \# 14364
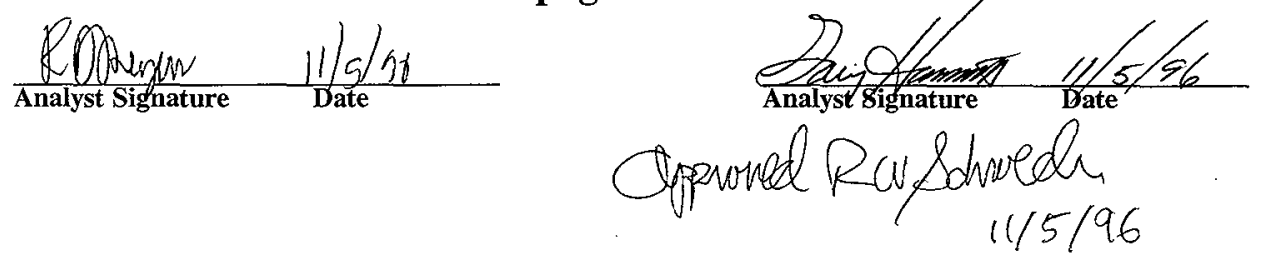

Data Entry Comments:

$.2005 \mathrm{~m} \mathrm{HNO}$

Units shown for $Q C$ (SPK \& STD) may not reflect the actual units. $D L=$ Detection Limit, $S=$ Worklist Slot Number, $R=$ Replicate Number,$A=$ Aliquot Code . 


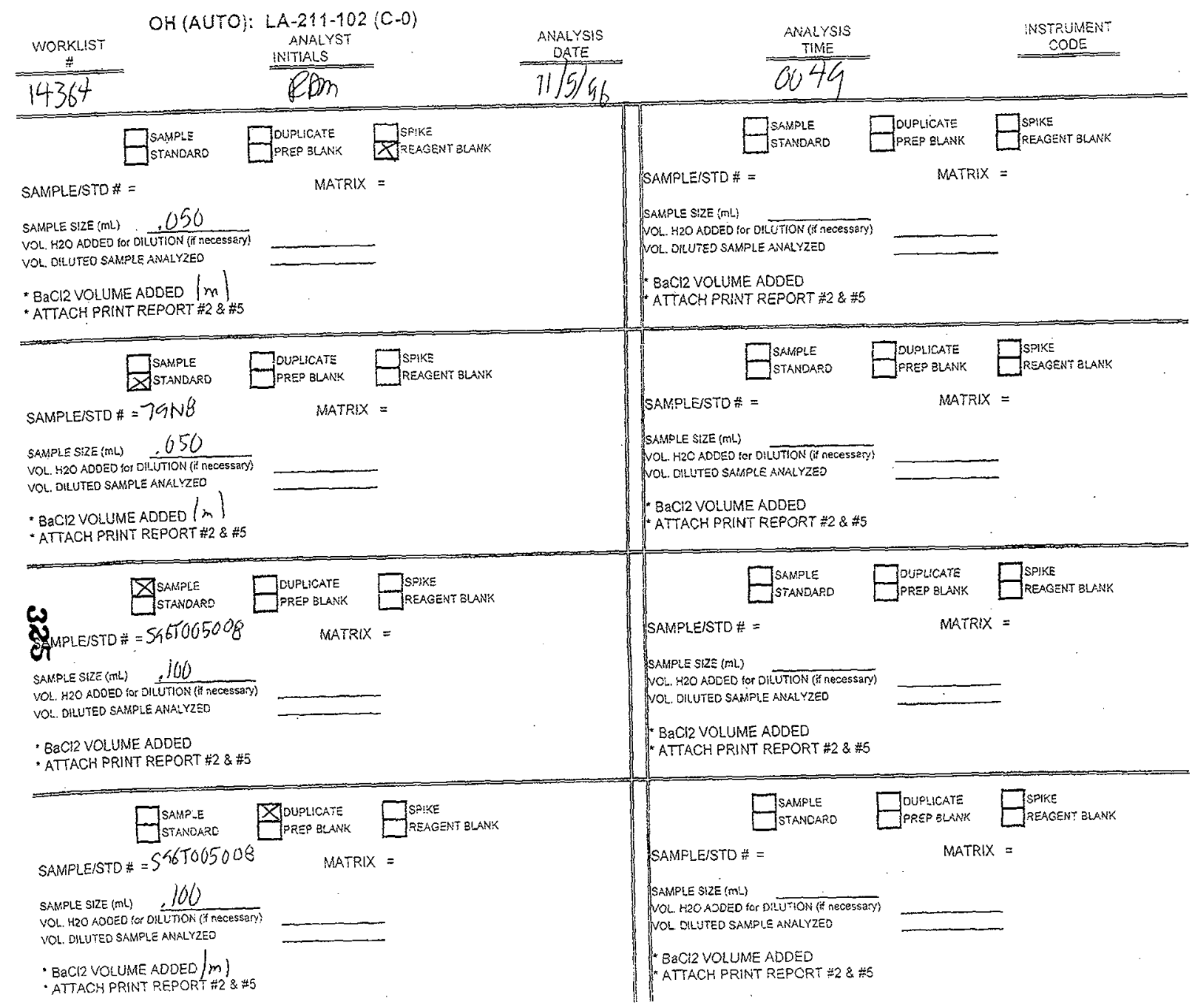




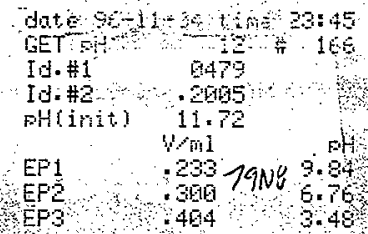

HNF-SD-WM-DP-214, REV. 0

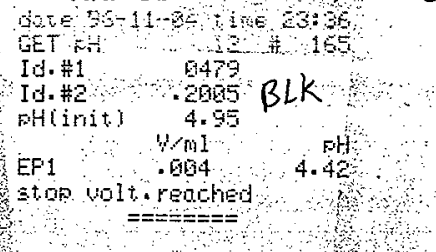

top volo redohed

$+2=====$

4

$\because 2$

$\therefore \therefore \because \because a$

date $90-11-04$ time 23

date $96-11-94$ time 2356

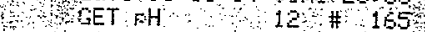

EET EH 12 \# 166

$1=1 \mathrm{gm} / \mathrm{div} \quad \mathrm{dPH}=1 \mathrm{div}$

x 1Gmlaiv . AsH=1/div start $Y$. $800 \mathrm{mI}$

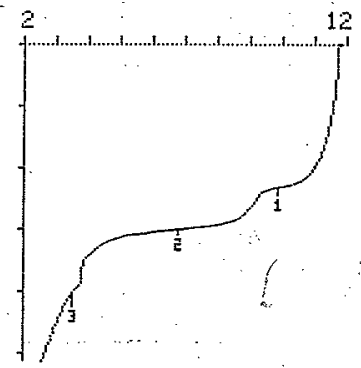

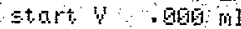

$2 \quad \therefore \quad 5$

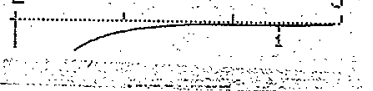

$\therefore-1$

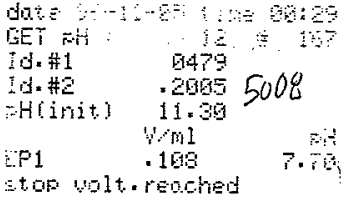

=ニニニニ=-=

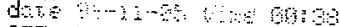

CET $\quad$ iz \# 168

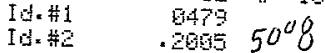

ohlinits 11.32 ohp

EF1 .114 6.97

Etor wol t. reoched

ニニニニニニニニ

nte $96-11-95$ t. ine 8014

LET OH 12 \# 189

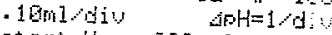

Etort $y$. $600 \mathrm{mI}$

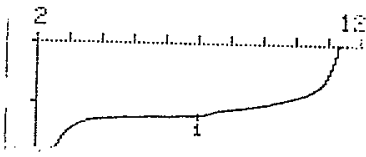




\begin{tabular}{|c|c|c|}
\hline \multicolumn{2}{|c|}{ OH (AUTO) : LA-211-102 (C-0) } & STANDARD \\
\hline S & Sample Size (mL) SS & 0.050 \\
\hline STANDARD & Concentration of $\mathrm{HNO}_{3}$ (Molarity) & 0.2005 \\
\hline Workistist & HNO3 Titrant at $\mathrm{OH}$ end-point in $\mathrm{mL}$ & 0.233 \\
\hline 14364 & Dilution Factor DF & \\
\hline STestoode & Concentration of $\mathrm{OH}$ in Sample (Molarity) & $9.34 \mathrm{E}-01$ \\
\hline $\mathrm{OH}-01$ & $\mathrm{OH}$ in Sample in $\mu \mathrm{g} / \mathrm{m} \mathrm{L}$ (PPM) & $1.59 E+04$ \\
\hline \multirow{2}{*}{\multicolumn{3}{|c|}{ LIOUID }} \\
\hline \multirow{2}{*}{\multicolumn{3}{|c|}{ LIQUID }} \\
\hline & & \\
\hline \multicolumn{3}{|l|}{ STD } \\
\hline \multicolumn{3}{|l|}{ I TStrumenteode } \\
\hline \multicolumn{3}{|l|}{ PHO1 } \\
\hline 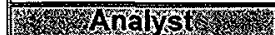 & \multirow{4}{*}{\multicolumn{2}{|c|}{$\begin{array}{l}\text { OH Molarity }=\left((\mathrm{mL} \text { HNO3 })^{*}(\mathrm{M} H \mathrm{HNO})\right) / \text { Sample Size in } \mathrm{mL} \mathrm{x}^{*} \text { Dilution Factor } \\
\mathrm{OH} \text { in } \mu \mathrm{g} / \mathrm{mL}=(\mathrm{OH} \text { MOLARITY })^{*}(17.0 \mathrm{~g} / \mathrm{mole})^{*}((1000000 \mu \mathrm{g} / \mathrm{g}) /(1000 \mathrm{~mL} / \mathrm{L}))\end{array}$}} \\
\hline RDM & & \\
\hline 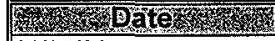 & & \\
\hline \multirow{2}{*}{$\frac{11 / 05 / 96}{11 / 2}$} & & \\
\hline & & STANDARD \\
\hline \multirow[t]{2}{*}{$12: 49 \mathrm{AM}$} & Concentration of $\mathrm{OH}$ in Sample (Molarity) & 9.34E-01 \\
\hline & $\mathrm{OH}$ in Sample in $\mu \mathrm{g} / \mathrm{mL}$ (PPM) & $1.59 \mathrm{E}+04$ \\
\hline
\end{tabular}

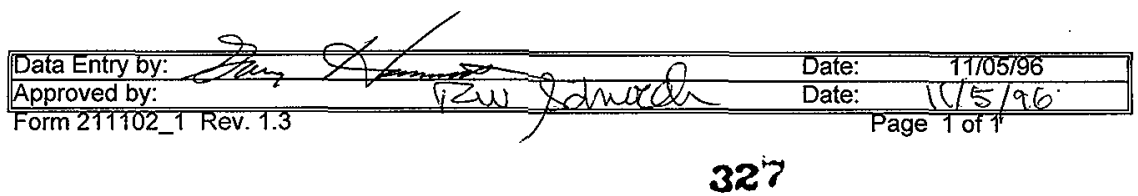




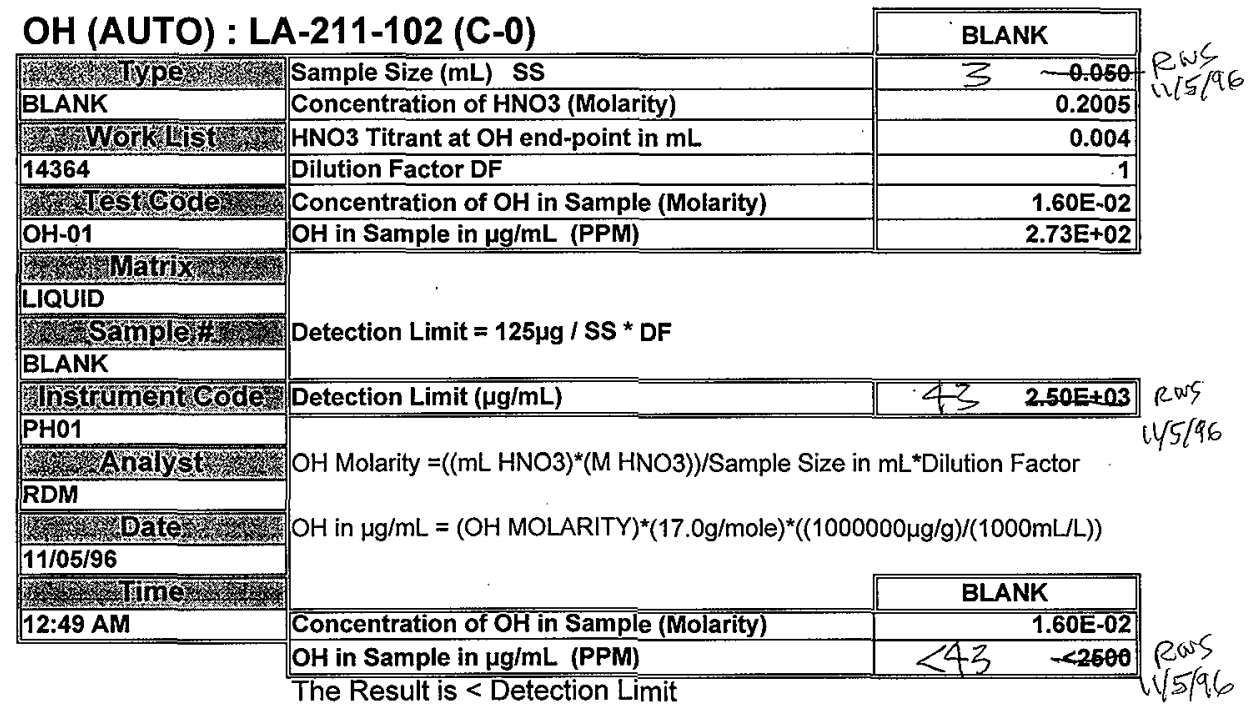

Data Entry by: Approved by: Form 217102_1 Rev. 1.3 


\begin{tabular}{|c|c|c|}
\hline \multicolumn{2}{|c|}{ OH (AUTO) : LA-211-102 (C-0) } & SAMPLE \\
\hline 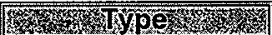 & Sample Size (mL) SS & $\mathbf{0 . 1 0 0}$ \\
\hline SAMPLE & Concentration of HNO3 (Molarity) & 0.2005 \\
\hline 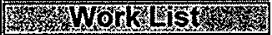 & HNO3 Titrant at $\mathrm{OH}$ end-point in $\mathrm{mL}$ & 0.108 \\
\hline $14364-2$ & Dilution Factor DF & \\
\hline Fis & Concentration of $\mathrm{OH}$ in Sample (Molarity) & 2.17E-01 \\
\hline $\mathrm{OH}-01$ & $\mathrm{OH}$ in Sample in $\mu \mathrm{g} / \mathrm{mL}$ (PPM) & $3.68 \mathrm{E}+03$ \\
\hline \multirow{2}{*}{ LIQUID } & \multirow{4}{*}{ Detection Limit $=125 \mu \mathrm{g} / \mathrm{SS} * \mathrm{DF}$} & \\
\hline & & \\
\hline Sampler & & \\
\hline $\begin{array}{l}\text { Sampler } \\
\text { S96T005008 }\end{array}$ & & \\
\hline \multirow{2}{*}{ 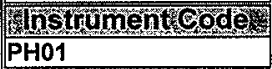 } & Detection Limit ( $\mu \mathrm{g} / \mathrm{mL}$ ) & $1.25 \mathrm{E}+03$ \\
\hline & \multirow{5}{*}{\multicolumn{2}{|c|}{$\begin{array}{l}\mathrm{OH} \text { Molarity }=\left((\mathrm{mL} \text { HNO3 })^{*}(\mathrm{M} H N O 3)\right) / \text { Sample Size in } \mathrm{mL} L^{*} \text { Dilution Factor } \\
\mathrm{OH} \text { in } \mu \mathrm{g} / \mathrm{mL}=(\mathrm{OH} \text { MOLARITY })^{*}(17.0 \mathrm{~g} / \mathrm{mole})^{*}((1000000 \mu \mathrm{g} / \mathrm{g}) /(1000 \mathrm{~mL} / \mathrm{L}))\end{array}$}} \\
\hline \multirow{2}{*}{ RDM } & & \\
\hline & & \\
\hline \multirow{2}{*}{$\begin{array}{l}\text { 18 Date } \\
11 / 05 / 96 \\
\end{array}$} & & \\
\hline & & \\
\hline \multirow{3}{*}{$\begin{array}{l}\text { 12:49 AM } \\
\end{array}$} & & SAMPLE \\
\hline & Concentration of OH in Sample (Molarity) & 2.17E-01 \\
\hline & $\mathrm{OH}$ in Sample in $\mu \mathrm{g} / \mathrm{mL}$ (PPM) & $3.68 \mathrm{E}+03$ \\
\hline
\end{tabular}




\begin{tabular}{|c|c|c|}
\hline \multicolumn{2}{|c|}{ OH (AUTO) : LA-211-102 (C-0) } & DUPLICATE \\
\hline Wha & Sample Size (mL) SS & 0.100 \\
\hline DUPLICATE & Concentration of HNO3 (Molarity) & 0.2005 \\
\hline Worrulst & HNO3 Titrant at OH end-point in $\mathrm{mL}$ & 0.114 \\
\hline 14364 & Dilution Factor DF & 1 \\
\hline Thesteode & Concentration of $\mathrm{OH}$ in Sample (Molarity) & 2.29E-01 \\
\hline $\mathrm{OH}-01$ & $\mathrm{OH}$ in Sample in $\mu \mathrm{g} / \mathrm{mL}$ (PPM) & $3.89 \mathrm{E}+03$ \\
\hline \multirow{2}{*}{\begin{tabular}{|l} 
Mattix \\
LIQUID
\end{tabular}} & \multirow{4}{*}{ Detection Limit $=125 \mu \mathrm{g} / \mathrm{SS}$ * DF } & \\
\hline & & \\
\hline 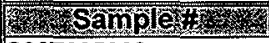 & & \\
\hline \multirow{2}{*}{$\begin{array}{l}5967005008 \\
\text { Thstrument }\end{array}$} & & \\
\hline & Detection Limit $(\mu \mathrm{g} / \mathrm{mL})$ & $1.25 \mathrm{E}+03$ \\
\hline \multirow{2}{*}{ PH01 } & \multirow{5}{*}{\multicolumn{2}{|c|}{$\begin{array}{l}\mathrm{OH} \text { Molarity }=\left((\mathrm{mL} \text { HNO3 })^{*}(\mathrm{M} H \mathrm{HO})\right) / \text { Sample Size in } \mathrm{mL}^{*} \text { Dilution Factor } \\
\mathrm{OH} \text { in } \mu \mathrm{g} / \mathrm{mL}=(\mathrm{OH} \text { MOLARITY })^{*}(17.0 \mathrm{~g} / \mathrm{mole})^{*}((1000000 \mu \mathrm{g} / \mathrm{g}) /(1000 \mathrm{~mL} / \mathrm{L}))\end{array}$}} \\
\hline & & \\
\hline \multirow{2}{*}{ RDM } & & \\
\hline & & \\
\hline $11 / 05 / 96$ & & \\
\hline \multirow{3}{*}{$\begin{array}{l}\text { 12:49 AM } \\
\end{array}$} & & DUPLICATE \\
\hline & Concentration of OH in Sample (Molarity) & $2.29 \mathrm{E}-01$ \\
\hline & $\mathrm{OH}$ in Sample in $\mu \mathrm{g} / \mathrm{mL}$ (PPM) & $3.89 \mathrm{E}+03$ \\
\hline
\end{tabular}

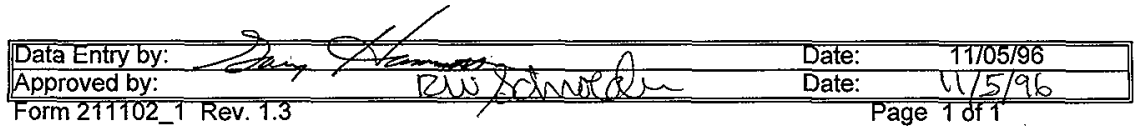




\section{Analyst: BAW Instrument: PH01 we 06695 Book\# 79 N8}

Method: LA-211-102 Rev/Mod C-O

Worklist Comment: AW-105 OH. RCJ

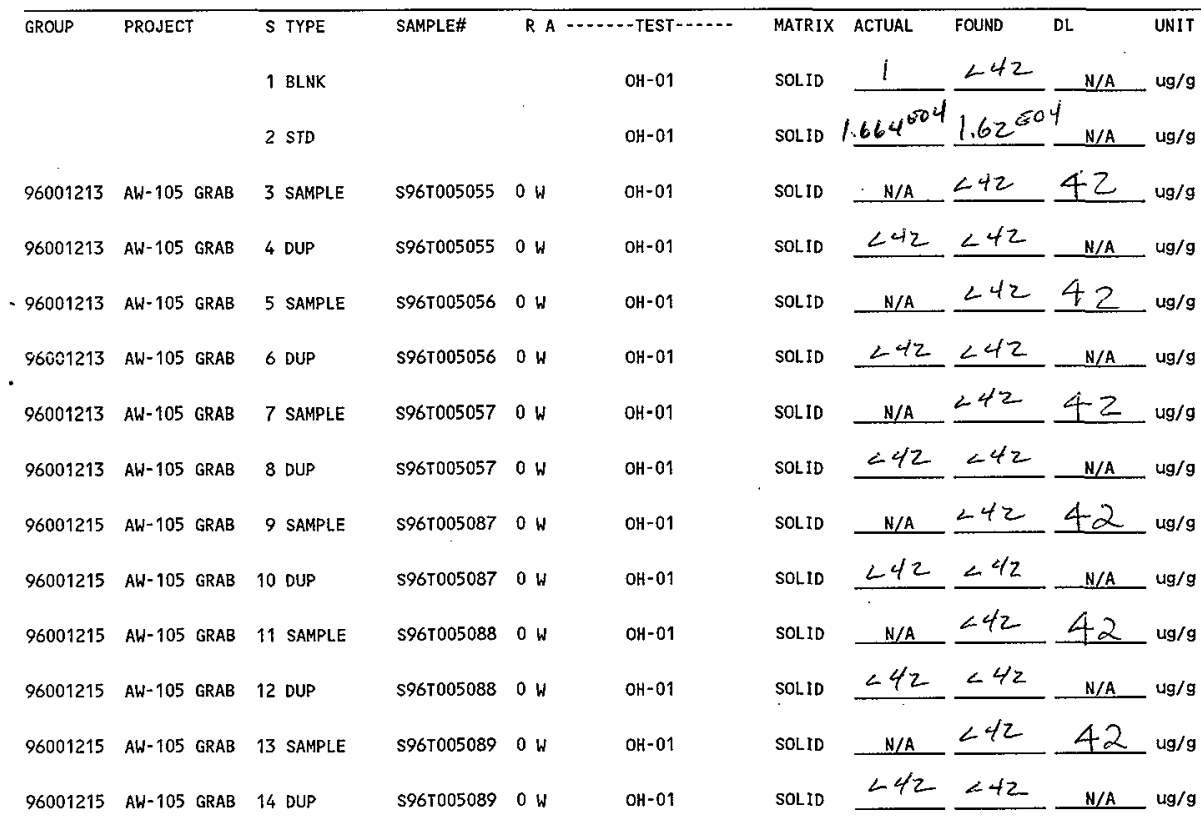

\section{Final page for worklist \# 14789}

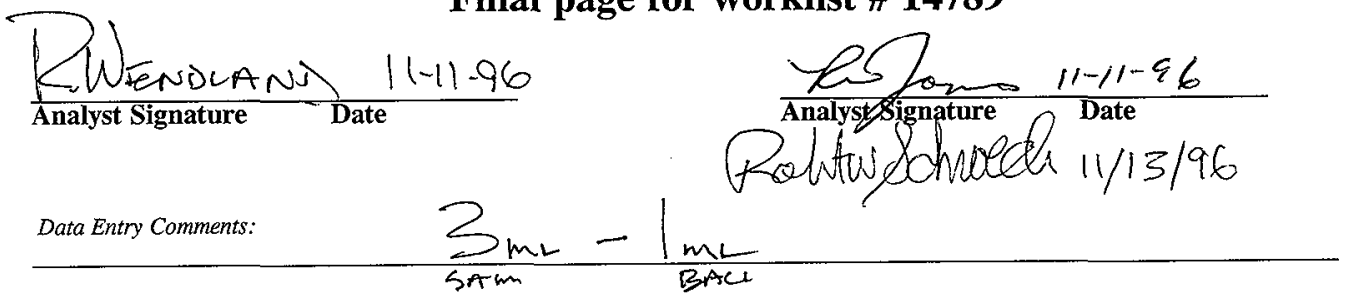

Units shown for $Q C$ (SPK \& STD) may not reflect the actual units. $D L=$ Detection Limit, $S=$ Worklist Slot Number, $R=$ Replicate Number, $A=$ Aliquot Code. 


\section{OH ANALYSIS}

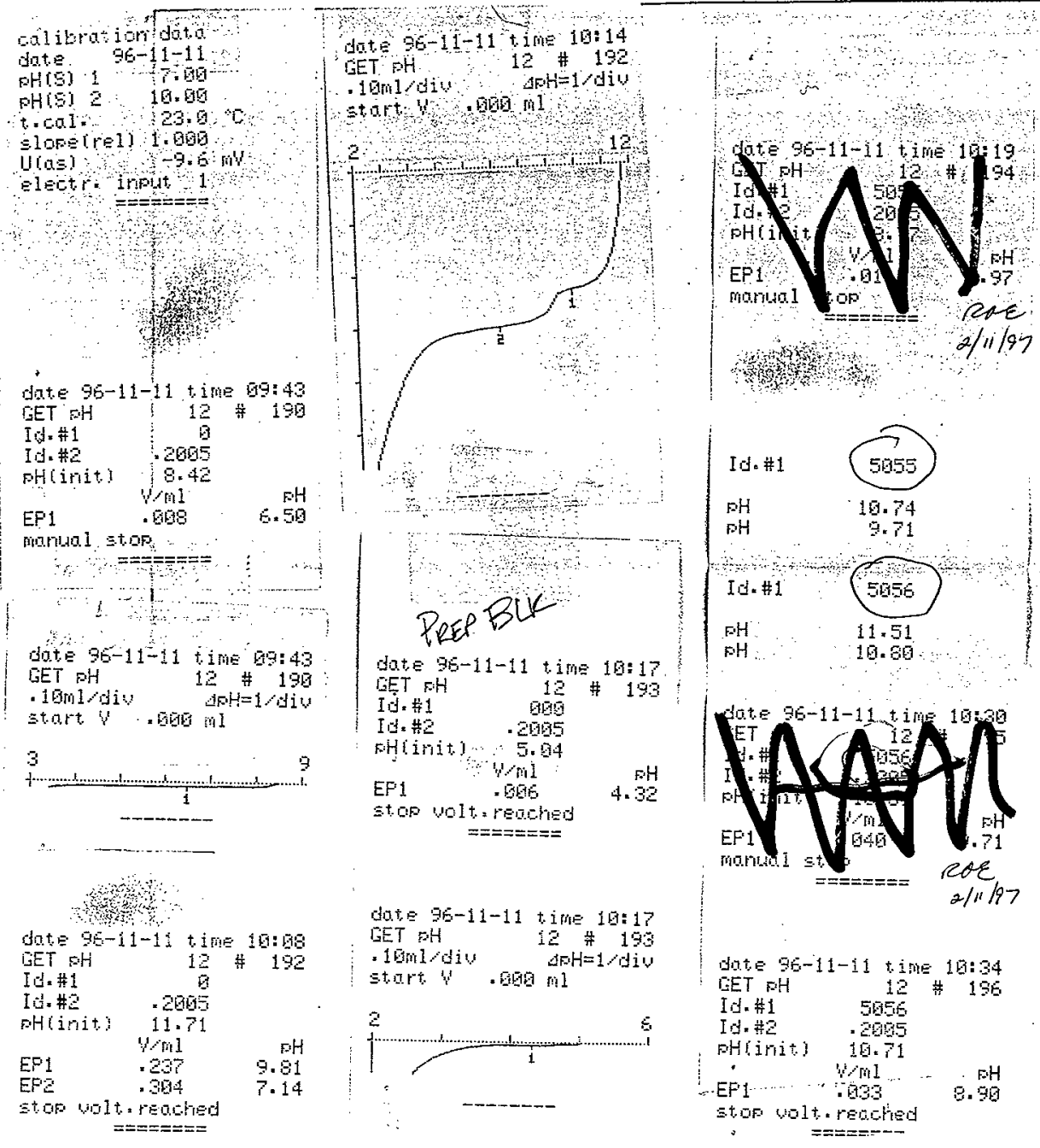




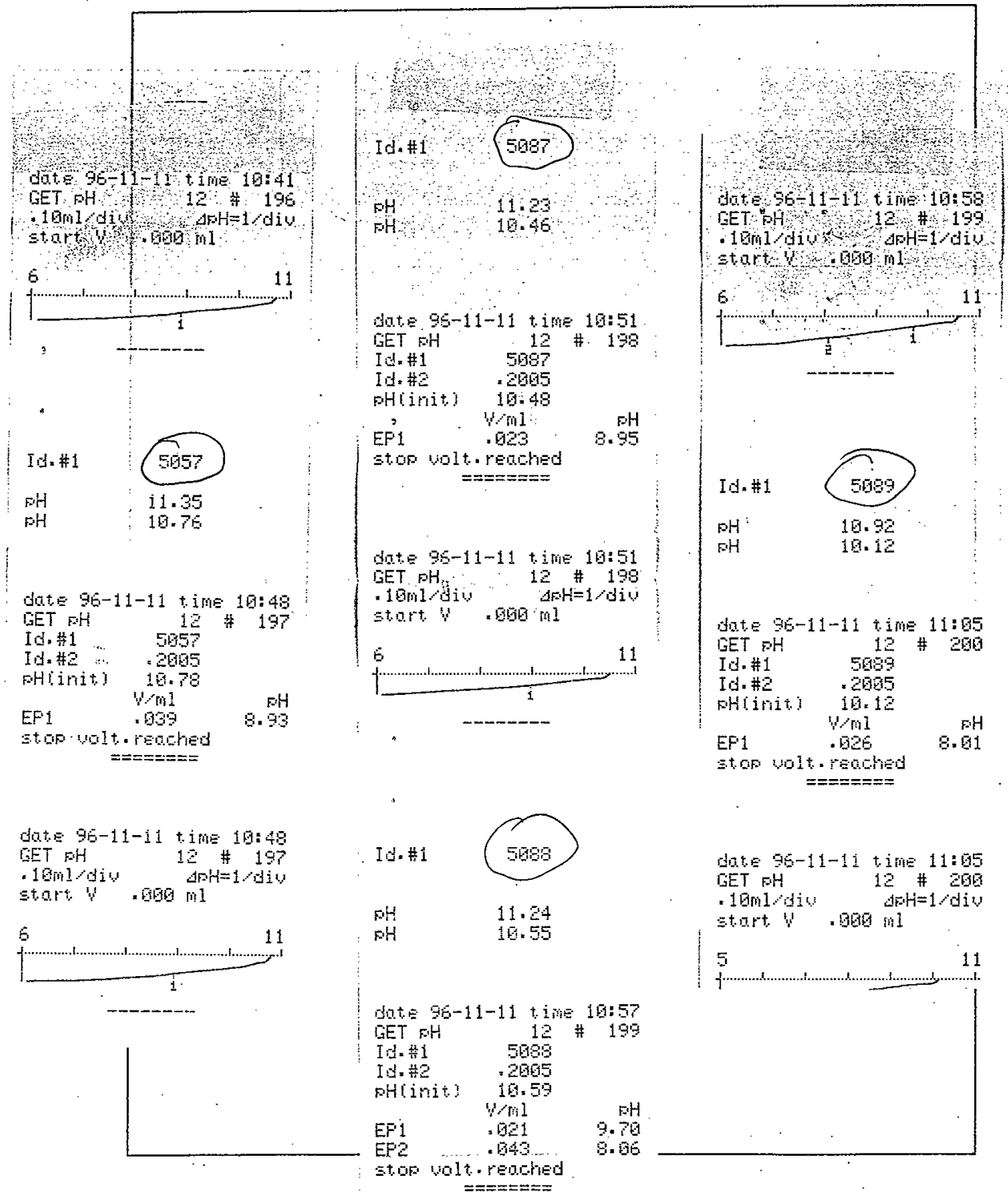


PLACE ANALYTICAL CARD IN BOX BELOW OR ATTACH TRAVELER

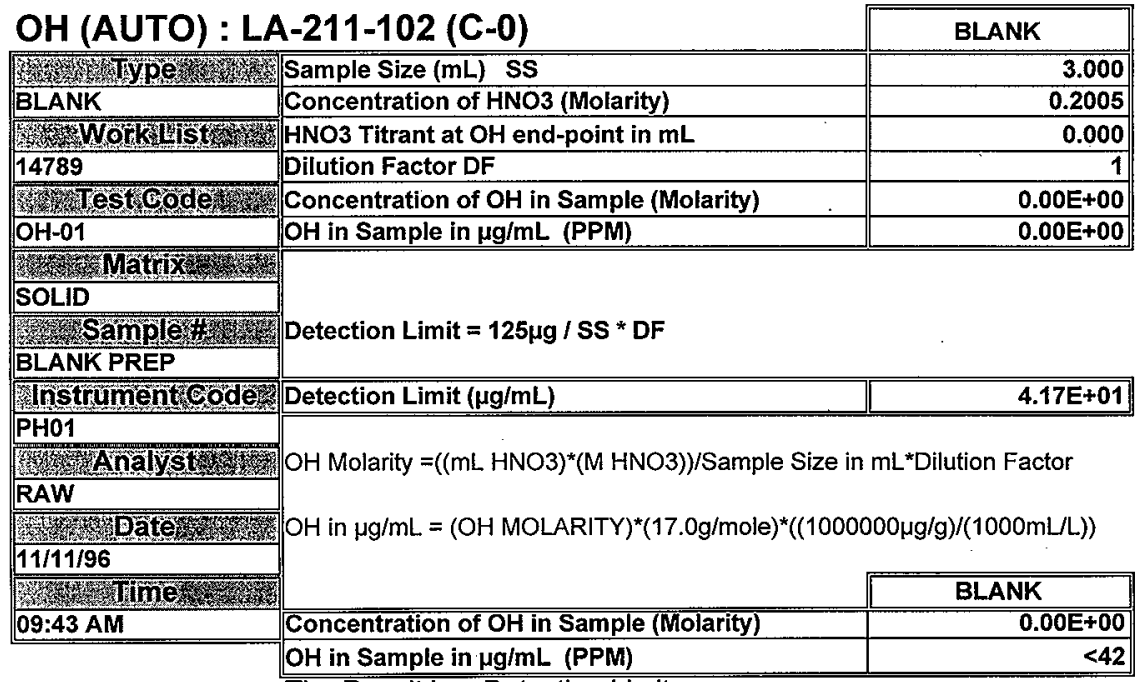

The Result is < Detection Limit 


\begin{tabular}{|c|c|c|}
\hline \multicolumn{2}{|c|}{ OH (AUTO) : LA-211-102 (C-0) } & STANDARD \\
\hline Yrivis & Sample Size $(\mathrm{mL})$ SS & 0.050 \\
\hline STANDARD & Concentration of HNO3 (Molarity) & 0.2005 \\
\hline 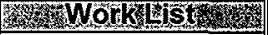 & HNO3 Titrant at $\mathrm{OH}$ end-point in $\mathrm{mL}$ & 0.237 \\
\hline 14789 & Dilution Factor DF & \\
\hline Wh & Concentration of OH in Sample (Molarity) & 9.50E-01 \\
\hline $\mathrm{OH}-01$ & $\mathrm{OH}$ in Sample in $\mu \mathrm{g} / \mathrm{mL}$ (PPM) & $1.62 \mathrm{E}+04$ \\
\hline \multirow{2}{*}{\multicolumn{3}{|c|}{ TIQUID }} \\
\hline & & \\
\hline \multicolumn{3}{|l|}{ Sample } \\
\hline \multicolumn{3}{|l|}{$79 N 8$} \\
\hline \multicolumn{3}{|l|}{ Instrumenteode } \\
\hline \multicolumn{3}{|l|}{ PHO1 } \\
\hline \multirow{2}{*}{\multicolumn{3}{|c|}{ RAW }} \\
\hline & & \\
\hline \multirow{2}{*}{\multicolumn{3}{|c|}{ 11/11/96 $\mathrm{OH}$ in $\mu \mathrm{g} / \mathrm{mL}=(\mathrm{OH} \text { MOLARITY })^{*}(17.0 \mathrm{~g} / \mathrm{mole})^{\star}((1000000 \mu \mathrm{g} / \mathrm{g}) /(1000 \mathrm{~mL} /))$}} \\
\hline & & \\
\hline \multicolumn{3}{|c|}{ 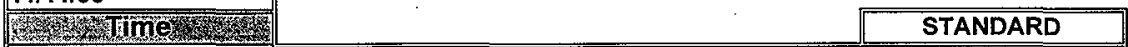 } \\
\hline \multirow[t]{2}{*}{$09: 43 \mathrm{AM}$} & Concentration of OH in Sample (Molarity) & $9.50 \mathrm{E}-01$ \\
\hline & $\mathrm{OH}$ in Sample in $\mu \mathrm{g} / \mathrm{mL}$ (PPM) & $1.62 \mathrm{E}+04$ \\
\hline
\end{tabular}

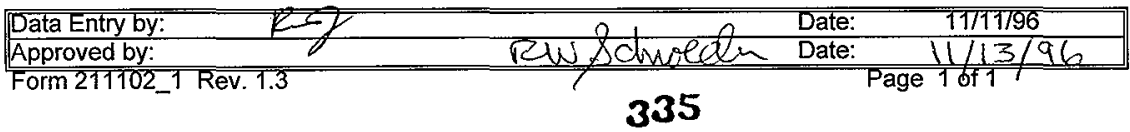




\begin{tabular}{|c|c|c|}
\hline \multicolumn{2}{|c|}{ OH (AUTO) : LA-211-102 (C-0) } & SAMPLE \\
\hline 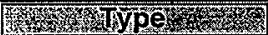 & Sample Size $(\mathrm{mL})$ SS & 3.000 \\
\hline SAMPLE & Concentration of HNO3 (Molarity) & 0.2005 \\
\hline Workists & HNO3 Titrant at $\mathrm{OH}$ end-point in $\mathrm{mL}$ & 0.000 \\
\hline 14789 & Dilution Factor DF & 1 \\
\hline hestreded & Concentration of $\mathrm{OH}$ in Sample (Molarity) & $0.00 \mathrm{E}+00$ \\
\hline $\mathrm{OH}-01$ & $\mathrm{OH}$ in Sample in $\mu \mathrm{g} / \mathrm{mL}$ (PPM) & $0.00 E+00$ \\
\hline \multirow{2}{*}{$\begin{array}{l}\text { SOLID } \\
\text { SOLAR }\end{array}$} & \multirow{4}{*}{ Detection Limit $=125 \mu \mathrm{g} / \mathrm{SS}$ * DF } & \\
\hline & & \\
\hline Samplery & & \\
\hline \multirow{2}{*}{$\begin{array}{l}\text { S96T005055 } \\
\text { instrut }\end{array}$} & & \\
\hline & Detection Limit $(\mathrm{\mu g} / \mathrm{mL})$ & $4.17 E+01$ \\
\hline PH01 & \multirow{5}{*}{\multicolumn{2}{|c|}{$\begin{array}{l}\text { OH Molarity }=\left((\mathrm{mL} H \mathrm{HO} 3)^{*}(\mathrm{M} H \mathrm{HO})\right) / \text { Sample Size in } \mathrm{mL}{ }^{*} \text { Dilution Factor } \\
\mathrm{OH} \text { in } \mu \mathrm{g} / \mathrm{mL}=(\mathrm{OH} \text { MOLARITY })^{*}(17.0 \mathrm{~g} / \mathrm{mole})^{*}((1000000 \mu \mathrm{g} / \mathrm{g}) /(1000 \mathrm{~mL} / \mathrm{L}))\end{array}$}} \\
\hline Analyst & & \\
\hline \multirow{4}{*}{ RAW } & & \\
\hline & & \\
\hline & & \\
\hline & & SAMPLE \\
\hline 09:43 AM & Concentration of $\mathrm{OH}$ in Sample (Molarity) & $0.00 \mathrm{E}+00$ \\
\hline & $\mathrm{OH}$ in Sample in $\mu \mathrm{g} / \mathrm{mL}$ (PPM) & $<42$ \\
\hline
\end{tabular}

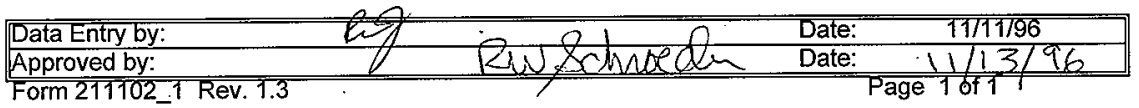
Form 211702_1 Rev. 1.3 
PLACE ANALYTICAL CARD IN BOX BELOW OR ATTACH TRAVELER

\begin{tabular}{|c|c|c|}
\hline \multicolumn{2}{|c|}{ OH (AUTO) : LA-211-102 (C-0) } & SAMPLE \\
\hline ris & Sample Size (mL) SS & 3.000 \\
\hline SAMPLE & Concentration of $\mathrm{HNO} 3$ (Molarity) & 0.2005 \\
\hline SWOKLISt & HNO3 Titrant at $\mathrm{OH}$ end-point in $\mathrm{mL}$ & 0.000 \\
\hline 14789 & Dilution Factor DF & 1 \\
\hline 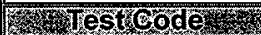 & Concentration of $\mathrm{OH}$ in Sample (Molarity) & $0.00 \mathrm{E}+00$ \\
\hline $\mathrm{OH}-01$ & $\mathrm{OH}$ in Sample in $\mu \mathrm{g} / \mathrm{mL}$ (PPM) & $0.00 \mathrm{E}+00$ \\
\hline
\end{tabular}

Kr.

SOLID

\begin{tabular}{|c|c|}
\hline \multirow{2}{*}{\multicolumn{2}{|c|}{ Sam }} \\
\hline & \\
\hline Instrumente & \\
\hline PHO1 & \\
\hline rralyst & \\
\hline RAW & \\
\hline ax & \\
\hline $11 / 11 / 96$ & \\
\hline 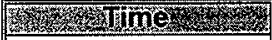 & \\
\hline 09:43 AM & \\
\hline
\end{tabular}

Detection Limit $=125 \mu \mathrm{g} / \mathrm{SS}$ * DF

Detection Limit $(\mu \mathrm{g} / \mathrm{mL})$

4.17E+01

$\mathrm{OH}$ Molarity $=\left((\mathrm{mL} H N O 3)^{*}(\mathrm{M} \mathrm{HNO3})\right) /$ Sample Size in $\mathrm{mL}$ * Dilution Factor

$\mathrm{OH}$ in $\mu \mathrm{g} / \mathrm{mL}=(\mathrm{OH} \text { MOLARITY })^{*}\left(17.0 \mathrm{~g} / \mathrm{mole}^{*}\right)^{*}((1000000 \mu \mathrm{g} / \mathrm{g}) /(1000 \mathrm{~mL} / \mathrm{L}))$

Concentration of OH in Sample (Molarity)

OH in Sample in $\mu \mathrm{g} / \mathrm{mL}$ (PPM)

SAMPLE

The Result is < Detection Limit

\begin{tabular}{|r|r|}
\cline { 2 - 2 } & \multicolumn{1}{|c|}{ SAMPLE } \\
\hline & $0.00 \mathrm{E}+00$ \\
\hline & $<42$ \\
\hline
\end{tabular}

1


HNF-SD-WM-DP-214, REV. 0

PLACE ANALYTICAL CARD IN BOX BELOW OR ATTACH TRAVELER

\begin{tabular}{|c|c|c|}
\hline \multirow{2}{*}{\multicolumn{2}{|c|}{ OH (AUTO) : LA-211-102 (C-0) }} & \multirow[b]{2}{*}{ UP } \\
\hline & & \\
\hline 被 & Sample Size $(\mathrm{mL})$ SS & 3.000 \\
\hline DUP & Concentration of $\mathrm{HNO} 3$ (Molarity) & 0.2005 \\
\hline 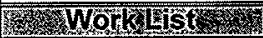 & HNO3 Titrant at $\mathrm{OH}$ end-point in $\mathrm{mL}$ & 0.000 \\
\hline 14789 & Dilution Factor DF. & 1 \\
\hline 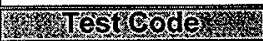 & Concentration of $\mathrm{OH}$ in Sample (Molarity) & $0.00 \mathrm{E}+00$ \\
\hline $\mathrm{OH}-01$ & $\mathrm{OH}$ in Sample in $\mu \mathrm{g} / \mathrm{mL}$ (PPM) & $0.00 \mathrm{E}+00$ \\
\hline \multirow{2}{*}{$\begin{array}{l}\text { SOLID } \\
\text { SOLATIX }\end{array}$} & \multirow{4}{*}{ Detection Limit $=125 \mu \mathrm{g} / \mathrm{SS}$ * DF } & \\
\hline & & \\
\hline Wrample & & \\
\hline \multirow{2}{*}{$\begin{array}{l}\text { S96T005056 } \\
\text { Whstrumenteode }\end{array}$} & & \\
\hline & Detection Limit $(\mu \mathrm{g} / \mathrm{mL})$ & 4.17E+01 \\
\hline $\mathrm{PH} 01$ & \multirow{5}{*}{\multicolumn{2}{|c|}{$\begin{array}{l}\mathrm{OH} \text { Molarity }=\left((\mathrm{mL} \text { HNO3 })^{*}(\mathrm{M} \text { HNO3) }) / \text { Sample Size in } \mathrm{mL}{ }^{*} \text { Dilution Factor }\right. \\
\mathrm{OH} \text { in } \mu \mathrm{g} / \mathrm{mL}=(\mathrm{OH} \text { MOLARITY })^{*}(17.0 \mathrm{~g} / \mathrm{mole})^{*}((100000 \mu \mathrm{g} / \mathrm{g}) /(1000 \mathrm{~mL} / \mathrm{L}))\end{array}$}} \\
\hline 36 & & \\
\hline RAW & & \\
\hline 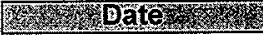 & & \\
\hline \multirow{2}{*}{$11 / 11 / 96$} & & \\
\hline & & UP \\
\hline \multirow[t]{2}{*}{$09: 43$ AM } & Concentration of $\mathrm{OH}$ in Sample (Molarity) & $0.00 E+00$ \\
\hline & $\mathrm{OH}$ in Sample in $\mu \mathrm{g} / \mathrm{mL}$ (PPM) & $<42$ \\
\hline
\end{tabular}

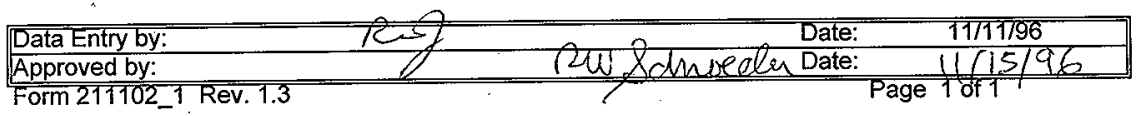


PLACE ANALYTICAL CARD IN BOX BELOW OR ATTACH TRAVELER

\begin{tabular}{|c|c|c|}
\hline \multicolumn{2}{|c|}{ OH (AUTO) : LA-211-102 (C-0) } & \multirow{2}{*}{$\begin{array}{l}\text { SAMPLE } \\
3000\end{array}$} \\
\hline 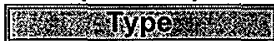 & Sample Size (mL) SS & \\
\hline SAMPLE & Concentration of $\mathrm{HNO}_{3}$ (Molarity) & 0.2005 \\
\hline 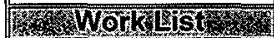 & HNO3 Titrant at $\mathrm{OH}$ end-point in $\mathrm{mL}$ & 0.000 \\
\hline 14789 & Dilution Factor DF & 1 \\
\hline 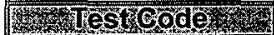 & Concentration of $\mathrm{OH}$ in Sample (Molarity) & $0.00 E+00$ \\
\hline $\mathrm{OH}-01$ & $\mathrm{OH}$ in Sample in $\mu \mathrm{g} / \mathrm{mL}$ (PPM) & $0.00 E+00$ \\
\hline
\end{tabular}

W.

SOLID

3.

S96T005057

Instriment \&oded Detection Limit $(\mu \mathrm{g} / \mathrm{mL})$

4.17E+01

\section{PH01}

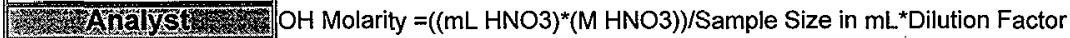

RAW

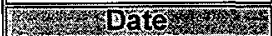

$11 / 11 / 96$

For

09:43 AM

Concentration of $\mathrm{OH}$ in Sample (Molarity)

$\mathrm{OH}$ in Sample in $\mu \mathrm{g} / \mathrm{mL}$ (PPM)

$\mathrm{OH}$ in $\mu \mathrm{g} / \mathrm{mL}=(\mathrm{OH} \text { MOLARITY })^{\star}(17.0 \mathrm{~g} / \mathrm{mole})^{\star}((1000000 \mu \mathrm{g} / \mathrm{g}) /(1000 \mathrm{~mL} / \mathrm{L}))$

The Result is < Detection Limit 
HNF-SD-WM-DP-214, REV. 0

PLACE ANALYTICAL CARD IN BOX BELOW OR ATTACH TRAVELER

\begin{tabular}{|c|c|c|}
\hline \multirow{2}{*}{\multicolumn{2}{|c|}{ OH (AUTO) : LA-211-102 (C-0) }} & \multirow[b]{2}{*}{ UP } \\
\hline & & \\
\hline 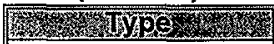 & Sample Size $(\mathrm{mL})$ SS & 3.000 \\
\hline DUP & Concentration of $\mathrm{HNO} 3$ (Molarity) & 0.2005 \\
\hline 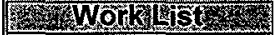 & HNO3 Titrant at $\mathrm{OH}$ end-point in $\mathrm{mL}$ & 0.000 \\
\hline 14789 & Dilution Factor DF & \\
\hline W Nesteder & Concentration of $\mathrm{OH}$ in Sample (Molarity) & $0.00 E+00$ \\
\hline $\mathrm{OH}-01$ & $\mathrm{OH}$ in Sample in $\mu \mathrm{g} / \mathrm{mL}$ (PPM) & $0.00 \mathrm{E}+00$ \\
\hline \multirow{2}{*}{$\begin{array}{l}\text { SOLID } \\
\text { Sathx }\end{array}$} & \multirow{4}{*}{ Detection Limit $=125 \mu \mathrm{g} / \mathrm{SS}$ * DF } & \\
\hline & & \\
\hline 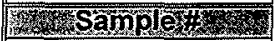 & & \\
\hline \multirow{2}{*}{$\begin{array}{l}\text { S96T005057 } \\
\text { Instrument Goded }\end{array}$} & & \\
\hline & Detection Limit $(\mu \mathrm{g} / \mathrm{mL})$ & 4.17E+01 \\
\hline $\mathrm{PH} 01$ & \multirow{5}{*}{\multicolumn{2}{|c|}{$\begin{array}{l}\mathrm{OH} \text { Molarity }=\left((\mathrm{mL} \text { HNO3 })^{*}(\mathrm{M} \text { HNO3) }) / \text { Sample Size in mL*Dilution Factor }\right. \\
\mathrm{OH} \text { in } \mu \mathrm{g} / \mathrm{mL}=(\mathrm{OH} \text { MOLARITY })^{*}(17.0 \mathrm{~g} / \mathrm{mole})^{*}((1000000 \mu \mathrm{g} / \mathrm{g}) /(1000 \mathrm{~mL} / \mathrm{L}))\end{array}$}} \\
\hline & & \\
\hline \multirow{2}{*}{$\begin{array}{l}\text { RAW } \\
\text { RW }\end{array}$} & & \\
\hline & & \\
\hline $11 / 11 / 96$ & & \\
\hline \multirow{2}{*}{ 09:43 AM } & & SUP \\
\hline & Concentration of $\mathrm{OH}$ in Sample (Molarity) & $0.00 \mathrm{E}+00$ \\
\hline & $\mathrm{OH}$ in Sample in $\mu \mathrm{g} / \mathrm{mL}$ (PPM) & $<42$ \\
\hline
\end{tabular}


PLACE ANALYTICAL CARD IN BOX BELOW OR ATTACH TRAVELER

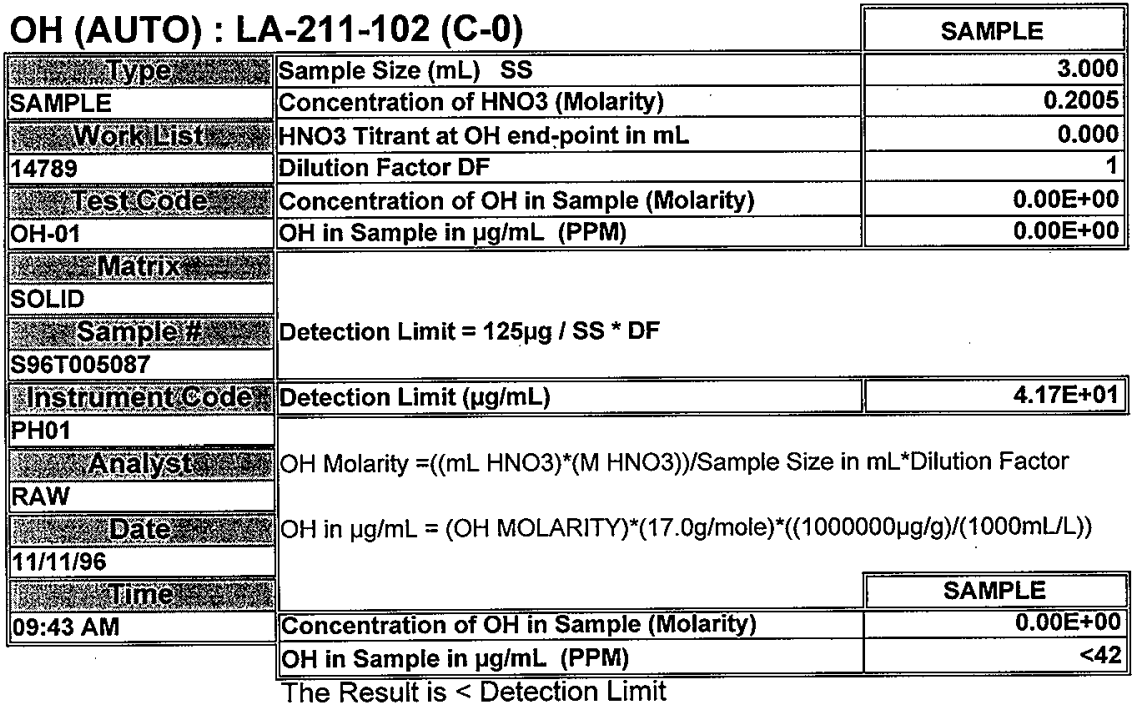

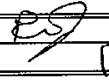


PLACE ANALYTICAL CARD IN BOX BELOW OR ATTACH TRAVELER

\begin{tabular}{|c|c|c|}
\hline \multirow{2}{*}{\multicolumn{2}{|c|}{ OH (AUTO) : LA-211-102 (C-0) }} & \multirow[b]{2}{*}{$\overline{\text { DUP }}$} \\
\hline & & \\
\hline 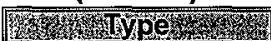 & Sample Size (mL) SS & 3.000 \\
\hline DUP & Concentration of $\mathrm{HNO} 3$ (Molarity) & 0.2005 \\
\hline 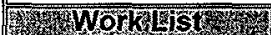 & HNO3 Titrant at $\mathrm{OH}$ end-point in $\mathrm{mL}$ & 0.000 \\
\hline 14789 & Dilution Factor DF & \\
\hline 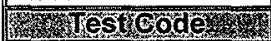 & Concentration of $\mathrm{OH}$ in Sample (Molarity) & $0.00 \mathrm{E}+00$ \\
\hline $\mathrm{OH}-01$ & $\mathrm{OH}$ in Sample in $\mu \mathrm{g} / \mathrm{mL}$ (PPM) & $0.00 \mathrm{E}+00$ \\
\hline \multirow{2}{*}{$\begin{array}{l}\text { SOLID } \\
\text { SOLAR }\end{array}$} & \multirow{4}{*}{ Detection Limit $=125 \mu \mathrm{g} / \mathrm{SS}$ * DF } & \\
\hline & & \\
\hline & & \\
\hline $\begin{array}{l}\text { Salmplent } \\
\text { S96T005087 }\end{array}$ & & \\
\hline Instrument Code & Detection Limit $(\mu \mathrm{g} / \mathrm{mL})$ & $4.17 \mathrm{E}+01$ \\
\hline $\mathrm{PH} 01$ & \multirow{3}{*}{\multicolumn{2}{|c|}{$\mathrm{OH}$ Molarity $=\left((\mathrm{mL} H \mathrm{HNO})^{*}(\mathrm{M} \mathrm{HNO})\right) /$ Sample Size in $\mathrm{mL}{ }^{*}$ Dilution Factor }} \\
\hline \multirow{2}{*}{ RAW } & & \\
\hline & & \\
\hline 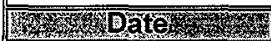 & \multicolumn{2}{|c|}{ 昜 $\mathrm{OH}$ in $\mu \mathrm{g} / \mathrm{mL}=(\mathrm{OH} \text { MOLARITY })^{*}(17.0 \mathrm{~g} / \mathrm{mole})^{*}((1000000 \mu \mathrm{g} / \mathrm{g}) /(1000 \mathrm{~mL} / \mathrm{L}))$} \\
\hline 11/11/96 & & \\
\hline \multirow{2}{*}{ 09:43 AM } & & DUP \\
\hline & Concentration of $\mathrm{OH}$ in Sample (Molarity) & $0.00 E+00$ \\
\hline & $\mathrm{OH}$ in Sample in $\mu \mathrm{g} / \mathrm{mL}$ (PPM) & $<42$ \\
\hline
\end{tabular}

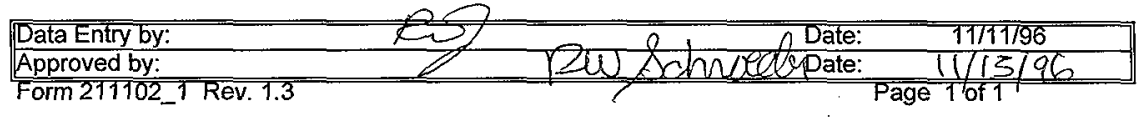




\begin{tabular}{|c|c|c|}
\hline \multicolumn{2}{|c|}{ OH (AUTO) : LA-211-102 (C-0) } & \multirow{2}{*}{ SAMPLE } \\
\hline 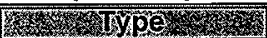 & Sample Size (mL) SS & \\
\hline SAMPLE & Concentration of HNO3 (Molarity) & 0.2005 \\
\hline 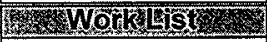 & HNO3 Titrant at $\mathrm{OH}$ end-point in $\mathrm{mL}$ & 0.000 \\
\hline 14789 & Dilution Factor DF & 1 \\
\hline 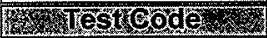 & Concentration of $\mathrm{OH}$ in Sample (Molarity) & $0.00 \mathrm{E}+00$ \\
\hline $\mathrm{OH}-01$ & $\mathrm{OH}$ in Sample in $\mu \mathrm{g} / \mathrm{mL}$ (PPM) & $0.00 \mathrm{E}+00$ \\
\hline $\begin{array}{l}\text { SOLID } \\
\text { SOLARX }\end{array}$ & \multirow{2}{*}{ Detection Limit $=125 \mu \mathrm{g} / \mathrm{SS}$ * DF } & . \\
\hline $\begin{array}{l}\text { Samplex } \\
\text { S96T005088 }\end{array}$ & & \\
\hline Iostrumentede & Detection Limit $(\mu \mathrm{g} / \mathrm{mL})$ & 4.17E+01 \\
\hline PH01 & \multirow{3}{*}{\multicolumn{2}{|c|}{$\mathrm{OH}$ Molarity $=\left((\mathrm{mL} \mathrm{HNO})^{\star}(\mathrm{M} \mathrm{HNO})\right) /$ Sample Size in $\mathrm{mL}^{*}$ Dilution Factor }} \\
\hline $\begin{array}{l}\text { RAW } \\
\text { RADalyst }\end{array}$ & & \\
\hline $\begin{array}{l}\text { Date } \\
11 / 11 / 96\end{array}$ & & \\
\hline (6) & & SAMPLE \\
\hline $09: 43 \mathrm{AM}$ & Concentration of $\mathrm{OH}$ in Sample (Molarity) & $0.00 \mathrm{E}+00$ \\
\hline & $\mathrm{OH}$ in Sample in $\mu \mathrm{g} / \mathrm{mL}$ (PPM) & $<42$ \\
\hline
\end{tabular}

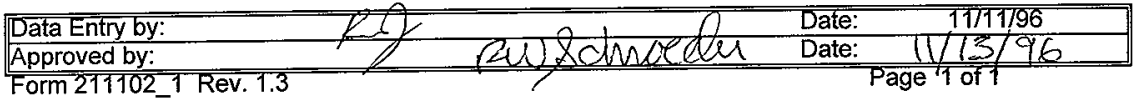


PLACE ANALYTICAL CARD IN BOX BELOW OR ATTACH TRAVELER

\begin{tabular}{|c|c|c|}
\hline \multicolumn{2}{|c|}{ OH (AUTO) : LA-211-102 (C-0) } & JuP \\
\hline 3. & Sample Size $(\mathrm{mL})$ SS & 3.000 \\
\hline DUP & Concentration of $\mathrm{HNO}_{3}$ (Molarity) & 0.2005 \\
\hline WorkILs & HNO3 Titrant at $\mathrm{OH}$ end-point in $\mathrm{mL}$ & 0.000 \\
\hline 14789 & Dilution Factor DF & 1 \\
\hline 26) & Concentration of $\mathrm{OH}$ in Sample (Molarity) & $0.00 \mathrm{E}+00$ \\
\hline $\mathrm{OH}-01$ & $\mathrm{OH}$ in Sample in $\mu \mathrm{g} / \mathrm{mL}$ (PPM) & $0.00 E+00$ \\
\hline \multirow{2}{*}{$\begin{array}{l}\text { Mathx } \\
\text { SOLID }\end{array}$} & \multirow[b]{3}{*}{ Detection Limit $=125 \mu \mathrm{g} / \mathrm{SS}$ * DF } & \\
\hline & & \\
\hline $\begin{array}{l}\text { Sample } \\
\text { S96T005088 }\end{array}$ & & \\
\hline Instrumentoode & Detection Limit $(\mu \mathrm{g} / \mathrm{mL})$ & 4.17E+01 \\
\hline \multirow{2}{*}{ PH01 } & \multirow{5}{*}{\multicolumn{2}{|c|}{$\begin{array}{l}\mathrm{OH} \text { Molarity }=\left((\mathrm{mL} H N O 3)^{*}(\mathrm{M} \mathrm{HNO})\right) / \text { Sample Size in } \mathrm{mL} \text { Dilution Factor } \\
\mathrm{OH} \text { in } \mu \mathrm{g} / \mathrm{mL}=(\mathrm{OH} \text { MOLARITY })^{*}(17.0 \mathrm{~g} / \mathrm{mole})^{*}((1000000 \mu \mathrm{g} / \mathrm{g}) /(1000 \mathrm{mLL}))\end{array}$}} \\
\hline & & \\
\hline RAW & & \\
\hline 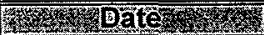 & & \\
\hline $11 / 11 / 96$ & & \\
\hline S1 & & DUP \\
\hline \multirow{2}{*}{$09: 43$ AM } & Concentration of $\mathrm{OH}$ in Sample (Molarity) & $0.00 \mathrm{E}+00$ \\
\hline & $\mathrm{OH}$ in Sample in $\mu \mathrm{g} / \mathrm{mL}$ (PPM) & $<42$ \\
\hline
\end{tabular}

L1

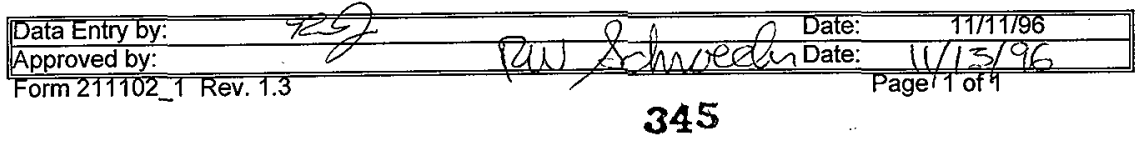




\begin{tabular}{|c|c|c|}
\hline \multicolumn{2}{|c|}{ OH (AUTO) : LA-211-102 (C-0) } & \multirow{2}{*}{$\frac{\text { SAMPLE }}{3.000}$} \\
\hline No & Sample Size $(\mathrm{mL}) \mathrm{SS}$ & \\
\hline SAMPLE & Concentration of $\mathrm{HNO} 3$ (Molarity) & 0.2005 \\
\hline Now Wortist & HNO3 Titrant at $\mathrm{OH}$ end-point in $\mathrm{mL}$ & 0.000 \\
\hline 14789 & Dilution Factor DF & \\
\hline Destoro & Concentration of OH in Sample (Molarity) & $0.00 \mathrm{E}+00$ \\
\hline $\mathrm{OH}-01$ & $\mathrm{OH}$ in Sample in $\mu \mathrm{g} / \mathrm{m} \mathrm{L}$ (PPM) & $0.00 E+00$ \\
\hline \multirow{2}{*}{ 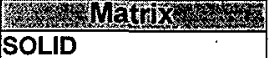 } & \multirow[b]{3}{*}{ Detection Limit $=125 \mathrm{\mu g} / \mathrm{SS}$ * DF } & \\
\hline & & \\
\hline $\begin{array}{l}\text { Sample } \\
\text { S96T005089 }\end{array}$ & & \\
\hline Instrumen $\mathrm{Code}$ & Detection Limit $(\mu \mathrm{g} / \mathrm{m} \mathrm{L})$ & $4.17 \mathrm{E}+01$ \\
\hline $\mathrm{PH} 01$ & \multirow{5}{*}{\multicolumn{2}{|c|}{$\begin{array}{l}\mathrm{OH} \text { Molarity }=\left((\mathrm{mL} H \mathrm{HO} 3)^{*}(\mathrm{M} H \mathrm{NO} 3)\right) / \mathrm{Sample} \text { Size in } \mathrm{mL} \mathrm{L}^{*} \text { Dilution Factor } \\
\mathrm{OH} \text { in } \mu \mathrm{g} / \mathrm{mL}=(\mathrm{OH} \text { MOLARITY })^{*}(17.0 \mathrm{~g} / \mathrm{mole})^{*}((1000000 \mu \mathrm{g} / \mathrm{g}) /(1000 \mathrm{~mL} / \mathrm{L}))\end{array}$}} \\
\hline 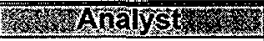 & & \\
\hline RAW & & \\
\hline 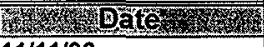 & & \\
\hline $11 / 11 / 96$ & & \\
\hline \multirow{3}{*}{$\begin{array}{l}\text { 19 } \\
09: 43 \mathrm{AM}\end{array}$} & & SAMPLE \\
\hline & Concentration of OH in Sample (Molarity) & $0.00 \mathrm{E}+00$ \\
\hline & $\mathrm{OH}$ in Sample in $\mu \mathrm{g} / \mathrm{mL}$ (PPM) & $<42$ \\
\hline
\end{tabular}

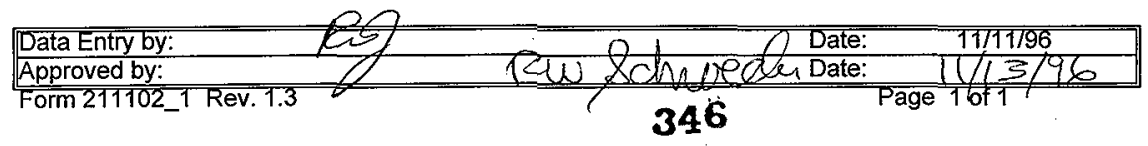




\begin{tabular}{|c|c|c|}
\hline \multicolumn{2}{|c|}{ OH (AUTO) : LA-211-102 (C-0) } & UP \\
\hline 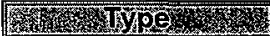 & Sample Size $(\mathrm{mL})$ SS & 3.000 \\
\hline DUP & Concentration of $\mathrm{HNO} 3$ (Molarity) & 0.2005 \\
\hline 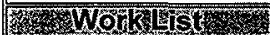 & HNO3 Titrant at $\mathrm{OH}$ end-point in $\mathrm{mL}$ & 0.000 \\
\hline 14789 & Dilution Factor DF & 1 \\
\hline Westrorde & Concentration of $\mathrm{OH}$ in Sample (Molarity) & $0.00 \mathrm{E}+00$ \\
\hline $\mathrm{OH}-01$ & OH in Sample in $\mu \mathrm{g} / \mathrm{mL}$ (PPM) & $0.00 E+00$ \\
\hline \multirow{2}{*}{$\begin{array}{l}\text { Wattrx } \\
\text { SOLID }\end{array}$} & \multirow[b]{3}{*}{ Detection Limit $=125 \mu \mathrm{g} / \mathrm{SS}$ * DF } & \\
\hline & & \\
\hline \multirow{2}{*}{$\begin{array}{l}\text { S96T005089 } \\
\text { Instrumenterde }\end{array}$} & & \\
\hline & Detection Limit $(\mu \mathrm{g} / \mathrm{mL})$ & 4.17E+01 \\
\hline \multirow{2}{*}{ 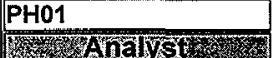 } & \multirow{5}{*}{\multicolumn{2}{|c|}{$\begin{array}{l}\mathrm{OH} \text { Molarity }=\left((\mathrm{mL} H N O 3)^{*}(\mathrm{M} H N O 3)\right) / \text { Sample Size in } \mathrm{mL} \text { Dilution Factor } \\
\mathrm{OH} \text { in } \mu \mathrm{g} / \mathrm{mL}=(\mathrm{OH} \text { MOLARITY })^{*}(17.0 \mathrm{~g} / \mathrm{mole})^{*}((1000000 \mu \mathrm{g} / \mathrm{g}) /(1000 \mathrm{~mL} \mathrm{~L}))\end{array}$}} \\
\hline & & \\
\hline \multirow{2}{*}{ RAW } & & \\
\hline & & \\
\hline \multirow{2}{*}{$11 / 11 / 96$} & & \\
\hline & & UP \\
\hline \multirow[t]{2}{*}{ 09:43 AM } & Concentration of OH in Sample (Molarity) & $0.00 \mathrm{E}+00$ \\
\hline & $\mathrm{OH}$ in Sample in $\mu \mathrm{g} / \mathrm{mL}$ (PPM) & $<42$ \\
\hline
\end{tabular}

The Result is < Detection Limit

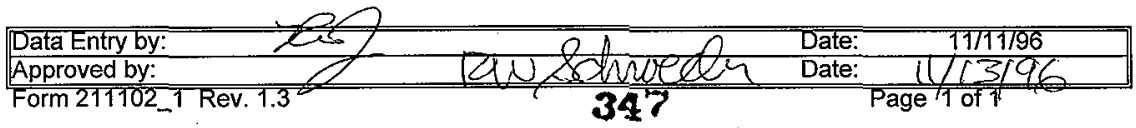




\section{LABCORE Completed Worklist Report for Worklist\# 13066}

Analyst: pjm

Instrument: $\mathrm{ICO} 2$

Book\# 14 N2OB

Method: $\angle A-533-105$ Rev/Mod $D-1$

Worklist Comment: AW-105 IC. RCJ

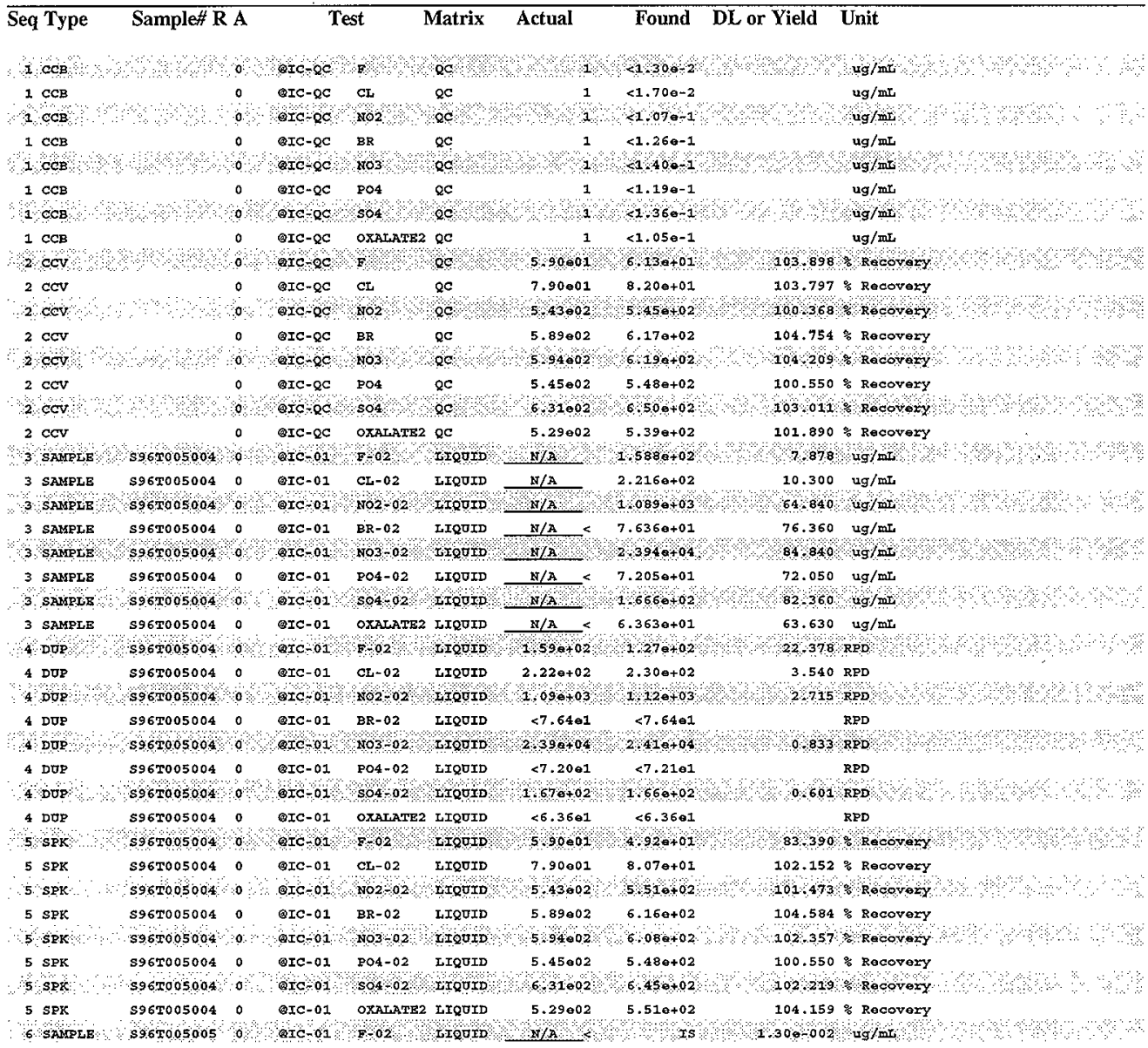




\section{LABCORE Completed Worklist Report for Worklist\# 13066}

\begin{tabular}{|c|c|c|c|c|c|c|c|c|c|}
\hline eq Type & Sample\# R & & & est & Matrix & Actual & Found & ield & Unit \\
\hline 6 SAMPLY & $\$ 96 \mathrm{~T} 005005$ & 0 & (1) 01 & CL- 02 & IIQUID & $N / 8<$ & Is & $1.700 \times 002$ & $u g / w$ \\
\hline 6 SAMPLE & 3967005005 & 0 & (arc- 01 & NO2-02 & LIQUTD & $\mathbb{N} / \mathrm{A}$ & Is & 0.107 & $\mathrm{ug} / \mathrm{s}$ \\
\hline 6 SAMPLU & $\$ 961005005$ & 0 & $6 \mathrm{LCO}$ & $\mathrm{BR}, \mathrm{O}$ & Lroos & 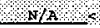 & ts & 0.26 & ug/m \\
\hline 6 SAMPLE & $596 \mathrm{~T} 005005$ & 0 & (बrC-0 1 & $\mathrm{NO3-02}$ & LIQUID & $N / A<$ & Is & 0.140 & $\mathrm{ug} / \mathrm{mar}$ \\
\hline 6 SAMPLE & s96roos005 & 0 & $0 x 0-0.2$ & 00402 & rroopo & $\mathrm{N} / \mathrm{A}, \mathrm{s}$ & Is & 0,19 & ug/m \\
\hline 6 SAMPLIS & S96T005005 & 0 & (ac-01 & $504-02$ & LIQUID & $N / A<$ & Is & 0.136 & $\mathrm{ug} / \mathrm{nt}$ \\
\hline 6 sAMLLE & S96T005005 & 0 & $\theta x<01$ & OXALAmE2 & 2 LTousp & $\mathrm{N} / \mathrm{A}, \mathrm{s}$ & rs & $0 / 05$ & $\mathrm{gg} / \mathrm{I}$ \\
\hline 7 DOP & S96T005005 & 0 & $c-01$ & $F-02$ & IIQUID & $<0.0000$ & $<I S$ & & RPD \\
\hline 7 DOP & 896005005 & 0 & $0 \pi c 01$ & 00.02 & I- 0 op & $<0.0000$ & $<\mathrm{ss}$ & & $\mathrm{RPD}$ \\
\hline 7 DOP & $\$ 96 \mathrm{~T} 005005$ & 0 & arc-01 & $\mathrm{NO2}-02$ & IIQTID & $<0.0000$ & $<I S$ & & RPD \\
\hline 7 DOP & S96T005005 & 0 & $a-01$ & $\mathrm{BR}-\mathrm{Z}$ & LToJpD & $<0,0000$ & \&ss & & $\mathrm{RPD}$ \\
\hline 7 DOP & $\$ 96 \% 005005$ & 0 & (2IC-01 & No3- 02 & IIQUID & $<0.0000$ & $<I S$ & & RPD \\
\hline $7 \mathrm{DOP}$ & S95T005005 & 0 & $0 r e-0 x$ & po4- -2 & 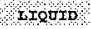 & 800000 & ss & & $\mathrm{RPD}$ \\
\hline 7 DOP & $596 \mathrm{~T} 005005$ & 0 & aIC-0I & $504-02$ & IIQUTD & $<0.0000$ & $<I s$ & & RPD \\
\hline 7 DOP & $396 \mathrm{~T} 005005$ & a & $\otimes I C<1$ & OxAGARE2 & 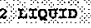 & 80,0000 & SIS & & RPD \\
\hline 8 SAMPLE & $596 \mathrm{~T} 005006$ & 0 & Dre-0I & $F-02$ & IIQDID & $\mathbb{N} / \mathrm{A}$ & $1.744 \mathrm{e}+02$ & 7.878 & $\mathrm{ug} / \mathrm{mi}$ \\
\hline B SAMELE & S96ro05006: & 0 & Qre- 01 & $c_{0} 02$ & IIOTID & $\triangle A$ & $2,2060+02$ & 10.300 & ug/mi \\
\hline 8 SAMPLE & $S 96 \mathrm{~T} 005006$ & 0 & (1) 1 - 01 & $502-02$ & EIQUID & $\mathrm{N} / \mathrm{A}$ & $1.122 \mathrm{e}+03$ & 64.840 & $\mathrm{ug} / \mathrm{mL}$ \\
\hline a sAMPLAB & $896 \mathrm{~T} 005006$ & 0 & Q $\mathbf{C}-0 \mathrm{I}$ & $\mathrm{BK}-2 \mathrm{z}$ & TIQOID & $\mathrm{N} / \mathrm{A}, \mathrm{s}$ & $7.6360+01$ & 76360 & ug $/ x$ \\
\hline 8 SAMPLE & $s 96$ & 0 & -01 & so3 & LIQUID & N/A & $2.4140+04$ & 84.840 & $\mathrm{ug} / \mathrm{mat}$ \\
\hline A SAMPL & S96 005006 & 6 & 001 & 0402 & - 10 OPD & $\mathrm{N} / \mathrm{A} \quad$ & $122050+01$ & 72050 & $\mathrm{ug} / \mathrm{mE}$ \\
\hline 8 SAMPLE & 5006 & 0 & $c-01$ & s04-02 & IIQUID & $\mathrm{N} / \mathrm{A}$ & $1.751 \mathrm{e}+02$ & 82.360 & $\mathrm{ug} / \mathrm{mz}$ \\
\hline A SAMRIE & 005006 & 0 & QIC- 6 & Oagang & $2+\because Q 0 I D$ & $\mathrm{~N} / \mathrm{A} / 4$ & $63630+01$ & 63630 & $\mathrm{ug} / \mathrm{mi}$ \\
\hline 9 DUP & 5006 & 0 & $c-01$ & F-02 & IIQDID & $3.74 \theta+02$ & $1.660+02$ & 4.706 & RPD \\
\hline 9 D०P & 05006 & 0 & exc-o1 & $\mathrm{et}$ & J Iodxo & $2,210+02$ & $2160+02$ & 2,28 & RPD \\
\hline 9 DUP & 05006 & 0 & $c-01$ & $\mathrm{ro2}-02$ & IXQUDD & $1.12 \theta+03$ & $1.100+03$ & 1.802 & RPD \\
\hline $9 \mathrm{DUE}$ & 5006 & 0 & & 102 & $\triangle I Q O M D$ & 70640 & 8648 & & $\mathrm{RPD}$ \\
\hline 9 Do & & 0 & & No3. & LIQUID & $2.41 e+04$ & $2.41 e+04$ & 0.000 & RPD \\
\hline ספד & 5006 & 0 & & & rnoणro & 87 & 67216 & 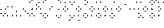 & $R P D$ \\
\hline & 5006 & 0 & & $504-02$ & IIQDID & $1.75 \theta+02$ & $1.89 e+02$ & 7.692 & RPD \\
\hline & s967005006 & & 01001 & & & & & & \\
\hline
\end{tabular}

\section{Comments Section:}

Comments for sample\# S96T005005 and test @IC-01 . Insufficient sample to run on WKL 13066 IC. jmf

\section{Final page for worklist\# 13066}

\section{$\begin{array}{llll}\text { Analyst Signature } & \text { Date } & \text { Analyst Signature } & \text { Date }\end{array}$}

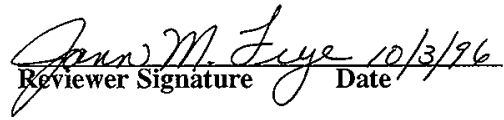

Units shown for $Q C(B L K / B K G)$ may not reflect the actual units. 


\section{LABCORE Data Entry Template for Worklist\# 13066}

Analyst: $P A M$ Instrument: IC00 ICOZ Book\# 14N20-B

Method: LA-533-105 Rev/Mod D-1

Worklist Comment: AW-105 IC. RCJ

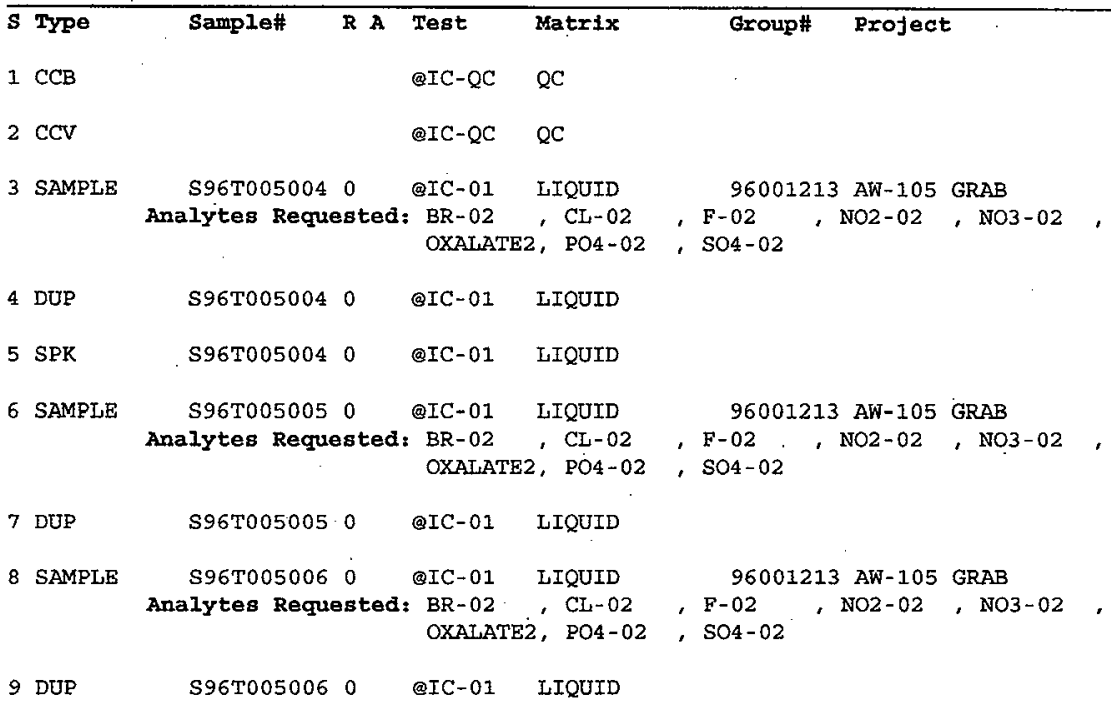

\section{Final page for worklist \# 13066}

\section{PsMcloum $9 / 29 / 96$}

Analyst Signature Date

Analyst Signature Date

$130663 p \cdot$. SV - added 2 extra files to can foo 6967005005 -witl

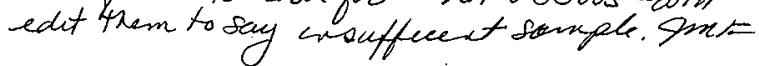

uploaded $10 / 3 / 269 m 1=$

Valudated $10 / 3 / 268 \mathrm{mF}$

Data Entry Comments:

5967005005 - no dample left in 22 m vial in mlip lid can. Could not find original sample to get anothes a liquot. Bsect 
HNF-SD-WM-DP-214, REV. 0

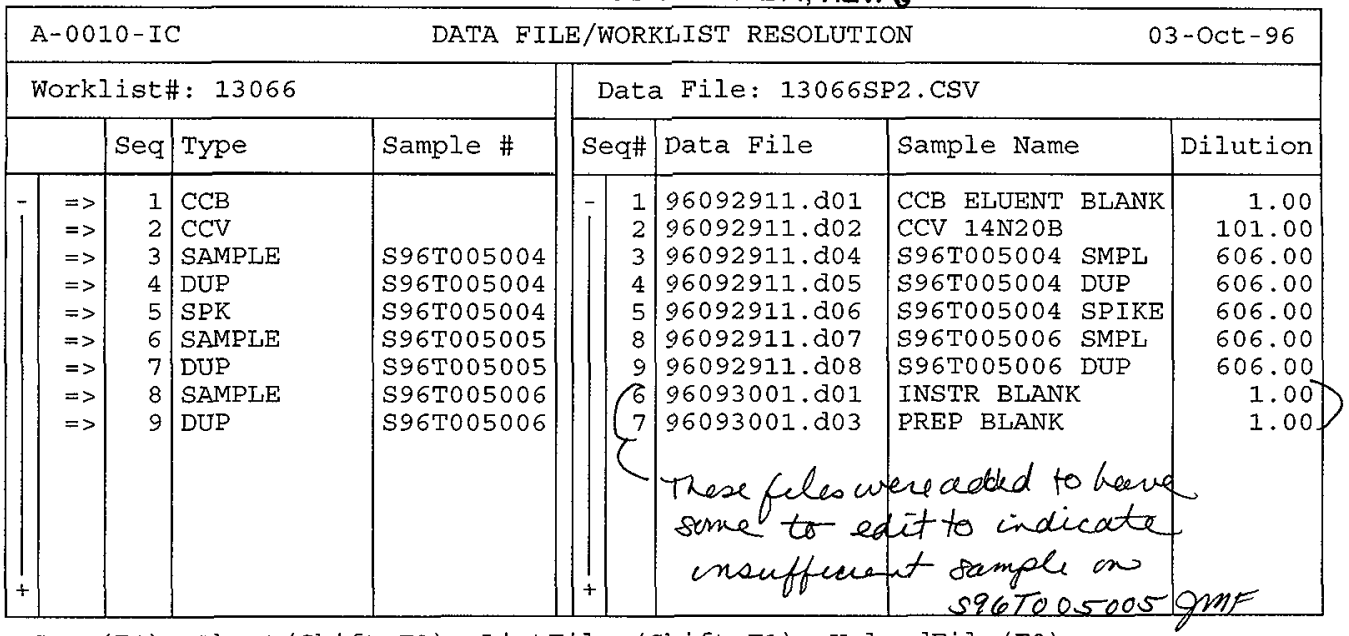

Save(F4) Abort(Shift-F3) ListFiles(Shift-F1) UploadFile(F8) 
Calibration Volume Dilution Points Rate start stop Area Reject

Cal-

External

$\mathbf{1}$

$1 \quad 3000 \quad 5 \mathrm{~Hz}$

$0.00 \quad 10.00$

200

$* * * * * * * * * * * * * * * * * * * * * * * * * * *$ Peak Report: All Peaks $* * * * * * * * * * * * * * * * * * * * * * * * * * * *$

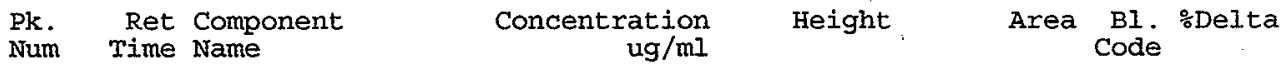

Totals

DIRECT:

File: 96092911.D01 Sample: CCB ELUENT BLANK

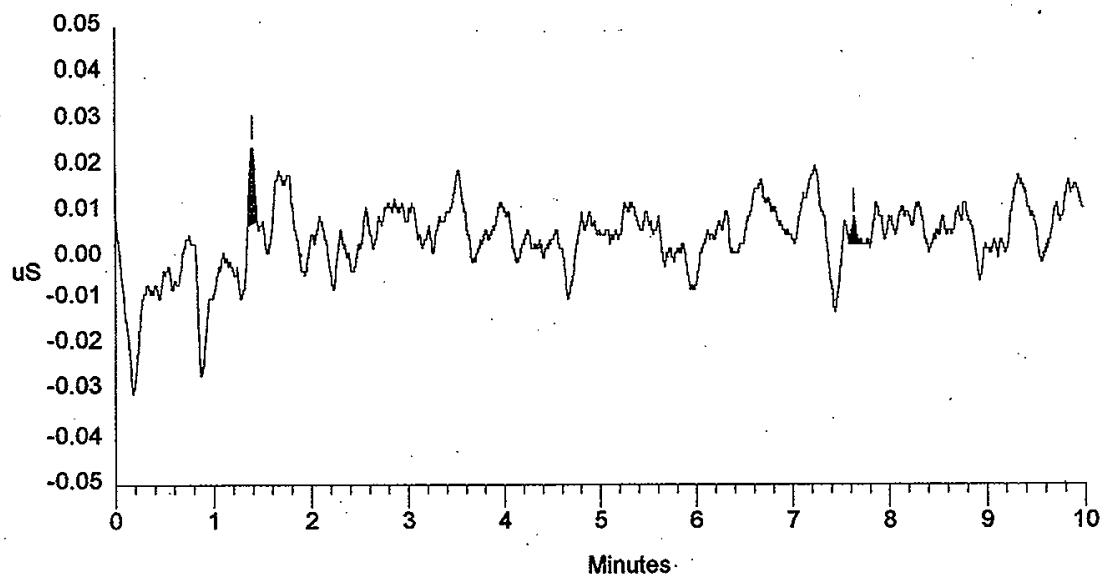




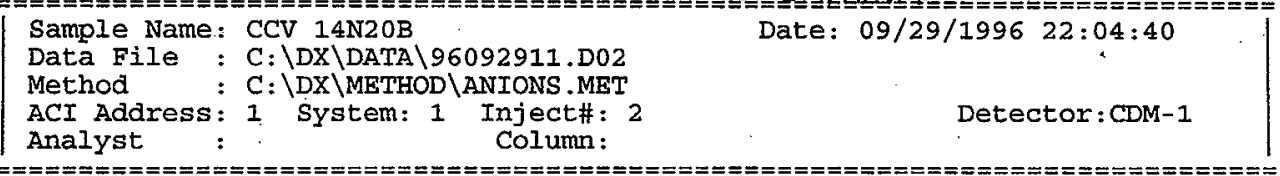

Calibration Volume Dilution Points Rate start stop Area Reject

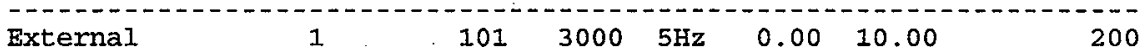

$* * * * * * * * * * * * * * * * * * * * * * * * * * *$ Peak Report: All Peaks $* * * * * * * * * * * * * * * * * * * * * * * * * * * *$

Pk. Ret Component Concentration Height Area Bl. $\%$ Delta Num Time Name . $\mathrm{ug} / \mathrm{ml}$ Code

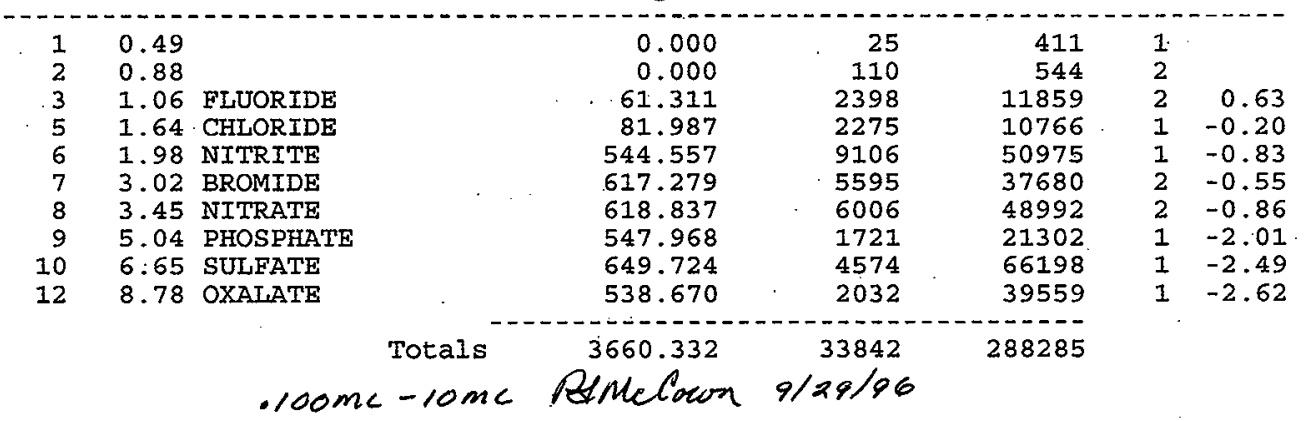

File: 96092911.D02 Sample: CCV 14N20B

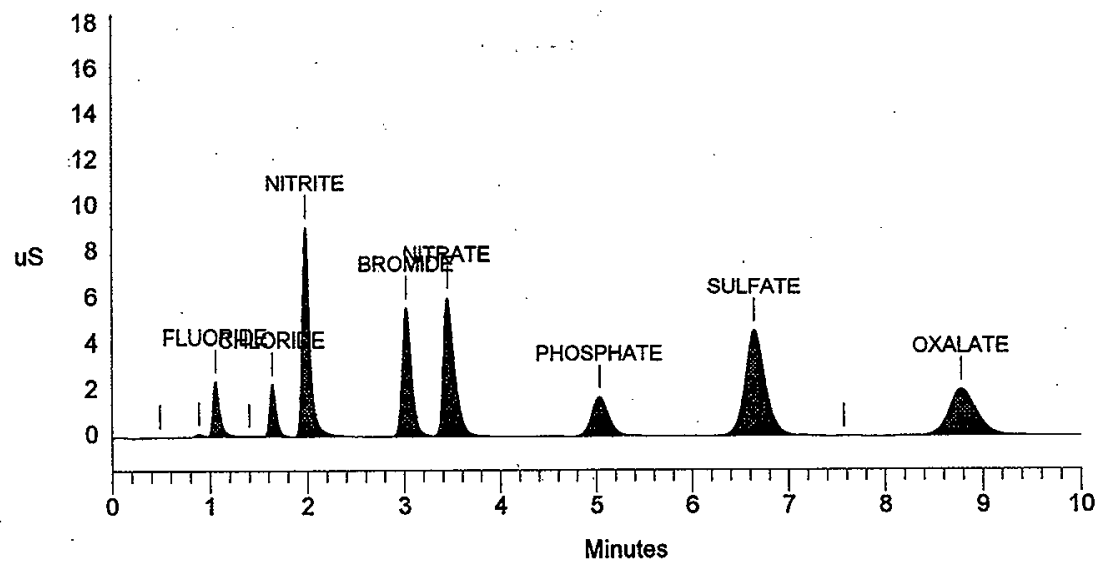

SIGNATURE ABOVE REPRESENTS CHEMICAL TECHNOLOGIST/CHEMIST THAY COMPLETEDNERIFIED THE CALIBRATION/ANALYSIS ON PAGES 353 TO 358 
Data Reprocessed on 10/02/1996 16:37:37

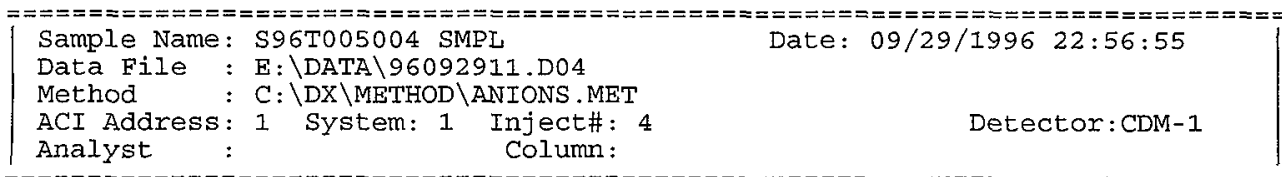

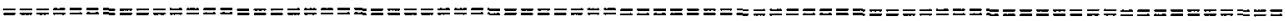

Calibration Volume Dilution Points Rate Start Stop Area Reject

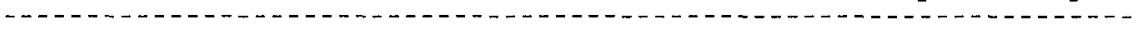

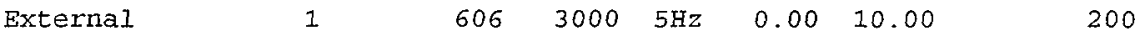

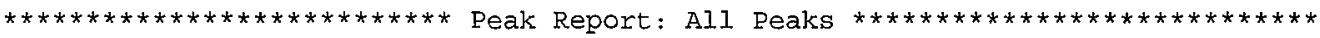

$\begin{array}{lrrr}\text { Pk. Ret Component } & \text { Concentration } & \text { Height } & \text { Area Bl. } \\ \text { Num } & \text { Time Name } / \mathrm{ml} & \text { Code }\end{array}$

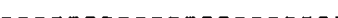

$\begin{array}{lll}2 & 0.88 & \\ 3 & 1.05 & \text { FLUORIDE } \\ 4 & 1.63 & \text { CHLORIDE } \\ 5 & 1.97 & \text { NITRITE } \\ 6 & 3.30 & \text { NITRATE } \\ 7 & 6.68 & \text { SULFATE }\end{array}$

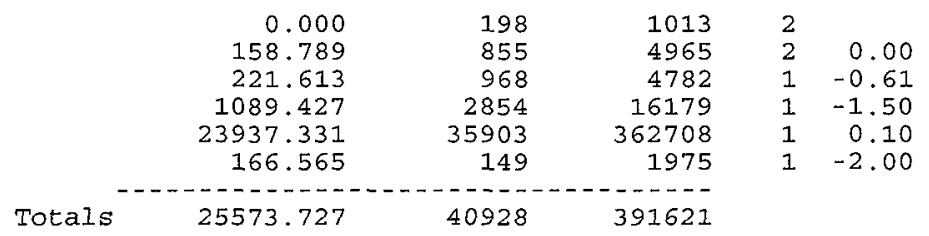

File: 96092911.D04 Sample: S96T005004 SMPL

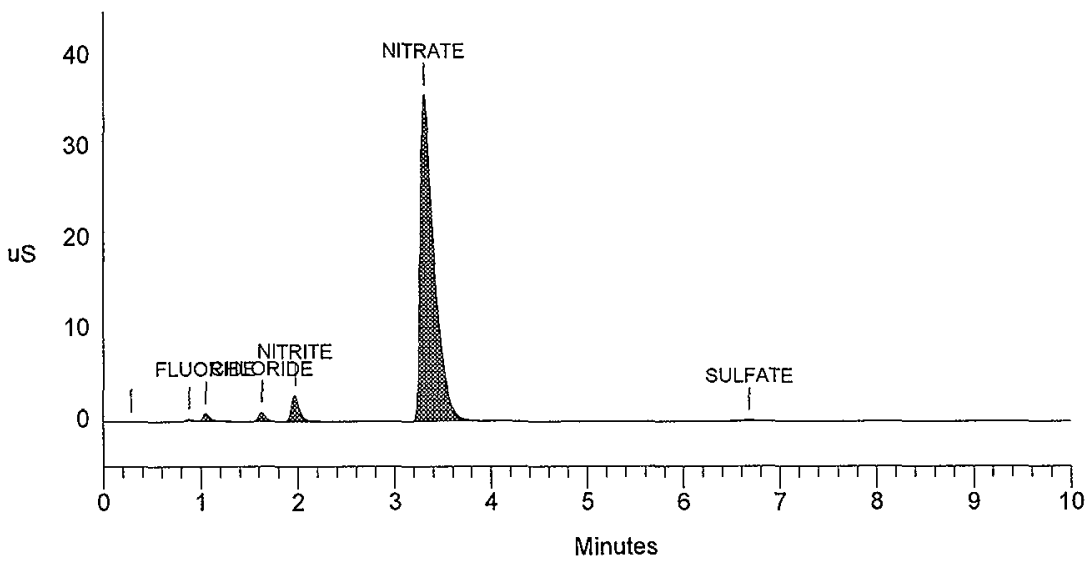


HNF-SD-WM-DP-214, REV. 0

Data Reprocessed On 10/02/1996 16:38:56

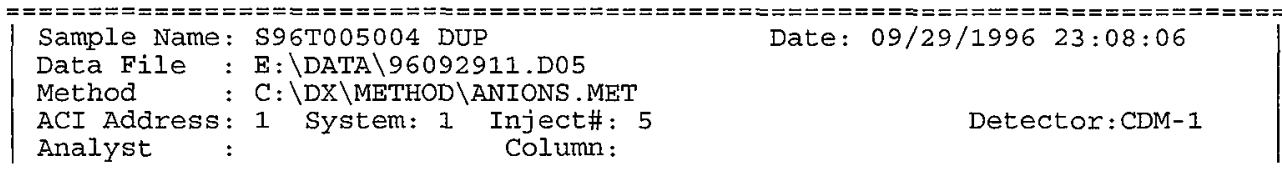

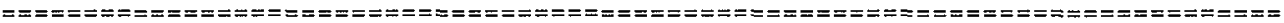

Calibration Volume Dilution Points Rate start stop Area Reject

$\begin{array}{llllllll}\text { External } & 1 & 606 & 3000 & 5 \mathrm{~Hz} & 0.00 & 10.00 & 200\end{array}$

$* * * * * * * * * * * * * * * * * * * * * * * * * * *$ Peak Report: All Peaks $* * * * * * * * * * * * * * * * * * * * * * * * * * * *$

$\begin{array}{lrrr}\text { Pk. Ret Component } & \text { Concentration } & \text { Height } & \text { Area } \\ \text { Num } & \text { B Time Name } & \text { Codelta }\end{array}$

2

3

41.63 CHLORIDE

$5 \quad 1.97$ NITRITE

$6 \quad 3.30$ NITRATE

76.68 SUIFATE

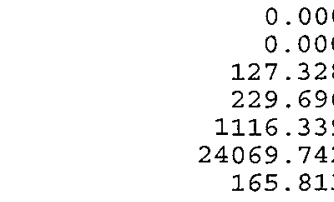

0.000

0.000

25

193

800

985

2850

35886

150

Totals

25708.917

40889

$320 \quad 1$

$912 \quad 2$

$3935 \quad 2$

4959

16606

365066

1962

1

20.00

$1-0.61$

$1-1.50$

$1 \quad 0.10$

$1-2.00$

File: 96092911.D05 Sample: S96T005004 DUP

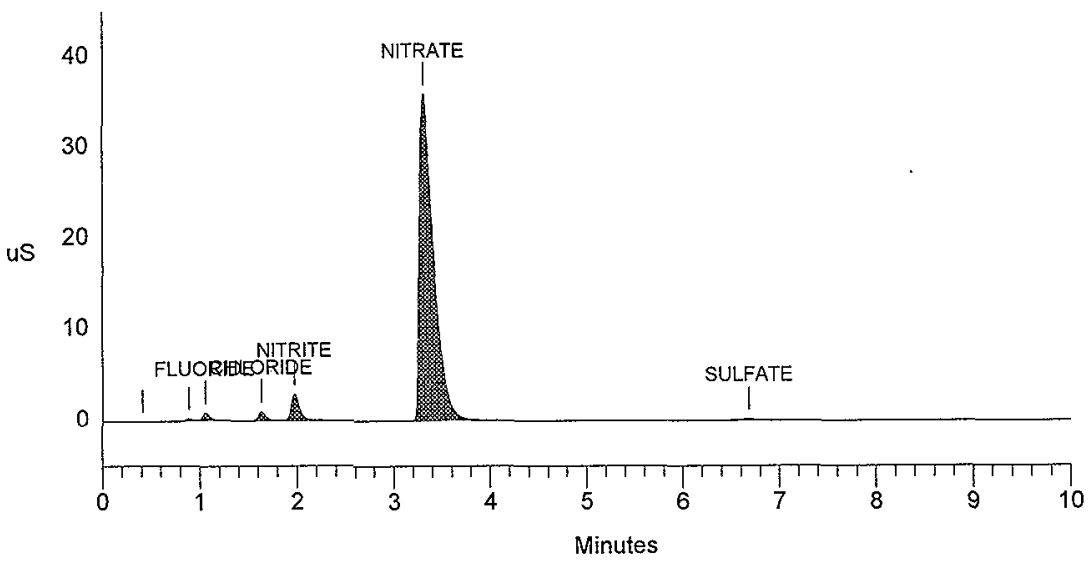


Data Reprocessed on 10/02/1945E-SD-WM-PP-214, REV. 0

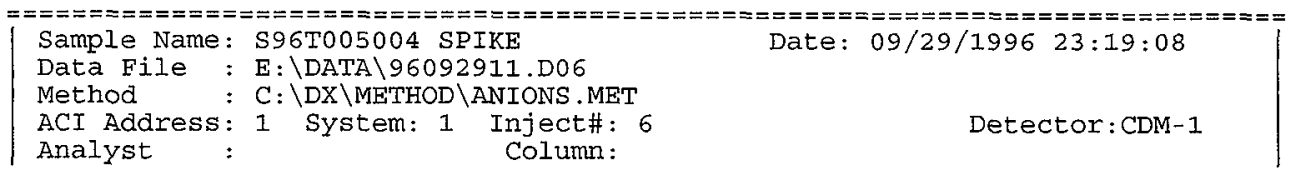

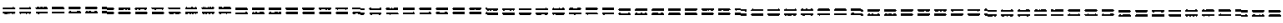

Calibration Volume Dilution Points Rate Start stop Area Reject

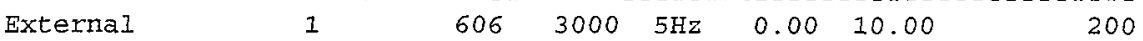

$\star * * * * * * * * * * * * * * * * * * * * * * * * * *$ Peak Report: All Peaks $* * * * * * * * * * * * * * * * * * * * * * * * * * * *$

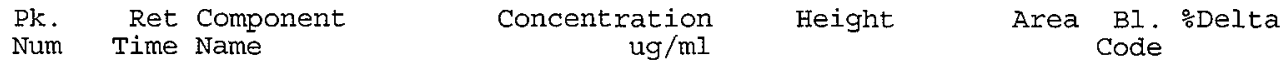

\begin{tabular}{|c|c|c|c|c|c|c|c|}
\hline 1 & 0.88 & & 0.000 & 261 & 1167 & 1 & \\
\hline 2 & 1.05 & FLUORIDE & 404.562 & 2799 & 13077 & 1 & 0.00 \\
\hline 3 & 1.63 & CHLORIDE & 625.146 & 2753 & 13751 & 2 & -0.6 \\
\hline 4 & 1.97 & NITRITE & 3845.128 & 10606 & 60283 & 2 & -1.5 \\
\hline 5 & 2.99 & BROMIDE & 3080.545 & 5033 & 31089 & 2 & -1.6 \\
\hline 6 & 3.28 & NITRATE & 26978.561 & 40133 & 418265 & 2 & 0.10 \\
\hline 7 & 5.04 & PHOSPHATE & 2739.031 & 1435 & 17609 & 1 & -2.01 \\
\hline 8 & 6.64 & SULFATE & 3393.161 & 3962 & 57353 & 1 & -2.59 \\
\hline 10 & 8.78 & OXALATE & 2756.846 & 1730 & 33594 & 1 & -2.70 \\
\hline
\end{tabular}

$\begin{array}{llll}\text { Totals } & 43822.979 & 68713 & 646187\end{array}$

File: 96092911.D06 Sample: S96T005004 SPIKE

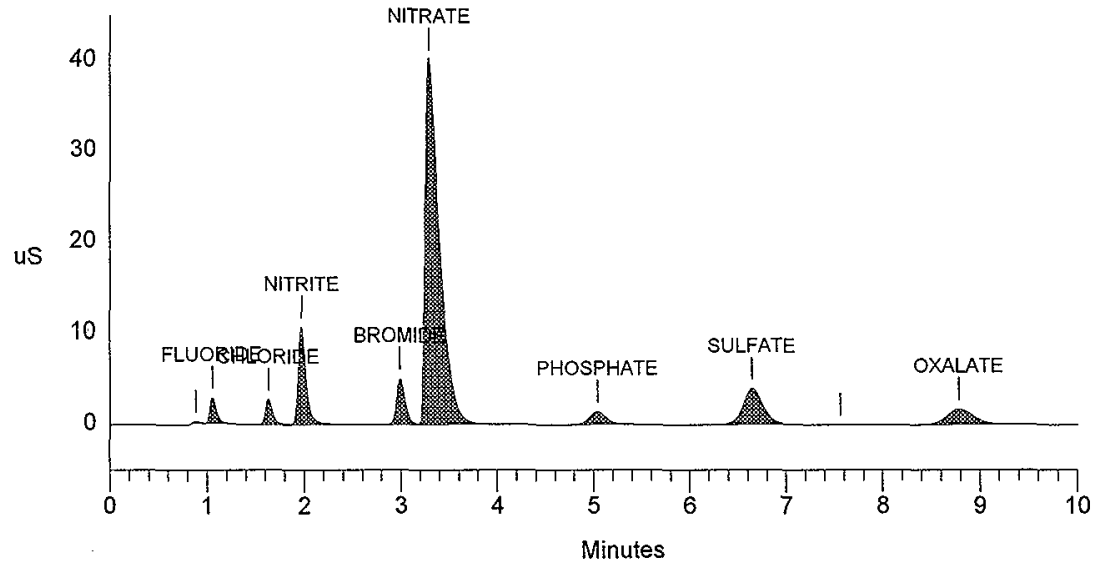


Data Reprocessed on 10/02/1996- HWM-DP-214, REV. O

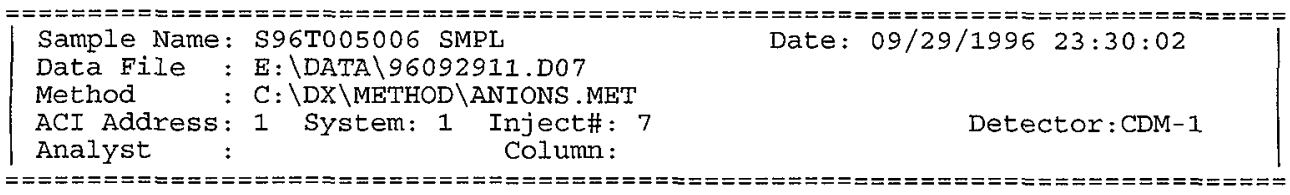

Calibration Volume Dilution Points Rate Start Stop Area Reject

\begin{tabular}{|c|}
\hline ternal \\
\hline
\end{tabular}

$\star * * * * * * * * * * * * * * * * * * * * * * * * * *$ Peak Report: All Peaks $* * * * * * * * * * * * * * * * * * * * * * * * * * * *$

Pk. Ret Component $\begin{gathered}\text { Concentration } \\ \mathrm{ug} / \mathrm{ml}\end{gathered}$
Num Time Name

\begin{tabular}{rrrrr}
0.000 & 200 & 1062 & 2 & \\
174.446 & 948 & 5478 & 2 & 0.00 \\
220.616 & 947 & 4760 & 2 & -0.61 \\
1122.153 & 2920 & 16698 & 2 & -1.50 \\
24140.753 & 36185 & 366333 & 1 & -0.10 \\
175.104 & 157 & 2119 & 1 & -2.00 \\
\hline & & 157 &
\end{tabular}

File: 96092911.D07 Sample: S96T005006 SMPL

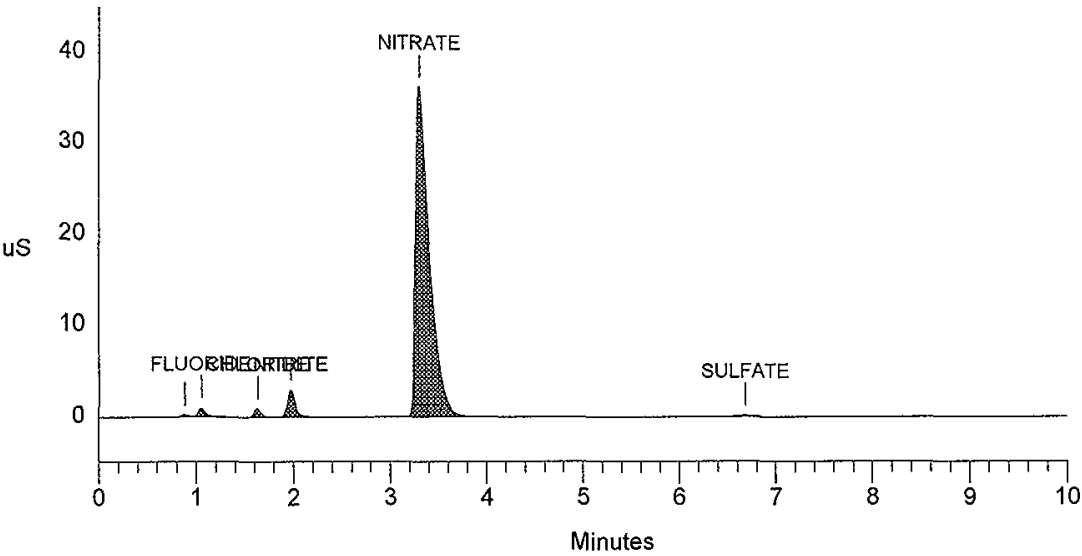


Data Reprocessed on 10/02/1996 16:42:52

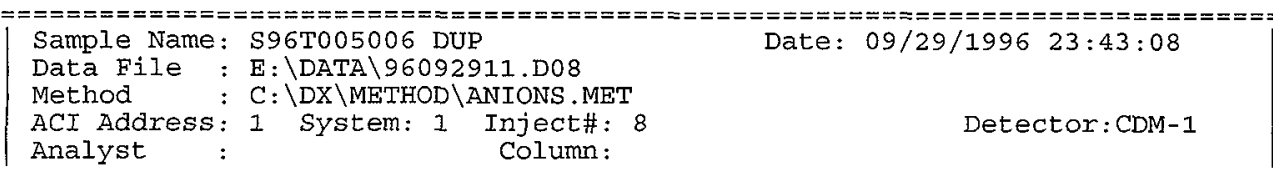

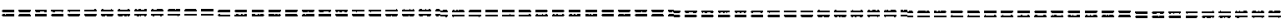

Calibration Volume Dilution Points Rate start stop Area Reject Cajubration Volume Dilution Points Rate Start

$\begin{array}{llllllll}\text { External } & 1 & 606 & 3000 & 5 \mathrm{~Hz} & 0.00 & 10.00 & 200\end{array}$

$* * * * * * * * * * * * * * * * * * * * * * * * * *$ Peak Report: All Peaks $* * * * * * * * * * * * * * * * * * * * * * * * * * * *$

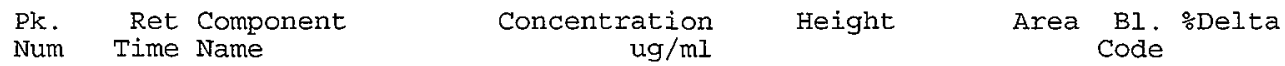

$\begin{array}{lll}1 & 0.39 & \\ 2 & 0.88 & \\ 3 & 1.05 & \text { FLUORIDE } \\ 4 & 1.63 & \text { CHLORIDE } \\ 5 & 1.97 & \text { NITRITE } \\ 6 & 3.30 & \text { NITRATE } \\ 7 & 6.68 & \text { SULFATE }\end{array}$

:

0.000
0.000
166.301
216.005
1103.274
24061.739
188.825

Totals

25736.143

18

203

924

951

2933

36063

166

41258

221

221
1049

5211

4658

16398

364924

2351 Code

200

File: 96092911.D08 Sample: S96T005006 DUP

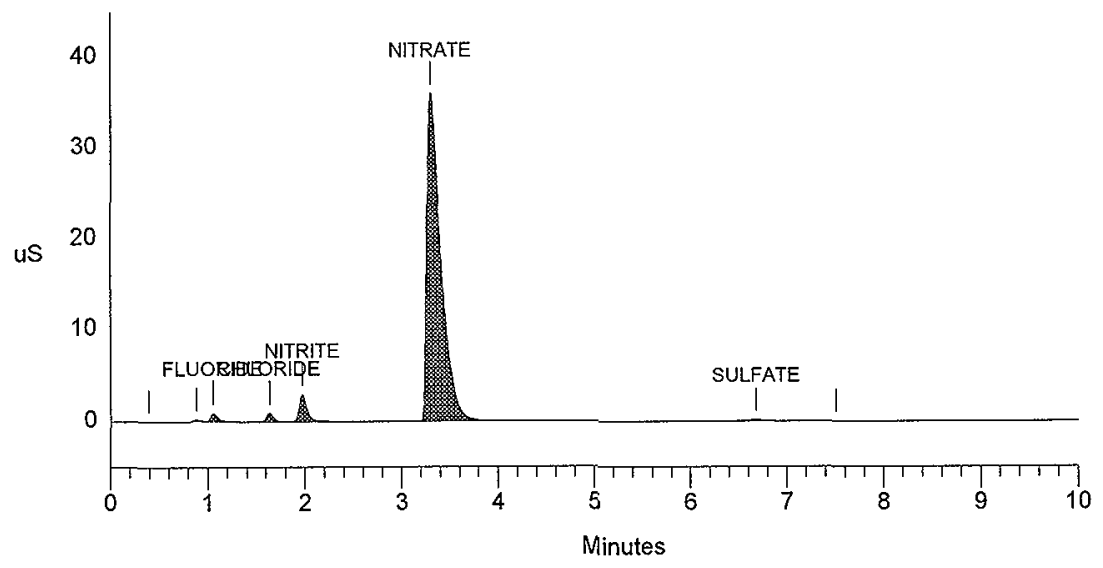




\section{LABCORE Completed Worklist Report for Worklist\# 13571}

Analyst: tam

Instrument: IC01

Book\#32N2OC

Method: $L A-533-105$ Rev/Mod $D-1$

Worklist Comment: Rerun AW-105 IC REPRINT. jmf

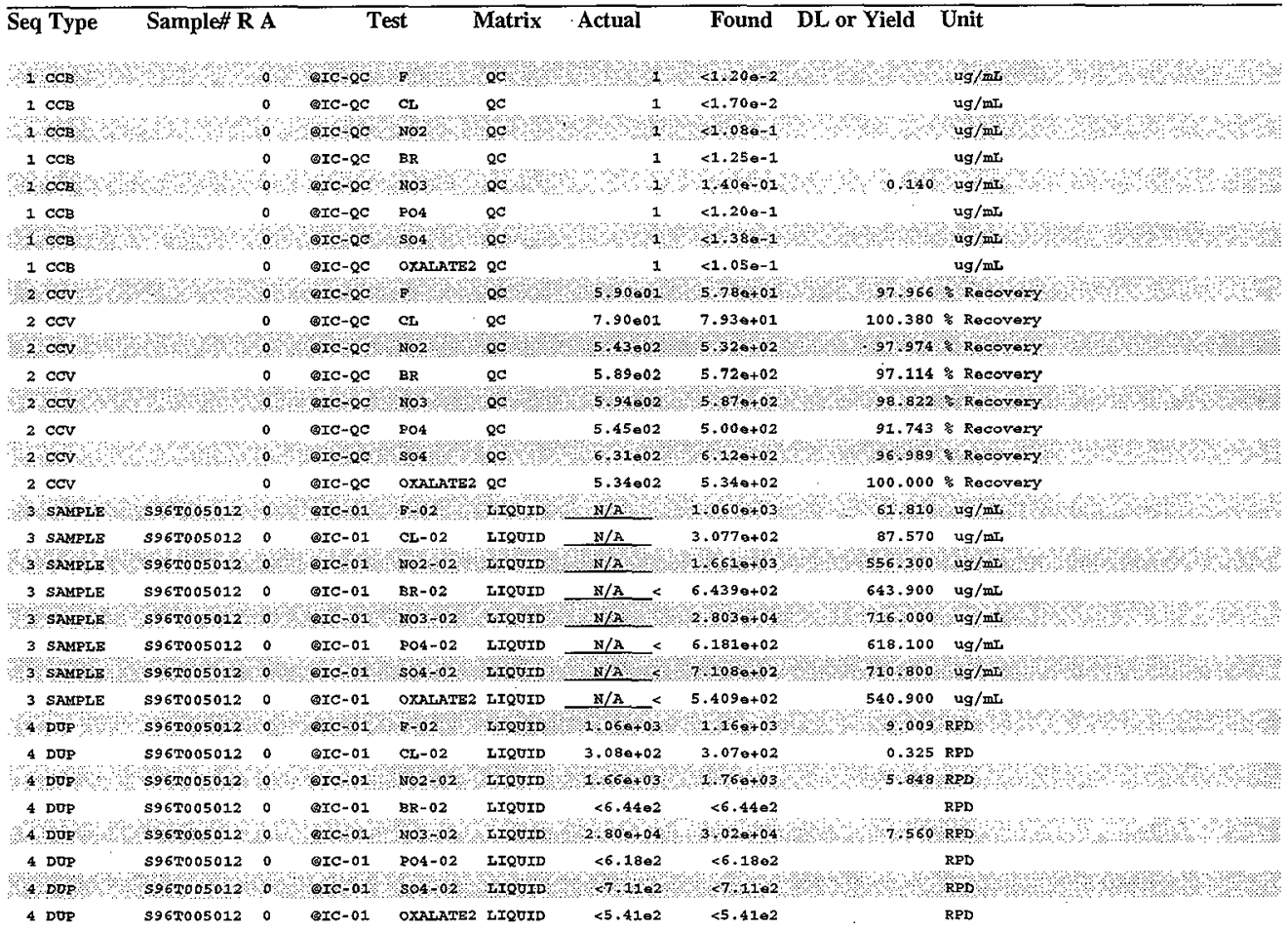

\section{Final page for worklist\# 13571}

Analyst Signature $\quad$ Date

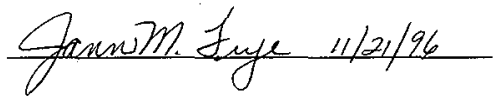


$11 / 21 / 9614: 16$

HNF-SD-WM-DP-214, REV. O

Page: 1

A-0004-1

LABCORE Data Entry Template for Worklist\# 13571

Analyst: $\quad$ TAM Instrument: IC0 ICO I_ Book\# 32N20C

Method: LA-533-105 Rev/Mod D-1

Worklist Comment: Rerun AW-105 IC REPRINT. jmf

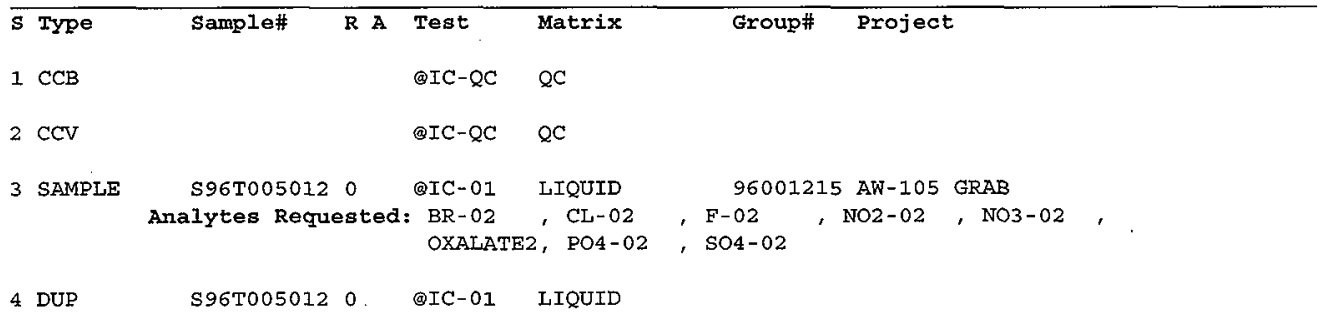

Final page for worklist \# 13571

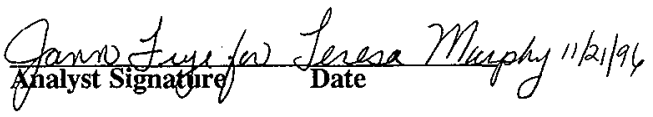

$\overline{\text { Analyst Signature }} \overline{\text { Date }}$

$1357 / \mathrm{NV}$. CS V

uploaded 112196

validated 1112196 Omfeye

Data Entry Comments:

$S=$ Worklist Slot Number, $R=$ Replicate Number, $A=$ Aliquot Code.

360 
HNF-SD-WM-DP-214, REV. 0

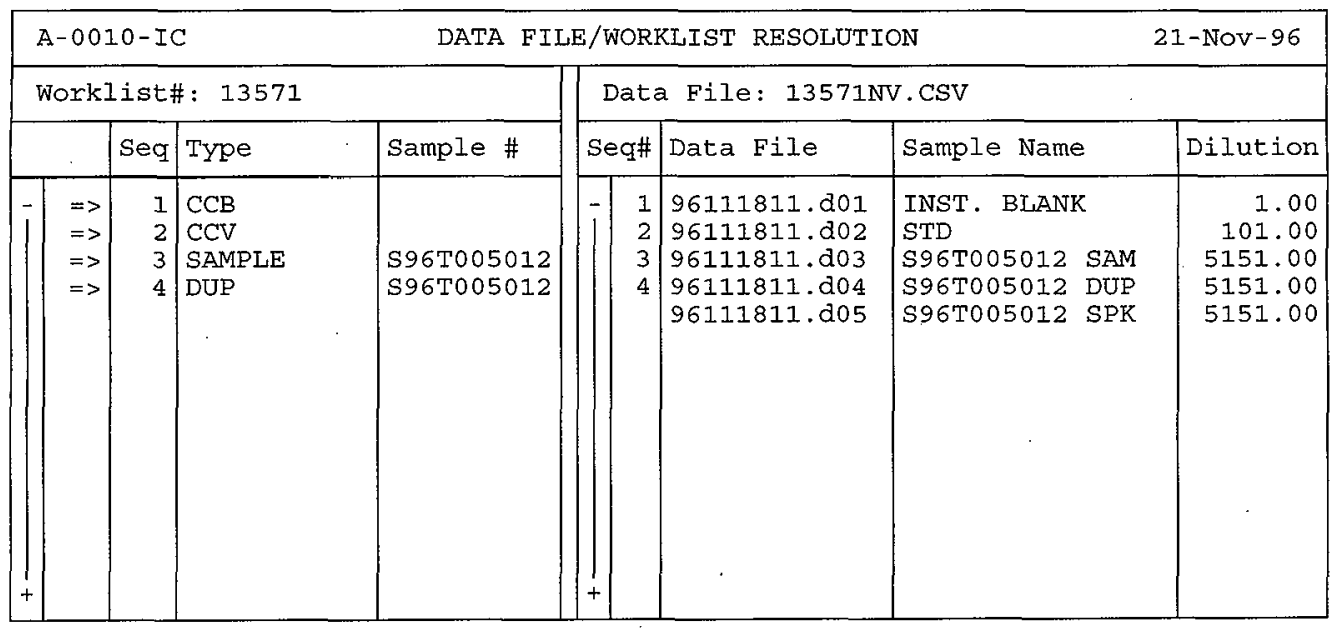

Save(F4) Abort(Shift-F3) ListFiles(Shift-F1) UploadFile(F8) 


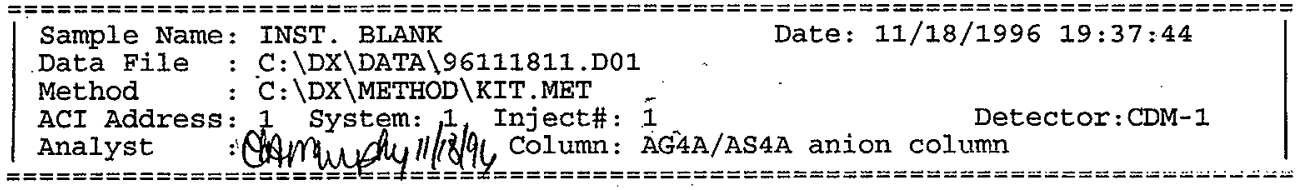

Calibration Volume Dilution Points Rate start stop Area Reject

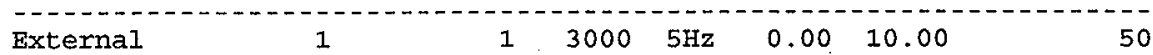

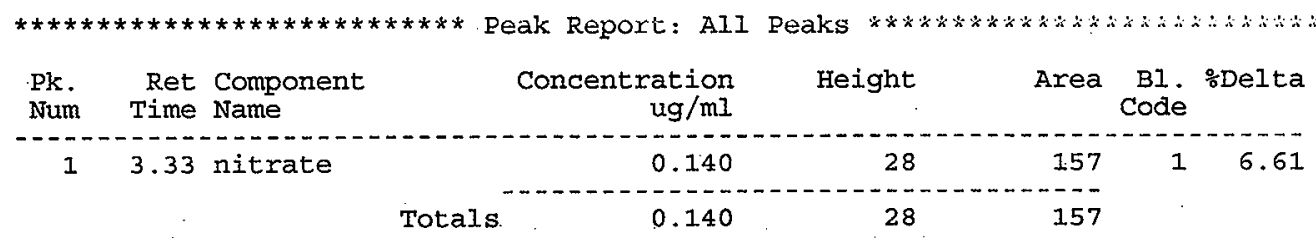

File: 96111811.D01 Sample: INST. DLAMrS

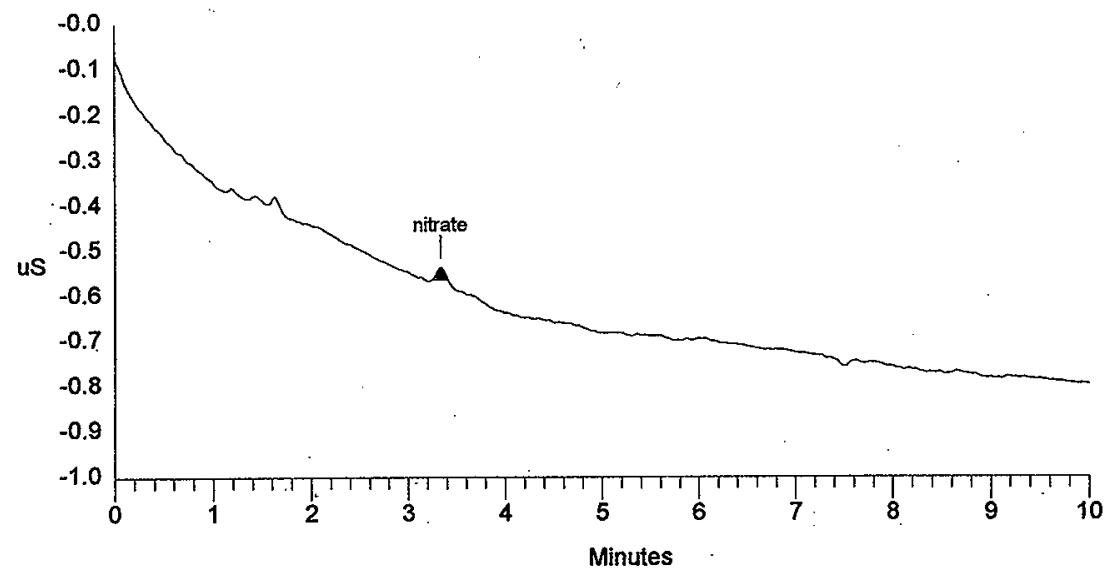

SISHATURE ABOVE REPRESENTS CHEMICAL TECHNOLOGIST/CHEMIST JHAT COMPLETEDIVERIFIED THE CALIBRATION/ANALYSIS ON PAGES 362 TO 3606 


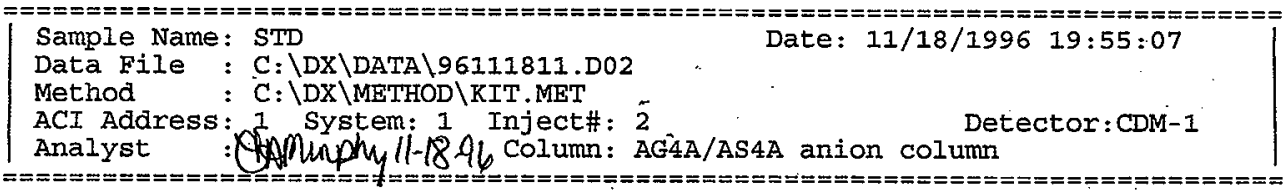

Calibration Volume Dilution Points Rate Start Stop Area Reject

External $\quad 1 \quad 101 \quad 3000 \quad 5 \mathrm{~Hz} .0 .00 \quad 10.00 \quad 50$

$\star * * * * * * * * * * * * * * * * * * * * * * * * * *$ Peak Report : All Peaks $* * * * * * * * * * * * * * * * * * * * * * * * * * * *$

Pk. Ret Component Concentration Height Area Bl. 5 Delta Num Time Name ug/ml Code

$\begin{array}{lll}1 & 0.91 \\ 2 & 1.08 \text { fluoride } \\ 3 & 1.61 \text { chloride } \\ 4 & 1.91 \text { nitrite } \\ 5 & 2.86 \text { bromide } \\ 6 & 3.25 \text { nitrate } \\ 7 & 4.64 \text { phosphate } \\ 8 & 6.03 \text { sulfate } \\ 9 & 7.89 \text { oxalate }\end{array}$

$\begin{array}{rrrr} & 0.000 & 32 & 109 \\ 57.841 & 1610 & 10019 \\ 79.341 & 1720 & 9230 \\ 532.170 & 6838 & 44958 \\ 571.751 & 4880 & 34179 \\ & 587.114 & 5442 & 45639 \\ & 499.552 & 1339 & 19525 \\ & 612.401 & 4171 & 65699 \\ \text { Totals } & 534.087 & 1974 & 42228 \\ & 3474.256 & 28008 & 271585\end{array}$

File: $96111811 . D 02$ Sample: STD

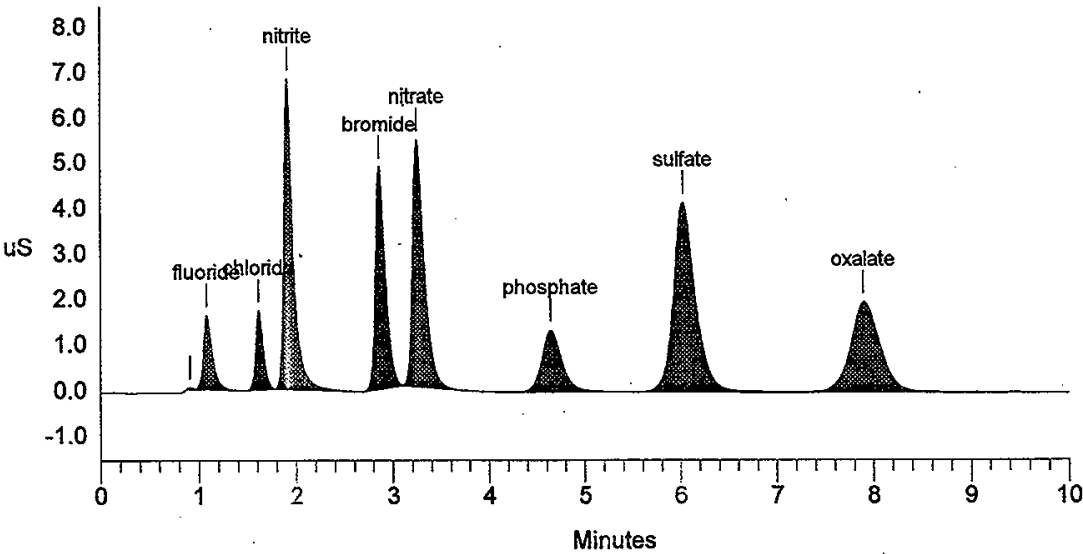


Sample Name: S96T005012 SAM

Data File : C: \DX\DATA \96111811.D03

Method : C: \DX\METHOD KIT.MET

ACI Address: 1 System: 1 Inject\#: $\overline{3}$

Analyst : Column: AG4A/AS4A anion column

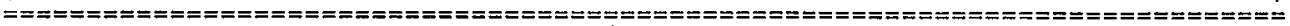

Calibration Volume Dilution Points Rate start stop Area Reject

External $\quad I \quad 5151 \quad 3000 \quad 5 \mathrm{~Hz} \quad 0.00 \quad 10.00 \quad 50$

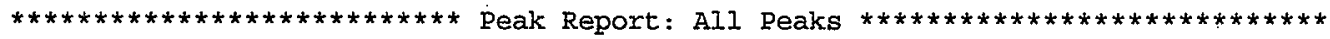

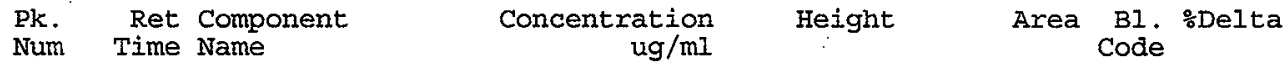

$\begin{array}{ll}2 & 1.09 \text { fluoride } \\ 3 & 1.63 \text { chloride } \\ 4 & 1.94 \text { nitrite } \\ 5 & 3.25 \text { nitrate } \\ 6 & 6.08 \text { sulfate }\end{array}$

\begin{tabular}{|c|c|c|}
\hline & $\begin{array}{r}1059.828 \\
307.681 \\
1661.274 \\
28025.981 \\
625.852\end{array}$ & $\begin{array}{r}522 \\
130 \\
273 \\
5044 \\
38\end{array}$ \\
\hline otals & 31680.616 & 6006 \\
\hline
\end{tabular}

File: 96111811.D03 Sample: S96T005012 SAM

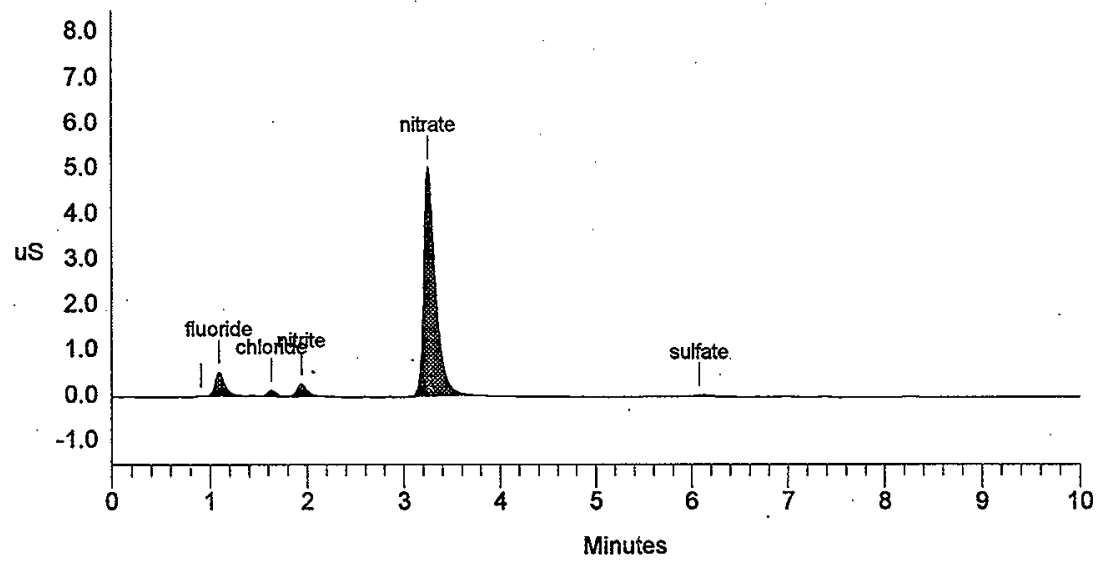


HNF-SD-WM-DP-214, REV. 0

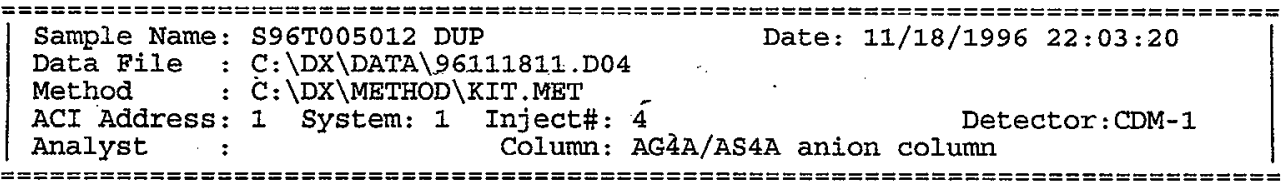

Calibration Volume Dilution Points Rate start stop Area Reject

Cxternal

$\begin{array}{llllllll}\text { External } & 1 & 5151 & 3000 & 5 \mathrm{~Hz} & 0.00 & 10.00 & 50\end{array}$

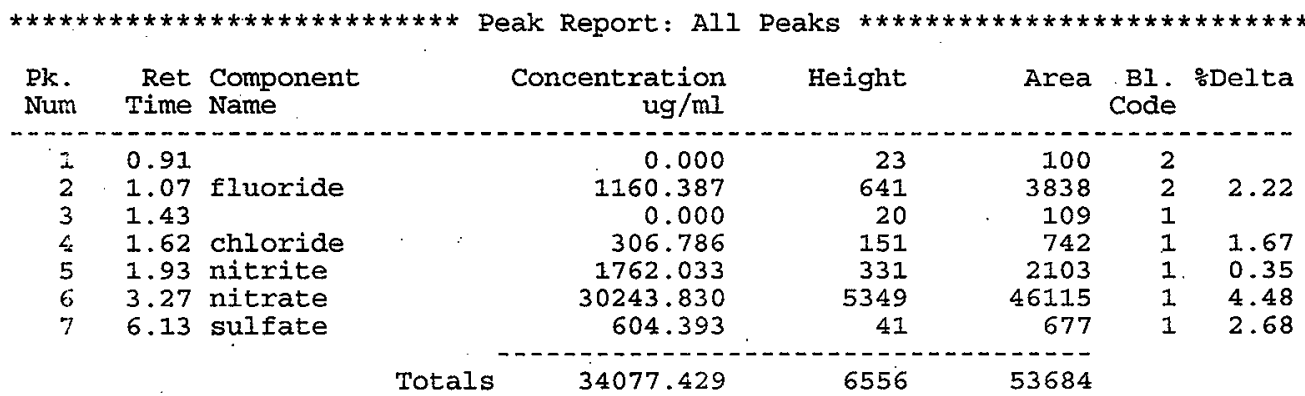

File: 96111811.D04 Sample: S96T005012 DUP

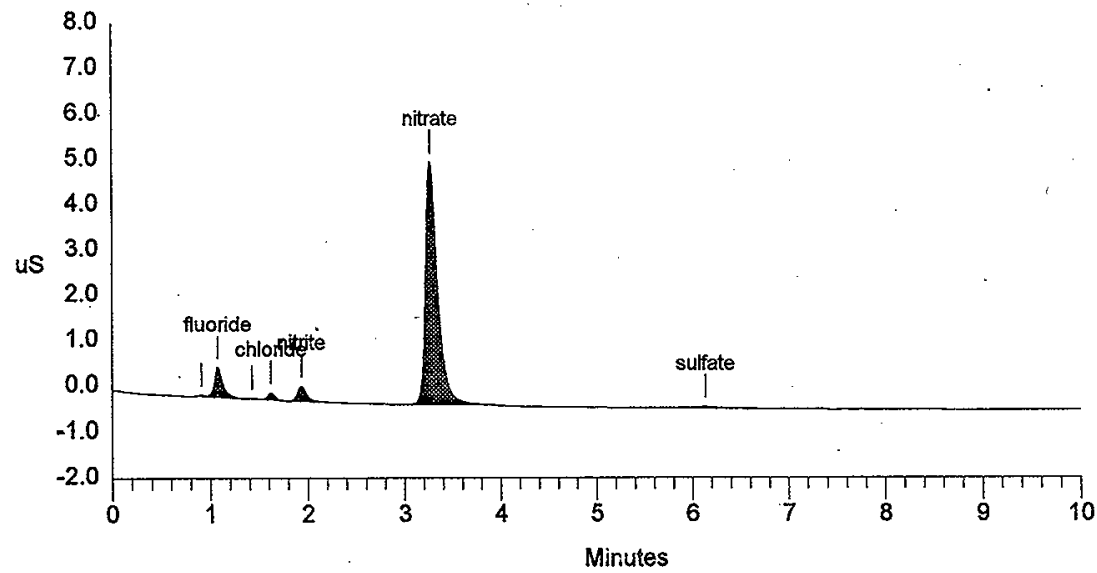


Calibration Volume Dilution Points Rate start Stop Area Reject

External $\quad 1 \quad 5151 \quad 3000 \quad 5 \mathrm{~Hz} 0.00 \quad 10.00 \quad 50$

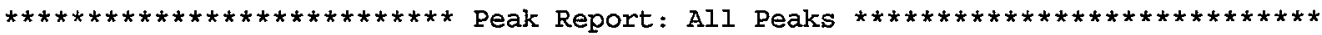

Pk. Ret Component
Num Time Name $\begin{gathered}\text { Concentration } \\ \text { ug/ml }\end{gathered}$ Height Area Bl. $\begin{gathered}\text { BDelta } \\ \text { Code }\end{gathered}$

Num Time Name

\begin{tabular}{|c|c|c|c|c|c|c|c|}
\hline $\begin{array}{l}2 \\
3\end{array}$ & $\begin{array}{l}1.09 \\
1.45\end{array}$ & fluoride & $\begin{array}{r}716.636 \\
0.000\end{array}$ & $\begin{array}{r}361 \\
21\end{array}$ & $\begin{array}{r}2327 \\
114\end{array}$ & $\begin{array}{l}2 \\
1\end{array}$ & 4.13 \\
\hline 4 & 1.64 & chloride & 260.961 & 121 & 640 & 1 & 2.93 \\
\hline 5 & 1.94 & nitrite & 1726.031 & 301 & 2043 & 1 & 0.69 \\
\hline 6 & 2.92 & bromide & 752.922 & 77 & 542 & 1 & 3.79 \\
\hline 7 & 3.28 & nitrate & 20222.615 & 3535 & 30361 & 1 & 4.90 \\
\hline 8 & 4.69 & phosphate & 242.762 & 18 & 213 & 1 & 2.33 \\
\hline 9 & 6.08 & sulfate & 1226.394 & 103 & 1976 & 1 & 1.79 \\
\hline 10 & 7.95 & oxalate & 671.597 & 29 & 672 & 1 & 2.05 \\
\hline
\end{tabular}

Totals $25819.9 \pm 8 \quad 4567 \quad 38886$

File: 96111811.D05 Sample: S96T005012 SPK

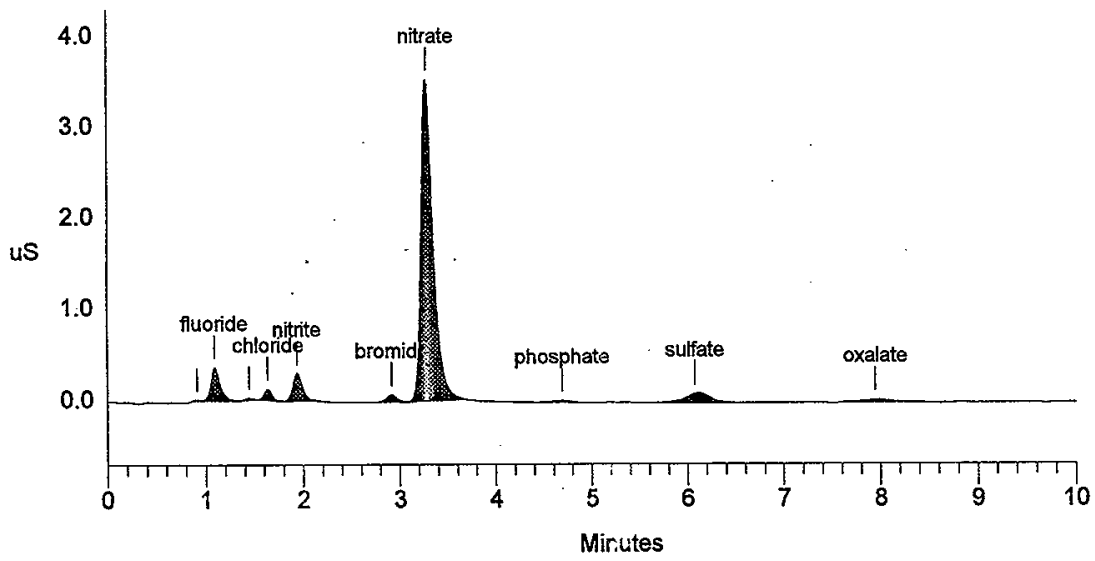




\section{LABCORE Completed Worklist Report for Worklist\# 13780}

Analyst: eal

Instrument: IC02

Book\# 32N20A

Method: $\angle A-533-10 S \operatorname{Rev} / \operatorname{Mod} D-1$

Worklist Comment: AW-105 IC. RCJ

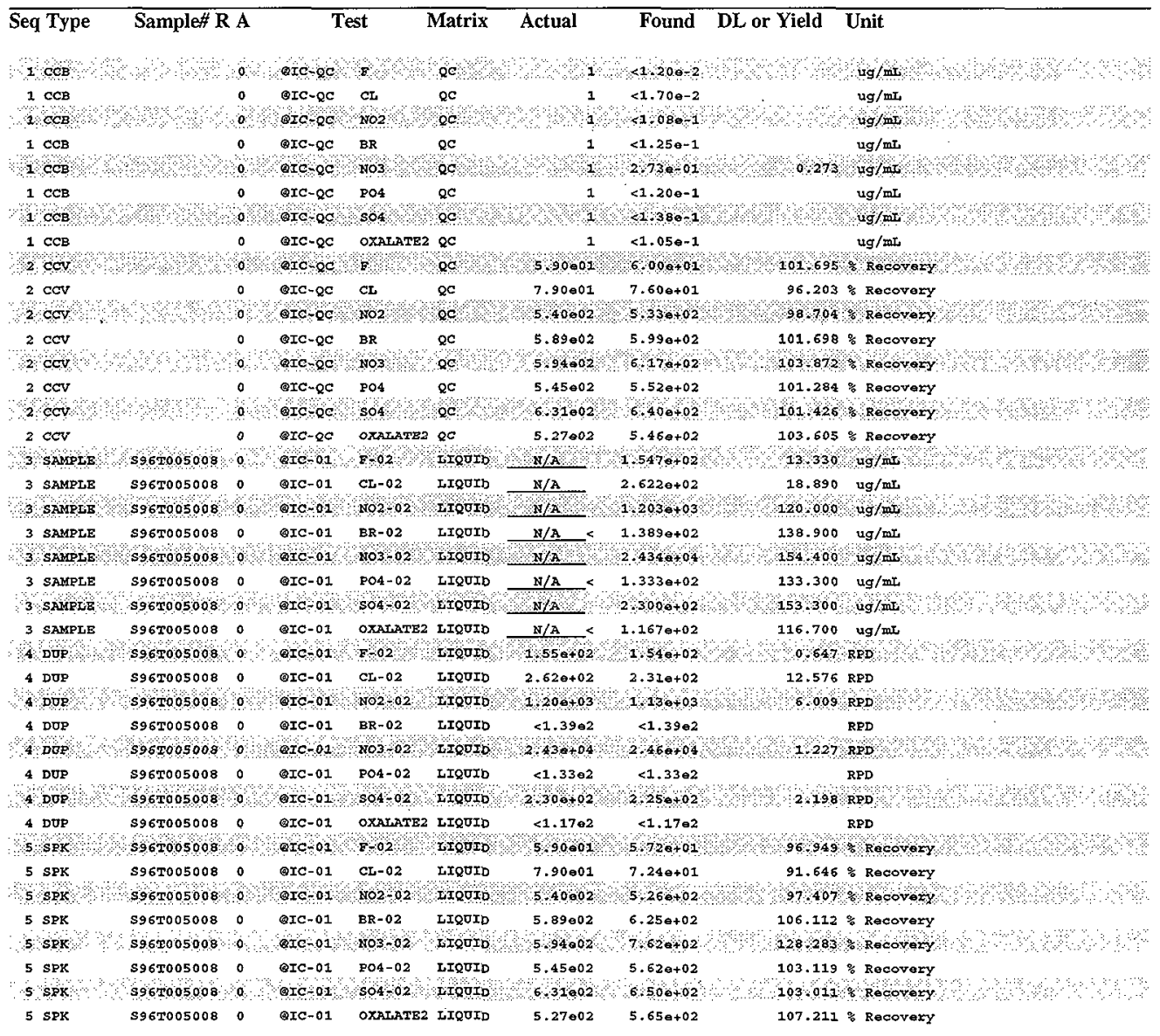




\section{LABCORE Completed Worklist Report for Worklist\# 13780}

\begin{tabular}{lllll}
\hline Seq Type Sample\#R A & Test & Matrix Actual Found DL or Yield Unit
\end{tabular}

\section{Final page for worklist\# 13780}

Analyst Signature Date Analyst Signature Date




\section{LABCORE Data Entry Template for Worklist\# 13780}

Analyst:

\&De Instrument: IC0 ICOZZ Book\# 32N20-A

Method: LA-533-105 Rev/Mod D.l

Worklist Comment: AW-105 IC. RCJ

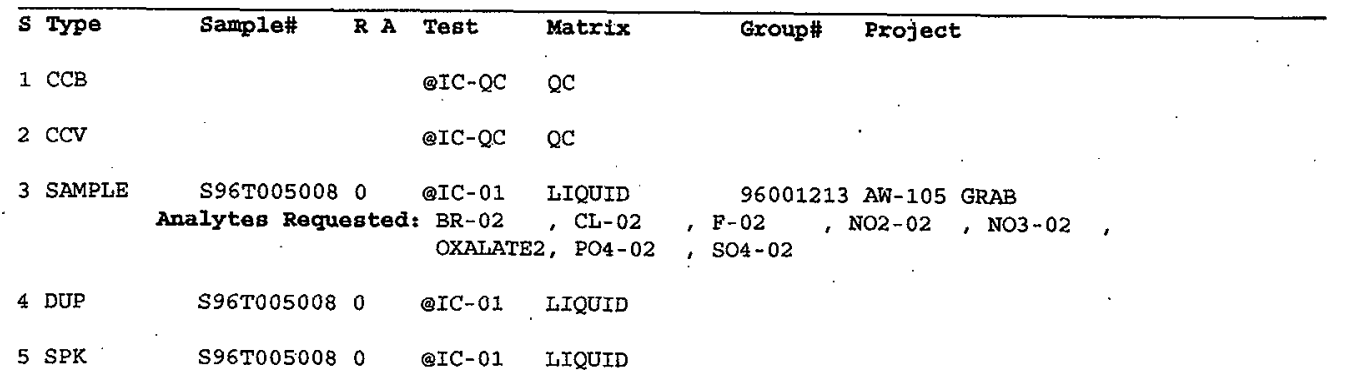

A 1020.96

Andlyst Signature Date

Analyst Signature Date

13780 oc.esy, sect

Inshell

liploadid $10 / 30 / 96$ ginfey

valedete $10 / 3 / 96$

Data Entry Comments:

$\bar{S}=$ Worklist Slot Number, $R=$ Replicate Number, $A=$ Aliquot Code. 
Data Reprocessed On 10/30/1996 14:42:50

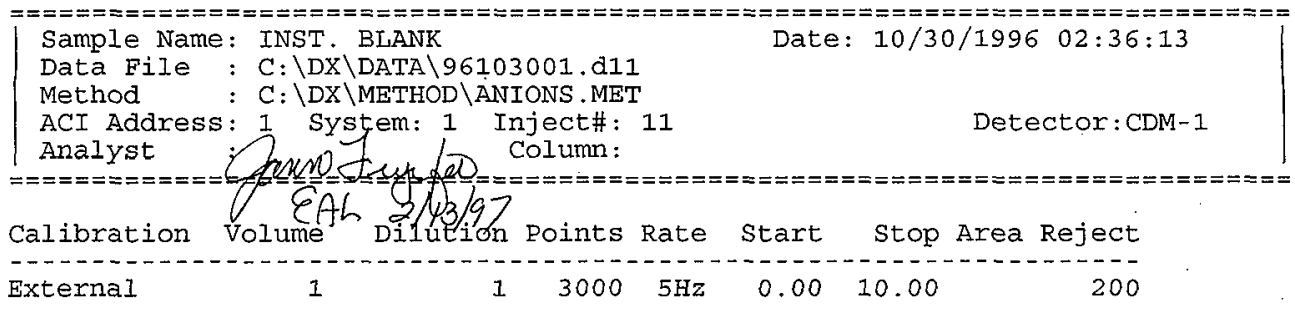

$* * * * * * * * * * * * * * * * * * * * * * * * * * *$ Peak Report: All Peaks $* * * * * * * * * * * * * * * * * * * * * * * * * * * *$

$\begin{array}{lrrr}\text { Pk. Ret Component } & \text { Concentration } & \text { Height } & \text { Area Bl. } \\ \text { Num Delta }\end{array}$

$4 \quad 3.47$ NITRATE

0.273

151

1050

$1-0.29$

Totals

0.273

151

1050

File: 96103001.d11 Sample: INST. BLANK

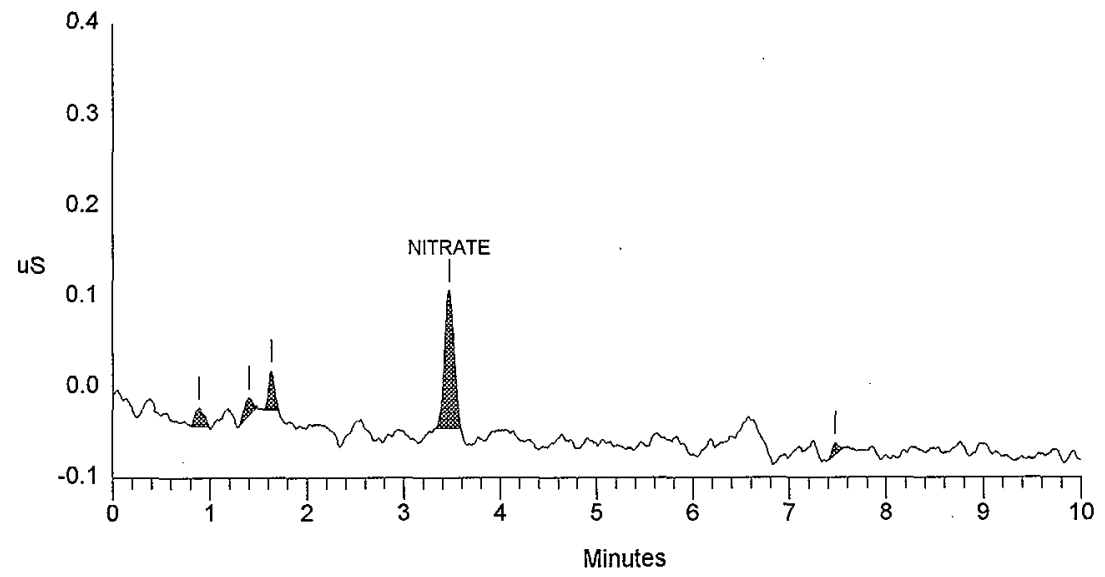

SIGNATURE ABOVE REPRESENTS CHEMICAL TECHNOLOGIST/CHEMIST THAT COMPLETEDNERIFIED THE CALIBRATION/ANALYSIS ON PAGES 270 TO 276 
Data Reprocessed on 10/30/1996 14:42:50

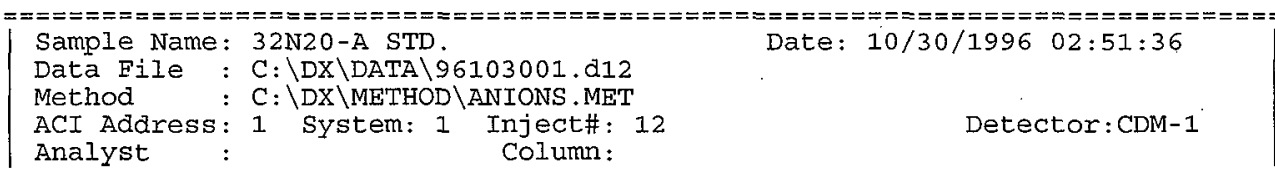

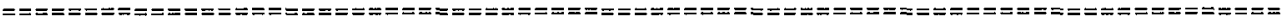

Calibration Volume Dilution Points Rate start stop Area Reject

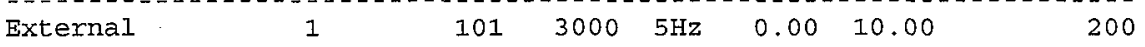

$* * * * * * * * * * * * * * * * * * * * * * * * * * *$ Peak Report: All Peaks $* * * * * * * \star * * * * * * * * * * * * * * * * * * * *$

$\begin{array}{lrrr}\text { Pk. Ret Component } & \text { Concentration } & \text { Height } & \text { Area Bl. } \\ \text { Num Delta } & \text { Time Name } / m l & \text { Code }\end{array}$

\begin{tabular}{|c|c|c|c|c|c|c|c|}
\hline 1 & 0.34 & & 0.000 & 23 & 258 & 1 & \\
\hline 2 & 0.88 & & 0.000 & 125 & 584 & 2 & \\
\hline 3 & 1.05 & FIUUORIDE & 60.042 & 2253 & 11606 & 2 & 0.00 \\
\hline 4 & 1.62 & CHLORIDE & 76.024 & 1979 & 9969 & 1 & -1.02 \\
\hline 5 & 1.96 & NITRITE & 533.088 & 8313 & 49868 & 1 & -1.83 \\
\hline 6 & 2.98 & BROMIDE & 598.933 & 5360 & 36512 & 2 & -1.86 \\
\hline 7 & 3.40 & NITRATE & 617.177 & 5923 & 48855 & 2 & -2.20 \\
\hline 8 & 4.94 & PHOSPHATE & 552.451 & 1723 & 21483 & 1 & -3.96 \\
\hline 9 & 6.52 & SULFATE & 639.581 & 4537 & 65129 & 1 & -4.35 \\
\hline 10 & 8.60 & OXALATE & 546.354 & 2065 & 40139 & 1 & -4.69 \\
\hline
\end{tabular}

Totals $\quad 3623.649 \quad 32301 \quad 284404$

File: 96103001.d12 Sample: 32N20-A STD.

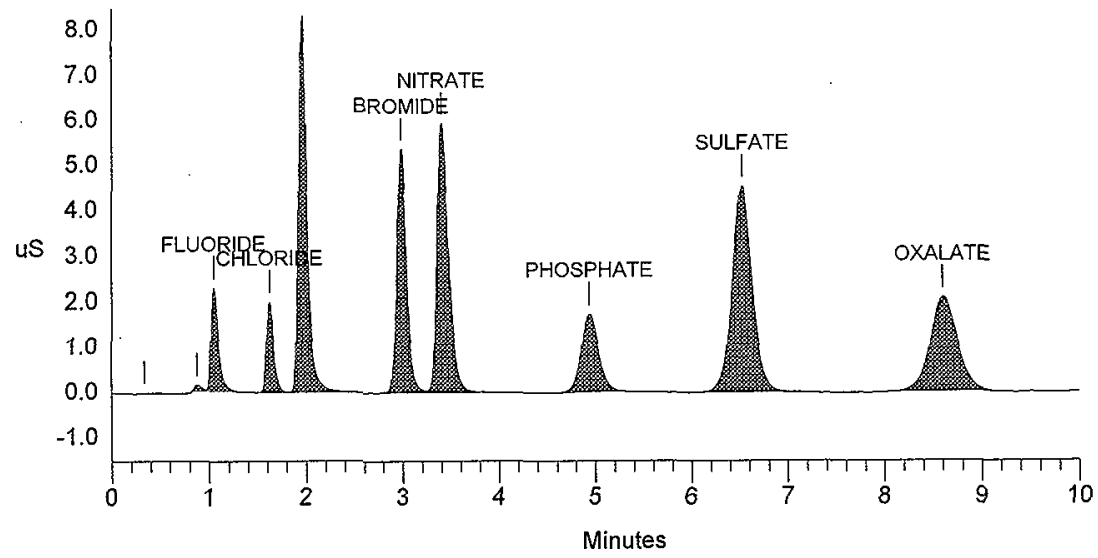


HNF-SD-WM-DP-214, REV. 0

Data Reprocessed On 10/30/1996 14:42:51

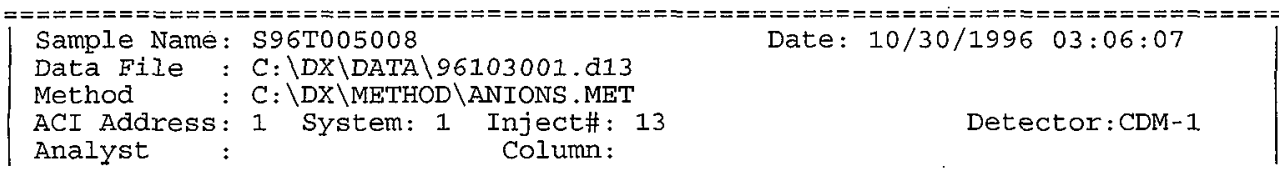

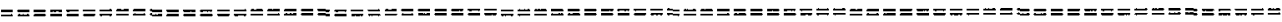

Calibration Volume Dilution Points Rate start Stop Area Reject

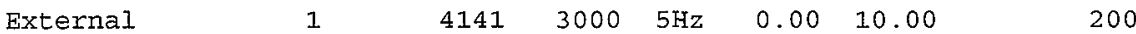

$\star * * * * * * * * * * * * * * * * * * * * * * * * * *$ Peak Report: All Peaks $* * * * * * * * * * * * * * * * * * * * * * * * * * * *$

Pk. Ret Component $\quad \begin{array}{r}\text { Concentration } \\ \text { ug/mI }\end{array}$
Num Time Name Height Area Bl.

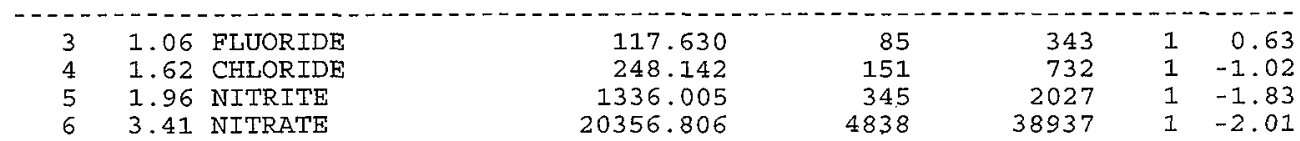

$\begin{array}{llll}\text { Totals } & 22058.582 & 5418 & 42039\end{array}$

File: 96103001.d13 Sample: S96T005008

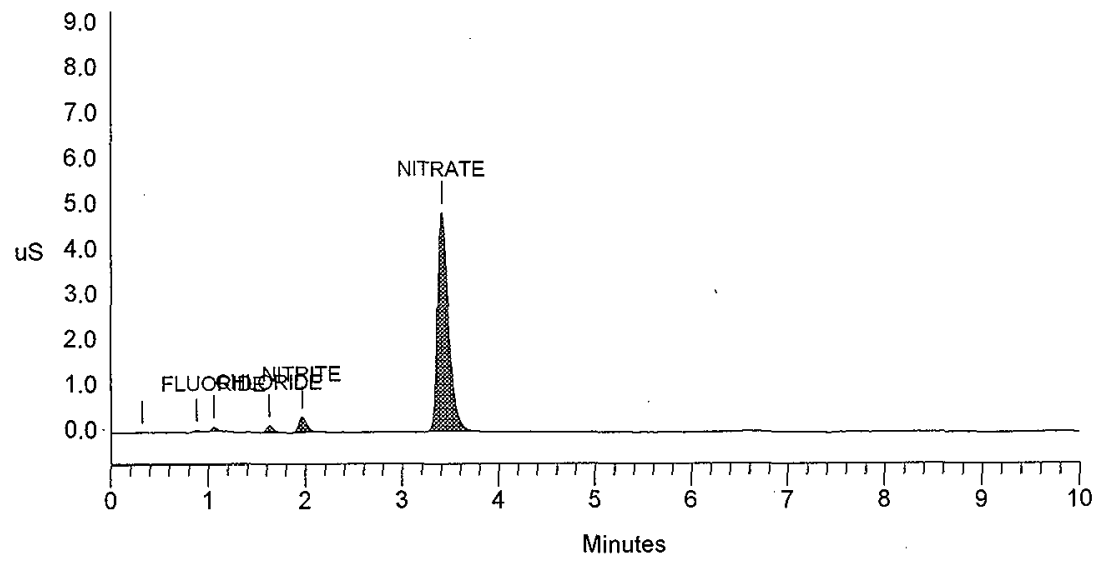


Data Reprocessed on 10/30/1996 14:42:51

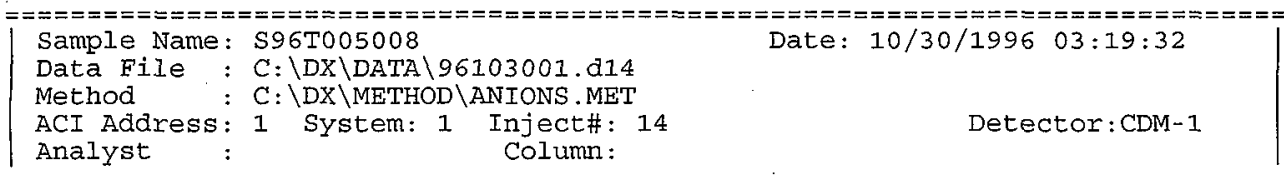

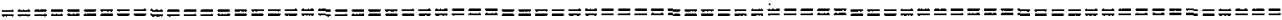

Calibration Volume Dilution Points Rate start stop Area Reject

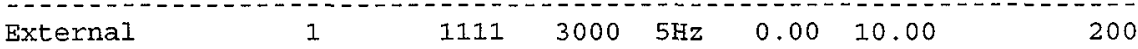

$* * * * * * * * * * * * * * * * * * * * * * * * * * *$ Peak Report: All Peaks $* * * * * * * * * * * * * * * * * * * * * * * * * * * *$

$\begin{array}{lrrr}\text { Pk. Ret Component } & \text { Concentration } & \text { Height } & \text { Area B } / \mathrm{ml} \\ \text { Num } & \text { Time Name } & \text { Code }\end{array}$

$\begin{array}{lll}2 & 0.88 & \\ 3 & 1.05 & \text { ELUORIDE } \\ 4 & 1.62 & \text { CHLORIDE } \\ 5 & 1.96 & \text { NITRITE } \\ 7 & 3.32 & \text { NITRATE } \\ 8 & 6.56 & \text { SULFATE }\end{array}$

$\begin{array}{rrrrr}0.000 & 161 & 921 & 2 & \\ 154.702 & 456 & 2531 & 2 & 0.00 \\ 262.229 & 546 & 3059 & 1 & -1.02 \\ 1202.888 & 1486 & 9310 & 1 & -2.17 \\ 24339.610 & 20377 & 187420 & 1 & -4.50 \\ 230.005 & 91 & 1282 & 1 & -3.86\end{array}$

Totals $26189.434 \quad 23118 \quad 204522$

\section{File: 96103001.d14 Sample: S96T005008}

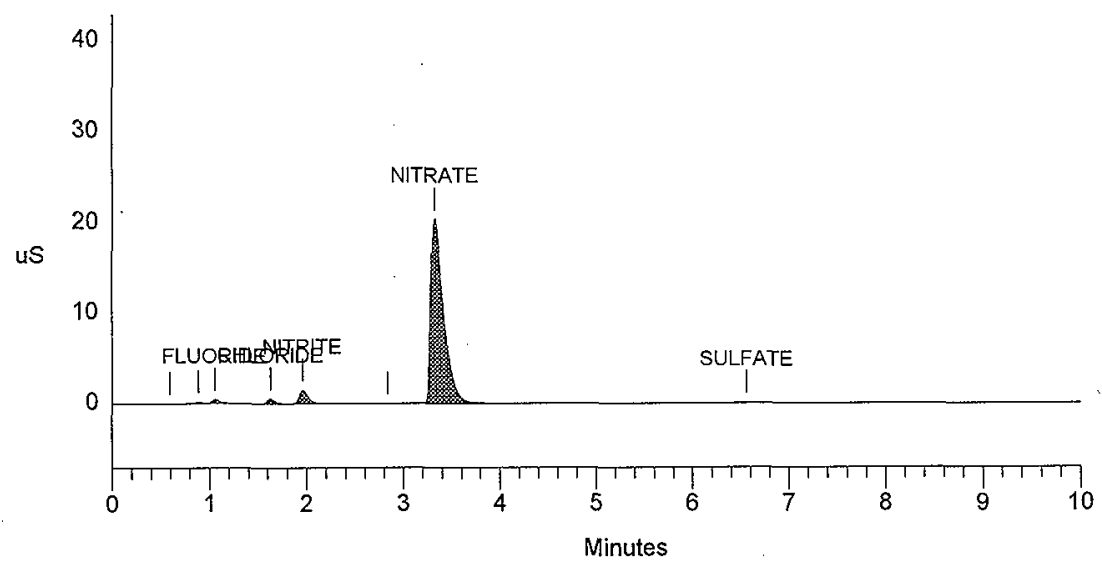


Data Reprocessed on 10/30/1996 14:42:51

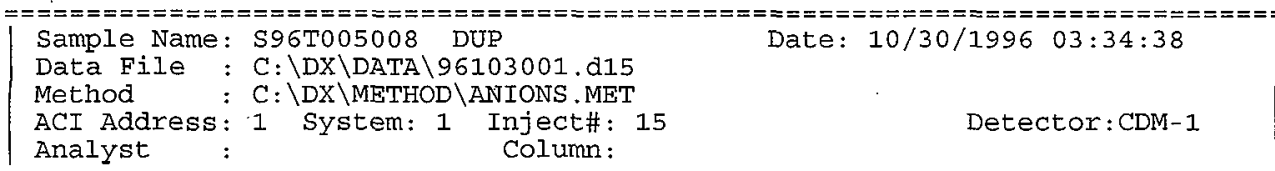

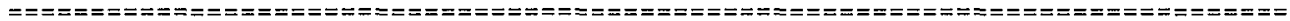

Calibration Volume Dilution Points Rate start stop Area Reject

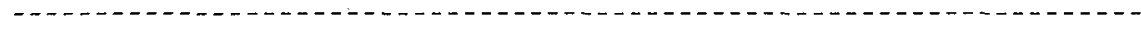

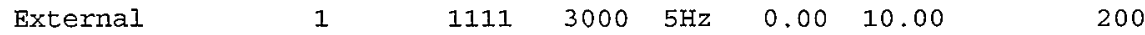

$* * * * * * * * * * * * * * * * * * * * * * * * * * *$ Peak Report: All Peaks $* * * * * * * * * * * * * * * * * * * * * * * * * * * *$

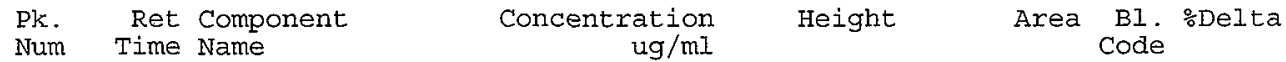

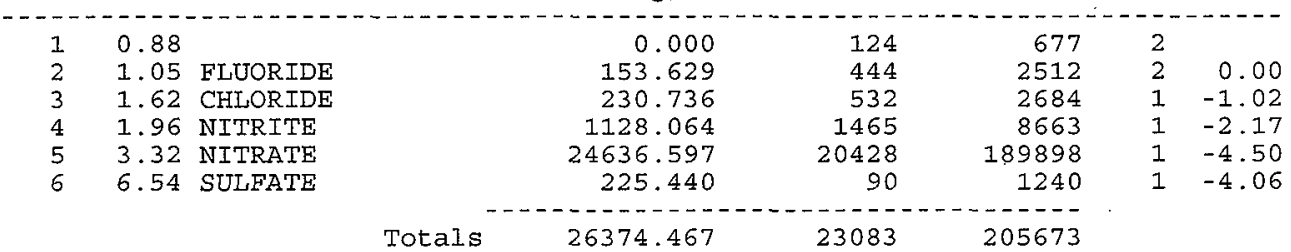

\section{File: $96103001 . d 15$ Sample: S96T005008 DUP}

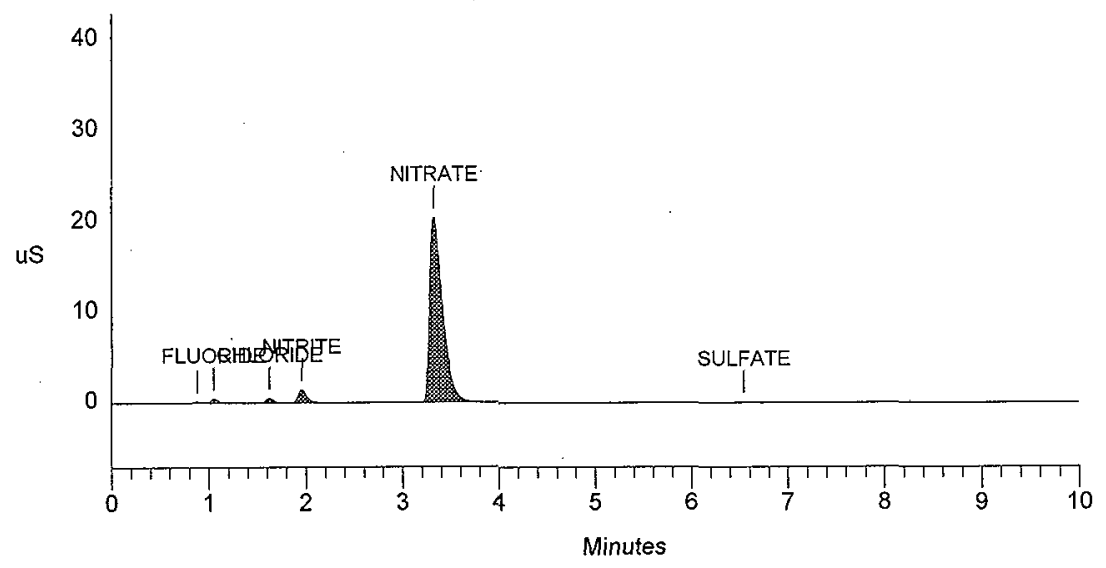




\section{HNF-SD-WM-DP-214, REV. 0}

Data Reprocessed On 10/30/1996 $14: 42: 52$

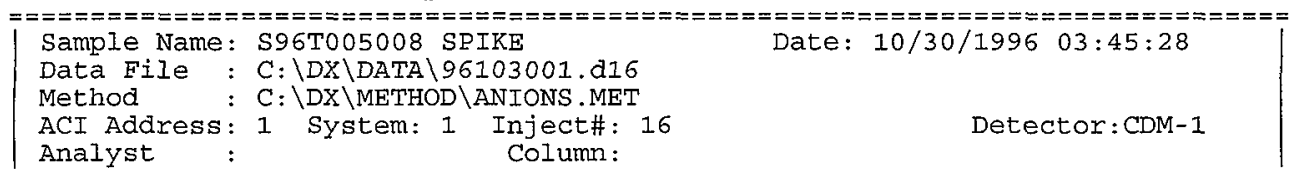

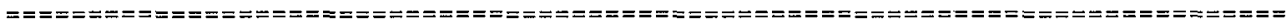

Calibration Volume Dilution Points Rate start stop Area Reject

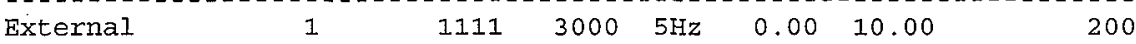

$\star * * * * * * * * * * * * * * * * * * * * * * * * * *$ Peak Report: All Peaks $* * * * * * * * * * * * * * * * * * * * * * * * * * * *$

$\begin{array}{lrrr}\text { Pk. Ret Component } & \text { Concentration } & \text { Height } & \text { Area Bl. } \\ \text { Num } & \text { Time Name } / \mathrm{ml} & \text { Code }\end{array}$

$1 \quad 0.88$

21.05 FLUORIDE

31.62 CHLORIDE

41.96 NITRITE

$5 \quad 2.96$ BROMIDE

$6 \quad 3.30$

$7 \quad 4.94$ PHOSPHATE

86.52 SULFATE

98.60 OXALATE

.

Totals

$\begin{array}{rrrrr}0.000 & 216 & 1003 & 2 & \\ 732.193 & 2547 & 12905 & 2 & 0.00 \\ 993.402 & 2319 & 11882 & 2 & -1.42 \\ 6515.706 & 9434 & 55589 & 2 & -2.17 \\ 6310.581 & 5239 & 34907 & 2 & -2.52 \\ 0.000 & 26162 & 253314 & 2 & \\ 5679.770 & 1614 & 20022 & 1 & -3.83 \\ 6797.265 & 4330 & 62852 & 1 & -4.45 \\ 5711.580 & 1972 & 38093 & 1 & -4.69 \\ ---19 .-496 & 53835 & 490569 & & \end{array}$

\section{File: 96103001.d16 Sample: S96T005008 SPIKE}

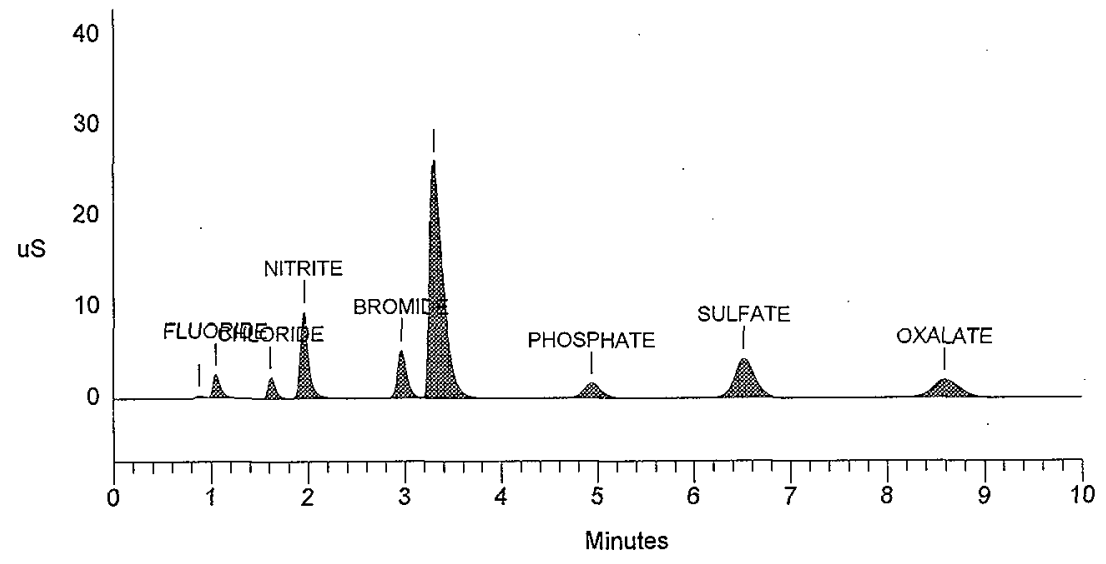


Data Reprocessed On 10/30/1996 14:50:00

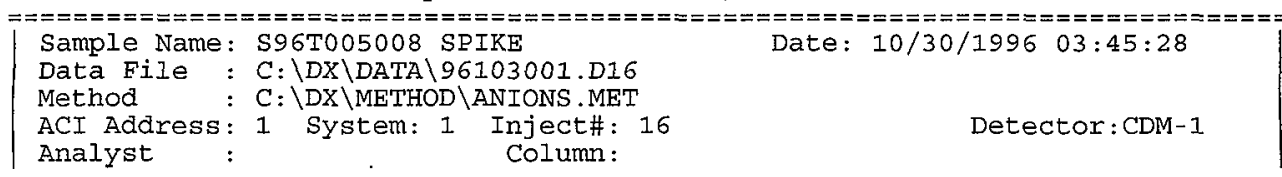

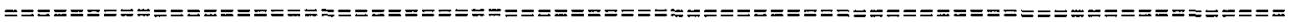

Calibration Volume Dilution Points Rate start stop Area Reject

External

External

$11113000 \quad 5 \mathrm{~Hz} \quad 0.00 \quad 10.00$

200

$\star * * * * * * * * * * * * * * * * * * * * * * * * * *$ Peak Report: All Peaks $* * * * * * * * * * * * * * * * * * * * * * * * * * * *$

Pk. Ret Component
Num Time Name

$1 \quad 0.88$

2. 1.05 FLUORIDE

31.62 CHLORIDE

41.96 NITRITE

$5 \quad 2.96$ BROMIDE

$6 \quad 3.30$ NITRATE

74.94 PHOSPHATE

86.52 SULFATE

98.60 OXALATE
Concentration $\mathrm{ug} / \mathrm{ml}$

.

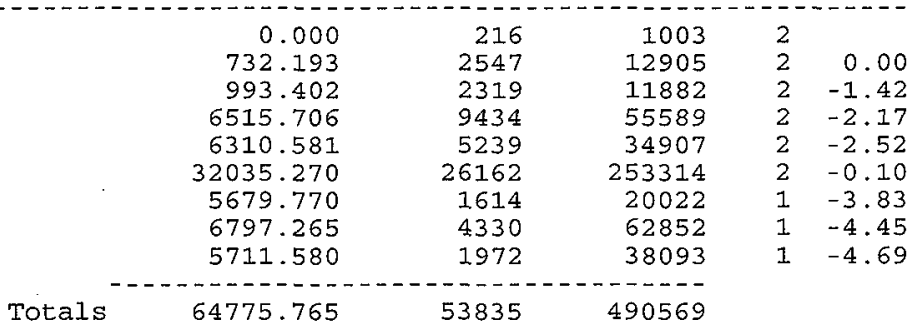

File: 96103001.D16 Sample: S96T005008 SPIKE

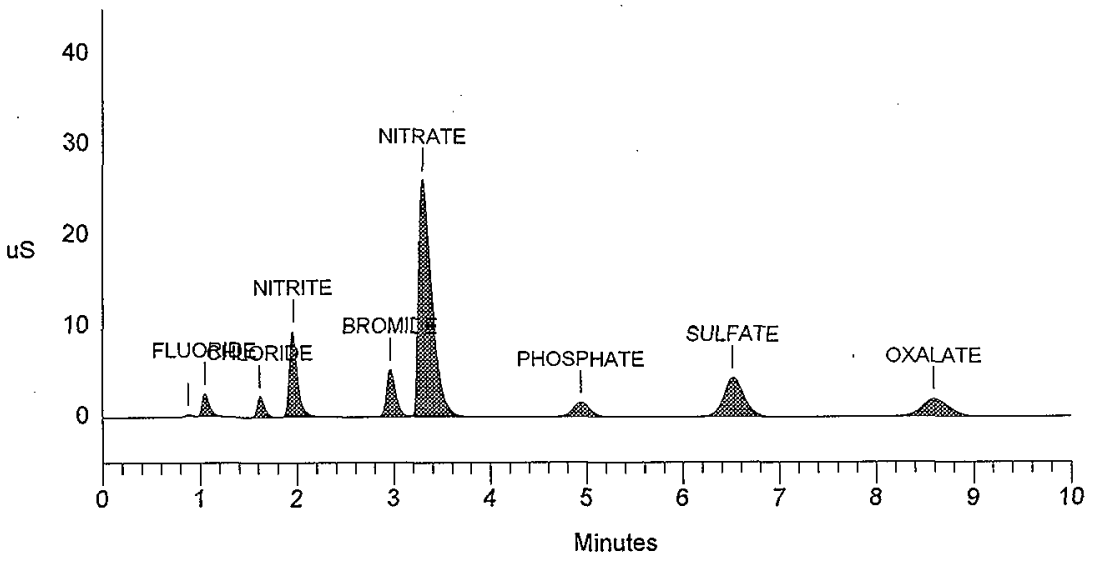




\section{LABCORE Completed Worklist Report for Worklist\# 13805}

Analyst: rdm

Instrument: IC01

Book\# $14 / 20 C$

Method: LA-033-105-Rev/Mod D-1

Worklist Comment: Rerun of AW-105 Ic. Try 5151 df.jmf

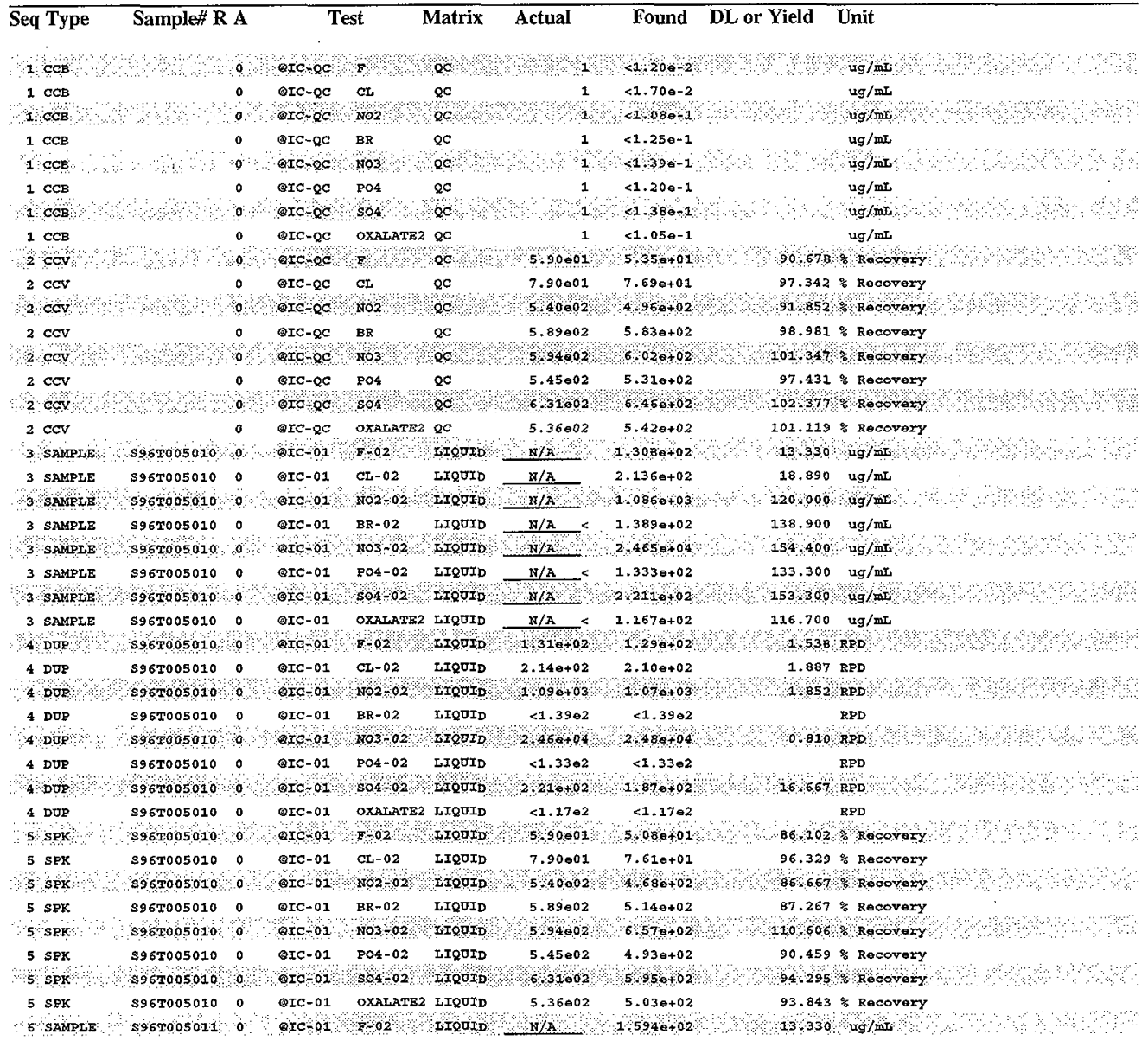




\section{LABCORE Completed Worklist Report for Worklist\# 13805}

\begin{tabular}{|c|c|c|c|}
\hline$\overline{\text { Seq }}$ & Type & Sample\# $\mathbf{R}$ & \\
\hline 6 & SAMPLE & S96T005011 & 0 \\
\hline 6 & SAMPLE & S96T005011 & \\
\hline 6 & SAMPE & 506700501 & \\
\hline 6 & SAMPLE & S96T005011 & \\
\hline 6 & SAMPLE & $\$ 961005011$ & \\
\hline 6 & SAMPLE & $596 \pi 005011$ & \\
\hline 6 & SAMPLB & $\$ 960005011$ & \\
\hline 7 & DUR & $596 \mathrm{~T} 005011$ & \\
\hline 7 & $D U D$ & $95 T 005011$ & \\
\hline 7 & DUP & $\$ 96 T 005011$ & 0 \\
\hline 7 & 0 & 906005011 & \\
\hline 7 & DUR & S96T005011 & 0 \\
\hline$?$ & DUR & 9909005011 & \\
\hline 7 & DOR & 5967005011 & \\
\hline & & 600501 & \\
\hline
\end{tabular}

\begin{tabular}{|c|c|c|c|c|}
\hline \multicolumn{2}{|c|}{ Test } & Matrix & Actual & Found \\
\hline (arc-01 & $\mathrm{CL}-02$ & IxQDID & $N / A$ & $2.5160+$ \\
\hline OIC-01 & $\mathrm{NO2}-02$ & IIQUID & $\mathrm{N} / \mathrm{A}$ & $1.2840+$ \\
\hline CIC 01 & $B R \quad 02$ & ITOUID & $N / x$ & 13890 \\
\hline (1C-01 & No3-02 & IIQOID & $\mathbb{N} / \mathrm{A}$ & $2.9180+0$ \\
\hline QIC- 01 & 00402 & IIODID & $N / A$ & $13338-$ \\
\hline QIC-01 & SO4-02 & IIQUID & $N / A$ & $2.1370+$ \\
\hline 0101 & $O X A \sim A T 2$ & $2 \mathrm{IrOOD}_{0}$ & $\mathrm{~N} / \mathrm{A}$ & $1-1670-$ \\
\hline (IC-01 & F- 02 & IIQUID & $1.590+02$ & $1.530+$ \\
\hline$Q 10-01$ & 0,02 & OTOPD & $2 / 520+02$ & $2,48 \theta+0$ \\
\hline $\operatorname{arc}-01$ & NO2-02 & IIQUID & $1.28 e+03$ & $1.250+0$ \\
\hline $9 r-01$ & $\mathrm{ER} 02$ & YQOUDD & $\begin{array}{r}3902 \\
\end{array}$ & $<139$ \\
\hline (IC-01 & No3-02 & LIQUID & $2.92 \theta+04$ & $2.85 e+0$ \\
\hline $010-03$ & Po 402 & Tyerro & 41,3302 & $<1 \div 33$ \\
\hline (i) 01 & $304-02$ & IIQOXD & $2.14 \theta+02$ & $2.14 a+0$ \\
\hline & & & & \\
\hline
\end{tabular}

\section{DL or Yield Unit}

$18.890 \mathrm{ug} / \mathrm{mr}$

$220.000 \mathrm{Lg} / \mathrm{mL}$

$138.900 \mathrm{ug} / \mathrm{mu}$

$154.400 \mathrm{ug} / \mathrm{mL}$

$133,300 \mathrm{Hg} / \mathrm{ms}$

$153.300 \mathrm{ug} / \mathrm{mL}$

$116700 \mathrm{hg} / \mathrm{mL}$

3.846 RPD

$1.600 \mathrm{RPD}$

2.372 RPD

RPD

2.426 RPD

0.000 PPD

RPD:

\section{Final page for worklist\# 13805}

Analyst Signature

Date

Analyst Signature

Date

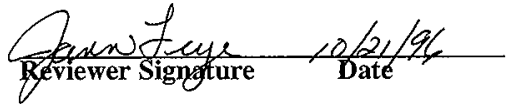

Units shown for $Q C$ (BLK/BKG) may not reflect the actual units. 


\section{Analyst: RoOn Instrument: $1 \mathrm{CO} I \mathrm{ICO} / \mathrm{Bok} \# 14 \mathrm{~N} 20 \mathrm{C}$}

Method: LA-533-105 Rev/Mod D-1

Worklist Comment: Rerun of AW-105 Ic. Try 5151 df.jmf

\begin{tabular}{|c|c|c|c|c|c|c|c|c|}
\hline S Type & Sample\# & R A & Test & Natrix & Group\# & Project & & \\
\hline $1 \mathrm{CCB}$ & & & @IC-QC & $Q C$ & & & & \\
\hline $2 \mathrm{CCV}$ & & & $\Theta I C-Q C$ & QC. & & & & \\
\hline 3 SAMPLE & S96T005010 & 0 & @IC -01 & IIQUID & 96001215 & $5 \mathrm{AW}-105$ & GRAB & \\
\hline & Analytes Reque & ested: & $\begin{array}{l}\text { BR-02 } \\
\text { OXALATE2 }\end{array}$ & $\begin{array}{l}\mathrm{CL}-02 \\
\therefore \mathrm{PO}-02\end{array}$ & $\left\{\begin{array}{l}\mathrm{F}-02 \\
\mathrm{SO}-\mathrm{-O} 2\end{array}\right.$ & NO2-02 & , No3-02 & , \\
\hline 4 DUP & S96T005010 & 0 & (a)-01 & I,IQUID & & & & \\
\hline 5 SPK & s96T005010 & 0 & @IC-01 & LIQUID & & & & \\
\hline \multirow[t]{2}{*}{ 6. SAMPLE } & S96T005011 & 0 & QIC-01 & LIQUID & 96001215 & 5 AW-105 & GRAB & \\
\hline & Analytes Reque & ested: & $\begin{array}{l}\text { BR-02 } \\
\text { OXAI_ATE2, }\end{array}$ & $\begin{array}{l}\mathrm{CL}-02 \\
\therefore \mathrm{PO}-02\end{array}$ & $\begin{array}{l}\mathrm{F}-02 \\
\mathrm{SO}-02-02\end{array}$ & NO2-02 & , $\mathrm{NO}-02$ & , \\
\hline
\end{tabular}

7 DUP

S96T005011 0 BIC-01 IIQUID

Final page for worklist \# 13805

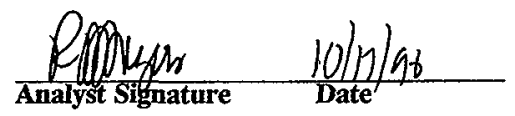

\section{Analyst Signature Date}

13805 oc esv

Inshell

cyploaded 10/21/96 omp

valedated $1021 / 96$ MmE

Data Entry Comments:

$S=$ Worklist Slot Number, $R=$ Replicate Number, $A=$ Aliquot Code. 
HNF-SD-WM-DP-214, REV. 0

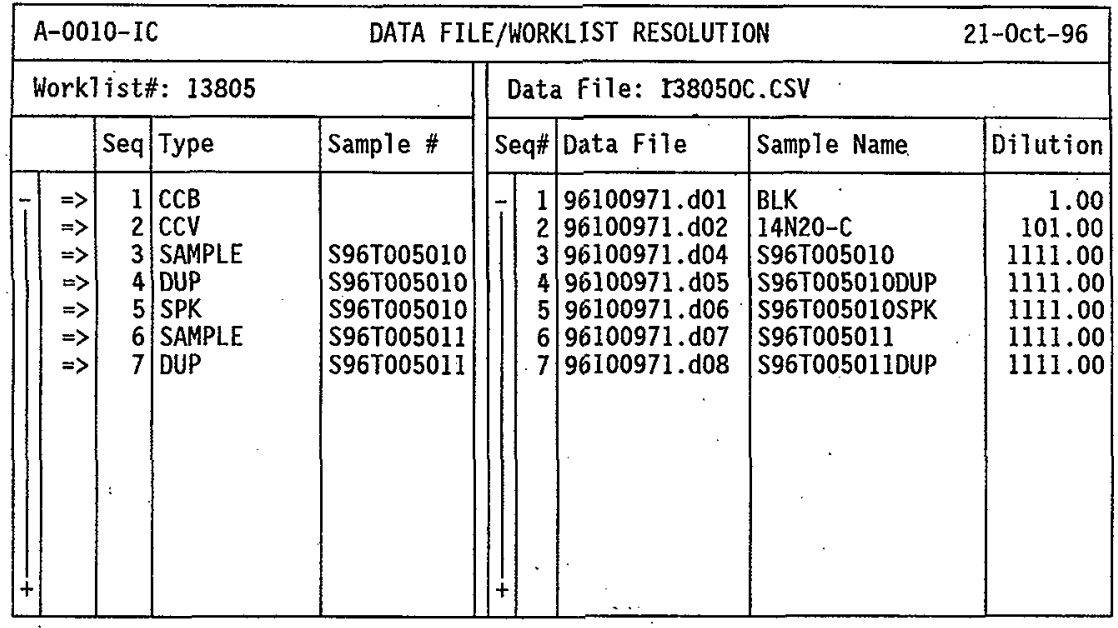

Save(F4) Abort(Shift-F3) ListFiles(Shift-F1) UploadFile(F8) 
Sample Name: BLK

Data File : C:\DX\DATA \96100971.D01

Method : C: \DX \METHOD $\backslash$ KIT.MET

ACI Address: 1 System: 1 Inject\# : 1
Date: $10 / 17 / 199619: 19: 39$

Analyst Coun 1 Column: AG4À/AS4A anion column

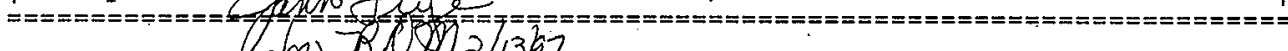
calibration volfme Dilution Pbints Rate start stop Area Reject

External 1

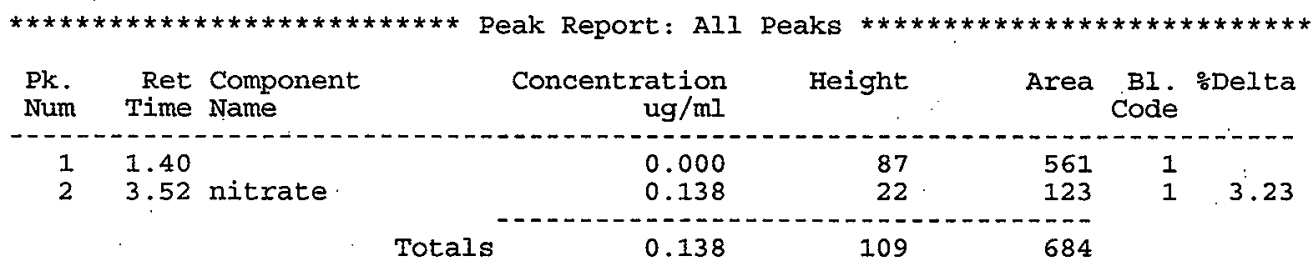

File: 96100971.D01 Sample: BLK

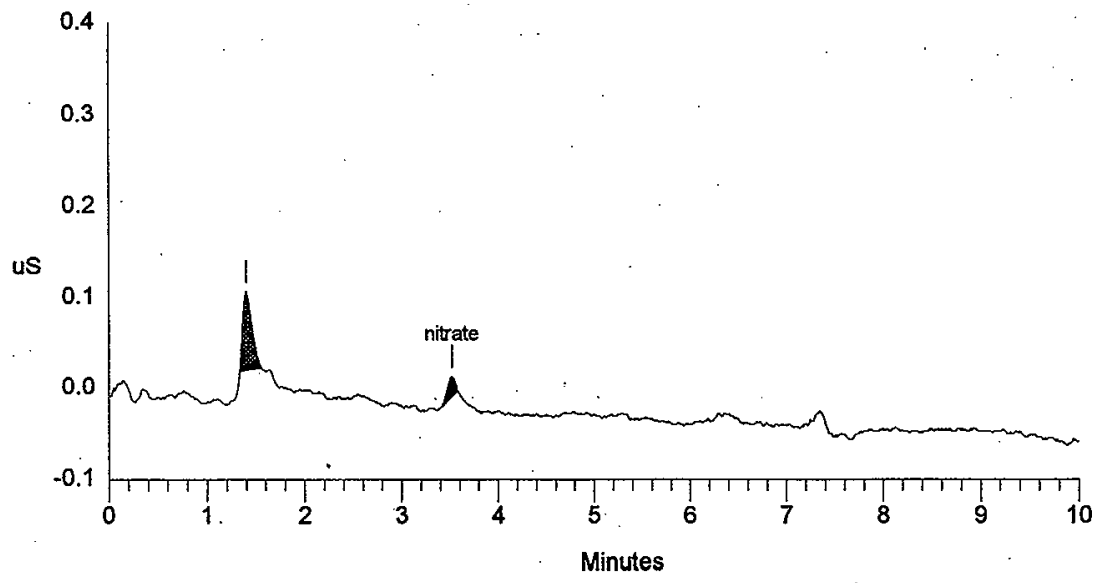

SIGNATURE ABOVE REPRESENTS CHEMICAL TECHNOLOGIST/GHEMIST THAT

COMPLETED/VERIFIED THE CALIBRATION/ANALYSIS ON PAGES 387 TO 387 . 


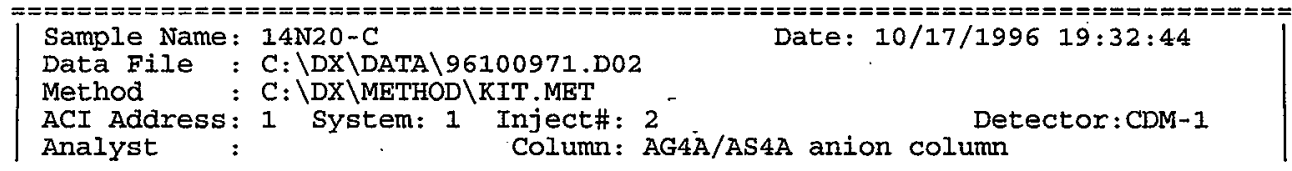

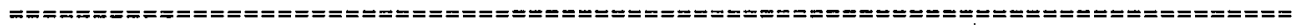

Calibration Volume Dilution Points Rate Start stop Area Reject

External $\quad 1 \quad 101 \quad 3000 \quad 5 \mathrm{~Hz} 0.00 \quad 10.00 \quad 50$

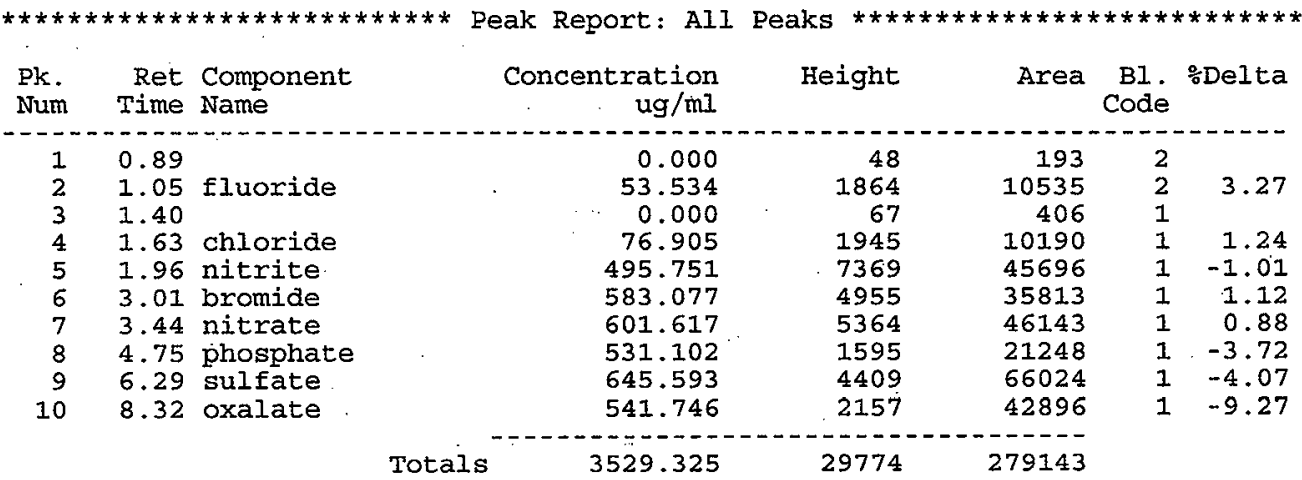

File: 96100971:D02 Sample: 14N20-C

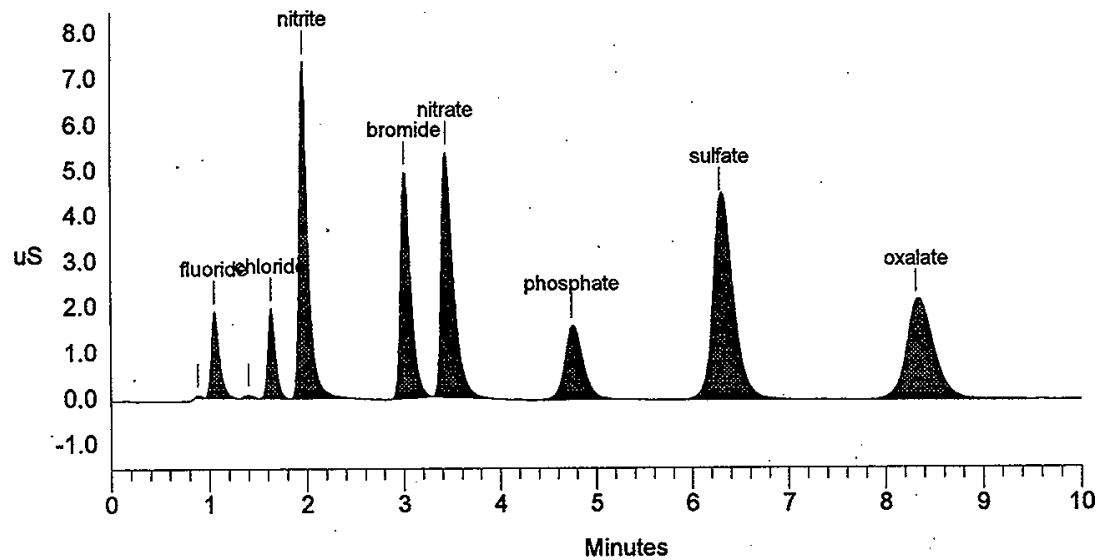




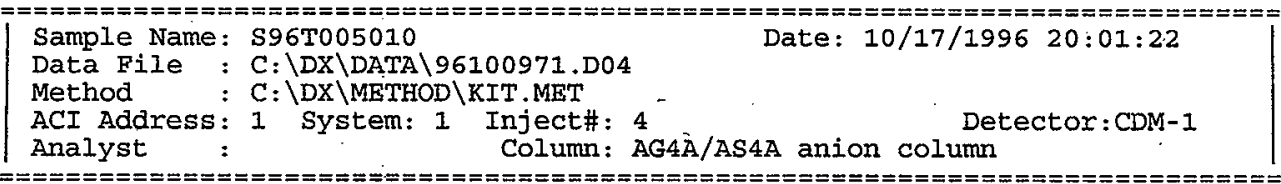

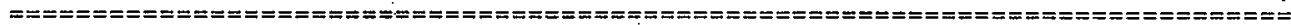

Calibration Volume Dilution Points Rate start stop Area Reject

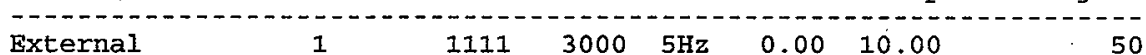

External

1

$\begin{array}{lllll}1111 & 3000 \quad 5 \mathrm{~Hz} & 0.00 & 10.00\end{array}$

50

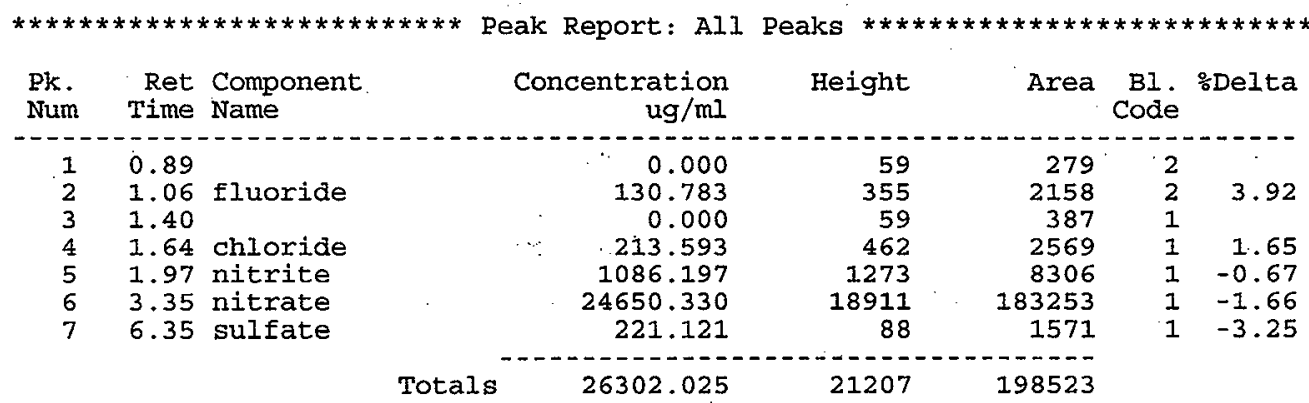

File: 96100971.D04 Sample: S96T005010

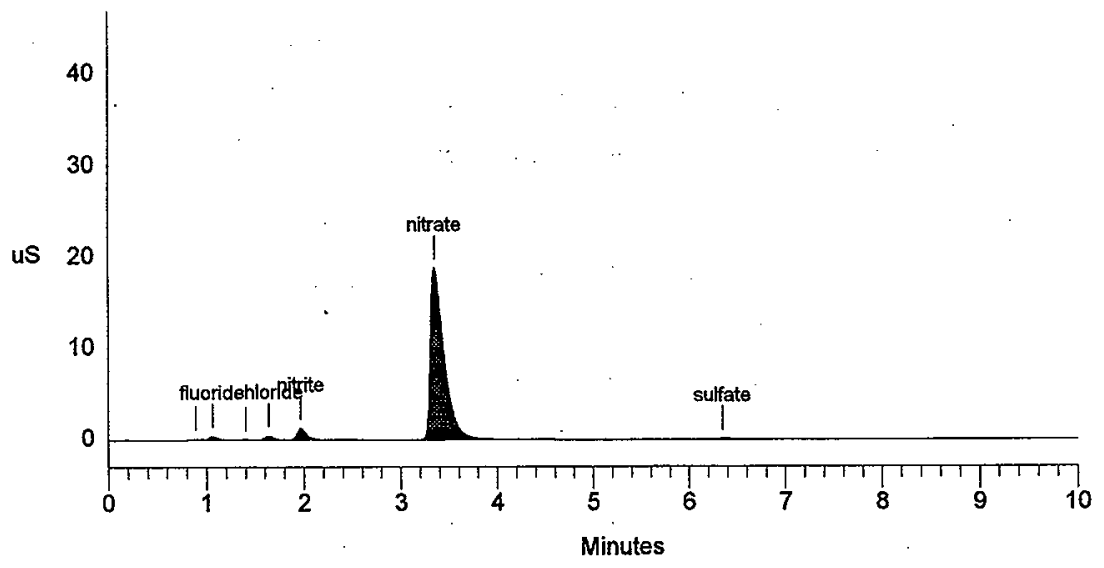




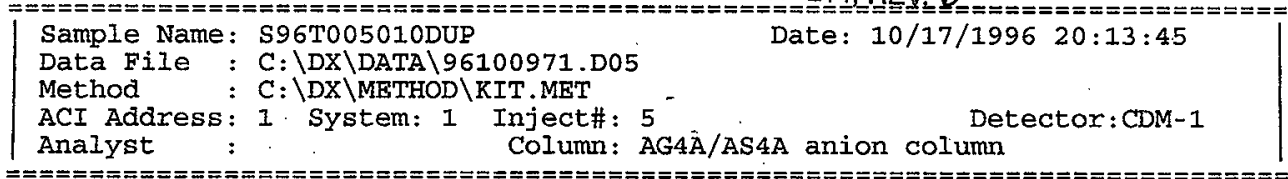

Calibration Volume Dilution Points Rate start stop Area Reject

Calibration

External

1

$\begin{array}{lllll}1111 & 3000 \quad 5 \mathrm{~Hz} & 0.00 \quad 10.00\end{array}$

50

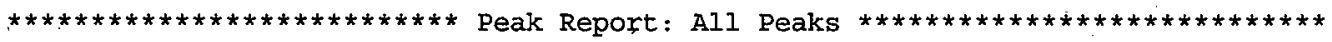

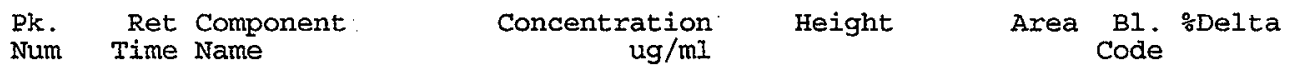

Num Time Name $\mathrm{ug} / \mathrm{mI}$

$\begin{array}{lll}1 & 0.89 & \\ 2 & 1.06 & \text { fluoride } \\ 3 & 1.39 & \\ 4 & 1.63 & \text { chloride } \\ 5 & 1.97 & \text { nitrite } \\ 6 & 3.35 & \text { nitrate } \\ 7 & 6.35 & \text { sulfate }\end{array}$

$\begin{array}{rrrrrr} & 0.000 & 59 & 286 & 2 & \\ 129.294 & 356 & 2131 & 2 & 3.92 \\ 0.000 & 210.072 & 58 & 357 & 1 & \\ & 1069.675 & 1269 & 2527 & 1 & 1.24 \\ & 24812.717 & 18902 & 184555 & 1 & -1.66 \\ & 187.173 & 85 & 1259 & 1 & -3.25 \\ \text { Totals } & 26408.932 & 21195 & 199280 & & \end{array}$

File: 96100971.D05 Sample: S96T005010DUP

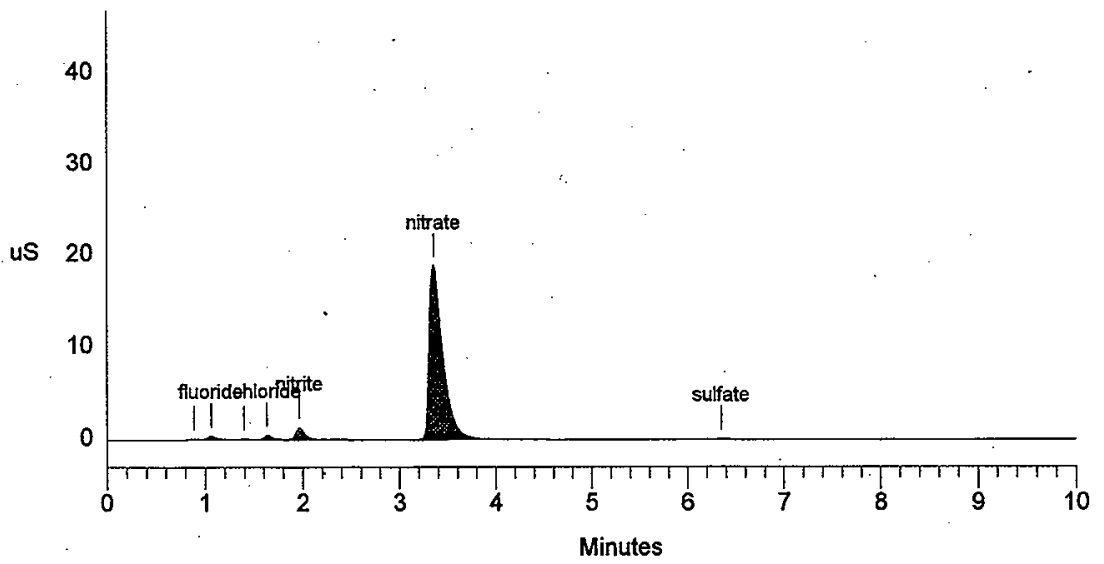




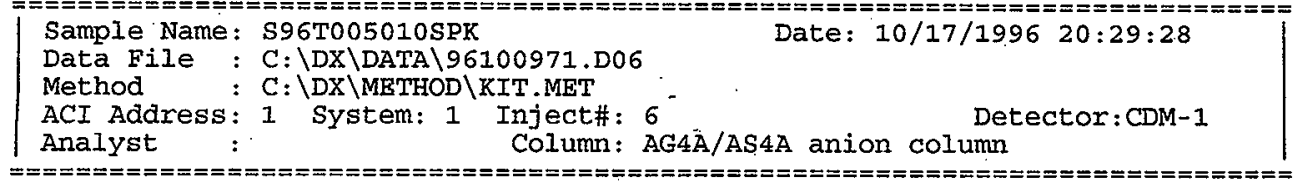

Calibration Volume Dilution Points Rate start stop Area Reject Caljbation

$\begin{array}{llllllll}\text { External } & 1 & 1111 & 3000 & 5 \mathrm{~Hz} & 0.00 & 10.00 & 50\end{array}$

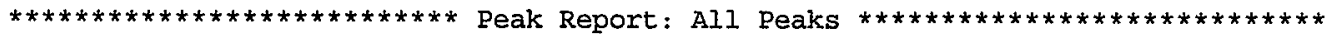

$\begin{array}{lrrr}\text { Pk. Ret Component } & \text { Concentration } & \text { Height } & \text { Area } \\ \text { Num } & \text { Bime Name } / \mathrm{ml} & & \text { Code }\end{array}$

Num Time Name

$\begin{array}{rll}1 & 0.89 \\ 2 & 1.05 & \text { fluoride } \\ 3 & 1.39 & \\ 4 & 1.63 & \text { chloride } \\ 5 & 1.95 & \text { nitrite } \\ 6 & 2.99 & \text { bromide } \\ 7 & 3.33 & \text { nitrate } \\ 8 & 4.75 & \text { phosphate } \\ 9 & 6.29 \text { sulfate } \\ 10 & 8.32 \text { oxalate }\end{array}$

$\begin{array}{rrrrr}6.000 & 100 & 418 & 2 & \\ 644.048 & 2009 & 11554 & 2 & 3.27 \\ 0.000 & 38 & 212 & 1 & \\ 982.305 & 2161 & 11854 & 1 & 0.83 \\ 5809.014 & 7743 & 48767 & 1 & -1.35 \\ 5196.137 & 4312 & 28790 & 1 & 0.45 \\ 31287.036 & 23881 & 237576 & 1 & -2.44 \\ 4980.313 & 1334 & 18073 & 1 & -3.72 \\ 6236.164 & 3805 & 57790 & 1 & -4.07 \\ 5079.410 & 1834 & 36485 & 1 & -9.27\end{array}$

Totals

60214.427

47216

451.519

File: 96100971.D06 Sample: S96T005010SPK

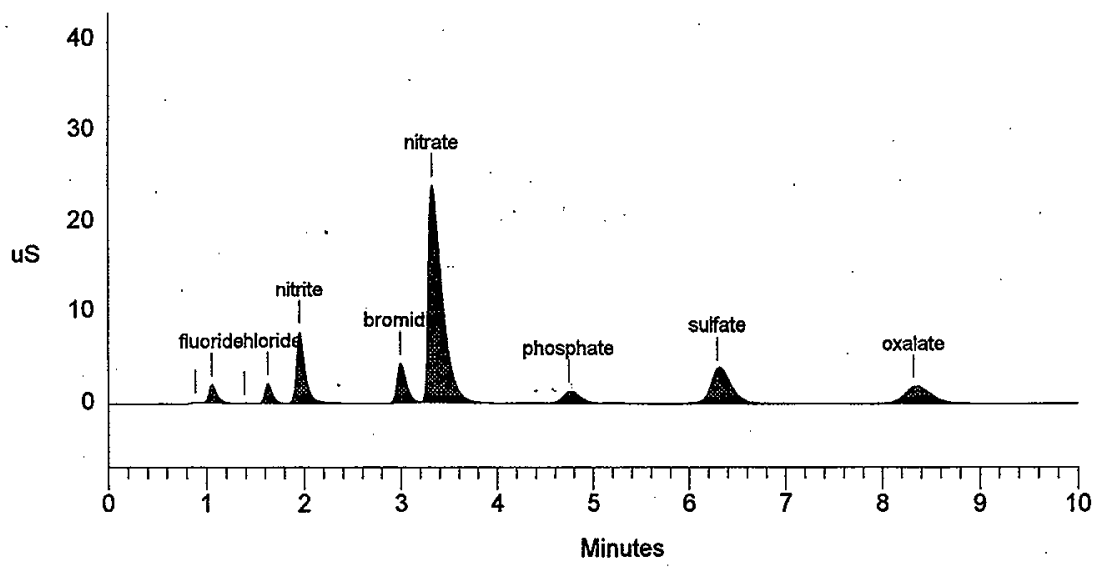




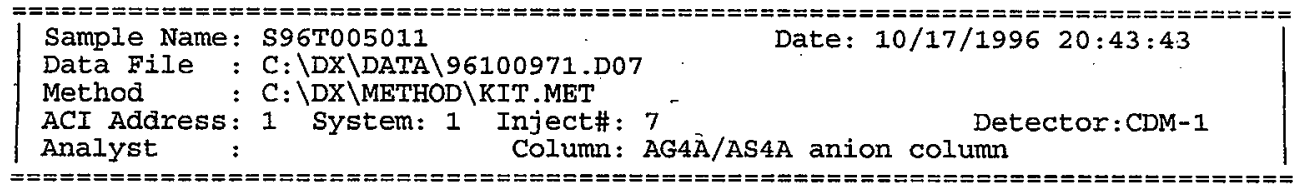

Calibration Volume Dilution Points Rate Start stop Area Reject

\begin{tabular}{|c|}
\hline External \\
\hline
\end{tabular}

$* * * * * * * * * * * * * * * * * * * * * * * * * * *$ Peak Report: All Peaks $* * * * * * * * * * * * * * * * * * * * * * * * * * * *$

$\begin{array}{lrrr}\text { Pk. Ret Component } & \text { Concentration } & \text { Height } & \text { Area Bl. } \\ \text { Num } & \text { Time Name } & \text { Code }\end{array}$

Num Time Name $\mathrm{ug} / \mathrm{ml}$

$\begin{array}{lll}1 & 0.89 & \\ 2 & 1.06 & \text { fluoride } \\ 3 & 1.39 & \\ 4 & 1.63 & \text { chloride } \\ 5 & 1.96 & \text { nitrite } \\ 6 & 3.33 & \text { nitrate } \\ 7 & 6: 35 & \text { sulfate }\end{array}$

\begin{tabular}{rrrrrr} 
& 0.000 & 68 & 303 & 2 & \\
159.405 & 451 & 2677 & 2 & 3.92 \\
0.000 & 46 & 299 & 1 & \\
251.631 & 555 & 3023 & 1 & 1.24 \\
& 2283.592 & 1546 & 9984 & 1 & -1.01 \\
& 29178.048 & 22457 & 220056 & 1 & -2.25 \\
& 213.732 & 93 & 1503 & 1 & -3.25 \\
\hline & 31086.408 & 25216 & 237846 & &
\end{tabular}

File: 96100971.D07 Sample: S96T005011

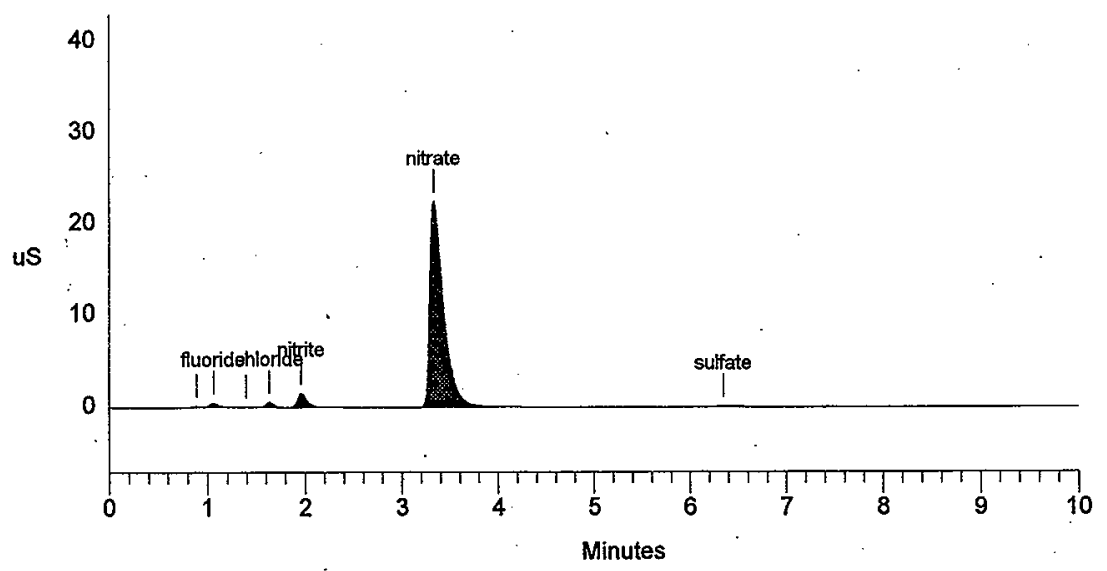


HNF-SD-WM-DP-214 $\mathrm{BEE}, \mathrm{Q}$

Sample Name: S96T005011DUP

Data File : C:\DX\DATA 96100971. D08

Method : C: \DX \METHOD \KIT.MET

ACI Address : 1 System: 1 Inject\# : 8

Analyst : Column: $A G 4 \bar{A} / A S 4 A$ anion column

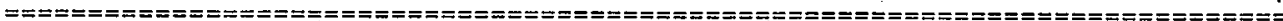

Calibration Volume Dilution Points Rate Start Stop Area Reject

Calibration Voluen

External

1

$\begin{array}{lllll}1111 & 3000 \quad 5 \mathrm{~Hz} & 0.00 & 10.00\end{array}$

50

$\star * * * * * * * * * * * * * * * * * * * * * * * * * *$ Peak Report All Peaks $* * * * * * * * * * * * * * * * * * * * * * * * * * * *$
Pk.
Ret Component
Concentration
Height
Area $\mathrm{Bl}$. :Delta
Num Time Name
$\mathrm{ug} / \mathrm{ml}$ Code

$\begin{array}{lll}1 & 0.89 & \\ 2 & 1.06 & \text { fluoride } \\ 3 & 1.40 & \\ 4 & 1.63 & \text { chloride } \\ 5 & 1.96 & \text { nitrite } \\ 6 & 3.33 & \text { nitrate } \\ 7 & 6.35 & \text { sulfate }\end{array}$

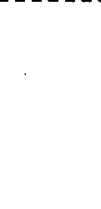

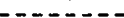

Totals

0.000

152.844

0.000

247.813

1248.969

28501.154

214.145

30364.925

64
433
58
547
1487
21769
93

24451

231882

File: 96100971.D08 Sample: S96T005011DUP

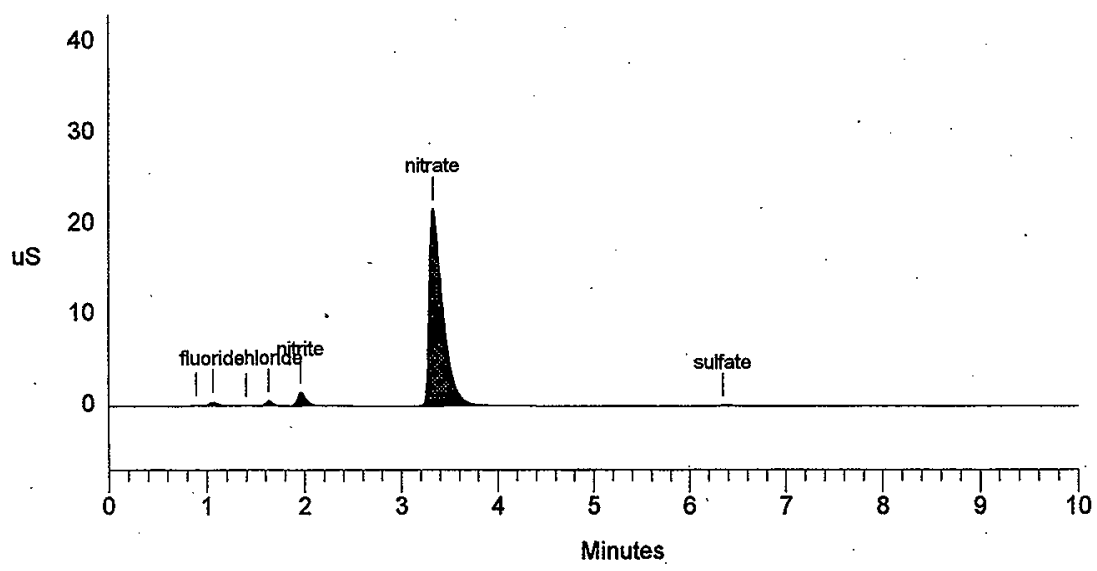


Method: LA-5 33-105Rev/Mod D-1

Worklist Comment: AW-105 IC. RCJ

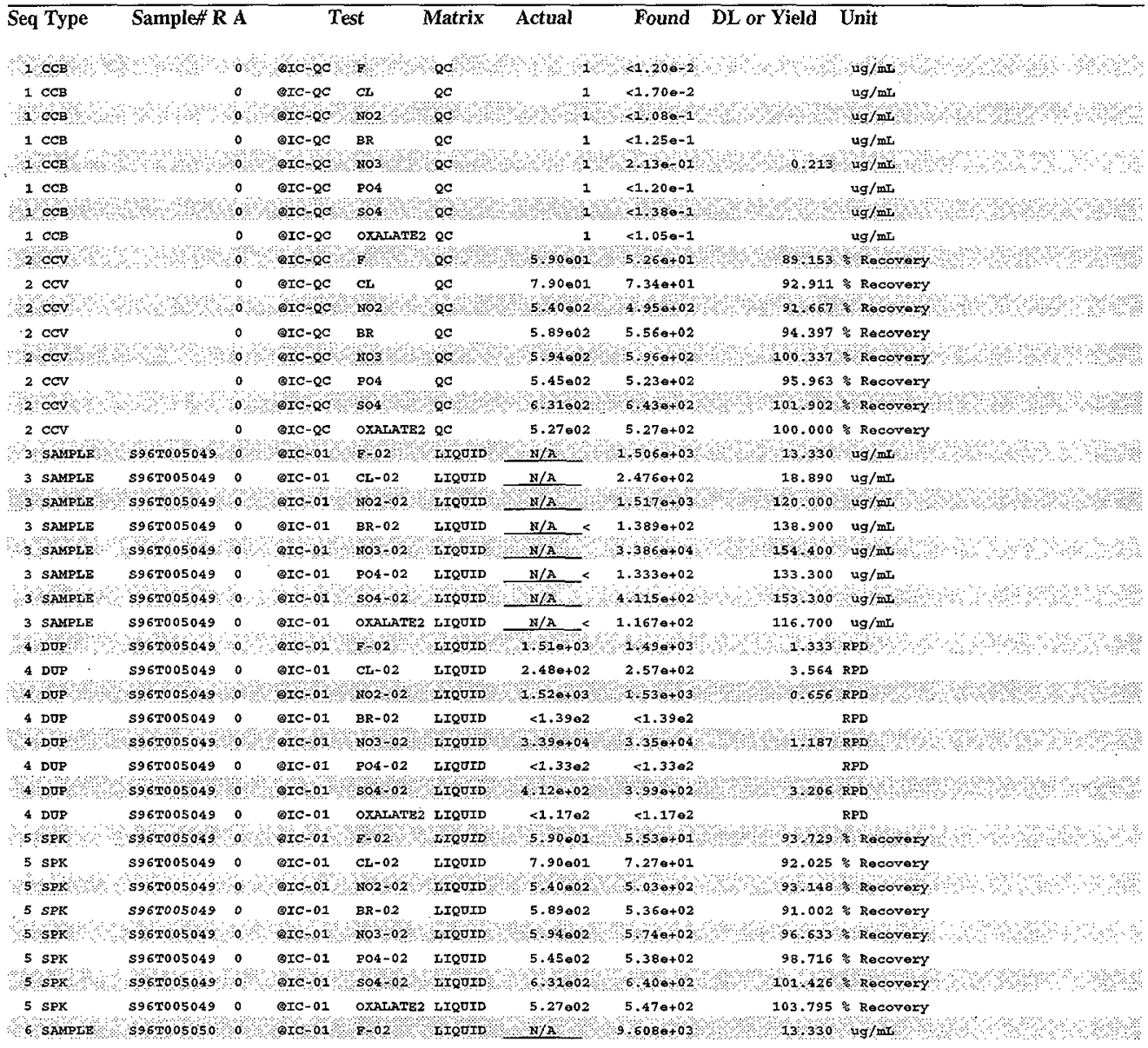




\section{LABCORE Completed Worklist Report for Worklist\# 13846}

\begin{tabular}{|c|c|}
\hline Seq Type & Sampleff $\mathbf{R}$ \\
\hline 6 SAMPLE & $896 \mathrm{~T} 005050$ \\
\hline 6 SAMPLE & $\$ 967005050$ \\
\hline 6 SAMPE & $596 T 005050$ \\
\hline 6 SAMPLE & S96T005050 \\
\hline 6 SAMPLE & $\$ 96 T 0.05050$ \\
\hline 6 SAMPLB & S96T005050 \\
\hline 6 SPMPSR & s967005050 \\
\hline 7 DUP & $S 96 T 005050$ \\
\hline 2 Dण्: & $\$ 96005050$ \\
\hline 7 DUP & S96T005050 \\
\hline 7 POP & $\$ 96005050$ \\
\hline 7 DUP & 5967005050 \\
\hline 7008 & $\$ 96 T 005050$ \\
\hline 7 DOP & 5967005050 \\
\hline & $\$ 967005050$ \\
\hline
\end{tabular}

\begin{tabular}{|c|c|c|c|}
\hline \multicolumn{2}{|c|}{ Test } & Matrix & Actual \\
\hline (1C-01 & CL-02 & ITQtrD & $\mathbb{N} / \mathrm{A}$ \\
\hline BIC-0I & $\mathrm{NO} 2-02$ & IIQUID & $\mathrm{N} / \mathrm{A}$ \\
\hline Oron & $\mathrm{BR} \bigcirc 02$ & magणro & $\mathrm{N} / \mathrm{A}$ \\
\hline QIC-01 & $\mathrm{NO3}-02$ & LIQUXD & $N / A$ \\
\hline $0 r 0,01$ & $004-02$ & r & $\mathrm{N} / \mathrm{A}$ \\
\hline BIC-0I & $504-02$ & IIQOID & $N / A$ \\
\hline Iaroi & OXATAKE2 & 2trooI & $y / 2$ \\
\hline OIC-01 & $F-02$ & LIQUTD & $9.610+03$ \\
\hline Qrcos & $c-02$ & SIODro & 3 tSeros \\
\hline QIC-01 & xо2- -02 & LIQOID & $4.640+03$ \\
\hline $9 I C-1$ & $\mathrm{BR}-02$ & $=T Q 010$ & $<<1,39 a$ \\
\hline QIC-01 & $\mathrm{No3}-02$ & LIQUID & $2.680+04$ \\
\hline $0 I C-02$ & $804-02$ & IIQDOD & $2898+0$ \\
\hline GIC-01 & $504-02$ & IIOUID & $1.550+03$ \\
\hline $9 I C-01$ & oxA $\mathrm{NAR}$ & IIOUID & $3200+02$ \\
\hline
\end{tabular}

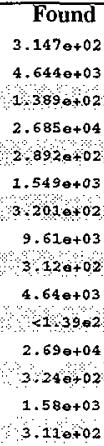

DL or Yield Unit

\section{Final page for worklist\# 13846}

Analyst Signature Date

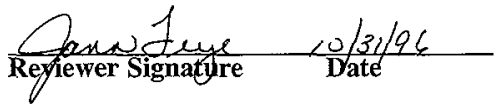




\section{LABCORE Data Entry Template for Worklist\# 13846}

Page: $\quad l$

\section{Analyst: $\quad K M$ Instrument: ICO TCOL Book\# $33200 \mathrm{~A}$}

Method: LA-533-105 Rev/Mod D-1

Worklist Comment: AW-105 IC. RCJ

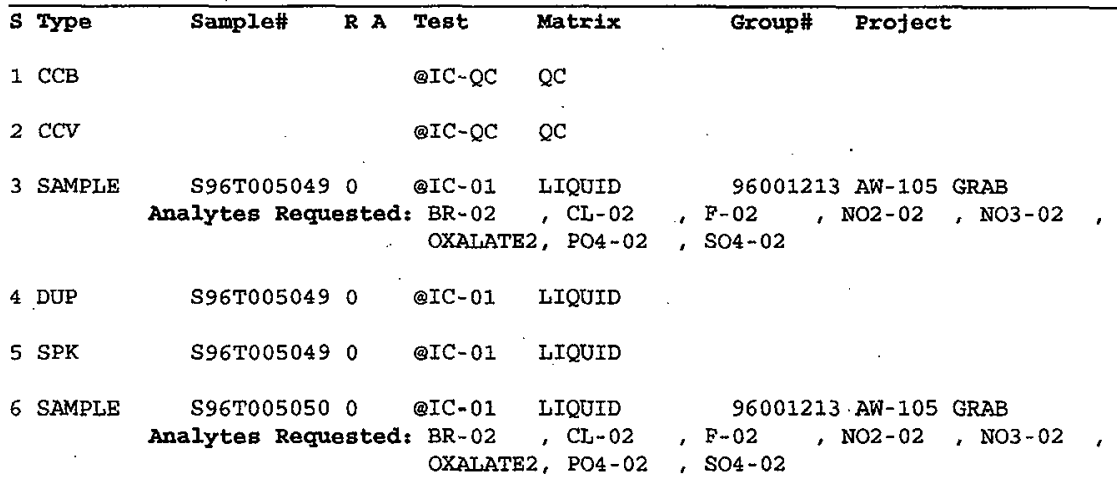

7 DUP

S96T0050500 QIC-01 LIQUID

Final page for worklist \# 13846

$10 \cdot 30-96$

Analyst Signature

Date
Analyst Signature

Date

$138460 \mathrm{cos}$

iploaded 10/31/96 Jmfuy

vale datal $10 \mathrm{kp} / 96$ omfup.

Data Entry Comments:

$S=$ Worklist Slot Number, $R=$ Replicate Number, $A=$ Aliquot Code. 
HNF-SD-WM-DP-214, REV. 0

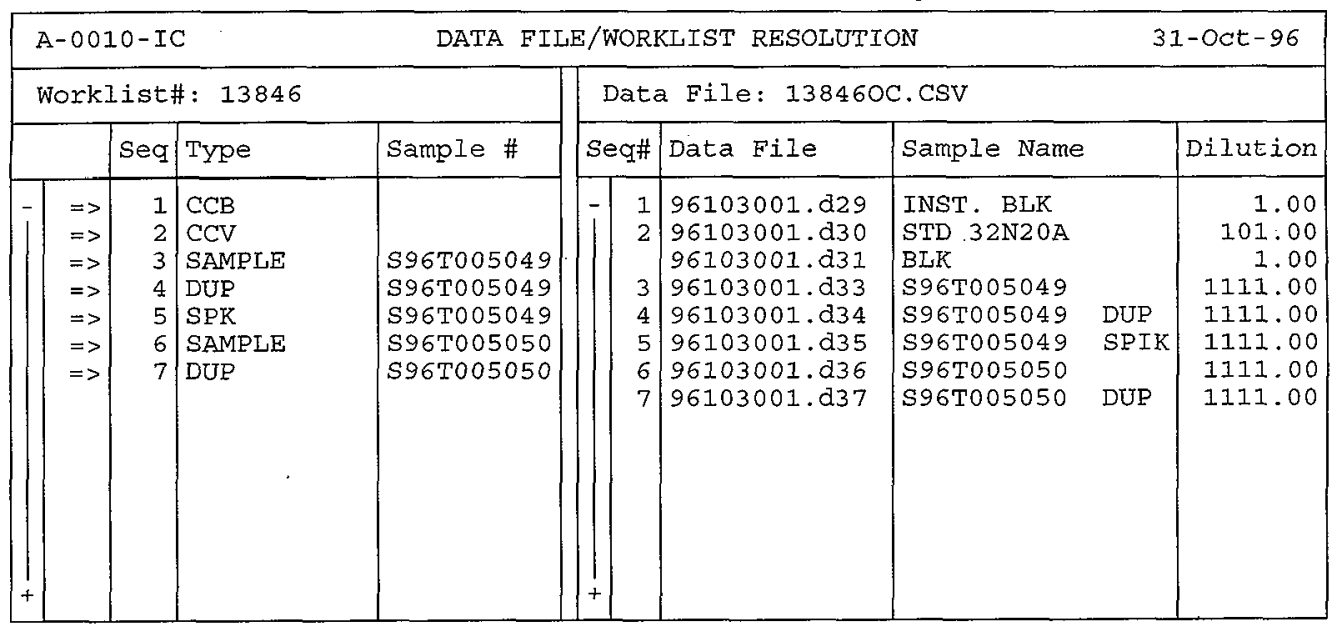

Save(F4) Abort(Shift-F3) ListFiles(Shift-F1) UploadFile(F8) 
Calibration Volume Dilution Points Rate start stop Area Reject

Cal

$\begin{array}{llllllll}\text { External } & 1 & 1 & 3000 & 5 \mathrm{~Hz} & 0.00 & 10.00 & 50\end{array}$

$\star * * * * * * * * * * * * * * * * * * * * * * * * * *$ Peak Report: AII Peaks $* * * * * * * * * * * * * * * * * * * * * * * * * * * *$

Pk. Ret Component Concentration Height Area Bl. $\%$ Delta

Num Time Name . .ug/mi Code

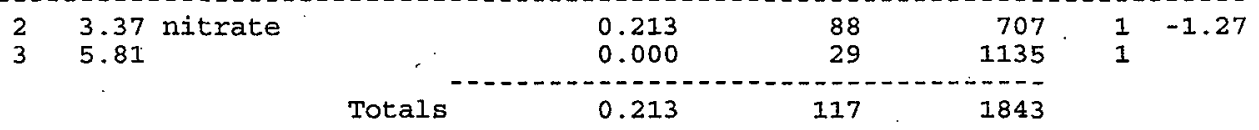

File: 96103001.D29 Sample: INST. BLK

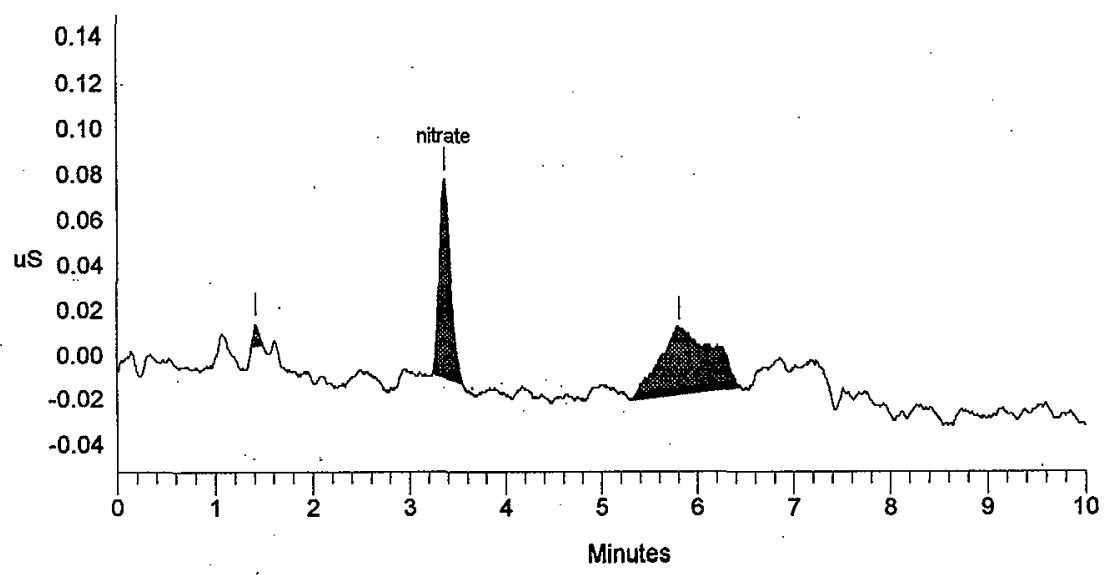

SIGNATURE ABOVE REPRESENTS CHEMICAL TECHNOLOGIST/CHEMIST IHAT COMPLETEDNVPIFIED THE CALIBRATIONANALYSIS ON PAGES 392 TO 398. 


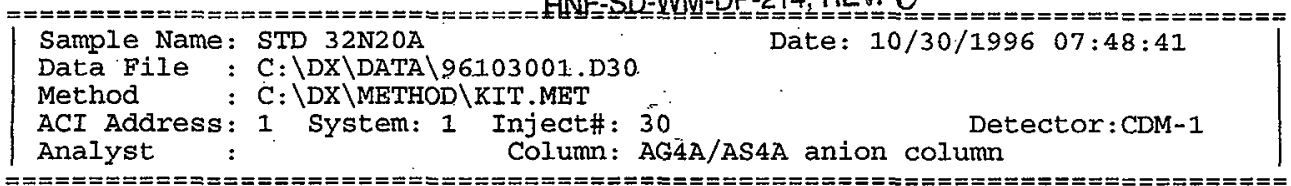

Calibration Volume Dilution Points Rate start stop Area Reject

Cal

$\begin{array}{llllllll}\text { External } & 1 & 101 & 3000 & 5 \mathrm{~Hz} & 0.00 & 10.00 & 50\end{array}$

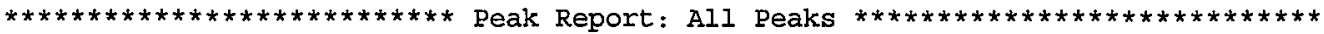

Pk. Ret Component Concentration Height Area Bl. $\%$ Delta

Num. Time Name

$\mathrm{ug} / \mathrm{ml}$ Code

$\begin{array}{lll}1 & 0.89 & \\ 2 & 1.06 & \text { fluoride } \\ 3 & 1.61 & \text { chloride } \\ 4 & 1.92 & \text { nitrite } \\ 5 & 2.89 & \text { bromide } \\ 6 & 3.30 & \text { nitrate } \\ 7 & 4.69 & \text { phosphate } \\ 8 & 6.13 & \text { sulfate } \\ 9 & 8.05 & \text { oxalate }\end{array}$

$\begin{array}{rrrrr}0.000 & 41 & 155 & 2 & \\ 52.615 & 1772 & 10348 & 2 & 3.92 \\ 73.401 & 1769 & 9721 & 1 & -0.41 \\ 495.065 & 7067 & 45631 & 1 & -3.03 \\ 555.924 & 4940 & 34086 & 1 & -2.91 \\ 596.126 & 5491 & 45706 & 1 & -3.23 \\ 523.016 & 1514 & 20919 & 1 & -4.80 \\ 642.675 & 4459 & 65718 & 1 & -6.50 \\ 526.660 & 1994 & 41685 & 1 & -1.91\end{array}$

Totals $\quad 3465.483 \quad 29046 \quad 273971$

File: 96103001.D30 Sample: STD 32N20A

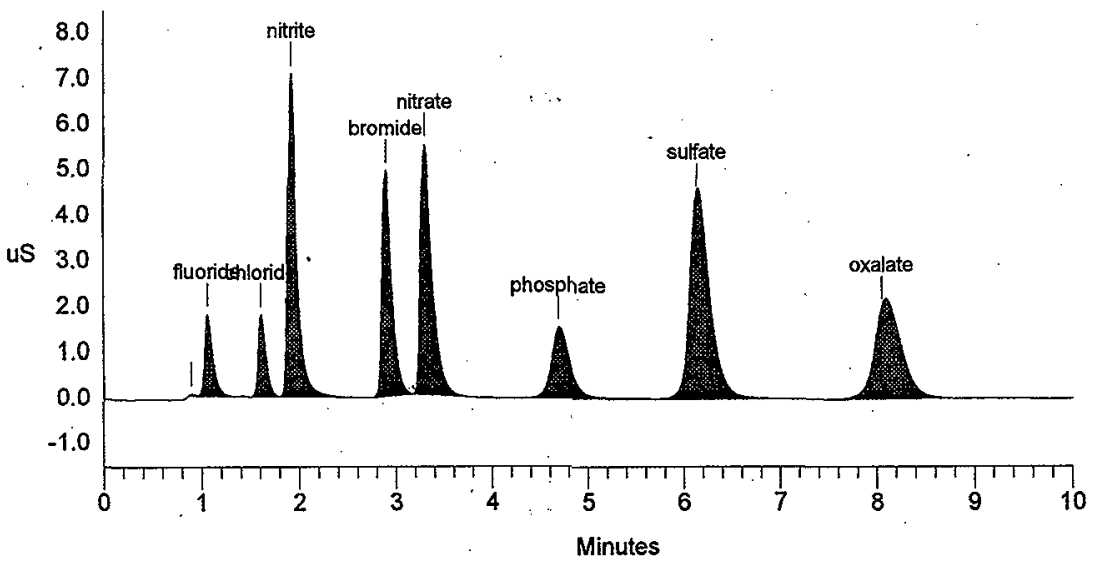




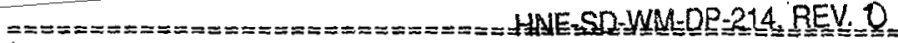

Sample Name: S96T005049

Data File : C: \DX\DATA $96103001 . D 33$

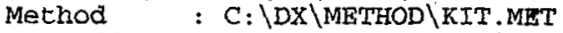

ACI Address: 1 System: 1 Inject\#: 33 Detector:CDM-1

Analyst : Column: AG4A/AS4A anion column

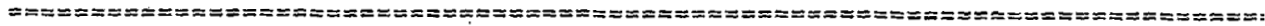

Calibration Volume Dilution Points Rate staxt Stop Area Reject

External $\quad 11113000 \quad 5 \mathrm{~Hz} 0.00 \quad 10.000$

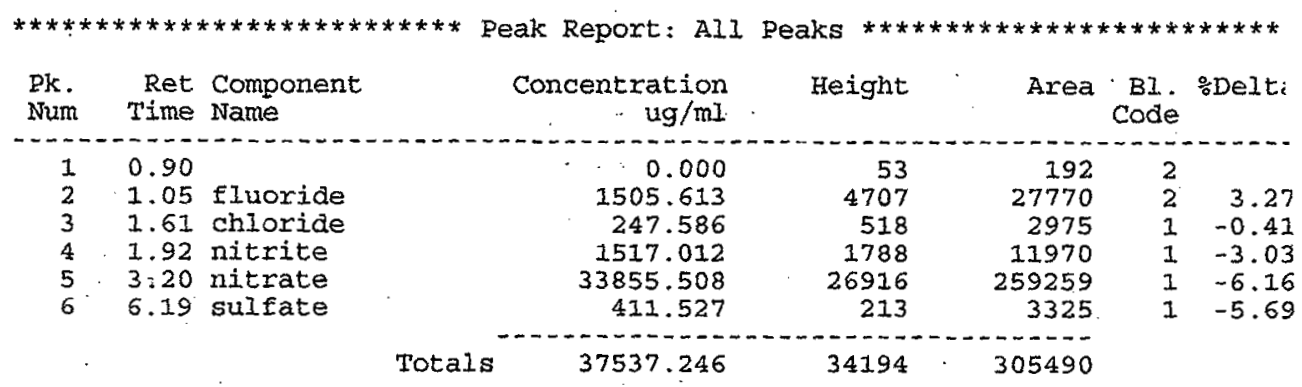

File: $96103001 . D 33$ Sample: S96T005049

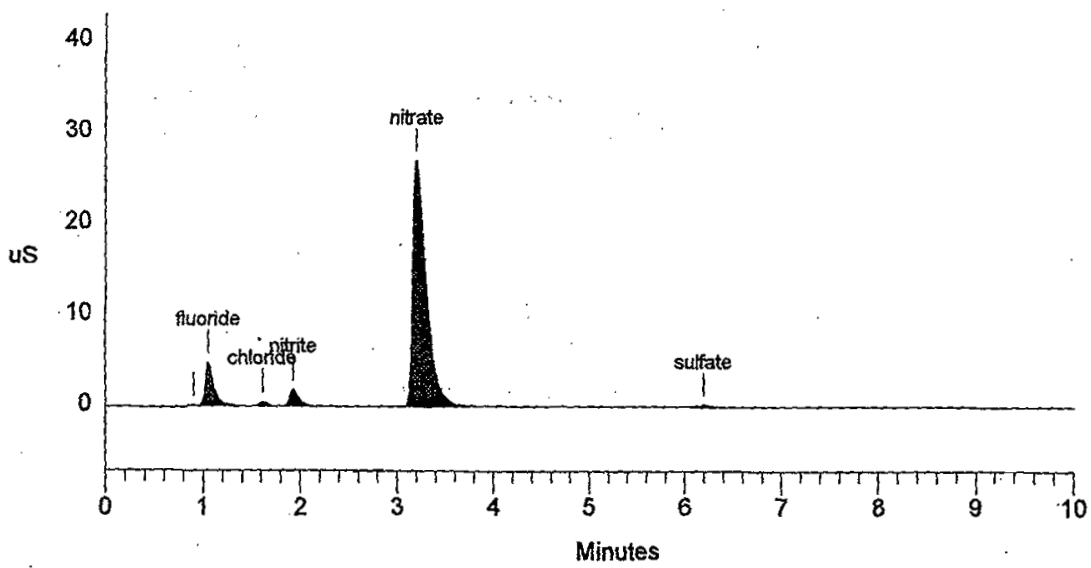


HNF-SD-WM-DP-214, REV : $\theta$

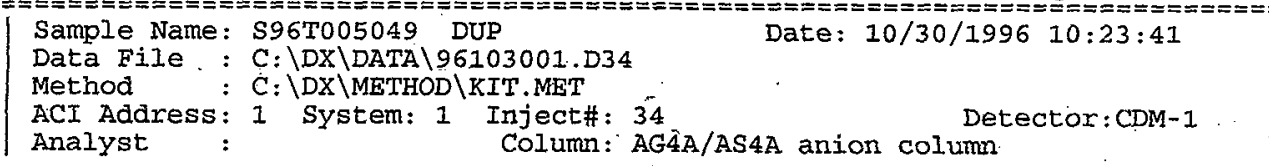

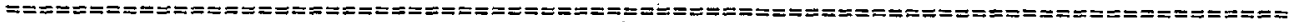

\begin{tabular}{lcccc} 
Calibration Volume Dilution Points Rate start Stop Area Reject \\
\hline External & 1 & $1111 \quad 3000 \quad 5 \mathrm{~Hz} 0.00 \quad 10.00$ & 50
\end{tabular}

$* * * * * * * * * * * * * * * * * * * * * * * * * * *$ Peak Report: All Peaks $* * * * * * * * * * * * * * * * * * * * * * * * * * * *$

Pk. Ret Component Concentration Height Area BI. $\frac{\circ}{D}$ lta Num Time Name ug/ml

10.90

21.05 fluoride

31.61 chloride

0.000

1493.597

257.015

1.92 nitrite

5. 3.20 nitrate

$6 \quad 6.19$ sulfate

$\begin{array}{rrrrrr} & 0.000 & 57 & 211 & 2 & \\ 1493.597 & 4733 & 27540 & 2 & 3.27 \\ 257.015 & 523 & 3087 & 1 & 0.00 \\ & 1528.045 & 1792 & 12064 & 1 & -3.03 \\ & 33498.360 & 26790 & 256220 & 1 & -6.16 \\ & 399.119 & 215 & 3210 & 1 & -5.69 \\ \text { Totals } & 37176.136 & 34110 & 302332 & & \end{array}$

File: 96103001.D34 Sample: S96T005049 DUP

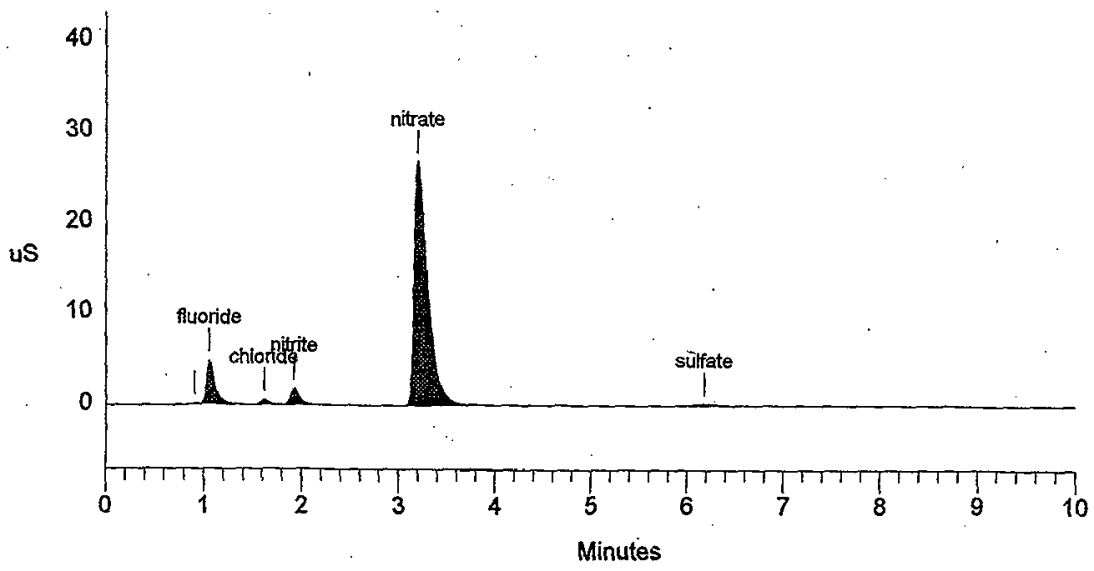


HNF-SD-WM-DP-214, REV. 0

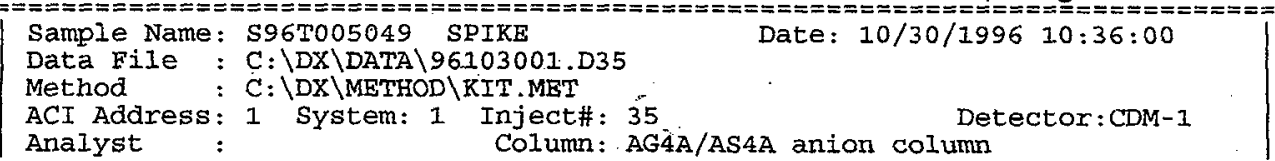

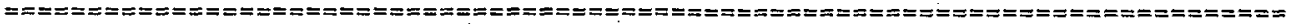

Calibration Volume Dilution Points Rate start stop Area Reject

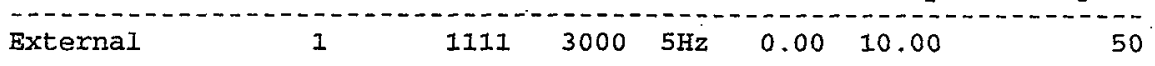

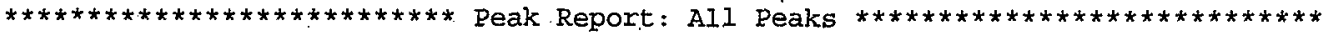

pk. Ret Component Concentration Height Area Bl. $\%$ Delta

Num Time Name $u g / m i$ Code

$\begin{array}{lll}1 & 0.89 & \\ 2 & 1.05 & \text { Eluoride } \\ 3 & 1.60 & \text { chloride } \\ 4 & 1.91 & \text { nitrite } \\ 5 & 2.89 & \text { bromide } \\ 6 & 3.19 & \text { nitrate } \\ 7 & 4.67 & \text { phosphate } \\ 8 & 6.08 & \text { sulfate } \\ 9 & 8.05 \text { oxalate }\end{array}$

$\begin{array}{rrrrrr}2063.742 & 89 & 351 & 2 & \\ 981.684 & 6735 & 38598 & 2 & 2.61 \\ 6593.295 & 8179 & 11847 & 1 & -0.83 \\ & 5413.240 & 4737 & 55551 & 1 & -3.37 \\ & 39650.701 & 31846 & 309679 & 1 & -6.55 \\ & 5431.212 & 1438 & 19732 & 1 & -5.34 \\ & 6881.147 & 3948 & 63924 & 1 & -7.32 \\ & 5526.378 & 2081 & 39740 & 1 & -1.91 \\ \text { Totals } & 72541.399 & 61853 & 569460 & & \end{array}$

File: 96103001.D35 Sample: S96T005049 SPIKE

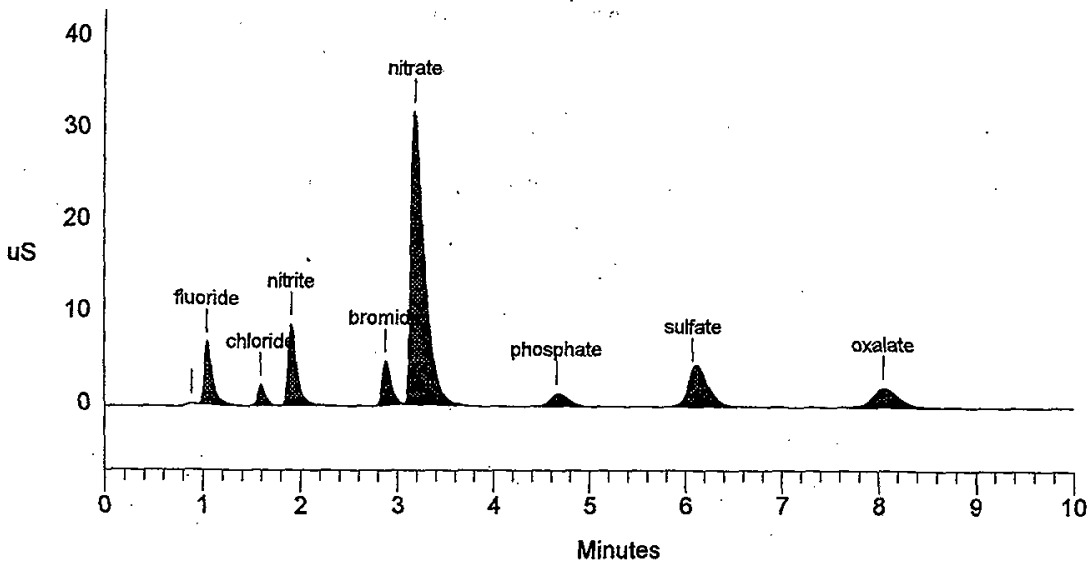


Calibration Volume Dilution Points Rate start stop Area Reject

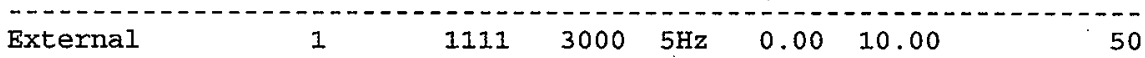

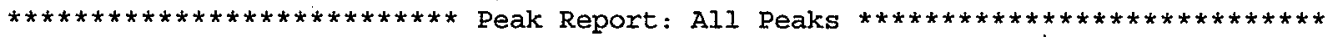

\begin{tabular}{|c|c|c|c|}
\hline $\begin{array}{l}\mathrm{Pk} \text {. } \\
\text { Num }\end{array}$ & $\begin{array}{l}\text { Ret } \\
\text { Time }\end{array}$ & $\begin{array}{l}\text { Component } \\
\text { Name }\end{array}$ & \\
\hline
\end{tabular}

-

10.90

21.05 fluoride

31.60 chloride

4 1.91 nitrite

$5 \quad 3.21$ nitrate

64.03

74.67 phosphate

86.13 sulfate

98.11 oxalate

.

0.000

$$
9607.629
$$

314.709

4643.916

26852.664

0.000

289.219

1548.701

320.053
70

41453

672

5996

21399

92

83

900

104

199
228048
3777

38724

201020

847

1045

13830

2081
2

$2 \quad 2.61$

$1-0.83$

$1 \quad-3.37$

1. -5.77

1

$1-5.34$

$1-6.50$

$1-1.26$

Totals

43576.892

70769

489571

- File: 96103001.D36 Sample: S96T005050

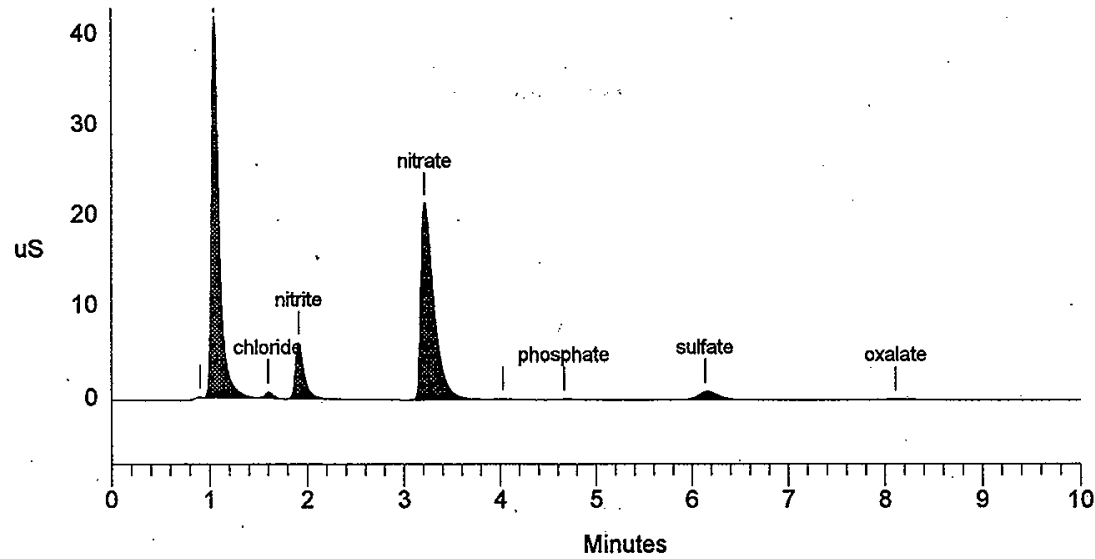

Minutes 
Calibration Volume Dilution Points Rate start stop Area Reject

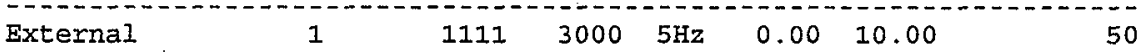

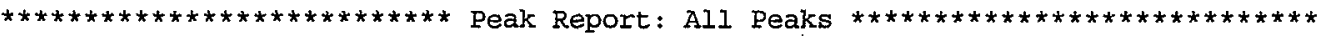

Pk. Ret Component Concentration Height Area BI. \%Delta Num Time Name ug/ml

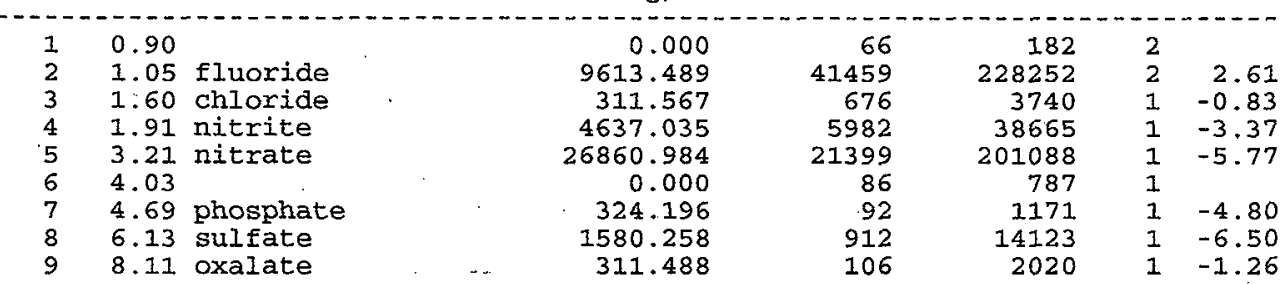

Totals

43639.017

70778

490026

File: 96103001.D37 Sample: S96T005050 DUP

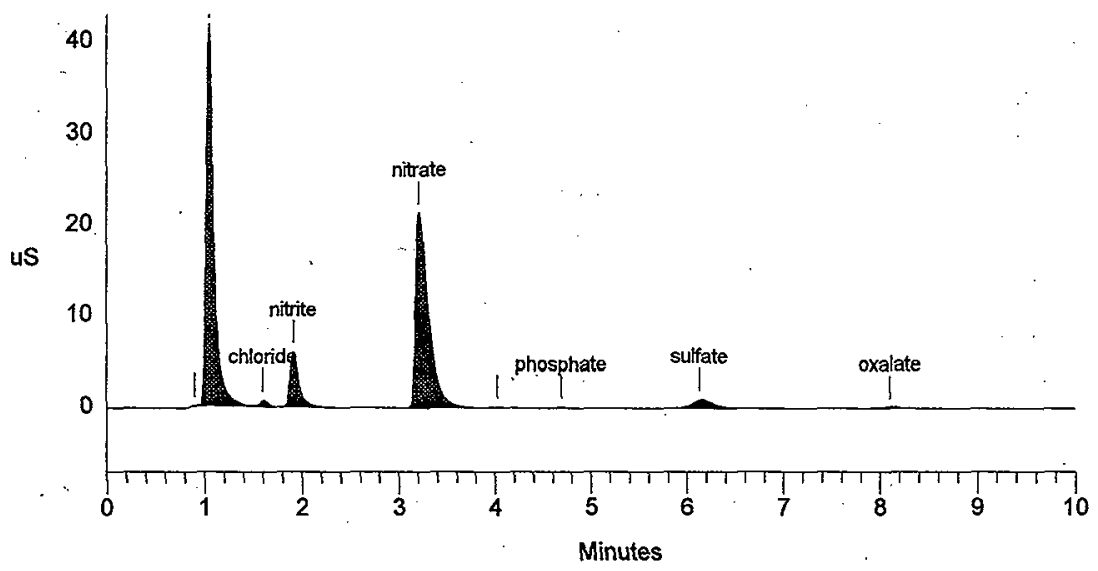




\section{LABCORE Completed Worklist Report for Worklist\# 13847}

Analyst: $\mathrm{krm}$

Instrument: IC01

Book\# 32N20/f

Method: $\angle A-533>105^{\operatorname{Rev} / \mathrm{Mod} D-1}$

Worklist Comment: AW-105 IC, USE .100ML-10ML SS. RCJ

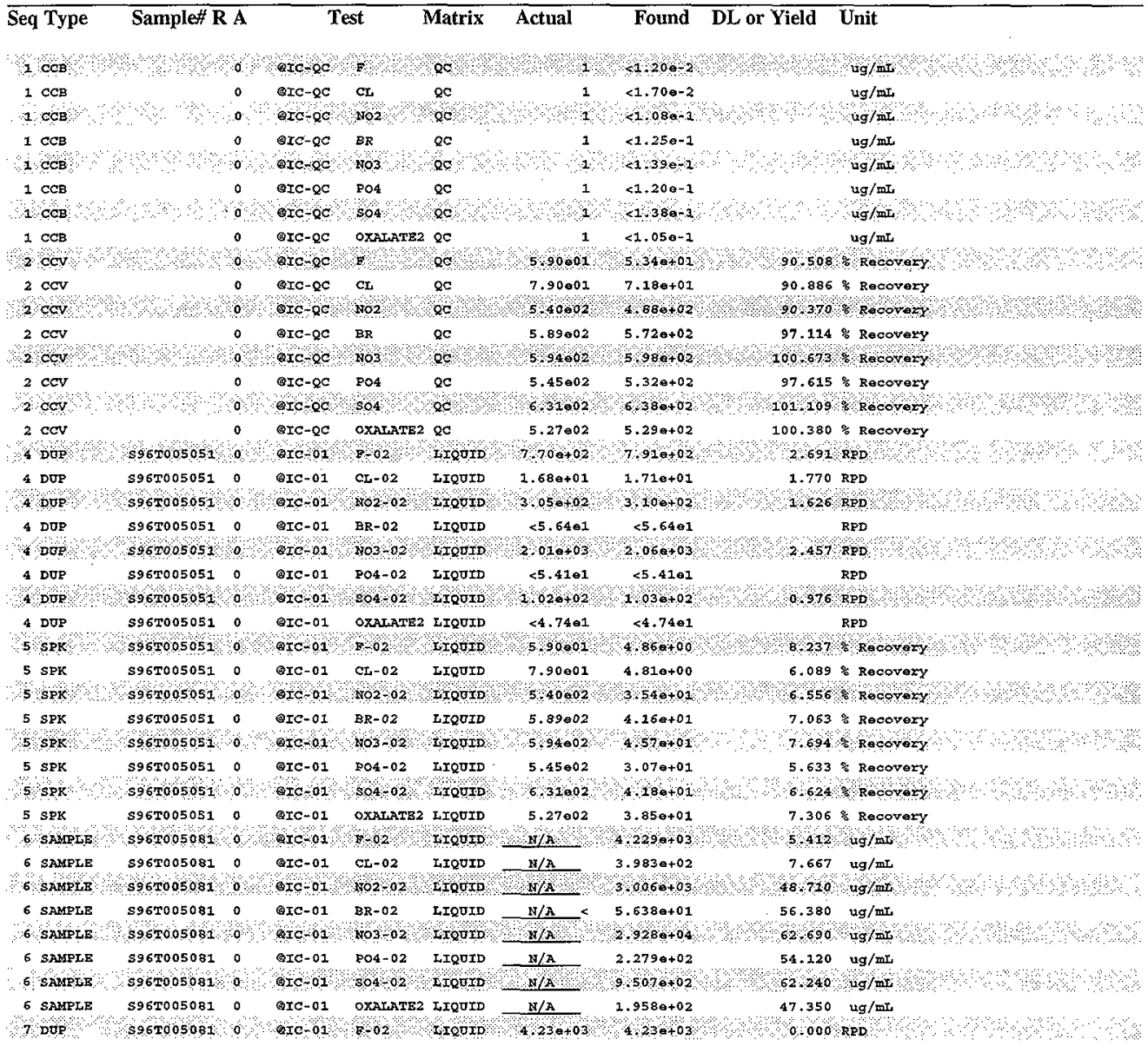




\section{LABCORE Completed Worklist Report for Worklist\# 13847}

\begin{tabular}{|c|c|c|c|c|c|c|c|c|c|}
\hline Seq Type & Sample\# & $A$ & & $\mathrm{st}$ & Matrix & Actual & Found & DL or Yield & Tnit \\
\hline 7 DUP & s96T005081 & 0 & QIC-01 & $\mathrm{CL}-02$ & LIQUID & $3.980+02$ & $3.939+02$ & 1.264 & RPD \\
\hline 7 DOP & S96T005081 & 0 & QIC-01 & $\mathrm{NO} 2-02$ & LIQDID & $3.01 e+03$ & $2.93 e+03$ & 2.694 & RPD \\
\hline 1) oup & s96moosos & 0 & $0 \mathrm{PC}-01$ & $\mathrm{BR}-02$ & LIOUID & $\$ 564$ a & -5 641 & & $\mathrm{RPD}$ \\
\hline 7 DOP & \$96T005081 & 0 & OIC-01 & No3-02 & LIQUID & $2.930+04$ & $2.91 \theta+04$ & 0.685 & RPD \\
\hline $1 /$ pup & $\$ 969005081$ & 0 & QIC 01 & $004-02$ & TIOUID & $2280+02$ & 2.23602 & 2217 & $\mathrm{RPD}$ \\
\hline 7 DUP & S96T005081 & 0 & $0 I C-01$ & SO4-02 & LIQOID & $9.510+02$ & $9.49 \theta+02$ & 0.211 & RPD \\
\hline T DUP & s96T0050B & 0 & QIC- 01 & OXALART & 2 LIROID & $1-0,6+02$ & $2,32 \Leftrightarrow+02$ & 16822 & pps: \\
\hline
\end{tabular}

\section{Final page for worklist\# 13847}

Analyst Signature Date

Date

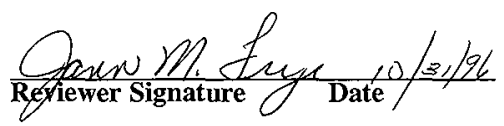


10/10/96 12:17

HNF-SD-WM-DP-214, REV. 0

Page: 1

A-0004-1

LABCORE Data Entry Template for Worklist\# 13847

$$
\begin{aligned}
& \text { Analyst: } \quad K R M \text { Instrument: ICO ICO) Book\# 32N20A } \\
& \text { Method: LA-533-105 Rev/Mod } D-1
\end{aligned}
$$

Worklist Comment: AW-105 IC, USE .100ML-10ML SS. RCJ

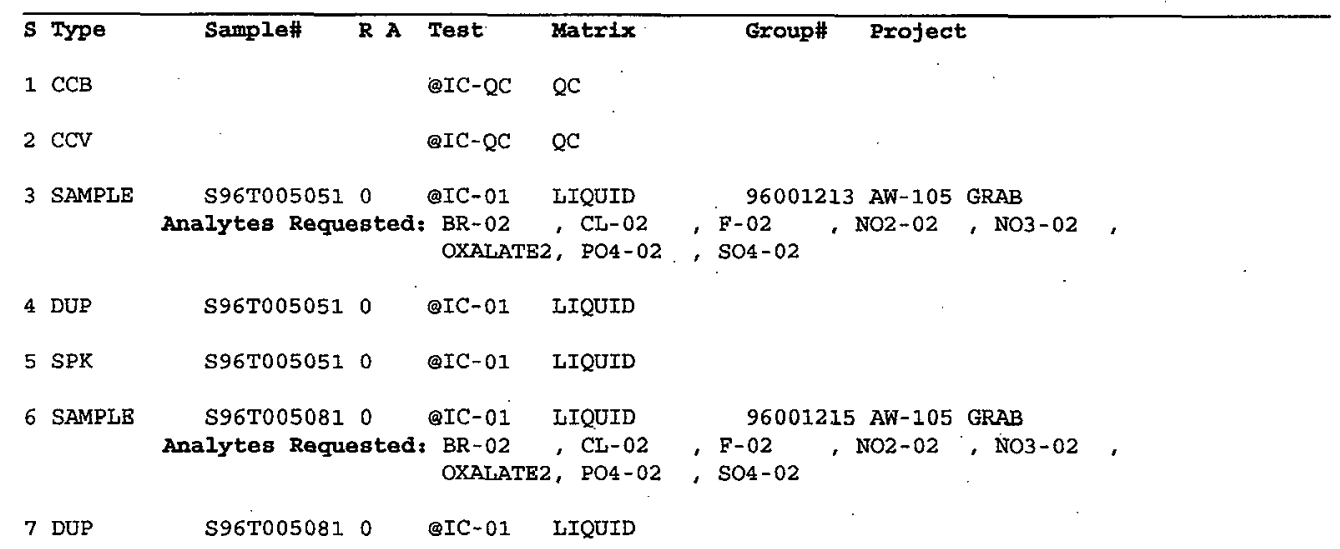

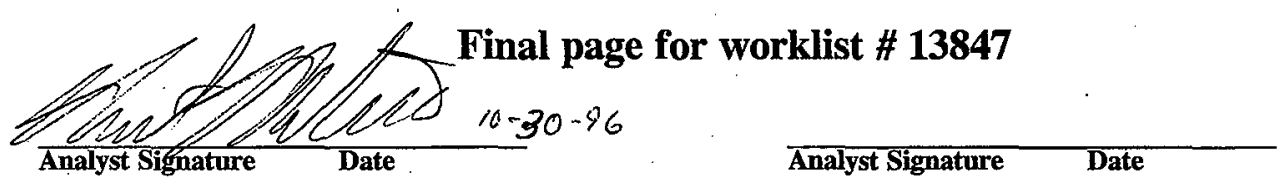

$138470 \mathrm{C} \cdot \mathrm{CSV}$

Uploaded 10/3196e OMF

S94T005051. Rejected Kecause speter was not dilutid puperly.

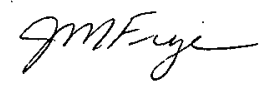

Re suen ON WKL 14522

Data Entry Comments:

$$
251 \text { DF }-.250-10-1000-10
$$

$S=$ Worklist Slot Number, $R=$ Replicate Number, $A=$ Aliquot Code 401 
HNF-SD-WM-DP-214, REV. 0

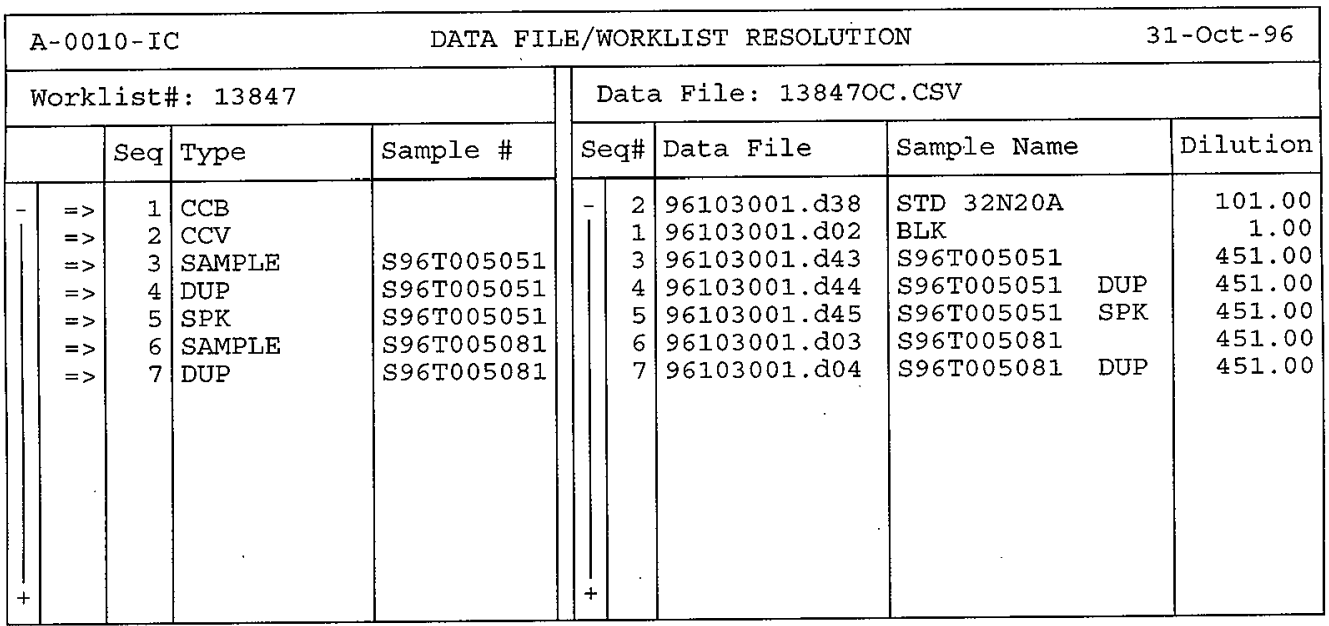

Save(F4) Abort(Shift-F3) IistFiles(Shift-F1) UploadFile(F8) 


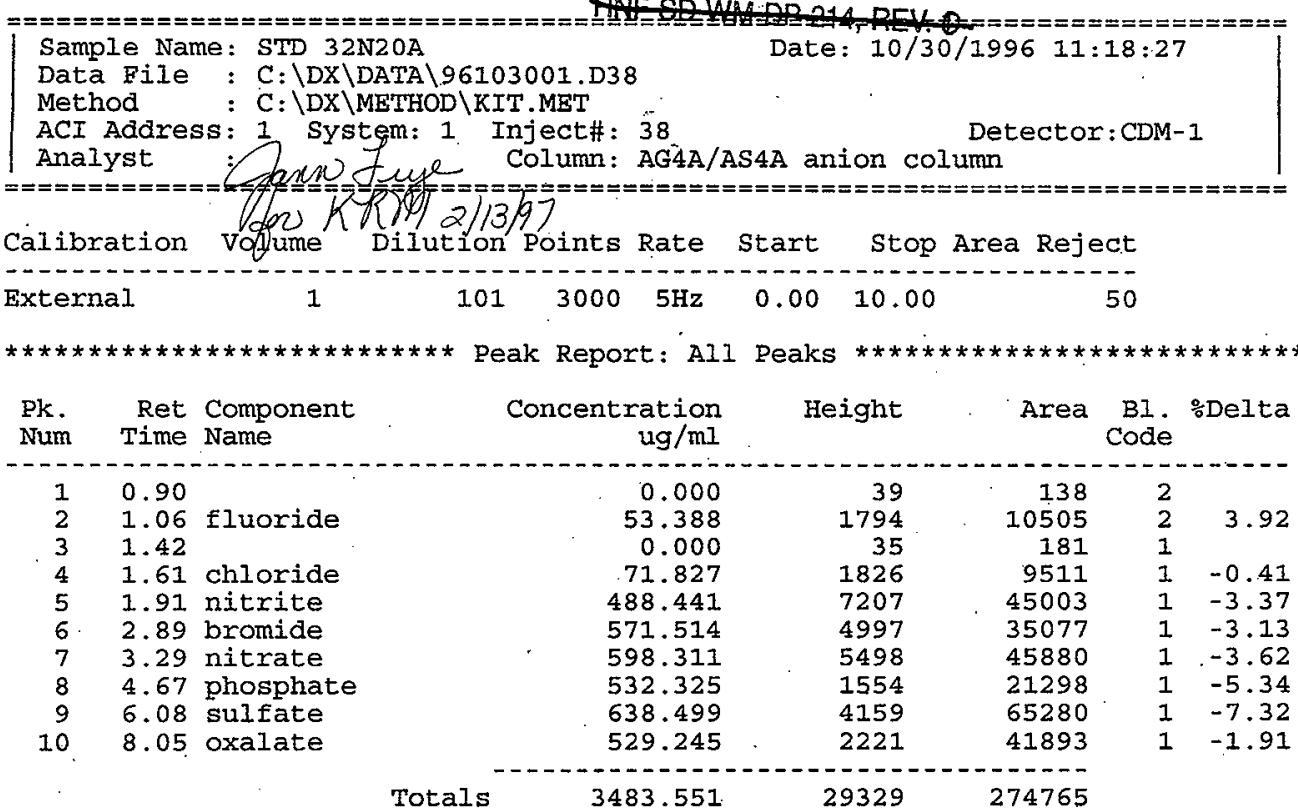

File: 96103001.D38 Sample: STD 32N20A

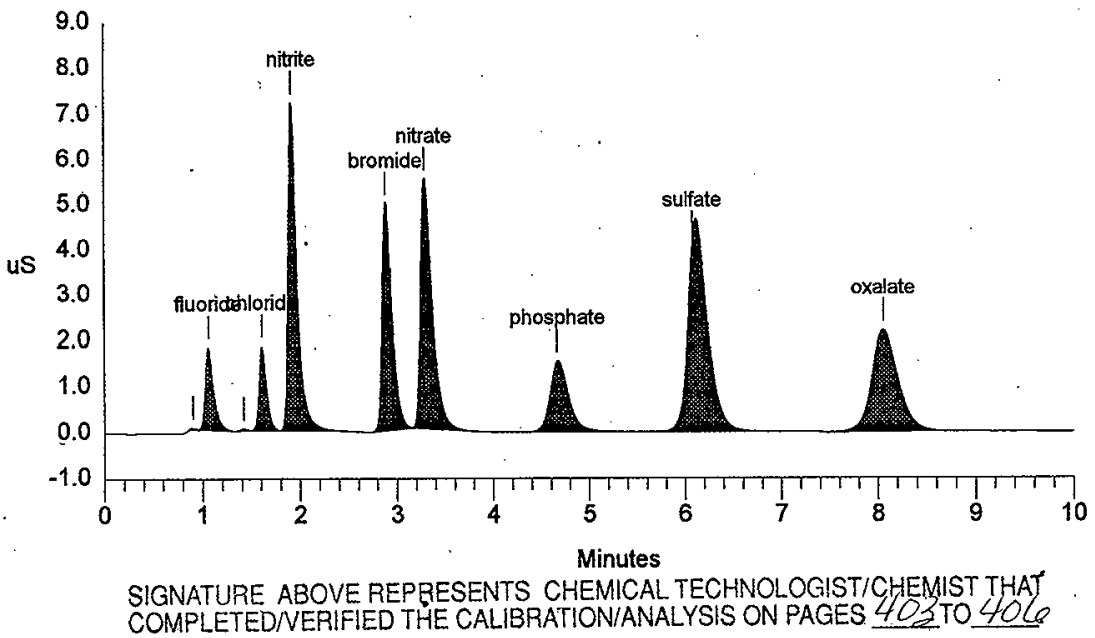


Calibration Volume Dilution Points Rate start Stop Area Reject

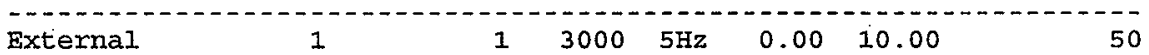

$* * * * * * * * * * * * * * * * * * * * * * * * * * *$ Peak Report: All Peaks $* * * * * * * * * * * * * * * * * * * * * * * * * * * *$

$\begin{array}{lrrr}\text { Pk. Ret Component } & \text { Concentration } & \text { Height } & \text { Area Bl. } \\ \text { Num } & \text { Time Name } & \text { Code }\end{array}$

$\begin{array}{ll}1 & \\ 1 & 3.85\end{array}$

$\begin{array}{ll}1 & 0.87 \\ 2 & 2.27 \\ 3 & 5.12 \text { phosphate }\end{array}$

\begin{tabular}{rrrrrr} 
& 0.000 & 82 & 488 & 1 & \\
& 0.000 & 678 & 5544 & 1 & \\
& 0.083 & 23 & 339 & 1 & 3.85 \\
\hline Totals & 0.083 & 782 & 6371 & &
\end{tabular}

File: 96103001.D02 Sample: BLK

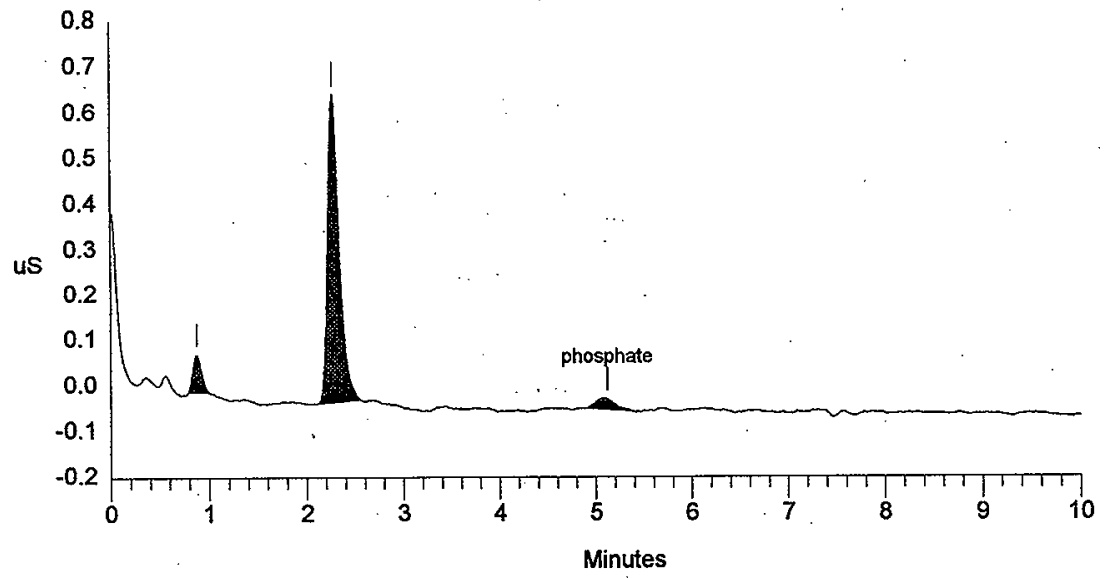


Data Reprocessed On 10/31/1996 10:04:12

HNF-SD-WM-DP-214, REV. 0

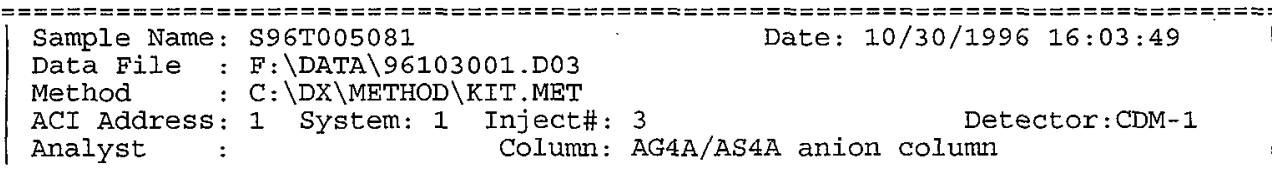

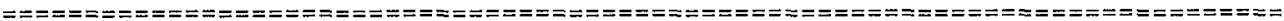

Calibration Volume Dilution Points Rate start Stop Area Reject

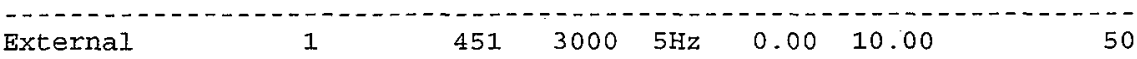

$* * * * * * * * * * * * * * * * * * * * * * * * * * *$ Peak Report: All Peaks $* * * * * * * * * * * * * * * * * * * * * * * * * * * *$

$\begin{array}{lrrr}\text { Pk. Ret Component } & \text { Concentration } & \text { Height } & \text { Area Bl. } \\ \text { Num } & \text { Time Name } & \text { Code }\end{array}$

$\begin{array}{lll}1 & 0.90 & \\ 2 & 1.05 & \text { fluoride } \\ 3 & 1.58 & \text { chloride } \\ 4 & 1.90 & \text { nitrite } \\ 5 & 3.09 & \text { nitrate } \\ 6 & 4.01 & \\ 7 & 4.64 & \text { phosphate } \\ 8 & 6.08 & \text { sulfate } \\ 9 & 8.00 & \text { oxalate }\end{array}$

$\begin{array}{rrrrr}0.000 & 138 & 421 & 2 & \\ 4229.315 & 46245 & 258118 & 2 & 2.61 \\ 398.266 & 2019 & 11840 & 1 & -2.07 \\ 3005.514 & 9531 & 62581 & 1 & -4.04 \\ 29276.223 & 58646 & 648071 & 1 & 0.11 \\ 0.000 & 89 & 846 & 1 & \\ 227.856 & 154 & 2021 & 1 & -5.88 \\ 950.653 & 1435 & 21194 & 1 & -7.32 \\ 195.773 & 162 & 3246 & 1 & -2.56\end{array}$

Totals $\quad 38283.599 \quad 118419 \quad 1008337$

File: $96103001 . D 03$ Sample: S96T005081

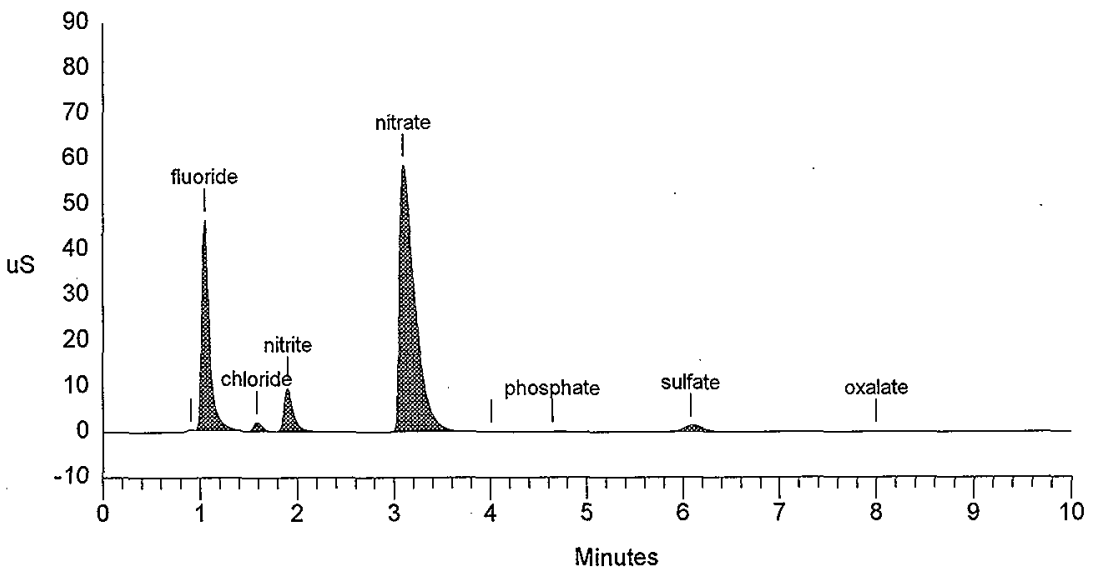


Data Reprocessed On 10/31/1996 10:05:30

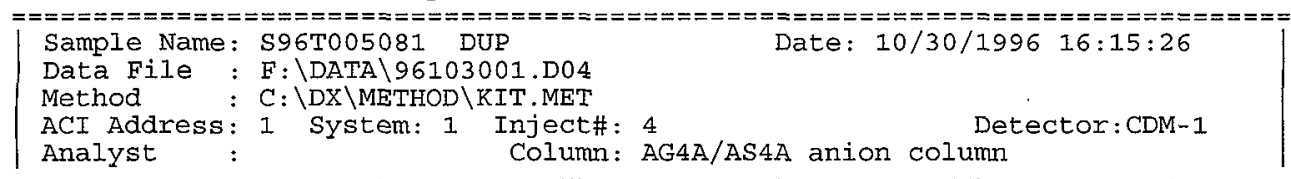

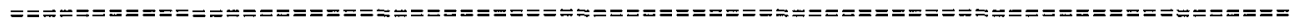

Calibration Volume Dilution Points Rate start stop Area Reject

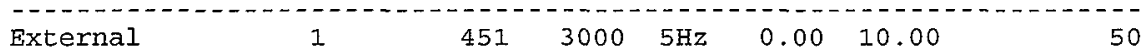

$* * * * * * * * * * * * * * * * * * * * * * * * * * *$ Peak Report: AII Peaks $* * * * * * * * * * * * * * * * * * * * * * * * * * * *$.

$\begin{array}{lrrr}\text { Pk. Ret Component } & \text { Concentration } & \text { Height } & \text { Area Bl. } \\ \text { Num } & \text { Time Name. } & \text { Code }\end{array}$

$\begin{array}{lll}1 & 0.90 \\ 2 & 1.05 & \text { fluoride } \\ 3 & 1.58 \text { chloride } \\ 4 & 1.90 \text { nitrite } \\ 5 & 3.10 \text { nitrate } \\ 6 & 4.00 & \\ 7 & 4.64 & \text { phosphate } \\ 8 & 6.08 & \text { sulfate } \\ 9 & 8.00 \text { oxalate }\end{array}$

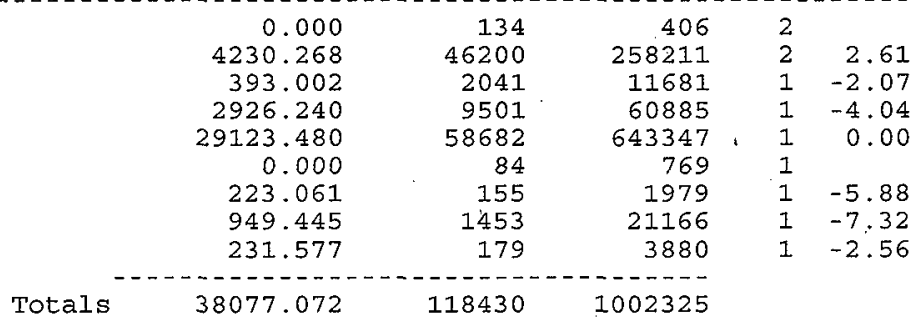

File: 96103001.D04 Sample: S96T005081 DUP

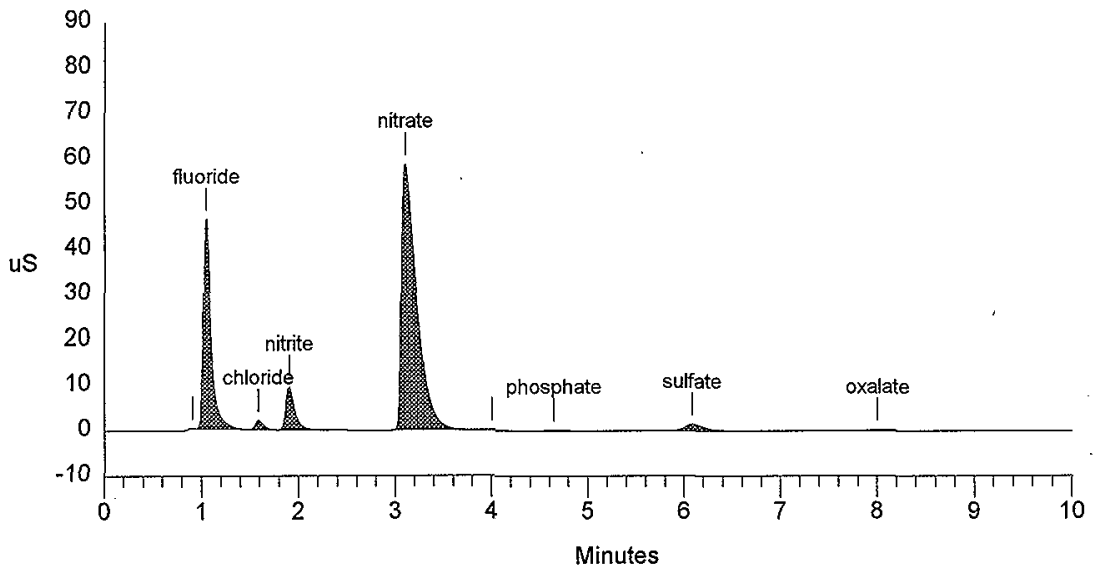




\section{LABCORE Completed Worklist Report for Worklist\# 13849}

Analyst: eal

Instrument: IC01

Book\# 32020 A

Method: $\quad L A-533-105$ Rev/Mod $D-1$

Worklist Comment: AW-105 IC, USE .100ML-10ML SS. RCJ

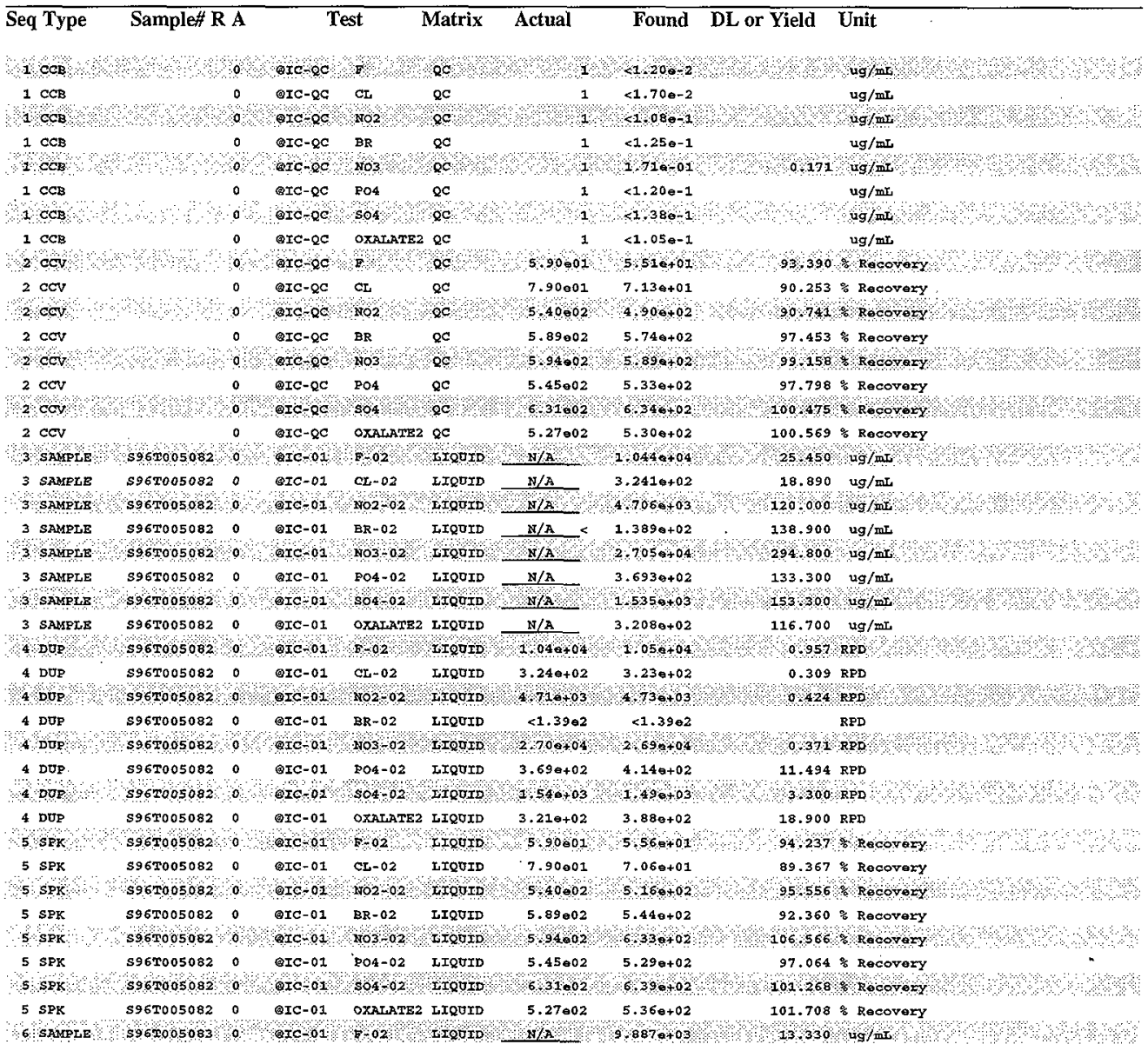

Units shown for $Q C(B L K / B K G)$ may not reflect the actual units. 


\section{LABCORE Completed Worklist Report for Worklist\# 13849}

\begin{tabular}{|c|c|c|c|c|c|c|c|c|c|c|}
\hline Seq & Type & Sample\# & & & & Matrix & Actual & Found & DL or Yield & Unit \\
\hline & SAMPLE & $\$ 96 \mathrm{~T} 0050 \mathrm{~B} 3$ & 0 & arc-01 & CL-02 & LIQUID & N/A & $2.9560+02$ & 18.890 & ug $/ \mathrm{n}$ \\
\hline 6 & SAMPLE & $596 T 005083$ & 0 & (a)- 01 & $\mathrm{NO} 2-02$ & IIQUID & $N / A$ & $3.941 e+03$ & 120.000 & ug/ \\
\hline 6 & SAMPIA & $\$ 967005083$ & 0 & arc- 01 & $\mathrm{BR}-02$ & ITOUI & $\mathrm{N} / \mathrm{A}$ & $1+380+02$ & 1384900 & ig \\
\hline 6 & SAMPLE & 5967005083 & 0 & QIc-01 & $\mathrm{NO3-02}$ & IrQUID & $N / A$ & $3.034 \mathrm{e}+04$ & 154.400 & ugf \\
\hline 6 & SAMPLP & 5967005083 & 0 & $Q I C-01$ & 00402 & TQLOLD & $\mathrm{A} / \mathrm{A}$ & $30310+02$ & 333300 & ug/ \\
\hline 6 & SAMPLE & 5967005083 & 0 & aIc-01 & $504-02$ & IIQUID & $N / A$ & $2.2390+03$ & 153.300 & $\mathbf{u g} / \mathrm{n}$ \\
\hline 6 & sAMPNE & $\$ 961005083$ & 0 & $0 \pm-01$ & OXARATEZ & $1 \mathrm{THOOO}$ & x/A & $2,7230+02$ & 116,200 & ugl \\
\hline 7 & DUP & $596 T 005083$ & 0 & axc-01 & F-02 & LIQUID & $9.89 \theta+03$ & $9.81 e+03$ & 0.812 & RPD \\
\hline 7 & DUR & $\$ 96 \mathrm{~T} 0050 \mathrm{~B}$ & 0 & $01 c-01$ & 0,02 & IIQU ro & $2,960+02$ & $2,980+02$ & 0.673 & RPD \\
\hline 7 & DUP & $596 T 005083$ & 0 & aIc-01 & $\mathrm{xO2-02}$ & IIQOID & $3.94 \theta+03$ & $3.92 e+03$ & 0.509 & RPD \\
\hline 7 & DUp & $\$ 969005083$ & 0 & $\triangle I C-01$ & $\mathrm{BR}-02$ & ATQYTD & $<1,3962$ & r 3902 & & RPD \\
\hline 7 & DEP & $596 \mathrm{~T} 005083$ & 0 & QIC-01 & ro3-02 & LIQUID & $3.03 e+04$ & $3.030+04$ & 0.000 & RPD \\
\hline 7 & pup & $\$ 96 T 005083$ & 0 & $a x-01$ & $004-02$ & IIQUID & $3936+02$ & $4.048+02$ & 2760 & RPD \\
\hline 7 & DUP & S96T005083 & 0 & QIC-01 & SO4-02 & LIQUID & $1.24 \theta+03$ & $1.280+03$ & 3.175 & RPD \\
\hline & & 962005083 & 0 & +01 & 00 & 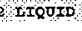 & $2-720002$ & $0.876+02$ & 5367 & RPD \\
\hline
\end{tabular}

\section{Final page for worklist\# 13849}

Analyst Signature $\quad$ Date




\section{LABCORE Data Entry Template for Worklist\# 13849}

Analyst:

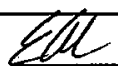

Instrument: IC

\section{(1)}

Method: LA-533-105 Rev/Mod D.

Book\# 32N20,A

Worklist Comment: AW-105 IC, USE .100ML-10ML SS. RCJ

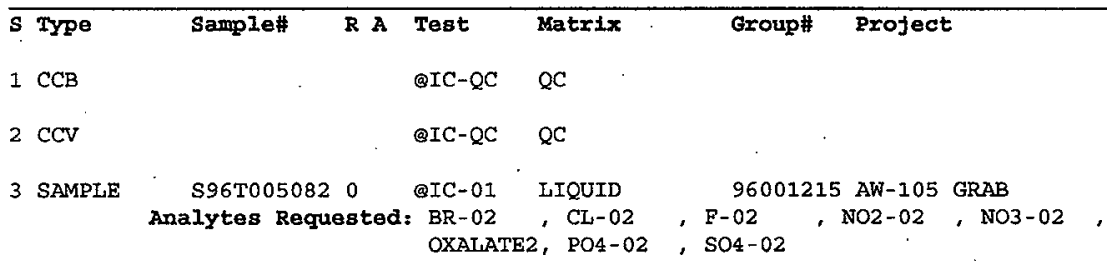
4 DUP
\$96T0050820
QIC-01
LIQUID
5 SPK
\$96T0050820
@IC-01 LIQUID
6 SAMPLE
S96T0050830
QIC-01
LIQUID
96001215 AW-105 GRAB
Analytes Requested: BR-02, $\mathrm{CL}-02$
OXALATE2, PO4-02
, F-02
, NO2-02, $\mathrm{NO3-02,}$

7 DUP

QIC-0I IIQUID

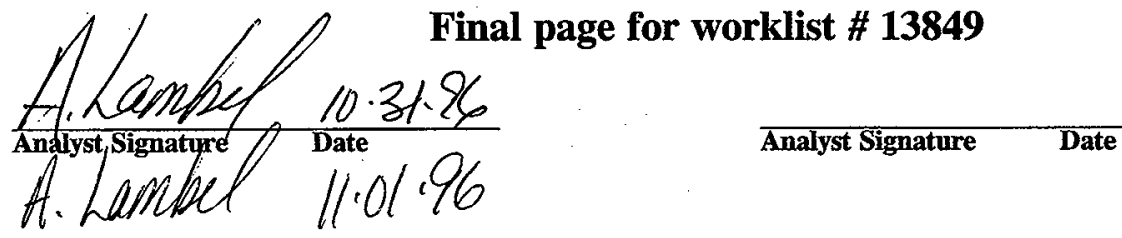

$13849 \mathrm{NV}, \mathrm{CSV}$

In rhele

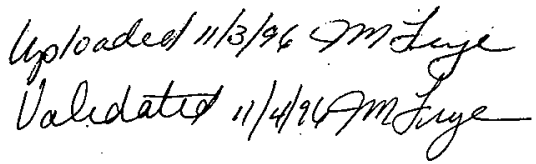

Data Entry Comments:

$S=$ Worklist Slot Number, $R=$ Replicate Number, $A=$ Aliquot Code. 
HNF-SD-WM-DP-214, REV. 0

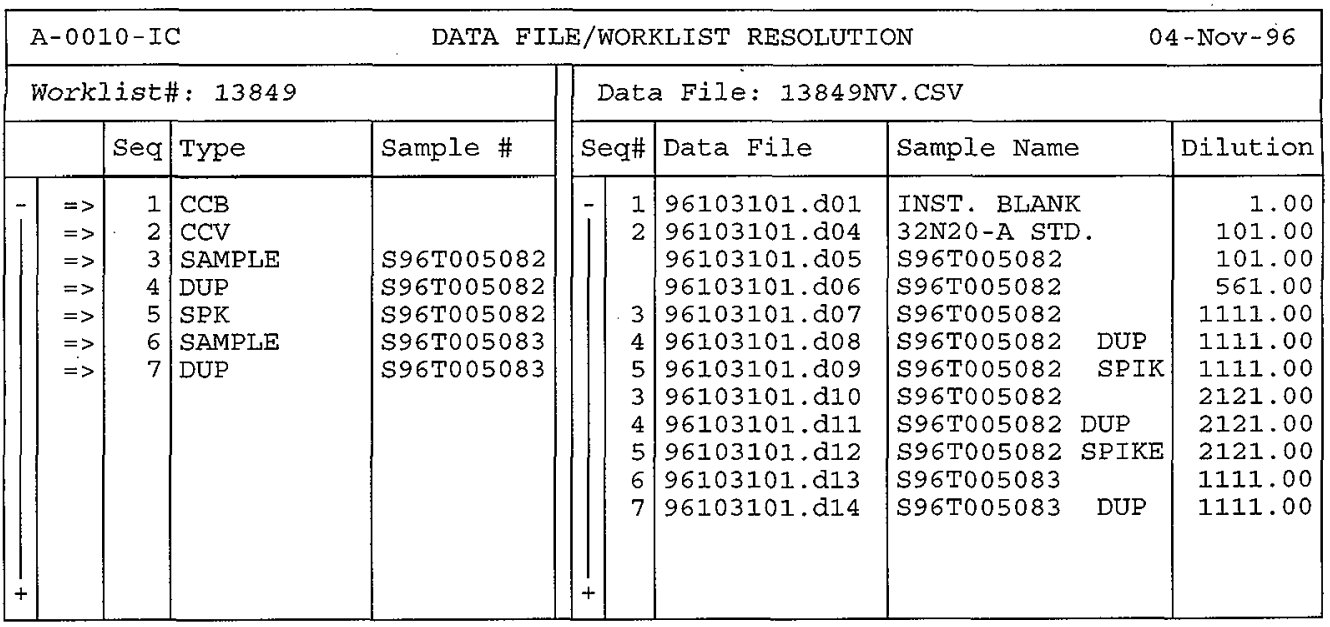

Save (F4) Abort(Shift-F3) ListFiles(Shift-FI) UploadFile(F8) 
HNF-SD-WM-DP-214, REV. 0

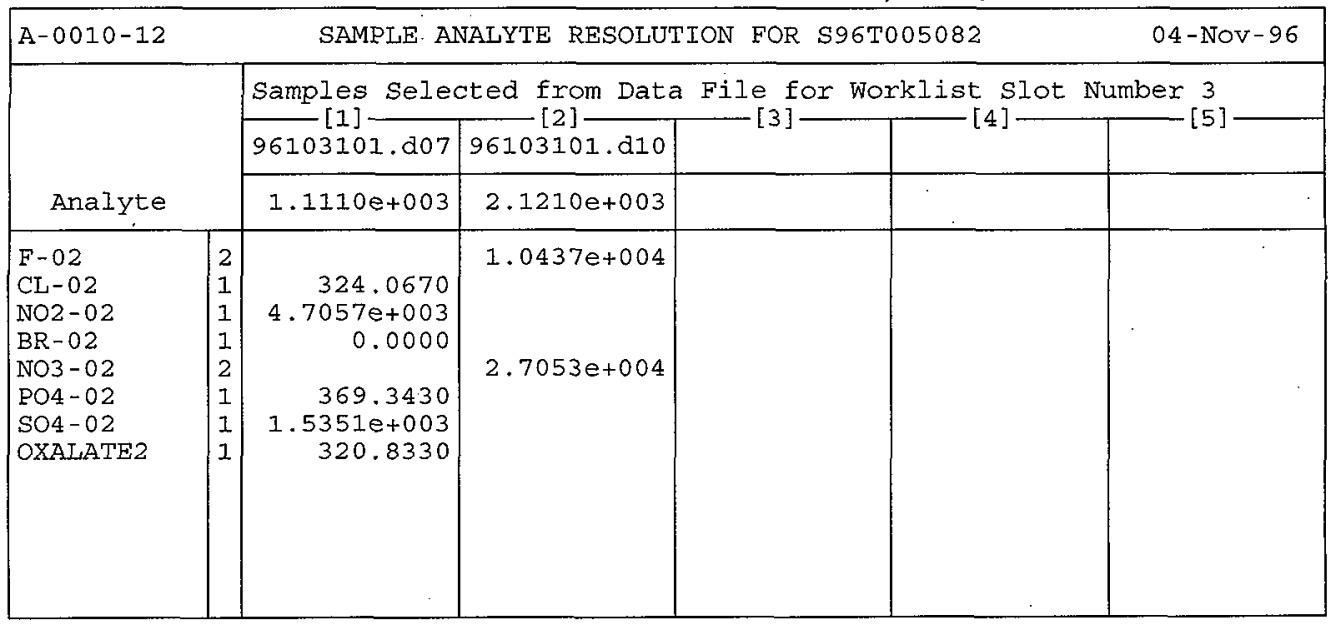

Done (F4) Abort (Shift-F3) UnPick(F7) 
HNF-SD-WM-DP-214, REV. O

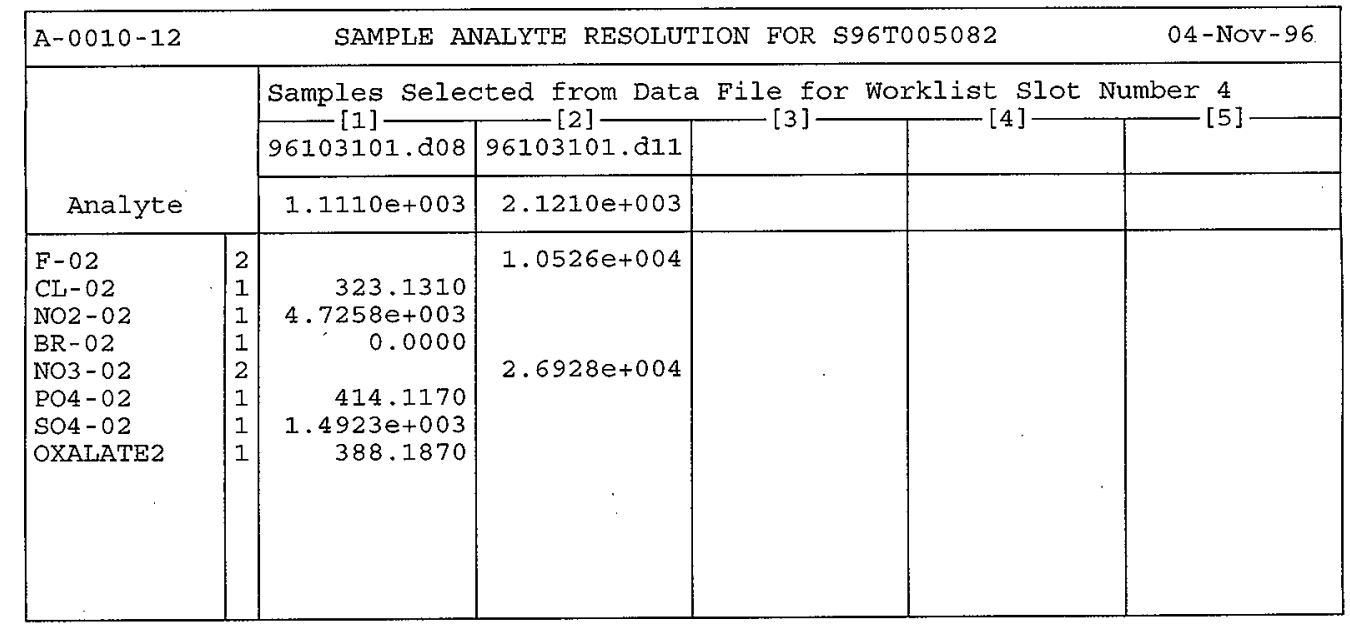

Done(F4) Abort(Shift-F3) UnPick(F7) 
HNF-SD-WM-DP-214, REV. 0

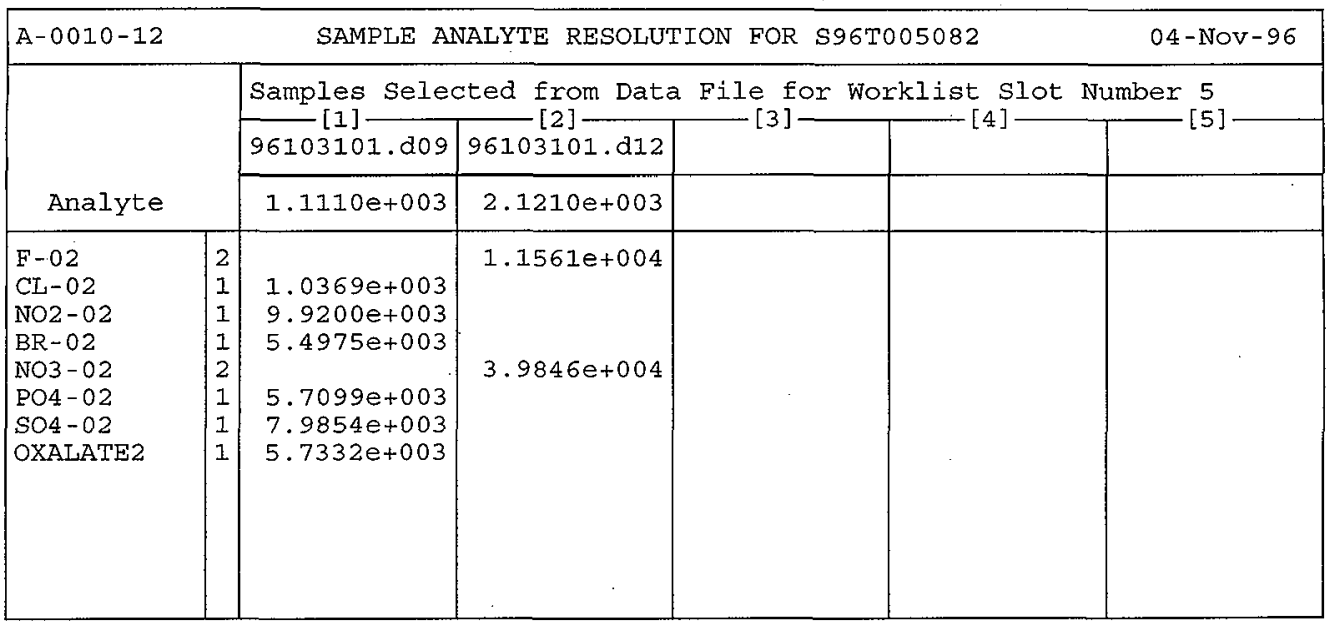

Done(F4) Abort(Shift-F3) UnPick(F7) 
HNF-SD-WM-DP-214, REV. 0

Data Reprocessed on 11/04/1996 10:02:45

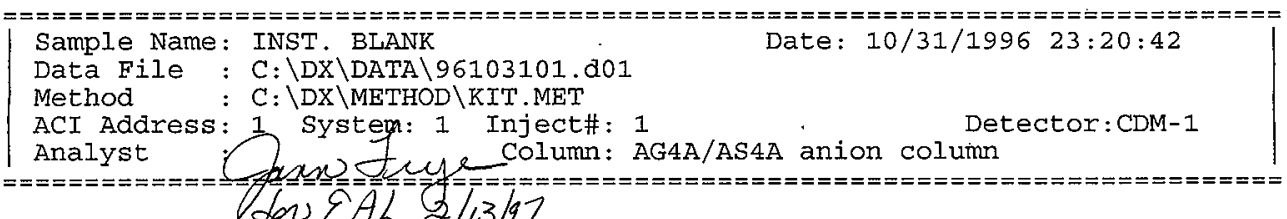

Calibration volum $\mathrm{CACH} / 3 / 97$

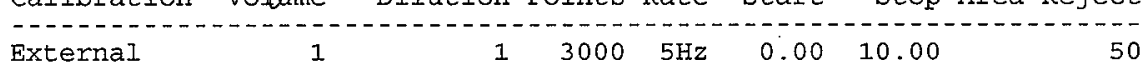

ExternaI

1

Peak Report: All Peaks

$\begin{array}{lrrr}\text { Pk. Ret Component } & \text { Concentration } & \text { Height } & \text { Area Bl. } \\ \text { Num } & \text { Time Name } & \text { Code }\end{array}$

0.000

0.000

0.171

53

27

56

0.171

137

293

107

375

1

1

1

Totals

File: 96103101.d01 Sample: INST. BLANK

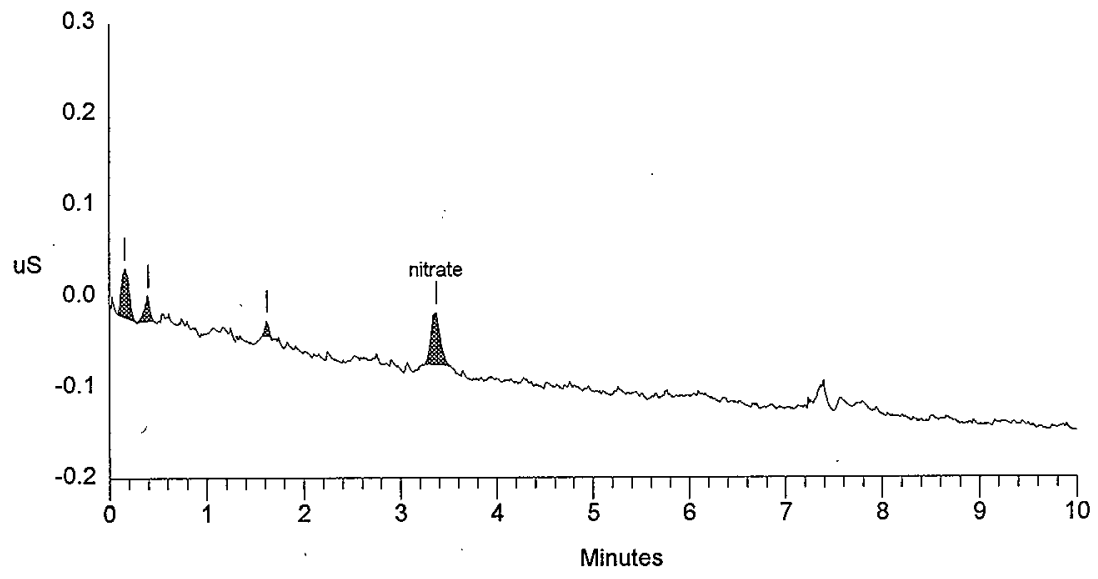

SIGNATURE ABOVE REPRESENTS CHEMICAL TECHNOLOGIST/CHEMIST THAT

COMPLETEDIVERIFIED THE CALIBRATION/ANALYSIS ON PAGES 414 TO 423. 
HNF-SD-WM-DP-214, REV. $O$

Data Reprocessed On 11/04/1996 10:02:48

Sample Name: 32N20-A STD.

Date: $11 / 01 / 199600: 18: 40$

Data File : C: \DX\DATA $\backslash 96103101$. d04

Method : C: \DX \METHOD $\backslash$ KIT.MET

ACI Address: 1 System: 1 Inject\# : 4

Analyst : Column: AG4A/AS4A anion column

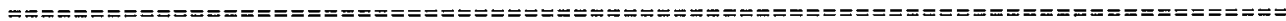

Calibration Volume Dilution Points Rate start stop Area Reject

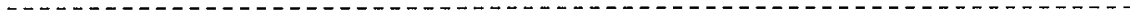

$\begin{array}{llllllll}\text { External } & 1 & 101 & 3000 & 5 \mathrm{~Hz} & 0.00 & 10.00 & 50\end{array}$

$* * * * * * * * * * * * * * * * * * * * * * * * * * *$ Peak Report: All Peaks $* * * * * * * * * * * * * * * * * * * * * * * * * * * *$

$\begin{array}{lrrr}\text { Pk. Ret Component } & \text { Concentration } & \text { Height } & \text { Area } \\ \text { Num } & \text { BI. } & \% \text { Delta } \\ \text { Code Name } & \end{array}$

Num Time Name

$\mathrm{ug} / \mathrm{mI}$

$\begin{array}{lll}1 & 0.90 & \\ 2 & 1.05 & \text { fluoride } \\ 3 & 1.60 & \text { chloride } \\ 4 & 1.91 & \text { nitrite } \\ 5 & 2.88 & \text { bromide } \\ 6 & 3.28 & \text { nitrate } \\ 7 & 4.59 & \text { phosphate } \\ 8 & 6.03 & \text { sulfate } \\ 9 & 7.95 \text { oxalate }\end{array}$

$\begin{array}{rrrrr}55.077 & 40 & 146 & 2 & \\ 51.340 & 1788 & 10848 & 2 & 3.27 \\ 489.784 & 7748 & 9445 & 1 & -0.83 \\ 573.748 & 4989 & 45130 & 1 & -3.70 \\ 589.019 & 5473 & 35219 & 1 & -3.36 \\ 532.694 & 1533 & 45141 & 1 & -3.81 \\ 633.645 & 4595 & 64313 & 1 & -6.96 \\ 530.121 & 2244 & 41963 & 1 & -3.13\end{array}$

Totals $\quad 3475.427 \quad 29495.273976$

File: 96103101.d04 Sample: 32N2O-A STD.

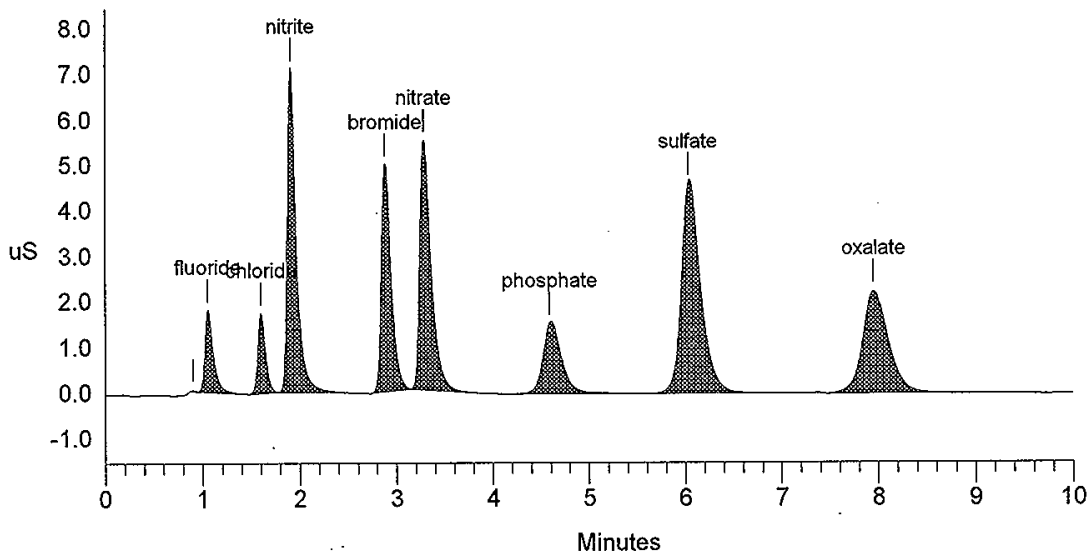


Data Reprocessed on 11/04/1996 HNF:SD-WWM-DP-214, REV. 0

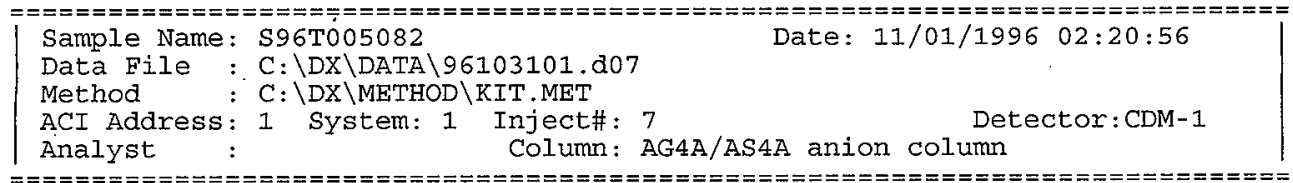

Calibration Volume Dilution Points Rate start stop Area Reject

C.-.-.-.

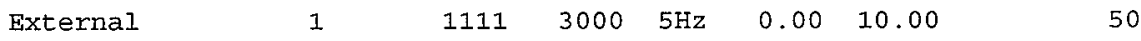

$* * * * * * * * * * * * * * * * * * * * * * * * * * *$ Peak Report: All Peaks $* * * * * * * * * * * * * * * * * * * * * * * * * * * *$

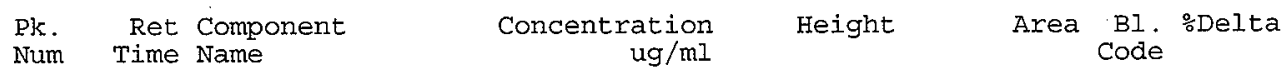

Num Time Name

$\mathrm{ug} / \mathrm{ml}$ Code

$\begin{array}{lll}1 & 0.90 & \\ 2 & 1.05 & \text { fluoride } \\ 3 & 1.60 & \text { chloride } \\ 4 & 1.91 & \text { nitrite } \\ 5 & 3.19 & \text { nitrate } \\ 6 & 3.99 & \\ 7 & 4.61 & \text { phosphate } \\ 8 & 6.08 & \text { sulfate } \\ 9 & 8.00 \text { oxalate }\end{array}$

$\begin{array}{rrrrrr}9.000 & 76 & 203 & 2 & \\ 9977.369 & 44208 & 241274 & 2 & 3.27 \\ 324.067 & 670 & 3889 & 1 & -0.83 \\ 4705.706 & 5967 & 39256 & 1 & -3.70 \\ 29356.621 & 23837 & 221530 & 1 & -6.35 \\ 0.000 & 72 & 666 & 1 & \\ & 369.343 & 102 & 1332 & 1 & -6.42 \\ & 1535.090 & 916 & 13704 & 1 & -7.32 \\ & 320.833 & 115 & 2087 & 1 & -2.56 \\ \text { Totals } & 46589.030 & 75962 & 523941 . & & \end{array}$

File: $96103101 . d 07$ Sample: S96T005082

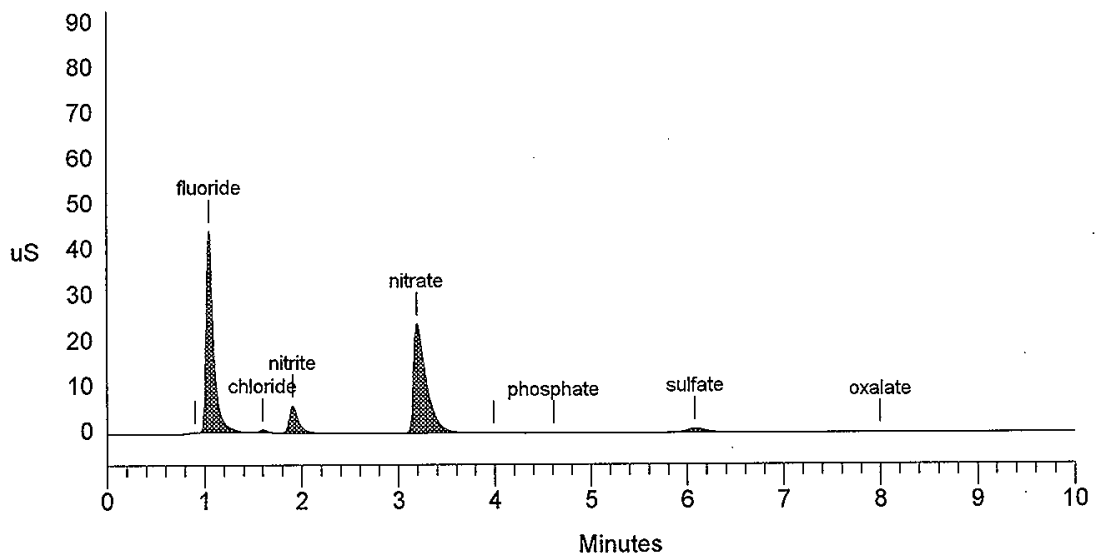


Data Reprocessed on 11/04/1996 10:02:55

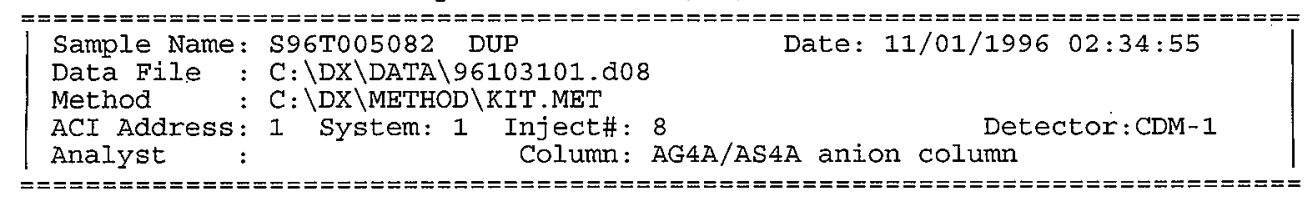

Calibration Volume Dilution Points Rate start Stop Area Reject

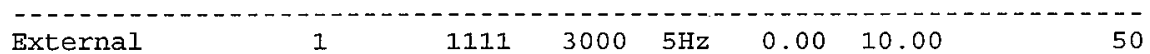

External

$\begin{array}{llllll}1 & 111 \mathrm{I} & 3000 & 5 \mathrm{~Hz} & 0.00 & 10.00\end{array}$

50

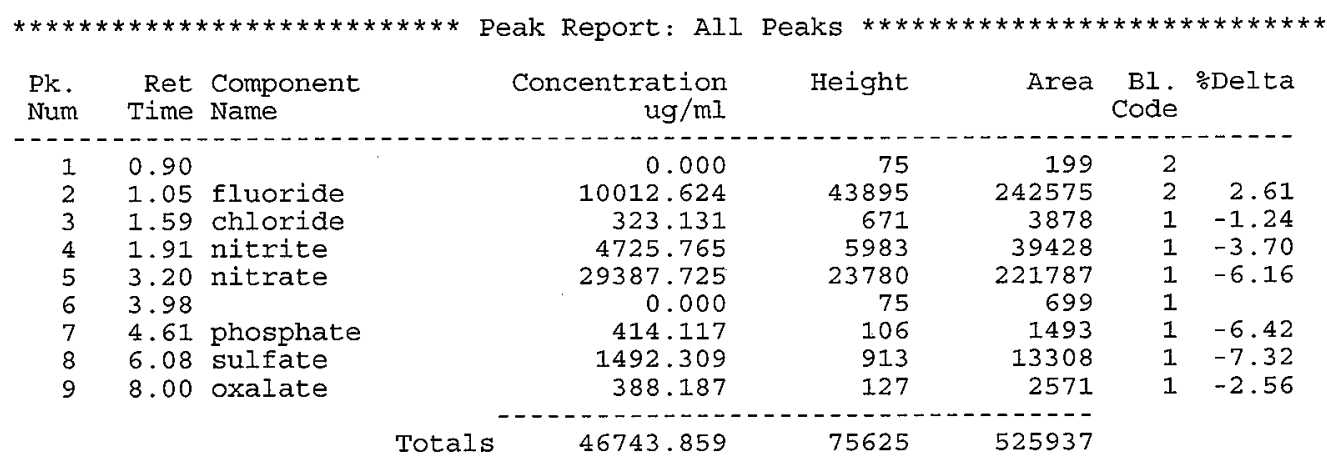

File: 96103101.d08 Sample: S96T005082 DUP

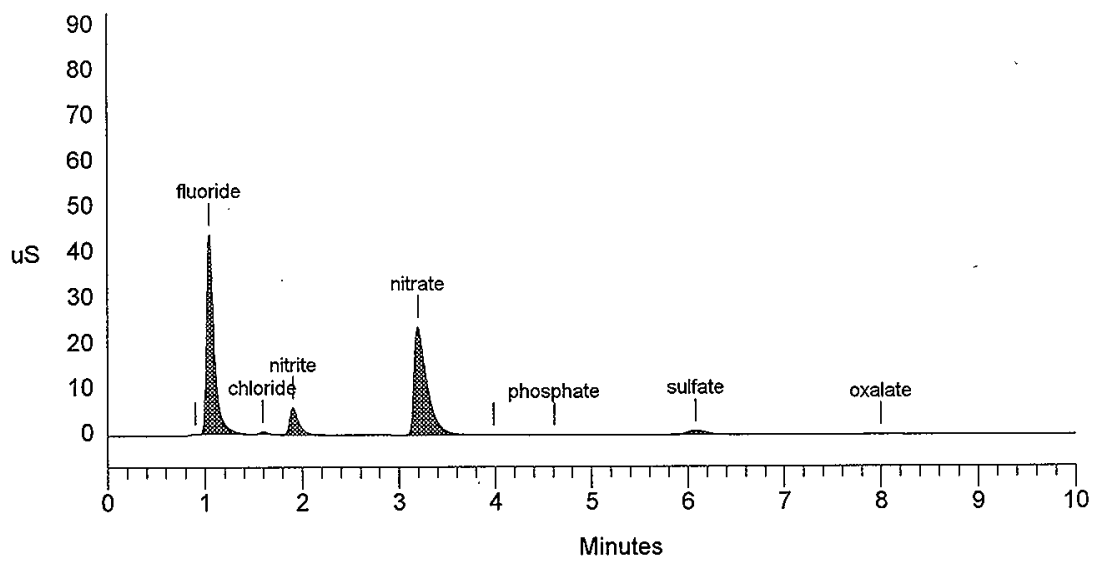


Data Reprocessed on 11/04/1996 10:02:57

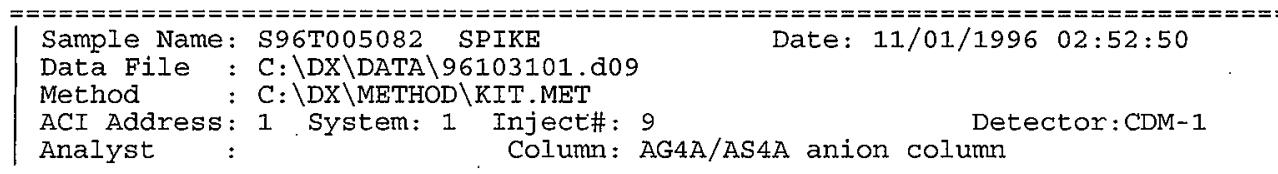

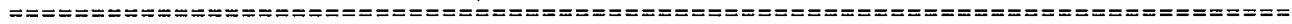

Calibration Volume Dilution Points Rate start stop Area Reject

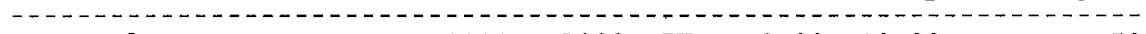

External

$\begin{array}{llllll}1 & 1111 & 3000 & 5 \mathrm{~Hz} & 0.00 & 10.00\end{array}$

50

$* * * * * * * * * * * * * * * * * * * * * * * * * * *$ Peak Report: All Peaks $* * * * * * * * * * * * * * * * * * * * * * * * * * * *$

$\begin{array}{lrrr}\text { Pk. Ret Component } & \text { Concentration } & \text { Height } & \text { Area Bl. } \\ \text { Num } & \text { Time Name } & \text { Code }\end{array}$

Num Time Name

$\begin{array}{rll}1 & 0.89 & \\ 2 & 1.05 & \text { Eluoride } \\ 3 & 1.59 & \text { chloride } \\ 4 & 1.90 & \text { nitrite } \\ 5 & 2.87 & \text { bromide } \\ 6 & 3.17 & \text { nitrate } \\ 7 & 3.98 & \\ 8 & 4.59 & \text { phosphate } \\ 9 & 6.03 & \text { sulfate } \\ 10 & 7.95 & \text { oxalate }\end{array}$

$\begin{array}{rrrrr}0.000 & 93 & 269 & 2 & \\ 10291.253 & 46101 & 253125 & 2 & 2.61 \\ 1036.923 & 2266 & 12523 & 1 & -1.65 \\ 9920.047 & 13475 & 84536 & 1 & -4.04 \\ 5497.536 & 4681 & 30524 & 1 & 0.12 \\ 35927.487 & 28849 & 277039 & 1 & -6.94 \\ 0.000 & 68 & 614 & 1 & \\ 5709.905 & 1528 & 20760 & 1 & -6.96 \\ 7985.353 & 5334 & 74474 & 1 & -8.13 \\ 5733.204 & 2181 & 41248 & 1 & -3.21\end{array}$

Totals

82101.708

104575

795112

File: 96103101.d09 Sample: S96T005082 SPIKE

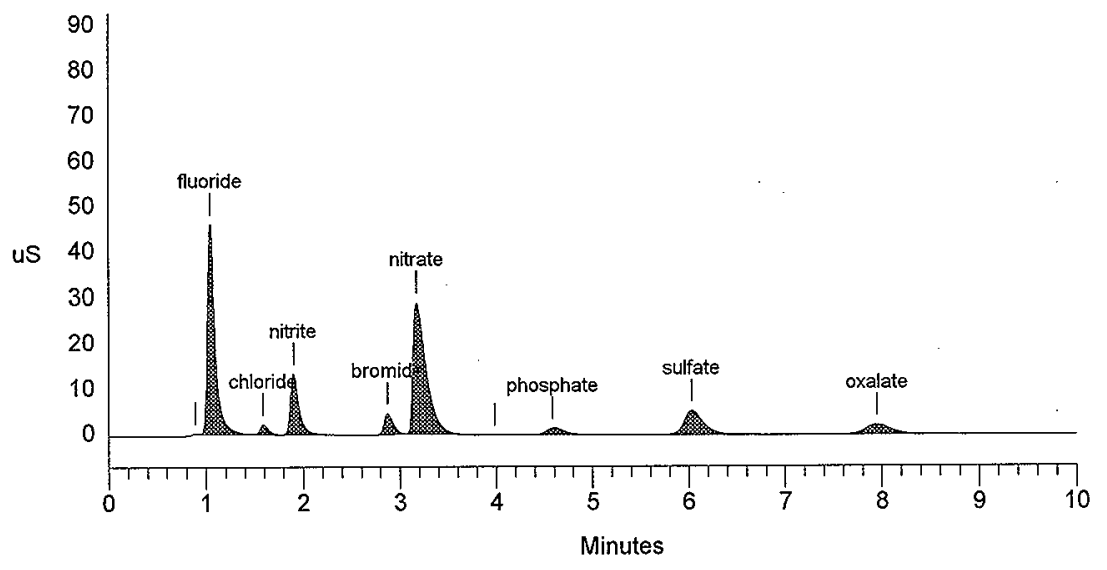


Data Reprocessed on 11/04/1996 10:03:00

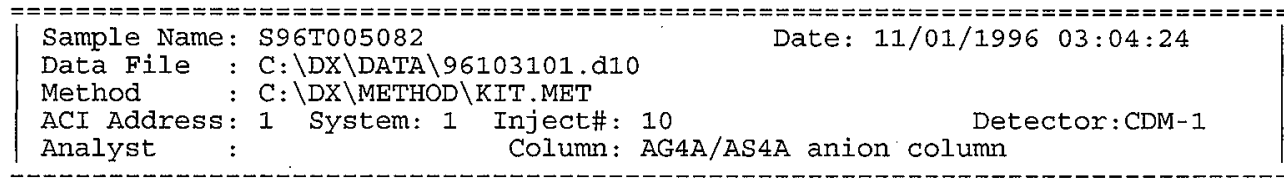

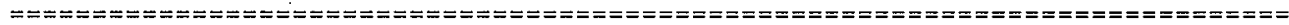

Calibration Volume Dilution Points Rate Start stop Area Reject

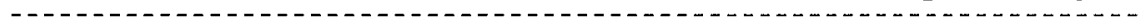

External

2121

3000

$5 \mathrm{~Hz}$

0.0010 .00

50

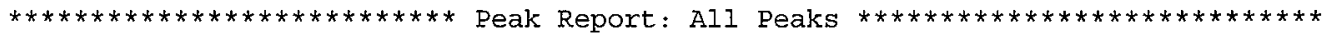
Pk. Ret Component
Concentration
Height
Area Bl. :Delta
Num Time Name
$\mathrm{ug} / \mathrm{mI}$ Code

$\begin{array}{lll}1 & 0.90 & \\ 2 & 1.05 & \text { fluoride } \\ 3 & 1.61 & \text { chloride } \\ 4 & 1.91 & \text { nitrite } \\ 5 & 3.24 & \text { nitrate } \\ 6 & 4.61 & \text { phosphate } \\ 7 & 6.08 & \text { sulfate } \\ 8 & 8.00 \text { oxalate }\end{array}$

0.000
10437.007
303.247
4338.684
27052.541
283.281
1524.928
306.956

44246.643

40

20610

348

2771

11633

44

445

53

Totals

35944

$125 \quad 2$

$\begin{array}{lll}111568 & 2 & 2.61\end{array}$

$\begin{array}{lll}1917 & 1 & -0.41\end{array}$

$\begin{array}{lll}18409 & 1 & -3.37\end{array}$

$\begin{array}{rrr}18409 & 1 & -3.37\end{array}$

$\begin{array}{rrr}540 & 1 & -6.42\end{array}$

$\begin{array}{lll}6895 & 1 & -7.32\end{array}$

$\begin{array}{lll}938 & 1 & -2.56\end{array}$

\section{File: $96103101 . d 10$ Sample: S96T005082}

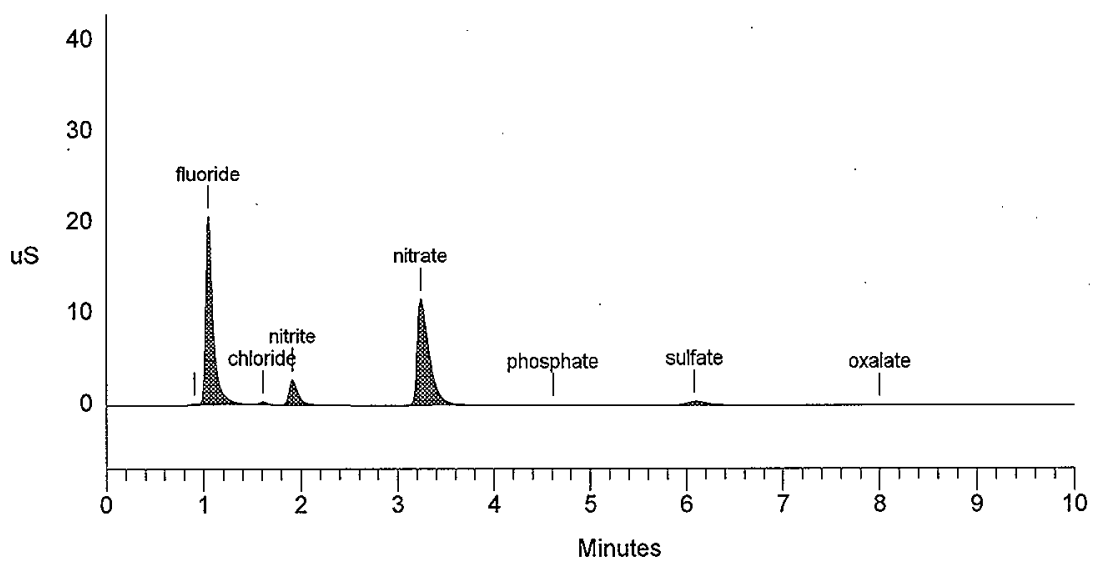


Data Reprocessed On 11/04/1996 10:03:02

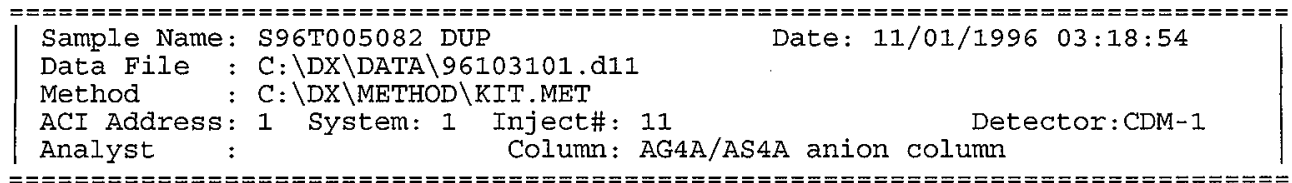

Calibration Volume Dilution Points Rate start stop Area Reject

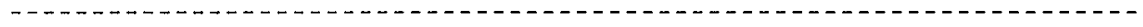

External

$\begin{array}{llllll}1 & 2121 & 3000 \quad 5 \mathrm{~Hz} & 0.00 & 10.00\end{array}$

50

$\star * * * * * * * * * * * * * * * * * * * * * * * * * *$ Peak Report: All Peaks $* * * * * * * * * * * * * * * * * * * * * * * * * * * *$

$\begin{array}{lrrr}\text { Pk. Ret Component } & \text { Concentration } & \text { Height } & \text { Area Bl. } \\ \text { Num } & \text { Timelta }\end{array}$

Num Time Name ug/m1

$\begin{array}{lll}1 & 0.90 & \\ 2 & 1.05 & \text { fluoride } \\ 3 & 1.61 & \text { chloride } \\ 4 & 1.91 & \text { nitrite } \\ 5 & 3.24 & \text { nitrate } \\ 6 & 3.98 & \\ 7 & 4.61 & \text { phosphate } \\ 8 & 6.08 & \text { sulfate } \\ 9 & 8.00 \text { oxalate }\end{array}$

$\begin{array}{rrrrr}0.000 & 44 & 128 & 2 & \\ 10525.893 & 20790 & 112668 & 2 & 2.61 \\ 329.166 & 364 & 2078 & 1 & -0.41 \\ 4405.737 & 2798 & 18708 & 1 & -3.37 \\ 26928.013 & 11710 & 101394 & 1 & -4.99 \\ 0.000 & 33 & 299 & 1 & \\ 408.495 & 55 & 775 & 1 & -6.42 \\ 1517.771 & 449 & 6861 & 1 & -7.32 \\ 357.038 & 61 & 1126 & 1 & -2.56\end{array}$

Totals

44472.114

36305

244038

File: 96103101.d11 Sample: S96T005082 DUP

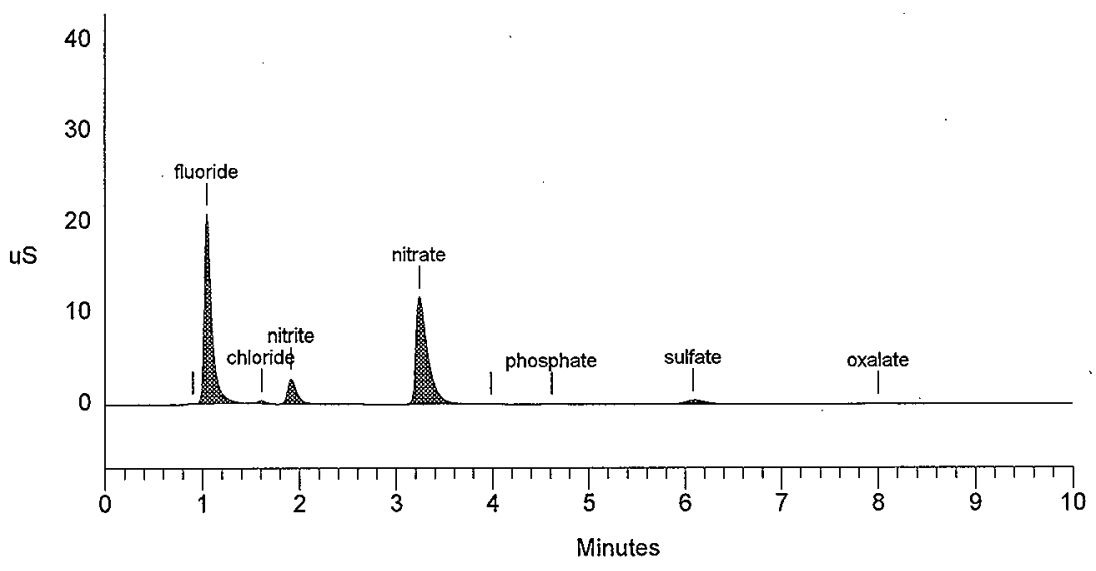


Data Reprocessed on 11/04/1996 09:46:25

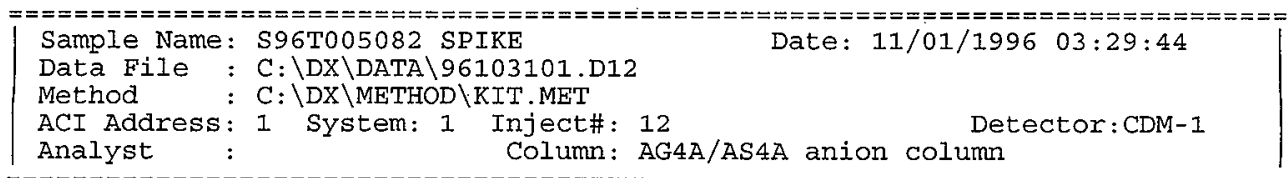

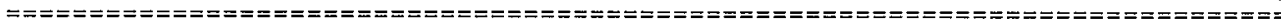

Calibration Volume Dilution Points Rate start stop Area Reject

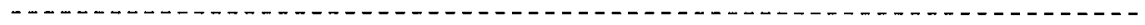

External

$1 \quad 2121 \quad 3000 \quad 5 \mathrm{~Hz} \quad 0.00 \quad 10.00$

50

$\star * * * * * * * * * * * * * * * * * * * * * * * * * *$ Peak Report: All Peaks $* * * * * * * * * * * * * * * * * * * * * * * * * * * *$

$\begin{array}{lrrr}\text { Pk. Ret Component } & \text { Concentration } & \text { Height } & \text { Area Bl. } \\ \text { Num Delta }\end{array}$

Num Time Name

$\begin{array}{rll}1 & 0.90 & \\ 2 & 1.05 & \text { fluoride } \\ 3 & 1.59 & \text { chloride } \\ 4 & 1.90 & \text { nitrite } \\ 5 & 2.87 \text { bromide } \\ 6 & 3.22 & \text { nitrate } \\ 7 & 3.98 & \\ 8 & 4.59 & \text { phosphate } \\ 9 & 6.03 & \text { sulfate } \\ 10 & 7.95 & \text { oxalate }\end{array}$

$\begin{array}{rrrrr}11560.758 & 65 & 194 & 2 & \\ 1742.603 & 2025 & 125739 & 2 & 2.61 \\ 14461.668 & 10086 & 64070 & 1 & -1.24 \\ 11285.038 & 4841 & 32908 & 1 & 0.12 \\ 39846.397 & 17189 & 153333 & 1 & -5.57 \\ 0.000 & 29 & 249 & 1 & \\ 10987.367 & 1521 & 20927 & 1 & -6.96 \\ 14253.444 & 4957 & 69505 & 1 & -8.13 \\ 10930.832 & 2199 & 41193 & 1 & -3.21\end{array}$

Totals

115068.108

65939

519122

File: 96103101.D12 Sample: S96T005082 SPIKE

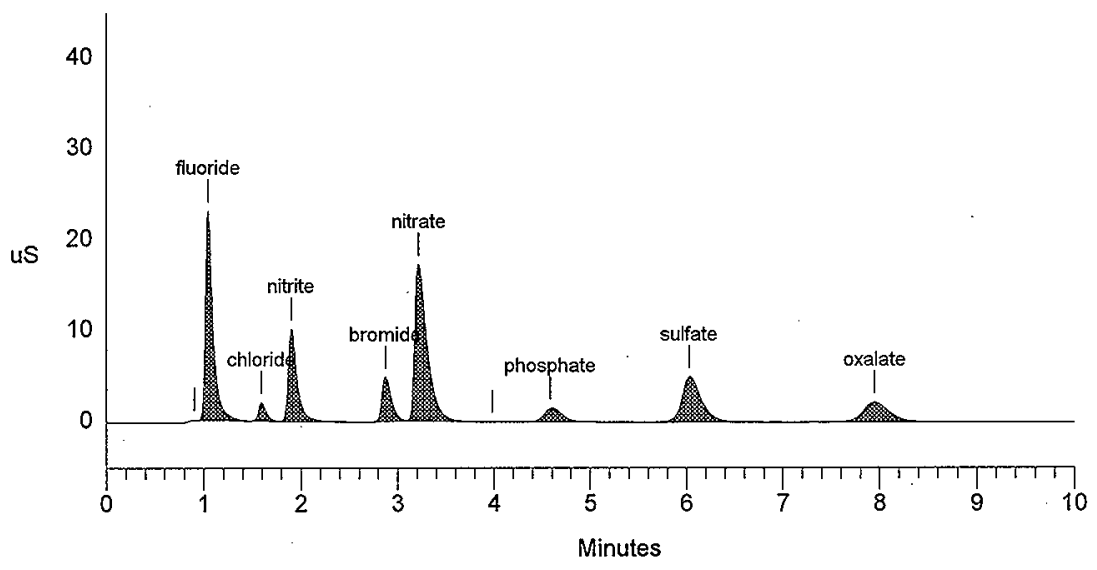


Data Reprocessed on 11/04/1996 10:03:06

HNF-SD-WM-DP-214, REV. 0
$03: 06$

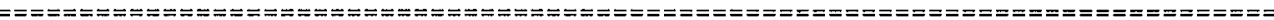

Sample Name: S96T005083

Data File : C:\DX\DATA \96103101.d13

Method : C: DXX \METHOD $\backslash$ KIT.MET

ACI Address: 1 System: 1 Inject\#: 13

Date: $11 / 01 / 199603: 40: 59$

Analyst : Column: AG4A/AS4A anion column

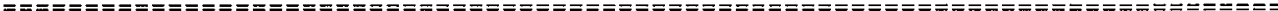

Calibration Volume Dilution Points Rate start stop Area Reject

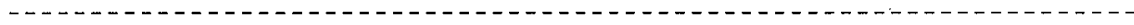

External

1

$11113000 \quad 5 \mathrm{~Hz} \quad 0.00 \quad 10.00$

50

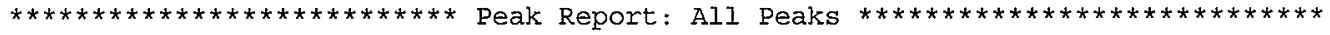

$\begin{array}{lrrr}\text { Pk. Ret Component } & \text { Concentration } & \text { Height } & \text { Area } \\ \text { Num Time Name } & \text { Codpelta }\end{array}$

Num Time Name $\mathrm{lg} / \mathrm{ml}$

$1 \quad 0.89$

1.05 fluoride

1. 60 chloride

0.000

9886.902

1.91 nitrite

295.584

3.19 nitrate

3.98

4.61 phosphate

3940.910

30338.728

0.000

393.070

6.08 sulfate

1238.866

272.269

63
43709
643
5025
24243
56
113
761
91

$\begin{array}{rrr}176 & 2 & \\ 237969 & 2 & 2.61 \\ 3548 & 1 & -0.83 \\ 32684 & 1 & -3.70 \\ 229667 & 1 & -6.35 \\ 512 & 1 & \\ 1418 & 1 & -6.42 \\ 10962 & 1 & -7.32 \\ 1738 & 1 & -2.56\end{array}$

Totals $\quad 46366.330$

74705

518674

File: 96103101.d13 Sample: S96T005083

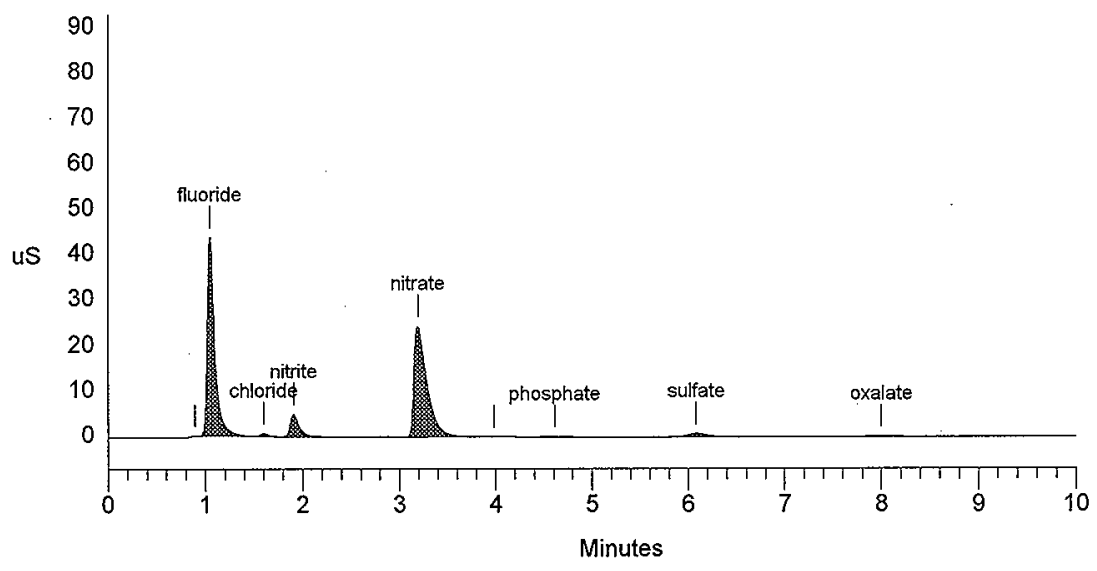


Data Reprocessed on 11/04/1996 10:HF:SQ-WM-DP-214, REV. 0

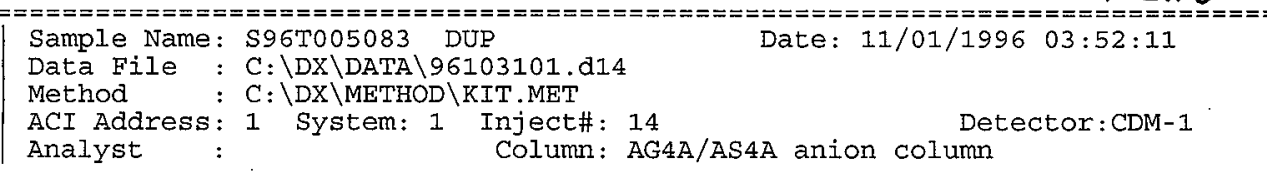

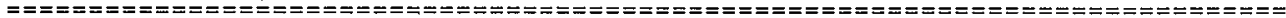

Calibration Volume Dilution Points Rate start stop Area Reject

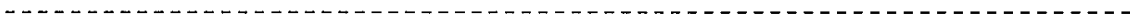

External

$1 \quad 1111 \quad 3000 \quad 5 \mathrm{~Hz} \quad 0.00 \quad 10.00$

50

$* * * * * * * * * * * * * * * * * * * * * * * * * * *$ Peak Report: All Peaks $* * * * * * * * * * * * * * * * * * * * * * * * * * * *$

$\begin{array}{lrrr}\text { Pk. Ret Component } & \text { Concentration } & \text { Height } & \text { Area Bl. } \\ \text { Num } & \text { Time Name } / \mathrm{ml} & & \text { Code }\end{array}$

$\begin{array}{lll}1 & 0.90 \\ 2 & 1.05 & \text { fluoride } \\ 3 & 1.60 \text { chloride } \\ 4 & 1.91 \text { nitrite } \\ 5 & 3.19 & \text { nitrate } \\ 6 & 3.98 & \\ 7 & 4.61 & \text { phosphate } \\ 8 & 6.08 \text { sulfate } \\ 9 & 8.00 \text { oxalate }\end{array}$

$\begin{array}{rrrrr}0.000 & 72 & 207 & 2 & \\ 9811.758 & 43533 & 235259 & 2 & 2.61 \\ 297.892 & 660 & 3576 & 1 & -0.83 \\ 3921.694 & 5042 & 32519 & 1 & -3.70 \\ 30306.992 & 24337 & 229404 & 1 & -6.55 \\ 0.000 & 58 & 539 & 1 & \\ 404.332 & 108 & 1458 & 1 & -6.42 \\ 1276.482 & 770 & 11310 & 1 & -7.32 \\ 286.531 & 92 & 1840 & 1 & -2.56\end{array}$

Totals $\quad 46305.681$

74672

516112

File: 96103101.d14 Sample: S96T005083 DUP

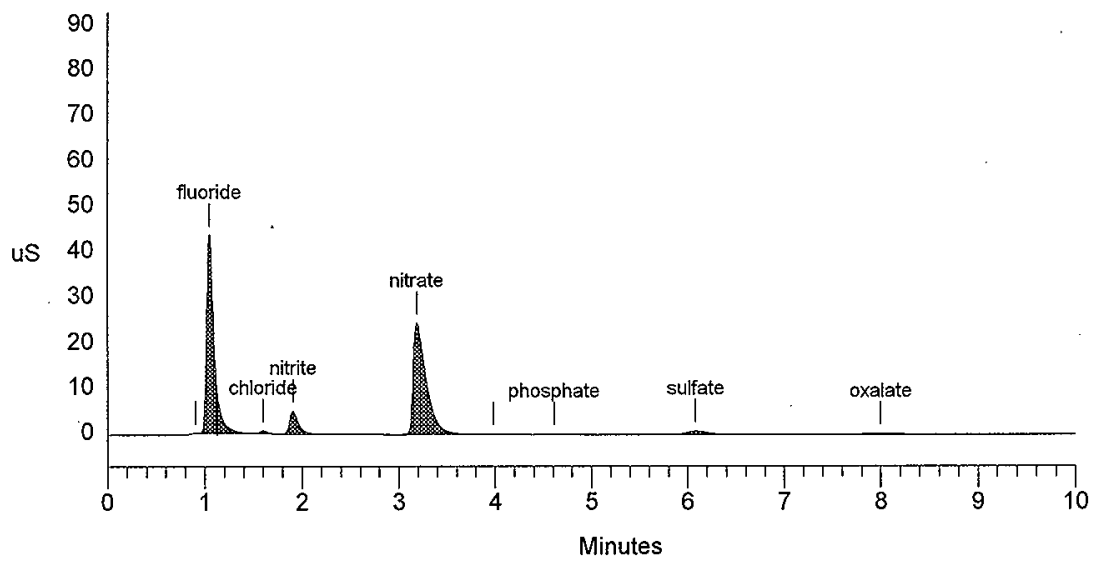




\section{LABCORE Completed Worklist Report for Worklist\# 14191}

Analyst: eal

Instrument: ICO1

Book\# 32N204

Method: $1-533-105$ Rev/Mod $D-1$

Worklist Comment: AW-105 IC. RCJ

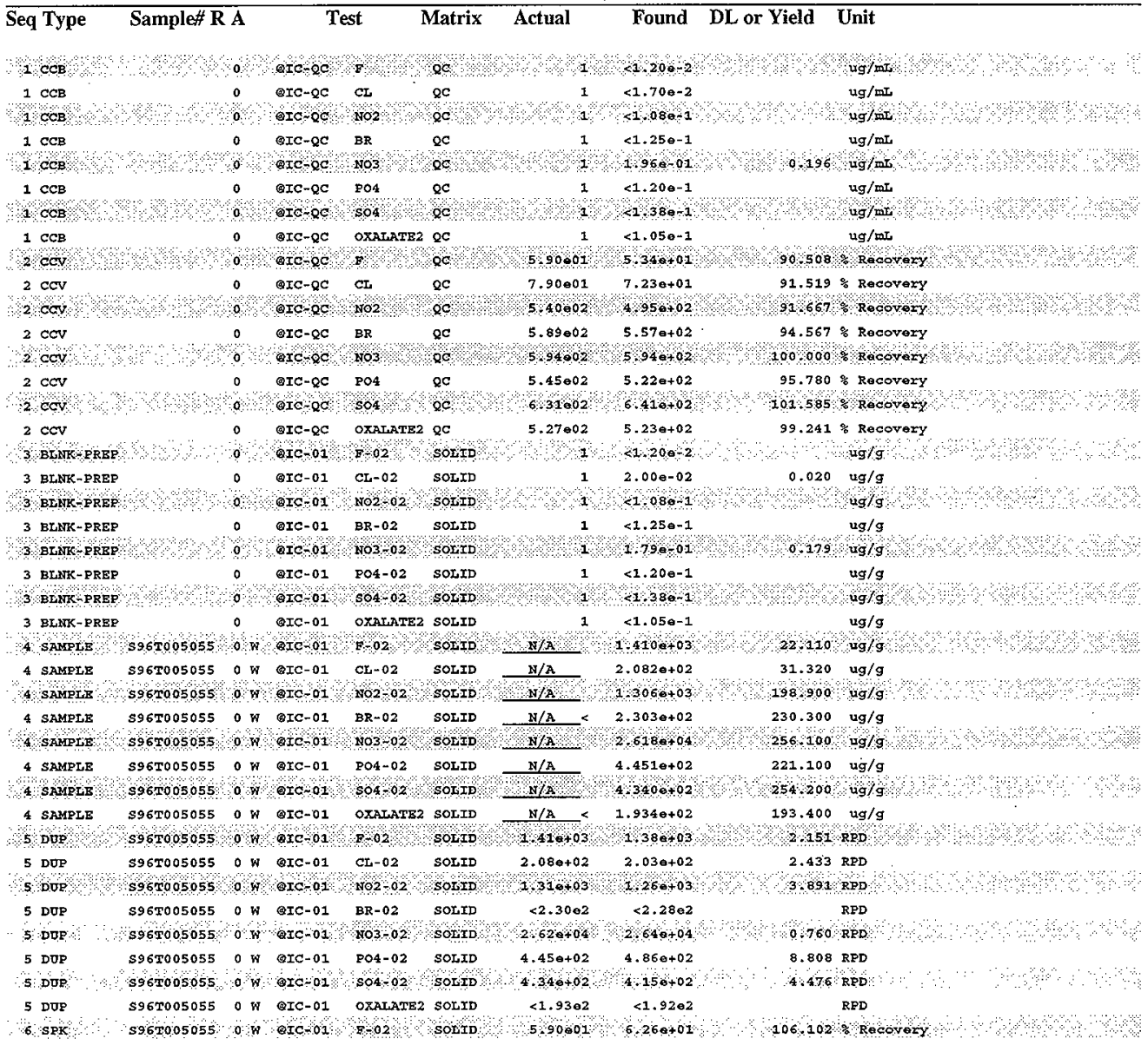




\section{LABCORE Completed Worklist Report for Worklist\# 14191}

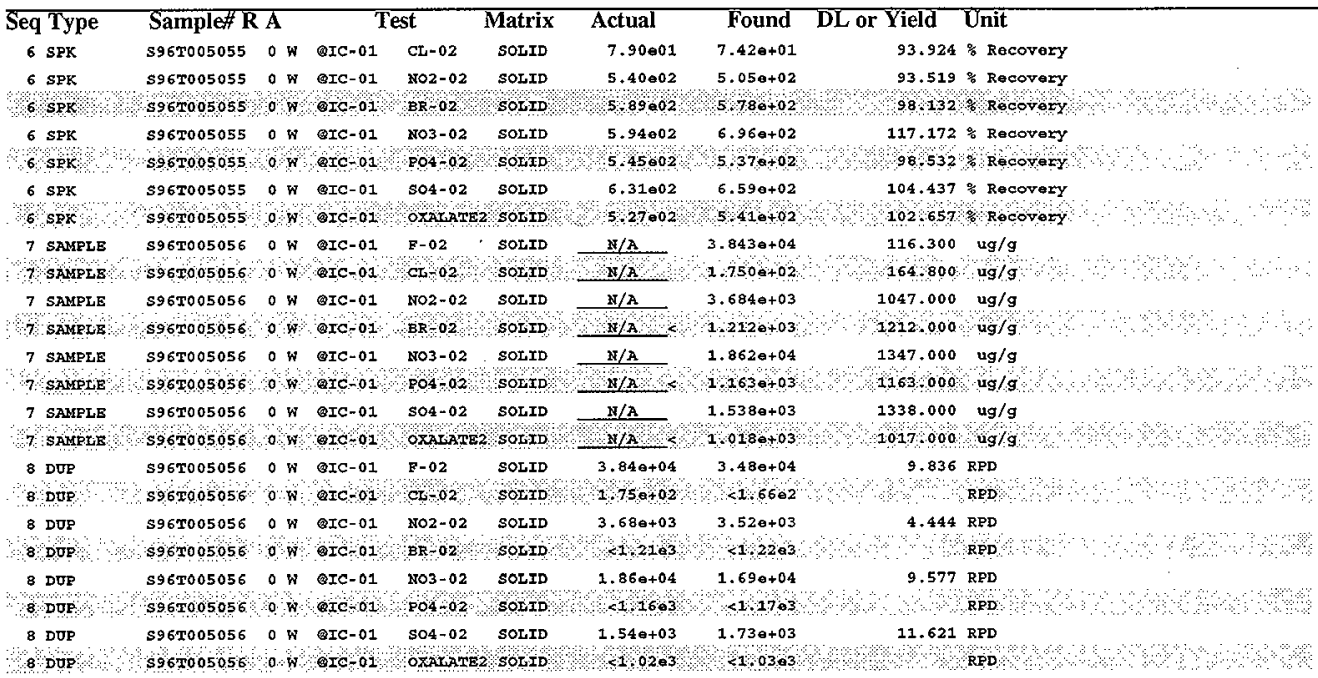

\section{Final page for worklist\# 14191}

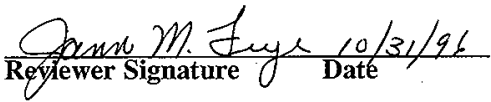




\section{LABCORE Data Entry Template for Worklist\# 14191}

Analyst: $\quad$ gal Instrument: ICo $\mathrm{ZCO}$ Book\# 32N20 $\rightarrow$

Method: LA-533-105 Rev/Mod D.l.

Worklist Comment: AW-105 IC. RCJ

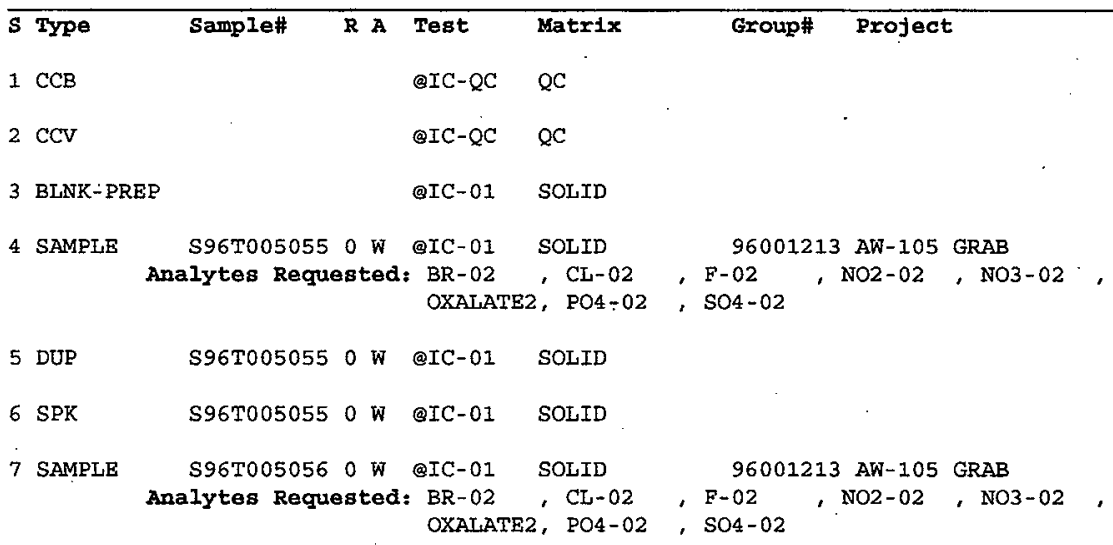

8 DUP S96T005056 0'W @IC-01 SOLID

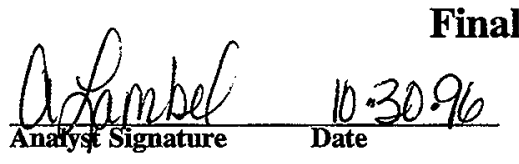

$1419100, \operatorname{csl}$

Upoloaded 10/31/96 Omptuye

Valudated 10 31/96 gmafux

Analyst Signature Date

Data Entry Comments:

$S=$ Worklist Slot Number, $R=$ Replicate Number, $A=$ Aliquot Code. 
HNF-SD-WM-DP-214, REV. 0

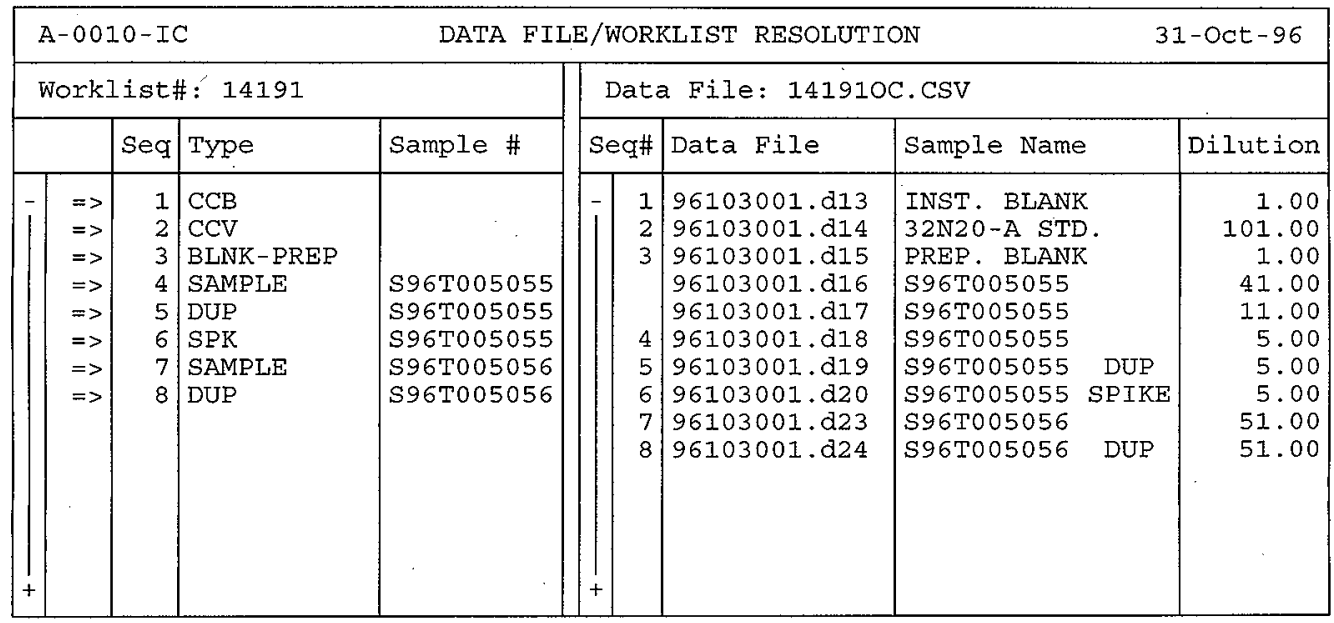

Save(F4) Abort(Shift-F3) ListFiles(Shift-F1) UploadFile(F8) 
$======================$
Sample Name: $32 \mathrm{~N} 20-\mathrm{A}$ STD.

Data File : C: \DX\DATA \96103001.D14

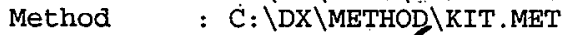

ACI Address: 1) Oystem: Inject\#: 14 Detector:CDM-1

Date: $10 / 30 / 1996 \quad 02: 50: 30$
Column: AG4A/AS4A anion column

Analyst

A. kenter

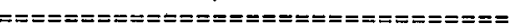

Calibration Volume Dilution Points Rate start stop Area Reject

External

1

$101 \quad 3000 \quad 5 \mathrm{~Hz}$

0.0010 .00

50

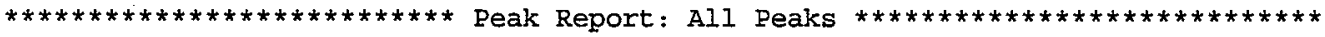

Pk. Ret Component $\quad \begin{array}{r}\text { Concentration } \\ \text { ug/ml }\end{array}$
Num Time Name Height

Num Time Name

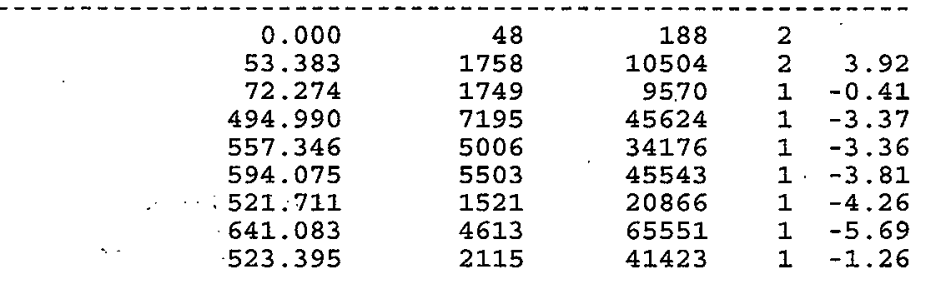

Totals $\quad 3458.258 \quad 29508 \quad 273447$.

File: 96103001.D14 Sample: 32N20-A STD.

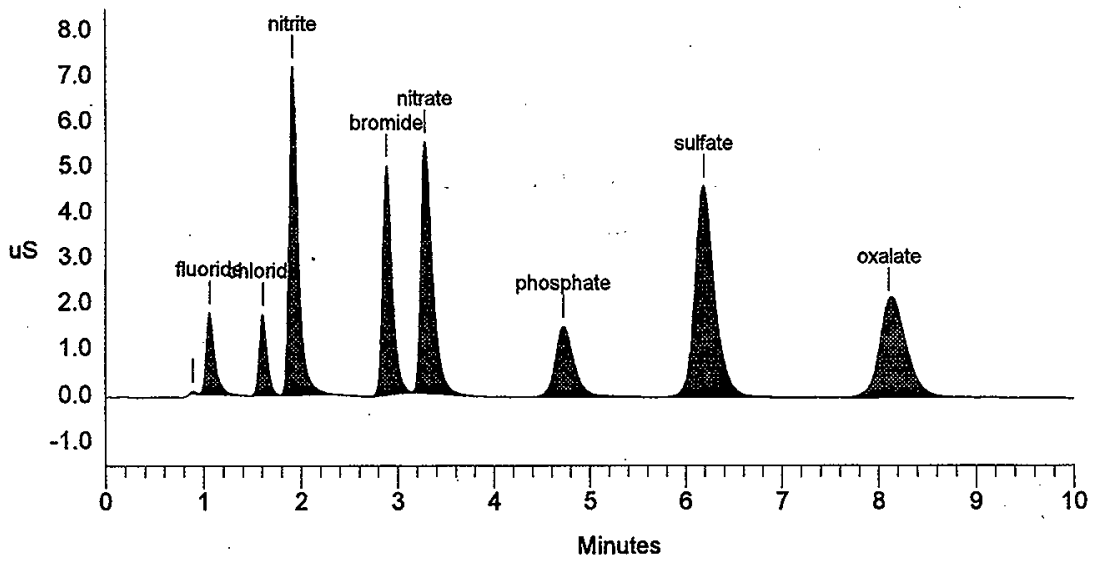

SICNATURE ABOVE REPRESENTS CHEMICAL TECHNOLOGIST/CHEMIST THAT

COMPLETEDVERIFIED THE CALIBRATION/ANALYSIS ON PAGES 128 TO 407. 
Sample Name: INST. BLANK

Data File : C:\DX\DATA \96103001.D13

Method : $\dot{C}: \backslash D X \backslash M E T H O D \backslash K I T . M E T$

ACI Address: 1 System: 1 Inject\#: $1 \hat{3}$
Column: AG4A/AS4A anion column
Date: $10 / 30 / 199602: 36: 41$

Detector: CDM-1

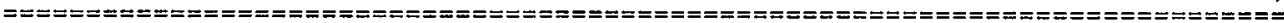

Calibration Volume Dilution Points Rate start stop Area Reject

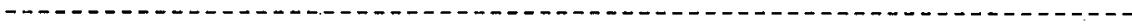

$\begin{array}{llllllll}\text { Bxternal } & 1 & 1 & 3000 & 5 \mathrm{~Hz} & 0.00 & 10.00 & 50\end{array}$

$\star * * * * * * * * * * * * * * * * * * * * * * * * * *$ Peak Report: All Peaks $* * * * * * * * * * * * * * * * * * * * * * * * * * * *$

Pk. Ret Component Concentration Height Area Bl. $\frac{\circ D e l t a}{}$

Num Time Name ug/ml code

$\begin{array}{rrlrrrr}1 & 1.42 & 0.000 & 16 & 79 & 1 & \\ 2 & 1.62 \text { chloride } & 0.003 & 18 & 75 & 1 & 0.41 \\ 3 & 3.36 \text { nitrate } & 0.196 & 78 & 575 & 1 & -1.47\end{array}$

Totals

0.200

112

729

File: 96103001.D13 Sample: INST. BLANK

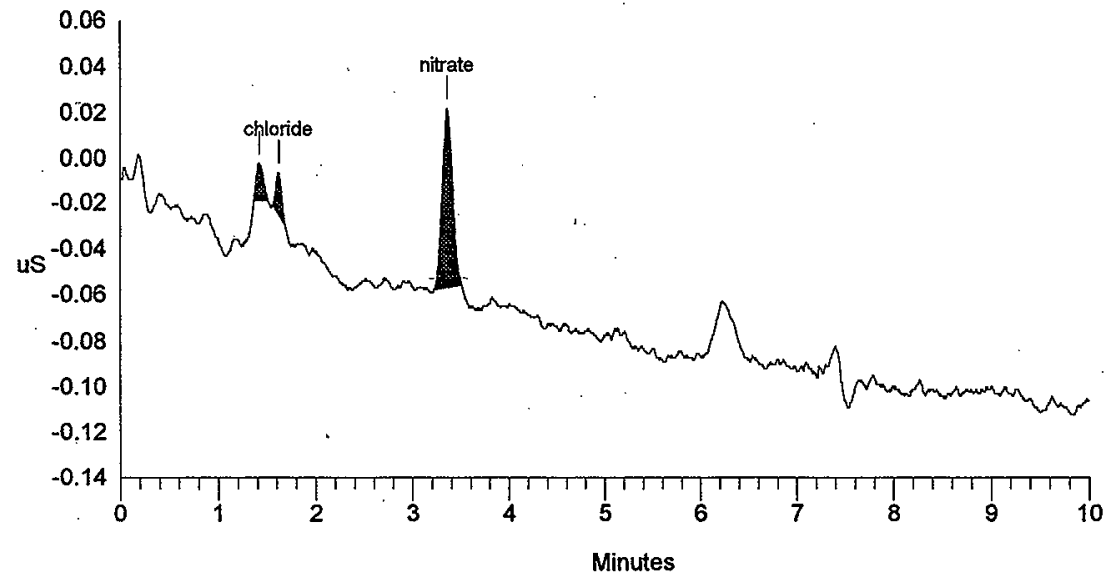




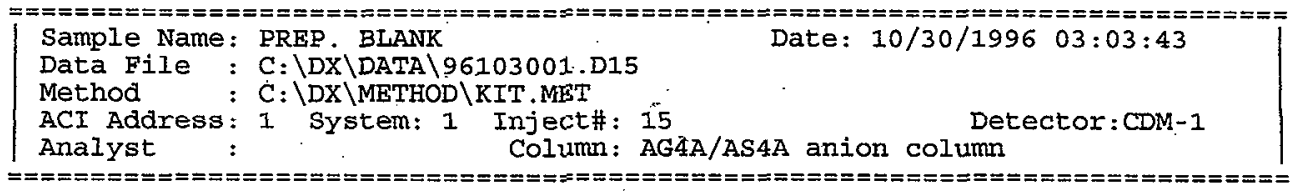

Calibration Volume Dilution Points Rate start stop Area Reject

\begin{tabular}{|c|}
\hline External \\
\hline
\end{tabular}

$* * * * * * * * * * * * * * * * * * * * * * * * * * *$ Peak Report: All Peaks $* * * * * * * * * * * * * * * * * * * * * * * * * * * *$ Pk. Ret Component $\quad$ Concentration
Num Time Name Height $\quad$ Area Bl. BDelta

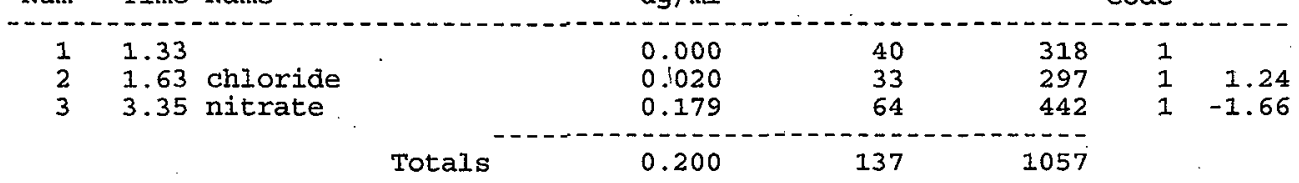

File: 96103001.D15 Sample: PREP. BLANK

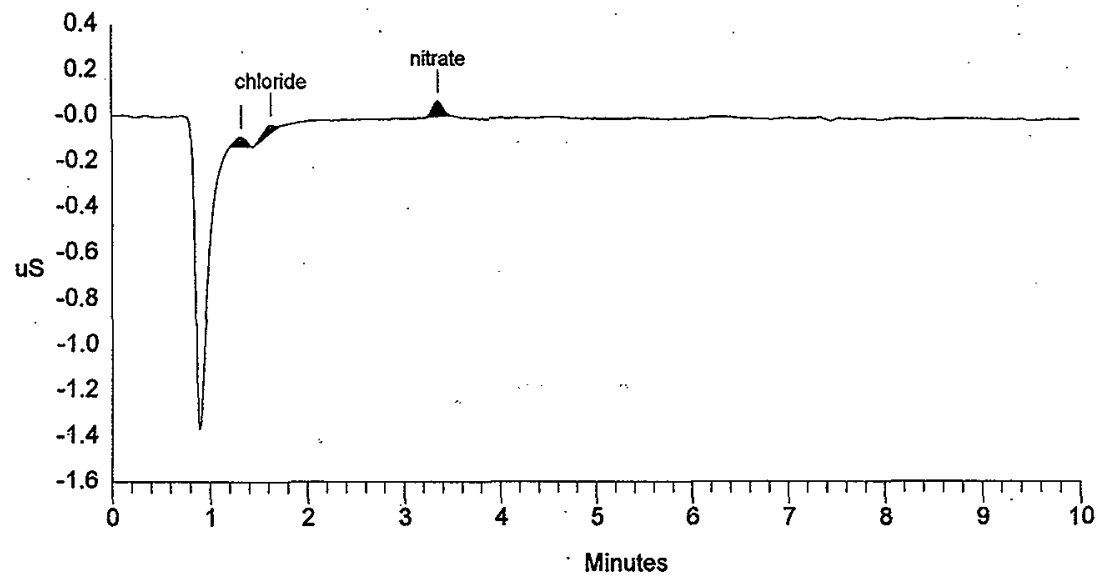

